\title{
Towards Synthesis of Simplified Analogues of Pateamine A
}

\author{
by \\ Tao Xu
}

A thesis submitted to the Victoria University of Wellington in fulfilment of the requirements for the degree of Doctor of Philosophy

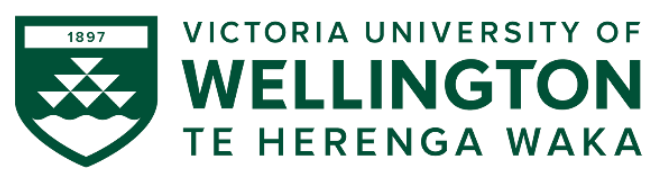

Victoria University of Wellington

2020 


\section{Abstract}

Pateamine A (22) is a natural product that was isolated from a marine sponge inhabiting the coast of New Zealand. It exhibits potent inhibition of protein synthesis and nonsensemediated mRNA decay through binding with eIF4A isoforms. Due to the scarcity of pateamine A (22) in the natural source and the low yield of total synthesis of pateamine A, it is necessary to prepare structurally simplified analogues which would allow further research on structure-activity relationships (SAR) of pateamine A (22).

Based on the structure-activity relationship studies reported by Romo and co-workers, a simplified triazole analogue $\mathbf{1 8 2}$ lacking methyl groups was synthesized by Hemi Cumming, a previous Ph.D. student who studied at Victoria University of Wellington. The antiproliferative activity of this analogue was found to be significantly lower than that of pateamine A, suggesting that the thiazole embedded within the molecule or the excised methyl groups are crucial for its potency.
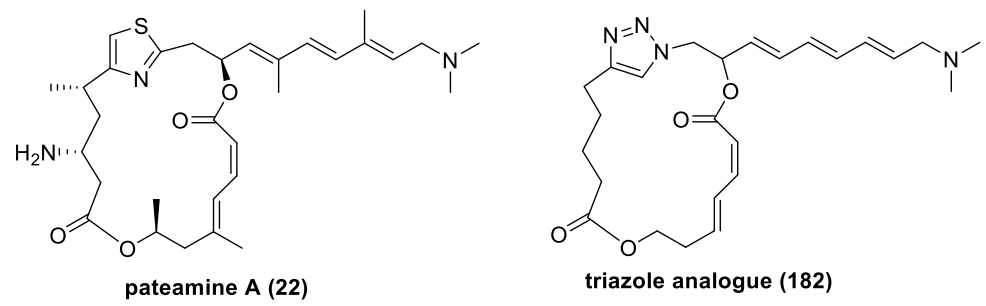

Therefore, to further explore the necessary features for its selective activity for eIF4A isoforms, new thiazole analogues 183 - 186 and triazole analogues (10S)-and (10R)analogue 187 were targeted in this project.
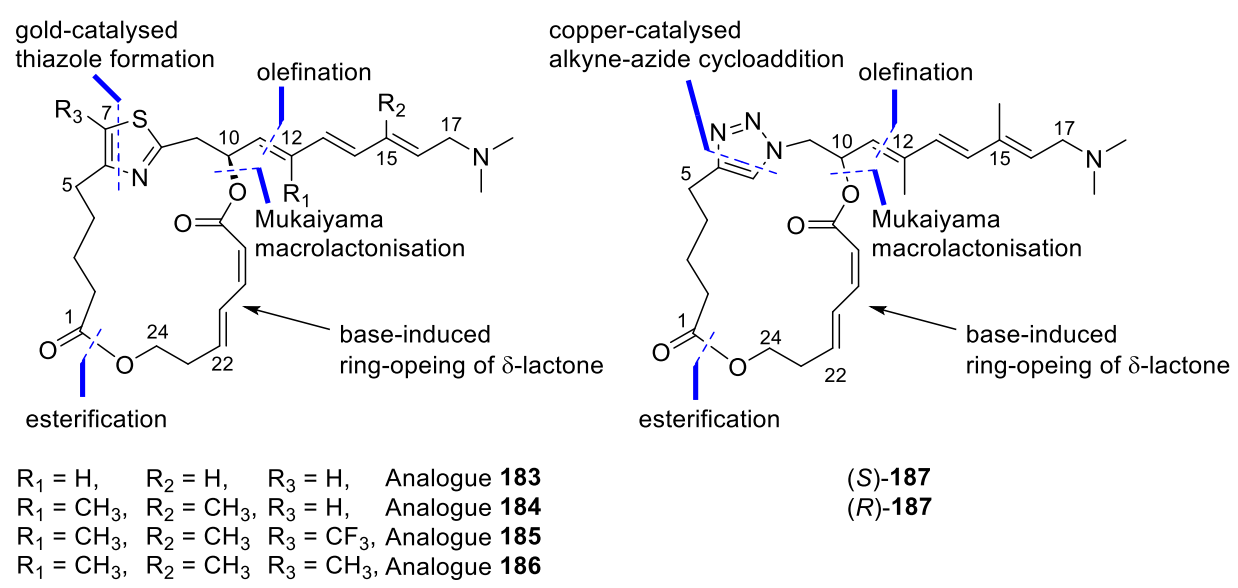
The preparation of the thiazole-containing macrocyclic core of analogues $\mathbf{1 8 3}$ and $\mathbf{1 8 4}$ was achieved. It features: (1) gold-catalysed thiazole formation through coupling between an alkyne fragment and a thioamide fragment; (2) preparation of the Z,E-dienoate moiety by base-induced ring-opening of a $\delta$-substituted- $\alpha, \beta$-unsaturated lactone; and (3) a modified Mukaiyama macrolactonisation. The synthesis of the triazole-containing macrocyclic core of (10S)-analogue 187 was completed. It features: (1) a coppercatalysed triazole formation through 1,3-dipolar cycloaddition between an alkyne fragment and an azide fragment; (2) preparation of the Z,E-dienoate moiety by baseinduced ring-opening of a $\delta$-substituted- $\alpha, \beta$-unsaturated lactone; and (3) a modified Mukaiyama macrolactonisation. Studies on the preparation of a side-chain fragment with suitable functionalities to allow coupling with the various macrocycles through olefination reactions were also conducted.

The attachment of the side-chain fragment onto the macrocyclic cores for the synthesis of the targeted analogues 183 and 184 and (10S)-analogue 187 will be investigated in the future work. These experimental results will inform the synthesis of new generation analogues to further study the key structures required for effective binding to the protein target eIF4A and selectivity between isoforms. 


\section{Acknowledgements}

In my PhD journey, I've encountered many unexpected challenges. Although it was filled with stress and frustration sometimes, it has also been very enjoyable and enriching. It is now very satisfying that I can finally be able to share what I have learnt and achieved in these few years and thank those who have helped me succeed.

First, I would like to thank my supervisors, Joanne Harvey and Paul Teesdale-Spittle. Thank you very much for your guidance, encouragement, patience and immense knowledge throughout these years. Paul, your inspiring ideas; and Joanne, your enthusiasm for chemistry, has helped me tremendously succeeding my goals. Also, I really appreciate your proofreading my thesis and invaluable suggestions.

I was very lucky to get financial support for most of my time of study. Many thanks to Worldwide Cancer Research for offering my scholarship, Faculty Strategic Research Grants from Faculty of Science and New Zealand Institute Travel Grant (allowing me to attend 22nd International Conference on Organic Synthesis at Florence) and Victoria PhD Submission Scholarship (allowing to survive in my last months). Big thanks again to Joanne for offering me a part-time research assistant job to help me get through all those days when my scholarship was running out.

Furthermore, I would like to acknowledge Ian Vorster for his help to keeping the NMR facilities working nicely and running NOE experiments for my samples, and Sophie and Ethan for their time running my samples on the mass spectrometer. I want to thank Sarah Andreassend for running samples on the $600 \mathrm{NMR}$.

To Chao, I probably wouldn't have been able to finish my Ph.D. project if you weren't by my side. Thank you for accompanying me when I was working in lab on the weekends to make me feel safe, and driving me to university or picking me up after working hours.

I'm really thankful for the members of "ECAT". Claire, Amira, Ethan. Thank you for being very supportive to me, and making the "boring" lab to be such a fun place to work. Also, big thanks to Claire Cuyamendous and Sarah Brown, for helping me out at the early days of my project, and contributing a lot to the pateamine project. 
To all the past and present members of our group - Jordan, Ben, Tom, Dan, Hedley, Sophie, Chris, Matt, Olly, thanks for all the great discussions and all the help.

To my mum and dad, thank you so much for encouraging me to pursue my future overseas and supporting me to complete this long and stressful journey. I'm so blessed that I can have you in my life. Everything I have and will achieve is because of you both. 
\begin{tabular}{l|l|} 
ABSTRACT & II
\end{tabular}

ACKNOWLEDGEMENTS IV

\begin{tabular}{ll} 
ABBREVIATIONS $\quad \mathrm{X}$ \\
\hline
\end{tabular}

CHAPTER ONE: INTRODUCTION 1

1.1 Natural Products and Analogues as Anticancer Agents 1

$\begin{array}{ll}\text { 1.2 Discovery of Pateamine A } & 10\end{array}$

$\begin{array}{ll}\text { 1.3 Biological Activity of Pateamine A } & 11\end{array}$

1.4 Syntheses of Pateamine A and Analogues 15

$\begin{array}{ll}\text { 1.4.1Total Syntheses of Pateamine A } & 15\end{array}$

$\begin{array}{ll}\text { 1.4.2 Pateamine A Analogues Synthesis } & 37\end{array}$

1.5 Structure-Activity Relationship Studies of PatA and Analogues 45

1.5.1 Studies on Immunosuppressive Activity of PatA Analogues 45

1.5.2 Studies on Anti-proliferative Activity of PatA Analogues 47

$\begin{array}{ll}1.5 .2 \text { Summary } & 51\end{array}$

1.6 Cumming's Synthesis of Triazole Analogue 52

1.7 Research Aim $\quad 54$

1.7.1 Analogue Design $\quad 54$

1.7.2 Retrosynthetic Analysis $\quad 56$

CHAPTER TWO: TOWARDS THE SYNTHESIS OF THIAZOLE ANALOGUE

WITH NON-METHYLATED SIDE CHAIN

2.1 Retrosynthesis

2.2 Synthesis of C8-C11 Thioamide (Fragment IV) 61

$\begin{array}{ll}2.2 .1 \text { Introduction } & 61\end{array}$

2.2.2 Initial Attempts Towards Synthesis of Nitrile 206

2.2.3 Preparation of Thioamide Fragment IV 66 
2.3.1 Introduction

2.3.2 Synthesis of Thiazole Fragment 232 Using Gold Catalysis

2.4 Coupling of Side Chain Fragment

2.4.1 Introduction

2.4.2 Preparation of Sulfone 277

2.4.3 Synthesis of Side Chain Fragment 297

2.5 Revised Synthesis of Sulfone 316

2.5.1 Attempted Synthesis of Thioamide 317

CHAPTER THREE: TOWARDS SYNTHESIS OF THIAZOLE ANALOGUE WITH METHYLATED SIDE CHAIN 
CHAPTER FOUR: TOWARDS SYNTHESIS OF TRIAZOLE ANALOGUE WITH METHYLATED SIDE CHAIN

$\begin{array}{ll}4.1 \text { Introduction } & 179\end{array}$

$\begin{array}{ll}\text { 4.1.1 Synthesis of Triazole Analogue } 182 & 179\end{array}$

4.1.2 Reintroduction of Methyl Groups to Side Chain 182

$\begin{array}{ll}\text { 4.2 Retrosynthetic Analysis } & 183\end{array}$

$\begin{array}{ll}4.3 \text { Attempted Preparation of Aldehyde }(R)-509 & 186\end{array}$

4.4 Revised Synthetic Route for Synthesis of Analogue (S)-187 188

$\begin{array}{ll}\text { 4.5 Construction of Macrocyclic Compound } 523 & 189\end{array}$

$\begin{array}{ll}\text { 4.6 Conclusion } & 195\end{array}$

CHAPTER FIVE: SUMMARY AND FUTURE WORK 197

$\begin{array}{ll}\text { 5.1 Summary } & 197\end{array}$

5.1.1 Attempted Synthesis of Thiazole Analogue $183 \quad 197$

5.1.2 Towards Synthesis of Thiazole Analogue $184 \quad 200$

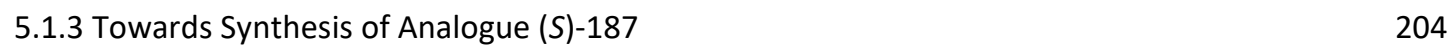

$\begin{array}{ll}\text { 5.1.4 Closing Remarks } & 205\end{array}$

5.2 Future Work 206

5.2.1 Synthesis of Analogues 184 and $187 \quad 206$

5.2.2 Role of the Thiazole and Methyl Groups on the Macrocycle 209

5.2.3 Role of Methyl Groups on Side Chain 211

5.2.4 Importance of the C-10 Configuration 212

$\begin{array}{ll}\text { CHAPTER SIX: EXPERIMENTAL } & 213\end{array}$

$\begin{array}{ll}\text { 6.1 General Experimental Details } & 213\end{array}$

6.2 Experimental for Chapter Two 215

$\begin{array}{ll}\text { 6.2.1 Preparation of Thioamide } 223 & 215\end{array}$

$\begin{array}{ll}\text { 6.2.2 Gold-catalysed Preparation of Thiazole } 276 & 224\end{array}$ 


\subsection{Experimental for Chapter Three}

6.3.1 Synthesis of Thioamide 382

6.3.2 Synthesis of Alkyne 364

6.3.4 Synthesis of Thiazoles 392, 393 and 394

6.3.5 Synthesis of Macrocyclic Alcohol 441

6.3.5 Synthesis of the Side Chain Fragments

\subsection{Experimental for Chapter Four}

6.4.1 Attempted Synthesis of Aldehyde (R)-509 


\section{Abbreviations}

18-crown-6

2D

$4 \AA$

$a q$.

Ac

BAIB

Boc

BRSM

c.f.

$c a$.

CAN

cat.

$\mathrm{Cbz}$

DBU

DCC

DCE

DCM

DDQ

DIAD

\section{1,4,7,10,13,16-Hexaoxacyclooctadecane}

Two-dimensional

4 Angstrom

Aqueous

Acetyl

(Diacetoxyiodo)benzene

tert-Butyloxycarbonyl

Based on recovered starting material

confer ("compare to")

circa ("approximately")

Ceric ammonium nitrate

Catalytic

Carboxybenzyl

1,8-Diazabicyclo[5.4.0]undec-7-ene

$N, N^{\prime}$-Dicyclohexylcarbodiimide

1,2-Dichloroethane

Dichloromethane

2,3-Dichloro-5,6-dicyano-1,4-benzoquinone

Diisopropyl azodicarboxylate 


\begin{tabular}{|c|c|}
\hline DIBAL-H & Diisobutylaluminium hydride \\
\hline DIPEA & $N, N$-Diisopropylethylamine \\
\hline DMAP & 4-Dimethylaminopyridine \\
\hline DME & 1,2-Dimethoxyethane \\
\hline $\mathrm{DMF}$ & $N, N$-Dimethylformamide \\
\hline DMP & Dess-Martin periodinane \\
\hline DMSO & Dimethyl sulfoxide \\
\hline e.e. & Enantiomeric excess \\
\hline e.g. & exempli gratia ("for example") \\
\hline EDCI & $N$-(3-Dimethylaminopropyl)- $N$ '-ethylcarbodiimide hydrochloride \\
\hline EJC & Exon junction complex \\
\hline equiv. & Equivalent \\
\hline $\mathrm{Et}$ & Ethyl \\
\hline $\mathrm{Et}_{2} \mathrm{O}$ & Diethyl ether \\
\hline EtOAc & Ethyl acetate \\
\hline gCOSY & Gradient correlation spectroscopy \\
\hline gem- & Geminal \\
\hline $\mathrm{h}$ & Hour(s) \\
\hline HMBC & Heteronuclear multiple bond correlation \\
\hline HSQC & Heteronuclear single quantum correlation \\
\hline HMPA & Hexamethylphosphoramide \\
\hline
\end{tabular}




\begin{tabular}{|c|c|}
\hline HRMS & High resolution mass spectrometry \\
\hline HWE & Horner-Wadsworth-Emmons \\
\hline $\mathrm{IC}_{50}$ & Inhibitory concentration for $50 \%$ effect \\
\hline IR & Infrared \\
\hline KHMDS & Potassium bis(trimethylsilyl)amide \\
\hline LiHMDS & Lithium bis(trimethylsilyl)amide \\
\hline M & Molar \\
\hline$m$-CPBA & 3-Chloroperoxybenzoic acid \\
\hline $\mathrm{Me}$ & Methyl \\
\hline MOM & Methoxymethyl \\
\hline MS & Molecular sieves \\
\hline Ms & Mesylate \\
\hline $\mathrm{MsOH}$ & Methanesulfonic acid \\
\hline MTPA & $\alpha$-Methoxy- $\alpha$-trifluoromethylphenylacetic acid \\
\hline MTT assay & $\begin{array}{l}\text { ((3-(4,5-Dimethylthiazol-2-yl)-2,5-diphenyltetrazolium bromide) } \\
\text { tetrazolium reduction assay }\end{array}$ \\
\hline NBS & $N$-Bromosuccinimide \\
\hline NMD & Nonsense-mediated decay \\
\hline NMR & Nuclear magnetic resonance \\
\hline NOE & Nuclear overhauser effect \\
\hline -OTf & Trifluoromethanesulfonate (triflate) \\
\hline
\end{tabular}


pet. ether

PG

$\mathrm{Ph}$

PKC

PMB

PPTS

$\operatorname{Pr}$

PT

$p$-TSA

quant.

$\mathrm{R}$

$\mathrm{R}_{f}$

$\mathrm{rt}$

TBAF

TBAI

TBDPS

TBS

TcBoc

TEA

TEMPO

TES
Petroleum ether

Unspecified protecting group

Phenyl

Protein kinase $\mathrm{C}$

para-Methoxybenzyl

Pyridinium para-toluenesulfonate

Propyl

Phenyl tetrazole

para-Toluenesulfonic acid

Quantitative

Unspecified Substituent

Retardation factor

Room temperature

Tetra- $n$-butylammonium fluoride

Tetra- $n$-butylammonium iodide

tert-butyldiphenyl silyl

tert-butyldimethyl silyl

Trichloro-tert-butoxy carbamate

Triethylamine

2,2,6,6-Tetramethylpiperidine 1-oxyl

Triethyl silyl 
TFA

TFAA

THF

TIPS

TLC

$\operatorname{Tr}$

Ts

UV

viz.
Trifluoroacetic acid

Trifluoroacetic anhydride

Tetrahydrofuran

Triisopropyl silyl

Thin-layer chromatography

Trityl

para-Toluenesulfonate (Tosyl)

Ultraviolet

videlicet ("namely") 



\section{Chapter One: Introduction}

This thesis presents efforts directed towards the synthesis of simplified analogues of the marine natural product, pateamine A. This chapter will begin by introducing the importance of marine-sourced natural products in the discovery of anticancer agents. The precedent for design and synthesis of structurally simplified analogues of marine natural products in drug development will be discussed. The discovery of pateamine A and its biological activity will be described. This is followed by a review of previous total syntheses of pateamine A, syntheses of its analogues, and the analysis of its structureactivity relationship. Finally, the research aim of this thesis and the retrosynthetic analysis of target analogues will be presented.

\subsection{Natural Products and Analogues as Anticancer Agents}

Natural products, by definition, are secondary metabolites naturally produced by plants, animals, and microbes. Historically, they have provided numerous agents of great value for medicines, such as aspirin (1), penicillin G (2), and paclitaxel (3) (Figure 1.1). ${ }^{1}$ Natural products are very important for drug discovery because they possess enormous structural and chemical diversity, and can exhibit diverse bioactivities. In addition, they are evolutionarily optimised to have drug-like properties with regard to absorption, distribution, metabolism, excretion, and toxicity (ADMET) characteristics, ${ }^{2}$ which influence the performance and pharmacological activity of a drug compound.<smiles>CC(=O)Oc1ccccc1C(=O)O</smiles>

Aspirin (1)

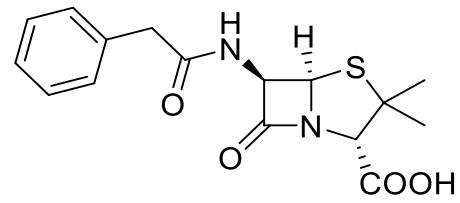

Penicillin G (2)

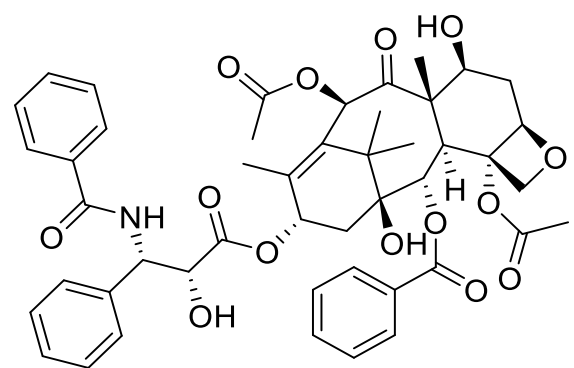

Paclitaxel (3)

Figure 1.1 Chemical structures of aspirin (1), penicillin G (2) and paclitaxel (3). ${ }^{1}$

Despite these advantages, it is undeniable that screening natural products for drug discovery is associated with some disadvantages. First, the process of isolation and characterisation of active compounds from a crude extract is time-consuming and 
sometimes lacking in efficiency for finding novel chemical entities due to the high rediscovery rate. Second, identifying a pharmacophore of a structurally complex natural product through structure-activity studies is very challenging. Last but most importantly, scale-up for process chemistry to deliver sufficient quantities of structurally complex natural products is a significant obstacle for medicinal chemists. ${ }^{3}$ Consequently, the major pharmaceutical companies have been less enthusiastic about participation in natural product research since the 1990s after the advent of high-throughput screening (HTS) and combinatorial chemistry, which can rapidly generate large libraries of novel compounds for various bioactivity screening through diversity-oriented syntheses. Disappointingly, high-throughput screening of large numbers of purposely synthesised compounds imbued with high randomness has failed to deliver new lead compounds in significant numbers. ${ }^{4}$ A scan of drugs approved by the U.S. FDA (and similar organisations) from 1981-2014 shows that only 3 approved drugs, sorafenib (4), ataluren (5), and vemurafenib (6) (Figure 1.2), could be ascribed to combinatorial chemistry. ${ }^{4}$ In contrast, about $50 \%$ of drugs in the scan are naturally derived or naturally inspired. In the case of cancer, $77 \%$ of the anticancer drugs from the late 1930 s to 2014 are related to natural products. ${ }^{4}$ This comprehensive analysis has demonstrated that natural products and structures related to natural products have played and will continue to play a very important role in the development of novel therapeutic treatments, which explains the recent trend of revisiting natural products for the discovery of new drug candidates in pharmaceutical companies and institutions. ${ }^{5}$

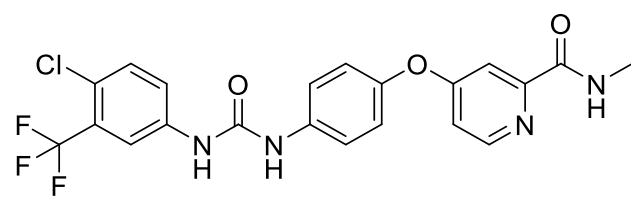

Sorafenib (4)

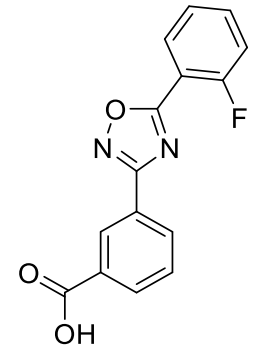

Atuluren (5)

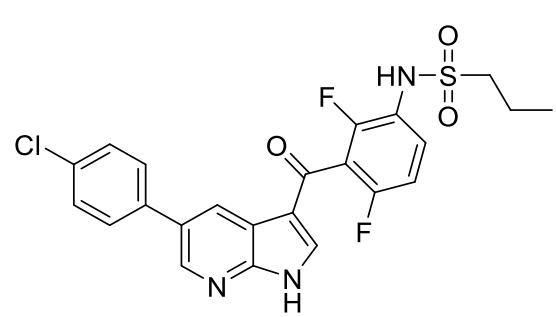

Vemurafenib (6)

Figure 1.2 Chemical structures of sorafenib (4), ataluren (5), and vemurafenib (6).

In this area of research, the exploration of new anticancer agents from organisms living in the sea, such as sponges, alga, ascidians, coral, etc., has attracted increasing interests. The oceans are vast and cover more than $70 \%$ of the surface of the earth, and marine species are estimated to approach one to two million species. However, the majority 
cohabit in the ocean fringe. Competition from such a high concentration of species in a narrow range of habitats makes them promotes greater evolutionary variation. Therefore, many species have evolved to be capable of producing chemicals to defend against predators, to suppress competing species, or to prey on other species for survival. ${ }^{6}$ These chemical adaptations involve a wide variety of primary and secondary metabolites, including nucleosides, sugars, steroids, terpenoids, alkaloids, polyketides and compounds of mixed structural origins, some of which exhibit potent anticancer effects. For example, didemnin B (7) (Figure 1.3), a cyclic depsipeptide isolated from the tunicate Trididemnum solidum living in the Caribbean area, showed in vivo cytotoxicity and could effectively increase the life-span of P388 leukemia-bearing mice. ${ }^{7,8}$ It was the first marine natural product that successfully entered clinical trials as an anticarcinoma agent, although clinical trials against breast cancer, small-cell lung cancer, central nervous system tumors, and several other cancers were suspended due to significant neuromuscular toxicity and no positive responses. ${ }^{9-19}$

Another good example of the development of marine-derived antitumor agents is ecteinascidin-743 (ET-743) (8) (Figure 1.3), the structure of which was published by Rinehart ${ }^{20}$ and $\mathrm{Wright}^{21}$ in 1990, 20 years after the first report about the antitumor activity of the extracts of the Caribbean tunicate Ecteinascidia turbinata. Its distinguishing structural characteristic is a 10-membered lactone containing a thioether bridge connecting the tetrahydroisoquinoline ring and the base scaffold. Ecteinascidins were found to display cytotoxicity against $\mathrm{L} 1210$ leukemia cells ( $\mathrm{IC}_{50}$ value of $0.5 \mathrm{ng} / \mathrm{mL}$ ) and to possess significant in vivo antitumor activity in several mice models bearing P388 leukemia, B16 melanoma and colon carcinoma $26 .{ }^{20}$ The mechanism of action of ET-743 is accredited to its covalent modification of DNA by acting as an alkylating agent, a property similar to another natural-product-based successful anticancer drug, anthramycin (9) (Figure 1.3). ${ }^{22}$ In 2007, ET-743, under the trade name Yondelis, was approved by the European Union for the treatment of patients with refractory soft tissue sarcoma, meaning that it became the first marine-derived marketable anticancer drug. 


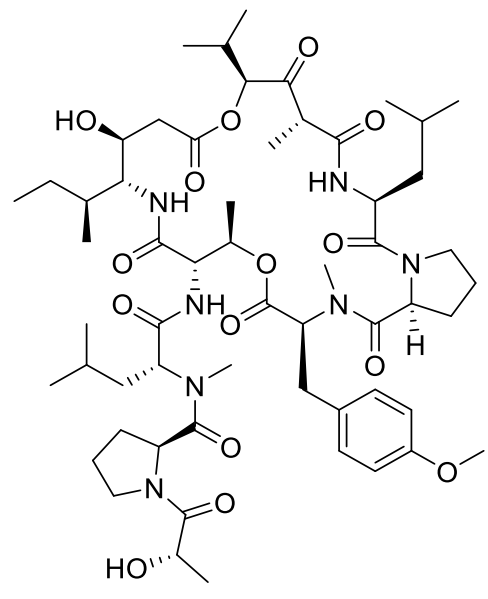

Didemnin B (7)

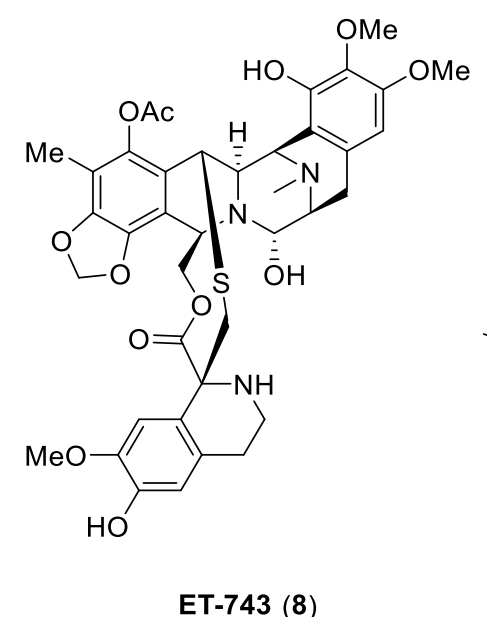

ET-743 (8)

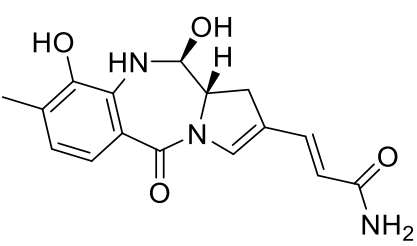

Anthramycin (9)

Figure 1.3 Chemical structures of didemnin B (7), ET-743 (8) and

anthramycin (9)..$^{8,20,21,23}$

Recently, marine bacteria have been considered as new potential sources for the discovery of drug leads. ${ }^{24}$ Actually, many compounds isolated from marine invertebrates are considered to be produced by as-yet-uncultured symbiotic marine bacteria in larger host organisms, such as marine sponges, marine hares, and tunicates. ${ }^{25}$ Metagenomics, or "genome mining", has provided a promising method to search for novel natural product skeletons from free-living marine bacteria or endosymbiotic marine bacteria. The exploration of metagenomics has shown that the genetic information related to the biosynthesis of bioactive metabolites could be "abstracted" from uncultured microbes and in some cases successfully heterologously expressed in cultivable host microorganisms to achieve the desired secondary metabolites. ${ }^{24}$

Nevertheless, it is obvious that a major difficulty for developing anticancer drugs from marine sources is to ensure a sustainable supply of adequate quantities of these rare compounds. Indeed, many marine natural products with interesting pharmacological properties can only be obtained in minute amounts. The extremely low yields of isolation and purification are already a forbidding challenge for preclinical and clinical trials with patients, not to mention the immense supply problem faced by the pharmaceutical industry once a bioactive compound successfully enters the market as a drug. To solve this pressing problem, several strategies appear to be feasible: 1. aquaculture of marine invertebrates; ${ }^{26}$ 2. cloning and heterologous expression of key biosynthetic gene clusters of marine microbes to facilitate large-scale fermentation for production; ${ }^{24}, 263$. semi- 
synthesis; 4. total synthesis. For example, the breakthrough in the supply of ET-743 (8) was achieved by PharmaMar through large-scale semi-synthesis starting from cyanosafracin B (10) (Figure 1.4), an antibiotic that can be obtained in bulk by fermentation of Pseudomonas fluorescens. ${ }^{27}$ Recently, Ma and co-workers reported the scalable 26-step total synthesis of ET-743 (8) starting from commercially available carbamate benzyloxy $(\mathrm{Cbz})$ protected $(S)$-tyrosine, which could also potentially solve the supply issue of this potent antitumor drug. ${ }^{28}$

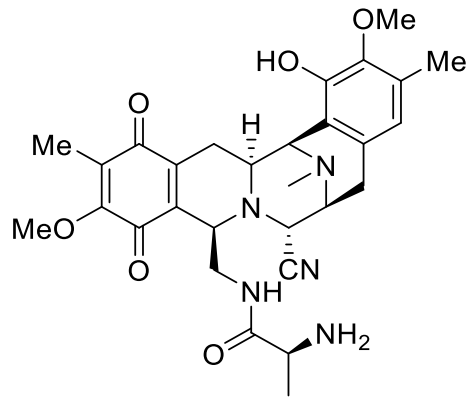

Cyanosafracin B (10)
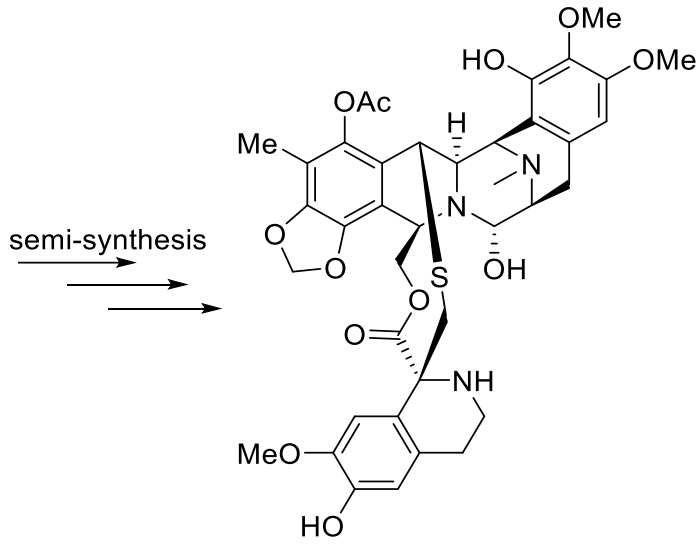

ET-743 (8)

Figure 1.4 Scalable semi-synthesis of ET-743 (8) from cyanosafracin B (10). ${ }^{27,29}$

Although the total synthesis of many marine natural products has been achieved, in many cases, it is not economically pragmatic for large-scale production because of the intrinsic structural complexity and low yields. Design and synthesis of analogues with simplified structures but retaining the crucial pharmacophores for target binding, can reduce the number of synthetic steps and improve the yield.

Halichondrin B (11) is a classic example of a complex marine natural product that was converted into a significantly simplified analogue eribulin mesylate (12) to produce a drug candidate (Figure 1.5). Halichondrin B is a polyether macrolide originally isolated from the marine sponge Halichondria okadai, ${ }^{30,31}$ which binds to tubulin and inhibits tubulin assembly, thereby, preventing cell division, and thus, tumor growth. ${ }^{32,33}$ However, the scarcity of this natural product hampered its sustainable supply for drug development. Its total synthesis required about 90 steps starting from commercially available starting materials,${ }^{34}$ which is obviously unfeasible for scale-up production. Mass culture of source sponges was also carried out and indeed provided halichondrin B, but it could only address partially the problem because the growth of sponges is highly dependent on 
environmental conditions. ${ }^{35}$ A significant breakthrough was the discovery and scale-up production of structurally simplified analogues, especially eribulin mesylate, ${ }^{36-38}$ which displays antiproliferative activity against a diverse variety of human tumor cells, ${ }^{39}$ and has undergone phase II and phase III clinical trials, ${ }^{40}$ and is approved for clinical use in treatment of metastatic breast cancer and liposarcoma.

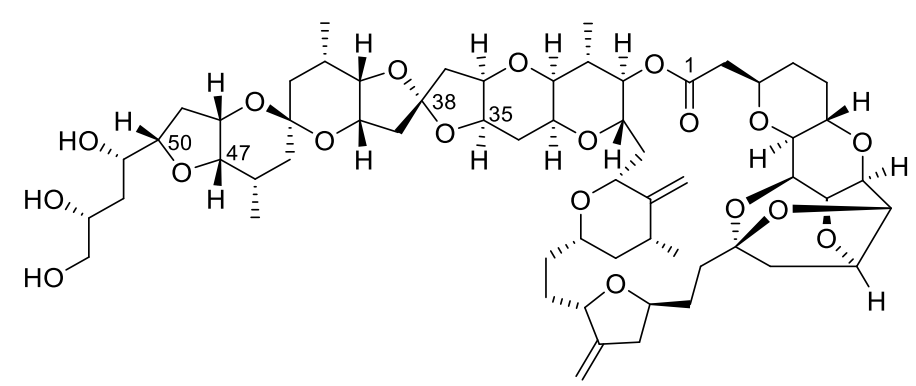

Halichondrin B (11)

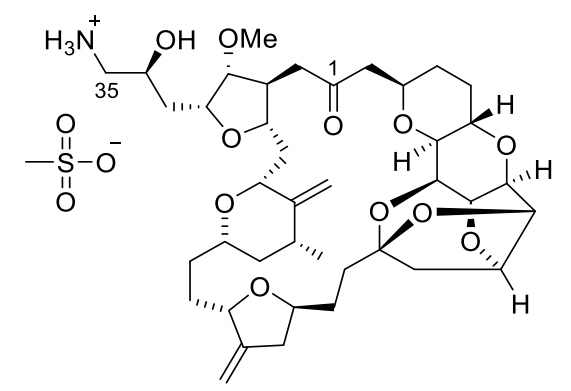

Eribulin mesylate (12)

Figure 1.5 Chemical structures of halichondrin B (11) and its analogue eribulin

$$
\text { mesylate (12). }{ }^{31,37}
$$

Another example of preparing analogues for clinical development is provided by bryostatin 1 and its synthetic analogue. Bryostatin 1 (13) (Figure 1.6), a 26-membered macrocyclic lactone, was produced by a symbiotic bacteria in marine bryozoan Bugula neritina ${ }^{41,42}$ Early studies have shown that bryostatin 1 exhibited remarkable anticancer activity, ${ }^{41}$ and it was identified as a modulator of protein kinase C (PKC). ${ }^{43}$ In addition, bryostatin 1 has shown promising activity related to other diseases, including stroke, ${ }^{44}$ diabetes, ${ }^{45}$ and Alzheimer's disease. ${ }^{46}$ This wide range of potentials in medicinal applications makes it an intriguing drug candidate. Unfortunately, the extremely low abundance of this compound in the source organism (18 $\mathrm{g}$ isolated from about 10,000 gallons of Bugula neritina $\mathrm{L})^{47}$ restricted its supply for preclinical and clinical studies. The reported total synthesis (52 total steps $)^{48}$ is not economically practical for scalable production, either. Hence, a simplified analogue was sought to resolve the supply problem. Based on computational modelling studies, C1, C19, and C26 oxygen atoms are suggested to be critical elements for the binding of bryostatin to PKC. ${ }^{49}$ Following this hypothesis, a bryostatin analogue (14) (Figure 1.6) was prepared in 27 steps for the longest linear sequence starting from $(R)-(+)$-methyl lactate. ${ }^{50}$ It can be seen that the important features have been maintained while the complexity of the skeleton has been reduced. As expected, this analogue has a similar affinity for PKC and shows significant 
levels of in vitro antiproliferative activity against several human tumour cell lines. ${ }^{51}$
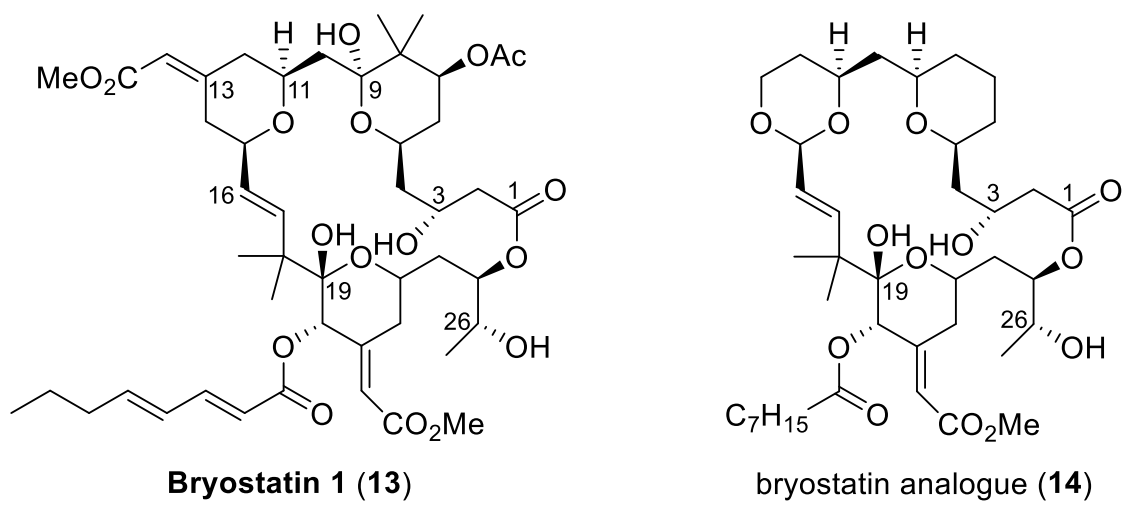

Figure 1.6 Chemical structures of bryostatin $1(\mathbf{1 3})$ and its analogue (14). ${ }^{41,52}$

The value of analogue preparation for drug development of marine natural products is not only to facilitate economical chemical synthesis for large-scale manufacture but also to provide solutions for another major question in developing anticancer drugs based on marine natural products, which is how to optimise structures to access new chemical entities with enhanced desirable biological and/or pharmacokinetic properties for human therapies.

Dolastatin 10 (15) and its analogue TZT-1027 (16) (Figure 1.7) provide a good example in this case. Dolastatin 10, isolated from the sea hare Dolabella auricularia ${ }^{53}$ is a very potent antimitotic pentapeptide originally produced by the marine cyanobacteria Symploca, which is the main diet of a sea hare species. ${ }^{54,55}$ Its absolute configuration was determined unequivocally through total synthesis. ${ }^{56}$ Further studies showed that dolastatin 10 inhibits microtubule assembly and tubulin polymerisation through binding to tubulin near the vinca binding site, resulting in cell cycle arrest and apoptosis. ${ }^{57-59}$ However, phase I and phase II clinical trials revealed that dolastatin could induce peripheral neuropathy and exhibit no clinically significant activity. ${ }^{60-62}$ Therefore, in efforts to reduce the clinical toxicity while maintaining the antitumor activity, a series of analogues were synthesised, of which TZT-1027 was the most promising molecule. ${ }^{63}$ The structural modification of TZT-1027 is the omission of the thiazole ring from the parent compound. Intermittent injections of TZT-1027 in mice showed it has equivalent or greater potency than dolastatin 10 against several cancer models. ${ }^{64}$ TZT-1027 shares its same mechanism of action with dolastatin 10 and it has been evaluated in phase I and phase II clinical trials in Japan, ${ }^{65,66}$ USA, ${ }^{67}$ and Europe. ${ }^{68}$ 

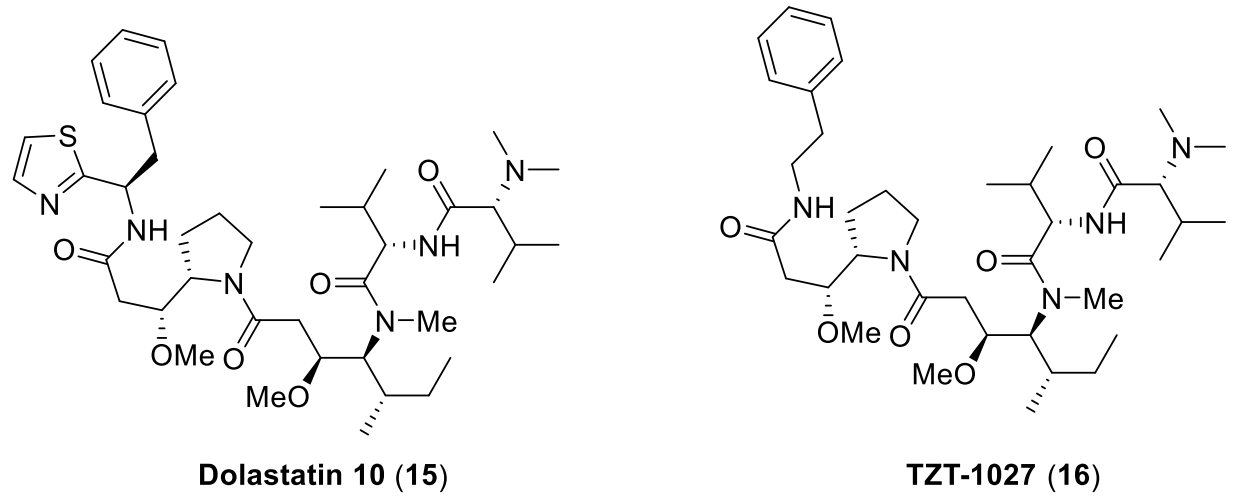

Figure 1.7 Chemical structures of dolastatin $10(\mathbf{1 5})$ and TZT-1027 (16). ${ }^{53,69}$

Another example of an analogue that provided improved biological activity is hemiasterlin (17) and its analogue HTI-286 (18) (Figure 1.8). Hemiasterlin was a tripeptide molecule isolated from marine sponge Hemiusrerellu minor, and it shows high cytotoxicity and antimitotic activity with an $\mathrm{IC}_{50}$ value of $c a .0 .01 \mu \mathrm{g} / \mathrm{ml}$ against P388 leukaemia cells. ${ }^{70}$ Similar to dolastatin 10 , hemiasterlin binds to the vinca binding site on tubulin..$^{70,71}$ To further explore the important portions of the molecule that are indispensable for the bioactivity, the $N$-methylindole was replaced by a phenyl group to afford a structurally simplified analogue HTI-286 (Figure 1.8), which displays greater in vitro potency than hemiasterlin against human mammary carcinoma MCF-7 cells. ${ }^{72}$ Furthermore, it has substantially less interaction with P-glycoproteins than other anticancer drugs, like paclitaxel and vinblastine. In this way, HTI-286 maintains high efficacy at inhibiting growth even in P-glycoprotein-mediated multi-drug resistant tumor cells. ${ }^{73}$<smiles>CC(=C[C@H](C(C)C)N(C)C(=O)[C@H](NC(=O)[C@H](N)C(C)(C)c1cn(C)c2ccccc12)C(C)(C)C)C(=O)O</smiles>

Hemiasterlin (17)<smiles>C/C(=C\[C@@H](C(C)C)N(C)C(=O)[C@H](NC(=O)[C@H](N)C(C)(C)c1ccccc1)C(C)(C)C)C(=O)O</smiles>

HTI-286 (18)

Figure 1.8 Chemical structures of hemiasterlin (17) and HIT-286 (18). ${ }^{70,73}$

Cryptophycin-52 (19) (Figure 1.9), an analogue of cryptophycin 1 (20) (Figure 1.9), gives an example of an analogue with improved pharmacological properties. Cryptophycin 1 was first isolated from cultured cyanobacteria Nostoc sp. strain ATCC $53789^{74}$ and then re-isolated from cyanobacteria Nostoc $s p$. strain GSV 224 by Moore $e t$ al..$^{75}$ It was found 
to be extremely potent against KB carcinoma and LoVo adenocarcinoma tumor cells. ${ }^{75}$ However, the ester functionalities of cryptophycin 1 are very susceptible to basic conditions, especially the ester bond shown in the box in Figure 1.9, which is readily hydrolysed at $\mathrm{pH}=11$ to afford cryptophycin-13 (21) (Figure 1.9) within one hour, suggesting that this ester bond may be susceptible to esterases. ${ }^{76}$ To reduce this susceptibility, a dimethyl substituted analogue, cryptophycin-52 (19), was developed by Eli Lilly. As expected, this modification improves its chemical stability. Interestingly, this modification also enhances its efficacy. Cryptophycin 52 displays exceptionally potent cytotoxicity against several human tumour cell lines, including leukaemia HL-60, mammary carcinoma MCF-7, and colon carcinoma HT-29 cells. ${ }^{77}$

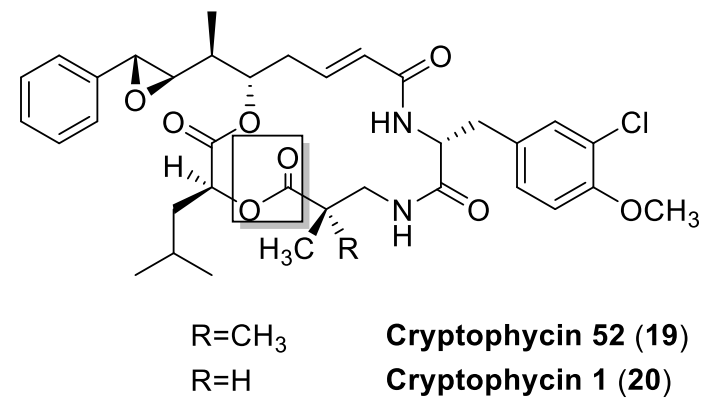

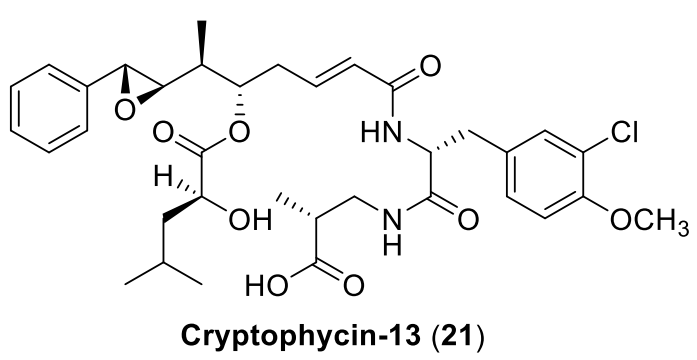

Figure 1.9 Chemical structures of hemiasterlin cryptophycin-52 (19), cryptophycin-1 (20) and crytophycin-13 (21). ${ }^{76}$

In summary, marine organisms have provided many promising antitumor agents with potential therapeutic applications. The continued exploration of marine natural products will continue to provide exciting drug leads due to advances in analytical spectroscopy and developments in metagenomics, which now allows researchers to probe bacteria with broad diversity from seawater, benthic sediment or in the marine invertebrate host. Simplification of complex marine natural products makes it possible to prepare analogues with limited loss of anticancer activity for pharmaceutical industry manufacture in a practical and economical way. Synthetic analogues also provide efficient methods to regulate the bioactivity of parent marine natural products to improve potency or pharmacology. 


\subsection{Discovery of Pateamine A}

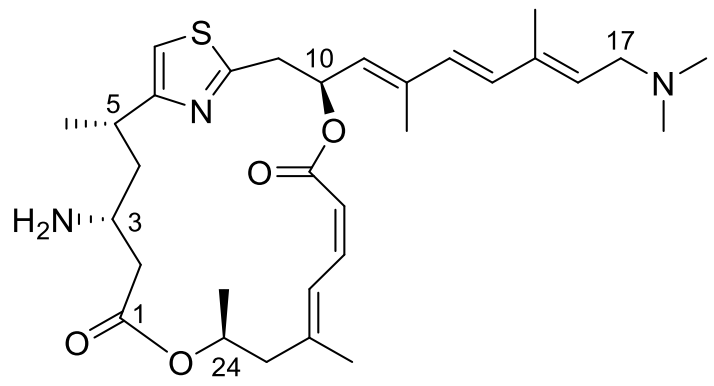

Pateamine A (22)

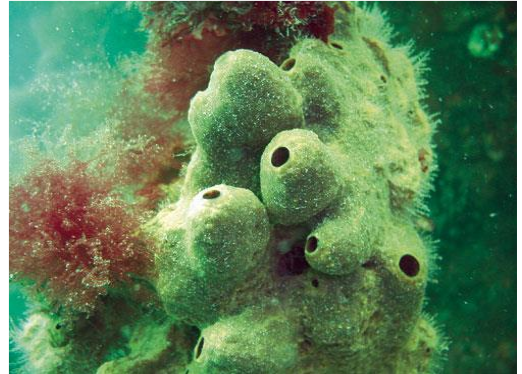

Mycale hentscheli

Figure 1.10 Structure of pateamine A (22) and a photo of the marine sponge Mycale hentscheli.

The isolation of pateamine A (22) (Figure 1.10), a macrodiolide, was first reported in 1991 from specimens of the marine sponge Mycale hentscheli, which were collected in Thompson Sound on the southwest coast of the South Island of New Zealand. ${ }^{78}$ Its unique structural features include a 2,4-disubstituted thiazole ring, and an isomerisation-prone $Z, E$-dienoate within a 19-membered bis-lactone macrocycle attached to an all-E trienylamine side chain.

The stereochemical assignment of C24 was then determined as $S$ by Romo's synthetic studies on the C18-C24 fragment. ${ }^{79}$ This assignment combined with modelling studies, extensive 2D-NMR experiments, and further chemical derivatisation provided the tentative stereochemical assignment as $3 R, 5 S, 10 S, 24 S .^{80,81}$ In 1998 , the first total synthesis by Romo's group finally unequivocally confirmed the absolute stereochemistry of pateamine $\mathrm{A}$ as $3 R, 5 S, 10 S, 24 S^{82}$

The original isolation by Northcote $e t$ al. was directed by cytotoxic activity of extracts from the marine sponge, resulting in the finding that pateamine A was a potent cytotoxin against fast-growing P388 leukemia murine cells with an $\mathrm{IC}_{50}$ value of $0.15 \mathrm{ng} / \mathrm{mL}$ based on an MTT assay. Interestingly, pateamine A was shown to be largely inactive against BSC-1 cells under static growth with an $\mathrm{IC}_{50}$ value of $300 \mathrm{ng} / \mathrm{mL} .^{78}$ This selectivity between proliferating cells and quiescent cells has suggested that pateamine A could act as a selective antitumor agent against different tumor cells. Pateamine A was also reported

\footnotetext{
* Photo taken from https://www.niwa.co.nz/sites/niwa.co.nz/files/w-a14-3handley.pdf
} 
to have in vitro antifungal activity in a preliminary screening assay. ${ }^{78}$ The abbreviated name 'PatA' will be used for the remainder of this thesis to represent pateamine A.

\subsection{Biological Activity of Pateamine A}

Early studies suggested PatA exerted its dose-dependent cytotoxic effects by an apoptotic mechanism. ${ }^{83}$ PatA was also found to display immunosuppressive activity through a signaling pathway involved in T-cell receptor-mediated IL-2 production. ${ }^{82,}{ }^{84}$ It is now established that PatA blocks protein synthesis through inhibition of translation initiation, thereby inducing programmed cell death. The primary binding target for PatA was identified as the eukaryotic initiation factor $4 \mathrm{~A}$ (eIF4A) family by two independent research groups, Bordeleau et al. and Liu et al. in $2005 .{ }^{85,86}$

The eIF4A family is a member of the DEAD-box protein family of RNA helicases, whose members are defined by the presence of nine highly conserved amino acid motifs. ${ }^{87}$ It plays a critical role in the translation initiation process, which is the rate-determining step for protein synthesis. In humans, the eIF4A family consists of three isoforms: eIF4AI, eIF4AII, and eIF4AIII. EIF4AI and eIF4AII are 90-95\% identical at the amino acid level and largely functionally equivalent in translation initiation. ${ }^{88-91}$ EIF4AIII shows $65 \%$ similarity to the other isoforms and exhibits a function distinct from eIF4I/II. ${ }^{92,93}$

The pivotal process at the initiation stage of cap-dependent eukaryotic translation is the recruitment of the $43 \mathrm{~S}$ ribosome subunit to the mRNA template. This process is accomplished through the concerted and systematic action of a number of proteins, including the eIF4F complex. ${ }^{94}$ This eIF4F complex is comprised of three subunits (as shown in the box in Figure 1.11): eIF4E, which binds with 5' cap structure on the mRNA $\left(\mathrm{m}^{7} \mathrm{GpppN}\right.$, where $\mathrm{N}$ is any nucleotide); eIF4AI/II, a protein that possesses RNAdependent ATPase activity and ATP-dependent RNA helicase activity; ${ }^{95}$ and eIF4G, an important scaffolding protein that mediates the interaction between eIF4AI/II and eIF4E. ${ }^{96}$ The eIF4AI/II is the most abundant initiation factor and it exists in two different forms: a free form (eIF4A $A_{f}$ ) and a complexed form (eIF4A $A_{c}$ ) as a subunit of eIF4F complex; and it is thought that there is a dynamic exchange between the two forms through the eIF4F complex during the initiation process (Figure 1.11). ${ }^{90}$ The complexed form eIF4 $\mathrm{c}$ is responsible for unwinding the local secondary structure of mRNA to facilitate the binding of the ribosome preinitiation complex (Figure 1.11). ${ }^{97}$ It was 
observed that eIF4A $\mathrm{A}_{\mathrm{c}}$ has a 20 -fold more efficient helicase activity than the free form of eIF4A, which suggested that the "active" conformation of eIF4A may be stabilised when bound with eIF4G. ${ }^{98}$

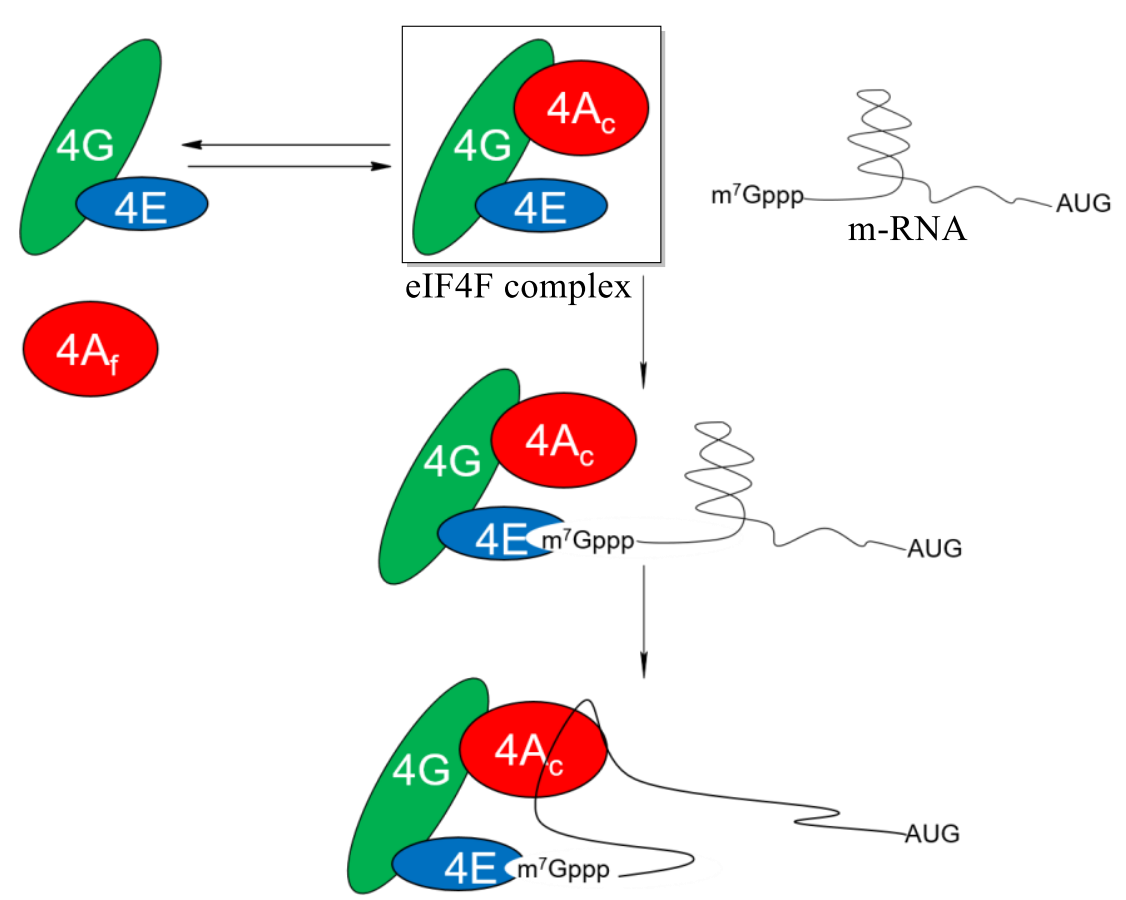

Figure 1.11 Reversible association of subunits of eIF4F complex and its function in the initiation process.$^{99}$

Key: 4A, 4G and 4E represent eIF4A, eIF4G and eIF4E, respectively. $4 \mathrm{~A}_{\mathrm{f}}$ and $4 \mathrm{~A}_{\mathrm{c}}$ represent the free and complexed forms of eIF4A. $\mathrm{m}^{7} \mathrm{GpppN}$ represents the cap structure of mRNA. AUG represents the start codon of mRNA.

The mode of action of PatA is that it specifically binds to the free form eIF4A $\mathrm{A}_{\mathrm{f}}$ (Figure 1.12). Interestingly, Pat $A$ also increases the ATPase and helicase activity of eIF4 $A_{f}$, which means that its ATP-stimulated RNA-binding activity is activated. ${ }^{85,} 86$ Consequently, eIF4A $\mathrm{A}_{\mathrm{f}}$ bound with PatA is sequestered by RNA and kept away from the interaction with eIF4G (Figure 1.12), which is necessary for the formation of the pivotal apparatus for cap-dependent translation initiation - the eIF4F complex. ${ }^{99}$ This would decrease the levels of eIF4 $4_{\mathrm{f}}$ available for recycling into the complexed form eIF4A $\mathrm{A}_{c}$. At high intracellular levels of PatA, this leads to an absence of eIF4 $\mathrm{A}_{c}$ and the consequent loss of eIF4F complex for cap-dependent translation initiation, causing the stalling of protein synthesis. ${ }^{99}$ 

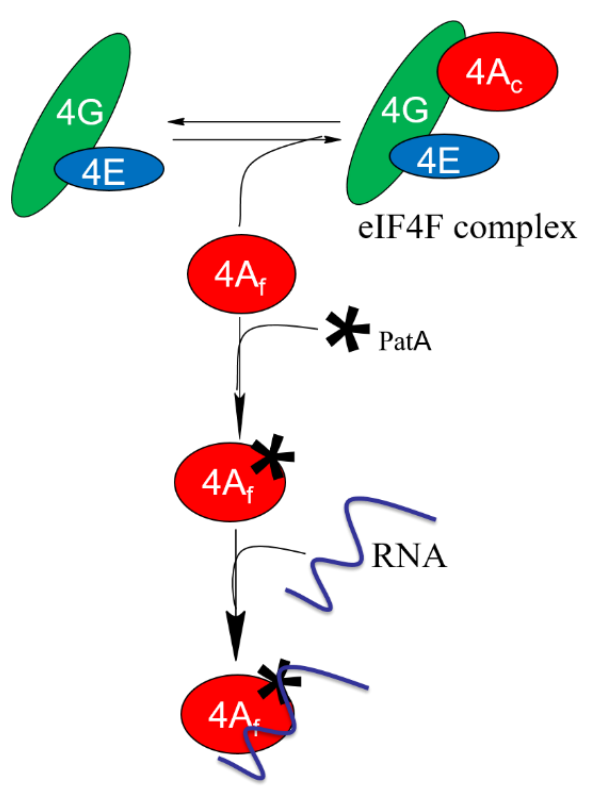

Figure 1.12 Proposed mode of action of PatA for inhibition of translation initiation. ${ }^{99}$

Key: See Figure 1.11.

EIF4AIII is a key component of the exon junction complex (EJC) core, which also includes Y14-MAGOH heterodimer and MLN51 (Figure 1.13). ${ }^{100}$ Within the EJC core, ATP-bound eIF4AIII and MLN51 directly bind with RNA while Y14-MAGOH heterodimer inhibits eIF4III ATPase activity and locks it into a conformation that can tightly clamp RNA (Figure 1.13). ${ }^{100,101}$ These four proteins are assembled onto mRNAs to form the EJC core in the nucleus during splicing and the EJC complex is deposited by spliceosomes at a fixed position located $20-24$ nucleotides upstream of exon-exon junctions. ${ }^{102}$ Afterwards, the EJC complex remains on spliced mRNAs and accompanies mRNAs as it is transported to the cytoplasm until it is eventually dissociated from mRNAs and disassembled during the first round of translation. ${ }^{103,104}$ During this period, the composition of the EJC is highly dynamic as the EJC core constitutes a binding platform for the recruitment of numerous EJC peripheral factors, most of which interact with the EJC core only transiently. ${ }^{105}$ This capacity grants the EJC a variety of functions in several post-transcriptional processes, including mRNA transport, translation and nonsense-mediated decay (NMD). ${ }^{106}$ 
This image is unavailable. Please consult the reference list for further details.

Figure 1.13 A mode of four proteins (eIF4AIII, Y14, MAGOH, and MLN51) forming the RNA-binding EJC core. ${ }^{107}$

NMD is a translation-dependent mRNA surveillance mechanism to maintain a high level of fidelity in gene expression, which is crucial for cellular homeostasis and survival. ${ }^{106}$ NMD specifically promotes the degradation of aberrant mRNAs containing premature termination codons (PTCs) to minimise the synthesis of dysfunctional or even harmful truncated proteins. ${ }^{108}$ In mammalian cells, NMD is mainly EJC-dependent. ${ }^{109}$

In addition to its aforementioned inhibitory activity in protein synthesis, PatA displays an inhibitory effect on NMD through its direct binding to eIF4AIII, which is independent of its inhibition of translation initiation. It was proposed that PatA may initiate the conformational change of eIF4AIII, causing the perturbation of the dynamic EJC-UPF1 interaction and the eventual inhibition of NMD ${ }^{110}$ Although the important role of the EJC in NMD is unveiled, a thorough understanding of the details of the mechanism are still lacking. ${ }^{106}$ In this circumstance, PatA may serve as a molecular probe to explore the mechanism of the EJC-dependent NMD. ${ }^{110}$

More recently, eIF4I/II and eIF4AIII were reported to be important for efficient replication of human cytomegalovirus (HCMV), suggesting that eIF4A family helicases may be a potential class of targets for the development of new therapeutics for viral diseases. ${ }^{111}$ As an inhibitor of both eIF4I/II and eIF4AIII, PatA displayed potent antiviral activity against different RNA and DNA viruses, raising the possibility that PatA may be used as a broad-spectrum antiviral drug. ${ }^{111}$ 


\subsection{Syntheses of Pateamine A and Analogues}

The high potency of PatA as a cytotoxin and its selective activity against cancer cell lines has garnered much attention from both chemists and biologists. ${ }^{112}$ A major concern in the development of PatA-based anticancer drugs is the difficulty in obtaining adequate amounts for preclinical and clinical trials due to its scarcity in the natural source Mycale sponges. Therefore, chemical synthesis is required to resolve the supply problem for further research.

\subsubsection{Total Syntheses of Pateamine A}

\subsubsection{Romo's Total Synthesis}

Romo and co-workers reported the first total synthesis of PatA in $1998{ }^{82}$ As shown in Figure 1.14, their synthetic strategy involved the initial construction of the macrocycle, followed by the attachment of the side chain fragment by Stille coupling reaction. The macrocycle was constructed by a combination of a Mitsunobu esterification and a $\beta$ lactam-based macrolactonisation. To minimise the risk of isomerisation, the $Z$, $E$-dienoate was delivered by a Lindlar reduction of the corresponding enyne. The 2,4-disubstituted thiazole ring was furnished by the Hantzsch thiazole synthesis between an $\alpha$-bromoketone and a thioamide fragment.

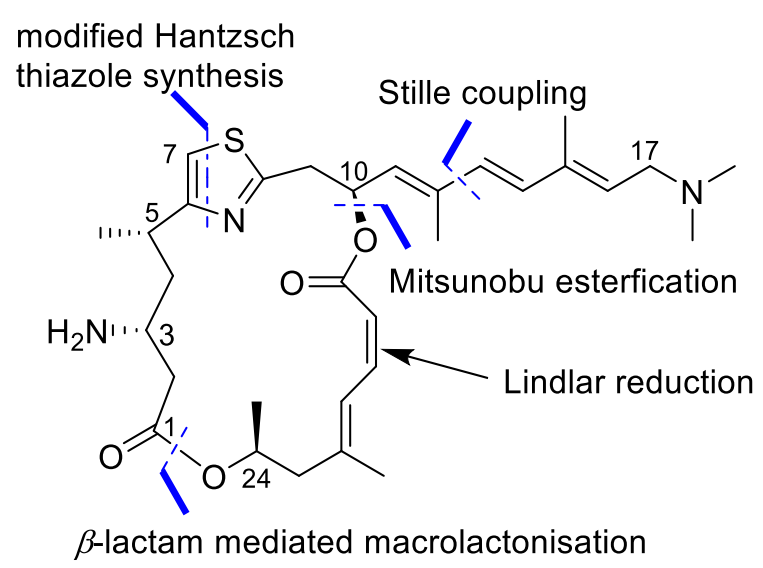

Figure 1.14 Key connections and features in Romo's synthetic strategy. ${ }^{82,84}$

Romo's total synthesis started with the preparation of three major fragments - the $\beta$ lactam fragment $\mathbf{2 3}$, the dienyl stannane fragment $\mathbf{2 4}$ and the enynoic acid fragment $\mathbf{2 5}$ (Figure 1.15). 

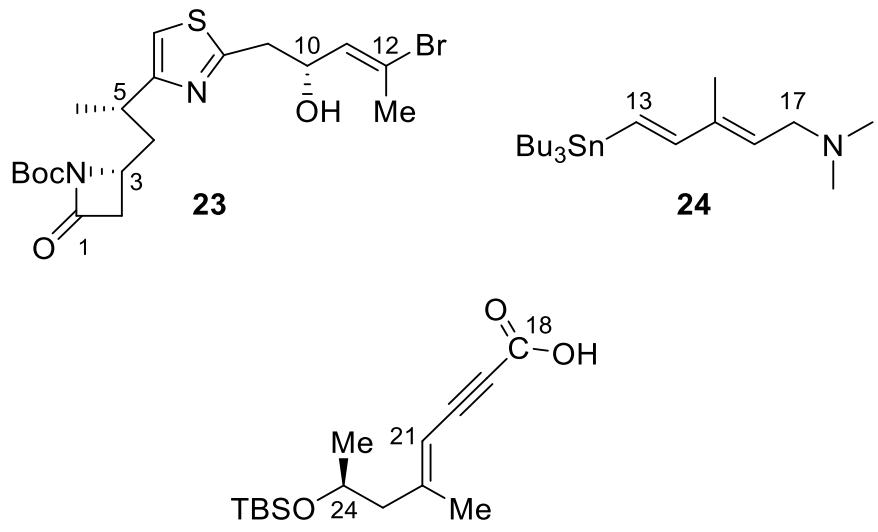

25

Figure 1.15 Major fragments in Romo's total synthesis of PatA.

\section{Preparation of $\beta$-Lactam Fragment 23}

As shown in Scheme 1.1, the preparation of fragment $\mathbf{2 3}$ started from a Nagao aldol reaction between thiazolidinone $\mathbf{2 6}$ and aldehyde 27 to afford alcohol 29 with good diastereoselectivity $(\mathrm{dr}>9: 1)$, via the presumed tin complex 28. The stereochemistry at C10 position (PatA numbering) was inverted when compared to PatA. Silyl protection and aminolysis with gaseous ammonia cleaved the auxiliary to afford the amide $\mathbf{3 1}$, which was converted to thioamide 32 with Belleau's reagent. Thiazole $\mathbf{3 3}$ was formed in an excellent yield following Meyers' modified Hantzsch conditions. ${ }^{113}$ Partial reduction of ester $\mathbf{3 3}$ to aldehyde $\mathbf{3 4}$ followed by a Horner-Wadsworth-Emmons reaction with an oxazolidinone-containing phosphonate $\mathbf{3 5}$ provided the homologated product $\mathbf{3 6}$. Compound 36 was treated with an excess of methyl magnesium bromide and an equivalent amount of copper(I) bromide dimethyl sulfide complex according to the method developed by Hruby ${ }^{114}$ to deliver the desired methyl adduct $\mathbf{3 8}$ in good diastereoselectivity $(\mathrm{dr}=6.4: 1)$. The selectivity was proposed to be induced by the formation of a $\mathrm{Cu}(\mathrm{I}) \pi$-olefin $\mathrm{Mg}$ (II) complex 37. The methyl group added from the less hindered side - the opposite side to the phenyl group of the auxiliary. The auxiliary was transamidated with $N, O$-dimethylhydroxylamine hydrochloride and trimethyl aluminum to provide Weinreb amide 39. During the process of transamidation, a crystalline byproduct, amide 40, was obtained as a result of the Weinreb amine attacking at the oxazolidinone carbonyl, which fortuitously allowed the verification of the stereochemistry at C5 and C10 position (PatA numbering) by X-ray crystallography. ${ }^{84}$ Weinreb amide 39 was partially reduced by diisobutylaluminium hydride (DIBAL-H) to deliver aldehyde $\mathbf{4 1}$. 

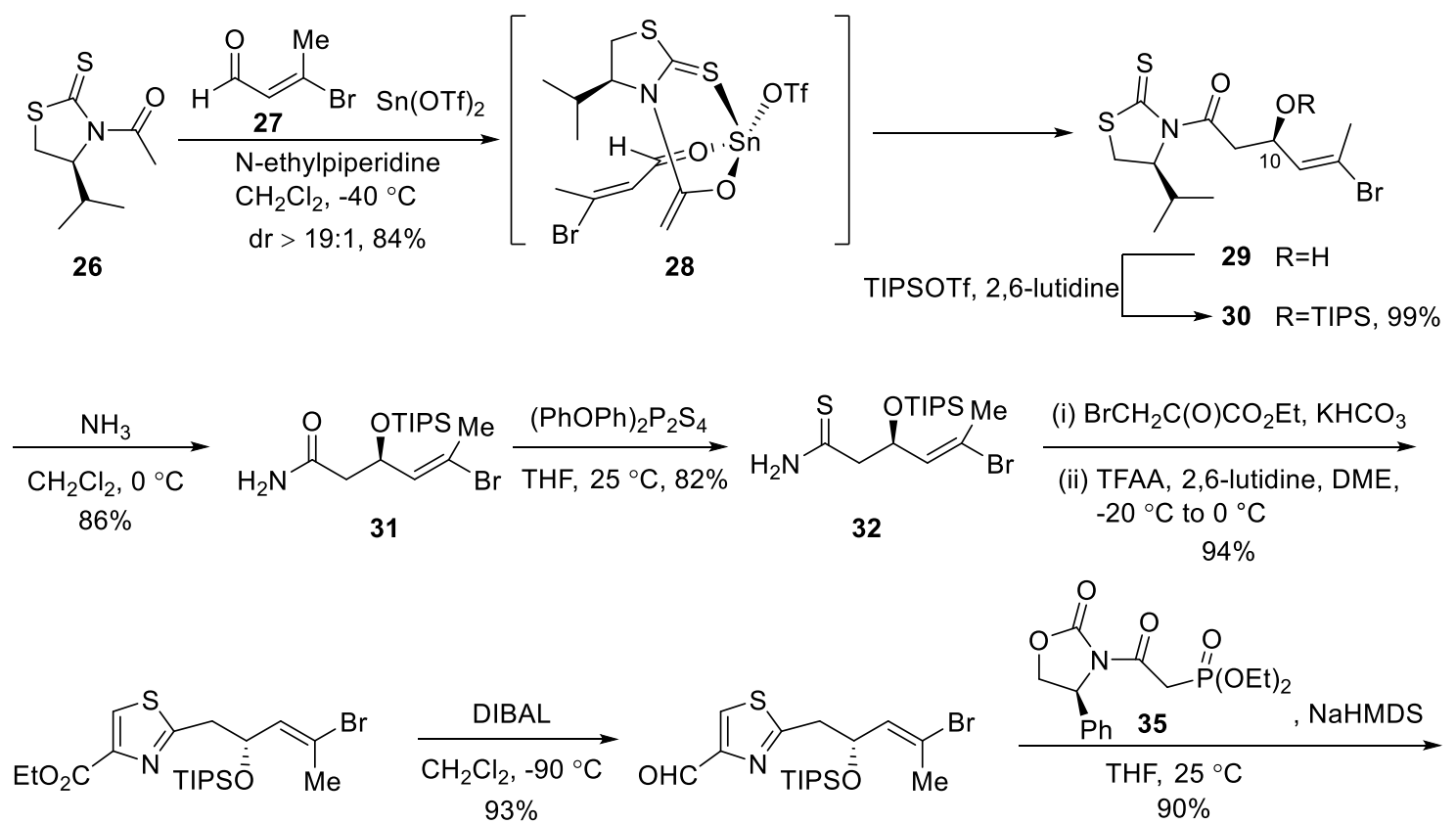

33

34<smiles>CC(Br)=CC(Cc1nc(C=CC(=O)N2C(=O)OC[C@H]2c2ccccc2)cs1)[PH2+][Mg]</smiles>

36

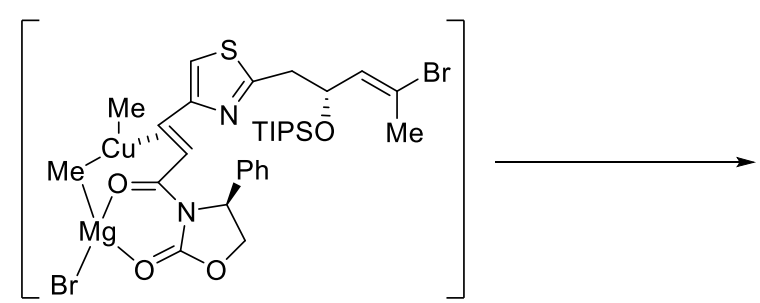

37

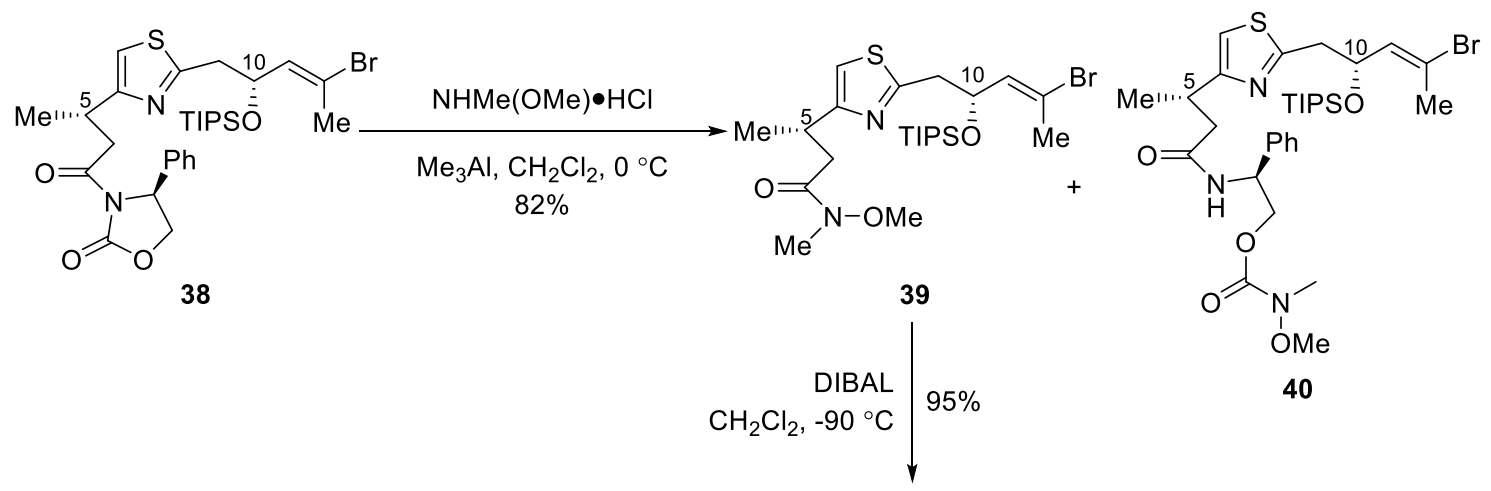

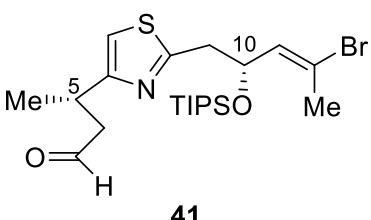

Scheme 1.1 Introduction of stereochemistry at C5 and C10 in the preparation of $\mathbf{4 1}$.

A second Nagao acetate aldol reaction between $\mathbf{4 1}$ and the $N$-acetylthiazolidinethione 26 afforded the alcohol 42 with high stereoselectivity ( $\mathrm{dr}>$ 19:1) (Scheme 1.2). Transamidation with $O$-benzylhydroxylamine hydrochloride cleaved the auxiliary to afford the $N$-benzyloxy amide 43. The following intramolecular Mitsunobu reaction 
inverted the configuration of $\mathrm{C} 3$ (PatA numbering) and gave the $\beta$-lactam 44 with the correct stereochemistry. In order to utilise a more compatible deprotection condition with later intermediates, the $\mathrm{N}-\mathrm{O}$ bond of the benzoyl-protected $\beta$-lactam was reductively cleaved by samarium(II) iodide $\left(\mathrm{SmI}_{2}\right)$ in the presence of water to afford the unprotected $\beta$-lactam 45, which was subsequently reprotected as the tert-butyl carbamate 46. Compound $\mathbf{4 6}$ was desilylated to give the desired $\beta$-lactam fragment 23.

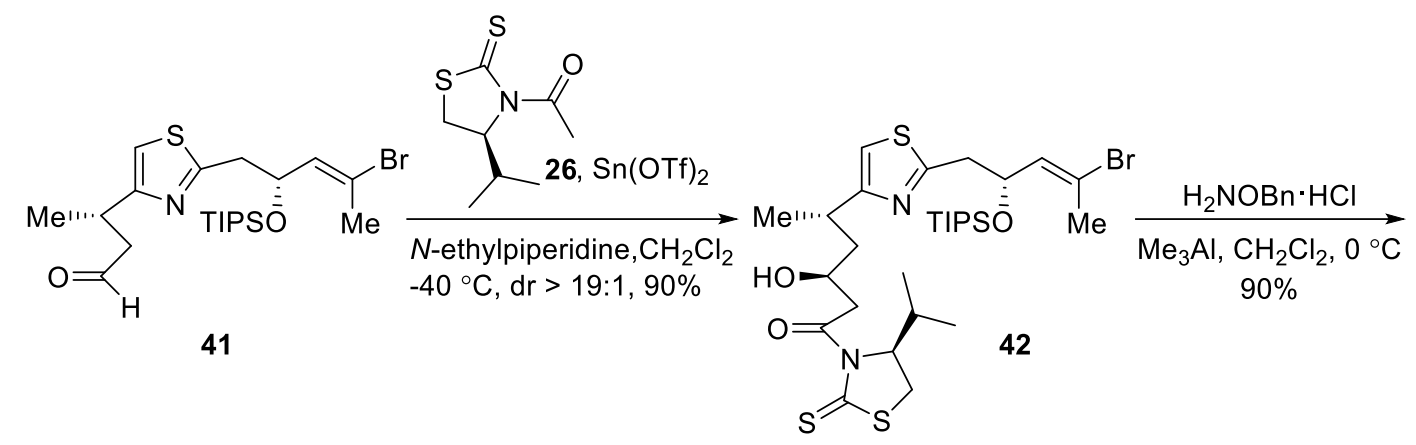

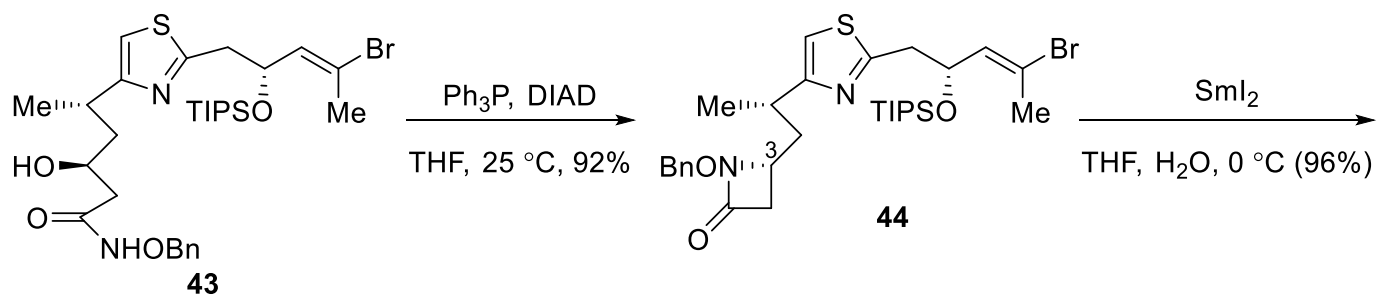

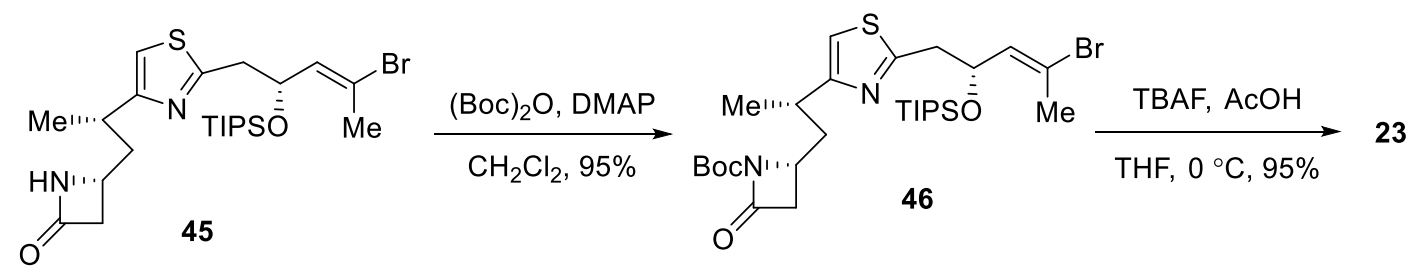

Scheme 1.2 Preparation of $\beta$-lactam fragment 23.

\section{Preparation of Dienyl Stannane Fragment 24}

The preparation of the dienyl stannane fragment $\mathbf{2 4}$ started with one-pot tosylation of the enyne alcohol 47 and substitution with dimethylamine (Scheme 1.3). The resulting alkyne 48 was stannylated, according to Aksela and Oehlschlager's method, ${ }^{115}$ to afford the desired stannane $\mathbf{2 4}$ for the intermolecular Stille coupling reaction. 

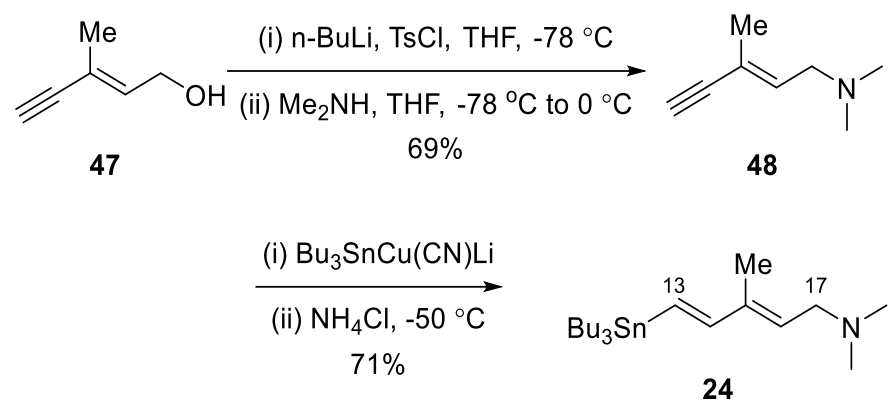

Scheme 1.3 Preparation of the stannane fragment 24 .

\section{Preparation of Enynoic Acid Fragment 25}

The preparation of the precursor for the E,Z-dienoate motif, the enynoic acid $\mathbf{2 5}$, is shown in Scheme 1.4. Ethyl acetoacetate 49 was asymmetrically hydrogenated by the modified Noyori method to set the desired stereochemical configuration at C24 (PatA numbering) with excellent enantioselectivity (94\% ee). Silyl protection and partial reduction afforded the aldehyde 52, which was subjected to the Corey-Fuchs procedure to provide the alkyne 54 via a dibromoalkene intermediate 53. ${ }^{116}$ Alkyne 54 was converted to the vinyl iodide $\mathbf{5 5}$ by a zirconium catalysed carboalumination/iodination sequence. Sonogashira coupling with propargyl alcohol delivered the enyne alcohol 56, which was subjected to sequential Swern and Pinnick oxidations to provide the pivotal enynoic acid 25. 


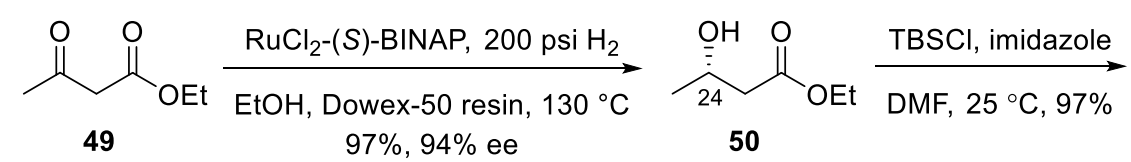<smiles>CCOC(=O)CC(C)O[AsH3-]</smiles>

51<smiles>CC(CC=C(Br)Br)O[SnH3]</smiles>

53

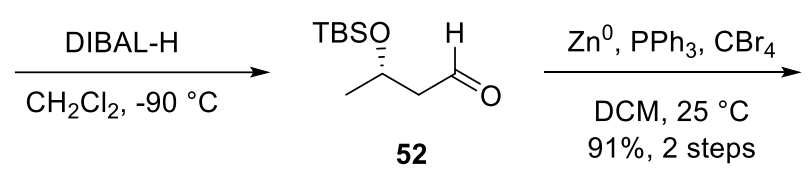

52

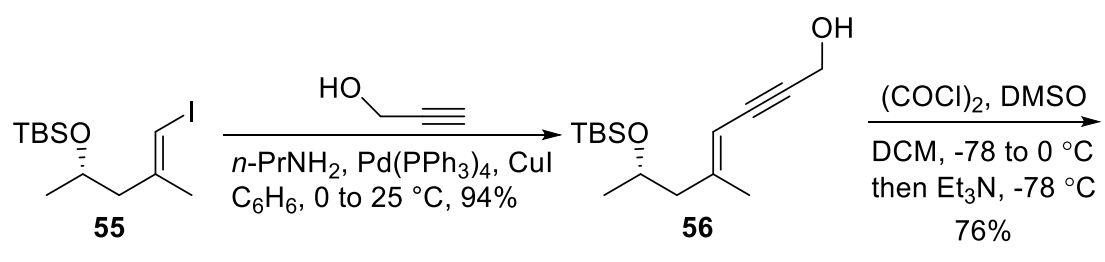

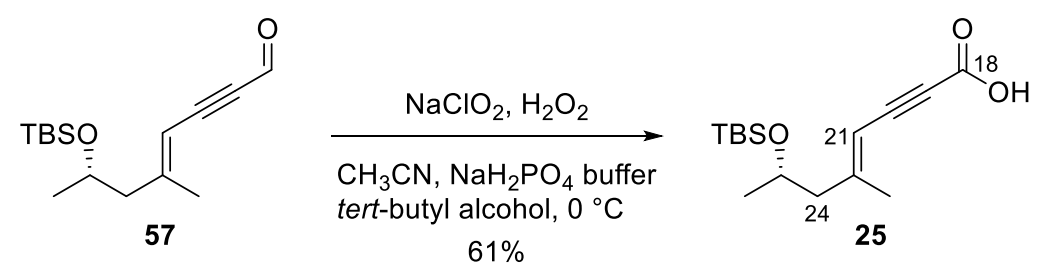

Scheme 1.4 Preparation of enynoic acid 25 as the precursor of $Z, E$-dienoate.

\section{Coupling of Fragments}

As shown in Scheme 1.5, $\beta$-lactam fragment 23 was coupled with enynoic acid 25 under Mitsunobu conditions to invert the configuration of C10 to afford the required $(S)$ configuration in PatA (Scheme 1.5). Desilylation of compound 58 with hydrogen fluoride-pyridine complex was followed by the intramolecular alcoholysis of the $\beta$-lactam using modified Palomo conditions to provide the macrocycle core $60 .{ }^{117}$ The initial attempt, as outlined with blue arrows, utilised Lindlar's catalyst to reduce the alkyne and the resulting $Z, E$-diene $\mathbf{6 1}$ was coupled with the dienyl stannane fragment $\mathbf{2 4}$ to provide the Boc-protected PatA 62 in 32\% yield. Unfortunately, deprotection of the Boc group under acidic conditions (e.g., 5 to 100 equiv of trifluoroacetic acid (TFA); $\mathrm{MeSO}_{3} \mathrm{H}$, $\mathrm{CH}_{2} \mathrm{Cl}_{2},-78{ }^{\circ} \mathrm{C}$; TESOTf, 2,6-lutidine, 0 to $25^{\circ} \mathrm{C}$ ) led to decomposition of PatA, which was plausibly caused by the elimination of the ester group at $\mathrm{C} 10 .{ }^{84} \mathrm{To}$ avoid using acidic conditions for the deprotection reaction, the trichloro-tert-butoxycarbamate (TcBoc) 
group, which could be removed under neutral conditions, was chosen as an alternative amine protecting group. Therefore, the tert-butyl carbamate group was removed and the free amine group was re-protected as a trichloro-tert-butoxycarbamate ( $\mathrm{TcBoc}$ ) protecting group. The resulting TcBoc-protected product underwent Lindlar reduction to provide the $Z, E$-diene $\mathbf{6 3}$. Stille coupling with $\mathbf{2 4}$ and treatment with a cadmium-lead couple to remove the TcBoc group from 64 eventually gave the PatA (22) in 24 steps (based on the longest linear sequence starting from aldehyde 27) with a $2.5 \%$ overall yield. 

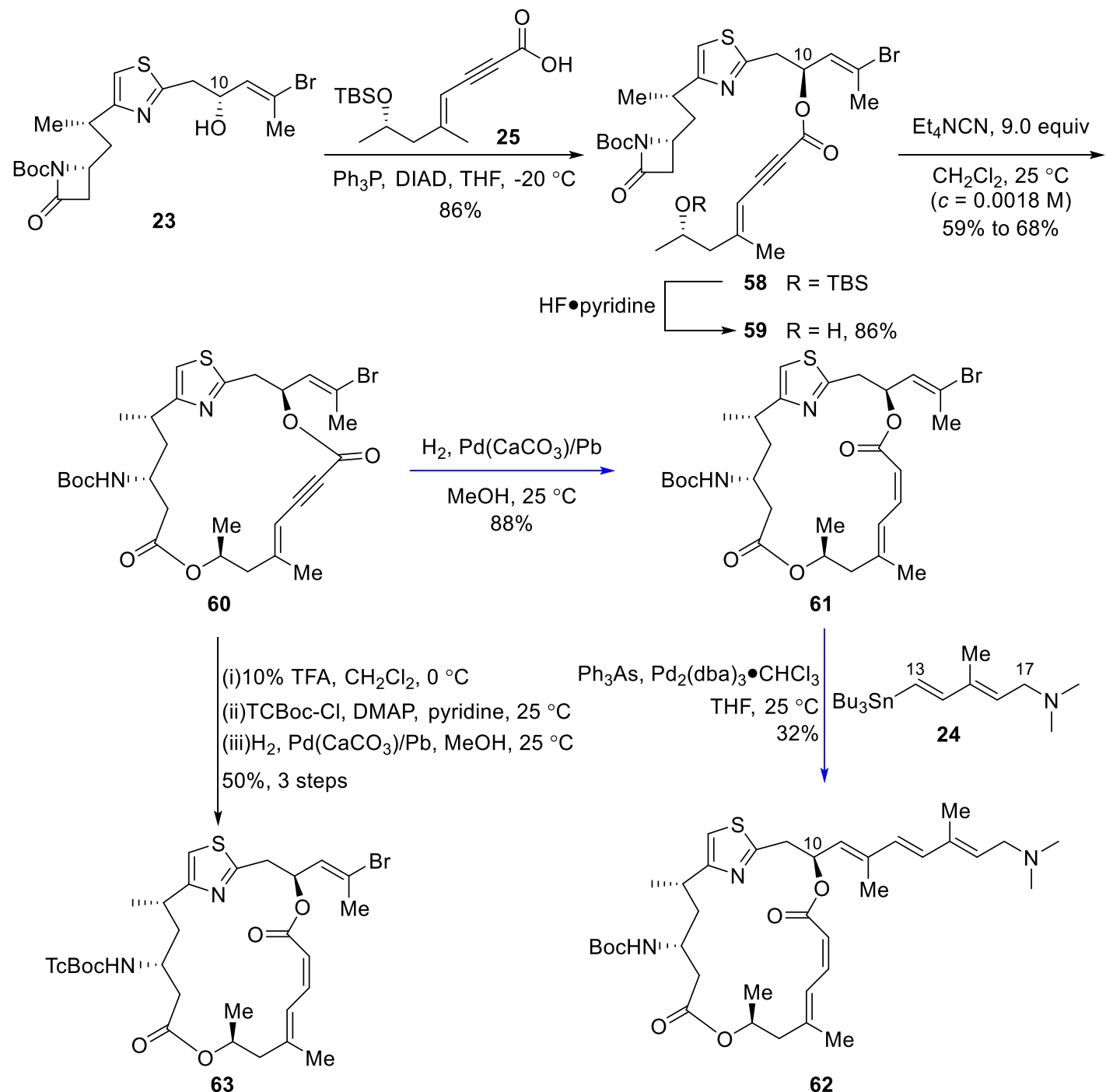

63

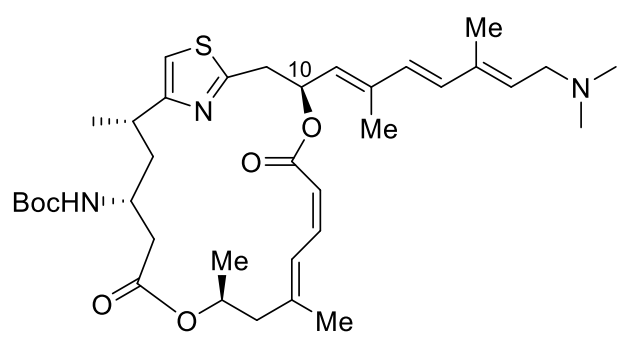

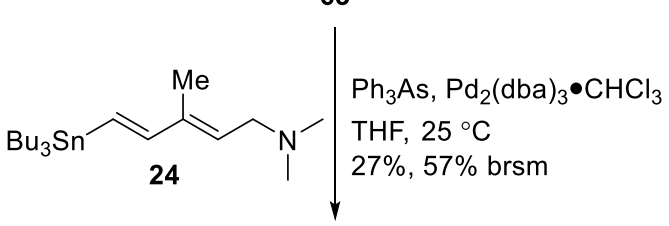

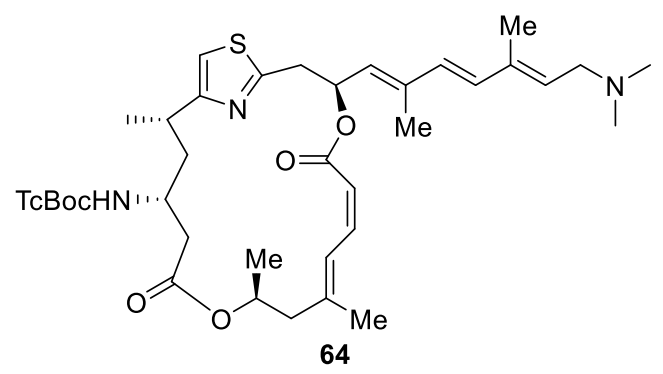

$\mathrm{Cd} / \mathrm{Pb}, 1 \mathrm{M} \mathrm{NH} \mathrm{H}_{4} \mathrm{OAC}$ THF, $25^{\circ} \mathrm{C}, 80 \%$

PatA (22)

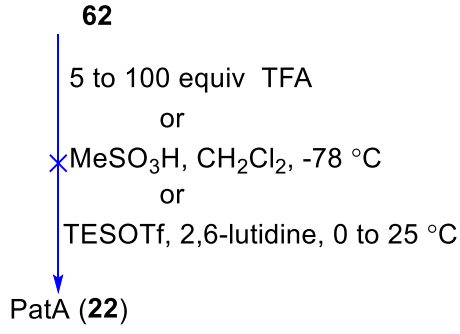

Scheme 1.5 Coupling of fragments 23,24 , and 25 to complete the synthesis of PatA. 


\subsubsection{Pattenden's Total Synthesis}

In 2000, Pattenden and co-workers disclosed their total synthesis of PatA based on a chiral pool approach. ${ }^{118}$ As shown in Figure 1.16, their synthetic strategy involved the initial formation of the macrocycle core via a combination of a sulfinimine directed aldol reaction, a Yamaguchi esterification, and an intramolecular Stille coupling. Similarly to Romo's approach, the construction of the thiazole fragment was accomplished by the modified Hantzsh thiazole synthesis. The side chain was elongated with a combination of Wittig homologation and intermolecular Stille coupling.

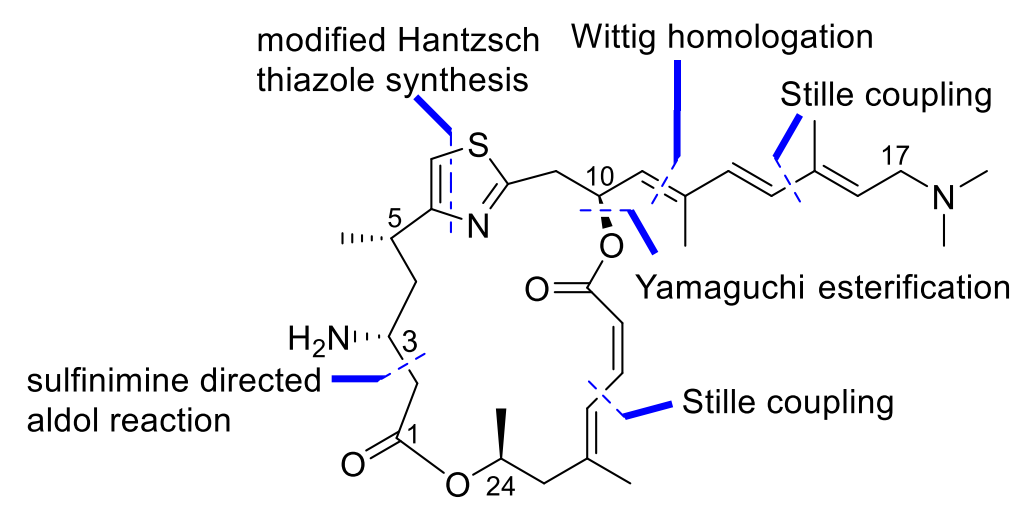

Figure 1.16 Key connections and features in Pattenden's synthetic strategy. ${ }^{118}$

Therefore, Pattenden's total synthesis utilised five smaller fragments 65, 66, 67, 68 and 69, and started from the preparation of sulfinimine fragment 65 and ester fragment 68 (Figure 1.17).

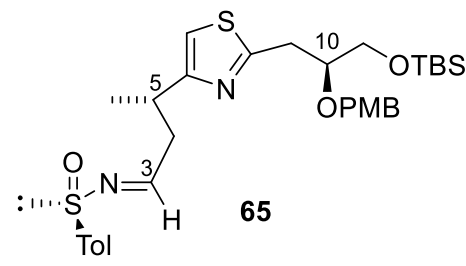

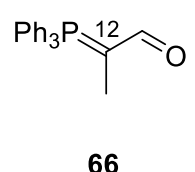

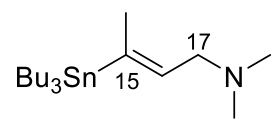

66 67<smiles>CC(=O)O[C@@H](C)C/C(C)=C/I</smiles>

68<smiles></smiles>

69

Figure 1.17 Major fragments in Pattenden's total synthesis. ${ }^{118}$ 


\section{Preparation of Sulfinimine Fragment 65}

The preparation of sulfinimine fragment $\mathbf{6 5}$ started with the construction of the thiazole ring. The preparation of thioamide $\mathbf{7 0}$ (Scheme 1.6) required for the Hantzsh thiazole synthesis began from commercially available L-malate 71, which sets the required $(S)$ configuration at the $\mathrm{C} 10$ position in PatA. Selective reduction of compound $\mathbf{7 1}$ by treatment with a combination of borane dimethyl sulfide and sodium borohydride provided diol 72, which was then subjected to a sequential mono-silyl protection and para-methoxybenzyl (PMB) protection procedure to afford the ester 74. Saponification of $\mathbf{7 4}$ and subsequent amidation via a mixed anhydride intermediate provided amide 76, and this underwent thionation with Lawesson's reagent to deliver thioamide $\mathbf{7 0 .}$
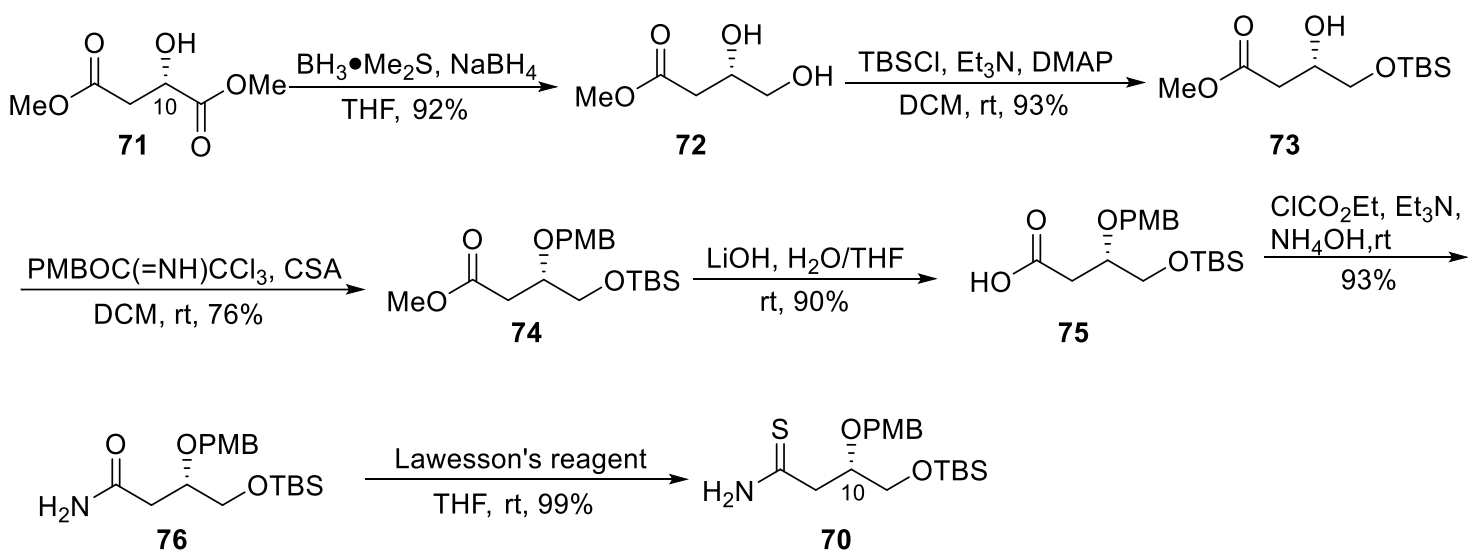

Scheme 1.6 Preparation of thioamide 70.

The synthesis of the $\alpha$-bromoketone partner 77 (Scheme 1.7) started with commercially available (S)-methyl 3-hydroxy-2-methylpropanoate $\mathbf{7 8}$, which provided the stereochemistry at C5 in PatA. Silylation of the alcohol group and treatment with $\mathrm{N}, \mathrm{O}$ dimethylhydroxylamine hydrochloride and trimethyl aluminum gave Weinreb amide 80. Conversion to methyl ketone $\mathbf{8 1}$ with a Grignard reagent followed by regioselective bromination mediated by the bulky base LiHMDS gave the $\alpha$-bromoketone 77 . 


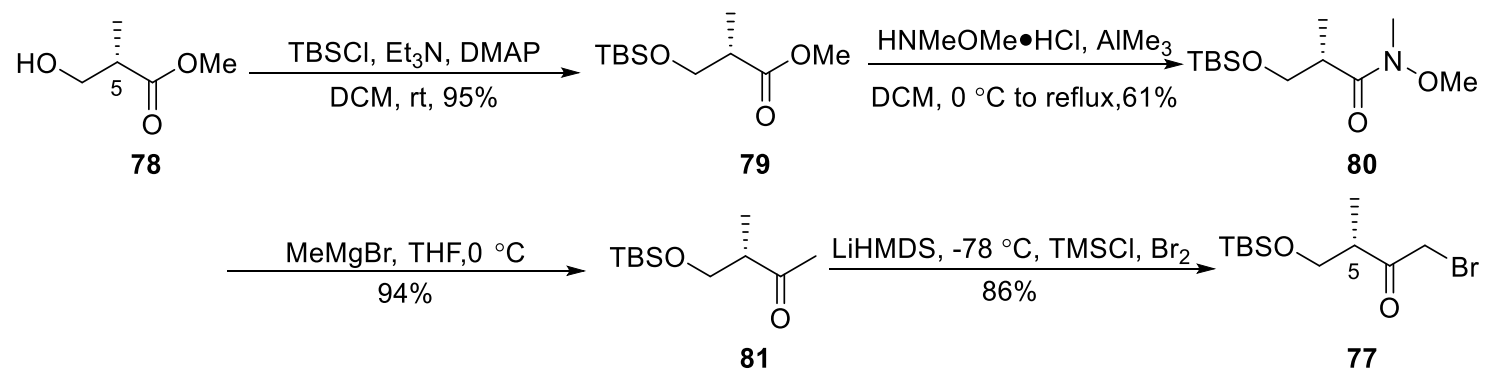

Scheme 1.7 Preparation of $\alpha$-bromoketone 77 .

As outlined in Scheme 1.8, the modified Hantzsch reaction following Meyers' conditions ${ }^{113}$ between $\mathbf{7 0}$ and $\mathbf{7 7}$ gave thiazole $\mathbf{8 2}$ in high optical purity. Regioselective desilylation of $\mathbf{8 2}$ followed by mesylation and cyanation with sodium cyanide afforded nitrile 84, which was reduced by DIBAL-H to provide aldehyde 85 . Treatment of 85 with $(R)$-p-toluenesulfinamide and titanium tetraethoxide led to the formation of the enantiopure sulfinimine $\mathbf{6 5}$.
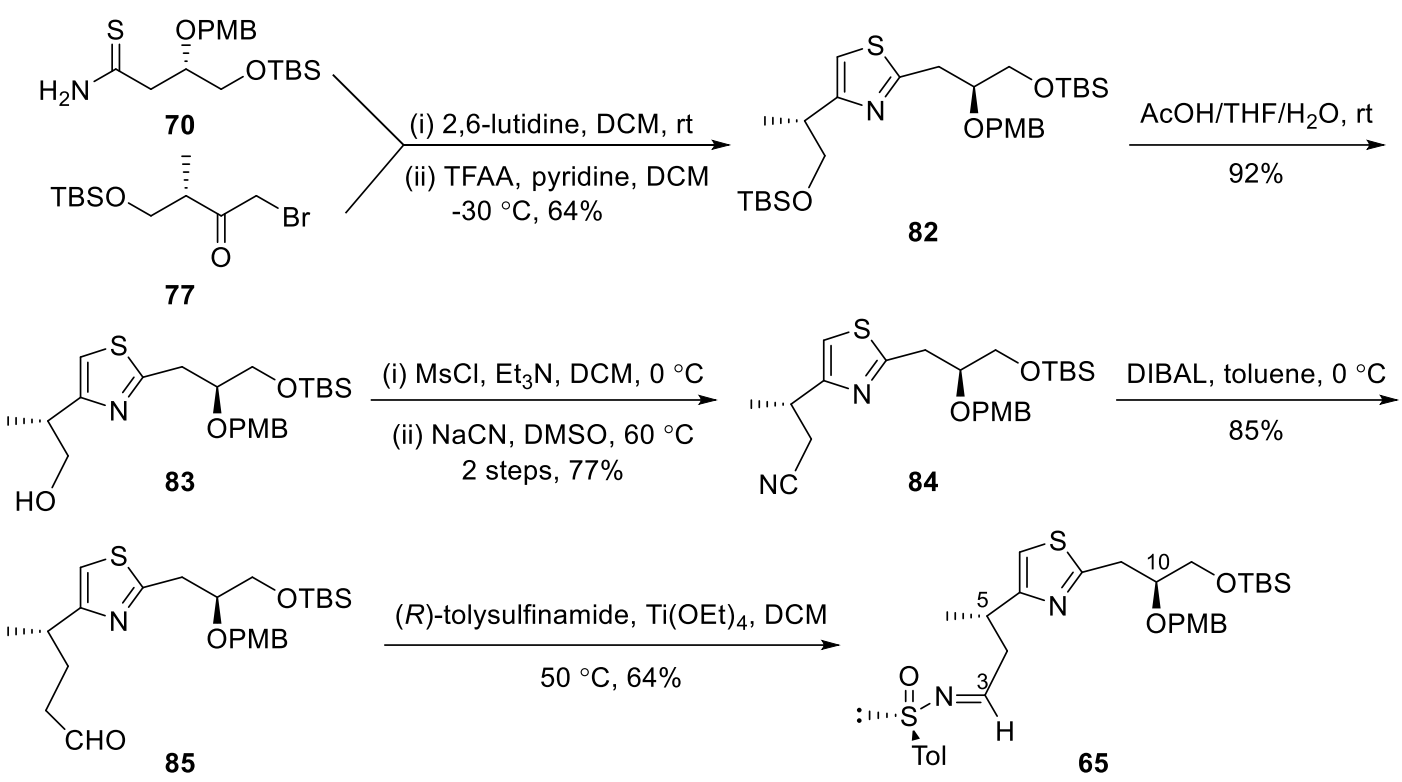

Scheme 1.8 Preparation of sulfinimine 65. 


\section{Preparation of Chiral Ester Fragment 68}

As shown in Scheme 1.9, epoxide ring-opening of the commercially available $(S)-(-)$ propylene oxide $\mathbf{8 6}$ by the ethylenediamine complex of lithium acetylide provided the chiral alkyne 87 in high enantiopurity, which sets the stereochemistry at C24 position (PatA numbering). Alkyne 87 then underwent a zirconium-catalysed carboalumination and iodination reaction to afford the iodide $\mathbf{8 8}$, which was then esterified to deliver the desired ester fragment $\mathbf{6 8} .{ }^{119}$ The yields for these three steps were not provided in the publication and the reagents for the esterification step were not specified either. ${ }^{119}$

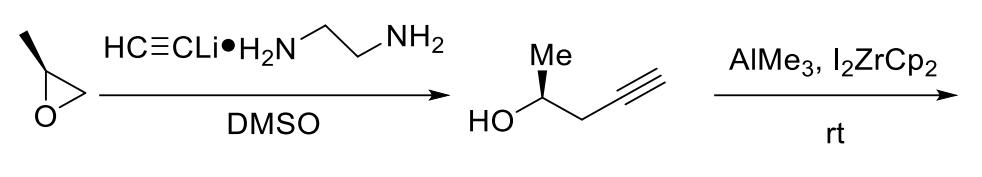

86

87

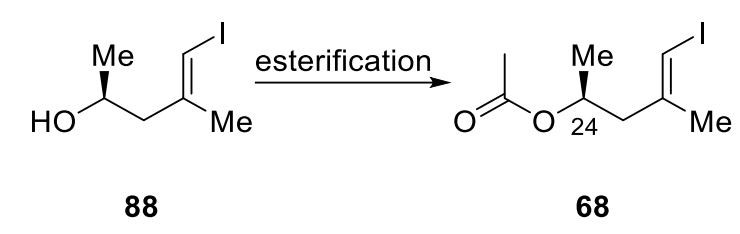

Scheme 1.9 Preparation of chiral alcohol fragment 68.

\section{Preparation of Macrocyclic Aldehyde 97}

As shown in Scheme 1.10, sulfinimine 65 was coupled with chiral ester 68 upon treatment with LiHMDS, via its derived enolate, to give substituted $\beta$-amino ester $\mathbf{8 9}$ in about $90 \%$ diastereoselectivity for the $(R)$-configuration at the $\mathrm{C} 3$ position (PatA numbering). Treatment with TFA-methanol cleaved the $p$-toluenesulfinyl group to give amine $\mathbf{9 0}$, which was protected with 2,2,2-trichloro-1,1-dimethylethyl chloroformate to provide the corresponding TcBoc carbamate 91. Removal of the $p$-methoxybenzyl (PMB) protecting group with 2,3-dichloro-5,6-dicyano-1,4-benzoquinone (DDQ), followed by esterification with Z-3-tri- $n$-butylstannylpropenoic acid 69 using the Yamaguchi activating reagent 93, provided the key stannane-iodide 94. Intramolecular Stille coupling furnished the 19-membered bis-lactone macrocycle 95 with the complete preservation of the desired $E / Z$ stereochemistry. Deprotection with tetra- $n$-butylammonium fluoride (TBAF) and acetic acid followed by oxidation with pyridine-buffered Dess-Martin periodinane afforded aldehyde 97. 

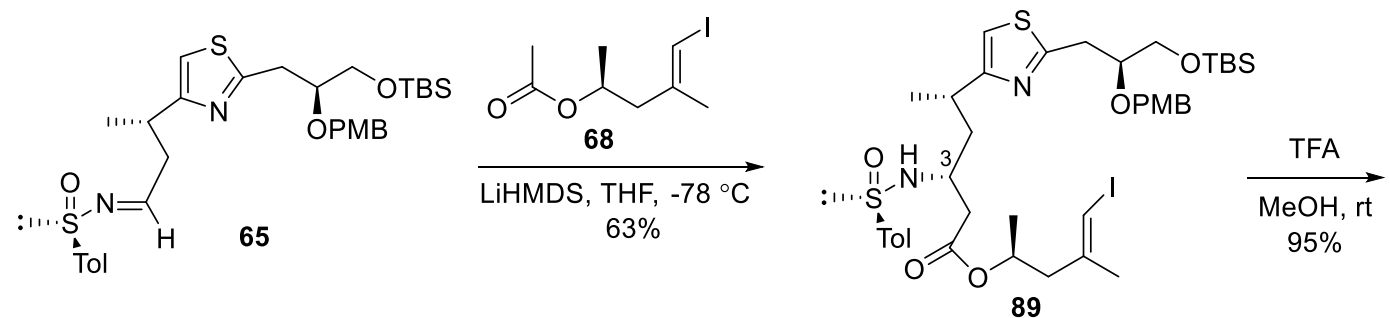

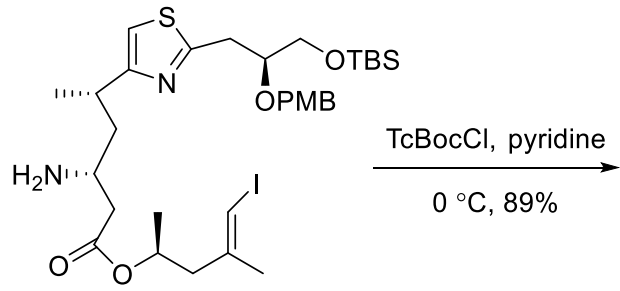

90

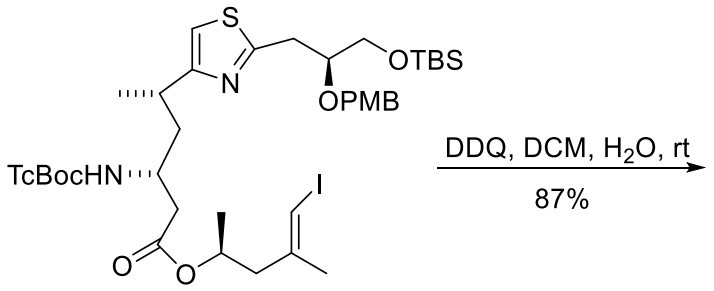

91

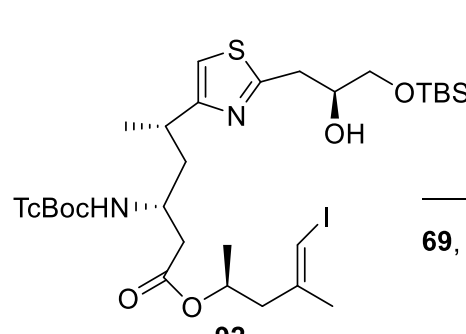

92<smiles>O=C(Cl)c1c(Cl)cc(Cl)cc1Cl</smiles>

69, $\mathrm{Et}_{3} \mathrm{~N}, \mathrm{DMAP}$, toluene, $-30{ }^{\circ} \mathrm{C}$ $67 \%$

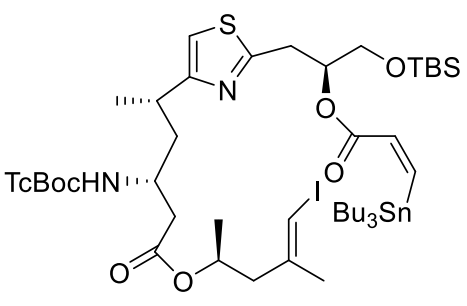

94

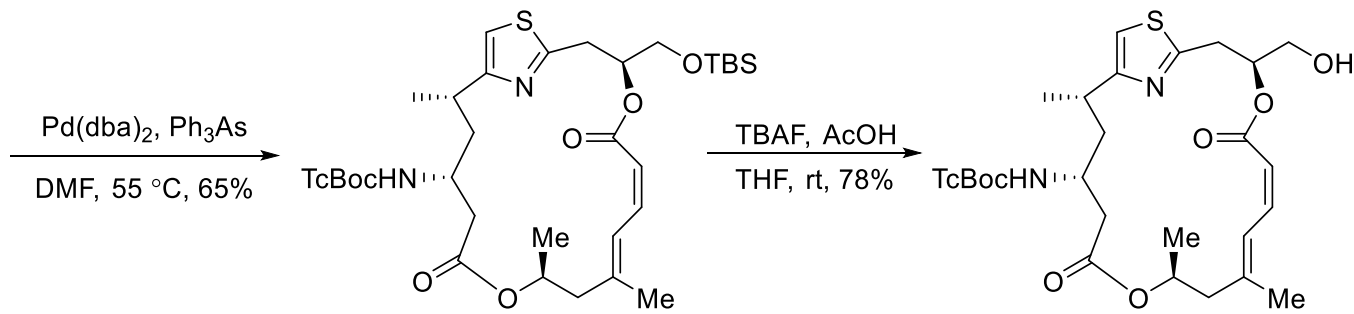

95

96

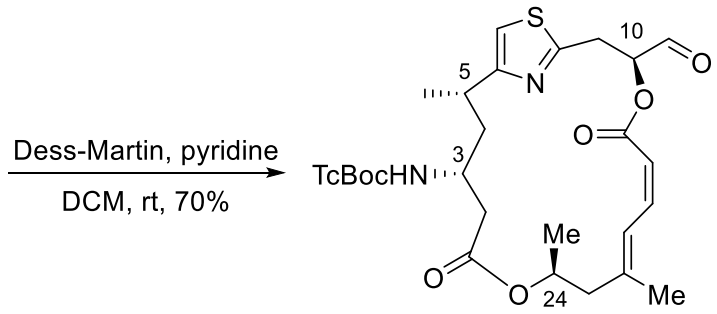

97

Scheme 1.10 Coupling of fragment 65,68 and 69 to furnish the macrocycle core.

\section{Attachment of the Side Chain Fragments 66 and 67}

Aldehyde 97 was homologated with 2-(triphenylphosphoranylidene)propionaldehyde 66 to exclusively provide $E$-unsaturated enal 98 (Scheme 1.11). Treatment of aldehyde 98 under Takai conditions, followed by Stille coupling with vinyl stannane 67, resulted in 
the formation of TcBoc-protected PatA 64. Finally, removal of the TcBoc protecting group using a $\mathrm{Cd} / \mathrm{Pb}$ couple with ammonium acetate afforded Pat $\mathrm{A}(\mathbf{2 2})$. This synthetic route provided PatA over 25 steps (the longest linear sequence from starting material 70) with an overall yield of $0.19 \%$.

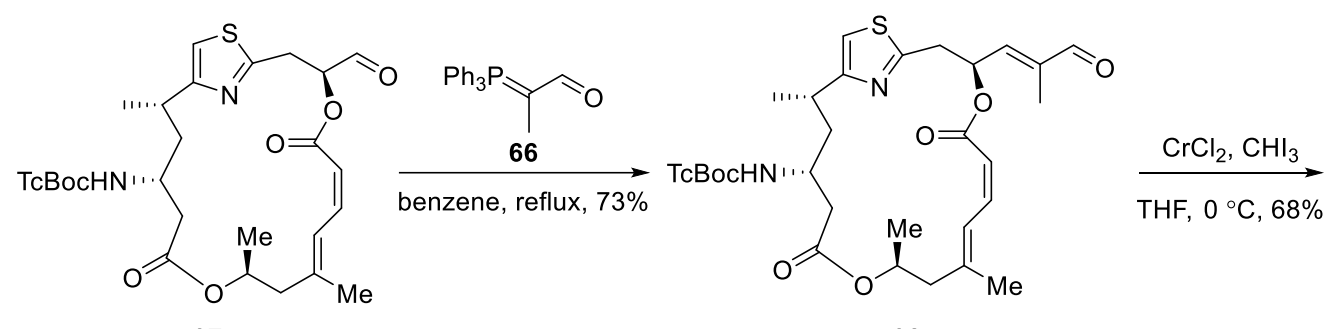

97

98

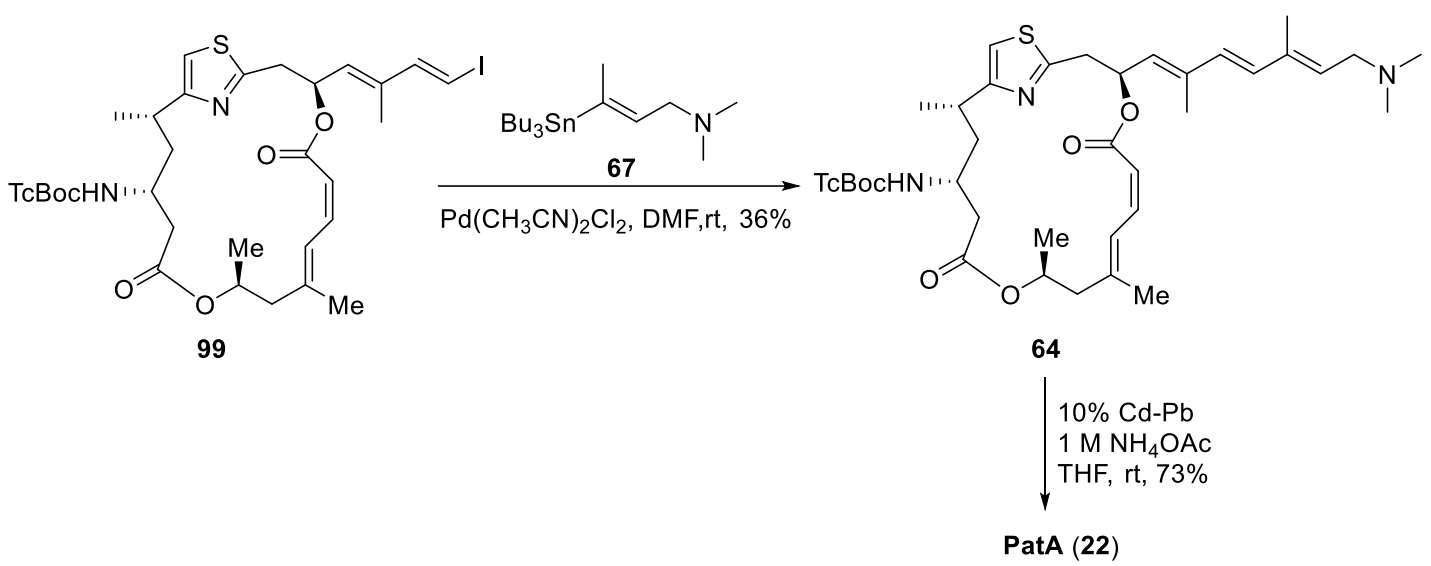

Scheme 1.11 Coupling of side chain fragments 66 and 67 to complete synthesis of PatA (22).

\subsubsection{Fürstner and Zhuo's Total Synthesis}

Recently, Fürstner and Zhuo reported their robust and efficient total synthesis of PatA, which centres on utilising iron-catalysed ring-opening of a 2-pyrone for the formation of the highly isomerisation-prone $Z, E$-dienoate motif (Figure 1.18 ). ${ }^{120}$ The macrocycle core was formed via a combination of Steglich esterification and modified Mukaiyama macrolactonisation. The attachment of the side chain followed the previous synthetic strategy reported by Pattenden and co-workers. ${ }^{118}$ 


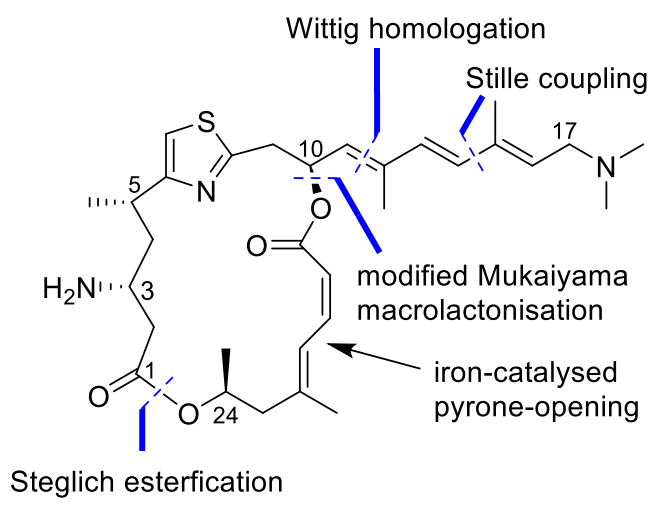

Figure 1.18 Key connections and features in Fürstner and Zhuo’s synthetic strategy. ${ }^{120}$

Fürstner's synthetic work started with the preparation of the two major fragments thiazole-containing fragment 100 and 2-pyrone fragment 101 (Figure 1.19).

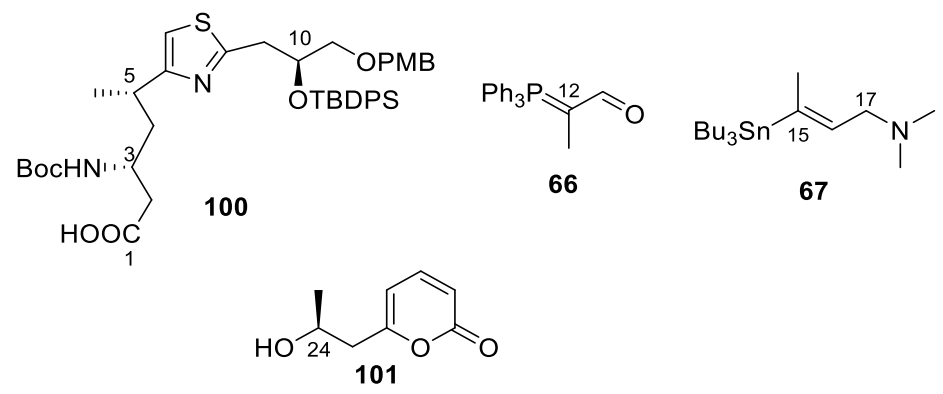

Figure 1.19 Major fragments in Fürstner and Zhuo’s total synthesis.

\section{Synthesis of Thiazole-containing Fragment 100}

In contrast to Romo and Pattenden's previous synthetic work, in which the 2,4disubstituted thiazole ring was formed using modified Hantsch conditions, dibromothiazole $\mathbf{1 0 2}$ was chosen as the starting material in Fürstner and Zhuo's total synthesis (Scheme 1.12). Dibromothiazole 102 was first treated with lithium chloride and activated zinc to form the organozinc species 103, which was quenched with allyl bromide in the presence of catalytic copper(I) cyanide to give the mono-allylated product 104. Vicinal boronate species $\mathbf{1 0 6}$ was then formed upon exposure to bis(pinacolato)diboron $\left(\mathrm{B}_{2} \mathrm{pin}_{2}\right)$ and a catalyst complex generated in situ from tris(dibenzylideneacetone)platinum(0) $\left(\mathrm{Pt}(\mathrm{dba})_{3}\right)$ and a chiral phosphonite ligand $\mathbf{1 0 5}$. The primary product 106 was then oxidised to deliver diol 107. This two-step/one-pot procedure introduced the correct stereochemical configuration at $\mathrm{C} 10$ (PatA numbering) with high enantioselectivity ( $91 \%$ e.e.). The diol 107 was then transformed into $p$ methoxybenzylidene acetal 108. Regioselective opening of the acetal 108 and subsequent 
silylation furnished thiazole $\mathbf{1 1 0}$.
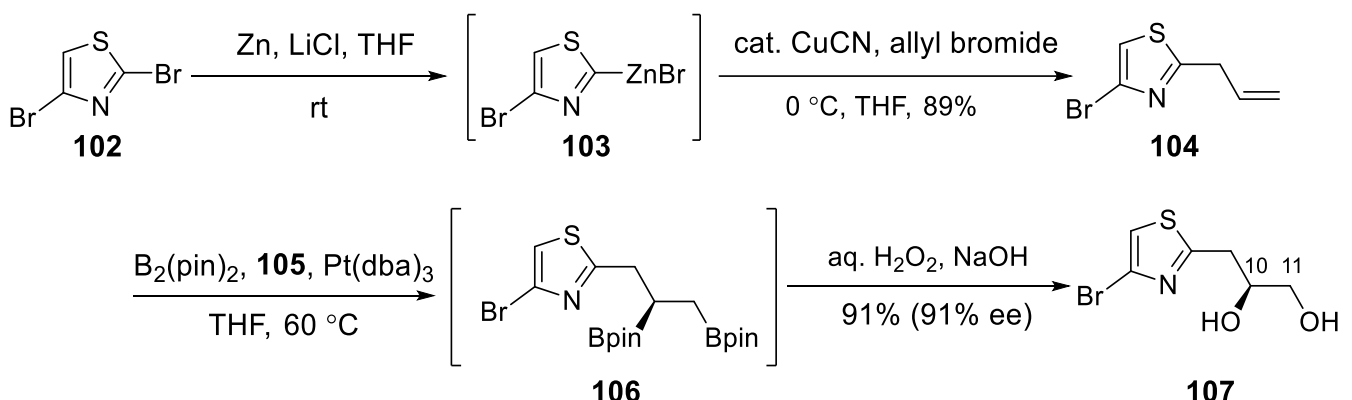

106

107
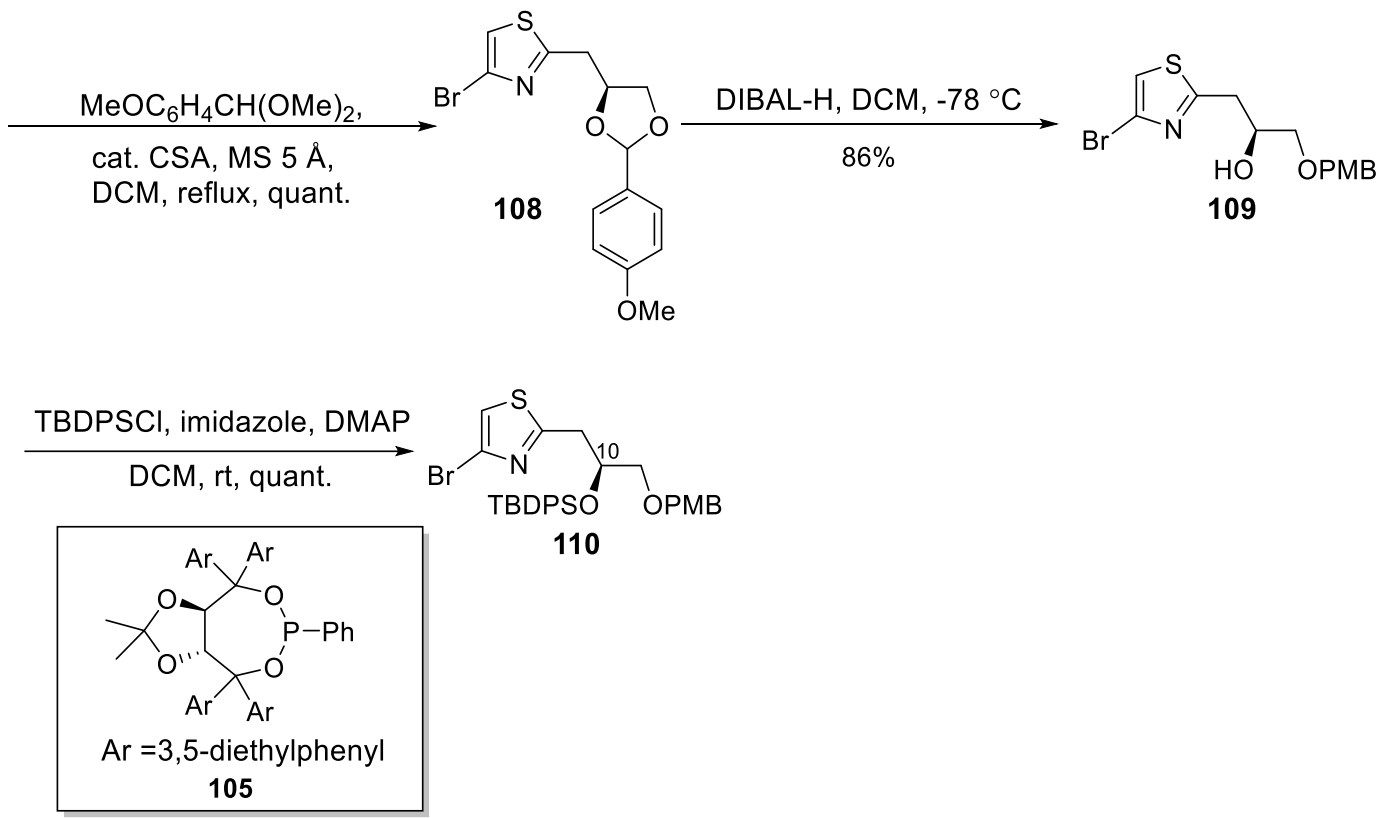

Scheme 1.12 Introduction of stereochemistry at C10 in preparation of thiazole $\mathbf{1 1 0 .}$

As shown in Scheme 1.13, the Suzuki coupling reaction between brominated thiazole 110 and the nucleophilic boronate partner 111, which was derived from alkynoate 113 via a stereoselective borylation reaction catalysed by the copper catalyst generated in situ from copper(I) iodide and xantphos 112, ${ }^{121}$ afforded enoate 114. Following the conditions of Lam and co-workers, ${ }^{122}$ the $\mathrm{C}=\mathrm{C}$ bond of compound $\mathbf{1 1 4}$ was asymmetrically reduced with phenylsilane in the presence of copper(II) acetate monohydrate and $(S, R)$-Josiphos 115 as the catalyst to set the stereochemical configuration at C5 (PatA numbering) with excellent diastereoselectivity ( $\mathrm{dr}>25 / 1)$. Ester 116 was then reduced to form aldehyde 117, which reacted with $9 H$-fluorenyl-9-amine to give the imine intermediate 118. 

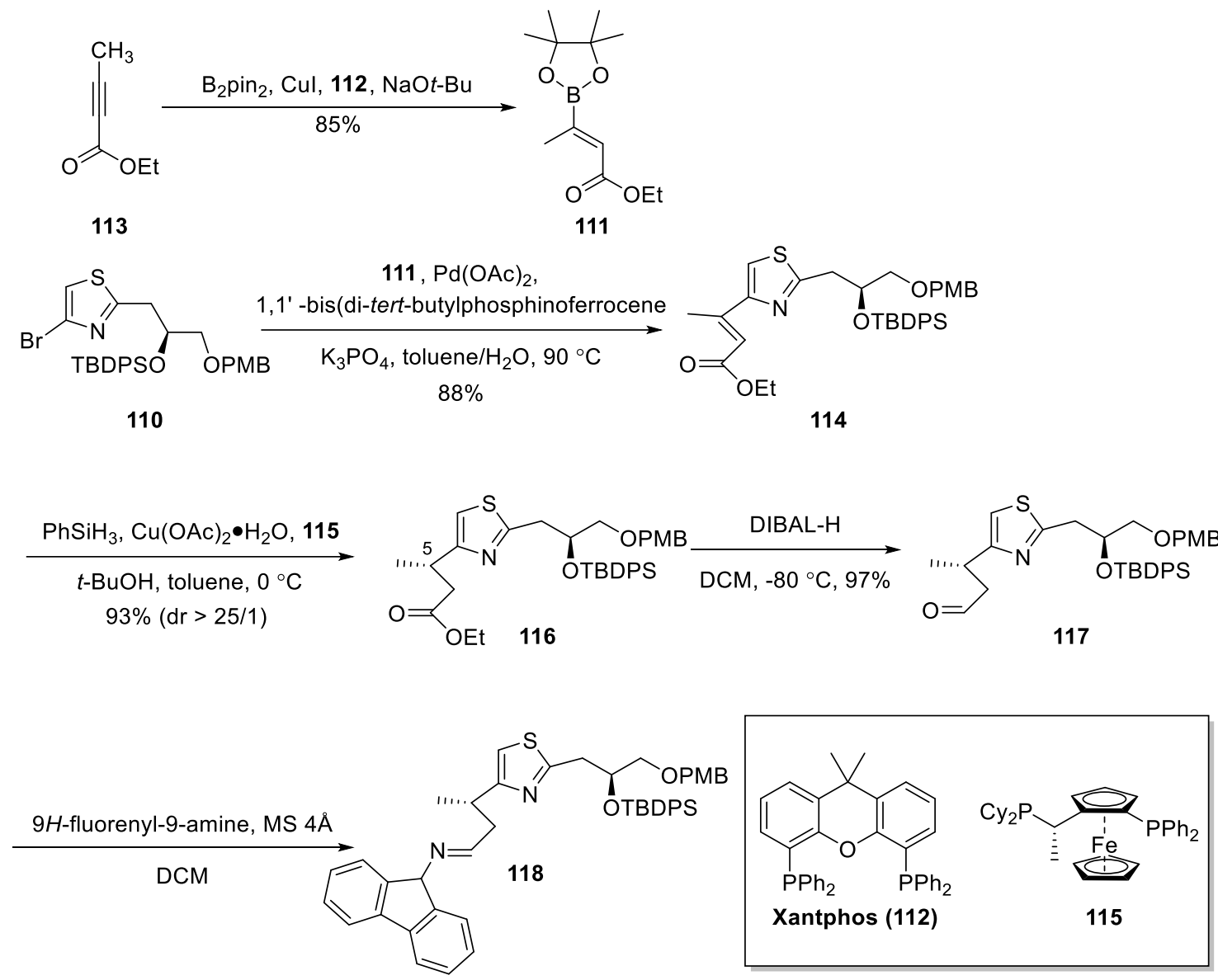

Scheme 1.13 Preparation of imine 118.

The fluorenyl imine $\mathbf{1 1 8}$ was then deprotonated to give a nucleophilic partner, which engaged in an iridium-catalysed asymmetric allylation reaction with compound $\mathbf{1 2 0}$ (Scheme 1.14). The resulting intermediate 121 underwent an aza-Cope rearrangement in THF at $50{ }^{\circ} \mathrm{C}$ to deliver imine 122. The imine 122 was hydrolysed by treatment with hydroxylamine hydrochloride to provide the resulting amine. This three-step sequence successfully afforded the desired $(R)$-configuration at the $\mathrm{C} 3$ position (PatA numbering) in excellent diastereoselectivity $(\mathrm{dr}>25: 1)$. The primary amino group was protected by using di-tert-butyl dicarbonate $\left((\mathrm{Boc})_{2} \mathrm{O}\right)$ and triethylamine. The $\mathrm{C}=\mathrm{C}$ bond of the Bocprotected product 123 was then cleaved under modified Lemieux-Johnson conditions to afford aldehyde 124, which underwent Pinnick oxidation to provide the desired thiazole fragment 100. 


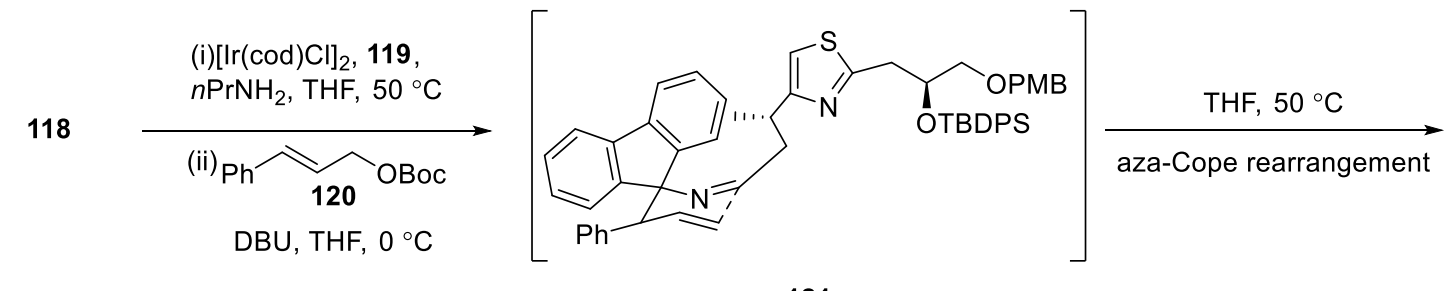

121
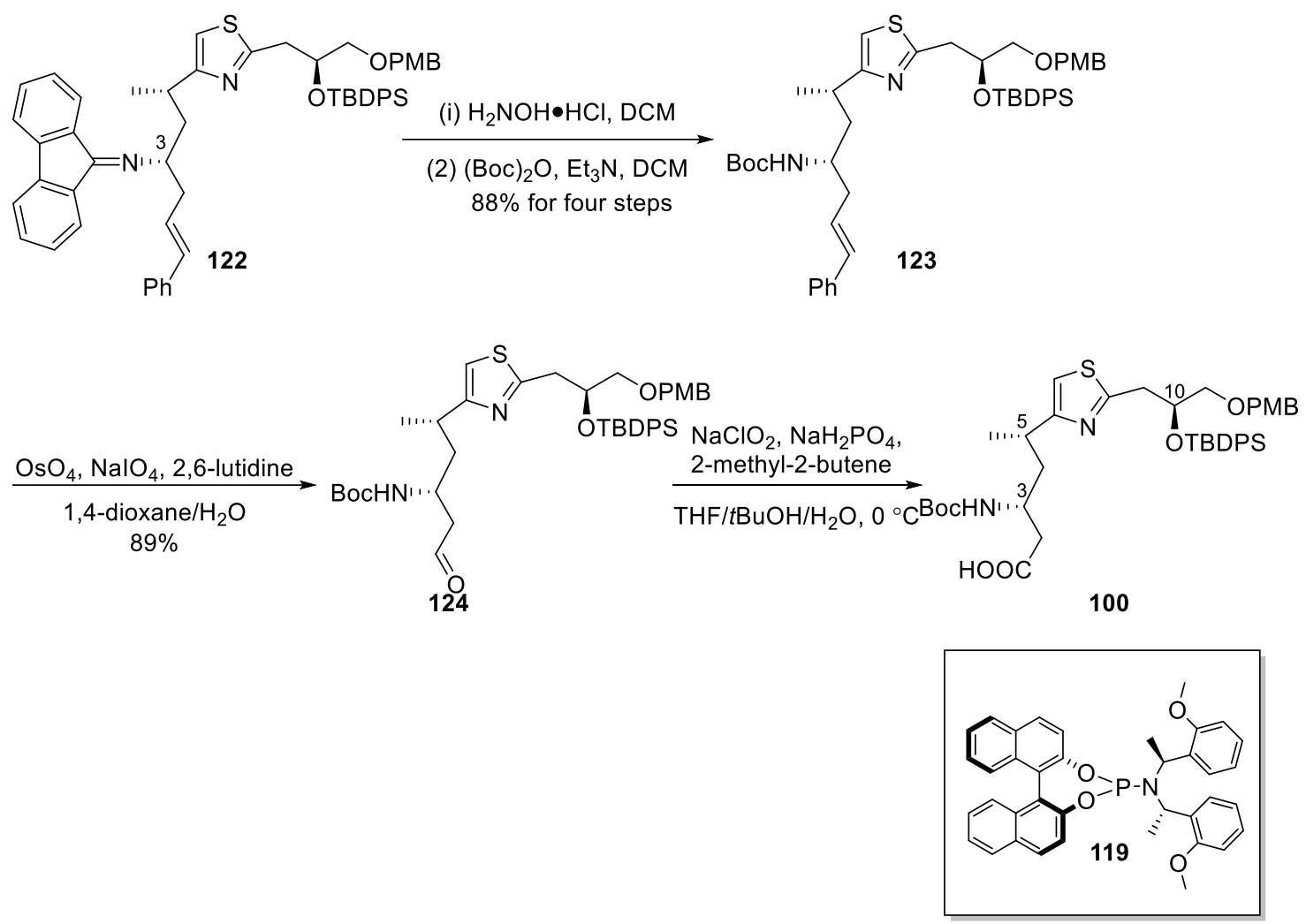

Scheme 1.14 Synthesis of thiazole fragment 100.

\section{Preparation of 2-Pyrone Fragment 101}

To introduce the desired stereochemistry at C24 position (PatA numbering), the preparation of the 2-pyrone fragment 101 started from $(S)$-(-)-propylene oxide 86 (Scheme 1.15), the same chiral starting material used in Pattenden's total synthesis. ${ }^{118}$ Similarly, epoxide 86 was first opened by the ethylenediamine complex of lithium acetylide to provide alkyne 87. Sonogashira coupling between the chiral alkyne 87 and the iodide 126, which was prepared from propiolate 125, delivered alcohol 127 in $93 \%$ yield. Gold-catalysed 6-endo-dig cyclisation of compound 127 provided the desired 2pyrone fragment 101. Fürstner and Zhuo mentioned that using the tert-butyl ester was very important to ensure the exclusive formation of a 2-pyrone rather than a 4-pyrone. 

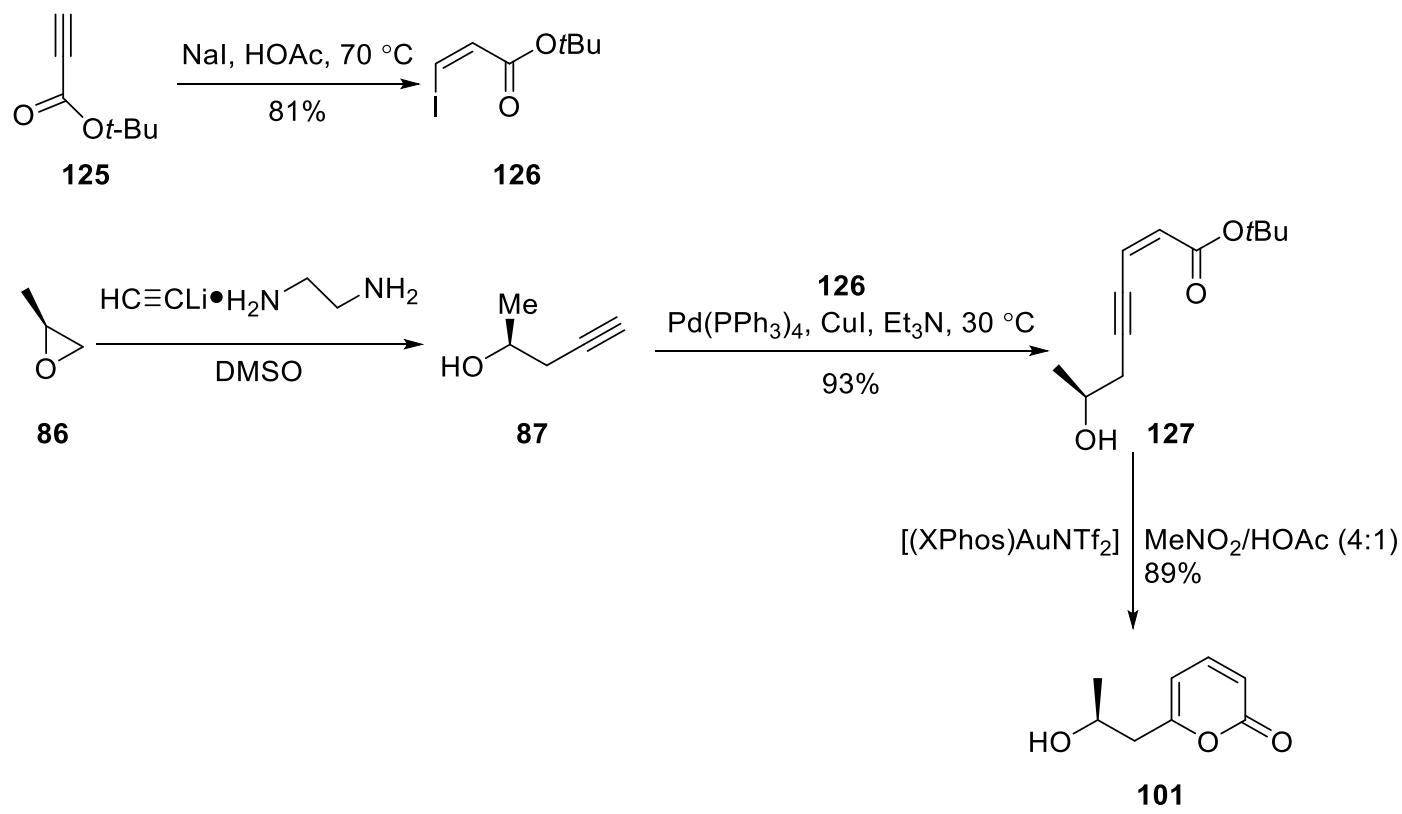

Scheme 1.15 Preparation of 2-pyrone fragment 101.

\section{Synthesis of the Macrocycle Core}

Modified Steglich esterification of the acid fragment $\mathbf{1 0 0}$ with the 2-pyrone fragment 101 provided compound 128 (Scheme 1.16). Methyl magnesium bromide opened the pyrone ring to give the isomerisation-prone $Z, E$-dienoic acid motif and install the methyl group at C22 position simultaneously in the presence of iron(III) acetylacetonate as the catalyst. Fürstner and co-workers reported that it was necessary to perform this reaction at $-60{ }^{\circ} \mathrm{C}$ to minimise the undesired attack of the Grignard reagent onto the -NHBoc group. The resulting Z,E-configured dienoic acid 129 was desilylated and the macrolactonisation of the resulting seco-acid under modified Mukaiyama conditions ${ }^{123}$ provided the macrocyclic compound $\mathbf{1 3 1}$ in good yield. Use of a Mukaiyama reagent 130, which was accompanied by a non-nucleophilic counterion, was critical to avoid massive isomerisation of the Z-double bond. Subsequent cleavage of the PMB-ether under treatment with DDQ provided alcohol 132. 

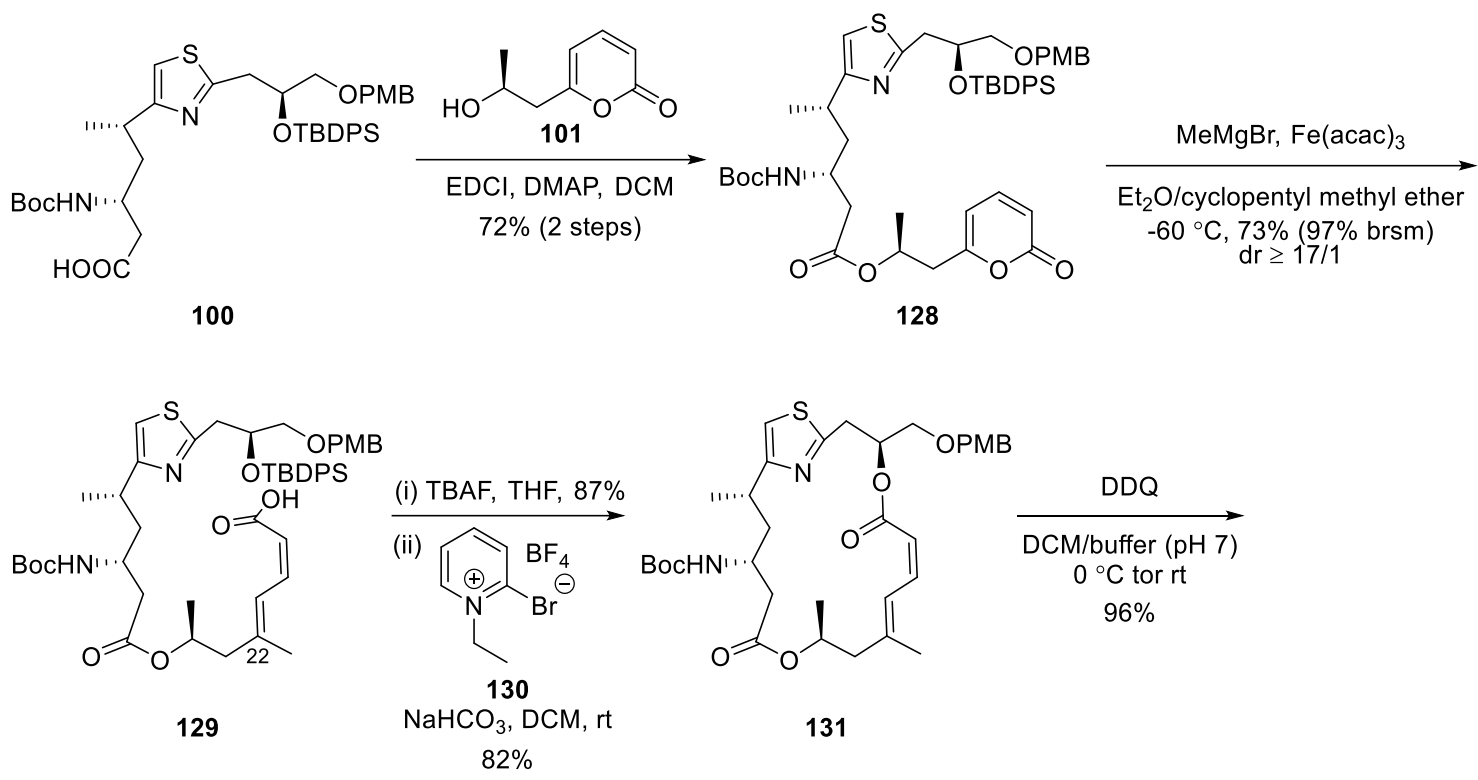

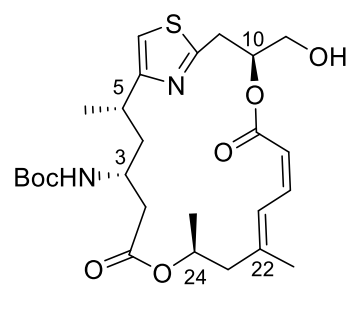

132

Scheme 1.16 Coupling of major fragments 100 and 101 to form macrocycle core.

\section{Attachment of Side Chain Fragments}

Oxidation of alcohol 132 under Parikh-Doering conditions provided aldehyde $\mathbf{1 3 3}$ (Scheme 1.17). Following Pattenden's previous synthetic work, ${ }^{118}$ Wittig reaction with stabilised ylide 66 provided homologated aldehyde 134, which was subjected to Takai conditions to afford iodide 135. According to the modified Stille-Migita protocol developed by Fürstner's research group, ${ }^{124}$ the coupling reaction with vinyl stannane 67 , in the presence of copper thiophene carboxylate (CuTC) and tetrakis(triphenylphosphine)palladium( 0$)$ as catalysts and tetra- $n$-butylammonium diphenylphosphinate $\left(\left[\mathrm{Ph}_{2} \mathrm{PO}_{2}\right]\left[\mathrm{NBu}_{4}\right]\right)$ as the neutral tin species scavenger, ${ }^{125}$ provided Boc-protected PatA 62 in $84 \%$ yield. Compared to the analogous Stille reactions performed in previous Romo's and Pattenden's total synthesis, ${ }^{82,118}$ the yield was improved considerably. Although Romo reported that attempts to cleave the -NHBoc group under acidic conditions resulted in decomposition, ${ }^{84}$ Fürstner and Zhuo reinvestigated this attractive possibility. Interestingly, they found the use of $\mathrm{HCl}$ in dioxane and ethyl acetate could cleave the Boc-protecting group cleanly without 
destroying this sensitive material and PatA (22) was obtained in reproducibly good yields.

This synthetic route provided PatA with 24 steps in the longest linear sequence from starting material dibromothiazole $\mathbf{1 0 2}$ and an overall yield of 5.3\%, which is much higher than the yields reported by Romo (2.5\%) and Pattenden (0.19\%).

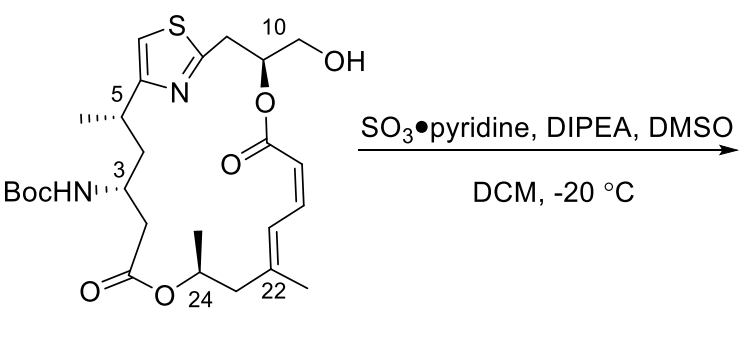

132<smiles>CC(C=O)=CC(Cc1nc([C@H](C)C[C@@H](CC(=O)OC(C)C)NC(=O)OCc2ccccc2)cs1)OC(=O)/C=C\C=C(/C)CC(C)C</smiles>

134

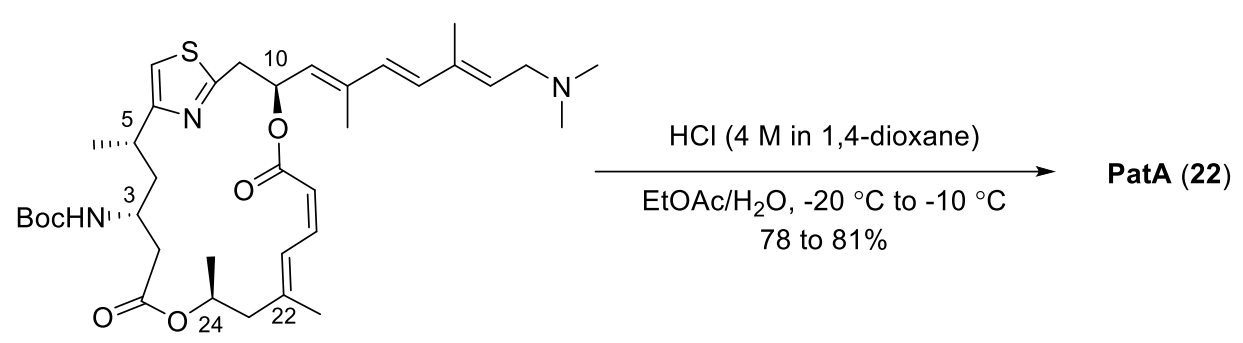

62

Scheme 1.17 Attachment of side chain fragments and deprotection of -NHBoc group in Fürstner and Zhuo's total synthesis.

\subsubsection{Comparison of Syntheses}

The common feature in Romo's, Pattenden's and Fürstner's strategies is that the bislactone macrocycle core is constructed prior to the attachment of the conjugated side chain. To form the macrocycle core, Romo used an intramolecular alcoholysis of the $\beta$ lactam intermediate with a 59-68\% yield (vide supra, Scheme 1.5), Pattenden employed a Stille coupling with a similar yield (65\%) (vide supra, Scheme 1.10), and Fürstner obtained a better yield ( $82 \%$ ) using modified Mukaiyama macrolactonisation conditions 
(vide supra, Scheme 1.16). In terms of the addition of the conjugated side chain, Pattenden and Fürstner both adopted a more linear reaction sequence, which involves the connection of smaller fragments through a combination of Wittig olefination, Takai reaction and Stille coupling (vide supra, Scheme 1.11 and Scheme 1.17). However, Fürstner obtained an improved yield due to the use of advanced Stille coupling conditions. To prepare the isomerisation-prone Z,E-dienoate motif, Romo utilised Sonogashira conditions to couple an $E$-vinyl iodide and an alkyne to form an enyne, the triple carboncarbon bond of which was reduced by Lindlar catalyst after the formation of the macrocycle to afford the Z,E-dienoate (vide supra, Scheme 1.11 and Scheme 1.17); Pattenden employed a Stille coupling between a $Z$-configured vinyl stannane and an $E$ configured vinyl iodide (vide supra, Scheme 1.10); and Fürstner used a 2-pyrone as the surrogate, which was unfolded under iron-catalysed ring-opening/cross-coupling conditions to provide the desired Z,E-dienoate (vide supra, Scheme 1.16).

PatA contains four chiral centres at C3, C5, C10 and C24, for which Romo and coworkers used auxiliary-based asymmetric aldol reactions to obtain chiral centres at $\mathrm{C} 3$ and $\mathrm{C10}$, an asymmetric hydrogenation in the presence of a chiral ligand to obtain the chiral centre at C24 and an auxiliary-based asymmetric methylation to introduce the desired stereochemistry at C5 (Figure 1.20). Fürstner and Zhuo utilised metal-catalysed asymmetric reactions with the involvement of chiral ligands to obtain three of these chiral centres (at C3, C5, and C10) and incorporated a commercially available chiral starting material to obtain the chiral centre at C24 (Figure 1.20). In contrast, Pattenden's group used the chiral pool strategy to introduce the stereochemistries at C5, C10, and C24, and an auxiliary-based aldol-like asymmetric reaction to obtain the desired stereocentre at C3 (Figure 1.20). 


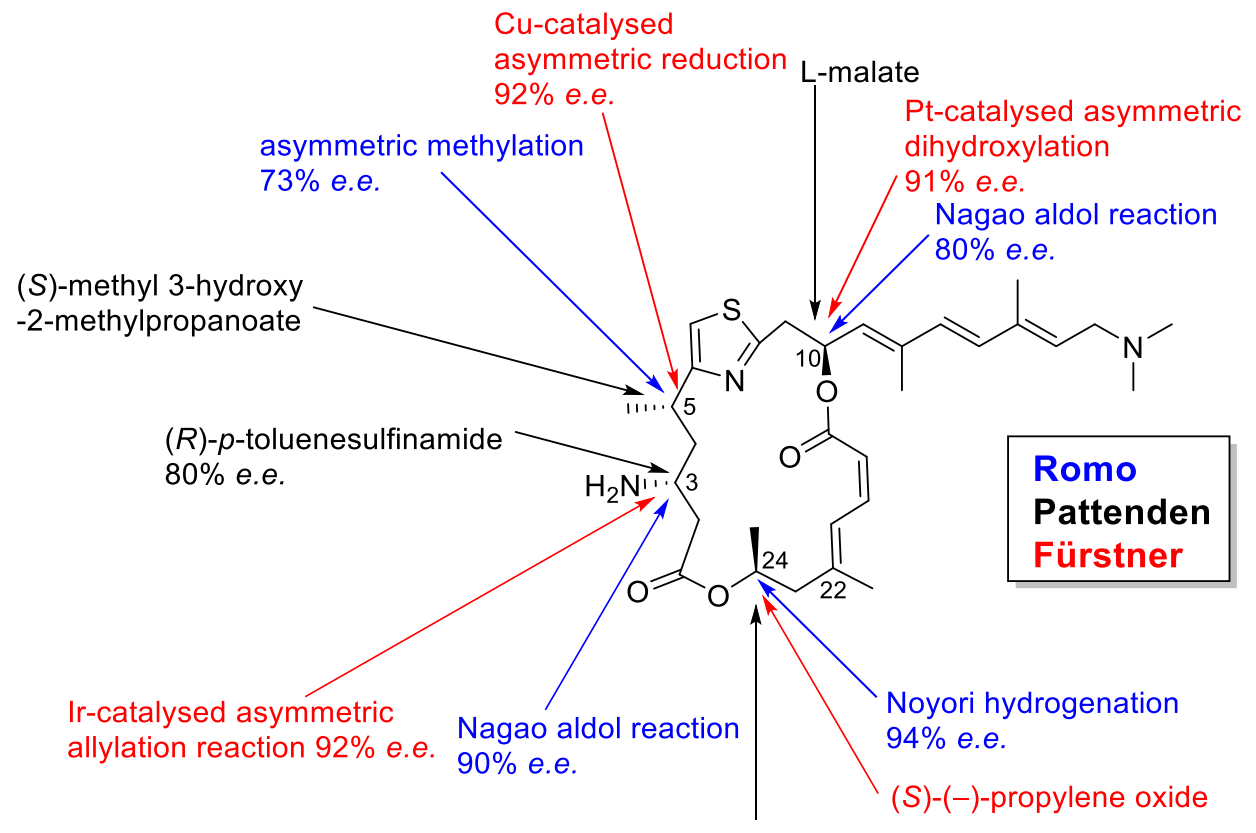

(S)-(-)-propylene oxide

Figure 1.20 Comparison of the introduction of stereochemistries in Romo's, Pattenden and Fürstner's total synthesis of PatA, showing obtained enantiomeric excess (e.e.).

\subsubsection{Pateamine A Analogues Synthesis}

\subsubsection{Romo's Synthesis of DMDA PatA Analogue}

Based on the structural analysis and preliminary molecular modelling studies of PatA, Romo hypothesised that PatA is comprised of two structural domains, a more flexible 'scaffolding domain' (C1 - C5) responsible for defining the conformation of the macrocycle, and the rigid highly unsaturated 'binding domain' (C6 - C24) crucial for protein binding (Figure 1.21). ${ }^{126}$ This hypothesis was also bolstered by Liu and coworkers' findings that the activity of Boc-protected PatA was only $5-10$ times lower than that of PatA in human mixed lymphocyte reaction. ${ }^{82}$ Accordingly, it was supposed that the C3-amino group, as well as the C5-methyl group, might be omitted without significant loss of activity. To provide more evidence for this hypothesis, Romo's laboratory used a similar strategy to that used in their first total synthesis of PatA and synthesised the simplified derivative des-methyl, des-amino PatA (DMDA PatA) 136 (Figure 1.21), which is devoid of the C3-amino group and C5-methyl group. 


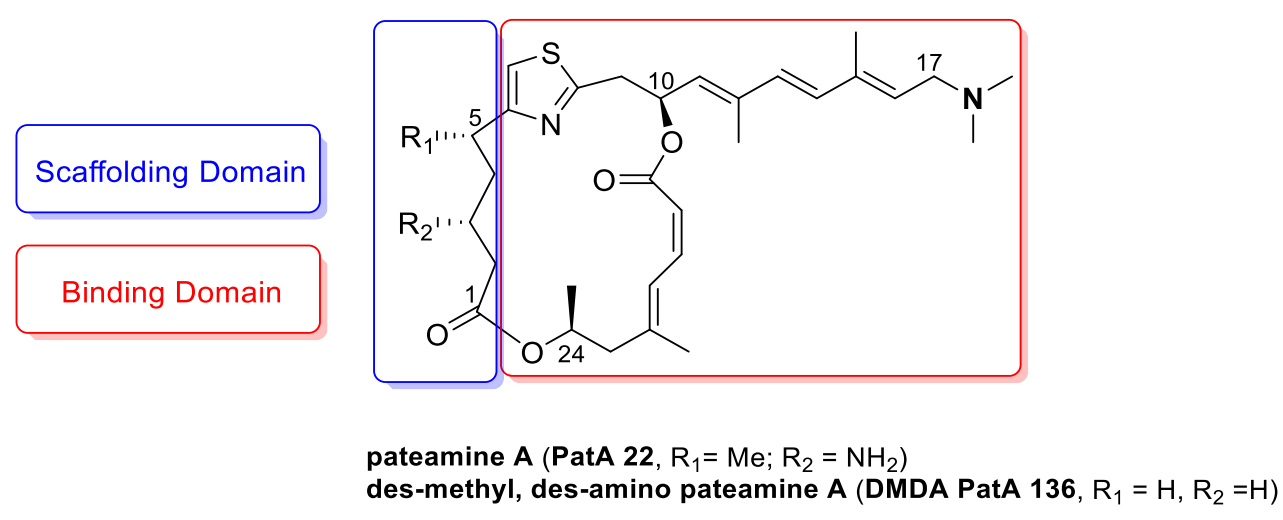

Figure 1.21 Structures of PatA (22) and DMDA PatA (42) showing hypothesised 'scaffolding domain'(blue box) and 'binding domain' (red box). ${ }^{127}$

Comparing to Romo and co-workers' synthetic strategy for PatA (Figure 1.14), the major two modifications in their strategy for DMDA PatA are firstly in the preparation of $\mathrm{C} 1$ C7 fragment and secondly in the macrolactonisation step, where a Yamaguchi esterification was applied instead of the $\beta$-lactam mediated macrolactonisation (Figure $1.22) .{ }^{126}$

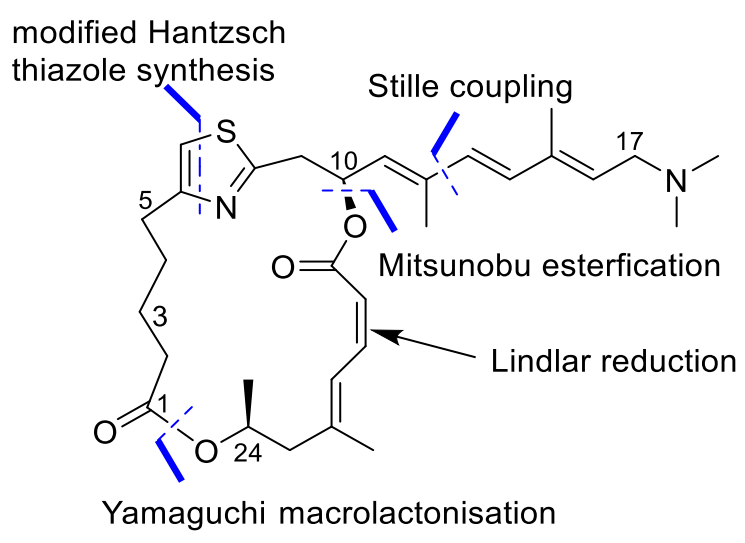

Figure 1.22 Key connections and features in Romo's synthetic strategy for DMDA

$$
\text { PatA. }{ }^{126}
$$

As shown in Scheme 1.18, the synthesis of DMDA PatA (136) started from esterification of commercially available 6-oxoheptanoic acid 137 with commercially available 2,2,2trichloroethanol, which served as the $\mathrm{C} 1-\mathrm{C} 7$ fragment. The resulting trichloroethyl ester 138 was regioselectively brominated to give $\alpha$-bromoketone 139. Modified Hantzsch thiazole coupling between 139 and thioamide 31, the same building block used in Romo's synthesis of PatA (Scheme 1.1), provided thiazole 141 via the formation of thiazoline intermediate 140. Subsequent desilylation gave secondary alcohol 142 with $R$ 
configuration at the $\mathrm{C} 10$ position (PatA numbering). Mitsunobu coupling with triisopropylsilyl (TIPS)-protected enynoic acid 143, which could be prepared using a similar sequence to that for acid $\mathbf{2 5}$ (Scheme 1.4), inverted the undesired $R$ configuration to the desired $S$ configuration and gave compound 144 . Subsequent desilylation provided alcohol 145.
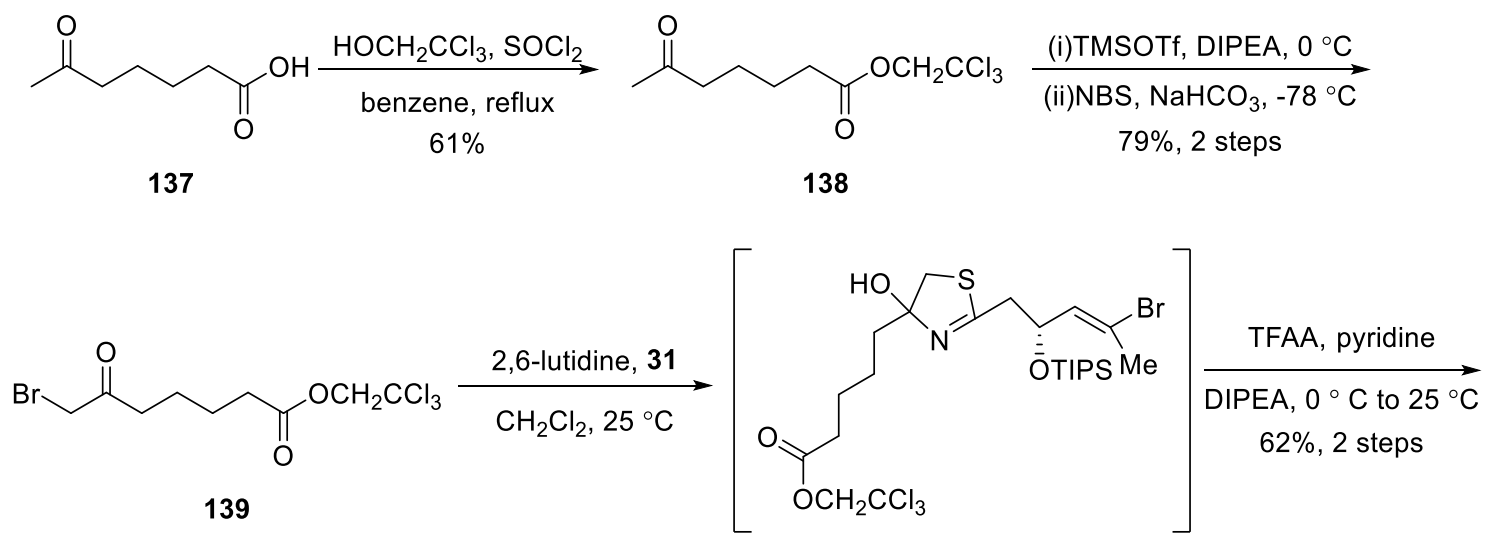

140

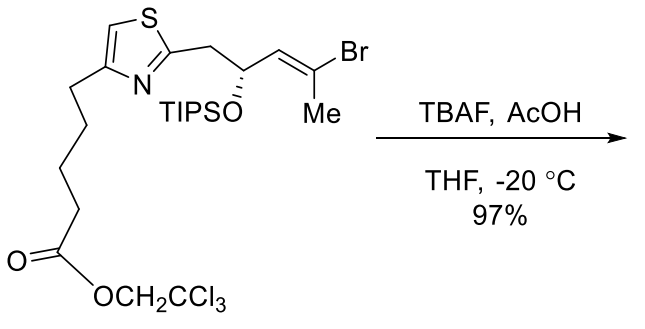

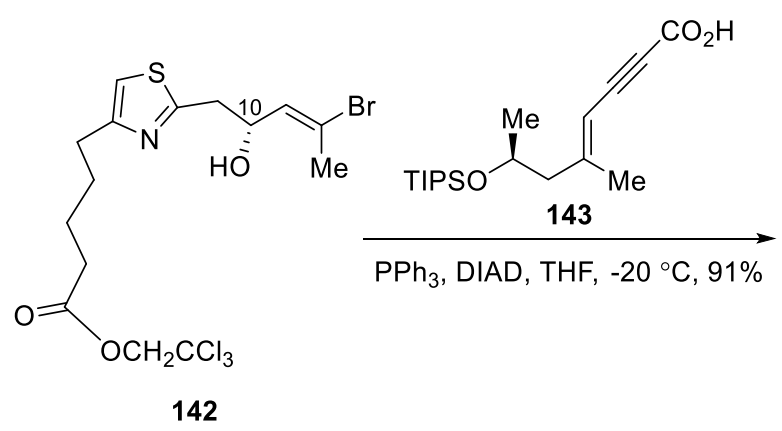

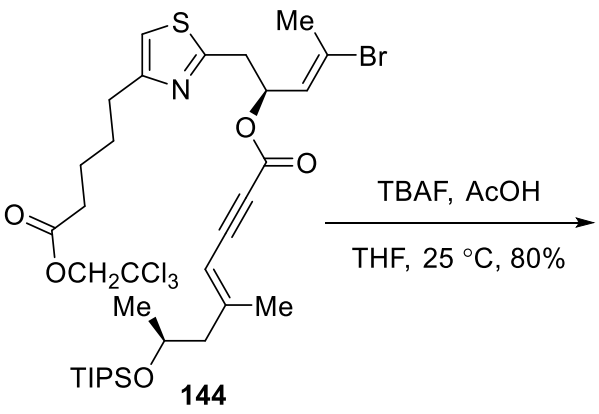

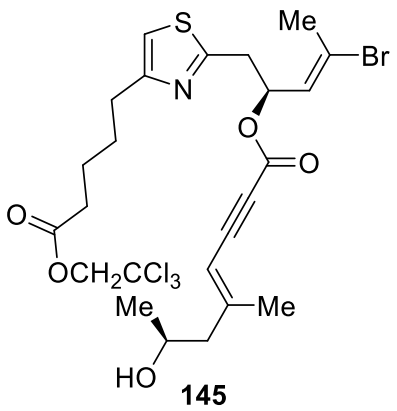

Scheme 1.18 Preparation of alcohol 145 in the synthesis of DMDA PatA.

Deprotection of the trichloroethyl ester provided compound 146, which set the stage for the lactonisation step (Scheme 1.19). Yamaguchi macrolactonisation followed by treatment with Lindlar's catalyst afforded the $Z, E$-dienoate 148. Stille coupling with 
dienyl stannane 24 delivered DMDA PatA (136).
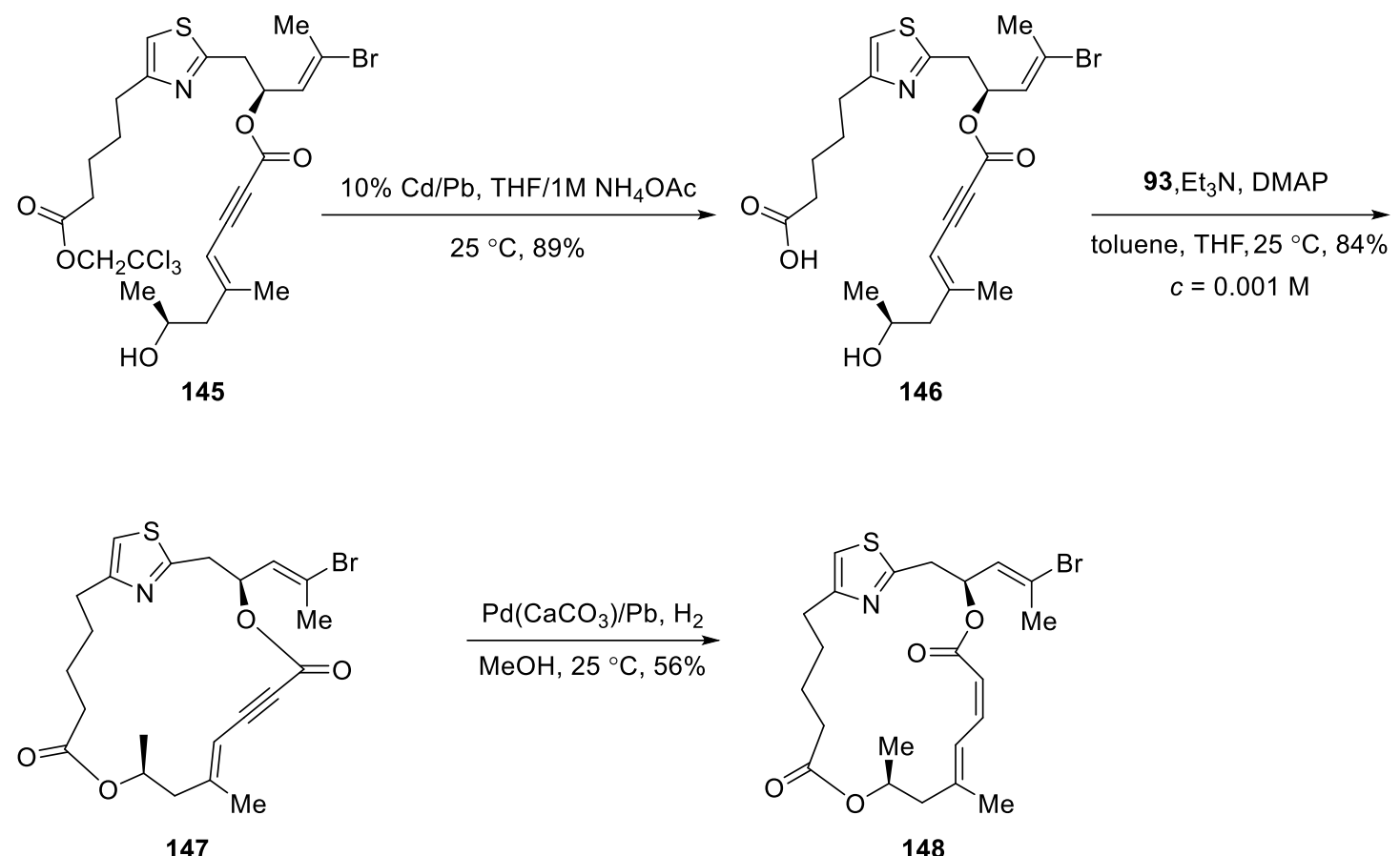

148
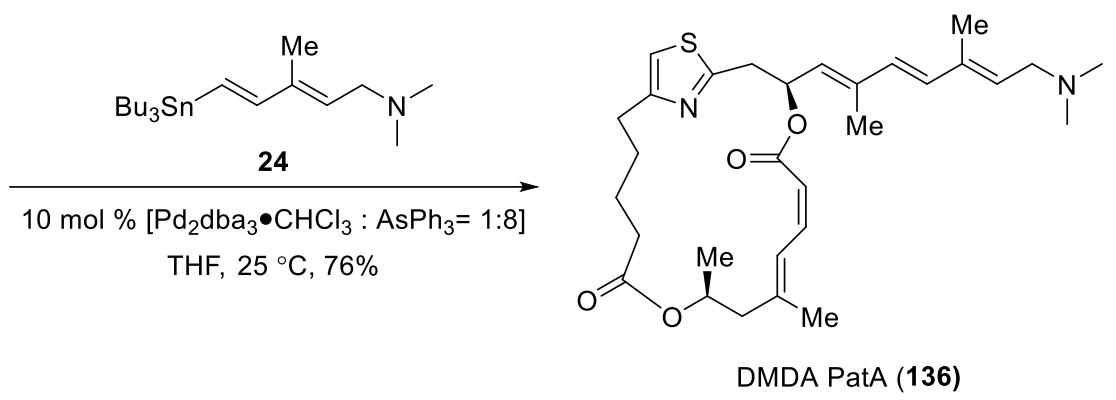

Scheme 1.19 Preparation of DMDA PatA.

This synthetic route for DMDA PatA is only 14 steps starting from aldehyde 25 ( $c f .24$ steps for Romo's synthesis of PatA) and the overall yield is $13 \%$ ( $c f .2 .5 \%$ for Romo's synthesis of PatA).

Apart from being chemically more accessible, DMDA PatA was also found to display similar potency relative to PatA in its ability to inhibit cell proliferation across a number of cancer lines, ${ }^{127}$ which provides direct evidence supporting the proposed scaffolding domain and binding domain hypothesis (Figure 1.21). In addition, it displays better chemical stability than PatA. ${ }^{126}$ Therefore, it is necessary to develop more efficient synthesis for this promising anticancer agent. 


\subsubsection{Fürstner and Zhuo's Synthesis of DMDA PatA}

In similar fashion to Fürstner and Zhuo's total synthesis of PatA, their synthesis of DMDA PatA utilised the diol fragment 107 and 2-pyrone fragment 101 (Scheme 1.20), the same building blocks derived from dibromothiazole 102 and (S)-(-)-propylene oxide 86, respectively (vide supra, Schemes 1.12 and 1.15 ). ${ }^{128} \mathrm{As}$ the amino group at $\mathrm{C} 3$ and the methyl group at $\mathrm{C} 5$ are excised in DMDA PatA, the commercially available 4-pentenoic acid 150 was chosen to serve as the $\mathrm{C} 1$ - C5 fragment. Yamaguchi esterification of 2pyrone 101 with acid 150 provided ester $\mathbf{1 5 1}$. Hydroboration of the terminal double bond of ester $\mathbf{1 5 1}$ gave the trialkylborane intermediate, which was coupled with mono-silylated thiazole $\mathbf{1 4 9}$ to afford compound 152. Interestingly, although a free hydroxyl group is present in compound 152, iron-catalysed ring-opening/cross-coupling reaction with excess methyl Grignard reagent proceeded well. The 2-pyrone ring was unfolded cleanly and the desired methylated Z,E-dienoic acid $\mathbf{1 5 3}$ was obtained in high isomeric purity. Modified Mukaiyama macrolactonisation, desilylation, and subsequent Parikh-Doering oxidation gave aldehyde 155, which was found to be rather sensitive and prone to epimerisation. Therefore, aldehyde 155 production was telescoped with Wittig olefination to provide homologated aldehyde 156. Treatment with Takai conditions and subsequent modified Stille-Migita coupling ${ }^{124}$ with fragment $\mathbf{6 7}$ afforded the complete DMDA PatA. Overall, this synthetic route for DMDA PatA is only 12 steps in the longest sequence ( $c f .24$ steps for Fürstner's synthesis of PatA) and the overall yield is 18\% ( $c f$. 5.3\% for Fürstner's synthesis of PatA). 


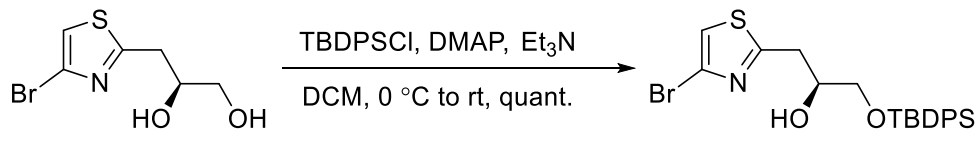

107

149

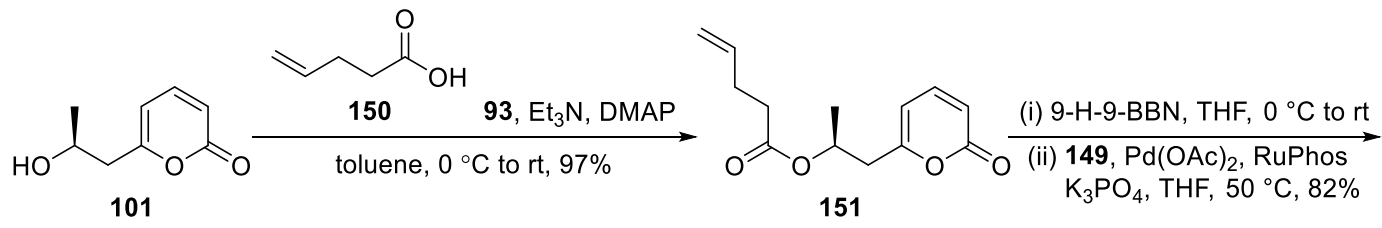

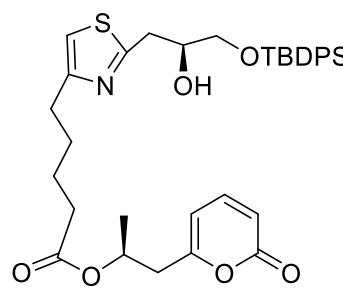

152<smiles>C/C(=C/C=C\C(=O)OC(C[O-])Cc1nc(CCCCCC(=O)OC(C)C)cs1)C[C@@H](C)O</smiles>

154

\section{$\underset{\mathrm{Et}_{2} \mathrm{O} / \text { cyclopentyl methyl ether, }-30^{\circ} \mathrm{C}}{\mathrm{MeMgBr}, \mathrm{Fe}(\mathrm{acac})_{3}}$}

151

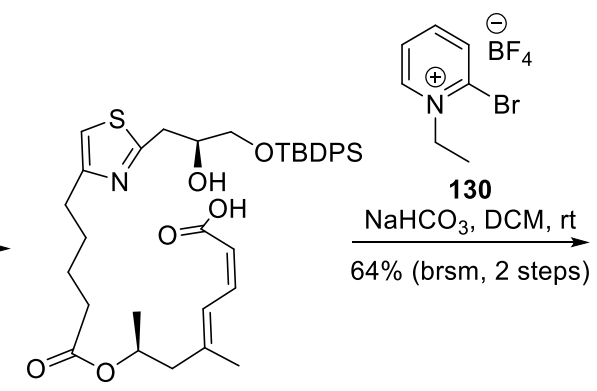

153

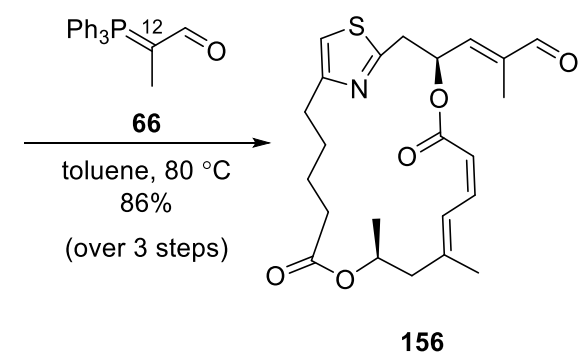

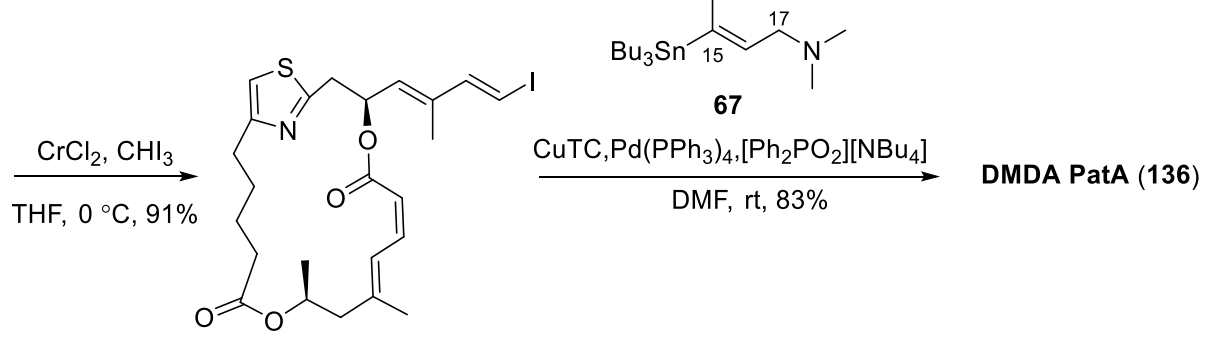

157

Scheme 1.20 Fürstner and Zhuo's synthesis of DMDA PatA. ${ }^{128}$

\subsubsection{Romo's Analogue Synthesis Based on PatA}

To explore the importance of the side chain in the identified 'binding domain' of PatA, Romo and co-workers synthesised analogues with minor structural variations on the side chain. To investigate whether or not the dienoate motif is essential for the bioactivity, they also synthesised enynoate analogues, in which the Z-olefin at C19 - C20 (PatA numbering) was replaced with a carbon-carbon triple bond. As C3-Boc protected PatA 62 was found to only have a 3-4 fold reduction in activity and to be more stable when 
compared to the parent natural product, ${ }^{82}$ the Boc-protecting groups were retained in all these derivatives. ${ }^{126}$

As shown in Scheme 1.21, synthesis of enynoate analogues $\mathbf{1 5 8}$ - 161 was completed through Stille coupling between the bis-lactone macrocyclic core $\mathbf{6 0}$ and a series of vinylstannanes, respectively. Analogues 162 - 164 with variant side chains were synthesised in a two-step sequence comprising Lindlar reduction of macrocycle $\mathbf{6 0}$ followed by attachment of a series of vinylstannanes to bromoalkene $\mathbf{6 1}$ through Stille coupling.

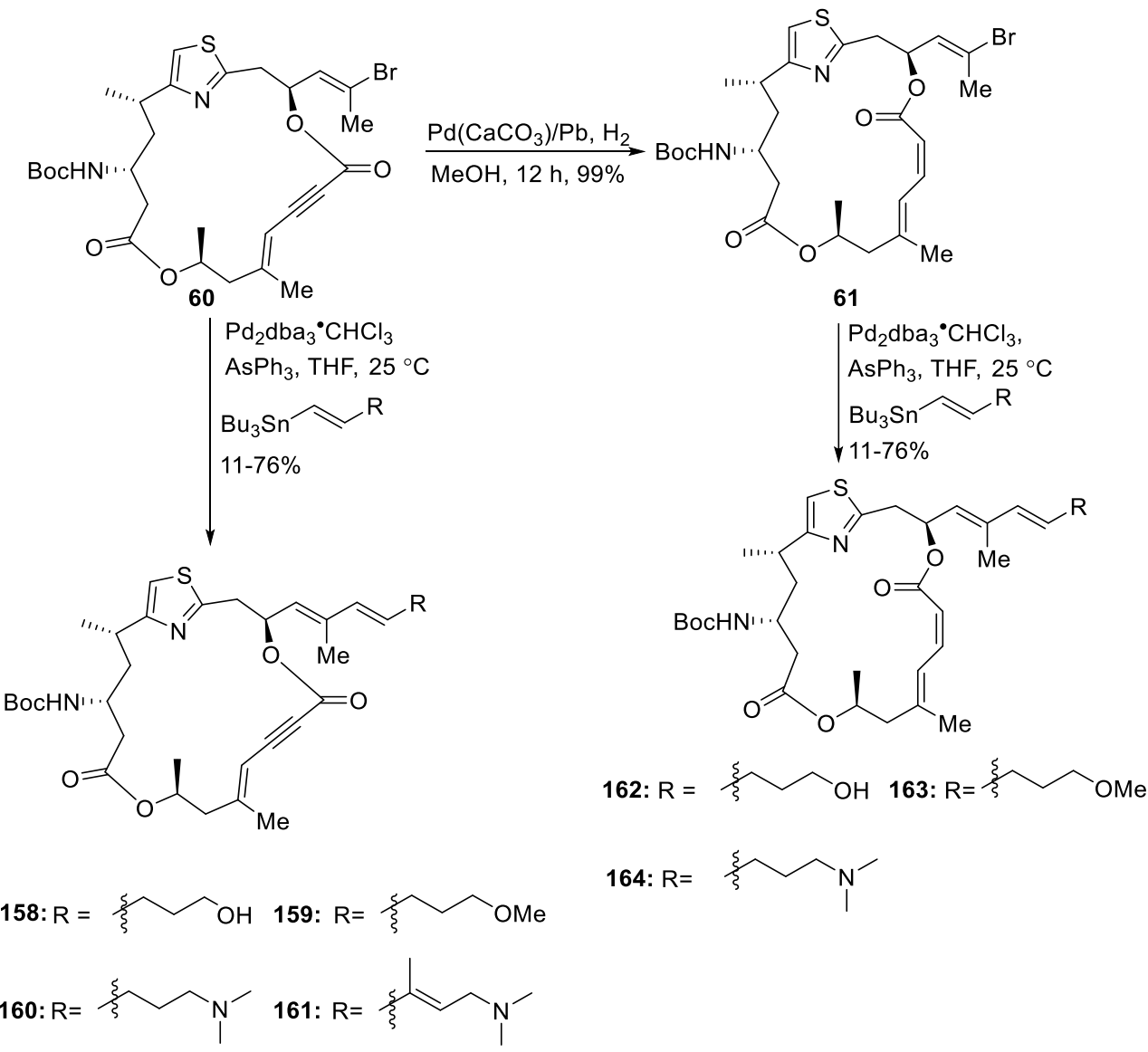

Scheme 1.21 Preparation of analogues 158 - 164 of PatA.

\subsubsection{Romo's Analogue Synthesis Based on DMDA PatA}

Given that the simplified derivative DMDA PatA is almost equipotent to Pat $\mathrm{A}$ and is simpler to synthesise, a series of analogues based on DMDA PatA were designed and synthesised in order to further examine the importance of the side chain and the diene system in the identified 'binding domain' of DMDA PatA. ${ }^{126,127}$ 
As shown in Scheme 1.22, the enyne macrocycle core 147 was reduced by Lindlar's catalyst under particular conditions to provide a mixture of $Z, E$-dienoate 148 and $E, E$ dienoate $165(Z, E: E, E=5: 1)$ that were separable by silica gel chromatography. To synthesise the analogue with an $E, E$-configured dienoate system, bromoalkene 165 was coupled with the vinylstannane 24 to provide analogue 166. Analogues 167 - 172 with structural variations in the side chain were synthesised through Stille coupling with the $Z$, E-dienoate macrocycle $\mathbf{1 4 8}$ and a series of vinyl stannanes. ${ }^{127}$

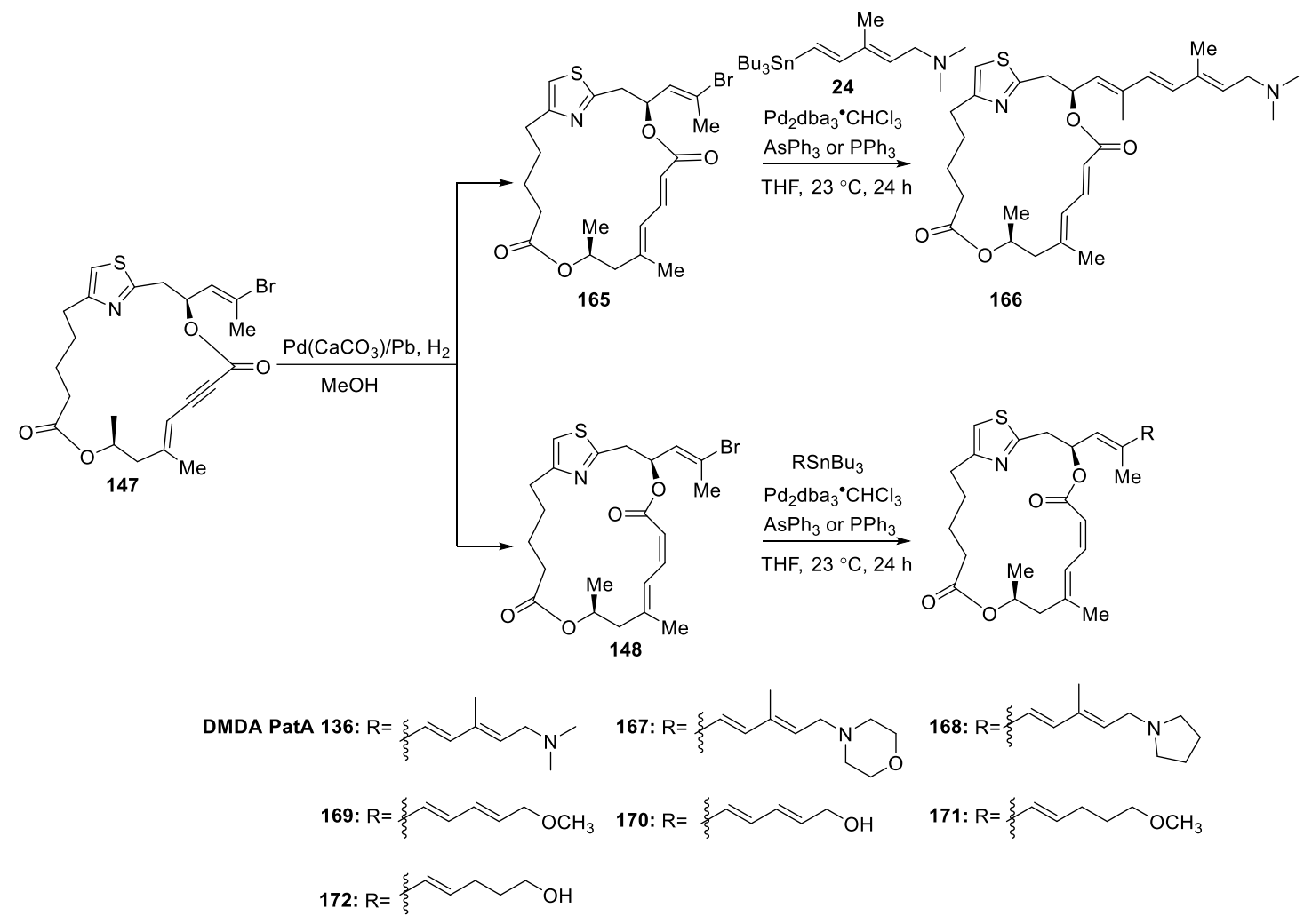

Scheme 1.22 Preparation of analogues 166 - 172 based on DMDA PatA.

Enynoate analogues of DMDA PatA 173 - 179 were prepared through the Stille coupling reaction between the enyne macrocycle 147 and a series of vinyl stannanes (Scheme 1.23). 

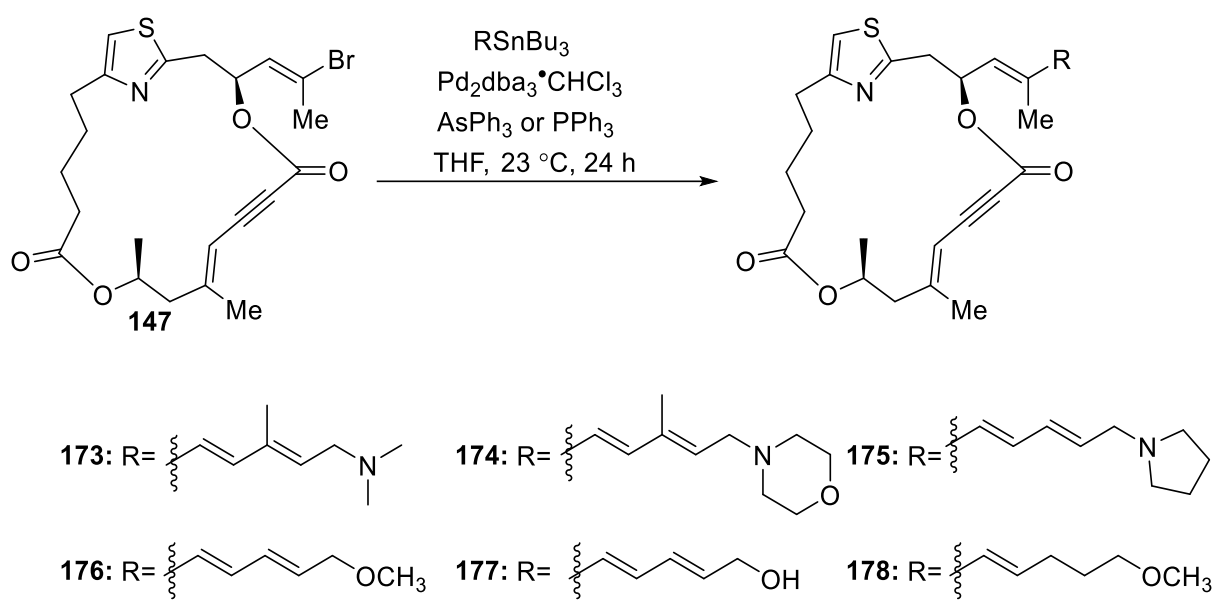

179: $\mathrm{R}=$

Scheme 1.23 Preparation of enynoate analogues 173 - 179 based on DMDA PatA.

\subsection{Structure-Activity Relationship Studies of PatA and Analogues}

\subsubsection{Studies on Immunosuppressive Activity of PatA Analogues}

Preliminary studies by Dr. Glynn Faircloth from PharmaMar found that PatA exerted potent inhibitory effects on both murine and human mixed lymphocyte reactions, suggesting it has immunosuppressive activity. ${ }^{82}$ Romo and co-workers found that PatA inhibited T-cell receptor-mediated IL-2 production, which led to inhibition in T cell activation. $^{84}$

In order to examine the effects of structural modifications on the immunosuppressive activity, analogues 158 - 164, two C3-acylated derivatives 180 and 181, and DMDA PatA 136 were tested in the same IL-2 gene assay as PatA. ${ }^{126}$ Although analogues listed in Table 1.1 were less active than PatA, DMDA PatA 136 (not listed in Table 1.1) exhibited greater potency $\left(\mathrm{IC}_{50} 0.8 \pm 0.3 \mathrm{nM}\right)$ when compared to PatA $22\left(\mathrm{IC}_{50} 4.0 \pm 0.94 \mathrm{nM}\right)$. Analogue 164 was less active than PatA 22, indicating the unsaturation or the methyl on the side chain is necessary for the potency. Enyne analogues with the partially saturated side chain 160 or the natural conjugated side chain 161 were not active. Conversely, enyne derivatives (158 and 159) with oxygen terminus in the side chain were found to retain a degree of activity in the IL-2 reporter gene assay (340 nM and $55 \mathrm{nM}$, respectively). This inconsistency may suggest that the oxygenated side chain has compensatory effects for the conformational change of the macrocycle caused by the 
introduction of the enyne motif. Another possibility is that $\mathbf{1 5 8}$ and $\mathbf{1 5 9}$ may display immunosuppressive activity through binding to a different protein target. ${ }^{126}$

The immune system is a surveillance mechanism that maintains homeostasis outside cells while NMD is a counterpart mechanism inside the cells. These two mechanisms are closely related. By degrading aberrant immunoglobulin (Ig) and T-cell receptor (TCR) transcripts generated from non-productively rearranged Ig and TCR genes, NMD largely decreases the production of dysfunctional or even deleterious molecules in lymphocytes, which is crucial for the survival of B and T lymphocytes. ${ }^{129}$ Therefore, inhibition of NMD would impact the immune system indirectly. Given that eIF4AIII plays a crucial role in NMD (refer to Section 1.3), the conformational change of eIF4AIII generated from binding with PatA would affect NMD and possibly cause immunosuppression. We proposed that these inhibitory activities in the IL-2 gene assay reported by Romo and coworkers may indirectly reveal the real activities of PatA and its analogues in inhibition of NMD. 


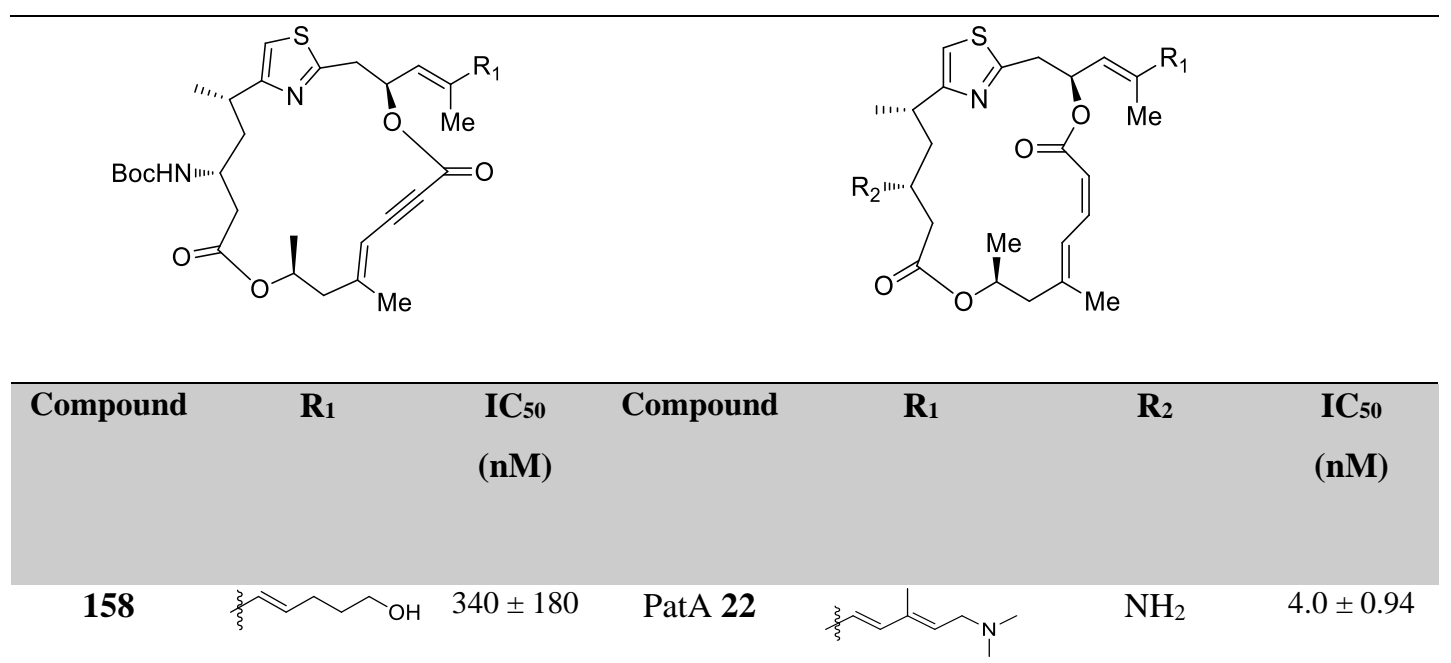

159
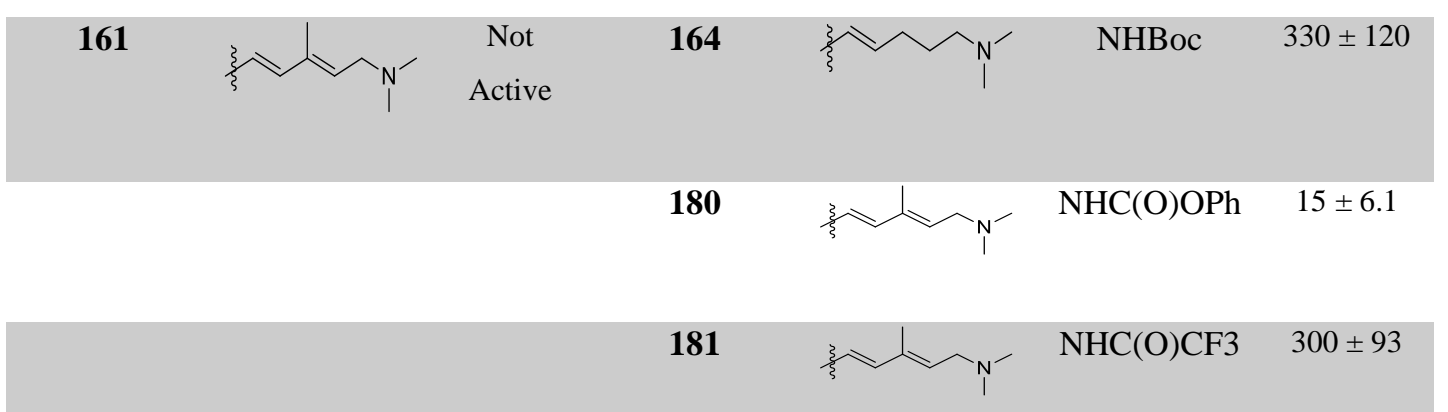

Table 1.1 Comparison of $\mathrm{IC}_{50}$ values for analogues of PatA from IL-2 reporter assay. ${ }^{126}$

\subsubsection{Studies on Anti-proliferative Activity of PatA Analogues}

Intriguingly, Romo and co-workers found that the structurally simplified analogue DMDA PatA was only slightly less active than PatA in all human cell lines tested for in vitro cytotoxicity (Hela (a cervical carcinoma cell line), RKO (a poorly differentiated colon carcinoma cell line), and Jurkat immortalised T cells)(Table 1.2). ${ }^{127}$ DMDA PatA exhibited similar potent anti-proliferative activity in the immortalised human keratinocyte cell line $\mathrm{HaCaT}$ and in primary proliferating bovine aortic endothelial (BAEC) cell line (Table 1.2). ${ }^{127}$ DMDA PatA was also found to have the same mechanism of action as the parental PatA. ${ }^{127}$ These results provided convincing evidence for the 'scaffolding' and 'binding' domain hypothesis (Figure 1.21). DMDA PatA is considered to be a promising natural product-based anticancer agent that requires further research for drug development. 


\begin{tabular}{|c|c|c|c|c|c|}
\hline \multirow[t]{2}{*}{ Compound } & \multicolumn{5}{|c|}{$\mathrm{IC}_{50}(\mathrm{nM})$} \\
\hline & HeLa & RKO & Jurkat T & HaCaT & BAEC \\
\hline PatA 22 & $0.72 \pm 0.88$ & $0.38 \pm 0.02$ & $0.60 \pm 0.02$ & $0.42 \pm 0.03$ & $0.5 \pm 0.04$ \\
\hline DMDA PatA 136 & $4.5 \pm 0.4$ & $1.2 \pm 0.05$ & $3.0 \pm 0.1$ & $0.9 \pm 0.1$ & $1.97 \pm 0.1$ \\
\hline
\end{tabular}

Table 1.2 IC $_{50}$ values for PatA 22 and DMDA PatA 42 from cell proliferation assays. ${ }^{127}$

Intensive studies on DMDA PatA showed that it exhibited little differential growth inhibitory activity in a panel of 32 human cancer cell lines including both solid tumours and hematologic malignancies. ${ }^{130}$ Surprisingly, DMDA PatA exerted anticancer effects in two different melanoma xenograft models (MDA-MB-435 and LOX), resulting in significant tumour regression, whereas it did not show a significant effect in either MiaPaca-2 pancreatic cancer or an HT-29 colon cancer xenograft model. ${ }^{130}$ Future studies are required to figure out why this analogue is indiscriminatively potent amongst a broad range of human cancer cell lines in vitro but is selectively effective in vivo. What is also encouraging is that DMDA PatA was found to retain its potency against P-glycoprotein (PgP)-overexpressing human uterine sarcoma cells, suggesting that it is insensitive to PgP-mediated drug efflux. ${ }^{130}$ More intriguingly, DMDA PatA at low nanomolar concentration has no cell-killing ability for quiescent human fibroblasts, indicating that the observed inhibitory activity against human cell growth results from inhibited proliferative processes rather than indiscriminative proliferation-independent cytotoxicity. ${ }^{130}$ Although the mechanism of action of DMDA PatA was considered to be the same as its parental counterpart, ${ }^{127}$ this simplified analogue was found to inhibit $S$ phase DNA synthesis in some cell lines, although giving no specific cell cycle blockade in others. Where observed, S-phase blockade is proposed to be through direct inhibition of DNA polymerases, which provides an additional impetus for further research on the mechanism of action of DMDA PatA. ${ }^{130}$

On the basis of the established research described above, DMDA PatA is considered to be an intriguing anticancer agent worthy of further structure-activity relationship (SAR) studies. 
To find out more information about DMDA PatA's structure-activity relationship, the side chain truncated analogues 147 and 148 and analogues 167 - 179 based on DMDA PatA were tested by Romo' research group for cell proliferation activity in two cell lines (HeLa and Jurkat T). ${ }^{127}$

As shown in Table 1.3, the brominated derivative 148 displays no activity for HeLa cells and Jurkat $\mathrm{T}$ cells even at the highest concentration tested, indicating that the trienyl amine side chain plays a crucial role in binding with the protein target. ${ }^{127}$ When the terminal $N, N$-dimethylamine group is replaced with other tertiary amines - a 6-membered morpholine ring (analogue 167) or a 5-membered pyrrolidine ring (analogue 168) - it is noteworthy that the morpholine substitution shows a greater loss of activity than the pyrrolidine substitution (400-fold loss for 167 in HeLa cells versus 2-fold loss for 168). The conjugate acid of pyrrolidine has a similar $\mathrm{pKa}$ value of approximately 11 to that of $N, N$-dimethylamine whereas the morpholine ring is significantly less basic, with a $\mathrm{pKa}$ value of 8.36 (conjugate acid). In addition, replacement of the $N, N$-dimethyl tertiary amine with ether and alcohol functionalities resulted in significant losses of activity (analogues 169 - 172). These results suggest that the basic terminal in the side chain is also important for the high potency. It is of interest that analogue $\mathbf{1 6 7}$ displays differential degrees of loss of activity in HeLa cells and Jurkat T cells, suggesting that replacement of the $N, N$-dimethyl tertiary amine with different basic substitutions may increase selectivity between different proliferating cell lines. ${ }^{127}$ Moreover, the different activity of $\mathbf{1 7 0}$ and $\mathbf{1 7 2}$ is due to their only structural difference - the loss of unsaturation at C15 and C16. Therefore, the rigidity generated from double bonds of the side chain may also be crucial for the potency. ${ }^{127}$

Analogues (173 - 179) with enyne structures demonstrate a drastic decrease in activity (Table 1.3). The reason for the loss of activity is likely to be that the enyne replacement results in a significant change in the preferred conformations of the macrocycle, which is critical for binding with eIF4A. ${ }^{127}$ Not surprisingly, E,E-dienoate analogue 166 (Scheme 1.22) with the alteration at the geometry of the olefin at C19-C20 in the dienoate unit, which was proposed to act as a Michael acceptor for biological nucleophiles, ${ }^{86,110,131}$ was found to be inactive in the tested cell lines. ${ }^{127}$ 


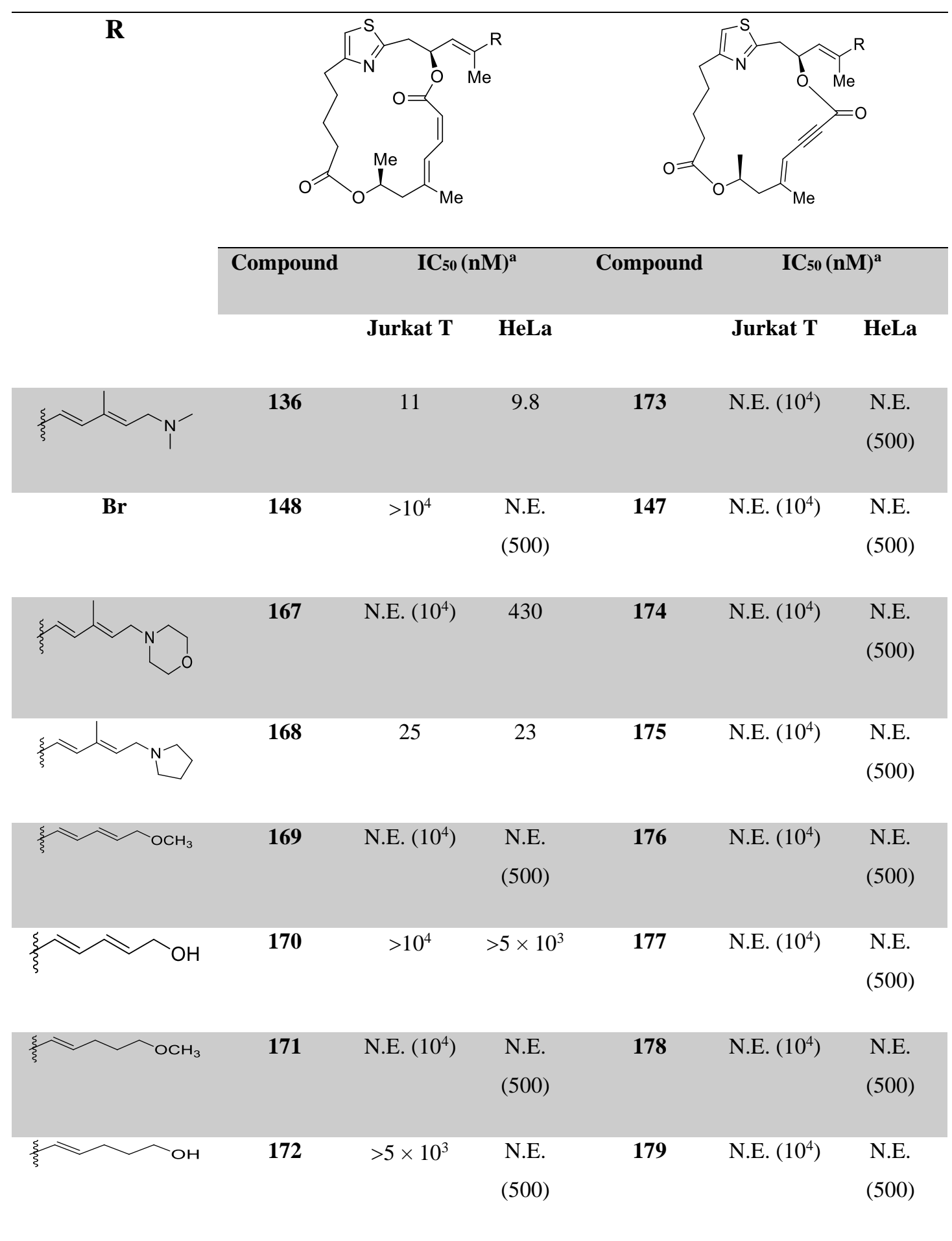

a'N.E.' means that no effect was observed up to the concentration tested shown in parentheses.

Table 1.3 Comparison of $\mathrm{IC}_{50}$ values for analogues of DMDA PatA from two different cell proliferation assays. ${ }^{127}$ 


\subsubsection{Summary}

In brief, based on the previous structure-activity studies, ${ }^{126,127}$ the crucial structures residing in the 'binding domain' for the potency of PatA (22)/DMDA PatA (136) include the basic terminus and the unsaturation in the side chain, the conformation of the macrocycle and the Z,E-dienoate motif. The roles of the heterocycle, the methyl groups on the side chain, and the methyl groups on the macrocycle core (at C22 and C24 position, PatA numbering) still need to be further examined.

Additionally, considering that DMDA PatA is slightly less potent than PatA in the antiproliferation assay but more potent than PatA in the IL-2 reporter gene assay, ${ }^{127}$ it is conceivable that the elimination of the C3 methyl and C5 amino may enhance the immunosuppressive activity while slightly weakening the anti-proliferative activity. When comparing the activity data of all analogues synthesised by Romo's group, an obvious discrepancy was that analogues $\mathbf{1 5 8}$ and $\mathbf{1 5 9}$ still demonstrated some activity in the IL-2 reporter assay (Table 1.1), ${ }^{126}$ but the derivatives 179 and 178 with the same enyne structure and the identical side chains did not display any significant anti-proliferative activity in tested cell lines (Table 1.3). ${ }^{127}$ Romo and co-workers proposed that one possibility is that PatA and its analogues only display cell growth inhibition at low concentrations but possess both IL-2 production inhibition activity and antiproliferative activity at higher concentrations. After eliminating anti-proliferative activity through structural modification, IL-2 transcriptional inhibition activity would become the primary biological activity. ${ }^{127}$ This led to the idea that it is possible to design an analogue of PatA that could regulate the immune system without any significant impact on cell proliferation, and vice versa.

In view of the interaction between NMD and the immune system, it is favourable to explore the possibility of synthesising an analogue that selectively binds to eIF4AIII over eIF4AI/II. This analogue would impact NMD directly and may act as a probe to further explore the NMD mechanism based on the EJC model. Furthermore, this may also provide potential regulatory molecules for the immune system and NMD, which may benefit further studies on treatments for autoimmune diseases (e.g., rheumatoid arthritis and lupus) $)^{132}$ and inherited genetic diseases related to NMD (e.g., $\beta$-thalassemia and Marfan syndrome). ${ }^{133}$ To achieve this goal, it is necessary to further explore PatA's mode of binding to different eIF4A isoforms - eIF4AI/II and eIF4AIII. 


\subsection{Cumming's Synthesis of Triazole Analogue}

To examine the role of the heterocycle ring in the binding process of PatA and to probe the possibility of selective binding to either eIF4AI/II or eIF4AIII isoforms, Hemi Cumming, a former Ph.D. student in our research group, designed and synthesised a simplified 1,2,3-triazole-containing analogue 182 (Figure 1.23) based on DMDA PatA. ${ }^{134}$ In this analogue, the thiazole ring embedded within the molecule was replaced with a triazole ring while the key structural features including the size of the macrocycle, the bis-lactone functionalities, the $Z, E$-dienoate, the all $E$-configured triene and the tertiary amine terminus were retained. Under the assumption that losses of methyl substituents on the side chain would not significantly impact target protein binding, the methyl groups at C12 and C15 (PatA numbering) were excised. To further reduce the structural complexity, the methyl groups at $\mathrm{C} 22$ and $\mathrm{C} 24$ (PatA numbering), which were proposed to be not critical for the biological activity, were also omitted. Based on the molecular modelling studies results collected by Cumming, there was no significant change in conformations of DMDA PatA and this simplified triazole analogue 182. ${ }^{135}$ However, calculation of electrostatic potential surface for DMDA PatA 136 and the triazole analogue 182 showed that the replacement of the thiazole ring with a triazole ring could cause significant differences in charge distribution in the macrocycle. ${ }^{135}$

As summarised in Figure 1.23, Cumming's synthesis of analogue 182 involved a JuliaKocienski olefination reaction between a sulfone and an aldehyde to connect the side chain fragment, a base-induced ring-opening reaction of a $\delta$-valerolactone to provide the E,Z-dienoate motif and a copper-catalysed azide-alkyne cycloaddition reaction to construct the triazole ring. Steglich esterification and Yamaguchi macrolactonisation were utilised to construct the macrocycle. The synthesis of the non-methylated side chain fragment from furan will be covered in Chapter 2 and the preparation of the $\alpha, \beta$ unsaturated $\delta$-lactone will be covered in Chapter 3. The remainder of the details of the synthesis will be covered in Chapter 4 . 


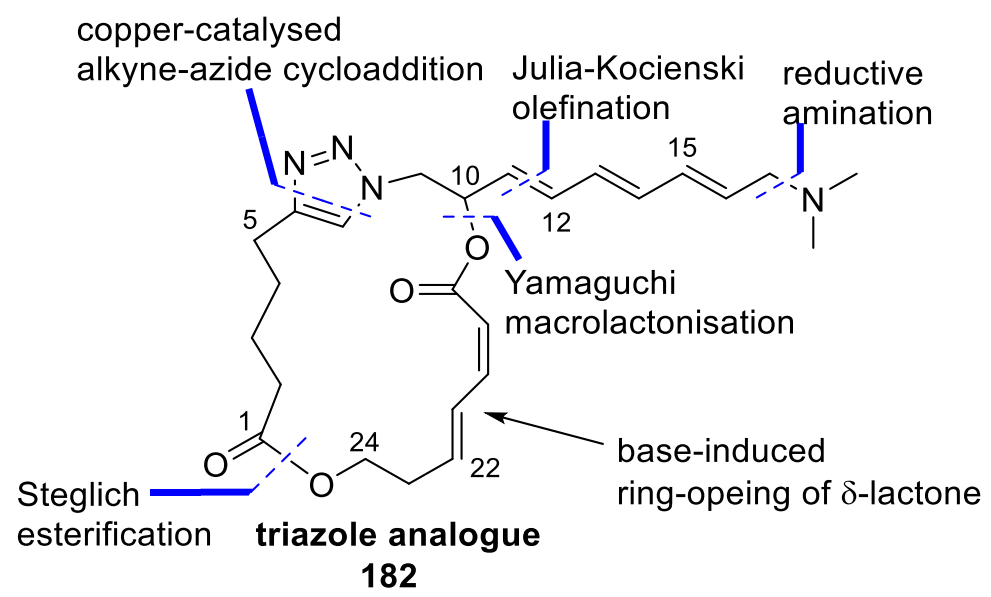

Figure 1.23 Key connections and features in Cumming's synthesis of triazole analogue 182. ${ }^{134}$

This triazole analogue 182 was tested in two cell lines: HL-60 (human acute myeloid leukaemia cells) and 1A9 (human ovarian carcinoma cells). Compared to DMDA PatA with an $\mathrm{IC}_{50}$ value of approximately $1.0 \mathrm{nM}$ in NALM-6 pre-B leukaemia cells, ${ }^{130}$ analogue 182 displayed a drastic reduction in anti-proliferative activity with an $\mathrm{IC}_{50}$ value of $34600 \mathrm{nM}$ and $51100 \mathrm{nM}$ in HL-60 and 1A9 cells respectively (Table 1.4). ${ }^{135}$ It is of note that analogue 182 was tested as a mixture of enantiomers, in which the (10S)enantiomer could be completely inactive. However, this still does not rationalise the significant loss in bioactivity relative to PatA/DMDA PatA. Due to the fact that several changes were made simultaneously in this analogue, namely thiazole replacement and removal of four methyl groups, it is difficult to assess the individual contributions to the reduction in the activity for each of the altered structural features.

\begin{tabular}{ccc}
\hline Compound & \multicolumn{2}{c}{ IC $_{50}(\mathbf{n M})$} \\
\cline { 2 - 3 } & HL-60 & $\mathbf{1 A 9}$ \\
Triazole Analogue $( \pm)-182$ & 34600 & 51100
\end{tabular}

Table 1.4 IC $_{50}$ values for analogue 182 based on MTT assay. ${ }^{135}$ 


\subsection{Research Aim}

The aim of this project is to design and synthesise new simplified PatA analogues as probes to further explore its mode of binding with eIF4A isoforms. There are two main facets of this research project: firstly, to synthesise new analogues of PatA with fewer structural modifications than Cumming's triazole analogue 182 to explore the structureactivity relationship of simplified PatA analogues and so reveal the individual contributions of the structure modifications to the significant reduction in activity; secondly, to examine the scope and efficiency of the novel synthetic methodology and develop new analogues which can be prepared in a more tractable synthetic route than the natural product.

\subsubsection{Analogue Design}

As shown in Figure 1.24, the conjugate acid of 2,5-dimethylthiazole has a $\mathrm{pKa}$ value of $3.91^{136}$ whereas the conjugate acid of 1-methyl-1,2,3-triazole has a significantly lower $\mathrm{pKa}$ value of 1.25. ${ }^{137}$ This means that the basicity of the nitrogen atom in 1,2,3-triazole is lower than the nitrogen atom in thiazole and therefore 1,2,3-triazole is not an easily protonable functional group when compared to thiazole. We considered that the protonation of the nitrogen atom of the thiazole ring residing in PatA is crucial for its efficient binding to eIF4A isoforms. The substitution of the thiazole for the triazole may affect its binding ability to the target protein, and this modification could be the major cause of the significant loss in the activity.
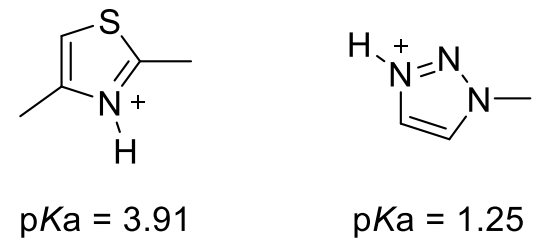

$\mathrm{pKa}=1.25$

Figure 1.24 Comparison of the $\mathrm{pKa}$ values of the conjugate acids of 2,5dimethylthiazole ${ }^{136}$ and 1-methyltriazole. ${ }^{137}$

To provide evidence for this hypothesis, it is necessary to reintroduce the thiazole ring into the analogue scaffold, and therefore, thiazole analogue 183 would be the first target (Figure 1.25) in this project. The comparison of activities of 1,2,3-triazole analogue 182 and thiazole analogue $\mathbf{1 8 3}$ would reveal whether the thiazole ring is the key requirement 
for binding to eIF4A.

Compared to the potent simplified analogue DMDA PatA (136), the other major change in the structure of triazole analogue $\mathbf{1 8 2}$ is the absence of the methyl groups at the C12, C15, C22 and C24 positions (PatA numbering). As the so-called 'magic' methyl effects have been proven to be very important in molecular recognition by bioreceptors in medicinal chemistry, ${ }^{138,139}$ we propose that the absence of methyl groups may also contribute to the reduction in the activity. To examine the importance of the methyl groups at the $\mathrm{C} 12$ and $\mathrm{C} 15$ positions, the 1,2,3-triazole analogue with the methylated side chain, analogue 187 (Figure 1.25), would also be prepared. Since analogues with the $(R)$ configuration at C10 (PatA numbering) have not yet been synthesised in Romo's previous structure-activity relationship studies, ${ }^{126,127}$ preparing a stereoisomeric analogue at C10 would be of interest. As triazoles are synthetically more accessible than thiazoles, we decided to prepare both enantiomers of analogue 187, namely the (10R)-analogue $(R)$ 187 and the (10S)-analogue $(S)$-187. The comparison of bioactivities of triazole analogues 182, $(R)-\mathbf{1 8 7}$ and $(S)-\mathbf{1 8 7}$ would provide information on the importance of the methyl groups on the side chain and the stereochemistry of C10 (PatA numbering).

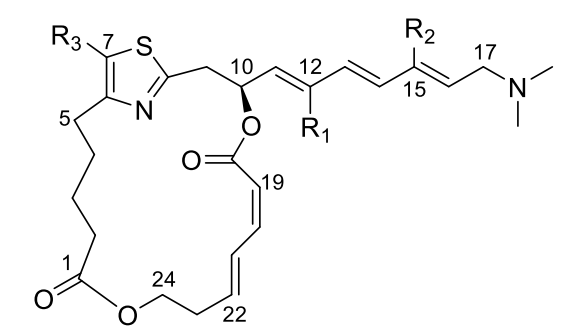

$\mathrm{R}_{1}=\mathrm{H}, \quad \mathrm{R}_{2}=\mathrm{H}, \quad \mathrm{R}_{3}=\mathrm{H}, \quad \mathbf{1 8 3}$

$\mathrm{R}_{1}=\mathrm{CH}_{3}, \mathrm{R}_{2}=\mathrm{CH}_{3}, \mathrm{R}_{3}=\mathrm{H}, \quad \mathbf{1 8 4}$

$\mathrm{R}_{1}=\mathrm{CH}_{3}, \mathrm{R}_{2}=\mathrm{CH}_{3} \quad \mathrm{R}_{3}=\mathrm{CF}_{3}, 185$

$\mathrm{R}_{1}=\mathrm{CH}_{3}, \quad \mathrm{R}_{2}=\mathrm{CH}_{3} \quad \mathrm{R}_{3}=\mathrm{CH}_{3}, 186$

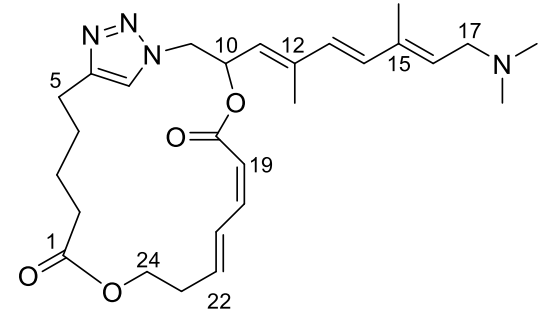

(R)-187

(S)-187

Figure 1.25 Chemical structures of targeted analogues $\mathbf{1 8 3}-\mathbf{1 8 7}$.

Based on the previous structure-activity studies showing that the modifications of the configuration of the E,Z-diene resulted in a drastic decrease in activity, ${ }^{126,127}$ we propose that the methyl at C22 may have modulatory effects when the diene acts as a Michael acceptor in the binding to the target protein..$^{86,110,131}$ In addition, the methyl group at the C24 chiral centre could modify the conformation of the macrocycle to that required for binding to the active site of the protein. To examine the importance of these two methyl groups on the macrocycle, thiazole analogue $\mathbf{1 8 4}$ with the methylated side chain of the 
natural product would also be prepared. Comparison of the bioactivities of analogue 184 and DMDA PatA (136) would disclose whether or not the removal of these two methyl groups is also responsible for the erosion of activity.

In this project, we would like to further explore the role of the heterocycle in the activity. To examine whether the basicity of the thiazole ring is important for binding to its protein target or not, one viable modification would be to introduce an electron-withdrawing group (e.g. a trifluoromethyl group - analogue 185) or an electron-donating group (e.g. a methyl group - analogue 186) on C7 (PatA numbering) to reduce or increase the basicity of the nitrogen atom of the thiazole ring. The difference in activities of analogues 184, 185 and 186 would provide more information on the importance of the heterocycle protonation in the activity.

It was hoped that a scaffold that possesses reduced structural complexity while retaining similar bioactivity relative to PatA could be finally determined through all these studies. This would provide a solid foundation for the future design of analogues.

\subsubsection{Retrosynthetic Analysis}

We envisioned a strategy that could allow the collective synthesis of our designed thiazole analogues 183 - 186 and triazole analogue 187. In other words, we wanted a synthesis of these analogues that would employ the same building blocks wherever possible.

Figure 1.26 shows our first-generation general retrosynthetic analysis of thiazole analogues 183 - 186 and triazole analogue 187. In order to converge retrosynthetically with the highly favoured cycloaddition strategy for forming triazoles by coupling an alkyne with an azide, ${ }^{140}$ a method that would similarly couple an alkyne with a thioamide in forming a thiazole was sought. We envisioned that the thiazole ring in analogues $\mathbf{1 8 3}$ - 186 could be formed via a gold-catalysed coupling reaction between the alkyne fragment (Fragment I) and an appropriate thioamide. ${ }^{141}$ The C7-substituent (PatA numbering) could be introduced onto the thiazole ring after its formation in the synthesis of analogues 185 and $\mathbf{1 8 6}$. The triazole ring in analogue 187 could be prepared by coppercatalysed cycloaddition between the same alkyne fragment (Fragment I) and an appropriate azide fragment (Fragment V). ${ }^{142,}{ }^{143}$ As Julia-Kocienski olefination (an olefination reaction between a phenyl tetrazolyl sulfone and an aldehyde or a ketone) has been utilised successfully to attach the side fragment in the synthesis of the triazole 
analogue 182, ${ }^{134}$ we decided to employ this olefination method to connect the nonmethylated side chain fragment (Fragment II) or the methylated side chain fragment (Fragment $\mathbf{I I}^{\prime}$ ) to further evaluate their performance. The E,Z-dienoate motifs in these analogues would be prepared by the base-induced ring-opening reaction of $\delta$-lactone (Fragment III), a methodology already used in the synthesis of the triazole analogue 182. ${ }^{134}$ The macrocyclic core of these analogues would be constructed by a combination of esterification and macrolactonisation, using appropriate conditions. Therefore, we envisioned the synthesis of thiazole analogues $\mathbf{1 8 3}$ - $\mathbf{1 8 6}$ would require fragment I, II or II', III and the thioamide IV (Figure 1.26), while the major fragments for the synthesis of triazole analogue $\mathbf{1 8 7}$ would include fragment I, II', III and the azide V (Figure 1.26). Since there are different orders in which these fragments could be coupled into the desired analogues, the coupling sequence would be investigated individually for different analogues. Our work towards the synthesis of thiazole analogues $183-186$ will be presented in chapter two and chapter three and the work towards the synthesis of triazole analogue 187 will be presented in chapter four. 


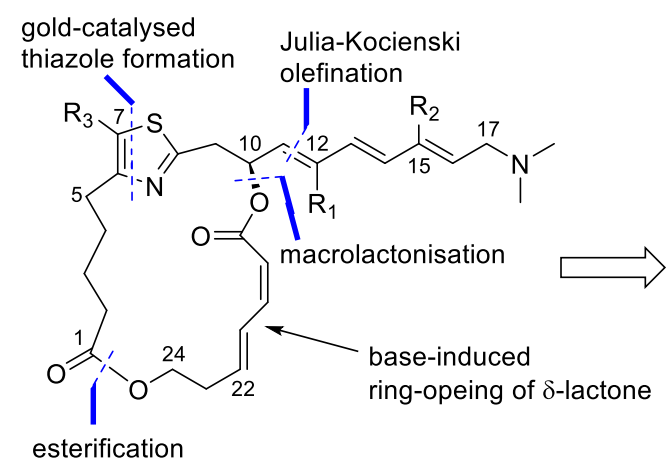

$\mathrm{R}_{1}=\mathrm{H}, \quad \mathrm{R}_{2}=\mathrm{H}, \quad \mathrm{R}_{3}=\mathrm{H}, \quad$ Analogue 183

$\mathrm{R}_{1}=\mathrm{CH}_{3}, \mathrm{R}_{2}=\mathrm{CH}_{3}, \mathrm{R}_{3}=\mathrm{H}, \quad$ Analogue 184

$\mathrm{R}_{1}=\mathrm{CH}_{3}, \mathrm{R}_{2}=\mathrm{CH}_{3} \mathrm{R}_{3}=\mathrm{CF}_{3}$, Analogue 185

$\mathrm{R}_{1}=\mathrm{CH}_{3}, \quad \mathrm{R}_{2}=\mathrm{CH}_{3} \quad \mathrm{R}_{3}=\mathrm{CH}_{3}$, Analogue 186

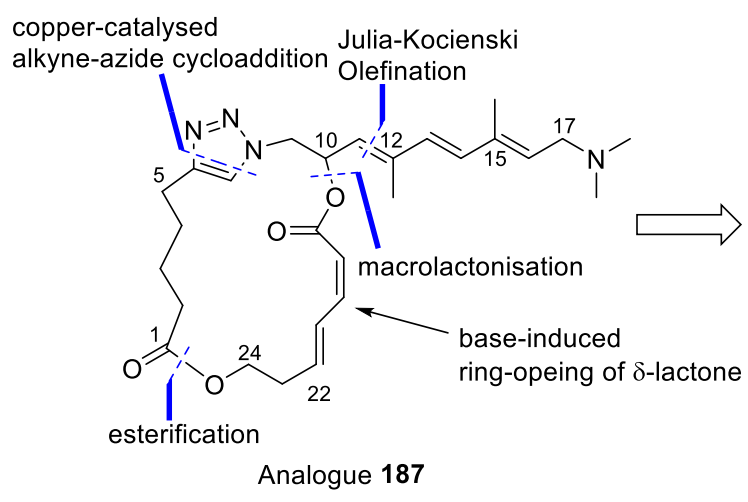

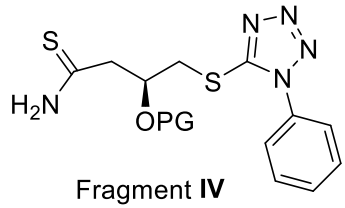

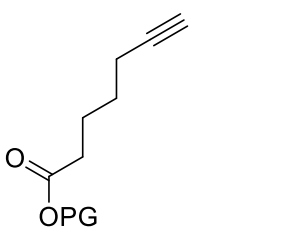

$\mathrm{O}_{\mathrm{H}}$

Fragment II<smiles>[X]C/C=C(/[O])C=CC(C)=O</smiles>

Fragment II'<smiles>O=C(O)OCCC1CC=CC(=O)O1</smiles>

Fragment III

Fragment I<smiles>NCC(CSc1nnnn1-c1ccccc1)OC(=O)OC(F)(F)F</smiles><smiles>[X]C/C=C(C)\C=C\C(C)=O</smiles><smiles>C#CCCCCC(=O)O[Na]</smiles>

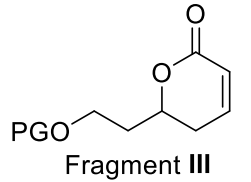

Fragment I

Figure 1.26 First generation retrosynthetic analysis of thiazole analogues $\mathbf{1 8 3}-\mathbf{1 8 6}$ and triazole analogue 187 


\section{Chapter Two: Towards the Synthesis of Thiazole Analogue with Non-Methylated Side Chain}

As discussed in Section 1.7.1, the synthesis of thiazole analogue 183 (Figure 1.23) with non-methylated side -chain could help to examine whether or not the absence of a thiazole ring in the triazole analogue $\mathbf{1 8 2}$ (Figure 1.23) is the primary cause of the observed significant loss in cytostatic activity. ${ }^{134}$ This chapter describes the work towards the synthesis of analogue $\mathbf{1 8 3}$

\subsection{Retrosynthesis}

Our proposed synthesis of analogue $\mathbf{1 8 3}$ (Scheme 2.1) was designed to be easily directed towards other five-membered heterocyclic analogues of PatA, such as those containing an oxazole or imidazole ring. Also, we envisioned that our synthesis could evaluate the performance of a new synthetic methodology for thiazole formation ${ }^{141}$ in a scaffold with structural complexity, which would expand the scope of its applicability.

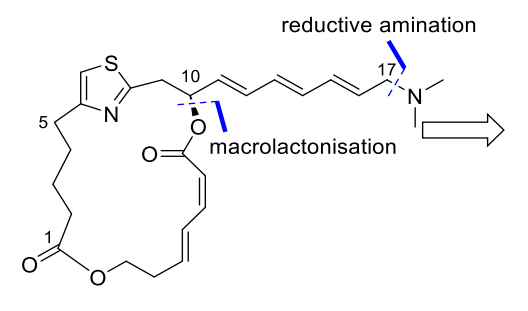

183
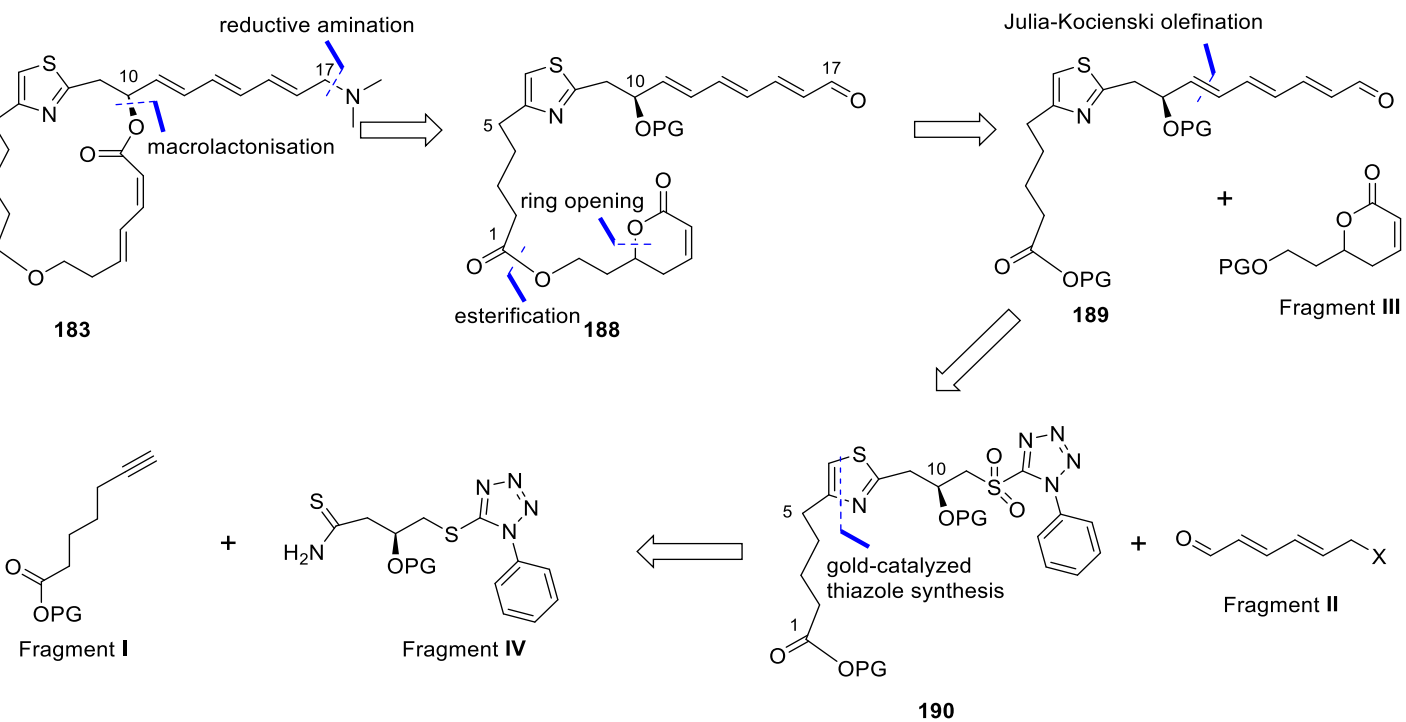

Scheme 2.1 Retrosynthesis of analogue $\mathbf{1 8 3}$.

As shown in Scheme 2.1, the E,Z-dienoate motif could be formed via a base-induced ring opening of $\delta$-valerolactone (Fragment III), a methodology well-established in Cumming's synthesis of the triazole analogue 182 (See Scheme 2.2 for a representative example). ${ }^{135}$ The conjugated aldehyde (Fragment II) could be attached to the macrocyclic core through a Julia-Kocienski olefination reaction. The thiazole ring could be prepared 
by a recently developed gold-catalysed coupling reaction between the terminal alkyne (Fragment I) and the primary thioamide (Fragment IV). ${ }^{141}$

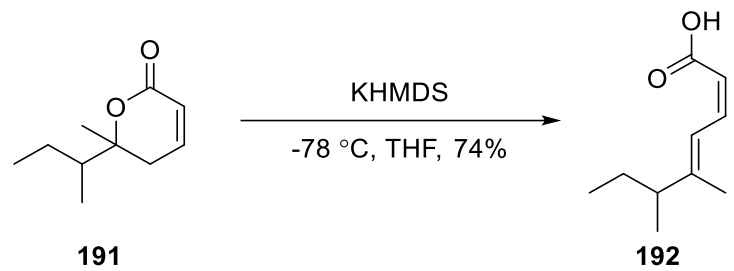

Scheme 2.2 Example of preparation of E,Z-dienoic acid via base-induced ring opening of $\alpha, \beta$-unsaturated $\delta$-lactone. ${ }^{135}$

These four fragments (I, II, III, IV) may be coupled in different orders to synthesise the thiazole analogue $\mathbf{1 8 3}$, and a coupling route that could minimise the occurrence of side reactions was sought. Julia-Kocienski reaction requires a strong base (e.g., KHMDS), which means that the esterification step to attach the E,Z-dienoate surrogate (Fragment III) onto 190 should be performed later than the olefination step. Given that the formation of alkene product 196 was observed by Cumming in the reaction between 193 and cinnamaldehyde 194 (Scheme 2.3), ${ }^{135}$ the macrolactonisation reaction should be performed after side chain attachment to avoid potential degradation resulting from $\beta$ elimination of the lactone functional group.

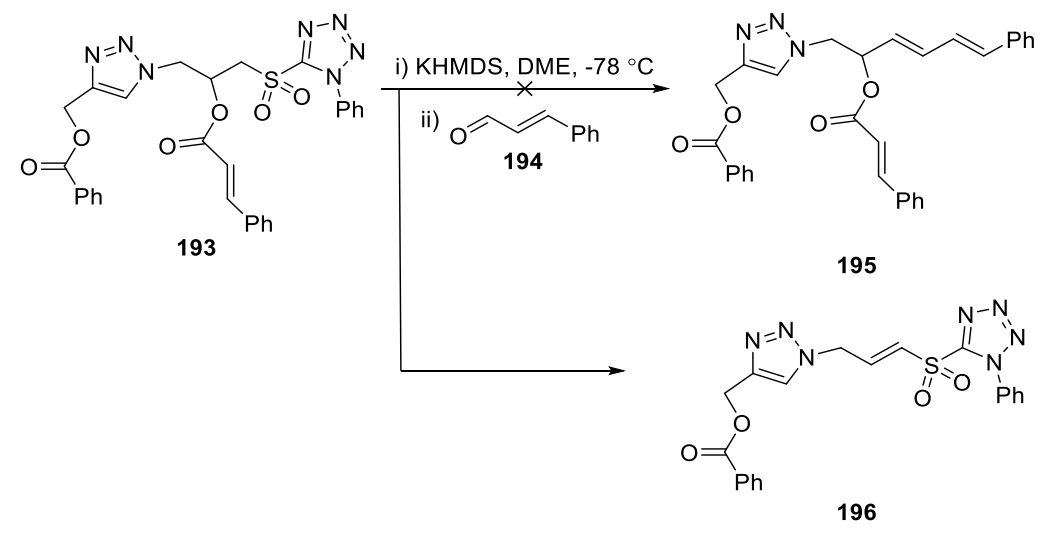

Scheme 2.3 Cumming's attempted Julia-Kocienski reaction with acyclic model substrate 193. ${ }^{135}$

As gold-alkene complexes have been extensively studied in the area of organometallic chemistry, ${ }^{144}$ presuming that the coordination between the gold-centred catalyst required for the thiazole formation and the multiple alkenes in fragment II might lead to 
undesirable side reactions, the coupling of fragment I and fragment IV should be carried out before Julia-Kocienski olefination. Finally, we envisioned the terminal tertiary amine group in the side chain could be generated from the reductive amination of an aldehyde intermediate in the final step using a mild boron reductant (e.g., tetramethylammonium triacetoxyborohydride). Hence, we planned to accomplish the construction of the 2,4disubstituted thiazole 190 in the first place. Preparation of fragments IV, I and, thiazole 190 will be first discussed in the following sections.

\subsection{Synthesis of C8-C11 Thioamide (Fragment IV)}

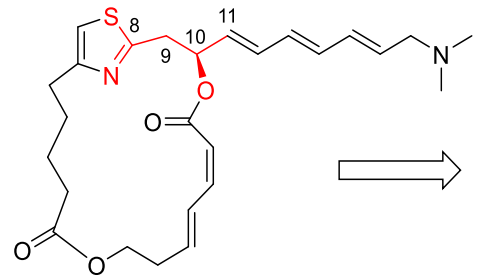

183

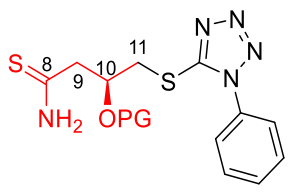

Fragment IV

The C8 - C11 fragment IV is a central linker involved in three attachment points to the proposed macrocycle analogue 183: at the thiazole core, the $E, Z$-dienoate and the conjugated side chain. Accordingly, it requires three functional groups, a thioamide, an alcohol, and a thioether that is readily oxidised to form a sulfone, on a four-carbon molecule, making it a densely functionalised compound.

\subsubsection{Introduction}

In general, thioamides are more reactive than their corresponding amides, and the sulfur atom in the $\mathrm{C}=\mathrm{S}$ group can act as an electrophile and a nucleophile because the sulfur atom and the carbon atom in thioamides have more similar electronegativities. ${ }^{145}$ The nitrogen atom in primary and secondary thioamides can also react with electrophiles (e.g., carbonyls). ${ }^{146}$ Therefore, thioamides have been increasingly recognised as valuable intermediates in the synthesis of a variety of functional groups ${ }^{147}$ and used as organosulfur ligands in transition metal-catalysed functionalisation to perform a range of reactions, including C-H activation, ${ }^{148}$ Suzuki-Miyaura coupling, ${ }^{149}$ and ruthenium catalysed hydrogenation. ${ }^{150}$

A range of different methods has been reported to prepare primary thioamides, ${ }^{151}$ which can be classified based on substrates. The first method, which has been called the 
Willgerodt-Kindler reaction, ${ }^{152}$ makes use of the direct incorporation of elemental sulfur to a combination of ketones and amines at high reaction temperatures (Figure 2.1a). Although various methods and variants (e.g., using sulfated polyborate ${ }^{153}$ or ferrous chloride ${ }^{154}$ as a catalyst) have been developed to allow for lower reaction temperatures, most of those conditions proceed well for aromatic ketone substrates but are highly problematic for the preparation of aliphatic thioamides. The second strategy employs thiohydrolysis of the corresponding nitriles to give primary thioamides (Figure 2.1b). Compounds bearing $\mathrm{P}=\mathrm{S}$ bonds, such as Lawesson's reagent 198 in combination with a Lewis acid, diphenylphosphinodithioic acid, ammonium phosphorodithioate, and alkali metal hydrogen sulfides or ammonium sulfide, are widely utilised in this type of reaction instead of elemental sulfur. In most cases, aromatic and aliphatic primary thioamides are obtained in reasonable yields. ${ }^{155}$ The third method is the exchange of the oxygen of amides for the sulfur atom of thioamides (Figure 2.1c). It is this third method that has attracted the most attention of the approaches to thioamide formation. A wide range of agents bearing $\mathrm{P}=\mathrm{S}$ compounds has been developed, such as Berzelius reagent $\left(\mathrm{P}_{4} \mathrm{~S}_{10}\right)$ 197, ${ }^{156}$ Davy's reagent ${ }^{157}$ and Lawesson's reagent $\mathbf{1 9 8},{ }^{158}$ and these have been extensively used in this one-step transformation of amides to thioamides.

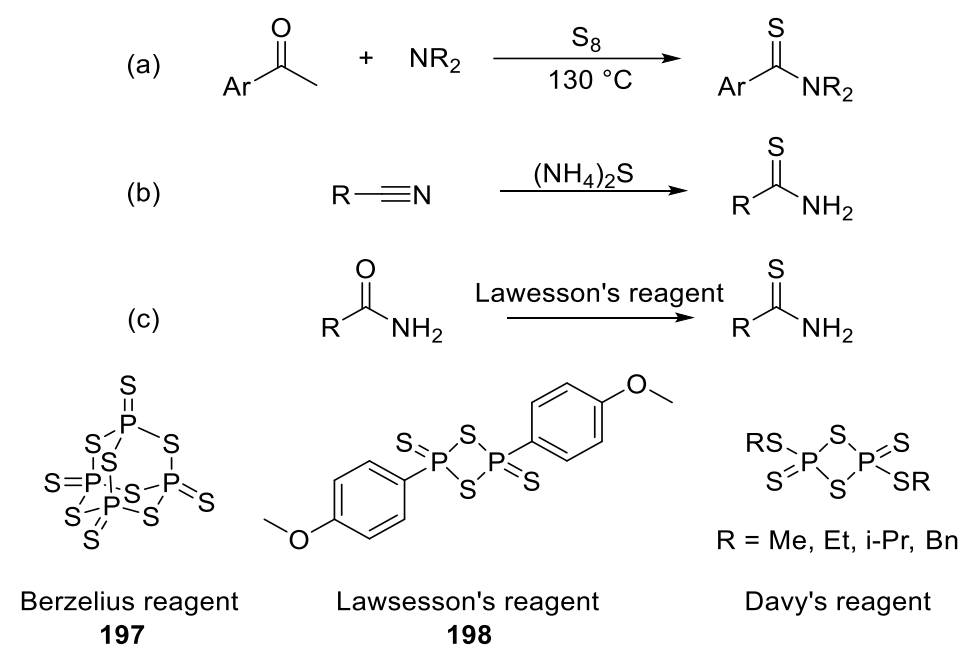

Figure 2.1 Examples of thioamide preparation using (a) ketones, (b) nitriles and (c) amides, and structures of Berzelius reagent 197, Lawesson's reagent 198 and Davy's reagent.

One of the most frequently used agents is Lawesson's reagent 198. Because of its commercial availability, mild reaction temperature, excellent functional group compatibility, and high efficiency and chemoselectivity, it has been widely applied in 
syntheses of complex natural products and biologically active molecules. Two representative examples demonstrating its excellent functional group selectivity and tolerance are shown in Scheme 2.4. ${ }^{159,} 160$

(a)

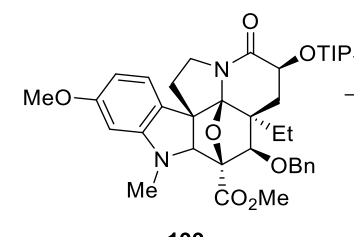

199

(b)

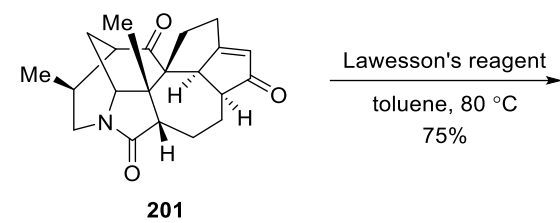

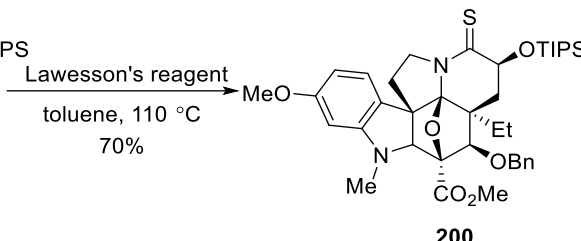

200
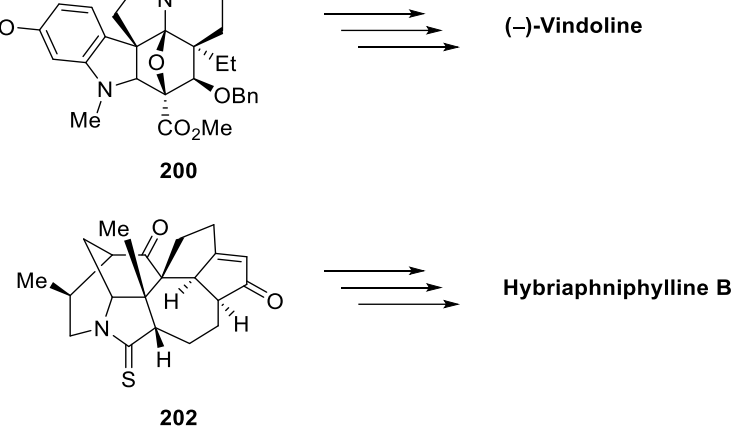

Scheme 2.4 Thionation using Lawesson's reagent in the total synthesis of (a) (-)-

$$
\text { vindoline }^{159} \text { and (b) hybriaphniphylline B. }{ }^{160}
$$

Lawesson's reagent 198 breaks down in an equilibrium process to the more active dithiophosphine $\mathbf{2 0 3}$, which reacts with primary amides to form a four-membered cyclic oxathiaphosphetane intermediate 204 (Scheme 2.5). Subsequent retro [2+2] cycloaddition liberates the thioxophosphine oxide $\mathbf{2 0 5}$ to provide the primary thioamide. ${ }^{161,162}$ Thermodynamically, the $\mathrm{P}=\mathrm{O}$ bond is more stable than the $\mathrm{P}=\mathrm{S}$ bond. This could be the most important driving force for the decomposition of the fourmembered intermediate 204. ${ }^{163}$

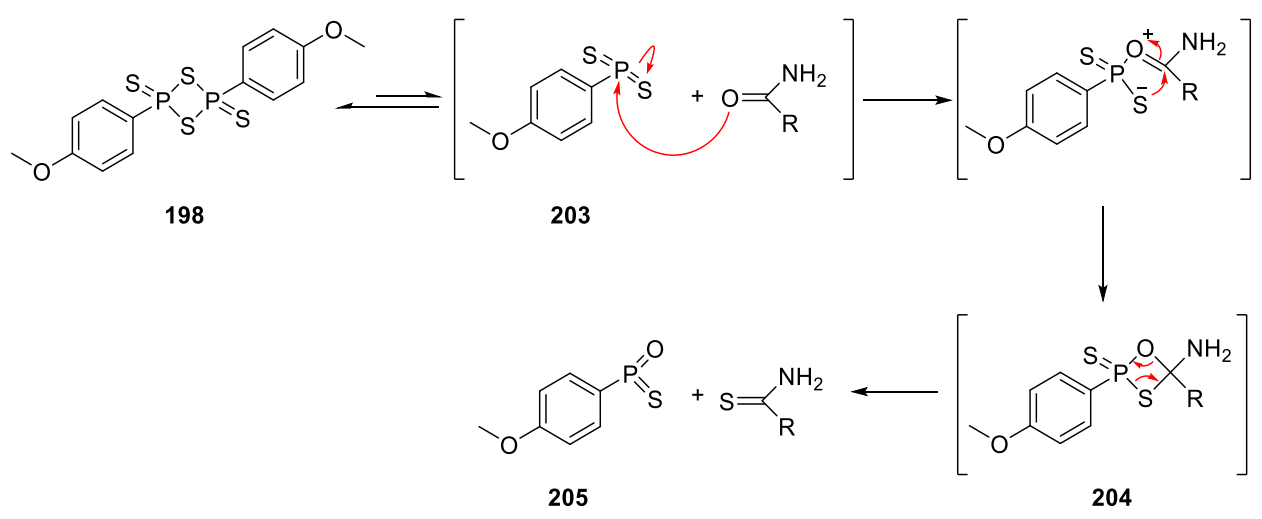

Scheme 2.5 Proposed reaction mechanism for thioamide formation with Lawesson's reagent.

With regards to the formation of the thioamide fragment $\mathbf{I V}$, the second and third methods were chosen for exploration because both of them are appropriate for aliphatic primary 
thioamide synthesis. Since nitriles could be easily hydrated to provide amides, ${ }^{164}$ nitrile 206 could serve as an intermediate for both strategies (Figure 2.2).

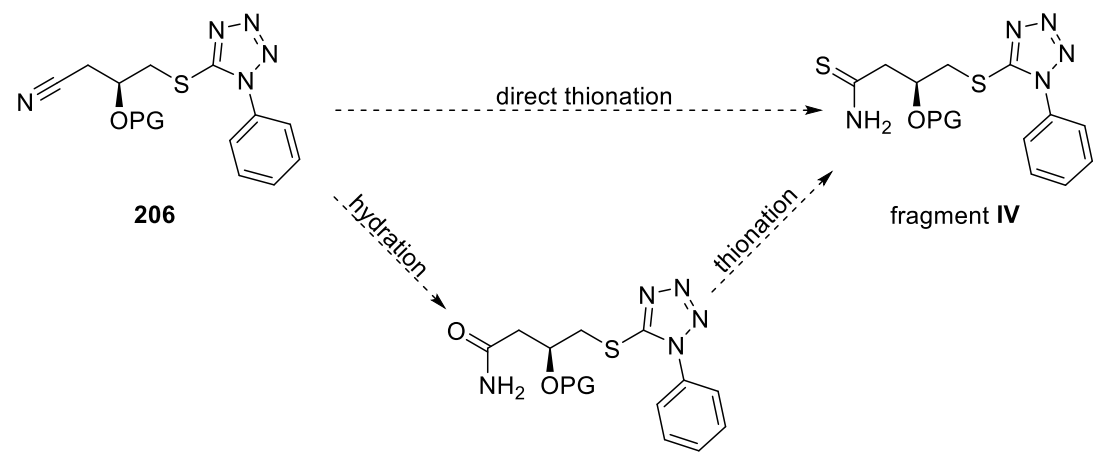

207

Figure 2.2 Two possible pathways to synthesise thioamide fragment IV from nitrile 206.

The following section describes the synthesis of the thioamide fragment IV utilising these two methods.

\subsubsection{Initial Attempts Towards Synthesis of Nitrile 206}

A literature search ${ }^{\dagger}$ suggested that the opening of epoxide $(S)$-209 (Scheme 2.6) with 1phenyl- $1 H$-tetrazole-5-thiol $\mathbf{2 1 0}$ as the nucleophile by using catalytic lithium bromide ${ }^{165}$ or Montmorillonite ${ }^{166}$ can provide the desired difunctional compound 211. Hydrolytic kinetic resolution of terminal epoxides using Jacobsen's catalyst has been demonstrated to be an efficient methodology to produce optically pure epoxides with a wide range of functional groups. ${ }^{167,168}$ Therefore, we envisioned that 3-butenenitrile $\mathbf{2 0 8}$ could serve as an appealing starting material for the desired nitrile intermediate $\mathbf{2 1 1}$ (Scheme 2.6).

†Using SciFinder database; https://scifinder.cas.org (accessed December 2015) 


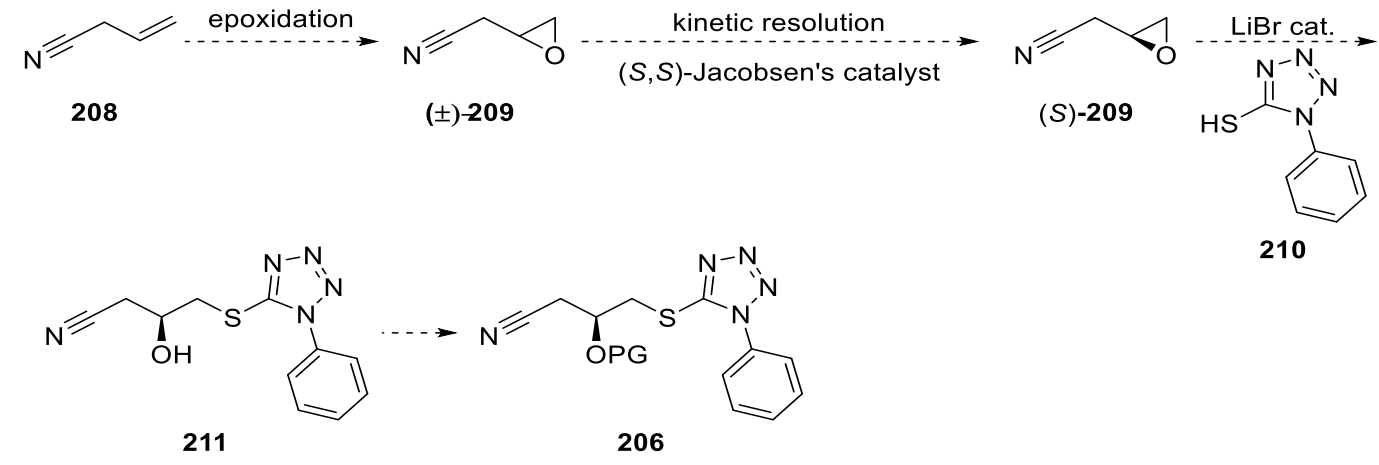

Scheme 2.6 Proposed synthetic route starting from 3-butenentrile 208.

The synthesis of epoxide ( \pm -209 by epoxidation of nitrile 208 had been reported with variable yields from $61 \%$ to $77 \%$ although the reaction requires a rather long reaction time (variable from two to fourteen days). ${ }^{169-171}$ Unfortunately, in our hands, the epoxidation was found to be sluggish; and it had not gone to completion with the continuous addition of excess oxidant over eight days (Scheme 2.7). Not surprisingly, the isolated yield after silica gel chromatography purification was only $20 \%$.

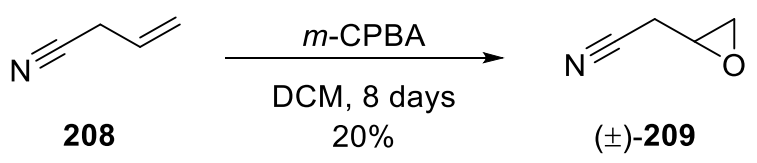

Scheme 2.7 Preparation of epoxide ( \pm )-209.

Alongside this work, postdoctoral research fellow Claire Cuyamendous in our research group also trialled Sharpless asymmetric dihydroxylation of nitrile 208 to synthesise chiral diol 212, which could also be an appealing precursor. However, the enantiomeric excess (e.e.) of obtained diol 212 was determined to be unsatisfactory by Mosher ester analysis (Scheme 2.8). ${ }^{172}$

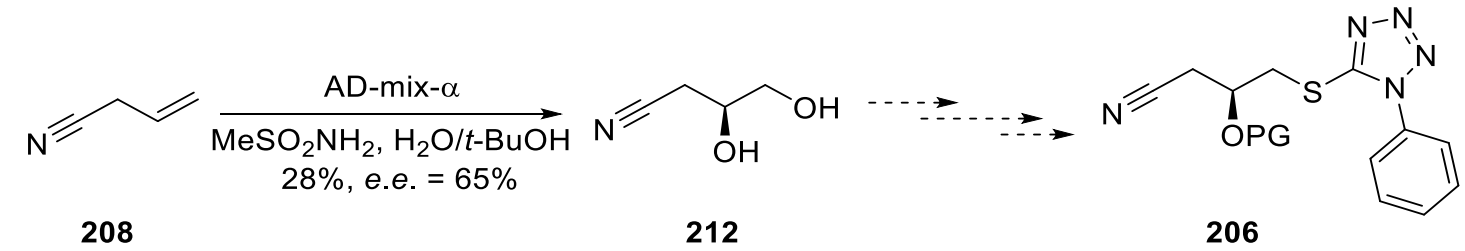

Scheme 2.8 Cuyamendous' preparation of diol 212.

This inability to effectively synthesise epoxide ( \pm )-209 and optically pure diol $\mathbf{2 1 2}$ prompted us to adopt a chiral pool strategy, a classic synthetic strategy starting from 
readily available enantiopure substances that allows efficient enantioselective synthesis.

The direct transformation of terminal epoxides into 1,3-dioxolanes, which are wellknown precursors for 1,2-diols and could be further converted into a wide range of multifunctional molecules, has been demonstrated using a wide range of Lewis acid catalysts. Accordingly, we envisioned $(S)$-epoxide 213, a readily available enantiopure compound, could be a convenient point of departure (Scheme 2.9).

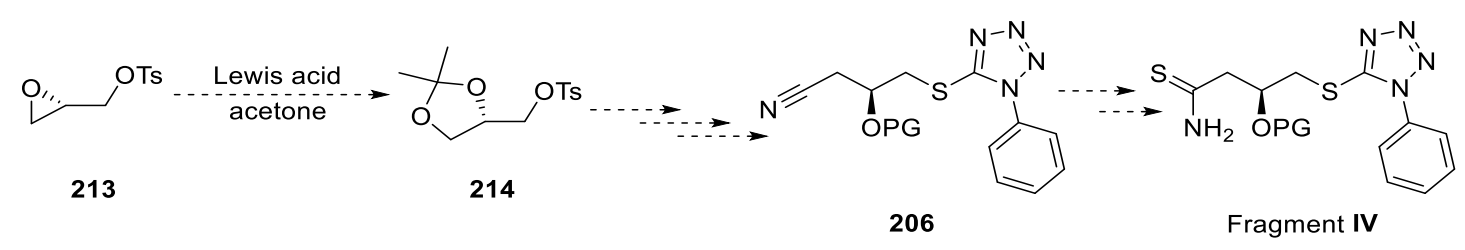

Scheme 2.9 Proposed synthetic route starting from enantiopure epoxide 213.

\subsubsection{Preparation of Thioamide Fragment IV}

\subsubsection{Preparation of Alcohol 217}

Direct transformation of the enantiopure $(S)$-epoxide 213 into acetonide 214 with acetone as the nucleophile, in the presence of several reported Lewis acid catalyst such as $\mathrm{BF}_{3} \cdot \mathrm{Et}_{2} \mathrm{O},{ }^{173} \mathrm{FeCl}_{3}{ }^{174}$, and $\mathrm{AlCl}_{3},{ }^{175}$ was explored (Scheme 2.10). In this work, $\mathrm{AlCl}_{3}$ was found to be the optimal catalyst to provide reproducible excellent yield for both smallscale (e.g., 0.1 gram) and large-scale (e.g., 10 grams) reactions. As acid-catalysed attack on an epoxide by acetone has been reported to result in partial or complete racemisation at the chiral carbon centre, the enantiomeric purity of the acetonide 214 was determined by Mosher ester analysis of compound 217, the product of a later step in the synthesis, ${ }^{176}$ and the stereochemical integrity was mostly preserved relative to the starting material 213. (see section 2.2.3.2 for details). Substitution of tosylate 214 by 1-phenyl-1H-tetrazole-5thiol 210, followed by deprotection with acetyl chloride in methanol, provided diol 216 in nearly quantitative yield in three steps. 


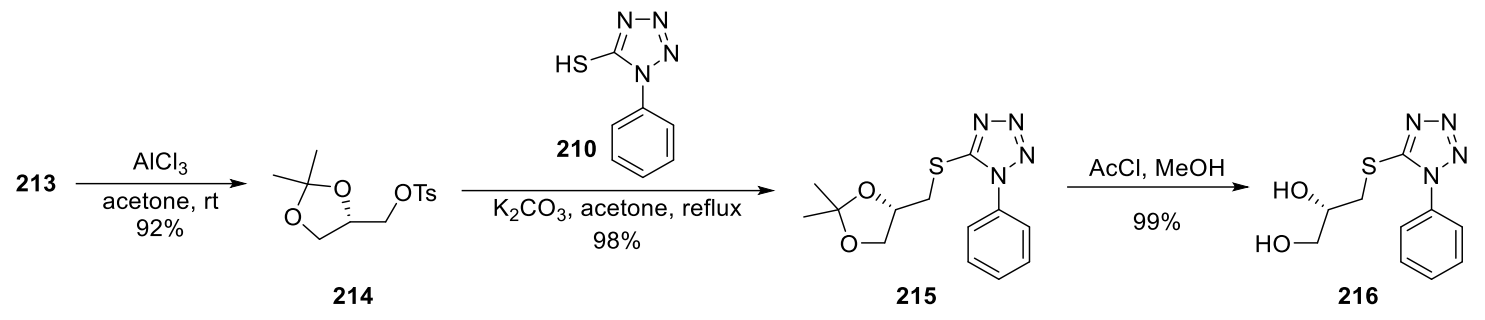

Scheme 2.10 Synthesis of diol 216.

Attempted selective tosylation of the primary alcohol in the diol 216 using triethylamine (TEA) and tosyl chloride resulted in a mixture of mono-tosylate 217 and bis-tosylate 218, as determined by ${ }^{1} \mathrm{H}$ NMR analysis of isolated products by silica gel chromatoghy (Table 2.1 , entry 1$)$. When catalytic dibutyl tin (IV) oxide $\left(\mathrm{Bu}_{2} \mathrm{SnO}\right)$ was used to promote the selectivity (as reported by Martinelli and co-workers), ${ }^{177}$ the mono-tosylate 217 was obtained as the sole product in excellent yield (entry 2 ).
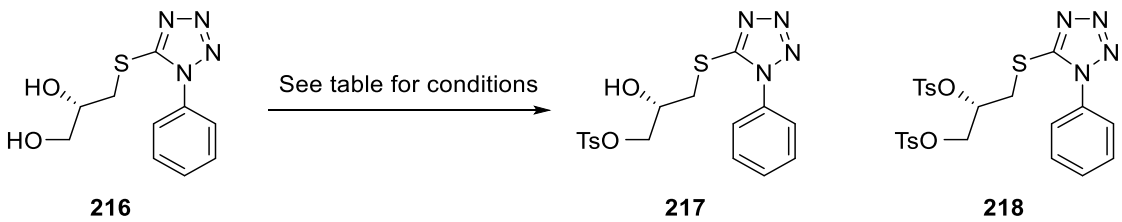

\section{$\begin{array}{llll}\text { Entry } & \text { Conditions (equiv.) } & \text { Ratio }^{\mathrm{b}} & \text { Yield }^{\mathrm{c}}(\%)\end{array}$ \\ $217: 218$}

\begin{tabular}{cccc}
\hline $\mathbf{1}$ & $\mathrm{TsCl}(1.0), \mathrm{Et}_{3} \mathrm{~N}(1.0), \mathrm{DCM}, 0{ }^{\circ} \mathrm{C}$ & $2: 1$ & $31 \%$ \\
\hline $\mathbf{2}$ & $\mathrm{TsCl}(1.0), \mathrm{Et}_{3} \mathrm{~N}(1.0), \mathrm{Bu}_{2} \mathrm{SnO}(0.2)$, & $1: 0$ & $93 \%$ \\
& $\mathrm{DCM}, \mathrm{rt}$ & & \\
\hline
\end{tabular}

${ }^{\mathrm{a}} \mathrm{rt}=$ room temperature. ${ }^{b}$ Determined by ${ }^{1} \mathrm{H}$ NMR spectroscopy on reaction product mixture. ${ }^{\text {I Isolated yield of } \mathbf{2 1 7} \text { after }}$ purification by silica chromatography.

Table 2.1 Reaction screening for selective tosylation of diol $\mathbf{2 1 6}$.

Characterised using 1D ${ }^{1} \mathrm{H}$ NMR spectroscopy, with peaks at $\left(500 \mathrm{MHz}, \mathrm{CDCl}_{3}\right) \delta 7.80-7.75(\mathrm{~m}, 2 \mathrm{H})$, $7.72-7.66(\mathrm{~m}, 2 \mathrm{H}), 7.61-7.55(\mathrm{~m}, 3 \mathrm{H}), 7.50-7.45(\mathrm{~m}, 2 \mathrm{H}), 7.37(\mathrm{~d}, J=8.0 \mathrm{~Hz}, 2 \mathrm{H}), 7.23-7.18(\mathrm{~m}$, $2 \mathrm{H}), 5.20-5.11(\mathrm{~m}, 1 \mathrm{H}), 4.30(\mathrm{~d}, J=3.5 \mathrm{~Hz}, 2 \mathrm{H}), 3.62(\mathrm{dd}, J=14.7,4.5 \mathrm{~Hz}, 1 \mathrm{H}), 3.41(\mathrm{dd}, J=14.7,8.2$ $\mathrm{Hz}, 1 \mathrm{H}), 2.47$ (s, 3H), 2.32 (s, 3H). 


\subsubsection{Mosher Ester Analysis of Alcohol 217}

The enantiomeric purity of the secondary alcohol 217 was assayed by ${ }^{1} \mathrm{H}$ NMR spectroscopy following the modified Mosher's method. ${ }^{172,} 178$ The alcohol 217 was acylated with $(S)-(+)-\alpha$-methoxy- $\alpha$-trifluoromethylphenylacetic acid and $(R)-(+)-\alpha-$ methoxy- $\alpha$-trifluoromethylphenylacetic acid to give Mosher ester $(S)$-MTPA-219 and (R)-MTPA-219, respectively. As shown in Table 2.2, the stereochemistry of the major enantiomer of the secondary alcohol $\mathbf{2 1 7}$ was confirmed to be $S$ configuration.

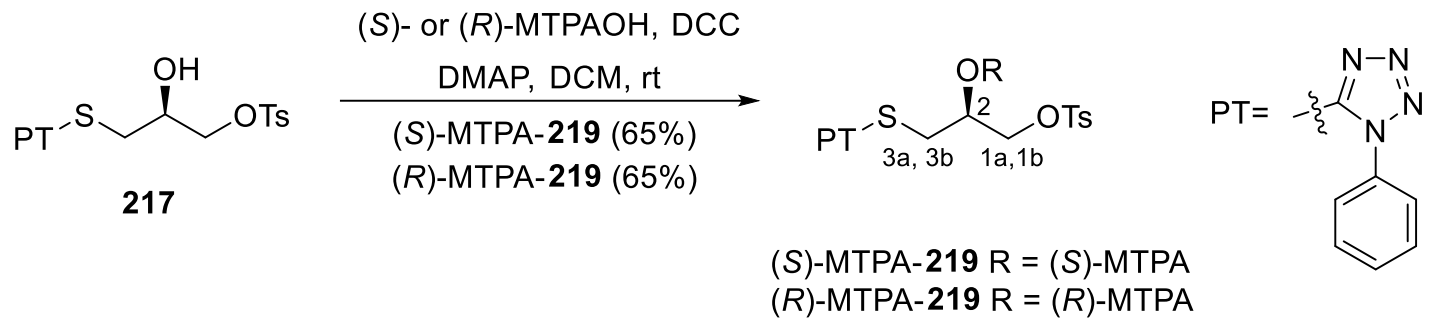

\begin{tabular}{cccc}
\hline Proton & $\begin{array}{c}\boldsymbol{\delta}(\boldsymbol{S}) \text {-MTPA ester } \\
(\mathbf{p p m})\end{array}$ & $\begin{array}{c}\boldsymbol{\delta}(\boldsymbol{R}) \text {-MTPA ester } \\
(\mathbf{p p m})\end{array}$ & $\begin{array}{c}\Delta \boldsymbol{\delta}^{\mathbf{S R}}=\boldsymbol{\delta} \mathbf{S}-\boldsymbol{\delta}_{\mathbf{R}} \\
(\mathbf{p p m})\end{array}$ \\
\hline $\mathbf{1 a}$ & 4.29 & 4.37 & -0.08 \\
\hline $\mathbf{1 b}$ & 4.24 & 4.28 & -0.04 \\
\hline $\mathbf{2}$ & 5.51 & 5.64 & -0.13 \\
\hline 3a & 3.80 & 3.72 & 0.08 \\
\hline 3b & 3.49 & 3.39 & 0.10 \\
\hline
\end{tabular}

Table 2.2 Analysis of $\Delta \delta^{\mathrm{SR}}$ data for diastereomeric MTPA ester derivatives of alcohol 217.

However, the ${ }^{1} \mathrm{H}$ NMR spectrum of the (S)-MTPA-219 obtained at $500 \mathrm{MHz}$ showed a doublet of doublets centred at $\delta 3.80$ with an integration of 1.00 and another doublet of doublets centred at $\delta 3.72$ with an integration of 0.04 (see Figure 2.3), indicating that the enantiomeric excess of the alcohol had decreased to about $92 \%$. Therefore, a small degree of racemisation has taken place, most likely in the direct transformation of the epoxide 213 into the acetonide 214. 

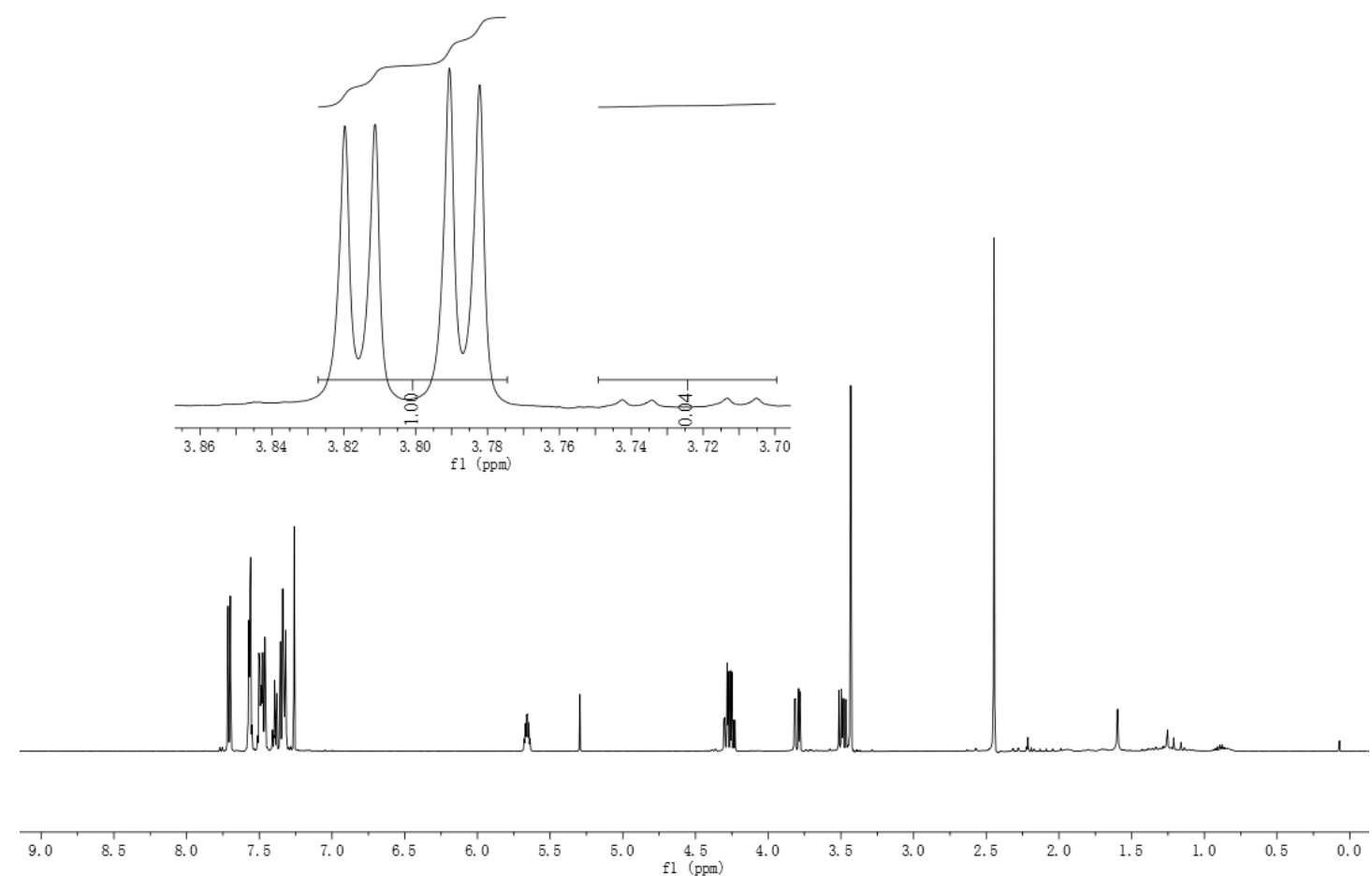

Figure 2.3 $500 \mathrm{MHz}{ }^{1} \mathrm{H}$ NMR spectrum of (S)-MTPA-219 and expansion of $\delta 3.70-$ 3.86 .

Presuming that opening of an epoxide by the carbonyl oxygen of a ketone goes via an $\mathrm{S}_{\mathrm{N}}$ 2-like mechanism, a nucleophilic attack at either carbon of the epoxide could result in the different enantiomers. As shown in mechanism A (Scheme 2.11), after coordination of the epoxide oxygen to $\mathrm{AlCl}_{3}$, the activated oxirane could be opened by the carbonyl oxygen of acetone at $\mathrm{C} 3$, and the dioxolane ring would form in a two-step manner to give the product 214 with enantiomeric integrity relative to the starting material 213. Alternatively, the selectively complexed epoxide could be attacked at the stereogenic C2 position with inversion of configuration to afford the oxiranium intermediate (mechanism B) and would give the enantiomeric product 220. As the $\mathrm{C} 3$ position is more electrondeficient and less sterically hindered than the $\mathrm{C} 2$ position, the nucleophilic $\mathrm{C} 3$ opening should be mostly preferential to the other, which matches experimental observation. 

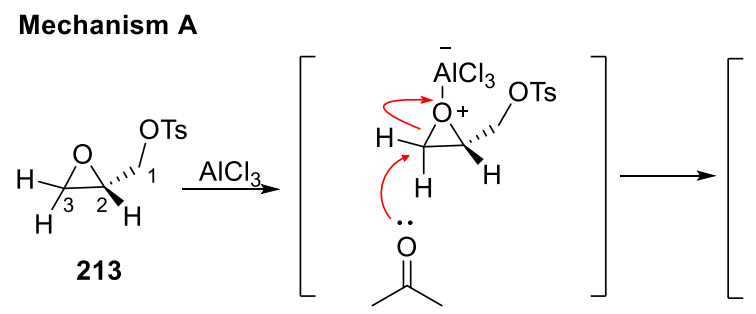<smiles>COC([O-])C(C[O+]=C(C)C)O[Na]</smiles>

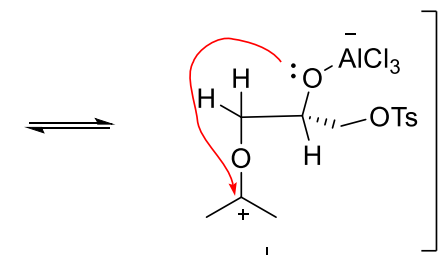<smiles>[Z10]C1COC(C)(C)O1</smiles><smiles>CC[C@@H]1COC(C)(C)[O+]1[O-]</smiles>

\section{Mechanism B}

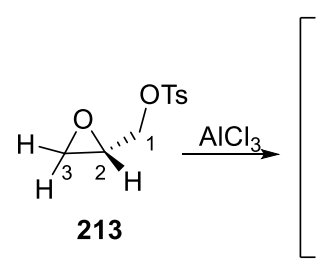

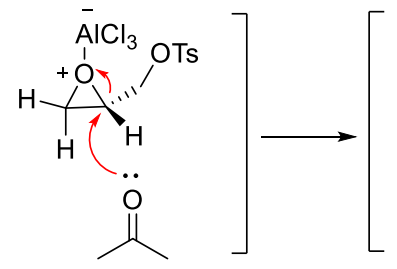

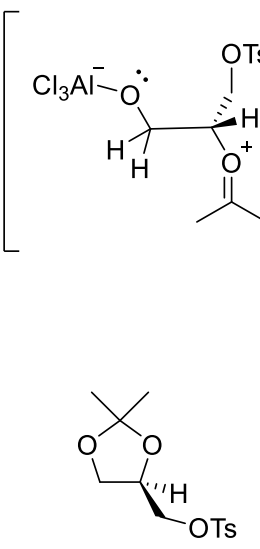

220

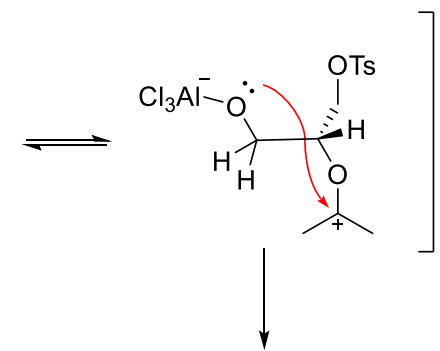<smiles>CC(C)CCC1O[Ge](C)OC1(C)C</smiles>

Scheme 2.11 Proposed mechanism for the observed partial racemisation in acetonide formation.

\subsubsection{Preparation of Nitrile 222}

Although the enantiomeric fidelity of epoxide 213 was not completely preserved, e.e. of alcohol 217 (92\%) was still acceptable. Thus, alcohol 217 was silylated using tertbutyldimethylsilyl chloride (TBSCl) to give compound 221 (Scheme 2.12).

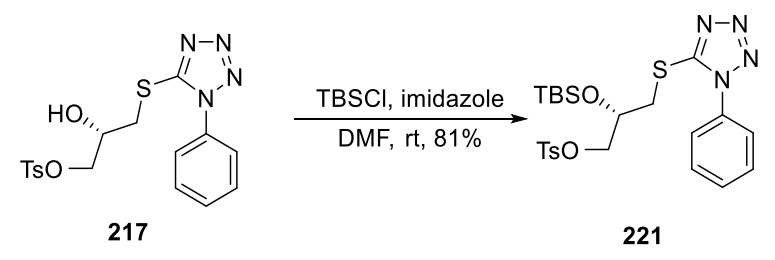

Scheme 2.12 Preparation of silyl ether 221.

The subsequent substitution to form nitrile $\mathbf{2 2 2}$ was initially attempted by treatment with potassium cyanide at $90{ }^{\circ} \mathrm{C}$ (Table 2.3, entry 1). Although tosylate 221 was consumed 
completely, nitrile $\mathbf{2 2 2}$ was obtained in poor yield, and a mixture of by-products was observed, one of which was revealed to be structurally related to phenyl tetrazole, ${ }^{\S}$ suggesting that phenyl tetrazole could possibly be cleaved by cyanide at high temperature. Lowering the reaction temperature to $60{ }^{\circ} \mathrm{C}$ did not reduce the formation of side products significantly, and it resulted in a mixture of nitrile 222 and tosylate 221 (entry 2), which was inseparable by silica gel chromatography, prompting the need to optimise reaction conditions to increase the conversion rate whilst minimising the side reactions. Assuming that the poor yield could be a result of potassium cyanide not being sufficiently homogeneous in the DMF solvent, we then trialled using phase transfer catalysts at different reaction temperatures (entries $3-7$ ). Increasing the equivalents of 18-crown-6 catalyst ( 0.3 to 3 equiv., entries $3-5)$ and lowering temperature $\left(60{ }^{\circ} \mathrm{C}\right.$ to $40{ }^{\circ} \mathrm{C}$, compare entries $3 \& 4$, and also entries $6 \& 7$ ) did improve the conversion rate, however, it did not suppress the side reactions significantly and the yield of nitrile $\mathbf{2 2 2}$ remained unsatisfying.

Given that the accessible cyanide sources were old legacy chemicals, it was plausible that the $\mathrm{KOH}$ impurity resulting from slow hydrolysis of $\mathrm{KCN}$ over long-term storage might be the cause of side reactions. Attention was then directed towards using LiCN prepared in situ by mixing acetone cyanohydrin and lithium hydride, ${ }^{179}$ which could also help to reduce the risk of direct cyanide exposure. This protocol did reduce the formation of the side product to some extent; however, it also resulted in a lower conversion rate even after prolonged reaction time (entries 8, 9 and 10). Since hexamethylphosphoramide (HMPA) was reported to be able to increase the reactivity of different organolithium reagents through the coordination between its three nitrogen atoms and lithium cation, ${ }^{180}$ we supposed that using HMPA as the co-solvent could increase the reactivity of LiCN. The conversion rate of compound $\mathbf{2 2 1}$ was indeed increased as expected (compare entries 8 and 11). Nonetheless, the attempt to enhance the conversion rate by raising reaction temperature resulted in poor yield (entry 12), which might be a consequence of increased cleaving of the phenyl tetrazole at the higher temperature. Presuming that the small amount of impurity (e.g., $\mathrm{LiOH}$ ) in the commercially available LiH from Sigma-Aldrich (purity 95\%) could potentially play an important role in the side reaction, using a mixture of LiHMDS and acetone cyanohydrin as a surrogate of high quality of LiCN was trialled

${ }^{8}$ Characterised using $1 \mathrm{D}{ }^{1} \mathrm{H}$ NMR with peaks at $\left(500 \mathrm{MHz}, \mathrm{CDCl}_{3}\right) \delta 8.99(\mathrm{~s}, 1 \mathrm{H}), 7.75-7.69(\mathrm{~m}, 2 \mathrm{H})$, $7.63-7.57(\mathrm{~m}, 2 \mathrm{H}), 7.57-7.52(\mathrm{~m}, 1 \mathrm{H})$. 
at $70{ }^{\circ} \mathrm{C}$. Gratifyingly, the yield was increased from $24 \%$ to $76 \%$ accompanied by the complete conversion of tosylate 221 (entry 13).

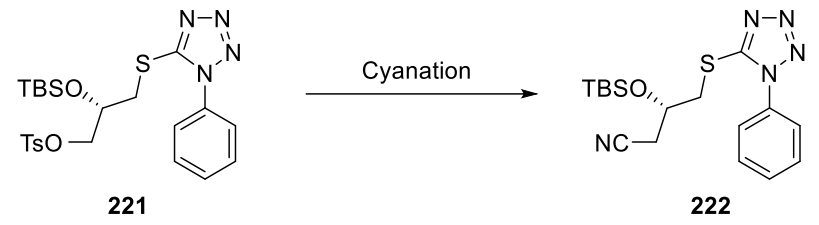

\begin{tabular}{|c|c|c|c|c|c|c|c|}
\hline Entry & $\begin{array}{l}\text { Cyanide } \\
\text { (equiv.) }\end{array}$ & Catalyst (equiv.) & Solvent & $\begin{array}{c}\mathbf{T} \\
\left({ }^{\circ} \mathrm{C}\right)\end{array}$ & $\begin{array}{l}\text { Time }^{c} \\
\text { (hrs) }\end{array}$ & $\begin{array}{c}\text { Ratio } \\
\text { 222:221 }\end{array}$ & $\begin{array}{c}\text { Yield } \\
(\%)\end{array}$ \\
\hline 1 & $\mathrm{KCN}(1.5)$ & - & DMF & 90 & $\mathrm{o} / \mathrm{n}$ & $1: 0$ & $7.0^{\mathrm{d}}$ \\
\hline 2 & $\mathrm{KCN}(1.5)$ & - & DMF & 60 & $\mathrm{o} / \mathrm{n}$ & $6: 1$ & $31^{\mathrm{e}}$ \\
\hline 3 & $\mathrm{KCN}(1.5)$ & 18-crown-6 (0.3) & DMF & 60 & $\mathrm{o} / \mathrm{n}$ & $4: 1$ & - \\
\hline 4 & $\mathrm{KCN}(1.5)$ & 18-crown-6 (0.3) & DMF & 40 & $\mathrm{o} / \mathrm{n}$ & $5: 1$ & - \\
\hline 5 & $\mathrm{KCN}(1.5)$ & 18-crown-6 (3) & DMF & 40 & $\mathrm{o} / \mathrm{n}$ & $30: 1$ & $46^{\mathrm{e}}$ \\
\hline 6 & $\mathrm{KCN}(1.5)$ & TBAI (0.3) & DMF & 60 & $\mathrm{o} / \mathrm{n}$ & $7: 1$ & $45^{\mathrm{e}}$ \\
\hline 7 & $\mathrm{KCN}(1.5)$ & TBAI (0.3) & DMF & 40 & $\mathrm{o} / \mathrm{n}$ & $20: 1$ & $48^{\mathrm{e}}$ \\
\hline $8^{a}$ & $\operatorname{LiCN}(4.4)$ & - & THF & 70 & $\mathrm{o} / \mathrm{n}$ & $1.1: 1$ & - \\
\hline $9^{\mathbf{a}}$ & $\operatorname{LiCN}(4.4)$ & - & THF & 70 & 34 & $2.5: 1$ & - \\
\hline $10^{\mathbf{a}}$ & $\operatorname{LiCN}(4.4)$ & - & THF & 70 & 72 & $3: 1$ & - \\
\hline $11^{a}$ & $\operatorname{LiCN}(2.0)$ & & HMPA/THF & $\mathrm{rt}$ & $\mathrm{o} / \mathrm{n}$ & $2: 1$ & - \\
\hline $12^{\mathrm{a}}$ & $\operatorname{LiCN}(2.0)$ & - & HMPA/THF & 50 & $\mathrm{o} / \mathrm{n}$ & $1: 0$ & $24^{\mathrm{d}}$ \\
\hline $13^{b}$ & $\mathrm{LiCN}(2.0)$ & & THF & 70 & $\mathbf{o} / \mathbf{n}$ & 1:0 & $76^{d}$ \\
\hline
\end{tabular}

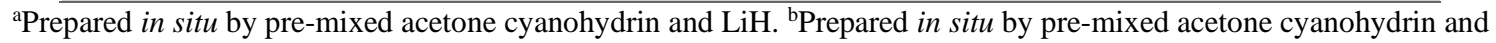
LiHMDS. This result was obtained by research assistant Sarah Andreassend. ${ }^{c} \mathrm{rt}=$ room temperature, $\mathrm{o} / \mathrm{n}=$ overnight (i.e. $16-20$ hours). ${ }^{\mathrm{d} D e t e r m i n e d ~ a f t e r ~ p u r i f i c a t i o n ~ b y ~ s i l i c a ~ g e l ~ c h r o m a t o g r a p h y . ~}{ }^{\mathrm{e}}$ Determined by ${ }^{1} \mathrm{H}$ NMR of an isolated mixture of $\mathbf{2 2 2}$ and $\mathbf{2 2 3}$ obtained by silica gel chromatography.

Table 2.3 Trialled conditions for the formation of nitrile $\mathbf{2 2 2}$.

\subsubsection{Preparation of Thioamide 223}

With nitrile 222 in hand, in order to make our synthetic sequence more atom-efficient, we first attempted thiohydrolysis of nitrile 222 using ammonium sulfide and pyridine, conditions reported by Spychala. ${ }^{181}$ A literature search suggested that chemical shifts of $\alpha$-protons of thioamides are normally downfield relative to those of nitriles. As chemical shifts of $\alpha$-protons of nitrile 222 are at $2.71 \mathrm{ppm}$, the $\alpha$-proton signals of desired thioamide 223 should move downfield. Unfortunately, no desired thionation product 223 was observed by ${ }^{1} \mathrm{H}$ NMR analysis of the crude material after work-up, and instead, a mixture of unidentified products was obtained (Table 2.4, entry 1). Following the conditions 
optimised by Schmid and co-workers, ${ }^{182}$ thiohydrolysis of nitrile 222 was then attempted with a combination of Lawesson's reagent and $\mathrm{BF}_{3} \cdot \mathrm{Et}_{2} \mathrm{O} .{ }^{1} \mathrm{H} \mathrm{NMR}$ analysis of the crude material after work-up showed that the proton signals at $2.71 \mathrm{ppm}$ disappeared completely while two new doublet of doublets peaks appeared at 3.09 and $2.98 \mathrm{ppm}$, respectively, suggesting that the conversion might be successful. However, the isolated product was

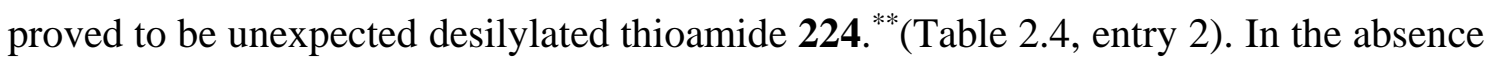
of success in the one-step preparation of the thioamide $\mathbf{2 2 3}$ from nitrile $\mathbf{2 2 2}$, we decided to move onto the alternative method comprising thionation of amides to prepare the desired thioamide.

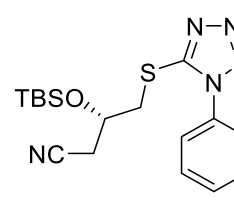

222

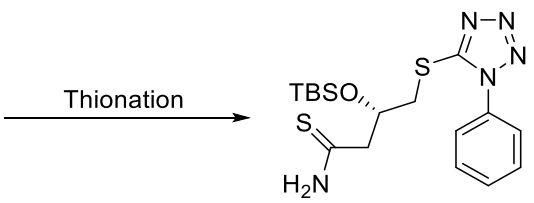

223

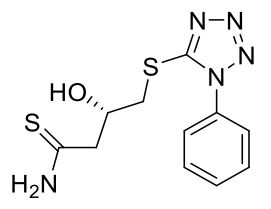

224

\section{Entry Conditions (equiv.) ${ }^{\mathrm{a}} \quad$ Result $^{\mathrm{b}}$}

\begin{tabular}{ccc}
\hline 1 & $\left(\mathrm{NH}_{4}\right)_{2} \mathrm{~S}(5.0)$, pyridine, $\mathrm{H}_{2} \mathrm{O}, \mathrm{rt}$ & $\begin{array}{c}\text { A mixture of unidentified } \\
\text { products }\end{array}$ \\
\hline $\mathbf{2}$ & $\begin{array}{c}\text { Lawesson's reagent }(1.5), \mathrm{BF}_{3} \cdot \mathrm{Et}_{2} \mathrm{O} \\
\text { (2.4), toluene-diethyl ether, } 50{ }^{\circ} \mathrm{C}\end{array}$ & $\mathbf{2 2 4} 57 \%$ yield \\
&
\end{tabular}

$\mathrm{a}_{\mathrm{rt}}=$ room temperature. ${ }^{\mathrm{b}}$ Isolated yield after purification by silica chromatography.

Table 2.4 Trialled conditions for one-step conversion of nitrile $\mathbf{2 2 2}$ to thiamide $\mathbf{2 2 3}$.

A well-known procedure for amide preparation involves treating nitriles with alkaline solutions of hydrogen peroxide. ${ }^{183}$ Thus, nitrile 222 was treated with a solution of hydrogen peroxide and potassium carbonate in ethanol (Table 2.5, entry1), with the hope that excess hydrogen peroxide would oxidise the sulfur atom to afford sulfone 226 simultaneously. This would be expedient, given the requirements of the intended JuliaKocienski reaction later in the sequence. Interestingly, only amide 225 accompanied by a tiny amount of inseparable sulfone $\mathbf{2 2 6}$ was obtained. Considering that amide $\mathbf{2 2 5}$ was

\footnotetext{
***This was characterised by $1 \mathrm{D}{ }^{1} \mathrm{H}$ NMR through observing the disappearance of peaks corresponding to the TBS group: at $\left(500 \mathrm{MHz}, \mathrm{CDCl}_{3}\right) \delta 8.12$ (br. s, $\left.1 \mathrm{H}\right), 7.72$ (br. s, $\left.1 \mathrm{H}\right), 7.61-7.54$ (m, 5H), 4.52 (ddt, $J$ $=8.0,6.9,3.5 \mathrm{~Hz}, 1 \mathrm{H}), 3.63(\mathrm{dd}, J=14.3,3.9 \mathrm{~Hz}, 1 \mathrm{H}), 3.51(\mathrm{dd}, J=14.2,6.8 \mathrm{~Hz}, 1 \mathrm{H}), 3.09(\mathrm{dd}, J=15.0$, $3.1 \mathrm{~Hz}, 1 \mathrm{H}), 2.98(\mathrm{dd}, J=15.0,8.3 \mathrm{~Hz}, 1 \mathrm{H})$.
} 
very polar based on observation by TLC analysis, it was plausible that the low yield was a consequence of significant material loss in the aqueous work-up procedure. Therefore, to circumvent this material recovery problem, the hydration of nitrile $\mathbf{2 2 2}$ using acetaldoxime (freshly prepared from hydroxylamine hydrochloride and sodium hydroxide, see Section 6.2.1 for experimental details) as the source of water was performed following mild conditions reported by Lee and co-workers. ${ }^{184}$ Upon completion, the solvent toluene was evaporated and the resulting crude product was directly purified by silica gel chromatography to provide amide 225 in good yield (entry 2).

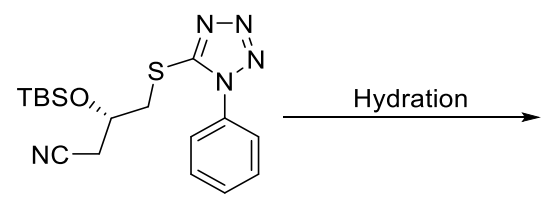

222

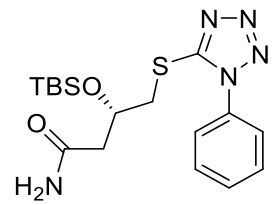

225

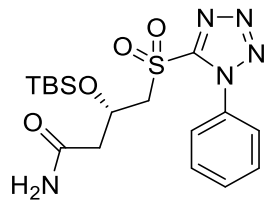

226

\begin{tabular}{ccc}
\hline Entry & Conditions (equiv.) & Result \\
\hline $\mathbf{1}^{\mathbf{b}}$ & $\mathrm{H}_{2} \mathrm{O}_{2}(15), \mathrm{K}_{2} \mathrm{CO}_{3}(5.0)$, ethanol, $0{ }^{\circ} \mathrm{C}$ to $\mathrm{rt}$ & $\mathbf{2 2 5} 26 \%$ yield \\
\hline $\mathbf{2}^{\mathbf{c}}$ & Acetaldoxime (15), $\mathrm{RhCl}\left(\mathrm{PPh}_{3}\right)_{3}$, toluene, $110{ }^{\circ} \mathrm{C}$ & $\mathbf{2 2 5} 88 \%$ yield
\end{tabular}

$\mathrm{a}_{\mathrm{rt}=\text { room temperature. }}{ }^{\mathrm{b}}$ Yield based on the calculation of an inseparable mixture of $\mathbf{2 2 5}$ and $\mathbf{2 2 6}$ after purification by silica chromatography. The ratio of $\mathbf{2 2 5}$ and $\mathbf{2 2 6}$ in the isolated mixture was determined to be 1:0.03 based on the ${ }^{1} \mathrm{H}$ NMR spectrum. 'Isolated yield after silica gel chromatography purification.

Table 2.5 Trialled conditions for hydration of nitrile $\mathbf{2 2 2}$.

The thionation of amide $\mathbf{2 2 5}$ was first trialled with Lawesson's reagent under heating (Table 2.6, entry 1). ${ }^{1} \mathrm{H}$ NMR analysis of the crude material after work-up showed that the $\alpha$-proton signals of amide 225 at 2.63 and 2.54 ppm disappeared completely while two new strongly downfield doublet of doublets peaks appeared at 3.67 and $3.49 \mathrm{ppm}$, indicating that the coversion of amide to thioamide should proceed well. However, thioamide $\mathbf{2 2 3}$ was obtained in only modest yield (23\%). In addition to it, the by-product nitrile 222 resulting from dehydration of amide 225 was also observed. A similar dehydration process has been reported by Robins and co-workers in their synthesis of nucleoside derivatives, ${ }^{185}$ where a mixture of thioamide 228 and nitrile 229 was obtained from the thionation reaction of amide 227 (Scheme 2.13a). Romo's research group also experienced the exclusive formation of nitrile product $\mathbf{2 3 0}$ resulting from dehydration in 
their attempt to synthesise thioamide intermediate $\mathbf{3 2}$ for Hantsch thiazole formation in their PatA synthesis (Scheme 2.13b) ${ }^{84}$ It was then later discovered that using Belleau's reagent 231 instead of Lawesson's reagent 198 could significantly reduce the formation of nitrile $\mathbf{2 3 0}$ and the desired thioamide $\mathbf{3 2}$ was obtained in high yield (vide supra, Scheme 1.1 in Section 1.4.1.1). ${ }^{82,84}$

(a)
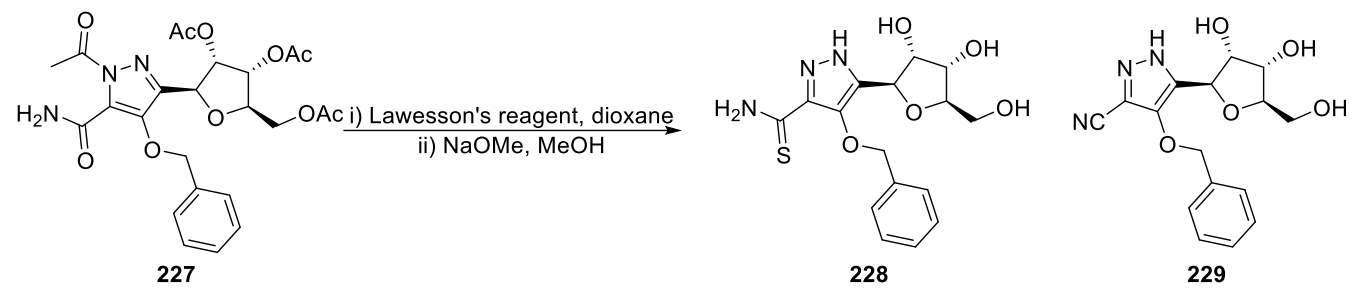

(b)

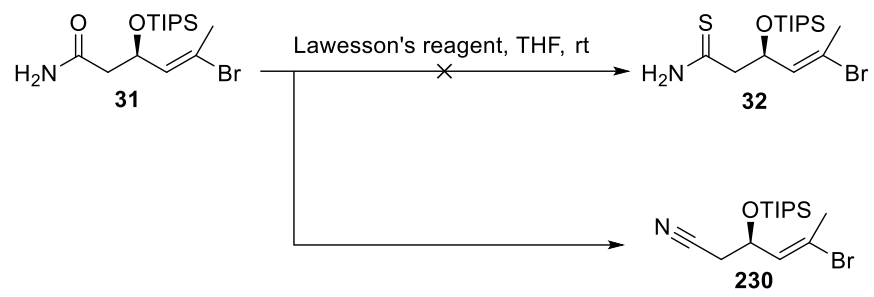

Scheme 2.13 Dehydration caused by the use of Lawesson's reagent reported by (a)

$$
\text { Robins }{ }^{185} \text { and (b) Romo. }{ }^{84}
$$

Therefore, Belleau's reagent 231 was prepared from diphenyl ether following Belleau and co-workers' original procedure ${ }^{186}$ and then tested. Although using Belleau's reagent 231 provided thioamide $\mathbf{2 2 3}$ as the sole product, the rate of the reaction decreased drastically (entry 2). Following Charette and Grenon's mild thionating conditions, ${ }^{187}$ a combination of ammonium sulfide, $\mathrm{Tf}_{2} \mathrm{O}$, and pyridine was also trialled, which gave no desired product but instead a mixture of unidentified products (entry 3). A comprehensive investigation revealed that Lawesson's reagent could decompose under heating due to polymerisation, ${ }^{188}$ which might be the cause of the low yield in entry 1 , therefore, we then decided to go back to test Lawesson's reagent at a lower reaction temperature. It is also worth noting that the typical impurity in the commercial Lawesson's reagent is $\mathrm{P}_{4} \mathrm{~S}_{10}$ (197), which has been utilised as a dehydrating reagent. ${ }^{189}$ Assuming that the $\mathrm{P}_{4} \mathrm{~S}_{10}$ impurity in the excess Lawesson's reagent could be the stimulus for the side reaction, we decided to reduce the equivalents of Lawesson's reagent to 0.54 equivalent and lower the reaction temperature to room temperature to suppress the dehydration (entry 4). This practice did reduce the formation of nitrile $\mathbf{2 2 2}$ and the yield was improved as well, prompting us to move forward to purify the reagent. Following the purification procedure 
in the original preparation reported by Lawesson and co-workers, ${ }^{190}$ the Lawesson's reagent purchased from suppliers was washed with anhydrous $\mathrm{Et}_{2} \mathrm{O}$ and anhydrous DCM, and then recrystallised from boiling anhydrous toluene. Gratifyingly, when purified Lawesson's reagent was used, full conversion of amide 225 was observed within 30 minutes, and thioamide $\mathbf{2 2 3}$ was obtained in $62 \%$ with a small amount of nitrile product $\mathbf{2 2 3}$ on both small-scale (entry 5) and gram-scale (entry 6) reactions, which were judged to be the optimal results.
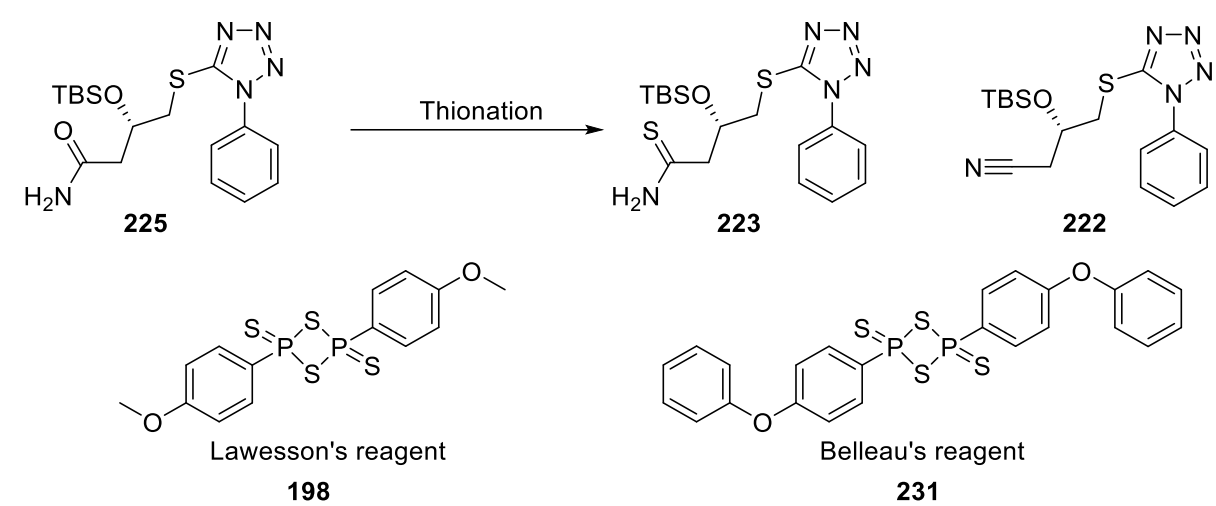

\begin{tabular}{|c|c|c|c|c|c|c|c|}
\hline Entry & $\begin{array}{l}\text { Reagent } \\
\text { (equiv.) }\end{array}$ & Solvent & $\mathbf{T}\left({ }^{\circ} \mathbf{C}\right)^{\mathrm{a}}$ & $\begin{array}{l}\text { Time } \\
\text { (h) }\end{array}$ & $\begin{array}{c}\text { Conversion } \\
(\%)\end{array}$ & $\begin{array}{c}\text { Ratio }^{\mathbf{b}} \\
\text { 223:222 }\end{array}$ & $\begin{array}{c}\text { Yield }^{\mathrm{c}} \\
(\%)\end{array}$ \\
\hline 1 & $198(3.0)$ & THF & 60 & 0.5 & 100 & $1.8: 1$ & 23 \\
\hline 2 & $231(0.7)$ & THF & $\mathrm{rt}$ & 48 & 69 & 1:0 & 21 \\
\hline 3 & $\begin{array}{c}\left(\mathrm{NH}_{4}\right)_{2} \mathrm{~S}(1.5) \\
\mathrm{Tf}_{2} \mathrm{O}(1.2) \\
\text { pyridine (3.0) }\end{array}$ & DCM & -40 to $\mathrm{rt}$ & 2 & 100 & - & 0 \\
\hline 4 & $198(0.54)$ & THF & $\mathrm{rt}$ & 48 & 66 & $5.6: 1$ & 39 \\
\hline $5^{d}$ & $198^{\mathrm{f}}(0.7)$ & THF & rt & 0.5 & 100 & 3.8:1 & 62 \\
\hline $\mathbf{6}^{\mathrm{e}}$ & $198^{\mathrm{f}}(0.7)$ & THF & rt & 0.5 & 100 & 4.4:1 & 62 \\
\hline
\end{tabular}

Table 2.6 Reaction screening for thionation of amide $\mathbf{2 2 5}$. 


\subsection{Synthesis of Thiazole Fragment 232}

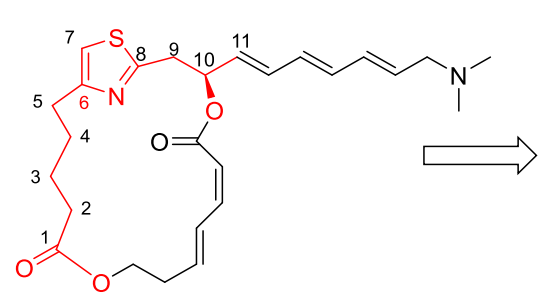

183
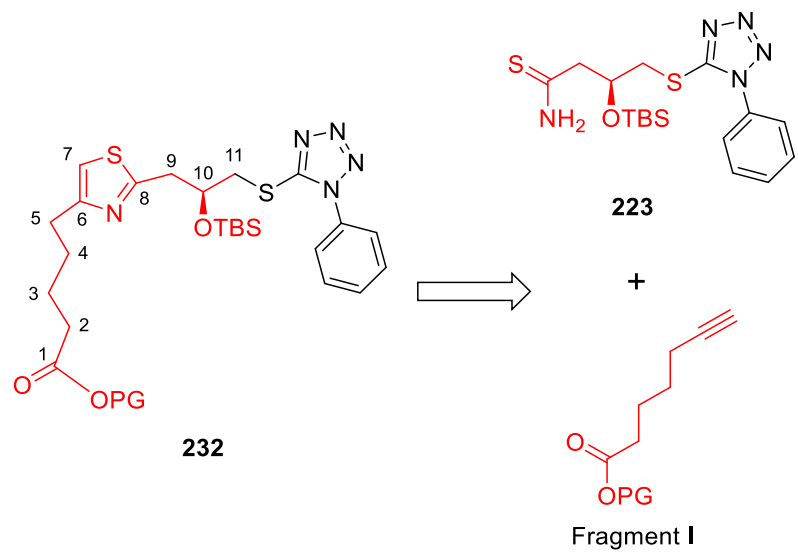

\subsubsection{Introduction}

Thiazoles are heterocyclic compounds possessing sites for both electrophilic substitution and nucleophilic substitution, meaning that they are able to provide ligand points for multiple types of bioreceptors. ${ }^{191}$ Hence, thiazoles are considered to be privileged scaffolds in drug discovery and a significant amount of drugs containing thiazole subunits are currently on the market or in clinical trials. ${ }^{192}$ Recent studies have revealed that substituents at position- 2 and position- 4 of a thiazole ring could significantly affect its molecular electrostatic potential surface, which plays a critical role in interactions between drugs and protein targets. ${ }^{193}$ Therefore, the 2,4-disubstituted thiazole motif has been considered to be an important pharmacophore in various active synthetic and natural products. ${ }^{194}$ Natural products containing 2,4-disubstituted thiazole subunits (see Figure 2.4 for some examples) have been reported to exhibit remarkably diverse biological activities, such as anticancer, antibacterial, antifungal, anti-inflammatory, anthelmintic and antihypertensive activities. ${ }^{195}$ 

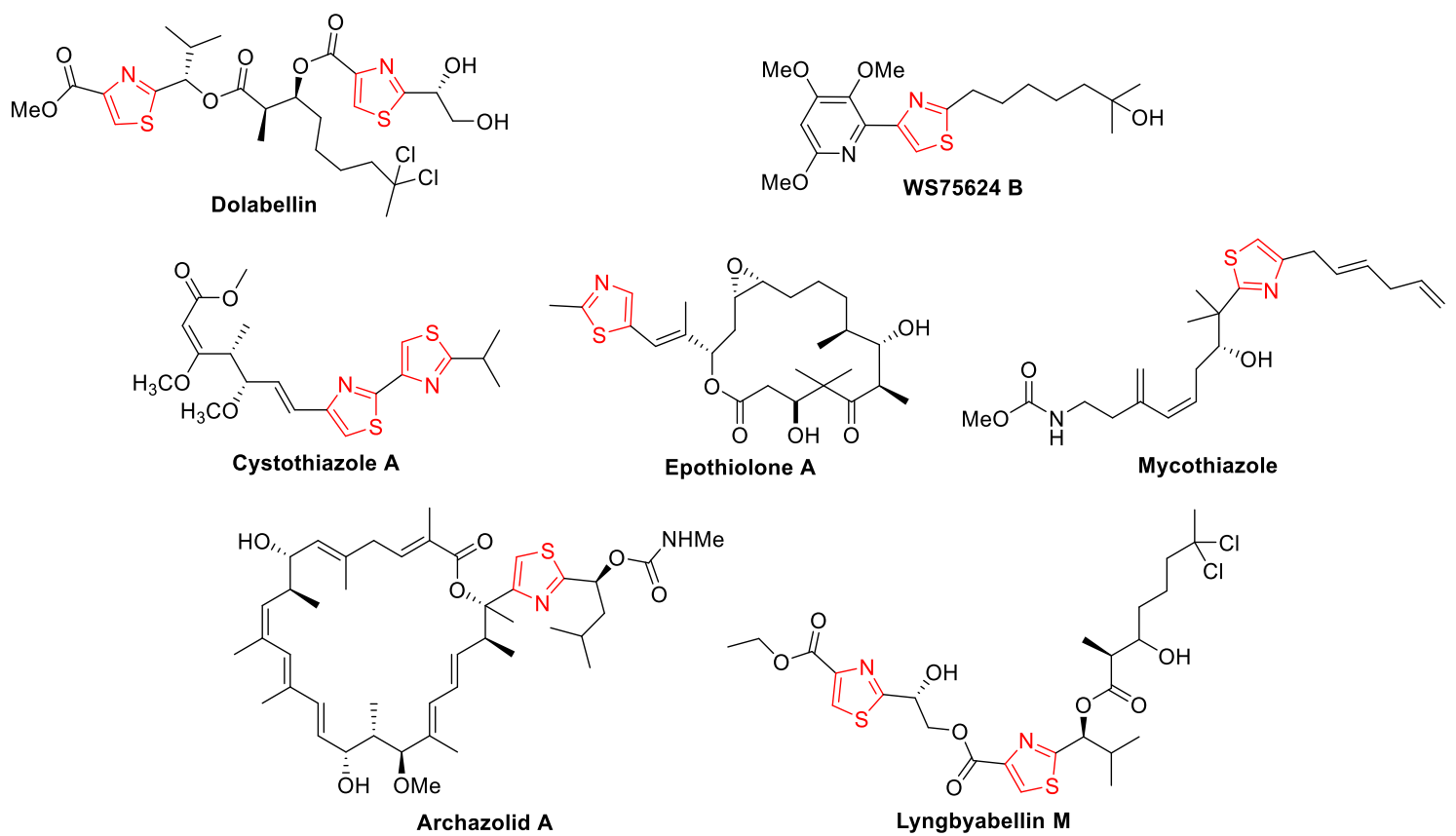

Figure 2.4 Examples of natural products containing 2,4-disubstituted thiazoles.

Given that compounds containing 2,4-disubstituted thiazoles display a wide range of pharmacological activities, this motif has attracted many synthetic endeavours. A range of synthetic methodologies has been developed and they can be roughly classed as follows: (a) Hantzsch method and modifications involving condensation between primary thioamides and $\alpha$-bromoketo esters; and (b) oxidation of thiazoline intermediates generated from condensation of vicinal amino thiols with various partners (e.g., aldehydes, cyanides, carboxylic acids or esters); and (c) transition-metal-catalysed cross-coupling reactions between halo-substituted thiazoles and appropriate partners (e.g., organoboron or organotin species).

The Hantzsch method has been used widely in the synthesis of organic molecules containing a 2,4-disubstituted thiazole, ${ }^{196}$ such as archazolid A, ${ }^{197}$ epothilone $\mathrm{A}^{198}$ and WS75624B ${ }^{199}$ (Scheme 2.14). 

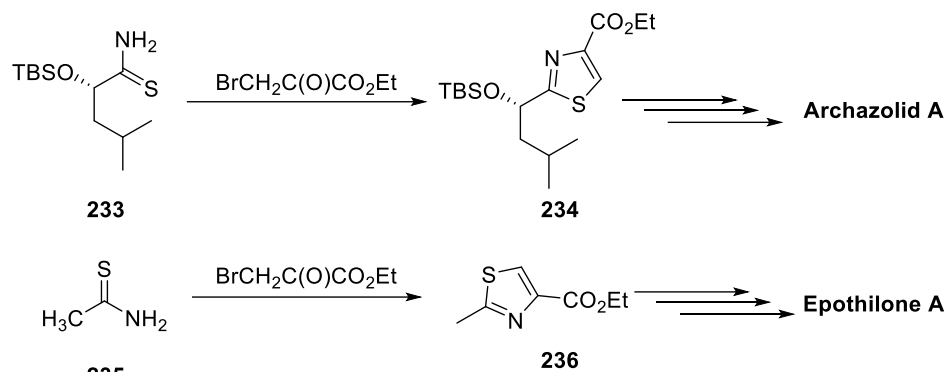

235

236

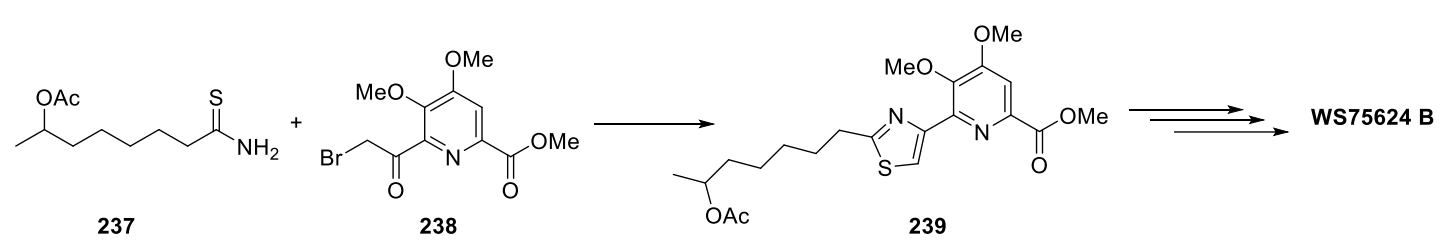

Scheme 2.14 Examples of application of the Hantzsch method in the total synthesis of natural products.

However, as shown in Scheme 2.15, the typical Hantzsch reaction concomitantly forms one equivalent of hydrogen bromide, which can cause significant loss of optical purity in substrates prone to racemisation via the formation of enamine intermediates. ${ }^{200}$
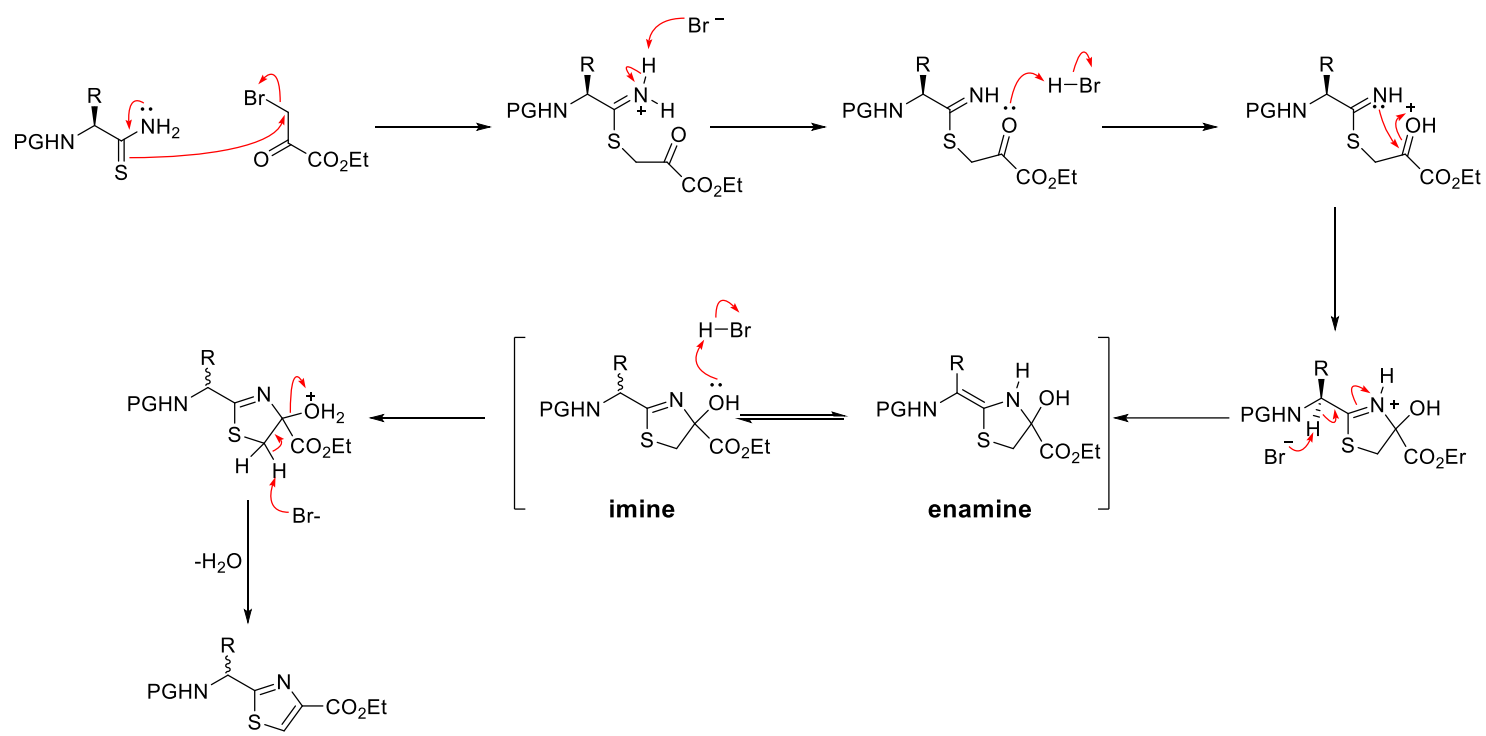

Scheme 2.15 Proposed mechanism of racemisation occurring in Hantzsch thiazole formation.

To overcome this racemisation problem and improve the suitability for chiral thiazole synthesis, a modified protocol using a two-step procedure, called the Holzapfel-MeyersNicolaou modification, ${ }^{113,201,202}$ has been developed. The first step of this modified method is the reaction between a thioamide and an $\alpha$-bromoketo ester in the presence of 
a mild base (e.g., $\mathrm{KHCO}_{3}$ or $\mathrm{NaHCO}_{3}$ ) as the acid scavenger to form a hydroxythiazoline intermediate. The second step is the activation of this intermediate by the addition of reagents (e.g, TFAA) to facilitate the necessary elimination. This method has been applied in the preparation of chiral thiazole building blocks with complete stereocontrol in the synthesis of natural products and their analogues (see Scheme 2.16 for examples). In the total synthesis of aeruginazole A, the cyclocondensation of thioamide $\mathbf{2 4 0}$ and ethyl bromopyruvate provided hydroxythiazoline intermediate $\mathbf{2 4 1}$, which was then treated by TFAA and 2,6-lutidine, following Meyers' conditions, to furnish thiazole building block 242. ${ }^{203}$ Under similar conditions, in the synthesis of thiostrepton, a combination of TFAA, pyridine, and triethylamine, instead of 2,6-lutidine, was used to promote the elimination step of hydroxythioazoline $\mathbf{2 4 4}$ and thiazole $\mathbf{2 4 5}$ was obtained in optically pure form. ${ }^{204}$
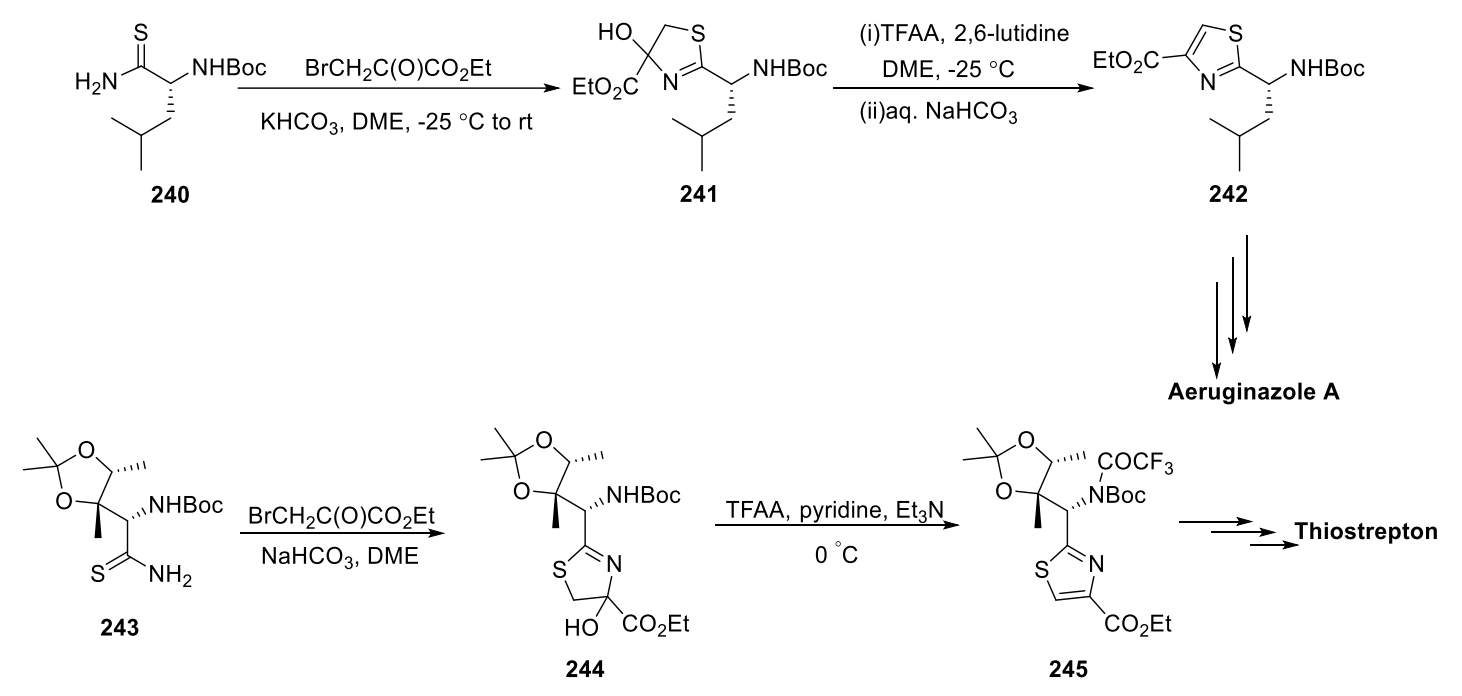

Scheme 2.16 Examples of application of Holzapfel-Meyers-Nicolaou modified method in the total synthesis of natural products.

Another method for the preparation of 2,4-disubstituted thiazoles is oxidation of the corresponding thiazoline precursors. From the biosynthetic point of view, thiazole moieties in natural products are derived from cysteine-containing peptides. ${ }^{205}$ Therefore, cysteine and its derivatives are often used as vicinal amino thiol agents to provide 2,4disubstituted thiazoline intermediates via condensation with suitable partners. Since the sulfur atoms of thiazolines are prone to oxidation by strong oxidants to afford undesired products, e.g., sulfones, sulfinic acids or sulfonic acids, only a few mild oxidation conditions, such as manganese dioxide and a combination of bromotrichloromethane and DBU, have been proven to be useful in this transformation of thiazolines into thiazoles. ${ }^{206}$ 
Similarly, racemisation could also occur in the formation of thiazolines when substrates prone to epimerisation are exposed to strong basic or acidic conditions, ${ }^{207,} 208$ which remains a major challenge for the stereocontrolled synthesis of 2,4-disubstituted thiazoles through this method. As shown in Scheme 2.17, this approach was utilised effectively in the synthesis of mycothiazole ${ }^{209}$ and cystothiazole A (Scheme 2.17). ${ }^{210}$

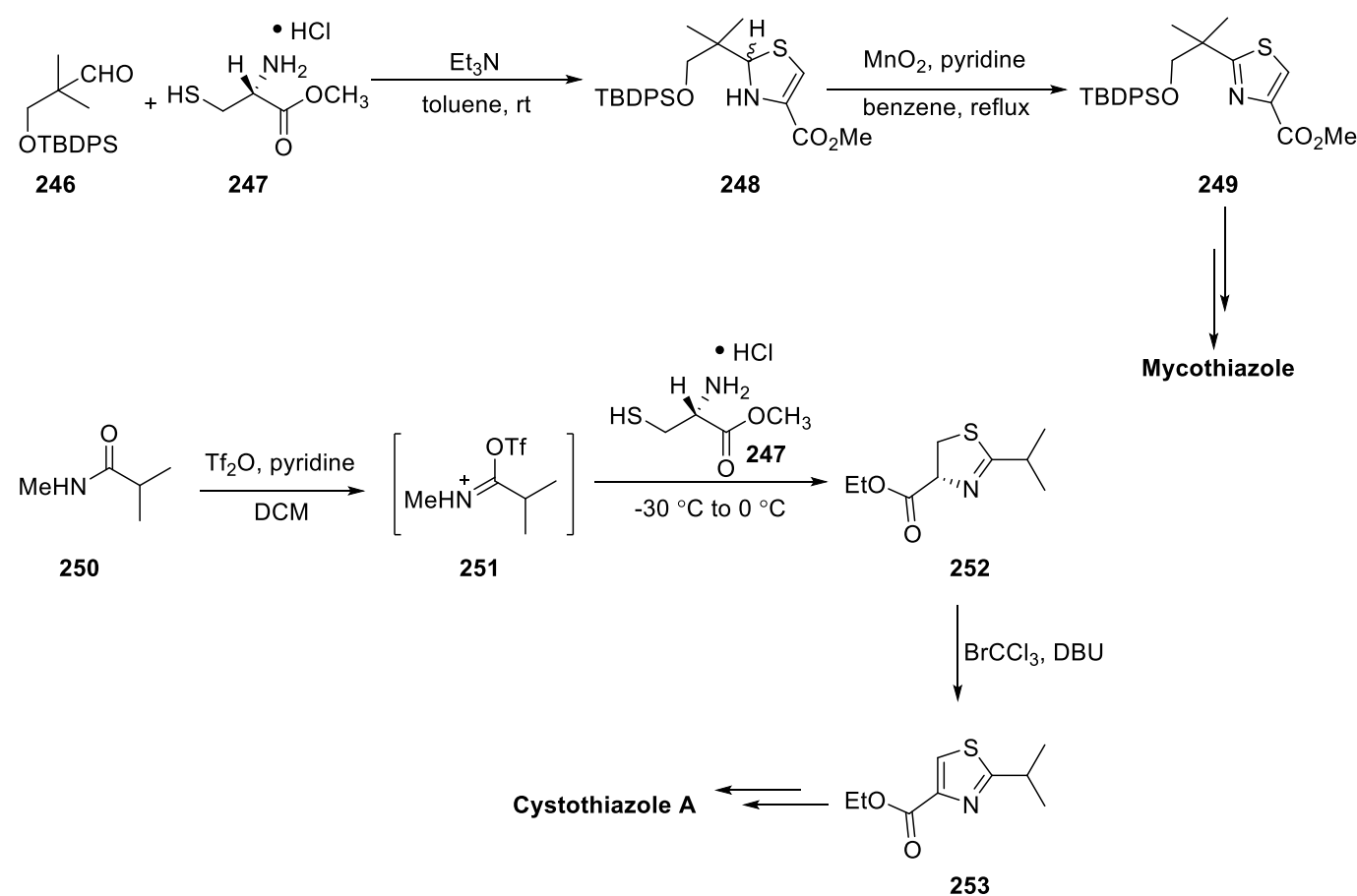

Scheme 2.17 Examples of thiazole formation via oxidation of thiazoline.

These two methods mentioned above have several disadvantages: (1) limited substrate scope; for example, Hantzsch and its modified methods require using $\alpha$-bromoketo esters as starting materials and they are normally prepared by bromination, which can cause serious environmental pollution; (2) time-loss and yield-loss associated with the isolation and purification of thiazoline intermediates in two-step sequences; and (3) potential risk of racemisation when chiral substrates are used. In order to overcome these disadvantages, a range of metal-catalysed methodologies to form 2,4-disubstituted thiazoles has been reported, such as copper-catalysed condensation from oximes, anhydrides and potassium thiocyanate, ${ }^{211}$ and iron-catalysed condensation from azides and potassium thiocyanate. ${ }^{212}$ Among all these studies, a novel gold-catalysed methodology for 2,4disubstituted thiazole formation via coupling between terminal alkynes and primary thioamides appeared attractive because of its versatility and good tolerance of a range of functional groups, such as chloroalkyl, silyl ether, alkene and cyclopropane. ${ }^{141}$ 
1,2,3-triazoles can be prepared from the well-known copper-catalysed 1,3-dipolar cycloaddition reactions between terminal alkynes and azides (Figure 2.5). ${ }^{142,143}$ Oxazoles can be formed through gold-catalysed coupling reactions between terminal alkynes and amides. $^{213}$ Imidazoles can be constructed through rhodium-catalysed transannulation reactions between nitriles and $N$-sulfonyl 1,2,3-triazoles, which can be prepared from reactions between terminal alkynes and sulfonyl azides. ${ }^{214}$ Therefore, a synthetic strategy for thiazole formation that relies on using terminal alkynes as building blocks enables the versatile generation of PatA heterocycle variants, such as oxazole and imidazolecontaining analogues. Also, to the best of our knowledge, this methodology has not been utilised yet in the total synthesis of natural products or analogues related to natural products. Thus, our synthetic work assesses the feasibility of this approach to thiazoles from terminal alkynes with multiple functionalities, expands the reaction scope further with chiral substrates, and enhances the use of the gold catalyst in the total synthesis of structurally complex natural products.

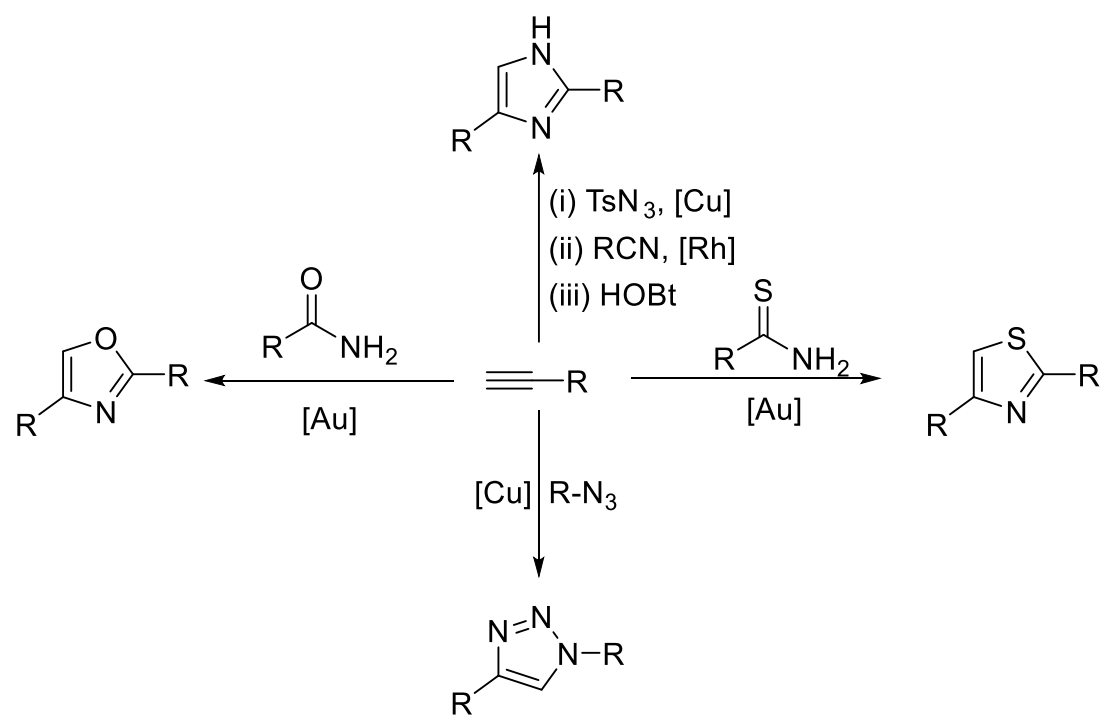

Figure 2.5 Representative of metal-catalysed preparations of thiazoles, oxazoles, imidazoles, and triazoles starting from terminal alkynes. 


\subsubsection{Synthesis of Thiazole Fragment 232 Using Gold Catalysis}

\subsubsection{Proposed Mechanism of Gold-catalysed Thiazole Formation}

In 2012, Zhang and co-workers reported that $\alpha$-oxo gold carbenes generated by oxidation of terminal alkynes were highly electrophilic and could even abstract halogen atoms from 1,2-dichloroethane or 1,2-dibromoethane to give $\alpha$-chloro/bromo ketones. ${ }^{215}$ As shown in Figure 2.6, it was later discovered that the electrophilicity of $\alpha$-oxo gold carbenes (A) could be tempered through the formation of a tricoordinated gold intermediate $\left(B / B^{\prime}\right)$, achieved with an electron-rich $P, N$-bidentate ligand such as di(1-adamantyl)-2morpholinophenylphosphine (Mol-DalPhos). This modulation of the electrophilicity allows chemoselective intermolecular trapping of gold carbenes by amides ${ }^{213}$ or carboxylic acids ${ }^{216}$ to form oxazoles or esters.

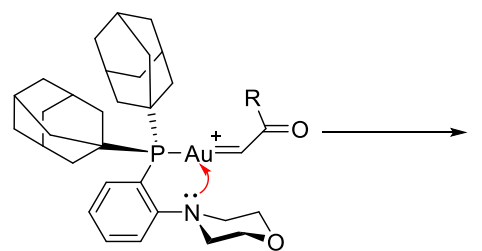

A

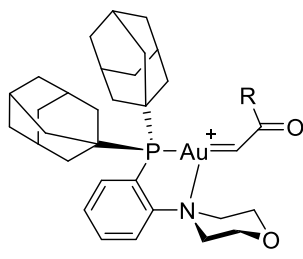

B

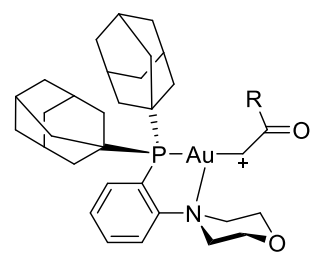

$B^{\prime}$

Figure 2.6 Proposed role of Mol-DalPhos in the stabilisation of the $\alpha$-oxo gold carbene, as presented by Zhang. ${ }^{213}$

Similarly, in the presence of Mol-DalPhosAuOMs, the tricoordinated gold carbene generated via intermolecular oxidation of terminal alkynes by 8-methylquinoline $\mathrm{N}$-oxide 254 could be trapped by methanesulfonic acid $(\mathrm{MsOH})$ to provide the methanesulfonyloxymethyl ketone intermediate, which could be reacted in situ with primary thioamide to give the 2,4-disubstituted thiazole by cyclocondensation (Scheme 2.18). 


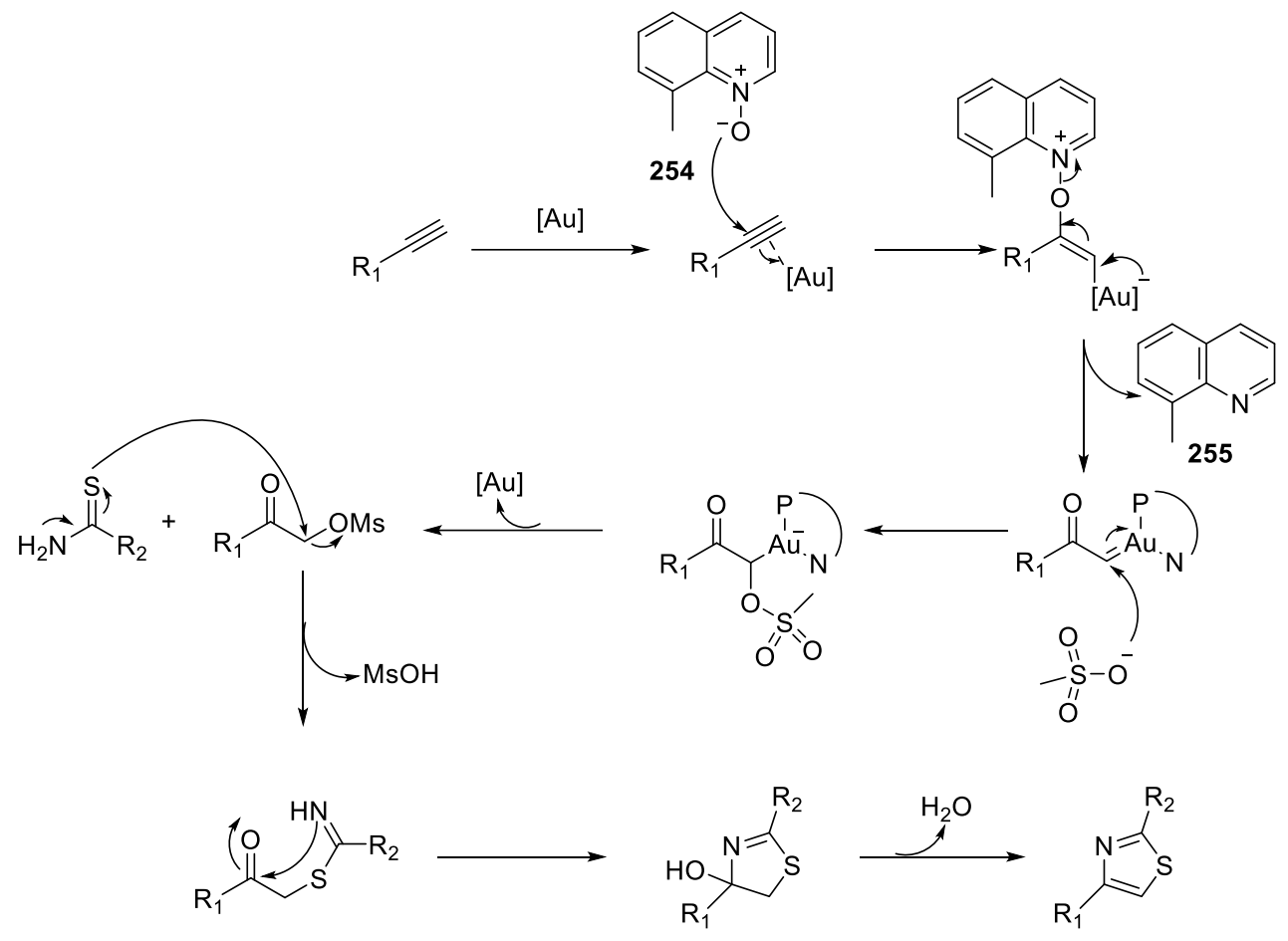

Scheme 2.18 Proposed mechanism of formation of 2,4-disubstituted thiazoles through trapping of an in-situ generated gold carbene by $\mathrm{MsO}^{-}$(modified from Zhang). ${ }^{141}$

\subsubsection{Synthesis of N-Oxide 254 and Mol-DalPhosAuOMs 257}

From a practical point of view, it is more cost-effective to prepare 8-methylquinoline $\mathrm{N}$ oxide 254 by oxidation of 8-methyl quinoline 255 than purchasing it from suppliers. ${ }^{\dagger \dagger}$ We first attempted using trifluoroperacetic acid, which was generated in situ from the reaction between urea hydrogen peroxide and trifluoroacetic anhydride in the presence of $\mathrm{K}_{2} \mathrm{CO}_{3},{ }^{217}$ to avoid the awkward purification associated with the use of $m$-CPBA. ${ }^{218}$ Unfortunately, these conditions were found to result in a sluggish reaction with little conversion, prompting us to go back to using the conventional oxidant $m$-CPBA to prepare $N$-oxide 254 for thiazole synthesis (Scheme 2.19). Following the procedure reported by Zhang and co-workers, ${ }^{141}$ Mol-DalPhosAuOMs 257 was prepared from commercially available Mol-DalPhosAuCl 256 in excellent yield (Scheme 2.19).

\footnotetext{
$\$(\mathrm{NZ}) 389$ for 5 grams of 8-methylquinoline $N$-Oxide 254, \$(NZ)67.50 for 5 grams of 8-methylquinoline. Prices taken from Sigma-Aldrich catalogue (February 2016). https://www.sigmaaldrich.com/newzealand.html
} 

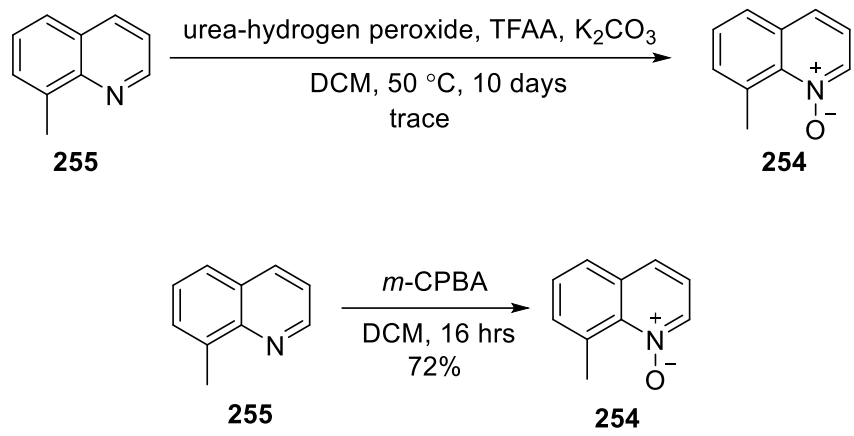

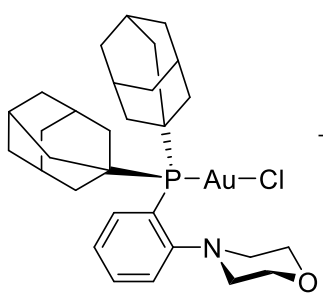

Mol-DalPhosAuCl

256

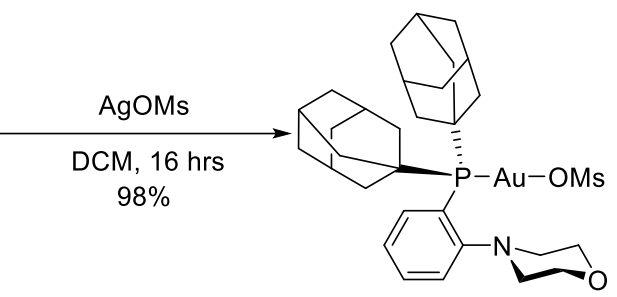

Mol-DalPhosAuOMs

257

Scheme 2.19 Preparation of 8-methyl quinoline $N$-oxide 254 and Mol-DalPhosAuOMs 257.

\subsubsection{Gold-catalysed Preparation of Thiazole 276}

As the $\alpha$-oxo gold carbene can be trapped with carboxylic acids, ${ }^{216}$ commercially available 6-heptynoic acid $\mathbf{2 5 8}$ was protected by esterification to afford the ethyl ester 259 (Scheme 2.19) which could serve as the C1 - C7 fragment (Fragment I, see Scheme 2.1) in the PatA analogue 183. In order to assess the compatibility of the ester alkyne substrate 259 with the gold-catalysed method, propanethioamide 261 was synthesised (Scheme 2.20) and used as the thioamide partner for model reactions. The one-pot thiazole formation reaction was performed following the conditions optimised by Zhang and co-workers. After the addition of the mixture of $\mathrm{MsOH}$ and $\mathrm{N}$-oxide 254 to the solution of alkyne 259 and Mol-DalPhosAuOMs by a syringe pump, the proton signals of the terminal alkyne at $1.95 \mathrm{ppm}$ disappeared, as determined by ${ }^{1} \mathrm{H}$ NMR analysis of the aliquot sample of the reaction mixture. This indicated that the alkyne substrate was consumed completely. Then propanethioamide $\mathbf{2 6 1}$ was added and the resulting mixture was heated at $40{ }^{\circ} \mathrm{C}$ for sixteen hours. To our delight, ${ }^{1} \mathrm{H}$ NMR analysis of the crude material showed an indicative singlet peak at $6.79 \mathrm{ppm}$, which was consistent with the reported proton signals of the 2,4-thiaozles prepared by Zhang and co-workers. ${ }^{141}$ This suggested that the formation of the desired thiazole should be successful. However, 
thiazole 262 was obtained in only $35 \%$ yield after purification by silica gel chromatography (Scheme 2.20).

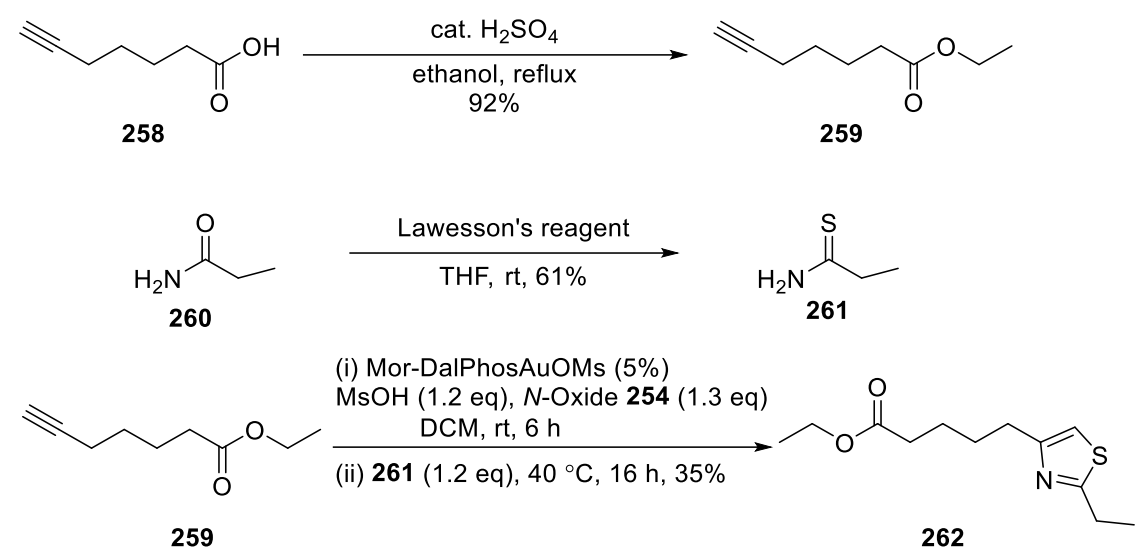

Scheme 2.20 Preparation of ester 259, propanethioamide 261 and thiazole 262.

Given that the oxygen atom of homopropargylic ether has been reported to be capable of trapping a gold carbene center, ${ }^{219}$ it was surmised that the nucleophilic oxygens in the ester functional group of alkyne $\mathbf{2 5 9}$ might also participate in intramolecular trapping of the gold carbene. To determine whether the low yield of thiazole 262 was attributed to the presence of the ester group in the alkyne substrate, 1-octyne 263 and alkyne $\mathbf{2 6 6}$, which was synthesised from 6-heptyn-1-ol 265 (Scheme 2.21), were chosen as substrates for comparative experiments. The gold-catalysed coupling reactions were carried out by postdoctoral fellow Claire Cuyamendous following the same conditions, and thiazole 264 and thiazole 267 accompanied with a small amount of inseparable unidentified byproducts were obtained in $49 \%$ and $34 \%$ yield, respectively (Scheme 2.21). 


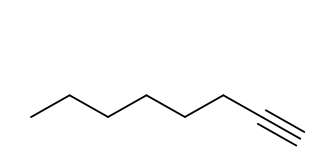

263

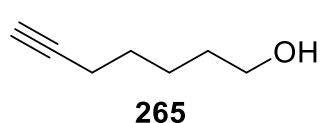

265

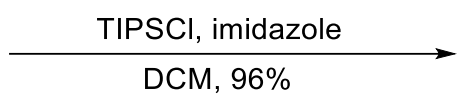

(i) Mor-DalPhosAuOMs (5\%)

$\mathrm{MsOH}$ (1.2 eq), $N$-oxide 254 (1.3 eq)

DCM, rt, $16 \mathrm{~h}$

(ii) 261 (1.2 eq), $40{ }^{\circ} \mathrm{C} 16 \mathrm{~h},<34 \%$

(i) Mor-DalPhosAuOMs (5\%)

DCM, rt, $16 \mathrm{~h}$

(ii) 261 (1.2 eq), $40{ }^{\circ} \mathrm{C}, 16 \mathrm{~h}, 49 \%$
$\mathrm{MsOH}$ (1.2 eq), $N$-oxide 254 (1.3 eq)

264

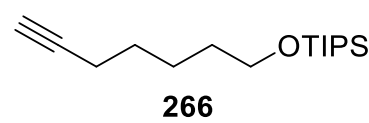

266

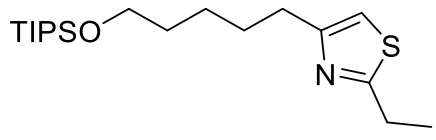

267

Scheme 2.21 Preparation of thiazoles 266 and 267.

Our yields for these reactions were lower than the reported yields of thiazoles prepared from alkynes with similar structural features (viz. thiazole 264 versus 270, thiazole 267 versus 272) (see Scheme 2.22). Therefore, we assumed that the introduction of a small amount of water from methanesulfonic acid, a notoriously hydroscopic chemical, could disrupt the generation of gold carbene, resulting in the low yields of thiazole formation.

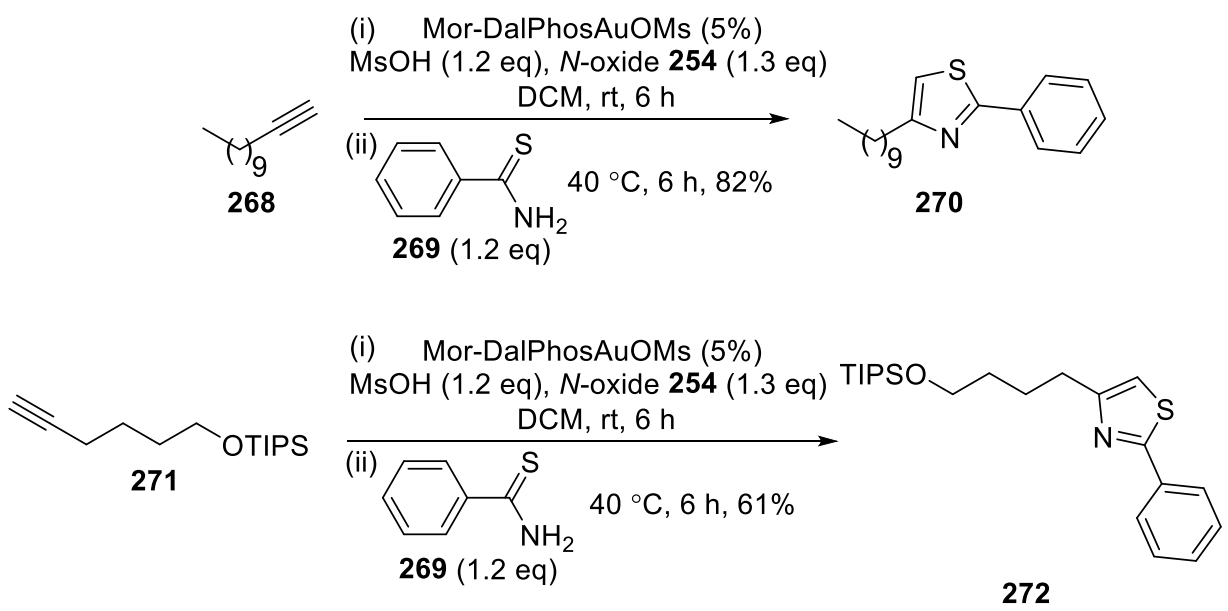

Scheme 2.22 Preparation of thiazole 270 and 272 reported by Zhang. ${ }^{141}$

Given that methanesulfonic acid is difficult to handle for small-scale reactions and is hard to purify, para-toluenesulfonic acid ( $p$-TSA) was considered to be a potential replacement, which was supported by the precedent literature about the formation of thiazoles via condensation reactions between $\alpha$-tosyloxy ketones and thioamides. ${ }^{220} p$-TSA was 
recrystallised from ethanol and water, and azeotropically dried with toluene to ensure that anhydrous $p$-TSA was used for the thiazole synthesis. Simultaneously, a new bottle of extra pure $\mathrm{MsOH}$ was purchased from Acros Organics, which was the exactly same supplier of $\mathrm{MsOH}$ for Zhang's work. As, in theory, $\alpha$-oxo gold carbenes only participate in the first stage - the formation of $\alpha$-mesyloxy ketones - experiments were stopped at the first stage to exclude the influence of thioamides. As shown in Table 2.7, using purified $p$-TSA did not give a better yield (entry 2). As expected, using a new bottle of $\mathrm{MsOH}$ provided a slight increase in the yield from $45 \%$ to $58 \%$ (compare entries 1 and 3 ), indicating that the water content level of $\mathrm{MsOH}$ was an influencing factor of the reaction yield.

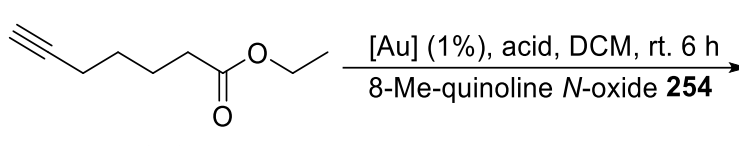

259

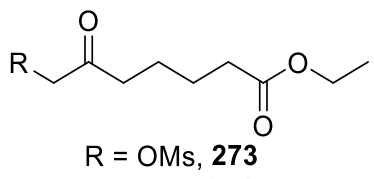

$\mathrm{R}=\mathrm{OTs}, 274$

\begin{tabular}{ccccc}
\hline Entry & [Au] & 254 (equiv.) & Acid (equiv.) & $\begin{array}{c}\text { Yield }^{\mathrm{a}} \\
(\mathbf{\%})\end{array}$ \\
\hline $\mathbf{1}$ & $\begin{array}{c}\text { Mor- } \\
\text { DalPhosAuOMs }\end{array}$ & 1.3 & $\mathrm{MsOH}^{\mathrm{b}}(1.2)$ & $\mathbf{2 7 3} 45$ \\
\hline $\mathbf{2}$ & $\begin{array}{c}\text { Mor- } \\
\text { DalPhosAuOMs }\end{array}$ & 1.3 & $p-\mathrm{TSA}^{\mathrm{c}}(1.2)$ & $\mathbf{2 7 4} 29$ \\
\hline $\mathbf{3}$ & $\begin{array}{c}\text { Mol- } \\
\text { DalPhosAuOMs }\end{array}$ & 1.3 & $\mathrm{MsOH}^{\mathrm{d}}(1.2)$ & $\mathbf{2 7 3} 58$ \\
\hline
\end{tabular}

${ }^{\mathrm{a}}$ Isolated yield after silica gel chromatography. ${ }^{\mathrm{b}}$ Old bottle of $\mathrm{MsOH}$ was used. ${ }^{\mathrm{c}}$ Recrystallised $p$-TSA was used. ${ }^{\mathrm{d}}$ New bottle of $\mathrm{MsOH}$ from Acros Organics was used.

Table 2.7 Investigation of gold-catalysed oxidation of alkyne $\mathbf{2 5 9}$ for using different acids.

As discussed by Zhang and co-workers, the gold carbene intermediate could be further oxidised to form an $\alpha$-ketoaldehyde by the remaining 8 -methylquinoline $N$-oxide. ${ }^{141}$ In order to avoid over-oxidation, it is necessary to add the mixture of oxidant and $\mathrm{MsOH}$ to the reaction vessel by a syringe pump to maintain a low concentration of the oxidant during the reaction, which could be a time-consuming process for a large-scale reaction. To overcome this deficiency, a modified one-pot methodology was developed by $\mathrm{Wu}$ and co-workers, in which ammonium sulfonate salt $\mathbf{2 7 5}$ was used as the trapping reagent for 
the gold carbene. ${ }^{221}$ The benefit of this methodology was that, except for the thioamides, other reaction components - alkyne, gold catalyst, oxidant, and ammonium sulfonate could be added into the reaction vessel at the same time. In addition, the low-acidity of the ammonium sulfonate makes this reaction more suitable for acid-sensitive substrates. Last, but not least, the yield was reported to be slightly improved over Zhang's results when using substrates $\mathbf{2 6 8}$ and $\mathbf{2 6 9}$ on a larger scale (86\% versus 82\%, see Scheme 2.22). Therefore, this new reaction system was tested with alkyne ester $\mathbf{2 5 9}$ and simple thioamide 261 in the hope that this could improve the yield of the thiazole formation.

To our dismay, using the modified methodology provided thiazole 262 in only $27 \%$ yield (Table 2.8, entry 1). Adding the solution of salt 275 and the oxidant 254 slowly by a syringe pump and raising the reaction temperature to $40{ }^{\circ} \mathrm{C}$ resulted in a drastic decrease in the yield (compare entries 1 and 2). It was not clear whether these uninspiring yields were due to changes in reaction temperature, addition rate or mesylate source. Therefore, previously optimised conditions using $\mathrm{MsOH}$ were replicated in the reaction between alkyne 259 and thioamide 261, and thiazole 262 was obtained in an improved yield (entry 3), albeit similar to those encountered by us earlier.

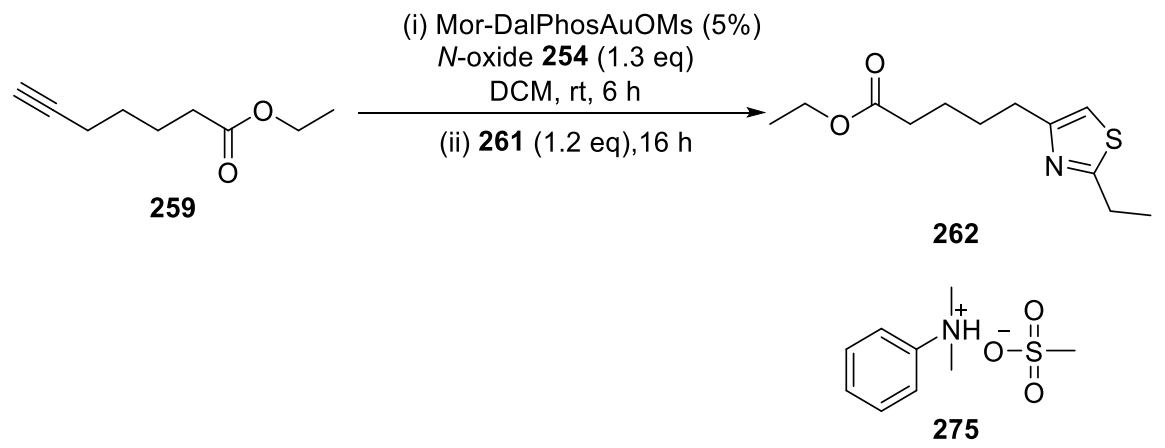

\begin{tabular}{ccccc}
\hline Entry & $\begin{array}{c}\text { Reagent } \\
\text { (equiv.) }\end{array}$ & $\begin{array}{c}\text { Addition via Syringe } \\
\text { Pump }\end{array}$ & $\mathbf{T}^{\mathbf{a}}\left({ }^{\mathbf{0}} \mathbf{C}\right)$ & Yield $^{\mathbf{b}}(\boldsymbol{\%})$ \\
\hline $\mathbf{1}^{\mathbf{c}}$ & $\mathbf{2 7 5}(1.2)$ & No & $\mathrm{rt}$ & 27 \\
\hline $\mathbf{2}^{\mathbf{c}}$ & $\mathbf{2 7 5}(1.2)$ & Yes & 40 & 3 \\
\hline $\mathbf{3}^{\mathbf{d}}$ & $\mathrm{MsOH}(1.2)$ & Yes & 40 & 53 \\
$\mathrm{a}_{\mathrm{rt}}=$ room temperature. & ${ }^{\mathrm{b}}$ Isolated yield of thiazole $\mathbf{2 6 2}$ after silica gel chromatography. ${ }^{\mathrm{c}}$ Salt $\mathbf{2 7 5}$ was prepared from \\
$N, N$-dimethylaniline and methanesulfonic acid. ${ }^{\mathrm{d}}$ New bottle of MsOH from Acros Organics was used.
\end{tabular}

Table 2.8 Reaction screening with different sources of $\mathrm{MsO}^{-}$. 
Since the effect of different solvents, which could play an important role in the performance of the gold catalyst, ${ }^{222}$ was not reported in Zhang's work, ${ }^{141}$ halogenated solvents-dichloromethane (DCM), dichloroethane (DCE), and chlorobenzene, which are widely used for gold catalysed oxidation of alkynes, were chosen for screening of conditions in the formation of thiazole 264 (Table 2.9).

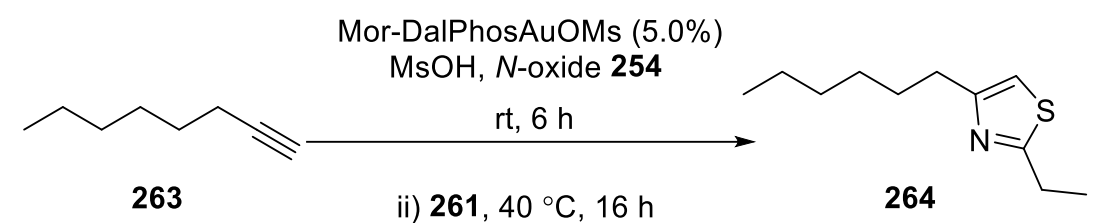

\begin{tabular}{|c|c|c|c|}
\hline Entry & $\mathrm{MsOH} / 254^{\text {a }}$ (equiv.) & Solvent & $\begin{array}{c}\text { Yield }^{b} \\
(\%)\end{array}$ \\
\hline 1 & $1.2 / 1.3$ & DCM & $62^{c}$ \\
\hline 2 & $1.2 / 1.3$ & DCE & 18 \\
\hline 3 & $1.2 / 1.3$ & $\mathrm{PhCl}$ & 54 \\
\hline
\end{tabular}

${ }^{\mathrm{a}} \mathrm{New}$ bottle of $\mathrm{MsOH}$ from Acros Organics was used. ${ }^{\mathrm{b}}$ Isolated yield after silica gel chromatography. ${ }^{\mathrm{c}}$ Yield was obtained by postdoctoral fellow Claire Cuyamendous.

Table 2.9 Reaction screening with different solvents.

From the results listed above, it was clear that DCM was the optimal solvent for this transformation, which was in agreement with $\mathrm{Wu}$ 's reported experimental observations in $2016 . .^{221}$

Despite the lack of major improvements in yield, we could still draw some useful information from all these optimisation attempts. It is worth noting that the yields of thiazole 262 (Table 2.8, entry 3) and mesylate 273 (Table 2.7, entry 3) are close to each other (53\% versus 58\%); and the yield of thiazole 264 (Table 2.9, entry 1) was significantly lower than the reported yield of thiazole 270 by Zhang (62\% versus $82 \%$ ). Therefore, we assumed that the possible cause of the inefficient catalytic results was that there are problems with the methodology when using aliphatic thioamides because the thioamide substrates used in Zhang and co-worker's reaction scope studies, apart from ethanethioamide and cyclohexanecarbothioamides, are all aromatic thioamides. Additionally, the interference of the two oxygen atoms of the ester functional group in alkyne 259 with the intermolecular trapping of $\alpha$-oxo gold carbene by $\mathrm{MsO}^{-}$could not be 
ruled out by our experimental results.

Nonetheless, our optimised conditions gave yields of up to $62 \%$ in the model reaction between alkyne 263 and thioamide 261 , which was sufficient to continue with their application to the preparation of desired thiazole $\mathbf{2 7 6}$.

The preparation of thiazole $\mathbf{2 7 6}$ was performed with modifications of Zhang's optimised conditions using functionalised thioamide 223 (Scheme 2.23, the loading of the gold catalyst was lowered to be $1 \mathrm{~mol} \%$ instead of $5 \mathrm{~mol} \%$ ). ${ }^{1} \mathrm{H}$ NMR investigation of the aliquot sample of the reaction mixture after 16-hours heating showed a sharp singlet peak at $6.25 \mathrm{ppm}$, which was considered to be an obvious sign of the formation of desired thiazole 276. To our delight, thiaozle 276 was obtained with a $61 \%$ yield, which was higher than the $30 \%$ yield of five steps required for the preparation of the $\alpha$-bromoketone 139 and thiazole 141 in Romo's synthesis of DMDA PatA (vide supra, Scheme 1.18 in Section 1.4.2.1). ${ }^{126}$

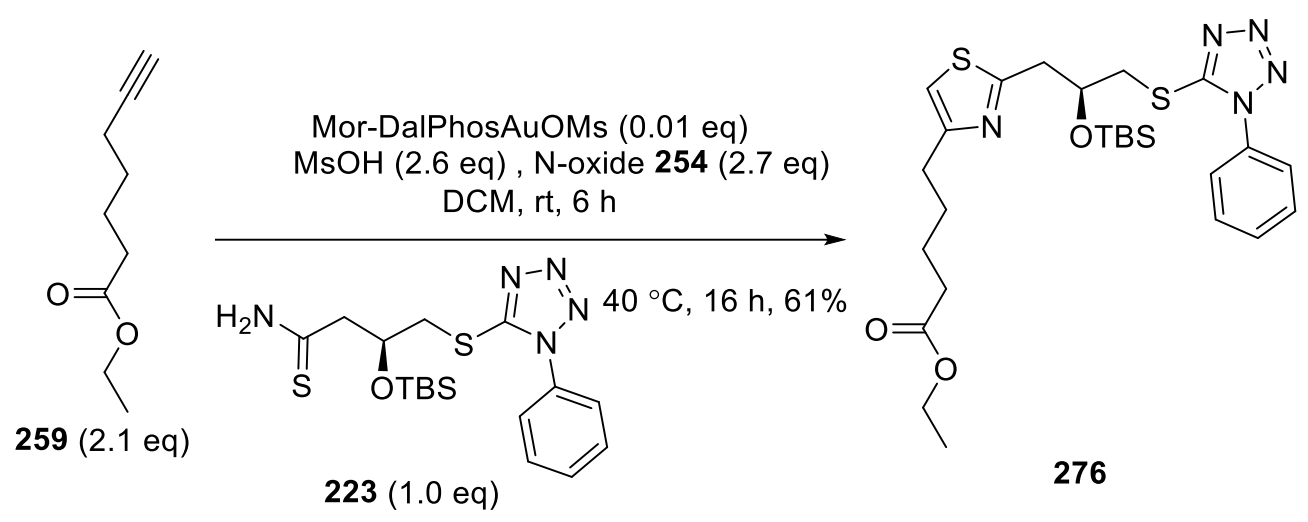

Scheme 2.23 Synthesis of thiazole 276 . 


\subsection{Coupling of Side Chain Fragment}
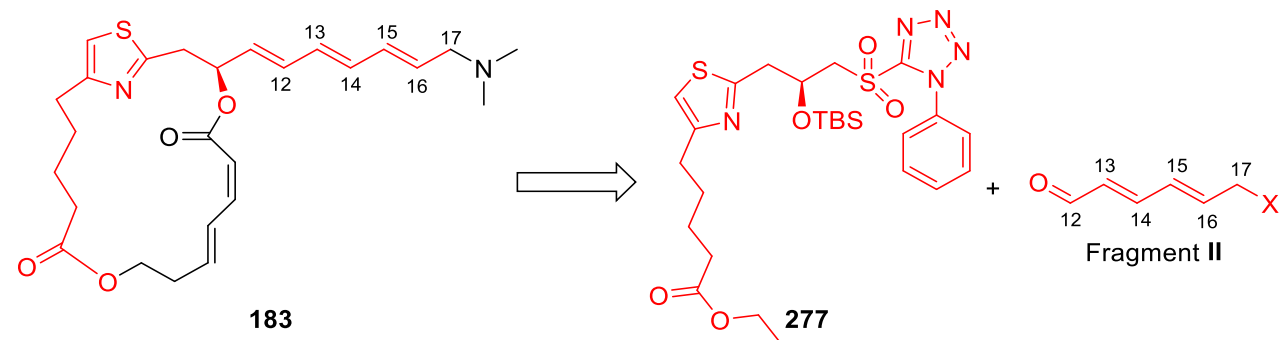

As discussed earlier in our retrosynthetic analysis in Section 2.1, Julia-Kocienski reaction between the thiazole-containing sulfone $\mathbf{2 7 7}$ and the fragment II was chosen for the attachment of the side chain fragment. 。

\subsubsection{Introduction}

The modified Julia-Kocienski reaction has emerged as one of the premiere alkene forming reactions in organic synthesis because of its tolerance of base-sensitive aldehyde substrates. ${ }^{223}$ As shown in Figure 2.7, the reaction mechanism begins with the metalation of phenyl tetrazolyl sulfone $\mathbf{2 7 8}$ with an appropriate metal base to give the nucleophilic sulfone anion 279, which couples with an aldehyde to form the $\beta$-alkoxysulfone (syn-280 or anti-280). This unstable intermediate then rearranges via a facile Smiles rearrangement to give sulfinate salt (281 or $\mathbf{2 8 2}$ ), which spontaneously eliminates sulfur dioxide and metallated phenyltetrazole to provide the alkene product. The $Z / E$-selectivity of the alkene product is highly dependent on the substrates, the base used to form the sulfone anion, the metal cation, and solvents in most cases. ${ }^{224}$ When larger metal cation $\left(\mathrm{K}^{+}\right)$and polar solvents are involved, the non-chelated open transition state becomes predominant and the transition state II is of lower energy because it lacks the unfavourable interaction between $\mathrm{R}$ and $\mathrm{R}^{\prime}$ which is present in the alternative transition state $\mathbf{I} .{ }^{225}$ Progression of the olefination process via the favoured transition state leads to the formation of antiintermediate alkoxide $\mathbf{2 8 0}$ and thence to the $E$-alkene product. In addition, the selectivity can also be influenced to some extent by the steric hindrance imparted by the phenyltetrazole in Smiles rearrangement (Figure 2.7). ${ }^{226}$ 


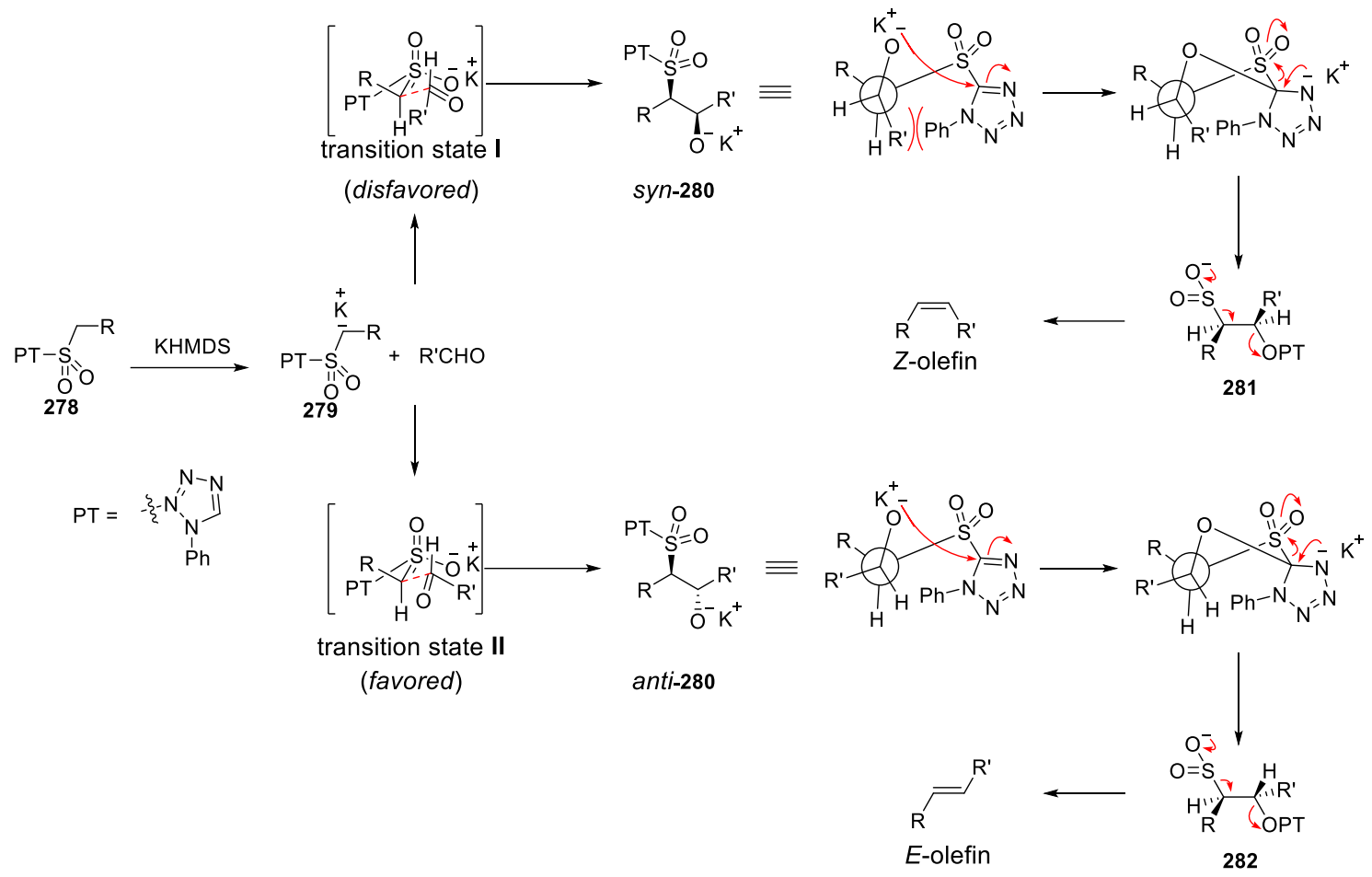

Figure 2.7 Proposed mechanism of Julia-Kocienski olefination and rationale for stereocontrol of olefins.

\subsubsection{Preparation of Sulfone 277}

With thiazole 276 in hand, we then set out to prepare sulfone 277 required for JuliaKocienski reaction. Thiazole $\mathbf{2 7 6}$ has two oxidisable sulfur atoms. We anticipated that the thioether sulfur atom would be the most prone to oxidation. In the preparation of sulfones from thioethers, up to 3 equivalents of $m$-CPBA are commonly used in the literature. However, to minimise unwanted oxidation at the sulfur atom of the thiazole ring, the oxidation of thioether $\mathbf{2 7 6}$ was first attempted with 2.0 equivalents of $m$-CPBA (Table 2.10 , entry 1). Interestingly, only the over-oxidised product 283 was isolated, ${ }^{\sharp}$ along with the recovery of unreacted starting material. The oxidation reaction was then tested in treatment with catalytic ammonium heptamolybdate and aqueous hydrogen peroxide as the terminal oxidant, a chemoselective oxidation method which has enjoyed widespread use for the synthesis of sulfones. ${ }^{227}$ Thankfully, the desired sulfone 277 was

$\$$ Characterised by HR-MS investigation. The base peak in the HR-MS spectrum of the isolated product was at $\mathrm{m} / \mathrm{z}=610.2181$, which matches with the calculated mass for compound $\mathbf{2 8 3}$ plus one hydrogen ion $\left(\mathrm{C}_{26} \mathrm{H}_{40} \mathrm{~N}_{5} \mathrm{O}_{6} \mathrm{~S}_{2} \mathrm{Si}^{+}[\mathrm{M}+\mathrm{H}]^{+} 610.2189\right)$. 
obtained as the sole product in $71 \%$ yield, albeit in a slower reaction rate (entry 2 ).

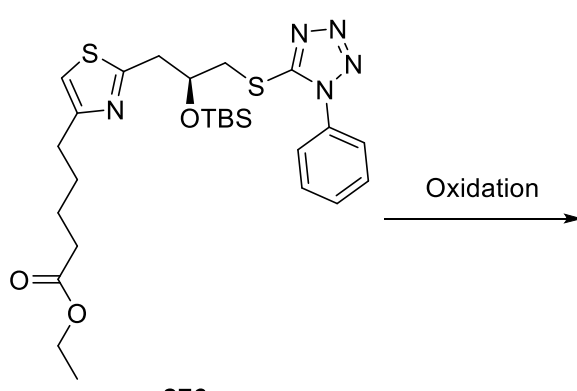

276

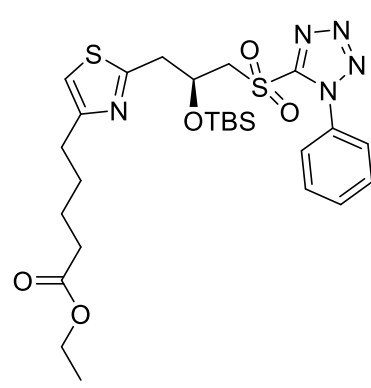

277

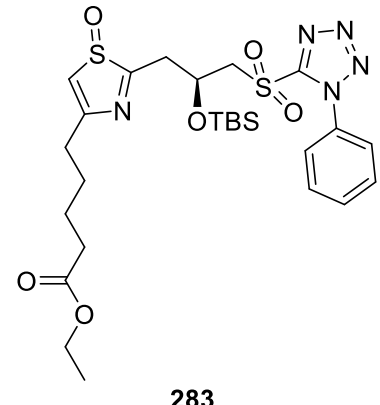

283

\begin{tabular}{|c|c|c|c|}
\hline Entry & Conditions (equiv.) ${ }^{\mathbf{a}}$ & Time (hrs) & Yield $^{b}$ \\
\hline 1 & $\begin{array}{c}m \text {-CPBA (2.0), } \mathrm{NaHCO} 3 \\
\text { (5.2), DCM, } 0{ }^{\circ} \mathrm{C} \text { to } \mathrm{rt}\end{array}$ & 18 & $28336 \%$ \\
\hline 2 & $\begin{array}{c}\mathrm{H}_{2} \mathrm{O}_{2}(30),\left(\mathrm{NH}_{4}\right)_{6} \mathrm{Mo}_{7} \mathrm{O}_{24} \\
(0.5) \text {, ethanol, } 0^{\circ} \mathrm{C} \text { to rt }\end{array}$ & 38 & $27771 \%$ \\
\hline
\end{tabular}

Table 2.10 Trialled conditions for the formation of sulfone 277 .

\subsubsection{Synthesis of Side Chain Fragment 297}

In Cumming's endeavours to synthesise thtriazole analogue 182 (see Figure 1.23 in Section 1.6), he found that reactions between sulfone 284 and aldehydes 285 or 287 under Julia-Kocienski olefination conditions did not provide desired triene products, but resulted in mixtures of degradation products (Scheme 2.24). He proposed that these failures were possibly due to the presence of an enolisable centre in these aldehyde substrates. Therefore, conjugated ester $\mathbf{2 8 9}$ was then trialled in his project under the same conditions and triene 290 was obtained with good $E$-selectivity. ${ }^{135}$ Informed by Cumming's work, we proposed that a non-enolisable conjugated fragment could serve as an appropriate substrate for Julia-Kocienski reaction in the synthesis of PatA analogue 183. 

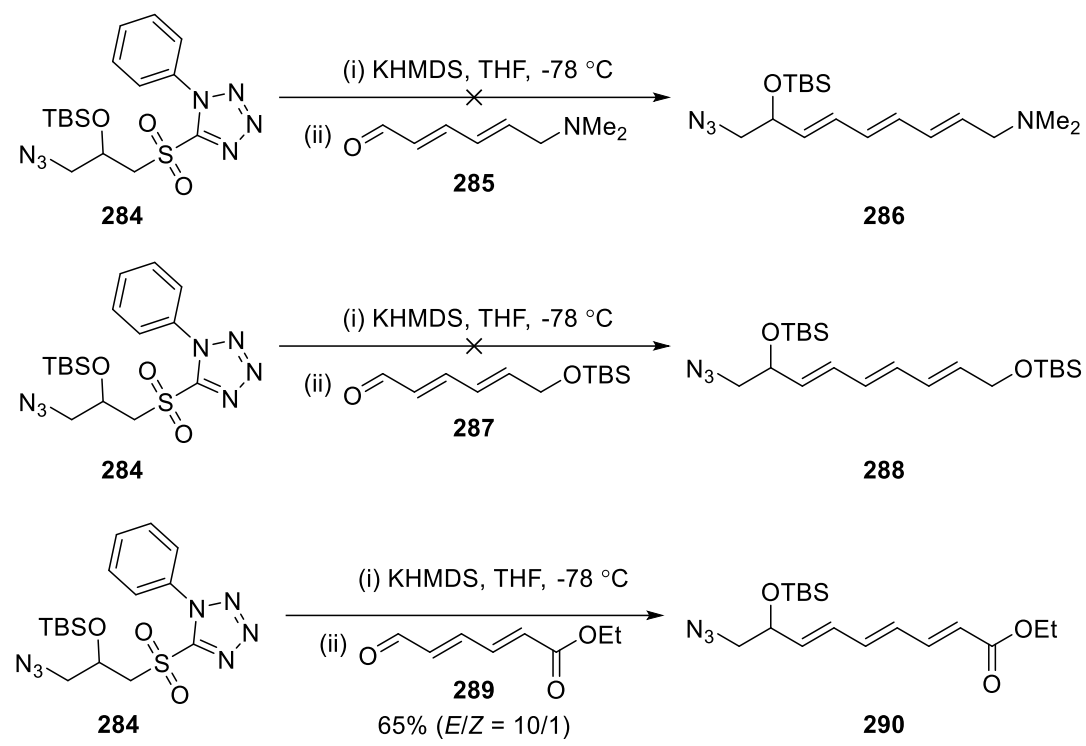

Scheme 2.24 Julia-Kocienski olefination with sulfone 284 and aldehydes in Cumming's previous work. $^{135}$

The synthetic strategy of the side chain fragment in Cumming's synthesis of the triazole analogue $\mathbf{1 8 2}^{134}$ was incorporated with modifications in the preparation of the desired fragment for coupling with sulfone 277. Cheap commercial available starting material furan 291 was treated with bromine and methanol to provide bis-dimethyl acetal 292 via oxidative ring-opening (Scheme 2.25), ${ }^{228}$ subsequent mono-deprotection catalysed by Amberlyst-15, a resin with strongly acidic sulfonic acid groups, in acetone and water gave aldehyde 293, and Wittig reaction with stabilised triphenylphosphoryl ylide 294 (prepared from 2-bromoacetate and triphenylphosphine, see Section 6.2.3 for experimental details) provided $E, E$-dienoate $\mathbf{2 9 5}$ as the major product in good yield. The attempted partial reduction of methyl ester 295 to aldehyde 297 using one equivalent of DIBAL-H resulted in a mixture of alcohol 296 and aldehyde 297. The methyl ester 295 was instead subjected to a two-step protocol. Reduction to the corresponding primary alcohol 296 occurred in 71\% yield. Considering that the acetal was sensitive to the acidic environment, pyridine was used as an acid scavenger in the following Dess-Martin oxidation, and the aldehyde $\mathbf{2 9 7}$ was obtained in $17 \%$ yield over 5 steps. It was proposed that enal 297 was unlikely to be able to enolise under Julia-Kocienski olefination conditions. Therefore, enal $\mathbf{2 9 7}$ was targeted as a potential candidate for the side chain fragment, which would also allow progress to the terminal dimethyl amine for PatA analogue synthesis. 


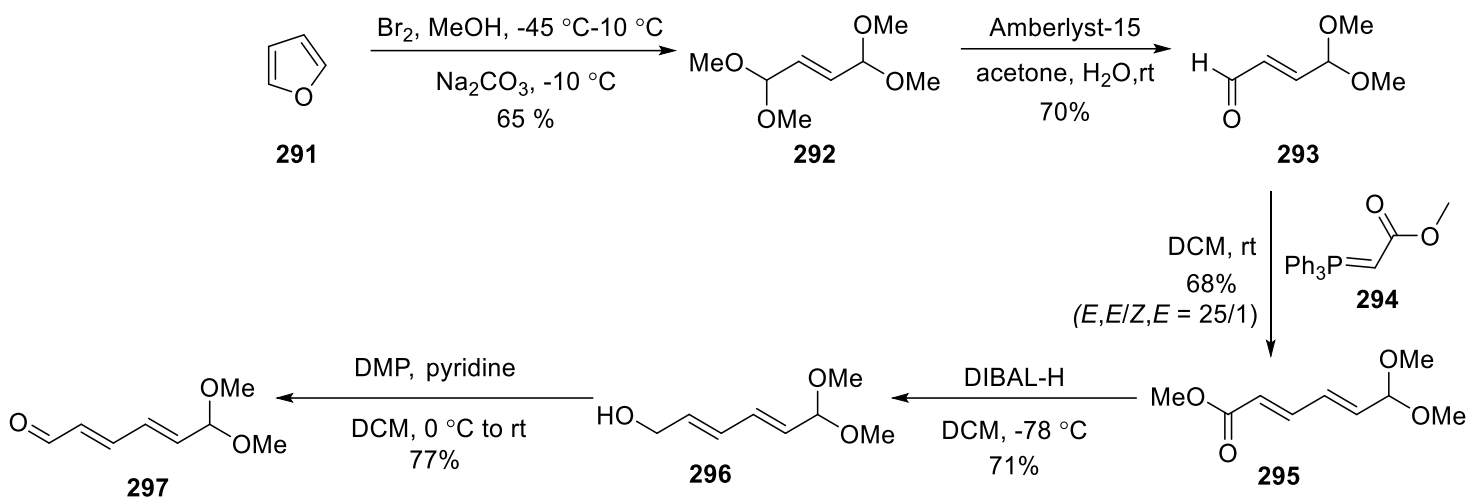

Scheme 2.25 Synthesis of enal 297.

It is worth mentioning that the acetal functional group of enal 297 rapidly deprotected in deuterated chloroform used for NMR analysis. This unexpected deprotection process was prompted by the tiny amount of $\mathrm{HCl}$ impurity generated by chloroform degradation in air, indicating that the terminal acetal could be converted into the terminal aldehyde when required by very mild acidic conditions. The acid-lability of the acetal would be beneficial for the late-stage progression of PatA analogues.

\subsubsection{Model Julia-Kocienski Olefination}

Given that there are few published examples of enals containing acetal functional groups that successfully couple in Julia-Kocienski reactions, sulfones 298 and 299 were prepared by oxidation of corresponding thioethers 215 and 222 with hydrogen peroxide and catalytic ammonium heptamolybdate (Scheme 2.26), which would allow assessment of the performance of enal 297 in model versions of this olefination method.
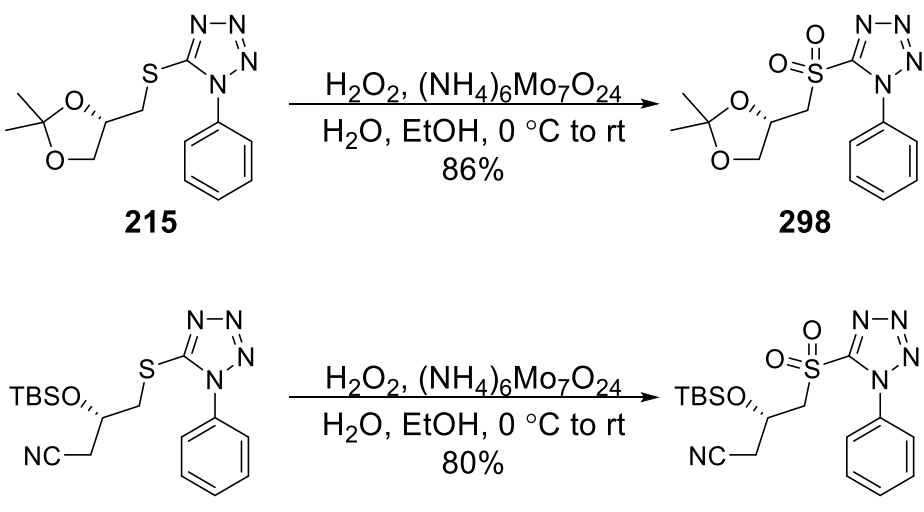

222

Scheme 2.26 Preparation of sulfone 298 and 299. 
Presuming that enal 297 could be unstable in a strongly basic environment, KHMDS used in pre-treating the sulfones was strictly limited to one equivalent to ensure that no excess KHMDS would be present in the reaction vessels when the enal 297 was added to couple with the deprotonated sulfone partners. Gratifyingly, the Julia-Kocienski reactions with sulfones 298 and 299 afforded triene products $\mathbf{3 0 0}$ and $\mathbf{3 0 1}$ in inseparable $(E, Z)$ mixtures with good $E$-selectivity, respectively (Scheme 2.27).
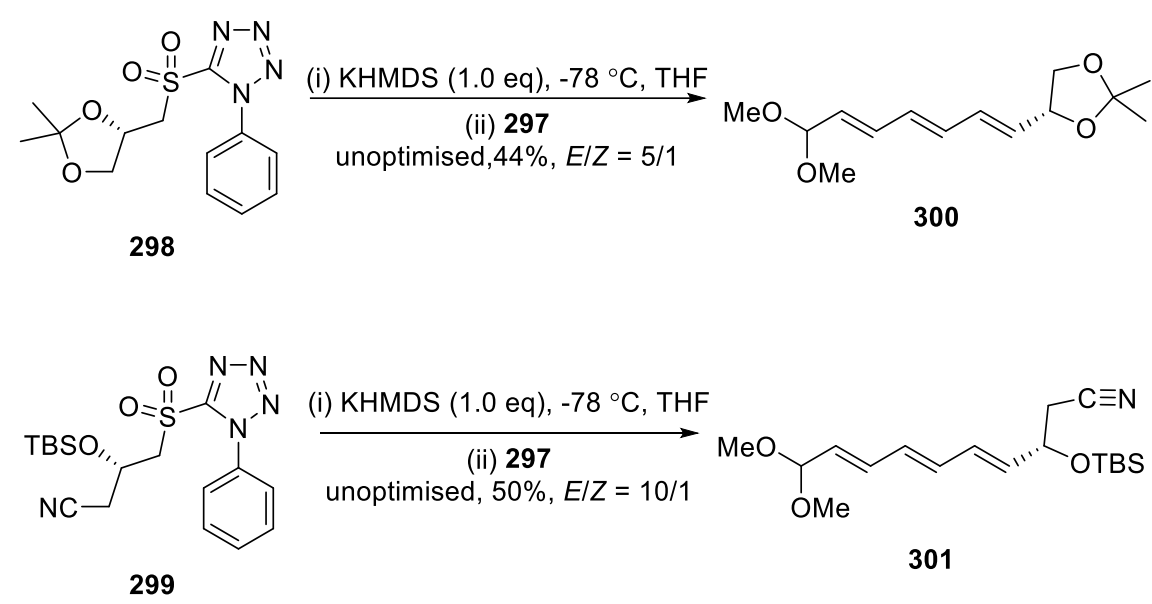

Scheme 2.27 Model Julia-Kocienski reactions with sulfones 298 and 299.

Encouraged by these successful results, we then attempted coupling sulfone 277 with enal 297 using the same conditions (Scheme 2.28). Disappointingly, instead of desired triene product 302, a mixture of unidentified products was obtained, which were possibly derived from degradation of enal 297.

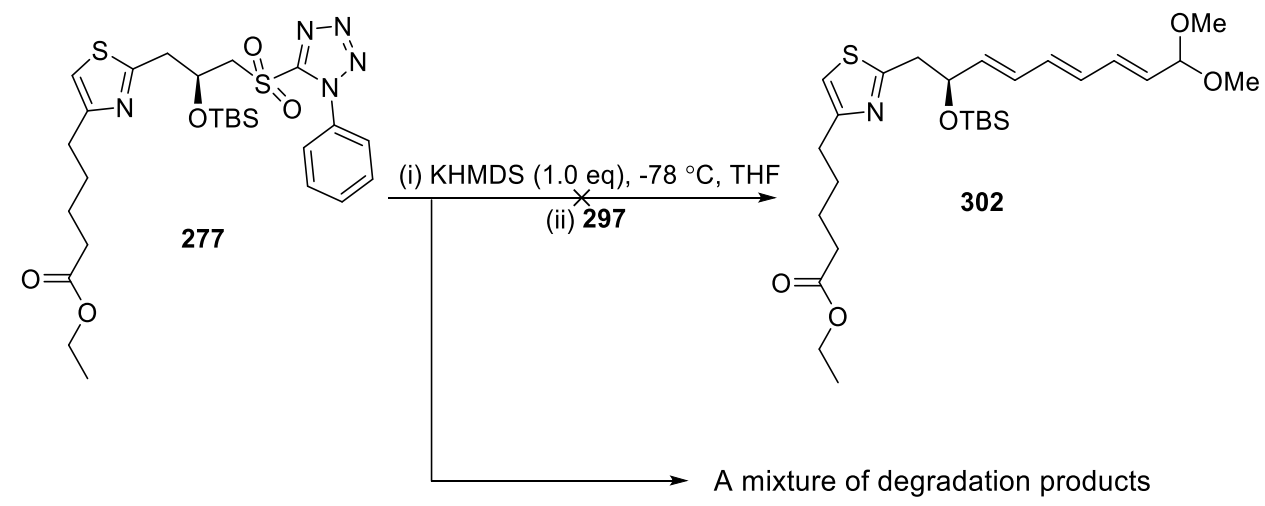

Scheme 2.28 Attempted Julia-Kocienski reaction with sulfone 277 and enal 297.

There are some reported examples of aldehydes containing 2,4-disubstituted thiazole moieties that were efficiently reacted with sulfone partners under Julia-Kocienski's 
optimised conditions, for example, the coupling of aldehyde $\mathbf{3 0 3}$ with sulfone 304 to provide diene $\mathbf{3 0 5}$ in the synthesis of epothilone (Scheme 2.29). ${ }^{229}$ Therefore, the interference of thiazole with the reaction between enal 297 and the sulfone anion derived from $\mathbf{2 7 7}$ was presumed not to be the major cause of the failure.

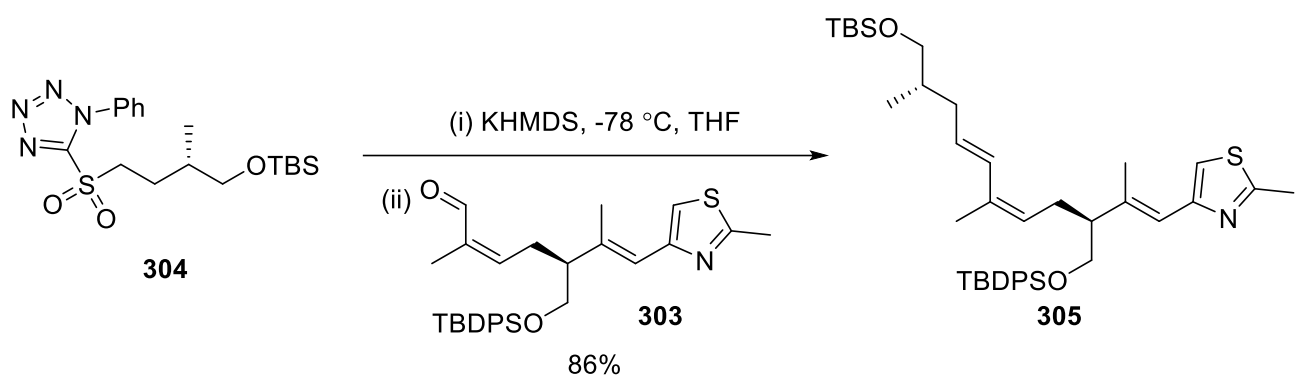

Scheme 2.29 Julia-Kocienski reaction of thiazole-containing aldehyde 303 in the synthesis of epothilone. ${ }^{229}$

It was of note that sulfone starting material 277 could be partially recovered and the JuliaKocienski reaction of TBS-protected sulfone 299 had provided the olefination product 301 with enal 297 in moderate yield (Scheme 2.27). It was then proposed that the steric effect of the protecting group in sulfone 277 could be enhanced dramatically by the presence of a relatively rigidified thiazole ring, which would make access of the bulky base KHMDS to the proton at the $\alpha$-position of the sulfone slow. Consequently, the nucleophilic sulfone anion was probably not produced in the first phase, and then the treatment of enal 297 with unreacted KHMDS could have led to significant degradation. Hence, we assumed that using a free hydroxy group or replacing the TBS group with a less bulky protecting group could benefit the deprotonation process. In view of the step economy, it would be ideal that the Julia-Kocienski reaction could work with an unprotected hydroxyl group.

To our delight, a scan through the literature disclosed that Omura and co-workers, en route to the synthesis of nafuredin, utilised sulfone $\mathbf{3 0 6}$ with a free hydroxyl group in their pivotal Julia-Kocienski reaction (Scheme 2.30). 


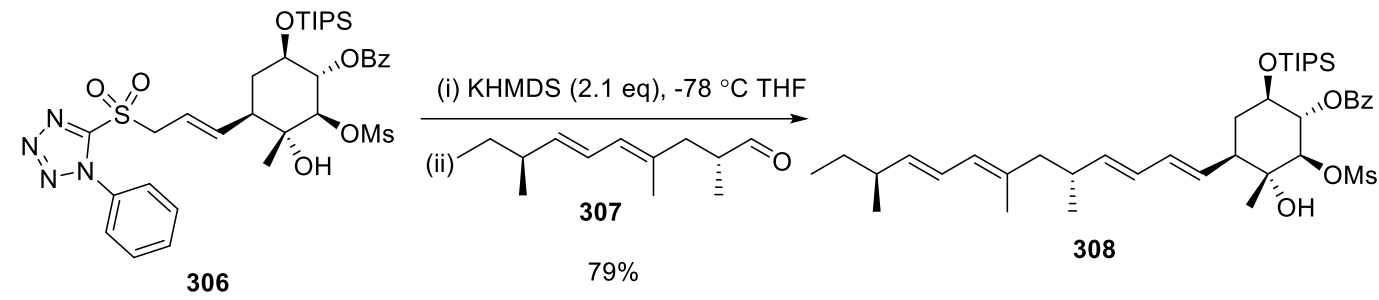

Scheme 2.30 Julia-Kocienski reaction with sulfone $\mathbf{3 0 6}$ containing free -OH group. ${ }^{230}$

Therefore, sulfone 277 was desilylated by HF-pyridine to provide sulfone $\mathbf{3 0 9}$ (Scheme 2.31). To test whether the removal of the TBS group was beneficial for the JuliaKocienski reaction, we attempted to prepare the deuterated sulfone 310 first, which would be unequivocal evidence that the efficiency of the deprotonation process had been improved. Interestingly, no desired deuterated product $\mathbf{3 1 0}$ was observed, and instead, allylic thiazole 311, the structure of which was determined by 2D NMR and HR-MS investigation, was obtained in moderate yield, (Scheme 2.31). This experimental result suggested that compound $\mathbf{3 0 9}$ containing a free -OH group was not an appropriate sulfone partner for coupling with enal fragment 297 under Julia-Kocienski conditions.

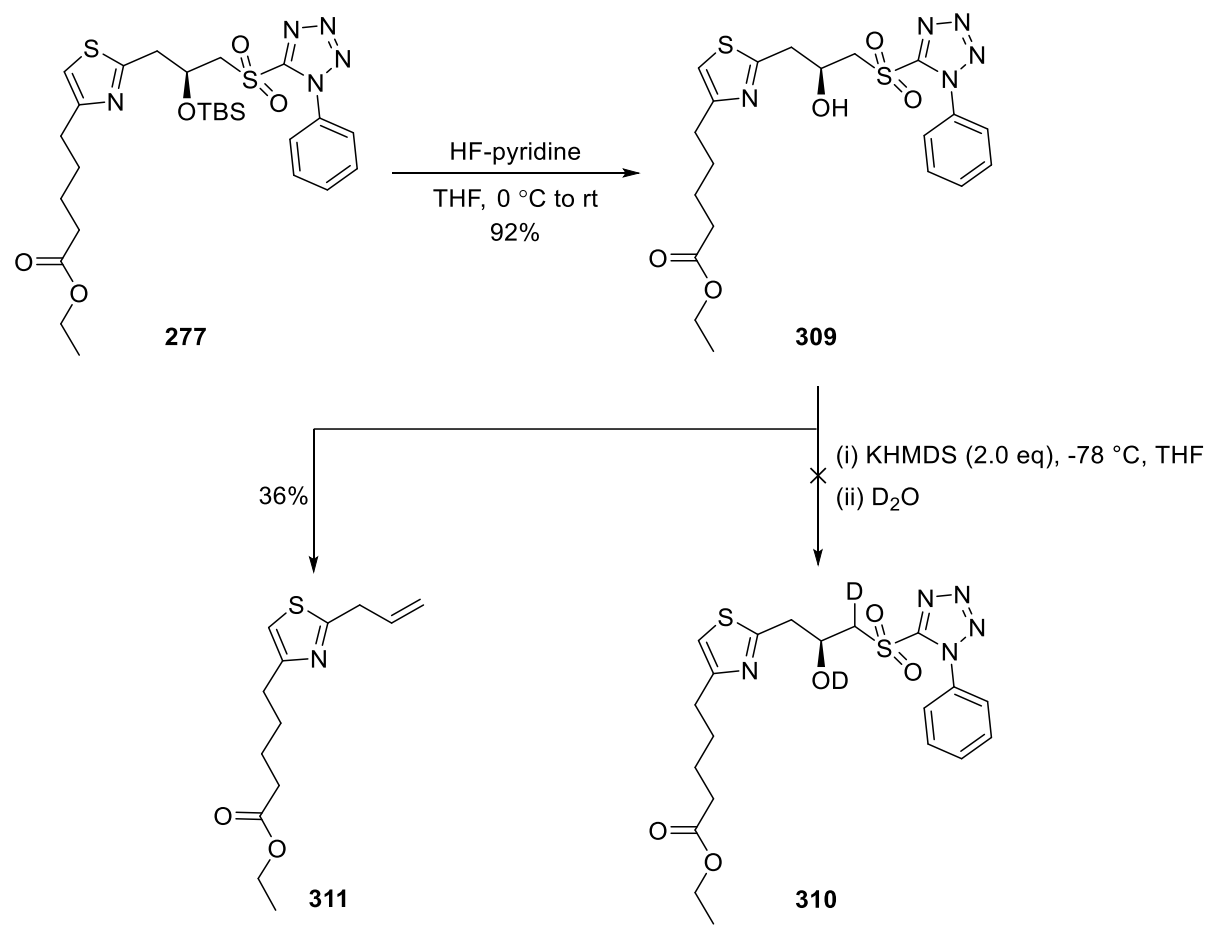

Scheme 2.31 Attempted preparation of deuterated sulfone $\mathbf{3 1 0}$.

On the basis of the proposed mechanism of Julia-Kocienski olefination, as shown in Scheme 2.32, we conceived that unstable $\beta$-alkoxide $\mathbf{3 1 2}$ derived from sulfone $\mathbf{3 0 9}$ may 
have experienced similar Smiles rearrangement via the formation of a spirocyclic intermediate $\mathbf{3 1 3}$ to give the unexpected eliminated thiazole $\mathbf{3 1 1}$.

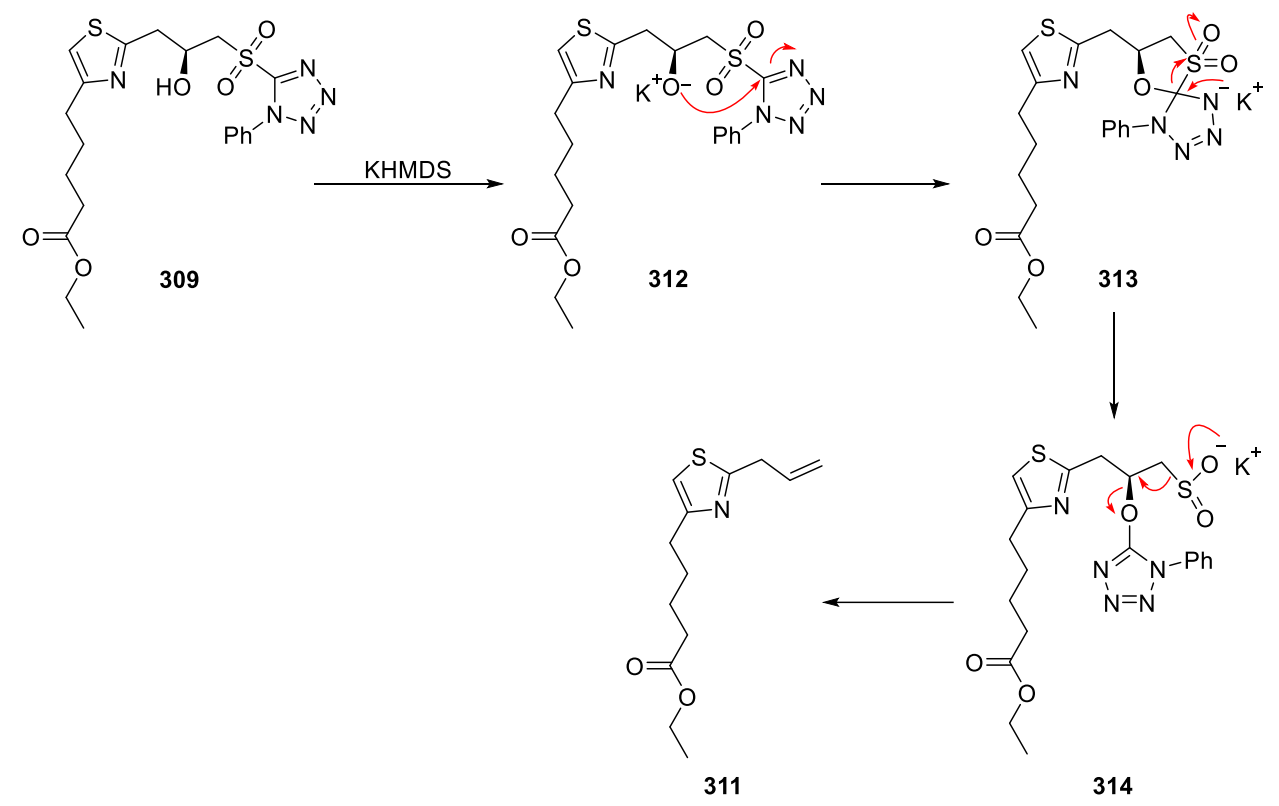

Scheme 2.32 Proposed mechanism for the formation of thiazole 311 .

To avoid the tedious deprotection-protection at this stage, we then opted to go back to replace the protecting group at an early stage (Scheme 2.33). Given that the methoxymethyl (MOM) group is considered to be less bulky than the TBS group, we attempted to prepare the methoxymethyl ether $\mathbf{3 1 5}$ following classic conditions ${ }^{231}$ for the preparation of methoxymethyl (MOM)-protected sulfone 316. However, this resulted in a mixture of unidentified products, with no sign of the desired compound $\mathbf{3 1 5}$ observed by ${ }^{1} \mathrm{H}$ NMR and HRMS analysis.

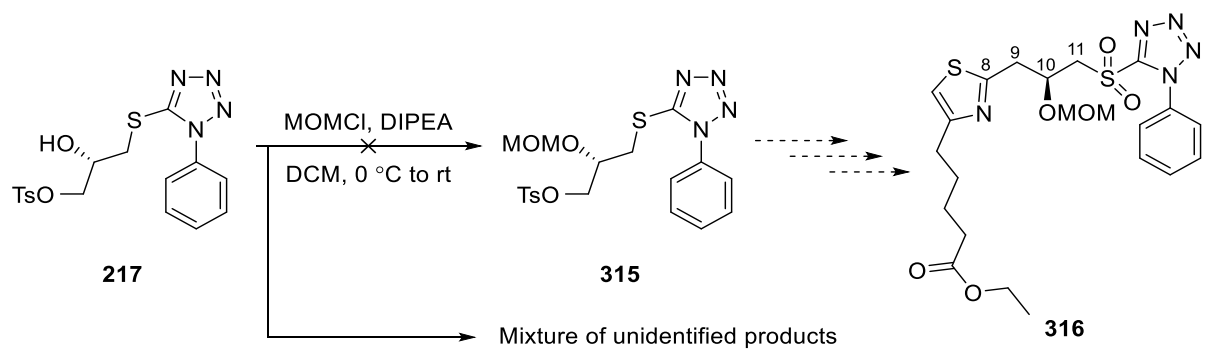

Scheme 2.33 Attempted preparation of methoxymethyl protected alcohol 315 for the synthesis of sulfone 316. 


\subsection{Revised Synthesis of Sulfone 316}

Given that the formation of methoxymethyl ether $\mathbf{3 1 5}$ in the early stage was unsuccessful, it was necessary to develop a new synthetic route for sulfone $\mathbf{3 1 6}$ which could be a suitable substrate for the Julia-Kocienski olefination. The present synthetic route utilised a chemist-unfriendly cyanation step for one-carbon homologation, which required an awkward procedure for quenching cyanide residue. Besides, the one-step conversion of epoxide 213 to acetonide 214 resulted in a degree of racemisation at the stereocentre based on Mosher analysis (vide supra, Section 2.2.3.2). To overcome these disadvantages, a chiral thioamide $\mathbf{3 1 7}$ that possesses sites for the connection with both alkyne fragment 259 and enal fragment 297 was targeted as a potential building block for the alternative route (Scheme 2.34).

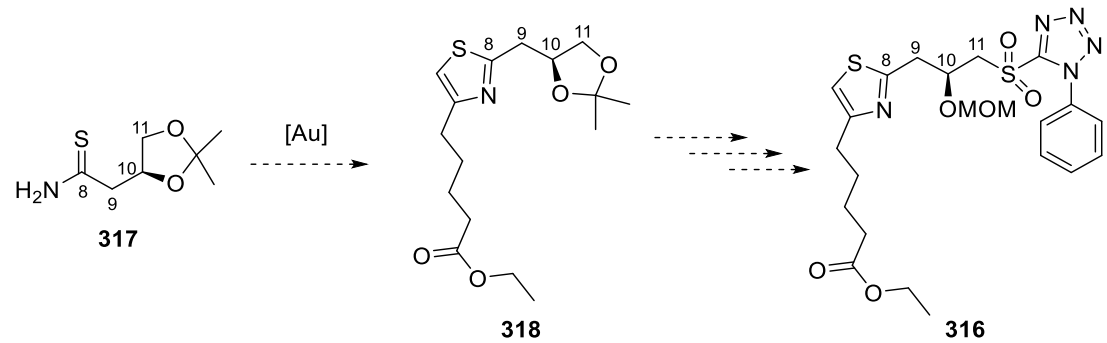

Scheme 2.34 Proposed synthetic route for sulfone 316 starting from thioamide 317.

\subsubsection{Attempted Synthesis of Thioamide 317}

In 2014, Snowden and co-workers reported that the transformation of aldehydes into onecarbon homologated amides could be achieved in two steps via a Jocic-Reeve type reaction. ${ }^{232,233}$ As shown in Scheme 2.35, the plausible reaction mechanism starts with the trichloromethylation of an aldehyde. The trichloromethyl carbinol intermediate is then slowly deprotonated by $\mathrm{NaOH}$ in a protic solvent to form a highly electrophilic gemdichloroepoxide 319, which is then quickly regioselectively substituted by the phenylseleno(triethyl)borate complex, a nucleophile prepared in situ by mixing diphenyl diselenide and $\mathrm{NaBH}_{4}$ in argon-purged ethanol. The opening of epoxide gives a reactive acid chloride intermediate, which allows for rapid acyl substitution by the available amine in the reaction system. The resultant $\alpha$-phenylselenoamide $\mathbf{3 2 0}$ slowly undergoes in situ deselenylation to afford the enolate intermediate, which is immediately protonated by the protic solvent to provide a one-carbon homologated amide product. 

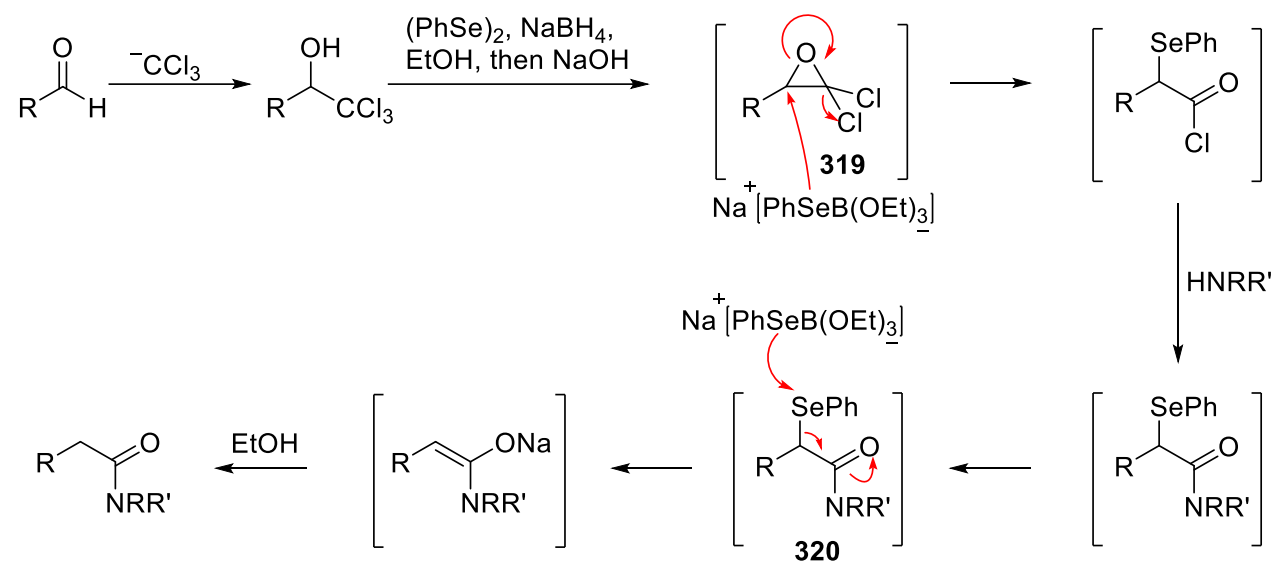

Scheme 2.35 Proposed mechanism of homologated amide formation via gemdicholoroepoxide by Snowden and co-workers. ${ }^{232}$

The typical transformation of aldehydes to one-carbon homologated amides involves reduction to primary alcohols, conversion to leaving groups, substitution by cyanides and nitrile hydration to amides. In comparison, the benefit of this novel method is that it can avoid the use of hazardous cyanide and furnish the transformation in two steps. In addition, based on Snowden and co-worker's experimental observations, diphenyl diselenide used in the reaction could be completely recovered after work-up procedures and the recycled diphenyl diselenide could be reused in the subsequent homologations without decrease in reaction efficiency, making this two-step reaction sequence to be an environmental benign methodology. Furthermore, this approach was found to have broad substrate scope and be capable of converting enolisable aldehydes into the corresponding one-carbon homologated amides without racemisation. For example, butanamide 321, a potential precursor for thioamide $\mathbf{3 1 7}$, was reported to be conveniently obtained in $51 \%$ yield with high optical purity (Scheme 2.36). Therefore, we opted to utilise this novel synthetic method, which would enable a more step-economic synthesis for thioamide $\mathbf{3 1 7}$.

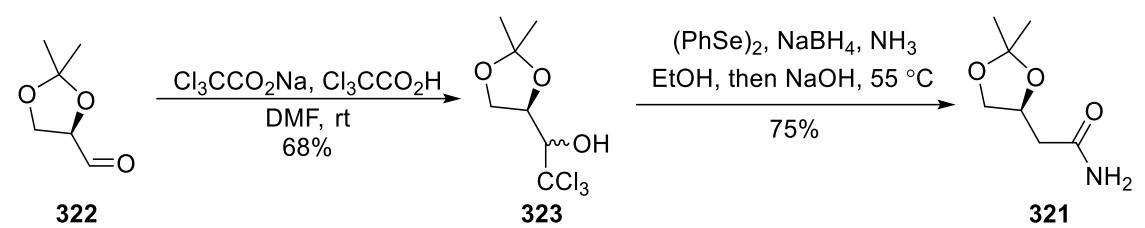

Scheme 2.36 Preparation of chiral amide 321 from aldehyde 322 reported by Snowden. ${ }^{232}$ 


\subsubsection{Preparation of Trichloromethyl Carbinol 323}

It was reported that the monomeric stability of chiral aldehyde $\mathbf{3 2 2}$ is highly dependent on storage conditions and its polymerisation process can cause a slight deviation in its optical purity. ${ }^{234}$ Also, from a practical point of view, it is more sustainable to prepare chiral aldehyde 322 from cheap starting material D-mannitol $\mathbf{3 2 4}^{\S \S}$ following the highly reliable two-step protocol reported by Schmid and co-workers. ${ }^{234}$ Thus, we decided to use freshly prepared aldehyde $\mathbf{3 2 2}$ rather than purchasing it from suppliers. As shown in Scheme 2.37, D-mannitol 324 was first ketalised with catalytic stannous chloride and 2,2dimethoxypropane to provide diacetonide $\mathbf{3 2 5}$, followed by oxidative cleavage, providing aldehyde 322. The high optical purity of aldehyde $\mathbf{3 2 2}$ was determined by the comparison between the observed specific rotation and reported specific rotation in previous literature (See section 6.2.3 for details).

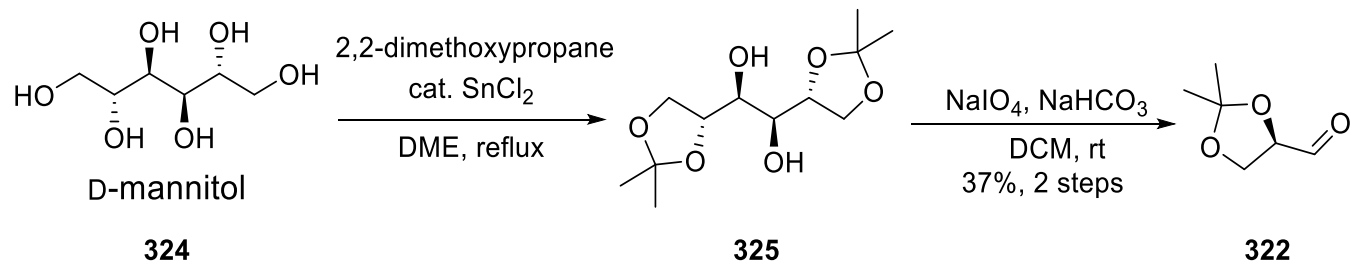

Scheme 2.37 Preparation of chiral aldehyde 322 from D-mannitol 324.

Based on the proposed mechanism by Snowden and co-workers (Scheme 2.35), neither the trichloromethyl carbinol nor the homologated amide should be enolisable in the basic reaction conditions as the stereogenic hydrogen in either compound lacks activation by an adjacent electron-attracting functional group (e.g., the carbonyl group in the aldehyde). Thus, in terms of stereochemical fidelity, the only step of concern was the trichloromethylation of aldehyde 322. As reported by Snowden, the mild buffered CoreyLink trichloromethylation protocol ${ }^{235}$ was utilised to retain the enantiomeric purity, giving a mixture of diastereomers $\mathbf{3 2 3}$ in $81 \%$ yield (Scheme 2.38).

\footnotetext{
${ }^{\S}$ Chiral aldehyde 322, \$(NZ)134 for 0.5 gram, compared to D-mannitol, \$(NZ)64 for 500 grams. Prices taken from Sigma-Aldrich catalogue (July 2017). https://www.sigmaaldrich.com/new-zealand.html
} 


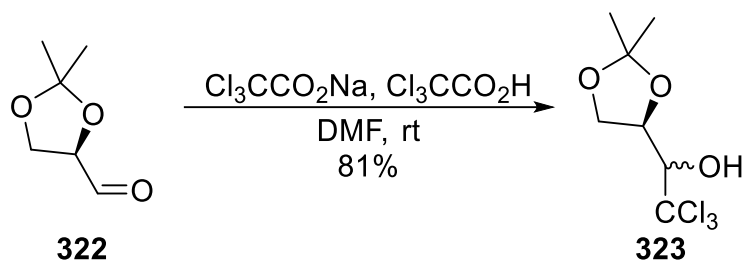

Scheme 2.38 Preparation of trichloromethyl carbinol 323.

\subsubsection{Preparation of Butanamide 321}

As reported in Snowden and co-workers' experimental procedures, ethanol saturated by gaseous ammonia was used as the reaction media in their synthesis of butanamide 321 . Given that an ammonia cylinder was unavailable in our laboratory and the reaction was performed in a sealed pressure tube, we initially reckoned that commercially available ammonia solution in ethanol could be a feasible substitute. Disappointingly, after three days, the reaction had not gone to completion even when excess ammonia solution (2.0 $\mathrm{M}$ in ethanol) was used, and the butanamide 321 was obtained in poor yield (Scheme 2.39), suggesting that the high concentration of ammonia could be essential to help outcompete other nucleophiles in the reaction system $\left(\mathrm{PhSeB}(\mathrm{OEt})_{3}^{-}, \mathrm{HO}^{-}, \mathrm{EtO}^{-}, \mathrm{Cl}^{-}\right)$. Therefore, exploring an alternative synthetic method for the amide $\mathbf{3 2 1}$ was deemed necessary.

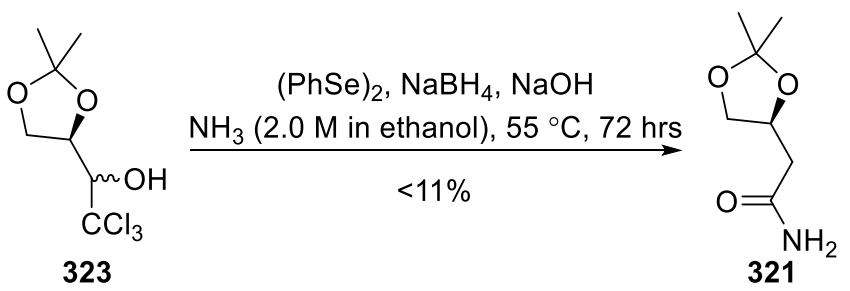

Scheme 2.39 One-step synthesis of amide 321.

A scan through Snowden and co-workers' research related to transformations of trichloromethyl carbinols revealed that they could be also converted to one-carbon homologated carboxylic acid in one-step via the similar Jocic-Reeve type mechanism. ${ }^{236}$ Motivated by the fact that methyl ester $\mathbf{3 2 6}$ was synthesised via carboxylic acid intermediate 327 with no detectable decrease in e.e. (Scheme 2.40), our attention was focused on a potential alternative approach to amide 321, which involved aminolysis of an anhydride intermediate. 


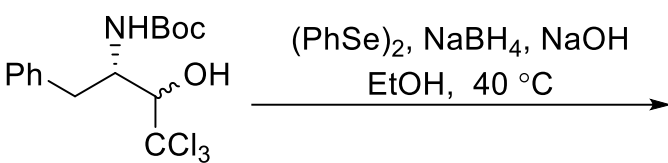

328

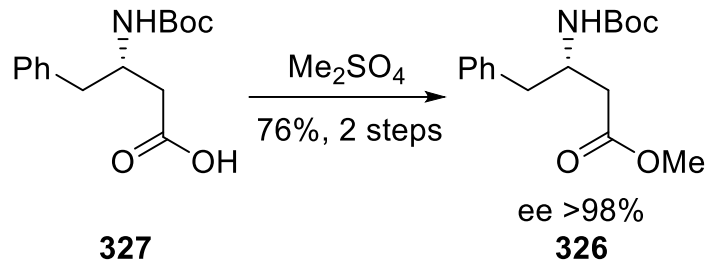

326

Scheme 2.40 Preparation of chiral methyl ester 326 reported by Snowden and co-

$$
\text { workers. }^{236}
$$

Following Snowden and co-workers' optimised conditions, ${ }^{236}$ trichloromethyl carbinol 323 was treated with a mixture of diphenyl diselenide, $\mathrm{NaBH}_{4}$ and $\mathrm{NaOH}$ in argon-purged ethanol at $40{ }^{\circ} \mathrm{C}$. The reported chemical shifts of $\alpha$-protons of carboxylic acid $\mathbf{3 2 9}$ ((300 $\left.\left.\mathrm{MHz}, \mathrm{CDCl}_{3}\right) \delta 2.71(\mathrm{dd}, J=16.2,6.7 \mathrm{~Hz}), 2.53(\mathrm{dd}, J=16.2,6.8 \mathrm{~Hz})\right)^{237}$ were used as the reference in monitoring the formation of the acid by ${ }^{1} \mathrm{H}$ NMR analysis of reaction aliquots. After three-day heating, although the starting material was consumed completely based on observation by TLC plates, there was no evidence of the formation of the desired acid $\mathbf{3 2 9}$ over the reaction period (Scheme 2.41).

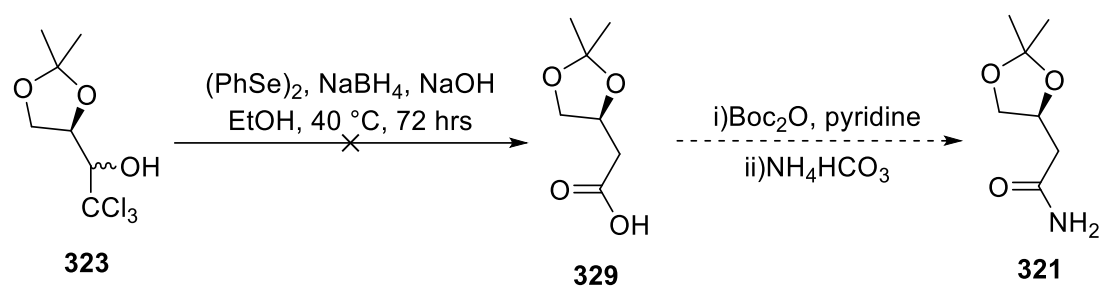

Scheme 2.41 Attempted synthesis of amide 321 via carboxylic acid intermediate 329.

With the failed preparation of the carboxylic acid 329 after multiple attempts, primary alcohol 330, which could be prepared via reductive homologation of the trichloromethyl carbinol 323, ${ }^{238}$ was targeted as the appropriate precursor for acid $\mathbf{3 2 9}$.

Gratifyingly, treatment with a mixture of $\mathrm{LiBH}_{4}$ and $\mathrm{NaOH}$ in isopropanol provided the alcohol 330 in $31 \%$ yield (Scheme 2.42). Although the yield obtained in our hands was significantly lower than the reported yield in the literature (31\% versus $75 \%),{ }^{238}$ it was proven to be reliable and reproducible on both small-scale (e.g., 0.1 gram) and large-scale (e.g., 1.4 gram) reactions. Subsequent mild oxidation reaction, in which a catalytic amount of TEMPO worked as the oxidant and a stoichiometric amount of BAIB was used to regenerate TEMPO, afforded carboxylic acid 329. ${ }^{239}$ The resultant carboxylic acid was then treated with $\mathrm{Boc}_{2} \mathrm{O}$ and pyridine to give an anhydride intermediate, which was 
inherently unstable and underwent aminolysis to provide desired amide $\mathbf{3 2 1}$.

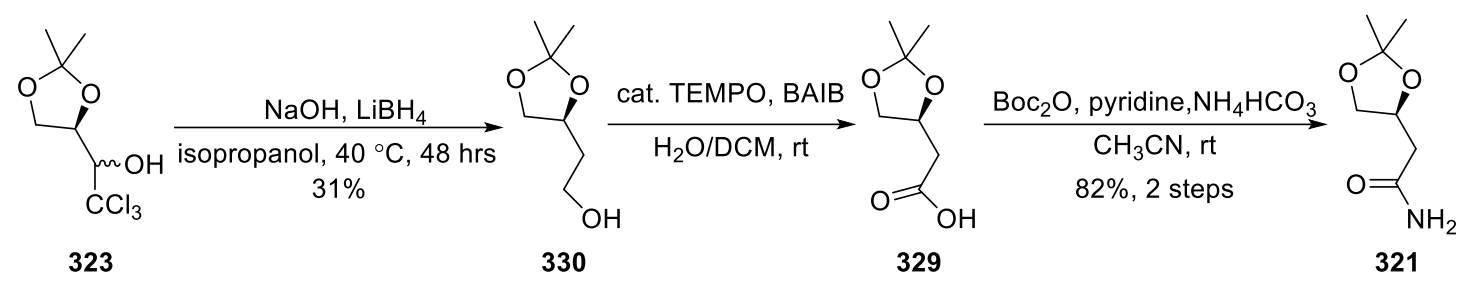

Scheme 2.42 Preparation of amide 321 via primary alcohol intermediate 330.

\subsubsection{Attempted Preparation of Thioamide 317}

As there were only a handful of reported examples in which amides containing an acetonide functional group were transformed into thioamides successfully (see Scheme 2.43 for one example), ${ }^{* * *}$ we were concerned about the compatibility of the acetonide functionality in amide $\mathbf{3 2 1}$ with Lawesson's reagent. To avoid possible side-reactions caused by unreacted Lawesson's reagent, we decided to minimise the equivalents of Lawesson's reagent. Therefore, the thionation of amide $\mathbf{3 2 1}$ was first attempted by treating with 0.55 equivalent of Lawesson's reagent. As the amide starting material and the by-product generated from Lawesson's reagent were indistinguishable on TLC plates, the reaction was monitored by intensive ${ }^{1} \mathrm{H}$ NMR analysis of the aliquot samples of the reaction mixture. After 12-hour stirring at room temperature, the $\alpha$-proton signals ofamide 321 at 2.55 and $2.47 \mathrm{ppm}$ disappeared, indicating the conversion was completed. The reaction was then quenched with saturated $\mathrm{NaHCO}_{3}$ solution and a thionation product was isolated in a moderate yield (Scheme 2.44). Whilst this product was initially believed to be the desired thioamide 317 based on ${ }^{1} \mathrm{H}$ NMR analysis, HR-MS investigation revealed that the major line with peak height of about $92 \%$ was at $\mathrm{m} / \mathrm{z}=192.0685$, which matches with the mass of the desired thioamide product plus the mass of one oxygen atom (Figure 2.8). This result suggested that the major component of the obtained product was, in fact, thioamide $S$-oxide 333, which is most likely the result of oxidation via the addition of atmospheric oxygen. Interestingly, Pattenden and co-workers reported that they successfully prepared thioamide $\mathbf{3 1 7}$ in their initial synthetic studies towards PatA, but they did not report any characterisation data. ${ }^{119}$ Therefore, we assumed that they also encountered this instability problem caused by aerobic oxidation. Given that the thionation reaction was performed under argon atmosphere in our hands, it was proposed

*** Using SciFinder database; https://scifinder.cas.org (accessed November 2017) 
that the aerobic oxidation occurred in the work-up procedure.

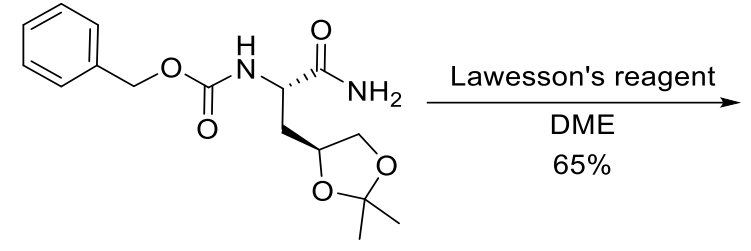

331<smiles>CC1(C)OCC(C[C@H](NC(=O)OCc2ccccc2)C(N)=S)O1</smiles>

332

Scheme 2.43 Example of thionation of amide with acetonide functionality. ${ }^{240}$<smiles>CC1(C)OCC(CC(N)=O)O1</smiles>

321
Lawesson's reagent $(0.55 \mathrm{eq})$ DCM, rt, 12 hrs

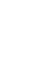

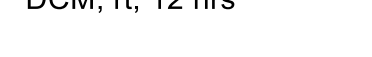

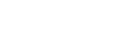

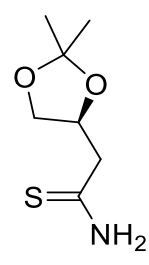

317

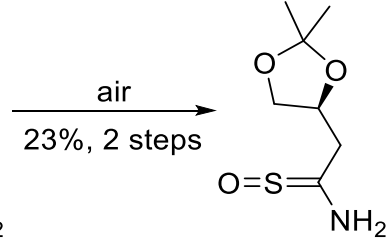

333

Scheme 2.44 Attempted preparation of thioamide 317 .

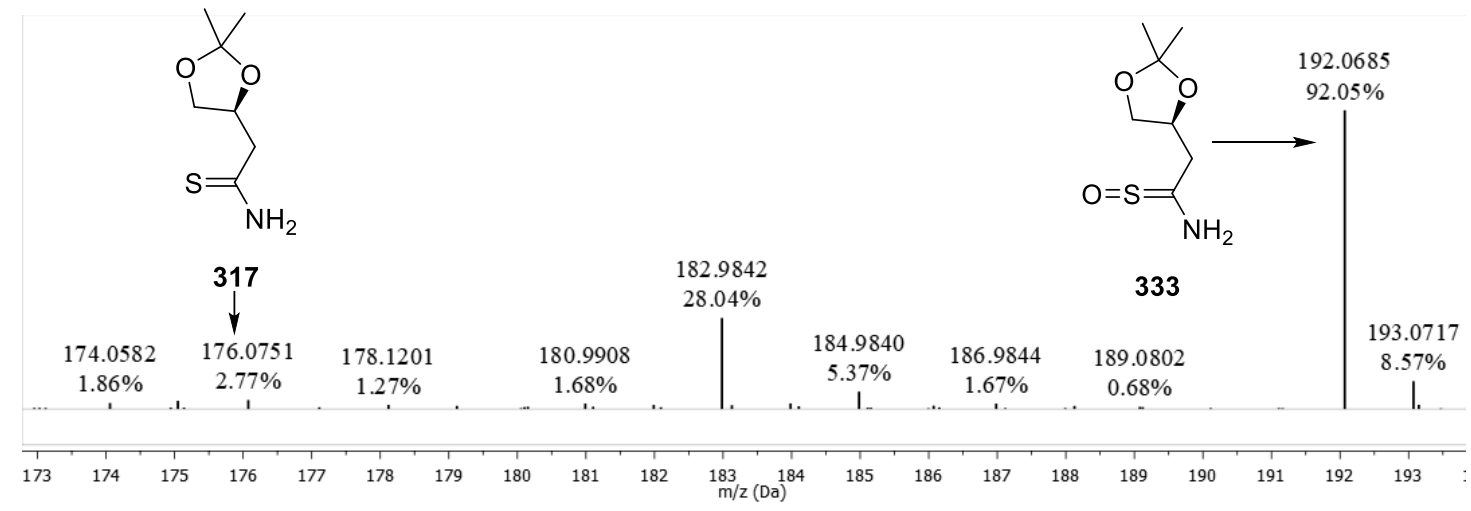

Figure 2.8 HR-MS of thioamide $S$-oxide 333 .

Mass of protonated thioamide 317 at $\mathrm{m} / \mathrm{z}=176.0751$, calculated for $\mathrm{C}_{7} \mathrm{H}_{14} \mathrm{NO}_{2} \mathrm{~S}^{+}$, 176.0740 and protonated thioamide $S$-oxide 333 at $\mathrm{m} / \mathrm{z}=192.0685$, calculated for $\mathrm{C}_{7} \mathrm{H}_{14} \mathrm{NO}_{3} \mathrm{~S}^{+}, 192.0689$.

As the photochemical $S$-oxidation of thiobenzamides by singlet oxygen is well established, ${ }^{241,242}$ it was conceived that the existence of singlet oxygen in air may be the cause of the oxidative degradation of thioamide 317. A mechanism for reactions between sulfides and singlet oxygen was proposed by Jensen and co-workers based on calculations, ${ }^{243}$ and the aerobic oxidation of thioamide $\mathbf{3 1 7}$ may undergo a similar process. Thus, as described in Scheme 2.45, thioamide 317 reacts readily with single oxygen to 
form a highly reactive thiadioxirane $\mathbf{3 3 5}$ via a diradical peroxysulfoxide intermediate $\mathbf{3 3 4}$, which then rapidly breaks down to provide thioamide S-oxide.

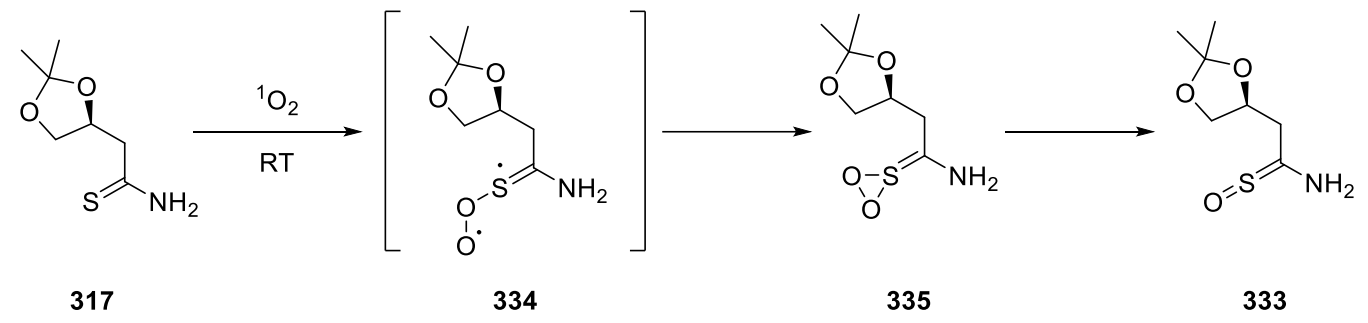

Scheme 2.45 Proposed mechanism of oxidation of thioamide 317 by singlet oxygen.

As shown in Table 2.11, the proton signal of thioacetamide $\mathbf{3 3 6}$ appears at $2.40 \mathrm{ppm}$ in deuterated DMSO ${ }^{\dagger \dagger}$ while the proton signal of thioacetamide $S$-oxide 337, reported by Sarma and Hanzlik, ${ }^{244}$ appears at 1.94 ppm in the same deuterated solvent. Therefore, the chemical shifts of protons at the $\alpha$-position of thioamide $S$-oxide $\mathbf{3 3 3}$ were supposed to also be shifted upfield comparing to those of desired thioamide $\mathbf{3 1 7}$.

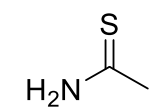

thioacetamide

336<smiles>CC(N)=O</smiles>

thioacetamide $S$-oxide

337

\begin{tabular}{llr}
\hline Compound & Thioacetamide 336 & $S$-oxide 337
\end{tabular}

${ }^{1} \mathbf{H}$ NMR $\boldsymbol{\delta}(\mathbf{p p m}) \quad 2.40, \mathrm{~s} \quad 1.94, \mathrm{~s}$

Table 2.11 Comparison of ${ }^{1} \mathrm{H}$ NMR of thioacetamide $\mathbf{3 3 6}$ and thioacetamide $S$-oxide 337 in DMSO-d 6.

In the literature, the chemical shifts of protons at the $\alpha$-position of thioamides appear at $2.7-3.7 \mathrm{ppm}$ in deuterated chloroform. The obtained proton shifts of the $\alpha$-position of thioamide $S$-oxide 333 in deuterated chloroform appears at 3.04 and $2.91 \mathrm{ppm}$. Therefore, we reviewed the $2.9-4.0 \mathrm{ppm}$ region in the ${ }^{1} \mathrm{H}$ NMR spectra of reaction aliquots at various time points of the reaction. Surprisingly, we could not find any evidence of the existence of thioamide 317. This suggested that the oxidation process occurred so rapidly that the sulfur atom of thioamide $\mathbf{3 1 7}$ was fully oxidised in the preparation of aliquot

\footnotetext{
${ }^{\dagger \dagger \dagger 1} \mathrm{H}$ NMR data obtained in our laboratory using thioacetamide purchased from suppliers.
} 
samples for NMR analysis. Such a rate of oxidation was unexpected because the photochemical oxidation of thioamide normally requires use of photosensitiser to speed up the reaction rate. ${ }^{241}$

However, in the interest of examining whether the oxidised thioamide $\mathbf{3 3 3}$ could be transformed into the thiazole $S$-oxide product $\mathbf{3 3 8}$, which could potentially allow access to a novel oxidised analogue of PatA, we then attempted to couple $S$-oxide $\mathbf{3 3 3}$ and the alkyne 259 following the gold-catalysed conditions reported by Zhang. ${ }^{141}$ Unfortunately, no sign of formation of the desired product was observed and only the mesylate intermediate 273 (vide supra, Table 2.7) was recovered (Scheme 2.46). Consequently, it would be reasonable to propose that the decreased nucleophilicity of the sulfur atom resulting from the electron-withdrawing $\mathrm{S}=\mathrm{O}$ bond renders it incapable of reacting in the cyclocondensation step of the thiazole formation.

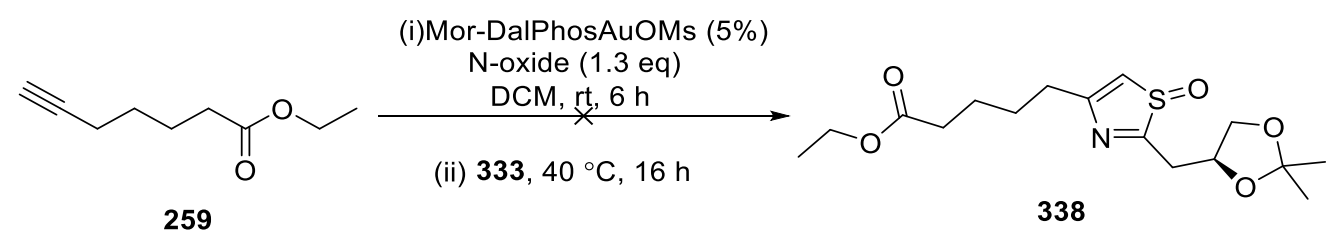

Scheme 2.46 Attempted synthesis of thiazole $S$-oxide 338 . 


\subsubsection{Attempted Preparation of Air-Stable Thioamides}

In light of the finding that the thioamide $\mathbf{3 1 7}$ was oxidised rapidly when exposed to air, attention was then focused on structural modifications of the thioamide which could potentially improve its stability. After reviewing structures of thioamides reported in Romo's ${ }^{82}$ and Pattenden's ${ }^{245}$ studies on the total synthesis of PatA, and 223 in our work (Figure 2.9), we hypothesised that the presence of bulky protecting groups, like TIPS and TBS, could be a stabilising factor against oxidation.

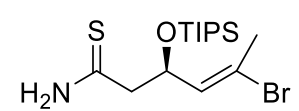

31

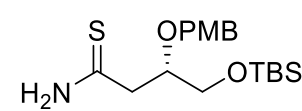

70

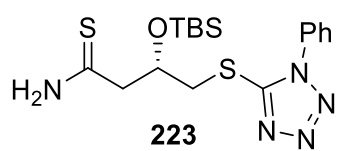

223

Figure 2.9 Structures of air-stable thioamides in Romo's, ${ }^{82}$ Pattenden's ${ }^{245}$ and this work.

To test whether the introduction of two TBS groups could protect the sulfur atom from the undesired oxidation, we set out to prepare thioamide 341. Amide 321 was reacted with acetic acid and water, followed by double-silylation to provide amide 340. Bissilylated amide 340 was then treated with Lawesson's reagent. (Scheme 2.47).

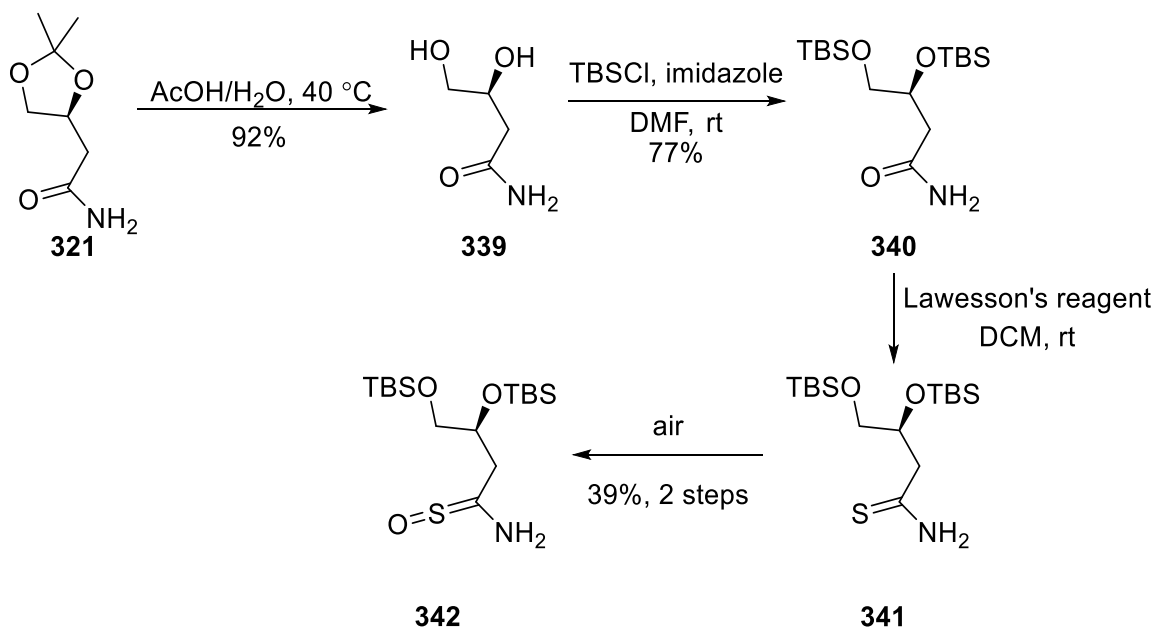

Scheme 2.47 Attempted preparation of thioamide 341 . 


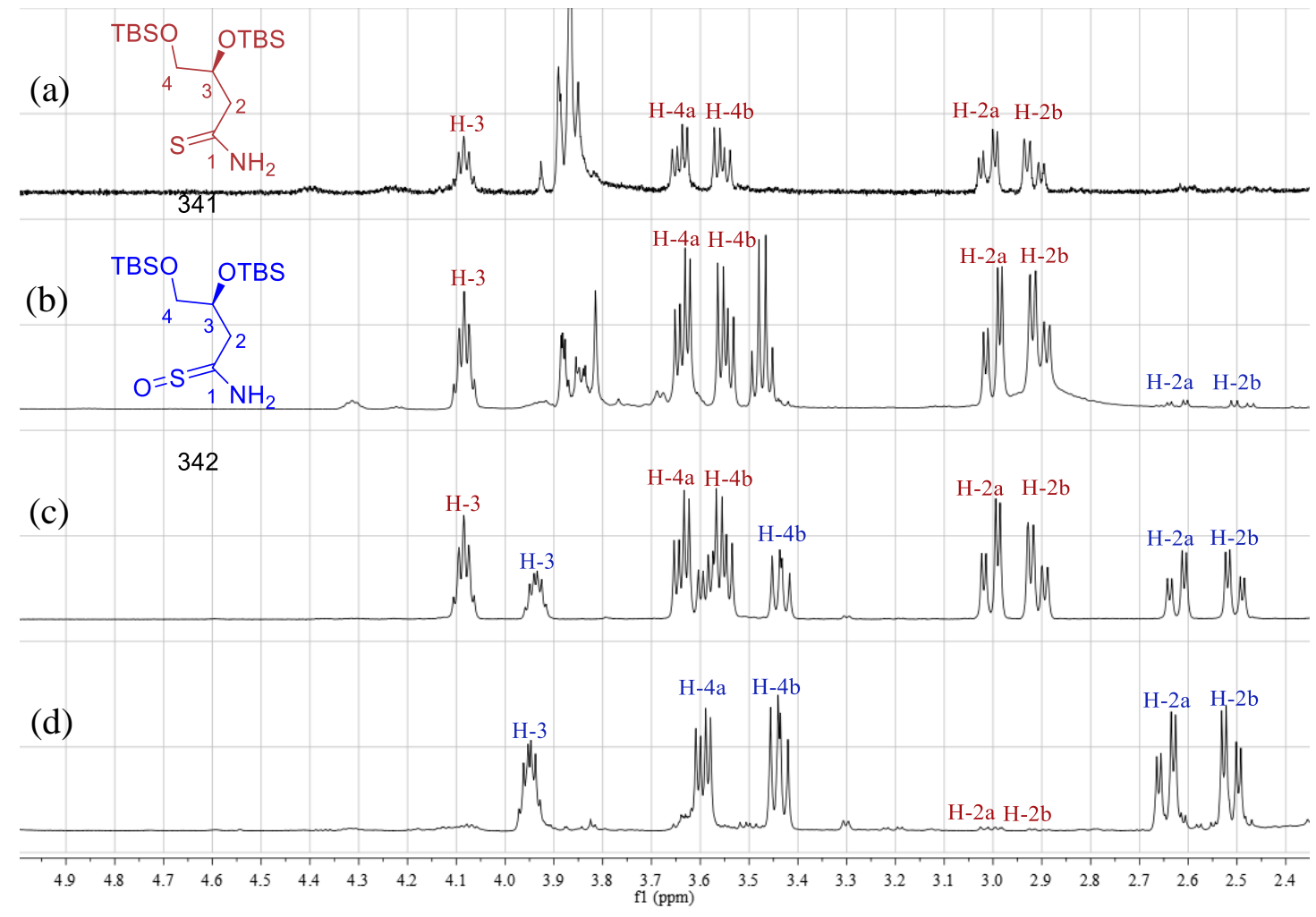

Figure 2.10 Observed oxidation of thioamide 341 based on analysis of ${ }^{1} \mathrm{H}$ NMR spectra of mixtures at different time points: (a) before quenching by aqueous $\mathrm{NaHCO}_{3}$ solution; (b) after extraction by ethyl acetate; (c) during purification by silica gel chromatography; and (d) post-column concentration under vacuum at room temperature. +

Gratifyingly, as shown in Figure 2.10, aerobic oxidation of the thioamide 341 was clearly slowed down to some extent based on intensive ${ }^{1} \mathrm{H}$ NMR investigation throughout the work-up and purification process. Consequently, it was reasonable to propose that replacing the TBS group with bulkier tert-butyldiphenylsilyl (TBDPS) protecting group would further retard the oxidation. The synthetic route for sulfone $\mathbf{3 1 6}$ was then modified to use a potential air-stable thioamide $\mathbf{3 4 3}$ as the major fragment, which could be prepared from amide 344 (Scheme 2.48).

$\$$ Thioamide 341 with peaks at $\left(500 \mathrm{MHz}, \mathrm{CDCl}_{3}\right) \delta 4.12-4.04(\mathrm{~m}), 3.64(\mathrm{dd}, J=10.3,5.1 \mathrm{~Hz}), 3.55(\mathrm{dd}$, $J=10.3,5.9 \mathrm{~Hz}), 3.00(\mathrm{dd}, J=14.4,4.5 \mathrm{~Hz})$ and $2.91(\mathrm{dd}, J=14.4,5.6 \mathrm{H})$, compared to thioamide $S$-oxide 342 with peaks at $\left(500 \mathrm{MHz}, \mathrm{CDCl}_{3}\right) \delta 3.97-3.90(\mathrm{~m}), 3.59(\mathrm{dd}, J=10.2,4.9 \mathrm{~Hz}), 3.43(\mathrm{dd}, J=10.1,7.8$ $\mathrm{Hz}), 2.62(\mathrm{dd}, J=15.0,4.5 \mathrm{~Hz})$, and $2.50(\mathrm{dd}, J=15.0,4.4 \mathrm{~Hz})$. 

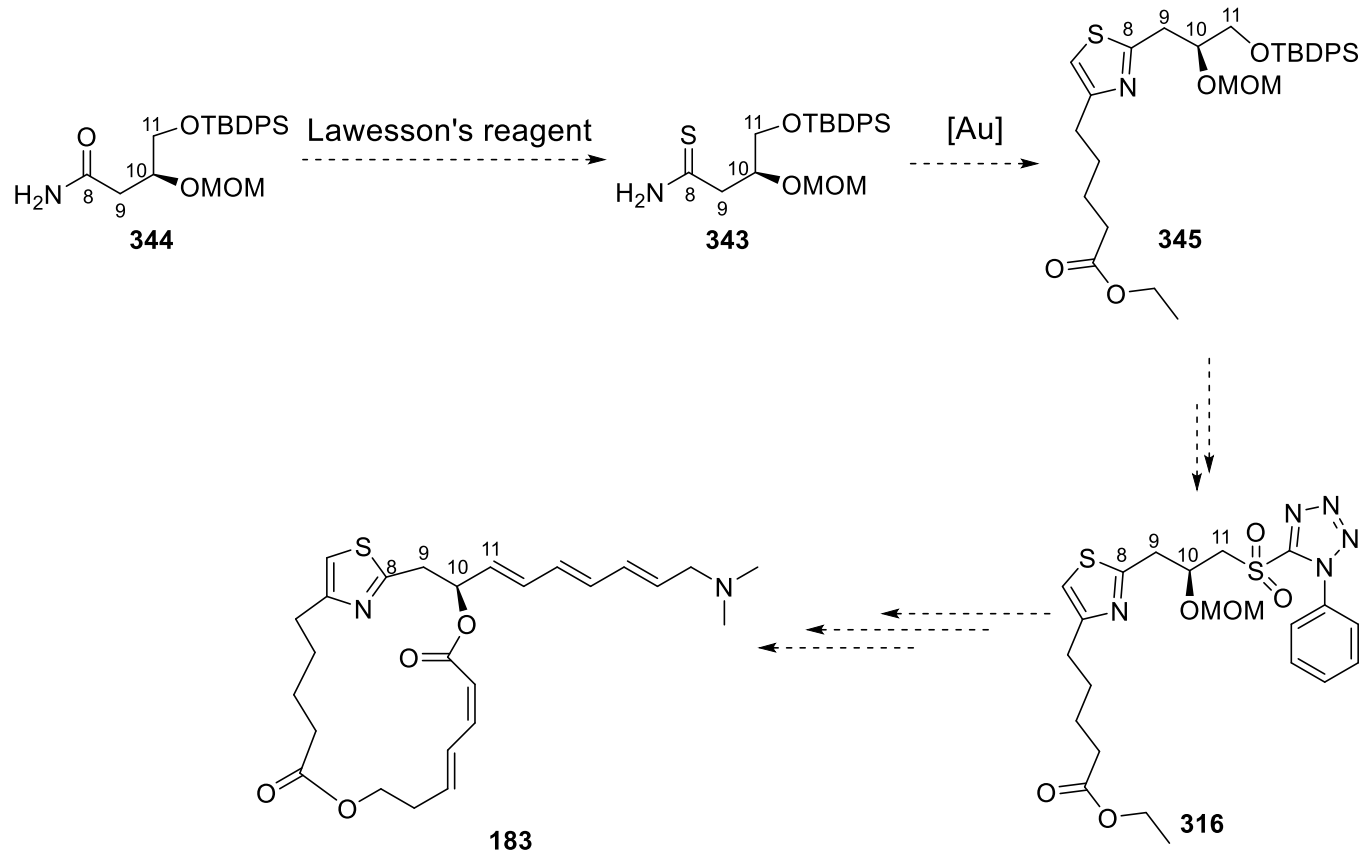

Scheme 2.48 Proposed synthetic route for PatA analogue 183 using thioamide 343.

It has been reported that the para-methoxybenzyl (PMB) protecting groups of amides could be cleaved by treatment with one-electron oxidising agent ceric ammonium nitrate, ${ }^{246}$ which could be compatible with the MOM protecting group. Therefore, to avoid the likely chemoselectivity issues with MOM protection between a primary amide and a secondary alcohol, we decided to use para-methoxybenzylamine $\left(\mathrm{PMBNH}_{2}\right)$ as opposed to ammonia in ethanol to prepare secondary amide 346 (Scheme 2.49). Another benefit is that the PMB-protecting group could also facilitate the reaction monitoring and purification process because of it being a chromophore easily visualised by UV on TLC plates.

To our delight, following Snowden and co-workers' optimised condition, ${ }^{232}$ the one-pot reaction provided amide 346 in reasonable yield. In addition, a minor product was isolated in isomerically pure form, and 2D NMR and HRMS investigations revealed it to be $\alpha$ substituted amide 347 (Scheme 2.49). 


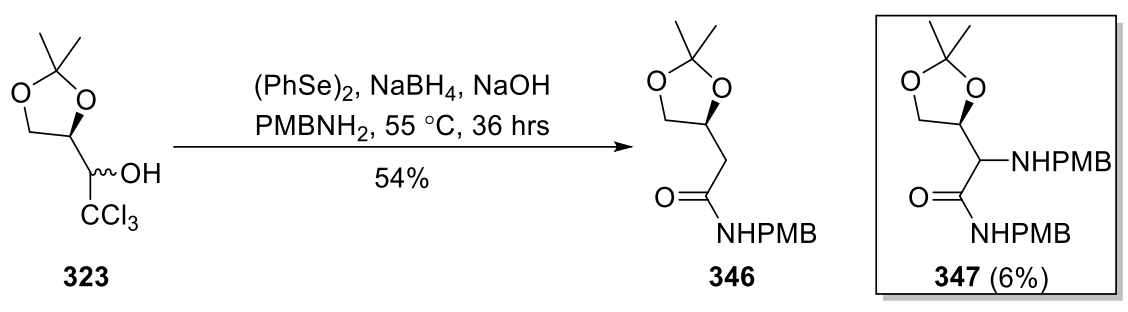

Scheme 2.49 Preparation of PMB-protected amide 346.

The formation of the side product $\mathbf{3 4 7}$ could be attributed to the nucleophilicity of the nitrogen atom resulting from the strong electron-donating effect of the PMB group. As shown in Scheme 2.50, $\mathrm{PMBNH}_{2}$ acts as a nucleophile to attack the highly electrophilic gem-dichloroepoxide intermediate at $\mathrm{C} 2$ to give the acid chloride intermediate, which rapidly reacted again with $\mathrm{PMBNH}_{2}$ to provide the substituted amide 347.

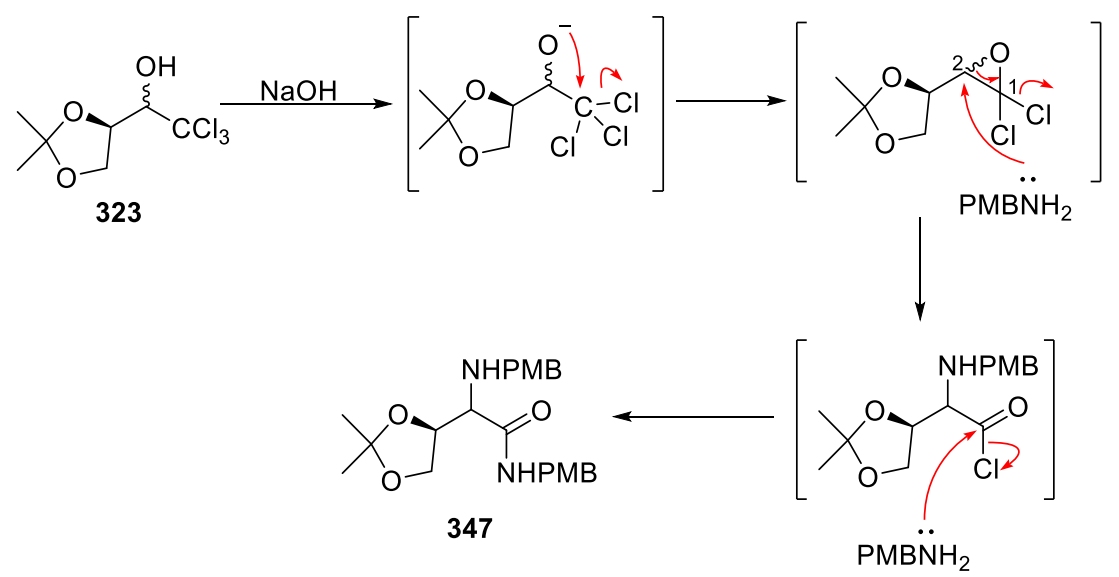

Scheme 2.50 Proposed mechanism for formation of $\alpha$-substituted amide 347 .

Since a mixture of diastereomers $\mathbf{3 2 3}$ was used in the reaction, it was conceived that a mixture of diastereomers of $\mathbf{3 4 7}$ would be generated through the proposed mechanism but only one stereoisomer was obtained. Considering that the other isomer of amide $\mathbf{3 4 7}$ might be lost in the purification process, the reaction was then repeated on a larger scale and intensive ${ }^{1} \mathrm{H}$ NMR experiments of aliquot samples were conducted. However, no evidence of formation of the other diastereomer of $\mathbf{3 4 7}$ was observed. This indicates stereospecificity in the by-product formation, which is possibly due to different reaction rates of epoxide formation from 323. To unambiguously determine the stereoselectivity of this concomitant side reaction, it would be necessary to assign the configuration of the new-forming chiral centre of the isolated by-product through chiral derivatisation of the primary amine, ${ }^{247}$ which could be readily prepared by selective removal of the PMB 
protecting group. However, due to time constraints, this was not further explored during the course of this Ph.D. project.

With the PMB-protected amide in hand, we then attempted to prepare amide $\mathbf{3 4 2}$, the precursor of thioamide $\mathbf{3 4 3}$. Amide $\mathbf{3 4 6}$ was first treated with acetic acid in water to give diol product 348 (Scheme 2.51).

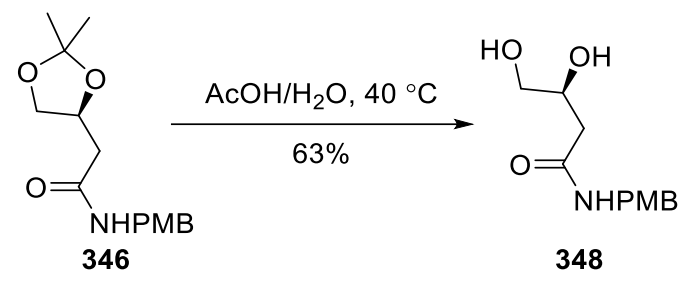

Scheme 2.51 Preparation of diol 348 .

${ }^{1} \mathrm{H}$ NMR spectrum of the diol product 348 obtained in deuterated chloroform showed a broadened singlet centred at $\delta 2.19$ with an integration of 1.00 and another broadened singlet centred at $\delta 4.00$ with an integration of 1.00 (see Figure 2.11), and the large downfield chemical shift was possibly due to the internal hydrogen bonding interaction between the secondary alcohol and the carbonyl group. It is well known that hydrogen bonding interactions can reduce the acidity of alcohols and inhibit their reactions with bases. ${ }^{248}$ Hence, this internal hydrogen bonding could be beneficial for the subsequent selective silylation of the primary $\mathrm{OH}$. 

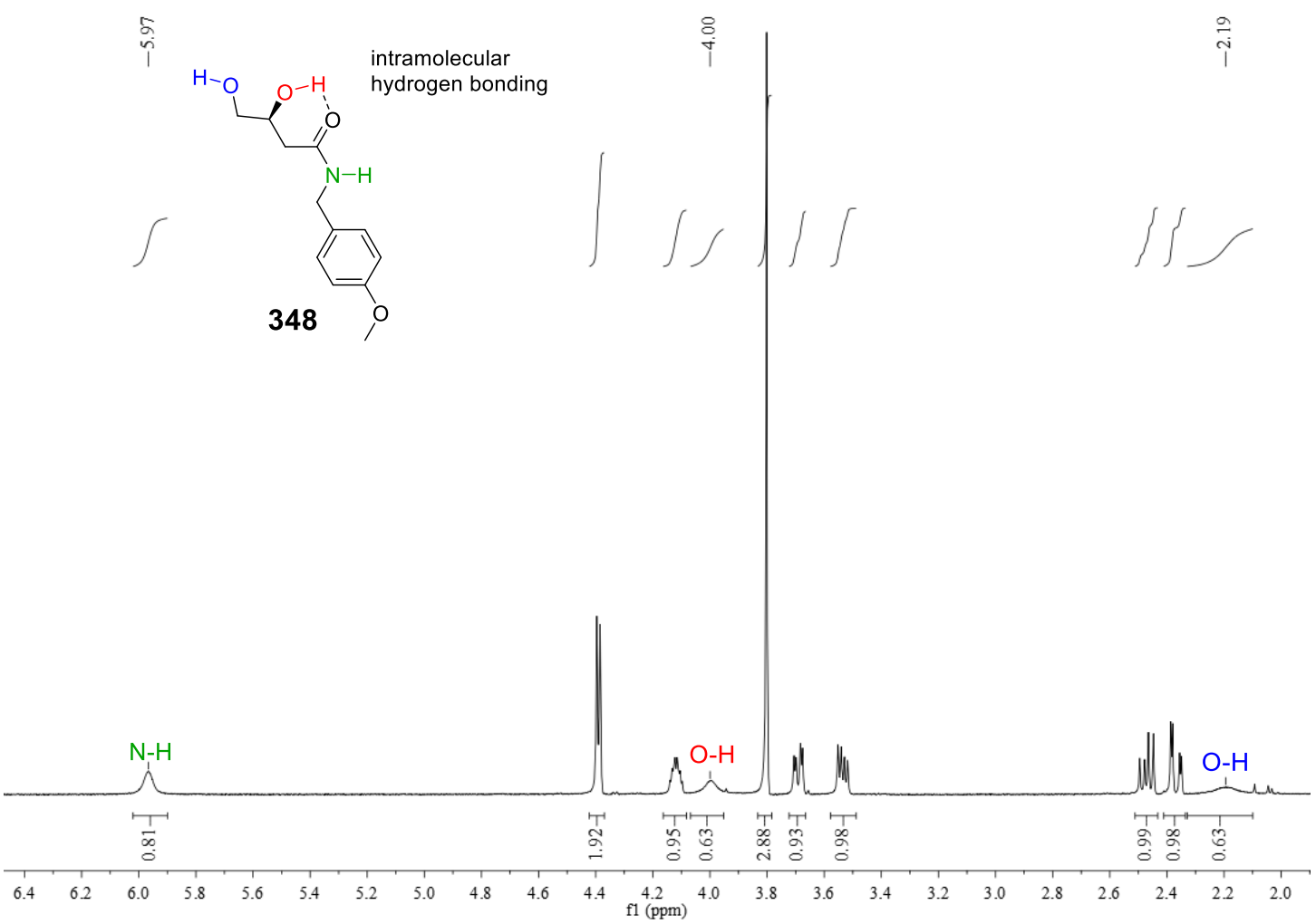

Figure 2.11 ${ }^{1} \mathrm{H}$ NMR evidence of possible internal hydrogen bonding interaction between secondary $\mathrm{OH}$ and carbonyl group in diol $348\left({ }^{1} \mathrm{H} \mathrm{NMR}\right.$ run in $\left.\mathrm{CDCl}_{3}\right)$. (Assigned using gCOSY NMR)

As the use of a nonpolar solvent would promote such intramolecular hydrogen bonding interaction and facilitate the selectivity, the diol $\mathbf{3 4 8}$ was initially treated with 1 equivalent of TBDPSCl and imidazole in DCM, a relatively nonpolar solvent. Unexpectedly, the desired mono-silylated product $\mathbf{3 4 9}$ was obtained in less than $3 \%$ yield with $70 \%$ recovery of the diol starting material. The poor yield was possibly because the polar diol was poorly solubilised in DCM. Given that selective TBDPS silylation of 1,2-diols has been used with great regularity in organic synthesis, we then changed to use a polar aprotic solvent DMF, which should sufficiently solubilise diol 348 via intermolecular hydrogen bonding, with the thought that the bulkiness of TBDPS could counteract the disrupted intramolecular hydrogen bonding. To our dismay, the reaction in DMF provided both mono-silylated product 349 and bis-silylated product $\mathbf{3 5 0}$ in poor yields (Scheme 2.52). However, no further optimisation was performed for this step at this stage, and we moved onto the next step to test the feasibility of this route. Protection of the secondary $\mathrm{OH}$ with $\mathrm{MOMCl}$ and DIPEA provided the product 351 in an uninspiring yield, which was attributed to the potential internal hydrogen bonding similar to that seen in diol $\mathbf{3 4 8}$. 
Repeating the reaction with more active methoxymethyl iodide, which was prepared in situ by mixing $\mathrm{MOMCl}$ and sodium iodide, at higher temperatures resulted in significant degradation of the substrate, with possible cleavage of the PMB protecting group observed and no evidence of forming any desired MOM ether. In light of the lack of highyielding steps, this route was not pursued.

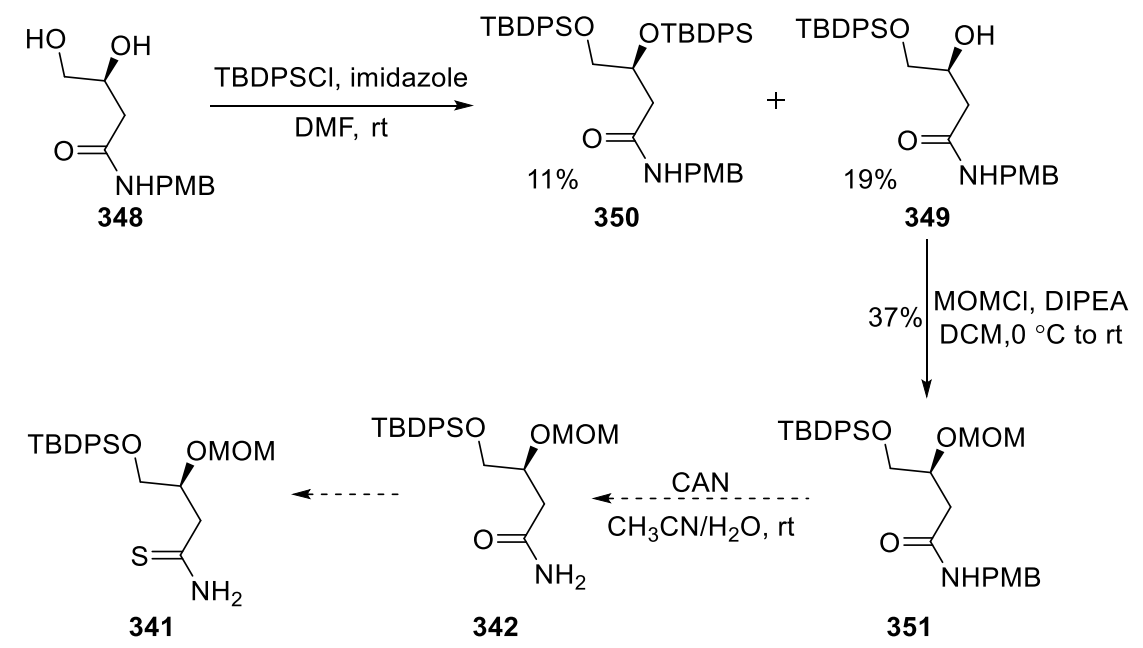

Scheme 2.52 Attempted preparation of thioamide 343 starting from diol $\mathbf{3 4 8}$.

The low yields of these two protection steps were possibly consequences of interference by the intramolecular hydrogen bonding between the secondary $\mathrm{OH}$ and the carbonyl group. As sulfur atoms are known to be poor hydrogen bond acceptors, ${ }^{246}$ we then attempted to transform the amide $\mathbf{3 4 6}$ into thioamide $\mathbf{3 5 2}$ with the hope that the absence of internal hydrogen bonding could improve the yields of the following protection steps. A literature search suggested that thionation reactions of protected amides normally require higher reaction temperatures (e.g., reflux in 1,2-dimethoxyethane or toluene). ${ }^{\S \S \S}$ To avoid the risk of degradation of the acetonide functional group, we first performed the reaction in refluxing DCM. But no significant reaction was observed. When the reaction was repeated in refluxing THF, a mixture of unreacted amide 346, and unidentified degradation products were obtained, with no sign of thioamide $\mathbf{3 5 2}$ or the $S$-oxide of thioamide 352 observed by HR-MS investigation (Scheme 2.53). This suggested that a further increase in the reaction temperature would possibly lead to significant degradation of the substrate instead of the formation of the desired thioamide.

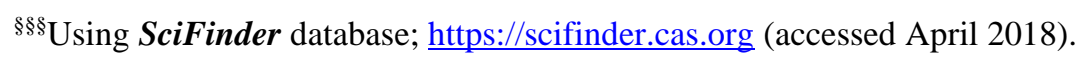




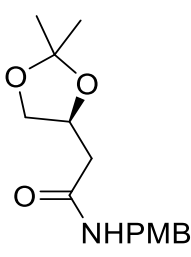

346

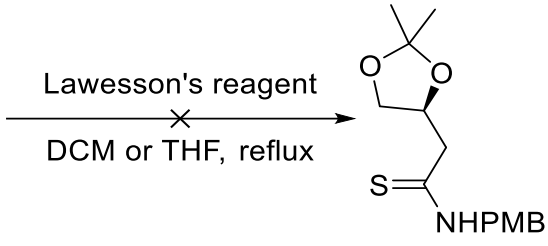

352

Scheme 2.53 Attempted preparation of thioamide $\mathbf{3 5 2}$.

With the absence of success in the preparation of the TBDPS-protected thioamide 352, we decided to move on to the phenyltetrazole functional group as a potential stabiliser for the sulfur atom, even though it is considered to be less bulky than TBDPS protective group. Thus, attempts were made to prepare thioamide 353 starting from mono-tosylation of diol 348. Surprisingly, when the diol was treated with catalytic $\mathrm{Bu}_{2} \mathrm{SnO}, \mathrm{TsCl}$ and 1 equivalent of $\mathrm{Et}_{3} \mathrm{~N}$, only the starting material 348 was recovered and no evidence of formation of mono-tosylate 354 was observed (Scheme 2.54) by HRMS investigation.

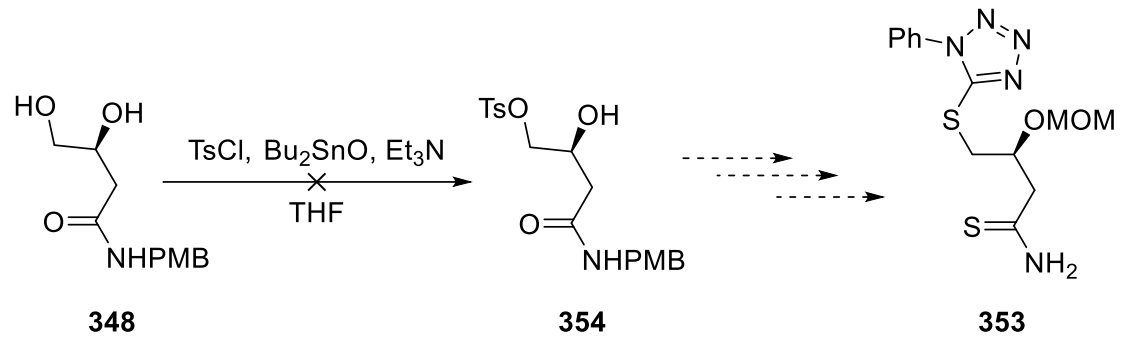

Scheme 2.54 Attempted preparation of mono-tosylated amide 354 .

Based on the proposed mechanism of $\mathrm{Bu}_{2} \mathrm{SnO}$ catalysed selective tosylation of diol (Scheme 2.55), ${ }^{249}$ the reaction proceeds via a dimeric species 355 , which can be sulfonylated by $p$ - $\mathrm{TsCl}$ to produce intermediates 356 and $357 . \mathrm{Et}_{3} \mathrm{~N}$ acts as a nucleophile and helps to break up dimer intermediates $\mathbf{3 5 6}$ and $\mathbf{3 5 7}$ to furnish intermediate $\mathbf{3 5 8}$. Thus, it was proposed that the sluggishness of the tosylation reaction of diol $\mathbf{3 4 8}$ was a consequence of the existence of the secondary amide functional group. Normally, the nitrogen atom on an amide is less nucleophilic than the nitrogen of an amine because the amide carbonyl group can provide the resonance stabilisation of the nitrogen lone pair electrons. However, the electron-donating effect of the PMB protective group could possibly make the nitrogen atom on the amide $\mathbf{3 4 8}$ more nucleophilic and to be able to interfere with the break-up process of dimeric intermediates, resulting in the incomplete catalytic cycle. 


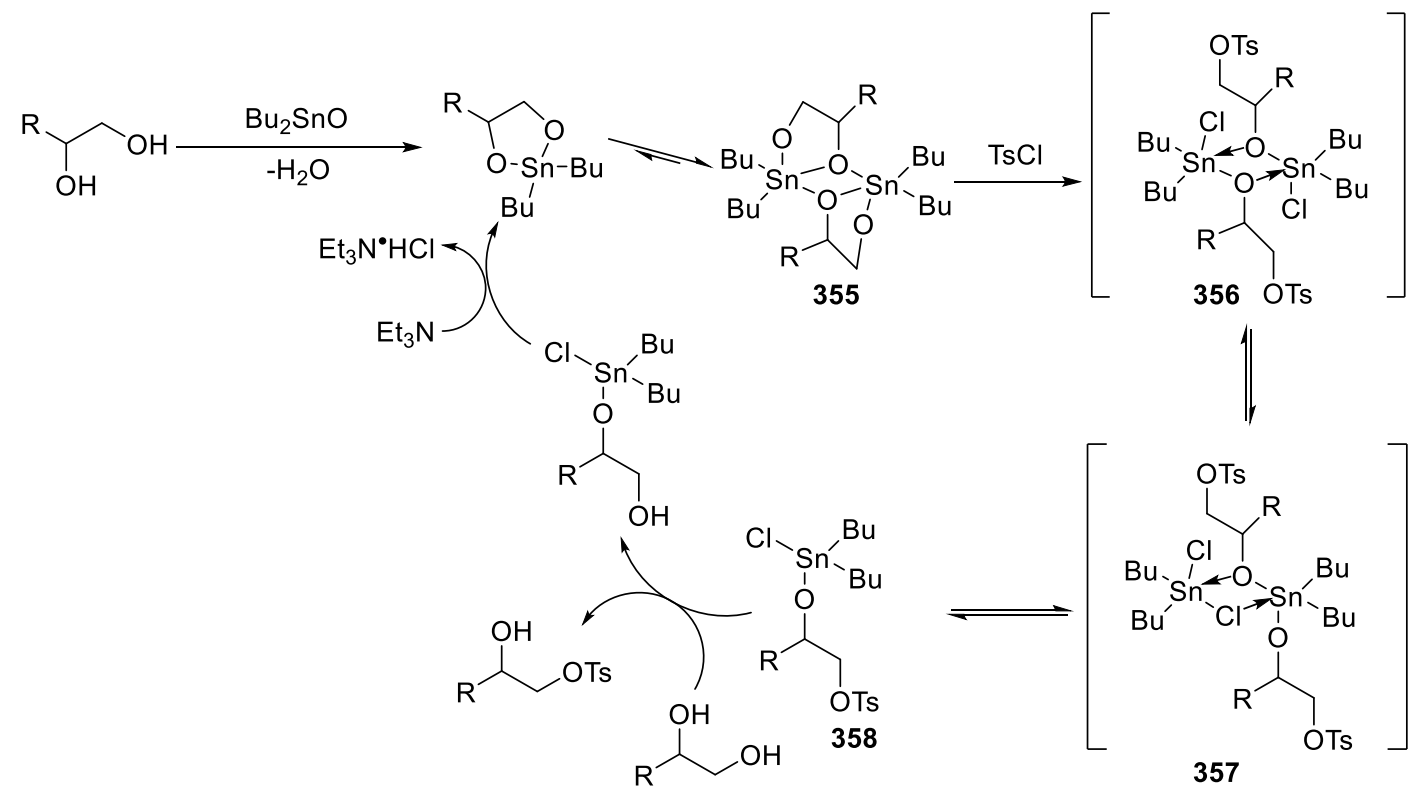

Scheme 2.55 Proposed mechanism of $\mathrm{Bu}_{2} \mathrm{SnO}$ catalysed tosylation by Martinelli and co-workers. ${ }^{249}$

\subsection{Revised Synthesis of Analogue 183}

Alongside our efforts to prepare mono-tosylated amide 354 and MOM-protected sulfone fragment 316, postdoctoral fellow Claire Cuyamendous in our group conducted extensive studies on model reactions between sulfone 284 and ketone 359 to test the feasibility of attaching the methylated side chain fragment (Fragment II', vide supra, Section 1.7.2) by Julia-Kocienski olefination, and found that the reaction was sluggish with no conversion (Scheme 2.56).

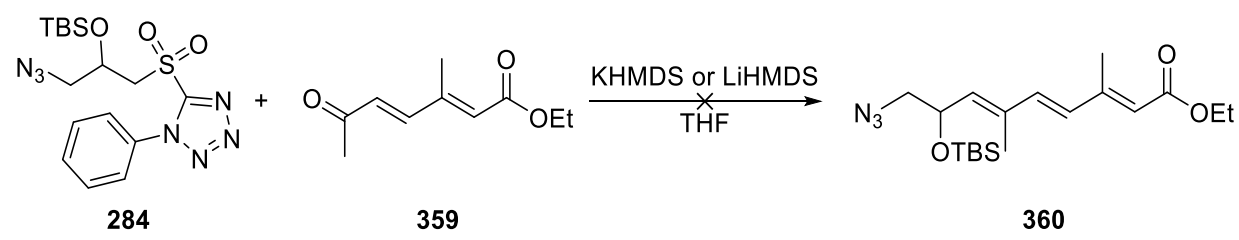

Scheme 2.56 Cuyamendous' attempted Julia-Kocienski reaction between sulfone 284 and ketone 359.

Based on this unsuccessful result, it was clear that an alternative strategy for attachment of the side chain fragments was necessary to facilitate the collective syntheses of $\mathrm{PaA}$ analogues 183 and 184. 
As shown in Scheme 2.57, to make the most of the existing results from our work, the revised route would involve performing a Wittig/Horner-Wadsworth-Emmons (HWE) olefination with the side chain fragment $\mathbf{3 6 1}$ or $\mathbf{3 6 2}$ and macrocyclic aldehyde $\mathbf{3 6 3}$. The thiazole core could be constructed via the gold-catalysed coupling between alkyne $\mathbf{3 6 4}$ and thioamide 365 . Alkyne 364 could be prepared via esterification between 6-heptynoic acid 258 and $\delta$-valerolactone 366. Owing to the fact that it is no longer necessary to introduce ester protecting groups to avoid inadvertent trapping of the reactive gold carbene with the acid functionality, this route can enable a degree of step economy. ${ }^{250}$

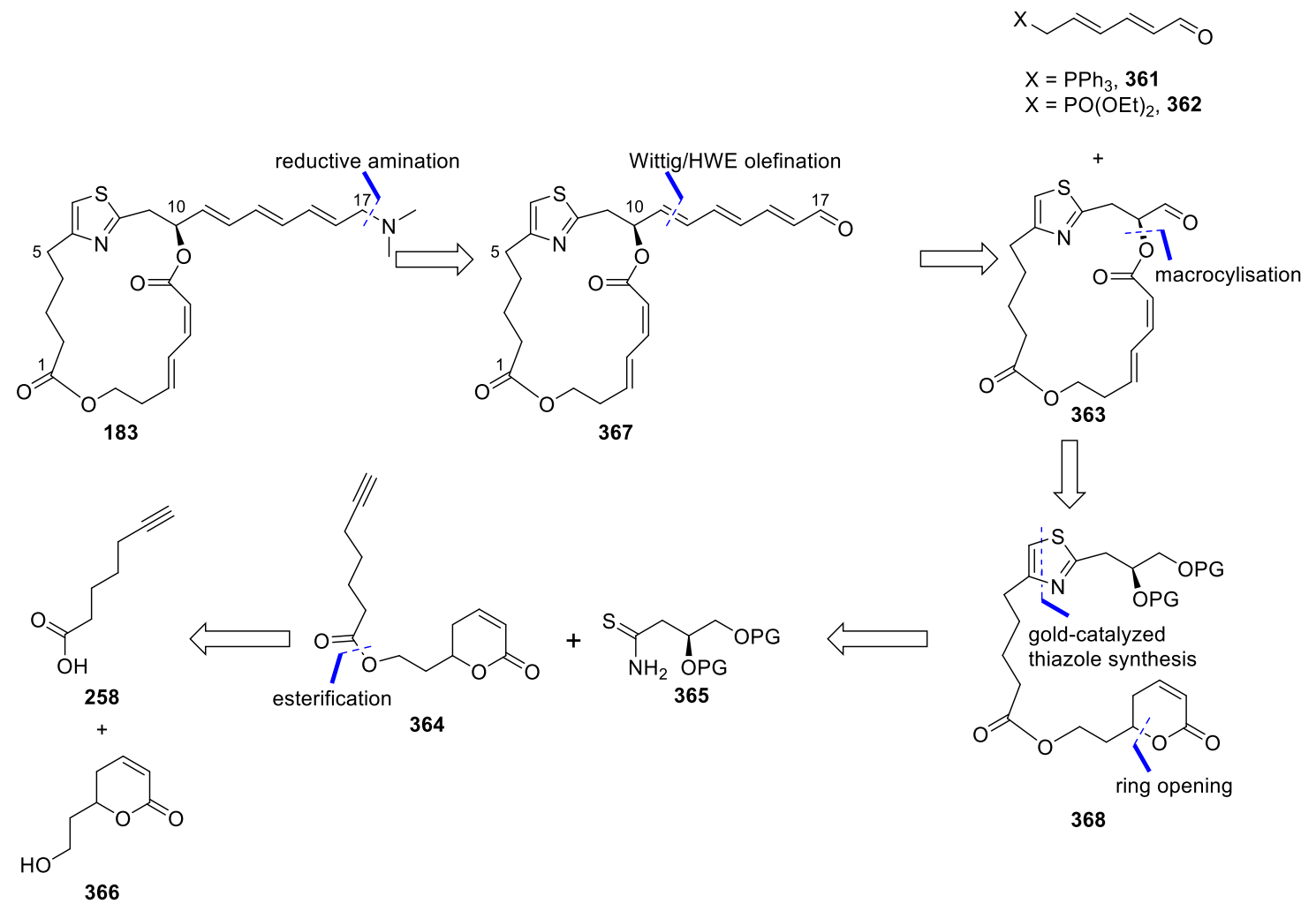

Scheme 2.57 Revised retrosynthetic analysis of PatA analogue $\mathbf{1 8 3 .}$

As the PatA analogue 184 (see Figure 1.25 in Section 1.7.1) with methylated side chain could provide more information about whether the two methyl groups at C22 and C24 (PatA numbering) on the macrocyclic core are critical for activity, we decided to prioritise the synthesis of analogue $\mathbf{1 8 4}$ rather than the fully non-methylated analogue $\mathbf{1 8 3}$. 


\subsection{Conclusion}

In conclusion, the key four-carbon fragment, namely thioamide $\mathbf{2 2 3}$, for the synthesis of thiazole analogue 183 was prepared from an enantiopure epoxide with a $28 \%$ yield over 8 steps. However, a degree of racemisation occurred in this reaction sequence, which was determined by Mosher analysis of alcohol intermediate 217. In the synthesis of nitrile intermediate 222, a range of reported conditions was screened for the substitution of tosylate 221 with a cyanide source. LiCN generated in situ from LiHMDS and acetone cyanohydrin was found to be effective in facilitating this transformation. In the thionation step, it was found the impurities of the purchased Lawesson's reagent could possibly be the cause of the dehydration side reaction and the yield was significantly improved by using further purified Lawesson's reagent.

After optimisation efforts to improve the yield of gold-catalysed thiazole formation, thiazole 276 was obtained in $61 \%$ yield. Thiazole-containing sulfone partner 277 required for Julia-Kocienski olefination was prepared successfully using catalytic ammonium heptamolybdate and aqueous hydrogen peroxide.

The side chain fragment 297 was prepared from furan with a 17\% yield over 5 steps. However, the reaction of sulfone 277 and aldehyde 297 under Julia-Kocienski reaction conditions resulted in a mixture of degradation products, which was possibly due to the steric hindrance of the TBS group. Attention was then focused on the preparation of an alternative four-carbon fragment, namely, acetonide-protected thioamide 317. Scalable preparation of amide 321, the precursor of thioamide 317, was achieved by employing a 6-step reaction sequence starting from D-mannitol 324. Thionation of amide 321 was then performed. Unfortunately, thioamide $\mathbf{3 1 7}$ was found to be very unstable and prone to oxidation by air.

It was hypothesised that introducing bulky protecting groups could potentially improve the stability of thioamides. Therefore, attempts to prepare thioamides 341 and 343 were carried out and were found to be unsuccessful. However, the results of these efforts revealed that the presence of bulky protecting groups in thioamides could slow down the aerobic oxidation.

As the Julia-Kocienski olefination was found to be unable to couple the methylated side chain fragment to the sulfone partner in model reactions, the synthetic route was revised 
in order to ensure the collective synthesis of targeted analogues. Wittig/HWE reaction would be utilised as opposed to Julia-Kocienski olefination in the new reaction sequence.

Since analogue 184 could give direct evidence of the importance of the methyl groups on the macrocycle, the synthesis of analogue $\mathbf{1 8 4}$ was prioritised. 


\section{Chapter Three: Towards Synthesis of Thiazole Analogue with Methylated Side Chain}

As outlined in Section 1.7.1, the synthesis of thiazole analogue 184 with the methylated side chain could help to test whether the two methyl groups at C22 and C24 methyl groups (PatA numbering) are critical for the binding process of PatA to its protein target. This chapter describes the work towards the synthesis of this analogue.

\subsection{Retrosynthetic Analysis}

As the structural difference between analogue 184 and analogue 183 is the side chain fragment, the synthetic route for analogue 184 could incorporate the same macrocyclic aldehyde intermediate $\mathbf{3 6 3}$, which was proposed in the retrosynthetic analysis in Section 2.6. As shown in Scheme 3.1, to make the most of our existing synthetic strategy, the thiazole core would be constructed through gold-catalysed thiazole formation using thioamide 365 and alkyne 364 as substrates. The $E$, Z-dienoic acid would be produced by base-induced ring opening of $\delta$-valerolactone 368 . The macrocycle would be furnished by using an appropriate macrolactonisation, and the methylated side chain fragment, which could be derived from 369 or 370 , would be coupled with the macrocyclic aldehyde 363 via Wittig/HWE olefination. The terminal tertiary amine group in the side chain would be introduced in the final step by reductive amination. 


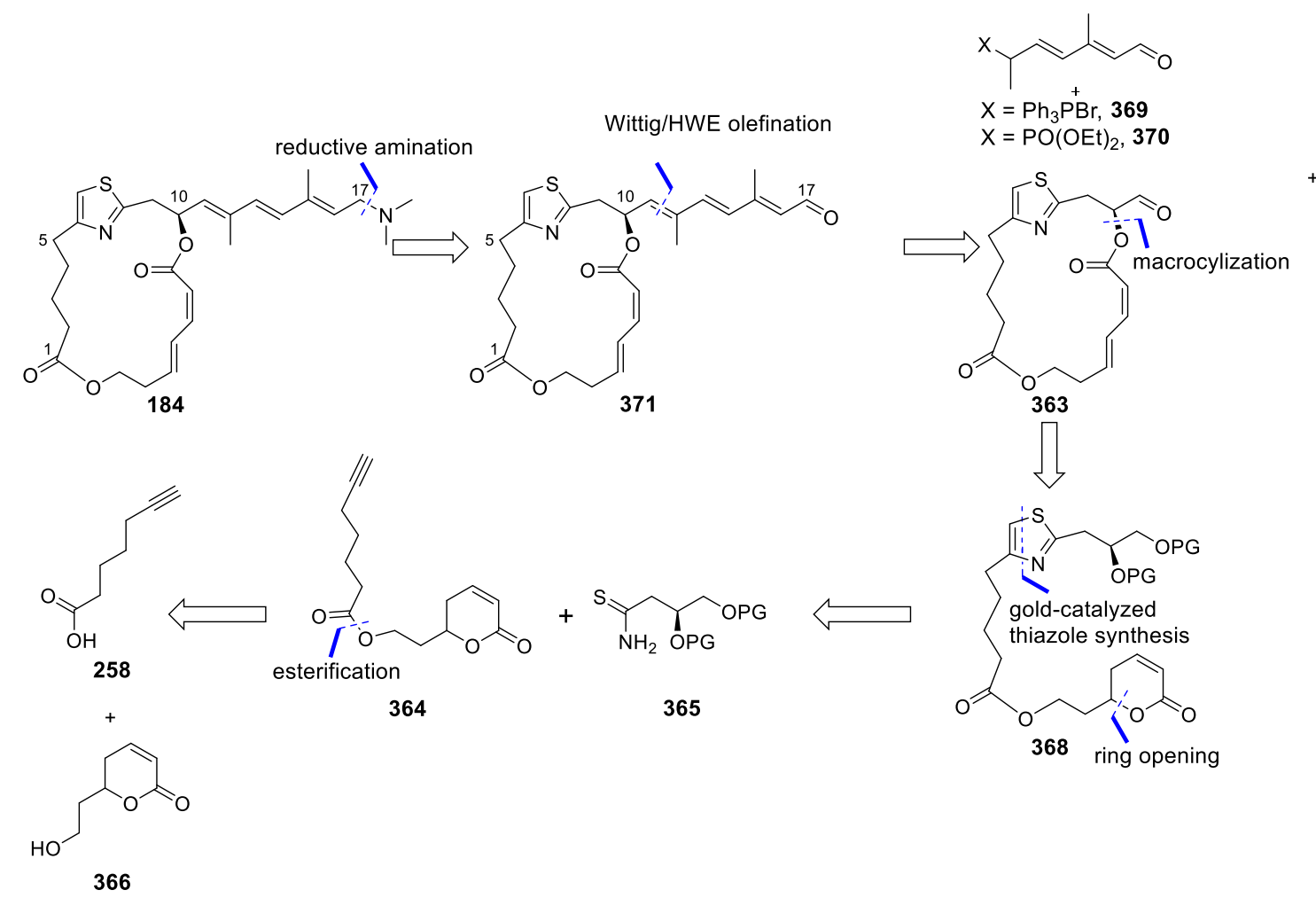

Scheme 3.1 Revised retrosynthetic analysis of PatA analogue $\mathbf{1 8 4}$.

Preparation of thioamide 365 and alkyne 364, construction of the macrocycle and synthesis of the side chain fragments will be discussed in the following sections.

\subsection{Preparation of Air-stable Thioamide 382}

Based on our observations that the aerobic oxidation of sulfur atoms in thioamides could be slowed by introducing bulky protective groups (vide supra, Section 2.5.2), compounds $\mathbf{3 7 2}$ and $\mathbf{3 7 3}$ were targeted as potential candidates for air-stable thioamides.
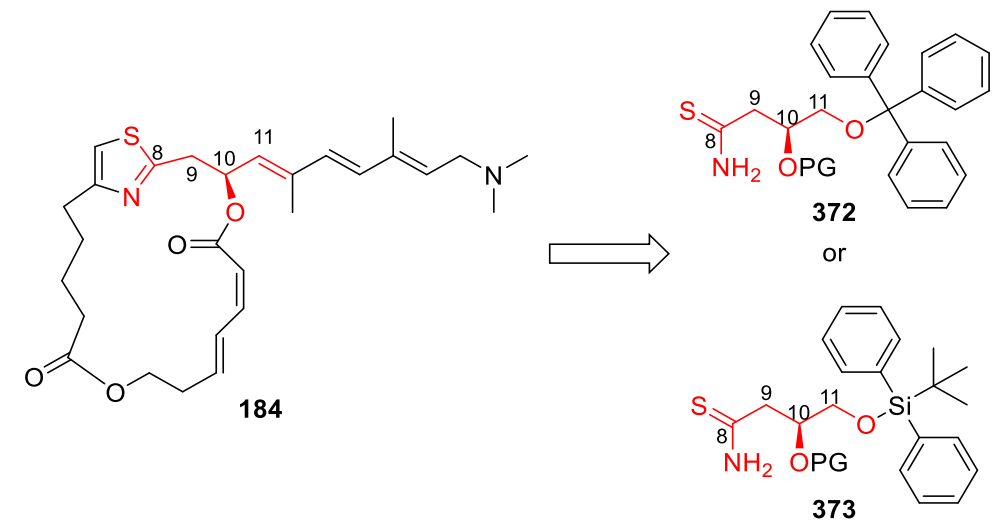

Figure 3.1 Proposed appropriate thioamides $\mathbf{3 7 2}$ and $\mathbf{3 7 3}$ for synthesis of analogue $\mathbf{1 8 4}$. 
As the trityl protecting group is considered to be bulkier than the TBDPS protecting group, we first attempted to prepare tritylated thioamide 372 starting from commercially available amide 339, which could be also prepared from D-mannitol 324 (vide supra, Section 2.5.1).

To ensure that the polar amide $\mathbf{3 3 9}$ was fully solubilised to accelerate the tritylation reaction, polar DMF was chosen as the solvent and mono-tritylation of amide $\mathbf{3 7 4}$ was achieved in excellent yield (Scheme 3.2). Considering that the potential hydrogen bonding interaction between the secondary $\mathrm{OH}$ and the carbonyl group could interfere with the subsequent silylation reaction, DMF was used again to disrupt the internal hydrogen-bonding and the desired product 375 was obtained in $73 \%$ yield. Thionation of amide 375 by treatment with Lawesson's reagent afforded a mixture of degradation products, one of which was identified to be predominately triphenylmethanol by comparison with literature NMR values. ${ }^{251}$ This suggested that the degradation was possibly caused by the incompatibility of the trityl ether with Lawesson's reagent.

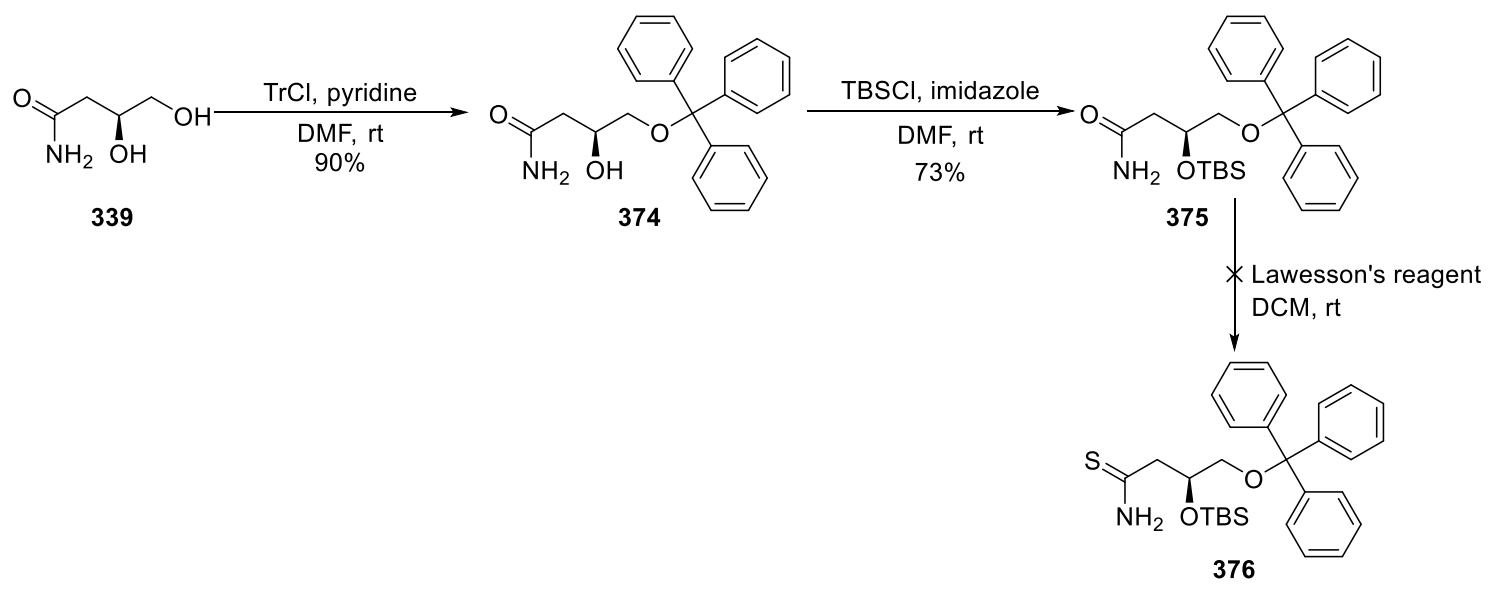

Scheme 3.2 Attempted preparation of thioamide 376 .

This unexpected failure prompted us to turn our attention to the synthesis of the TBDPSprotected thioamide 373. As discussed in Section 2.5.2, we encountered difficulties in mono-silylation of amide 348, which was possibly due to the intramolecular hydrogen bonding. Assuming that mono-silylation of amide 339 would not provide good regioselectivity between primary $\mathrm{OH}$ and secondary $\mathrm{OH}$, we opted to start from selective desilylation of amide $\mathbf{3 4 0}$ for preparation of thioamide $\mathbf{3 7 3}$ because a substantial amount of amide $\mathbf{3 4 0}$ was available from previous work (see Section 2.5.2 for the details for the preparation of amide 340) 
The bis-TBS protected amide 340 was treated with ( \pm )-10-camphorsulfonic acid (CSA) and the formation of desired amide 377 was observed by ${ }^{1} \mathrm{H}$ NMR analysis of reaction aliquots. However, it rapidly isomerised to amide 378 when saturated $\mathrm{NaHCO}_{3}$ aqueous solution was used to quench the reaction (See Figure 3.2 for comparison of ${ }^{1} \mathrm{H}$ NMR of amide 377 and 378). This isomerisation presumably occurred via a pentacoordinate silicon intermediate $\mathbf{3 7 9}^{252}$ and could be explained by less molecular crowding and the formation of the internal hydrogen bonding between the $-\mathrm{NH}_{2}$ and the secondary $\mathrm{OH}$ or between the carbonyl group and the secondary $\mathrm{OH}$. Given that the presence of a base is requisite for silylation reactions, this undesired 1,2-silyl migration prompted by basic conditions would possibly also occur in TBDPS protection of TBS-protected amide 377. Thus, we abandoned this approach and decided to move on to preparing thioamide $\mathbf{3 7 3}$ from unprotected amide 339.

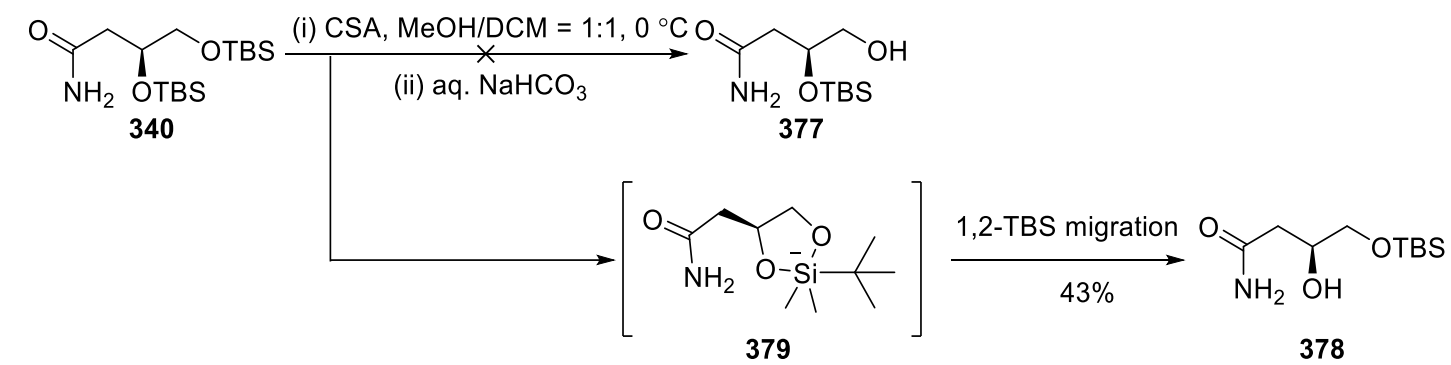

Scheme 3.3 Attempted selective desilylation of amide $\mathbf{3 4 0}$. 

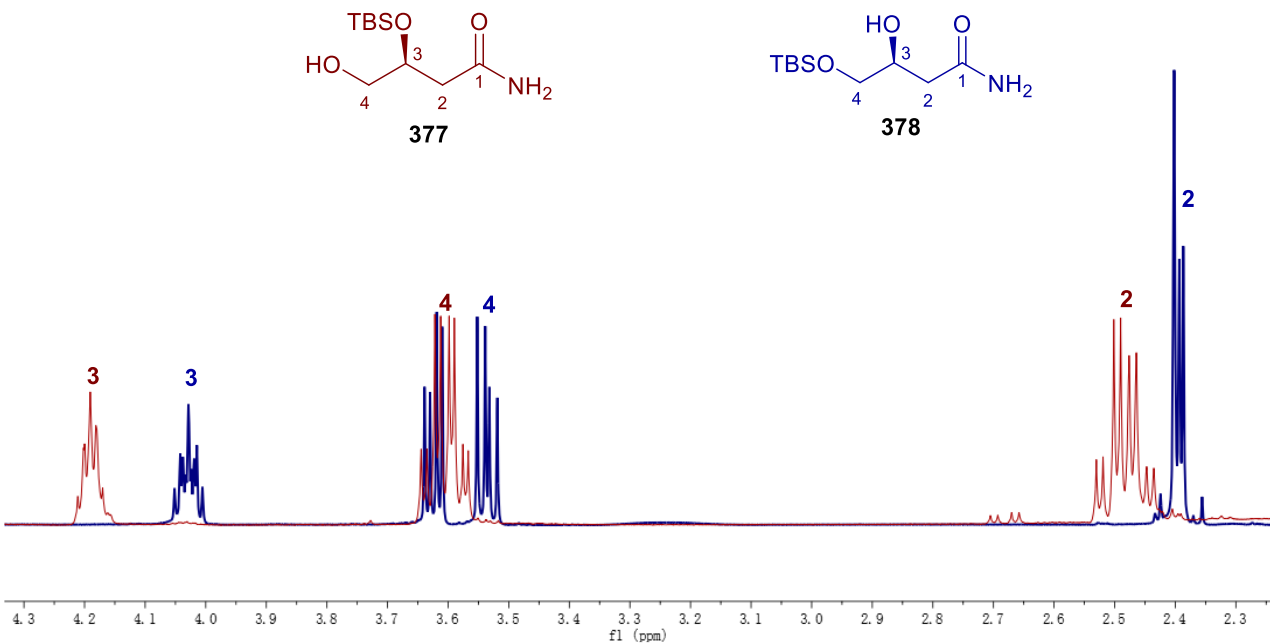

Figure 3.2 Comparison of $\delta 4.3-2.3$ ppm region in ${ }^{1} \mathrm{H}$ NMR of amide 377 (in maroon) and $\mathbf{3 7 8}$ (in blue).

Gratifyingly, initial treatment of amide 339 with one equivalent of TBDPSCl in DMF provided the desired mono-silylated amide $\mathbf{3 8 0}$ as the sole product, albeit in $26 \%$ yield (Scheme 3.4). It was proposed that the uninspiring yield was a consequence of substantial material loss in the aqueous work-up procedure to remove DMF. For this reason, in subsequent attempts, DMF was removed by distillation under reduced pressure and the yield was improved to $67 \%$. Subsequent silylation by treatment with TBSCl produced the desired amide $\mathbf{3 8 1}$ in excellent yield. However, the reaction was sluggish, prompting us to seek an alternative less bulky silyl protecting group for the secondary $\mathrm{OH}$. Given that the trityl protecting group could be cleaved under treatment with Lawesson's reagent, it was reasonable to propose that the trimethylsilyl (TMS) group was not compatible with the following thionation step. Because the triethylsilyl (TES) group was more susceptible to acid hydrolysis when compared to TBS, which could potentially facilitate desilylation

Based on analysis of the ${ }^{1} \mathrm{H}$ NMR and gCosy spectra. Amide $377\left(500 \mathrm{MHz}, \mathrm{CDCl}_{3}\right) \delta 4.19(\mathrm{tt}, J=5.8$, $4.6 \mathrm{~Hz}, 1 \mathrm{H}, \mathrm{H}-3), 3.63$ (dd, $J=11.4,4.8 \mathrm{~Hz}, 1 \mathrm{H}, \mathrm{H}-4), 3.58$ (dd, $J=11.4,4.3 \mathrm{~Hz}, 1 \mathrm{H}, \mathrm{H}-4), 2.51$ (dd, $J=$ $14.5,5.6 \mathrm{~Hz}, 1 \mathrm{H}, \mathrm{H}-2), 2.46(\mathrm{dd}, J=14.5,5.9 \mathrm{~Hz}, 1 \mathrm{H}, \mathrm{H}-2)$, compared to amide $378\left(500 \mathrm{MHz}, \mathrm{CDCl}_{3}\right) \delta$ 4.03 (tt, $J=6.9,4.7 \mathrm{~Hz}, 1 \mathrm{H}, \mathrm{H}-3), 3.62$ (dd, $J=10.0,4.6 \mathrm{~Hz}, 1 \mathrm{H}, \mathrm{H}-4), 3.54$ (dd, $J=10.0,6.6 \mathrm{~Hz}, 1 \mathrm{H}, \mathrm{H}-$ 4), $2.41-2.38(\mathrm{~m}, 2 \mathrm{H}, \mathrm{H}-2)$. 
under milder conditions before the macrolactonisation step, the seemingly less bulky TES group was chosen to substitute TBS. Unfortunately, treatment of amide $\mathbf{3 8 0}$ with TESCl and imidazole for 7 days at $35{ }^{\circ} \mathrm{C}$ resulted in no significant reaction, suggesting that the TES group was possibly effectively bulkier than the TBS group in this circumstance.

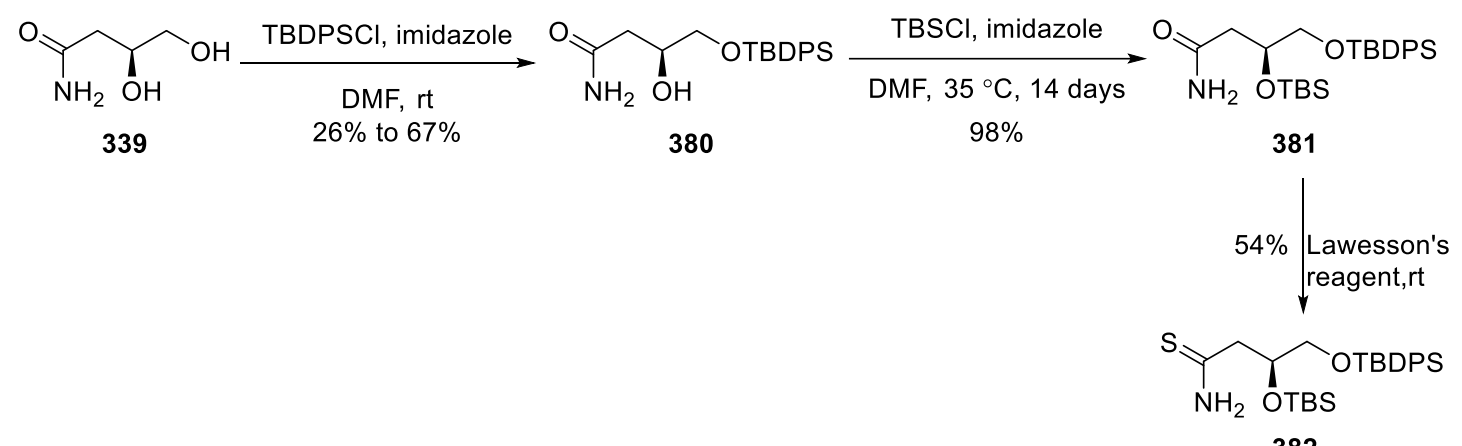

Scheme 3.4 Preparation of thioamide 382.

Thionation of amide $\mathbf{3 8 1}$ by Lawesson's reagent was performed at room temperature under argon atmosphere. As the amide starting material $\mathbf{3 8 1}$ and the by-product generated from Lawesson's reagent were indistinguishable on TLC plates, the reaction was monitored by ${ }^{1} \mathrm{H}$ NMR analysis of the reaction aliquots. After 30 -minute stirring at room temperature, the $\alpha$-proton signals of amide 381 at 2.66 and $2.42 \mathrm{ppm}$ disappeared, indicating the conversion was completed. The reaction was then quenched and the desired thioamide 382 was isolated by silica gel chromatography. However, as shown in Figure 3.3a, partial oxidation by air was still observed by ${ }^{1} \mathrm{H}$ NMR analysis after product fractions from column chromatography being concentrated under reduced pressure at room temperature (18 to $20^{\circ} \mathrm{C}$ ). Despite that, it was clear that oxidation rate of thioamide 382 was slower than that of the bis-TBS protected thioamide 341 (see Section 2.5.2 for observed oxidation of thioamide 341). Assuming that the concentrated product was more susceptible to oxidation by air than when dissolved (e.g., in column fractions), we then performed solvent evaporation of column fractions at $10{ }^{\circ} \mathrm{C}$ under vacuum, and used nitrogen to backfill the rotary evaporator when opening the stopcock on the rotary evaporator after column fractions had completed evaporating to reduce exposure of the concentrated sample to air. Gratifying, after taking these protective measures, we obtained thioamide 382 in high purity (Figure 3.3b), albeit in moderate yield (54\%). 


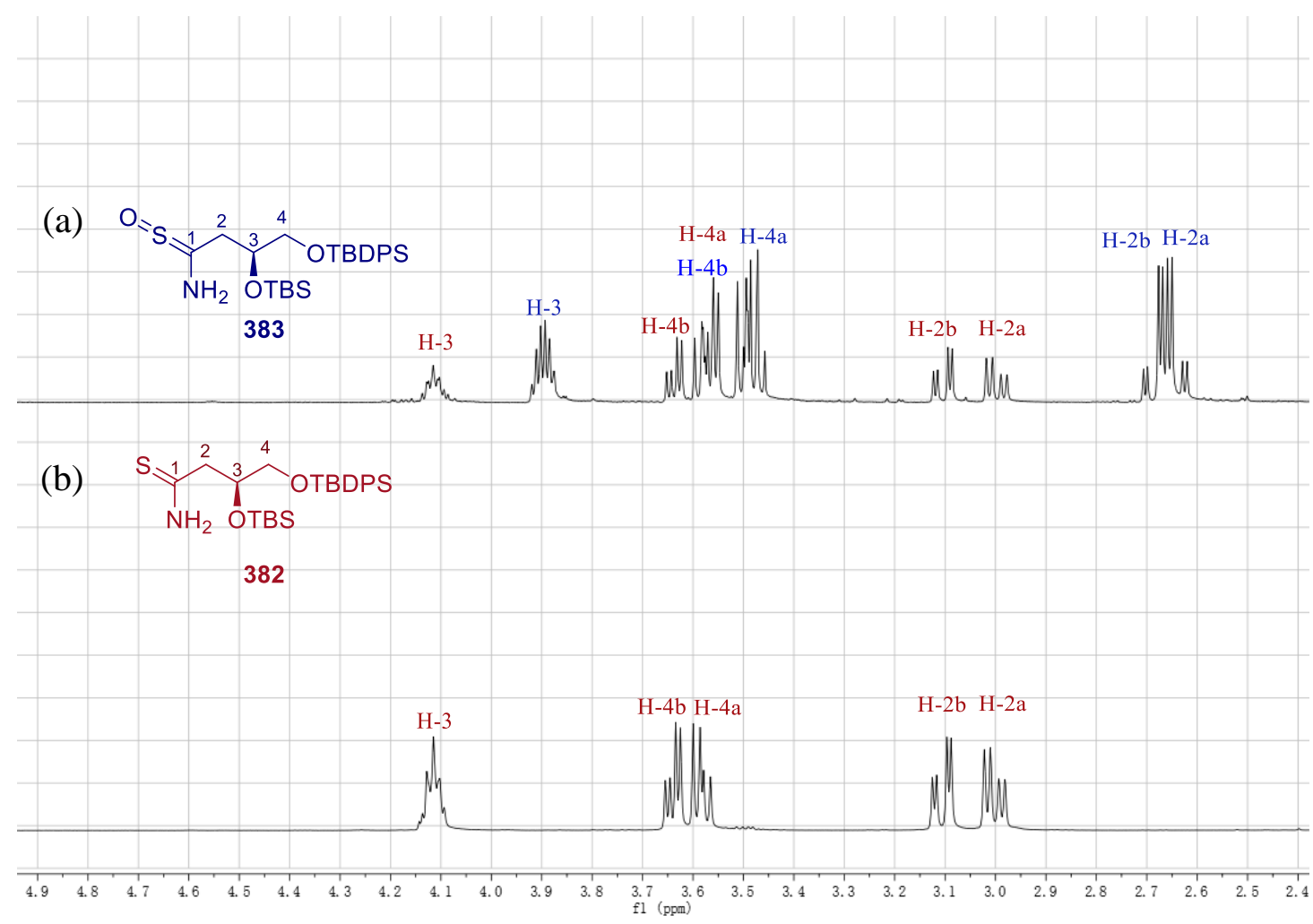

Figure 3.3 Comparison of ${ }^{1} \mathrm{H}$ NMR spectra of obtained thioamide 382 samples: (a) without exclusion of air in the concentration of column fractions after chromatography; and (b) under an inert atmosphere in the concentration of column fractions after chromatography. ${ }^{\dagger \dagger \dagger \dagger}$

In summary, trityl-protected thioamide $\mathbf{3 7 6}$ and TBDPS-protected thioamide $\mathbf{3 8 2}$ were chosen as potential candidates for air-stable thioamides. Efforts to prepare thioamide 376 by thionation of trityl-protected amide $\mathbf{3 7 5}$ were unsuccessful due to the incompatibility of the trityl ether with Lawesson's reagent. In order to prepare thioamide 382, selective desilylation of bis-TBS protected amide $\mathbf{3 4 0}$ was performed and it was found the desired amide 377 rapidly isomerised to amide $\mathbf{3 7 8}$ under mild basic conditions. Thioamide 382 was then prepared from amide 339 in three steps. However, it was necessary to take extra measures to exclude exposure to air in the concentration of product

$\Pi$ Hased on analysis of the ${ }^{1} \mathrm{H}$ NMR and gCosy spectra. Thioamide $S$-oxide $383\left(500 \mathrm{MHz}, \mathrm{CDCl}_{3}\right) \delta$ 3.93 - 3.86 (m, H-3), 3.57 (dd, $J=10.3,4.8 \mathrm{~Hz}, \mathrm{H}-4 \mathrm{~b}$ ), 3.49 (dd, $J=12.3,7.9 \mathrm{~Hz}, \mathrm{H}-4 \mathrm{a}$ ), 2.69 (dd, $J=14.9$, $4.0 \mathrm{~Hz}, \mathrm{H}-2 \mathrm{~b}$ ), 2.64 (dd, $J=14.9,4.6 \mathrm{~Hz}, \mathrm{H}-2 \mathrm{a}$ )., compared to thioamide $382\left(500 \mathrm{MHz}, \mathrm{CDCl}_{3}\right) \delta 4.14-$ 4.08 (m, H-3), 3.64 (dd, $J=10.3,4.6 \mathrm{~Hz}, \mathrm{H}-4 \mathrm{~b}$ ), 3.58 (dd, $J=10.4,6.8 \mathrm{~Hz}, \mathrm{H}-4 \mathrm{a}$ ), 3.10 (dd, $J=14.4,4.1$ $\mathrm{Hz}, \mathrm{H}-2 \mathrm{~b}$ ) and 3.00 (dd, $J=14.4,6.0 \mathrm{~Hz}, \mathrm{H}-2 \mathrm{a})$. 
fractions after chromatography to ensure that thioamide $\mathbf{3 8 2}$ was obtained in high purity.

\subsection{Preparation of Alkyne 364}

As mentioned in Section 3.1, alkyne 364 could react with thioamide 382 to furnish the thiazole ring through a gold-catalysed coupling reaction. The $\delta$-lactone motif of the alkyne could be unfolded to provide the E,Z-dienoic acid under basic conditions. This fragment could provide most of the skeleton of the macrocycle core for the synthesis of PatA analogue 184 (Figure 3.4) and it has been previously utilised in Cumming's synthesis of triazole analogue 182 (see Figure 1.23 in Section 1.6). ${ }^{134}$ Thus, we largely followed the reported procedures for its synthesis.

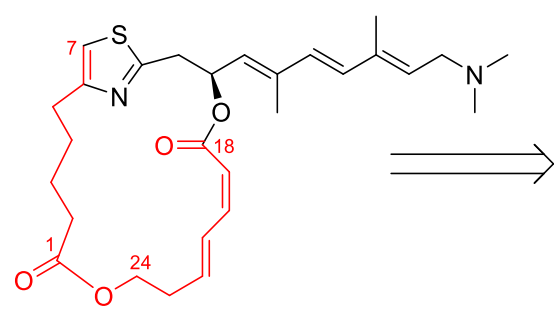

184

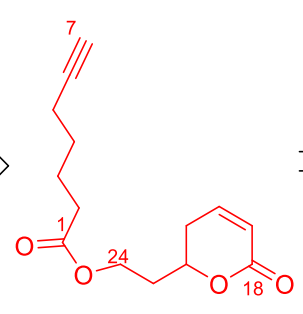

364

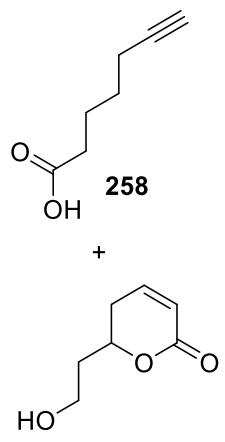

366

Figure 3.4 Proposed alkyne fragment $\mathbf{3 6 4}$ for the synthesis of PatA analogue $\mathbf{1 8 4}$.

As shown in Scheme 3.5, in the presence of catalytic p-TSA, commercially available 1,3propanediol 384 was reacted with para-anisaldehyde in a Dean-Stark apparatus to provide acetal 385. Subsequent reductive cleavage of acetal by DIBAL-H at $-78{ }^{\circ} \mathrm{C}$ afforded mono-alcohol 386, which was then subjected to typical Swern conditions to provide aldehyde 387. The one-pot reaction of aldehyde 387 with allylmagnesium bromide and acryloyl chloride gave diene $\mathbf{3 8 8}$ in $81 \%$ yield on a modest scale (e.g., 0.59 gram). However, when the reaction was repeated on a larger scale (e.g., 5 gram), it was noticed that the purified diene $\mathbf{3 8 8}$ polymerised when the product fractions after chromatography purification were heated at 40 to $50{ }^{\circ} \mathrm{C}$ on a rotary evaporator to remove solvent for a prolonged period of time. In addition, the purified diene $\mathbf{3 8 8}$ from the smallscale reaction was found to polymerise slowly even on storage in the freezer at $-20{ }^{\circ} \mathrm{C}$. The polymer was largely insoluble in deuterated solvents, and therefore, the nature of the polymerised products could not be determined by NMR techniques. 


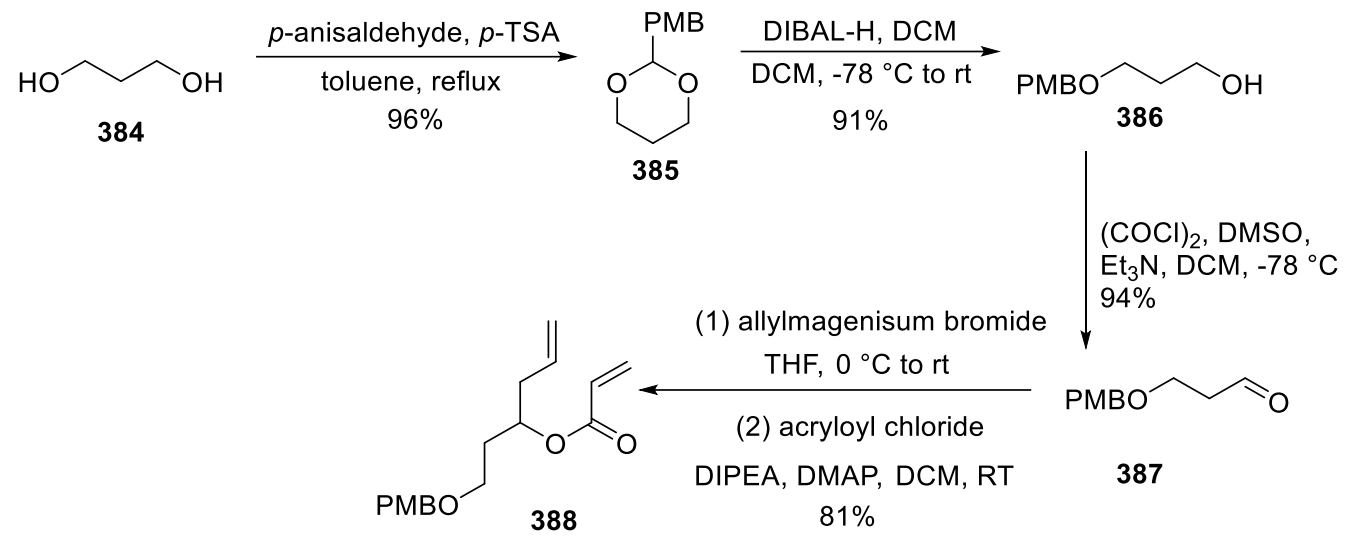

Scheme 3.5 Preparation of diene $\mathbf{3 8 8}$ starting from 1,3-propanediol 384

Since various options for the formation of acrylate polymers, including anionic, cationic and radical polymerisation, have been widely reported and they have been utilised in numerous applications, like plastics and adhesives industry, ${ }^{253}$ we initially proposed that self-initiated spontaneous thermal polymerisation without the addition of radical initiators was possibly the cause of the observed polymerisation process. ${ }^{254,255}$ However, this type of radical polymerisation of acrylates normally requires employing high temperature $\left(>100{ }^{\circ} \mathrm{C}\right) .{ }^{255}$ It seems unlikely that diene $\mathbf{3 8 8}$ could polymerise rapidly at moderate temperatures $\left(e . g, 40\right.$ to $\left.50{ }^{\circ} \mathrm{C}\right)$ without the involvement of radical initiators.

Instead, we proposed that an alternative potential cause for the degradation of the diene 388 was the acid lability of PMB protective group. A catalytic amount of $\mathrm{HCl}$ might be introduced to column fractions by sand used in preparing columns, which was washed by acid in production and could contain up to $0.2 \% \mathrm{HCl}$ according to its recorded manufacturing parameter. This could cause small amounts of deprotection of product $\mathbf{3 8 8}$, which could lead to polymerisation by oxa-Michael addition of the unprotected alcohol 389 to the acrylate of the lactone (Figure 3.5). 


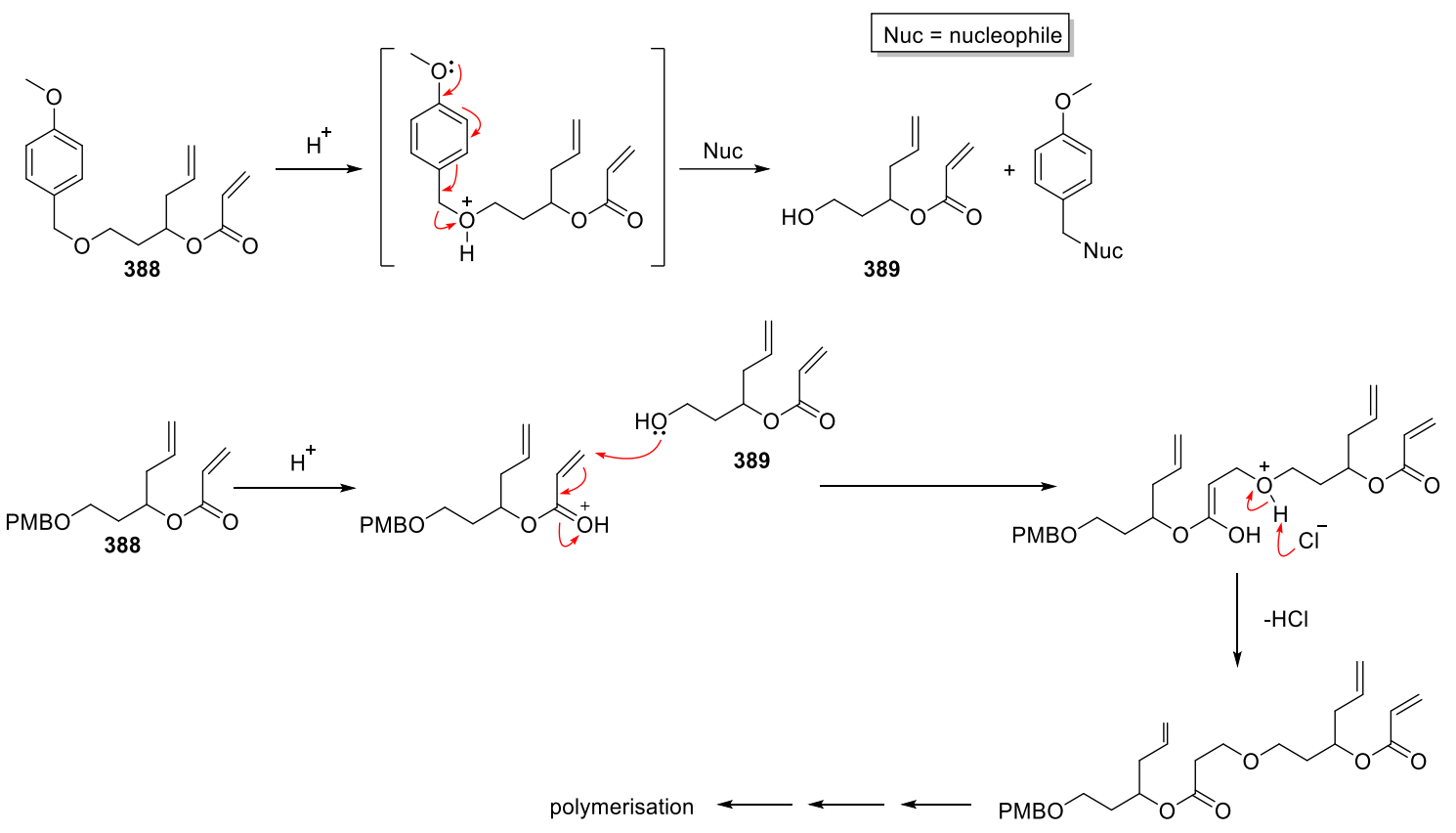

Figure 3.5 Proposed polymerisation of diene $\mathbf{3 8 8}$ caused by cleavage of the PMB group.

In view of the above discussion, acid-washed sand was replaced with neutral sodium sulfate in the following preparation of columns for purification of diene $\mathbf{3 8 8}$. To our delight, it seemed that this practice did prevent the occurrence of polymerisation, because the pure monomeric diene $\mathbf{3 8 8}$ was obtained on a 5-gram scale with $80 \%$ yield even after the concentrated diene was heated at 40 to $50{ }^{\circ} \mathrm{C}$ on the rotary evaporator for a relatively prolonged period of time.

Diene 388 then underwent ring-closing metathesis in the catalysis of Grubbs' $2^{\text {nd }}$ generation catalyst 390 to afford $\delta$-lactone 391 (Scheme 3.6). Cleavage of the PMB ether of lactone using DDQ provided alcohol 366, which was then coupled with hept-6-ynoic acid 258 under modified Steglich esterification conditions to afford the desired alkyne 364. The yield of alkyne over 7 steps was $30 \%$, which was lower than the $43 \%$ global yield reported by Cumming. ${ }^{134}$ It is worth mentioning that alkyne 364 was obtained in racemic form and both isomers were used for the following reaction sequence because the chiral centre will be removed by elimination in the base-induced ring-opening reaction of the $\delta$-lactone. 


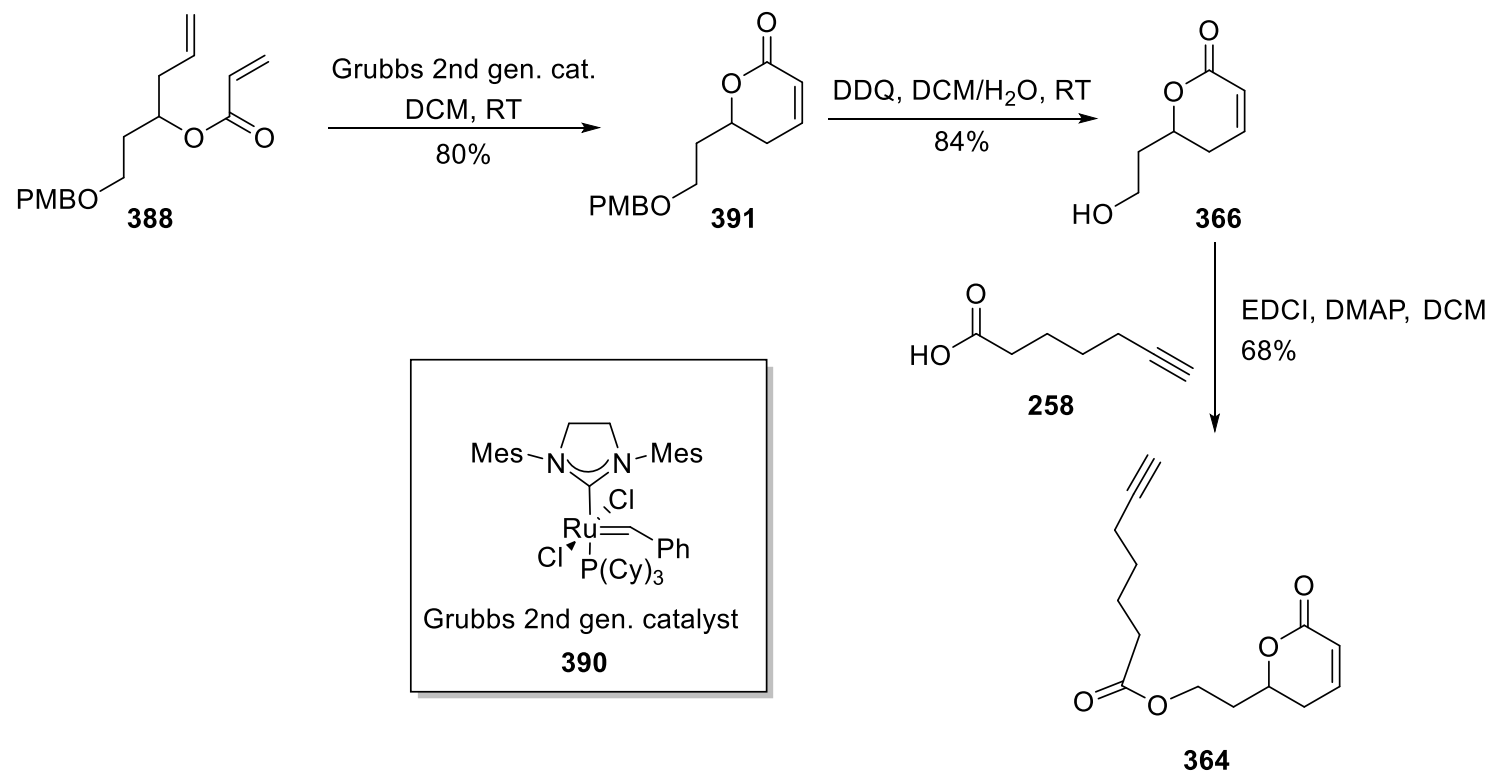

Scheme 3.6 Preparation of alkyne 364 .

\subsection{Preparation of Thiazole And C7-Substituted Thiazole}

\subsubsection{Preparation of Thiazoles 392 and 393}

The use of a gold-catalysed formation of the thiazole was discussed in Section 2.3.2. As gold alkene complexes have attracted considerable attention in the area of organometallic chemistry, ${ }^{144}$ we were concerned whether the conjugated $\mathrm{C}=\mathrm{C}$ double bond or the two oxygen atoms in the $\delta$-lactone motif of alkyne 364 could interference with the function of the gold catalyst. In light of the fact that the bis-silylated thioamide $\mathbf{3 8 2}$ could be partially oxidised to form the corresponding thioamide $S$-oxide $\mathbf{3 8 3}$ after a short exposure to air at room temperature $\left(18\right.$ to $20^{\circ} \mathrm{C}$ ), we were also concerned about its stability when heated at $40{ }^{\circ} \mathrm{C}$ with a mesylate intermediate in the cyclocondensation stage of the goldcatalysed thiazole formation. Thus, a model reaction using alkyne $\mathbf{2 5 9}$ was first performed to test the compatibility of the thioamide $\mathbf{3 8 2}$ under gold-catalysed conditions. This model reaction was conducted under the optimised conditions (see Section 2.3.2.3). As excess oxidant $N$-oxide 254 was present in the reaction vessel, we were concerned that this could lead to the formation of the undesired thioamide $S$-oxide 383 , which could significantly reduce the efficiency of this transformation. Therefore, intensive ${ }^{1} \mathrm{H}$ NMR analysis of the reaction aliquots was performed to detect the oxidised by-product after thioamide $\mathbf{3}$ was added to the reaction mixture. To our delight, the $\alpha$-proton signals of thioamide $S$-oxide 383 at $2.69 \mathrm{ppm}$ and $2.64 \mathrm{ppm}$ were not observed throughout the heating period, 
indicating that the oxidative degradation of thioamide $\mathbf{3 8 2}$ did not occur. Thiazole $\mathbf{3 9 2}$ was obtained in $61 \%$ yield and no degradation products of thioamide $\mathbf{3 8 2}$ were observed in this model reaction (Scheme 3.7). This satisfying result suggested that thioamide 382 could serve as a good substrate in this transformation.

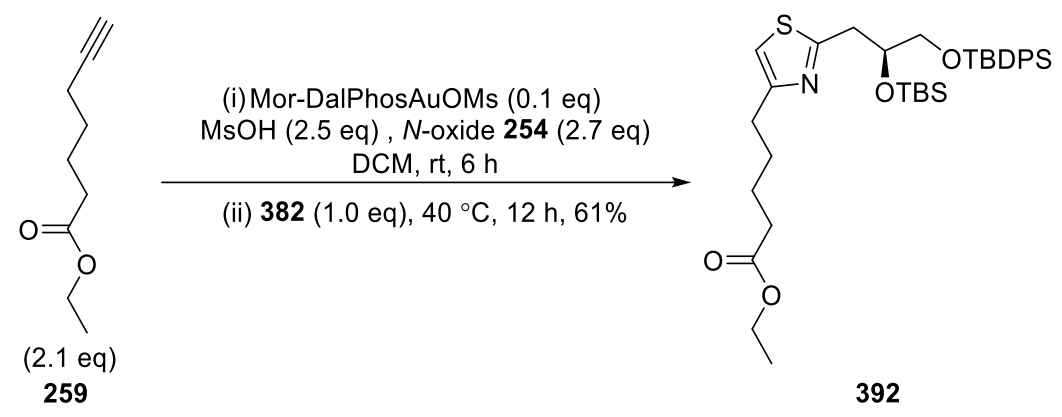

Scheme 3.7 Preparation of thiazole 392.

The gold-catalysed coupling between alkyne $\mathbf{3 6 4}$ and thioamide $\mathbf{3 8 2}$ was first performed on 0.1-gram scale and thiazole 393 was obtained in $60 \%$ yield, suggesting that the presence of the lactone motif might have little effect on the performance of the gold carbene. Thus, to test the applicability of this transformation for alkyne $\mathbf{3 6 4}$ on a larger scale, we subjected 0.86-gram of alkyne 364 to the one-pot protocol over 10 hours, following previously optimised conditions used for the preparation of thiazole 276 (see Section 2.3.2.3). As the catalyst loading was lowered to be $1 \%$ in this reaction, the consumption rate of thioamide 382 was expectedly slower. ${ }^{1} \mathrm{H}$ NMR analysis of the reaction aliquots at intervals indicated that the integration of the $\alpha$-proton signals of thioamide 382 at 3.64 and 3.58 ppm was decreasing while the integration of the proton signal of the thiazole ring at $6.80 \mathrm{ppm}$ was increasing, suggesting that the reaction proceeded smoothly under argon atmosphere. Pleasingly, thiazole 392 was obtained in $52 \%$ yield (BRSM yield 57\%), with the recovery of a small amount of unreacted thioamide 382 (Scheme 3.8). It is worth mentioning that the diastereomers of thiazole 392 are inseparable by flash chromatography and the isolated product was a mixture of diastereomers. 


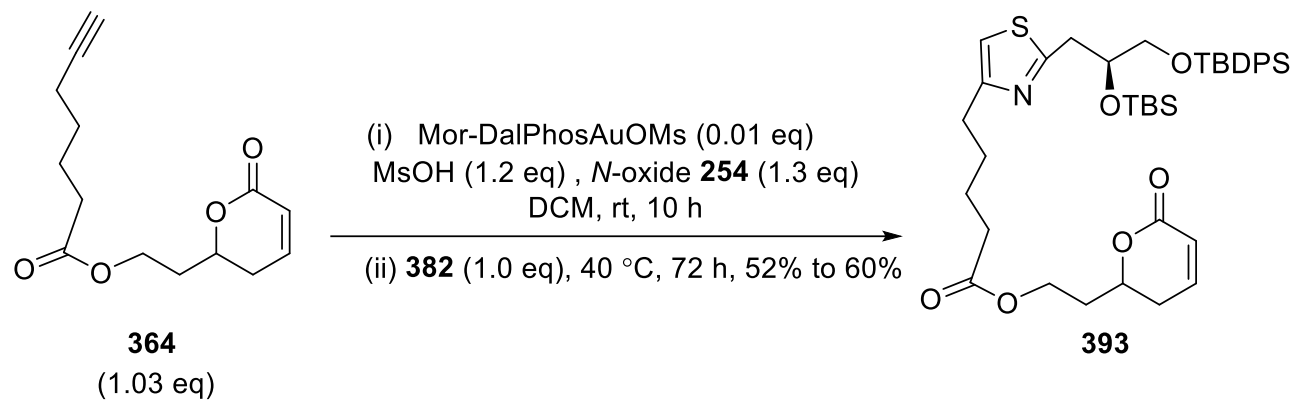

Scheme 3.8 Preparation of thiazole 393.

\subsubsection{Preparation of C-7 Substituted Thiazole}

As discussed in Section 1.7.1, we are interested in examining the effects of different substituents of the thiazole ring in PatA on its binding to the protein target. With the thiazole 392 in hand, we were able to explore the feasibility of modifications on the thiazole ring.

\subsubsection{Preparation of Brominated Thiazole 394}

We conceived that a bromo group at the C-5 position (equivalent to the C-7 position of PatA) could be a useful handle to allow the further introduction of an electronwithdrawing or electron-donating group to the thiazole ring for the preparation of PatA analogue 185 or 186 (see Section 1.7.1 for structures). As reflected by the resonance structures of thiazole (Figure 3.6), the C-5 position in the present system of thiazole 392 is the most reactive site for electrophilic substutition. ${ }^{257}$

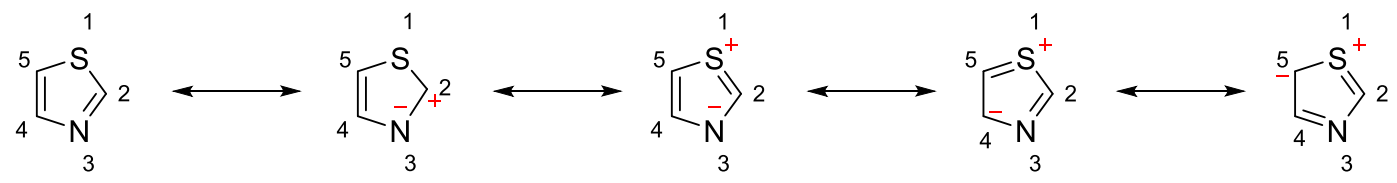

Figure 3.6 Resonance structures of thiazole. (Modified from Pola, 2016) ${ }^{258}$

A literature search $+*$ suggested that the electrophilic phenyl bromination by $N$ bromosuccinimide (NBS) normally requires the addition of activation reagents (e.g., Lewis acid). Thus, we had little concern about the interference of the phenyl groups of the TBDPS protective group in the regioselective bromination of thiazole $\mathbf{3 9 2}$ by solely using NBS. However, the reaction with one equivalent of NBS provided brominated

Mt Using SciFinder database; https://scifinder.cas.org (accessed July 2018). 
thiazole 394 in only moderate yield (54\%) (Scheme 3.9) with an unidentified by-product.

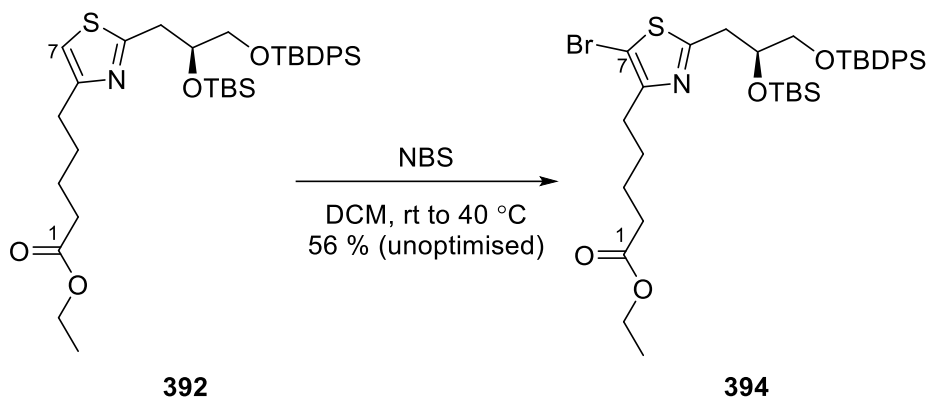

Scheme 3.9 Preparation of brominated thiazole 394.

\subsubsection{Attempted Preparation of Trifluoromethylated Thiazole 398}

As aromatic compounds bearing trifluoromethyl $\left(\mathrm{CF}_{3}\right)$ groups have been considered to be privileged motifs in drug discovery because of their unique properties arising from the electron-withdrawing nature, lipophilicity and metabolic stability of $\mathrm{CF}_{3}$ group, ${ }^{259}$ a significant number of copper-catalysed methodologies have been developed to achieve formation of aryl- $\mathrm{CF}_{3}$ bonds, most of which involve formation of reactive $\left[\mathrm{CuCF}_{3}\right]$ species in trifluoromethylation processes and are mainly limited to aryl iodide substrates. ${ }^{260,261}$ A scan of the literature (see Scheme 3.10 for a selected example) showed that palladium-based methodologies for trifluoromethylation could be appropriate for the preparation of $\mathrm{CF}_{3}$-substituted thiazole 395 from brominated thiazole 394 .

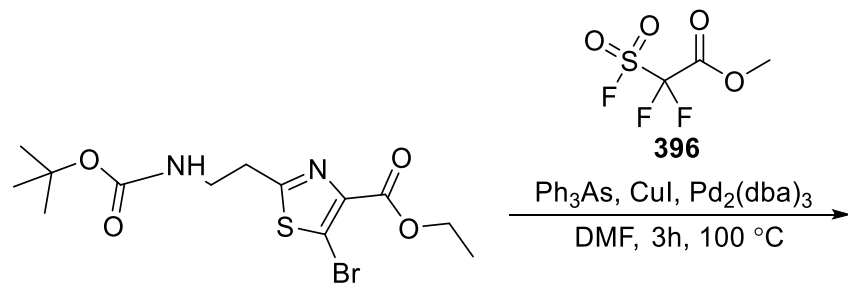

395

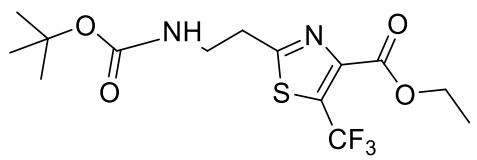

397

Scheme 3.10 Selected example of trifluoromethylation of brominated thiazole. ${ }^{262}$

Following the condition used in the synthesis of compound 397, methyl fluorosulfonyldifluoroacetate 396 was chosen as the $\mathrm{CF}_{3}$ source, which decarboxylates in the presence of $\mathrm{Cu}(\mathrm{I})$ iodide and releases difluorocarbene and fluoride ion to form $\mathrm{CF}_{3}{ }^{-}$ in situ. As shown in Scheme 3.11, trifluoromethylation of thiazole 394 with $\operatorname{Pd}_{2}\left(\mathrm{dba}_{3}\right.$ catalysis in DMF resulted in no significant reaction, with the recovery of bromothiazole 394. 


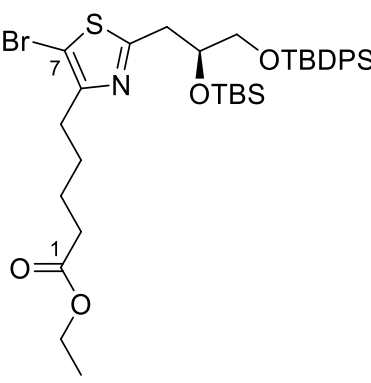

394

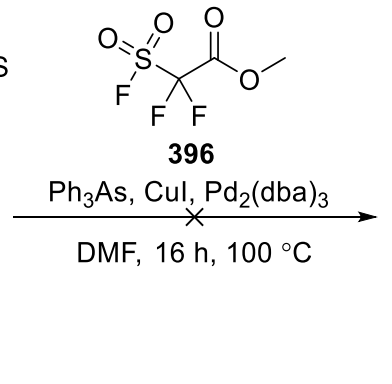

DMF, $16 \mathrm{~h}, 100^{\circ} \mathrm{C}$

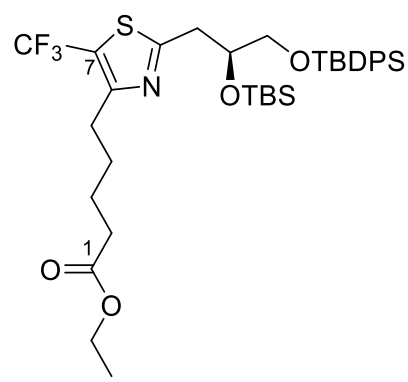

398

Scheme 3.11 Attempted preparation of trifluoromethlyated thiazole 398 .

The Pd- $\mathrm{CF}_{3}$ bond in the $\left[\mathrm{Pd}(\mathrm{II})(\mathrm{Aryl})\left(\mathrm{CF}_{3}\right)\right]$ complex has been reported to be relatively inert in the reductive elimination step of the catalytic cycle (Figure 3.7). ${ }^{261}$ For example, Marshall and Grushin discovered that the reductive elimination step required heating in xylene at $145{ }^{\circ} \mathrm{C}$ to produce $\mathrm{PhCF}_{3}$ slowly in their studies on the reactivity of several diphosphine-stabilised trifluoromethyl palladium phenyl complexes. ${ }^{263}$ To overcome this limitation, one major strategy is to use sterically and electronically modified phosphine ligands, such as Brettphos (compound 399), ${ }^{260}$ and Xantphos (compound 115, Figure $3.7)^{264}$

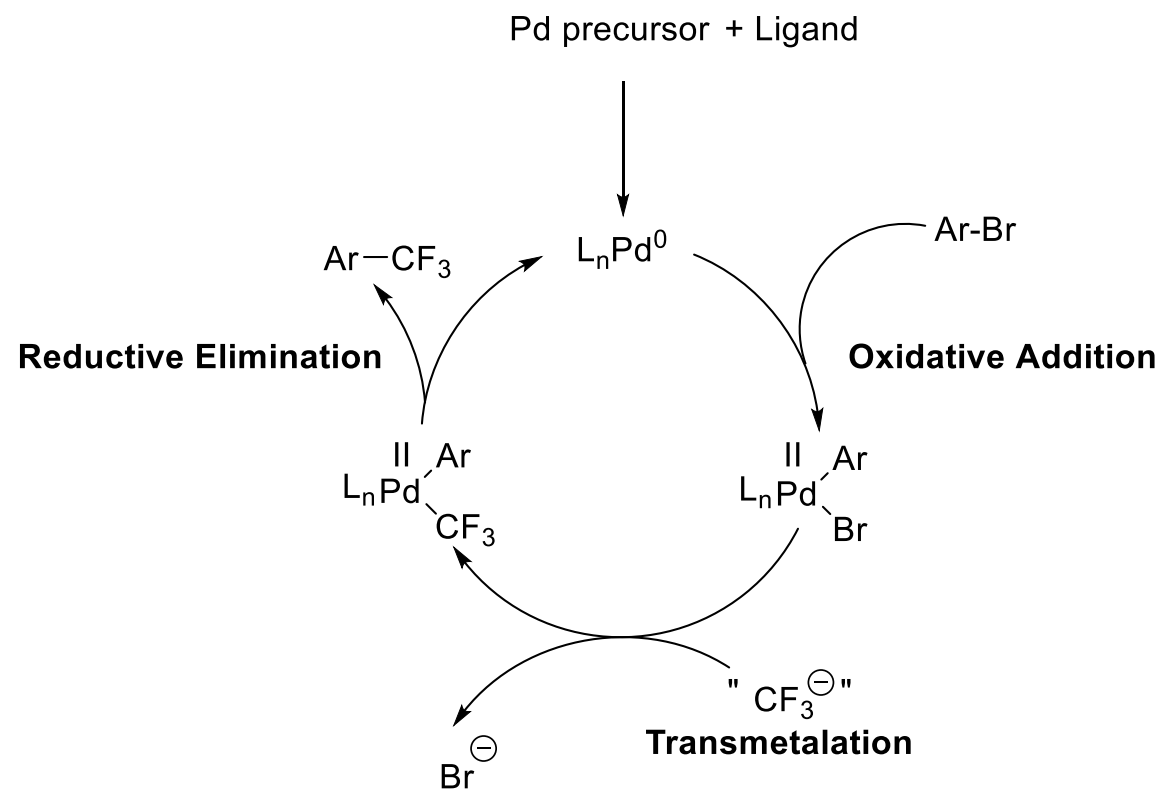

Figure 3.7 Generalised catalytic cycle for trifluoromethylation of aryl bromide $(\mathrm{L}=$ ligand; Ar =aryl) $\left(\right.$ modified from Buchwald et al). ${ }^{260}$ 


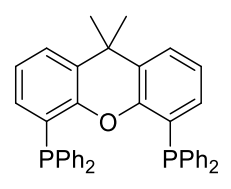

Xantphos (112)

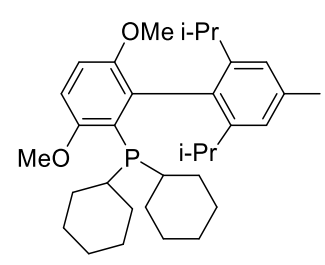

Brettphos (399)

Figure 3.8 Structures of Xantphos (112) and Brettphos (399).

It has been reported that a possible side reaction associated with the inertness of the reductive elimination step is the dehalogenation of the halide substrate. ${ }^{265}{ }^{1} \mathrm{H}$ NMR and HR-MS analysis of the reaction aliquots was performed again to detect the formation of the hydrodebrominated product-thiazole 392, which would potentially provide further insights into the cause of the sluggishness of the trifluoromethylation reaction. However, no evidence of the formation of thiazole $\mathbf{3 9 2}$ was observed. This raised that the possibility that the failure of the reaction might not be merely due to the activation barrier in the reductive elimination step. The problem with the oxidative addition of the brominated thiazole to the palladium( $(0)$ species might also play a role. Therefore, an obvious step forward is to assess the effectiveness of the conditions used in the trifluoromethylation of thiazole 394 by performing model reactions with simple brominated aromatic substrates (e.g., bromobenzene). If these model reactions did provide good results, the next step would be screening for reported combinations of ligand, palladium catalyst and $\mathrm{CF}_{3}$ source to facilitate reductive elimination of the $\mathrm{Pd}-\mathrm{CF}_{3}$ bond. However, due to time constraints, this was not further explored during the course of this Ph.D. project.

\subsubsection{Summary}

The gold-catalysed coupling between alkyne 259 and thioamide 382 was performed as the model reaction and thiazole 392 was obtained in $61 \%$ yield, suggesting thioamide 382 was compatible with the optimised conditions for the thiazole formation. The coupling reaction between alkyne 364 and thioamide 382 was then carried out and thiazole 393 was obtained in 52\% - 60\% yield. C7-brominated thiazole 394 was prepared in moderate yield in order to access $\mathrm{C} 7$-substituted PatA analogues. The preparation of $\mathrm{CF}_{3}$ substituted thiazole 395 by trifluoromethylation of thiazole 394 was unsuccessful, necessitating the need for optimising reaction conditions in the future work. 


\subsection{Construction of macrocycle}

\subsubsection{Synthesis of $E, Z$-Dienoic Acid}

\subsubsection{Introduction}

Although structures containing a carbonyl group conjugated to one or more alkenes frequently occur in macrocyclic lactone nature products, ${ }^{266}$ the presence of the $Z, E$ dienoate moiety is relatively rare compared to its counterpart E,E-dienoate. Usually, "isolated" $Z, E$-isomers require more energy to form than "isolated" $E, E$-isomers for steric reasons. However, in a complicated chemical environment, the situation may be different. For example, in a macrolide where the constraints of the macrocycle may favour one of the alkenes being $Z$-configuration, so the energy of the entire molecule with the $Z, E$-diene may be lower than that with the E,E-diene. The selected examples of natural products possessing Z,E-dienoate motif are presented in Figure 3.9, which include roridin E, dictyostatin, macrolactin A, zampanolide and salarin C. All of these compounds except roridin E (an extract from the black mold-Stachybotrys chartarum) ${ }^{267}$ are isolated from marine microorganisms.

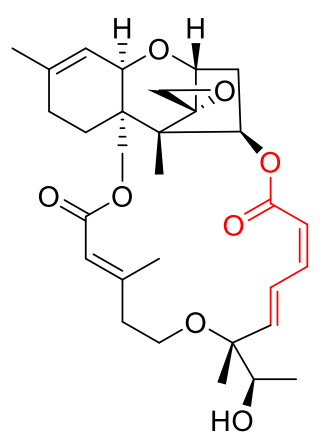

Roridin E

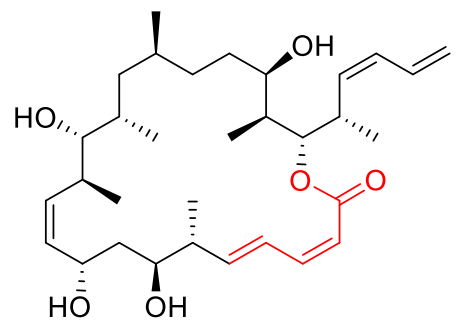

Dictyostatin<smiles></smiles>

Macrolactin A<smiles></smiles>

Zampanolide

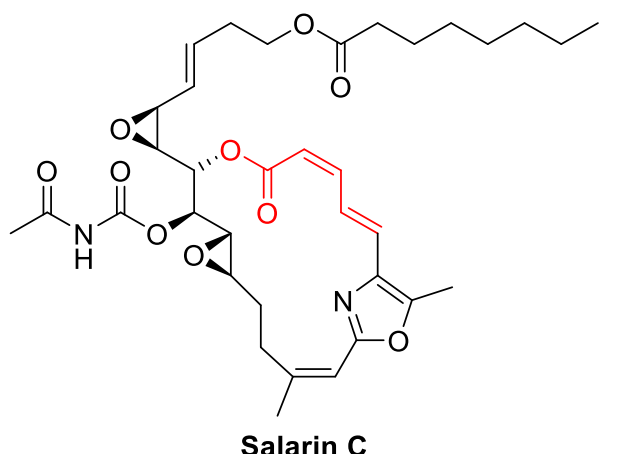

Salarin C

Figure 3.9 Examples of natural products possessing $Z, E$-dienoate motif. 
The major challenge in the synthesis of $Z, E$-dienoates is their tendency to isomerise to energetically more favoured $E, E$-dienoates. The most reliable methodologies for the stereoselective preparation of $Z, E$-dienoates can be roughly classified as follows: (a) cross-coupling reactions between an $E$ - or $Z$-vinyl stannane and a $Z$ - or $E$-vinyl halide, which has been utilised in Pattenden's total synthesis of PatA (vide supra, Section 1.4.1.2); (b) Z-selective olefination reactions, like Still-Gennari olefination and its modifications; (c) Sonogashira coupling reaction between an $E$-vinyl iodide and an alkyne, followed by subsequent selective hydrogenation of the alkyne by Lindlar catalyst, which has been applied in Romo's synthesis of PatA and its analogue DMDA PatA (vide supra, Section 1.4.1.1 and Section 1.4.2.1); and (d) the base-induced ring opening of $\alpha, \beta$-unsaturated lactone.

For example, in the synthesis of dictyostatin, to prevent the risk of isomerisation, $E$-vinyl iodide 400 was coupled with terminal alkyne $\mathbf{4 0 1}$ under Sonogashira conditions, whereby the resultant alkyne $\mathbf{4 0 2}$ could be reduced to the $Z$-alkene by Lindlar catalyst to give $Z, E$ dienoate 403. ${ }^{268}$

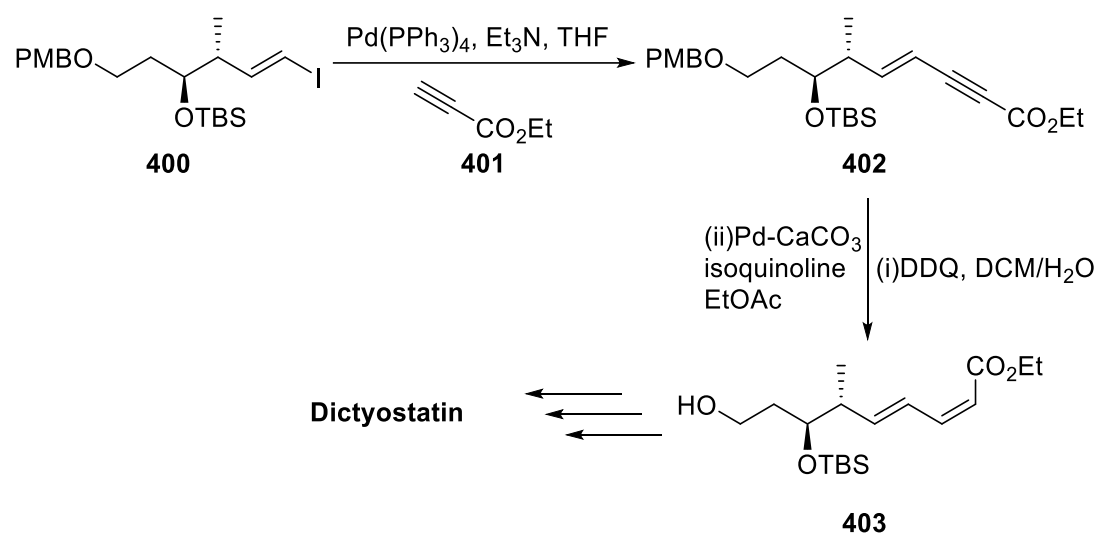

Scheme 3.12 Preparation of $Z, E$-dienoate 403 through a combination of Sonogashira coupling and Lindlar reduction in synthesis of dictyostatin. ${ }^{268}$

An alternative method to prepare $Z, E$-dienoic acid moieties is base-induced ring opening of $\alpha, \beta$-unsaturated $\delta$-lactones. This was first reported by Eisner and co-workers when they discovered that treatment of racemic parasorbic acid 404 with methanolic sodium methoxide yielded $(2 Z, 4 E)$-hexadienoic acid 405 (Scheme 3.13a). ${ }^{269}$ Following their discovery, it has been reported that other bases, such as $t$-BuOK, ${ }^{270} \mathrm{MeSNa},{ }^{271} \mathrm{NaOH},{ }^{272}$ and LiHMDS, ${ }^{273}$ can also induce this transformation. This method has been used in the 
synthesis of the ansamycins (Scheme 3.13b) ${ }^{271}$ and saliniketal A (Scheme 3.13c). ${ }^{273}$ As shown in Scheme 3.13, the most intriguing features of this reaction are its complete stereospecificity in these examples.

(a)

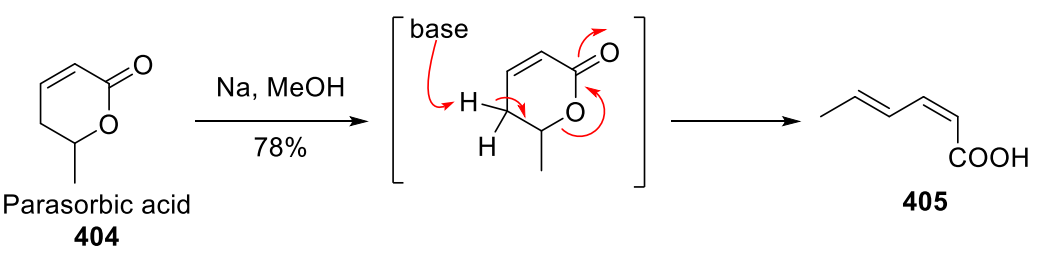

(b)

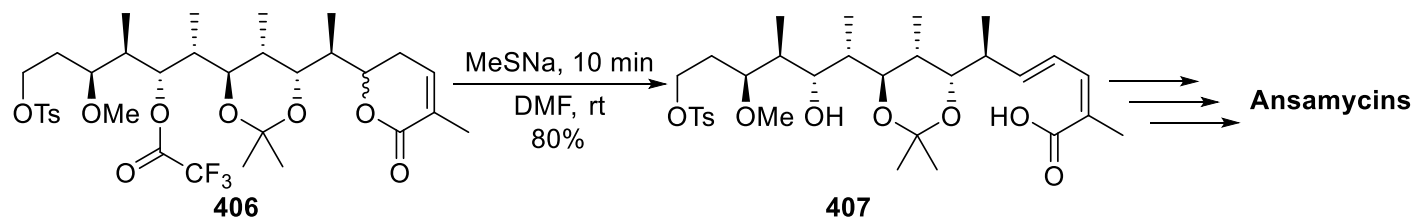

(c)

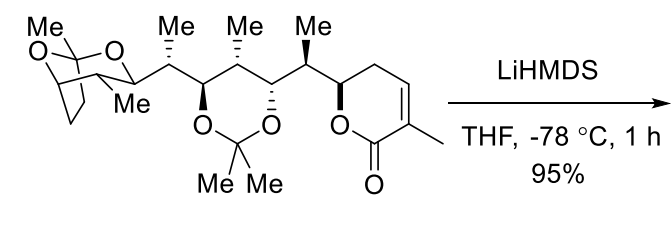

408

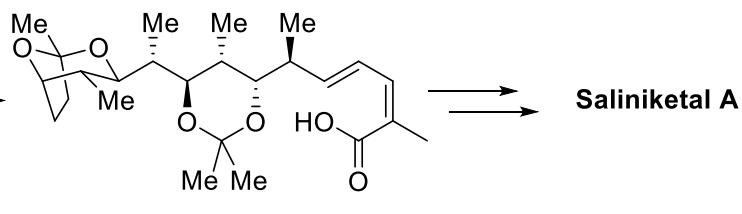

409

Scheme 3.13 Ring-opening reactions of $\alpha, \beta$-unsaturated lactones induced by stong base: (a) of parasorbic acid; ${ }^{269}$ (b) in the synthesis of ansamycins; ${ }^{271}$ and (c) in the synthesis

$$
\text { of saliniketal. }{ }^{273}
$$

However, functional groups susceptible to strong basic conditions are not compatible with the conditions mentioned above. In an effort to search much milder conditions, Oishi and co-workers found that the mild base TBAF could act effectively in this transformation. ${ }^{274}$ For example, the acetoxyl groups in compound $\mathbf{4 1 0}$ remained completely intact in the ring-opening reaction induced by TBAF (Scheme 3.14a). This method was also used effectively towards the synthesis of leustroducsin B (Scheme 3.14b). ${ }^{275}$ As TBAF has enjoyed widespread use for desilylation, ${ }^{276}$ silyl protecting groups are not compatible with this method. 
(a)

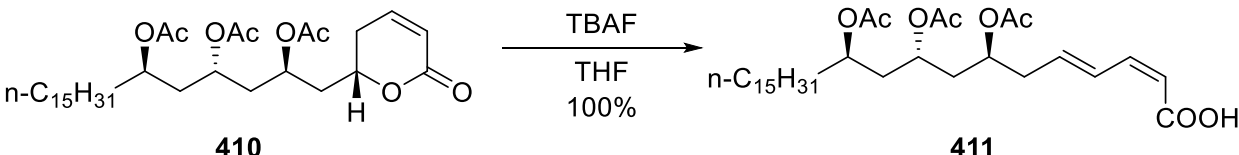

410

(b)

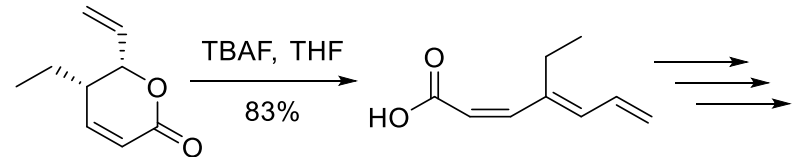

412

413

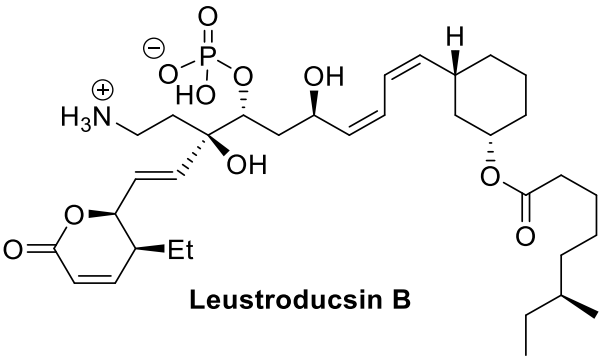

Scheme 3.14 TBAF induced ring-opening reactions of lactones: (a) in preparation of triacylated compound $\mathbf{4 1 1} ;{ }^{274}$ and (b) towards the synthesis of leustroducsin B. ${ }^{275}$

Despite these reported excellent stereoselectivities and good yields for $Z, E$-dienoic acids, this method has not attracted lots of attention from the synthetic community. Therefore, to explore the practicality of this method in the synthesis of PatA triazole analogue $\mathbf{1 8 2}$ (vide supra, Section 1.6) lacking a methyl group at the $\mathrm{C} 22$ position, previous $\mathrm{Ph} . \mathrm{D}$. student Hemmi Cumming in our research group conducted extensive studies on ringopening reactions of $\alpha, \beta$-unsaturated lactones, and found that the treatment of lactones 364 and 391 with TBAF provided the desired E,Z-dienoic acids 414 and 415 as single isomers, respectively.
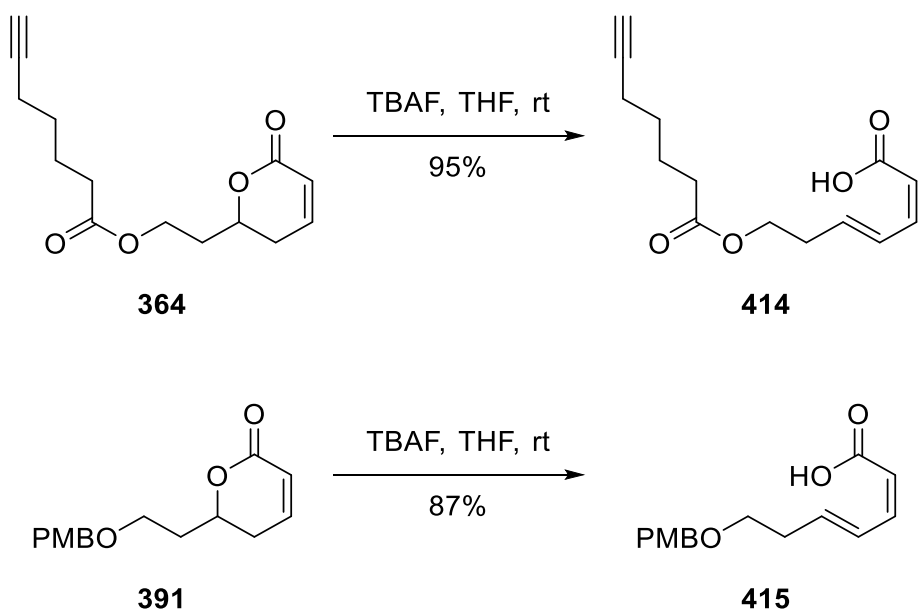

Scheme 3.15 TBAF induced ring-opening reactions of lactone 364 and 391 conducted by Cumming. ${ }^{135}$

\subsubsection{Initial Synthesis of E,Z-Dienoic Acid 419}

In light of the excellent stereoselectivities and yields obtained in the preparation of $E, Z$ - 
dienoic acids 414 and 415 through ring opening of lactones 364 and 391 (Scheme 3.15), we decided to adopt this base-induced ring-opening strategy in the preparation of E,Zdienoic acid 419 required for the macrolactonisation step.

Although treatment with TBAF could theoretically cleave the silyl protecting groups simultaneously with the lactone ring-opening reaction, we were concerned that this onepot protocol could cause significant side-reactions on the basis of Cumming's discovery that the treatment of lactone $\mathbf{4 1 6}$ with TBAF led to the formation of a considerable amount of degradation products (Scheme 3.16). Thus, we decided to first test the validity of using KHMDS for the lactone ring-opening transformation.

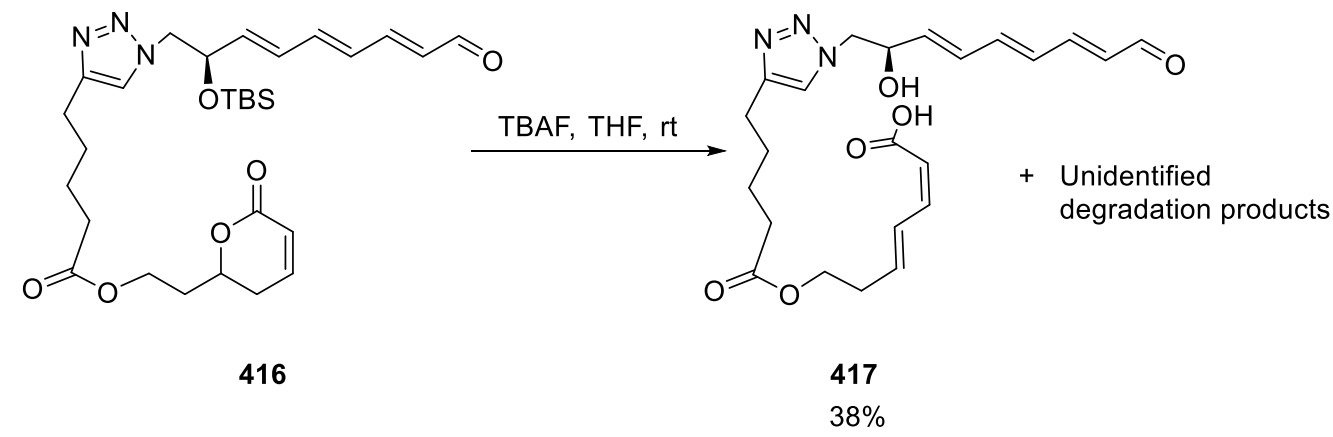

Scheme 3.16 Simultaneous silyl ether deprotection and lactone opening of $\mathbf{4 1 6}$ by treatment with TBAF reported by Cumming. ${ }^{135}$

In 1966, Landesberg and Olofson conducted extensive studies to assess the electron distribution in thiazoles as depicted by the resonance structures in Figure 3.6 by deuteration experiments and discovered that the proton at C-2 position and C-5 position (thiazole numbering) of the unsubstituted thiazole were deprotonated at the same rate under treatment with sodium methoxide in deuterated methanol. ${ }^{277}$ This means that the acidity of the proton of the thiazole in compound $\mathbf{3 9 3}$ needs to be taken into account when performing the base-induced ring-opening reaction. Nonetheless, the $\delta$-position of the lactone was likely to be more acidic than the proton on the thiazole, and therefore reactions occurring on the thiazole ring might be minimised by limiting the equivalents of KHMDS used in the ring-opening reaction. This should avoid potential side-reactions caused by the nucleophilic attack of the deprotonated thiazole. Initial treatment with 1.1 equivalent of KHMDS (Table 3.1, entry 1) at $-78^{\circ} \mathrm{C}$ led to incomplete conversion of the lactone 393. However, the coupling constants of the alkenes of the isolated dienoic acid product were consistent with the reported characterisation data of intermediate $\mathbf{1 5 3}$ in 
Fürstner's synthesis of DMDA PatA (vide supra, Section 1.4.2.2), ${ }^{128}$ indicating the product was the desired $Z, E$-dienoic acid $\mathbf{4 1 8}$. The isolated yield was $43 \%$, and no other geometrical isomer was observed by ${ }^{1} \mathrm{H}$ NMR analysis. Pleasingly, increasing the usage of KHMDS to 1.3 equivalent successfully drove the reaction to completion and improved the yield to $60 \%$ (Table 3.1, entry 2).

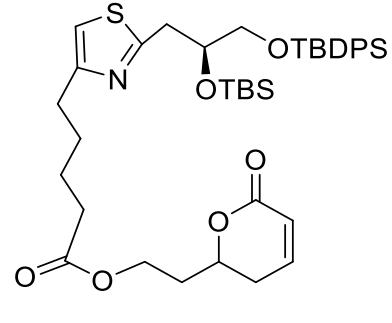

393

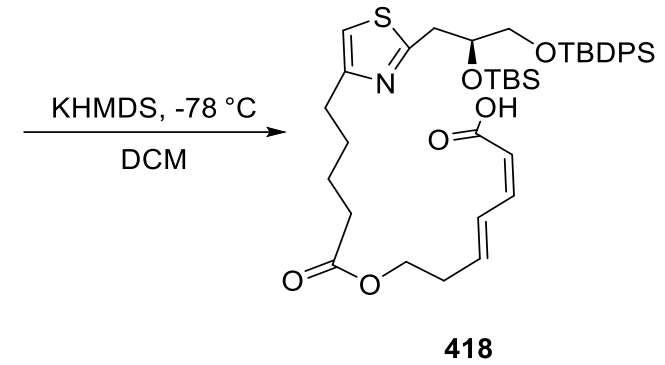

418

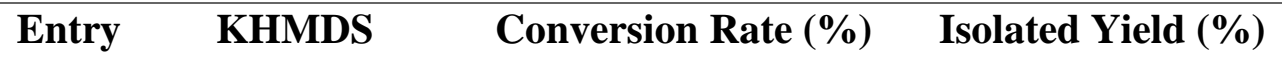 (equiv.)}

\begin{tabular}{llcc}
\hline $\mathbf{1}$ & 1.1 & 77 & 43 \\
\hline $\mathbf{2}$ & 1.3 & 100 & 60
\end{tabular}

Table 3.1 Optimisation of the base-induced ring opening of lactone 393 .

With Z,E-dienoic acid $\mathbf{4 1 8}$ in hand, we were then able to test conditions for chemoselective cleavage of the secondary TBS silyl group. As indicated by Table 3.2, the difference between the lability of primary TBS and TBDPS silyl ethers in acid is greater than that between their fluoride reactivity. ${ }^{278,279}$ Although these results were obtained with primary silyl ethers, in contrast to the secondary TBS group in the present system, it was reasonable to assume that acid-mediated desilylation could differentiate more effectively between the secondary TBS silyl ether and the primary TBDPS silyl ether than fluoride-induced desilylation. Thus, we then set out to screen for acid methods that allow removal of a secondary TBS ether in the presence of a primary TBDPS ether. 
Table 3.2 Comparison of acidic hydrolytic lability and fluoride reactivity of primary TBS and TBDPS silyl ethers. ${ }^{278,279}$

\begin{tabular}{ccc}
\hline Silyl Ether & $\begin{array}{c}\text { Half-Lives with } \\
\mathbf{1 \%} \mathbf{~ H C l - M e O H ,} \\
\mathbf{2 5}{ }^{\mathbf{0}} \mathbf{C}\end{array}$ & $\begin{array}{c}\text { Half-Lives with } \\
\text { TBAF, 25 }{ }^{\mathbf{o}} \mathbf{C}^{\mathbf{a}}\end{array}$ \\
\hline$n-\mathrm{C}_{6} \mathrm{H}_{13} \mathrm{OTBS}$ & $\leq 1 \mathrm{~min}$ & - \\
$n-\mathrm{C}_{6} \mathrm{H}_{13} \mathrm{OTBDPS}$ & $225 \mathrm{~min}$ & - \\
$n-\mathrm{C}_{12} \mathrm{H}_{25} \mathrm{OTBS}$ & - & $140 \mathrm{~h}$ \\
$n-\mathrm{C}_{12} \mathrm{H}_{25} \mathrm{OTBDPS}$ & - & $375 \mathrm{~h}$
\end{tabular}

${ }^{\mathrm{a}} 0.01 \mathrm{M}$ in substrate containing TBAF (6 equiv.), DCM/THF (9:1). Half-Lives were determined by ${ }^{1} \mathrm{H}$ NMR monitoring quenched reaction aliquots.

A range of acidic conditions that may be suitable for the desired chemoselectivity has been reported. These included the use of $p$-TSA, ${ }^{280}$ camphorsulfonic acid, ${ }^{281}$ a mixture of $\mathrm{AcOH}-\mathrm{H}_{2} \mathrm{O}-\mathrm{THF}(3: 1: 1),{ }^{282} \mathrm{HCl}$ in $\mathrm{MeOH},{ }^{283}$ and pyridinium $p$-toluenesulfonate (PPTS) (rt or heat). ${ }^{284}$ As we were concerned that the treatment of compound $\mathbf{4 1 8}$ with more strongly acidic conditions could lead to degradation of $Z, E$-dienoic acid, we decided to first test the weakly acidic salt PPTS in the selective desilylation reaction.

Following the original conditions of Blair and co-workers, ${ }^{284}$ the removal of the TBS group was first attempted with 2 equivalents of PPTS in $\mathrm{MeOH}$ at room temperature, but after 1 day, only little consumption of the starting material was observed (Table 3.3, entry 1). This suggested that we needed to seriously consider the impacts of the basicity of the thiazole ring in the following experiments. ${ }^{285} \mathrm{We}$ then proposed that increasing the equivalents of PPTS could drive the reaction to completion. However, using 4 equivalents of PPTS at room temperature resulted in no significant reaction neither (entry 2). Raising the reaction temperature to $55^{\circ} \mathrm{C}$ did provide desired product 419 , but after 1 day the reaction had not gone to completion (entry 3). Increasing PPTS equivalents at $55{ }^{\circ} \mathrm{C}$ did not drive the reaction to completion, nor did it appreciably improve the yield (entry 4). 

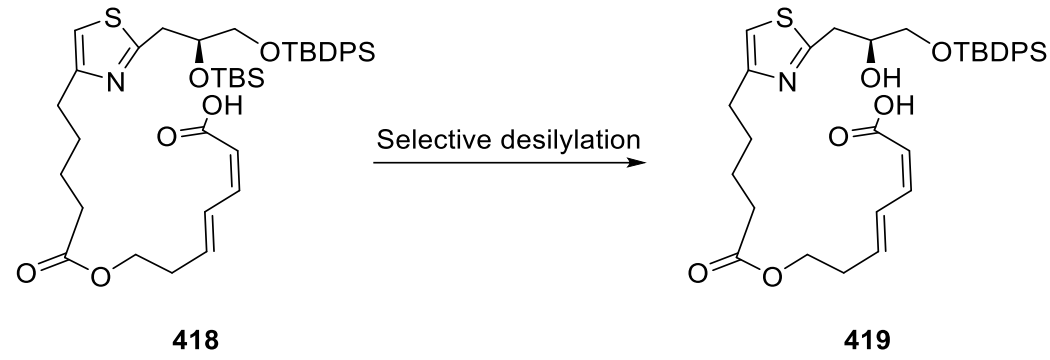

\begin{tabular}{ccccccc}
\hline Entry $^{\mathbf{a}}$ & $\begin{array}{c}\text { Reagent } \\
\text { (equiv.) }\end{array}$ & Solvent & $\mathbf{T}\left({ }^{\mathbf{0}} \mathbf{C}\right)^{\mathbf{b}}$ & Time (h) & $\begin{array}{c}\text { Consumption } \\
\text { Rate of SM }^{\mathbf{c}}(\boldsymbol{\%})\end{array}$ & Yield $^{\mathbf{d}}(\boldsymbol{\%})$ \\
\hline $\mathbf{1}$ & PPTS (2.0) & $\mathrm{MeOH}$ & $\mathrm{rt}$ & 27 & little & - \\
\hline $\mathbf{2}$ & PPTS (4.0) & $\mathrm{MeOH}$ & $\mathrm{rt}$ & 27 & little & - \\
\hline $\mathbf{3}$ & PPTS (4.0) & $\mathrm{MeOH}$ & 55 & 27 & 68 & $27(\mathrm{BRSM} 40)$ \\
\hline $\mathbf{4}$ & PPTS (10.0) & $\mathrm{MeOH}$ & 55 & 27 & 71 & $30(\mathrm{BRSM} 43)$ \\
\hline
\end{tabular}

${ }^{\mathrm{a}}$ All reactions were carried out at concentrations of $0.014 \mathrm{M}$. ${ }^{\mathrm{b}} \mathrm{rt}=$ room temperature. ${ }^{\mathrm{c}} \mathrm{SM}=$ starting material. Consumption rate was calculated based on the amount of recovered bis-silylated starting material after purification by silica gel chromatography. ${ }^{\mathrm{d}}$ Isolated yields of $\mathbf{4 1 9}$ purified by silica gel chromatography. (BRSM yields are in parentheses).

Table 3.3 Trialled conditions for selective desilylation of compound $\mathbf{4 1 8}$.

HR-MS investigation of the aliquot samples from the reaction mixture after 27 hours heating at $55{ }^{\circ} \mathrm{C}$ revealed that it contained a mixture of the bis-silylated starting material $418\left([\mathrm{M}-\mathrm{H}]^{-}\right.$ion at $\left.\mathrm{m} / \mathrm{z}=734.3372\right)$, desired TBS-deprotected product $419\left([\mathrm{M}-\mathrm{H}]^{-}\right.$ion at $\mathrm{m} / \mathrm{z}=620.2525)$, undesired TBDPS-deprotected product $420\left([\mathrm{M}-\mathrm{H}]^{-}\right.$ion at $\mathrm{m} / \mathrm{z}=$ 496.2168) and bis-desilylated product $421\left([\mathrm{M}-\mathrm{H}]^{-}\right.$ion at $\left.\mathrm{m} / \mathrm{z}=382.1339\right)$ (see Figure 3.10 for HR-MS spectrum). 


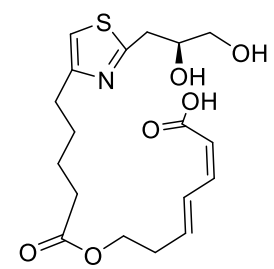

421

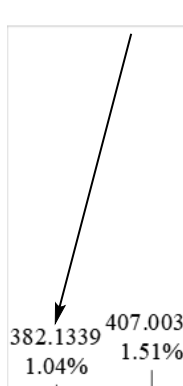

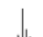

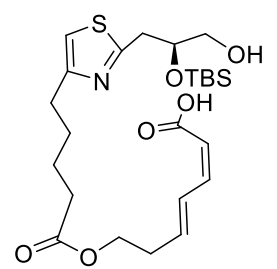

420

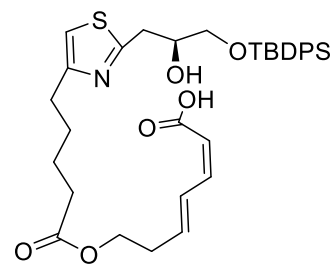

419

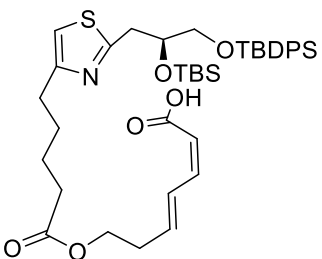

418

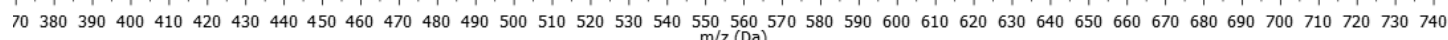

Figure 3.10 HR-MS for the reaction mixture of mono-desilylation of $\mathbf{4 1 8}$ using 10 equivalents of PPTS. ${ }^{\S \S \S}$

Given that the formation of doubly desilylated product $\mathbf{4 2 1}$ was detected by HR-MS, attention then turned to the possibility of recovery of compound $\mathbf{4 2 1}$ and reprotection of the primary hydroxyl. This would potentially provide more desired product $\mathbf{4 1 9}$. However, there was a risk that the use of base (e.g., imidazole or pyridine) in selective re-silylation of the primary $\mathrm{OH}$ of product $\mathbf{4 2 1}$ could cause isomerisation of the $Z, E$-dienoic acid. We attempted to recover product $\mathbf{4 2 1}$ from the PPTS reaction described above. Unfortunately, bis-desilylated product $\mathbf{4 2 1}$ was lost while being purified by flash silica gel chromatography due to its high polarity.

The overall yield of these two steps for the preparation of $Z, E$-dienoic acid $\mathbf{4 1 9}$ was only $18 \%$, which prompted us to explore a more efficient synthetic route.

\subsubsection{Alternative Routes for Synthesis of E,Z-dienoic Acid 419}

As shown in Scheme 3.17, an alternative two-step approach was then considered, in which the TBS removal would be performed ahead of the lactone opening. This would

$\S \S \S \S[\mathrm{M}-\mathrm{H}]^{-}$peak of compound 418 (at 734.3372, calculated for $\mathrm{C}_{40} \mathrm{H}_{56} \mathrm{NO}_{6} \mathrm{SSi}_{2}{ }^{-}, 734.3372$ ), compound 419 (at 620.2525, calculated for $\mathrm{C}_{34} \mathrm{H}_{42} \mathrm{NO}_{6} \mathrm{SSi}^{-}, 620.2508$ ), compound 420 (at 496.2168, calculated for $\mathrm{C}_{24} \mathrm{H}_{38} \mathrm{NO}_{6} \mathrm{SSi}^{-}, 496.2195$ ) and compound 421 (at 382.1339, calculated for $\mathrm{C}_{18} \mathrm{H}_{24} \mathrm{NO}_{6} \mathrm{~S}^{-}, 382.1330$ ). 
allow more options for the acid-mediated selective removal of the secondary TBS silyl ether.

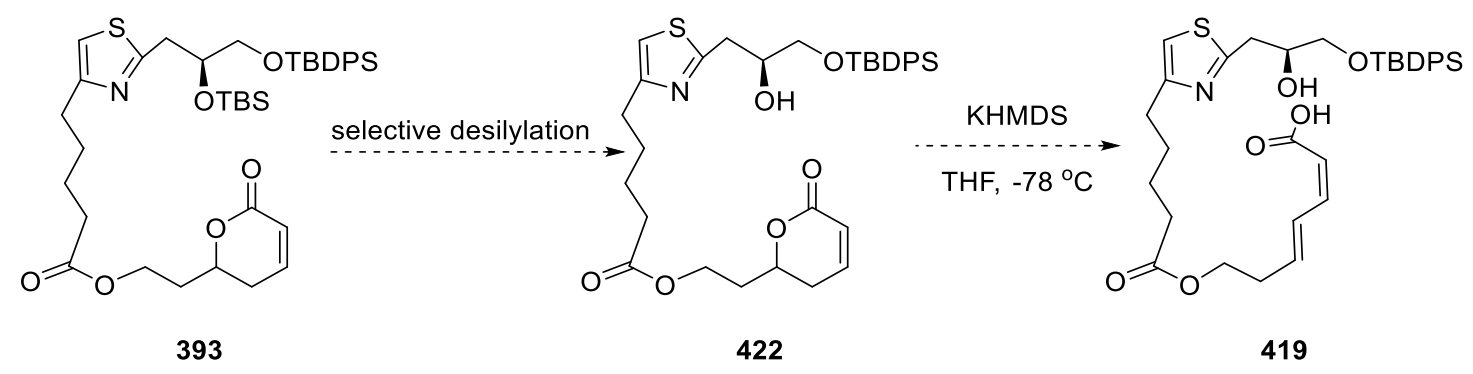

Scheme 3.17 Proposed alternative two-step approach to the synthesis of seco-acid 419.

The desilylation of compound $\mathbf{3 9 3}$ was first trialled with a catalytic amount of strong acid p-TSA in MeOH and DCM at room temperature (Table 3.4, entry 1). ${ }^{286}$ Surprisingly, little consumption of the starting material was observed after over-night stirring. Increasing $p$ TSA equivalents provided only a trace amount of desired product 422 (entry 2). Instead, a diol product $\mathbf{4 2 3}$ resulting from the removal of both silyl ethers was obtained as the major product. Attention was then directed towards use of milder acidic conditions. Treatment with a mixture of $\mathrm{AcOH}, \mathrm{H}_{2} \mathrm{O}$, and THF at room temperature resulted in no significant reaction (entry 3). Performing the reaction at $50{ }^{\circ} \mathrm{C}$ cleanly provided the desired product $\mathbf{4 2 2}$ as the sole product, albeit with the recovery of substantial amounts of the starting material (entry 4). Efforts to drive the reaction to completion by extending the reaction time did prompt the consumption of the starting material (entries 5 and 6) but also resulted in substantial formation of diol product 423. Not surprisingly, the isolated yields of compound $\mathbf{4 2 2}$ were not improved significantly. Two unconventional conditions for selective removal of secondary TBS silyl ether were then explored: one used the oxidising reagent $\mathrm{NaIO}_{4}$ in $\mathrm{THF},{ }^{287}$ the second used a 1:1 mixture of TBAF and $\mathrm{BF}_{3} \cdot \mathrm{Et}_{2} \mathrm{O}$ in anhydrous acetonitrile. ${ }^{288}$ Unfortunately, these two conditions were found to be sluggish with little consumption of starting material (entries 7 and 8). Given that reactions with mixtures of $\mathrm{AcOH}, \mathrm{H}_{2} \mathrm{O}$ and THF under prolonged heating in fact provided mixtures of mono-silylated product $\mathbf{4 2 2}$ and the diol $\mathbf{4 2 3}$, along with unreacted starting material, we then opted to prepare compound $\mathbf{4 2 2}$ by full deprotection, followed by monoprotection of the primary position, with the hope that this could provide a better overall yield (albeit with more steps). 


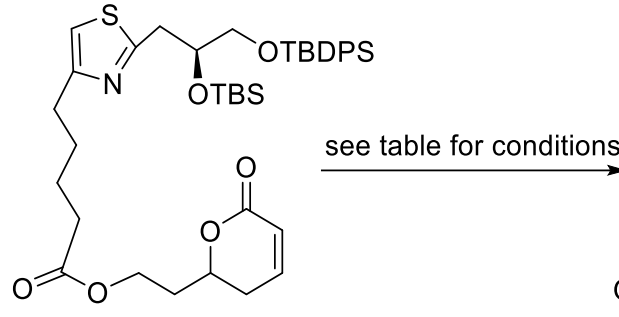

393

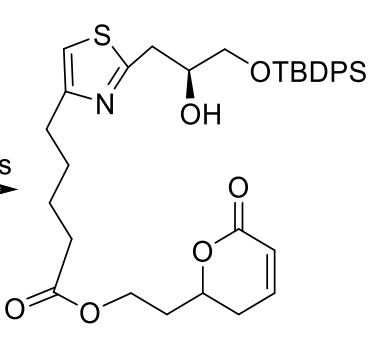

422

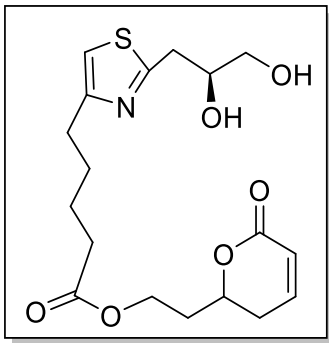

423

\begin{tabular}{|c|c|c|c|c|c|c|}
\hline Entry & $\begin{array}{l}\text { Reagent } \\
\text { (equiv.) }\end{array}$ & Solvent & $\begin{array}{c}\mathrm{T} \\
\left({ }^{\circ} \mathbf{C}\right)^{\mathrm{a}}\end{array}$ & $\begin{array}{c}\text { Time } \\
\text { (h) }\end{array}$ & $\begin{array}{c}\text { Consumption } \\
\text { Rate }^{\mathrm{b}}(\%)\end{array}$ & Yield $^{\mathrm{c}}(\%)$ \\
\hline 1 & $p$-TSA $(0.3)$ & MeOH-DCM & $\mathrm{rt}$ & 18 & little & - \\
\hline 2 & $p$-TSA (2.0) & MeOH-DCM & $\mathrm{rt}$ & 18 & 44 & 422, trace \\
\hline 3 & $\mathrm{AcOH}$ & $\mathrm{THF}-\mathrm{H}_{2} \mathrm{O}$ & $\mathrm{rt}$ & 18 & little & - \\
\hline 4 & $\mathrm{AcOH}$ & THF- $\mathrm{H}_{2} \mathrm{O}$ & 50 & 3 & 33 & $\begin{array}{c}\text { 422, } 30(\text { BRSM } \\
100)\end{array}$ \\
\hline 5 & $\mathrm{AcOH}$ & THF- $\mathrm{H}_{2} \mathrm{O}$ & 50 & 9 & 76 & 422, 26 (BRSM 35) \\
\hline 6 & $\mathrm{AcOH}$ & THF- $\mathrm{H}_{2} \mathrm{O}$ & 50 & 23 & 88 & 422, 34 (BRSM 39) \\
\hline 7 & $\mathrm{NaIO} 4$ & $\mathrm{EtOH}$ & $\mathrm{rt}$ & 18 & - & - \\
\hline 8 & $\mathrm{BF}_{3}-\mathrm{TBAF}$ & $\mathrm{MeCN}$ & $\mathrm{rt}$ & 18 & - & - \\
\hline
\end{tabular}

${ }^{\mathrm{a}} \mathrm{rt}=$ room temperature. ${ }^{\mathrm{b}}$ Calculation based on the amount of recovered bis-silylated starting material 393 after purification by silica gel chromatography. ${ }^{\mathrm{c}}$ Yields of purified products by silica gel chromatography. (BRSM yields are in parentheses).

Table 3.4 Trialled conditions for selective desilylation of compound 393.

As shown in Scheme 3.18, desilylation of compound 393 by treatment with HF-pyridine provided diol $\mathbf{4 2 3}$ in uninspiring 57\% yield. Assuming that this moderate yield was a result of material loss in the aqueous quench with aqueous saturated $\mathrm{NaHCO}_{3}$ solution, given that the polarity (and likely hydrophilicity) of the diol, we then used trimethylsilanol to quench excess HF instead. But this modification did not give a better yield. Since sufficient diol was obtained for the next step, no further optimisation was performed. We then moved on to perform selective silyl protection of the primary hydroxyl group. The classic conditions using TBDPSCl and imidazole were first trialled. ${ }^{289}$ Surprisingly, a mixture of unidentified degradation products was obtained, with no sign of the desired product observed by ${ }^{1} \mathrm{H}$ NMR analysis. Assuming that the unexpected degradation was induced by deprotonation of the highly acidic $\delta$-proton of the lactone by imidazole, we repeated the reaction with TBDPSCl and pyridine, a base considered to be about 100 times less basic than imidazole. ${ }^{290}$ To our dismay, this reaction 
again provided a mixture of degradation products.

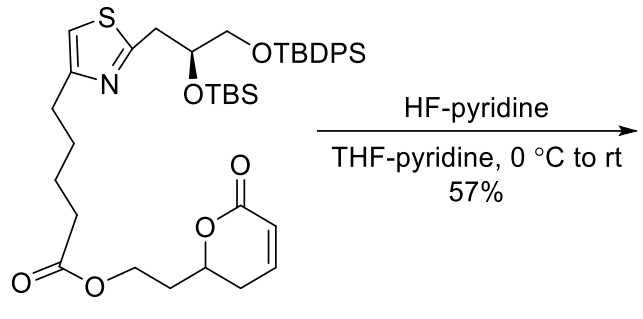

393

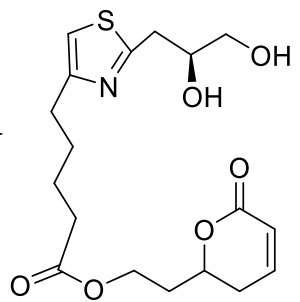

423

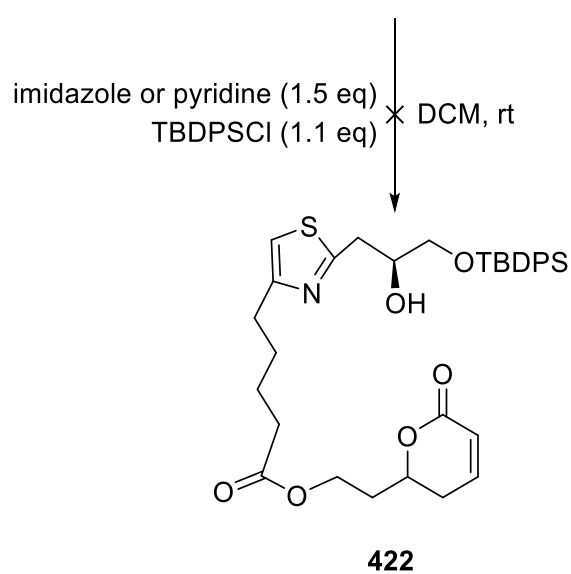

Scheme 3.18 Attempted preparation of compound $\mathbf{4 2 2}$ via diol intermediate $\mathbf{4 2 3}$.

This inability to prepare compound $\mathbf{4 2 2}$ by selective protection of diol $\mathbf{4 2 3}$ prompted us to return to selective desilylation of $\mathbf{3 9 3}$ (Table 3.4, Entry 3 ) in order to access compound 419. After obtaining sufficient material by using this method, we then performed the lactone opening reaction using 2.3 equivalents of KHMDS at $-78{ }^{\circ} \mathrm{C}$ (Scheme 3.19). Unexpectedly, this reaction provided seco-acid $\mathbf{4 1 9}$ in only $36 \%$ yield, accompanied by a mixture of unidentified by-products. The obtained overall yield for these two steps was $11 \%$, which was lower than the yield obtained in the initial synthetic route described in Section 3.5.1.2. 

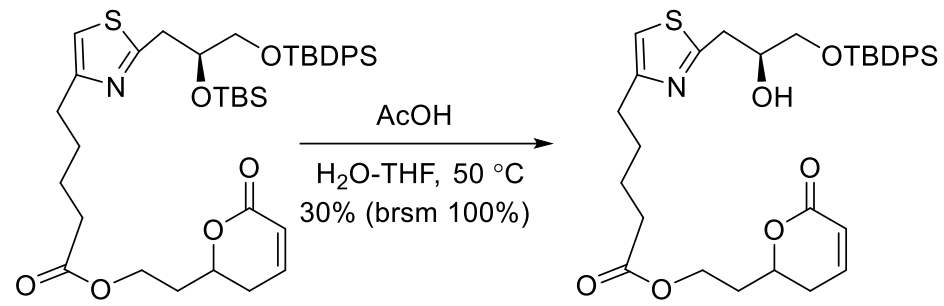

393

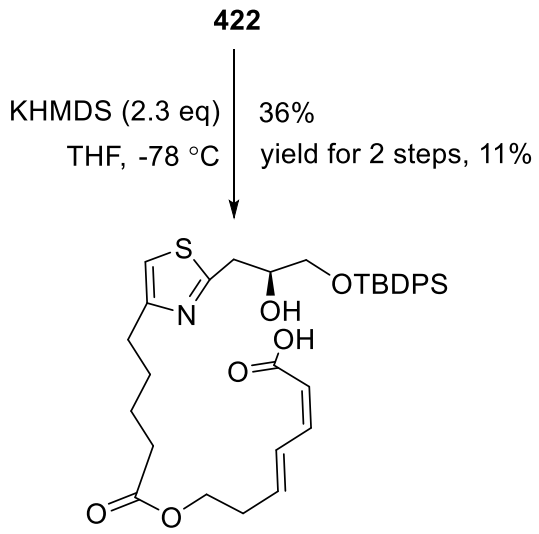

419

Scheme 3.19 Preparation of seco-acid 419 via mono-silylated intermediate $\mathbf{4 2 2}$.

The poor yield then led us to re-consider the feasibility of a one-pot protocol for the preparation of seco-acid 421. The problem associated with the purification of polar compound 421 would likely be resolved by using reversed-phase chromatography. Thus, we then carried out the double desilylation reaction using 3 equivalents of TBAF in THF with the hope that it would allow the base-induced lactone opening and the silyl cleavage to occur simultaneously (Scheme 3.20). Unfortunately, a mixture of degradation products was obtained, with no sign of compound $\mathbf{4 2 1}$ observed by ${ }^{1} \mathrm{H}$ NMR investigation.

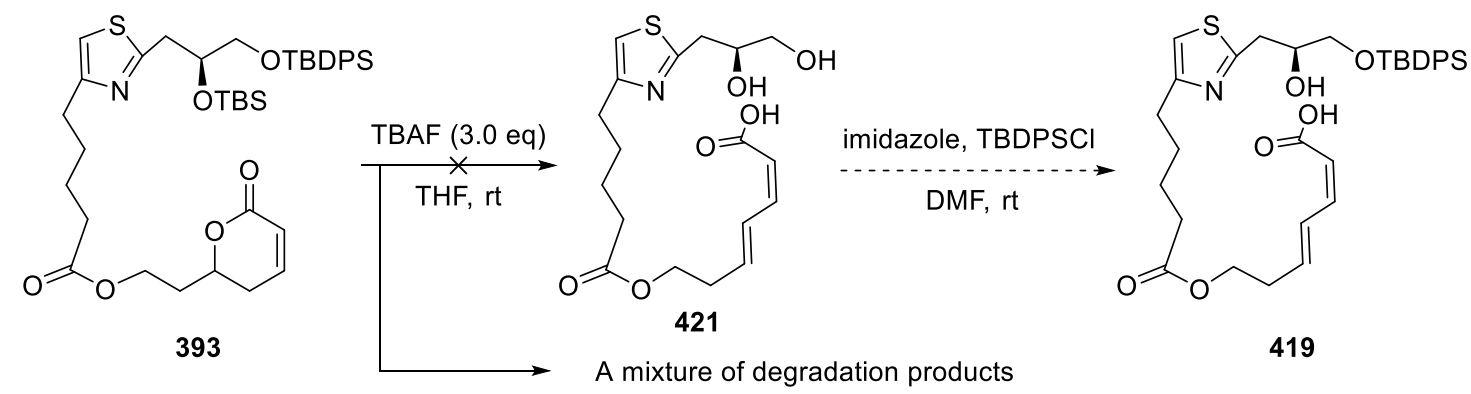

Scheme 3.20 Attempted preparation of seco-acid 419 via intermediate 421.

Based on experimental results obtained in our endeavours described above, the preparation of seco-acid $\mathbf{4 1 9}$ will require further optimisation. As TBAF has been used to 
chemoselectively cleave the secondary TBS ether in the presence of the primary TBDPS ether in the preparation of diol $\mathbf{4 2 5}$ towards the synthesis of lytophilippine A (Scheme $3.21),{ }^{291}$ fluoride-induced desilylation could be another feasible method for the synthesis of seco-acid 419. Strict control of the equivalents of TBAF and slow addition of TBAF by a syringe pump at a lower temperature could potentially provide an improved yield for seco-acid 419.

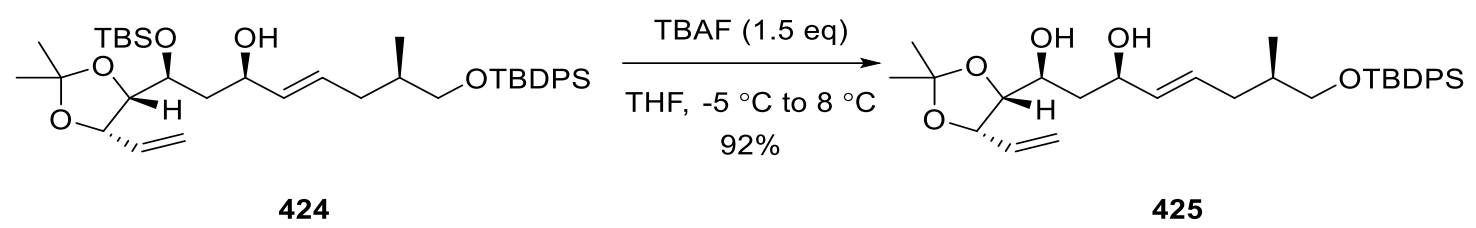

Scheme 3.21 TBAF-induced chemoselective cleavage of TBS ether reported by Gille and Hiersemann. ${ }^{291}$

Nonetheless, a sufficient quantity of seco-acid 419 had been stockpiled using the initial synthetic route (vide supra, Section 3.5.1.2) for exploring conditions for the construction of the macrocycle.

\subsubsection{Summary}

The initial KHMDS-induced ring opening of bis-silylated $\delta$-lactone 393 provided the desired Z,E-dienoic acid $\mathbf{4 1 8}$ at an acceptable yield. Attempts to selectively remove the TBS group of acid 418 under treatment with PPTS (vide supra, Table 3.4). provided secoacid 419 in an uninspiring yield, necessitating the need for developing alternative routes to improve the yield. Full removal of silyl protecting groups and subsequent re-protection of the primary -OH group of diol $\mathbf{4 2 1}$ resulted in a mixture of unidentified products, which was possibly due to the high acidity of the proton residing in the lactone motif. Selective cleavage of the TBS group of lactone 393, followed by base-induced ring opening of resulting product $\mathbf{4 2 2}$ with the presence of a free -OH group, afforded the seco-acid 419 in a reduced yield when compared to the initial route. The TBAF-mediated one-pot approach to prepare diol 421, which could be potentially transformed into seco-acid $\mathbf{4 1 9}$ through selective silylation, was unsuccessful. Therefore, the preparation of seco-acid 419 requires further investigation. 


\subsubsection{Macrolatonisation}

\subsubsection{Introduction}

Macrocyclic lactones are common and broadly found in natural products from terrestrial and marine sources ${ }^{292}$ and present a remarkably diverse range of medicinal properties, such as antibiotic, anticancer or antitumoral, antifungal, and immunosuppressive. ${ }^{293}$ Thus, it is not surprising that macrocyclic lactone natural products have served as intriguing targets to drive organic chemists to develop new macrocyclisation methodologies for their synthesis. Even though a significant number of diverse macrocyclisation approaches have been reported over the years, such as HWE olefination, ${ }^{294}$ intramolecular ring-closing metathesis, ${ }^{295}$ palladium-catalysed carbon-carbon cross-coupling (e.g, Stille coupling), ${ }^{296}$ and chromium-induced Nozaki-Hiyama-Takai-Kishi reaction, ${ }^{297}$ the macrolactonisation of seco-acids still remains one of the most often used protocols.

According to Illuminati and Mandolini's comprehensive kinetic investigations of lactonisation with different ring sizes,${ }^{298}$ there are three factors directing the $\kappa_{\text {intra }} / \kappa_{\text {inter }}$ ratio in the macrolactonisation: the activation energy (enthalpy), decreased degree of freedom (entropy) and the possibility of end-end encounters. The activation energy is the energy that needs to be provided to overcome the strain energy, which arises from geometric deviations of bond angles (Baeyer strain), steric hindrance due to imperfect staggered conformation (Pitzer strain) and transannular strains due to repulsive interactions between atoms, when a bifunctional open chain precursor is converted to the transition state for the ring to be formed. The internal rotation of single bonds is constrained as the lactone is being formed, meaning that the level of disorder in the system is reduced, and this is entropically disfavoured. As the ring size gets larger, the proportion of conformers that include end-to-end encounters typically decreases

As shown in Figure 3.11, for medium-sized rings $(n=8 \sim 12)$, enthalpic factors from the increased strain energy, which decreases the ability to adopt an ideal bond angle and form the reactive conformation, in combination with the entropic factors, make the formation of medium-sized rings the most difficult to achieve. For macrocyclic lactone rings ( $\mathrm{n}>$ 13), although the ring strain is low (enthalpic factor), the entropic factors increases and the probability of the chain terminals encountering each other also decreases. However, the intermolecular reaction may also suffer a similar entropy penalty. Therefore, the 
$\kappa_{\text {intral }} / \kappa_{\text {inter }}$ ratio remains relatively constant.

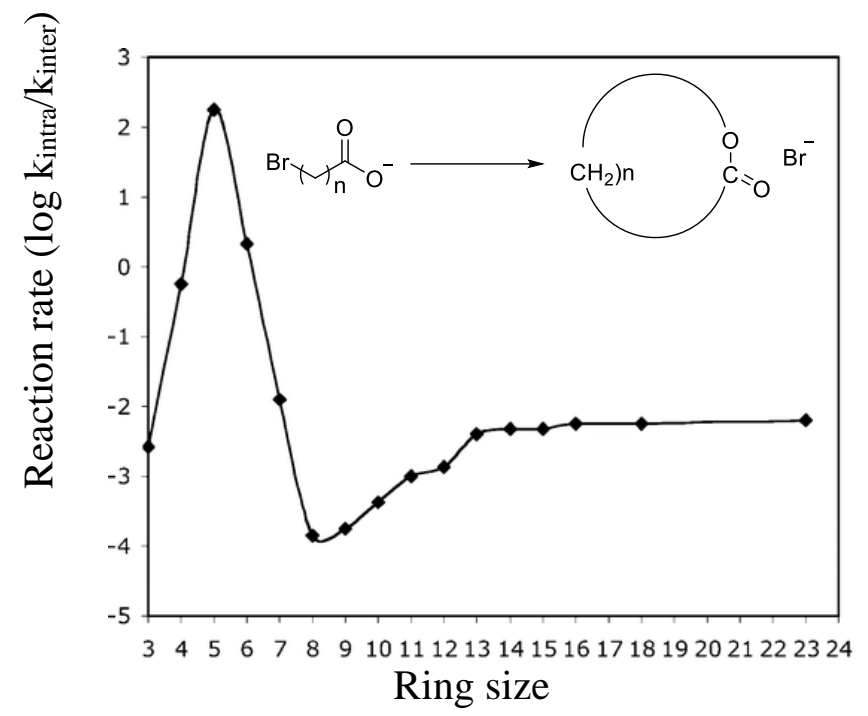

Figure 3.11 Reactivity profile for lactone formation. ${ }^{298}$

Therefore, to favour intramolecular reactions in the competition between intra- and intermolecular reactions, high-dilution conditions were first introduced by Ruggli and Ziegler, ${ }^{299}$ which requires adding the substrate at a slow rate, for example by a syringe pump, to a large volume of solvent.

Many reagents and conditions have been developed for the purpose of accelerating the macrolactonisations, most of which work by means of either "acid activation" (path a in Figure 3.12) or "carboxylate attack" on a leaving group (path b in Figure 3.12).

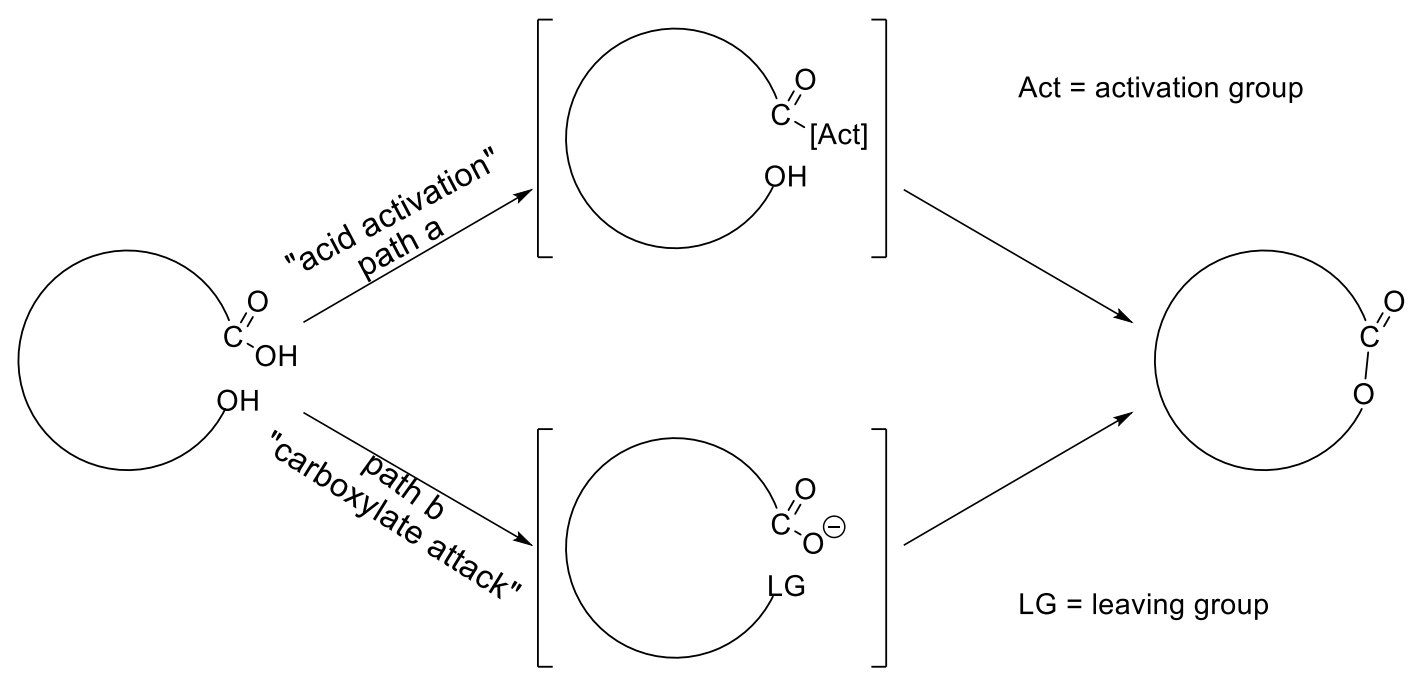

Figure 3.12 Representative scheme of macrolactonisation by "acid activation" or “carboxylate attack" (Modified from Campagne et al.) ${ }^{300}$ 
Some general macrolactonisation reactions/reagents and their mechanistic intermediates are summarised in Figure 3.13. Each of the reactions exemplifies their respective category of macrolactonisation reactions/reagents, of which a number of variations and modifications have been developed and applied successfully in the synthesis of macrocyclic natural products and their analogues. ${ }^{300}$ 


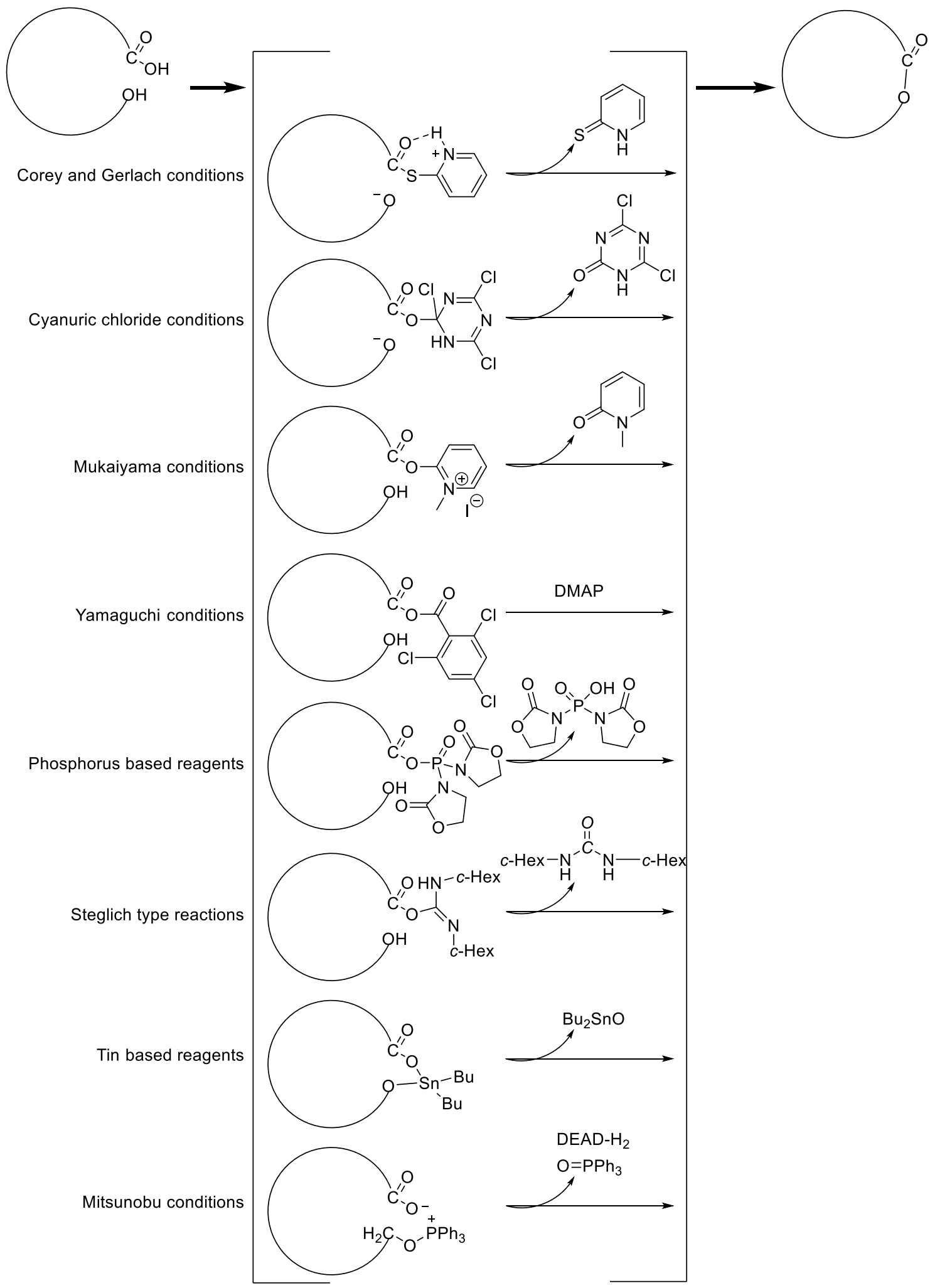

Figure 3.13 Summary of selected macrolactonisation methods.

Among all these methods, the original Yamaguchi reaction, which uses 2,4,6trichlorobenzoyl chloride $\mathbf{9 3}$ as "acid activator", ${ }^{301}$ remains the most popular method for construction of macrocyclic lactones and has been utilised with success in many synthetic 
approaches. ${ }^{300}$ As shown in Scheme 3.22, the carboxylic acid first reacts with Yamaguchi reagent 93 in the presence of triethylamine to provide the mixed anhydride. The anhydride is then added slowly by a syringe pump to a highly diluted solution of DMAP at high temperatures. DMAP acts as a stronger nucleophile than the alcohol and attacks the anhydride to afford the reactive amide intermediate, which reacts rapidly with the alcohol to form the macrocyclic lactone.

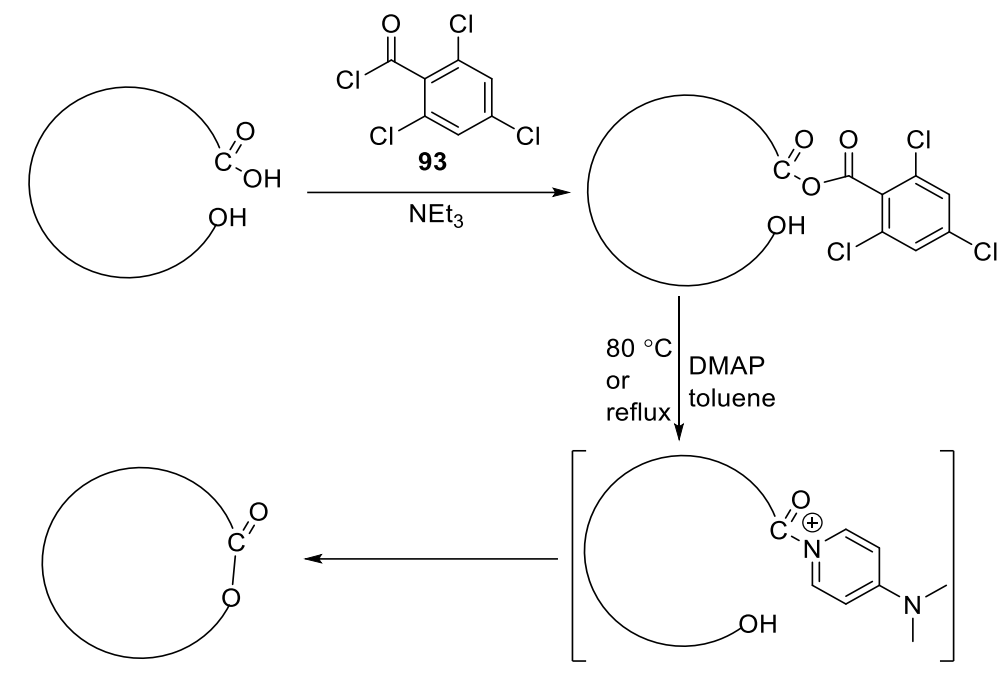

Scheme 3.22 Proposed mechanism of Yamaguchi macrolactonisation. ${ }^{301}$

When Yamaguchi procedure is used for lactonisation of an E,Z-dienoic acid and an alcohol, significant isomerisation can sometimes occur. For example, in the synthesis of macrolactin A analogue reported by Takemoto et al. (Scheme 3.23), the Yamaguchi lactonisation step afforded the undesired $(2 E, 4 E)$-lactone $\mathbf{4 2 7}$ as the sole product, instead of the desired $(2 Z, 4 E)$-lactone. ${ }^{302}$

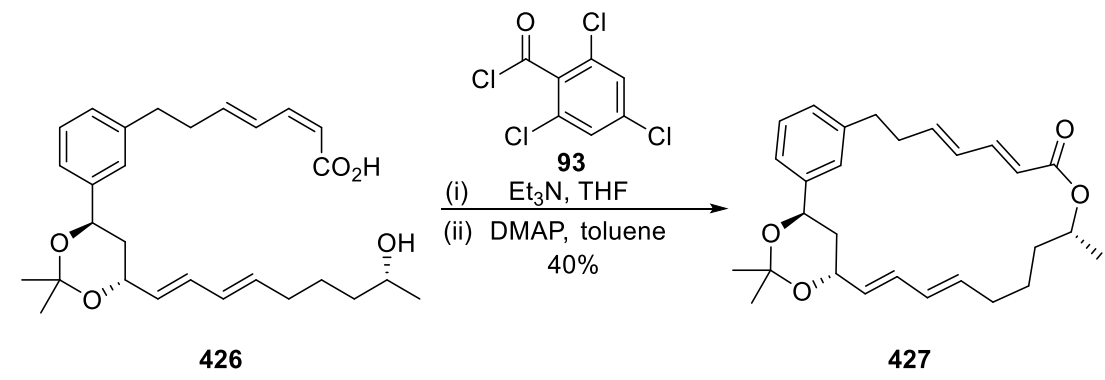

Scheme 3.23 Isomerisation in lactonisation in synthesis of macrolactin A analogue. ${ }^{302}$

Considering that the isomerisation could be partially attributed to the high reaction temperature required in the second step, Yonemitsu further developed a modification of 
Yamaguchi conditions where an excess amount of $\operatorname{DMAP}(e . g ., 10$ equivalents) is directly added to the solution of preformed anhydride intermediates at room temperature. ${ }^{303}$ In the synthesis of dictyostatin, the macrolactonisation of seco-acid was conducted under these modified conditions and the $(2 Z, 4 E)$-lactone $\mathbf{4 2 9}$ was obtained in $78 \%$ yield, accompanied by $5-10 \%$ of the $(2 E, 4 E)$-lactone isomer (Scheme 3.24$){ }^{304}$

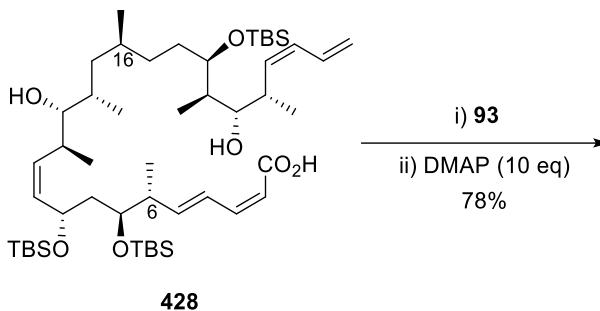

428

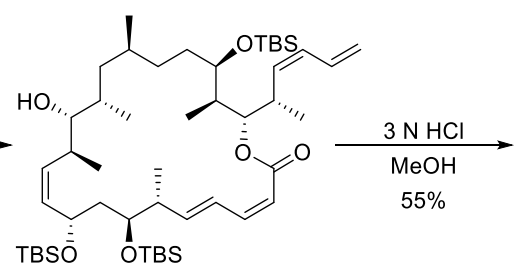

429

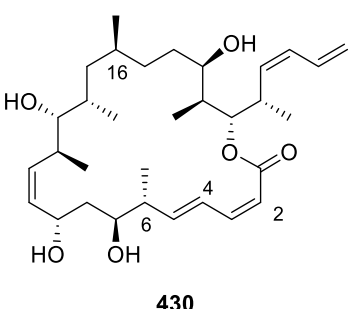

Dictyostatin

Scheme 3.24 Use of Yonemitsu's modified conditions in synthesis of dictyostatin. ${ }^{304}$

Similarly, the Shiina reaction, a variation of the Yamaguchi reaction, can be also performed with an excess of DMAP at room temperature. In the synthesis of dideoxysalarin $\mathrm{C}$ recently reported by Altmann and Schrof, the desired macrocycle $\mathbf{4 3 3}$ was constructed under Shina conditions with only about $7 \%$ of isomerised $(2 E, 4 E)$ dienoate byproduct observed based on ${ }^{1} \mathrm{H}$ NMR analysis. ${ }^{305}$

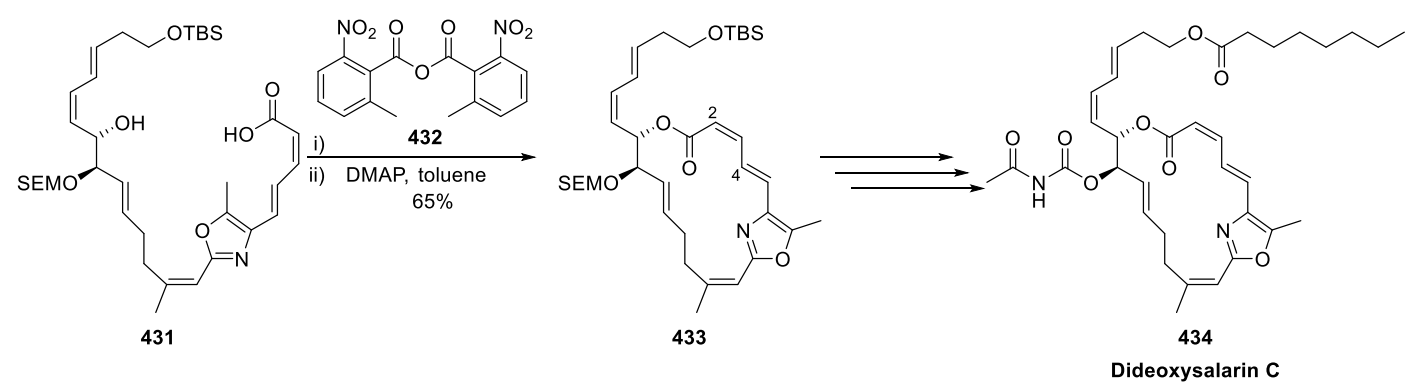

Scheme 3.25 Shiina reaction towards the synthesis of dideoxysalarin C. ${ }^{305}$

Interestingly, in the synthesis of an analogue of dictyostatin, the Shinna reaction on secoacid 435 provided a 60/40 ratio of Z,E-product 436 and E,E-product 437 (Scheme 3.26). ${ }^{306}$ 


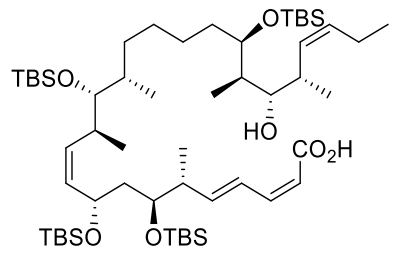

435

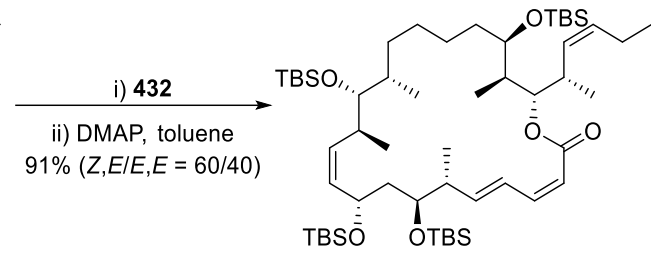

436

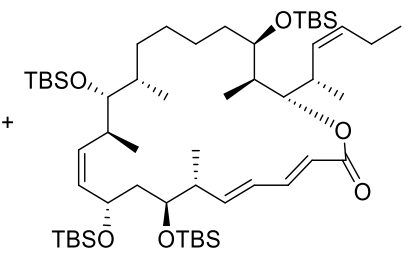

437

Scheme 3.26 Isomerisation in Shiina reaction in the synthesis of dictyostatin analogue. $^{306}$

The isomerisation observed in the classic Yamaguchi reaction and its modifications (e.g., Yonemitsu's and Shiina's conditions) was proposed to be a consequence of a reversible nucleophilic aza-Michael-type addition of DMAP onto the activated acid intermediate. ${ }^{307}$ Therefore, in the synthesis of sorangicin A, the modified Mukaiyama reagent 2-bromo1-ethyl-pyridinium tetrafluoroborate $\mathbf{1 3 0},{ }^{308}$ which possesses a non-nucleophilic counterion, was tested for the lactonisation step (Scheme 3.27). It was anticipated that this could minimise the isomerisation of the trienoacid $\mathbf{4 3 8}$. The desired product $\mathbf{4 3 9}$ was obtained in $80 \%$ yield without significant isomerisation. ${ }^{123}$ This suggests that this coupling reagent could be useful for the synthesis of other macrocyclic lactones containing isomerisation-prone $E, Z$-dienoate motif.

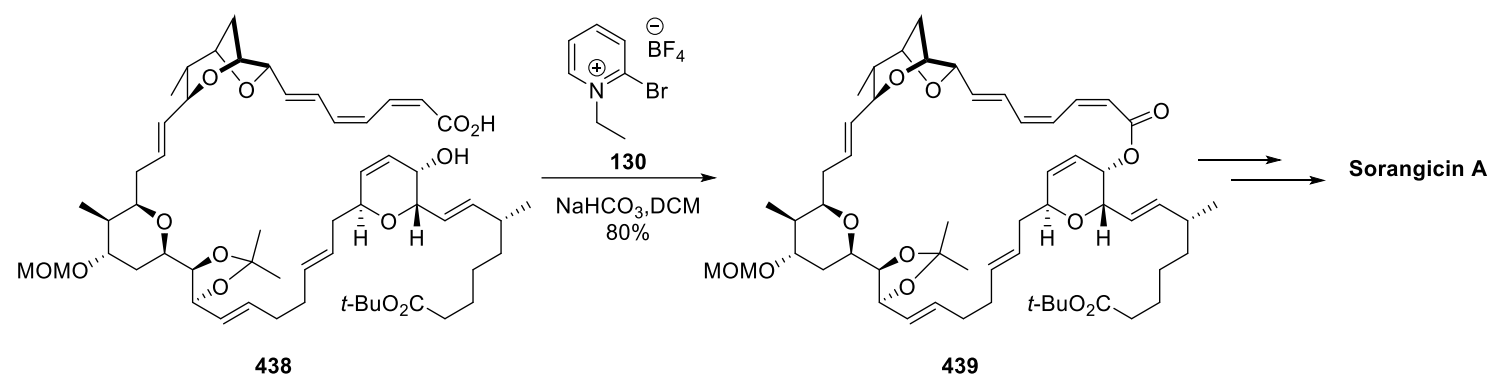

Scheme 3.27 Macrolactonisation in the total synthesis of sorangicin A under modified Mukaiyama conditions. ${ }^{123}$

\subsubsection{Preparation of Macrocyclic Alcohol 441}

As mentioned in Section 1.4.1.3 and 1.4.2.2, in Fürstner's synthetic work towards PatA and DMDA-PatA, only the modified Mukaiyama protocol proved to give reproducibly good yields for the lactonisation step, whereas other conventional reagents/conditions provided substantial amounts of undesired isomerised products. ${ }^{120}$ Based on their experimental results, the modified Mukaiyama reagent $\mathbf{1 3 0}$ was chosen for the 
macrolactonisation step in our synthesis.

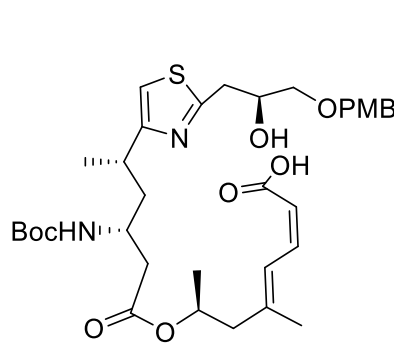

129

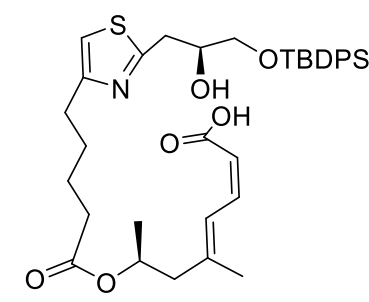

153

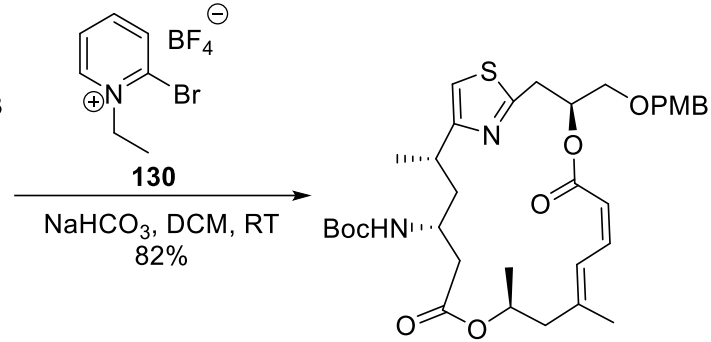

131
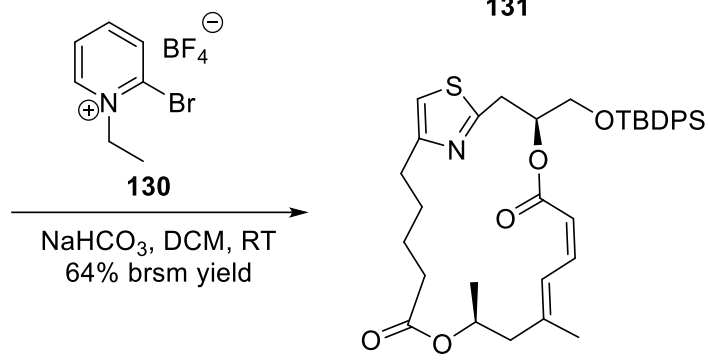

154

Scheme 3.28 Macrolactonisation under modified Mukaiyama conditions in synthesis of

$$
\text { PatA and DMDA PatA. }{ }^{120}
$$

Following the conditions reported by Fürstner and co-workers, highly diluted seco-acid 419 in DCM $\left(c=0.56 \times 10^{-3} \mathrm{M}\right)$ was treated with excess $\mathrm{NaHCO}_{3}$ and reagent 130 in a dark environment, and gratifyingly, the desired Z,E-configured macrocycle 440 was obtained as the sole product in $75 \%$ yield, with no geometric isomer observed by ${ }^{1} \mathrm{H} \mathrm{NMR}$ analysis (Scheme 3.29). The coupling constants of the alkenes of compound $\mathbf{4 4 0}$ were consistent with the reported characterisation data of the macrocyclic compound $\mathbf{1 5 4}$ in Fürstner's synthesis of DMDA PatA (vide supra, Section 1.4.2.2). ${ }^{128}$ Subsequent desilylation of compound $\mathbf{4 4 0}$ with buffered TBAF provided primary alcohol $\mathbf{4 4 1}$ in good yield. 


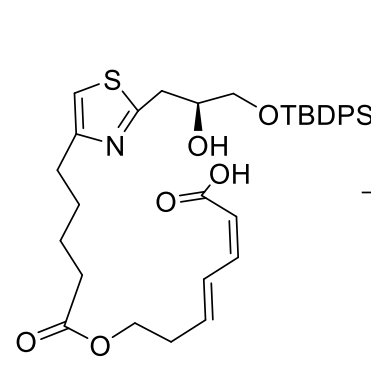

419<smiles>COC(C=O)Cc1nc(CCCCC(=O)OCC/C=C\C=C/C(=O)O)cs1</smiles>

363
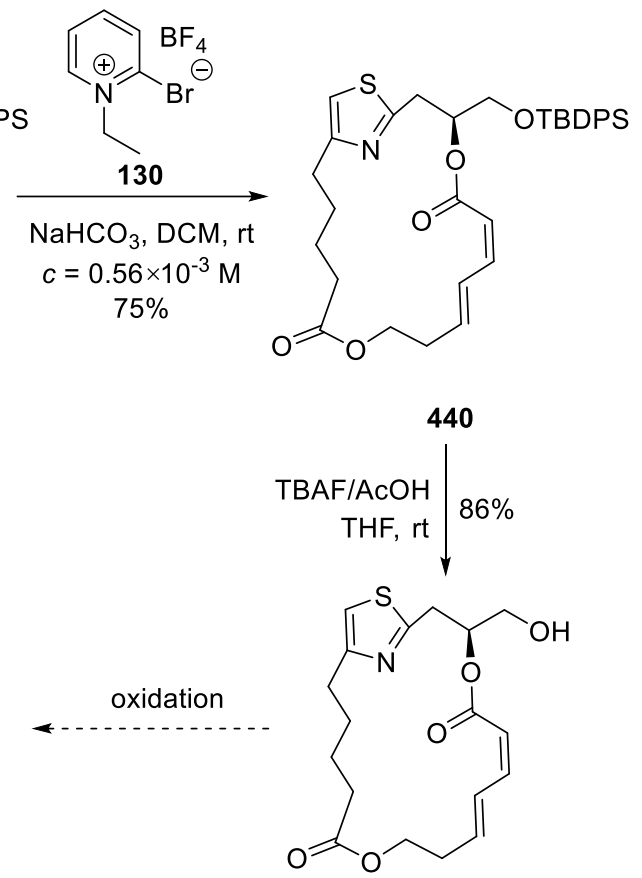

441

Scheme 3.29 Preparation of macrocylic alcohol 441.

Macrocyclic alcohol 441 was prepared in 12 steps as the longest linear sequence (15 steps total), starting from commercially available chemicals, with an overall yield of $3 \%$. The selective deprotection for compound $\mathbf{4 1 8}$ is the major hurdle to overcome. Aside from this step, the overall yield would be $10 \%$.

As aldehyde 363 prepared from the alcohol 441 is structurally similar to aldehyde $\mathbf{1 5 5}$, an intermediate in Fürstner's synthesis of DMDA PatA (refer to Section 1.4.2.2), ${ }^{128}$ it is presumed to be rather sensitive and prone to epimerisation on storage. We then decided to stockpile the alcohol $\mathbf{4 4 1}$ and not to perform the oxidation reaction until the side chain fragment was ready for coupling with it. Therefore, at this stage, we moved on to preparing the methylated side chain fragment. 


\subsection{Synthesis of Side Chain Fragment}

\subsubsection{Wittig Olefination and Horner-Wadsworth-Emmons Reaction}

Wittig olefination employs the reaction between a phosphonium ylide and an aldehyde or a ketone for alkene synthesis. Triphenylphosphonium ylides are the most often used reagents because of their good air-stability and easy preparation. ${ }^{309}$ As shown in Scheme 3.30, the stereochemical outcome of Wittig reactions using triphenylphosphonium ylides is highly dependent on whether or not they are stabilised. In the case of unstabilised ylides, the reaction proceeds under kinetic control. The puckered transition state TS-442 forms at an early stage to minimise steric interactions between $R_{1}$ and $R_{2}$ (1,2-interaction) and between $\mathrm{R}_{2}$ and $\mathrm{Ph}$ (1,3-interaction). The resulting oxaphosphetane intermediate cis-443 eliminates triphenylphosphine oxide to give the Z-olefin. ${ }^{310}$ By contrast, the addition of ylides stabilised by electron-withdrawing functional groups (e.g., alkoxycarbonyl or cyano group) to aldehydes or ketones preferentially proceeds through a late planar transition state TS-442' to relieve the steric interaction between $\mathrm{R}_{2}$ and $\mathrm{Ph}$ and the dipoledipole interaction. The resulting oxaphosphetane trans $\mathbf{- 4 4 3}$ provides an $E$-olefin. ${ }^{310,311}$

(a)
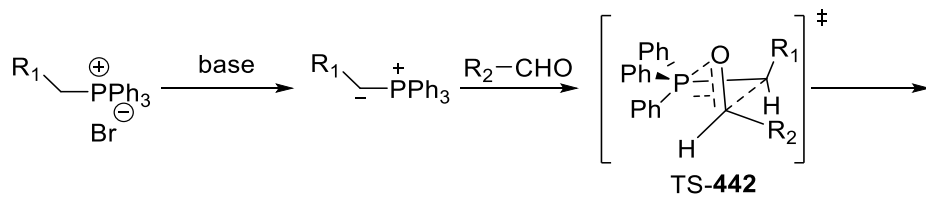

(b)

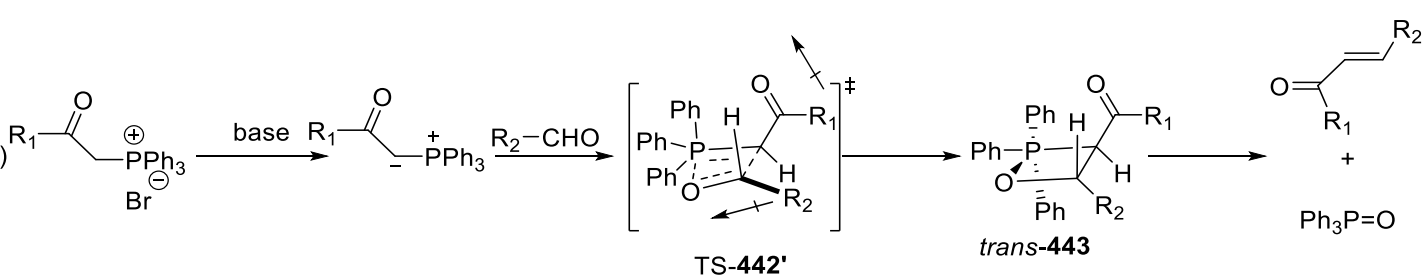

Scheme 3.30 Preferred transition state based on Vedejs model for: (a) non-stabilised triphenylphosphonium ylides; and (b) stabilised triphenylphosphonium ylides. ${ }^{310,311}$

Generally speaking, Wittig reactions using ylides semi-stabilised by functional groups (e.g., vinyl or aryl group) provides mixtures of $Z$ - and $E$-olefins, and the ratio of $Z$ - and E-olefins varies on individual cases. The stereochemical outcomes of representative Wittig reactions using vinyl triphenylphosphonium ylides are shown in Scheme 3.31. 
(a)<smiles>CC(=O)OC1CC(CC=O)OO1</smiles>

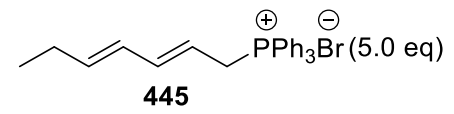

(b)<smiles>C/C(=C\CC[C@H](C)CC=O)CO</smiles>

447
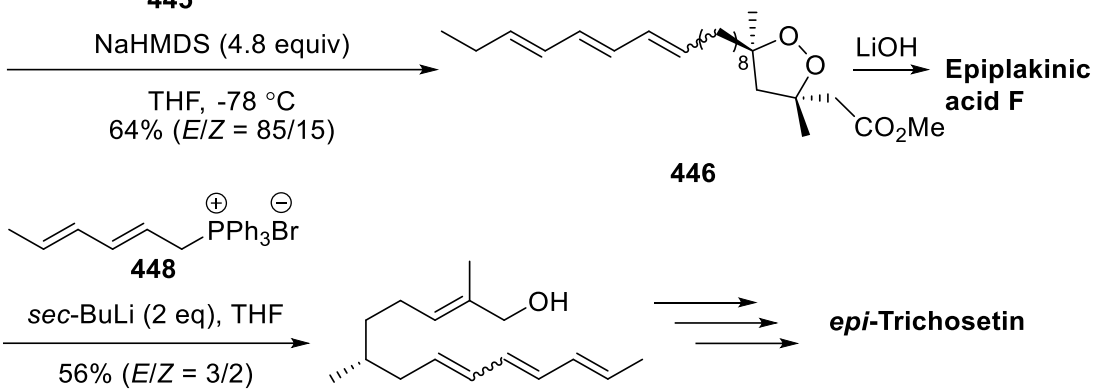

449

Scheme 3.31 Use of vinyl triphenylphosphonium ylide in the synthesis of (a) epiplakinic acid $\mathrm{F} ;{ }^{312}$ and (b) epi-trichosetin. ${ }^{313}$

It was found that the $E$-selectivity of Wittig reactions with unstabilised ylides could be dramatically boosted by using trialkylphosphines in place of triphenylphosphines. ${ }^{314}$ For example, in the synthesis of (+)-ratjadone, tributylphosphonium salt 450, after deprotonation, underwent Wittig reaction between the resulting unstabilised ylide and aldehyde $\mathbf{4 5 1}$ afforded $E$-isomer $\mathbf{4 5 2}$ as the sole product (Scheme 3.32). ${ }^{315}$

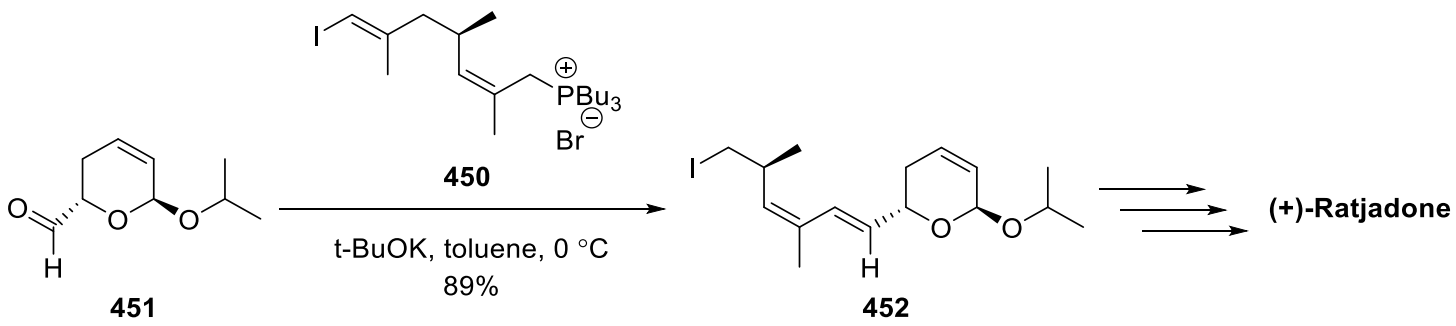

Scheme 3.32 Use of tributylphosphonium salt for Wittig olefination. ${ }^{315}$

One of the most important modifications of Wittig reaction is the Horner-WadsworthEmmons (HWE) reaction, and its mechanism is closely related to that of Wittig reaction of stabilised ylides. ${ }^{316}$ In the proposed mechanism (Scheme 3.33), the addition of the stabilised phosphonate anion to the carbonyl group provides a mixture of isomeric $\beta$ oxido phosphonates, which close to form cis- and trans-oxaphosphetanes 453. Both cisand trans-oxaphosphetane intermediates then rapidly eliminate dialkylphosphate to afford $Z$-and $E$-olefins. It should be pointed out that the presence of electron-withdrawing groups (e.g., ester, cyano, sulfonyl, vinyl, or aryl) on the phosphonate $\alpha$-centre is essential for the deprotonation process and the decomposition of the $\beta$-oxido phosphonate intermediate. ${ }^{317}$ As the initial addition and the formation of the four-membered cyclic 
intermediates are under equilibrium conditions, the ratio of $Z$ - and $E$-olefins is controlled by both kinetic and thermodynamic factors. Generally speaking, the conditions that favour the $E$-selectivity include increased bulkiness of phosphonates and $\alpha$-substitution of aldehydes, higher reaction temperature, and use of lithium or sodium as the counterion. $^{317}$

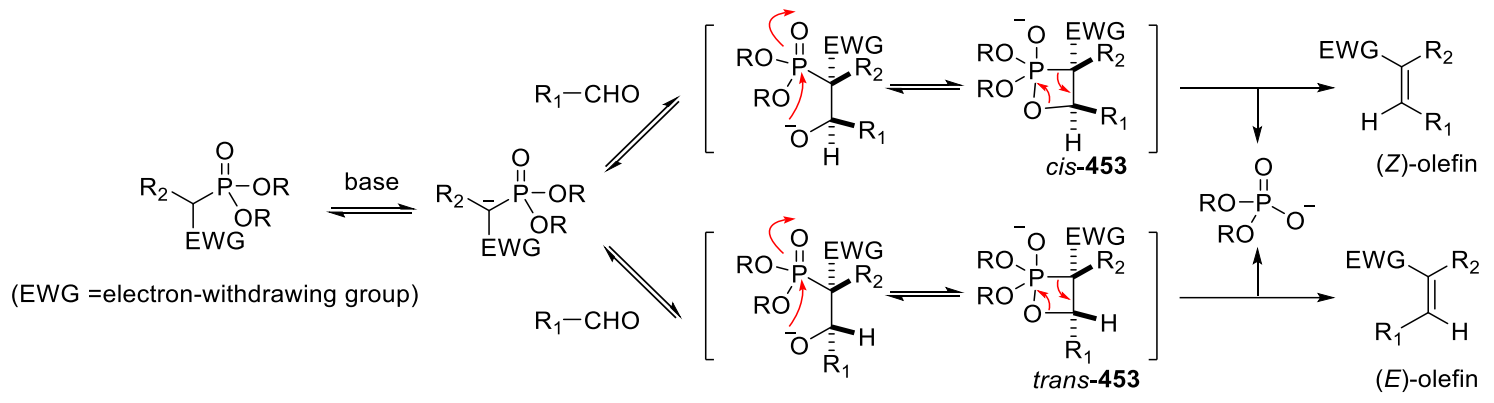

Scheme 3.33 Proposed mechanism of HWE reaction.

The HWE reaction has a number of advantages over Wittig olefination, one of which is that phosphonate ylides are more nucleophilic but less basic than comparable phosphorus ylides derived from phosphonium salts. Therefore, they have less difficulty in reacting with sterically hindered carbonyl groups and broader substrate range. ${ }^{318}$ However, aldehyde substrates with stereocentres adjacent to carbonyl groups may be prone to epimerisation under treatment of strong anionic bases in the HWE reaction. To obviate the use of any kind of ionic bases, modified protocols have been reported, which employ non-ionic weaker bases such as bicyclic guanidine base $\mathbf{4 5 4 ^ { 3 1 9 }}$ and bicyclic triaminophosphine compound $\mathrm{P}\left(i-\mathrm{BuNCH}_{2} \mathrm{CH}_{2}\right)_{3} \mathrm{~N}$ (455) (Figure 3.14). ${ }^{320}$<smiles>C1CN=C2NCCCN2C1</smiles>

454

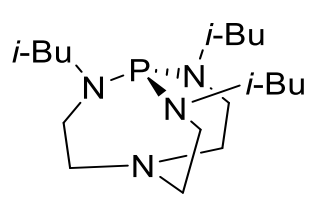

bicyclic triaminophosphine $\mathbf{4 5 5}$

Figure 3.14 Structures of $\mathbf{4 5 4}$ and bicyclic triaminophosphine $\mathbf{4 5 5}$. 


\subsubsection{Preparation of Side Chain Fragments}

As described in Section 3.1, the terminal tertiary amine functional group would be introduced by reductive amination of the terminal aldehyde and the methylated side chain would be attached to the macrocyclic core with a Wittig/HWE reaction. Since intramolecular Wittig reactions of phosphoryl ylides and carbonyl groups and intramolecular HWE reactions of phosphorylated aldehydes have been used widely for the construction of various five-, six-, seven-membered rings and macrocycles, ${ }^{321}$ it is necessary to protect the terminal aldehyde to avoid self-condensation. The frequently used protective method is to transform the terminal aldehyde to an acetal, ${ }^{322}$ and the other reported method is to temporarily mask the aldehyde as a relatively unstable imine, ${ }^{323}$ which could be easily converted back to the aldehyde in the purification process by silica gel chromatography (see Scheme 3.34 for examples).

(a)

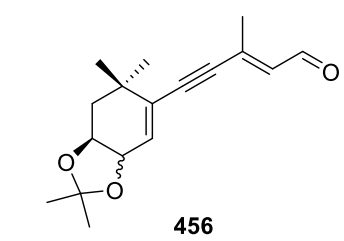

(c)<smiles>CC(=O)OC(C)=CC=CC(C)=CC=O</smiles>

459

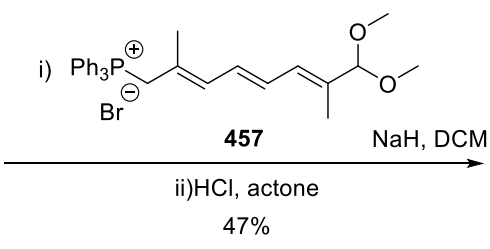

i) $\overbrace{0}$

$>460$

ii) LiHMDS, $-78^{\circ} \mathrm{C}$ to $\mathrm{rt}$

iii) silica gel

$75 \%, E / Z=96 / 4$

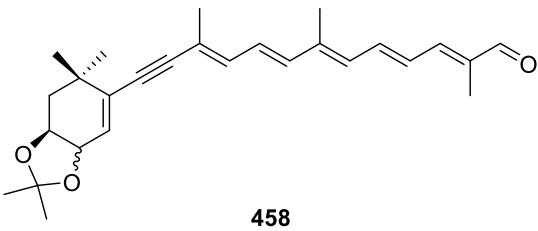

458

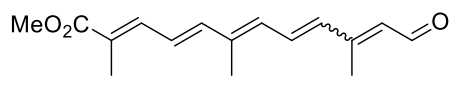

461

Scheme 3.34 Examples of protection of aldehydes in Wittig/HWE reactions using acetals $^{322}$ or imines. ${ }^{323}$

Therefore, as shown in Scheme 3.35, we proposed that compound 463 and 464 with an acetal and an imine functional groups, respectively, could be prepared from the brominated unsaturated ester $\mathbf{4 6 2}$ and could serve as a Wittig/HWE reagent for appending the side chain onto the macrocycle. 


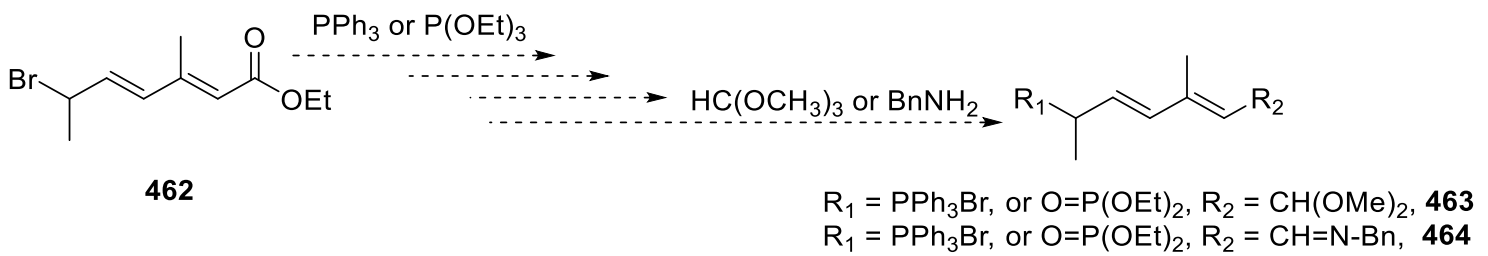

Scheme 3.35 Proposed preparation of methylated side chain fragments for Wittig/HWE olefination.

In 1999, Collignon and co-workers reported a methodology for the preparation of dienal compounds. ${ }^{324}$ It involves the reaction between an aldehyde and a tetrabutylammonium dienolate, which is generated in situ from $O$-triisopropylsilylated $(E, E)$-enol ether under treatment with TBAF (see Scheme 3.36 for a representative example). Based on their experimental results, this reaction showed high $E$-selectivity within non-conjugated aliphatic aldehydes. To the best of our knowledge, this methodology has not yet been utilised in synthetic work since its publication. Therefore, we were interested in testing the feasibility of using this method to attach the methylated side chain onto the macrocycle core.

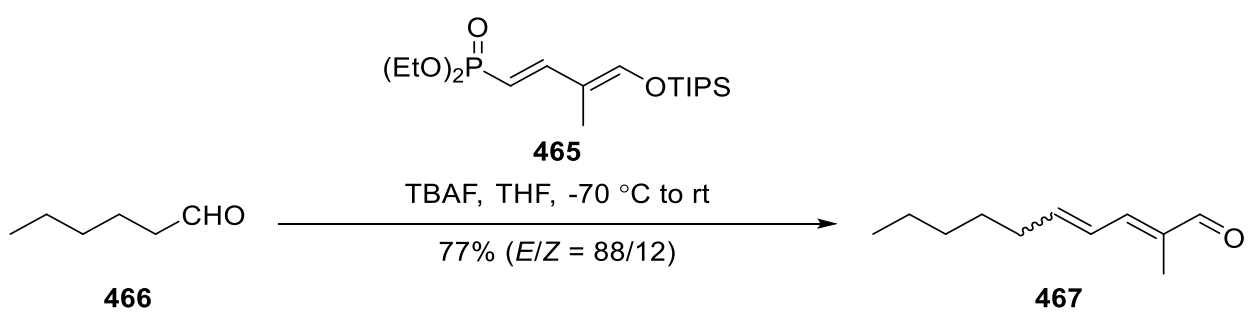

Scheme 3.36 Use of enol silyl ether 465 in preparation of dienal $467 .^{324}$

The preparation of brominated ester $\mathbf{4 6 2}$ largely followed a synthetic route designed by postdoctoral fellow Claire Cuyamendous in our research group. As shown in Scheme 3.37, the HWE reagent 468 required for this route was first prepared. This was achieved by Michaelis-Arbuzov reaction of triethylphosphite $\left(\mathrm{P}(\mathrm{OEt})_{3}\right)$ and iodoacetone, which was generated in situ by iodide substitution of chloroacetone 469. ${ }^{325}$ Wittig reaction of hydroxyacetone 470 with stabilised triphenyl phosphorane 471 provided $E$ - $\alpha, \beta$ unsaturated ester 472. Subsequent allylic oxidation by active manganese dioxide, which was freshly prepared from activated carbon and potassium permanganate (see Section 6.3.5 for experimental details), afforded aldehyde 473. Initial small-scale HWE reaction of aldehyde 473 with the phosphonate 468 in an aqueous potassium carbonate solution 
gave desired methyl ketone $\mathbf{3 5 9}$ in almost quantitative yield. However, when the reaction was scaled up, the yield of the ketone decreased and a minor product was observed. 2D NMR investigation revealed it to be compound $\mathbf{4 7 4}$, which is generated from the aldol reaction between ketone $\mathbf{3 5 9}$ and the aldehyde starting material. The formation of this undesired product was probably prompted by excess $\mathrm{K}_{2} \mathrm{CO}_{3}$ and the higher concentration of the product ketone $\mathbf{3 5 9}$ in the reaction vessel. Therefore, lowering the equivalents of $\mathrm{K}_{2} \mathrm{CO}_{3}$ and performing the reaction in a more diluted manner should reduce the formation of the aldol product and improve the yield for large-scale reactions. Nonetheless, a reasonable yield of ketone $\mathbf{3 5 9}$ was obtained, and the optimisation was therefore not attempted.
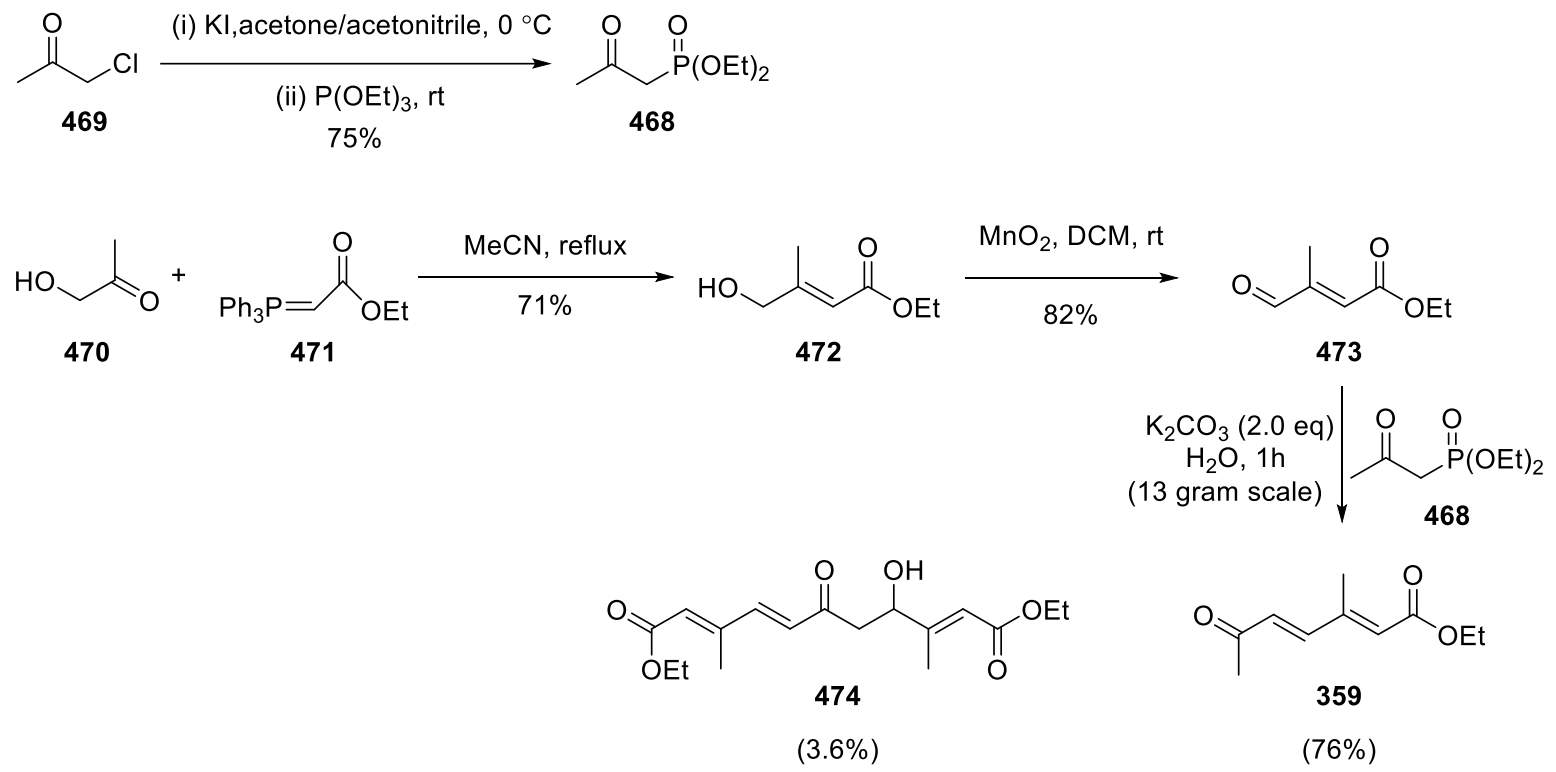

Scheme 3.37 Preparation of methyl ketone 359.

Selective 1,2-reduction of methyl ketone 359 under Luche conditions provided allylic alcohol 475 in almost quantitative yield (Scheme 3.38). ${ }^{326}$ The conversion of allylic alcohol 475 into allylic bromide 462 was achieved by using phosphorus(III) bromide $\left(\mathrm{PBr}_{3}\right)$ as the brominating reagent. It is worth mentioning that the slow addition of $\mathrm{PBr}_{3}$ by a syringe pump at a lower temperature was essential for the excellent yield. Not surprisingly, it was found that the allylic bromide was quite unstable and it could degrade significantly even on storage at $-18{ }^{\circ} \mathrm{C}$. Hence, the bromide needs to be used for the next step immediately. 


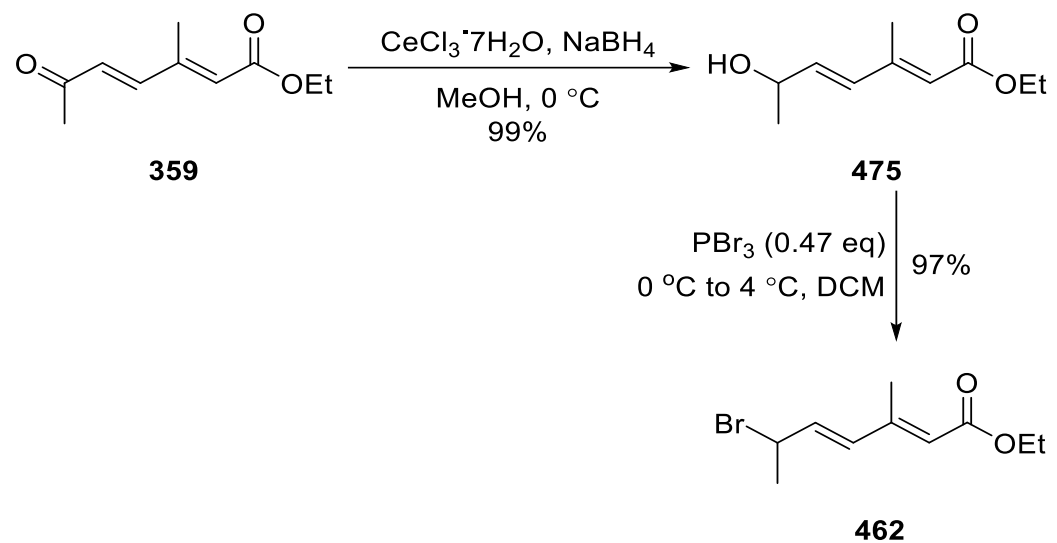

Scheme 3.38 Preparation of bromide 462.

The bromide 462 was then reacted with triphenylphosphine $\left(\mathrm{PPh}_{3}\right)$ at room temperature in toluene to provide the triphenylphosphonium salt $\mathbf{4 7 6}$ in $68 \%$ yield (Scheme 3.39).

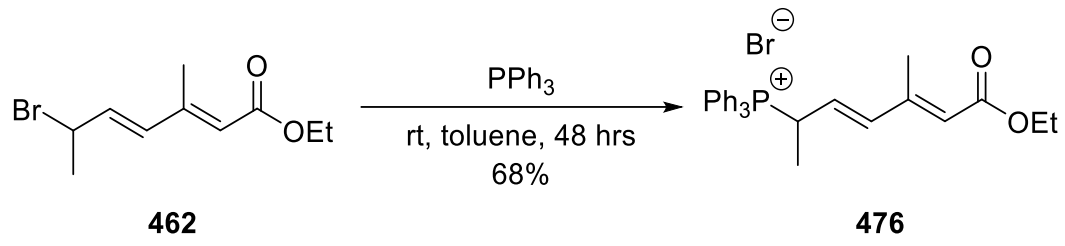

Scheme 3.39 Synthesis of triphenylphosphonium salt 476.

Because the phosphorus ylide generated by deprotonation of phosphonium salt $\mathbf{4 7 6}$ could be stabilised by the terminal ester group through the conjugated system, we assumed that the Wittig reaction should have good E-selectivity based on Vedejs' model (refer to Section 3.6.1). To investigate its stereochemical outcome, we chose the chiral aldehyde 322 as the substrate for the model reaction. The reaction was carried out by pre-treating phosphonium salt 476 with $\mathrm{BuLi}$ at $-40{ }^{\circ} \mathrm{C}$ followed by the addition of aldehyde 322 , resulting in an inseparable 62/38 ratio of the desired E,E,E-trienoate 477 and the undesired E,E,Z-trienoate $\mathbf{4 7 8}$ (Scheme 3.40). 


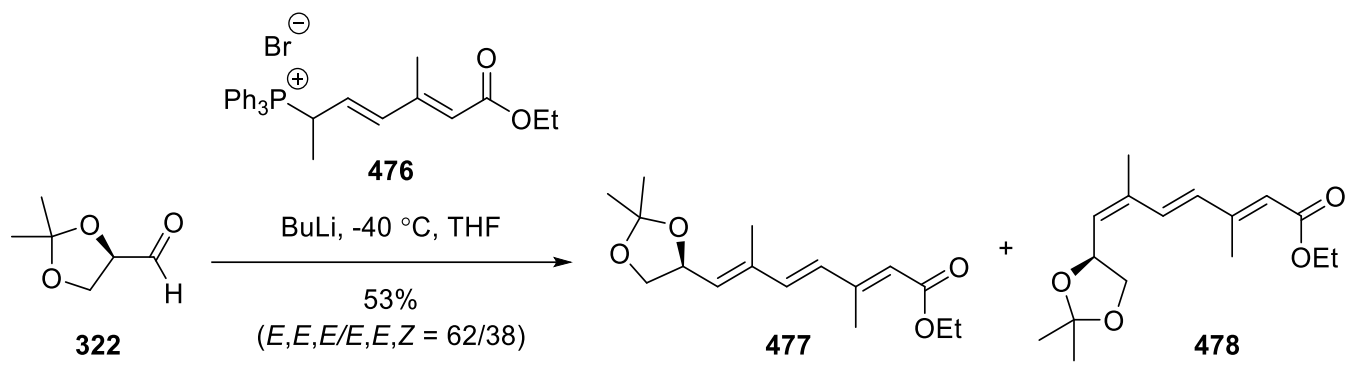

Scheme 3.40 Preparation of trienoate 477 and 478.

Assignments of proton peaks of the mixture of stereoisomers (Figure 3.15) were made on the basis of COSY, HSQC and HMBC correlations. The COSY spectrum enabled connectivity within the protonated fragments of each isomer to be determined (Figure 3.16). A structural subunit of $\mathrm{C} 7 / \mathrm{C} 8 / \mathrm{C} 9$ could be established as an isolated spin-system according to the $1 \mathrm{H}-1 \mathrm{H}$ COSY correlations of $\mathrm{H} 7 / \mathrm{H} 8 / \mathrm{H} 9$. The HMBC correlations from $\mathrm{H} 2$ to $\mathrm{C} 1, \mathrm{C} 3$ and $\mathrm{C} 13$, from $\mathrm{H} 4$ to $\mathrm{C} 2, \mathrm{C} 3$ and $\mathrm{C} 13$, and from $\mathrm{H} 5$ to $\mathrm{C} 3$ and $\mathrm{C} 6$, in combination with the HMBC correlations from $\mathrm{H} 7$ to $\mathrm{C} 6, \mathrm{C} 9$ and $\mathrm{C} 14$, successfully established the connectivity throughout the full carbon chain for each compound (Figure $3.17)$. 

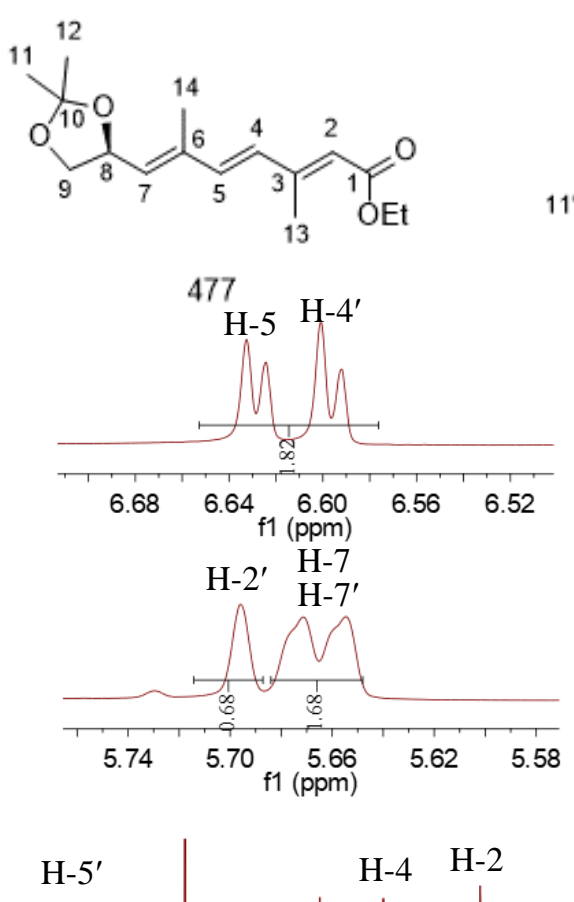
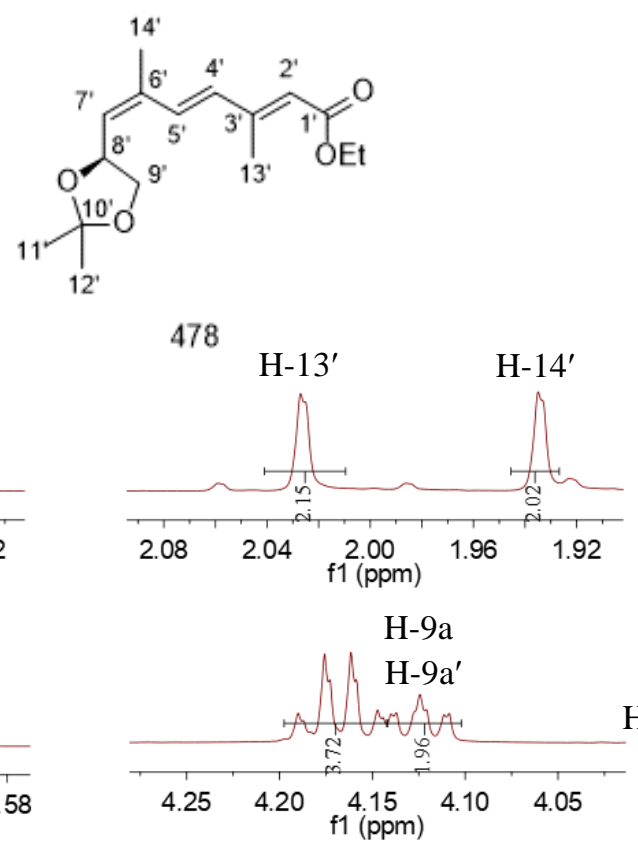

H-13 H-14

$\mathrm{H}-9 \mathrm{~b}$

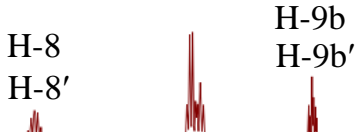

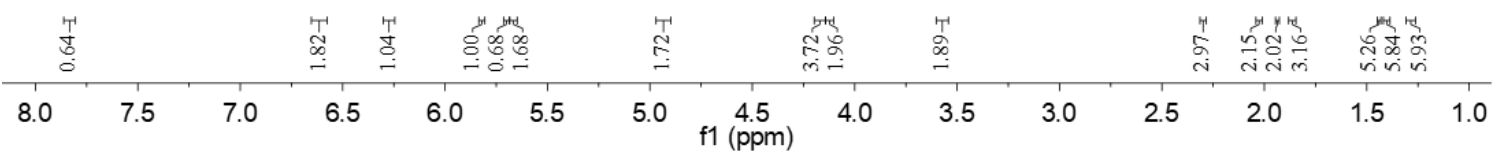

Figure 3.15 Assignment of ${ }^{1} \mathrm{H}$ NMR of the mixture of trienoate 477 and 478 in $\mathrm{CDCl}_{3}$

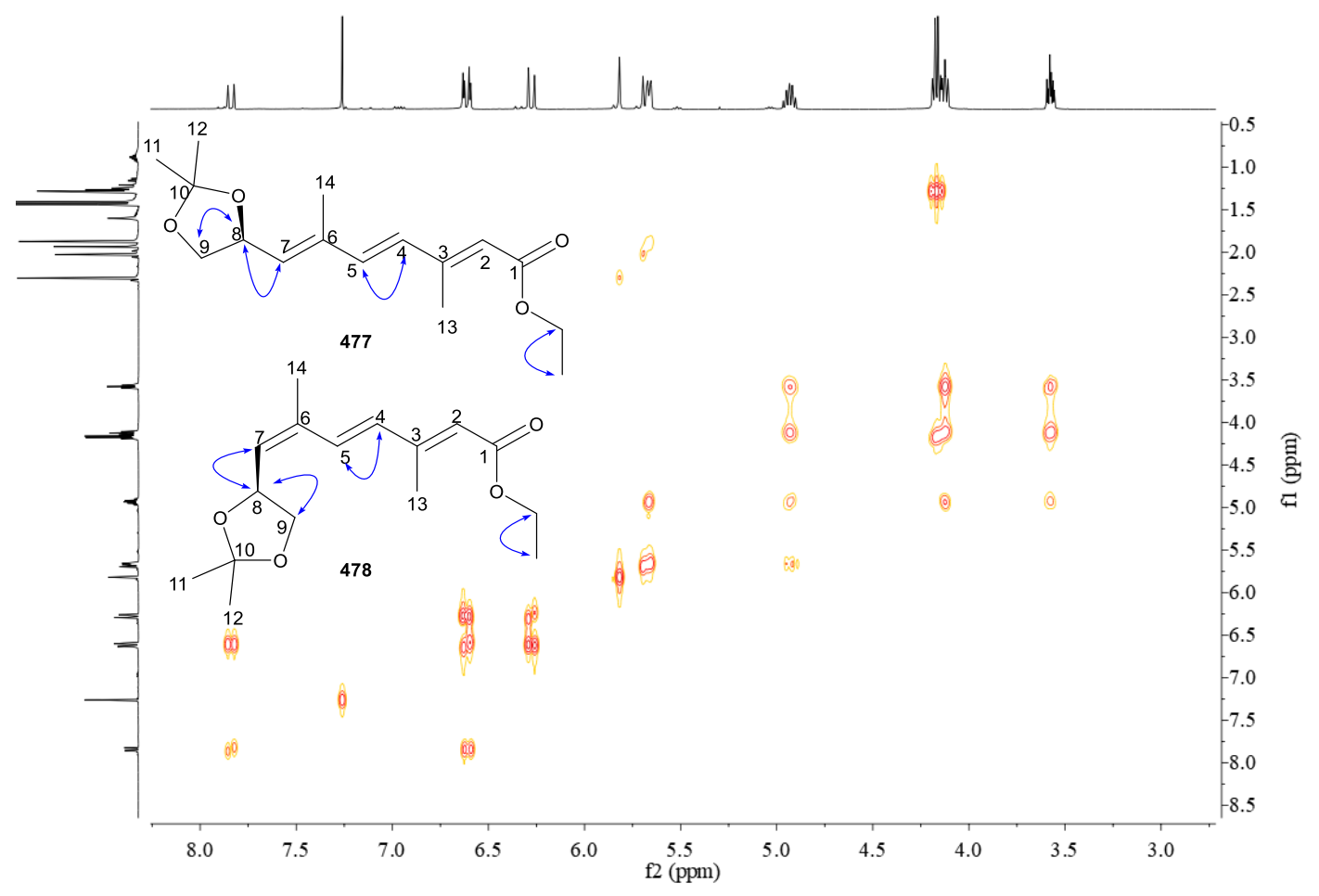

Figure 3.16 COSY correlations in structural elucidation of compounds $\mathbf{4 7 7}$ and $\mathbf{4 7 8}$ 

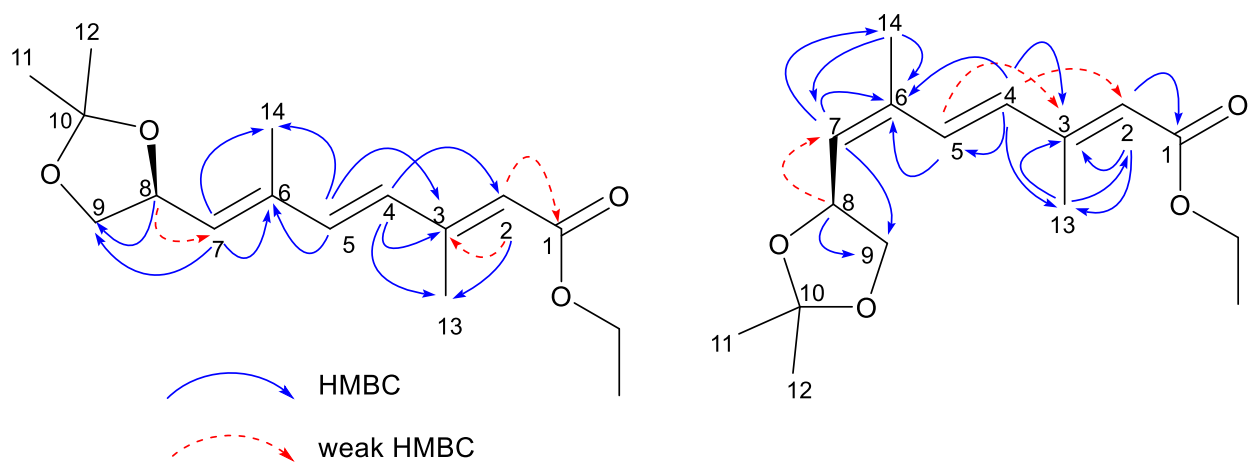

Figure 3.17 Key HMBC correlations in structural elucidation of compounds 477 and 478

To distinguish the two stereoisomers of the trienoate, it was necessary to perform NOE experiments because the newly-formed double bond was trisubstituted. Unfortunately, the chemical shifts of the protons (between 5.68 and $5.64 \mathrm{ppm}$ ) at $\mathrm{C} 7$ for the two stereoisomers completely overlap in the ${ }^{1} \mathrm{H}$ NMR spectrum (Figure 3.15). Nevertheless, attempts were made to distinguish and assign the two stereoisomers by NOE correlations. 
(a)
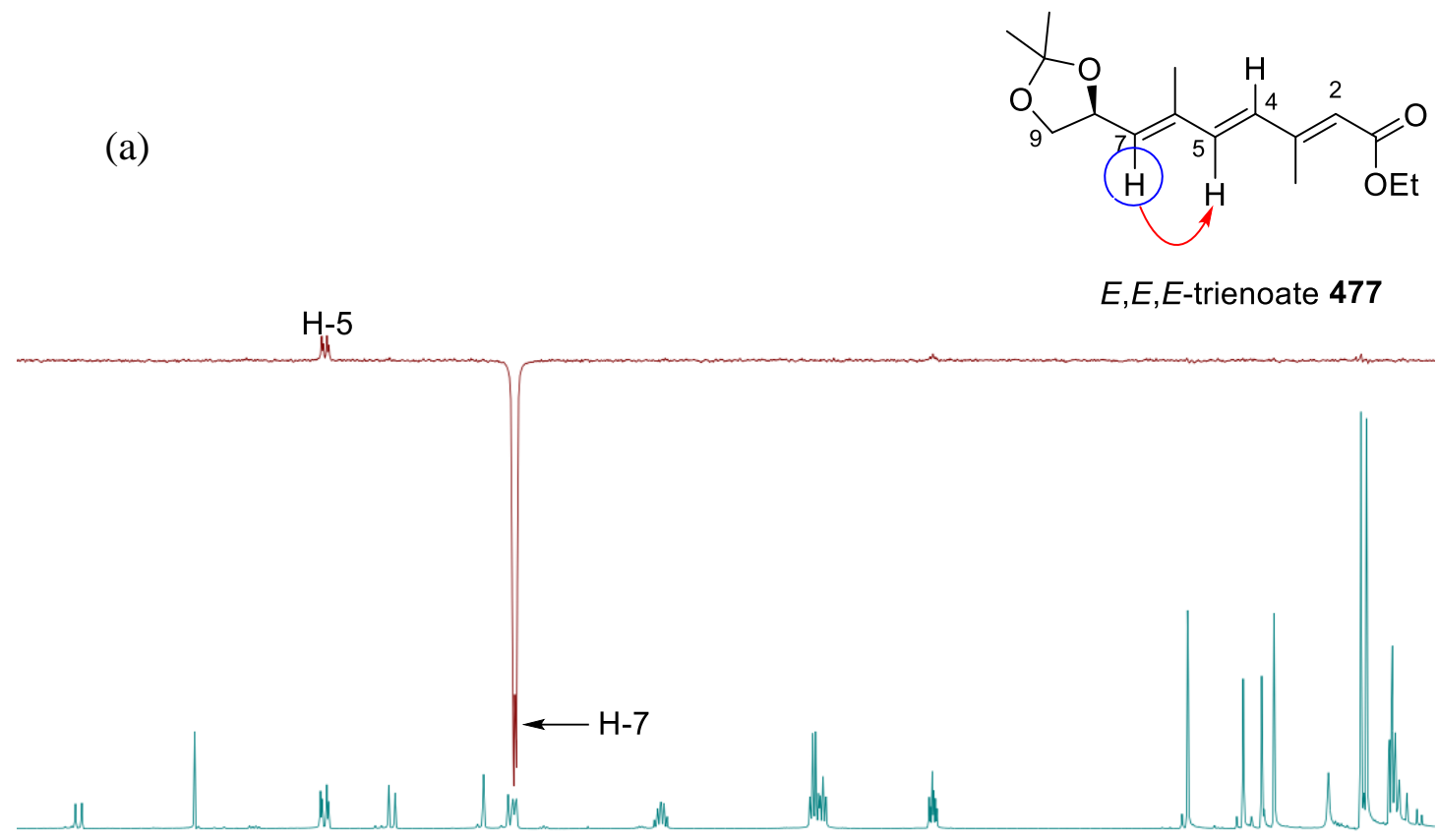

$\begin{array}{llllllllllllllllllllllllllllllllllllllllllllllllll}8.0 & 7.8 & 7.6 & 7.4 & 7.2 & 7.0 & 6.8 & 6.6 & 6.4 & 6.2 & 6.0 & 5.8 & 5.6 & 5.4 & 5.2 & 5.0 & 4.8 & 4.6 & 4.4 & 4.2 & 4.0 & 3.8 & 3.6 & 3.4 & 3.2 & 3.0 & 2.8 & 2.6 & 2.4 & 2.2 & 2.0 & 1.8 & 1.6 & 1.4 & 1.2\end{array}$

(b)
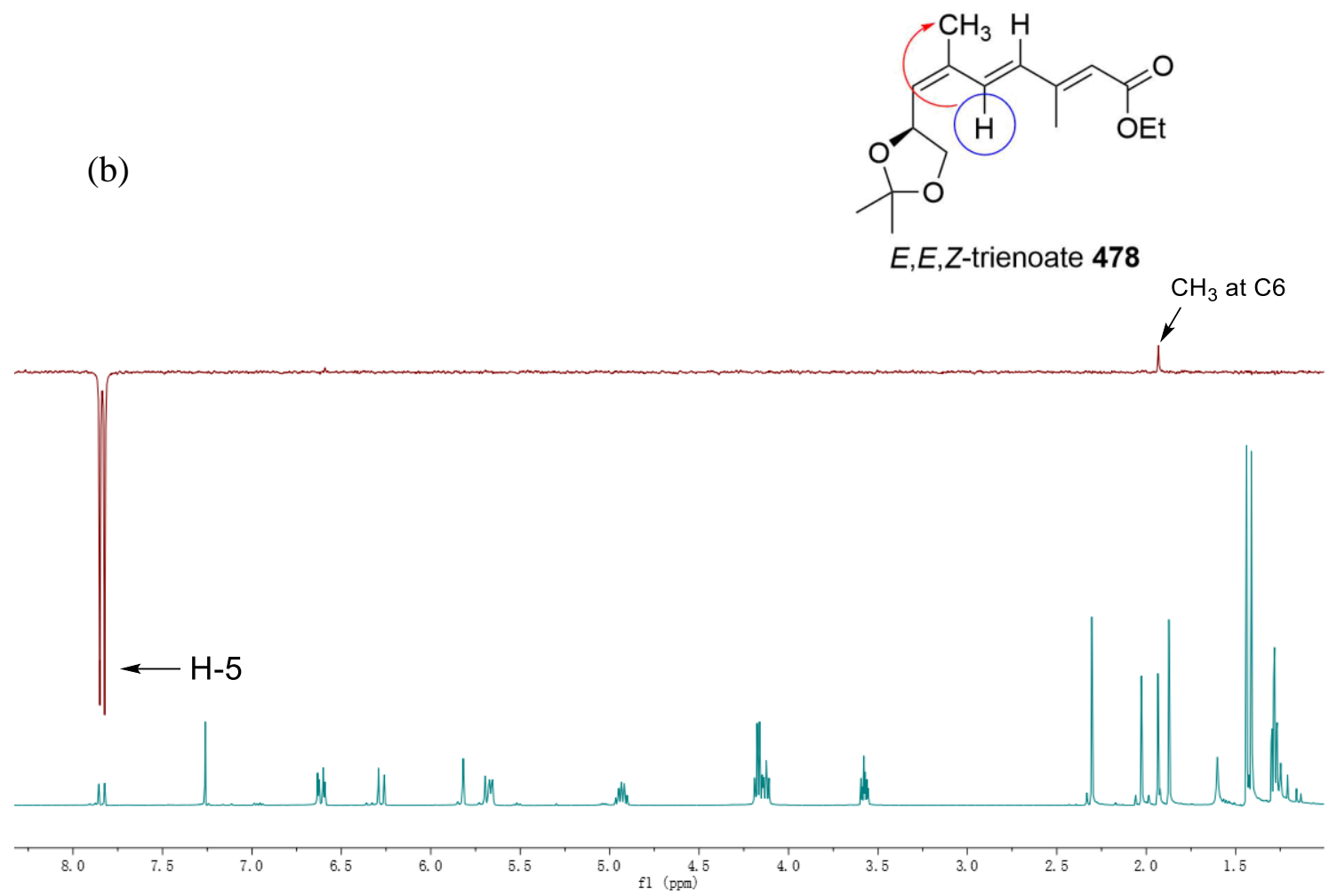
(c)

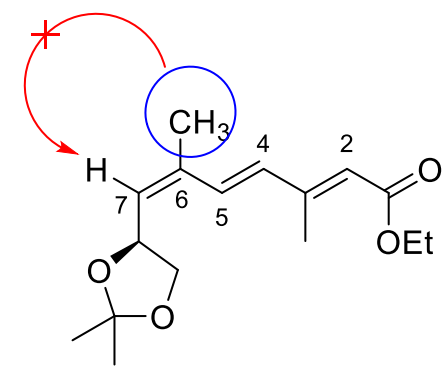

E,E,Z-trienoate $\mathbf{4 7 8}$

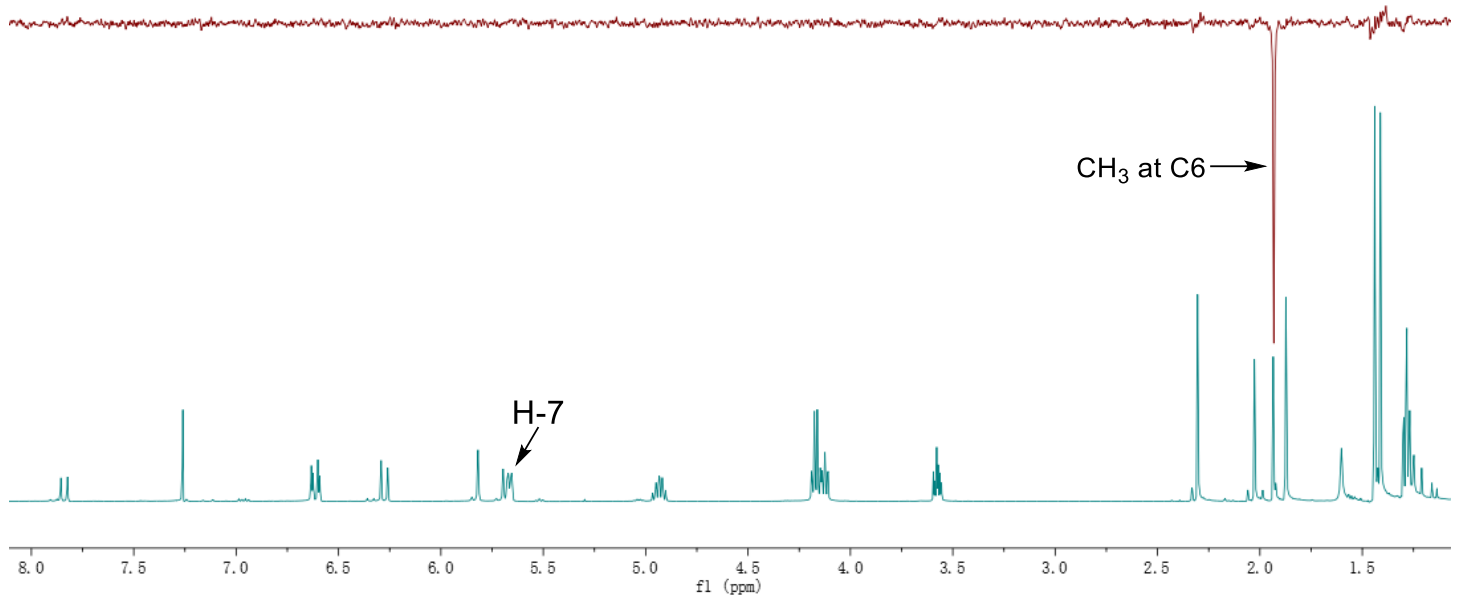

Figure 3.18 NOE correlations (in maroon) and ${ }^{1} \mathrm{H}$ NMR spectrum (in blue) of the mixture of trienoate 477 and 478 in $\mathrm{CDCl}_{3}$. The irradiated proton is shown in the blue circle and any NOE correlation observed is shown with a red arrow.

As shown in Figure 3.18a, irradiation at $\delta 5.68 \mathrm{ppm}(\mathrm{H} 7)$ caused enhancement of the signal of the C5 proton (at $\delta 6.61 \mathrm{ppm}$ ) of the major isomer, identifying that the isomer as $E, E, E$-trienoate 477. An irradiation of the $\mathrm{C} 5$ proton of the minor isomer at $\delta 7.84 \mathrm{ppm}$ caused enhancement of the signal of the C6-methyl group (at $\delta 1.93 \mathrm{ppm}$ ), which is not helpful in identification of the configuration of the $\mathrm{C}-\mathrm{C}$ double bond (Figure 3.18b). An irradiation of the C6-methyl group of the minor isomer at $\delta 1.93 \mathrm{ppm}$ did not provide the expected correlation to the $\mathrm{C} 7$ proton (Figure 3.18c). However, we were confident that the minor isomer is E,E,Z-trienoate 478 based on 2D NMR analysis. It is also worth mentioning that the chemical shifts of the $\mathrm{C} 5$ proton allowed some additional confidence in these assignments. The signal of $\mathrm{H} 5$ in the major isomer is at $\delta 6.63 \mathrm{ppm}$ whereas for the minor isomer is at $\delta 7.84 \mathrm{ppm}$ (Figure 3.15). The significant downfield shift in the minor isomer is consistent with the assignment as E,E,Z-trienoate $\mathbf{4 7 8}$ as a result of the through-space deshielding effect due to the electronegative oxygen atom in the acetonide (Figure 3.19). 


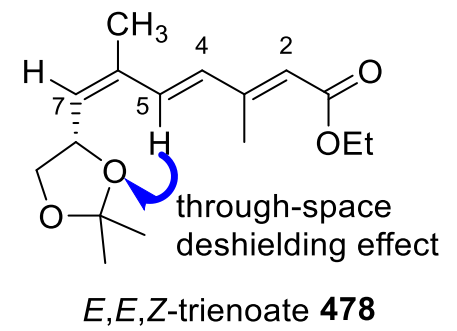

Figure 3.19 Proposed deshielding effect due to the oxygen atom of the acetonide in E,E,Z-trienoate $\mathbf{4 7 8}$.

In view of the moderate $E$-selectivity $(E / Z=62 / 38)$ of the Wittig reaction with triphenylphosphonium salt 476, we assumed that the triphenylphosphoryl ylide with a terminal acetal or imine group would afford a similar stereochemical outcome. Whether the use of a trialkylphosphonium salt would improve the $E$-selectivity would need to be further examined. However, an HWE strategy was first pursued.

As a phosphoryl aldehyde can serve as the common intermediate in the synthesis of the phosphoryl acetal or phosphoryl imine for HWE reaction and a phosphoryl silyl ether substrate for the TBAF-mediated aldol condensation, we decided to prioritise preparing phosphonate $\mathbf{4 8 1}$ at this stage. This would be synthesised by an Arbuzov reaction between bromide 462 and triethylphosphite.

The Arbuzov reaction is a traditional method to prepare organophosphonates from the corresponding halides. ${ }^{327}$ In the proposed mechanism (Scheme 3.41), the reaction begins with the $\mathrm{S}_{\mathrm{N}} 2$ nucleophilic attack on the alkyl group of the alkyl halide by the triethylphosphite to provide a phosphonium intermediate 479. A second $\mathrm{S}_{\mathrm{N}} 2$ reaction of the displaced halide anion with the phosphonium intermediate 479 resulted in the formation of a phosphonate product $\mathbf{4 8 0}$ accompanied by an alkyl halide. ${ }^{328}$ Despite its prevalence, it requires high reaction temperatures (e.g., $140-180{ }^{\circ} \mathrm{C}$ ) for an extended period of time for the activation of the reaction. ${ }^{328,329}$ Therefore, we were concerned that the degradation of the unstable allylic bromide $\mathbf{4 6 2}$ would likely occur under heating at such high temperature, leading to the formation of unexpected by-products. In addition, traditional Arbuzov reactions with alkyl bromides and triethylphosphite concomitantly generate one equivalent of volatile and toxic ethyl bromide, which is not chemist-friendly. The resulting ethyl bromide could also react with the phosphite at such high temperatures to reduce the reaction efficiency. Therefore, direct conversion of alcohol 475 into 
corresponding phosphonate $\mathbf{4 8 1}$ under mild conditions would be attractive as this could limit the risk of degradation and potentially give a higher yield.

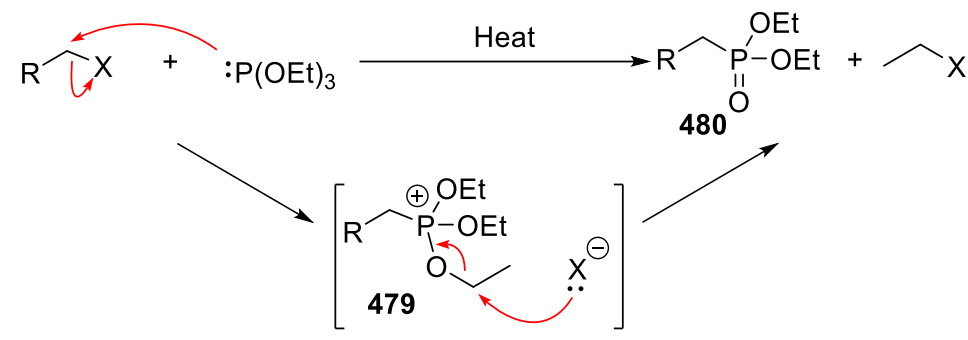

Scheme 3.41 Proposed mechanism of the Arbuzov reaction between alkyl halide and triethylphosphite. ${ }^{327,328}$

Following the conditions of Iranpoor and co-workers, ${ }^{330}$ the direct conversion of allylic alcohol 475 to phosphonate 481 was attempted with a combination of $\mathrm{PPh}_{3}$, DDQ and $\mathrm{P}(\mathrm{OEt})_{3}$ (Table 3.5, entry 1). However, no obvious evidence of formation of the desired phosphonate product 481 was obtained by ${ }^{1} \mathrm{H}$ NMR and HR-MS investigation. As TBAI has been efficiently used as a catalyst in this type of direct transformation, ${ }^{331}$ the reaction was repeated with the presence of TBAI at $125^{\circ} \mathrm{C}$ (entry 2 ) but a mixture of unidentified by-products was obtained. The inability to prepare phosphonate $\mathbf{4 8 1}$ by alcohol-based Michaelis-Arbuzov reaction prompted us to go back to attempt the traditional Arbuzov reaction. In order to reduce the risk of degradation of bromide 462, the reaction was first carried out with $\mathrm{P}(\mathrm{OEt})_{3}$ at reflux in toluene (at $110^{\circ} \mathrm{C}$ ) as opposed to $140-180{ }^{\circ} \mathrm{C}$ (entry 3). However, only a trace amount of the desired phosphonate was obtained after two-days heating. Repeating the reaction in refluxing $m$-xylene resulted in the degradation of the substrate, with the elimination of the allylic bromide observed (Entry 4). However, the desired product was obtained in $26 \%$ yield. We assumed that performing the reaction neat could potentially accelerate the desired transformation and therefore limit the degradation or elimination of the bromide substrate. Satisfyingly, when excess triethylphosphite was used as the solvent, the yield of phosphonate $\mathbf{4 8 1}$ improved to 50\% (entry 5). 


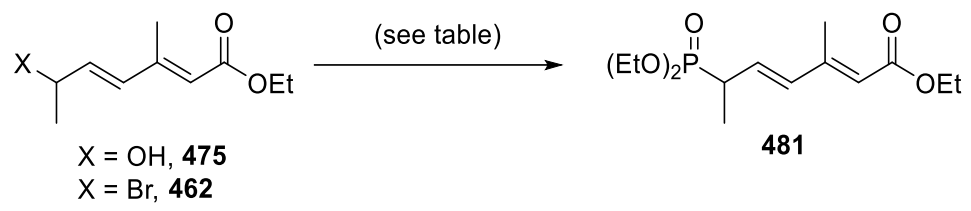

\begin{tabular}{lclc}
\hline Entry & $\mathbf{X}$ & \multicolumn{1}{c}{ Conditions $^{\mathbf{a}}$ (equiv.) } & Yield $^{\mathbf{b}}(\boldsymbol{\%})$ \\
\hline $\mathbf{1}$ & $\mathrm{OH}$ & $\mathrm{P}(\mathrm{OEt})_{3}(2.0), \mathrm{PPh}_{3}(2.0), \mathrm{DDQ}(2.0), \mathrm{MeCN}, 80^{\circ} \mathrm{C}, \mathrm{o} / \mathrm{n}$ & No product \\
\hline $\mathbf{2}^{\mathbf{c}}$ & $\mathrm{OH}$ & $\mathrm{P}(\mathrm{OEt})_{3}(1.5), \mathrm{TBAI}(0.02)$, neat, $125^{\circ} \mathrm{C}, \mathrm{o} / \mathrm{n}$ & No product \\
\hline $\mathbf{3}$ & $\mathrm{Br}$ & $\mathrm{P}(\mathrm{OEt})_{3}(1.0)$, toluene, reflux, $2 \mathrm{~d}$ & trace \\
\hline $\mathbf{4}$ & $\mathrm{Br}$ & $\mathrm{P}(\mathrm{OEt})_{3}(1.0), m$-xylene, $140{ }^{\circ} \mathrm{C}, \mathrm{o} / \mathrm{n}$ & 50 \\
\hline $\mathbf{5}^{\mathbf{c}}$ & $\mathrm{Br}$ & $\mathrm{P}(\mathrm{OEt})_{3}(3.0)$, neat, $140^{\circ} \mathrm{C}, \mathrm{o} / \mathrm{n}$ & 50 \\
\hline
\end{tabular}

${ }^{\mathrm{a}} \mathrm{O} / \mathrm{n}=$ overnight (i.e. $16-20$ hours). ${ }^{\mathrm{b}}$ Isolated yields following silica gel chromatography. ${ }^{\mathrm{c}} \mathrm{P}(\mathrm{OEt})_{3}$ was used as solvent.

Table 3.5 Reaction screening for preparation of phosphonate $\mathbf{4 8 1}$.

As shown in Scheme 3.42, the phosphoryl ester 481 was then reduced by DIBAL-H to provide alcohol $\mathbf{4 8 2}$ in almost quantitative yield. Subsequent allylic oxidation by active manganese dioxide afforded phosphoryl aldehyde 483 smoothly.

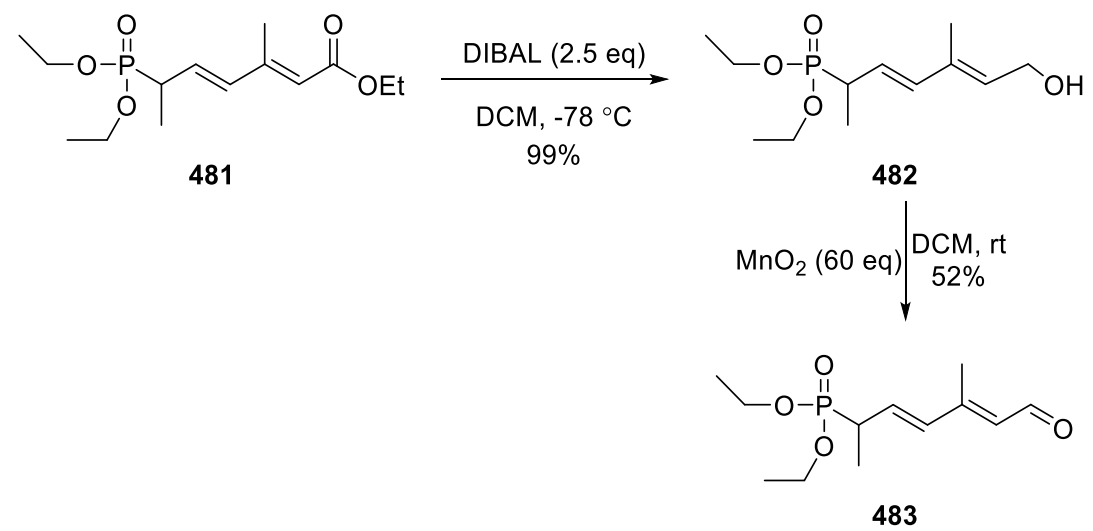

Scheme 3.42 Preparation of phosphoryl aldehyde 483.

With aldehyde $\mathbf{4 8 3}$ in hand, we then attempted to prepare phosphoryl silyl ether $\mathbf{4 8 5}$. Following the procedure reported by Collignon and co-workers, ${ }^{324}$ aldehyde 483 was deprotonated by KHMDS at $-78{ }^{\circ} \mathrm{C}$ to $0{ }^{\circ} \mathrm{C}$ to form the potassium dienolate 484 . This intermediate displayed low solubility in THF, which matches Collignon's experimental observation. ${ }^{324}$ Subsequent reaction between TIPSCl and dienolate intermediate 484 afforded silyl ether $\mathbf{4 8 5}$ (Scheme 3.43). NMR analysis showed that the coupling constant between $\mathrm{C} 6$ proton and $\mathrm{C} 7$ proton ${ }^{3} \mathrm{~J}$ is $11.9 \mathrm{~Hz}$, which is not a solid evidence to support 
the desired E-configuration. At this point, the time constraints limited further investigation on optimisations to improve the yield and thorough NOE experiments to confirm the configuration of the double bonds of compound $\mathbf{4 8 5}$.

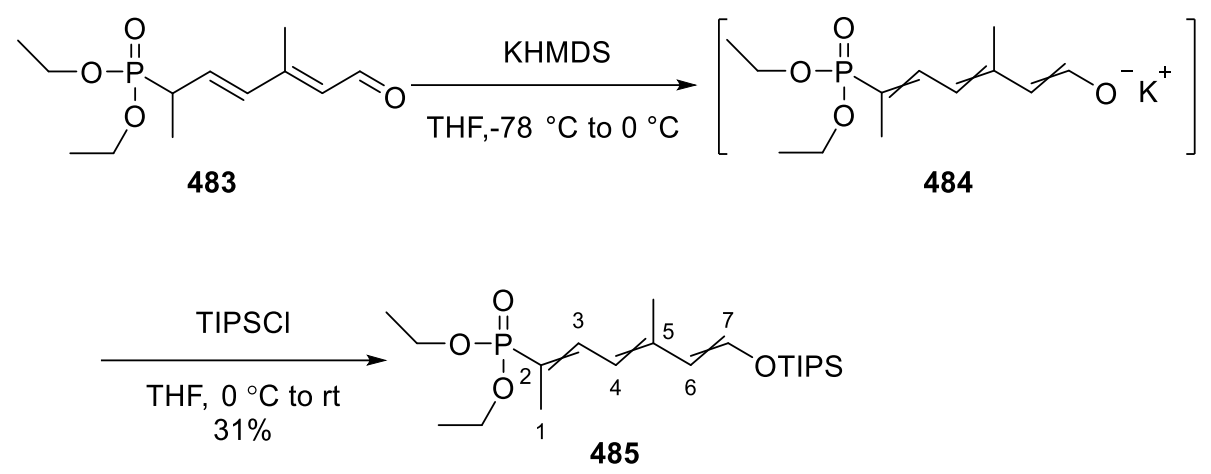

Scheme 3.43 Preparation of phosphoryl silyl ether $\mathbf{4 8 5}$.

\subsection{Conclusion}

In conclusion, the three major fragments, namely thioamide 382, alkyne 364 and phosphoryl silyl ether $\mathbf{4 8 5}$, were prepared for the synthesis of thiazole analogue $\mathbf{1 8 4}$. The C8 - C11 fragment thioamide $\mathbf{3 8 2}$ was prepared from commercially available amide $\mathbf{3 3 9}$ with a $35 \%$ yield over 3 steps and the formation of the thioamide $(S)$-oxide impurity by air could be avoided by taking extra measures in the post-purification stage. The alkyne fragment was efficiently accessed from 1,3-propanediol with a 30\% yield over 7 steps. In preparation of alkyne 364, it was found that the observed polymerisation of diene intermediate $\mathbf{3 8 8}$ could be possibly attributed to the inadvertent introduction of acid from sand used for column preparation. The side chain-fragment phosphoryl silyl ether $\mathbf{4 8 5}$ was prepared from hydroxyacetone in an $8 \%$ yield over 8 steps and the yield could be potentially further improved after optimising conditions for the formation of the silyl ether.

The one-pot gold-catalysed coupling between alkyne 364 and thioamide 382 provided the desired thiazole 393 in an acceptable yield. KHMDS-induced ring opening of the lactone residing in compound 393 provided the desired Z,E-dienoic acid $\mathbf{4 1 8}$ smoothly. However, the subsequent selective desilylation step was found to be low-yielding, there, several alternative routes, including performing the desilylation step prior to the lactone opening, were trialled. Unfortunately, these efforts did not improve the yield significantly. This 
means that the preparation of seco-acid 419 will require further investigation. The macrolactonisation of compound $\mathbf{4 1 9}$ under modified Mukaiyama conditions, followed by fluoride-induced desilylation, provided macrocyclic alcohol 441 efficiently. To complete the synthesis of analogue 184, TBAF-mediated aldol-like condensation between silyl ether $\mathbf{4 8 5}$ and the aldehyde derived from alcohol $\mathbf{4 4 1}$ will be performed.

In order to prepare $\mathrm{CF}_{3}$-substituted thiazole analogue 185 , attempts to access $\mathrm{CF}_{3}$ substituted thiazole 395 via brominate thiazole intermediate 394 were made. Unfortunately, the initial palladium-catalysed trimethylation reaction was unsuccessful. Further investigation of appropriate conditions will be conducted. 


\section{Chapter Four: Towards Synthesis of Triazole Analogue with Methylated Side Chain}

\subsection{Introduction}

\subsubsection{Synthesis of Triazole Analogue 182}

As mentioned in Section 1.6, the key features of the synthesis of the racemic analogue 182 included Julia-Kocienski olefination to attach the non-methylated side chain fragment, lactone ring-opening to provide the $Z, E$-dienoate and a copper-catalysed alkyne-azide cycloaddition (CuAAC) to form the triazole ring. Therefore, three major fragments - sulfone fragment 284, aldehyde fragment 289, and alkyne fragment 414 were utilised in Cumming's synthesis of analogue $\mathbf{1 8 2} .{ }^{134}$
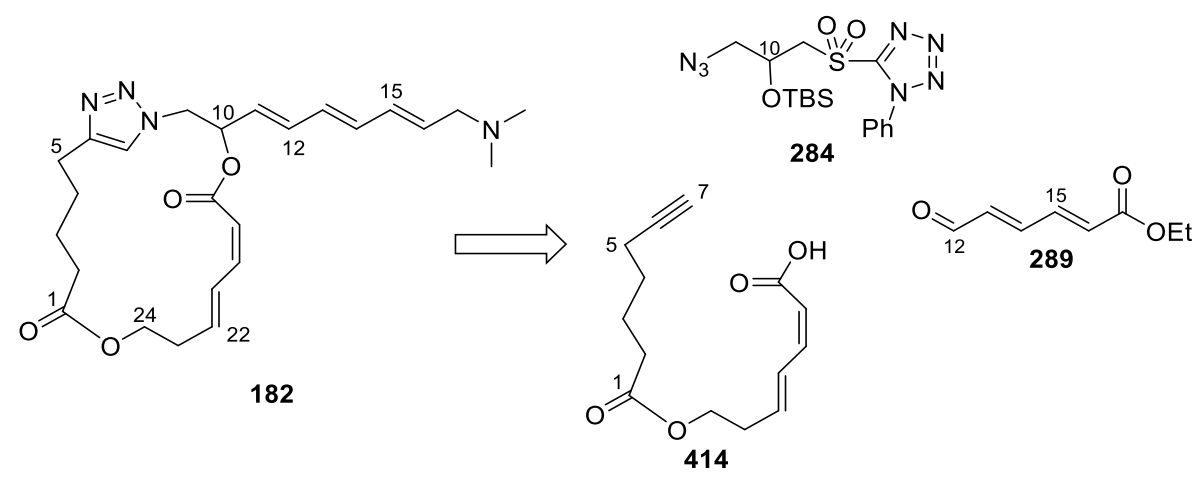

Figure 4.1 Major fragment in Cumming's synthesis of analogue $\mathbf{1 8 2}$.

\section{Preparation of Sulfone Fragment 284}

The synthesis of sulfone fragment $\mathbf{2 8 4}$ began with the ring opening of 2(chloromethyl)oxirane $\mathbf{4 8 6}$ with 1-phenyl-1H-tetrazole-5-thiol 210, giving chloroalkylthioether $\mathbf{4 8 7}$. The alkyl chloride was then substituted by sodium azide to afford compound 488. Subsequent silylation, followed by oxidation of the resulting thioether 489, provided sulfone fragment $\mathbf{2 8 4}$ (Scheme 4.1). 


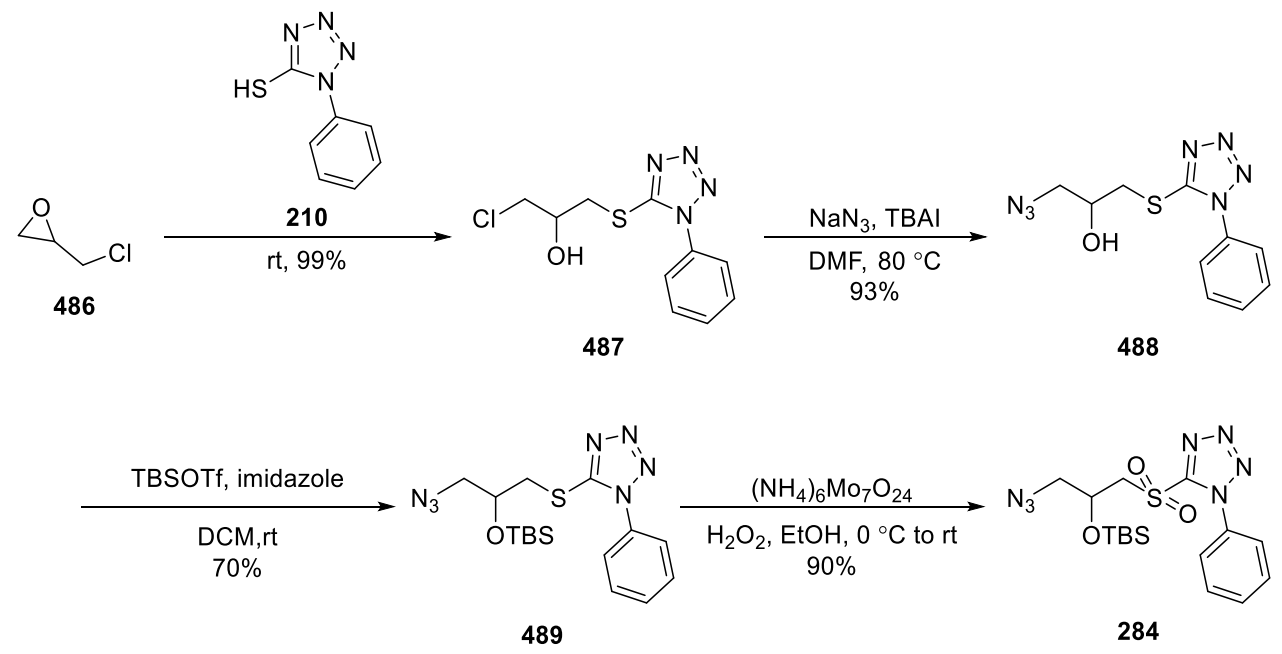

Scheme 4.1 Preparation of sulfone 284 in the synthesis of analogue $\mathbf{1 8 2}$.

\section{Preparation of Aldehyde Fragment 289}

The key acetal intermediate $\mathbf{2 9 3}$ for the synthesis of aldehyde fragment $\mathbf{2 8 9}$ was prepared from furan in 2 steps, which has been described in the synthesis of enal 297 (refer to Section 2.3.3). Wittig reaction with stabilised ylide 490 provided E,E-dienoate 491. Acetal cleavage under treatment with Amberlyst-15, a solid-supported acidic resin, at 60 ${ }^{\circ} \mathrm{C}$ provided the desired dienal $\mathbf{2 8 9}$ (Scheme 4.2).

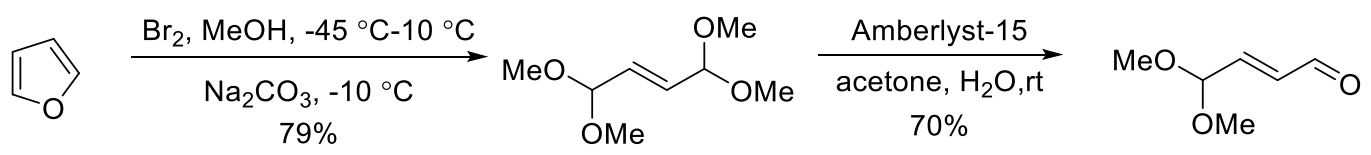

291

292<smiles>CCOC(=O)/C=C/C=C/C=O</smiles>

289

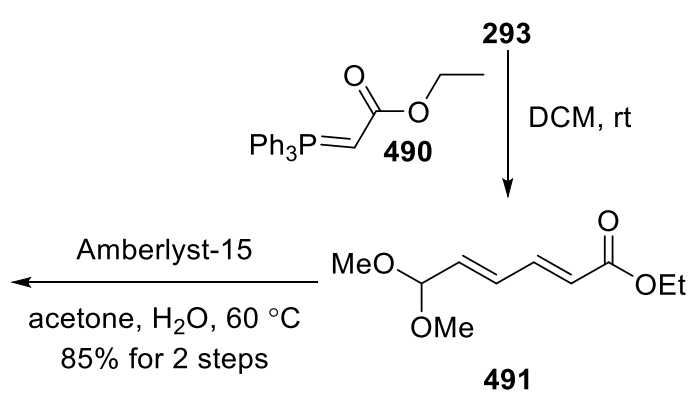

Scheme 4.2 Synthesis of aldehyde 289 from furan 291.

\section{Preparation of Alkyne Fragment 414}

Alkyne fragment 414 was prepared from the TBAF-induced ring opening of alkyne 364 (Scheme 4.3), which was synthesised from the Steglich esterification of 6-heptynoic acid 258 with alcohol 366 (refer to Section 3.3 for the details of the synthesis of $\mathbf{3 6 6}$ from 1,3propanediol). 


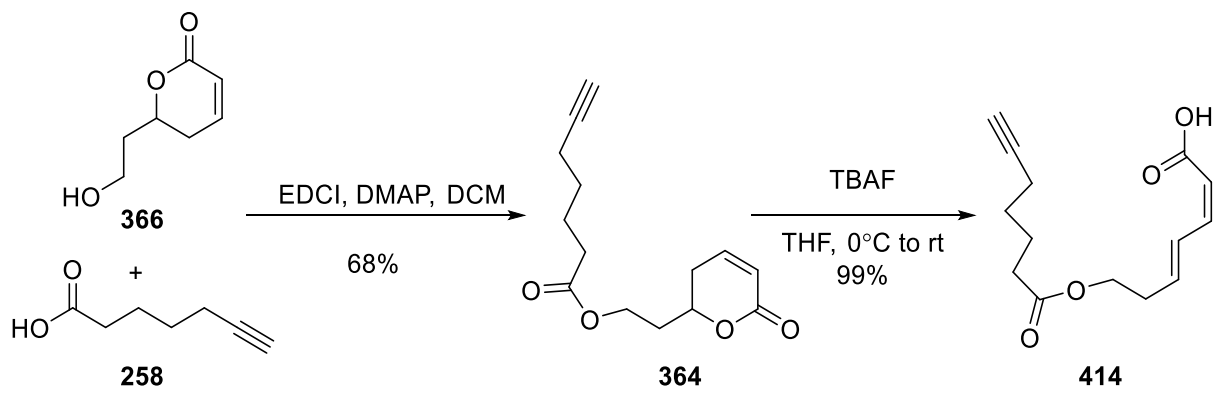

Scheme 4.3 Preparation of alkyne fragment 414 .

\section{Coupling of Fragments to Complete Analogue 182}

The side chain fragment enal $\mathbf{2 8 9}$ was coupled with sulfone $\mathbf{2 8 4}$ through Julia-Kocienski olefination to provide trienoate 290 (Scheme 4.4). Ester 290 was reduced by DIBAL-H to give alcohol 492, which was subjected to Parikh-Doering conditions to afford trienal 493. As prolonged treatment of trienal 493 under acidic conditions could potentially lead to the formation of a teraene product through the elimination of the hydroxyl group of desilylated product 494, treatment with $\mathrm{HCl}$ in methanol was only conducted for 2 hours and the silyl ether starting material was recovered and recycled in subsequent desilylation reactions. Azid-containing seco-acid 417 was prepared via a CuAAC reaction between azide 494 and alkyne fragment 414 (vide supra, Scheme 4.3). In the following Yamaguchi macrolactonisation step, to minimise the risk of isomerisation of the $Z, E$-dienoic acid, a catalytic amount of DMAP was added to the highly-diluted anhydride intermediate at $78{ }^{\circ} \mathrm{C}$, which was generated from the reaction with the activating reagent $\mathbf{9 3}$. Although the use of these modified Yamaguchi conditions did prevent the isomerisation, the desired macrocyclic compound $\mathbf{4 9 5}$ was obtained in only 13\% yield. Reductive amination of $\mathbf{4 9 5}$ with dimethyl amine and tetramethylammonium triacetoxyborohydride provided racemic triazole analogue $\mathbf{1 8 2}$.

Cumming's synthetic route provided triazole analogue 182 over 11 steps for the longest linear sequence but the overall yield is only $2.2 \%$. It is worth mentioning that the overall yield before the Yamaguchi macrolactonisation step is $31 \%$ and the overall yield after that is only $4 \%$. Therefore, the macrolactonisation step is the major hurdle to improve the yield. Based on the good results obtained in the macrolatonisation step in Fürstner's synthesis of PatA and DMDA PatA (refer to Section 1.4.1.3 and 1.4.2.2), use of the modified Mukaiyama macrolactonisation methodology would potentially enhance the yield for the synthesis of this type of PatA analogues. 

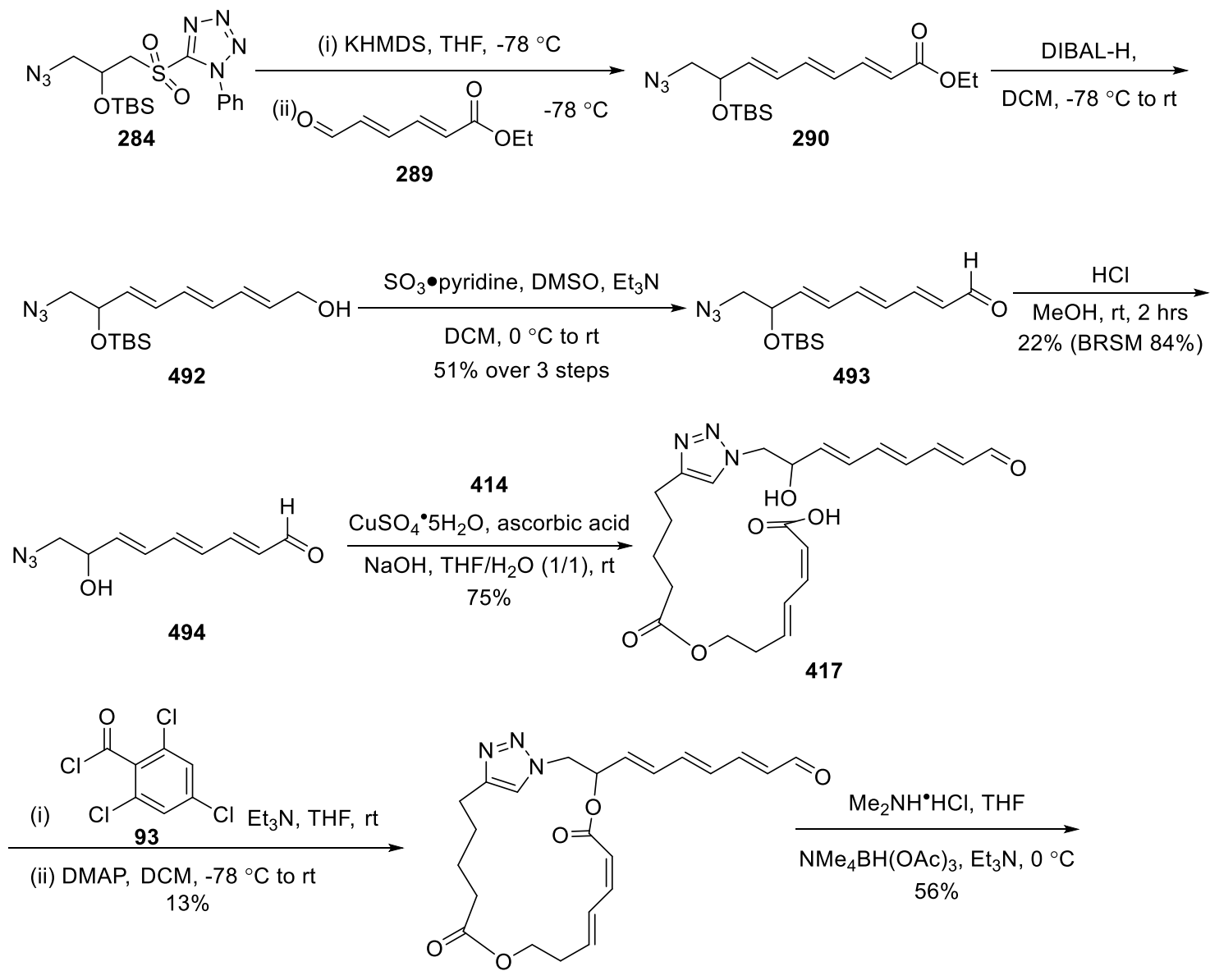

495

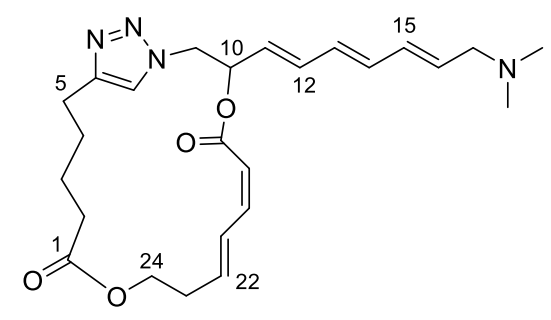

182

Scheme 4.4 Completion of synthesis of analogue 182 .

\subsubsection{Reintroduction of Methyl Groups to Side Chain}

As oxidative degradation of compound 496, containing the non-methylated triene side chain fragment, was observed by Cumming in his synthetic work on the triazole analogue 182 (Scheme 4.5), ${ }^{135}$ we proposed that the presence of the methyl groups on the side chain could stabilise PatA and prevent its rapid oxidative degradation inside cells, which may be advantageous in its binding to the protein target. Additionally, the methyl group at C12 (PatA numbering) may generate an advantageous restriction on the conformation of the rigid side chain in relation to the macrocycle. 


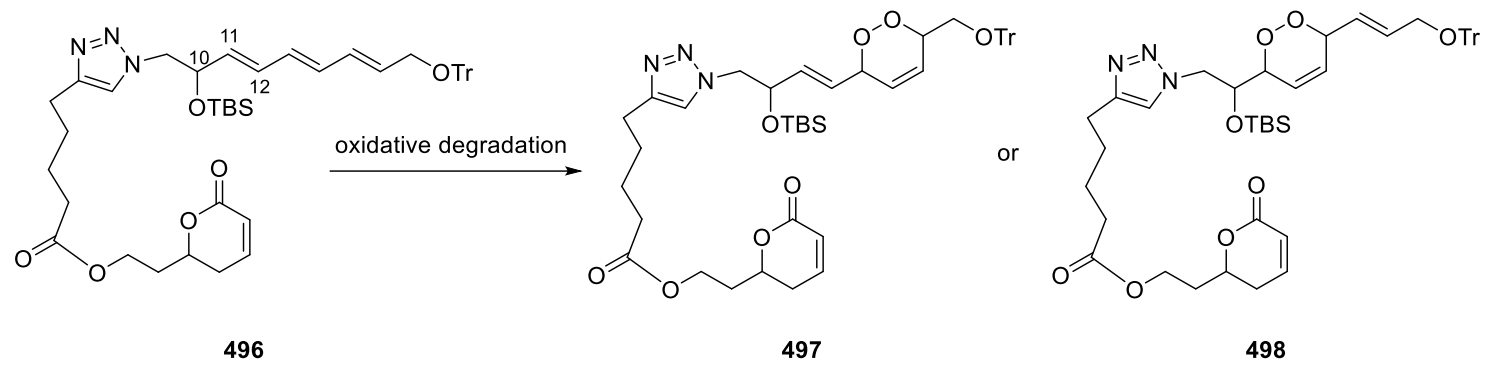

Scheme 4.5 Proposed degradation of compound 496 caused by autoxidation. ${ }^{135}$

As mentioned in Section 1.7.1, investigating the difference between the biological activity of Cumming's triazole analogue $\mathbf{1 8 2}$ and that of a triazole analogue with the methylated side chain would ascertain whether the reintroduction of the methyl groups at C12 and C15 (PatA numbering) could enhance the inhibitory activity. This chapter describes studies towards the synthesis of a novel triazole analogue that we carried out alongside the synthesis of thiazole analogue $\mathbf{1 8 4}$ described in chapter three.

\subsection{Retrosynthetic Analysis}

In a variation of Cumming's strategy in the synthesis of analogue 182, the reaction sequence for the synthesis of analogue 187 would use modified Mukaiyama macrolatonisation as opposed to Yamaguchi macrolatonisation to improve the overall yield. Also, the side chain-fragment would be attached through a Wittig/HWE reaction instead of the Julia-Kocienski olefination.

As shown in Scheme 4.6, the terminal tertiary amine group in the side chain would be introduced in the final step by reductive amination of aldehyde 499. The side chain fragment 476 or $\mathbf{4 8 1}$ would be incorporated into the molecule prior to the construction of the macrocycle. The triazole ring would be synthesised by a CuAAC after the formation of the E,Z-dienoic acid. Trienoate fragment $\mathbf{5 0 0}$ could be prepared from a Wittig/HWE reaction between azide $\mathbf{5 0 1}$ and a triphenylphosphonium salt $\mathbf{4 7 6}$ or a phosphoryl ester 481 (see Section 3.6.2 for their synthetic routes). Alkyne fragment 414 could be prepared by a base-induced ring-opening reaction of the lactone 364 (see Section 3.3 for its synthetic route). The macrocycle 499 would be conveniently constructed through a combination of the modified Mukaiyama macrocyclisation and the CuAAC reaction. 


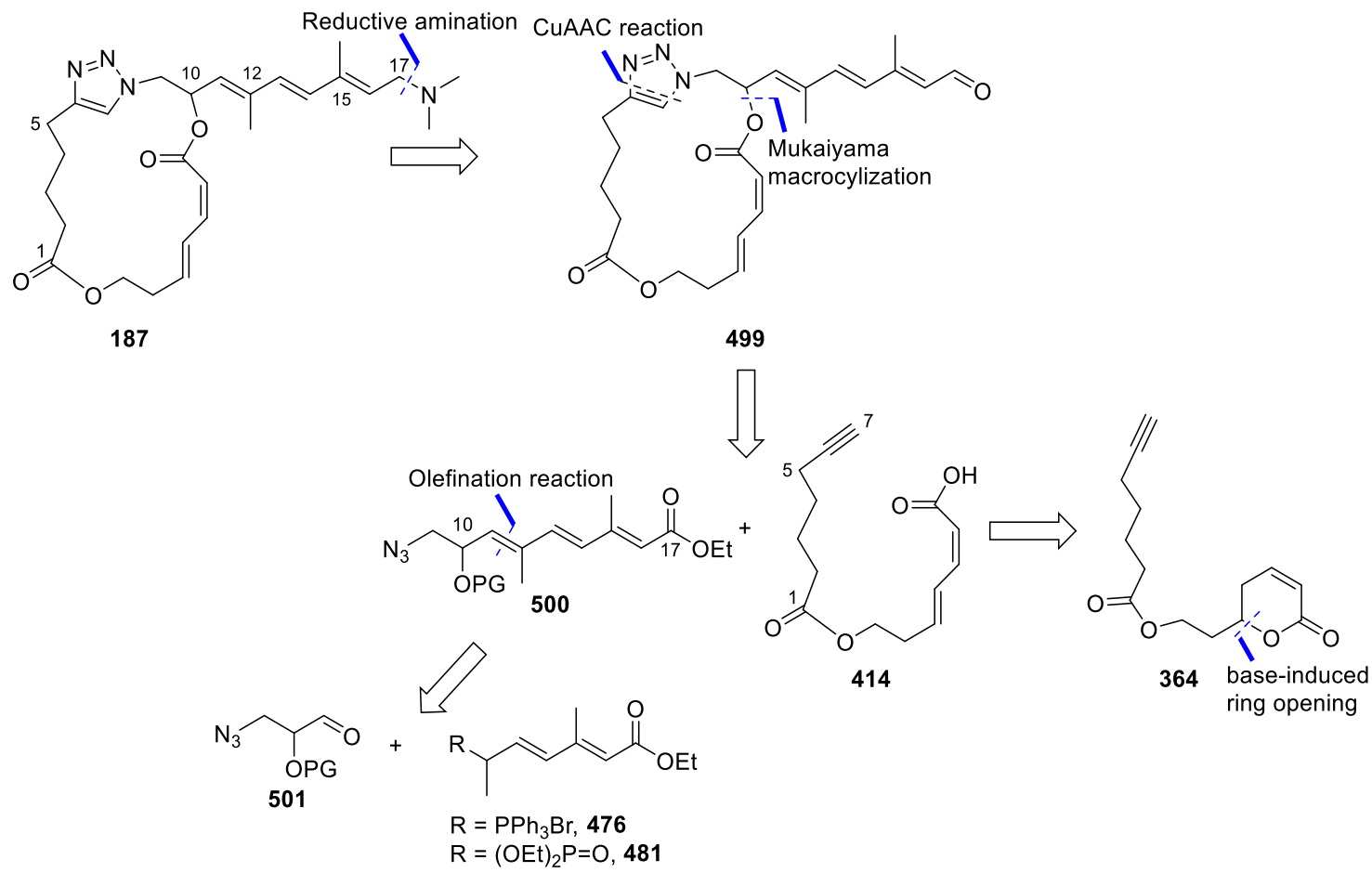

Scheme 4.6 Retrosynthetic analysis of triazole analogue 187.

The CuAAC reaction is a variant of the Huisgen 1,3-dipolar cycloaddition and was independently discovered by Medal ${ }^{143}$ and Sharpless ${ }^{142}$ in 2002 . It is a robust reaction that displays extraordinary tolerance towards a wide range of functionalities. ${ }^{140}$ Therefore, it has been utilised in various fields such as drug discovery, ${ }^{332}$ biochemistry, ${ }^{333}$ and polymer chemistry. ${ }^{334}$ It has been identified as one of the reactions that fulfil the prerequisite criteria of "click chemistry", a terminology introduced by Sharpless and coworkers to describe reactions that are high-yielding, tolerant to a wide range of functionalities, able to generate only by-products which can be removed by nonchromatographic methods, simple to conduct (ideally, the reaction should not be sensitive to water or oxygen) and can be performed without solvents or in benign solvents (such as water). ${ }^{335}$

Although Cumming proposed that the stereochemistry of $\mathrm{C} 10$ (PatA numbering) is essential for the binding process and that the stereoisomeric analogues of $\mathrm{C} 10$ could be completely inactive, ${ }^{135}$ there were no structure-relationship studies on the configuration of $\mathrm{C} 10$ reported in the literature. Therefore, in order to compare the bioactivity of stereoisomers at C10, we chose to prepare both enantiomers of the triazole analogue 187.

Based on Cumming's unpublished experimental results, ${ }^{135}$ attempts to construct the 
macrocycle and simultaneously invert the configuration of C-10 using an intramolecular Mitsunobu reaction would result in significant degradation of substrates. Therefore, it would be attractive to use a switchable starting material for the preparation of $(R)$ - and $(S)$-187. We envisioned that $(S)$-solketal 502, a readily available enantiopure building block, could serve the purpose because it could be conveniently transformed to chiral aldehyde $(S)$-501 or $(R)$-501. via a few simple steps (Scheme 4.7).

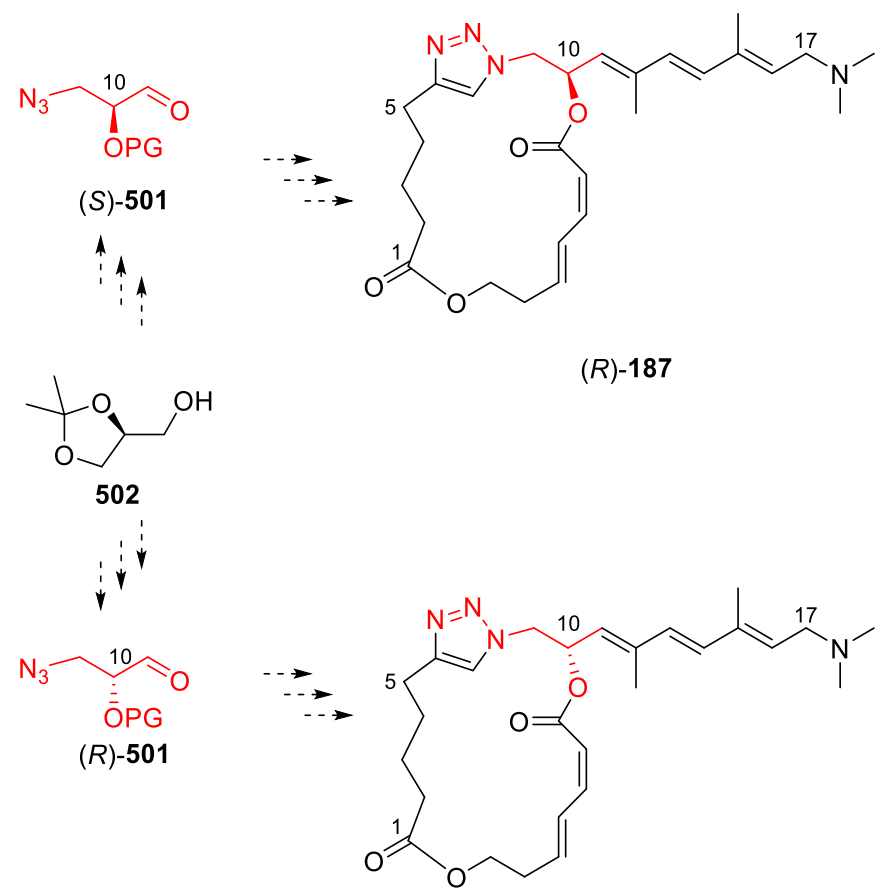

$(S)-187$

Scheme 4.7 Proposed synthesis of (R)-187 and $(S)$-187 from the same starting material $(S)$-solketal $\mathbf{5 0 2}$.

As the preparation of $(R)$-501 from $(S)$-solketal 502 requires fewer steps than that of $(S)$ $\mathbf{5 0 1}$, it would be more likely to succeed. In addition, because enantiomers exhibit identical behaviours in achiral chemical reactions, the synthesis of analogue $(S)-\mathbf{1 8 7}$ would be informative for the synthesis of $(R)-\mathbf{1 8 7}$ analogue. Therefore, attempts to synthesise analogue $(S)$-187 from aldehyde $(R)$-501 were first performed. 


\subsection{Attempted Preparation of Aldehyde (R)-509}

Our attempts to synthesise MOM-protected aldehyde $(R)-\mathbf{5 0 9}$ began with TBDPS protection of $(S)$-soketal $\mathbf{5 0 2}$ (Scheme 4.8). Subsequent cleavage of the acetonide group in silyl ether $\mathbf{5 0 3}$ was achieved by reflux with $90 \%$ acetic acid. Tosylate $\mathbf{5 0 5}$ was formed via the $\mathrm{Bu}_{2} \mathrm{SnO}$-catalysed selective tosylation of diol 504. Subsequent substitution of the tosylate by sodium azide was performed in DMF at $90{ }^{\circ} \mathrm{C}$. MOM-protection of the secondary $\mathrm{OH}$ of azide $\mathbf{5 0 6}$ using methoxymethyl chloride and DIPEA provided MOM ether 507, which was then treated with TBAF to give primary alcohol 508. Buffered DessMartin oxidation was used in our first attempt to prepare chiral aldehyde $(R)$-509. After one-hour stirring at room temperature, ${ }^{1} \mathrm{H}$ NMR analysis of aliquot samples of the reaction mixture showed that the signals of the $\alpha$-protons of the -OH group of $\mathbf{5 0 8}$ at 3.68 and 3.63 ppm completely disappeared while a sharp singlet peak at $9.71 \mathrm{ppm}$ appeared, suggesting that the oxidation of the alcohol $\mathbf{5 0 8}$ to the desired aldehyde ${ }^{* * * * *}$ had a good conversion rate. However, purification of the crude product by silica gel flash chromatography to remove impurities generated from Dess-Martin periodinane provided only a mixture of unidentified by-products. 2D TLC analysis of the crude sample was then performed, and it was found that aldehyde $(R)-\mathbf{5 0 9}$ rapidly decomposed on silica.

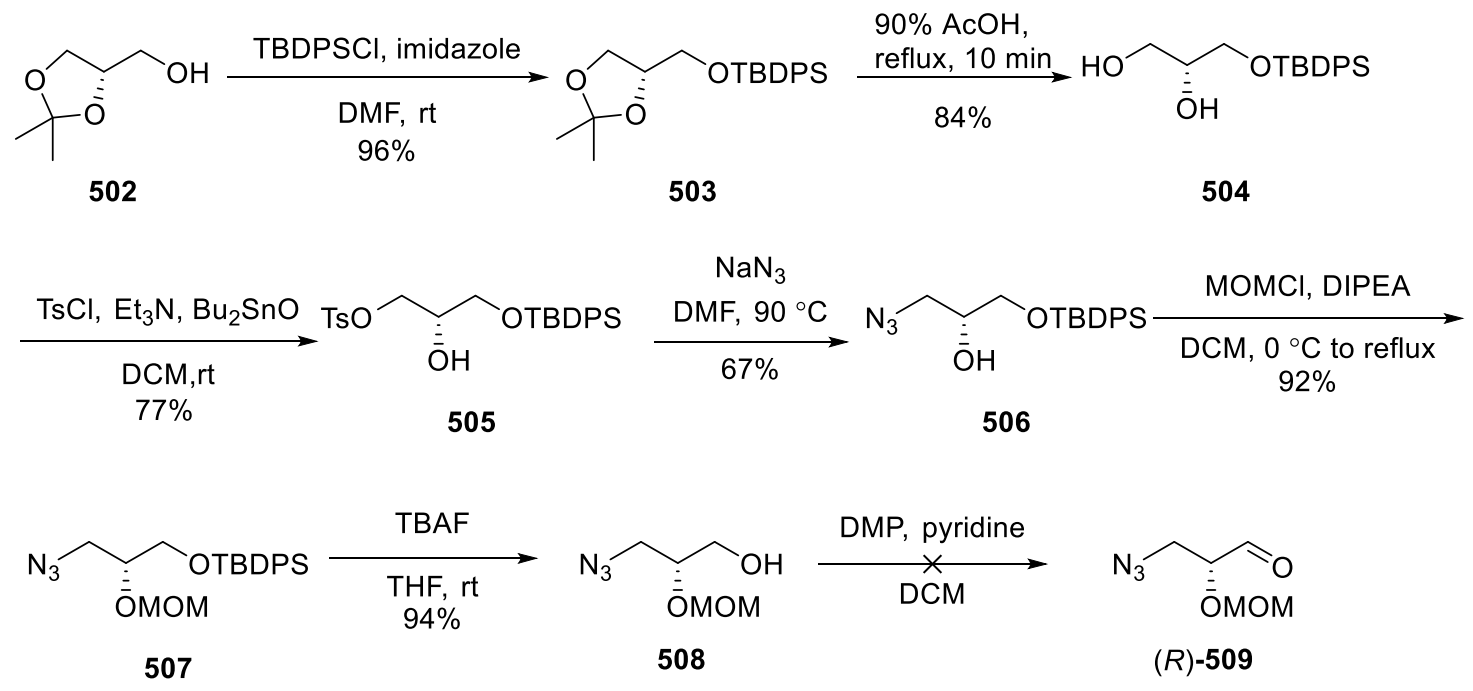

Decomposition on silica gel

Scheme 4.8 Attempted preparation of aldehyde $(R)$-490 from $(S)$-solketal 489.

This was based on analysis of the ${ }^{1} \mathrm{H}$ NMR and gCosy spectra of the crude mixture. With peaks at $\left(500 \mathrm{MHz}, \mathrm{CDCl}_{3}\right) \delta 9.71(\mathrm{~s}, 1 \mathrm{H}), 4.83(\mathrm{~s}, 2 \mathrm{H}), 4.13-4.09(\mathrm{~m}, 1 \mathrm{H}), 3.59-3.55(\mathrm{~m}, 2 \mathrm{H}), 3.48(\mathrm{~s}, 3 \mathrm{H})$. 
A scan through the literature revealed that aldehyde $\mathbf{5 1 0}$ with a similar structure to $(R)$ 509 was reported by Fürstner and co-workers to be sensitive to silica gel. Instead, activated magnesium silicates for chromatography, like Florisil, were used for its purification (Scheme 4.9). ${ }^{336}$ As this type of chromatography material was unavailable in our laboratory, we decided to seek an alternative method to provide the aldehyde in pure form which does not require using chromatographic purification.

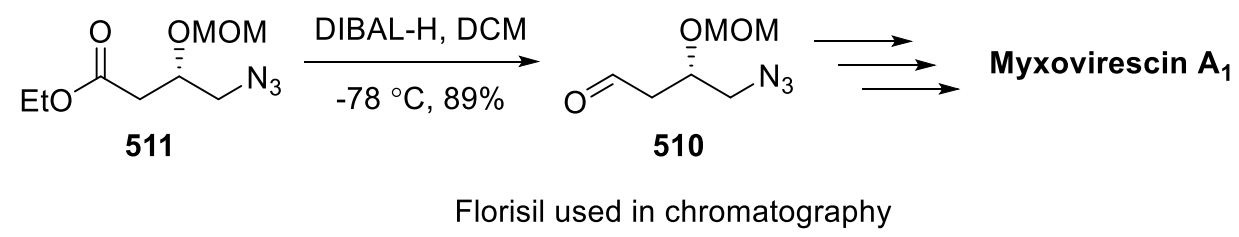

Scheme 4.9 Preparation of aldehyde in the total synthesis of myxovirescin A1. ${ }^{336}$

In 2001, Giacomelli and co-workers reported that primary alcohols could be efficiently oxidised to the corresponding aldehydes by trichloroisocyanuric acid at room temperature in the presence of catalytic TEMPO. ${ }^{337}$ This mild method was very attractive to us because it provided chiral aldehydes with $\alpha$-stereocenters in high purity after a simple aqueous work-up procedure. Therefore, oxidation of alcohol 508 was performed following their conditions. Unfortunately, in addition to the desired product, a minor unidentified product was observed in the crude sample after work-up. Purification of the aldehyde $(R)$-509 by distillation was then attempted. Not surprisingly, it was found that aldehyde degraded significantly in the distillation process when it was heated at $40-45$ ${ }^{\circ} \mathrm{C}$ for a relatively prolonged period of time. As purification of the aldehyde proved to be problematic, the oxidation of alcohol $\mathbf{5 0 8}$ under Giacomelli's conditions was repeated, and the crude aldehyde was used directly after work-up for the Wittig reaction with triphenylphosphonium salt $\mathbf{4 7 6}$ (Scheme 4.10). To our dismay, no sign of the desired product 512 was observed, and instead, a mixture of unidentified products was obtained. This result was possibly due to the instability of the aldehyde in the strongly basic conditions. 

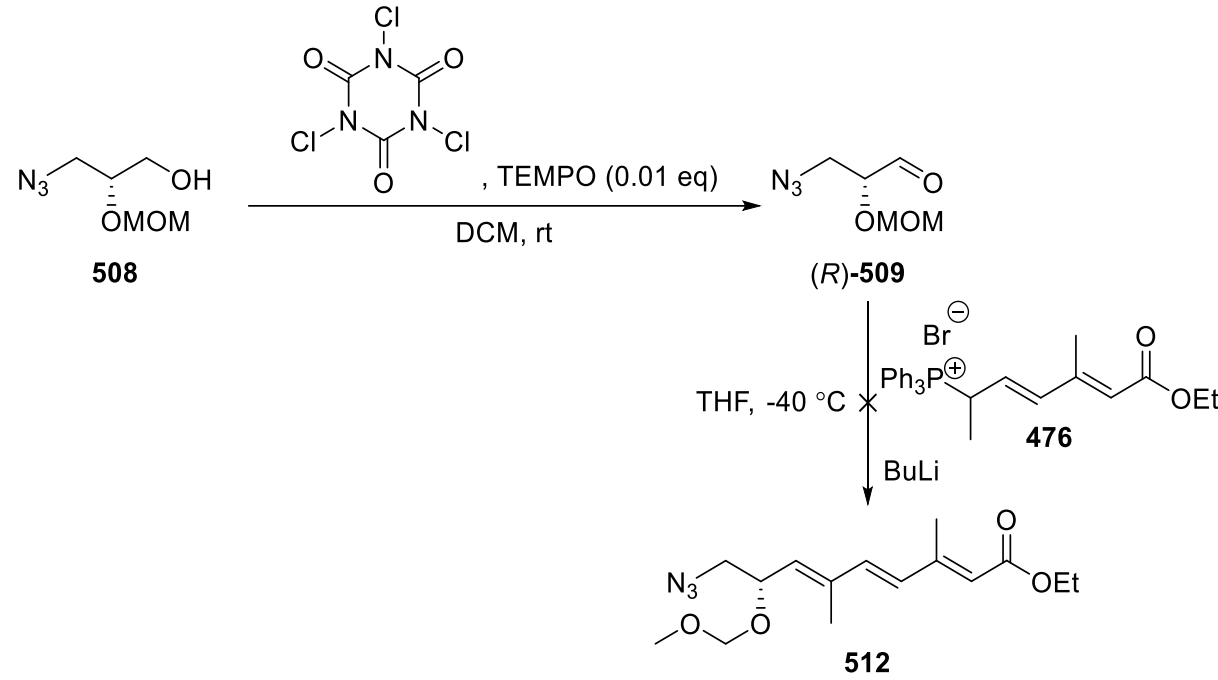

Scheme 4.10 Attempted preparation of trienoate 512 via crude aldehyde (R)-509.

The inability to purify the aldehyde $(R)-\mathbf{5 0 9}$, as well as its potential instability under basic conditions required for Wittig/HWE olefination, led us to abandon this approach. A new synthetic route to triazole analogues $(S)$ - and $(R)-\mathbf{1 8 7}$ that aligned with the synthesis of the thiazole analogue 184 was designed. In this new design, the macrocyclic core would be constructed prior to the attachment of the side chain fragment.

\subsection{Revised Synthetic Route for Synthesis of Analogue (S)-187}

The revised route for the synthesis of $(S)$-187 would involve a macrocyclic aldehyde partner and a side chain phosphonate (Scheme 4.11). This would mean performing the CuAAC reaction between mono-silylated azide 506 and alkyne 414. The triazolecontaining macrocycle 513 would be constructed at an early stage via modified Mukaiyama macrocyclisation. The olefination reaction between triazole-containing macrocyclic aldehyde $\mathbf{5 1 3}$ and an appropriate derivative of phosphoryl aldehyde $\mathbf{4 8 3}$ would be carried out at a late stage to attach the side chain. 


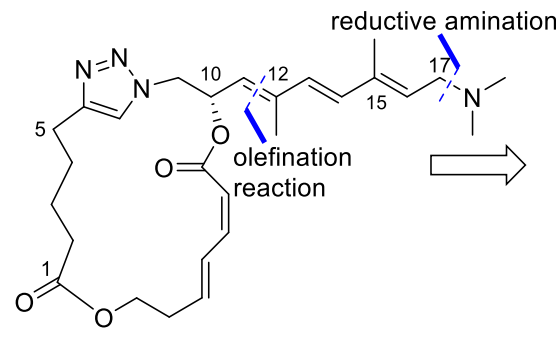

(S)-187

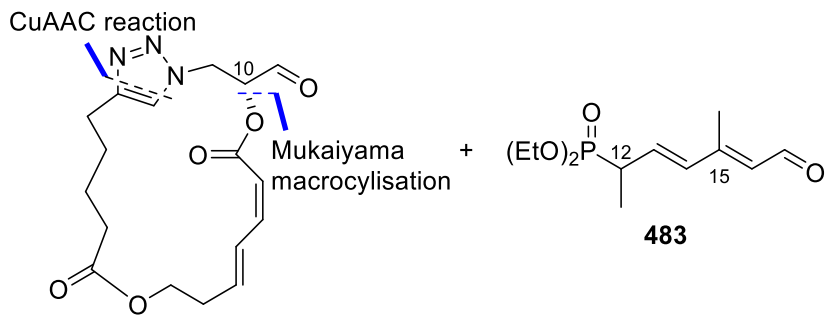

513

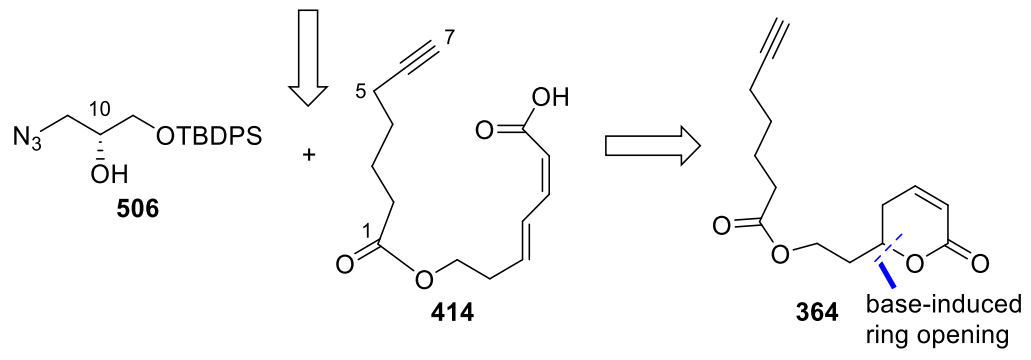

Scheme 4.11 Revised retrosynthetic analysis of triazole analogue $(S)-\mathbf{1 8 7}$.

\subsection{Construction of Macrocyclic Compound 523}

Attention was first turned to the construction of the macrocycle core. The ring opening of lactone 364 facilitated by treatment with TBAF gave $Z, E$-dienoic acid 414 in almost quantitative yield, which was subsequently coupled with azide $\mathbf{5 0 6}$ via a CuAAC process to provide seco-acid $\mathbf{5 1 4}$ (Scheme 4.12).
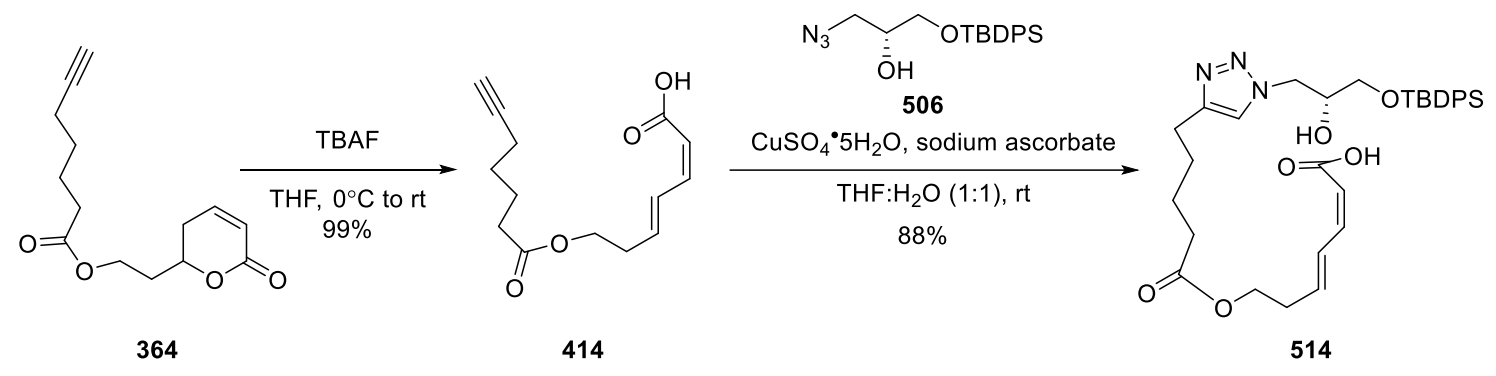

Scheme 4.12 Preparation of seco-acid 514.

Performing Mukaiyama lactonisation of seco-acid 514 following conditions optimised for the synthesis of thiazole-containing macrocycle 440 (see Scheme 3.29 in Section 3.5.2.2) afforded triazole-containing macrocycle 515 (see entry 1 of Table 4.2 for detailed conditions) in a $40 \%$ yield (Scheme 4.13) and no geometric isomer was observed by ${ }^{1} \mathrm{H}$ NMR analysis. However, in addition to the desired product, an unexpected by-product 516 was observed by ${ }^{1} \mathrm{H}$ NMR analysis of the aliquot samples and was successfully 
isolated by silica gel chromatography.

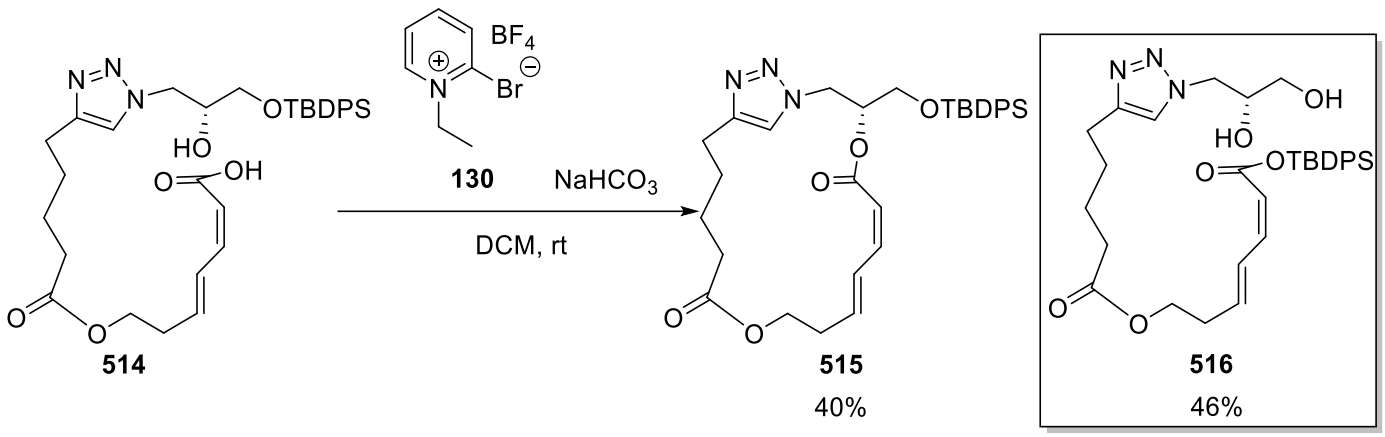

Scheme 4.13 Preparation of triazole-containing macrocycle 515.

Careful investigation of the 2D NMR spectra of both seco-acid starting material $\mathbf{5 1 4}$ and the by-product 516 revealed that the changes of proton and carbon chemical shifts, compared to the starting material, mainly appeared in the $\mathrm{C} 11$ to $\mathrm{C} 15$ region (Table 4.1) and especially, the signal of the carbon at the C11 position was significantly shifted upfield from $169.6 \mathrm{ppm}$ to $161.6 \mathrm{ppm}$. However, it was of note that there were no significant changes in the coupling constants of the alkenes, suggesting that the $Z, E$-diene motif should be completely retained.
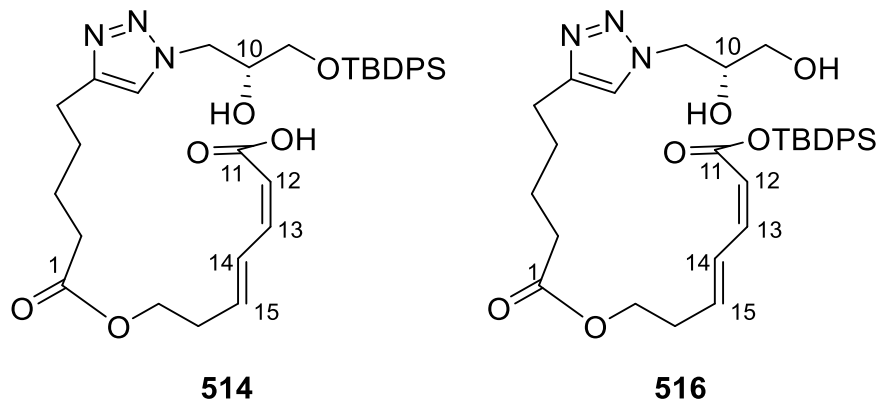

\begin{tabular}{|c|c|c|c|c|}
\hline Position & $\begin{array}{c}\text { Compound } 514 \\
{ }^{1} \mathrm{H} \text { NMR } \\
\delta(\mathrm{ppm}) / J(\mathrm{~Hz})\end{array}$ & $\begin{array}{c}\text { Compound } 516 \\
{ }^{1} \mathrm{H} \text { NMR } \\
\delta(\mathrm{ppm}) / J(\mathrm{~Hz})\end{array}$ & $\begin{array}{c}\text { Compound } 514 \\
{ }^{13} \text { C NMR } \\
\delta \text { (ppm) }\end{array}$ & $\begin{array}{c}\text { Compound } 516 \\
{ }^{13} \text { C NMR } \\
\delta \text { (ppm) }\end{array}$ \\
\hline 11 & - & - & 169.6 & 161.6 \\
\hline 12 & $5.63, \mathrm{~d}, 11.3$ & $5.62, \mathrm{~d}, 11.1$ & 116.9 & 115.0 \\
\hline 13 & $6.60, t, 11.3$ & $6.72, t, 11.2$ & 145.5 & 149.0 \\
\hline 14 & overlapped & overlapped & 129.4 & 129.2 \\
\hline 15 & $6.04, \mathrm{dt}, 14.3,6.7$ & $6.14, \mathrm{dt}, 14.6,7.1$ & 140.1 & 143.1 \\
\hline
\end{tabular}

Table 4.1 Comparison of selected ${ }^{1} \mathrm{H}$ NMR and ${ }^{13} \mathrm{C}$ NMR data of seco-acid 514 with that of silyl ester $\mathbf{5 1 6}$. 
A literature search of TBDPS esters showed that the chemical shifts of carbonyl groups of silyl ester products are normally shifted upfield when compared to those of carbonyl groups of the corresponding carboxylic acids (See Figure 4.2 for selected examples). This was consistent with the observations in the NMR comparison listed in Table 4.1, raising the idea that the silyl migration had led to the formation of product $\mathbf{5 1 6}$.

(a)

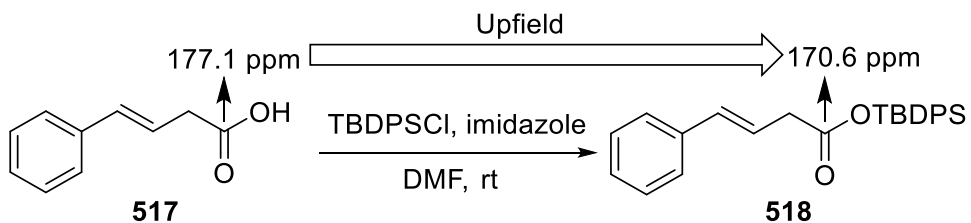

(b)

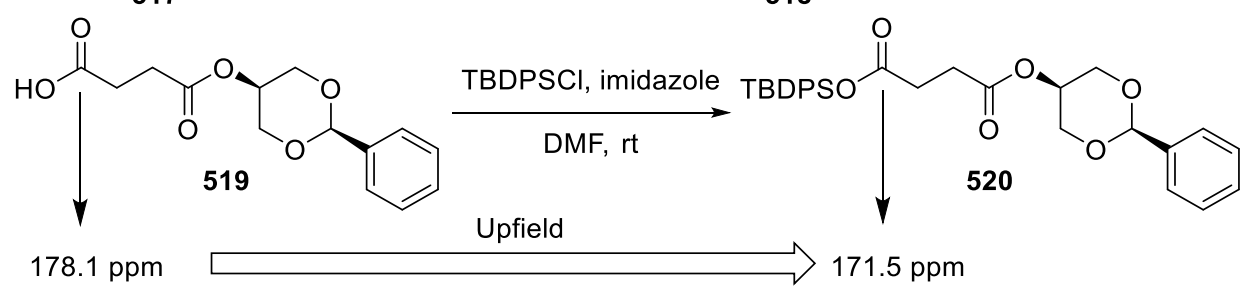

Figure 4.2 Examples of reported upfield chemical shifts of carbonyl groups in preparation of silyl esters. ${ }^{338,339}$

HR-MS analysis showed a minor line with peak height of about $20 \%$ at $\mathrm{m} / \mathrm{z}=606.2974$, which did match with the calculated mass of silyl ester product 516 plus one hydrogen ion (Figure 4.3). However, the obtained base peak at $\mathrm{m} / \mathrm{z}=597.2922$ was not explainable in terms of reasonable fragmentation of the molecule of silyl ester $\mathbf{5 1 6}$ or seco-acid starting material $\mathbf{5 1 4}$ (the calculated mass of $\mathbf{5 1 4}$ is 605.2921 ). 


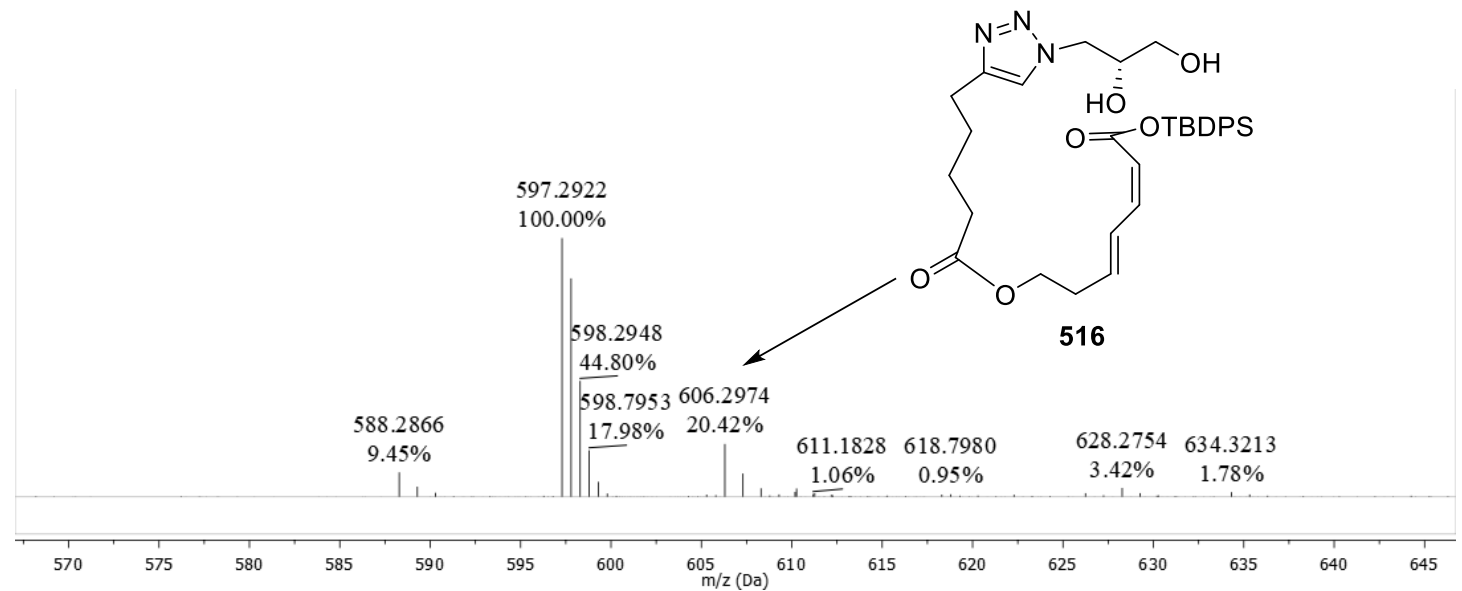

Figure 4.3 HR-MS of by-product 516. . $^{\dagger+1 \dagger}$

Acetylation of the isolated by-product was then performed using acetic anhydride and pyridine, with the hope that NMR and HR-MS analysis of the resulting bis-acylated product 521 could help to fully confirm that the by-product was the proposed silyl ester 516 (Scheme 4.14).
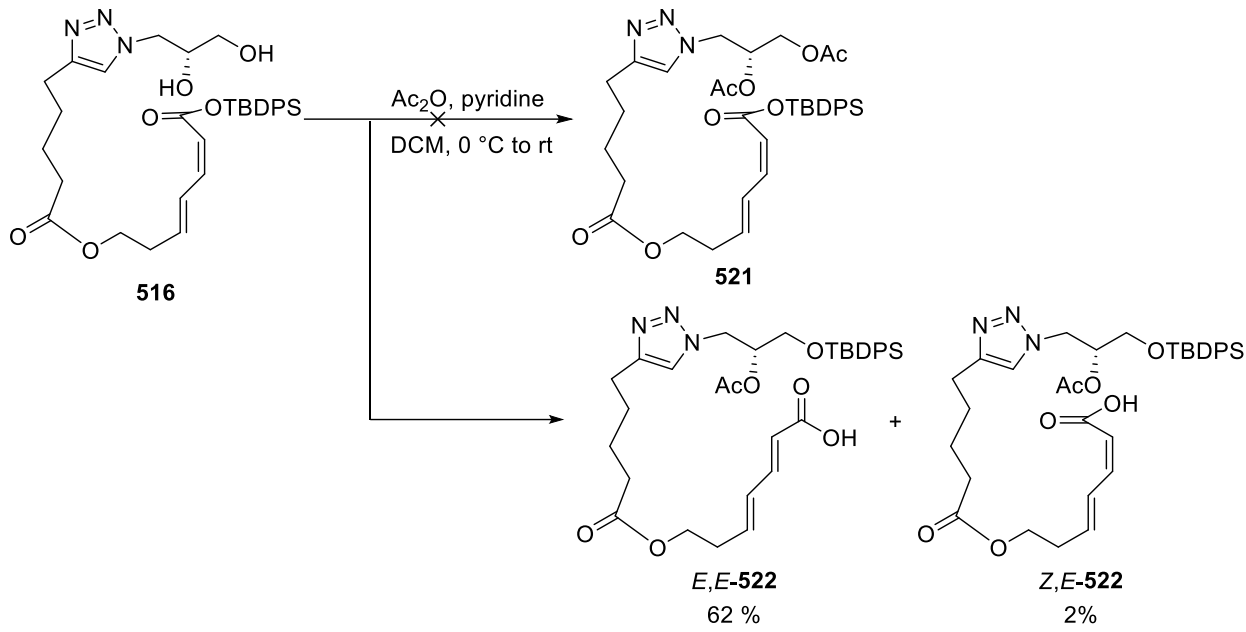

Scheme 4.14 Attempted preparation of bis-acylated product 521.

Interestingly, based on 2D NMR analysis and HR-MS investigation, mono-acylated products $E, E-\mathbf{5 2 2}$ and $Z, E-522$ were obtained instead. The rational explanation for this experimental result was that the TBDPS group rapidly migrated from the carboxylic acid

tit† Mass of protonated compound $\mathbf{5 1 6}$ is at $\mathrm{m} / \mathrm{z}=606.2974$ while the calculated mass for silyl ester product 516 plus one hydrogen ion $\left(\mathrm{C}_{33} \mathrm{H}_{44} \mathrm{~N}_{3} \mathrm{O}_{6} \mathrm{SSi}^{+}\right)$is 606.2994. 
group back to the primary $\mathrm{OH}$ group under treatment with pyridine, meaning that the proposed structure of the by-product $\mathbf{5 1 6}$ was correct. It is also worth mentioning that $E, E-522$ was obtained as the major product while only a trace amount of Z,E-522 was isolated. As 4-DMAP has been proposed to be a major cause of the observed isomerisation in esterification reactions of $Z, E$-dienoic acids (see Section 3.5.2.1), the significant isomerisation in this acetylation reaction could possibly be triggered by the reversible nucleophilic addition of pyridine onto the mixed anhydride intermediate. These two stereoisomers were clearly distinguished by ${ }^{1} \mathrm{H}$ NMR spectroscopy in the coupling constant of $\alpha$-proton of the unsaturated moiety $(J=15.6 \mathrm{~Hz}$ and $11.4 \mathrm{~Hz}$, respectively) (see Figure 4.4 for the comparison of the proton signals in the alkene region) and their chemical shifts of the alkene protons also matches with the reported characteristic signal shifts of dienoate isomers synthesised by Romo and co-workers in their structural studies of PatA. ${ }^{79}$

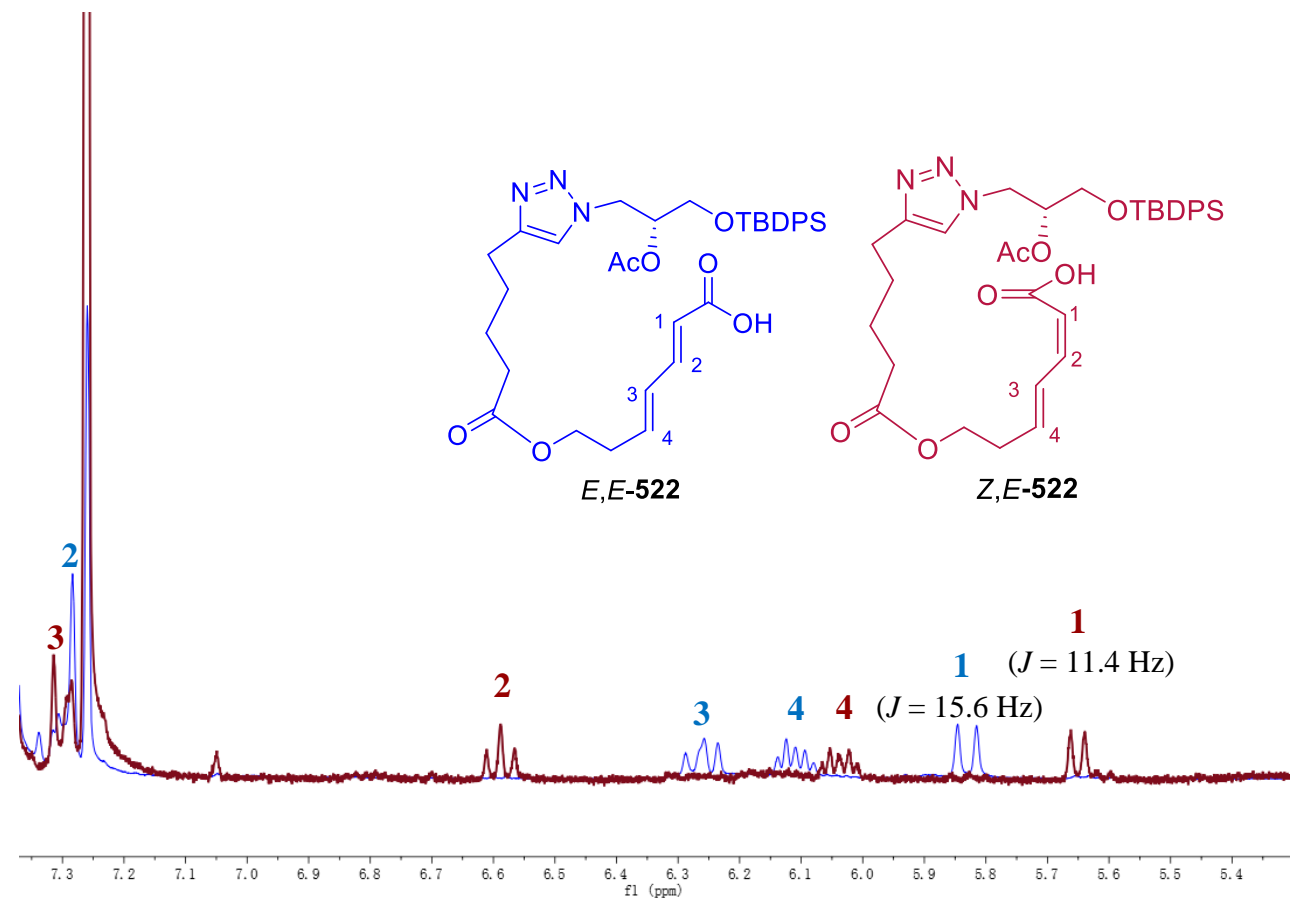

Figure 4.4 Comparison of the alkene region (5.45 ppm - 7.35 ppm) for the ${ }^{1} \mathrm{H}$ NMR spectra of Z,E-522 (in maroon) and E,E-522 (in blue). (Assigned by gCOSY NMR). ${ }^{+\ldots+t}$

H+Z,E-522 with alkene peaks at $\left(500 \mathrm{MHz}, \mathrm{CDCl}_{3}\right) \delta 7.32-7.27(\mathrm{~m}, 1 \mathrm{H}, \mathrm{H}-3), 6.59(\mathrm{t}, J=11.4 \mathrm{~Hz}, 1 \mathrm{H}$, H-2), 6.04 (dt, $J=13.9,6.3 \mathrm{~Hz}, 1 \mathrm{H}, \mathrm{H}-4)$, and 5.65 (d, $J=11.4 \mathrm{~Hz}, 1 \mathrm{H}, \mathrm{H}-1$ ), compared to E,E-522 with alkene peaks at $\left(500 \mathrm{MHz}, \mathrm{CDCl}_{3}\right) \delta 7.35-7.27$ (overlapped, $\left.\mathrm{m}, 1 \mathrm{H}, \mathrm{H}-2\right), 6.26(\mathrm{dd}, J=15.2,11.8 \mathrm{~Hz}, 1 \mathrm{H}$, H-3), $6.14-6.06$ (m, 1H, H-4), and 5.83 (d, $J=15.6 \mathrm{~Hz}, 1 \mathrm{H}, \mathrm{H}-1)$. 
In order to improve the synthetic yield of the desired macrocycle 515, it was necessary to optimise the macrolactonisation conditions to suppress the silyl-migration side reaction. Considering the silyl migration may plausibly have been caused by the presence of excess $\mathrm{NaHCO}_{3}$ (246 equivalents) in the macrolactonisation, it was proposed that reducing the equivalents of the base used in the reaction could potentially reduce the formation of silyl ester 516. However, lowering the amount of $\mathrm{NaHCO}_{3}$ (246 to 17 equiv.) and Mukaiyama reagent (24.6 to 15 equiv.) drastically decreased conversion and provided a $5 / 1$ ratio of seco-acid starting material 514 and the undesired by-product 516 (Table 4.2, entry 2), which could be a consequence of the extremely low solubility of $\mathrm{NaHCO}_{3}$ in $\mathrm{DCM}$. It was then assumed that maintaining a continuously low concentration of base in the reaction system in a controllable manner should favour the desired lactonisation reaction and limit the side reaction simultaneously because the reaction between the secondary alcohol group and the activated acid intermediate might proceed faster than the basemediated silyl-migration between the primary alcohol group and the free acid group. Therefore, a soluble base DIPEA was then trialled, and it was added slowly by a syringe pump to the reaction mixture of seco-acid $\mathbf{5 1 4}$ and the activating reagent $\mathbf{1 3 0}$ (Table 4.2, entry 3). Surprisingly, silyl ester $\mathbf{5 1 6}$ was obtained as the sole product. These experimental results suggested that igh-yielding macrolactonisation conditions of secoacid 514 would require further investigation. Nonetheless, a sufficient quantity of macrolactone $\mathbf{5 1 5}$ had been stockpiled for exploring further elaboration.
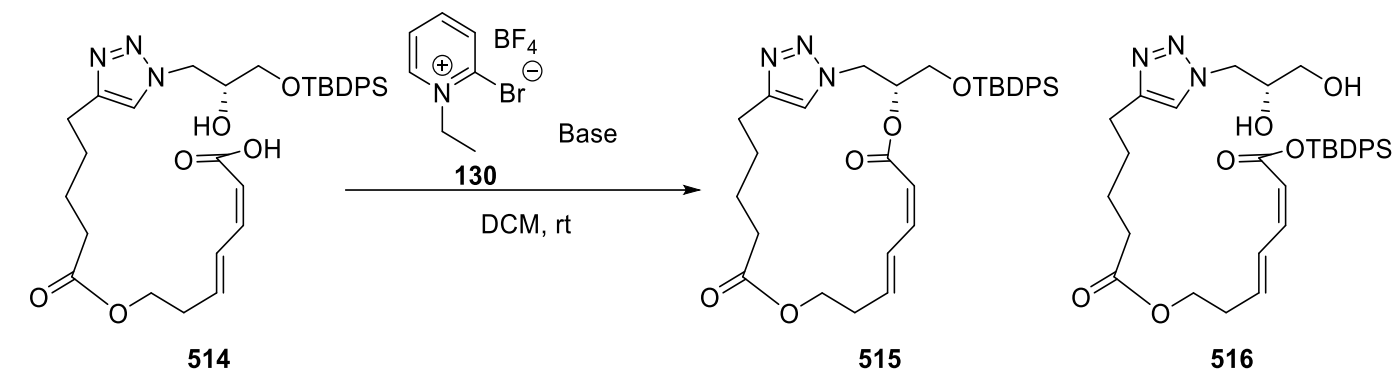

\begin{tabular}{|c|c|c|c|c|c|}
\hline Entry $^{a}$ & Compound (equiv.) & Base (equiv.) & $\begin{array}{c}\text { Ratio }^{b} \\
514: 515: 510\end{array}$ & Time (h) & Yield $^{c}(\%)$ \\
\hline 1 & 24.6 & $\mathrm{NaHCO}_{3}(246)$ & $0: 1: 1.15$ & 16 & 40 \\
\hline 2 & 15 & $\mathrm{NaHCO}_{3}(17)$ & $5: 0: 1$ & 16 & - \\
\hline 2 & 15 & DIPEA (2) & $0: 0: 1$ & 16 & - \\
\hline
\end{tabular}

Table 4.2 Trialled conditions for macrolactonisation of seco-acid $\mathbf{5 1 4}$. 
Desilylation of TBDPS silyl ether $\mathbf{5 1 5}$ using buffered TBAF afforded primary alcohol $\mathbf{5 1}$ in good yield (Scheme 4.15). At this point, the time constraints limited further investigations on the oxidation of the alcohol $\mathbf{5 2 3}$ to the aldehyde $\mathbf{5 1 3}$ and the attachment of the side chain fragment on the macrocyclic core.

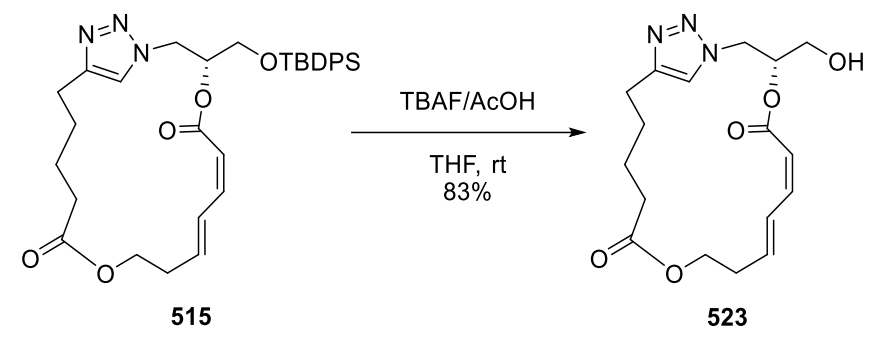

Scheme 4.15 Preparation of macrocyclic alcohol 523.

\subsection{Conclusion}

In conclusion, the initial synthetic route to analogue $(S)-\mathbf{1 8 7}$, which involves using aldehyde $(R)-\mathbf{5 0 9}$ as the key fragment, was unsuccessful and a revised synthetic route, in which macrocyclic alcohol $\mathbf{5 2 3}$ acts as the pivotal intermediate, was pursued.

Alcohol 523 was prepared in 11 steps as the longest linear sequence (15 steps total), starting from commercially available chemicals, with an overall yield of $9 \%$. However, in the macrolactonisation step, an unexpected silyl ester by-product $\mathbf{5 1 6}$ was observed. To minimise the formation of this by-product, several reaction conditions were trialled. Unfortunately, these attempts were unsuccessful in reducing the by-product. Therefore, it is necessary to further optimise conditions in order to enhance the yield of the macrocyclic compound $\mathbf{5 1 5}$.

To complete the synthesis of analogue $(S)$-187, coupling of the side chain fragment to the macrocycle core would require further investigation. 


\section{Chapter Five: Summary and Future Work}

\subsection{Summary}

Pateamine A has potent antiproliferative activity in cancer cell lines. The mechanism of action of PatA involves binding to the eukaryotic initiation factor 4A (eIF4A) to inhibit the initiation of protein synthesis, thereby inducing programmed cell death.

To further explore the structural features that are most important for its binding to eIF4A protein targets, thiazole analogues 183, 184, 185, 186 and triazole analogue $(S)-187$ and (R)-187 were designed (Figure 5.1). The work towards the synthesis of analogues $\mathbf{1 8 3}$, 184 and $(S)$-187 was first carried out, with the hope that this could be informative for the synthesis of a new generation of analogues.

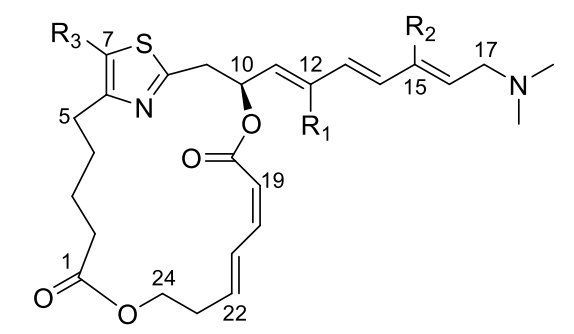

$\mathrm{R}_{1}=\mathrm{H}, \quad \mathrm{R}_{2}=\mathrm{H}, \quad \mathrm{R}_{3}=\mathrm{H}, \quad 183$

$\mathrm{R}_{1}=\mathrm{CH}_{3}, \mathrm{R}_{2}=\mathrm{CH}_{3}, \mathrm{R}_{3}=\mathrm{H}, \quad \mathbf{1 8 4}$

$\mathrm{R}_{1}=\mathrm{CH}_{3}, \quad \mathrm{R}_{2}=\mathrm{CH}_{3} \quad \mathrm{R}_{3}=\mathrm{CF}_{3}, \mathbf{1 8 5}$

$\mathrm{R}_{1}=\mathrm{CH}_{3}, \mathrm{R}_{2}=\mathrm{CH}_{3} \quad \mathrm{R}_{3}=\mathrm{CH}_{3}, \mathbf{1 8 6}$

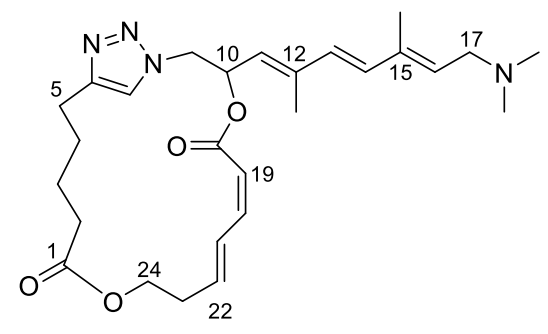

(10S)-187

$(10 R)-187$

Figure 5.1 Chemical structures of targeted analogues $183-187$

\subsubsection{Attempted Synthesis of Thiazole Analogue 183}

Our strategy for the synthesis of analogue $\mathbf{1 8 3}$ was to construct the thiazole core by a gold-catalysed coupling reaction between a thioamide and an alkyne. The initial method tested to connect the non-methylated side chain fragment was Julia-Kocienski olefination. Three fragments were successfully prepared for this purpose (Figure 5.2). 

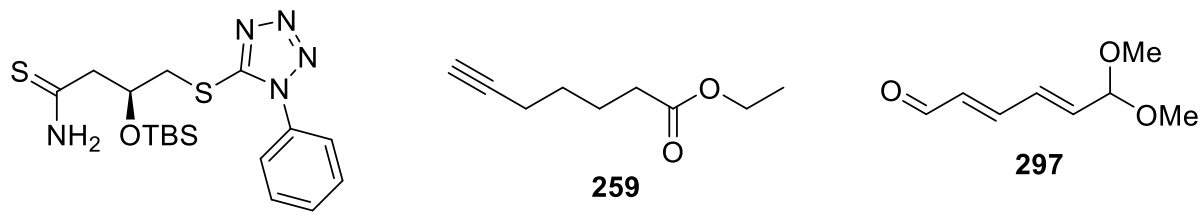

223

Figure 5.2 Three fragments prepared based on the initial synthetic strategy for analogue

183.

Thioamide fragment $\mathbf{2 2 3}$ was first coupled with the alkyne fragment $\mathbf{2 5 9}$ under optimised gold-catalysed conditions and the desired thiazole 276 was obtained in acceptable yield (Scheme 5.1a). However, the coupling of sulfone $\mathbf{2 7 7}$ with the side chain fragment aldehyde 297 by Julia-Kocienski olefination resulted in a mixture of degradation products with no sign of formation of the desired triene product 302. This failure was possibly due to the steric effects from the TBS protecting group (Scheme 5.1b).

(a)
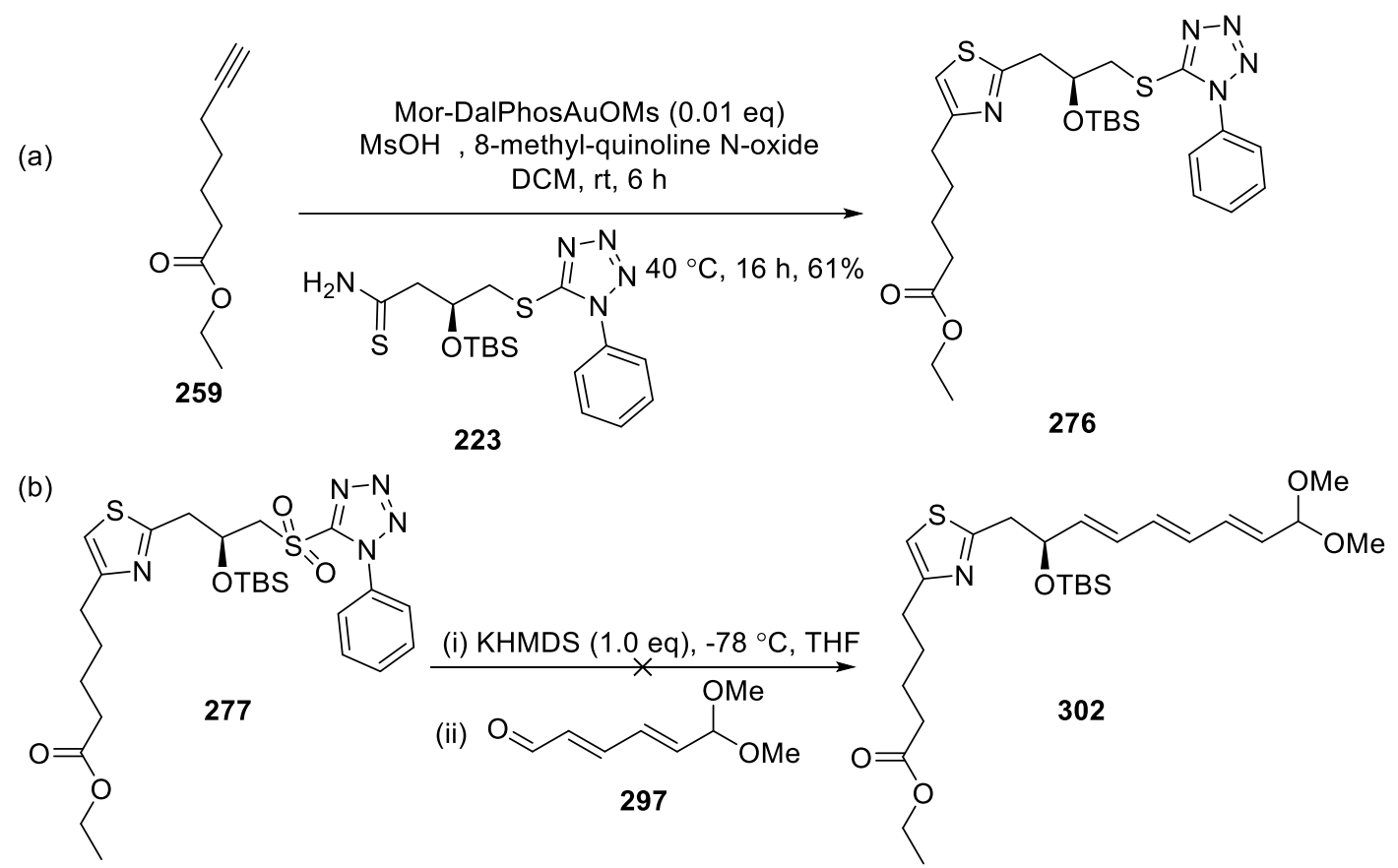

Scheme 5.1 Coupling of fragments for the synthesis of analogue 183: (a) gold-catalysed thiazole formation; (b) Julia-Kocienski olefination

As the MOM group was considered to be less bulky than the TBS group, MOM-protected sulfone $\mathbf{3 1 6}$ was chosen as the alternative sulfone partner for the Julia-Kocienski reaction with enal 297. We proposed that sulfone $\mathbf{3 1 6}$ could be prepared from thioamide $\mathbf{3 1 7}$ (Scheme 5.2). The amide precursor 321 was successfully prepared from aldehyde $\mathbf{3 2 2}$ in 
four steps (Scheme 5.3). To our dismay, the thionation of $\mathbf{3 2 1}$ only provided the undesired thioamide $(S)$-oxide 333 , as determined by HR-MS investigation. This appeared to be due to the oxidation of the thioamide $\mathbf{3 1 9}$ when exposed to air.

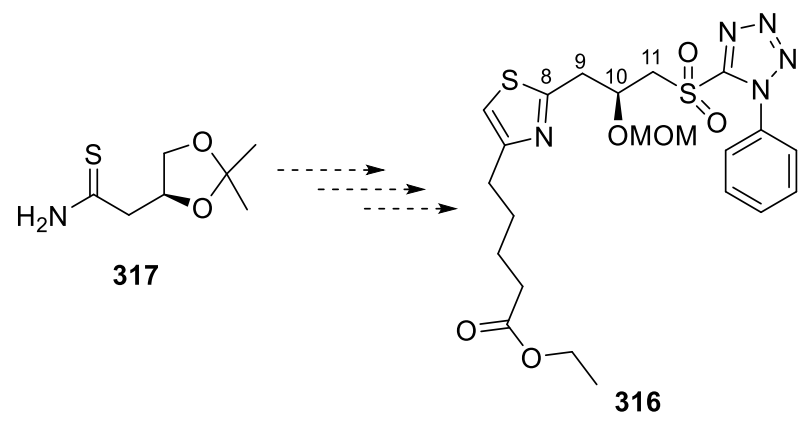

Scheme 5.2 Proposed synthesis of $\mathbf{3 1 6}$ from thioamide 317.

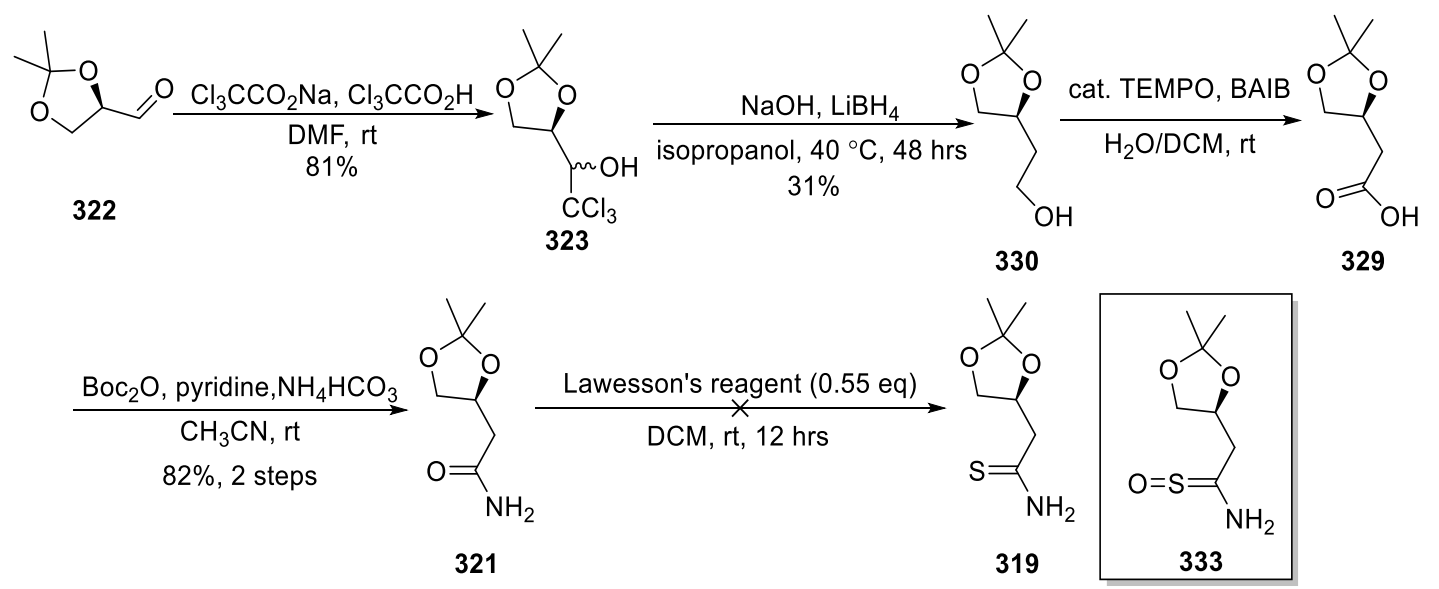

Scheme 5.3 Attempted synthesis of thioamide 319 .

As introducing bulky protecting groups could potentially retard the undesired oxidation, attempts to prepare bis-TBS protected thioamide 341 (Figure 5.3) were carried out first and it was found that the undesired oxidation was indeed slowed down to some extent by the presence of TBS groups. Therefore, TBDPS-protected thioamide 343 (Figure 5.3) was then targeted as a bulkier alternative that might be even more resistant to oxidation. The attempted preparation of thioamide 343 started from PMB-protected amide 346 (Scheme 5.4). However, this synthetic route was eventually abandoned because of the unacceptable yields in the reaction sequence. 


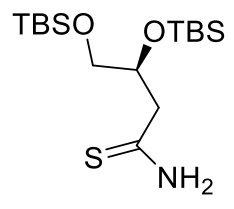

341

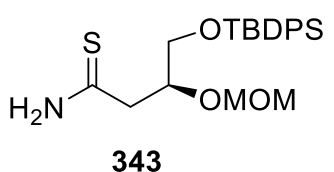

343

Figure 5.3 Targeted thioamides with bulky protecting groups.

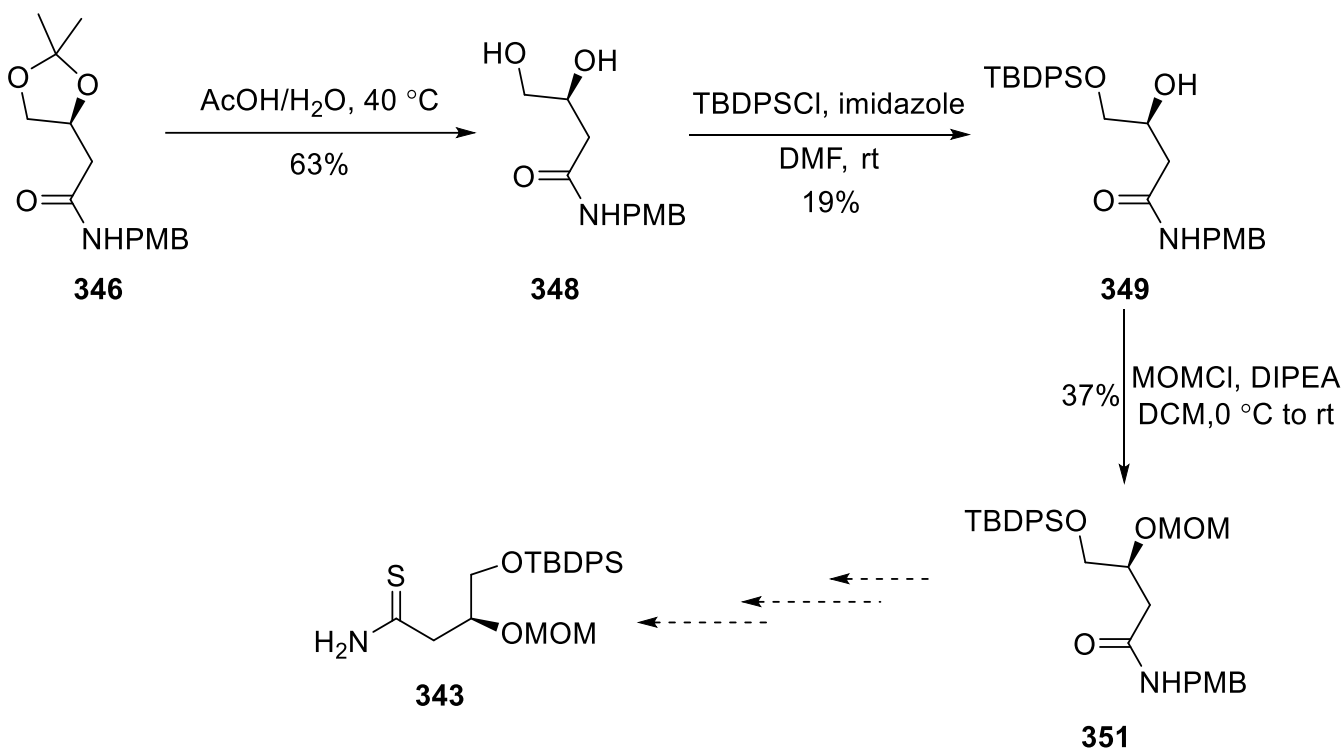

Scheme 5.4 Attempted synthesis of thioamide 343.

As the Julia-Kocienski olefination was found to be ineffective for coupling the methylated side chain fragment to the sulfone partner in model reactions, we decided to change our initial synthetic strategy and to use Wittig/HWE olefination for the attachment of the side chain in order to ensure the collective synthesis of targeted analogues. As the side chain methylated analogue 184 (Figure 5.1) could provide more specific information about the importance of the methyl groups on the macrocyclic core, the synthesis of analogue $\mathbf{1 8 4}$ was prioritised.

\subsubsection{Towards Synthesis of Thiazole Analogue 184}

In preparing thiazole analogue $\mathbf{1 8 4}$, an alternative strategy was employed, whereby the macrocycle would be constructed prior to attachment of the side chain. The macrocyclic core of thiazole analogue $\mathbf{1 8 4}$ was constructed by a combination of gold-catalysed thiazole formation, base-induced ring opening of $\alpha, \beta$-unsaturated- $\delta$-lactone, and modified Mukaiyama macrolactonisation. The thioamide fragment $\mathbf{3 8 2}$ required for gold-catalysed 
formation was prepared from amide 339 with a 35\% yield over 3 steps (Scheme 5.5), and it was found that bis-silylated thioamide $\mathbf{3 8 2}$ could be obtained in high purity after taking extra measures at the post-column stage to exclude air exposure. Alkyne fragment 364 was prepared successfully from 1,3-propanediol in 8 steps following Cumming's reported synthetic strategy. ${ }^{134}$

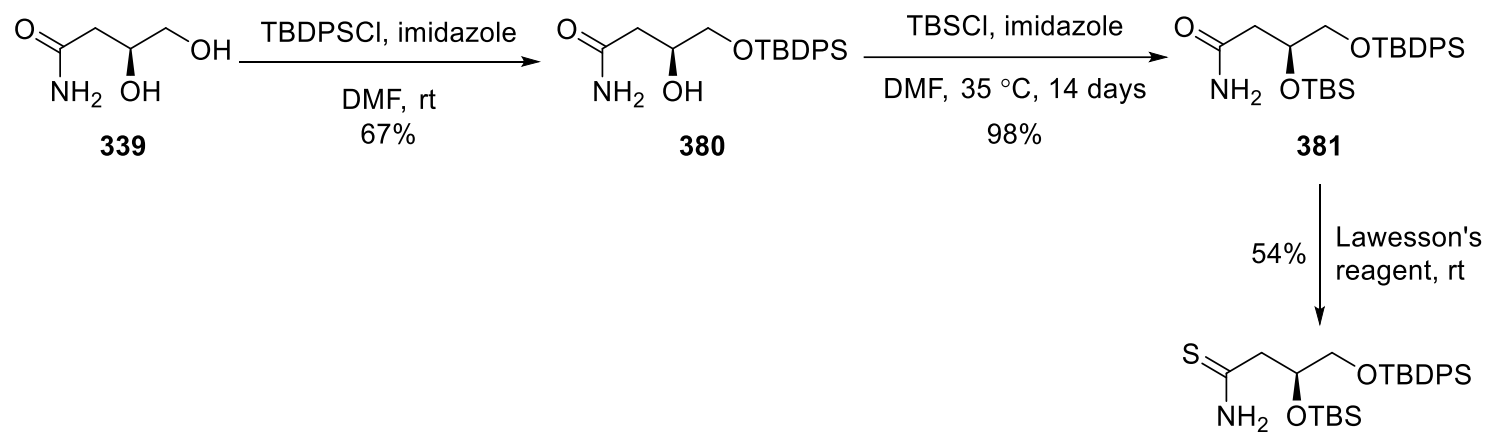

382

Scheme 5.5 Synthesis of thioamide 382 .

Macrocyclic alcohol 441 was successfully synthesised from the alkyne fragment 364 in 6 steps (Scheme 5.6). However, the overall yield of 441 was only 10\%. The low-yielding selective desilylation step to form $\mathbf{4 1 9}$ was the major hurdle to overcome. Therefore, the optimisation for this step will require further investigation. Several alternative synthetic routes were then trialled. However, these efforts did not enhance the yield. 


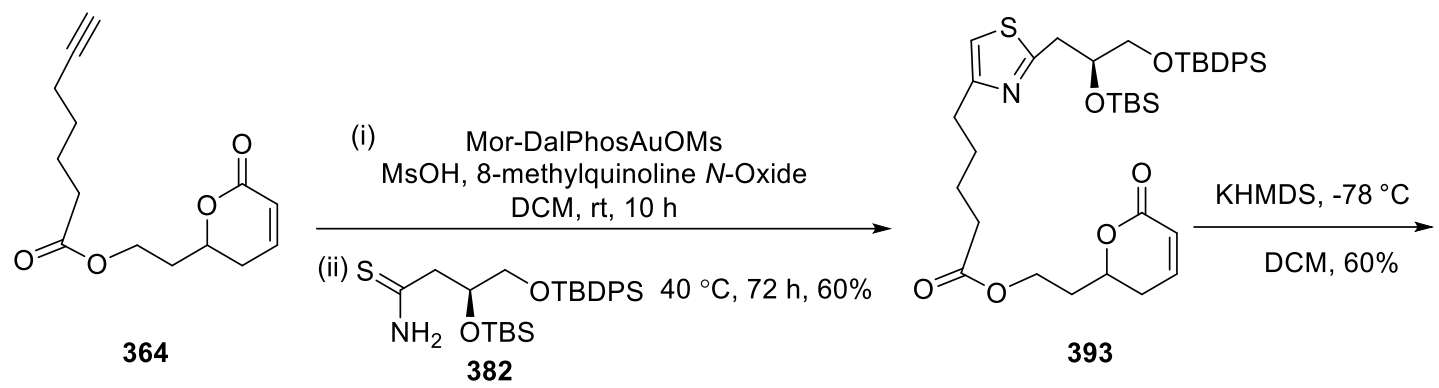

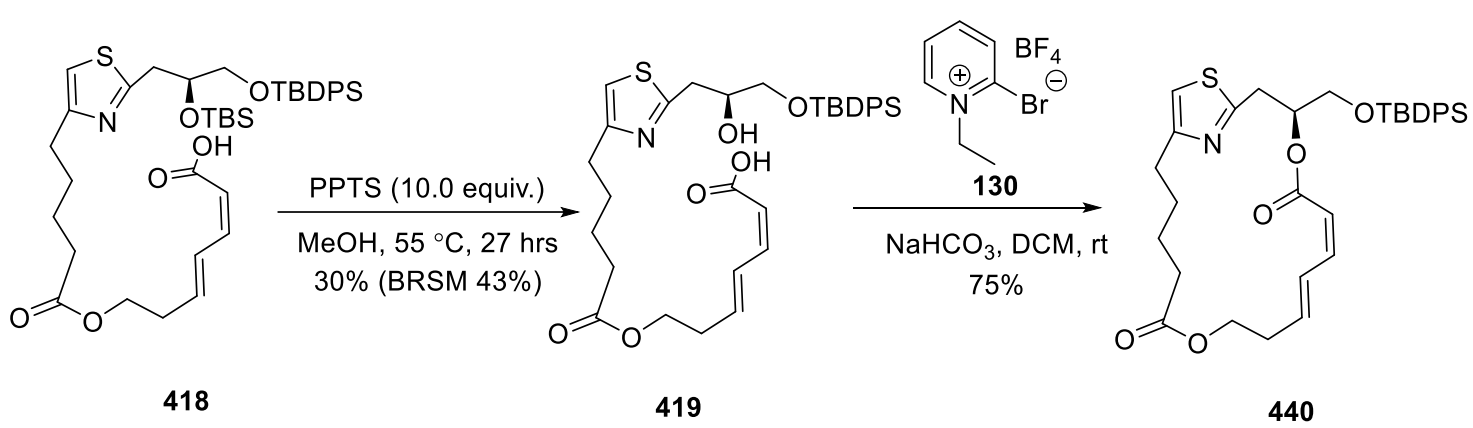

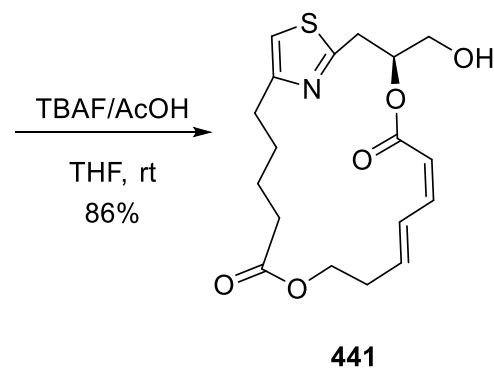

Scheme 5.6 Preparation of the thiazole-containing macrocycle $\mathbf{4 4 1}$ for the synthesis of analogue 184 .

C7-brominated thiazole 394, a useful intermediate for introducing modifications onto the C7-position, was also prepared successfully (Scheme 5.7). In order to prepare $\mathrm{CF}_{3}$ substituted analogue 185, a palladium-catalysed trifluoromethylation of 394 was carried out. However, none of the desired product $\mathbf{3 9 5}$ was obtained. This failure was possibly due to the choice of palladium catalyst or reagents. Therefore, the screening for the appropriate combination of palladium catalyst, ligand, and $\mathrm{CF}_{3}$ source would also require further investigation. 


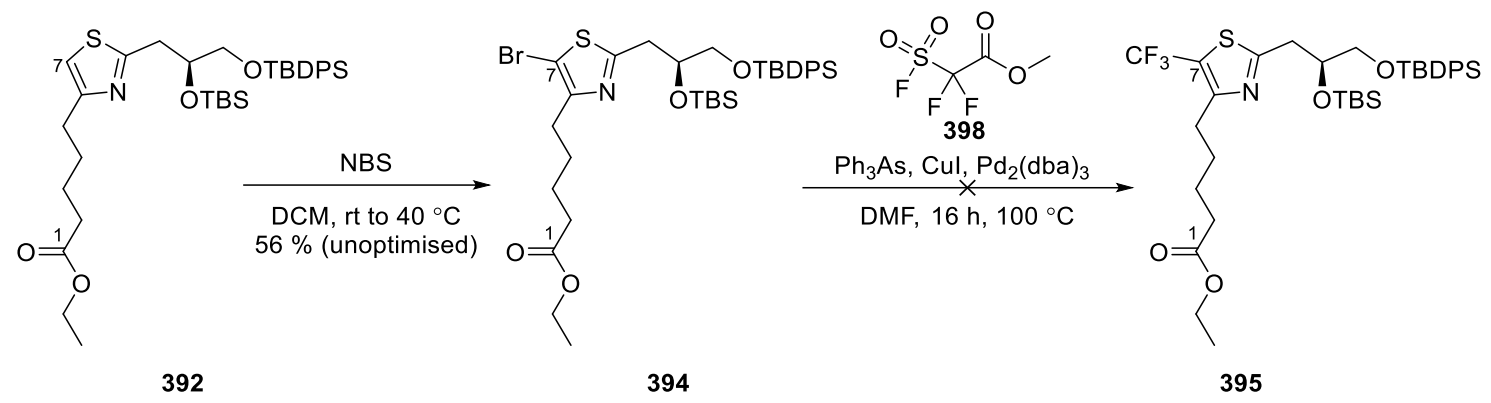

Scheme 5.7 Attempted preparation of $\mathrm{CF}_{3}$-substituted thiazole 395 .

The strategy for the coupling of the side chain fragment through Wittig olefination was first explored. Triphenylphosphonium salt $\mathbf{4 7 6}$ was successfully synthesised from hydroxyacetone $\mathbf{4 7 0}$ with a 56\% yield over 6 steps (Scheme 5.8). Aldehyde 322 was used as a model substrate to test the stereochemical outcome of the Wittig reaction with the phosphonium salt 476. Unfortunately, the model reaction provided a $62 / 38$ ratio of $E, E, E-$ trienoate $\mathbf{4 7 7}$ and $E, E, Z$-trienoate $\mathbf{4 7 8}$ products.

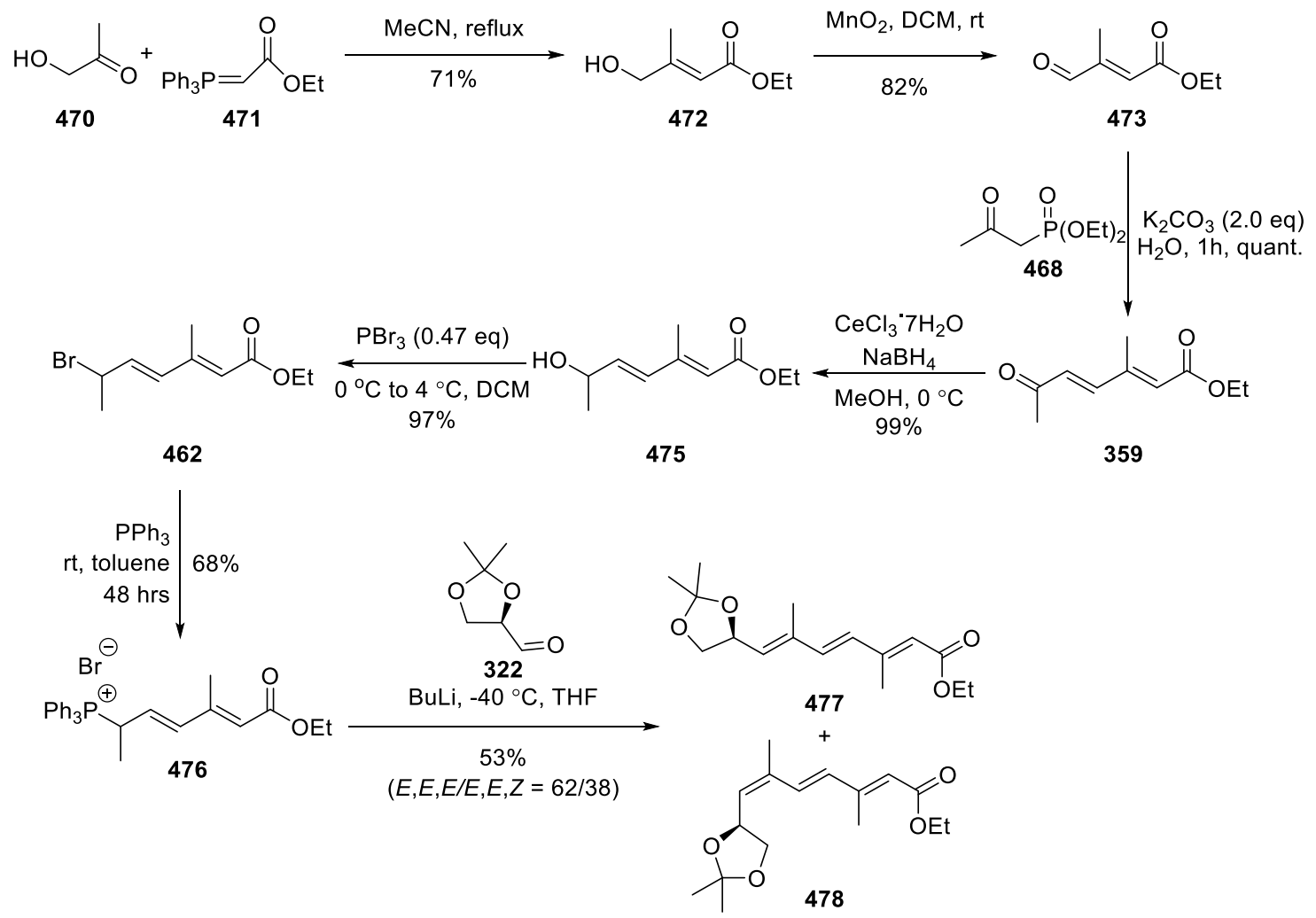

Scheme 5.8 Preparation of phosphonium salt $\mathbf{4 7 6}$ and trienoates 477 and $\mathbf{4 7 8}$.

This moderate $E$-selectivity prompted us to consider using HWE olefination or TBAF- 
mediated aldol condensation ${ }^{324}$ for the connection with the side chain. Therefore, phosphoryl aldehyde $\mathbf{4 8 3}$, an intermediate could serve in both strategies, was prepared successfully (Scheme 5.9). Since the TBAF-mediated aldol condensation has not yet been applied in synthetic work towards any natural products or their analogues, the preparation of the requisite enol silyl ether $\mathbf{4 8 5}$ was prioritised in order to test this route. Although NMR analysis suggested that the reaction of aldehyde $\mathbf{4 8 3}$ with KHMDS and TIPSCl did provide silyl ether product $\mathbf{4 8 5}$ in moderate conversion, full confirmation of the double bond configuration would require further NOE experiments on more material. After that, the coupling between the silyl ether $\mathbf{4 8 5}$ and the macrocycle core by the TBAF-mediated aldol condensation could be performed.

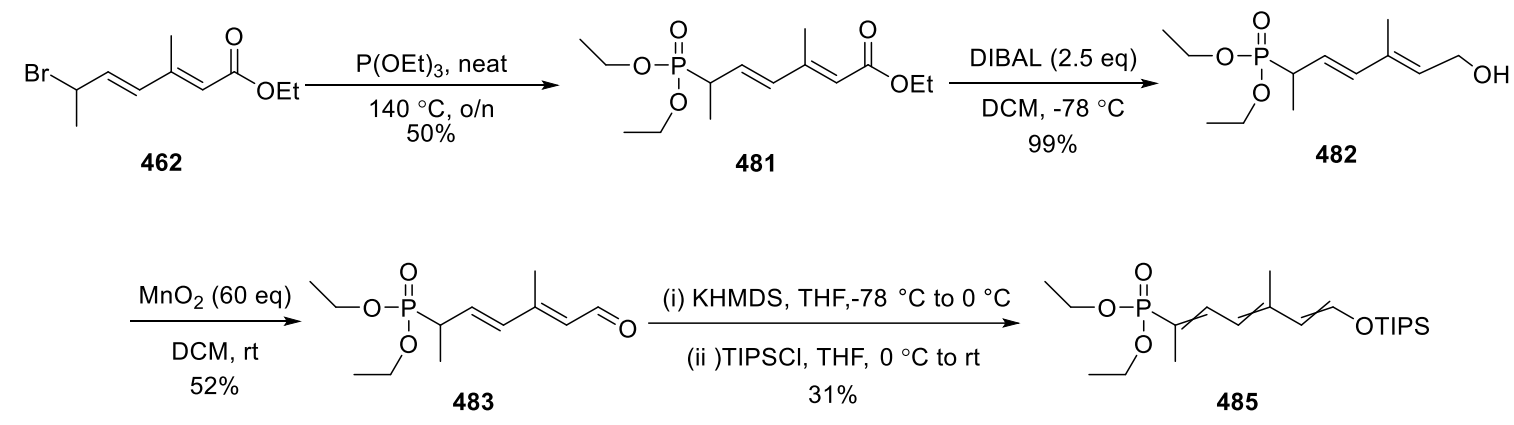

Scheme 5.9 Synthesis of phosphoryl silyl ether $\mathbf{4 8 5}$.

\subsubsection{Towards Synthesis of Analogue (S)-187}

As the synthesis of triazole analogue $(S)$-187 could employ the same lactone fragment 364 and side chain fragment 485 as with thiazole analogue 184, attention was focused on the preparation of the azide fragment for the construction of the triazole ring by coppercatalysed azide-alkyne cycloaddition. In our initial synthetic strategy, the coupling between the side chain fragment and the azide fragment was performed prior to the construction of the macrocycle. Therefore, the preparation of azide fragment $(R)-\mathbf{5 0 9}$ starting from (S)-solketal $\mathbf{5 0 2}$ was first performed (vide supra, Section 4.3). However, it was found out that aldehyde $(R)-\mathbf{5 0 9}$ degraded significantly when purified by silica gel flash chromatography or by distillation. This inability to purify the aldehyde $(R)-509$ led us to consider using the crude material for the model Wittig reaction with triphenylphosphonium salt 476. To our dismay, no desired product was observed, which was possibly due to the instability of the aldehyde in strongly basic conditions. The lack of success in the Wittig reaction, as well as the difficulty in the purification, prompted us 
to return to the alternative strategy, in which the macrocycle is prepared before attachment of the side chain, which could allow the collective synthesis of all the desired analogues. Macrocyclic alcohol 523 was then prepared from alkyne fragment 414 and azide fragment 506 in three steps (Scheme 5.10).

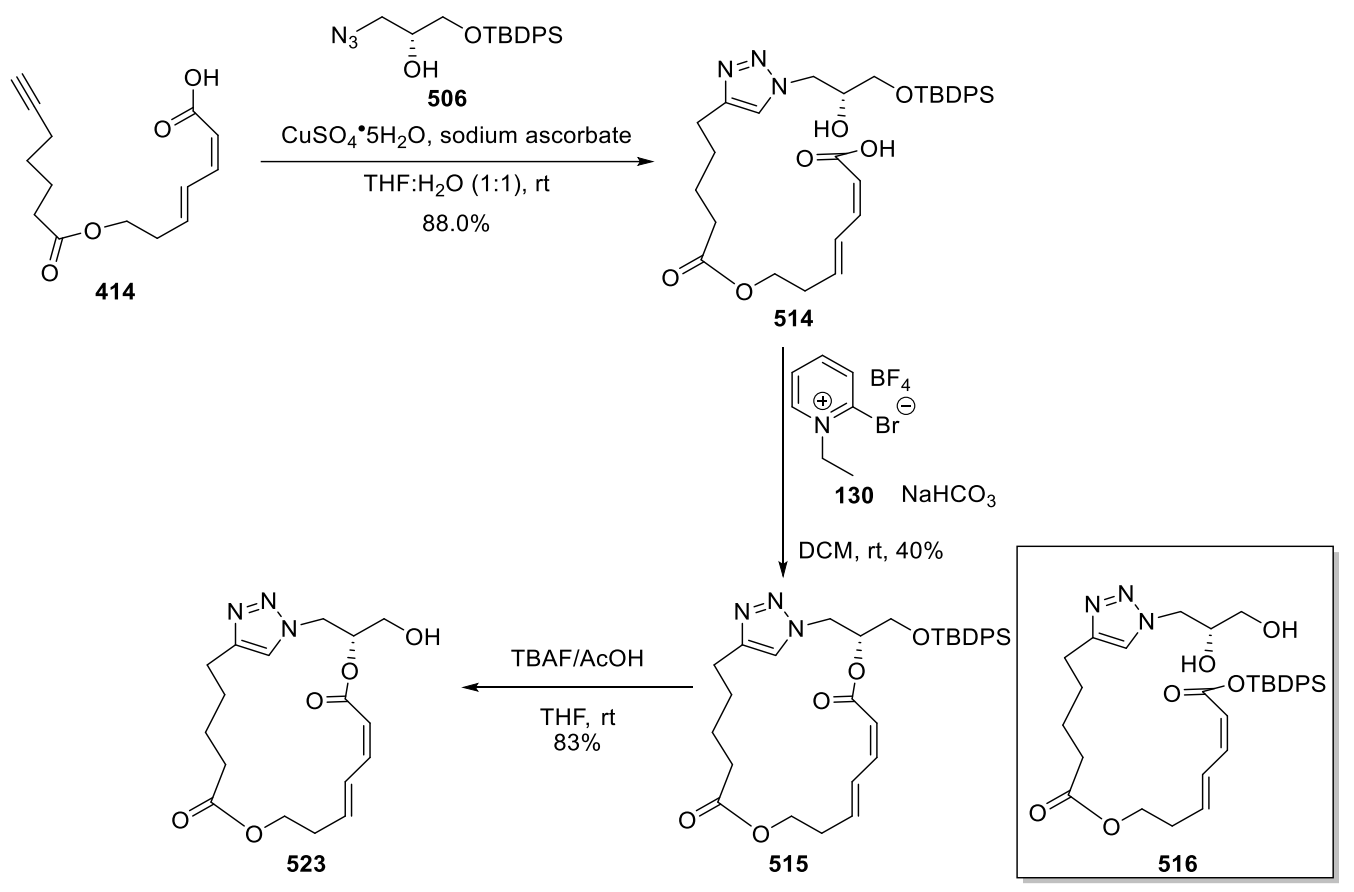

Scheme 5.10 Preparation of the triazole-containing macrocycle $\mathbf{5 1 5}$ for the synthesis of analogue $(S)-187$

An interesting by-product, silyl ester 516, was observed in the macrolactonisation step and its structure is consistent with the outcomes of NMR analysis and preparation of the acetate derivatives $E, E-522$ and $E, Z-522$ To synthesise triazole analogue $(S)-\mathbf{1 8 7}$, coupling of the aldehyde derived from $\mathbf{5 2 3}$ with the side chain fragment by TBAFmediated aldol condensation or HWE olefination would require further investigation.

\subsubsection{Closing Remarks}

The established synthetic route to the thiazole-containing macrocyclic alcohol $\mathbf{4 4 0}$ has 11 steps and the overall yield from the commercially available chemicals was $3.5 \%$. Compared to Fürstner and Zhuo's synthetic route for macrocyclic intermediate 154 with similar structures, although our route has a longer reaction sequence and a lower overall yield, the major strength of this route is the use of an alkyne fragment for the construction of the thiazole ring, which could allow us to conveniently harmonise synthetic routes to 
access different heterocyclic rings, like triazoles and oxazoles. This advantage has been demonstrated by the successful preparation of triazole-containing macrocycle $\mathbf{5 1 5}$.

The synthesis of the methylated side chain fragment, phosphoryl silyl ether $\mathbf{4 8 5}$, or an alternative derivative from phosphoryl aldehyde 483, was completed in 6 steps. ${ }^{\S \S \S \S}$ Compared to Pattenden's established step-wise strategy to connect the side chain, our synthetic route employs relatively benign reagents and conditions, and does not involve using chromium-mediated or tin-mediated coupling reactions This means that it could be more environmentally friendly and be easily scaled up. Also, our strategy could potentially allow us to attach the side chain as a whole fragment, making the synthesis of targeted analogues more convergent.

\subsection{Future Work}

The overall aim of this project was to synthesise thiazole analogues $183,184,185,186$ and triazole analgoues $(S)-\mathbf{1 8 7}$ and $(R)-\mathbf{1 8 7}$, which will help to examine the individual roles of the thiazole ring, the methyl groups on the side chain (at $\mathrm{C} 12$ and $\mathrm{C} 15$ ), and the methyl groups on the macrocycle in binding to the protein target of PatA. As the synthesis of thiazole-containing macrocycle 441, triazole-containing macrocycle 515 and the side chain fragment $\mathbf{4 8 5}$ have been achieved, future work will first focus on the synthesis of analogue 184 and $(S)-187$ and $(R)-187$.

\subsubsection{Synthesis of Analogues 184 and 187}

Thorough NOE experiments will be first performed in order to fully confirm that the double bonds of ilyl ether $\mathbf{4 8 5}$ are all $E$-configured. If silyl ether $\mathbf{4 8 5}$ is proven to have the desired E,E,E-configuration, macrocyclic alcohol 441 will be oxidised to provide the aldehyde 363. As Fürstner and Zhuo reported that the oxidation under optimised ParikhDoering conditions provided the macrocyclic aldehyde 155 in reproducibly good yield (vide supra, Section 1.4.2.2), ${ }^{128}$ the oxidation step will initially utilise their conditions. The validity of using TBAF-mediated aldol condensation between phosphoryl silyl ether 485 and aldehyde 363 to attach the side chain will then be tested (Scheme 5.11). If the aldol condensation is unsuccessful or the E-selectivity of the reaction is not satisfying, phosphoryl aldehyde 483 will be converted into phosphoryl acetal 524 or phosphoryl

$\S \S \S \S \S$ Taking into account that ethyl (E)-3-methyl-4-oxobut-2-enoate $\mathbf{4 7 3}$ is commercially available. 
imine 525 and the HWE protocol to couple the side chain would then be tested (Scheme 5.12). Given that the macrocyclic aldehyde $\mathbf{3 6 3}$ is possibly prone to epimerisation (vide supra, section 3.5.2.2), the use of relatively weaker non-ionic bases (see Figure 3.14 for examples) will be also investigated to minimise the risk of epimerisation. After suitable conditions for the coupling are found, analogue 184 will be produced by reductive amination of the aldehyde intermediate 371 using a mild boron reductant (e.g., tetramethylammonium triacetoxyborohydride). If neither of these attempts to couple the side chain fragment with the macrocyclic core provides the desired aldehyde 371, Pattenden's established step-wise strategy to connect the side chain fragment will be incorporated and the efficiency of the optimised conditions for the reaction sequence reported by Fürstner and Zhuo ${ }^{128}$ will also be examined. The synthesis of analogue $\mathbf{1 8 4}$ will be informative for the synthesis of the following other analogues.
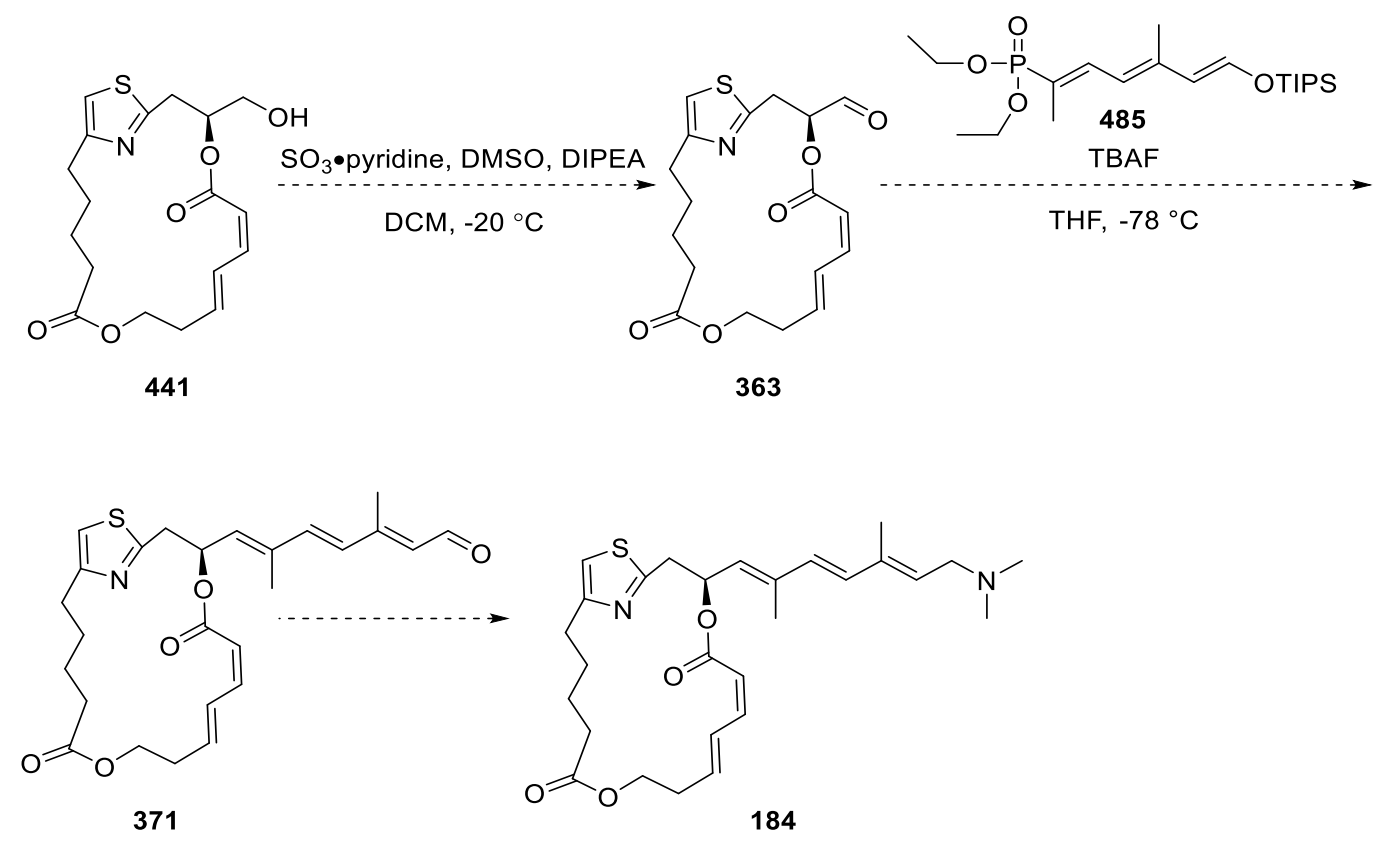

Scheme 5.11 Proposed synthetic route for analogue 184 by TBAF-mediated aldol condensation. 


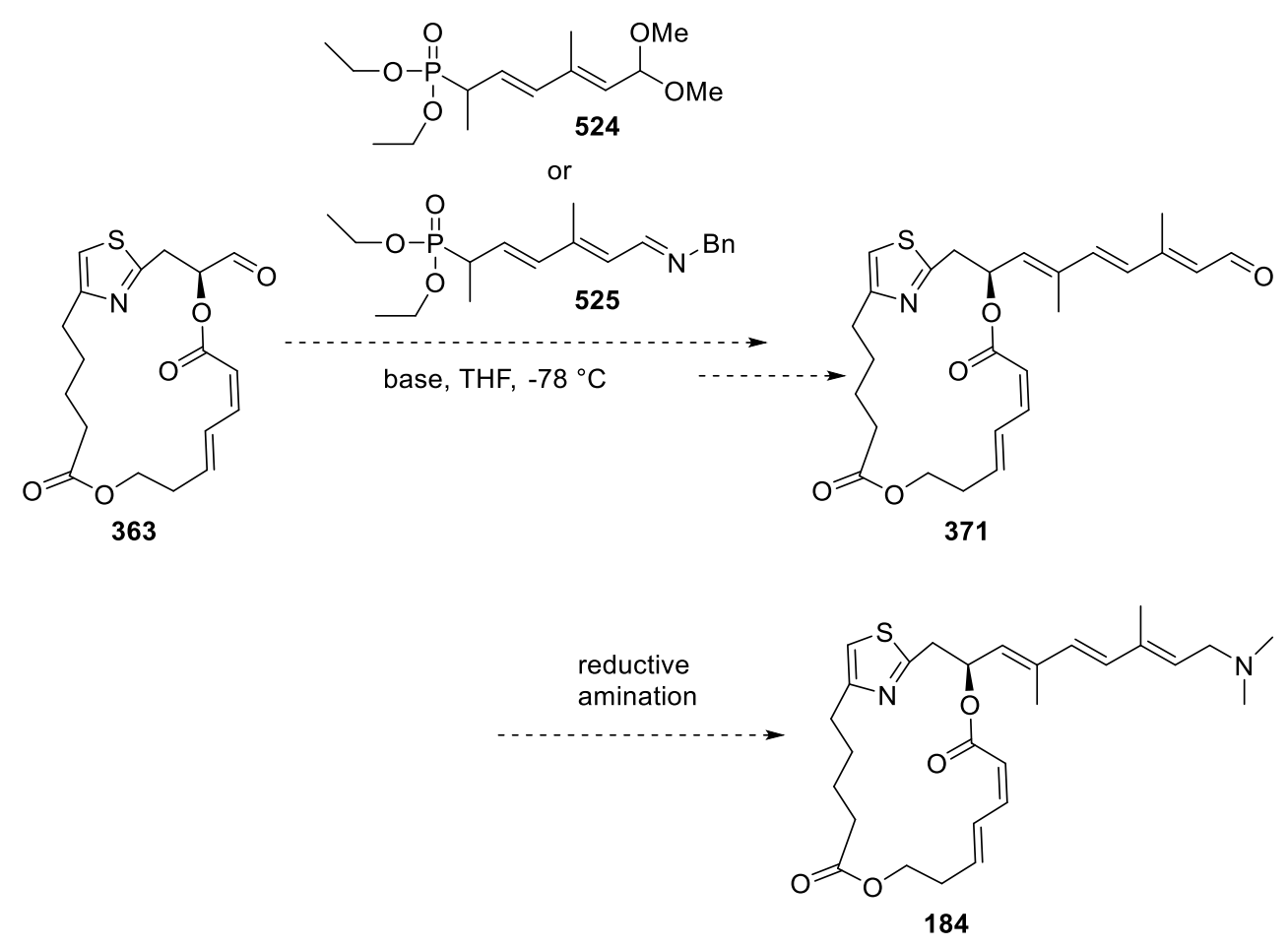

Scheme 5.12 Proposed synthetic route for analogue 184 by HWE olefination.

The synthesis of analogue $(S)$-187 from macrocyclic alcohol $\mathbf{5 2 3}$ could potentially follow a similar reaction sequence as analogue 184. However, optimisation for suitable conditions to achieve good results for each step may be required.

In terms of the synthesis of analogue $(R)-\mathbf{1 8 7}$, the key intermediate macrocylic alcohol $\mathbf{5 3 0}$ could be synthesised from $(S)$-solketal $\mathbf{5 0 2}$ following a similar synthetic route as $(S)$ 187 (Scheme 5.13), which has been discussed in Section 4.5. The attachment of the side chain-fragment could be achieved using exactly the same conditions as for analogue $(S)$ 187. 


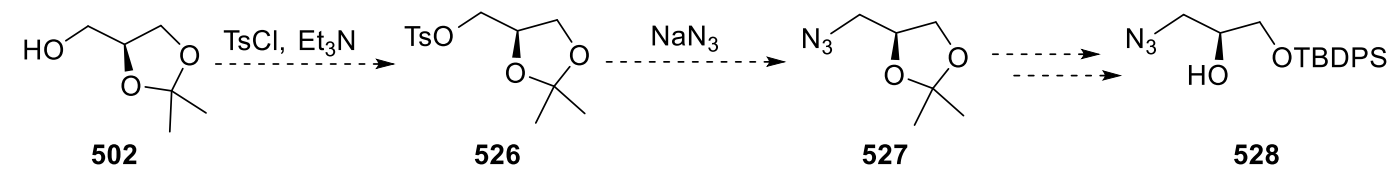

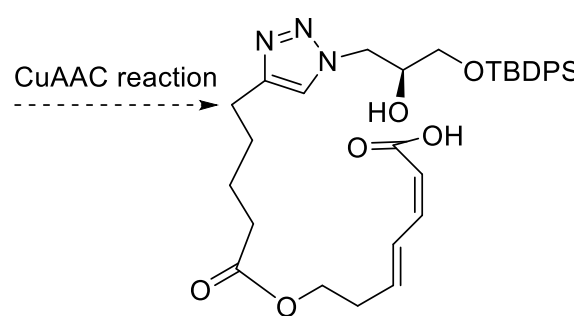

529

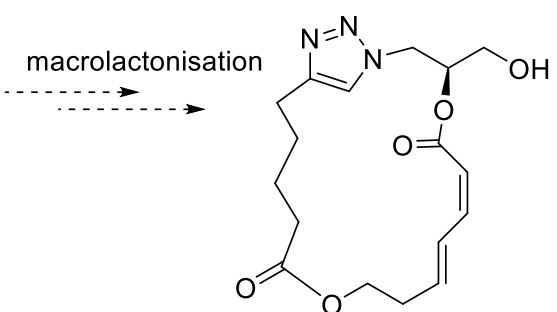

530

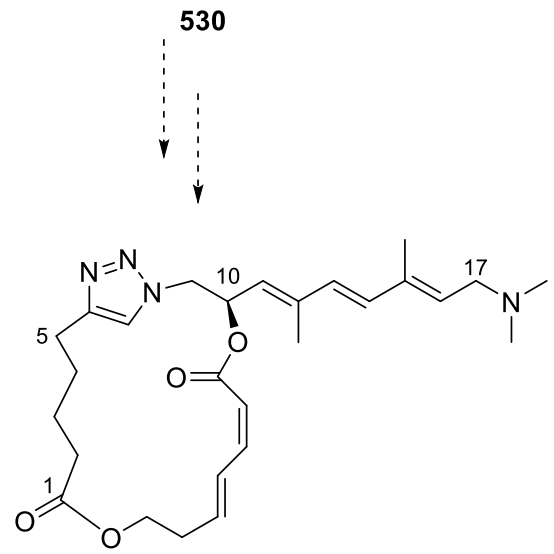

(R)-187

Scheme 5.13 Proposed synthetic route for the synthesis of analogue (R)-187 via macrocyclic alcohol $\mathbf{5 3 0}$ intermediate.

\subsubsection{Role of the Thiazole and Methyl Groups on the Macrocycle}

A comparison of the activities of analogues 184 and $(R)-\mathbf{1 8 7}$ will reveal whether the thiazole ring is critical for binding to the protein target eIF4A. If analogue $\mathbf{1 8 4}$ does indeed exhibit enhanced activity relative to $(R)-\mathbf{1 8 7}$, this would raise the idea that the protonability or the electron distribution of the heterocycle ring may play an important role in binding to eIF4A. This means modifications on the thiazole ring that could impact its basicity or electron distribution could potentially modulate the activity. Preparation and testing of C7-substituted analogues $\mathbf{1 8 5}$ and $\mathbf{1 8 6}$ will address this issue. To introduce the $\mathrm{CF}_{3}$ group to the thiazole, the palladium or copper-catalysed conditions for the trifluoromethylation of brominated or iodinated thiazole (394 or 531) will be further investigated. A methyl group will be incorporated into the molecule through a two-step protocol. The halogenated thiazole (394 or 531) will first undergo a lithium-halide 
exchange reaction and the resulting organolithium species can be trapped by methyl iodide. Analogues $\mathbf{1 8 5}$ and $\mathbf{1 8 6}$ will be synthesised from the intermediates $\mathbf{3 9 5}$ and 532, respectively (Scheme 5.14).

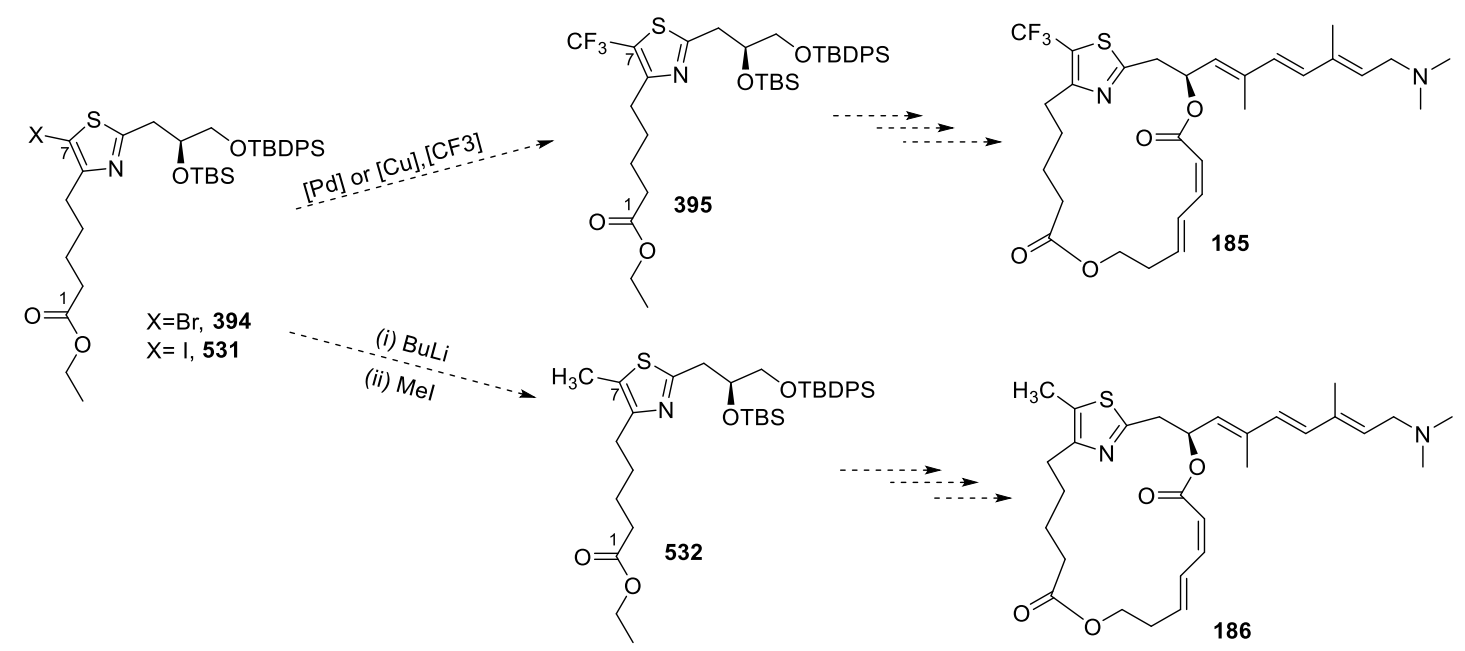

Scheme 5.14 Introduction of trifluoromethyl or methyl group to thiazole ring.

If the activity of analogue $\mathbf{1 8 4}$ is significantly reduced when compared to that of DMDA PatA, this would mean the omitted methyl groups on the macrocycle (at the C22 and C24 position) are required for potent activity. As the Z,E-dienoate was proposed to act as a Michael acceptor in binding to eIF4A, 86, 110, 131 the presence of modifications at the C22 position could modulate its Michael accepting ability. This could be further investigated by the preparation of analogues with a methyl group or a trifluoromethyl group at C22 (Figure 5.4). The influence of the methyl group at the $\mathrm{C} 24$ position in favouring an active conformation of the macrocycle required for the binding could be examined by the preparation of stereoisomers of an analogue with a C24 methyl group (Figure 5.5). Synthesis of an analogue with varying C24 substitution, including a gem-dimethyl group, could be also of interest. Under these circumstances, the potential to substitute the thiazole for the more synthetically facile triazole will then be reinvestigated. 


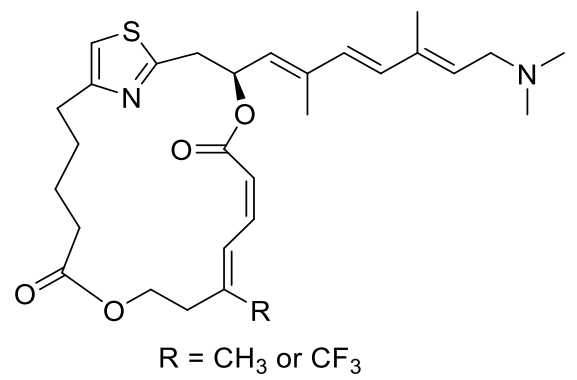

Figure 5.4 Pateamine analogues with modifications at the C22 position.

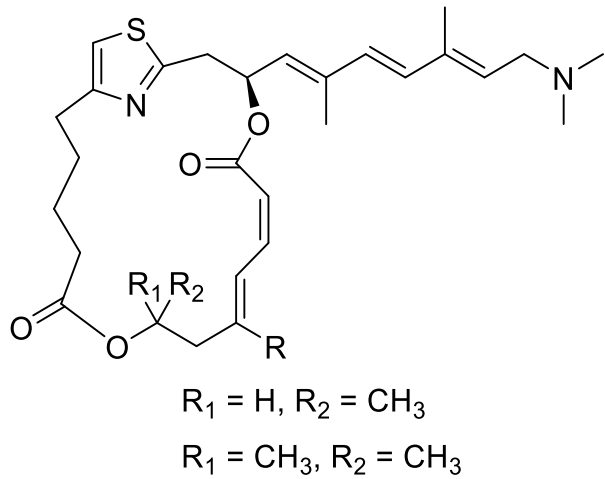

Figure 5.5 Pateamine analogues with modifications at the $\mathrm{C} 24$ position.

\subsubsection{Role of Methyl Groups on Side Chain}

A comparison of the activities of analogues $(R)-\mathbf{1 8 7}$ and 182 will potentially provide evidence on whether the reintroduction of the methyl groups on the side chain is advantageous. If analogue $(R)-\mathbf{1 8 7}$ does not display significantly improved activity relative to analogue 182, this would mean that the presence of methyl groups at the $\mathrm{C} 12$ and $\mathrm{C} 15$ position may play only a minor role in biological activity. The preparation of thiazole-containing analogue 183 will be informative in addressing this problem. As depicted in Scheme 5.15, the existing synthetic route for enal 297 (vide supra, Section 2.3.3) could be incorporated in the preparation. 

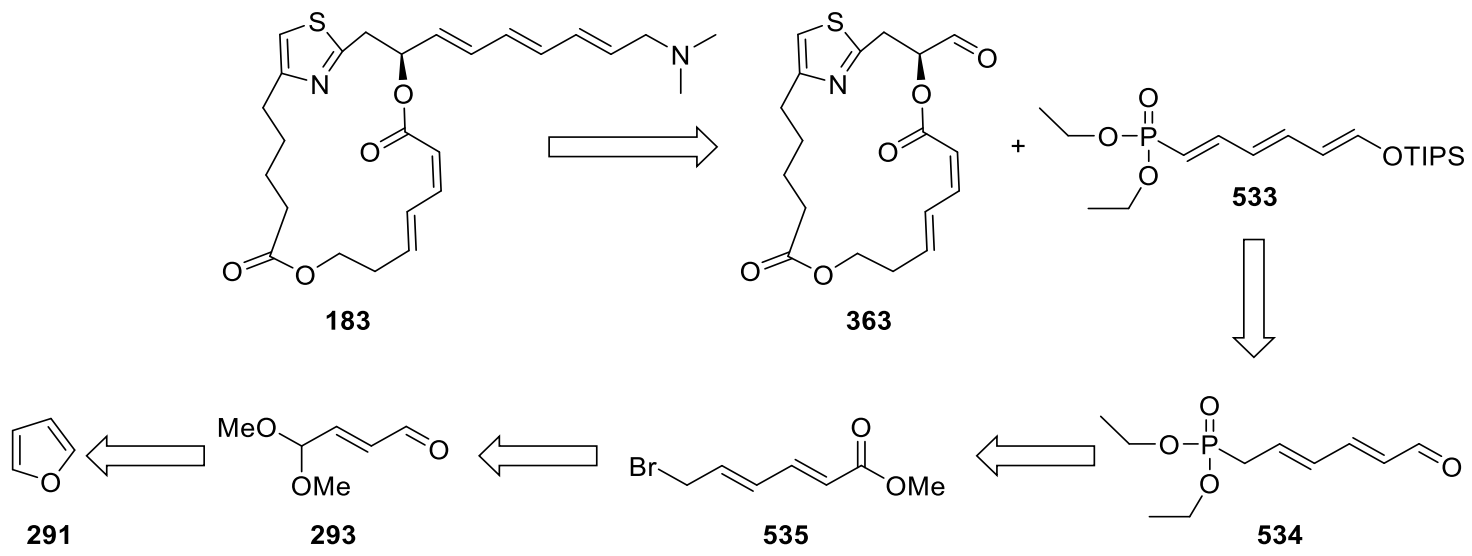

Scheme 5.15 Retrosynthetic analysis for analogue 184

\subsubsection{Importance of the $\mathrm{C}-10$ Configuration}

A comparison of the activities of analogues $(R)-\mathbf{1 8 7}$ and $(S)$-187 would give information about the role of the stereochemical configuration at $\mathrm{C} 10$ in binding to the protein target. If these two analogues show similar activities in tested cell lines, that would suggest that the configuration of C10 may not be crucial in biological activity. Preparation of a thiazole analogue with an $(R)$-configuration at the $\mathrm{C} 10$ position could provide more direct evidence (Figure 5.6). The synthetic route for this analogue will start from the four-carbon amide building block with the $(R)$-configuration, namely $(R)$-amide 339 and utilise the identical reaction sequence that used for the synthesis of analogue $\mathbf{1 8 4}$.

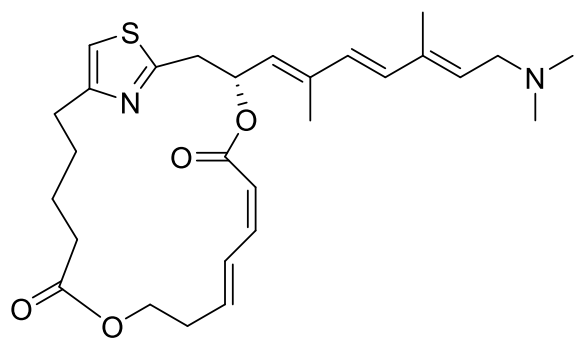

$(R)-184$

Figure 5.6 Pateamine analogue with $(R)$-configuration at $\mathrm{C} 10$ position.

These future works discussed above will provide further insights into the relationship between the structure and the activity of pateamine A, which would provide a solid foundation for the design of a new generation of analogues. 


\section{Chapter Six: Experimental}

\subsection{General Experimental Details}

Unless stated otherwise, all reactions were carried out in oven- or heat gun-dried glassware, using anhydrous solvents under argon atmosphere and standard syringe techniques. THF, DCM, Et $2 \mathrm{O}$, and toluene were purified using Innovative Technology's PureSolv system. Other solvents were purified by distillation over the following desiccating reagents and were transferred under argon: acetone (boron trioxide), isopropanol (dried and stored over MS $4 \AA$ ); dimethyl sulfoxide (DMSO), dichloroethane (DCE), chlorobenzene, acetonitrile, and 1,2-dimethoxyethane (DME) were distilled over calcium hydride. Anhydrous (99.8\%) N,N-dimethylformamide (DMF) containing MS 4 $\AA$ was purchased from Sigma-Aldrich.

Unless stated otherwise, all commercially available compounds (Alfa Aesar, BDH Chemicals, Sigma-Aldrich, AKSci, Acros Organics) were used as received. Triethylamine $\left(\mathrm{Et}_{3} \mathrm{~N}\right)$ and 2,2-dimethoxypropane were purified by distillation over calcium hydride. Furan was purified by distillation over sodium. Anhydrous paratoluenesulfonic acid was obtained by recrystallisation from ethanol and water and azeotropic desiccation with toluene. Lawesson's reagent was purified by washing with anhydrous $\mathrm{Et}_{2} \mathrm{O}$ and anhydrous DCM and recrystallisation from anhydrous toluene. Trichloroacetic acid was purified by recrystallisation from hexane. Organic solvents were removed by rotary evaporation with water bath temperature between $40{ }^{\circ} \mathrm{C}$ and $50{ }^{\circ} \mathrm{C}$ unless otherwise stated.

The reaction progress was monitored using TLC plates (Macherey-Nagel pre-coated plates (POLYGRAM®SIL/UV254)) and visualised by UV fluorescence quenching (254 $\mathrm{nm}$ ), in combination with phosphomolybdic acid (PMA), cerium ammonium molybdate and potassium permanganate $\left(\mathrm{KMNO}_{4}\right)$ dip. Purification of products via flash chromatography was conducted using a column filled with silica gel 60 (220 - 240 mesh) as the matrix, obtained from Pure Science Ltd, with solvent systems as indicated. Celite used for filtration was obtained from Pure Science Ltd. ${ }^{1} \mathrm{H}$ and ${ }^{13} \mathrm{C}$ NMR spectra were recorded on a Varian Unity Inova 500 spectrometer or a JEOL 500 spectrometer (operating at $500 \mathrm{MHz}$ for ${ }^{1} \mathrm{H} \mathrm{NMR}$ and $125 \mathrm{MHz}$ for ${ }^{13} \mathrm{C}$ NMR), or on a Varian Unity 
Inova 600 spectrometer or a JEOL 600 spectrometer (operating at $600 \mathrm{MHz}$ for ${ }^{1} \mathrm{H} \mathrm{NMR}$ and $150 \mathrm{MHz}$ for $\left.{ }^{13} \mathrm{C} \mathrm{NMR}\right)$ in the solvents indicated. All chemical shifts $(\delta)$ are given in ppm relative to tetramethylsilane (TMS), coupling constants $(J)$ in $\mathrm{Hz}$ and multiplicity (s = singlet, $\mathrm{d}=$ doublet, $\mathrm{t}=$ triplet, $\mathrm{q}=$ quartet, quin = quintet, $\mathrm{m}=$ multiplet, $\mathrm{br}=$ broad). The solvent signals were used as references and the chemical shifts converted to the TMS scale $\left(\mathrm{CDCl}_{3}: \delta \mathrm{C}=77.0 \mathrm{ppm}\right.$; residual $\mathrm{CHCl}_{3}$ in $\mathrm{CDCl}_{3}: \delta \mathrm{H}=7.26 \mathrm{ppm} ; \mathrm{C}_{6} \mathrm{D}_{6}: \delta \mathrm{C}=$ 128.06 ppm; residual $\mathrm{C}_{6} \mathrm{D}_{5} \mathrm{H}: \delta \mathrm{H}=7.16 \mathrm{ppm} ;\left(\mathrm{CD}_{3}\right)_{2} \mathrm{CO}: \delta \mathrm{C}=29.84,206.26 \mathrm{ppm}$; residual $\left.\left(\mathrm{CD}_{3}\right) \mathrm{CD}_{2} \mathrm{HCO}: \delta \mathrm{H}=2.05 \mathrm{ppm}\right)$. Melting points were determined using a Gallenkamp or Digimelt SRS melting point apparatus. Optical rotations were measured at the sodium D line on an Autopol II polarimeter from Rudolph Research Analytical and are quoted as specific rotation based on the equation $[\alpha]_{D}{ }^{\mathrm{T}}=\alpha \times 100 / \mathrm{c} \times 1$ (where $\alpha$ is rotation in degrees, $\mathrm{c}$ is concentration in $\mathrm{g} / 100 \mathrm{~mL}, 1=1 \mathrm{dm}$ ). Infrared spectra were obtained on a Bruker Alpha Platinum-ATR. High-resolution mass spectrometry was conducted on a 6530 Accurate Mass Q-TOF LC/MS instrument (Agilent Technologies). 


\subsection{Experimental for Chapter Two}

\subsubsection{Preparation of Thioamide 223}

Preparation of 2-(oxiran-2-yl)acetonitrile, 209

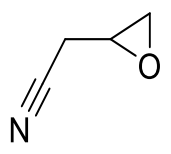

To a solution of nitrile $\mathbf{2 0 8}$ (5.0 g, $60.0 \mathrm{mmol}, 1.0$ equiv) in DCM (50 mL) was added 3chloroperbenzoic acid (13.4 g, purity $77 \%, 60.0 \mathrm{mmol}, 1.0$ equiv.) at room temperature. The reaction mixture was stirred at room temperature while 3-chloroperbenzoic acid (500 $\mathrm{mg}, 2.2 \mathrm{mmol}, 0.037$ equiv.) was added to the reaction mixture each day for a total of 8 days. A saturated aqueous solution of $\mathrm{NaHSO}_{3}(30 \mathrm{~mL})$ was added to reduce the unreacted 3-chloroperbenzoic acid and the resulting mixture was stirred vigorously for 20 minutes. Then the organic layers were separated, washed with saturated aqueous $\mathrm{NaHCO}_{3}(10 \times$ $50 \mathrm{~mL}$ ), dried over anhydrous $\mathrm{Na}_{2} \mathrm{SO} 4$, filtered, and evaporated under reduced pressure. The residue was then purified by silica gel flash chromatography (pet. ether/EtOAc $=2: 1$ ) to afford the title compound as a colourless oil (1.0 g, 20\%). $\mathbf{R}_{f}$ : 0.10 (pet. ether/EtOAc = 10:1). ${ }^{1} \mathbf{H}$ NMR (500 MHz, $\left.\mathrm{CDCl}_{3}\right), \delta: 3.15-3.07$ (m, $\left.1 \mathrm{H}\right), 2.77$ (t, $\left.1 \mathrm{H}, J=4.3 \mathrm{~Hz}\right)$, 2.70-2.56 (m, $3 \mathrm{H}) .{ }^{13} \mathrm{C}$ NMR (125 MHz, $\left.\mathrm{CDCl}_{3}\right)$, $\delta: 115.4,46.6,46.1,21.1$. These characterisation data match those reported in the literature. ${ }^{170}$

\section{Preparation of $(S)$-(2,2-dimethyl-1,3-dioxolan-4-yl)methyl 4-}

methylbenzenesulfonate, 214

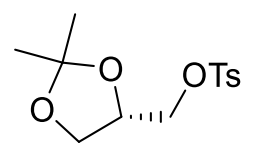

To a solution of (2S)-(+)-glycidyl tosylate 213 (3.18 g, $13.9 \mathrm{mmol}, 1$ equiv.) in dry acetone $(14 \mathrm{~mL})$ was added aluminium chloride $(58.0 \mathrm{mg}, 0.435 \mathrm{mmol}, 0.03$ equiv.), and the solution was stirred at room temperature for 1 hour. The reaction mixture was then cooled to $2{ }^{\circ} \mathrm{C}$ and added $30 \mathrm{~mL}$ saturated $\mathrm{NaHCO}_{3}$ aqueous solution slowly. The resulting mixture was then extracted with ethyl acetate $(3 \times 50 \mathrm{~mL})$. The combined organic fractions were then dried over anhydrous $\mathrm{Na}_{2} \mathrm{SO}_{4}$, filtered, and then concentrated under reduced pressure. The crude material was purified by silica gel flash 
chromatography (pet. ether/EtOAc $=5: 1)$ to provide the title compound $(7.00 \mathrm{~g}, 92 \%)$ as a colorless oil. $\mathbf{R}_{f}: 0.50$ (pet. ether/EtOAc $=2: 1$ ). Melting point: $69-72{ }^{\circ} \mathrm{C} .{ }^{1} \mathbf{H}$ NMR $\left(500 \mathrm{MHz}, \mathrm{CDCl}_{3}\right): \delta 7.83-7.78(\mathrm{~m}, 2 \mathrm{H}), 7.40-7.33(\mathrm{~m}, 2 \mathrm{H}), 4.30-4.24(\mathrm{~m}, 1 \mathrm{H}), 4.06$ - $4.00(\mathrm{~m}, 2 \mathrm{H}), 3.97$ (dd, $J=10.1,6.2 \mathrm{~Hz}, 1 \mathrm{H}), 3.77$ (dd, $J=8.8,5.1 \mathrm{~Hz}, 1 \mathrm{H}), 2.45$ (s, 3H), 1.34 (s, 3H), 1.31 (s, 3H). ${ }^{13} \mathbf{C}$ NMR (125 MHz, $\left.\mathrm{CDCl}_{3}\right): \delta$ 145.1, 132.6, 129.9, 128.0, 110.1, 72.9, 69.5, 66.2, 26.7, 25.2, 21.7. FTIR (thin film) $v 2987,1598,1495,1455,1359$, 1257, 1213, 1189, 1175, 1095, 1054, 974, 788, 664, $554 \mathrm{~cm}^{-1}$. HRMS (ESI): Calculated for $\mathrm{C}_{13} \mathrm{H}_{19} \mathrm{O}_{5} \mathrm{~S}^{+}[\mathrm{M}+\mathrm{H}]^{+}$287.0948; found 287.0951. $[\alpha]_{D}{ }^{20}+3.0(c=1.00$, EtOH $)$ (literature $\left.[\alpha]_{D}^{20}+4.5(c 1.00 \mathrm{EtOH})\right)$. These characterisation data match those reported in the literature. ${ }^{340}$

\section{Preparation of $(S)-5-(((2,2-d i m e t h y l-1,3-d i o x o l a n-4-y l) m e t h y l) t h i o)-1-p h e n y l-1 H$ -} tetrazole, 215

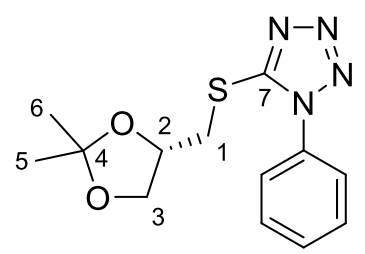

To a solution of tosylate 214 (8.15 g, 28.5 mmol, 1 equiv.) and 1-phenyl-1H-tetrazole-5thiol 210 (15.66 g, $85.5 \mathrm{mmol}, 3.0$ equiv.) in acetone $(250 \mathrm{~mL})$ was added $\mathrm{K}_{2} \mathrm{CO}_{3}$ (19.7 g, $0.142 \mathrm{mmol}, 5.0$ equiv.) at room temperature. The reaction mixture was refluxed for 16 hours and then evaporated to remove solvent under vacuum. The residue was partitioned between ethyl acetate and water. The aqueous phase was extracted with diethyl ether $(4 \times 100 \mathrm{~mL})$. The combined organic phase was washed with brine, dried over anhydrous $\mathrm{Na}_{2} \mathrm{SO}_{4}$, filtered and then concentrated under reduced pressure. The crude material was purified by silica gel flash chromatography (pet. ether/EtOAc $=5: 1$ ) to provide the title compound $(8.14 \mathrm{~g}, 98 \%)$. $\mathbf{R}_{f}$. 0.48 (pet. ether/EtOAc $=2: 1$ ). ${ }^{1} \mathbf{H}$ NMR $\left(500 \mathrm{MHz}, \mathrm{CDCl}_{3}\right): \delta 7.62-7.49(\mathrm{~m}, 5 \mathrm{H}), 4.61-4.51(\mathrm{~m}, 1 \mathrm{H}, \mathrm{H}-2), 4.20-4.13(\mathrm{~m}, 1 \mathrm{H}$, H-3a), 3.81 (dd, $J=8.6,5.6 \mathrm{~Hz}, 1 \mathrm{H}, \mathrm{H}-3 \mathrm{~b}), 3.67$ (dd, $J=13.6,4.5 \mathrm{~Hz}, 1 \mathrm{H}, \mathrm{H}-1 \mathrm{a}), 3.51$ (dd, $J=13.5,7.1 \mathrm{~Hz}, 1 \mathrm{H}, \mathrm{H}-1 \mathrm{~b}), 1.44$ (s, 3H, H-5 or H-6), 1.35 (s, 3H, H-6 or H-5). ${ }^{13} \mathrm{C}$ NMR (125 MHz, $\left.\mathrm{CDCl}_{3}\right): \delta 153.9$ (C-7), 133.5, 130.1, 129.8, 123.7, 110.0 (C-4), 73.7 (C-2), 68.3 (C-3), 36.6 (C-1), 26.8 (C-5 or C-6), 25.2 (C-6 or C-5). FTIR (thin film) $v$ 2981, 2933, 2883, 1596, 1501, 1460, 1389, 1377, 1366, 1280, 1245, 1213, 1190, 1175, 1150, 1090, 1081, 1073, 1051, 1015, 1000, 976, 960, 918, 869, 818, 785, 766, 736, 710, 
694, 685, 555, 510, 462, 447 $\mathrm{cm}^{-1}$. HRMS (ESI): Calculated for $\mathrm{C}_{13} \mathrm{H}_{17} \mathrm{~N}_{4} \mathrm{O}_{2} \mathrm{~S}^{+}[\mathrm{M}+\mathrm{H}]^{+}$ 293.1067; found 293.1070. $[\alpha]_{D}^{25}-360$ ( $\left.c=0.375, \mathrm{DCM}\right)$.

Preparation of (S)-3-((1-phenyl-1H-tetrazol-5-yl)thio)propane-1,2-diol, 216<smiles>OC(O)[C@H](O)Sc1nnnn1-c1ccccc1</smiles>

To a solution of acetonide 215 (5.12 g, $17.5 \mathrm{mmol}, 1.0$ equiv.) in methanol (67 mL) was added acetyl chloride $(0.37 \mathrm{~mL}, 5.25 \mathrm{mmol}, 0.3$ equiv. $)$ at $2{ }^{\circ} \mathrm{C}$. Then the reaction mixture was stirred at room temperature for 24 hours. Then the reaction mixture was evaporated under reduced vacuum and the residue was purified by a short silica plug (pet. ether/EtOAc $=1: 2)$ to provide the title compound $(4.4 \mathrm{~g}, 99.5 \%)$ as a colourless oil. $\mathbf{R}_{f}$ : 0.13 (pet. ether/EtOAc = 1:2). ${ }^{1} \mathbf{H}$ NMR $\left(500 \mathrm{MHz}, \mathrm{CDCl}_{3}\right): \delta 7.65-7.52(\mathrm{~m}, 5 \mathrm{H}), 4.16$ - 4.07 (m, 1H, H-2), 3.81 - 3.68 (m, 2H, H-1), 3.60 (dd, J=14.5, 5.6 Hz, 1H, H-3a), 3.47 (dd, $J=14.5,6.1 \mathrm{~Hz}, 1 \mathrm{H}, \mathrm{H}-3 \mathrm{~b}), 3.27(\mathrm{~d}, J=6.1 \mathrm{~Hz}, 1 \mathrm{H}, \mathrm{OH}), 3.01(\mathrm{t}, J=6.5 \mathrm{~Hz}, 1 \mathrm{H}$, $\mathrm{OH}) .{ }^{13} \mathbf{C}$ NMR $\left(125 \mathrm{MHz}, \mathrm{CDCl}_{3}\right): \delta 155.0(\mathrm{C}-4), 130.5,129.9,123.9,71.1(\mathrm{C}-2), 63.9$ (C-1), 35.9 (C-3). FTIR (thin film) v 3432, 3396, 3069, 2935, 2882, 1596, 1499, 1461, 1410, 1388, 1318, 1284, 1243, 1176, 1093, 1074, 1038, 1016, 761, 694, $549 \mathrm{~cm}^{-1}$. HRMS (ESI): Calculated for $\mathrm{C}_{10} \mathrm{H}_{13} \mathrm{~N}_{4} \mathrm{O}_{2} \mathrm{~S}^{+}[\mathrm{M}+\mathrm{H}]^{+} 253.0754$; found 253.0756. $[\alpha]_{D}^{23}+3.7(c=$ $\left.1.16, \mathrm{CHCl}_{3}\right)$.

Preparation of $(S)$-2-hydroxy-3-((1-phenyl-1H-tetrazol-5-yl)thio)propyl 4methylbenzenesulfonate, 217

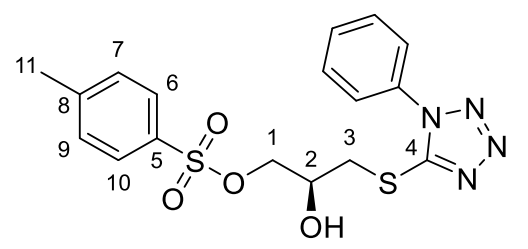

To a solution of diol 216 (4.53 g, 17.9 mmol, 1.0 equiv.) in anhydrous DCM (150 mL) were added dibutyltin oxide ( $0.918 \mathrm{~g}, 3.59 \mathrm{mmol}, 0.2$ equiv.) at room temperature. The resulting mixture was stirred at room temperature for 1 hour. Triethylamine $(2.50 \mathrm{~mL}$, 17.9 mmol, 1.0 equiv.) was added dropwise to the reaction mixture. Then 4- 
toluenesulfonyl chloride (3.5 g, $17.9 \mathrm{mmol}, 1.0$ equiv.) in anhydrous DCM (50 mL) was added to the reaction mixture by a syringe pump over $1 \mathrm{hr}$. The reaction mixture was stirred for 8 hours. The reaction was added water $(35 \mathrm{~mL})$ and the aqueous phase was extracted with ethyl acetate $(3 \times 50 \mathrm{~mL})$. The combined organic phase was washed with brine, dried over anhydrous $\mathrm{Na}_{2} \mathrm{SO}_{4}$, filtered and evaporated under vacuum. The crude material was purified by silica gel flash chromatography (pet. ether/EtOAc $=2: 1$ ) to provide the title compound $(6.76 \mathrm{~g}, 93 \%)$ as a foamy gel. $\mathbf{R}_{\boldsymbol{f}}: 0.63$ (pet. ether/EtOAc $=$ 1:2). ${ }^{1} \mathbf{H}$ NMR (500 MHz, $\left.\mathrm{CDCl}_{3}\right) \delta 7.84-7.78$ (m, 2H, H-6, H-10), $7.62-7.53(\mathrm{~m}, 5 \mathrm{H})$, $7.36(\mathrm{~d}, J=8.0 \mathrm{~Hz}, 2 \mathrm{H}, \mathrm{H}-7, \mathrm{H}-9), 4.36-4.29$ (m, 1H, H-2), 4.14 (dd, J = 5.4, $1.9 \mathrm{~Hz}$, 2H, H-1), 3.59 (dd, $J=14.6,3.6 \mathrm{~Hz}, 1 \mathrm{H}, \mathrm{H}-3 \mathrm{a}), 3.44$ (dd, $J=14.6,7.0 \mathrm{~Hz}, 1 \mathrm{H}, \mathrm{H}-3 \mathrm{~b}$ ), 2.45 (s, 3H, H-11). ${ }^{13} \mathrm{C}$ NMR (125 MHz, $\mathrm{CDCl}_{3}$ ) $\delta 154.4$ (C-4), 145.3 (C-5), 133.3, 132.3 (C-8), 130.4, 130.0 (C-7, C-9), 129.9, 128.0 (C-6, C-10), 123.8, 71.1 (C-2), 68.6 (C-1), 36.1 (C-3), 21.7 (C-11). FTIR (thin film) v 3255, 3065, 2922, 1595, 1501, 1448, 1418, 1384, 1331, 1289, 1213, 1172, 1121, 1089, 1064, 1033, 1010, 816, 762, 712, 681, 608, $567 \mathrm{~cm}^{-1}$. HRMS (ESI): Calculated for $\mathrm{C}_{17} \mathrm{H}_{19} \mathrm{~N}_{4} \mathrm{O}_{4} \mathrm{~S}_{2}{ }^{+}[\mathrm{M}+\mathrm{H}]^{+}$407.0842; found 407.0839. $[\alpha]_{D}^{23}-46(c=0.385, \mathrm{DCM})$.

Preparation of $(R)-1-((1-p h e n y l-1 H$-tetrazol-5-yl)thio)-3-(tosyloxy)propan-2-yl $(S)$ 3,3,3-trifluoro-2-methoxy-2-phenylpropanoate, (S)-MTPA-219

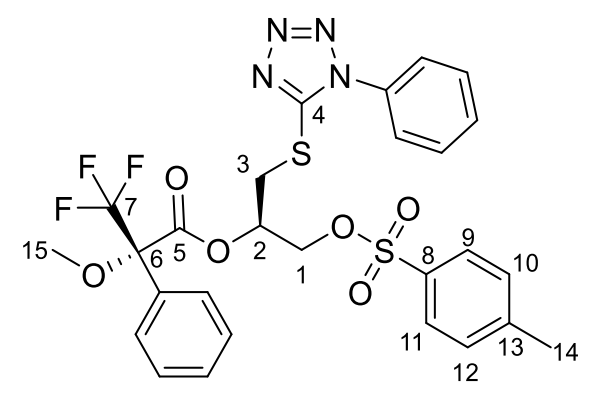

To a solution of tosylate 217 (23.2 mg, $0.057 \mathrm{mmol}, 1.0$ equiv.) in anhydrous DCM (3.0 $\mathrm{mL}$ ) was added sequentially $(S)-(+)-\alpha$-methoxy- $\alpha$-trifluoromethylphenylacetic acid (46.0 mg, 0.182 mmol, 3.2 equiv.), $N, N$-dicyclohexylcarbodiimide (112.3 mg, 0. mmol, 8.8 equiv.) and 4-dimethylaminopyridine (24.2 $\mathrm{mg}, 0.198 \mathrm{mmol}, 3.0$ equiv.). The reaction mixture was stirred at room temperature for 5 days. $2 \mathrm{~mL}$ waster was added the reaction mixture. Then the aqueous phase was extracted with ethyl acetate $(3 \times 5 \mathrm{~mL})$. The combined organic phase was washed with brine, dried over anhydrous $\mathrm{Na}_{2} \mathrm{SO}_{4}$, filtered and evaporated under vacuum. The crude material was purified by silica gel flash 
chromatography (pet. ether/EtOAc $=3: 1)$ to provide the title compound $(25.7 \mathrm{mg}, 65 \%)$ as a colourless oil. $\mathbf{R}_{f}$ : 0.77 (pet. ether/EtOAc = 1:1). ${ }^{1} \mathbf{H} \mathbf{~ N M R}\left(500 \mathrm{MHz}, \mathrm{CDCl}_{3}\right.$, represents peaks from major diastereomer) $\delta 7.73-7.69(\mathrm{~m}, 2 \mathrm{H}, \mathrm{H}-9, \mathrm{H}-11), 7.59-7.54$ (m, 3H), $7.52-7.48(\mathrm{~m}, 2 \mathrm{H}), 7.48-7.45(\mathrm{~m}, 2 \mathrm{H}), 7.42-7.37(\mathrm{~m}, 1 \mathrm{H}), 7.37-7.31$ (m, 4H, overlapped, H-10, H-12), $5.69-5.63(\mathrm{~m}, 1 \mathrm{H}, \mathrm{H}-2), 4.29$ (dd, $J=11.2,3.2 \mathrm{~Hz}, 1 \mathrm{H}, \mathrm{H}-$ 1a), 4.24 (dd, $J=11.2,4.7 \mathrm{~Hz}, 1 \mathrm{H}, \mathrm{H}-1 \mathrm{~b}$ ), 3.80 (dd, $J=14.6,4.2 \mathrm{~Hz}, 1 \mathrm{H}, \mathrm{H}-3 \mathrm{a}$ ), 3.43 (s, 3H, H-15), 3.49 (dd, $J=14.6,8.4$ Hz, 1H, H-3b), 2.45 (s, 3H, H-14). ${ }^{13}$ C NMR (125 MHz, $\mathrm{CDCl}_{3}$, represents peaks from major diastereomer) $\delta 165.9(\mathrm{C}-5), 152.8(\mathrm{C}-4), 145.4(\mathrm{C}-$ 8), 133.2, 131.9, 131.3 (C-13), 130.4, 130.0 (C-10, C-12), 129.9, 129.8, 128.5, 128.0 (C9, C-11), 127.4, 122.9 (q, $\left.{ }^{1} J_{\mathrm{C}-\mathrm{F}}=287.1 \mathrm{~Hz}, \mathrm{C}-7\right), 123.8,84.8\left(\mathrm{q},{ }^{2} J_{\mathrm{C}-\mathrm{F}}=27.8 \mathrm{~Hz}, \mathrm{C}-6\right)$, 71.1 (C-2), 68.0 (C-1), 55.5 (C-15), 32.8 (C-3), 21.7 (C-14). FTIR (thin film) $v 2952$, 1753, 1597, 1500, 1451, 1366, 1268, 1240, 1189, 1174, 1120, 1107, 1096, 1081, 1052, 1015, 989, 932, 814, 791, 762, 732, 717, 695, 666, 611, $553 \mathrm{~cm}^{-1}$. HRMS (ESI): Calculated for $\mathrm{C}_{27} \mathrm{H}_{26} \mathrm{~F}_{3} \mathrm{~N}_{4} \mathrm{O}_{6} \mathrm{~S}_{2}{ }^{+}[\mathrm{M}+\mathrm{H}]^{+}$623.1246; found 623.1224. $[\alpha]_{D}{ }^{24}+17.5(c=$ $1.29, \mathrm{DCM})$.

Preparation of $(R)-1-((1-p h e n y l-1 H$-tetrazol-5-yl)thio)-3-(tosyloxy)propan-2-yl $(R)$ 3,3,3-trifluoro-2-methoxy-2-phenylpropanoate, $(R)$-MTPA-219

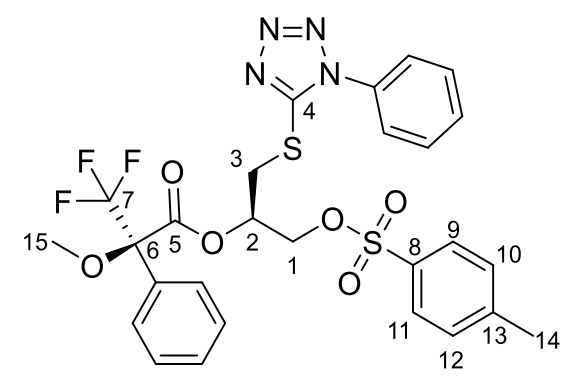

To a solution of tosylate 217 (27.0 mg, $0.066 \mathrm{mmol}, 1.0$ equiv.) in anhydrous DCM (3.0 $\mathrm{mL}$ ) was added sequentially $(R)-(+)-\alpha$-methoxy- $\alpha$-trifluoromethylphenylacetic acid (63.2 mg, $0.25 \mathrm{mmol}, 3.79$ equiv.), $N, N^{\prime}$-dicyclohexylcarbodiimide (119.5 mg, 0.579 mmol, 8.8 equiv.) and 4-dimethylaminopyridine ( $24.2 \mathrm{mg}, 0.198 \mathrm{mmol}, 3.0$ equiv.). The reaction mixture was stirred at room temperature for 5 days. $2 \mathrm{~mL}$ waster was added the reaction mixture. Then the aqueous phase was extracted with ethyl acetate $(3 \times 5 \mathrm{~mL})$. The combined organic phase was washed with brine, dried over anhydrous $\mathrm{Na}_{2} \mathrm{SO}_{4}$, filtered and evaporated under vacuum. The crude material was purified by silica gel flash chromatography (pet. ether/EtOAc $=3: 1)$ to provide the title compound $(26.7 \mathrm{mg}$, 
$65 \%)$ as a colourless oil.

$\mathbf{R}_{f}: 0.77$ (pet. ether/EtOAc $\left.=1: 1\right) .{ }^{1} \mathbf{H}$ NMR $\left(500 \mathrm{MHz}, \mathrm{CDCl}_{3}\right.$, represents peaks from major diastereomer) $\delta 7.79-7.74(\mathrm{~m}, 2 \mathrm{H}, \mathrm{H}-9, \mathrm{H}-11), 7.60-7.55(\mathrm{~m}, 3 \mathrm{H}), 7.49-7.46$ (m, 2H), 7.46 - 7.43 (m, 2H), 7.37 - 7.32 (m, 3H, overlapped, H-10, H-12), 7.31 - 7.26 (m, 2H), $5.67-5.61$ (m, 1H, H-2), 4.37 (dd, $J=11.3,2.8 \mathrm{~Hz}, 1 \mathrm{H}, \mathrm{H}-1 \mathrm{a}), 4.28$ (dd, $J=$ 11.3, 5.1 Hz, 1H, H-1b), 3.72 (dd, $J=14.5,4.1 \mathrm{~Hz}, 1 \mathrm{H}, \mathrm{H}-3 \mathrm{a}$ ), 3.49 (s, 3H, H-15), 3.39 (dd, $J=14.5,8.4 \mathrm{~Hz}, 1 \mathrm{H}, \mathrm{H}-3 \mathrm{~b}), 2.45$ (s, 3H, H-14). ${ }^{13} \mathrm{C} \mathrm{NMR}\left(125 \mathrm{MHz}, \mathrm{CDCl}_{3}\right.$, represents peaks from major diastereomer) $\delta 165.9$ (C-5), 152.9 (C-4), $145.6(\mathrm{C}-8)$, 133.2, 131.9, 131.6, 130.4, 130.1 (C-10, C-12), 129.9 (C-13), 129.7, 128.4, 128.0 (C-9, C-11), $127.2123 .8,123.1$ (q, $\left.{ }^{1} J_{\mathrm{C}-\mathrm{F}}=287.2 \mathrm{~Hz}, \mathrm{C}-7\right), 84.6\left(\mathrm{q},{ }^{2} J_{\mathrm{C}-\mathrm{F}}=27.7 \mathrm{~Hz}, \mathrm{C}-6\right), 71.0$ (C-2), 68.3 (C-1), 55.7 (C-15), 32.8 (C-3), 21.7 (C-14). FTIR (thin film) v2952, 1753, 1597, 1500, 1451, 1366, 1268, 1238, 1189, 1174, 1120, 1107, 1096, 1081, 1052, 1015, 988, 932, 814, 762, 731, 717, 694, 666, 648, $553 \mathrm{~cm}^{-1}$. HRMS (ESI): Calculated for $\mathrm{C}_{27} \mathrm{H}_{26} \mathrm{~F}_{3} \mathrm{~N}_{4} \mathrm{O}_{6} \mathrm{~S}_{2}{ }^{+}[\mathrm{M}+\mathrm{H}]^{+}$623.1246; found 623.1225. $[\alpha]_{D^{24}}+63$ ( $c=1.34$, DCM).

\section{Preparation of $(S)$-2-((tert-butyldimethylsilyl)oxy)-3-((1-phenyl-1H-tetrazol-5- yl)thio)propyl 4-methylbenzenesulfonate, 221}

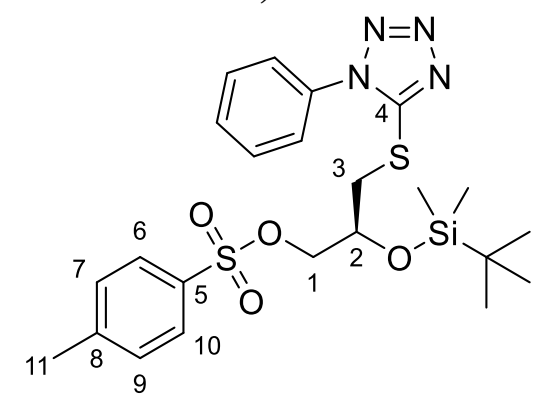

To a solution of tosylate 217 (2.86 g, 7.04 mmol, 1.0 equiv.), imidazole ( $2.88 \mathrm{~g}, 42.2$ mmol, 6.0 equiv.) and 4-dimethylaminopyridine (91.4 mg, $0.704 \mathrm{mmol}, 0.1$ equiv.) in anhydrous DMF (30 mL) was added tert-butyldimethylsilyl chloride (3.18 g, $21.1 \mathrm{mmol}$, 3.0 equiv.). The reaction mixture was stirred at room temperature for 16 hours. Then the reaction mixture was diluted with ethyl acetate $(100 \mathrm{~mL})$, and washed with water $(3 \times 50$ $\mathrm{mL})$. The combined aqueous phase was extracted with ethyl acetate $(3 \times 50 \mathrm{~mL})$. The combined organic phase was washed with brine, dried over anhydrous $\mathrm{Na}_{2} \mathrm{SO}_{4}$, filtered and evaporated under vacuum. The crude material was purified by silica gel flash chromatography (pet. ether/EtOAc $=5: 1$ ) to provide the title compound (2.96 g, yield $81 \%$ ) as a white crystal. $\mathbf{R}_{f}: 0.50$ (pet. ether/EtOAc $=2: 1$ ). Melting point: $122-123{ }^{\circ} \mathrm{C}$. ${ }^{1} \mathbf{H}$ NMR (500 MHz, $\mathrm{CDCl}_{3}$ ): $\delta 7.81$ - 7.75 (m, 2H, H-6, H-10), 7.61 - 7.51 (m, 5H), 7.32 
(d, $J=8.0 \mathrm{~Hz}, 2 \mathrm{H}, \mathrm{H}-7, \mathrm{H}-9$ ), 4.32 (quin, $J=5.2 \mathrm{~Hz}, 1 \mathrm{H}, \mathrm{H}-2$ ), $4.09-4.02$ (m, 2H, H-1), $3.53(\mathrm{dd}, J=13.7,5.5 \mathrm{~Hz}, 2 \mathrm{H}, \mathrm{H}-3 \mathrm{a}), 3.45$ (dd, $J=13.7,6.0 \mathrm{~Hz}, 2 \mathrm{H}, \mathrm{H}-3 \mathrm{~b}$ ), 2.43 (s, 3H, $\mathrm{H}-11), 0.81$ (s, 9H), 0.023 (s, 3H), 0.018 (s, 3H). $\left.{ }^{13} \mathbf{C ~ N M R ~ ( 1 2 5 ~ M H z , ~} \mathrm{CDCl}_{3}\right): \delta 153.8$ (C-4), 145.1 (C-5), 133.2, 132.5 (C-8), 130.3, 129.91, 129.86 (C-7, C-9), 128.0 (C-6, C10), 71.2 (C-2), 68.2 (C-1), 36.6 (C-3), 25.6, 21.7 (C-11), 17.9, -4.77, -4.85. FTIR (solid) $v 2951,2929,2890,2855,1597,1500,1473,1460,1446,1423,1397,1358,1307,1294$, 1277, 1255, 1189, 1172, 1110, 1090, 1041, 1017, 997, 976, 929, 865, 841, 824, 809, 778, 764, 738, 692, 666, 609, 573, 553, 489, $469 \mathrm{~cm}^{-1}$. HRMS (ESI): Calculated for $\mathrm{C}_{23} \mathrm{H}_{33} \mathrm{~N}_{4} \mathrm{O}_{4} \mathrm{~S}_{2} \mathrm{Si}^{+}[\mathrm{M}+\mathrm{H}]^{+}$521.1712; found 521.1735. $[\alpha]_{D}{ }^{18}+276(c=0.25, \mathrm{DCM})$.

Preparation of $(S)-3-(($ tert-butyldimethylsilyl)oxy)-4-((1-phenyl-1H-tetrazol-5yl)thio)butanenitrile, 222

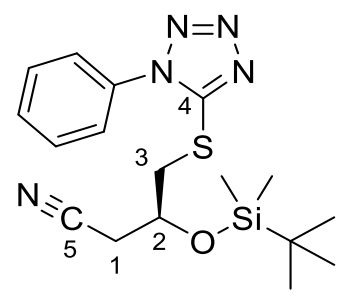

To a solution of silyl ether 221 (104 mg, $0.20 \mathrm{mmol}, 1.0$ equiv.) and 18-crown-6 (151 mg, $0.57 \mathrm{mmol}, 2.85$ equiv.) in anhydrous acetonitrile $(4 \mathrm{~mL})$ was added potassium cyanide (43.7 mg, $0.67 \mathrm{mmol}, 3.35$ equiv.) at room temperature. The reaction mixture was heated at $40{ }^{\circ} \mathrm{C}$ for 20 hours under argon atmosphere. The reaction mixture was diluted with ethyl acetate $(10 \mathrm{~mL})$ and was added saturated aqueous $\mathrm{NaHCO}_{3}$ solution $(5 \mathrm{ml})$. The aqueous phase was extracted by ethyl acetate $(3 \times 10 \mathrm{~mL})$. The combined organic phase was washed with brine, dried over anhydrous $\mathrm{Na}_{2} \mathrm{SO}_{4}$, filtered and evaporated under vacuum. The crude material was purified by silica gel flash chromatography (pet. ether/EtOAc $=4: 1)$ to provide the title compound $(34.7 \mathrm{mg}$, yield $46 \%)$ as a white solid. $\mathbf{R}_{f}: 0.45$ (pet. ether/EtOAc = 3:1). Melting point: $108-109^{\circ} \mathrm{C}^{1} \mathbf{H}$ NMR $(500 \mathrm{MHz}$, $\left.\mathrm{CDCl}_{3}\right): \delta 7.62-7.53(\mathrm{~m}, 5 \mathrm{H}), 4.51-4.45(\mathrm{~m}, 1 \mathrm{H}, \mathrm{H}-2), 3.60(\mathrm{dd}, J=13.9,5.7 \mathrm{~Hz}, 1 \mathrm{H}$, H-3a), 3.48 (dd, $J=13.9,6.0 \mathrm{~Hz}, 1 \mathrm{H}, \mathrm{H}-3 \mathrm{~b}$ ), 2.71 (dd, $J=4.9,1.3 \mathrm{~Hz}, 2 \mathrm{H}, \mathrm{H}-1$ ), 0.90 (s, 9H), 0.15 (s, 3H), 0.12 (s, 3H). ${ }^{13}$ C NMR (125 MHz, $\left.\mathrm{CDCl}_{3}\right)$ : $\delta 153.6$ (C-4), 133.4, 130.4, 130.0, 123.8, 116.7 (C-5), 66.5 (C-2), 38.8 (C-3), 25.6, 25.5 (C-1), 17.9, -4.6, -4.8. FTIR (thin film) $v 2954,2929,2887,2856,1597,1499,1471,1463,1411,1388,1363,1279$, 1251, 1099, 1075, 1014, 938, 838, 806, 779, 760, 712, 687, 665, $552 \mathrm{~cm}^{-1}$. HRMS (ESI): Calculated for $\mathrm{C}_{17} \mathrm{H}_{26} \mathrm{~N}_{5} \mathrm{OSSi}^{+}[\mathrm{M}+\mathrm{H}]^{+} 376.1627$; found 376.1622. $[\alpha]_{D}{ }^{18}+93.3(c=0.15$, 
DCM).

\section{Preparation of cis- and trans-acetaldoxime mixture}<smiles>CC=NO</smiles>

A cold solution of sodium hydroxide ( $21.0 \mathrm{~g}, 0.53 \mathrm{~mol}$ in $60 \mathrm{~mL}$ distilled water, 1.5 equiv.) was added slowly to an aqueous solution of hydroxylamine hydrochloride (50 g, $0.7 \mathrm{~mol}$ in $100 \mathrm{ml}$ distilled water). Then acetaldehyde $(20 \mathrm{~mL}, 0.35 \mathrm{~mol})$ was added slowly to the reaction mixture. The resulting mixture was stirred at $100{ }^{\circ} \mathrm{C}$ for 3 hours and then cooled to room temperature. The reaction mixture was extracted by diethyl ether $(4 \times 100 \mathrm{~mL})$. The combined organic phase was washed with brine, dried over anhydrous $\mathrm{Na}_{2} \mathrm{SO}_{4}$, filtered and evaporated under atmospheric pressure. The crude material was purified by distillation to provide the title compound (18.5 g, yield 89\%) as a colourless crystal. ${ }^{1} \mathbf{H}$ NMR (500 MHz, $\mathrm{CDCl}_{3}$ ): 8.57 (br. s, 1H), 8.19 (br. s, 1H), 7.46 (q, J=5.8 Hz, 1H), 6.84 $(\mathrm{q}, J=5.5 \mathrm{~Hz}, 1 \mathrm{H}), 1.88(\mathrm{~d}, J=5.5 \mathrm{~Hz}, 3 \mathrm{H}), 1.86(\mathrm{~d}, J=5.8 \mathrm{~Hz}, 3 \mathrm{H}) .{ }^{13} \mathbf{C}$ NMR $(125$ $\left.\mathrm{MHz}, \mathrm{CDCl}_{3}\right): \delta 148.1,147.8,15.1,11.1$. These characterisation data match those reported in the literature. ${ }^{341}$

\section{Preparation of (S)-3-((tert-butyldimethylsilyl)oxy)-4-((1-phenyl-1H-tetrazol-5-} yl)thio)butanamide, 225

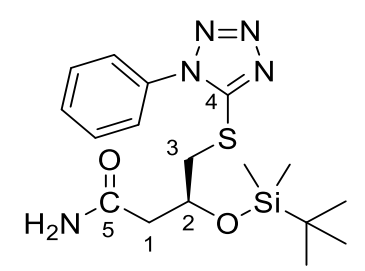

To an $20 \mathrm{~mL}$ vial were added nitrile 222 (0.927 g, 2.47 mmol, 1.0 equiv.), freshly prepared acetaldoxime (0.729 g, $12.4 \mathrm{mmol}, 5.0$ equiv.), $\mathrm{RhCl}\left(\mathrm{PPh}_{3}\right)_{3}(0.026 \mathrm{~g}, 0.01$ equiv.) and anhydrous toluene $(8 \mathrm{~mL})$. The reaction mixture was vigorously stirred at $110{ }^{\circ} \mathrm{C}$ under argon atmosphere in the sealed vial for 22 hours. Then solvent was removed under vacuum. The crude material was purified by silica gel flash chromatography (pet. ether/EtOAc $=1: 1)$ to provide the title compound $(855 \mathrm{mg}$, yield $88 \%)$ as a white solid. $\mathbf{R}_{f}: 0.11$ (pet. ether/EtOAc $\left.=1: 1\right)$. Melting point: $78-79^{\circ} \mathrm{C} .{ }^{1} \mathbf{H} \mathbf{N M R}\left(500 \mathrm{MHz}, \mathrm{CDCl}_{3}\right)$ : $\delta 7.62-7.51$ (m, 5H), 6.26 (br. s, $1 \mathrm{H}, \mathrm{N}-\mathrm{H}$ ), 5.48 (br. s, $1 \mathrm{H}, \mathrm{N}-\mathrm{H}$ ), 4.54 (quin, $J=5.5 \mathrm{~Hz}$, 
1H, H-2), , 3.64 (dd, $J=13.7,5.5 \mathrm{~Hz}, 1 \mathrm{H}, \mathrm{H}-3 \mathrm{a}), 3.52$ (dd, $J=13.7,6.2 \mathrm{~Hz}, 1 \mathrm{H}, \mathrm{H}-3 \mathrm{~b}$ ), $2.63(\mathrm{dd}, J=14.9,5.2 \mathrm{~Hz}, 1 \mathrm{H}, \mathrm{H}-1 \mathrm{a}), 2.54$ (dd, $J=14.9,5.4 \mathrm{~Hz}, 1 \mathrm{H}, \mathrm{H}-1 \mathrm{~b}), 0.88$ (s, 9H), $0.13(\mathrm{~s}, 3 \mathrm{H}), 0.12(\mathrm{~s}, 3 \mathrm{H}) .{ }^{13} \mathbf{C}$ NMR $\left(125 \mathrm{MHz}, \mathrm{CDCl}_{3}\right): \delta 172.1$ (C-5), $154.2(\mathrm{C}-4)$, 133.5, 130.3, 129.9, 123.8, 67.7 (C-2), 42.5 (C-1), 39.1 (C-3), 25.7, 18.0, -4.6, -4.8. FTIR (solid) v3389, 3339, 3197, 2954, 2929, 2887, 2856, 1673, 1615, 1598, 1499, 1471, 1463, 1407, 1389, 1362, 1335, 1251, 1162, 1090, 1015, 955, 838, 811, 779, 761, 694, 667, 573, $557 \mathrm{~cm}^{-1}$. HRMS (ESI): Calculated for $\mathrm{C}_{17} \mathrm{H}_{28} \mathrm{~N}_{5} \mathrm{O}_{2} \mathrm{SSi}^{+}[\mathrm{M}+\mathrm{H}]^{+}$394.1728; found 394.1726. $[\alpha]_{D}^{23}+165(c=1.65, \mathrm{DCM})$.

Preparation of Belleau's Reagent (2,4-bis(4-phenoxyphenyl)-1,3,2,4dithiadiphosphetane 2,4-disulfide), ${ }^{342} 231$

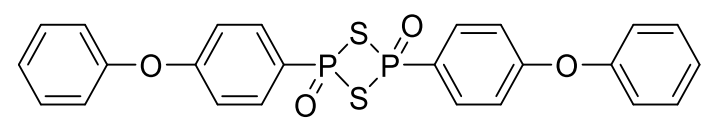

231

Phosphorus pentasulfide (6.94 g, $0.016 \mathrm{mmol}, 0.1$ equiv.) and diphenyl ether $(25 \mathrm{~mL}$, $0.16 \mathrm{mmol}, 1.0$ equiv.) were stirred at $160{ }^{\circ} \mathrm{C}$ under argon atmosphere for 9 hours. The reaction mixture was then stirred at room temperature for 15 hours. The yellow precipitate was filtered, washed with anhydrous toluene, and recrystallised from anhydrous toluene $(60 \mathrm{~mL})$ to afford the title compound as a yellow powder (3.16 g, yield 37\%). Melting point: $187-190{ }^{\circ} \mathrm{C}$ (sealed tube). This characterisation data match those reported in the literature. ${ }^{186,342}$

Preparation of (S)-3-((tert-butyldimethylsilyl)oxy)-4-((1-phenyl-1H-tetrazol-5yl)thio)butanethioamide, 223

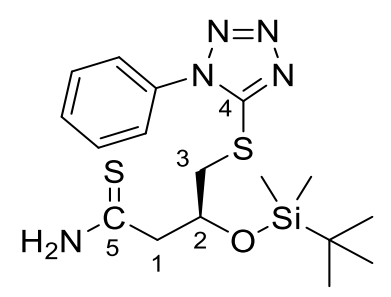

To a solution of amide 225 (1.025 g, $13.7 \mathrm{mmol}, 1.0$ equiv.) in anhydrous THF (50 mL) was added Lawesson's reagent (3.78 g, $9.5 \mathrm{mmol}, 0.7$ equiv.). The reaction mixture was stirred at room temperature for 30 minutes. $50 \mathrm{ml}$ saturated aqueous $\mathrm{NaHCO}_{3}$ solution was added to the reaction mixture and the aqueous phase was extracted with ethyl acetate 
$(3 \times 100 \mathrm{~mL})$. The combined organic phase was washed with brine, dried over anhydrous $\mathrm{Na}_{2} \mathrm{SO}_{4}$, filtered and evaporated under vacuum. The crude material was purified by silica gel flash chromatography (pet. ether/EtOAc $=3: 1)$ to provide the title compound $(0.661$ g, yield 62\%) as a yellow oil. $\mathbf{R}_{f}: 0.4$ (pet. ether/EtOAc $\left.=1: 1\right) .{ }^{1} \mathbf{H}$ NMR $(500 \mathrm{MHz}$, $\left.\mathrm{CDCl}_{3}\right): \delta 8.16$ (br.s, $\left.1 \mathrm{H}, \mathrm{N}-\mathrm{H}\right), 7.62-7.52(\mathrm{~m}, 5 \mathrm{H}), 7.49$ (br.s, $\left.1 \mathrm{H},-\mathrm{NH}\right), 4.61-4.56$ (m, 1H, H-2), 3.67 (dd, $J=13.9,4.8 \mathrm{~Hz}, 1 \mathrm{H}, \mathrm{H}-1, \mathrm{H}-1 \mathrm{a}), 3.49$ (dd, $J=13.9,6.5 \mathrm{~Hz}, 1 \mathrm{H}, \mathrm{H}-$ 1b), 3.13 (ddd, $J=14.4,4.6,1.0 \mathrm{~Hz}, 1 \mathrm{H}, \mathrm{H}-3 \mathrm{a}$ ), 3.07 (dd, $J=14.5,5.4 \mathrm{~Hz}, 1 \mathrm{H}, \mathrm{H}-3 \mathrm{~b}$ ), 0.89 (s, 9H), 0.165 (s, 3H), 0.162 (s, 3H). $\left.{ }^{13} \mathbf{C ~ N M R ~ ( 1 2 5 ~ M H z , ~} \mathrm{CDCl}_{3}\right): \delta 205.8,154.4$, $133.4,130.3,129.9,123.8,69.7,50.8,38.5,25.8,17.9,-4.6,-4.8$. FTIR (thin film) $v$ 3307, 3182, 2953, 2928, 2885, 2855, 1620, 1597, 1499, 1461, 1408, 1388, 1361, 1333, 1316, 1279, 1250, 1209, 1146, 1088, 1014, 939, 917, 836, 809, 778, 730, 692, 663, 552, $465 \mathrm{~cm}^{-1}$. HRMS (ESI): Calculated for $\mathrm{C}_{17} \mathrm{H}_{28} \mathrm{~N}_{5} \mathrm{OS}_{2} \mathrm{Si}^{+}[\mathrm{M}+\mathrm{H}]^{+} 410.1505$; found 410.1479. $[\alpha]_{D}{ }^{18}-317(c=0.23, \mathrm{DCM})$.

\subsubsection{Gold-catalysed Preparation of Thiazole 276}

\section{Preparation of 8-methylquinoline $\mathrm{N}$-oxide, 254}<smiles>Cc1cccc2ccc[n+]([O-])c12</smiles>

To a solution of 8-methylquinoline 255 (1.58 g, $11.0 \mathrm{mmol}, 1.0$ equiv.) in DCM (110 mL) was added 3-chloroperbenzoic acid (3.3 g, 77\% purity, $14.3 \mathrm{mmol}, 1.3$ equiv.). The reaction mixture was stirred at room temperature in dark for 16 hours. Then the reaction mixture was washed with $1 \mathrm{M} \mathrm{KOH}$ aqueous solution $(4 \times 50 \mathrm{~mL})$. The combined aqueous phase was extracted with DCM $(6 \times 100 \mathrm{~mL})$. The combined organic phase was washed with brine, dried over anhydrous $\mathrm{Na}_{2} \mathrm{SO}_{4}$, filtered and evaporated under vacuum. The crude material was purified by silica gel flash chromatography ( $\mathrm{DCM} / \mathrm{MeOH}=30: 1)$ to provide the title compound $(1.268 \mathrm{~g}, 72 \%)$ as a brown oil that solidified upon standing at room temperature. $\mathbf{R}_{f}: 0.29(\mathrm{DCM} / \mathrm{MeOH}=10: 1) .{ }^{1} \mathbf{H} \mathbf{~ N M R}\left(500 \mathrm{MHz}, \mathrm{CDCl}_{3}\right): \delta 8.40$ $(\mathrm{dd}, J=6.1,1.1 \mathrm{~Hz}, 1 \mathrm{H}), 7.67-7.62(\mathrm{~m}, 2 \mathrm{H}), 7.46-7.40$ (m, 2H), 7.18 (dd, J = 8.4, 6.0 $\mathrm{Hz}, 1 \mathrm{H}), 3.19$ (s, 3H). ${ }^{\mathbf{1 3}} \mathrm{C}$ NMR (125 MHz, $\left.\mathrm{CDCl}_{3}\right): \delta 141.5,137.2,133.7,133.3,132.5$, $128.1,126.8,126.3,120.7,24.9$. These characterisation data match those reported in the literature. ${ }^{343}$ 


\section{Preparation of Mor-Dal Phos AuOMs, 257}

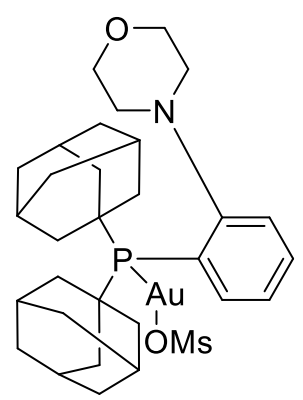

To a suspension of Mor-DalPhos $\mathrm{AuCl} 256$ (50mg, $0.072 \mathrm{mmol}, 1.0$ equiv.) in $5 \mathrm{~mL}$ anhydrous DCM was added silver methanesulfonate $(16.5 \mathrm{mg} \mathrm{mmol}, 0.08 \mathrm{mmol}, 1.1$ equiv.). The mixture was stirred for 1 hour at room temperature under argon atmosphere and the reaction mixture was allowed to settle for $5 \mathrm{~min}$ after which silver chloride all precipitated in the bottom. After passing through a Teflon syringe filter $(0.2 \mu \mathrm{m})$, the solvent was evaporated off under reduced pressure to give the desired gold complex MorDalPhos AuOMs in (52.9 mg, 98\% yield) as an off-white powder. ${ }^{1} \mathbf{H}$ NMR (500 MHz, $\left.\mathrm{CDCl}_{3}\right): \delta 7.81-7.75(\mathrm{~m}, 1 \mathrm{H}), 7.66-7.56(\mathrm{~m}, 2 \mathrm{H}), 7.38-7.31(\mathrm{~m}, 1 \mathrm{H}), 4.43(\mathrm{td}, \mathrm{J}=$ 11.5, 2.0 Hz, 2H), $3.86(\mathrm{dd}, \mathrm{J}=11.5,2.4 \mathrm{~Hz}, 2 \mathrm{H}), 3.12$ (td, J = 11.5, $3.2 \mathrm{~Hz}, 2 \mathrm{H}), 2.98$ (s, $3 \mathrm{H}), 2.66(\mathrm{~d}, \mathrm{~J}=11.5 \mathrm{~Hz}, 2 \mathrm{H}), 2.24-2.16(\mathrm{~m}, 6 \mathrm{H}), 2.13-2.05(\mathrm{~m}, 6 \mathrm{H}), 2.04-1.97(\mathrm{~m}$, $6 \mathrm{H}), 1.68(\mathrm{~s}, 12 \mathrm{H}) .{ }^{13} \mathrm{C}$ NMR $\left(125 \mathrm{MHz}, \mathrm{CDCl}_{3}\right): \delta 158.5\left(\mathrm{~d}, J_{\mathrm{C}-\mathrm{P}}=4.6 \mathrm{~Hz}\right), 134.5\left(\mathrm{~d}, J_{\mathrm{C}-}\right.$ $\mathrm{P}=2.5 \mathrm{~Hz}), 132.9\left(\mathrm{~d}, J_{\mathrm{C}-\mathrm{P}}=1.7 \mathrm{~Hz}\right), 127.2\left(\mathrm{~d}, J_{\mathrm{C}-\mathrm{P}}=4.9 \mathrm{~Hz}\right), 125.1\left(\mathrm{~d}, J_{\mathrm{C}-\mathrm{P}}=6.8 \mathrm{~Hz}\right)$, $121.0\left(\mathrm{~d}, J_{\mathrm{C}-\mathrm{P}}=48.4 \mathrm{~Hz}\right), 66.3,54.5,42.3\left(\mathrm{~d}, J_{\mathrm{C}-\mathrm{P}}=2.5 \mathrm{~Hz}\right), 39.4,36.2\left(\mathrm{~d}, J_{\mathrm{C}-\mathrm{P}}=1.3 \mathrm{~Hz}\right)$, $28.5\left(\mathrm{~d}, J_{\mathrm{C}-\mathrm{P}}=10.0 \mathrm{~Hz}\right)$. These characterisation data match those reported in the literature. $^{141}$

\section{Preparation of ethyl hept-6-ynoate, 259}<smiles>C#CCCCCC(=O)OCC</smiles>

To an oven-dried $20 \mathrm{~mL}$ round-bottomed flask outfitted with a stir bar, a condenser, a gas inlet adapter and a rubber septum, absolute ethanol (5.0 mL), hept-6-ynoic acid 258 (99.7 $\mathrm{mg}, 0.79 \mathrm{mmol}$ ) and concentrated $\mathrm{H}_{2} \mathrm{SO}_{4}$ (1 drop) were added sequentially. The mixture was refluxed for $3 \mathrm{~h}$ before cooling to room temperature and the solvent removed under reduced pressure. The crude product was diluted with diethyl ether $(50 \mathrm{~mL})$, washed with saturated $\mathrm{NaHCO}_{3}(5 \mathrm{~mL})$ and water $(5 \mathrm{~mL})$. The organic layers were dried over 
magnesium sulfate, filtered and the solvent removed under reduced pressure. The residue was then purified by flash silica gel chromatography (pet. ether/Et ${ }_{2} \mathrm{O}=10 / 1$ ) to afford the title compound (112 mg, 92\%) as a colourless oil. $\mathbf{R}_{f}: 0.43$ (pet. ether/EtOAc $\left.=5: 1\right) .{ }^{1} \mathbf{H}$ NMR (500 MHz, $\left.\mathrm{CDCl}_{3}\right): \delta 4.13(\mathrm{q}, J=7.1 \mathrm{~Hz}, 2 \mathrm{H}), 2.32(\mathrm{t}, J=7.4 \mathrm{~Hz}, 2 \mathrm{H}), 2.21$ (td, $J$ $=7.0,2.6 \mathrm{~Hz}, 2 \mathrm{H}), 1.95(\mathrm{t}, J=2.7 \mathrm{~Hz}, 1 \mathrm{H}), 1.79-1.71(\mathrm{~m}, 2 \mathrm{H}), 1.60-1.53(\mathrm{~m}, 2 \mathrm{H})$, $1.25(\mathrm{t}, J=7.1 \mathrm{~Hz}, 3 \mathrm{H}) .{ }^{13} \mathrm{C}$ NMR $\left(125 \mathrm{MHz}, \mathrm{CDCl}_{3}\right): \delta 173.4,84.0,68.6,60.3,33.8$, $27.9,24.0,18.1,14.3$. These characterisation data match those reported in the literature. ${ }^{344}$

\section{Preparation of propanethioamide, 261}

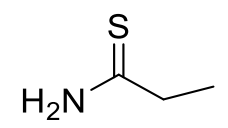

To a milky suspension of purified Lawesson's reagent (3.78 g, $9.5 \mathrm{mmol}, 0.7$ equiv.) in anhydrous THF (50 mL) was added propionamide 260 (1.025 g, $13.5 \mathrm{mmol}, 1.0$ equiv.). The reaction mixture was stirred at room temperature under argon atmosphere for 30 minutes. Saturated aqueous $\mathrm{NaHCO}_{3}$ solution $(70 \mathrm{~mL})$ was added to the reaction mixture. The combined aqueous phase was extracted with ethyl acetate $(3 \times 100 \mathrm{~mL})$. The combined organic phase was washed with brine, dried over anhydrous $\mathrm{Na}_{2} \mathrm{SO}_{4}$, filtered and evaporated under vacuum. The crude material was purified by silica gel flash chromatography (pet. ether/EtOAc $=1: 1)$ to provide the title compound $(0.761 \mathrm{~g}, 61 \%)$ as a yellow solid. Melting point: $40^{\circ} \mathrm{C}-41^{\circ} \mathrm{C} \mathbf{R}_{f}: 0.4$ (pet. ether/EtOAc $=1: 1$ ). ${ }^{1} \mathbf{H}$ NMR $\left(500 \mathrm{MHz}, \mathrm{CDCl}_{3}\right.$ ): $\delta 7.59$ (br. s, 1H), 6.86 (br. s, 1H), 2.69 (q, J = 7.5 Hz, 2H), 1.31 (t, J $=7.5 \mathrm{~Hz}, 3 \mathrm{H}) .{ }^{13} \mathbf{C}$ NMR $\left(125 \mathrm{MHz}, \mathrm{CDCl}_{3}\right): \delta 212.2,38.4,13.3$. These characterisation data match those reported in the literature. ${ }^{345}$

\section{Preparation of 2-ethyl-4-hexylthiazole, 264}

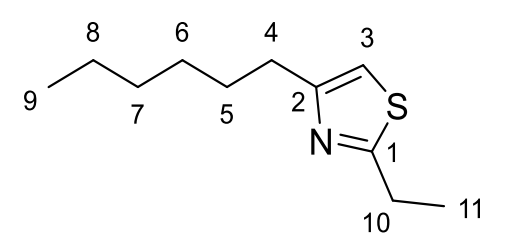

To a solution of Mol-DalPhosAuOMs 257 (17 mg, $0.023 \mathrm{mmol}, 0.05$ equiv.) in deoxygenated anhydrous DCM (0.9 mL) was added 1-octyne 263 (70 mg, $0.45 \mathrm{mmol}, 1.0$ equiv.). The resulting mixture was stirred at room temperature under argon atmosphere for 15 minutes. Then a solution of 8-methylquinoline $\mathrm{N}$-oxide 254 (94 mg, $0.59 \mathrm{mmol}$, 1.3 equiv.) and methanesulfonic acid ( $35 \mu \mathrm{L}, 0.54 \mathrm{mmol}, 1.2$ equiv.) in deoxygenated 
anhydrous DCM $(0.9 \mathrm{~mL})$ was added to the reaction mixture via a syringe pump under argon atmosphere in 5 hours. Upon completion, the reaction mixture was further stirred for 1 hour before propanethioamide 261 (48 mg, $0.54 \mathrm{mmol}, 1.2$ equiv.) in anhydrous $\operatorname{DCM}(0.5 \mathrm{~mL})$ adding. The resulting mixture was stirred at $40{ }^{\circ} \mathrm{C}$ in a sealed vial for 16 hours before concentration under reduced pressure. The crude material was purified by silica gel flash chromatography (pet. ether/EtOAc $=10: 1$ ) to provide the title compound (77.6 mg, yield $62 \%$ ) as a yellow oil. $\mathbf{R}_{f}: 0.47$ (pet. ether/EtOAc $\left.=5: 1\right) .{ }^{1} \mathbf{H}$ NMR (500 $\mathrm{MHz}_{\mathrm{CDCl}}$ ): $\delta 6.69$ (s, 1H, H-3), 3.00 (q, $\left.J=7.6 \mathrm{~Hz}, 2 \mathrm{H}, \mathrm{H}-10\right), 2.72(\mathrm{t}, J=7.3 \mathrm{~Hz}, 2 \mathrm{H}$, H-4), $1.72-1.65$ (m, 2H, H-5), 1.36 (t, J = 7.6 Hz, 3H, H-11, overlapped), 1.40 - 1.27 (m, 6H, overlapped, H-6, H-7, H-8), 0.88 (t, $J=7.0 \mathrm{~Hz}, 3 \mathrm{H}, \mathrm{H}-9) .{ }^{13} \mathbf{C}$ NMR (125 MHz, $\left.\mathrm{CDCl}_{3}\right): \delta 172.2(\mathrm{C}-1), 157.2$ (C-2), 111.3 (C-3), 31.62 (C-7), 31.58 (C-4), 29.2 (C-5), 29.0 (C-6), 26.9 (C-10), 22.6 (C-8), 14.3 (C-11), 14.0 (C-9). FTIR (thin film) v 2956, 2925, 2855, 1522, 1457, 1376, 1320,1260, 1182, 1131, 1096, 1049, 970, 829, 804, 726. HRMS (ESI): Calculated for $\mathrm{C}_{11} \mathrm{H}_{20} \mathrm{NS}^{+}[\mathrm{M}+\mathrm{H}]^{+}$198.1311; found 198.1317.

\section{Preparation of ethyl 7-((methylsulfonyl)oxy)-6-oxoheptanoate, 273}

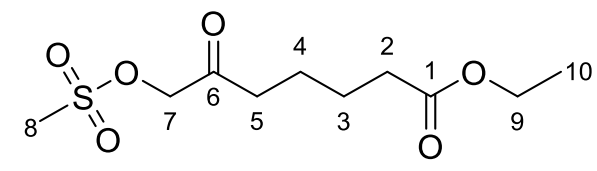

To a solution of alkyne 259 (49.2 $\mathrm{mg}, 0.324 \mathrm{mmol}, 1.0$ equiv.) in deoxygenated DCM (0.65 mL) was added Mol-DalPhosAuOMs 257 (2.4 mg, 0.00324, 0.01 equiv.) under argon atmosphere and the resulting mixture was stirred for 15 minutes. The resulting mixture was added a solution of 8-methylquinoline $N$-oxide 254 (67 mg, $0.421 \mathrm{mmol}, 1.3$ equiv.) and anhydrous methanesulfonic acid (25 $\mu \mathrm{L}, 0.3 \mathrm{mmol}, 1.2$ equiv.) in deoxygenated DCM $(0.65 \mathrm{~mL})$ was added by a syringe pump under argon atmosphere in 5 hours. Upon completion, the reaction mixture was stirred for 1 hour before concentration under reduced pressure. The crude material was purified by silica gel flash chromatography (pet. ether/EtOAc $=2: 1)$ to provide the title compound $(38 \mathrm{mg}, 45 \%)$ as a yellow oil. $\mathbf{R}_{f}$ : 0.45 (pet. ether/EtOAc = 1:1). ${ }^{1} \mathbf{H} \mathbf{N M R}\left(500 \mathrm{MHz}, \mathrm{CDCl}_{3}\right): \delta 4.78$ (s, 2H, H-7), 4.13 (q, J = 7.1 Hz, 2H, H-9), 3.20 (s, 3H, H-8), 2.50 (t, J = 6.9 Hz, 2H, H-5), 2.32 (t, J = 6.8 Hz, 2H, H-2), $1.71-1.60$ (m, 4H, H-3, H-4), 1.25 (t, J = 7.1 Hz, 3H, H10). $\left.{ }^{13} \mathrm{C} \mathrm{NMR} \mathrm{(125} \mathrm{MHz,} \mathrm{CDCl}_{3}\right): \delta 202.5$ (C-6), 173.2 (C-1), 71.5 (C-7), 60.4 (C-9), 38.9 (C-8), 38.2 (C-5), 33.9 (C-2), 24.2(C-4), 22.4 (C-3), 14.2 (C-10). FTIR (thin film) 
$v 2939,1723,1414,1352,1253,1173,1109,1060,1010,969,809,765,728,526 \mathrm{~cm}^{-1}$.

HRMS (ESI): Calculated for $\mathrm{C}_{10} \mathrm{H}_{22} \mathrm{NO}_{6} \mathrm{~S}^{+}\left[\mathrm{M}+\mathrm{NH}_{4}\right]^{+} 284.1168$; found 284.1163

Preparation of ethyl 6-oxo-7-(tosyloxy)heptanoate, 274

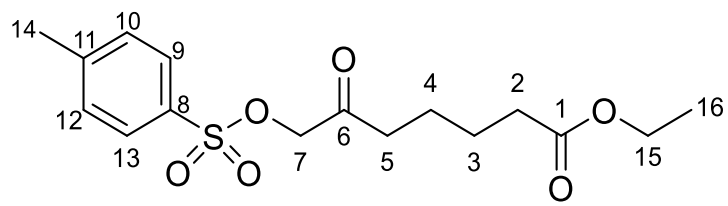

To a solution of ester 259 (49.9 mg, $0.324 \mathrm{mmol}, 1.0$ equiv.) in deoxygenated DCM (0.65 $\mathrm{mL}$ ) was added Mol-DalPhosAuOMs 257 (2.4 mg, 0.00324, 0.01 equiv.) under argon atmosphere and the resulting mixture was stirred for 15 minutes. The resulting mixture was added a solution of 8-methylquinoline $N$-oxide 254 (67 mg, $0.421 \mathrm{mmol}, 1.3$ equiv.) and $p$-toluenesulfonic acid (67 mg, $0.3 \mathrm{mmol}, 1.2$ equiv.) in deoxygenated DCM (0.65 $\mathrm{mL}$ ) was added by a syringe pump under argon atmosphere in 5 hours. Upon completion, the reaction mixture was stirred for 1 hour before concentration under reduced pressure. The crude material was purified by silica gel flash chromatography (pet. ether/EtOAc $=$ 4:1) to provide the title compound (32 mg, 29\%) as a colourless oil. $\mathbf{R}_{f}$ : 0.70 (pet. ether/EtOAc = 1:1). ${ }^{1} \mathbf{H}$ NMR $\left(500 \mathrm{MHz}, \mathrm{CDCl}_{3}\right): \delta 7.84-7.78(\mathrm{~m}, 2 \mathrm{H}, \mathrm{H}-9, \mathrm{H}-13), 7.40$ - 7.35 (m, 2H, H-10, H-12), 4.48 (s, 2H, H-7), 4.11 (q, J = 7.1 Hz, 2H, H-15), 2.53 (t, $J$ $=6.8 \mathrm{~Hz}, 2 \mathrm{H}, \mathrm{H}-5), 2.46$ (s, 3H, H-14), 2.34 (t, J=6.8 Hz, 2H, H-2), $1.62-1.57$ (m, 4H, H-3, H-4), 1.25 (t, $J=7.1 \mathrm{~Hz}, 3 \mathrm{H}, \mathrm{H}-16) .{ }^{13} \mathbf{C} \mathbf{N M R}\left(125 \mathrm{MHz}, \mathrm{CDCl}_{3}\right): \delta 202.8$ (C-6), 173.2 (C-1), 145.5 (C-8), 132.9 (C-11), 130.1 (C-10, C-12), 128.8 (C-9, C-13), 71.8 (C7), 60.4 (C-15), 38.6 (C-5), 33.9 (C-2), 24.2(C-4), 22.2 (C-3), 21.7 (C-14), 14.2 (C-16). FTIR (thin film) v 2937, 1729, 1449, 1369, 1292, 1253, 1190, 1177, 1096, 1005, 817, 774, 667, $554 \mathrm{~cm}^{-1}$. HRMS (ESI): Calculated for $\mathrm{C}_{16} \mathrm{H}_{22} \mathrm{NaO}_{6} \mathrm{~S}^{+}[\mathrm{M}+\mathrm{H}]^{+}$365.1035; found 365.1042 . 
Preparation of $N, N$-dimethylbenzenaminium methanesulfonate, 275<smiles>C[NH2+]c1ccccc1</smiles>

Methanesulfonic acid (1.60 mL, $24.7 \mathrm{mmol}, 1.0$ equiv.) was dropped to a solution of $\mathrm{N}, \mathrm{N}$ dimethylaniline (3.20 ml, $25.2 \mathrm{mmol}, 1.02$ equiv.) in ethanol (50 mL) over 15 minutes. The reaction mixture was then stirred at room temperature for 30 minutes. Ethanol was evaporated under reduced pressure and the crude material was recrystallised in DCM to afford the title compound (1.4 g, yield $26 \%)$ as a white solid. ${ }^{1} \mathbf{H} \mathbf{~ N M R}\left(500 \mathrm{MHz}, \mathrm{CDCl}_{3}\right)$ : $7.64-7.59(\mathrm{~m}, 2 \mathrm{H}), 7.58-7.46(\mathrm{~m}, 3 \mathrm{H}), 3.26(\mathrm{~s}, 6 \mathrm{H}), 2.90$ (s, 3H). ${ }^{13} \mathbf{C}$ NMR (125 MHz, $\mathrm{CDCl}_{3}$ ): $\delta 142.9,130.7,130.3,120.6,46.9,39.4$. FTIR (solid) $v 3036,2961,2976,2519$, 2479, 1598, 1515, 1499, 1475, 1429, 1410, 1337, 1222, 1200, 1179, 1164, 1146, 1133, $1076,1028,995,962,912,901,765,690,616,578,552,522 \mathrm{~cm}^{-1}$.

\section{Preparation of ethyl 5-(2-ethylthiazol-4-yl)pentanoate, 262}

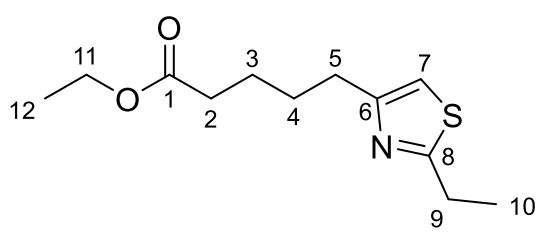

Method A (Use $\mathrm{MsOH}$ as $\mathrm{MsO}^{-}$source and syringe pump for addition)

To a solution of Mol-DalPhosAuOMs 257 (27.5 mg, $0.036 \mathrm{mmol}, 0.05$ equiv.) in deoxygenated anhydrous DCM (1.4 mL) was added alkyne 259 (111 mg, 0.718 mmol, 1.0 equiv.). The resulting mixture was stirred at room temperature under argon atmosphere for 15 minutes. Then a solution of 8-methylquinoline $N$-oxide 254 (148 $\mathrm{mg}$, $0.93 \mathrm{mmol}, 1.30$ equiv.) and methanesulfonic acid ( $56 \mu \mathrm{L}, 0.862 \mathrm{mmol}, 1.2$ equiv.) in deoxygenated anhydrous DCM $(1.4 \mathrm{~mL})$ was added to the reaction mixture via a syringe pump under argon atmosphere in 5 hours. Upon completion, the reaction mixture was further stirred for 1 hour before propanethioamide 261 (74.2 mg, 0.83 mmol, 1.16 equiv. $)$ in anhydrous DCM $(0.8 \mathrm{~mL})$ adding. The resulting mixture was stirred at $40^{\circ} \mathrm{C}$ in a sealed vial for 16 hours before concentration under reduced pressure. The crude material was purified by silica gel flash chromatography (pet. ether/EtOAc $=5: 1)$ to provide the title compound $(90.8 \mathrm{mg}$, yield $53 \%)$ as a yellow oil. $\mathbf{R}_{f} .0 .39$ (pet. ether/EtOAc = 5:1). ${ }^{1} \mathbf{H}$ NMR $\left(500 \mathrm{MHz}, \mathrm{CDCl}_{3}\right): \delta 6.73(\mathrm{~s}, 1 \mathrm{H}, \mathrm{H}-7)$, 
$4.12(\mathrm{q}, J=7.1 \mathrm{~Hz}, 2 \mathrm{H}, \mathrm{H}-11), 3.00(\mathrm{q}, J=7.6 \mathrm{~Hz}, 2 \mathrm{H}, \mathrm{H}-9), 2.75$ (t, $J=7.0 \mathrm{~Hz}, 2 \mathrm{H}, \mathrm{H}-$ 5), 2.33 (t, $J=7.2 \mathrm{~Hz}, 2 \mathrm{H}, \mathrm{H}-2), 1.78-1.66$ (m, 4H, H-3, H-4), 1.37 (t, $J=7.6 \mathrm{~Hz}, 3 \mathrm{H}$, $\mathrm{H}-10), 1.25(\mathrm{t}, J=7.1 \mathrm{~Hz}, 3 \mathrm{H}, \mathrm{H}-12) .{ }^{13} \mathrm{C}$ NMR (125 MHz, $\left.\mathrm{CDCl}_{3}\right): \delta 173.7(\mathrm{C}-1)$, 172.4 (C-8), 156.5 (C-6), 111.8 (C-7), 60.3 (C-11), 34.2 (C-2), 31.3 (C-5), 28.7 (C-4), 27.0 (C-9), 24.6 (C-3), 14.33 (C-10), 14.27 (C-12). FTIR (thin film) v2975, 2935, 2870, 1731, 1522, 1458, 1373, 1350, 1313, 1254, 1178, 1140, 1096, 1029, 968, 938, 859, 791, 735. HRMS (ESI): Calculated for $\mathrm{C}_{12} \mathrm{H}_{20} \mathrm{NO}_{2} \mathrm{~S}^{+}[\mathrm{M}+\mathrm{H}]^{+} 242.1209$; found 242.1212 .

Method B (Use salt 275 as $\mathrm{MsO}^{-}$source)

To a solution of Mol-DalPhosAuOMs 257 (36.6 mg, $0.048 \mathrm{mmol}, 0.05$ equiv.) in deoxygenated anhydrous DCM (1.0 mL) was added alkyne 259 (148.3 mg, 0.96 mmol, 1.0 equiv.), 8-methylquinoline $N$-oxide 254 (183 mg, 1.15 mmol, 1.20 equiv.) and $N, N$ dimethylbenzenaminium methanesulfonate 275 ( $248 \mathrm{mg}, 1.14 \mathrm{mmol}, 1.18$ equiv.) in turn. The resulting reaction mixture was stirred at room temperature under argon atmosphere for 6 hours, then propanethioamide 261 (108 mg, $1.21 \mathrm{mmol}, 1.26$ equiv.) was added to the reaction mixture. The resulting mixture was stirred at room temperature for 16 hours before concentration under reduced pressure. The crude material was purified by silica gel flash chromatography (pet. ether/EtOAc $=5: 1)$ to provide the title compound $(63 \mathrm{mg}$, yield $27 \%$ ) as a yellow oil.

Method C (Use 275 as $\mathrm{MsO}^{-}$source and syringe pump for addition)

To a solution of Mol-DalPhosAuOMs 257 (13.0 mg, $0.017 \mathrm{mmol}, 0.05$ equiv.) in deoxygenated anhydrous DCM (0.7 mL) was added alkyne 259 (50 mg, $0.324 \mathrm{mmol}, 1.0$ equiv.). The resulting mixture was stirred at room temperature for under argon atmosphere 15 minutes. Then a solution of 8-methylquinoline $N$-oxide 254 (61 mg, 0.39 mmol, 1.2 equiv.) and $N, N$-dimethylbenzenaminium methanesulfonate 275 (85 $\mathrm{mg}, 0.39$ mmol, 1.2 equiv.) in deoxygenated anhydrous DCM $(4.0 \mathrm{~mL})$ was added to the reaction mixture via a syringe pump under argon atmosphere in 5 hours. Upon completion, the reaction mixture was further stirred for 1 hour before propanethioamide $\mathbf{2 6 1}$ (35 mg, 0.39 mmol, 1.21 equiv. $)$ in anhydrous DCM $(0.4 \mathrm{~mL})$ adding. The resulting mixture was stirred at $40{ }^{\circ} \mathrm{C}$ in a sealed vial for 16 hours before concentration under reduced pressure. The crude material was purified by silica gel flash chromatography (pet. ether/EtOAc $=5: 1$ ) to provide the title compound (2.9 $\mathrm{mg}$, yield $3 \%)$ as a yellow oil. 


\section{Preparation of ethyl $(S)-5-(2-(2-((t e r t-b u t y l d i m e t h y l s i l y l) o x y)-3-((1-p h e n y l-1 H$ -} tetrazol-5-yl)thio)propyl)thiazol-4-yl)pentanoate, 276

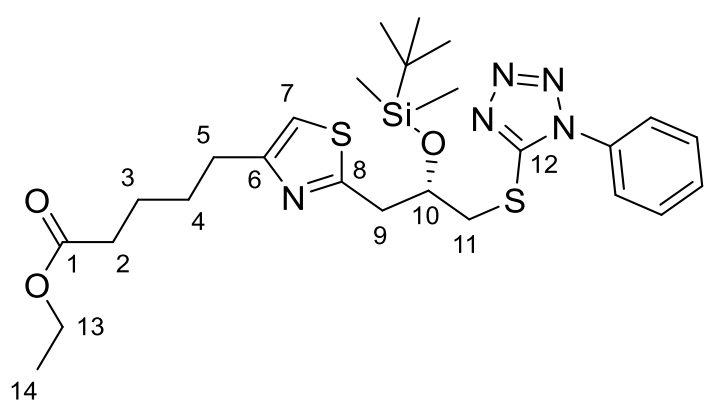

To a solution of Mol-DalPhosAuOMs 257 (35.4 mg, $0.047 \mathrm{mmol}, 0.11$ equiv.) in deoxygenated anhydrous DCM (2.40 mL) was added alkyne 259 (137 mg, 0.889 mmol, 2.1 equiv.). The resulting mixture was stirred at room temperature for 15 minutes. Then a solution of 8-methylquinoline $N$-oxide 254 (185 mg, $1.16 \mathrm{mmol}, 2.73$ equiv.) and methanesulfonic acid ( $74 \mu \mathrm{L}, 1.14 \mathrm{mmol}, 2.68$ equiv.) in deoxygenated anhydrous DCM $(2.40 \mathrm{~mL})$ was added to the reaction mixture via a syringe pump in 5 hours under argon atmosphere. Upon completion, the reaction mixture was further stirred for 1 hour before propanethioamide 223 (174 mg, $0.425 \mathrm{mmol}, 1.0$ equiv.) in anhydrous DCM (2.40 mL) adding. The resulting mixture was stirred at $40{ }^{\circ} \mathrm{C}$ in a sealed vial for 20 hours under argon atmosphere before concentration under reduced pressure. The crude material was purified by silica gel flash chromatography (pet. ether/EtOAc $=4: 1$ ) to provide the title compound (148 mg, yield 61\%) as a yellow oil. $\mathbf{R}_{f}$ : 0.33 (pet. ether/EtOAc $=3: 1$ ). ${ }^{1} \mathbf{H}$ NMR (500 MHz, $\left.\mathrm{CDCl}_{3}\right): \delta 7.61-7.52(\mathrm{~m}, 5 \mathrm{H}), 6.77$ (s, 1H, H-7), 4.55 (quin, $J=5.6$ $\mathrm{Hz}, 1 \mathrm{H}, \mathrm{H}-10), 4.11$ (q, $J=7.1 \mathrm{~Hz}, 2 \mathrm{H}, \mathrm{H}-13), 3.59$ (dd, $J=13.4,5.1 \mathrm{~Hz}, 1 \mathrm{H}, \mathrm{H}-11 \mathrm{a})$, 3.51 (dd, $J=13.4,6.0 \mathrm{~Hz}, 1 \mathrm{H}, \mathrm{H}-11 \mathrm{~b}), 3.27$ (d, $J=5.3 \mathrm{~Hz}, 2 \mathrm{H}, \mathrm{H}-9), 2.74$ (t, $J=7.1 \mathrm{~Hz}$, 2H, H-5), 2.32 (t, J = 7.0 Hz, 2H, H-2), $1.76-1.64$ (m, 4H, H-3, H-4), 1.24 (t, J = 7.1 $\mathrm{Hz}, 3 \mathrm{H}, \mathrm{H}-14), 0.83$ (s, 9H), 0.06 (s, 3H), -0.10 (s, 3H). ${ }^{13} \mathbf{C ~ N M R ~ ( 1 2 5 ~ M H z , ~} \mathrm{CDCl}_{3}$ ): $\delta$ 173.6 (C-1), 165.1 (C-8), 156.7 (C-6), 154.3 (C-12), 133.6 , 130.1, 129.8 , 123.8, 113.1 (C-7), 69.8 (C-10), 60.2 (C-13), 39.8 (C-9), 39.3 (C-11), 34.1 (C-2), 31.1 (C-5), 28.7 (C6), 25.7, 24.6 (C-5), 18.0 , 14.2 (C-14), -4.7, -5.0. FTIR (thin film) v3104, 3068, 3050, 2950, 2918, 1732, 1534, 1462, 1426, 1407, 1275, 1190, 1043, 923, 792, 735. HRMS (ESI): Calculated for $\mathrm{C}_{26} \mathrm{H}_{40} \mathrm{~N}_{5} \mathrm{OS}_{2} \mathrm{Si}^{+}[\mathrm{M}+\mathrm{H}]^{+} 562.2336$; found 562.2344. $[\alpha]_{D}^{21}-4.5(c$ $\left.=0.56, \mathrm{CHCl}_{3}\right)$. 


\subsubsection{Julia-Kocienski Coupling with Side Chain}

Preparation of ethyl (S)-5-(2-(2-((tert-butyldimethylsilyl)oxy)-3-((1-phenyl-1Htetrazol-5-yl)sulfonyl)propyl)thiazol-4-yl)pentanoate, 277

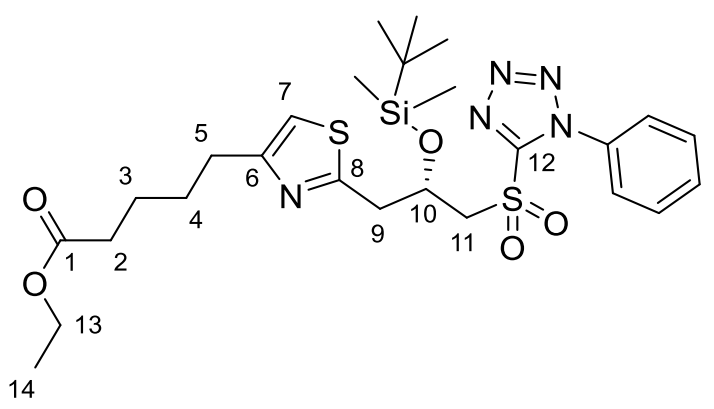

To a solution of thioether 276 (120 mg, $0.21 \mathrm{mmol}, 1.0$ equiv.) in EtOH (5 mL) was added the prepared oxidant solution of $\left(\mathrm{NH}_{4}\right)_{6} \mathrm{Mo}_{7} \mathrm{O}_{24}(135 \mathrm{mg}, 0.109 \mathrm{mmol}, 0.50$ equiv. $)$ in $\mathrm{H}_{2} \mathrm{O}_{2}$ (0.7 mL, 30\% in water, $6.3 \mathrm{mmol}, 30$ equiv.), and the yellow reaction mixture was stirred for 38 hours at room temperature. Saturated aqueous $\mathrm{Na}_{2} \mathrm{~S}_{2} \mathrm{O}_{3}$ solution $(5 \mathrm{~mL})$ was added slowly then ethanol solvent was removed by evaporation under vacuum. The residual aqueous phase was extracted with ethyl acetate $(3 \times 5 \mathrm{~mL})$. The combined organic phase was washed with brine, dried over anhydrous $\mathrm{Na}_{2} \mathrm{SO}_{4}$, filtered, concentrated under reduced pressure. The crude material was purified by flash silica gel chromatography (pet. ether/EtOAc $=3 / 1)$ to afford the title compound $(89.1 \mathrm{mg}, 71 \%$ yield) as a colourless oil. $\mathbf{R}_{f}$ : 0.22 (pet. ether/EtOAc = 3:1). ${ }^{1} \mathbf{H} \mathbf{N M R}\left(500 \mathrm{MHz}, \mathrm{CDCl}_{3}\right)$ : $\delta 7.69-7.64$ (m, 2H), $7.64-7.56$ (m, 3H), 6.80 (s, 1H, H-7), 4.87 - 4.81 (m, 1H, H-10), 4.14 (dd, $J=10.9,4.1 \mathrm{~Hz}, 1 \mathrm{H}, \mathrm{H}-11 \mathrm{a}), 4.11$ (q, $J=7.1 \mathrm{~Hz}, 2 \mathrm{H}, \mathrm{H}-13$ ), 4.01 (dd, $J=14.9$, $6.4 \mathrm{~Hz}, 1 \mathrm{H}, \mathrm{H}-11 \mathrm{~b}), 3.34$ (d, J=5.7 Hz, 2H, H-9), 2.75 (t, J= $7.3 \mathrm{~Hz}, 2 \mathrm{H}, \mathrm{H}-5$ ), 2.33 (t, $J=7.1 \mathrm{~Hz}, 2 \mathrm{H}, \mathrm{H}-2), 1.77-1.63$ (m, 4H, H-3, H-4), 1.24 (t, $J=7.1 \mathrm{~Hz}, 3 \mathrm{H}, \mathrm{H}-14), 0.82$ (s, 9H), 0.04 (s, 3H), 0.01 (s, 3H). $\left.{ }^{13} \mathbf{C ~ N M R ~ ( 1 2 5 ~ M H z , ~} \mathrm{CDCl}_{3}\right): \delta 173.6(\mathrm{C}-1), 163.5$ (C-8), 157.1 (C-6), 154.1 (C-12), 133.1, 131.4, 129.7, 125.2, 113.3 (C-7), 66.4 (C-10), 61.4 (C-11), 60.2 (C-13), 40.7 (C-9), 34.1 (C-2), 31.1 (C-5), 28.6 (C-4), 25.7, 24.5 (C-3), 17.8, 14.3 (C-14), -4.8, -4.9. FTIR (thin film) v 3111, 3072, 2930, 2857, 1730, 1595, 1521, 1498, 1463, 1420, 1343, 1299, 1255, 1154, 1122, 1086, 1045, 1015, 937, 918, 839, 827, 810, 779, 762, 688, 662, 621, 569, 523, 484, 451. HRMS (ESI): Calculated for $\mathrm{C}_{26} \mathrm{H}_{40} \mathrm{~N}_{5} \mathrm{O}_{5} \mathrm{~S}_{2} \mathrm{Si}^{+}[\mathrm{M}+\mathrm{H}]^{+}$594.2235; found 594.2242. $[\alpha]_{D}{ }^{18}-41.2(c=0.17, \mathrm{DCM})$. 


\section{Preparation of ethyl 5-(2-((S)-2-((tert-butyldimethylsilyl)oxy)-3-((1-phenyl-1H-} tetrazol-5-yl)sulfonyl)propyl)-1-oxidothiazol-4-yl)pentanoate, 283

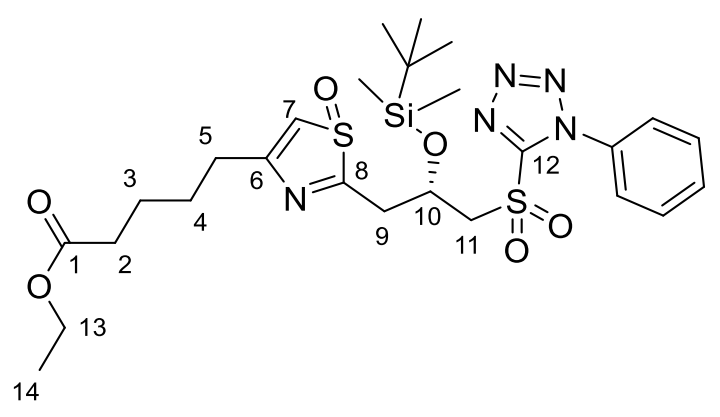

To a stirred solution of thioether $\mathbf{2 7 6}$ (25.3 mg, $0.045 \mathrm{mmol}, 1.0$ equiv.) in DCM (1.5 mL) were added NaHCO3 (19.7 mg, $0.234 \mathrm{mmol}, 5.2$ equiv.) and 3-chloroperbenzoic acid (20.2 mg, 77\% purity, $0.09 \mathrm{mmol}, 2.0$ equiv.) at $0{ }^{\circ} \mathrm{C}$. The suspension was stirred at room temperature for 18 hours. Saturated aqueous $\mathrm{Na}_{2} \mathrm{~S}_{2} \mathrm{O}_{3}$ solution $(2 \mathrm{~mL})$ was added slowly then ethanol solvent was removed by evaporation under vacuum. The residual aqueous phase was extracted with ethyl acetate $(3 \times 5 \mathrm{~mL})$. The combined organic phase was washed with brine, dried over anhydrous $\mathrm{Na}_{2} \mathrm{SO}_{4}$, filtered, concentrated under reduced pressure. The crude material was purified by flash silica gel chromatography using ethyl acetate to afford the title compound as the major product $(10.0 \mathrm{mg}, 36 \%$ yield) as a colourless oil. $\mathbf{R}_{\boldsymbol{f}} .0 .40$ (ethyl acetate). ${ }^{1} \mathbf{H}$ NMR (500 $\left.\mathrm{MHz} \mathrm{CDCl}_{3}\right): \delta 7.70-7.66$ (m, 2H, H-16, H-20), 7.64 - 7.56 (m, 3H, H-17, H-18, H-19), 7.01 (s, 1H, H-7), 5.02 - 4.97 (m, 1H, H-10), 4.20 (dd, $J=15.1,4.5 \mathrm{~Hz}, 1 \mathrm{H}, \mathrm{H}-11 \mathrm{a}), 4.13$ (q, $J=7.1 \mathrm{~Hz}, 2 \mathrm{H}, \mathrm{H}-13$ ), 3.92 (dd, $J=15.1,6.1 \mathrm{~Hz}, 1 \mathrm{H}, \mathrm{H}-11 \mathrm{~b}), 3.61$ (dd, $J=15.1,4.6 \mathrm{~Hz}, 1 \mathrm{H}, \mathrm{H}-9 \mathrm{a}), 3.26$ (dd, $J$ = 15.1, 5.9 Hz, 1H, H-9b), $2.82-2.73$ (m, 2H, H-5), 2.37 (t, J = 6.8 Hz, 2H, H-2), 1.79 - 1.71 (m, 4H, H-3, H-4), 1.25 (t, J = 7.1 Hz, 3H, H-14), 0.84 (s, 9H, H-23, H-24, H-25), 0.07 (s, 3H, H-21), 0.04 (s, 3H, H-22). ${ }^{13}$ C NMR (125 MHz, $\left.\mathrm{CDCl}_{3}\right): \delta 173.5$ (C-1), 154.0 (C-12), 149.0 (C-6), 141.4 (C-8), 133.0, 131.4, 129.6, 125.2, 110.7 (C-7), 64.5 (C-10), 61.3 (C-11), 60.4 (C-13), 35.0 (C-9), 33.8 (C-2), 26.8 (C-5), 25.9 (C-4), 25.7, 24.3 (C-3), 17.8, 14.3 (C-14), -4.9, -5.0. FTIR (thin film) v 3107, 2930, 2857, 1728, 1595, 1498, 1462, 1423, 1344, 1253, 1180, 1152, 1111, 1077, 939, 902, 838, 826, 809, 779, 763, 735, 689, 662, 622, 567, 522, 478, 462. HRMS (ESI): Calculated for $\mathrm{C}_{26} \mathrm{H}_{40} \mathrm{~N}_{5} \mathrm{O}_{6} \mathrm{~S}_{2} \mathrm{Si}^{+}$ $[\mathrm{M}+\mathrm{H}]^{+}$610.2189; found 610.2181. $[\alpha]_{D}{ }^{16}+60.9(c=0.45, \mathrm{DCM})$. 


\section{Preparation of $(E)$-1,1,4,4-tetramethoxybut-2-ene, 292}<smiles>COC(C)/C=C/C(OC)OC</smiles>

Freshly distilled furan (18.0 mL, 0.233 mol, 1.0 equiv.) and anhydrous $\mathrm{MeOH}(95 \mathrm{~mL})$ was placed in a $500 \mathrm{~mL}$ three-necked flask and cooled to $-45^{\circ} \mathrm{C}$ under argon atmosphere. A solution of bromine (14.9 mL, 0.289 mol, 1.24 equiv.) in anhydrous $\mathrm{MeOH}$ (95 mL) was added dropwise at such a rate that the temperature did not exceed $-25^{\circ} \mathrm{C}$. At the end of the addition, the reaction mixture was stirred for $2 \mathrm{~h}$ at $-10{ }^{\circ} \mathrm{C}$. Powdered anhydrous $\mathrm{Na}_{2} \mathrm{CO}_{3}$ (76.5 g, $0.722 \mathrm{~mol}, 3.1$ equiv.) was added portion-wise over a period of about 30 $\min$ at $-10{ }^{\circ} \mathrm{C}$ and then the reaction mixture was stirred for $18 \mathrm{~h}$ at room temperature. The mixture was then filtered and the solvent was removed under vacuum. The residue was purified by distillation $\left(65-70{ }^{\circ} \mathrm{C}, 1 \mathrm{mmHg}\right)$ to afford the title compound $(31.8 \mathrm{~g}, 65 \%)$ as a yellow oil. ${ }^{1} \mathbf{H}$ NMR (500 MHz, $\mathrm{CDCl}_{3}$ ): $\delta 5.78$ (br.s, 2H), 4.80 (br.s, 2H), 3.29 (s, 12H). ${ }^{13} \mathrm{C}$ NMR $\left(125 \mathrm{MHz}, \mathrm{CDCl}_{3}\right): \delta 130.9,101.8,52.6$. These characterisation data match those reported in the literature. ${ }^{346}$

\section{Preparation of $(E)-4,4-d i m e t h o x y b u t-2-e n a l, ~ 293$}<smiles>COC(C)/C=C/C=O</smiles>

To a solution of $(E)-1,1,4,4$-tetramethoxybut-2-ene 292 (8.20 g, $46.5 \mathrm{mmol}, 1.0$ equiv.) in acetone $(150 \mathrm{~mL})$ was added distilled water $(2.5 \mathrm{~mL})$, followed by Amberlyst-15 (1.71 g). The reaction mixture was stirred vigorously at room temperature for 10 minutes. Then the acidic resin was removed by filtration and the filtrate was concentrated under reduced pressure. The crude material was purified by flash silica gel chromatography (pet. ether/ $\left.\mathrm{Et}_{2} \mathrm{O} / \mathrm{Et}_{3} \mathrm{~N}=5 / 1 / 0.05\right)$ to afford the title compound $(4.23 \mathrm{~g}, 70 \%)$ as a yellow oil. $\mathbf{R}_{f}: 0.38$ (pet. ether/Et $\left.2 \mathrm{O}=5: 1\right) .{ }^{1} \mathbf{H} \mathbf{N M R}\left(500 \mathrm{MHz}, \mathrm{CDCl}_{3}\right) \delta 9.63(\mathrm{~d}, J=7.8 \mathrm{~Hz}, 1 \mathrm{H})$, $6.63(\mathrm{dd}, J=15.9,3.9 \mathrm{~Hz}, 1 \mathrm{H}), 6.37(\mathrm{ddd}, J=15.9,7.8,1.3 \mathrm{~Hz}, 2 \mathrm{H}), 5.05(\mathrm{dd}, J=3.9$, $1.3 \mathrm{~Hz}, 1 \mathrm{H}), 3.36(\mathrm{~s}, 6 \mathrm{H}) .{ }^{13} \mathbf{C}$ NMR $\left(125 \mathrm{MHz}, \mathrm{CDCl}_{3}\right): \delta 193.2,150.4,134.3,100.5$, 53.1. These characterisation data match those reported in the literature. ${ }^{134}$ 


\section{Preparation of methyl 2-(triphenyl- $\lambda^{5}$-phosphaneylidene)acetate, 294}

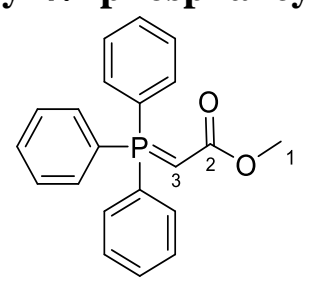

A solution of methyl bromoacetate $(5.0 \mathrm{~g}, 0.0327 \mathrm{~mol}, 1.0$ equiv.) and triphenylphosphine (9.0 g, 0.0340 mol, 1.04 equiv.) was heated at reflux for 4 hours. Then the reaction mixture was cooled to room temperature. The precipitate was separated by filtration and washed with cold $\mathrm{Et}_{2} \mathrm{O}(3 \times 50 \mathrm{~mL})$. The collected phosphonium salt was re-dissolved in DCM (150 mL) and distilled water (100 mL). 2M NaOH aqueous solution was added and the resulting biphasic mixture was stirred at room temperature 12 hours. The organic phase was separated and the aqueous phase was extracted with DCM $(3 \times 100 \mathrm{~mL})$. The combined organic phase was washed with brine, dried over anhydrous $\mathrm{Na}_{2} \mathrm{SO}_{4}$, filtered, concentrated under reduced pressure and dried under oil pump vacuum to afford the title compound (10.4 g, 95\%) as a white powder. ${ }^{1} \mathbf{H} \mathbf{~ N M R}\left(500 \mathrm{MHz}, \mathrm{CDCl}_{3}\right) \delta 7.70-7.60$ (m, 6H), $7.57-7.50$ (m, 3H), 7.46 (m, 6H), 3.52 (br. s, 3H, H-1), 2.90 (br. s, 1H, H-3). ${ }^{13} \mathrm{C} \mathrm{NMR}\left(125 \mathrm{MHz}, \mathrm{CDCl}_{3}\right): \delta 171.7(\mathrm{C}-2), 132.9\left(\mathrm{~d}, J_{\mathrm{C}-\mathrm{P}}=10.0 \mathrm{~Hz}\right), 132.1\left(\mathrm{~d}, J_{\mathrm{C}-\mathrm{P}}=\right.$ $10.0 \mathrm{~Hz}), 131.9\left(\mathrm{~d}, J_{\mathrm{C}-\mathrm{P}}=2.5 \mathrm{~Hz}\right), 131.9,128.7\left(\mathrm{~d}, J_{\mathrm{C}-\mathrm{P}}=12.5 \mathrm{~Hz}\right), 128.5\left(\mathrm{~d}, J_{\mathrm{C}-\mathrm{P}}=12.5\right.$ $\mathrm{Hz}), 49.8(\mathrm{C}-1), 29.7$ (d, $\left.J_{\mathrm{C}-\mathrm{P}}=127.9 \mathrm{~Hz}, \mathrm{C}-3\right)$. These characterisation data match those reported in the literature. ${ }^{347}$

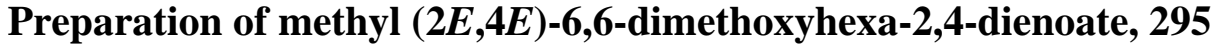

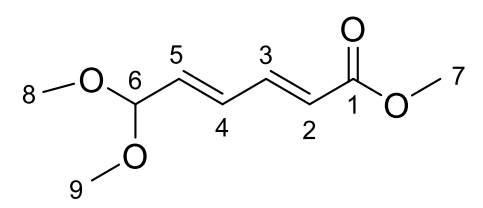

To a solution of aldehyde 293 (3.03 g, $23 \mathrm{mmol}, 1.0$ equiv.) in anhydrous DCM (20 mL) was added phosphorane $(8.0 \mathrm{~g}, 23.9 \mathrm{mmol}, 1.04$ equiv.). Then the reaction mixture was stirred at room temperature under argon atmosphere for 16 hours. Solvent was evaporated and the crude material was then purified by flash silica gel chromatography (pet. ether/EtOAc/Et $\left.{ }_{3} \mathrm{~N}=10: 1: 0.1\right)$ to afford the title compound $(2.95 \mathrm{~g}, 68 \%)$ as a yellow oil. $\mathbf{R}_{\boldsymbol{f}}: 0.47$ (pet. ether/EtOAc = 4:1). ${ }^{1} \mathbf{H}$ NMR $\left(500 \mathrm{MHz}, \mathrm{CDCl}_{3}\right): \delta 7.28(\mathrm{ddd}, J=15.4$, 11.2, $0.4 \mathrm{~Hz}, 1 \mathrm{H}, \mathrm{H}-3), 6.48$ (dddd, $J=15.5,11.2,1.2,0.7 \mathrm{~Hz}, 1 \mathrm{H}, \mathrm{H}-4), 6.00$ (dd, $J=$ 
15.5, 4.3 Hz, 1H, H-5), 5.96 (dd, $J=15.4,0.4 \mathrm{~Hz}, 1 \mathrm{H}, \mathrm{H}-2), 4.91$ (dd, $J=4.3,1.2 \mathrm{~Hz}$, 1H, H-6), 3.75 (s, 3H, H-7), 3.33 (s, 6H, H-8, H-9). $\left.{ }^{13} \mathbf{C ~ N M R ~ ( 1 2 5 ~ M H z , ~} \mathrm{CDCl}_{3}\right): \delta 167.1$ (C-1), 143.2 (C-3), 137.6 (C-5), 131.0 (C-4), 122.7 (C-2), 101.3 (C-6), 52.7 (C-8, C-9), 51.7 (C-7). FTIR (thin film) v 2951, 2832, 1719, 1651, 1619, 1436, 1359, 1313, 1267, 1234, 1190, 1174, 1126, 1051, 1002, 956, 911, 875, $723 \mathrm{~cm}^{-1}$. HRMS (ESI): Calculated for $\mathrm{C}_{8} \mathrm{H}_{11} \mathrm{O}_{3}{ }^{+}\left[\mathrm{M}-\mathrm{OCH}_{3}\right]^{+}$155.0703; found 155.0699.

\section{Preparation of (2E,4E)-6,6-dimethoxyhexa-2,4-dien-1-ol, 296}

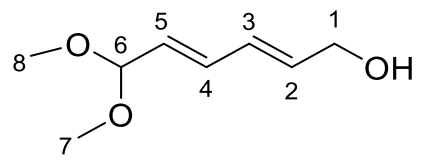

To a solution of methyl ester 295 (1.62 g, 8.7 mmol, 1.0 equiv.) in anhydrous DCM (87 $\mathrm{mL}$ ) was added a solution of diisobutylaluminium hydride $(31.0 \mathrm{~mL}, 1.0 \mathrm{M}$ in cyclohexane, $31.0 \mathrm{mmol}, 3.56$ equiv.) slowly at $-78^{\circ} \mathrm{C}$. The reaction mixture was stirred at $-78{ }^{\circ} \mathrm{C}$ for 6 hours and then quenched by addition of saturated aqueous potassium sodium tartrate $(80 \mathrm{~mL})$. The resulting mixture was stirred at room temperature for 2 hours and the aqueous phase was extracted with ethyl acetate $(3 \times 80 \mathrm{~mL})$. The combined organic phase was washed with brine, dried over anhydrous $\mathrm{Na}_{2} \mathrm{SO}_{4}$, filtered, concentrated under reduced pressure. The crude material was purified by flash silica gel chromatography (pet. ether/EtOAc/Et $3 \mathrm{~N}=2 / 1 / 0.02)$ to afford the title compound ( $0.97 \mathrm{~g}$, $71 \%)$ as a yellow oil. $\mathbf{R}_{f}: 0.38$ (pet. ether/EtOAc $\left.=2: 1\right) .{ }^{1} \mathbf{H} \mathbf{~ N M R}\left(500 \mathrm{MHz}, \mathrm{CDCl}_{3}\right): \delta$ 6.37 (dd, $J=15.2,10.7,1 \mathrm{H}, \mathrm{H}-4), 6.28$ (ddt, $J=15.4,10.5,1.6 \mathrm{~Hz}, 1 \mathrm{H}, \mathrm{H}-3), 5.92$ (dt, $J$ $=15.1,5.7 \mathrm{~Hz}, 1 \mathrm{H}, \mathrm{H}-2), 5.64(\mathrm{dd}, J=15.3,4.8 \mathrm{~Hz}, 1 \mathrm{H}, \mathrm{H}-5), 4.84$ (d, $J=4.8 \mathrm{~Hz}, 1 \mathrm{H}$, H-6), 4.21 (t, J = 5.6 Hz, 2H, H-1), 3.32 (s, 6H, H-7, H-8). ${ }^{13} \mathbf{C ~ N M R ~ ( 1 2 5 ~ M H z , ~} \mathrm{CDCl}_{3}$ ): $\delta 134.0$ (C-2), 132.9 (C-4), 129.9 (C-3), 129.4 (C-5), 102.4 (C-6), 63.2 (C-1), 52.6 (C-7, C-8). FTIR (thin film) v 3394, 2991, 2936, 1830, 1663, 1629, 1447, 1350, 1298, 1190, 1128, 1072, 1045, 990, 952, 905, 849, 586, $487 \mathrm{~cm}^{-1}$. HRMS (ESI): Calculated for $\mathrm{C}_{7} \mathrm{H}_{11} \mathrm{O}_{2}{ }^{+}\left[\mathrm{M}-\mathrm{OCH}_{3}\right]^{+}$127.0754; found 127.0750. 
Preparation of $(2 E, 4 E)-6,6-$ dimethoxyhexa-2,4-dienal, 297

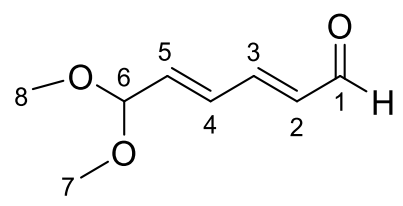

To a solution of alcohol 296 ( $0.21 \mathrm{~g}, 1.3 \mathrm{mmol}, 1.0$ equiv.) in wet DCM (17 mL) was added sequentially pyridine (0.36 mL, $4.55 \mathrm{mmol}, 3.5$ equiv.) and Dess-Martin periodinane $\left(0.75 \mathrm{~g}, 1.76 \mathrm{mmol}, 1.3 \mathrm{equiv}\right.$.) at $2{ }^{\circ} \mathrm{C}$. Then the reaction mixture was stirred at $2-4{ }^{\circ} \mathrm{C}$ for 2 hours. The reaction mixture was diluted with $\mathrm{Et}_{2} \mathrm{O}(15 \mathrm{~mL})$ and added a mixture of saturated aqueous solution of $\mathrm{Na}_{2} \mathrm{~S}_{2} \mathrm{O} 3$ and $\mathrm{NaHCO}_{3}\left(\mathrm{Na}_{2} \mathrm{~S}_{2} \mathrm{O}_{3} / \mathrm{NaHCO}_{3}=1 / 1\right.$, $15 \mathrm{~mL}$ ). The resulting mixture was stirred vigorously for 30 minutes and then the aqueous phase was extracted with $\mathrm{Et}_{2} \mathrm{O}(3 \times 15 \mathrm{~mL})$. The combined organic phase was washed with brine, dried over anhydrous $\mathrm{Na}_{2} \mathrm{SO}_{4}$, filtered, concentrated under reduced pressure. The crude material was purified by flash silica gel chromatography (pet. ether/Et ${ }_{2} \mathrm{O} / \mathrm{Et}_{3} \mathrm{~N}$ $=4 / 1 / 0.04)$ to afford the title compound $(0.159 \mathrm{~g}, 76 \%)$ as a yellow oil. $\mathbf{R}_{f}: 0.11$ (pet. ether/EtOAc = 9:1). ${ }^{1} \mathbf{H}$ NMR $\left(500 \mathrm{MHz}, \mathrm{C}_{6} \mathrm{D}_{6}\right) \delta 9.30(\mathrm{~d}, J=7.8 \mathrm{~Hz}, 1 \mathrm{H}, \mathrm{H}-1), 6.34-$ 6.25 (m, 1H, H-3), 6.25 - 6.17 (m, 1H, H-4), 5.88 (ddd, $J=14.9,7.8,2.8 \mathrm{~Hz}, 1 \mathrm{H}, \mathrm{H}-2$ ), $5.71(\mathrm{dt}, J=15.2,4.6 \mathrm{~Hz}, 1 \mathrm{H}, \mathrm{H}-5), 4.64$ (d, $J=4.3 \mathrm{~Hz}, 1 \mathrm{H}, \mathrm{H}-6), 3.07$ (d, $J=1.4 \mathrm{~Hz}$, 6H, H-7, H-8). ${ }^{13}$ C NMR (125 MHz, C6 $\mathrm{D}_{6}$ ) $\delta 192.5$ (C-1), 149.2 (C-3), 139.3 (C-5), 133.3 (C-2), 131.2 (C-4), 101.3 (C-6), 52.2 (C-7, C-8). FTIR (thin film) v2937, 2830, 1680, 1647, 1602, 1445, 1350, 1188, 1155, 1130, 1103, 1049, 1012, 989, 954, 909, 866, 565, $536 \mathrm{~cm}^{-1}$. HRMS (ESI): Calculated for $\mathrm{C}_{7} \mathrm{H}_{8} \mathrm{O}_{2}{ }^{+}\left[\mathrm{M}-\mathrm{OCH}_{3}\right]^{+}$125.0597; found 125.0590.

\section{Preparation of (S)-5-(((2,2-dimethyl-1,3-dioxolan-4-yl)methyl)sulfonyl)-1-phenyl-}

$1 \mathrm{H}$-tetrazole, 298

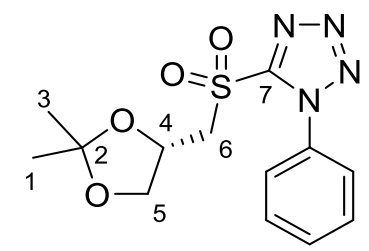

To a solution of thioether 215 ( $0.35 \mathrm{~g}, 1.18 \mathrm{mmol}, 1.0$ equiv.) in EtOH (12 mL) was added the prepared oxidant solution of $\left(\mathrm{NH}_{4}\right)_{6} \mathrm{Mo}_{7} \mathrm{O}_{24}(0.34 \mathrm{~g}, 0.275 \mathrm{mmol}, 0.23$ equiv. $)$ in $\mathrm{H}_{2} \mathrm{O}_{2}$ ( $0.7 \mathrm{~mL}, 30 \%$ in water, $6.18 \mathrm{mmol}, 5.2$ equiv.), and the yellow reaction mixture was stirred for 20 hours at room temperature. Saturated aqueous $\mathrm{Na}_{2} \mathrm{~S}_{2} \mathrm{O}_{3}$ solution $(5 \mathrm{~mL})$ was 
added then ethanol solvent was removed by evaporation under vacuum. The residual aqueous phase was extracted with ethyl acetate $(3 \times 15 \mathrm{~mL})$. The combined organic phase was washed with brine, dried over anhydrous $\mathrm{Na}_{2} \mathrm{SO}_{4}$, filtered, concentrated under reduced pressure. The crude material was purified by flash silica gel chromatography (pet. ether/EtOAc $=2 / 1)$ to afford the title compound $(0.33 \mathrm{~g}, 86 \%)$ as a colourless oil that solidified upon standing at room temperature. $\mathbf{R}_{f}: 0.73$ (pet. ether/EtOAc $=1: 1$ ). ${ }^{1} \mathbf{H}$ NMR $\left(500 \mathrm{MHz}, \mathrm{CDCl}_{3}\right): \delta 7.67-7.56(\mathrm{~m}, 5 \mathrm{H}), 4.67$ (ddt, $\left.J=7.6,6.1,5.0 \mathrm{~Hz} 1 \mathrm{H}, \mathrm{H}-4\right), 4.19$ (dd, $J=8.9,6.1 \mathrm{~Hz}, 1 \mathrm{H}, \mathrm{H}-5 \mathrm{a}$ ), 4.06 (dd, $J=14.7,7.6 \mathrm{~Hz}, 1 \mathrm{H}, \mathrm{H}-6 \mathrm{a}), 3.85$ (dd, $J=9.0$, $5.2 \mathrm{~Hz}, 1 \mathrm{H}, \mathrm{H}-5 \mathrm{~b}$ ), 3.75 (dd, $J=14.7,4.9 \mathrm{~Hz}, 1 \mathrm{H}, \mathrm{H}-6 \mathrm{~b}$ ), 1.32 (s, 3H, H-3), 1.30 (s, 3H, $\mathrm{H}-1) .{ }^{13} \mathrm{C}$ NMR $\left(125 \mathrm{MHz}, \mathrm{CDCl}_{3}\right): \delta 153.3$ (C-7), 133.0, 131.5, 129.6, 125.5, $110.8(\mathrm{C}-$ 2), 69.6 (C-4), 68.4 (C-5), 59.9 (C-6), 26.6 (C-3), 25.2 (C-1). FTIR (thin film) v 2992, 2914, 1496, 1463, 1379, 1370, 1348, 1338, 1319, 1258, 1229, 1184, 1174, 1146, 1107 , 1090, 1081, 1076, 1059, 1021, 1014, 997, 985, 963, 919, 874, 825, 818, 787, 775, 693, 651, 574, 539, 511, 476, 444, $423 \mathrm{~cm}^{-1}$. HRMS (ESI): Calculated for $\mathrm{C}_{13} \mathrm{H}_{16} \mathrm{~N}_{4} \mathrm{NaO}_{4} \mathrm{~S}^{+}$ $[\mathrm{M}+\mathrm{Na}]^{+}$347.0790; found 347.0788. $[\alpha]_{D}^{24}+21.4(c=0.37, \mathrm{DCM})$

\section{Preparation of $(S)-3-((t e r t-b u t y l d i m e t h y l s i l y l) o x y)-4-((1-p h e n y l-1 H$-tetrazol-5-} yl)sulfonyl)butanenitrile, 299

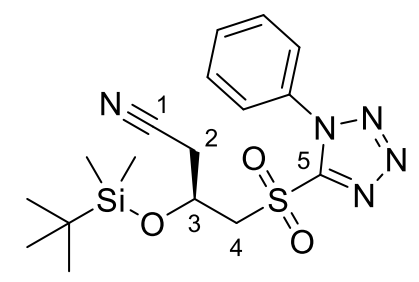

To a solution of nitrile 222 (102.6 mg, $0.273 \mathrm{mmol}, 1.0$ equiv.) in EtOH (3 mL) was added was added a solution of $\left(\mathrm{NH}_{4}\right)_{6} \mathrm{Mo}_{7} \mathrm{O}_{24}\left(31.4 \mathrm{mg}, 0.025 \mathrm{mmol}, 0.09\right.$ equiv.) in $\mathrm{H}_{2} \mathrm{O}_{2}$ (314 $\mu \mathrm{L}, 30 \%$ in water, $2.67 \mathrm{mmol}, 9.8$ equiv.) at $2{ }^{\circ} \mathrm{C}$, and the yellow reaction mixture was stirred at room temperature for 16 hours. Saturated aqueous $\mathrm{Na}_{2} \mathrm{~S}_{2} \mathrm{O}_{3}$ solution $(5 \mathrm{~mL})$ was added then ethanol solvent was removed by evaporation under vacuum. The residual aqueous phase was extracted with ethyl acetate $(3 \times 5 \mathrm{~mL})$. The combined organic phase was washed with brine, dried over anhydrous $\mathrm{Na}_{2} \mathrm{SO}_{4}$, filtered, concentrated under reduced pressure. The crude material was purified by flash silica gel chromatography (pet. ether/EtOAc $=4 / 1)$ to afford the title compound $(88.9 \mathrm{mg}, 80 \%)$ as a colourless oil that solidified upon standing at room temperature. $\mathbf{R}_{f}: 0.48$ (pet. ether/EtOAc $=2: 1$ ). ${ }^{1} \mathbf{H}$ NMR $\left(500 \mathrm{MHz}, \mathrm{CDCl}_{3}\right): \delta 7.70-7.60(\mathrm{~m}, 5 \mathrm{H}), 4.75-4.69(\mathrm{~m}, 1 \mathrm{H}, \mathrm{H}-3), 4.15$ (dd, $J=15.0$, 
$4.8 \mathrm{~Hz}, 1 \mathrm{H}, \mathrm{H}-4 \mathrm{a}), 3.98$ (dd, $J=15.0,6.7 \mathrm{~Hz}, 1 \mathrm{H}, \mathrm{H}-4 \mathrm{~b}), 2.89-2.78$ (m, 2H, H-2), 0.90 (s, 9H), 0.18 (s, 3H), 0.15 (s, 3H). $\left.{ }^{13} \mathbf{C ~ N M R ~ ( 1 2 5 ~ M H z , ~} \mathrm{CDCl}_{3}\right): \delta 153.6(\mathrm{C}-5), 132.8$, 131.7, 129.9, 125.0, 116.0 (C-1), 63.3 (C-3), 61.1 (C-4), 26.7 (C-2), 25.5, 17.8, -4.80, 4.97. FTIR (thin film) v 3402, 2956, 2929, 2858, 1497, 1472, 1463, 1412, 1372, 1344, 1297, 1256, 1220, 1155, 1103, 1068, 1015, 940, 841, 824, 808, 782, 763, 724, 688, 673, 646, 630, 603, 579, 534, $516 \mathrm{~cm}^{-1}$. HRMS (ESI): Calculated for $\mathrm{C}_{17} \mathrm{H}_{25} \mathrm{~N}_{5} \mathrm{NaO}_{3} \mathrm{SSi}^{+}$ $[\mathrm{M}+\mathrm{Na}]^{+} 430.1340$; found 430.1341. $[\alpha]_{D}{ }^{26}+74.8(c=0.05, \mathrm{DCM})$.

Preparation of $(R)-4-((2 E, 4 E, 6 E)-8,8$-dimethoxyocta-2,4,6-trien-1-yl)-2,2-dimethyl1,3-dioxolane, 300

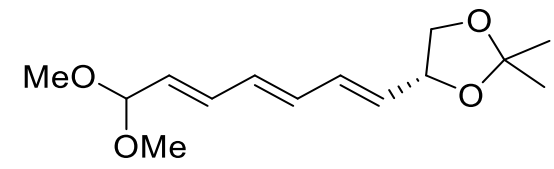

To a solution of sulfone 298 (41.0 mg, $0.126 \mathrm{mmol}, 1.0$ equiv.) in anhydrous THF (1.2 $\mathrm{mL}$ ) was added potassium bis(trimethylsilyl)amide ( $0.28 \mathrm{~mL}, 0.5 \mathrm{M}$ in toluene, 1.1 equiv.) at $-78{ }^{\circ} \mathrm{C}$ under argon atmosphere. The resulting mixture was stirred at $-78{ }^{\circ} \mathrm{C}$ for 15 minutes, then a solution of aldehyde $(28.0 \mathrm{mg}, 0.179 \mathrm{mmol}, 1.3$ equiv.) in anhydrous THF was added slowly. After that, the resulting mixture was allowed to slowly warm up to $20{ }^{\circ} \mathrm{C}$ over 2 hours. The reaction was then quenched by addition of saturated aqueous $\mathrm{NaHCO}_{3}$ solution $(2 \mathrm{~mL})$. The aqueous layers were separated and extracted with ethyl acetate $(3 \times 5 \mathrm{~mL})$. The combined organic phase was washed with brine, dried over anhydrous $\mathrm{Na}_{2} \mathrm{SO}_{4}$, filtered, concentrated under reduced pressure. The crude material was purified by flash silica gel chromatography (pet. ether/EtOAc/Et $3 \mathrm{~N}=20 / 1 / 0.02$ ) to afford the title compound as a mixture of $E, E, E$ - and $E, E, Z$-isomers $(18.7 \mathrm{mg}, 44 \%, E, E, E / E, E, Z$ = 5/1). $\mathbf{R}_{f}: 0.11$ (pet. ether/EtOAc = 9:1). ${ }^{1} \mathbf{H} \mathbf{~ N M R}\left(500 \mathrm{MHz}, \mathrm{CDCl}_{3}\right.$, represents peaks from $E, E, E$-isomer) $\delta 6.45-6.29(\mathrm{~m}, 2 \mathrm{H}), 6.29-6.18(\mathrm{~m}, 2 \mathrm{H}), 5.71-5.64(\mathrm{~m}$, overlapped, 1H), 5.66 (overlapped, dd, $J=15.5,4.8 \mathrm{~Hz}, 1 \mathrm{H}$ ), 4.85 (dd, $J=4.9,1.1 \mathrm{~Hz}$, $1 \mathrm{H}), 4.58-4.52(\mathrm{~m}, 1 \mathrm{H}), 4.10(\mathrm{dd}, J=8.2,6.2 \mathrm{~Hz}, 1 \mathrm{H}), 3.59(\mathrm{dd}, J=15.9,7.9 \mathrm{~Hz} 1 \mathrm{H})$, 3.32 (s, 6H), 1.43 (s, 3H), 1.39 (s, 3H). ${ }^{13} \mathbf{C} \mathbf{~ N M R}\left(125 \mathrm{MHz}, \mathrm{CDCl}_{3}\right) \delta 133.3,133.1$, 131.1, 130.5, 129.9, 129.1, 109.4, 76.8, 69.4, 52.6, 45.7, 26.7, 25.9. FTIR (thin film) $v$ 2955, 2924, 2854, 1460, 1378, 1263, 1123, 1059, 1000, $963 \mathrm{~cm}^{-1}$. HRMS (ESI): Calculated for $\mathrm{C}_{13} \mathrm{H}_{19} \mathrm{O}_{3}{ }^{+}\left[\mathrm{M}-\mathrm{OCH}_{3}\right]^{+} 223.1329$; found 223.1330. $[\alpha]_{D}{ }^{24}-52.1(c=0.135$, DCM). 


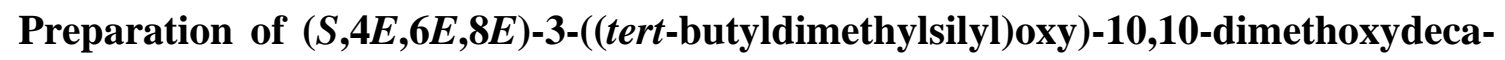
4,6,8-trienenitrile, 301

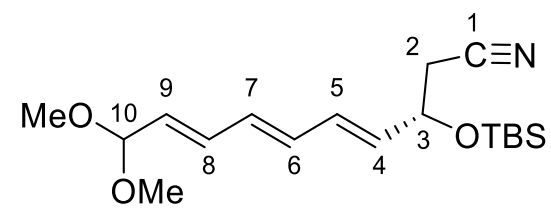

To a solution of sulfone 299 (88.9 mg, $0.218 \mathrm{mmol}, 1.0$ equiv.) in anhydrous THF (7.3 $\mathrm{mL}$ ) was added potassium bis(trimethylsilyl)amide $(444 \mu \mathrm{L}, 0.5 \mathrm{M}$ in toluene, 0.222 mmol, 1.02 equiv.) at $-78{ }^{\circ} \mathrm{C}$ under argon atmosphere. The resulting mixture was stirred at $-78{ }^{\circ} \mathrm{C}$ for 15 minutes, then a solution of aldehyde (34.0 mg, $0.262 \mathrm{mmol}, 1.2$ equiv.) in anhydrous THF was added slowly. After that, the resulting mixture was allowed to slowly warm up to $-10{ }^{\circ} \mathrm{C}$ over 4 hours. The reaction was then quenched by addition of saturated aqueous $\mathrm{NaHCO}_{3}$ solution $(4.5 \mathrm{~mL})$. The aqueous layers were separated and extracted with ethyl acetate $(3 \times 5 \mathrm{~mL})$. The combined organic phase was washed with brine, dried over anhydrous $\mathrm{Na}_{2} \mathrm{SO}_{4}$, filtered, concentrated under reduced pressure. The crude material was purified by flash silica gel chromatography (pet. ether/EtOAc/Et $3 \mathrm{~N}=$ 20/1/0.02) to afford the title compound as a mixture of E,E,E- and E,E,Z-isomers (36.6 $\mathrm{mg}, 50 \%, E, E, E / E, E, Z=10 / 1) . \mathbf{R}_{f}: 0.23$ (pet. ether/EtOAc = 5:1). ${ }^{1} \mathbf{H} \mathbf{N M R}(500 \mathrm{MHz}$, $\mathrm{C}_{6} \mathrm{D}_{6}$, represents peaks from $E, E, E$-isomer) $\delta 6.45$ (ddd, $\left.J=15.5,10.6,1.1 \mathrm{~Hz}, 1 \mathrm{H}, \mathrm{H}-8\right)$, 6.12 - 5.94 (m, 3H, H-5, H-6, H-7), 5.68 (dd, $J=15.5,4.5$ Hz, 1H, H-9), 5.30 (dd, $J=$ 14.8, 6.6 Hz, 1H, H-4), 4.85 (dd, $J=4.4,1.1 \mathrm{~Hz}, 1 \mathrm{H}, \mathrm{H}-10), 3.99$ - 3.94 (m, 1H, H-3), 3.17 (s, 6H), 1.81 (dd, $J=16.4,7.0 \mathrm{~Hz}, 1 \mathrm{H}, \mathrm{H}-2 \mathrm{a}), 1.72$ (dd, $J=16.4,5.0 \mathrm{~Hz}, 1 \mathrm{H}, \mathrm{H}-2 \mathrm{~b})$, 0.96 (s, 9H), 0.06 (s, 3H), -0.01 (s, 3H). ${ }^{13} \mathrm{C} \mathrm{NMR}\left(125 \mathrm{MHz}, \mathrm{C}_{6} \mathrm{D}_{6}\right) \delta 134.0(\mathrm{C}-4), 133.34$ (C-7 or C-8), 133.31 (C-8 or C-7), 132.8 (C-5), 131.6 (C-6), 131.4 (C-9), 117.1 (C-1), 102.1 (C-10), 69.5 (C-3), 52.1, 27.0 (C-2), 25.9, 18.3, -4.4, -4.9. FTIR (thin film) v2954, 2929, 2857, 1463, 1361, 1349, 1255, 1190, 1153, 1122, 1074, 1053, 1001, 940, 908, 880, 837, 809, 779, $687 \mathrm{~cm}^{-1}$. HRMS (ESI): Calculated for $\mathrm{C}_{17} \mathrm{H}_{28} \mathrm{NO}_{2} \mathrm{Si}^{+}\left[\mathrm{M}-\mathrm{OCH}_{3}\right]^{+}$ 306.1884; found 306.1894. $[\alpha]_{D}{ }^{22}+57.3(c=0.39$, DCM $)$ 


\section{Preparation of ethyl (S)-5-(2-(2-hydroxy-3-((1-phenyl-1H-tetrazol-5- yl)sulfonyl)propyl)thiazol-4-yl)pentanoate, 309}

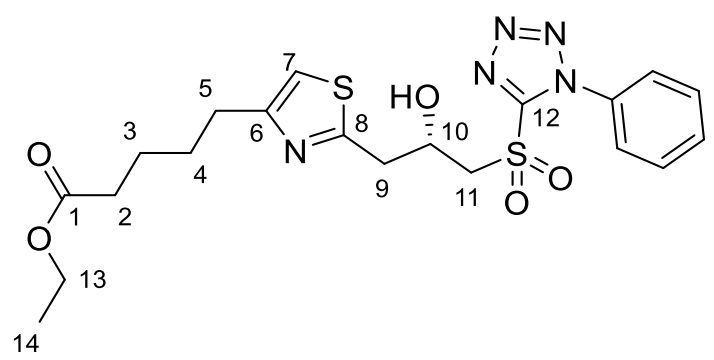

To a solution of sulfone 277 (31.7 mg, $0.052 \mathrm{mmol}, 1.0$ equiv.) in anhydrous THF (0.1 $\mathrm{mL})$ was added a solution of HF-pyridine ( $135 \mu \mathrm{L}, 5.15 \mathrm{mmol}, 99$ equiv.) in anhydrous THF $(0.5 \mathrm{~mL})$ dropwise at $0{ }^{\circ} \mathrm{C}$. The reaction mixture was stirred for 2 days at room temperature and $2.0 \mathrm{~mL}$ saturated $\mathrm{NaHCO}_{3}$ aqueous solution was added. Then the aqueous phase was extracted with ethyl acetate $(3 \times 5 \mathrm{~mL})$. The combined organic phase was washed with brine, dried over anhydrous $\mathrm{Na}_{2} \mathrm{SO}_{4}$, filtered, concentrated under reduced pressure. The combined organic phase was washed with brine, dried over anhydrous $\mathrm{Na}_{2} \mathrm{SO}_{4}$, filtered, concentrated under reduced pressure. The crude material was purified by flash silica gel chromatography (pet. ether/EtOAc $=2 / 1$ ) to afford the title compound (23.2 mg, 92\% yield) as a colourless oil. $\mathbf{R}_{\boldsymbol{f}}$. 0.44 (pet. ether/EtOAc $\left.=1: 1\right) .{ }^{1} \mathbf{H}$ NMR (600 MHz, CDCl 3 ): $\delta 7.72-7.55$ (m, 5H, H-16, H-17, H-18, H-19, H-20), 6.81 (s, 1H, H-7), $4.78-4.70$ (m, 1H, H-10), 4.11 (q, $J=7.1 \mathrm{~Hz}, 2 \mathrm{H}, \mathrm{H}-13$ ), 3.95 (dd, $J=15.0$, $8.7 \mathrm{~Hz}, 1 \mathrm{H}, \mathrm{H}-11 \mathrm{a}), 3.78$ (dd, $J=15.0,3.2 \mathrm{~Hz}, 1 \mathrm{H}, \mathrm{H}-11 \mathrm{~b}), 3.28$ (dd, $J=15.7,3.8 \mathrm{~Hz}$, 1H, H-9a), 3.18 (dd, $J=15.7,7.3 \mathrm{~Hz}, 1 \mathrm{H}, \mathrm{H}-9 \mathrm{~b}), 2.74$ (t, $J=7.2 \mathrm{~Hz}, 2 \mathrm{H}, \mathrm{H}-5$ ), 2.34 (t, $J$ $=7.1 \mathrm{~Hz}, 2 \mathrm{H}, \mathrm{H}-2), 1.76-1.62(\mathrm{~m}, 4 \mathrm{H}, \mathrm{H}-3, \mathrm{H}-4), 1.24$ (t, $J=7.1 \mathrm{~Hz}, 3 \mathrm{H}, \mathrm{H}-14) .{ }^{13} \mathrm{C}$ NMR (150 MHz, $\mathrm{CDCl}_{3}$ ): $\delta 173.6$ (C-1), 164.8 (C-8), 156.9 (C-6), 154.0 (C-12), 133.0, 131.5, 129.5, 125.6, 112.9 (C-7), 65.5 (C-10), 61.2 (C-11), 60.3 (C-13), 37.9 (C-9), 34.0 (C-2), 30.9 (C-5), 28.4 (C-4), 24.4 (C-3), 14.2 (C-14). FTIR (thin film) v 3397, 3105, 2937, 2864, 1769, 1728, 1694, 1595, 1523, 1498, 1461, 1434, 1348, 1311, 1280, 1246, 1176, 1152, 1112, 1076, 1032, 1018, 979, 926, 885, 844, 831, 803, 764, 725, 689, 633, 581, 536, 489, $450 \mathrm{~cm}^{-1}$. HRMS (ESI): Calculated for $\mathrm{C}_{20} \mathrm{H}_{26} \mathrm{~N}_{5} \mathrm{O}_{5} \mathrm{~S}_{2}{ }^{+}[\mathrm{M}+\mathrm{H}]^{+} 480.1370$; found 480.1376. $[\alpha]_{D}{ }^{20}+7.5\left(c=0.99, \mathrm{CHCl}_{3}\right)$. 


\section{Preparation of ethyl 5-(2-allylthiazol-4-yl)pentanoate, 311}

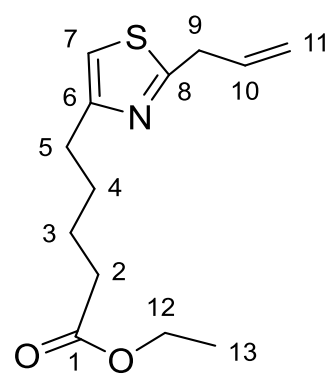

To a solution of alcohol $309(6.8 \mathrm{mg}, 0.014 \mathrm{mmol}, 1.0$ equiv. $)$ in anhydrous THF $(0.5 \mathrm{~mL})$ at $-78{ }^{\circ} \mathrm{C}$ was added KHMDS $(51 \mu \mathrm{L}, 0.5 \mathrm{M}$ in toluene, $0.026 \mathrm{mmol}, 1.8$ equiv.) under argon. Then the reaction mixture was stirred at $-78{ }^{\circ} \mathrm{C}$ for 30 minutes. Deuterium oxide $(20 \mu \mathrm{L})$ was added to the reaction mixture at $-78^{\circ} \mathrm{C}$ and the reaction mixture was stirred at $-78{ }^{\circ} \mathrm{C}$ for 2 hours. The reaction was quenched with $1 \mathrm{~mL}$ saturated aqueous $\mathrm{NH}_{4} \mathrm{Cl}$ solution and then extracted with ethyl acetate $(3 \times 5 \mathrm{~mL})$. The combined organic phase was washed with brine, dried over anhydrous $\mathrm{Na}_{2} \mathrm{SO}_{4}$, filtered, concentrated under reduced pressure. The crude material was purified by flash silica gel chromatography (pet. ether/EtOAc $=3 / 1)$ to afford the title compound $(1.3 \mathrm{mg}, 36 \%$ yield $)$ as a colourless oil. $\mathbf{R}_{f}: 0.50$ (pet. ether/EtOAc = 2:1). ${ }^{1} \mathbf{H}$ NMR $\left(500 \mathrm{MHz}, \mathrm{CDCl}_{3}\right): \delta 6.78(\mathrm{~s}, 1 \mathrm{H}, \mathrm{H}-7), 6.04$ (ddt, $J=16.9,10.0,6.8 \mathrm{~Hz}, 1 \mathrm{H}, \mathrm{H}-10), 5.30-5.17$ (m, 2H, H-11), 4.12 (q, $J=7.2 \mathrm{~Hz}$, 2H, H-12), 3.74 (dt, J = 6.9, 1.4 Hz, 1H, H-9), 2.77 (t, $J=7.1 \mathrm{~Hz}, 2 \mathrm{H}, \mathrm{H}-5$ ), 2.33 (t, $J=$ $7.2 \mathrm{~Hz}, 2 \mathrm{H}, \mathrm{H}-2), 1.78-1.65$ (m, 4H, H-3,H-4), 1.25 (t, $J=7.0 \mathrm{~Hz}, 3 \mathrm{H}, \mathrm{H}-13) .{ }^{13} \mathbf{C}$ NMR (125 MHz, $\left.\mathrm{CDCl}_{3}\right): \delta 173.7$ (C-1), 168.7 (C-8), 156.9 (C-6), 134.0 (C-10), 118.2 (C-11), 112.6 (C-7), 60.3 (C-12), 37.8 (H-9), 34.1 (C-2), 31.2 (C-5), 29.7 (grease peak), 28.7 (C4), 24.6 (C-3), 14.3 (C-13). FTIR (thin film) v2918, 2850, 1731, 1639, 1522, 1461, 1374, 1350, 1299, 1231, 1178, 1136, 1094, 1027, 920, 864, 795, 734, 584, $553 \mathrm{~cm}^{-1}$. HRMS (ESI): Calculated for $\mathrm{C}_{13} \mathrm{H}_{20} \mathrm{NO}_{2} \mathrm{~S}^{+}[\mathrm{M}+\mathrm{H}]^{+} 254.1209$; found 254.1212 


\subsubsection{Towards Synthesis of Thioamide 317}

Preparation of $(R)$-2,2-dimethyl-1,3-dioxolane-4-carbaldehyde, 322

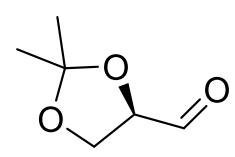

To an oven-dried $50 \mathrm{~mL}$ two-necked round bottom flask equipped with a stir bar and reflux condenser was added D-mannitol 324 (5.0 g, 27.5 mmol, 1.0 equiv.), anhydrous 1,2-dimethoxyethane $(12.0 \mathrm{ml})$ and anhydrous 2,2-dimethoxypropane $(8.0 \mathrm{~mL})$. To this stirred mixture was added $\mathrm{SnCl}_{2}$ (5 mg, 0.001 equiv.) and the reaction mixture was heated at $75{ }^{\circ} \mathrm{C}$ until a clear solution was obtained (ca. 1 hour). The reaction mixture was held at $75{ }^{\circ} \mathrm{C}$ for 30 minutes, then cooled to room temperature and was added pyridine $(6 \mu \mathrm{L}$, $0.075 \mathrm{mmol}, 0.002$ equiv.). The solvents were removed under reduce pressure (contents heated to $\left.80-90{ }^{\circ} \mathrm{C}\right)$. The crude material was slurried in DCM $(36 \mathrm{~mL})$ at room temperature for 1 hour and filtered. The filtrate was evaporated under reduced pressure to afford diacetonide 325 as a white solid (6.3 g, yield 87\%), which was used for the next step without further purification.

To a $100 \mathrm{~mL}$ two-necked round bottom flask equipped with a stir bar and thermometer was added diacetonide 325 (6.3 g, 24 mmol, 1.0 equiv.) in DCM (57 mL). Saturated aqueous $\mathrm{NaHCO}_{3}(2.27 \mathrm{~mL})$ was then added to the flask, maintaining the temperature at or below $25{ }^{\circ} \mathrm{C} . \mathrm{NaIO}_{4}(9.85 \mathrm{~g}, 46 \mathrm{mmol}, 1.9$ equiv. $)$ was added over a 10-min period with vigorous stirring. The reaction was stirred for 2 hours while the temperature was maintained below $30^{\circ} \mathrm{C}$. The solids were then removed by filtration and the filtrate was evaporated under atmospheric pressure at a temperature of $55{ }^{\circ} \mathrm{C}$. The residual oil was transferred to a smaller vessel and distilled at $90 \mathrm{mmHg}$ to afford the title compound as a colourless oil (2.83 g, yield 43\%). Boiling point: $100-105{ }^{\circ} \mathrm{C}(88-90 \mathrm{mmHg}) .{ }^{1} \mathbf{H}$ NMR (500 MHz, $\left.\mathrm{CDCl}_{3}\right): \delta 9.72(\mathrm{dd}, J=1.8 \mathrm{~Hz}, 0.2 \mathrm{~Hz}, 2 \mathrm{H}), 4.39(\mathrm{ddd}, J=7.5,4.7,1.9$ $\mathrm{Hz}, 1 \mathrm{H}), 4.18(\mathrm{dd}, J=8.8,7.5 \mathrm{~Hz}, 1 \mathrm{H}), 4.11(\mathrm{dd}, J=8.8,4.7 \mathrm{~Hz}, 1 \mathrm{H}), 1.49$ (s, 3H), 1.42 (s, 3H). ${ }^{13} \mathrm{C}$ NMR $\left(125 \mathrm{MHz}, \mathrm{CDCl}_{3}\right): 201.9,111.3,79.9,65.6,26.3,25.1 .[\alpha]_{D}{ }^{20}+61(c$ $=1.25$, benzene) (literature $[\alpha]_{D}{ }^{20}+63.3(c=1.25$, benzene). These characterisation data match those reported in the literature. ${ }^{234}$ 
Preparation of 2,2,2-trichloro-1-((R)-2,2-dimethyl-1,3-dioxolan-4-yl)ethan-1-ol, 323<smiles>CC1(C)OCC([C@H](O)C(Cl)(Cl)Cl)O1</smiles>

To a $0{ }^{\circ} \mathrm{C}$ solution of sodium trichloroacetate $(3.89 \mathrm{~g}, 21 \mathrm{mmol}, 1.5$ equiv.) and trichloroacetic acid (3.41 g, $21 \mathrm{mmol}, 1.5$ equiv.) in anhydrous DMF (28 mL) was added aldehyde 322 (1.83 g, 14 mmol, 1.0 equiv.) dissolved in anhydrous DMF (14 mL). After stirring for $15 \mathrm{~min}$, the mixture was warmed to room temperature then stirred rapidly for 17 hours. Upon completion, the mixture was diluted with $80 \mathrm{~mL}$ of EtOAc and washed with saturated aqueous $\mathrm{NaHCO}_{3}$ solution $(3 \times 40 \mathrm{~mL})$. Precipitated solids were filtered and rinsed three times with EtOAc $(3 \times 40 \mathrm{~mL})$. The aqueous phase was extracted with EtOAc $(3 \times 40 \mathrm{~mL})$. The combined organic phase was dried with anhydrous $\mathrm{Na}_{2} \mathrm{SO}_{4}$, filtered and evaporated under reduced pressure. The crude material was purified by flash chromatography through a plug of silica gel using 9:1 hexanes/EtOAc as eluent. The title compound was obtained as a mixture of $(S, R)-\mathbf{3 2 3}$ and $(R, R)-\mathbf{3 2 3}(2.89 \mathrm{~g}$, yield $81 \%)$. A small sample was further purified by silica gel flash chromatography (pet. Ether/EtOAc $=10 / 1)$ to characterise $(S, R)$-isomer and $(R, R)$-isomer.

$(S, R)-323$ : a colourless oil. $\boldsymbol{R}_{\boldsymbol{f}}: 0.57$ (pet. ether/EtOAc $\left.=5: 1\right) .{ }^{1} \mathbf{H} \mathbf{~ N M R}\left(500 \mathrm{MHz}, \mathrm{CDCl}_{3}\right)$ : $\delta 4.58(\mathrm{td}, J=6.8,2.4 \mathrm{~Hz}, 1 \mathrm{H}), 4.25(\mathrm{dd}, J=8.5,6.6 \mathrm{~Hz}, 1 \mathrm{H}), 4.01-3.96(\mathrm{~m}, 1 \mathrm{H}), 3.91$ $(\mathrm{dd}, J=8.5,7.1 \mathrm{~Hz}, 1 \mathrm{H}), 3.80-3.66(\mathrm{~m}, 1 \mathrm{H}), 1.49$ (s, 3H), 1.44 (s, 3H). ${ }^{13}$ C NMR (125 $\left.\mathrm{MHz}, \mathrm{CDCl}_{3}\right): 110.9,101.3,81.1,73.2,68.4,64.7,26.0,25.7$. These characterisation data match those reported in the literature. ${ }^{232}$

$(R, R)-323$ : a white solid. Rf: 0.48 (pet. ether/EtOAc = 5:1). ${ }^{1} \mathbf{H} \mathbf{~ N M R}\left(500 \mathrm{MHz}, \mathrm{CDCl}_{3}\right)$ : $\delta 4.60(\mathrm{td}, J=6.4,3.3 \mathrm{~Hz}, 1 \mathrm{H}), 4.37(\mathrm{~d}, J=3.3 \mathrm{~Hz}, 1 \mathrm{H}), 4.27(\mathrm{dd}, J=8.8,6.3 \mathrm{~Hz}, 1 \mathrm{H})$, 4.10 (dd, $J=8.8,6.5 \mathrm{~Hz}, 1 \mathrm{H}), 3.06$ (br.s, $1 \mathrm{H}), 1.48$ (s, 3H), 1.40 (s, 3H). ${ }^{13}$ C NMR (125 $\left.\mathrm{MHz}, \mathrm{CDCl}_{3}\right): 109.2,100.6,82.3,75.1,64.7,26.3,25.3$. These characterisation data match those reported in the literature. ${ }^{232}$ 


\section{Preparation of (S)-2-(2,2-dimethyl-1,3-dioxolan-4-yl)ethan-1-ol, 330}

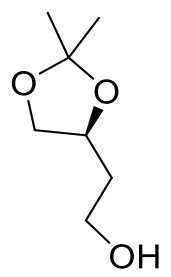

A mixture of diastereomers $(S, R)-\mathbf{3 2 3}$ and $(R, R)-\mathbf{3 2 3}(1.36 \mathrm{~g}, 5.48 \mathrm{mmol}, 1.0$ equiv.) was added to an oven-dried $100 \mathrm{~mL}$ two-necked round-bottom flask equipped with a magnetic stir bar and a reflux condenser under argon. Anhydrous deoxygenated isopropanol (20 $\mathrm{mL}$, purged for 30 minutes with argon) was then added, followed by slow addition of $\mathrm{LiBH}_{4}(2.0 \mathrm{M}$ in THF, $12 \mathrm{~mL}, 24 \mathrm{mmol}, 4.4$ equiv.) and rapid addition of freshly powdered $\mathrm{NaOH}(0.66 \mathrm{~g}, 16.4 \mathrm{mmol}, 3.0$ equiv.) under a blanket of argon. The reaction mixture was heated in a $40{ }^{\circ} \mathrm{C}$ oil bath and allowed to stir at that temperature while being monitored by TLC for the consumption of starting material. It is worth noting that the consumption rate of $(R, R)$-isomer was much faster than $(S, R)$-isomer. The corresponding isopropyl ester intermediate appeared by TLC analysis during the reaction as a spot with a high $R_{f}$ value $\left(R_{f}=0.9\right.$, pet. ether/EtOAc $\left.=5: 1\right)$. It was critical for this intermediate to disappear prior to quenching the reaction to ensure the intermediate was converted to the desired product completely. After 48 hours, the reaction mixture was cooled to room temperature and quenched with saturated aqueous $\mathrm{NH}_{4} \mathrm{Cl}$ solution $(30 \mathrm{~mL})$. The resulting aqueous phase was saturated with solid $\mathrm{NaCl}$ and then extracted with ethyl acetate $(5 \times$ $30 \mathrm{~mL}$ ). The combined organic phase was dried over anhydrous $\mathrm{Na}_{2} \mathrm{SO}_{4}$, filtered, and concentrated under reduced pressure. The crude material was purified by flash silica gel chromatography (pet. ether/EtOAc $=1 / 1)$ to afford the title compound $(244 \mathrm{mg}$, yield $31 \%)$ as a colourless oil. $\mathbf{R}_{f}: 0.17$ (pet. ether/EtOAc $\left.=2: 1\right) .{ }^{1} \mathbf{H} \mathbf{~ N M R}\left(500 \mathrm{MHz}, \mathrm{CDCl}_{3}\right)$ : $\delta 4.32-4.24(\mathrm{~m}, 1 \mathrm{H}), 4.10(\mathrm{dd}, J=8.1,6.0 \mathrm{~Hz}, 1 \mathrm{H}), 3.81(\mathrm{td}, J=5.9,2.1 \mathrm{~Hz}, 2 \mathrm{H}), 3.60$ $(\mathrm{dd}, J=8.1,7.3 \mathrm{~Hz}, 1 \mathrm{H}), 1.85-1.80(\mathrm{~m}, 2 \mathrm{H}), 1.43(\mathrm{~s}, 3 \mathrm{H}), 1.37(\mathrm{~s}, 3 \mathrm{H}) .{ }^{13} \mathbf{C}$ NMR $(125$ $\left.\mathrm{MHz}, \mathrm{CDCl}_{3}\right): \delta 109.2,75.2,69.3,60.5,35.6,26.9,25.6 .[\propto]_{D}{ }^{23}+1.08\left(c=1.24, \mathrm{CHCl}_{3}\right)$ (literature $\left.[\alpha]_{D}^{20}+2.45\left(c=1.1, \mathrm{CHCl}_{3}\right)\right)^{238}$. These characterisation data match those reported in the literature. ${ }^{238}$ 
Preparation of (S)-2-(2,2-dimethyl-1,3-dioxolan-4-yl)acetamide, 321

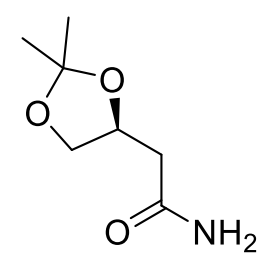

Method A (One-step protocol from alcohol 323)

To a $50 \mathrm{~mL}$ dry pressure tube temporarily fit with a rubber septum and equipped with a magnetic stir bar was added diphenyl diselenide (161.4 mg, $0.517 \mathrm{mmol}, 1.29$ equiv.). Ammonia solution (2.0 $\mathrm{M}$ in ethanol, $1.6 \mathrm{~mL}, 8.0$ equiv.) and $\mathrm{NaBH}_{4}$ (43 mg, $1.12 \mathrm{mmol}$, 2.8 equiv.) were sequentially rapidly added under argon atmosphere. Shortly after the addition, the initial yellow solution turned to be colourless solution, indicating that diphenyl diselenide was completely reduced. The solution was further stirred at room temperature for 25 minutes, then a mixture of diastereomers $(S, R)-\mathbf{3 2 3}$ and $(R, R)-\mathbf{3 2 3}$ (99 $\mathrm{mg}, 0.40 \mathrm{mmol}, 1.0$ equiv.) was added, followed by the addition of freshly powdered $\mathrm{NaOH}$ (43 mg, $1.08 \mathrm{mmol}, 2.7$ equiv.) under argon. The rubber septum was then quickly replaced with a Teflon screw cap and the solution was heated in a $55^{\circ} \mathrm{C}$ oil bath for 36 hours. The ethanolic ammonia was removed by evaporation under vacuum and the amorphous solids were dissolved in $5 \mathrm{~mL}$ ethyl acetate and $5 \mathrm{~mL}$ saturated aqueous $\mathrm{NH}_{4} \mathrm{Cl}$. The resulting solution was cooled to $0{ }^{\circ} \mathrm{C}$ and was carefully adjusted to $\mathrm{pH} 4$ with $1 \mathrm{~N} \mathrm{HCl}$ and then the resulting solution was saturated by the addition of solid $\mathrm{NaCl}$. The mixture was then extracted with ethyl acetate $(6 \times 5 \mathrm{~mL})$. The combined organic layers were dried with anhydrous $\mathrm{Na}_{2} \mathrm{SO}_{4}$ and then concentrated by rotary evaporation. The crude material was purified by flash chromatography through a small plug of triethylamine-saturated silica gel $(\mathrm{EtOAc} / \mathrm{MeOH}=10 / 1)$ to afford the title compound as a white solid (7.1 mg, yield 11\%). $\boldsymbol{R}_{\boldsymbol{f}}: 0.44\left(\right.$ EtOAc/MeOH = 9:1). ${ }^{1} \mathbf{H}$ NMR $(500 \mathrm{MHz}$, $\left.\mathrm{CDCl}_{3}\right) \delta 6.08$ (br. s, $\left.1 \mathrm{H}\right), 5.48$ (br. s, $\left.1 \mathrm{H}\right), 4.47-4.39$ (m, 1H), 4.15 (dd, $J=8.4,6.0 \mathrm{~Hz}$, 1H), $3.63(\mathrm{dd}, J=8.4,6.8 \mathrm{~Hz}, 1 \mathrm{H}), 2.55(\mathrm{dd}, J=15.4,7.9 \mathrm{~Hz}, 1 \mathrm{H}), 2.47$ (dd, $J=15.3$, $4.5 \mathrm{~Hz}, 1 \mathrm{H}), 1.44(\mathrm{~s}, 2 \mathrm{H}), 1.37$ (s, 3H). ${ }^{13} \mathbf{C ~ N M R}\left(126 \mathrm{MHz}, \mathrm{CDCl}_{3}\right) \delta 172.3,109.5,72.2$, $69.1,40.1,26.9,25.5$. These characterisation data match those reported in the literature. ${ }^{232}$

Method B (Two-step protocol from alcohol 330)

To a solution of alcohol 330 (195.3 mg, $1.34 \mathrm{mmol}, 1.0$ equiv.) in $\mathrm{H}_{2} \mathrm{O}-\mathrm{CH}_{3} \mathrm{CN}$ (v:v = 1:1, $10 \mathrm{~mL}$ ) were added TEMPO (65 mg, $0.4 \mathrm{mmol}, 0.3$ equiv.) and BAIB (1.30 g, 4.02 mmol, 3.0 equiv.) at $0{ }^{\circ} \mathrm{C}$. Then the reaction mixture was allowed to warm to room 
temperature and stirred at room temperature for 24 hours. The reaction was then quenched by addition of saturated aqueous $\mathrm{Na}_{2} \mathrm{~S}_{2} \mathrm{O} 3$ solution $(5 \mathrm{~mL})$ and the resulting mixture was stirred at room temperature for 1 hour. The resulting aqueous phase was saturated with solid $\mathrm{NaCl}$ and then extracted with ethyl acetate $(7 \times 5 \mathrm{~mL})$. The combined organic phase was dried over anhydrous $\mathrm{Na}_{2} \mathrm{SO}_{4}$, filtered, and concentrated under reduced pressure to afford the crude carboxylic acid $\mathbf{3 2 9}$ as a yellow oil, which was used directly for the next step without further purification.

The crude carboxylic acid 329 (1.0 equiv.) was dissolved in acetonitrile (12 mL) and then pyridine ( $0.43 \mathrm{~mL}, 5.36 \mathrm{mmol}, 4.0$ equiv.), di-tert-butylbicarbonate (583 mg, $2.68 \mathrm{mmol}$, 2.0 equiv.) and $\mathrm{NH}_{4} \mathrm{HCO}_{3}(219 \mathrm{mg}, 2.68 \mathrm{mmol}, 2.0$ equiv.) were added at room temperature. The reaction mixture was stirred for 14 hours. The reaction mixture was concentrated under reduced pressure and the crude material was purified by silica flash chromatography (EtOAc/MeOH = 20/1) to afford the title compound as a white solid (174 mg, two-step yield 82\%). Melting point: $105-106^{\circ} \mathrm{C}$. $\mathbf{R}_{\boldsymbol{f}}: 0.45$ (EtOAc/MeOH = 10/1). ${ }^{1} \mathbf{H}$ NMR (500 MHz, $\left.\mathrm{CDCl}_{3}\right): \delta 6.10$ (br. s, 1H), 5.73 (br. s, 1H), $4.46-4.40(\mathrm{~m}, 1 \mathrm{H})$, $4.15(\mathrm{dd}, J=8.4,6.0 \mathrm{~Hz}, 1 \mathrm{H}), 3.63(\mathrm{dd}, J=8.4,6.8 \mathrm{~Hz}, 1 \mathrm{H}), 2.55(\mathrm{dd}, J=15.4,7.9 \mathrm{~Hz}$, 1H), $2.48(\mathrm{dd}, J=15.4,4.6 \mathrm{~Hz}, 1 \mathrm{H}), 1.44$ (s, 3H), 1.38 (s, 3H). ${ }^{\mathbf{1 3}} \mathbf{C}$ NMR (125 MHz, $\mathrm{CDCl}_{3}$ ): $\delta 172.5,109.5,72.3,69.1,40.1,26.9,25.5$. FTIR (solid) $v$ 3360, 3179, 2991, 2940, 2899, 2858, 1656, 1631, 1431, 1420, 1371, 1328, 1256, 1236, 1213, 1157, 1135, 1097, 1064, 1042, 970, 924, 899, 842, 700, 659, 644, 618, 583, 566, 513, $431 \mathrm{~cm}^{-1}$. HRMS (ESI): Calculated for $\mathrm{C}_{7} \mathrm{H}_{13} \mathrm{NaO}_{3} \mathrm{~N}^{+}[\mathrm{M}+\mathrm{Na}]^{+} 182.0793$; found 182.0785. $[\alpha]_{D}{ }^{25}$ $-17.1\left(c=1.03, \mathrm{CHCl}_{3}\right)$ (literature $\left.[\alpha]_{D}{ }^{24}-15.4\left(c=1.0, \mathrm{CHCl}_{3}\right)\right)$. These characterisation data match those reported in the literature. ${ }^{232}$ 


\section{Preparation of (S)-(1-amino-2-(2,2-dimethyl-1,3-dioxolan-4-yl)ethylidene)- $\lambda^{4}$ -}

sulfanone, 333

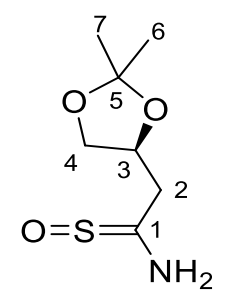

Lawesson's reagent (freshly re-purified by washing with $\mathrm{DCM}$ and $\mathrm{Et}_{2} \mathrm{O}, 36.5 \mathrm{mg}, 0.09$ mmol, 0.55 equiv.) was added to a solution of amide ( $25.6 \mathrm{mg}, 0.16 \mathrm{mmol}, 1.0$ equiv.) in anhydrous DCM. The reaction mixture was stirred vigorously under argon atmosphere at room temperature for 12 hours. Saturated aqueous $\mathrm{NaHCO}_{3}$ solution $(3 \mathrm{~mL})$ was added to the reaction mixture and the aqueous phase was extracted with ethyl acetate $(3 \times 5 \mathrm{~mL})$. The combined organic phase was dried over anhydrous $\mathrm{Na}_{2} \mathrm{SO}_{4}$, filtered, and then concentrated under reduced pressure. The crude material was purified by silica flash chromatography (pet. Ether/EtOAc $=2 / 1$ ) to afford the title compound as a yellow oil (7.2 mg, yield 23\%). $\mathbf{R}_{f}: 0.43$ (pet. Ether/EtOAc = 1/1). ${ }^{1} \mathbf{H} \mathbf{N M R}\left(500 \mathrm{MHz}, \mathrm{CDCl}_{3}\right): \delta$ 7.77 (br. s, 1H, N-H), 7.54 (br. s, 1H, N-H), 4.46 (dtd, $J=8.5,6.5,3.3$ Hz, 1H, H-3), 4.14 (dd, $J=8.4,6.1 \mathrm{~Hz}, 1 \mathrm{H}, \mathrm{H}-4 \mathrm{a}$ ), 3.65 (dd, $J=8.4,6.7 \mathrm{~Hz}, 1 \mathrm{H}, \mathrm{H}-4 \mathrm{~b}$ ), 3.04 (ddd, $J=15.3$, 3.3, 1.0 Hz, 1H, H-2a), 2.91 (dd, $J=15.3,8.4$ Hz, 1H, H-2b), 1.45 (s, 3H, H-6 or H-7), 1.37 (s, 3H, H-7 or H-6). ${ }^{13}$ C NMR (125 MHz, $\left.\mathrm{CDCl}_{3}\right)$ : $\delta 206.1$ (C-1), $109.9(\mathrm{C}-5), 73.8$ (C-3), 68.6 (C-4), 48.8 (C-2), 27.0 (C-6 or C-7), 25.5 (C-7 or C-6). FTIR (solid) v 3312, 3188, 2984, 2933, 1654, 1636, 1629, 1438, 1420, 1381, 1372, 1324, 1249, 1214, 1152, 1106, 1060, 965,929, 884, 832, $512 \mathrm{~cm}^{-1}$. HRMS (ESI): Calculated for $\mathrm{C}_{7} \mathrm{H}_{14} \mathrm{NO}_{3} \mathrm{~S}^{+}$ $[\mathrm{M}+\mathrm{H}]^{+}$192.0689; found 192.0685. $[\alpha]_{D}^{22}-67.5(c=0.215, \mathrm{DCM})$.

\subsubsection{Attempted Preparation of Air-stable Thioamides}

\section{Preparation of $(S)$-3,4-dihydroxybutanamide, 339}

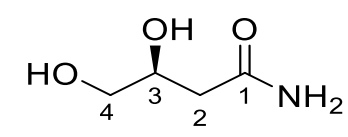

Amide 321 (25.4 mg, $0.091 \mathrm{mmol}, 1.0$ equiv.) was dissolved in a mixture of acetic acid $(0.10 \mathrm{~mL})$ and water $(25 \mu \mathrm{L})$. Then the reaction mixture was stirred at $40{ }^{\circ} \mathrm{C}$ for 2 hours under argon. The reaction mixture was then cooled to room temperature and saturated $\mathrm{NaHCO}_{3}$ aqueous solution $(1 \mathrm{~mL})$ was slowly added. All solvents were evaporated under reduced pressure at $45{ }^{\circ} \mathrm{C}$ and the solids was slurried with acetone and filtered. The filtrate 
was evaporated to afford the title compound as a white solid $(17.5 \mathrm{mg}$, yield $92 \%) .{ }^{1} \mathrm{H}$ NMR ((CD $\left.)_{2} \mathrm{CO}, 500 \mathrm{MHz}\right): \delta 6.94$ (br. s, $\left.1 \mathrm{H},-\mathrm{NH}\right), 6.30$ (br. s, $\left.1 \mathrm{H},-\mathrm{NH}\right), 3.93-3.87$ (m, 1H, H-3), 3.42 (d, $J=5.4$ Hz, 2H, H-4), 3.26 (s, 1H, -OH), 2.37 (dd, 1H, $J=15.1$, $4.0 \mathrm{~Hz}, \mathrm{H}-2 \mathrm{a}), 2.25$ (dd, $1 \mathrm{H}, J=15.0,8.3 \mathrm{~Hz}, \mathrm{H}-2 \mathrm{~b}) ;{ }^{13} \mathrm{C} \mathrm{NMR}\left(\left(\mathrm{CD}_{3}\right)_{2} \mathrm{CO}, 125 \mathrm{MHz}\right): \delta$ 174.8 (C-1), 69.9 (C-3), 66.5 (C-4), 39.4 (C-2). These characterisation data match those reported in the literature. ${ }^{232}$

\section{Preparation of $(S)-3,4-b i s((t e r t-b u t y l d i m e t h y l s i l y l) o x y)$ butanamide, 339}

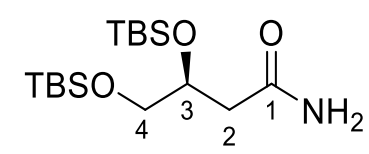

Imidazole (525 mg, $7.71 \mathrm{mmol}, 8.0$ equiv.) and tert-butyldimethylsilyl chloride ( $859 \mathrm{mg}$, $5.7 \mathrm{mmol}, 6.0$ equiv.) were added to a solution of amide 339 (100 mg, $0.95 \mathrm{mmol}, 1.0$ equiv.) in $5.0 \mathrm{~mL}$ anhydrous DMF. The reaction mixture was stirred at room temperature for 16 hours, and the reaction was diluted with $10 \mathrm{~mL}$ ethyl acetate, and washed 3 times with $5 \mathrm{~mL}$ of water. The combined aqueous phase was then extracted with extracted with ethyl acetate $(3 \times 5 \mathrm{~mL})$. The combined organic layers were dried with anhydrous $\mathrm{Na}_{2} \mathrm{SO}_{4}$, filtered, and then concentrated by rotary evaporation. The crude material was purified by flash silica chromatography (pet. Ether/EtOAc $=3 / 1$ ) to afford the title compound as a yellow oil (254 mg, yield 77\%). Rf: 0.43 (pet. Ether/EtOAc = 2/1). ${ }^{\mathbf{1}} \mathbf{H}$ NMR (500 MHz, $\mathrm{CDCl}_{3}$ ): $\delta 6.21$ (br. s, $\left.1 \mathrm{H}, \mathrm{N}-\mathrm{H}\right), 5.41$ (br. s, $\left.1 \mathrm{H}, \mathrm{N}-\mathrm{H}\right), 4.10-4.03$ (m, 1H, H-3), 3.62 (dd, $J=10.1,5.3 \mathrm{~Hz}, 1 \mathrm{H}, \mathrm{H}-4 \mathrm{a}), 3.50$ (dd, $J=10.2,6.4 \mathrm{~Hz}, 1 \mathrm{H}, \mathrm{H}-4 \mathrm{~b}), 2.57$ (dd, $J=14.8,4.4$ Hz, 1H, H-2a), 2.34 (dd, J = 14.7, 6.1 Hz, 1H, H-2b), 0.89 (s, 18H), 0.10 (s, 6H), 0.054 (s, 3H), 0.049 (s, 3H). ${ }^{13} \mathrm{C}$ NMR (125 MHz, $\left.\mathrm{CDCl}_{3}\right)$ : 173.6 (C-1), 70.4 (C-4), 66.5 (C-4), 40.9 (C-2), 25.9, 25.8, 18.3, 18.0, -4.53, -5.01, -5.43, -5.44. FTIR (thin film) $v 3324$, 3192, 2954, 2929, 2886, 2857, 1668, 1616, 1472, 1463, 1403, 1361, 1253, 1115, 1080, 1005, 982, 939, 830, 811, 775, 728, 667, 573, $452 \mathrm{~cm}^{-1}$. HRMS (ESI): Calculated for $\mathrm{C}_{16} \mathrm{H}_{38} \mathrm{NO}_{3} \mathrm{Si}_{2}{ }^{+}[\mathrm{M}+\mathrm{H}]^{+}$348.2385; found 348.2396. $[\alpha]_{D}^{25}-9.5(c=3.81$, EtOAc). 
Preparation of $(S)$-(1-amino-3,4-bis((tert-butyldimethylsilyl)oxy)butylidene)- $\lambda^{4}$ sulfanone, 340

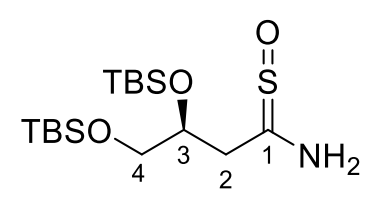

Lawesson's reagent ( $27.2 \mathrm{mg}, 0.067 \mathrm{mmol}, 0.7$ equiv.) was added to a solution of amide 339 (28.8 mg, $0.083 \mathrm{mmol}, 1.0$ equiv.) in anhydrous DCM $(0.8 \mathrm{~mL})$ under argon. The reaction mixture was stirred vigorously at room temperature for 30 minutes. The reaction was quenched with $2 \mathrm{~mL}$ aqueous saturated $\mathrm{NaHCO}_{3}$ solution and the aqueous solution was extracted with diethyl ether $(3 \times 5 \mathrm{~mL})$. The combined organic layers were dried with anhydrous $\mathrm{Na}_{2} \mathrm{SO}_{4}$, filtered and then concentrated by rotary evaporation. The crude material was purified by a short silica plug (pet. Ether/Et ${ }_{2} \mathrm{O}=5 / 1$ ). The fractions were collected and evaporated at room temperature under vacuum to afford the title compound as a colourless oil (12.3 mg, yield 39\%). ${ }^{1} \mathbf{H}$ NMR (500 MHz, $\left.\mathrm{CDCl}_{3}\right): \delta 7.51$ (br. s, $2 \mathrm{H}$, N-H), $4.01-3.93$ (m, 1H, H-3), 3.61 (dd, $J=10.2,4.9$ Hz, 1H, H-4a), 3.46 (dd, $J=10.1$, $7.7 \mathrm{~Hz}, 1 \mathrm{H}, \mathrm{H}-4 \mathrm{~b}), 2.67$ (dd, $J=14.9,4.4 \mathrm{~Hz}, 1 \mathrm{H}, \mathrm{H}-2 \mathrm{a}), 2.53$ (dd, $J=14.9,4.6 \mathrm{~Hz}, 1 \mathrm{H}$, H-2b), 0.92 (s, 9H), 0.91 (s, 9H), 0.13 (s, 3H), 0.11 (s, 3H), 0.09 (s, 3H), 0.08 (s, 3H). ${ }^{13} \mathbf{C}$ NMR (125 MHz, $\mathrm{CDCl}_{3}$ ): 191.0 (C-1), 71.53 (C-3), 65.29 (C-4), 32.41 (C-2), 25.84, 25.76, 18.20, 17.95, -4.65, -4.96, -5.41, -5.46. FTIR (thin film) $v 2953,2928,2856,1470$, 1463, 1361, 1253, 1112, 1078, 1004, 936, 834, 809, 776, 721, $669 \mathrm{~cm}^{-1}$. HRMS (ESI): Calculated for $\mathrm{C}_{16} \mathrm{H}_{38} \mathrm{NO}_{3} \mathrm{SSi}_{2}{ }^{+}[\mathrm{M}+\mathrm{H}]^{+} 380.2105$; found 380.2116. $[\alpha]_{D}^{22}+19.4(c=$ $0.57, \mathrm{DCM})$. 


\section{Preparation of (S)-2-(2,2-dimethyl-1,3-dioxolan-4-yl)- $N$-(4-}

methoxybenzyl)acetamide, 346

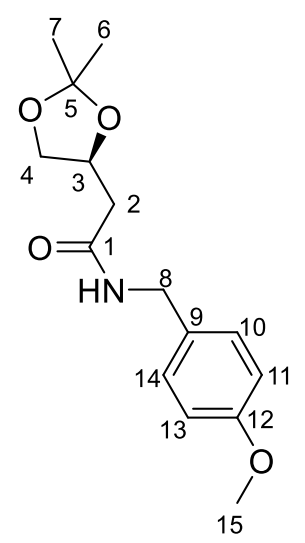

Diphenyl diselenide (491 mg, $1.57 \mathrm{mmol}, 1.3$ equiv.) was added to a dry round-bottom flask equipped with stir bar under a blanket of argon. Deoxygenated absolute ethanol (purged for 30 minutes with argon) was then added, followed by the rapid addition of $\mathrm{NaBH}_{4}$ (133 mg, $3.5 \mathrm{mmol}, 2.87$ equiv.). Shortly after the addition, the initial yellow solution turned colourless, indicating that diphenyl diselenide was completely reduced. The solution stirred at room temperature for 30 minutes, then a mixture of diastereomers (304 mg, $1.21 \mathrm{mmol}, 1.0$ equiv.), freshly powdered $\mathrm{NaOH}$ (110 mg, $3.02 \mathrm{mmol}, 2.5$ equiv.) and 4-methoxybenzylamine ( $0.19 \mathrm{~mL}, 1.45 \mathrm{mmol}, 1.2$ equiv.) were added sequentially under argon. The mixture was heated in a $55{ }^{\circ} \mathrm{C}$ oil bath and allowed to react at this temperature for 36 hours while monitoring by TLC for consumption of starting material. Upon completion, ethanol was removed by rotary evaporation under reduced pressure and the amorphous orange solids were dissolved in $5 \mathrm{~mL}$ saturated $\mathrm{NH}_{4} \mathrm{Cl}$ and extracted with ethyl acetate $(5 \times 10 \mathrm{~mL})$. The combined organic layers were dried with anhydrous $\mathrm{Na}_{2} \mathrm{SO}_{4}$, filtered, and then concentrated by rotary evaporation. The crude material was purified by flash silica chromatography (pet. Ether/EtOAc $=1 / 2$ ) to afford the desired product 346 as a yellow oil (183 mg, yield 54\%) and the by-product 347 as a yellow oil (28.3 mg, yield 6\%).

346: $\mathbf{R}_{\boldsymbol{f}}$. 0.6 (pet. Ether/EtOAc = 1/4). ${ }^{1} \mathbf{H}$ NMR $\left(500 \mathrm{MHz}, \mathrm{CDCl}_{3}\right): \delta 7.23-7.18(\mathrm{~m}$, 2H, H-10, H-14), 6.89 - 6.84 (m, 2H, H-11, H-13), 6.25 (br. s, 1H, N-H), $4.47-4.41$ (m, $1 \mathrm{H}, \mathrm{H}-3), 4.39$ (dd, $J=5.6,1.7 \mathrm{~Hz}, 2 \mathrm{H}, \mathrm{H}-8), 4.14$ (dd, $J=8.3,6.0 \mathrm{~Hz}, 1 \mathrm{H}, \mathrm{H}-4 \mathrm{a}), 3.80$ (s, 3H, H-15), 3.63 (dd, $J=8.3,6.9 \mathrm{~Hz}, 1 \mathrm{H}, \mathrm{H}-4 \mathrm{~b}), 2.51$ (dd, $J=6.2,3.2 \mathrm{~Hz}, 2 \mathrm{H}, \mathrm{H}-2$ ), 1.38 (s, 3H, H-6 or H-7), 1.35 (s, 3H, H-7 or H-6). ${ }^{13} \mathbf{C ~ N M R ~ ( 1 2 5 ~ M H z , ~ C D C l ~} 3$ ): $\delta 169.7$ (C-1), 159.0 (C-12), 130.2 (C-9), 129.0 (C-10, C-14), 114.0 (C-11, C-13), 109.4 (C-5), 72.5 (C-3), 69.1 (C-4), 55.3 (C-15), 43.0 (C-8), 40.5 (C-2), 26.9 (C-6 or C-7), 25.5 (C-7 
or C-6). FTIR (thin film) v3305, 2985, 2934, 1643, 1613, 1585, 1542, 1511, 1458, 1441, 1370, 1301, 1244, 1176, 1154, 1111, 1062, 1032, 832, 587, $513 \mathrm{~cm}^{-1}$. HRMS (ESI): Calculated for $\mathrm{C}_{15} \mathrm{H}_{22} \mathrm{NO}_{4}{ }^{+}[\mathrm{M}+\mathrm{H}]^{+}$280.1543; found 280.1541. $[\alpha]_{D}{ }^{26}-10.3(c=0.39$, DCM).

2-((S)-2,2-Dimethyl-1,3-dioxolan-4-yl)- $N$-(4-methoxybenzyl)-2-((4methoxybenzyl)amino)acetamide, 347

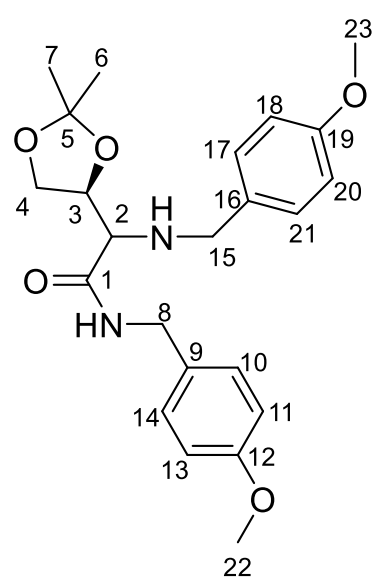

$\mathbf{R}_{f:} 0.78$ (pet. Ether/EtOAc = 1/4). ${ }^{1} \mathbf{H} \mathbf{N M R}\left(500 \mathrm{MHz}, \mathrm{CDCl}_{3}\right): \delta 7.71(\mathrm{t}, J=5.6 \mathrm{~Hz}, 1 \mathrm{H}$, N-H), 7.20 - 7.16 (m, 2H, H-10, H-14), $7.14-7.10$ (m, 2H, H-17, H-21), $6.89-6.84$ (m, 2H, H-11, H-13), 6.84 - 6.80 (m, 2H, H-18, H-20), 4.40 (dd, J=14.6, 6.1 Hz, 1H, H-8a), 4.33 (dd, $J=14.6,5.7 \mathrm{~Hz}, 1 \mathrm{H}, \mathrm{H}-8 \mathrm{~b}$ ), $4.20-4.15$ (m, 1H, H-3), 4.03 (dd, $J=6.0,1.3 \mathrm{~Hz}$, 2H, H-4), 3.80 (s, 3H, H-22 or H-23), 3.79 (s, 3H, H-22 or H-23), 3.70 (d, J=13.0 Hz, 1H, H-15a), 3.61 (d, J=13.0 Hz, 1H, H-15b), 3.19 (d, $J=7.2 \mathrm{~Hz}, 1 \mathrm{H}, \mathrm{H}-2), 1.36$ (s, 3H, H-6 or H-7), 1.32 (s, 3H, H-7 or H-6). ${ }^{13}$ C NMR (125 MHz, CDCl 3 ): $\delta 171.2(\mathrm{C}-1), 158.9$ (C-12), 158.8 (C-19), 131.3 (C-6), 130.3 (C-9), 129.4 (C-17, C-21), 128.9 (C-10, C-14), 114.1 (C-11, C-13), 113.9 (C-18, C-20), 109.4 (C-5), 76.2 (C-3), 67.1 (C-4), 64.8 (C-2), 55.29 (C-22 or C-23), 55.27 (C-22 or C-23), 52.2 (C-15), 42.5 (C-8), 26.6 (C-6 or C-7), 25.2 (C-7 or C-6). FTIR (thin film) v 3337, 2986, 2932, 2836, 1664, 1612, 1585, 1511, 1461, 1441, 1371, 1301, 1245, 1176, 1154, 1111, 1067, 1034, 820, 755, 572, $514 \mathrm{~cm}^{-1}$. HRMS (ESI): Calculated for $\mathrm{C}_{23} \mathrm{H}_{31} \mathrm{~N}_{2} \mathrm{O}_{5}{ }^{+}[\mathrm{M}+\mathrm{H}]^{+}$415.2233; found 415.2221. $[\alpha]_{D}{ }^{26}$ $+19.7(c=1.42, \mathrm{DCM})$. 


\section{Preparation of $(S)$-3,4-dihydroxy- $N$-(4-methoxybenzyl)butanamide, 348}

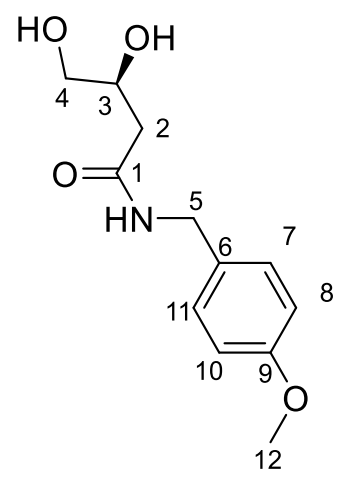

Amide 346 ( $84.7 \mathrm{mg}, 0.3 \mathrm{mmol}, 1.0$ equiv.) was dissolved in a mixture of acetic acid $(0.24 \mathrm{~mL})$ and water $(0.06 \mathrm{~mL})$. Then the reaction mixture was stirred at $40{ }^{\circ} \mathrm{C}$ for 2 hours. Then acetic acid and water was evaporated under reduced pressure. The crude material was purified by flash silica chromatography $(\mathrm{EtOAc} / \mathrm{MeOH}=10 / 1)$ to afford the title compound as a white solid (45.7 mg, yield 63\%). Melting point: $117-118{ }^{\circ} \mathrm{C}$. $\mathbf{R}_{f}: 0.25$ $(\mathrm{EtOAc} / \mathrm{MeOH}=10 / 1) .{ }^{1} \mathbf{H} \mathbf{~ N M R}\left(500 \mathrm{MHz}, \mathrm{CDCl}_{3}\right): \delta 7.23-7.19(\mathrm{~m}, 2 \mathrm{H}, \mathrm{H}-7, \mathrm{H}-11)$, $6.90-6.85$ (m, 2H, H-8, H-10), 5.97 (br. s, 1H, N-H), 4.39 (d, J = 5.6 Hz, 2H, H-5), 4.15 - 4.08 (m, 1H, H-3), 4.00 (br. s, 1H, -OH), 3.80 (s, 3H, H-12), 3.69 (dd, J=11.2, 3.7 Hz, 1H, H-4a), 3.53 (dd, $J=11.2,5.6 \mathrm{~Hz}, 1 \mathrm{H}, \mathrm{H}-4 \mathrm{~b}$ ), 2.47 (dd, $J=15.4,8.7 \mathrm{~Hz}, 1 \mathrm{H}, \mathrm{H}-2 \mathrm{a}$ ), 2.37 (dd, $J=15.4,3.3 \mathrm{~Hz}, 1 \mathrm{H}, \mathrm{H}-2 \mathrm{~b}), 2.19$ (br. s, $1 \mathrm{H},-\mathrm{OH}) .{ }^{13} \mathbf{C ~ N M R}\left(125 \mathrm{MHz}, \mathrm{CDCl}_{3}\right)$ : $\delta 171.8$ (C-1), 159.2 (C-9), 129.8 (C-6), 129.2 (C-7, C-11), 114.2 (C8, C-10), 68.8 (C-3), 65.9 (C-4), 55.3 (C-12), 43.1 (C-5), 38.6 (C-2). FTIR (thin film) v 3303, 1651, 1631, 1588, 1546, 1515, 1464, 1456, 1441, 1414, 1328, 1305, 1252, 1223, 1181, 1099, 1072 , 1034, 820, 809, 745, $585 \mathrm{~cm}^{-1}$. HRMS (ESI): Calculated for $\mathrm{C}_{12} \mathrm{H}_{18} \mathrm{NO}_{4}{ }^{+}[\mathrm{M}+\mathrm{H}]^{+}$ 240.1230; found 240.1225. $[\alpha]_{D}{ }^{22}-15.6(c=0.45, \mathrm{MeOH})$. 
Preparation of (S)-4-((tert-butyldiphenylsilyl)oxy)-3-hydroxy- $N$-(4methoxybenzyl)butanamide, 349

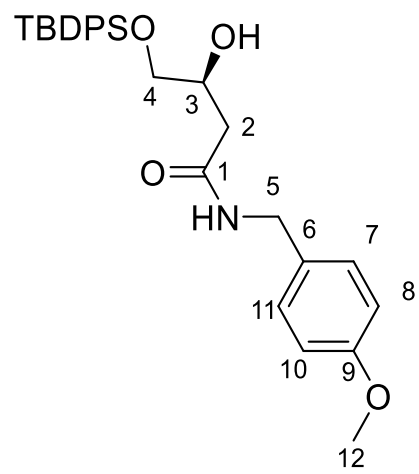

Imidazole (7.9 mg, $0.116 \mathrm{mmol}, 1.45$ equiv.) and TBDPSCl (23 $\mu \mathrm{L}, 0.088 \mathrm{mmol}, 1.05$ equiv.) were added to a solution of amide 348 (20 mg, $0.08 \mathrm{mmol}, 1.0$ equiv.) in $0.8 \mathrm{~mL}$ anhydrous DMF. The reaction mixture was stirred at room temperature for 16 hours, and the reaction was diluted with $5 \mathrm{~mL}$ ethyl acetate, and washed 3 times with $3 \mathrm{~mL}$ of water. The combined aqueous phase was then extracted with extracted with ethyl acetate $(3 \times 3$ $\mathrm{mL}$ ). The combined organic layers were dried with anhydrous $\mathrm{Na}_{2} \mathrm{SO}_{4}$, filtered, and then concentrated by rotary evaporation. The crude material was purified by flash silica chromatography to afford the title compound $\mathbf{3 4 9}$ as a colourless oil (7.6 mg, yield 19\%) and bis-TBDPS side-product $\mathbf{3 5 0}$ as a colourless oil $(6.5 \mathrm{mg}$, yield 11\%).

349: $\mathbf{R}_{f}: 0.34$ (pet. Ether/EtOAc = 1/1). ${ }^{1} \mathbf{H}$ NMR $\left(500 \mathrm{MHz}, \mathrm{CDCl}_{3}\right): \delta 7.65-7.61(\mathrm{~m}$, $4 \mathrm{H}), 7.46-7.34(\mathrm{~m}, 6 \mathrm{H}), 7.23-7.18$ (m, 2H, H-7, H-11), $6.89-6.84$ (m, 2H, H-8, H10), 6.38 (t, $J=5.7 \mathrm{~Hz}, 1 \mathrm{H},-\mathrm{NH}), 4.37$ (d, $J=5.6 \mathrm{~Hz}, 2 \mathrm{H}, \mathrm{H}-5), 4.16-4.08$ (m, 1H, H3), 3.80 (s, 3H, H-12), 3.64 (dd, $J=10.2,4.9 \mathrm{~Hz}, 1 \mathrm{H}, \mathrm{H}-4 \mathrm{a}), 3.60$ (dd, $J=10.2,6.5 \mathrm{~Hz}$, 1H, H-4b), $2.44-2.34$ (m, 2H, H-2), 2.08 (s, 1H, -OH), 1.05 (s, 9H). ${ }^{13}$ C NMR (125 $\mathrm{MHz}, \mathrm{CDCl}_{3}$ ): 171.3 (C-1), 159.0 (C-9), 135.5, 132.9, 130.1 (C-6), 129.9, 129.1 (C-7, C11), 127.8, 114.1 (C-8, C-10), 69.1 (C-3), 67.1 (C-4), 55.3 (C-12), 43.0 (C-5), 39.3 (C-2), 26.8, 19.2. FTIR (thin film) v 3309, 2954, 2930, 2857, 1654, 1642, 1613, 1588, 1577, 1560, 1542, 1535, 1513, 1487, 1462, 1438, 1428, 1390, 1362, 1302, 1248, 1175, 1112, 1076, 1035, 998, 823, 741, 702, 692, 614, 504, $489 \mathrm{~cm}^{-1}$. HRMS (ESI): Calculated for $\mathrm{C}_{28} \mathrm{H}_{36} \mathrm{NO}_{4} \mathrm{Si}^{+}[\mathrm{M}+\mathrm{H}]^{+}$478.2408; found 478.2359. $[\alpha]_{D}^{26}-11.2(c=0.37, \mathrm{DCM})$. 
(S)-3,4-Bis((tert-butyldiphenylsilyl)oxy)- $N$-(4-methoxybenzyl)butanamide, 350

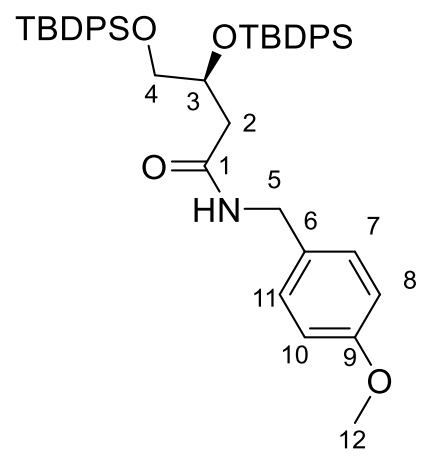

$\mathbf{R}_{f}: 0.50$ (pet. Ether/EtOAc = 3/1). ${ }^{1} \mathbf{H}$ NMR $\left(500 \mathrm{MHz}, \mathrm{CDCl}_{3}\right): \delta 7.60-7.45(\mathrm{~m}, 8 \mathrm{H})$, $7.43-7.34(\mathrm{~m}, 4 \mathrm{H}), 7.33-7.23(\mathrm{~m}, 8 \mathrm{H}), 7.12-7.06$ (m, 2H, H-7, H-11), $6.82-6.77$ (m, $2 \mathrm{H}, \mathrm{H}-8, \mathrm{H}-10), 6.23$ (t, $J=5.4 \mathrm{~Hz}, 1 \mathrm{H}, \mathrm{N}-\mathrm{H}), 4.30$ (dd, $J=14.3,5.6 \mathrm{~Hz}, 1 \mathrm{H}, \mathrm{H}-5 \mathrm{a}), 4.22$ (dd, $J=14.7,5.2 \mathrm{~Hz}, 1 \mathrm{H}, \mathrm{H}-5 \mathrm{~b}), 4.23-4.16$ (m, 1H, H-3), 3.79 (s, 3H, H-12), 3.57 3.53 (m, 2H, H-4), 2.57 (dd, $J=14.8,5.1 \mathrm{~Hz}, 1 \mathrm{H}, \mathrm{H}-2 \mathrm{a}), 2.43$ (dd, $J=14.8,5.2 \mathrm{~Hz}, 1 \mathrm{H}$, H-2b), 0.97 (s, 9H), 0.95 (s, 9H). ${ }^{13}$ C NMR (125 MHz, CDCl 3 ): 170.3 (C-1), 159.0 (C9), 135.8, 135.7 135.6, 135.5, 133.3, 133.22, 133.21, 133.11, 130.4 (C-6), 129.9, 129.7, 129.60, 129.57, 129.4 (C-7, C-11), 127.7, 127.64, 127.63, 127.59, 114.0 (C-8, C-10), 71.2 (C-3), 66.3 (C-4), 55.3 (C-12), 43.0 (C-5), 40.7 (C-2), 26.9, 26.8, 19.2, 19.1. FTIR (thin film) $v 3307,3070,3049,2929,2856,1649,1613,1588,1512,1463,1427,1390,1361$, 1302, 1248, 1176, 1111, 1082, 1037, 998, 938, 821, 739, 701, 612, 505, 489, $431 \mathrm{~cm}^{-1}$. HRMS (ESI) Calculated for $\mathrm{C}_{44} \mathrm{H}_{54} \mathrm{NO}_{4} \mathrm{Si}_{2}{ }^{+}[\mathrm{M}+\mathrm{H}]^{+}$716.3586; found 716.3573. $[\alpha]_{D}{ }^{26}$ $+140.2(c=0.21, \mathrm{DCM})$

Preparation of $(S)-4-(($ tert-butyldiphenylsilyl)oxy)- $N$-(4-methoxybenzyl)-3(methoxymethoxy)butanamide, 351

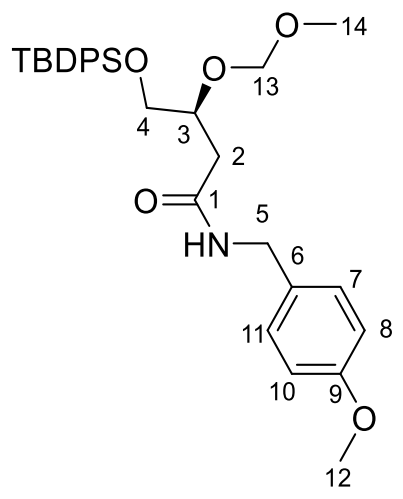

To a solution of amide $\mathbf{3 4 9}$ (6.7 mg, $0.014 \mathrm{mmol}, 1.0$ equiv.) in anhydrous DCM (0.8 mL) were added sequentially a solution of $N, N$-diisopropylethylamine $(185 \mu \mathrm{L}, 1.06 \mathrm{mmol}$, 75 equiv. $)$ in anhydrous DCM $(1.1 \mathrm{~mL})$ and a solution of methoxymethyl chloride $(57 \mu \mathrm{L}$, 
technical grade $90 \%$ purity, $0.67 \mathrm{mmol}, 48$ equiv. $)$ in anhydrous DCM $(0.75 \mathrm{~mL})$ at $2{ }^{\circ} \mathrm{C}$. Then the reaction mixture was stirred at room temperature for 66 hours. Saturated aqueous $\mathrm{NaHCO}_{3}$ solution $(2 \mathrm{~mL})$ was added slowly and then the resulting mixture was stirred vigorously at room temperature for 15 minutes to destroy residual methoxymethyl chloride. The aqueous phase was extracted with ethyl acetate $(3 \times 5 \mathrm{~mL})$. The combined organic phase was washed with brine, dried over anhydrous $\mathrm{Na}_{2} \mathrm{SO}_{4}$, filtered, concentrated under reduced pressure. The crude material was purified by flash silica gel chromatography (pet. ether/EtOAc $=3 / 1)$ to afford the title compound $(4.8 \mathrm{mg}, 37 \%$ yield) as a colourless oil. $\mathbf{R}_{f}: 0.65$ (pet. Ether/EtOAc $\left.=1 / 1\right) .{ }^{1} \mathbf{H} \mathbf{~ N M R}\left(500 \mathrm{MHz}, \mathrm{CDCl}_{3}\right): \delta$ $7.67-7.60(\mathrm{~m}, 4 \mathrm{H}), 7.45-7.34(\mathrm{~m}, 6 \mathrm{H}), 7.12-7.06(\mathrm{~m}, 2 \mathrm{H}, \mathrm{H}-7, \mathrm{H}-11), 6.88-6.80$ (m, 2H, H-8, H-10), 6.24 (t, J = 5.0 Hz, 1H, N-H), 4.66 - 4.60 (m, 2H, H-13), 4.36 (d, J = 5.5 Hz, 2H, H-5), 4.12 - 4.06 (m, 1H, H-3), 3.79 (s, 3H, H-12), 3.71 (dd, J=10.8, 5.1 Hz, 1H, H-4a), 3.66 (dd, $J=10.7,5.0 \mathrm{~Hz}, 1 \mathrm{H}, \mathrm{H}-4 \mathrm{~b}$ ), 3.22 (s, 3H, H-14), 2.59 (dd, $J=14.9$, $3.9 \mathrm{~Hz}, 1 \mathrm{H}, \mathrm{H}-2 \mathrm{a}), 2.45$ (dd, $J=15.0,8.2 \mathrm{~Hz}, 1 \mathrm{H}, \mathrm{H}-2 \mathrm{~b}), 1.04$ (s, 9H). ${ }^{13} \mathrm{C}$ NMR (125 $\mathrm{MHz}_{\mathrm{CDCl}}$ ): 170.4 (C-1), 159.0 (C-9), 135.5, 133.1, 130.3 (C-6), 129.8, 129.2 (C-7, C11), 127.7, 114.0 (C-8, C-10), 96.4 (C-13), 75.3 (C-3), 65.6 (C-4), 55.6 (C-14), 55.3 (C12), 43.1 (C-5), 39.5 (C-2), 26.8, 19.2. FTIR (thin film) v3320, 2927, 2854, 1641, 1559, 1513, 1462, 1451, 1428, 1302, 1248, 1176, 1150, 1112, 1034, 823, 741, 702, 614, 505, $491 \mathrm{~cm}^{-1}$. HRMS (ESI): Calculated for $\mathrm{C}_{30} \mathrm{H}_{40} \mathrm{NO}_{5} \mathrm{Si}_{2}{ }^{+}[\mathrm{M}+\mathrm{H}]^{+}$522.2670; found 522.2688. $[\alpha]_{D}{ }^{25}-6.6(c=0.24, \mathrm{DCM})$ 


\subsection{Experimental for Chapter Three}

\subsubsection{Synthesis of Thioamide 382}

\section{Preparation of (S)-3-hydroxy-4-(trityloxy)butanamide, 374}

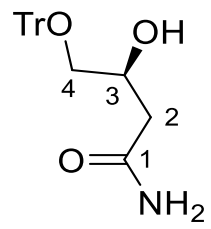

Amide 339 (64.8 mg, $0.61 \mathrm{mmol}, 1.0$ equiv.) and trityl chloride (208 mg, $0.74 \mathrm{mmol}, 1.2$ equiv.) were dissolved in anhydrous DMF (1 mL) under argon atmosphere. Then anhydrous pyridine $(0.15 \mathrm{~mL})$ was added to the solution, which was stirred at room temperature for 27 hours. The reaction was quenched by addition of ice water $(1.5 \mathrm{~mL})$, and the resulting mixture was diluted with $10 \mathrm{~mL}$ ethyl acetate and washed 3 times with $3 \mathrm{~mL}$ of water. The combined aqueous phase was then extracted with ethyl acetate $(3 \times 5$ $\mathrm{mL}$ ). The combined organic layers were dried with anhydrous $\mathrm{Na}_{2} \mathrm{SO}_{4}$, filtered, and then concentrated by rotary evaporation. The crude material was purified by flash silica chromatography (pet. Ether/EtOAc $=1 / 4$ ) to afford the desired product as white crystalline solid (201 mg, yield 90\%). $\mathbf{R}_{\boldsymbol{f}} .0 .39$ (pet. Ether/EtOAc = 1/4). ${ }^{\mathbf{1}} \mathbf{H}$ NMR (500 $\mathrm{MHz} \mathrm{CDCl}_{3}$ ): $\delta 7.44-7.39(\mathrm{~m}, 6 \mathrm{H}), 7.33-7.22$ (m, 9H), 6.03 (br. s, 1H), 5.39 (br. s, $1 \mathrm{H}), 4.21-4.15(\mathrm{~m}, 1 \mathrm{H}), 3.19-3.16(\mathrm{~m}, 2 \mathrm{H}), 2.42-2.39(\mathrm{~m}, 2 \mathrm{H}) .{ }^{13} \mathbf{C}$ NMR (125 MHz, $\left.\mathrm{CDCl}_{3}\right): 174.1,143.6,128.6,127.7,127.1,86.8,67.7,66.7,39.2$. These characterisation data match those reported in the literature. ${ }^{348}$

\section{Preparation of (S)-3-((tert-butyldimethylsilyl)oxy)-4-(trityloxy)butanamide, 375}

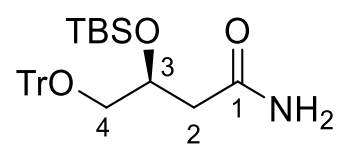

Imidazole (120 mg, $1.77 \mathrm{mmol}, 3.18$ equiv.) and tert-butyldimethylsilyl chloride (119 mg, $0.79 \mathrm{mmol}, 1.42$ equiv.) were added to a solution of amide 374 (201 mg, $0.556 \mathrm{mmol}, 1.0$ equiv.) in $1.0 \mathrm{~mL}$ anhydrous DMF. The reaction mixture was stirred at room temperature for 16 hours, and the reaction was quenched by addition of $2 \mathrm{~mL}$ of distilled water. The resulting mixture was diluted with $10 \mathrm{~mL}$ ethyl acetate, and washed 3 times with $3 \mathrm{~mL}$ of water. The combined aqueous phase was then extracted with extracted with ethyl acetate $(3 \times 5 \mathrm{~mL})$. The combined organic layers were dried with anhydrous $\mathrm{Na}_{2} \mathrm{SO}_{4}$, filtered, 
and then concentrated by rotary evaporation. The crude material was purified by flash silica chromatography (pet. Ether/EtOAc $=3 / 1$ ) to afford the title compound as a colourless oil (193 mg, yield 73\%). $\mathbf{R}_{\boldsymbol{f}}$ : 0.59 (pet. Ether/EtOAc = 1/1). ${ }^{\mathbf{1}} \mathbf{H}$ NMR (500 $\left.\mathrm{MHz} \mathrm{CDCl}_{3}\right): \delta 7.46-7.41(\mathrm{~m}, 6 \mathrm{H}), 7.32-7.27(\mathrm{~m}, 6 \mathrm{H}), 7.25-7.20(\mathrm{~m}, 3 \mathrm{H}), 6.06$ (br.s, 1H, N-H), 5.29 (br. s, 1H, N-H), 4.16 (tt, $J=6.4,4.7$ Hz, 1H, H-3), 3.15 (dd, $J=9.4,4.9$ Hz, 1H, H-4a), 3.09 (dd, J = 9.4, 6.4 Hz, 1H, H-4b), 2.68 (dd, $J=14.6,4.5 \mathrm{~Hz}, 1 \mathrm{H}, \mathrm{H}-$ 2a), 2.42 (dd, $J=14.6,6.4 \mathrm{~Hz}, 1 \mathrm{H}, \mathrm{H}-2 \mathrm{~b}), 0.84$ (s, 9H), 0.03 (s, 3H), -0.04 (s, 3H). ${ }^{13} \mathrm{C}$ NMR (125 MHz, $\left.\mathrm{CDCl}_{3}\right)$ : 173.6, 143.8, 128.6, 127.8, 127.0, 86.7, 69.0, 66.6, 41.6, 25.7, 17.9, -4.7, -5.1. FTIR (thin film) v 3338, 3187, 3059, 3032, 2953, 2928, 2884, 2856, 1671, 1598, 1490, 1471, 1463, 1448, 1402, 1361, 1253, 1220, 1184, 1154, 1074, 1033, 1000, 954, 899, 832, 812, 776, 746, 704, 646, 632, 574, 532, $501 \mathrm{~cm}^{-1}$. HRMS (ESI): Calculated for $\mathrm{C}_{2} \mathrm{H}_{37} \mathrm{NNaO}_{3} \mathrm{Si}^{+}[\mathrm{M}+\mathrm{H}]^{+} 498.2440$; found 498.2433. $[\alpha]_{D}{ }^{25}-8.1(c=1.36$, DCM).

\section{Preparation of $(S)$-4-((tert-butyldimethylsilyl)oxy)-3-hydroxybutanamide, 378}<smiles>CC(C)OCC(O)CC(N)=O</smiles>

To a solution of amide 339 (75.8 mg, $0.22 \mathrm{mmol}, 1.0$ equiv.) in MeOH-DCM (v/v = 1/1, $2.4 \mathrm{~mL}$ ) was added ( \pm )-10-camphorsulfonic acid (51 mg, $0.22 \mathrm{mmol}, 1.0$ equiv.) over 5 portions over 1 minute at $0{ }^{\circ} \mathrm{C}$. The reaction mixture was stirred at $0{ }^{\circ} \mathrm{C}$ for 20 minutes and then added $1 \mathrm{~mL}$ saturated aqueous $\mathrm{NaHCO}_{3}$ solution. All solvents were then evaporated under reduced pressure. The solid residue was slurried with $5 \mathrm{~mL}$ ethyl acetate and filtered. The filtrate was evaporated and the crude material was then purified by silica flash chromatography (pet. Ether/EtOAc $=1 / 4$ ) to afford the title compound as a colourless oil. $\mathbf{R}_{\boldsymbol{f}}$ : 0.22 (pet. Ether/EtOAc = 1/2). ${ }^{1} \mathbf{H} \mathbf{N M R}\left(500 \mathrm{MHz}, \mathrm{CDCl}_{3}\right) \delta 6.25(\mathrm{~s}$, $1 \mathrm{H}, \mathrm{N}-\mathrm{H}), 5.51$ (s, 1H, N-H), 4.03 (tt, $J=6.9,4.7 \mathrm{~Hz}, 1 \mathrm{H}, \mathrm{H}-3), 3.62$ (dd, $J=10.0,4.6$ Hz, 1H, H-4a), 3.54 (dd, $J=10.0,6.6$ Hz, 1H, H-4b), $2.41-2.38$ (m, 2H, H-2), 0.90 (s, 9H), 0.08 (s, 3H). ${ }^{13} \mathrm{C}$ NMR (125 MHz, $\left.\mathrm{CDCl}_{3}\right) \delta 173.9$ (C-1), 68.7 (C-3), 66.3 (C-4), 38.9 (C-2), 25.8, 18.3, -5.40, -5.43. FTIR (solid) v3371, 3205, 3125, 2952, 2926, 2877, 2854, 1678, 1656, 1620, 1468, 1435, 1410, 1393, 1362, 1348, 1321, 1279, 1254, 1201, 1120, 1105, 1075, 1047, 1007, 938, 888, 834, 779, 684, 667, 574, 508, 457, $446 \mathrm{~cm}^{-1}$. HRMS (ESI): Calculated for $\mathrm{C}_{10} \mathrm{H}_{24} \mathrm{NO}_{3} \mathrm{Si}^{+}[\mathrm{M}+\mathrm{H}]^{+} 234.1520$; found 234.1504. $[\alpha]_{D}{ }^{21}$ $+104(c=0.084, \mathrm{MeOH})$. 
Preparation of $(S)-4-(($ tert-butyldiphenylsilyl)oxy)-3-hydroxybutanamide, 380<smiles>CC(C)OC(C)CC(N)=O</smiles>

Imidazole (400 mg, $5.87 \mathrm{mmol}, 1.55$ equiv.) and tert-butyldiphenylchlorosilane (1.04 mL, $4.00 \mathrm{mmol}, 1.05$ equiv.) were added to a solution of amide 339 (400 mg, $3.8 \mathrm{mmol}, 1.0$ equiv.) in $3.8 \mathrm{~mL}$ anhydrous DMF. The reaction mixture was stirred at room temperature for 24 hours, and the reaction was quenched with distilled water $(5 \mathrm{~mL})$. Then DMF and water was removed at $60{ }^{\circ} \mathrm{C}$ by oil pump. The residue was then purified by flash silica chromatography (pet. ether/EtOAc $=1 / 2$ ) to afford the title compound as a colourless oil (911 mg, yield 67\%). $\mathbf{R}_{f}: 0.23$ (pet. Ether/EtOAc = 1/1). ${ }^{1} \mathbf{H} \mathbf{~ N M R}\left(500 \mathrm{MHz}, \mathrm{CDCl}_{3}\right): \delta$ $7.67-7.60$ (m, 4H), $7.47-7.36$ (m, 6H), 6.21 (br. s, 1H, N-H), 5.72 (br. s, 1H,N-H), 4.16 -4.05 (m, 1H, H-3), 3.66 (dd, $J=10.2,4.6 \mathrm{~Hz}, 1 \mathrm{H}, \mathrm{H}-4 \mathrm{a}), 3.60$ (dd, $J=10.2,6.6 \mathrm{~Hz}, 1 \mathrm{H}$, H-4b), 3.26 (br. s, O-H), $2.42-2.37$ (m, 2H, H-2), 1.07 (s, 9H). ${ }^{13}$ C NMR (125 MHz, $\mathrm{CDCl}_{3}$ ): 174.2 (C-1), 135.4, 132.81, 132.79, 129.946, 129.938, 127.8, 68.8 (C-3), 67.0 (C-4), 38.8 (C-2), 26.8, 19.2. FTIR (thin film) v 3338, 3202, 2956, 2930, 2857, 1663, 1617, 1589, 1472, 1462, 1427, 1391, 1361, 1111, 1070, 1007, 998, 823, 803, 740, 700, 621, 613, 504, $489 \mathrm{~cm}^{-1}$. HRMS (ESI): Calculated for $\mathrm{C}_{30} \mathrm{H}_{40} \mathrm{NO}_{5} \mathrm{Si}_{2}{ }^{+}[\mathrm{M}+\mathrm{H}]^{+} 358.1833$; found 358.1838. $[\alpha]_{D}^{25}-24.4(c=0.41, \mathrm{DCM})$.

\section{Preparation of $(S)-3-(($ tert-butyldimethylsilyl)oxy)-4-((tert-}

\section{butyldiphenylsilyl)oxy)butanamide, 381}

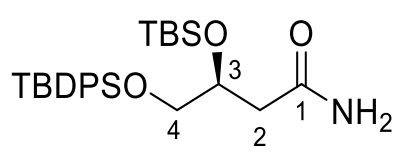

Imidazole (5.44 g, $79.3 \mathrm{mmol}, 6.0$ equiv.) and tert-butyldimethylsilyl chloride (5.97 g, 39.6 mmol, 3.0 equiv.) were added to a solution of amide $\mathbf{3 8 0}(911 \mathrm{mg}, 2.55 \mathrm{mmol}, 1.0$ equiv.) in $50 \mathrm{~mL}$ anhydrous DMF. The reaction mixture was stirred at $30-35^{\circ} \mathrm{C}$ for 14 days under nitrogen atmosphere. Then reaction mixture was quenched by addition of 50 $\mathrm{mL}$ ice-water. Then DMF and water was removed at $60^{\circ} \mathrm{C}$ by oil pump. The residue was re-dissolved in ethyl acetate $(200 \mathrm{~mL})$, and washed with distilled water $(3 \times 50 \mathrm{~mL})$. The combined aqueous phase was then extracted with ethyl acetate $(3 \times 50 \mathrm{~mL})$. The combined organic phase was dried with anhydrous $\mathrm{Na}_{2} \mathrm{SO}_{4}$, filtered, and then concentrated by rotary evaporation. The crude material was purified by flash silica chromatography (pet. ether/EtOAc $=2 / 1$ ) to afford the title compound as a colorless oil 
(6.12 g, yield 98\%). $\mathbf{R}_{f}: 0.30$ (pet. Ether/EtOAc = 2/1). ${ }^{1} \mathbf{H}$ NMR $\left(500 \mathrm{MHz}, \mathrm{CDCl}_{3}\right): \delta$ $7.68-7.62$ (m, 4H), $7.46-7.34$ (m, 6H), 6.11 (br. s, 1H, N-H), 5.29 (br. s, 1H,N-H), 4.15 -4.05 (m, 1H, H-3), 3.63 (dd, $J=10.2,4.8 \mathrm{~Hz}, 1 \mathrm{H}, \mathrm{H}-4 \mathrm{a}), 3.54$ (dd, $J=10.1,7.0 \mathrm{~Hz}, 1 \mathrm{H}$, H-4b), 2.66 (dd, $J=14.8,4.1 \mathrm{~Hz}, 1 \mathrm{H}, \mathrm{H}-2 \mathrm{a}), 2.42$ (dd, $J=14.8,6.3 \mathrm{~Hz}, 1 \mathrm{H}, \mathrm{H}-2 \mathrm{~b}$ ), 1.04 (s, 9H), 0.83 (s, 9H), 0.03 (s, 3H), -0.06 (s, 3H). ${ }^{13} \mathbf{C ~ N M R ~ ( 1 2 5 ~ M H z , ~ C D C l ~} 3$ ): $173.4(\mathrm{C}-$ 1), 135.6, 135.5, 133.2, 133.1, 129.8, 129.7, 127.74, 127.73, 70.2 (C-3), 66.9 (C-4), 41.0 (C-2), 26.8, 25.7, 19.2, 17.9, -4.7, -5.1. FTIR (thin film) v3327, 3190, 3072, 2954, 2929, 2890, 2857, 1670, 1612, 1590, 1472, 1463, 1427, 1390, 1361, 1253, 1189, 1110, 1075, 999, 983, 964, 939, 824, 804, 777, 739, 700, 665, 613, 504, $490 \mathrm{~cm}^{-1}$. HRMS (ESI):

Calculated for $\mathrm{C}_{26} \mathrm{H}_{42} \mathrm{NO}_{3} \mathrm{Si}_{2}{ }^{+}[\mathrm{M}+\mathrm{H}]^{+} 472.2698$; found 472.2704. $[\alpha]_{D}{ }^{25}+71.7(c=0.35$, DCM)

\section{Preparation of (S)-3-((tert-butyldimethylsilyl)oxy)-4-((tert-}

butyldiphenylsilyl)oxy)butanethioamide, 382

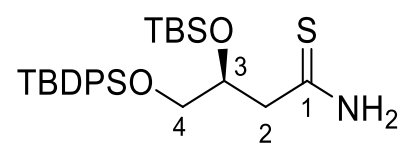

Lawesson's reagent ( $1.54 \mathrm{~g}, 3.82 \mathrm{mmol}, 0.6$ equiv.) was added to a solution of amide $\mathbf{3 8 1}$ (3.06 g, $6.36 \mathrm{mmol}, 1.0$ equiv.) in anhydrous deoxygenated DCM (63 mL) under argon atmosphere. The reaction mixture was stirred vigorously at room temperature for 20 minutes. Upon completion, the reaction mixture was directly loaded onto a short silica plug and purified using DCM as eluent rapidly. The fractions were immediately collected and evaporated at $10{ }^{\circ} \mathrm{C}$ under vacuum to afford the title compound as a yellow oil (1.71 g, yield 54\%). $\mathbf{R}_{f}$ : 0.37 (DCM).

Notes: Tendency towards oxidation by air was observed. Upon purification, the product should be stored in the freezer at $-20{ }^{\circ} \mathrm{C}$ under argon atmosphere.

${ }^{1} \mathbf{H}$ NMR (500 MHz, $\mathrm{CDCl}_{3}$ ): $\delta 7.76$ (br. s, 1H, N-H), $7.69-7.63$ (m, 4H), $7.46-7.36$ (m, 7H), $4.14-4.08$ (m, 1H, H-3), 3.64 (dd, $J=10.3,4.7 \mathrm{~Hz}, 1 \mathrm{H}, \mathrm{H}-4 \mathrm{a}), 3.58$ (dd, $J=$ 10.4, $6.8 \mathrm{~Hz}, 1 \mathrm{H}, \mathrm{H}-4 \mathrm{~b}), 3.11$ (dd, $J=14.4,4.1 \mathrm{~Hz}, 1 \mathrm{H}, \mathrm{H}-2 \mathrm{a}), 3.00$ (dd, $J=14.4,6.0 \mathrm{~Hz}$, 1H, H-2b), 1.06 (s, 9H), 0.83 (s, 9H), 0.04 (s, 3H), -0.06 (s, 3H). ${ }^{13}$ C NMR (125 MHz, $\left.\mathrm{CDCl}_{3}\right): \delta 207.7(\mathrm{C}-1), 135.6,135.5,133.1,132.9,129.81,129.77,127.77,127.74,72.3$ (C-3), 66.4 (C-4), 49.7 (C-2), 26.8, 25.8, 19.2, 17.9, -4.7, -5.0. FTIR (thin film) v 3290, 3169, 3071, 2954, 2929, 2887, 2856, 1618, 1471, 1462, 1427, 1406, 1390, 1361, 1325, $1255,1188,1111,1071,998,939,834,823,803,778,739,701,690,665,622,613,505$, 
$492 \mathrm{~cm}^{-1}$. HRMS (ESI): Calculated for $\mathrm{C}_{26} \mathrm{H}_{42} \mathrm{NO}_{2} \mathrm{SSi}_{2}{ }^{+}[\mathrm{M}+\mathrm{H}]^{+}$488.2469; found 488.2500. $[\alpha]_{D}^{25}-97.8(c=0.92, \mathrm{DCM})$.

\subsubsection{Synthesis of Alkyne 364}

Preparation of 2-(4-methoxybenzyl)-1,3-dioxane, 385<smiles></smiles>

To a solution of 1,3-propanediol $384(6.3 \mathrm{~mL}, 87.3 \mathrm{mmol}, 1.2$ equiv.) and $p$-anisaldehyde ( $8.8 \mathrm{~mL}, 72.7 \mathrm{mmol}, 1.0$ equiv.) in toluene $(50 \mathrm{~mL})$ was added $p$-toluenesulfonic acid (114 mg, 0.008 equiv.) as a catalyst. The reaction mixture was refluxed at $110{ }^{\circ} \mathrm{C}$ in a Dean-Stark apparatus for 17 hours. The reaction mixture was then allowed to cool to room temperature and was quenched by addition of saturated aqueous $\mathrm{NaHCO}_{3}$ solution $(2 \mathrm{~mL})$. The resulting mixture was washed with distilled water $(50 \mathrm{~mL})$, and the aqueous fractions were re-extracted with was extracted with diethyl ether $(2 \times 50 \mathrm{~mL})$. The combined organic phase was dried with anhydrous $\mathrm{Na}_{2} \mathrm{SO}_{4}$, filtered, and then concentrated by rotary evaporation. The crude material was purified by flash silica chromatography (pet. ether/EtOAc $=8 / 1)$ to afford the provide the title compound as a brown crystal $(12.6 \mathrm{~g}$, yield 90\%). $\mathbf{R}_{f}: 0.38$ (pet. ether/EtOAc = 5:1). ${ }^{1} \mathbf{H} \mathbf{N M R}\left(500 \mathrm{MHz}, \mathrm{CDCl}_{3}\right): \delta 7.45-7.40$ $(\mathrm{m}, 2 \mathrm{H}), 6.92-6.88(\mathrm{~m}, 2 \mathrm{H}), 5.46(\mathrm{~s}, 1 \mathrm{H}), 4.30-4.24(\mathrm{~m}, 2 \mathrm{H}), 4.03-3.96(\mathrm{~m}, 2 \mathrm{H}), 3.80$ $(\mathrm{s}, 3 \mathrm{H}), 2.24(J=13.5,12.4,5.0 \mathrm{~Hz}, 1 \mathrm{H}), 1.46(\mathrm{dtt}, J=13.5,2.7,1.5 \mathrm{~Hz}, 1 \mathrm{H}) .{ }^{13} \mathbf{C ~ N M R}$ $\left(125 \mathrm{MHz}, \mathrm{CDCl}_{3}\right): \delta 159.9,131.3,127.2,113.4,101.6,67.4,55.3,25.7$. These characterisation data match those reported in the literature. ${ }^{349}$

\section{Preparation of 3-((4-methoxybenzyl)oxy)propan-1-ol, 386}

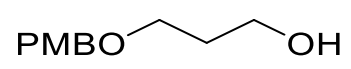

To a solution of acetal 385 (12.6 g, 64.9 mmol, 1.0 equiv.) in anhydrous DCM (400 mL) at $-78^{\circ} \mathrm{C}$ was a solution of diisobutylaluminium hydride $(129.8 \mathrm{~mL}, 1.0 \mathrm{M}$ in cyclohexane, $129.8 \mathrm{mmol}, 2.0$ equiv.) slowly at $-78^{\circ} \mathrm{C}$. The reaction mixture was allowed to warm to room temperature slowly over 2 hours and stirred at room temperature for 16 hours. The reaction was then quenched by addition of a saturated aqueous solution of potassium sodium tartrate $(270 \mathrm{~mL})$ and the resulting mixture was stirred at room temperature for 2 hours until the cloudy suspension turned to be a clear mixture. The aqueous phase was separated and extracted with ethyl acetate $(3 \times 100 \mathrm{~mL})$. The combined organic phase 
was dried with anhydrous $\mathrm{Na}_{2} \mathrm{SO}_{4}$, filtered, and then concentrated by rotary evaporation to afford the provide the pure title compound as a colourless oil (11.6 g, yield 91\%), which was used for the next step without further purification. $\mathbf{R}_{f}: 0.32$ (pet. ether/EtOAc $=1: 1$ ). ${ }^{1} \mathbf{H}$ NMR $\left(500 \mathrm{MHz}, \mathrm{CDCl}_{3}\right): \delta 7.27(\mathrm{~d}, J=8.7 \mathrm{~Hz}, 2 \mathrm{H}), 6.90(\mathrm{~d}, J=8.6 \mathrm{~Hz}, 2 \mathrm{H}), 4.47$ $(\mathrm{s}, 2 \mathrm{H}), 3.82(\mathrm{~s}, 3 \mathrm{H}), 3.79(\mathrm{t}, J=5.6 \mathrm{~Hz}, 2 \mathrm{H}), 3.66(\mathrm{t}, J=5.8 \mathrm{~Hz}, 2 \mathrm{H}), 1.87$ (quin, $J=5.7$ $\mathrm{Hz}, 2 \mathrm{H}) .{ }^{13} \mathrm{C}$ NMR $\left(125 \mathrm{MHz}, \mathrm{CDCl}_{3}\right): \delta 159.2,130.1,129.3,113.8,72.9,69.2,62.1$, $55.3,32.0$. These characterisation data match those reported in the literature. ${ }^{349}$

\section{Preparation of 3-((4-methoxybenzyl)oxy)propanal, 387}

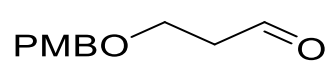

To a solution of anhydrous dimethyl sulfoxide (17.0 mL, $0.234 \mathrm{~mol}, 4.0$ equiv.) in anhydrous DCM (300 mL) at $-78^{\circ} \mathrm{C}$ was added freshly distilled oxalyl chloride $(10.0 \mathrm{~mL}$, 0.118 mol, 2.0 equiv.). The reaction mixture was stirred at that temperature for 30 minutes, then a solution of alcohol 386 (11.6 g, 0.059 mol, 1.0 equiv.) in anhydrous DCM (80 mL) was added at $-78{ }^{\circ} \mathrm{C}$. Then anhydrous triethylamine $(33 \mathrm{~mL}, 0.237 \mathrm{mmol}, 4.0$ equiv.) was added to the reaction mixture in 5 portions over 30 minutes at $-78{ }^{\circ} \mathrm{C}$. The reaction mixture was maintained at that temperature for 3 hours, and then was allowed to warm to room temperature slowly over 2 hours. Then the reaction mixture was stirred at room temperature overnight and quenched by addition of $300 \mathrm{~mL}$ of saturated aqueous $\mathrm{NH}_{4} \mathrm{Cl}$ solution. The aqueous phase was then separated and extracted with DCM $(3 \times 100 \mathrm{~mL})$. The combined organic phase was dried with anhydrous $\mathrm{Na}_{2} \mathrm{SO}_{4}$, filtered, and then concentrated by rotary evaporation. The crude material was purified by flash silica chromatography (pet. ether/EtOAc $=5 / 1$ ) to afford the provide the title compound as a yellow oil (10.8 g, yield 94\%). $\mathbf{R}_{\boldsymbol{f}}$. 0.25 (pet. ether/EtOAc = 5:1). ${ }^{1} \mathbf{H}$ NMR (500 MHz, $\left.\mathrm{CDCl}_{3}\right): \delta 9.78(\mathrm{t}, J=1.8 \mathrm{~Hz}, 1 \mathrm{H}), 7.26-7.24(\mathrm{~m}, 2 \mathrm{H}), 6.90-6.84(\mathrm{~m}, 2 \mathrm{H}), 4.46(\mathrm{~s}, 2 \mathrm{H})$, $3.80(\mathrm{~s}, 3 \mathrm{H}), 3.78(\mathrm{t}, J=6.1 \mathrm{~Hz}, 5 \mathrm{H}), 2.68(\mathrm{td}, J=6.1,1.8 \mathrm{~Hz}, 2 \mathrm{H}) .{ }^{13} \mathbf{C ~ N M R}(125 \mathrm{MHz}$, $\left.\mathrm{CDCl}_{3}\right): \delta 201.3,159.3,129.9,129.3,113.8,72.9,63.5,55.2,43.8$. These characterisation data match those reported in the literature. ${ }^{350}$ 


\section{Preparation of 1-((4-methoxybenzyl)oxy)hex-5-en-3-yl acrylate, 388}

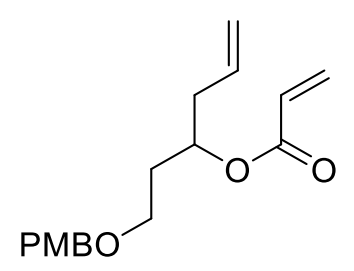

To a solution of aldehyde 387 ( $0.487 \mathrm{~g}, 2.51 \mathrm{mmol}, 1.0$ equiv.) in anhydrous THF ( $25 \mathrm{~mL})$ at $2{ }^{\circ} \mathrm{C}$ was added dropwise allylmagnesium bromide solution $(3.0 \mathrm{~mL}, 1.0 \mathrm{M}$ in diethyl ether, 1.2 equiv.). The reaction mixture was allowed to warm up to room temperature and stirred at room temperature overnight. Then anhydrous $N, N$-diisopropylethylamine (1.7 $\mathrm{mL}, 10.0 \mathrm{mmol}, 4.0$ equiv.) was added at room temperature and then the reaction mixture was cooled to $2{ }^{\circ} \mathrm{C}$, followed by addition of acryloyl chloride (1.2 mL, $15.1 \mathrm{mmol}$. 6.0 equiv.) and 4-dimethylaminopyridine (94 mg, $0.75 \mathrm{mmol}, 0.3$ equiv.). The resulting mixture was stirred at room temperature for 3 hours. The reaction was then quenched by the addition of $15 \mathrm{~mL}$ saturated aqueous solution of $\mathrm{NH}_{4} \mathrm{Cl}$ and the aqueous phase was then separated and extracted with DCM $(3 \times 100 \mathrm{~mL})$. The combined organic phase was dried with anhydrous $\mathrm{Na}_{2} \mathrm{SO}_{4}$, filtered, and then concentrated by rotary evaporation. The crude material was purified by flash silica chromatography (pet. ether/diethyl ether = 10/1). (Note: Acid-washed sand was replaced with $\mathrm{Na}_{2} \mathrm{SO}_{4}$ in the preparation of column.) The column fractions were concentrated at room temperature under reduced pressure to afford the provide the title compound as a colourless oil (0.59 g, yield 80\%). $\mathbf{R}_{f}$ : 0.43 (pet. ether/EtOAc = 5:1). ${ }^{1} \mathbf{H}$ NMR $\left(500 \mathrm{MHz}, \mathrm{CDCl}_{3}\right): \delta 7.25-7.22(\mathrm{~m}, 2 \mathrm{H}), 6.90-6.81(\mathrm{~m}$, 2H), $6.37(\mathrm{dd}, J=17.3,1.4 \mathrm{~Hz}, 1 \mathrm{H}), 6.08(\mathrm{dd}, J=17.3,10.4 \mathrm{~Hz}, 1 \mathrm{H}), 5.82-5.70$ (m, $2 \mathrm{H}), 5.21-5.13(\mathrm{~m}, 1 \mathrm{H}), 5.10-5.02(\mathrm{~m}, 2 \mathrm{H}), 4.40(\mathrm{~s}, 2 \mathrm{H}), 3.80(\mathrm{~s}, 3 \mathrm{H}), 3.52-3.42(\mathrm{~m}$, 2H), $2.45-2.32(\mathrm{~m}, 2 \mathrm{H}), 1.94-1.84(\mathrm{~m}, 2 \mathrm{H}) .{ }^{13} \mathbf{C ~ N M R}\left(125 \mathrm{MHz}, \mathrm{CDCl}_{3}\right): \delta$ 165.6, 159.1, 133.4, 130.5, 130.4, 129.3, 128.7, 118.0, 113.8, 72.7, 71.0, 66.2, 55.3, 38.8, 33.8. These characterisation data match those reported in the literature. ${ }^{351}$ 


\section{Preparation of 6-(2-((4-methoxybenzyl)oxy)ethyl)-5,6-dihydro-2H-pyran-2-one,}

391<smiles>CCCCCC1CC=CC(=O)O1</smiles>

To a solution of diene 388 (1.81 g, $6.2 \mathrm{mmol}, 1.0$ equiv.) in anhydrous DCM (500 mL) was added Grubbs $2^{\text {nd }}$ generation catalyst 390 (341 mg, $0.4 \mathrm{mmol}, 0.065$ equiv.). The reaction mixture was stirred at room temperature under argon atmosphere in dark for 21 hours. The solvent was then removed under reduced pressure. The crude material was purified by flash silica chromatography (pet. ether/ethyl acetate $=2 / 1$ ) to provide the title compound as a colourless oil $(1.31 \mathrm{~g}$, yield $80 \%) . \mathbf{R}_{f}$ : 0.37 (pet. ether/EtOAc $\left.=1: 1\right) .{ }^{1} \mathbf{H}$ NMR (500 MHz, $\left.\mathrm{CDCl}_{3}\right): \delta 7.26-7.21(\mathrm{~m}, 2 \mathrm{H}), 6.91-6.84(\mathrm{~m}, 3 \mathrm{H}), 6.02(\mathrm{dt}, J=9.8$, $1.7 \mathrm{~Hz}, 1 \mathrm{H}), 4.66-4.59(\mathrm{~m}, 1 \mathrm{H}), 4.44$ (q, $J=11.4 \mathrm{~Hz}, 2 \mathrm{H}), 3.80(\mathrm{~s}, 3 \mathrm{H}), 3.70-3.64(\mathrm{~m}$, $1 \mathrm{H}), 3.63-3.58(\mathrm{~m}, 1 \mathrm{H}), 2.38-2.33(\mathrm{~m}, 2 \mathrm{H}), 2.10-2.02(\mathrm{~m}, 1 \mathrm{H}), 1.98-1.90(\mathrm{~m}, 1 \mathrm{H})$. ${ }^{13} \mathrm{C}$ NMR (125 MHz, $\left.\mathrm{CDCl}_{3}\right): \delta 164.4,159.3,145.1,130.2,129.3,121.4,113.8,75.3$, $72.3,65.3,55.3,35.1,29.6$. These characterisation data match those reported in the literature. $^{134}$

\section{Preparation of 6-(2-hydroxyethyl)-5,6-dihydro-2H-pyran-2-one, 366}<smiles>O=C1C=CCC(CCO)O1</smiles>

DDQ (212 mg, $0.934 \mathrm{mmol}, 1.2$ equiv.) was added a solution of lactone 391 (204 mg, $0.78 \mathrm{mmol}, 1.0$ equiv. $)$ in a mixture of DCM $(16.2 \mathrm{~mL})$ and distilled water $(1.8 \mathrm{~mL})$. The reaction mixture was stirred at room temperature for 1 hour. The reaction mixture was then filtered through filter paper and the filter paper was rinsed with DCM a couple of times. The aqueous phase was separated and extracted with DCM $(3 \times 5 \mathrm{~mL})$. The combined organic phase was dried with anhydrous $\mathrm{Na}_{2} \mathrm{SO}_{4}$, filtered, and then concentrated by rotary evaporation. The crude material was purified by flash silica chromatography using ethyl acetate as eluent to afford the title compound as a red oil (89.3 mg, yield 81\%). $\mathbf{R}_{f}: 0.10$ (ethyl acetate). ${ }^{1} \mathbf{H} \mathbf{~ N M R ~ ( 5 0 0 ~ M H z , ~} \mathrm{CDCl}_{3}$ ): $\delta 6.91$ (dt, $J$ 
$=9.6,4.3 \mathrm{~Hz}, 1 \mathrm{H}), 6.04(\mathrm{dt}, J=9.8,1.8 \mathrm{~Hz}, 1 \mathrm{H}), 4.71-4.65(\mathrm{~m}, 1 \mathrm{H}), 3.94-3.88(\mathrm{~m}$, $1 \mathrm{H}), 3.87-3.81(\mathrm{~m}, 1 \mathrm{H}), 2.42-2.38(\mathrm{~m}, 2 \mathrm{H}), 2.09-2.00(\mathrm{~m}, 1 \mathrm{H}), 1.96-1.88(\mathrm{~m}, 1 \mathrm{H})$. ${ }^{13} \mathrm{C}$ NMR (125 MHz, $\left.\mathrm{CDCl}_{3}\right): \delta 164.3,145.2,121.4,75.6,58.6,37.3,29.7$. These characterisation data match those reported in the literature. ${ }^{352}$

\section{Preparation of $(2 Z, 4 E)$-7-(hept-6-ynoyloxy)hepta-2,4-dienoic acid, 364}

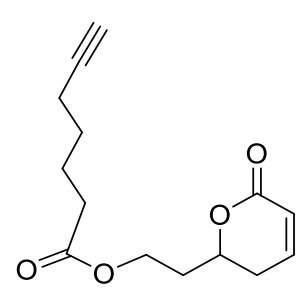

To a solution of alcohol 366 (1.66 g, 11.7 mmol, 1.0 equiv.) and hept-6-ynoic acid 258 (1.76 g, $14.0 \mathrm{mmol}, 1.2$ equiv.) in anhydrous DCM (120 mL) was added subsequentially EDCI (2.68 g, $14.0 \mathrm{mmol}, 1.2$ equiv.) and 4-dimethylaminopyridine (0.427 g, $3.5 \mathrm{mmol}$, 0.3 equiv.) at $1-2{ }^{\circ} \mathrm{C}$. Then the reaction mixture was stirred at room temperature overnight. Upon completion, the reaction was quenched by addition of $75 \mathrm{~mL}$ of saturated aqueous $\mathrm{NH}_{4} \mathrm{Cl}$ solution. The aqueous phase was then separated and extracted with ethyl acetate $(3 \times 50 \mathrm{~mL})$. The combined organic phase was dried with anhydrous $\mathrm{Na}_{2} \mathrm{SO}_{4}$, filtered, and then concentrated by rotary evaporation. The crude material was purified by flash silica chromatography (pet. ether/ethyl acetate $=3 / 2$ ) to afford the title compound as a yellow oil (1.98 g, yield 68\%). $\mathbf{R}_{\boldsymbol{f}}: 0.37$ (pet. ether/EtOAc $\left.=1: 1\right) .{ }^{1} \mathbf{H}$ NMR (500 $\mathrm{MHz}_{\mathrm{CDCl}}$ ): $\delta 6.90(\mathrm{ddd}, J=9.6,6.8,3.7 \mathrm{~Hz}, 1 \mathrm{H}), 6.04(\mathrm{dt}, J=9.8,1.7 \mathrm{~Hz}, 1 \mathrm{H}), 4.60$ $-4.53(\mathrm{~m}, 1 \mathrm{H}), 4.34-4.28(\mathrm{~m}, 1 \mathrm{H}), 4.27-4.21(\mathrm{~m}, 1 \mathrm{H}), 2.41-2.36(\mathrm{~m}, 2 \mathrm{H}), 2.34$ (t, $J$ $=7.5 \mathrm{~Hz}, 2 \mathrm{H}), 2.21(\mathrm{td}, J=7.0,2.6 \mathrm{~Hz}, 2 \mathrm{H}), 2.16-2.08(\mathrm{~m}, 1 \mathrm{H}), 2.06-1.96(\mathrm{~m}, 1 \mathrm{H})$, $1.95(\mathrm{t}, J=2.6 \mathrm{~Hz}, 1 \mathrm{H}), 1.78-1.70(\mathrm{~m}, 2 \mathrm{H}), 1.61-1.52(\mathrm{~m}, 2 \mathrm{H}) .{ }^{13} \mathbf{C}$ NMR $(125 \mathrm{MHz}$, $\left.\mathrm{CDCl}_{3}\right): \delta 173.2,163.9,144.7,121.5,83.9,74.7,68.6,59.9,34.0,33.6,29.4,27.8,23.9$, 18.1. These characterisation data match those reported in the literature. ${ }^{134}$ 


\subsubsection{Synthesis of Thiazoles 392, 393 and 394}

\section{Preparation of ethyl (S)-5-(2-(2-((tert-butyldimethylsilyl)oxy)-3-((tert-}

butyldiphenylsilyl)oxy)propyl)thiazol-4-yl)pentanoate, 392

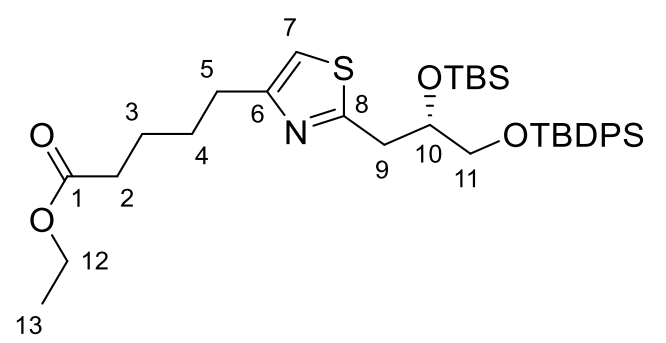

To a solution of Mol-DalPhosAuOMs 257 (2.9 mg, $0.00385 \mathrm{mmol}, 0.1$ equiv.) in anhydrous deoxygenated DCM $(0.2 \mathrm{~mL})$ was added alkyne $259(12 \mathrm{mg}, 0.0735 \mathrm{mmol}$, 2.1 equiv.). The resulting mixture was stirred at room temperature under argon atmosphere for 15 minutes. Then a solution of 8-methylquinoline $N$-oxide 254 (17.0 $\mathrm{mg}$, $0.106 \mathrm{mmol}, 2.74$ equiv.) and methanesulfonic acid ( $5.5 \mu \mathrm{L}, 0.085 \mathrm{mmol}, 2.52$ equiv.) in degassed anhydrous DCM $(0.2 \mathrm{~mL})$ was added to the reaction mixture via a syringe pump under argon atmosphere in 5 hours. Upon completion, the reaction mixture was further stirred for 1 hour before a solution of thioamide $382(11.9 \mathrm{mg}, 0.024 \mathrm{mmol}, 1.0 \mathrm{eq})$ in deoxygenated anhydrous DCM $(0.8 \mathrm{~mL})$ adding. The resulting mixture was stirred at 45 ${ }^{\circ} \mathrm{C}$ (oil bath temperature) in a sealed vial under argon atmosphere for 12 hours before concentration under reduced pressure. The crude material was purified by silica gel flash chromatography (pet. ether/EtOAc $=10: 1)$ to provide the title compound $(9.5 \mathrm{mg}$, yield $61 \%)$ as a colourless oil. $\mathbf{R}_{f}: 0.60$ (pet. ether/EtOAc $\left.=5: 1\right) .{ }^{1} \mathbf{H} \mathbf{N M R}\left(500 \mathrm{MHz}, \mathrm{CDCl}_{3}\right)$ : $\delta 7.69-7.62$ (m, 4H), 7.45 - 7.34 (m, 6H), 6.74 (s, 1H, H-7), 4.18 - 4.11 (m, overlapped, $3 \mathrm{H}, \mathrm{H}-10, \mathrm{H}-12), 3.63$ (dd, $J=10.2,4.7 \mathrm{~Hz}, 1 \mathrm{H}, \mathrm{H}-11 \mathrm{a}), 3.54$ (dd, $J=10.2,6.7 \mathrm{~Hz}, 1 \mathrm{H}$, H-11b), 3.39 (dd, $J=14.5,4.0$ Hz, 1H, H-9a), 3.11 (dd, $J=14.5,7.8$ Hz, 1H, H-9b), 2.75 (t, $J=7.2 \mathrm{~Hz}, 2 \mathrm{H}, \mathrm{H}-5), 2.33$ (t, $J=7.0 \mathrm{~Hz}, 2 \mathrm{H}, \mathrm{H}-2), 1.77-1.66$ (m, 4H, H-3,H-4), 1.25 $(\mathrm{t}, J=7.1 \mathrm{~Hz}, 3 \mathrm{H}, \mathrm{H}-13), 1.05$ (s, 9H), $0.76(\mathrm{~s}, 9 \mathrm{H}),-0.15$ (s, 3H), -0.25 (s, 3H). ${ }^{13} \mathbf{C ~ N M R}$ (125 MHz, $\left.\mathrm{CDCl}_{3}\right): \delta 173.7$ (C-1), 167.1 (C-8), 156.4 (C-6), 135.62, 135.59, 133.30, 129.67, 129.65, 127.684, 127.678, 112.5 (C-7), 72.8 (C-10), 67.4 (C-11), 60.3 (C-12), 38.6 (C-9), 34.2 (C-2), 31.2 (C-5), 28.8 (C-4), 26.9, 25.8, 24.6 (C-3), 19.2, 18.0, 14.3 (C13), -4.8, -5.3. FTIR (thin film) v2954, 2929, 2857, 1735, 1472, 1462, 1428, 1254, 1186, 1112, 1075, 836, 824, 805, 777, 740, 702, 690, 505, $493 \mathrm{~cm}^{-1}$. HRMS (ESI): Calculated for $\mathrm{C}_{35} \mathrm{H}_{54} \mathrm{NO}_{4} \mathrm{SSi}_{2}{ }^{+}[\mathrm{M}+\mathrm{H}]^{+}$640.3307; found 640.3266. $[\alpha]_{D}^{26}-111.1(c=0.45, \mathrm{DCM})$. 
Preparation of 2-(6-oxo-3,6-dihydro-2H-pyran-2-yl)ethyl 5-(2-((S)-2-((tertbutyldimethylsilyl)oxy)-3-((tert-butyldiphenylsilyl)oxy)propyl)thiazol-4yl)pentanoate, 393

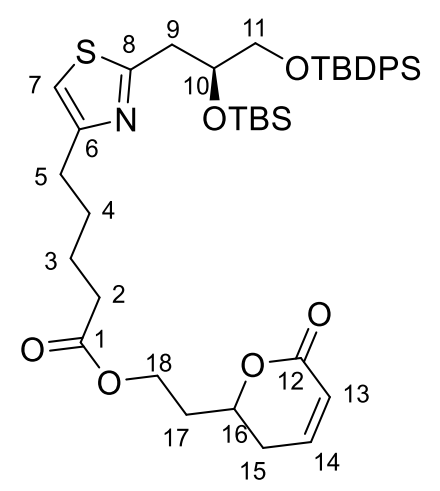

To a solution of Mol-DalPhosAuOMs 257 (26.0 mg, $0.0344 \mathrm{mmol}, 0.01$ equiv.) in anhydrous deoxygenated DCM (7.0 mL) was added alkyne 364 (862 mg, 3.44 mmol, 1.03 equiv.). The resulting mixture was stirred at room temperature under argon atmosphere for 30 minutes. Then a solution of 8-methylquinoline $N$-oxide 254 (712 mg, $4.47 \mathrm{mmol}$, 1.34 equiv.) and methanesulfonic acid (268 $\mu \mathrm{L}, 4.13 \mathrm{mmol}, 1.24$ equiv.) in degassed anhydrous DCM $(6.9 \mathrm{~mL})$ was added to the reaction mixture via a syringe pump under argon atmosphere in 9 hours. Upon completion, the reaction mixture was further stirred for 1 hour before a solution of thioamide 382 (1.63 g, $3.34 \mathrm{mmol}, 1.0$ equiv.) in deoxygenated anhydrous deoxygenated DCM $(22 \mathrm{~mL})$ adding. The resulting mixture was stirred at $45^{\circ} \mathrm{C}$ in a sealed vial for 72 hours under argon atmosphere before concentration under reduced pressure. The crude material was purified by silica gel flash chromatography (pet. ether/EtOAc $=3: 2)$ to provide the title compound $(1.31 \mathrm{~g}$, yield $52 \%$, BRSM yield 57\%) as a yellow oil. $\mathbf{R}_{f}: 0.52$ (pet. ether/EtOAc $\left.=1: 1\right) .{ }^{1} \mathbf{H}$ NMR (500 $\left.\mathrm{MHz}, \mathrm{CDCl}_{3}\right): \delta 7.69-7.62(\mathrm{~m}, 4 \mathrm{H}), 7.46-7.33(\mathrm{~m}, 6 \mathrm{H}), 6.94-6.82(\mathrm{~m}, 1 \mathrm{H}, \mathrm{H}-14)$, 6.75 (s, 1H, H-7), 6.04 (dt, J = 9.9, 1.7 Hz, 1H, H-13), 4.61 - 4.50 (m, 1H, H-16), 4.35 4.22 (m, 2H, H-18), 4.17 - 4.09 (m, 1H, H-10), 3.63 (dd, J = 10.2, 4.7 Hz, 1H, H-11a), $3.53(\mathrm{dd}, J=10.2,6.7 \mathrm{~Hz}, 1 \mathrm{H}, \mathrm{H}-11 \mathrm{~b}), 3.39$ (dd, $J=14.5,3.9 \mathrm{~Hz}, 1 \mathrm{H}, \mathrm{H}-9 \mathrm{a}), 3.11$ (dd, $J$ $=14.6,7.8 \mathrm{~Hz}, 1 \mathrm{H}, \mathrm{H}-9 \mathrm{~b}), 2.75$ (t, $J=7.1 \mathrm{~Hz}, 2 \mathrm{H}, \mathrm{H}-5), 2.43-2.31$ (m, 2H, H-15), 2.34 (t, J=6.9 Hz, 2H, H-2), $2.16-2.09$ (m, 1H, H-17a), $2.04-1.95$ (m, 1H, H-17b), $1.78-$ 1.64 (m, 4H, H-3, H-4), 1.05 (s, 9H), 0.76 (s, 9H), -0.15 (s, 3H), -0.25 (s, 3H). ${ }^{13}$ C NMR (125 MHz, $\mathrm{CDCl}_{3}$ ): $\delta 173.4$ (C-1), 167.2 (C-8), 163.9 (C-12), 156.3 (C-6), 144.73, 144.72 (C-14), 135.59, 135.56, 133.4, 133.3, 129.65, 129.63, 127.66, 127.65, 121.5 (C-13), 112.6 (C-7), 74.7 (C-16), 72.7 (C-10), 67.3 (C-11), 59.8 (C-18), 38.6 (C-9), 34.02 (C-17), 33.97 (C-2), 31.2 (C-5), 29.4 (C-15), 28.7 (C-4), 26.8, 25.7, 24.5 (C-3), 19.2, 17.9, -4.8, -5.3. 
FTIR (thin film) v 2953, 2929, 2856, 1731, 1472, 1462, 1428, 1389, 1361, 1248, 1155, 1111, 1061, 1006, 998, 938, 835, 822, 776, 739, 701, 690, 662, 622, 614, 505, $489 \mathrm{~cm}^{-1}$. HRMS (ESI): Calculated for $\mathrm{C}_{40} \mathrm{H}_{58} \mathrm{NO}_{6} \mathrm{SSi}_{2}{ }^{+}[\mathrm{M}+\mathrm{H}]^{+} 736.3518$; found 736.3494. $[\alpha]_{D}{ }^{25}$ $-14.3(c=1.05, \mathrm{DCM})$.

\section{Preparation of ethyl $(S)-5-(5-b r o m o-2-(2-((t e r t-b u t y l d i m e t h y l s i l y l) o x y)-3-((t e r t-$}

\section{butyldiphenylsilyl)oxy)propyl)thiazol-4-yl)pentanoate, 394}

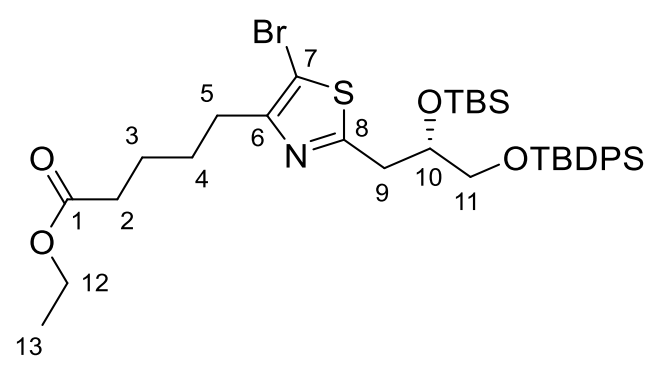

To a solution of thiazole 392 (7.6 mg, $0.012 \mathrm{mmol}, 1.0$ equiv.) in anhydrous DCM (0.2 $\mathrm{mL}$ ) was added $N$-bromosuccinimide ( $2.1 \mathrm{mg}, 0.012 \mathrm{mmol}, 1.0$ equiv.). The reaction mixture was stirred at room temperature for 20 hours. Solvent was then removed under reduced pressure and at $40{ }^{\circ} \mathrm{C}$, the crude material was purified by flash silica chromatography (pet. ether/EtOAc $=13 / 1$ ) to afford the title compound as a colourless oil (4.8 mg, yield 56\%). $\mathbf{R}_{f}: 0.57$ (pet. ether/EtOAc = 5:1). ${ }^{1} \mathbf{H} \mathbf{N M R}\left(500 \mathrm{MHz}, \mathrm{CDCl}_{3}\right)$ : $\delta 7.67-7.60(\mathrm{~m}, 4 \mathrm{H}), 7.45-7.34(\mathrm{~m}, 6 \mathrm{H}), 4.14-4.08$ (m, overlapped, 3H, H-10, H-12), 3.60 (dd, $J=10.2,4.7 \mathrm{~Hz}, 1 \mathrm{H}, \mathrm{H}-11 \mathrm{a}), 3.51$ (dd, $J=10.2,7.0 \mathrm{~Hz}, 1 \mathrm{H}, \mathrm{H}-11 \mathrm{~b}), 3.30$ (dd, $J=14.7,4.1 \mathrm{~Hz}, 1 \mathrm{H}, \mathrm{H}-9 \mathrm{a}), 3.11$ (dd, $J=14.7,7.0 \mathrm{~Hz}, 1 \mathrm{H}, \mathrm{H}-9 \mathrm{~b}), 2.74-2.67$ (m, 2H, H-5), $2.35-2.30$ (m, 2H, H-2), $1.72-1.65$ (m, 4H, H-3, H-4), 1.25 (t, J=7.1 Hz, 3H, H-13), 1.04 (s, 9H), 0.79 (s, 9H), -0.13 (s, 3H), -0.18 (s, 3H). ${ }^{13}$ C NMR (125 MHz, $\mathrm{CDCl}_{3}$ ): $\delta 173.6$ (C-1), 166.9 (C-8), 154.4 (C-6), 135.56, 135.53, 133.6, 129.70, 129.68, 127.69, 127.67, 103.0 (C-7), 72.2 (C-10), 66.8 (C-22), 60.2 (C-12), 39.0 (C-9), 34.1 (C2), 29.1 (C-5), 28.3 (C-4), 26.8, 25.7, 24.6 (C-3), 19.2, 18.0, 14.3 (C-13), -4.8, -5.2. FTIR (thin film) $v 2953,2929,2857,1735,1472,1462,1428,1361,1256,1184,1112,1080$, 1007, 837, 824, 804, 777, 738, 702, 692, 504, $492 \mathrm{~cm}^{-1}$. HRMS (ESI): Calculated for $\mathrm{C}_{35} \mathrm{H}_{53} \mathrm{BrNO}_{4} \mathrm{SSi}_{2}{ }^{+}[\mathrm{M}+\mathrm{H}]^{+}$718.2412; found 718.2239. $[\alpha]_{D}{ }^{24}-9.9(c=0.24, \mathrm{DCM})$. 


\subsubsection{Synthesis of Macrocyclic Alcohol 441}

Preparation of $(2 Z, 4 E)-7-((5-(2-((S)-2-(($ tert-butyldimethylsilyl)oxy)-3-((tertbutyldiphenylsilyl)oxy)propyl)thiazol-4-yl)pentanoyl)oxy)hepta-2,4-dienoic acid, 418

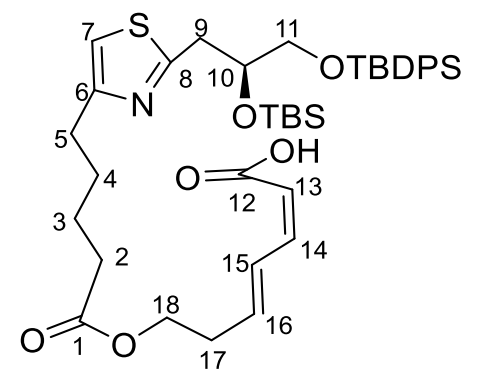

Potassium bis(trimethylsilyl)amide solution $(145 \mu \mathrm{L}, 1.0 \mathrm{M}$ in THF, $0.145 \mathrm{mmol}, 1.3$ equiv.) was added dropwise to a solution of thiazole 393 ( $82.2 \mathrm{mg}, 0.112 \mathrm{mmol}, 1.0$ equiv.) in anhydrous THF $(2.0 \mathrm{~mL})$ at $-78^{\circ} \mathrm{C}$. The reaction mixture was stirred at that temperature under argon atmosphere for 2 hours. Aqueous sulfuric acid (193 $\mu \mathrm{L}, 2$ vol\%) was added to the reaction mixture dropwise at $-78^{\circ} \mathrm{C}$, and then the resulting mixture was allowed to warm up to room temperature and stir at room temperature for 5 minutes. The mixture was then added ethyl actate $(6 \mathrm{~mL})$ and saturated brine $(2 \mathrm{~mL})$. The aqueous phase was extracted by ethyl acetate $(3 \times 5 \mathrm{~mL})$. The combined organic phase was dried with anhydrous $\mathrm{Na}_{2} \mathrm{SO}_{4}$, filtered, and then concentrated by rotary evaporation. The crude material was purified by flash silica chromatography (pet. ether/EtOAc $=4 / 1$ ) to afford the title compound as a colourless oil $\left(49.0 \mathrm{mg}\right.$, yield 60\%). $\mathbf{R}_{f}: 0.27$ (pet. ether/EtOAc $=$ 5:1). ${ }^{1} \mathbf{H}$ NMR $\left(500 \mathrm{MHz}, \mathrm{CDCl}_{3}\right): \delta 7.68-7.64(\mathrm{~m}, 4 \mathrm{H}), 7.45-7.35$ (m, overlapped, $7 \mathrm{H}), 6.75$ (s, 1H, H-7), 6.58 (t, $J=11.3 \mathrm{~Hz}, 1 \mathrm{H}, \mathrm{H}-14), 6.04$ (dt, $J=15.5,6.4 \mathrm{~Hz}, 1 \mathrm{H}, \mathrm{H}-$ 16), $5.62(\mathrm{~d}, J=11.4 \mathrm{~Hz}, 1 \mathrm{H}, \mathrm{H}-13), 4.20$ (t, $J=5.7 \mathrm{~Hz}, 2 \mathrm{H}, \mathrm{H}-18), 4.14(\mathrm{tt}, J=7.9,4.2$ Hz, 1H, H-10), 3.62 (dd, $J=10.2,4.5 \mathrm{~Hz}, 1 \mathrm{H}, \mathrm{H}-11 \mathrm{a}), 3.53$ (dd, $J=10.2,6.9 \mathrm{~Hz}, 1 \mathrm{H}, \mathrm{H}-$ 11b), 3.47 (dd, $J=14.5,3.9 \mathrm{~Hz}, 1 \mathrm{H}, \mathrm{H}-9 \mathrm{a}), 3.19$ (dd, $J=14.4,7.9 \mathrm{~Hz}, 1 \mathrm{H}, \mathrm{H}-9 \mathrm{~b}$ ), 2.77 (t, $J=6.8 \mathrm{~Hz}, 2 \mathrm{H}, \mathrm{H}-5), 2.54-2.48$ (m, 2H, H-17), 2.34 (t, $J=6.7 \mathrm{~Hz}, 2 \mathrm{H}, \mathrm{H}-2), 1.75-$ 1.68 (m, 4H, H-3, H-4), 1.05 (s, 9H), 0.75 (s, 9H), -0.17 (s, 3H), -0.25 (s, 3H). ${ }^{13}$ C NMR (125 MHz, $\mathrm{CDCl}_{3}$ ): $\delta 173.5$ (C-1), 169.6 (C-12), 168.1 (C-8), 156.1 (C-6), 144.6 (C-14), 139.3 (C-16), 135.59, 135.58, 133.4, 133.3, 129.65, 129.62, 129.2 (C-15), 127.67, 127.66, 117.3 (C-13), 112.9 (C-7), 72.6 (C-10), 67.2 (C-11), 62.4 (C-18), 38.1 (C-9), 34.2 (C-2), 31.8 (C-17), 30.7 (C-5), 29.2 (C-3), 26.8, 25.8, 24.6 (C-4), 19.2, 17.9, -4.9, -5.4. FTIR 
(thin film) $v 3070,3050,2953,2929,2893,2857,1736,1695,1640,1602,1525,1471$, $1463,1428,1389,1361,1252,1186,1112,1076,999,965,940,836,825,805,777,740$, 703, 614, 505, $492 \mathrm{~cm}^{-1}$. HRMS (ESI): Calculated for $\mathrm{C}_{40} \mathrm{H}_{58} \mathrm{NO}_{6} \mathrm{SSi}_{2}{ }^{+}[\mathrm{M}+\mathrm{H}]^{+} 736.3518$; found 735.3446. $[\alpha]_{D}^{23}-13.7(c=0.99, \mathrm{DCM})$.

\section{Preparation of $(2 Z, 4 E)-7-((5-(2-((S)-3-(($ tert-butyldiphenylsilyl)oxy)-2-}

\section{hydroxypropyl)thiazol-4-yl)pentanoyl)oxy)hepta-2,4-dienoic acid, 419}

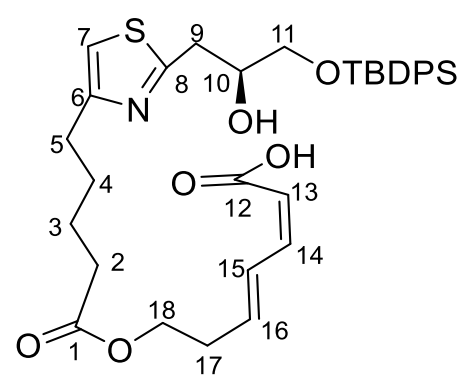

Method A: (Use bis-silylated compound 418 as the starting material)

Pyridinium $p$-toluenesulfonate (156 mg, $0.63 \mathrm{mmol}, 10.0$ equiv.) was added to a solution of acid 418 (46 mg, $0.063 \mathrm{mmol}, 1.0$ equiv.) in $\mathrm{MeOH}$ (4.5 mL). The reaction mixture was stirred at $45-50{ }^{\circ} \mathrm{C}$ for 27 hours. Upon completion, the reaction mixture was concentrated under reduced pressure and the residue was directly loaded onto a silica column and purified by flash chromatography using pet. ether/EtOAc $=2 / 1$ as eluent to afford the title compound as a colourless oil (11.9 mg, yield 30\%, BRSM 43\%). $\mathbf{R}_{f}$ : 0.56 (pet. ether/EtOAc = 1/1). ${ }^{1} \mathbf{H} \mathbf{N M R}\left(500 \mathrm{MHz}, \mathrm{CDCl}_{3}\right): \delta 7.68-7.62(\mathrm{~m}, 4 \mathrm{H}), 7.45-7.32$ (m, overlapped, 7H), 6.79 (s, 1H, H-7), 6.59 (t, $J=11.4$ Hz, 1H, H-14), 6.06 (dt, $J=15.1$, $6.2 \mathrm{~Hz}, 1 \mathrm{H}, \mathrm{H}-16), 5.59$ (d, $J=11.4 \mathrm{~Hz}, 1 \mathrm{H}, \mathrm{H}-13), 4.22$ (t, $J=5.8 \mathrm{~Hz}, 2 \mathrm{H}, \mathrm{H}-18), 4.15$ -4.07 (m, 1H, H-10), 3.72 (dd, $J=10.3,5.5 \mathrm{~Hz}, 1 \mathrm{H}, \mathrm{H}-11 \mathrm{a}), 3.67$ (dd, $J=9.7,5.3 \mathrm{~Hz}$, 2H, H-11b), 3.31 (dd, $J=15.6,3.1 \mathrm{~Hz}, 1 \mathrm{H}, \mathrm{H}-9 \mathrm{a}), 3.20$ (dd, $J=15.1,8.7 \mathrm{~Hz}, 1 \mathrm{H}, \mathrm{H}-9 \mathrm{~b}$ ), $2.74(\mathrm{t}, J=7.0 \mathrm{~Hz}, 2 \mathrm{H}, \mathrm{H}-5), 2.55-2.49$ (m, 2H, H-17), 2.35 (t, $J=6.6 \mathrm{~Hz}, 2 \mathrm{H}, \mathrm{H}-2)$, $1.76-1.63$ (m, 4H, H-3, H-4), 1.07 (s, 9H). ${ }^{13} \mathbf{C ~ N M R ~ ( 1 2 5 ~ M H z , ~ C D C l ~} 3$ ): $\delta 173.6(\mathrm{C}-$ 1), 170.1 (C-12), 167.7 (C-8), 156.1 (C-6), 145.4 (C-14), 140.0 (C-16), 135.52, 135.51, 133.10, 133.07, 129.76, 129.75, 129.1 (C-15), 127.74, 127.72, 116.8 (C-13), 112.8(C-7), 71.3 (C-10), 66.8 (C-11), 62.5 (C-18), 36.3 (C-9), 34.0 (C-2), 31.9 (C-17), 30.6 (C-5), 28.8 (C-4), 26.8, 24.4 (C-3), 19.2. FTIR (thin film) v3391, 3069, 2929, 2857, 1733, 1640, 1602, 1525, 1461, 1428, 1389, 1361, 1185, 1112, 999, 964, 939, 859, 824, 741, 703, 614, 593, 506, 434, $418 \mathrm{~cm}^{-1}$. HRMS (ESI): Calculated for $\mathrm{C}_{40} \mathrm{H}_{58} \mathrm{NO}_{6} \mathrm{SSi}_{2}{ }^{+}[\mathrm{M}+\mathrm{H}]^{+} 622.2659$; found $622.2625 .[\alpha]_{D}{ }^{24}-9.0(c=0.69, \mathrm{DCM})$. 
Method B: (Use mono-silylated compound $\mathbf{4 2 2}$ as the starting material)

Potassium bis(trimethylsilyl)amide solution $(88 \mu \mathrm{L}, 0.5 \mathrm{M}$ in toluene, $0.044 \mathrm{mmol}, 2.3$ equiv.) was added dropwise to a solution of thiazole 422 (12 mg, $0.019 \mathrm{mmol}, 1.0$ equiv.) in anhydrous $\mathrm{THF}(0.8 \mathrm{~mL})$ at $-78^{\circ} \mathrm{C}$. The reaction mixture was stirred at that temperature under argon atmosphere for 1 hours. Aqueous sulfuric acid $(60 \mu \mathrm{L}, 2$ vol\%) was added to the reaction mixture dropwise at $-78{ }^{\circ} \mathrm{C}$, and then the resulting mixture was allowed to warm up to room temperature and stir at room temperature for 5 minutes. The mixture was then added ethyl acetate $(3 \mathrm{~mL})$ and saturated brine $(3 \mathrm{~mL})$. The aqueous phase was extracted by ethyl acetate $(3 \times 5 \mathrm{~mL})$. The combined organic phase was dried with anhydrous $\mathrm{Na}_{2} \mathrm{SO}_{4}$, filtered, and then concentrated by rotary evaporation. The crude material was purified by flash silica chromatography (pet. ether/EtOAc $=4 / 1$ ) to afford the title compound as a colourless oil $(4.3 \mathrm{mg}$, yield $36 \%)$. $\mathbf{R}_{f}: 0.65$ (pet. ether/EtOAc $=$ 2:3). ${ }^{1} \mathbf{H}$ NMR $\left(500 \mathrm{MHz}, \mathrm{CDCl}_{3}\right): \delta 7.68-7.62(\mathrm{~m}, 4 \mathrm{H}), 7.47$ - 7.34 (m, overlapped, 7H), 6.80 (s, 1H, H-7), 6.58 (t, $J=11.4$ Hz, 1H, H-14), 6.05 (dt, J=13.9, $6.2 \mathrm{~Hz}, 1 \mathrm{H}, \mathrm{H}-$ 16), 5.59 (d, $J=11.4 \mathrm{~Hz}, 1 \mathrm{H}, \mathrm{H}-13), 4.22$ (t, $J=6.0 \mathrm{~Hz}, 2 \mathrm{H}, \mathrm{H}-18), 4.15-4.08$ (m, 1H, H-10), 3.72 (dd, $J=10.4,5.2 \mathrm{~Hz}, 1 \mathrm{H}, \mathrm{H}-11 \mathrm{a}), 3.67$ (dd, $J=9.7,5.3 \mathrm{~Hz}, 2 \mathrm{H}, \mathrm{H}-11 \mathrm{~b}$ ), 3.31 (dd, $J=15.6,3.1 \mathrm{~Hz}, 1 \mathrm{H}, \mathrm{H}-9 \mathrm{a}), 3.21$ (dd, $J=15.1,8.7 \mathrm{~Hz}, 1 \mathrm{H}, \mathrm{H}-9 \mathrm{~b}), 2.74$ (t, $J=7.0$ $\mathrm{Hz}, 2 \mathrm{H}, \mathrm{H}-5), 2.55-2.49$ (m, 2H, H-17), 2.35 (t, $J=6.8 \mathrm{~Hz}, 2 \mathrm{H}, \mathrm{H}-2), 1.76-1.63$ (m, 4H, H-3, H-4), 1.07 (s, 9H). ${ }^{13}$ C NMR (125 MHz, $\left.\mathrm{CDCl}_{3}\right)$ : $\delta 173.6$ (C-1), 170.1 (C-12), 167.7 (C-8), 156.1 (C-6), 145.4 (C-14), 140.0 (C-16), 135.52, 135.51, 133.10, 133.07, 129.76, 129.75, 129.1 (C-15), 127.74, 127.72, 116.8 (C-13), 112.8(C-7), 71.3 (C-10), 66.8 (C-11), 62.5 (C-18), 36.3 (C-9), 34.0 (C-2), 31.9 (C-17), 30.6 (C-5), 28.8 (C-4), 26.8, $24.4(\mathrm{C}-3), 19.2$. 


\section{Preparation of 2-(6-oxo-3,6-dihydro-2H-pyran-2-yl)ethyl 5-(2-((S)-3-((tert-}

\section{butyldiphenylsilyl)oxy)-2-hydroxypropyl)thiazol-4-yl)pentanoate, 422}

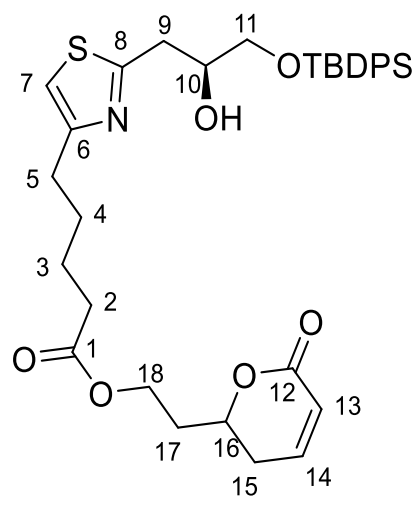

Acetic acid ( $0.25 \mathrm{~mL}, 4.43 \mathrm{mmol}, 200$ equiv.) was added in one portion to a biphasic

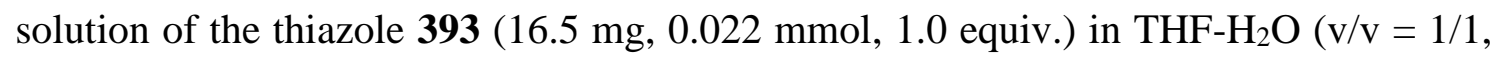
$0.25 \mathrm{~mL}$ ) and the mixture was stirred at $50{ }^{\circ} \mathrm{C}$ for 3 hours. Then the reaction mixture was allowed to cool down to $0{ }^{\circ} \mathrm{C}$ and quenched by slow addition of saturated $\mathrm{NaHCO}_{3}$ solution $(10 \mathrm{~mL})$. Then the aqueous phase was separated and extracted by ethyl acetate $(3 \times 5 \mathrm{~mL})$. The combined organic phase was dried with anhydrous $\mathrm{Na}_{2} \mathrm{SO}_{4}$, filtered, and then concentrated by rotary evaporation. The crude material was purified by flash silica chromatography (pet. ether/EtOAc $=1 / 2$ ) to afford the title compound as a colourless oil (4.2 mg, yield 30\%, BRSM yield 100\%). Rf: 0.14 (pet. ether/EtOAc = 1:1). ${ }^{1} \mathbf{H}$ NMR $\left(500 \mathrm{MHz}, \mathrm{CDCl}_{3}\right): \delta 7.67-7.62(\mathrm{~m}, 4 \mathrm{H}), 7.46-7.34(\mathrm{~m}, 6 \mathrm{H}), 6.90-6.85(\mathrm{~m}, 1 \mathrm{H}, \mathrm{H}-$ 14), 6.84 (s, 1H, H-7), 6.03 (d, J=9.7 Hz, 1H, H-13), $4.61-4.51$ (m, 1H, H-16), 4.33 4.20 (m, 2H, H-18), 4.17 - 4.10 (m, 1H, H-10), 3.71 (dd, $J=9.9,5.7$ Hz, 1H, H-11a), 3.66 (dd, $J=9.9,5.7$ Hz, 1H, H-11b), 3.34 (d, $J=14.7$ Hz, 1H, H-9a), 3.20 (dd, $J=15.1$, $8.4 \mathrm{~Hz}, 1 \mathrm{H}, \mathrm{H}-9 \mathrm{~b}), 2.78$ (t, J=7.1 Hz, 2H, H-5), 2.43 - 2.28 (m, overlapped, 4H, H-2, H15), 2.15 - 2.05 (m, 1H, H-17a), 2.03 - 1.94 (m, 1H, H-17b), 1.77 - 1.61 (m, 4H, H-3, $\mathrm{H}-4), 1.07$ (s, 9H). ${ }^{13} \mathrm{C}$ NMR (125 MHz, $\left.\mathrm{CDCl}_{3}\right): \delta 173.3$ (C-1), 168.1 (C-8), 163.9 (C12), 154.9 (C-6), 144.8 (C-14), 135.5, 133.0, 129.8, 127.8, 121.5 (C-13), 113.1 (C-7), 74.7 (C-16), 70.9 (C-10), 66.6 (C-11), 59.9 (C-18), 35.9 (C-9), 34.0 (C-17), 33.8 (C-2), 30.3 (C-5), 29.4(C-15), 28.3 (C-4), 26.8, 24.3 (C-3), 19.2. FTIR (thin film) v3353, 3071, 3049, 2930, 2857, 1735, 1731, 1701, 1697, 1654, 1648, 1523, 1472, 1460, 1427, 1389, 1362, 1307, 1245, 1178, 1154, 1112, 1059, 1041, 998, 958, 862, 820, 741, 703, 691, 622, 615, 554, 505, 490, 468, 451, $434 \mathrm{~cm}^{-1}$. HRMS (ESI): Calculated for $\mathrm{C}_{34} \mathrm{H}_{44} \mathrm{NO}_{6} \mathrm{SSi}_{2}{ }^{+}$ $[\mathrm{M}+\mathrm{H}]^{+}$622.2653; found 622.2667. $[\alpha]_{D}^{21}-6.4(c=0.55, \mathrm{DCM})$. 


\section{Preparation of 2-(6-0xo-3,6-dihydro-2H-pyran-2-yl)ethyl 5-(2-((S)-2,3-} dihydroxypropyl)thiazol-4-yl)pentanoate, 423

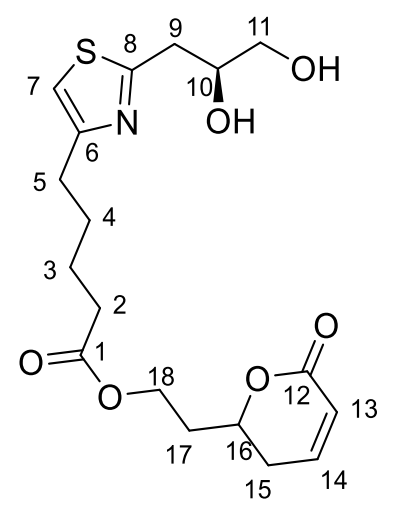

To a solution of 393 (106 mg, $0.144 \mathrm{mmol}, 1.0$ equiv.) in THF-pyridine (v/v = 4/1, 6.25 $\mathrm{mL})$ was added HF-pyridine $(375 \mu \mathrm{L})$ at $0{ }^{\circ} \mathrm{C}$. Then the reaction mixture was stirred for 17 hours at room temperature. Then the reaction was quenched by slow addition of saturated $\mathrm{NaHCO}_{3}$ aqueous solution $(5 \mathrm{~mL})$ at ice-water bath. Then the aqueous layers were separated and extracted by ethyl acetate $(3 \times 10 \mathrm{~mL})$. The combined organic phase was dried with anhydrous $\mathrm{Na}_{2} \mathrm{SO}_{4}$, filtered, and then concentrated by rotary evaporation. The crude material was purified by flash silica chromatography (EtOAc/ $\mathrm{MeOH}=20 / 1$ ) to afford the title compound as a colourless oil (31.6 mg, yield 57\%). $\mathbf{R}_{\boldsymbol{f}}$ : 0.5 $(\mathrm{EtOAc} / \mathrm{MeOH}=10: 1) .{ }^{1} \mathbf{H}$ NMR $\left(500 \mathrm{MHz}, \mathrm{CDCl}_{3}\right) \delta 6.89(\mathrm{dt}, \mathrm{J}=9.2,3.8 \mathrm{~Hz}, 1 \mathrm{H}, \mathrm{H}-$ 14), 6.78 (s, 1H, H-7), 6.04 (d, $J=10.2 \mathrm{~Hz}, 1 \mathrm{H}, \mathrm{H}-13), 4.56$ (tt, $J=9.5,5.2 \mathrm{~Hz}, 1 \mathrm{H}, \mathrm{H}-$ 16), $4.33-4.19$ (m, 2H, H-18), $4.17-4.06$ (m, 1H, H-10), 3.72 (dd, $J=11.5,3.8 \mathrm{~Hz}, 1 \mathrm{H}$, H-11a), 3.61 (dd, $J=11.4,5.6 \mathrm{~Hz}, 1 \mathrm{H}, \mathrm{H}-11 \mathrm{~b}), 3.14$ (d, J = 6.0 Hz, 2H, H-9), 2.75 (t, $J=$ $7.3 \mathrm{~Hz}, 2 \mathrm{H}, \mathrm{H}-5), 2.40-2.36$ (m, 2H, H-15), 2.34 (t, J = $7.2 \mathrm{~Hz}, 2 \mathrm{H}, \mathrm{H}-2), 2.16-2.06$ (m, 1H, H-17a), 2.03 - 1.94 (m, 1H, H-17b), 1.74 - 1.58 (m, 4H, H-3, H-4). ${ }^{13}$ C NMR (125 MHz, $\mathrm{CDCl}_{3}$ ) $\delta 173.4$ (C-1), 167.2 (C-8), 164.0 (C-12), 156.4 (C-6), 144.9 (C-14), 121.5 (C-13), 112.6 (C-7), 74.8 (C-16), 70.8 (C-10), 65.7 (C-11), 59.9 (C-18), 36.0 (C9), 34.0 (C-17), 33.9 (C-2), 30.8 (C-5), 29.4 (C-15), 28.5 (C-4), 24.3 (C-3). FTIR (thin film) v3356, 2929, 2867, 1719, 1523, 1459, 1422, 1390, 1362, 1251, 1178, 1125, 1093, 1039, 961, $820 \mathrm{~cm}^{-1}$. HRMS (ESI): Calculated for $\mathrm{C}_{18} \mathrm{H}_{26} \mathrm{NO}_{6} \mathrm{~S}^{+}[\mathrm{M}+\mathrm{H}]^{+} 384.1475$; found 384.1485. $[\alpha]_{D}^{22}+5.2(c=0.42, \mathrm{DCM})$. 


\section{Preparation of $\left(S, 1^{2} Z, 6 Z, 8 E\right)-3-(((t e r t-b u t y l d i p h e n y l s i l y l) o x y)$ methyl)-4,12-dioxa-}

1(2,4)-thiazolacycloheptadecaphane-6,8-diene-5,13-dione, 440

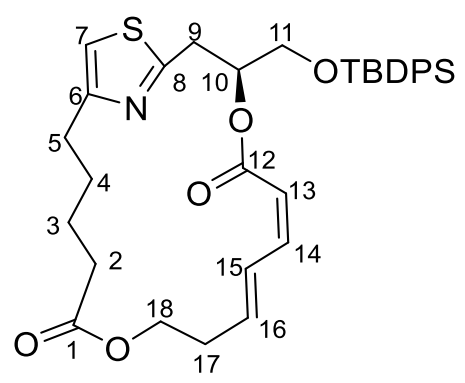

To a mixture of acid 419 (12.9 mg, $0.0203 \mathrm{mmol}, 1.0$ equiv.) and solid $\mathrm{NaHCO}_{3}$ (419 mg, 4.99 mmol, 246 equiv.) in anhydrous DCM (36 mL) was added 2-bromo-1-ethylpyridinium tetrafluoroborate 130 (141 $\mathrm{mg}, 0.50 \mathrm{mmol}, 24.6$ equiv.). The reaction mixture was stirred in dark under argon atmosphere at room temperature for 19 hours. The reaction was quenched by addition of distilled water $(3.9 \mathrm{~mL})$. The aqueous phase was separated and extracted by ethyl acetate $(4 \times 8 \mathrm{~mL})$. The combined organic phase was dried with anhydrous $\mathrm{Na}_{2} \mathrm{SO}_{4}$, filtered, and then concentrated by rotary evaporation. The crude material was purified by flash silica chromatography (pet. ether/EtOAc $=2 / 1$ ) to afford the title compound as a colorless oil $(9.4 \mathrm{mg}$, yield $75 \%) . \mathbf{R}_{f}: 0.73$ (pet. ether/EtOAc = 1:1). ${ }^{1} \mathbf{H}$ NMR $\left(600 \mathrm{MHz}, \mathrm{CDCl}_{3}\right): \delta 7.70-7.64(\mathrm{~m}, 4 \mathrm{H}), 7.45-7.36(\mathrm{~m}$, 6H), 7.19 (dd, $J=15.4,11.3 \mathrm{~Hz}, 1 \mathrm{H}, \mathrm{H}-15), 6.73$ (s, 1H, H-7), 6.46 (t, $J=11.4 \mathrm{~Hz}, 1 \mathrm{H}$, H-14), 5.93 (ddd, $J=15.3,8.9,5.6 \mathrm{~Hz}, 1 \mathrm{H}, \mathrm{H}-16$ ), 5.51 - 5.47 (m, overlapped, 2H, H-10, H-13), 4.26 (td, $J=10.6,10.1,2.8$ Hz, 1H, H-18a), 4.22 - 4.18 (m, 1H, H-18b), 3.84 (dd, $J=10.8,5.1 \mathrm{~Hz}, 1 \mathrm{H}, \mathrm{H}-11 \mathrm{a}), 3.79$ (dd, $J=10.8,4.8 \mathrm{~Hz}, 1 \mathrm{H}, \mathrm{H}-11 \mathrm{~b}), 3.33$ (dd, $J=14.6$, $2.7 \mathrm{~Hz}, 1 \mathrm{H}, \mathrm{H}-9 \mathrm{a}), 3.25$ (dd, $J=14.7,11.0 \mathrm{~Hz}, 1 \mathrm{H}, \mathrm{H}-9 \mathrm{~b}), 2.76$ (dt, $J=13.7,6.3 \mathrm{~Hz}, 1 \mathrm{H}$, H-5a), 2.64 (dt, $J=15.0,7.7$ Hz, 1H, H-5b), $2.52-2.45$ (m, 1H, H-17a), $2.44-2.36$ (m, 1H, H-17b), 2.29 (t, $J=7.6 \mathrm{~Hz}, 2 \mathrm{H}, \mathrm{H}-2), 1.76$ (dt, $J=14.5,7.5 \mathrm{~Hz}, 1 \mathrm{H}, \mathrm{H}-4 \mathrm{a}), 1.66$ (dt, $J=13.9,6.8 \mathrm{~Hz}, 1 \mathrm{H}, \mathrm{H}-4 \mathrm{~b}), 1.56-1.49$ (m, 2H, H-3), 1.07 (s, 9H). ${ }^{13} \mathbf{C}$ NMR (150 MHz, $\mathrm{CDCl}_{3}$ ): $\delta 173.4$ (C-1), 165.8 (C-12), 164.7 (C-8), 156.6 (C-6), 144.8 (C-14), 140.1 (C16), 135.61, 135.56, 133.13, 133.10, 129.8, 129.5 (C-15), 127.8, 127.7, 116.1 (C-13), 113.1 (C-7), 72.7 (C-10), 65.3 (C-11), 61.8 (C-18), 35.2 (C-9), 34.6 (C-2), 32.6 (C-17), 30.8 (C-5), 28.2 (C-4), 26.8, 24.1 (C-3), 19.3. FTIR (thin film) v2954, 2931, 2858, 1723, 1640, 1602, 1523, 1472, 1461, 1428, 1388, 1361, 1260, 1228, 1173, 1114, 1091, 998, $964,823,742,703,615,505 \mathrm{~cm}^{-1}$. HRMS (ESI): Calculated for $\mathrm{C}_{34} \mathrm{H}_{42} \mathrm{NO}_{5} \mathrm{SSi}_{2}{ }^{+}[\mathrm{M}+\mathrm{H}]^{+}$ 604.2547; found 604.2519. [ $\alpha]_{D}^{25}-32.5$ ( $\left.c=0.07, \mathrm{DCM}\right)$. 


\section{Preparation of $(S, 12 Z, 6 Z, 8 E)-3-(h y d r o x y m e t h y l)-4,12-d i o x a-1(2,4)-$}

thiazolacycloheptadecaphane-6,8-diene-5,13-dione, 441

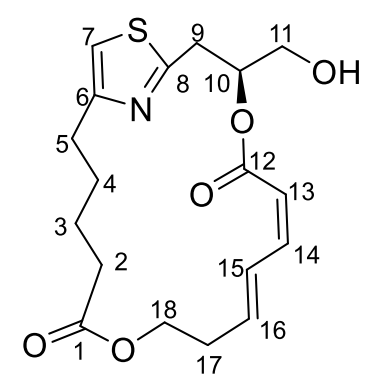

To a solution of macrocyclic compound 440 (35.7 mg, $0.059 \mathrm{mmol}, 1.0$ equiv.) and acetic acid (16.4 $\mu \mathrm{L}, 0.286 \mathrm{mmol}, 4.85$ equiv.) in anhydrous THF (3.0 mL) was slowly added tetrabutylammonium fluoride (118 $\mu \mathrm{L}, 1.0 \mathrm{M}$ in THF, $0.118 \mathrm{mmol}, 2.0$ equiv.) over 20 minutes at $0{ }^{\circ} \mathrm{C}$. Then the reaction mixture was allowed to warm up to room temperature and stirred at room temperature for 17 hours. The reaction mixture was then diluted with ethyl acetate $(20 \mathrm{~mL})$ and added saturated aqueous $\mathrm{NaHCO}_{3}$ solution $(2 \mathrm{~mL})$. The aqueous phase was separated and extracted by ethyl acetate $(3 \times 5 \mathrm{~mL})$. The combined organic phase was dried with anhydrous $\mathrm{Na}_{2} \mathrm{SO}_{4}$, filtered, and then concentrated by rotary evaporation. The crude material was purified by flash silica chromatography (pet. ether/EtOAc $=1 / 2)$ to afford the title compound $(18.5 \mathrm{mg}$, yield $86 \%)$ as a colourless oil. $\mathbf{R}_{f}: 0.25$ (pet. ether/EtOAc $\left.=1 / 2\right) .{ }^{1} \mathbf{H}$ NMR $\left(500 \mathrm{MHz}, \mathrm{CDCl}_{3}\right) \delta 7.17(\mathrm{dd}, J=15.7,11.7$ Hz, 1H, H-15), 6.73 (s, 1H, H-7), 6.48 (t, $J=11.3$ Hz, 1H, H-14), 5.95 (ddd, $J=15.0,8.7$, $5.6 \mathrm{~Hz}, 1 \mathrm{H}, \mathrm{H}-16), 5.51$ (d, $J=11.3 \mathrm{~Hz}, 1 \mathrm{H}, \mathrm{H}-13), 5.49$ - 5.44 (m, 1H, H-10), 4.30 4.25 (m, 1H, H-18a), 4.20 - 4.15 (m, 1H, H-18b), 3.86 (dd, J=11.9, 4.0 Hz, 1H, H-11a), $3.81(\mathrm{dd}, J=11.9,5.3 \mathrm{~Hz}, 1 \mathrm{H}, \mathrm{H}-11 \mathrm{~b}), 3.30-3.26$ (m, 2H, H-9), 2.78 (dt, $J=13.2,6.3$ Hz, 1H, H-5a), 2.64 (dt, J = 14.8, 7.6 Hz, 1H, H-5b), 2.53 - 2.37 (m, 2H, H-17), 2.28 (t, $\mathrm{J}=7.6 \mathrm{~Hz}, 2 \mathrm{H}, \mathrm{H}-2), 1.81-1.60$ (m, 2H, H-4), $1.52-1.48$ (m, 2H, H-3). ${ }^{13} \mathbf{C}$ NMR (125 $\left.\mathrm{MHz}, \mathrm{CDCl}_{3}\right) \delta 173.4$ (C-1), 165.3 (C-12), 164.9 (C-8), 156.7 (C-6), 145.3 (C-14), 140.6 (C-16), 129.2 (C-15), 115.6 (C-13), 113.2 (C-7), 73.1 (C-10), 64.4(C-11), 61.7 (C-18), 34.7 (C-9), 34.6 (C-2), 32.5 (C-17), 30.8 (C-5), 28.2 (C-4), 24.0 (C-3). FTIR (thin film) $v 3355,2923,2854,1719,1639,1602,1524,1458,1420,1380,1260,1172,1131,1057$, 1002, 964, 817, $759 \mathrm{~cm}^{-1}$. HRMS (ESI): Calculated for $\mathrm{C}_{18} \mathrm{H}_{24} \mathrm{NO}_{5} \mathrm{~S}^{+}[\mathrm{M}+\mathrm{H}]^{+} 366.1370$; found 366.1346. $[\alpha]_{D}{ }^{25}-137(c=0.125, \mathrm{DCM})$. 


\subsubsection{Synthesis of the Side Chain Fragments}

\section{Preparation of diethyl (2-oxopropyl)phosphonate, 468}

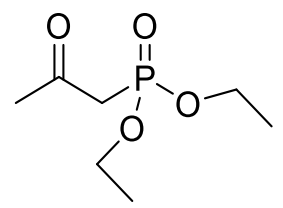

To a stirred suspension of potassium iodide $(22.9 \mathrm{~g}, 138 \mathrm{mmol}, 1.1$ equiv.) in acetone/acetonitrile $(50 \mathrm{~mL} / 50 \mathrm{~mL})$ was added chloroacetone 469 (10 mL, $125 \mathrm{mmol}, 1$ equiv.) The mixture was stirred in dark at room temperature for 1 hour, then triethyl phosphite (23.8 mL, $138 \mathrm{mmol}, 1$ equiv.) was added dropwise over $30 \mathrm{~min}$ via a dropping funnel. The mixture was stirred at room temperature for 13 hours, then filtered through a plug of Celite and the filtrate was concentrated under reduced pressure. The crude material was then dissolved in $100 \mathrm{~mL}$ distilled water and the solution was carefully adjusted to $\mathrm{pH} 9$ to 10 with solid lithium carbonate. Then the aqueous solution was washed with DCM-hexane $(\mathrm{v} / \mathrm{v}=5 / 95,3 \times 100 \mathrm{~mL})$ and then extracted with EtOAc $(5 \times$ $100 \mathrm{~mL}$ ). The combined EtOAc extracts were washed with brine, dried over $\mathrm{Na}_{2} \mathrm{SO}_{4}$ filtered and then concentrated under vacuum to afford the title compound as a yellow oil $\left(18.3 \mathrm{~g}\right.$, yield 75\%). ${ }^{1} \mathbf{H}$ NMR $\left(500 \mathrm{MHz}, \mathrm{CDCl}_{3}\right) \delta 4.19-4.10(\mathrm{~m}, 4 \mathrm{H}), 3.09\left(\mathrm{~d}, J_{\mathrm{CH}-\mathrm{P}}=\right.$ $22.9 \mathrm{~Hz}, 2 \mathrm{H}), 2.32(\mathrm{~s}, 3 \mathrm{H}), 1.34(\mathrm{t}, J=7.1 \mathrm{~Hz}, 6 \mathrm{H}) .{ }^{13} \mathbf{C} \mathbf{N M R}\left(125 \mathrm{MHz}, \mathrm{CDCl}_{3}\right) \delta 199.7$ $\left(\mathrm{d}, J_{\mathrm{C}-\mathrm{P}}=6.3 \mathrm{~Hz}\right), 62.4,62.3,43.10\left(\mathrm{~d}, J_{\mathrm{C}-\mathrm{P}}=127 \mathrm{~Hz}\right), 31.1,16.10,16.0$. FTIR (thin film) $v 2984,1713,1392,1361,1249,1163,1098,1018,957,853,820,790,591,531,494$, $459 \mathrm{~cm}^{-1}$. HRMS (ESI): Calculated for $\mathrm{C}_{7} \mathrm{H}_{16} \mathrm{O}_{4} \mathrm{P}^{+}[\mathrm{M}+\mathrm{H}]^{+}$195.0781; found 195.0776 . These characterisation data match those reported in the literature. ${ }^{353}$

\section{Preparation of ethyl (E)-4-hydroxy-3-methylbut-2-enoate, 472}<smiles>CCOC(=O)C=C(C)CO</smiles>

To a solution of alcohol 470 (3.3 mL, purity $90 \%, 43.6 \mathrm{mmol}, 1.0$ equiv.) in acetonitrile $(100 \mathrm{~mL})$ was added ethyl 2-(triphenyl- $\lambda^{5}$-phosphaneylidene)acetate 471 (18.3 g, 52.4 mmol, 1.2 equiv.) under nitrogen atmosphere. The reaction was heated at $70{ }^{\circ} \mathrm{C}$ for 24 hours and then concentrated under vacuum at room temperature. The residue was slurried with $\mathrm{Et}_{2} \mathrm{O}$ for 1 hour and then filtered. The filtrate was then concentrated under vacuum and the crude material was purified by flash silica chromatography (pet. ether/ethyl acetate $=3 / 1)$ to afford the title compound as a colourless oil $(4.46 \mathrm{~g}$, yield $71 \%)$. (Note: 
The title compound was volatile, therefore the solvents of the product fractions after column chromatography were evaporated at room temperature) $\mathbf{R}_{\boldsymbol{f}}$ : 0.60 (pet. ether/EtOAc = 1:1). ${ }^{1} \mathbf{H}$ NMR $\left(500 \mathrm{MHz}, \mathrm{CDCl}_{3}\right) \delta 6.01-5.96(\mathrm{~m}, 1 \mathrm{H}), 4.17(\mathrm{q}, J=7.2$ $\mathrm{Hz}, 2 \mathrm{H}), 4.16-4.12(\mathrm{~m}, 2 \mathrm{H}), 2.09$ (s, 3H), $1.28(\mathrm{t}, J=7.1 \mathrm{~Hz}, 3 \mathrm{H}) .{ }^{13} \mathbf{C}$ NMR (125 MHz, $\left.\mathrm{CDCl}_{3}\right): \delta 166.9,157.4,113.5,66.9,59.7,15.5,14.2$. These characterisation data match those reported in the literature. ${ }^{354}$

\section{Preparation of active manganese dioxide from activated carbon ${ }^{325}$}

A solution of potassium permanganate (10 g, $0.063 \mathrm{mmol}, 1.0$ equiv.) in distilled water $(250 \mathrm{~mL})$ contained in a one-litre beaker was heated to the boiling point and removed from the source of heat, and then treated with activated carbon $(3.13 \mathrm{~g}, 0.261,4.1$ equiv.) portionwise over 10 minutes. After the complete addition of the activated carbon, the mixture was boiled for another 5 minutes until the purple colour was completely discharged. Then the resulting black suspension was allowed to stand at room temperature for 15 minutes and filtered on a Büchner funnel. The precipitate was washed water $(4 \times$ $50 \mathrm{~mL}$ ) and spread out to dry in the air. The air-dried material was then dried in an oven at $105-110^{\circ}$ for 24 hours to afford the oxidant $(7.07 \mathrm{~g})$ as a fine powder which could be used directly without grinding.

\section{Preparation of ethyl (E)-3-methyl-4-oxobut-2-enoate, 473}<smiles>CCOC(=O)/C=C(\C)C=O</smiles>

To a solution of alcohol 472 (1.02 g, $7.07 \mathrm{mmol}, 1.0$ equiv.) in anhydrous DCM (140 mL) was added freshly prepared active manganese dioxide (7.07 g, $71.5 \mathrm{mmol}, 10.1$ equiv.). Then the reaction mixture was stirred vigorously at room temperature for 1 hour. The reaction mixture was then filtered through a Celite pad and the filtrate was evaporated at room temperature to afford the title product as a yellow oil $(0.84 \mathrm{~g}$, yield $82 \%)$. ${ }^{1} \mathbf{H}$ NMR $\left(500 \mathrm{MHz}, \mathrm{CDCl}_{3}\right) \delta 9.54(\mathrm{~s}, 1 \mathrm{H}), 6.49(\mathrm{q}, J=1.8 \mathrm{~Hz}, 1 \mathrm{H}), 4.26(\mathrm{q}, J=6.8 \mathrm{~Hz}, 2 \mathrm{H}), 2.15$ $(\mathrm{d}, J=1.2 \mathrm{~Hz}, 3 \mathrm{H}), 1.33(\mathrm{t}, J=7.2 \mathrm{~Hz}, 3 \mathrm{H}) .{ }^{13} \mathbf{C ~ N M R}\left(125 \mathrm{MHz}, \mathrm{CDCl}_{3}\right): \delta 194.8,165.7$, $150.6,135.8,61.3,14.4,11.0$. These characterisation data match those reported in the literature. ${ }^{354}$ 
Preparation of ethyl (2E,4E)-3-methyl-6-oxohepta-2, 4-dienoate, 359

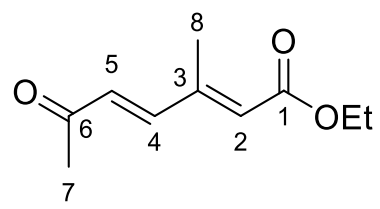

To a solution of aldehyde 473 (13.4 g, 0.094 mol, 1.0 equiv.) in distilled water (187 mL) was added diethyl (2-oxopropyl)phosphonate 468 (18.3 g, 0.094 mol, 1.0 equiv.) and potassium carbonate $(26.0 \mathrm{~g}, 0.188 \mathrm{~mol}, 2.0$ equiv.). The reaction mixture was stirred at room temperature for 18 hours. The reaction mixture was then extracted with $\operatorname{Et}_{2} \mathrm{O}(3 \times$ $150 \mathrm{~mL}$ ). The combined organic phase was washed with brine, dried over anhydrous $\mathrm{Na}_{2} \mathrm{SO}_{4}$, filtered, and then concentrated by rotary evaporation. The crude material was purified by flash silica chromatography (pet. ether/EtOAc $=9 / 1$ to 3/1) to afford the title compound 359 (13.0 g, yield 76\%) as a white solid and the by-product 474 (1.10g, yield $3.6 \%$ ) generated from the undesired aldol reaction as a yellow oil.

359: $\mathbf{R}_{f}: 0.50$ (pet. ether/EtOAc $\left.=1: 1\right)$. Melting point: $48-50{ }^{\circ} \mathrm{C} .{ }^{1} \mathbf{H}$ NMR $(500 \mathrm{MHz}$, $\left.\mathrm{CDCl}_{3}\right) \delta 7.10(\mathrm{~d}, J=15.0 \mathrm{~Hz}, 1 \mathrm{H}, \mathrm{H}-4), 6.45$ (d, $\left.J=15.0 \mathrm{~Hz}, 1 \mathrm{H}, \mathrm{H}-5\right), 6.07$ (s, 1H, H2), 4.20 (q, $J=7.1 \mathrm{~Hz}, 2 \mathrm{H}), 2.33$ (s, 3H, H-7), 2.29 (d, $J=1.2 \mathrm{~Hz}, 3 \mathrm{H}, \mathrm{H}-8), 1.30$ (t, $J=$ $7.1 \mathrm{~Hz}, 3 \mathrm{H}) .{ }^{13} \mathrm{C}$ NMR $\left(125 \mathrm{MHz}, \mathrm{CDCl}_{3}\right) \delta 198.2$ (C-6), 166.1 (C-1), 149.3 (C-3), 145.7 (C4), 131.4 (C-5), 126.4 (C-2), 60.5, 28.1 (C-7), 14.4, 13.8 (C-8). FTIR (thin film) v2982, 1712, 1668, 1638, 1605, 1367, 1247, 1230, 1160, 1117, 1045, 985, 893, 836, 602, 537 $\mathrm{cm}^{-1}$. HRMS (ESI): Calculated for $\mathrm{C}_{10} \mathrm{H}_{15} \mathrm{O}_{3}{ }^{+}[\mathrm{M}+\mathrm{H}]^{+}$183.1016; found 183.1015.

Diethyl (2E,4E,9E)-8-hydroxy-3,9-dimethyl-6-oxoundeca-2,4,9-trienedioate, 474<smiles>CCOC(=O)C=C(C)C=CC(=O)CC(O)C(C)=CC(=O)OCC</smiles>

$\mathbf{R}_{f}: 0.50$ (pet. ether/EtOAc = 1:1). ${ }^{1} \mathbf{H}$ NMR $\left(500 \mathrm{MHz}, \mathrm{CDCl}_{3}\right) \delta 7.16(\mathrm{~d}, J=15.0 \mathrm{~Hz}$, 1H, H-4), 6.47 (d, J = 15.0 Hz, 1H, H-5), $6.10-6.08$ (m, 1H, H-2), $6.05-6.02$ (m, 1H, H-10), 4.60 (d, J = 8.3 Hz, 1H, H-8), 4.23 - 4.12 (m, 4H), 3.28 (br. s, 1H, OH), 2.93 2.86 (m, 1H, H-7a), 2.80 (dd, $J=17.3,9.3 \mathrm{~Hz}, 1 \mathrm{H}, \mathrm{H}-7 \mathrm{~b}$ ), 2.27 (d, $J=5.0 \mathrm{~Hz}, 3 \mathrm{H}, \mathrm{H}-12$ ), $2.13(\mathrm{~d}, J=5.0 \mathrm{~Hz}, 3 \mathrm{H}, \mathrm{H}-13), 1.31-1.24(\mathrm{~m}, 6 \mathrm{H}) .{ }^{13} \mathbf{C} \mathbf{N M R}\left(125 \mathrm{MHz}, \mathrm{CDCl}_{3}\right) \delta 199.4$ (C-6), 166.8 (C-11), 166.0 (C-1), 157.5 (C-9), 148.8 (C-3), 146.3 (C-4), 130.2 (C-5), 127.3 (C-2), 115.8 (C-10), 72.0 (C-8), 60.5, 59.9, 45.8 (C-7), 15.4 (C-13), 14.4, 14.3, 13.7 (C-12). FTIR (thin film) v3483, 2981, 1710, 1654, 1597, 1444, 1367, 1353, 1277, 1226, 
1152, 1093, 1041, 979, 875, 825, $538 \mathrm{~cm}^{-1}$. HRMS (ESI): Calculated for $\mathrm{C}_{17} \mathrm{H}_{24} \mathrm{NaO}_{6}{ }^{+}$ $[\mathrm{M}+\mathrm{Na}]^{+} 347.1471$; found 347.1477 .

Preparation of ethyl $(2 E, 4 E)$-6-hydroxy-3-methylhepta-2,4-dienoate, 475

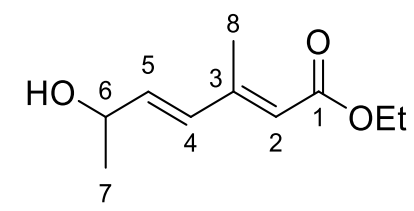

To a solution of ketone 359 (13.0 g, 71.3 mmol, 1.0 equiv.) and cerium (III) chloride heptahydrate (26.8 g, $72.0 \mathrm{mmol}, 1.01$ equiv.) in methanol $(185 \mathrm{~mL})$ was added sodium borohydride ( $1.89 \mathrm{~g}, 49.9 \mathrm{mmol}, 0.7$ equiv.) at $0{ }^{\circ} \mathrm{C}$. Then the reaction mixture was stirred at $0{ }^{\circ} \mathrm{C}$ for 2 hours and then quenched by slow addition of distilled water $(300 \mathrm{~mL})$. The organic solvent was then removed under reduce pressure and the resulting aqueous mixture was extracted with ethyl acetate $(3 \times 150 \mathrm{~mL})$. The combined organic phase was washed with brine, dried over anhydrous $\mathrm{Na}_{2} \mathrm{SO}_{4}$, filtered, and then concentrated by rotary evaporation to afford the title compound (13.0 g, yield 99\%) as a colourless oil, which was used directly for the next step without further purification. ${ }^{1} \mathbf{H} \mathbf{~ N M R}(500 \mathrm{MHz}$, $\left.\mathrm{CDCl}_{3}\right) \delta 6.26(\mathrm{~d}, J=15.7 \mathrm{~Hz}, 1 \mathrm{H}, \mathrm{H}-4), 6.11$ (dd, $\left.J=15.7,5.9 \mathrm{~Hz}, 1 \mathrm{H}, \mathrm{H}-5\right), 5.76$ (s, 1H, H-2), 4.43 (quintet, $J=6.2 \mathrm{~Hz}, 1 \mathrm{H}, \mathrm{H}-6), 4.16$ (q, $J=7.0 \mathrm{~Hz}, 2 \mathrm{H}$ ), 2.26 (s, 3H, H-8), 1.89 (br. s, $1 \mathrm{H}, \mathrm{OH}), 1.32$ (d, $J=6.4 \mathrm{~Hz}, 3 \mathrm{H}, \mathrm{H}-7), 1.27$ (t, $J=7.0 \mathrm{~Hz}, 3 \mathrm{H}) .{ }^{13} \mathbf{C ~ N M R}$ (125 MHz, $\left.\mathrm{CDCl}_{3}\right) \delta 167.0$ (C-1), 151.5 (C-3), 139.2 (C-5), 132.4 (C-4), 119.6 (C-2), 68.4 (C-6), 59.8, 23.3 (C-7), 14.3, 13.8 (C-8). FTIR (thin film) v3401, 2976, 2930, 1708, 1692, 1638, 1611, 1445, 1387, 1366, 1351, 1231, 1226, 1146, 1094, 1039, 966, 942, 875, 827, 545, 462, $419 \mathrm{~cm}^{-1}$. HRMS (ESI): Calculated for $\mathrm{C}_{10} \mathrm{H}_{17} \mathrm{O}_{3}{ }^{+}[\mathrm{M}+\mathrm{H}]^{+}$185.1172; found 185.1185 .

\section{Preparation of ethyl (2E,4E)-6-bromo-3-methylhepta-2,4-dienoate, 462}

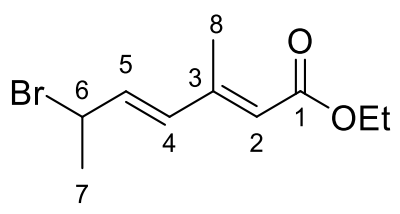

To a solution of alcohol 475 (3.81 g, 0.207 mol, 1.0 equiv.) in anhydrous DCM (5.8 mL) was added dropwise a solution of phosphorus tribromide $(0.89 \mathrm{~mL}, 9.5 \mathrm{mmol}, 0.46$ equiv. $)$ in anhydrous DCM $(5.8 \mathrm{~mL})$ by syringe pump over 30 minutes at $1-2{ }^{\circ} \mathrm{C}$. Then the reaction mixture was stirred at that temperature for 3 hours. Upon completion, $60 \mathrm{~mL}$ 
saturated aqueous $\mathrm{NaHCO}_{3}$ solution was added slowly to the reaction mixture at $0-4{ }^{\circ} \mathrm{C}$. The aqueous phase was then separated and extracted with DCM $(3 \times 50 \mathrm{~mL})$. The combined organic phase was dried with anhydrous $\mathrm{Na}_{2} \mathrm{SO}_{4}$, filtered, and then concentrated by rotary evaporation at room temperature to afford the pure title compound as a yellow oil (4.96 g, yield 97\%), which was used for the next step without further purification. ${ }^{1} \mathbf{H}$ NMR $\left(500 \mathrm{MHz}, \mathrm{CDCl}_{3}\right): \delta 6.30-6.20(\mathrm{~m}, 2 \mathrm{H}, \mathrm{H}-4, \mathrm{H}-5), 5.80(\mathrm{~s}, 1 \mathrm{H}$, H-2), $4.81-4.74$ (m, 1H, H-6), 4.18 (q, J=7.1 Hz, 2H), 2.27 (s, 3H, H-8), 1.84 (d, J= $6.5 \mathrm{~Hz}, 3 \mathrm{H}, \mathrm{H}-7), 1.29$ (t, $J=7.0 \mathrm{~Hz}, 3 \mathrm{H}) .{ }^{13} \mathbf{C ~ N M R}\left(125 \mathrm{MHz}, \mathrm{CDCl}_{3}\right): \delta 166.6(\mathrm{C}-1)$, 150.5 (C-3), 137.0 (C-5), 133.8 (C-4), 120.9 (C-2), 59.9, 48.4 (C-6), 25.7 (C-7), 14.3, 13.8 (C-8). FTIR (thin film) v 2979, 1707, 1634, 1612, 1442, 1367, 1354, 1275, 1236, 1148, 1095, 1074, 1042, 1019, 1004, 962, 879, 845, 614, 571, $452 \mathrm{~cm}^{-1}$. HRMS (ESI): Calculated for $\mathrm{C}_{10} \mathrm{H}_{16} \mathrm{BrO}_{2}^{+}[\mathrm{M}+\mathrm{H}]^{+}$247.0328; found 247.0417 .

\section{Preparation of ((3E,5E)-7-ethoxy-5-methyl-7-oxohepta-3,5-dien-2-}

\section{yl)triphenylphosphonium bromide, 476}

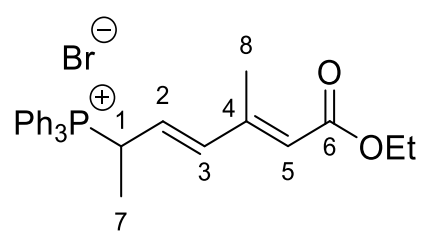

To a solution of bromide 462 (349 mg, $1.41 \mathrm{mmol}, 1.0$ equiv.) in toluene (1.7 mL) was added triphenylphosphine (378 $\mathrm{mg}, 1.43 \mathrm{mmol}, 1.01$ equiv.). The reaction mixture was stirred at room temperature for 48 hours. The percipitate was filtered and washed with $\mathrm{Et}_{2} \mathrm{O}(3 \times 3 \mathrm{~mL})$ to provide the title compound as a white solid. ${ }^{1} \mathbf{H}$ NMR (500 MHz, $\left.\mathrm{CDCl}_{3}\right): \delta 8.08-8.01(\mathrm{~m}, 6 \mathrm{H}), 7.80-7.74(\mathrm{~m}, 3 \mathrm{H}), 7.70-7.74$ (m, 6H), 6.79 (dp, $J=15.2,7.5 \mathrm{~Hz}, 1 \mathrm{H}, \mathrm{H}-1), 6.65$ (dd, $J=15.6,4.9 \mathrm{~Hz}, 1 \mathrm{H}, \mathrm{H}-3)$, 5.74 (s, 1H, H-5), 5.70 (dd, $J=15.3,8.0 \mathrm{~Hz}, 1 \mathrm{H}, \mathrm{H}-2), 4.11$ (q, $J=7.1 \mathrm{~Hz}, 2 \mathrm{H}), 2.02$ (s, 3H, H-8), 1.46 (dd, $J=19.0,6.7 \mathrm{~Hz}, 3 \mathrm{H}, \mathrm{H}-7), 1.26$ (t, $J=7.4 \mathrm{~Hz}, 3 \mathrm{H}) .{ }^{13} \mathbf{C}$ NMR $\left(125 \mathrm{MHz}, \mathrm{CDCl}_{3}\right): \delta 166.7\left(\mathrm{~d}, J_{\mathrm{C}-\mathrm{P}}=1.8 \mathrm{~Hz}, \mathrm{C}-6\right), 149.4\left(\mathrm{~d}, J_{\mathrm{C}-\mathrm{P}}=4.3 \mathrm{~Hz}, \mathrm{C}-4\right)$, $141.2\left(\mathrm{~d}, J_{\mathrm{C}-\mathrm{P}}=13.2 \mathrm{~Hz}, \mathrm{C}-3\right), 134.8\left(\mathrm{~d}, J_{\mathrm{C}-\mathrm{P}}=3.2 \mathrm{~Hz}\right), 134.1\left(\mathrm{~d}, J_{\mathrm{C}-\mathrm{P}}=9.3 \mathrm{~Hz}\right), 130.3$ $\left(\mathrm{d}, J_{\mathrm{C}-\mathrm{P}}=12.2 \mathrm{~Hz}\right), 126.3\left(\mathrm{~d}, J_{\mathrm{C}-\mathrm{P}}=7.7 \mathrm{~Hz}, \mathrm{C}-2\right), 121.8\left(\mathrm{~d}, J_{\mathrm{C}-\mathrm{P}}=4.0 \mathrm{~Hz}, \mathrm{C}-5\right), 117.6$ $\left(\mathrm{d}, J_{\mathrm{C}-\mathrm{P}}=82.5 \mathrm{~Hz}\right), 59.9,30.4\left(\mathrm{~d}, J_{\mathrm{C}-\mathrm{P}}=44.6 \mathrm{~Hz}, \mathrm{C}-1\right), 14.7\left(\mathrm{~d}, J_{\mathrm{C}-\mathrm{P}}=3.0 \mathrm{~Hz}, \mathrm{C}-7\right)$, 14.2, 13.5 (C-8). $\delta$. FTIR (thin film) $v$ 3035, 3009, 2979, 2929, 2817, 1705, 1630, 1608, 1588, 1487, 1459, 1438, 1399, 1365, 1318, 1300, 1286, 1240, 1155, 1110, 1092, 1040, 985, 954, 887, 839, 754, 723, 711, 690, 663, 637, 550, 531, 521, 508, 
488, 469, 458, $446 \mathrm{~cm}^{-1}$. HRMS (ESI): Calculated for $\mathrm{C}_{28} \mathrm{H}_{30} \mathrm{O}_{2} \mathrm{P}[\mathrm{M}]^{+}$429.1983; found 429.1988 .

\section{Preparation of trienoate 477 and 478}

To a solution of phosphonium salt 476 (78 $\mathrm{mg}, 0.154 \mathrm{mmol}, 2.0$ equiv.) in anhydrous THF (1.8 mL) was added $n$-BuLi $(69 \mu \mathrm{L}, 2.0 \mathrm{M}$ in cyclohexane, $0.139 \mathrm{mmol}, 1.8$ equiv.) dropwise at $-50^{\circ} \mathrm{C}$. Then the reaction mixture was allowed to room temperature at stirred at room temperature for 2 hours. The resulting orange solution was cooled to $-40{ }^{\circ} \mathrm{C}$ prior to the addition of a solution of aldehyde $\mathbf{3 2 2}$ (10 mg, $0.077 \mathrm{mmol}, 1.0$ equiv.) in anhydrous THF $(0.5 \mathrm{~mL})$. The reaction mixture was then allowed to warm up to $-4{ }^{\circ} \mathrm{C}$ over 2 hours. The reaction was then quenched with saturated $\mathrm{NH}_{4} \mathrm{Cl}$ solution $(4 \mathrm{~mL})$ and extracted with $\mathrm{Et}_{2} \mathrm{O}(3 \times 10 \mathrm{~mL})$. The combined organic phase was washed with brine, dried over anhydrous $\mathrm{Na}_{2} \mathrm{SO}_{4}$, filtered, and then concentrated by rotary evaporation. The crude material was purified by flash silica chromatography (pet. ether/EtOAc $=10 / 1$ ) to afford a 62/38 ratio of 477 and $\mathbf{4 7 8}(11.4 \mathrm{mg}$, yield 53\%) as a yellow oil.

Ethyl (2E,4E,6E)-7-((S)-2,2-dimethyl-1,3-dioxolan-4-yl)-3,6-dimethylhepta-2,4,6trienoate, 477

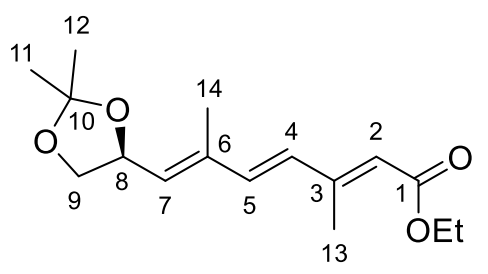

${ }^{1} \mathbf{H}$ NMR (500 MHz, $\left.\mathrm{CDCl}_{3}\right): \delta 6.61(\mathrm{~d}, J=15.8 \mathrm{~Hz}, 1 \mathrm{H}, \mathrm{H}-5), 6.27$ (d, $J=15.8 \mathrm{~Hz}, 1 \mathrm{H}$, H-4), 5.82 (s, 1H, H-2), 5.68 - 5.64 (m, overlapped, 1H, H-7), 4.97 - 4.90 (m, overlapped, 1H, H-8), 4.17 (q, 2H), $4.14-4.10$ (m, overlapped, 1H, H-9a), 3.60 - 3.55 (m, overlapped, 1H, H-9b), 2.30 (s, 3H, H-13), 1.87 (s, 3H, H-14), 1.44 (s, overlapped, 3H, H-11 or H12), 1.41 (s, overlapped, 3H, H-11 or H-12), 1.28 (t, $J=7.2 \mathrm{~Hz}) \cdot{ }^{13} \mathbf{C}$ NMR (125 MHz, $\mathrm{CDCl}_{3}$ ): $\delta 167.0$ (C-1), 152.0 (C-3), 137.8 (C-5), 137.4 (C-6), 132.3 (C-7), 131.9 (C-4), 119.8 (C-2), 109.3 (C-10), 72.8 (C-8), 69.33 (C-9), 59.7, 26.7 (C-11 or C-12), 25.95 (C12 or C-11), 14.3, 13.7 (C-13), 12.93 (C-14). 


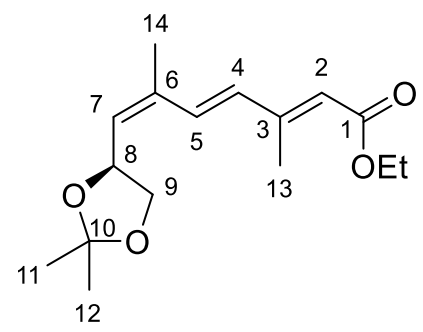

${ }^{1} \mathbf{H}$ NMR $\left(500 \mathrm{MHz}, \mathrm{CDCl}_{3}\right): \delta 7.84(\mathrm{~d}, J=16.2 \mathrm{~Hz}, 1 \mathrm{H}, \mathrm{H}-5), 6.61(\mathrm{~d}, J=16.2 \mathrm{~Hz}, 1 \mathrm{H}$, H-4), 5.70 (s, 1H, H-2), 5.68 - 5.64 (m, overlapped, 1H, H-7), 4.97 - 4.90 (m, overlapped, $1 \mathrm{H}, \mathrm{H}-8), 4.16$ (q, $J=7.2 \mathrm{~Hz}, 2 \mathrm{H}$ ), $4.14-4.10$ (m, overlapped, 1H, H-9a), $3.60-3.55$ (m, overlapped, 1H, H-9b), 2.02 (s, 3H, H-13), 1.93 (s, 3H, H-14), 1.44 (s, overlapped, $3 \mathrm{H}, \mathrm{H}-11$ or H-12), 1.41 (s, overlapped, $3 \mathrm{H}, \mathrm{H}-11$ or $\mathrm{H}-12), 1.29(\mathrm{t}, J=7.2 \mathrm{~Hz}) .{ }^{13} \mathbf{C ~ N M R}$ (125 MHz, $\left.\mathrm{CDCl}_{3}\right): \delta 166.4$ (C-1), 150.8 (C-3), 139.1 (C-4), 138.3 (C-6), 132.5 (C-7), 126.1 (C-5), 117.7 (C-2), 109.30 (C-10), 72.89 (C-8), 69.34 (C-9), 59.7, 26.7 (C-11 or C12), 25.92 (C-12 or C-11), 20.9 (C-13), 13.7, 12.97 (C-14).

Preparation of ethyl $(2 E, 4 E)-6$-(diethoxyphosphoryl)-3-methylhepta-2,4-dienoate, 481

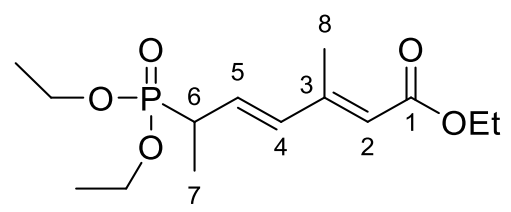

A mixture of bromide 462 (17.5 g, 71.0 mmol, 1.0 equiv.) and triethyl phosphite (36.5 $\mathrm{ml}, 213 \mathrm{mmol}, 3.0$ equiv.) was heated at $140{ }^{\circ} \mathrm{C}$ with vigorous stirring under argon atmosphere for 23 hours. Then the unreacted triethyl phosphite was removed by oil pump at $70{ }^{\circ} \mathrm{C}$. The residue was purified by flash silica chromatography (pet. ether/EtOAc $=$ 1:1) to afford the title compound (10.8 g, yield 50\%) as a yellow oil. $\mathbf{R}_{f}: 0.20$ (pet. ether/EtOAc = 1:1). ${ }^{1} \mathbf{H}$ NMR $\left(500 \mathrm{MHz}, \mathrm{CDCl}_{3}\right): \delta 6.20(\mathrm{dd}, J=15.8,4.6 \mathrm{~Hz}, 1 \mathrm{H}, \mathrm{H}-4)$, $6.12-6.05$ (m, 1H, H-5), 5.74 (s, 1H, H-2), 4.16 (q, J=6.9 Hz, 2H), $4.14-4.04$ (m, 4H), $2.76(\mathrm{dp}, J=21.9,7.1 \mathrm{~Hz}, 1 \mathrm{H}, \mathrm{H}-6), 2.27$ (s, 3H, H-8), 1.37 - 1.22 (m, 12H, overlapped, $\mathrm{H}-7) .{ }^{13} \mathrm{C}$ NMR $\left(125 \mathrm{MHz}, \mathrm{CDCl}_{3}\right): \delta 167.0\left(\mathrm{~d}, J_{\mathrm{C}-\mathrm{P}}=1.3 \mathrm{~Hz}, \mathrm{C}-1\right), 151.5\left(\mathrm{~d}, J_{\mathrm{C}-\mathrm{P}}=3.8\right.$ $\mathrm{Hz}, \mathrm{C}-3), 135.8$ (d, $\left.J_{\mathrm{C}-\mathrm{P}}=13.8 \mathrm{~Hz}, \mathrm{C}-4\right), 132.2$ (d, $\left.J_{\mathrm{C}-\mathrm{P}}=10.0 \mathrm{~Hz}, \mathrm{C}-5\right), 119.5$ (d, $J_{\mathrm{C}-\mathrm{P}}=$ $2.5 \mathrm{~Hz}, \mathrm{C}-2), 62.4\left(\mathrm{~d}, J_{\mathrm{C}-\mathrm{P}}=6.3 \mathrm{~Hz}\right), 62.2\left(\mathrm{~d}, J_{\mathrm{C}-\mathrm{P}}=7.5 \mathrm{~Hz}\right), 59.9,36.3\left(\mathrm{~d}, J_{\mathrm{C}-\mathrm{P}}=139 \mathrm{~Hz}\right.$, C-6), $16.59\left(\mathrm{~d}, J_{\mathrm{C}-\mathrm{P}}=0.9 \mathrm{~Hz}\right), 16.55\left(\mathrm{~d}, J_{\mathrm{C}-\mathrm{P}}=0.9 \mathrm{~Hz}\right), 14.4,13.90\left(\mathrm{~d}, J_{\mathrm{C}-\mathrm{P}}=6.3 \mathrm{~Hz}, \mathrm{C}-7\right)$, 
13.89 (C-8). FTIR (thin film) v2981, 2936, 1708, 1635, 1612, 1444, 1392, 1367, 1353, 1237, 1152, 1096, 1046, 1019, 959, 878, 836, 785, 700, 560, 500, $467 \mathrm{~cm}^{-1}$. HRMS (ESI): Calculated for $\mathrm{C}_{14} \mathrm{H}_{26} \mathrm{O}_{5} \mathrm{P}^{+}[\mathrm{M}+\mathrm{H}]^{+} 305.1512$; found 305.1516 .

\section{Preparation of diethyl ((3E,5E)-7-hydroxy-5-methylhepta-3,5-dien-2-} yl)phosphonate, 482

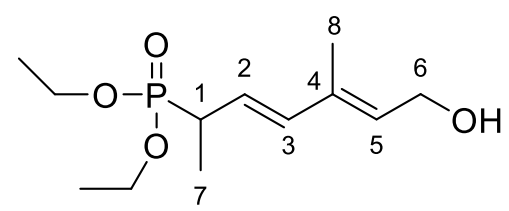

To a solution of ester 481 (521 mg, $1.71 \mathrm{mmol}, 1.0$ equiv.) in anhydrous DCM (30 mL) was added slowly diisobutylaluminium hydride ( $4.3 \mathrm{~mL}, 1.0 \mathrm{M}$ in cyclohexane, 2.5 equiv.) at $-78{ }^{\circ} \mathrm{C}$. Then the reaction mixture was stirred at $-78{ }^{\circ} \mathrm{C}$ for 1 hour and then quenched by addition of saturated aqueous potassium sodium tartrate $(40 \mathrm{~mL})$ solution. The resulting mixture was stirred at room temperature for 2 hours and the aqueous phase was extracted with ethyl acetate $(10 \times 20 \mathrm{~mL})$. The combined organic phase was washed with brine, dried over anhydrous $\mathrm{Na}_{2} \mathrm{SO}_{4}$, filtered, concentrated under reduced pressure to afford the title compound (446 mg, yield 99\%) as a yellow oil. ${ }^{1} \mathbf{H}$ NMR (500 MHz, $\left.\mathrm{CDCl}_{3}\right): \delta 6.18(\mathrm{dd}, J=15.7,4.5 \mathrm{~Hz}, 1 \mathrm{H}, \mathrm{H}-3), 5.70-5.61$ (m, 2H, H-2, H-5), 4.28 (d, $J$ $=6.8 \mathrm{~Hz}, 2 \mathrm{H}, \mathrm{H}-6), 4.13-4.01(\mathrm{~m}, 4 \mathrm{H}), 2.70(\mathrm{dp}, J=21.9,7.1 \mathrm{~Hz}, 1 \mathrm{H}, \mathrm{H}-1), 1.79(\mathrm{~s}, 3 \mathrm{H}$, H-8), 1.36 - 1.26 (m, 12H, overlapped, H-7). $\left.{ }^{13} \mathbf{C ~ N M R ~ ( 1 2 5 ~ M H z , ~} \mathrm{CDCl}_{3}\right): \delta 136.4$ (d, $\left.J_{\mathrm{C}-\mathrm{P}}=13.6 \mathrm{~Hz}, \mathrm{C}-3\right), 135.7\left(\mathrm{~d}, J_{\mathrm{C}-\mathrm{P}}=4.1 \mathrm{~Hz}, \mathrm{C}-4\right), 129.9\left(\mathrm{~d}, J_{\mathrm{C}-\mathrm{P}}=3.8 \mathrm{~Hz}, \mathrm{C}-5\right), 125.3$ (d, $\left.J_{\mathrm{C}-\mathrm{P}}=10.3 \mathrm{~Hz}, \mathrm{C}-2\right), 62.22(\mathrm{~d}, J=7.2 \mathrm{~Hz}), 61.98(\mathrm{~d}, J=7.0 \mathrm{~Hz}), 59.3(\mathrm{C}-6), 35.8\left(\mathrm{~d}, J_{\mathrm{C}-}\right.$ $\mathrm{P}=139.7 \mathrm{~Hz}, \mathrm{C}-1), 16.52,16.48,14.1(\mathrm{~d}, J=6.2 \mathrm{~Hz}, \mathrm{C}-7), 12.6(\mathrm{C}-8)$. FTIR (thin film) $v 3390,2980,2935,1444,1391,1228,1163,1096,1049,1018,960,771,700,560,525$, $480 \mathrm{~cm}^{-1}$. HRMS (ESI): Calculated for $\mathrm{C}_{14} \mathrm{H}_{24} \mathrm{O}_{4} \mathrm{P}^{+}[\mathrm{M}+\mathrm{H}]^{+}$263.1407; found 263.1413. 


\section{Preparation of diethyl $((3 E, 5 E)-5$-methyl-7-oxohepta-3,5-dien-2-yl)phosphonate,}

483

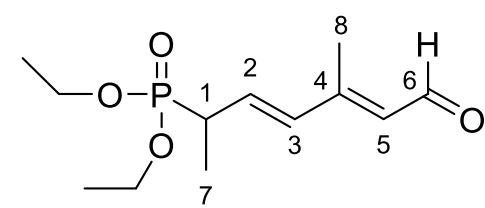

To solution of alcohol 482 (161 mg, 0.377 mmol, 1.0 equiv.) in anhydrous DCM (30 mL) was added freshly prepared active manganese dioxide (1.86 g, $21.4 \mathrm{mmol}, 57$ equiv.). The reaction mixture was stirred vigorously at room temperature for 14 hours. Then the reaction mixture was filtered by a Büchner funnel and the precipitate was rinsed with ethyl acetate $(3 \times 30 \mathrm{~mL})$. The combined filtrate was then concentrated under reduced pressure at $30{ }^{\circ} \mathrm{C}$ to afford the title compound (84 mg, yield $\left.52 \%\right)$ as a yellow oil. ${ }^{1} \mathbf{H}$ NMR $\left(500 \mathrm{MHz}, \mathrm{CDCl}_{3}\right): \delta 10.12(\mathrm{~d}, J=7.8 \mathrm{~Hz}, 1 \mathrm{H}, \mathrm{H}-6), 6.34-6.22$ (m, 2H, H-2, H3), 5.93 (d, $J=7.7 \mathrm{~Hz}, 1 \mathrm{H}, \mathrm{H}-5), 4.16-4.04$ (m, 4H), $2.88-2.74$ (m, 1H, H-1, d. quintet), 2.27 (s, 3H, H-8), $1.42-1.28$ (m, 9H, overlapped, H-7). $\left.{ }^{13} \mathbf{C ~ N M R ~ ( 1 2 5 ~ M H z , ~} \mathrm{CDCl}_{3}\right): \delta$ $191.4(\mathrm{C}-6), 153.7$ (d, $\left.J_{\mathrm{C}-\mathrm{P}}=3.4 \mathrm{~Hz}, \mathrm{C}-4\right), 135.3$ (d, $\left.J_{\mathrm{C}-\mathrm{P}}=10.9 \mathrm{~Hz}, \mathrm{C}-3\right), 134.2$ (d, $J_{\mathrm{C}-\mathrm{P}}=$ $8.7 \mathrm{~Hz}, \mathrm{C}-2), 129.6\left(\mathrm{~d}, J_{\mathrm{C}-\mathrm{P}}=2.5 \mathrm{~Hz}, \mathrm{C}-5\right), 62.3\left(\mathrm{~d}, J_{\mathrm{C}-\mathrm{P}}=5.8 \mathrm{~Hz}\right), 62.2\left(\mathrm{~d}, J_{\mathrm{C}-\mathrm{P}}=5.6 \mathrm{~Hz}\right)$, $36.4\left(\mathrm{~d}, J_{\mathrm{C}-\mathrm{P}}=115.5 \mathrm{~Hz}, \mathrm{C}-1\right), 16.5\left(\mathrm{~d}, J_{\mathrm{C}-\mathrm{P}}=4.7 \mathrm{~Hz}\right), 16.3\left(\mathrm{~d}, J_{\mathrm{C}-\mathrm{P}}=3.7 \mathrm{~Hz}\right), 13.7\left(\mathrm{~d}, J_{\mathrm{C}-}\right.$ $\mathrm{P}=5.2 \mathrm{~Hz}, \mathrm{C}-7$ ), 13.1 (C-8). FTIR (thin film) v 2982, 2934, 1663, 1629, 1598, 1443, 1389, 1241, 1207, 1162, 1112, 1048, 1017, 960, 774, 731, 702, 564, 538, $484 \mathrm{~cm}^{-1}$. HRMS (ESI): Calculated for $\mathrm{C}_{12} \mathrm{H}_{21} \mathrm{O}_{4} \mathrm{P}^{+}[\mathrm{M}+\mathrm{H}]^{+}$260.1177; found 260.1147.

\section{Preparation of diethyl $((2 E, 4 E, 6 E)-5$-methyl-7-((triisopropylsilyl)oxy)hepta-2,4,6-} trien-2-yl)phosphonate, 485

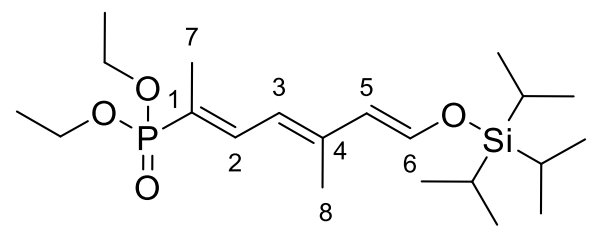

To a solution of KHMDS ( $40 \mu \mathrm{L}, 0.5$ in toluene, $0.020 \mathrm{mmol}, 1.0$ equiv.) in anhydrous THF (0.2 mL) was added a solution of aldehyde 483 (5.2 mg, $0.020 \mathrm{mmol}, 1.0$ equiv.) in anhydrous THF $(0.2 \mathrm{~mL})$ at $-78^{\circ} \mathrm{C}$. Then resulting mixture was stirred at $0{ }^{\circ} \mathrm{C}$ for 30 minutes, followed by dropwise addition of a solution of triisopropylsilyl chloride $(5 \mu \mathrm{L}$, $0.024 \mathrm{mmol}, 1.22$ equiv. $)$ in anhydrous $\operatorname{THF}(0.1 \mathrm{~mL})$ at $-78^{\circ} \mathrm{C}$. Then the reaction mixture was allowed to be stirred at room temperature for 30 minutes and then hydrolysed at $0{ }^{\circ} \mathrm{C}$ with distilled water $(0.2 \mathrm{~mL})$. Then the reaction mixture was diluted with ethyl acetate $(2$ 
$\mathrm{mL})$ and saturated $\mathrm{NH}_{4} \mathrm{Cl}$ aqueous solution $(2 \mathrm{~mL})$. The aqueous phase was separated and extracted with ethyl acetate $(3 \times 5 \mathrm{~mL})$. The combined organic phase was washed with brine, dried over anhydrous $\mathrm{Na}_{2} \mathrm{SO}_{4}$, filtered, concentrated under reduced pressure. The crude material was purified by flash silica chromatography (pet. ether/EtOAc $=2: 1$ ) to afford the title compound (3.7 mg, yield 31\%) as a colourless oil. $\mathbf{R}_{f}$ : 0.43 (pet. ether/EtOAc = 2:1). ${ }^{1} \mathbf{H}$ NMR $\left(500 \mathrm{MHz}, \mathrm{CDCl}_{3}\right) \delta 7.33(\mathrm{ddd}, J=22.8,11.9,1.6 \mathrm{~Hz}, 1 \mathrm{H}$, H-2), 6.84 (d, $J=11.9 \mathrm{~Hz}, 1 \mathrm{H}, \mathrm{H}-6), 6.09$ (d, $J=12.0 \mathrm{~Hz}, 1 \mathrm{H}, \mathrm{H}-5), 4.14-3.97$ (m, 4H), 1.93 (s, 3H, H-8), 1.87 (dd, $J=15.1,1.4 \mathrm{~Hz}, 2 \mathrm{H}, \mathrm{H}-7$ ), 1.31 (t, $J=7.0 \mathrm{~Hz}, 6 \mathrm{H}$ ), 1.22 $1.14(\mathrm{~m}, 3 \mathrm{H}), 1.10-1.03(\mathrm{~m}, 18 \mathrm{H}) .{ }^{13} \mathbf{C}$ NMR (125 MHz, CDCl $) \delta 145.2(\mathrm{C}-6), 140.7$ $(\mathrm{C}-4), 138.5\left(\mathrm{~d}, J_{\mathrm{C}-\mathrm{P}}=12.0 \mathrm{~Hz}, \mathrm{C}-2\right), 120.3\left(\mathrm{~d}, J_{\mathrm{C}-\mathrm{P}}=22.4 \mathrm{~Hz}, \mathrm{C}-5\right), 120.2$ (d, $J_{\mathrm{C}-\mathrm{P}}=182.4$ Hz, C-1), 117.9 (C-3), 61.45, 61.41, 17.64, 16.37, 16.32, 13.2 (C-8), 12.7 (d, $J_{\mathrm{C}-\mathrm{P}}=9.8$ Hz, C-7), 11.9. (FTIR (thin film) v 2943, 2868, 1689, 1632, 1603, 1463, 1390, 1243, 1236, 1183, 1167, 1121, 1098, 1053, 1024, 967, 882, 798, 686, 558, $534 \mathrm{~cm}^{-1}$. HRMS (ESI): Calculated for $\mathrm{C}_{21} \mathrm{H}_{42} \mathrm{O}_{4} \mathrm{PSi}^{+}[\mathrm{M}+\mathrm{H}]^{+}$417.2584; found 417.2584. 


\subsection{Experimental for Chapter Four}

\subsubsection{Attempted Synthesis of Aldehyde (R)-509}

\section{Preparation of $(R)$-tert-butyl((2,2-dimethyl-1,3-dioxolan-4-}

yl)methoxy)diphenylsilane, 503

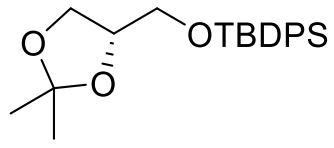

To a solution of $(S)$-solketal $502(3.00 \mathrm{~g}, 22.7 \mathrm{mmol}, 1.0$ equiv.) in anhydrous DMF (10 $\mathrm{mL})$ was added imidazole (3.63 g, $53.3 \mathrm{mmol}, 2.35$ equiv.) and tertbutyldiphenylchlorosilane $(6.86 \mathrm{~g}, 25.0 \mathrm{mmol}, 1.1$ equiv.) slowly at room temperature. Then the reaction mixture was stirred at room temperature for 1.5 hour. Upon completion, the reaction was diluted in ethyl acetate $(200 \mathrm{~mL})$ and then washed with distilled water $(3 \times 80 \mathrm{~mL})$. The combined aqueous layers were then separated and extracted with ethyl acetate $(3 \times 20 \mathrm{~mL})$. The combined organic phase was dried with anhydrous $\mathrm{Na}_{2} \mathrm{SO}_{4}$, filtered, and then concentrated by rotary evaporation. The crude material was purified by flash silica chromatography (pet. ether/ethyl acetate $=50 / 1$ ) to afford the title compound as a light-yellow oil (8.0 g, yield 96\%). $\mathbf{R}_{f}$ : 0.59 (pet. ether/EtOAc $\left.=10: 1\right) .{ }^{1} \mathbf{H}$ NMR (500 $\left.\mathrm{MHz}, \mathrm{CDCl}_{3}\right) \delta 7.69-7.63(\mathrm{~m}, 4 \mathrm{H}), 7.45-7.35(\mathrm{~m}, 6 \mathrm{H}), 4.24-4.17(\mathrm{~m}, 1 \mathrm{H}), 4.07$ (dd, $J=8.2,6.4 \mathrm{~Hz}, 1 \mathrm{H}), 3.91(\mathrm{dd}, J=8.2,6.0 \mathrm{~Hz}, 1 \mathrm{H}), 3.73(\mathrm{dd}, J=10.4,4.5 \mathrm{~Hz}, 1 \mathrm{H}), 3.65$ $(\mathrm{dd}, J=10.4,6.5 \mathrm{~Hz}, 1 \mathrm{H}), 1.39$ (s, 3H), 1.36 (s, 3H), 1.05 (s, 9H). ${ }^{13} \mathbf{C}$ NMR $(125 \mathrm{MHz}$, $\left.\mathrm{CDCl}_{3}\right): \delta 136.0,135.2,133.8,130.2,130.1,128.1,109.6,67.3,65.0,27.2,27.1,27.0$, $25.9,19.7$. These characterisation data match those reported in the literature. ${ }^{355}$

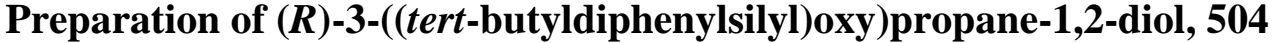

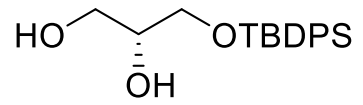

Silyl ether compound $\mathbf{5 0 3}$ (8.09 g, $21.8 \mathrm{mmol}, 1.0$ equiv.) was mixed with $100 \mathrm{~mL}$ of $90 \%$ $\mathrm{AcOH}$. After heating to reflux for $10 \mathrm{~min}$, the solution was allowed to cool to room temperature slowly. The solvent was removed under vacuum and the resulting oil was purified by flash silica chromatography (dichloromethane/methanol/acetic acid $=95 / 5 / 3$ ) to afford an off-white crystal (6.12 g, yield 84\%). $\mathbf{R}_{f}: 0.22(\mathrm{DCM} / \mathrm{MeOH}=50: 1) .{ }^{1} \mathbf{H}$ NMR $\left(500 \mathrm{MHz}, \mathrm{CDCl}_{3}\right) \delta 7.67-7.65(\mathrm{~m}, 4 \mathrm{H}), 7.47-7.37(\mathrm{~m}, 6 \mathrm{H}), 3.84-3.77(\mathrm{~m}$, 1H), $3.76-3.60(\mathrm{~m}, 4 \mathrm{H}), 2.56(\mathrm{~d}, J=5.0 \mathrm{~Hz}, 1 \mathrm{H}), 1.96$ (br. s, $1 \mathrm{H}), 1.07$ (s, 9H). ${ }^{13} \mathrm{C}$ 
NMR (125 MHz, $\left.\mathrm{CDCl}_{3}\right): \delta 135.5,132.9,130.0,127.9,71.9,65.2,63.9,26.9,19.2$. These characterisation data match those reported in the literature. ${ }^{356}$

\section{Preparation of (S)-3-((tert-butyldiphenylsilyl)oxy)-2-hydroxypropyl 4-} methylbenzenesulfonate, 505

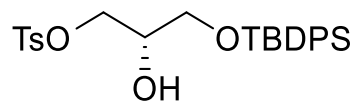

Diol compound 504 (512 mg, $1.55 \mathrm{mmol}, 1.0$ equiv.), 4-toluenesulfonyl chloride (295mg, $1.55 \mathrm{mmol}, 1.0$ equiv.) and dibutyltin oxide ( $77 \mathrm{mg}, 0.31 \mathrm{mmol}, 0.2$ equiv.) were mixed in a $50 \mathrm{~mL}$ round-bottom flask under nitrogen atmosphere. Then anhydrous DCM (15 $\mathrm{mL})$ and triethylamine $(0.22 \mathrm{ml}, 1.55 \mathrm{mmol}, 1.0$ equiv. $)$ were added to the flask. The reaction mixture was stirred at room temperature for 20 hours. Distilled water $(10 \mathrm{~mL})$ was then added to the reaction mixture and the aqueous layer was separated and extracted with distilled ethyl acetate $(3 \times 10 \mathrm{~mL})$. The combined organic phase was dried with anhydrous $\mathrm{Na}_{2} \mathrm{SO}_{4}$, filtered, and then concentrated by rotary evaporation. The crude material was purified by flash silica chromatography (pet. ether/ethyl acetate $=4 / 1$ ) to afford the title compound as a colourless oil (576 mg, yield 77\%). $\mathbf{R}_{\boldsymbol{f}}$ : 0.50 (pet. ether/EtOAc = 3:1). ${ }^{1} \mathbf{H}$ NMR $\left(500 \mathrm{MHz}, \mathrm{CDCl}_{3}\right) \delta 7.82-7.75(\mathrm{~m}, 2 \mathrm{H}), 7.82-7.75(\mathrm{~m}$, 4H), $7.47-7.42(\mathrm{~m}, 2 \mathrm{H}), 7.41-7.36(\mathrm{~m}, 4 \mathrm{H}), 7.34-7.30(\mathrm{~m}, 2 \mathrm{H}), 4.14(\mathrm{dd}, J=10.1$, $5.0 \mathrm{~Hz}, 1 \mathrm{H}), 4.06(\mathrm{dd}, J=10.1,5.9 \mathrm{~Hz}, 1 \mathrm{H}), 3.92-3.86(\mathrm{~m}, 1 \mathrm{H}), 3.66(\mathrm{~d}, J=5.1 \mathrm{~Hz}$, 2H), $2.44(\mathrm{~s}, 3 \mathrm{H}), 1.01(\mathrm{~s}, 9 \mathrm{H}) .{ }^{13} \mathbf{C} \mathbf{N M R}\left(125 \mathrm{MHz}, \mathrm{CDCl}_{3}\right): \delta 135.5,130.0,129.9$, $128.0,127.9,70.3,69.5,63.7,26.7,21.69,19.2$. These characterisation data match those reported in the literature. ${ }^{357}$

\section{Preparation of (R)-1-azido-3-((tert-butyldiphenylsilyl)oxy)propan-2-ol, 506}<smiles>NC[C@@H](O)CO[PbH2]</smiles>

To a solution of tosylate $\mathbf{5 0 5}(6.70 \mathrm{~g}, 13.8 \mathrm{mmol}, 1.0$ equiv. $)$ in anhydrous DMF (40 mL) was added sodium azide $(2.24 \mathrm{~g}, 34.6 \mathrm{mmol}, 2.5$ equiv. $)$ at room temperature. Then the reaction mixture was stirred at $90{ }^{\circ} \mathrm{C}$ for 23 hours. Then the reaction mixture was cooled down to room temperature and poured into $100 \mathrm{~mL}$ ice-cold water. The aqueous layers were extracted with diethyl ether $(3 \times 100 \mathrm{~mL})$. The combined organic phase was washed with brine, dried with anhydrous $\mathrm{Na}_{2} \mathrm{SO}_{4}$, filtered, and then concentrated by rotary evaporation. The crude material was purified by flash silica chromatography (pet. 
ether/ethyl acetate $=8 / 1)$ to afford the title compound as a colourless oil $(3.12 \mathrm{~g}$, yield 64\%). $\mathbf{R}_{f}: 0.53$ (pet. ether/EtOAc = 3:1). ${ }^{1} \mathbf{H}$ NMR $\left(500 \mathrm{MHz}, \mathrm{CDCl}_{3}\right) \delta 7.67-7.61(\mathrm{~m}$, 4H), $7.48-7.37(\mathrm{~m}, 6 \mathrm{H}), 3.87$ (sextet, $J=5.0 \mathrm{~Hz}, 1 \mathrm{H}), 3.71-3.63(\mathrm{~m}, 2 \mathrm{H}), 3.37(\mathrm{~d}, J=$ $5.0 \mathrm{~Hz}, 2 \mathrm{H}), 1.07$ (s, 9H). ${ }^{13} \mathrm{C}$ NMR $\left(125 \mathrm{MHz}, \mathrm{CDCl}_{3}\right) \delta 135.5,132.7,130.0,127.9$, 70.9, 64.9, 53.2, 26.8, 19.2. FTIR (thin film) v3443, 3071, 2930, 2858, 2099, 1589, 1472, $1427,1391,1362,1264,1189,1112,1105,1006,998,937,873,823,800,739,700,622$, 614, 554, 504, 487, $437 \mathrm{~cm}^{-1}$. HRMS (ESI): Calculated for $\mathrm{C}_{19} \mathrm{H}_{25} \mathrm{~N}_{3} \mathrm{NaO}_{2} \mathrm{Si}^{+}[\mathrm{M}+\mathrm{Na}]^{+}$ 378.1614; found 378.1642. $[\alpha]_{D}^{26}+16(c=1.57$, DCM). These characterisation data match those reported in the literature. ${ }^{358}$

\section{Preparation of $(R)-5$-(azidomethyl)-9,9-dimethyl-8,8-diphenyl-2,4,7-trioxa-8-} siladecane, 507

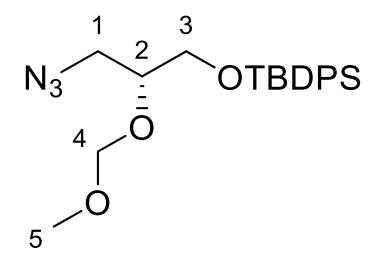

To a solution of azide 506 ( $3.12 \mathrm{~g}, 8.8 \mathrm{mmol}, 1.0$ equiv.) and 4-DMAP (0.43 g, $3.52 \mathrm{mmol}$, 0.4 equiv. $)$ in anhydrous DCM $(88 \mathrm{~mL})$ were sequentially added $N, N$ diisopropylethylamine (23 mL, $132 \mathrm{mmol}, 15$ equiv.) and $\mathrm{MOMCl}(8.25 \mathrm{~mL}$, purity $90 \%$, $88 \mathrm{mmol}, 10.0$ equiv.) at $0{ }^{\circ} \mathrm{C}$. Then the reaction mixture was allowed to warm up to room temperature and stirred at room temperature for 17 hours. Then the reaction mixture was heated at reflux for 6 hours. The reaction was quenched by addition of $20 \mathrm{~mL} \mathrm{NaHCO}_{3}$ aqueous solution. Then the aqueous layers were separated and extracted with diethyl ether $(3 \times 30 \mathrm{~mL})$. The combined organic phase was washed with brine, dried with anhydrous $\mathrm{Na}_{2} \mathrm{SO}_{4}$, filtered, and then concentrated by rotary evaporation. The crude material was purified by flash silica chromatography (pet. ether/ethyl acetate $=14 / 1$ ) to afford the title compound as a colourless oil (3.22 g, yield 92\%). $\mathbf{R}_{f}$ : 0.28 (pet. ether/EtOAc $\left.=10: 1\right) .{ }^{1} \mathbf{H}$ NMR $\left(500 \mathrm{MHz}, \mathrm{CDCl}_{3}\right) \delta 7.68$ - $7.62(\mathrm{~m}, 4 \mathrm{H}), 7.48$ - $7.37(\mathrm{~m}, 6 \mathrm{H}), 4.66$ - $4.62(\mathrm{~m}, 2 \mathrm{H}$, H-4), 3.82 - 3.77 (m, 1H, H-2), 3.73 (dd, $J=10.6,4.9$ Hz, 1H, H-3a), 3.68 (dd, $J=10.6$, $6.4 \mathrm{~Hz}, 1 \mathrm{H}, \mathrm{H}-3 \mathrm{~b}$ ), 3.52 (dd, $J=12.8,3.8 \mathrm{~Hz}, 1 \mathrm{H}, \mathrm{H}-1 \mathrm{a}), 3.44$ (dd, $J=12.8,6.1 \mathrm{~Hz}, 1 \mathrm{H}$, H-1b), 3.33 (s, 3H, H-5), 1.05 (s, 9H). ${ }^{13} \mathbf{C}$ NMR (125 MHz, $\left.\mathrm{CDCl}_{3}\right) \delta 135.6,135.5,133.1$, 133.0, 129.8, 127.78, 127.76, 96.1 (C-4), 76.5 (C-2), 63.4 (C-3), 55.6 (C-5), 52.3 (C-1), 26.8, 19.2. FTIR (thin film) v2931, 2888, 2858, 2098, 1472, 1428, 1281, 1154, 1105, 
1030, 998, 919, 823, 794, 739, 700, 622, 614, 504, $488 \mathrm{~cm}^{-1}$. HRMS (ESI): Calculated for $\mathrm{C}_{21} \mathrm{H}_{30} \mathrm{~N}_{3} \mathrm{O}_{3} \mathrm{Si}^{+}[\mathrm{M}+\mathrm{H}]^{+} 400.2051$; found 400.2050. $[\alpha]_{D}{ }^{26}+16(c=1.24, \mathrm{DCM})$.

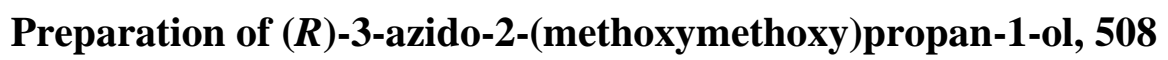

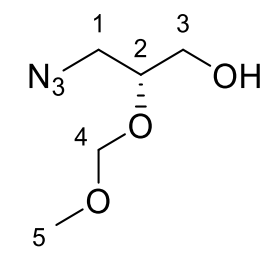

To a solution of azide $\mathbf{5 0 7}(307 \mathrm{mg}, 0.77 \mathrm{mmol}, 1.0$ equiv.) in anhydrous THF (10 mL) was added tetrabutylammonium fluoride (1.50 mL, 1.0 M in THF, $1.50 \mathrm{mmol}, 1.95$ equiv.) at room temperature and the reaction mixture was stirred at room temperature for 2 hours. Saturated aqueous $\mathrm{NH}_{4} \mathrm{Cl}$ solution $(10 \mathrm{~mL})$ was then added to the reaction mixture and the resulting mixture was stirred for 10 minutes. The aqueous phase was then separated and extracted with ethyl acetate $(3 \times 10 \mathrm{~mL})$. The combined organic phase was washed with brine, dried with anhydrous $\mathrm{Na}_{2} \mathrm{SO}_{4}$, filtered, and then concentrated by rotary evaporation. The crude material was purified by flash silica chromatography (pet. ether/ethyl acetate $=2 / 1$ ) to afford the title compound as a colourless oil (117 mg, yield 94\%). $\mathbf{R}_{f}: 0.16$ (pet. ether/EtOAc = 2:1). ${ }^{1} \mathbf{H}$ NMR $\left(500 \mathrm{MHz}, \mathrm{CDCl}_{3}\right) \delta 4.79-4.74(\mathrm{~m}$, 2H, H-4), $3.80-3.73$ (m, 1H, H-2), 3.68 (dd, $J=12.0,2.9$ Hz, 1H, H-3a), 3.63 (dd, $J=$ 11.9, 6.1 Hz, 1H, H-3b), 3.45 (s, 3H, H-5), 3.42 (dd, J = 12.9, 6.4 Hz, 1H, H-1a), 3.37 (dd, $J=12.9,4.7 \mathrm{~Hz}, 1 \mathrm{H}, \mathrm{H}-1 \mathrm{~b}), 2.59$ (br. s, $1 \mathrm{H},-\mathrm{OH}) .{ }^{13} \mathbf{C ~ N M R}\left(125 \mathrm{MHz}, \mathrm{CDCl}_{3}\right) \delta$ 96.9 (C-4), 79.5 (C-2), 63.2 (C-3), 55.9 (C-5), 51.8 (C-1). FTIR (thin film) v3423, 2927, 2096, 1443, 1280, 1212, 1151, 1105, 1022, 917, 811, 621, $554 \mathrm{~cm}^{-1}$. HRMS (ESI): Calculated for $\mathrm{C}_{21} \mathrm{H}_{30} \mathrm{~N}_{3} \mathrm{O}_{3} \mathrm{Si}^{+}[\mathrm{M}+\mathrm{H}]^{+}$162.0873; found 162.1010. [ $\left.\alpha\right]_{D}{ }^{21}-56.7(c=0.97$, DCM). 


\subsubsection{Synthesis of Macrocyclic Alcohol 523}

\section{Preparation of $(2 Z, 4 E)$-7-(hept-6-ynoyloxy)hepta-2,4-dienoic acid, 414}

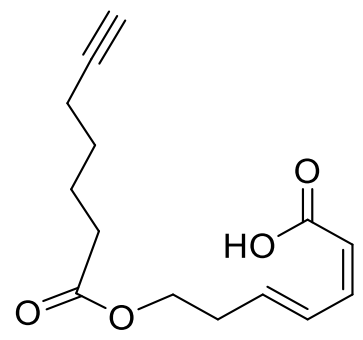

To a solution of lactone 364 (60.0 mg, $0.240 \mathrm{mmol}, 1.0$ equiv.) in THF (4.4 mL) was added a solution of tetrabutylammonium fluoride $(0.96 \mathrm{~mL}, 1.0 \mathrm{M}$ in THF, $0.96 \mathrm{mmol}$, 2.0 equiv.) at $0{ }^{\circ} \mathrm{C}$. The reaction mixture was stirred at room temperature for 2 hours. Then the reaction mixture was washed with $2 \% \mathrm{H}_{2} \mathrm{SO} 4$ aqueous solution $(3 \times 20 \mathrm{~mL})$. The organic phase was washed with brine, dried with anhydrous $\mathrm{Na}_{2} \mathrm{SO}_{4}$, filtered, and then concentrated by rotary evaporation to afford the title compound as a colourless oil (43 mg, yield 99\%). $\mathbf{R}_{f}: 0.64$ (pet. ether/EtOAc = 1:1). ${ }^{1} \mathbf{H}$ NMR $\left(500 \mathrm{MHz}, \mathrm{CDCl}_{3}\right) \delta 7.41$ $(\mathrm{dd}, J=15.6,11.1 \mathrm{~Hz}, 1 \mathrm{H}), 6.65(\mathrm{t}, J=11.3 \mathrm{~Hz}, 1 \mathrm{H}), 6.06(\mathrm{dd}, J=15.0,7.2 \mathrm{~Hz}, 1 \mathrm{H})$, $5.65(\mathrm{~d}, J=11.3 \mathrm{~Hz}, 1 \mathrm{H}), 4.18(\mathrm{t}, J=6.5 \mathrm{~Hz}, 2 \mathrm{H}), 2.55(\mathrm{dt}, J=6.9,6.6 \mathrm{~Hz}, 2 \mathrm{H}), 2.34(\mathrm{t}$, $J=7.3 \mathrm{~Hz}, 2 \mathrm{H}), 2.21(\mathrm{t}, J=7.0 \mathrm{~Hz}, 2 \mathrm{H}), 1.96-1.93(\mathrm{~m}, 1 \mathrm{H}), 1.74$ (quin, $J=6.9 \mathrm{~Hz}, 2 \mathrm{H}$ ), 1.56 (quin, $J=7.0 \mathrm{~Hz}, 2 \mathrm{H}$ ). ${ }^{13} \mathrm{C}$ NMR $\left(125 \mathrm{MHz}, \mathrm{CDCl}_{3}\right)$ 173.4, 171.5, 146.6, 140.9, $129.1,116.0,83.9,68.6,62.8,33.7,32.3,27.8,23.9,18.1$. These characterisation data match those reported in the literature. ${ }^{134}$

Preparation of $\quad(2 Z, 4 E)-7-((5-(1-((R)-3-(($ tert-butyldiphenylsilyl)oxy)-2hydroxypropyl)-1H-1,2,3-triazol-4-yl)pentanoyl)oxy)hepta-2,4-dienoic acid, 514

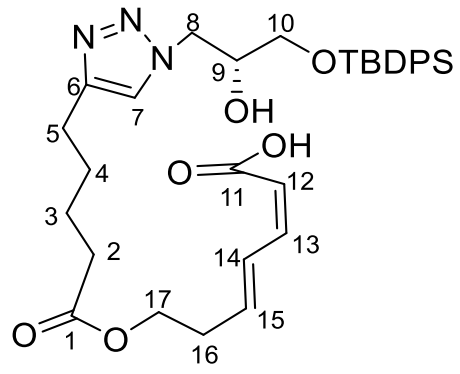

To a solution of CuSO4 (2.0 mg, $0.008 \mathrm{mmol}, 0.09$ equiv.) and sodium ascorbate ( $7.3 \mathrm{mg}$, 0.037 mmol, 0.5 equiv. $)$ in distilled water $(2.0 \mathrm{~mL})$ was added a solution of alkyne $\mathbf{4 1 4}$ (22.2 mg, $0.088 \mathrm{mmol}, 1.1$ equiv.) and azide 506 (28.8 mg, $0.081 \mathrm{mmol}, 1.0$ equiv.) in THF $(2.0 \mathrm{~mL})$. The reaction mixture was stirred at room temperature in dark for 12 hours. Then the reaction mixture was directly extracted with ethyl acetate $(3 \times 5 \mathrm{~mL})$. The 
combined organic phase was washed with brine, dried with anhydrous $\mathrm{Na}_{2} \mathrm{SO}_{4}$, filtered, and then concentrated by rotary evaporation. The crude material was purified by flash silica chromatography (pet. ether/ethyl acetate $=1 / 2$ ) to afford the title compound as a colourless oil (43 mg, yield 88\%). $\mathbf{R}_{f}$ : 0.24 (pet. ether/EtOAc = 1:1). ${ }^{1} \mathbf{H}$ NMR (500 MHz, $\left.\mathrm{CDCl}_{3}\right) \delta 7.66-7.60(\mathrm{~m}, 4 \mathrm{H}), 7.47-7.36(\mathrm{~m}, 8 \mathrm{H}$, overlapped, H-7, H-14), 6.60 (t, $J=$ $11.3 \mathrm{~Hz}, 1 \mathrm{H}, \mathrm{H}-13$ ), 6.04 (dt, $J=14.3,6.7 \mathrm{~Hz}, 1 \mathrm{H}, \mathrm{H}-15), 5.63$ (d, $J=11.3 \mathrm{~Hz}, 1 \mathrm{H}, \mathrm{H}-$ 12), 4.53 (d, $J=14.0 \mathrm{~Hz}, 1 \mathrm{H}, \mathrm{H}-8 \mathrm{a}), 4.32$ (dd, $J=13.9,7.7 \mathrm{~Hz}, 1 \mathrm{H}, \mathrm{H}-8 \mathrm{~b}), 4.19$ (t, $J=$ $5.7 \mathrm{~Hz}, 2 \mathrm{H}, \mathrm{H}-17), 4.16$ - 4.09 (m, 1H, H-9), 3.70 - 3.60 (m, 2H, H-10), 2.73 - 2.66 (m, 2H, H-5), $2.56-2.48$ (m, 2H, H-16), $2.38-2.29$ (m, 2H, H-2), $1.72-1.60$ (m, 4H, H-3, $\mathrm{H}-4), 1.07$ (s, 9H). ${ }^{13} \mathrm{C}$ NMR (125 MHz, CDCl $) 173.7$ (C-1), 169.9 (C-11), 147.6 (C-6), 145.5 (C-13), 140.1 (C-15), 135.68, 135.67, 132.81, 132.78, 130.2, 129.4 (C-14), 128.1, 122.6 (C-7), 116.9 (C-12), 70.9 (C-9), 65.1 (C-10), 62.7 (C-17), 53.0 (C-8), 34.1 (C-2), 32.2 (C-16), 28.9 (C-4 or C-3), 27.0, 25.2 (C-5), 24.5 (C-3 or C-4), 19.4. FTIR (thin film) $v 3493,3156,2929,2858,1708,1639,1601,1427,1361,1219,1179,1111,1065,998$, 964, 823, 741, 701, 690, 614, 504, $489 \mathrm{~cm}^{-1}$. HRMS (ESI): Calculated for $\mathrm{C}_{33} \mathrm{H}_{43} \mathrm{~N}_{3} \mathrm{NaO}_{6} \mathrm{Si}^{+}[\mathrm{M}+\mathrm{Na}]^{+}$628.2819; found 628.2860. $[\alpha]_{D}{ }^{25}+7.8(c=1.02, \mathrm{DCM})$.

Preparation of $(14 Z, 3 R, 6 Z, 8 E)-3-\left(\left((t e r t\right.\right.$-butyldiphenylsilyl)oxy $)$ methyl)-1 ${ }^{1} H-4,12$ dioxa-1(1,4)-triazolacycloheptadecaphane-6,8-diene-5,13-dione, 515

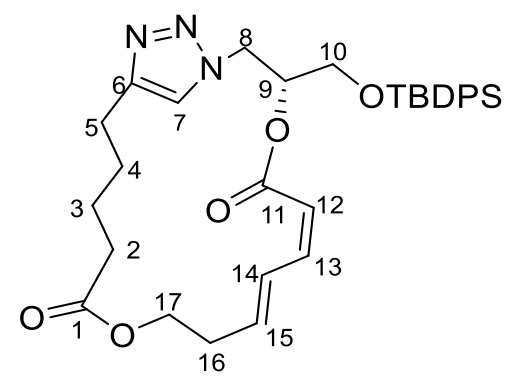

To a mixture of acid 514 (21.0 mg, 0.0347 mmol, 1.0 equiv.) and solid $\mathrm{NaHCO}_{3}$ (717 mg, 8.5 mmol, 246 equiv.) in anhydrous DCM (80 mL) was added 2-bromo-1-ethylpyridinium tetrafluoroborate ( $233 \mathrm{mg}, 0.85 \mathrm{mmol}, 24.6$ equiv.). The reaction mixture was stirred in dark under argon atmosphere at room temperature for 23 hours. The reaction was quenched by addition of distilled water $(3.6 \mathrm{~mL})$. The aqueous phase was separated and extracted ethyl acetate $(3 \times 5 \mathrm{~mL})$. The combined organic phase was dried with anhydrous $\mathrm{Na}_{2} \mathrm{SO}_{4}$, filtered, and then concentrated by rotary evaporation. The crude material was purified by flash silica chromatography (pet. ether/EtOAc $=2 / 1$ to pet. 
ether/EtOAc $=1 / 3)$ to afford the title compound 515 as a colourless oil $(8.1 \mathrm{mg}$, yield $40 \%)$ and the silyl ester by-product 516 (9.6 mg, yield 46\%) as a colourless oil.

515, $\mathbf{R}_{\boldsymbol{f}}: 0.34$ (pet. ether/EtOAc = 1:1). ${ }^{1} \mathbf{H}$ NMR $\left(500 \mathrm{MHz}, \mathrm{CDCl}_{3}\right): \delta 7.70-7.61(\mathrm{~m}$, 4H), $7.48-7.36$ (m, 6H), 7.24 (s, 1H, H-7), 7.15 (t, J=15.0 Hz, 1H, H-14), 6.54 (t, J= $11.2 \mathrm{~Hz}, 1 \mathrm{H}, \mathrm{H}-13$ ), 5.99 (dt, $J=14.8,7.2 \mathrm{~Hz}, 1 \mathrm{H}, \mathrm{H}-15), 5.50$ (d, $J=10.0 \mathrm{~Hz}, 1 \mathrm{H}, \mathrm{H}-$ 12), 5.49 - 5.42 (overlapped, m, 1H, H-9), 4.72 (d, $J=14.5 \mathrm{~Hz}, 1 \mathrm{H}, \mathrm{H}-8 \mathrm{a}$ ), 4.56 (dd, $J=$ 13.9, 10.6 Hz, 1H, H-8b), 4.30 (t, J=10.3 Hz, 1H, H-17a), 4.24 - 4.14 (m, 1H, H-17b), 3.87 (dd, $J=11.1,4.0 \mathrm{~Hz}, 1 \mathrm{H}, \mathrm{H}-10 \mathrm{a}), 3.81$ (dd, $J=11.0,4.6 \mathrm{~Hz}, 1 \mathrm{H}, \mathrm{H}-10 \mathrm{~b}$ ), 2.73 (dt, $J=14.4,7.0 \mathrm{~Hz}, 1 \mathrm{H}, \mathrm{H}-5 \mathrm{a}), 2.63$ (dt, $J=14.6,7.1 \mathrm{~Hz}, 1 \mathrm{H}, \mathrm{H}-5 \mathrm{~b}), 2.56-2.47$ (m, 1H, H16a), 2.47 - 2.39 (m, 1H, H-16b), 2.28 (dtd, $J=21.5,14.6,14.0,7.0 \mathrm{~Hz}, 2 \mathrm{H}, \mathrm{H}-2), 1.74$ - 1.61 (m, 2H, H-4, overlapped), $1.61-1.51$ (m, 2H, H-3, overlapped), 1.08 (s, 9H). ${ }^{13} \mathrm{C}$ NMR (125 MHz, $\mathrm{CDCl}_{3}$ ): 173.6 (C-1), 164.6 (C-11), 146.8 (C-6), 146.4 (C-13), 142.0 (C-15), 135.76, 135.69, 132.88, 132.84, 130.20, 130.18, 128.6 (C-14), 128.09, 128.05, 121.5 (C-7), 114.9 (C-12), 110.2, 71.2 (C-9), 63.8 (C-10), 62.0 (C-17), 51.5 (C-8), 34.9 (C-2), 34.0, 33.2 (C-16), 28.8 (C-4), 26.7, 25.4 (C-5), 24.5 (C-3), 19.5. FTIR (thin film) $v 2957,2930,2858,1725,1640,1602,1472,1460,1428,1389,1362,1308,1261,1168$, 1126, 1114, 1050, 998, 965, 823, 779, 742, 703, 622, 615, 505, 495, 490, 475, 468, 451, $433 \mathrm{~cm}^{-1}$. HRMS (ESI): Calculated for $\mathrm{C}_{33} \mathrm{H}_{42} \mathrm{~N}_{3} \mathrm{O}_{5} \mathrm{SSi}^{+}[\mathrm{M}+\mathrm{H}]^{+}$588.2888; found 588.2892. $[\alpha]_{D}^{23}+23.8(c=0.41, \mathrm{DCM})$.

Tert-butyldiphenylsilyl

(2Z,4E)-7-((5-(1-((R)-2,3-dihydroxypropyl)-1H-1,2,3triazol-4-yl)pentanoyl)oxy)hepta-2,4-dienoate, 516

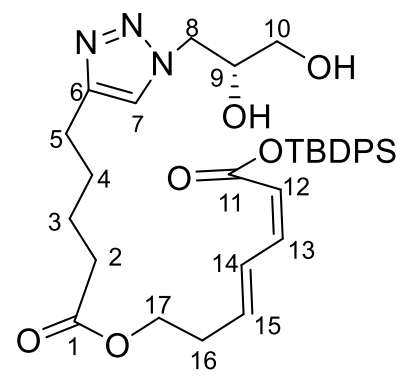

$\mathbf{R}_{f}: 0.10$ (pet. ether/EtOAc = 1:1). ${ }^{1} \mathbf{H}$ NMR $\left(500 \mathrm{MHz}, \mathrm{CDCl}_{3}\right): \delta 7.66-7.56(\mathrm{~m}, 4 \mathrm{H})$, 7.48 - 7.34 (m, 8H, overlapped, H-7, H-14), 6.72 (t, $J=11.2$ Hz, 1H, H-13), 6.14 (dt, $J$ $=14.6,7.1 \mathrm{~Hz}, 1 \mathrm{H}, \mathrm{H}-15), 5.62$ (d, $J=11.1 \mathrm{~Hz}, 1 \mathrm{H}, \mathrm{H}-12), 4.52$ (d, $J=14.2 \mathrm{~Hz}, 1 \mathrm{H}, \mathrm{H}-$ 8a), 4.32 (dd, $J=13.6,6.9 \mathrm{~Hz}, 1 \mathrm{H}, \mathrm{H}-8 \mathrm{~b}), 4.17$ (t, $J=6.1 \mathrm{~Hz}, 2 \mathrm{H}, \mathrm{H}-17), 4.14-4.07$ (m, 
1H, H-9), $3.70-3.59$ (m, 2H, H-10), $2.73-2.67$ (m, 2H, H-5), $2.59-2.48$ (m, 2H, H16), 2.36 - 2.30 (m, 2H, H-2), 1.73 - 1.59 (m, 4H, H-3,H-4), 1.07 (s, 9H). ${ }^{13}$ C NMR (125 $\mathrm{MHz}, \mathrm{CDCl}_{3}$ ): 173.6 (C-1), 161.6 (C-11), 149.0 (C-13), 147.7 (C-6), 143.1 (C-15), 135.7, 132.82, 132.79, 130.2, 129.2 (C-14), 128.1, 122.4 (C-7), 115.0 (C-12), 70.9 (C-9), 65.0 (C-10), 62.8 (C-17), 52.8 (C-8), 34.1 (C-2), 32.7 (C-16), 28.9 (C-4), 27.0, 25.4 (C-5), 24.6 (C-3), 19.4. FTIR (thin film) v 3386, 3234, 3153, 3069, 2930, 2857, 1773, 1734, 1654, 1636, 1589, 1560, 1472, 1461, 1428, 1391, 1361, 1308, 1258, 1214, 1112, 1034, 964, 930, 888, 824, 743, 703, 691, 663, 615, 589, 546, 507, 466, 454, 448, 442, $431 \mathrm{~cm}^{-}$ 1. HRMS (ESI): Calculated for $\mathrm{C}_{33} \mathrm{H}_{44} \mathrm{~N}_{3} \mathrm{O}_{6} \mathrm{SSi}^{+}[\mathrm{M}+\mathrm{H}]^{+}$606.2994; found 606.2974 . $[\alpha]_{D}^{23}+5.6(c=0.48, \mathrm{DCM})$.

\section{Preparation of $(2 E, 4 E)-7-((5-(1-((R)-2-a c e t o x y-3-((t e r t-$}

\section{butyldiphenylsilyl)oxy)propyl)-1H-1,2,3-triazol-4-yl)pentanoyl)oxy)hepta-2,4-} dienoic acid, $E, E-522$

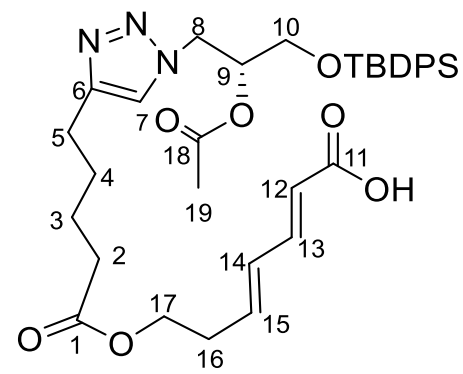

To a solution of diol $\mathbf{5 1 6}(11.3 \mathrm{mg}, 0.019 \mathrm{mmol}, 1.0$ equiv.) in DCM (1.0 mL) was added pyridine $(60 \mu \mathrm{L}, 0.72 \mathrm{mmol}, 38$ equiv.) and acetyl chloride $(26 \mu \mathrm{L}, 0.36 \mathrm{mmol}, 20.0$ equiv.) at $0{ }^{\circ} \mathrm{C}$. Then the reaction mixture was stirred at room temperature for 4 days. The reaction was then quenched by addition of saturated aqueous $\mathrm{NaHCO}_{3}$ solution $(2 \mathrm{~mL})$. The aqueous phase was separated and extracted ethyl acetate $(3 \times 5 \mathrm{~mL})$. The combined organic phase was dried with anhydrous $\mathrm{Na}_{2} \mathrm{SO}_{4}$, filtered, and then concentrated by rotary evaporation. The crude material was purified by flash silica chromatography (pet. ether/EtOAc $=3 / 2)$ to afford the title compound as a colourless oil $(7.5 \mathrm{mg}$, yield $62 \%)$. $\mathbf{R}_{f} .0 .12$ (pet. ether/EtOAc = 1:1). ${ }^{1} \mathbf{H}$ NMR $\left(500 \mathrm{MHz}, \mathrm{CDCl}_{3}\right): \delta 7.68-7.56(\mathrm{~m}, 4 \mathrm{H})$, $7.48-7.35$ (m, 6H), 7.35 - 7.27 (overlapped, m, 1H, H-13), 7.28 (s, 1H, H-7), 6.26 (dd, $J=15.2,11.8 \mathrm{~Hz}, 1 \mathrm{H}, \mathrm{H}-14), 6.14-6.06$ (m, 1H, H-15), 5.83 (d, $J=15.6 \mathrm{~Hz}, 1 \mathrm{H}, \mathrm{H}-12)$, $5.26-5.18$ (m, 1H, H-9), 4.68 (dd, $J=14.3,4.4$ Hz, 1H, H-8a), 4.60 (dd, $J=14.3,7.4$ Hz, 1H, H-8b), 4.16 (t, J = 6.6 Hz, 2H, H-17), 3.77 - 3.66 (m, 2H, H-10), 2.76 - 2.66 (m, 2H, H-5), $2.56-2.46$ (m, 2H, H-16), 2.34 (t, $J=5.8$ Hz, 2H, H-2), 1.98 (s, 3H, H-19), 
$1.75-1.61$ (m, 4H, H-3, H-4), 1.08 (s, 9H). ${ }^{13} \mathbf{C}$ NMR (125 MHz, CDCl $)$ : 173.4 (C-1), 170.2 (C-11), 169.9 (C-18), 147.8 (C-6), 146.3 (C-13), 134.0 (C-15), 135.54, 135.46, 132.66, 132.64, 130.4 (C-14), 130.0, 127.86, 127.83, 121.5 (C-7), 119.4 (C-12), 72.1 (C9), 62.6 (C-17), 62.4 (C-10), 49.8 (C-8), 33.9 (C-2), 32.3 (C-16), 28.8 (C-4), 26.8, 25.2 (C-5), 24.4 (C-3), 20.8 (C-19), 19.3. FTIR (thin film) v 3140, 2955, 2930, 2858, 1735, 1701, 1697, 1643, 1589, 1461, 1428, 1389, 1374, 1301, 1230, 1185, 1133, 1113, 1059, 998, 823, 803, 742, 703, 692, 505, $492 \mathrm{~cm}^{-1}$. HRMS (ESI): Calculated for $\mathrm{C}_{35} \mathrm{H}_{46} \mathrm{~N}_{3} \mathrm{O}_{7} \mathrm{Si}^{+}$ $[\mathrm{M}+\mathrm{H}]^{+}$648.3100; found 648.3108. $[\alpha]_{D}^{23}+11(c=0.33$, DCM $)$.

\section{Preparation of $\left(1^{4} Z, 3 R, 6 Z, 8 E\right)-3$-(hydroxymethyl)-1 ${ }^{1} H-4,12-d i o x a-1(1,4)-$}

triazolacycloheptadecaphane-6,8-diene-5,13-dione, 523

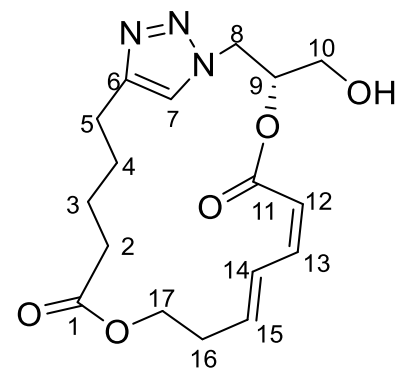

To a solution of compound 515 (34.4 mg, 0.059 mmol, 1.0 equiv.) and acetic acid (16 $\mu \mathrm{L}$, 0.280 mmol, 4.78 equiv. $)$ in anhydrous THF $(0.8 \mathrm{~mL})$ was slowly added tetrabutylammonium fluoride ( $116 \mu \mathrm{L}, 1.0 \mathrm{M}$ in THF, $0.116 \mathrm{mmol}, 2.0$ equiv.) over 20 minutes at $0{ }^{\circ} \mathrm{C}$. Then the reaction mixture was allowed to warm up to room temperature and stirred at room temperature for 28 hours. The reaction mixture was then diluted with ethyl acetate $(10 \mathrm{~mL})$ and added saturated aqueous $\mathrm{NaHCO}_{3}$ solution $(5 \mathrm{~mL})$. The aqueous phase was separated and extracted ethyl acetate $(3 \times 5 \mathrm{~mL})$. The combined organic phase was dried with anhydrous $\mathrm{Na}_{2} \mathrm{SO}_{4}$, filtered, and then concentrated by rotary evaporation. The crude material was purified by flash silica chromatography $(\mathrm{DCM} / \mathrm{MeOH}=20 / 1)$ to afford the title compound $(16.9 \mathrm{mg}$, yield $83 \%)$ as a colourless oil. Rf: $0.25(\mathrm{DCM} / \mathrm{MeOH}=10 / 1) .{ }^{1} \mathbf{H} \mathbf{~ N M R}\left(500 \mathrm{MHz}, \mathrm{CDCl}_{3}\right) \delta 7.24$ (s, 1H, H-7), 7.13 - 7.07 (m, 1H, H-14), 6.55 (t, $J=11.4 \mathrm{~Hz}, 1 \mathrm{H}, \mathrm{H}-13$ ), 6.00 (ddd, $J=14.8,8.8,5.3 \mathrm{~Hz}$, 1H, H-15), 5.54 (d, $J=11.3 \mathrm{~Hz}, 1 \mathrm{H}, \mathrm{H}-12$ ), 5.43 (dtd, $J=9.6,4.8,2.6 \mathrm{~Hz}, 1 \mathrm{H}, \mathrm{H}-9$ ), 4.70 (dd, $J=14.6,2.6 \mathrm{~Hz}, 1 \mathrm{H}, \mathrm{H}-8 \mathrm{a}), 4.59$ (dd, $J=14.6,9.7 \mathrm{~Hz}, 1 \mathrm{H}, \mathrm{H}-8 \mathrm{~b}), 4.33-4.27$ (m, 1H, H-17a), $4.23-4.18$ (m, 1H, H-17b), 3.86 (dd, $J=12.0,4.6$ Hz, 1H, H-10a), 3.82 (dd, $J=12.0,4.9 \mathrm{~Hz}, 1 \mathrm{H}, \mathrm{H}-10 \mathrm{~b}), 2.72(\mathrm{dt}, J=14.3,6.9 \mathrm{~Hz}, 1 \mathrm{H}, \mathrm{H}-5 \mathrm{a}), 2.63$ (dt, $J=14.8,7.2$ 
Hz, 1H, H-5b), 2.57 - 2.48 (m, 1H, H-16a), $2.47-2.40$ (m, 1H, H-16b), 2.35 - 2.22 (m, 2H, H-2), 1.70 - 1.62 (m, 2H, H-4), 1.58 - 1.51 (m, 2H, H-3). ${ }^{13}$ C NMR (125 MHz, $\left.\mathrm{CDCl}_{3}\right) \delta 173.5(\mathrm{C}-1), 164.6(\mathrm{C}-11), 147.8$ (C-6), 146.6 (C-13), 142.3 (C-15), 128.5 (C14), 121.7 (C-7), 114.7 (C-12), 71.5 (C-9), 62.3 (C-10), 62.0 (C-17), 50.9 (C-8), 34.9 (C2), 33.2 (C-16), 28.7 (C-4), 25.3 (C-5), 24.5 (C-3). FTIR (thin film) v3355, 2923, 2854, $1719,1639,1602,1524,1458,1420,1380,1260,1172,1131,1057,1002,964,817,759$ $\mathrm{cm}^{-1}$. HRMS (ESI): Calculated for $\mathrm{C}_{17} \mathrm{H}_{24} \mathrm{~N}_{3} \mathrm{O}_{5}{ }^{+}[\mathrm{M}+\mathrm{H}]^{+}$350.1710; found need re-do. $[\alpha]_{D}{ }^{25}-137(c=0.125, \mathrm{DCM})$. 


\section{NMR Spectra of Novel Compounds}

\section{Spectra of Chapter Two}

${ }^{1} \mathrm{H}$ NMR $\left(500 \mathrm{MHz}, \mathrm{CDCl}_{3}\right)$

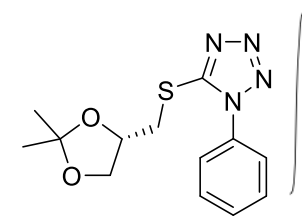

215

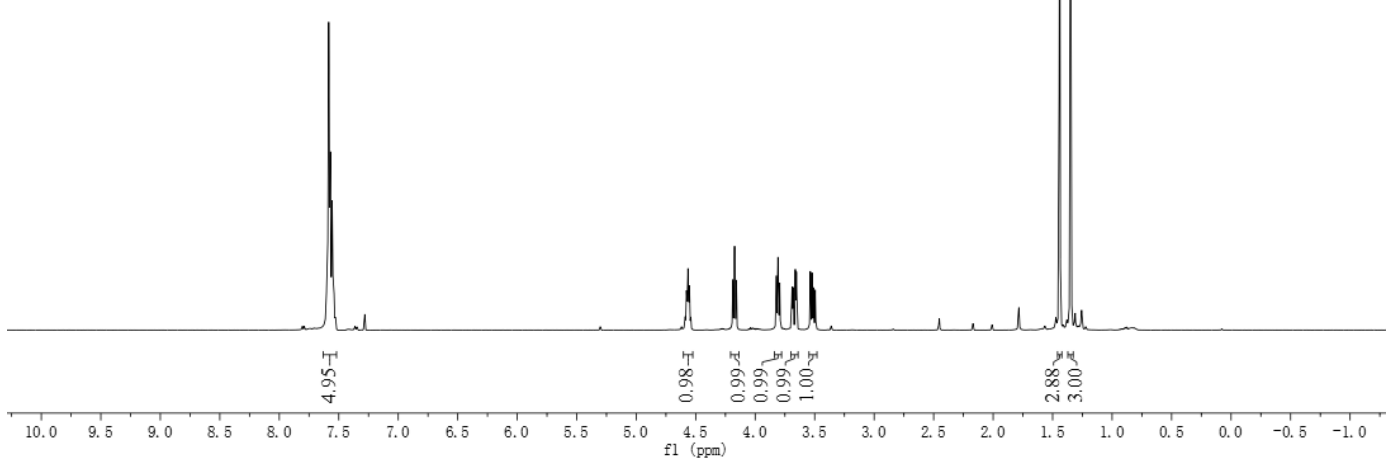

${ }^{13} \mathrm{C}$ NMR $\left(125 \mathrm{MHz}, \mathrm{CDCl}_{3}\right)$
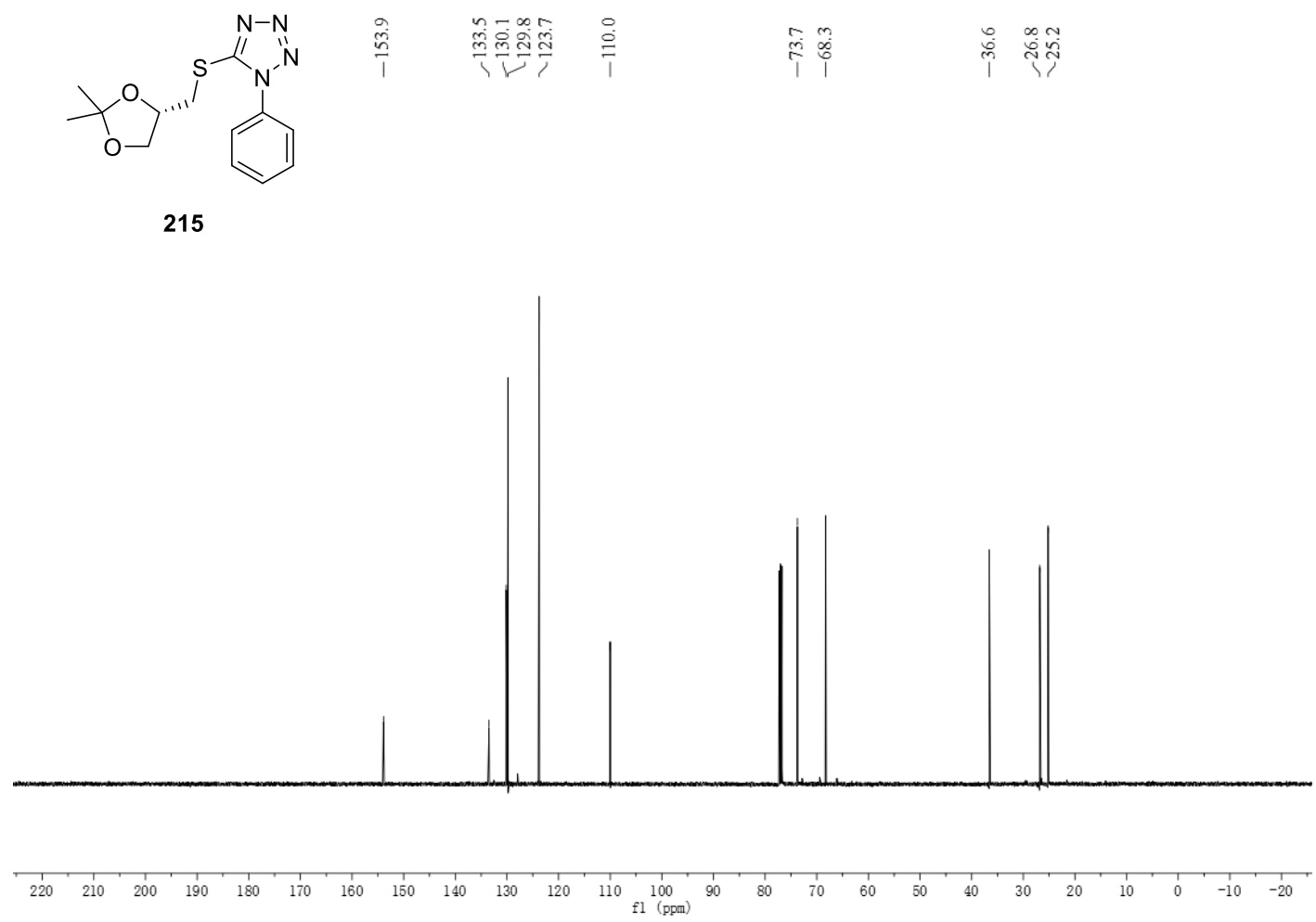
${ }^{1} \mathrm{H}$ NMR $\left(500 \mathrm{MHz}, \mathrm{CDCl}_{3}\right)$

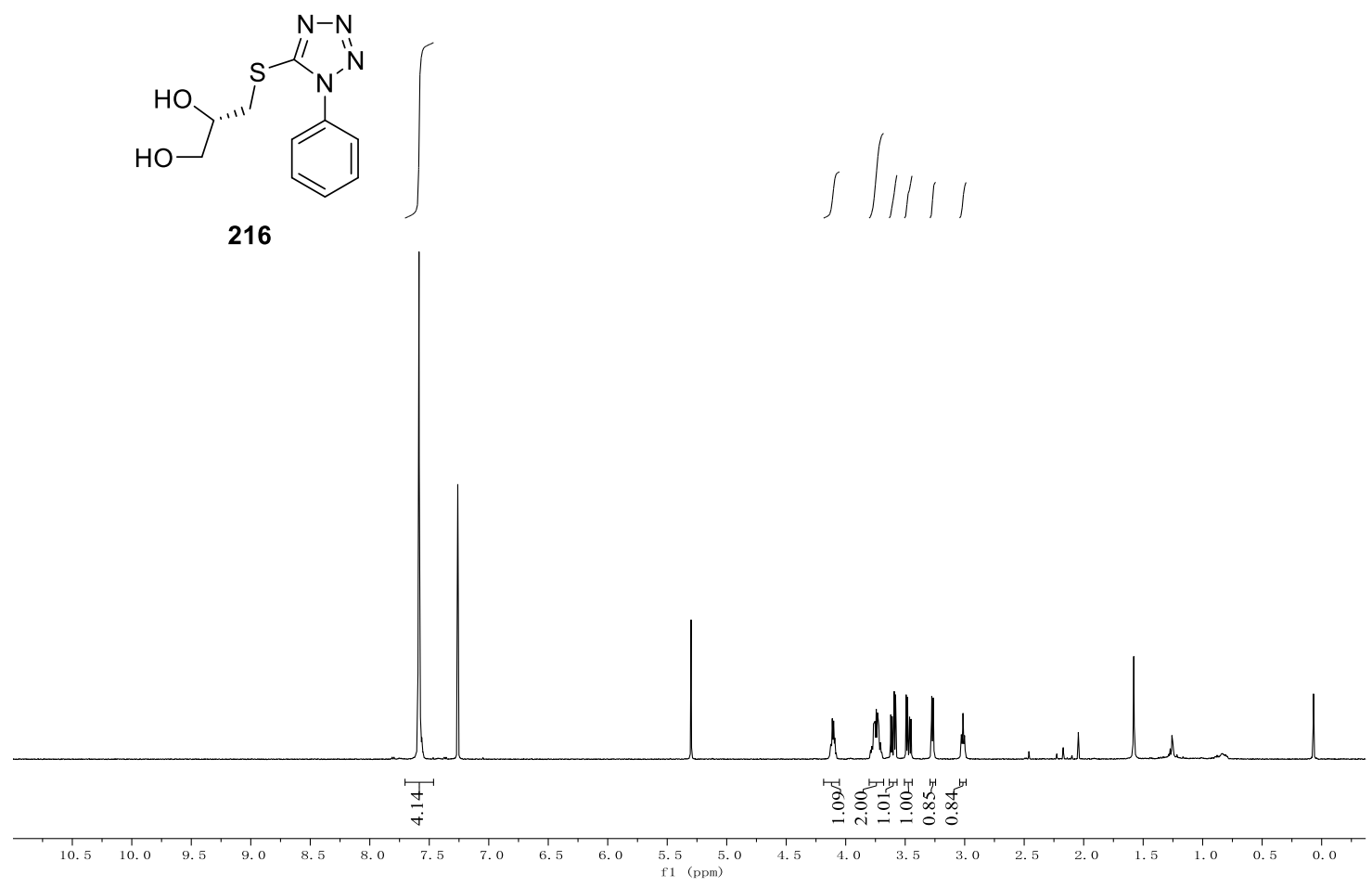

${ }^{13} \mathrm{C} \mathrm{NMR}\left(125 \mathrm{MHz}, \mathrm{CDCl}_{3}\right)$<smiles>OC[C@H](O)Sc1nnnn1-c1ccccc1</smiles>

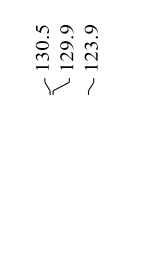

$\mid \begin{array}{ll}\vec{z} & 0 \\ 1 & 1 \\ 1 & 1\end{array}$ $\stackrel{\substack{\infty \\ \dot{c}}}{i}$ 


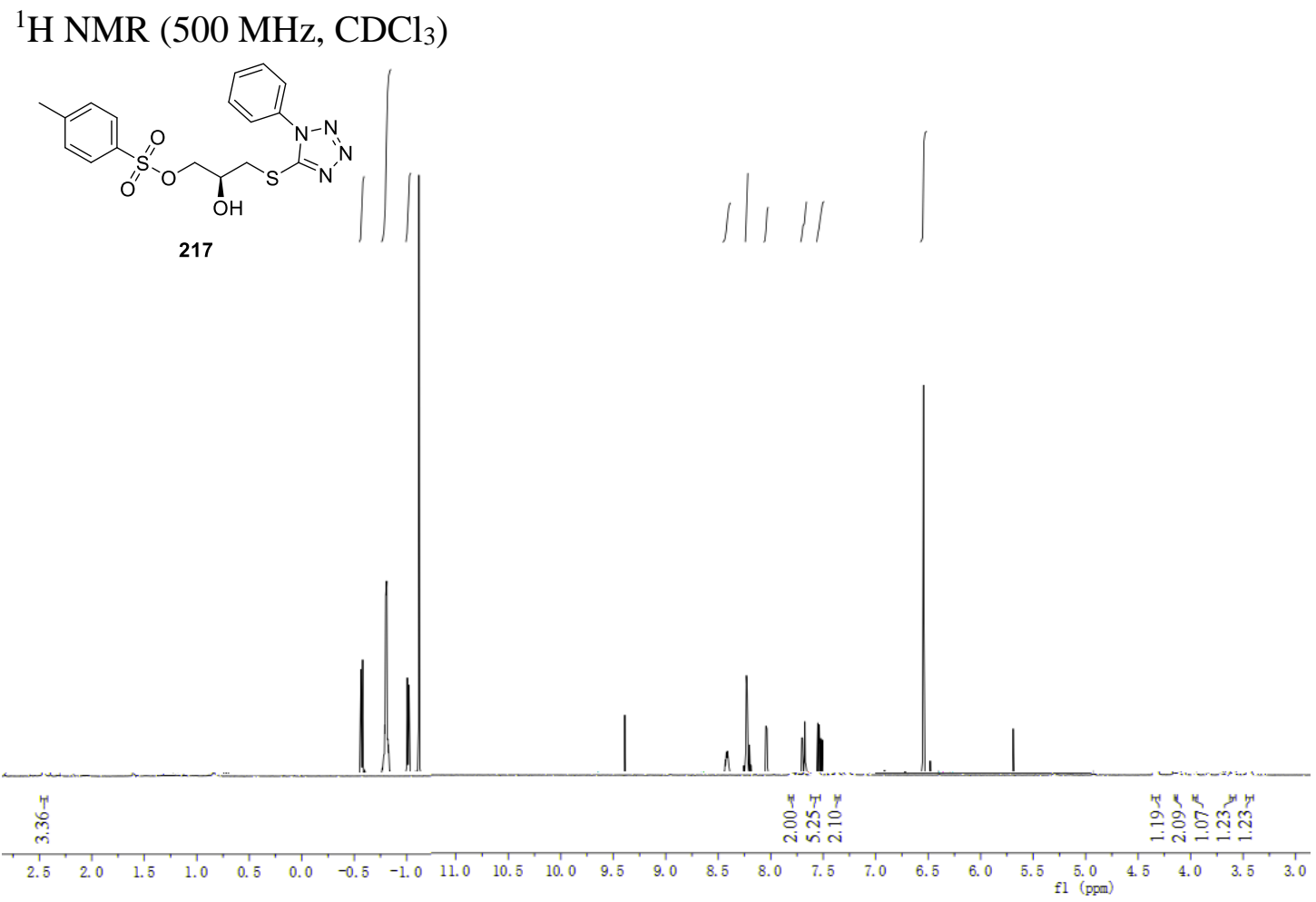

${ }^{13} \mathrm{C} \mathrm{NMR}\left(125 \mathrm{MHz}, \quad \mathrm{CDCl}_{3}\right)$
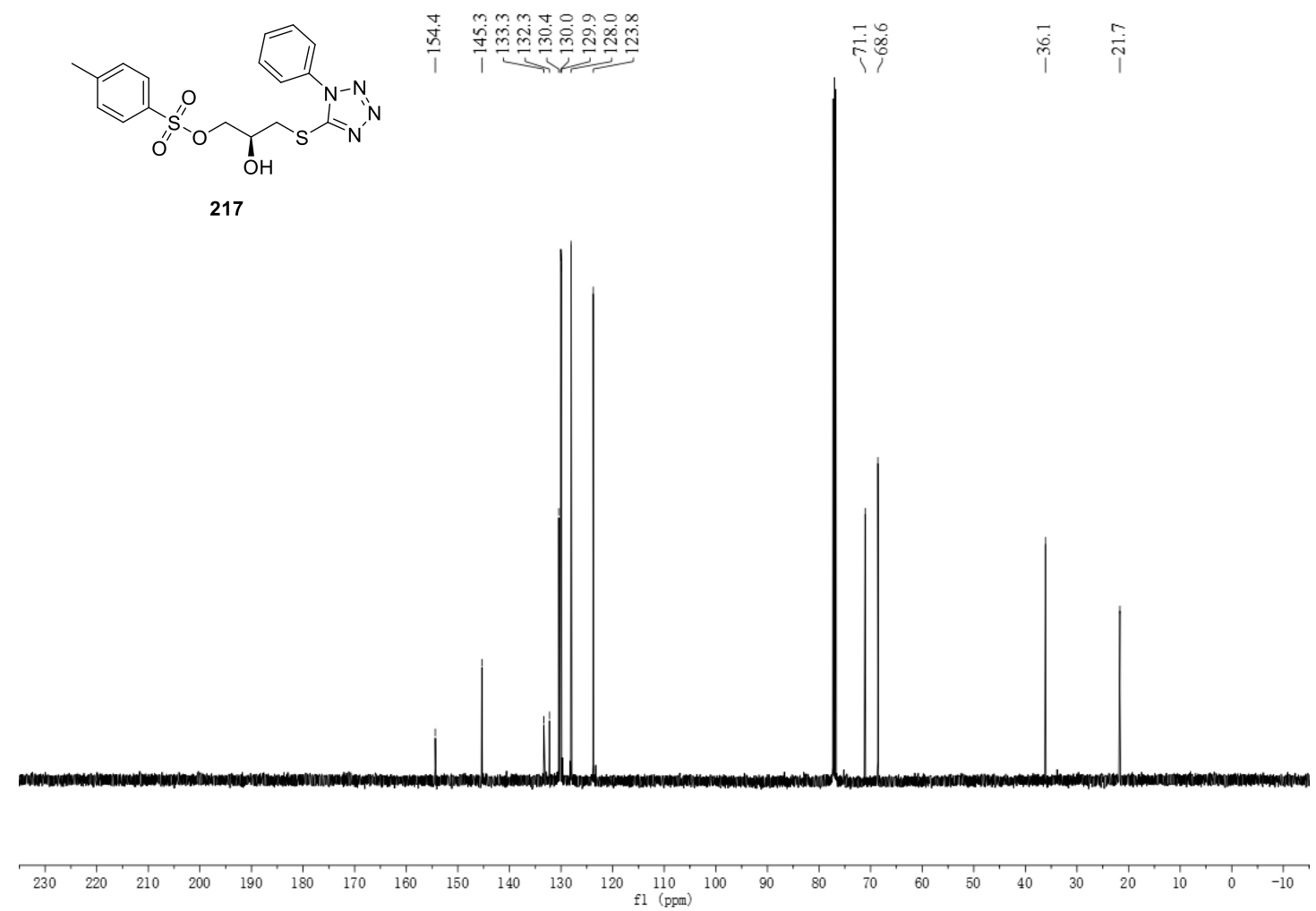
${ }^{1} \mathrm{H}$ NMR (500 MHz, $\left.\mathrm{CDCl}_{3}\right)$
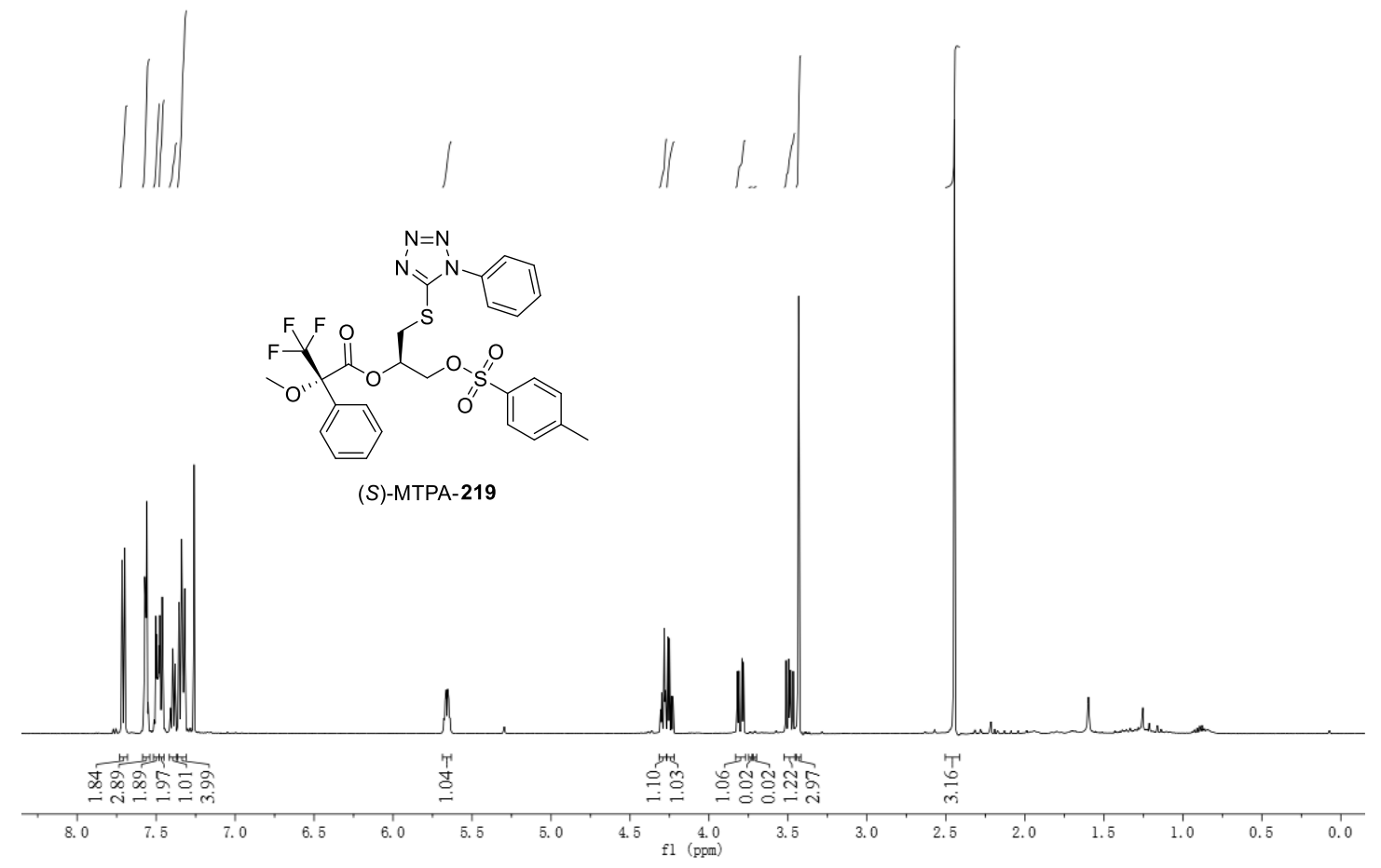

(S)-MTPA-219

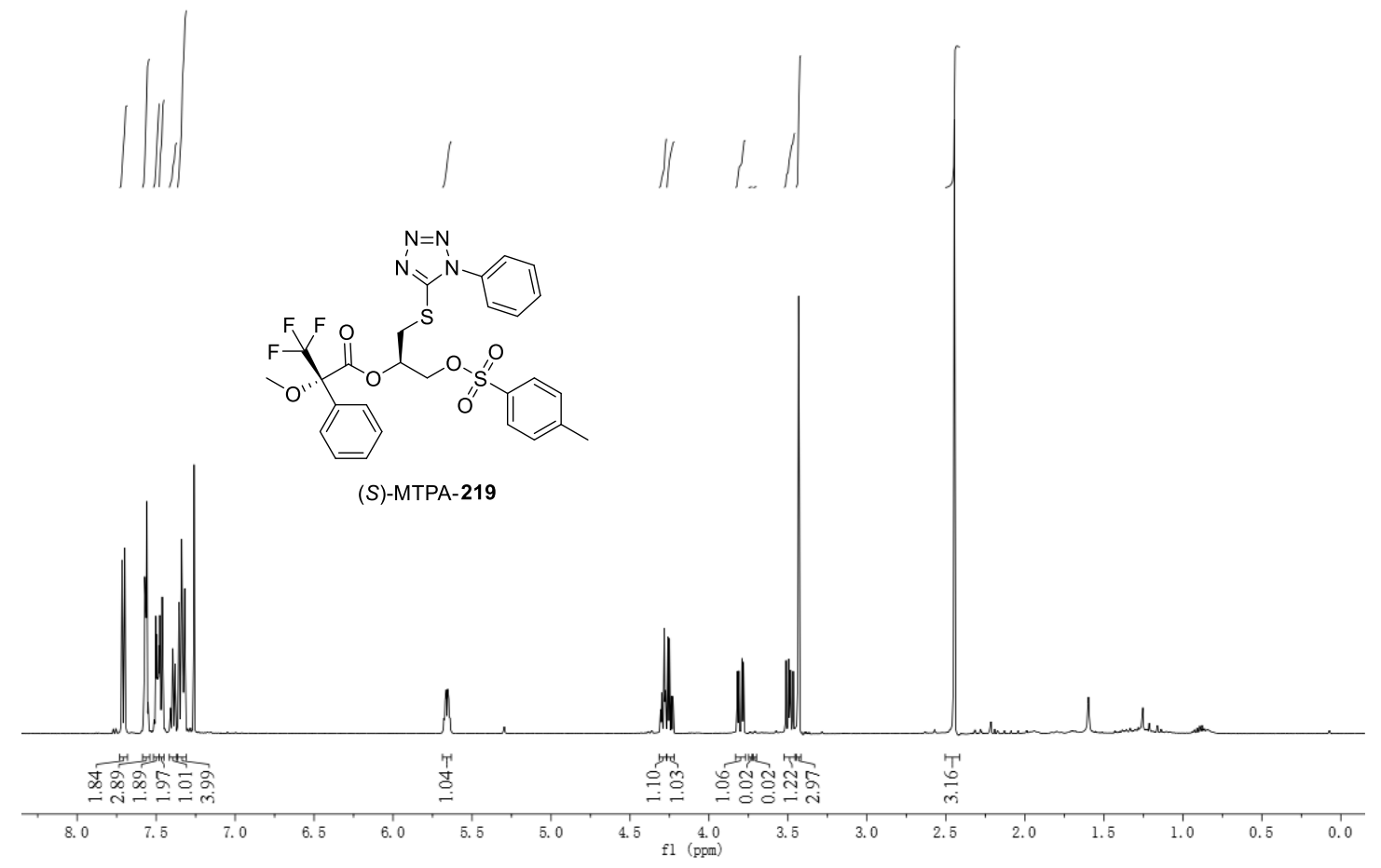

${ }^{13} \mathrm{C}$ NMR $\left(125 \mathrm{MHz}, \mathrm{CDCl}_{3}\right)$

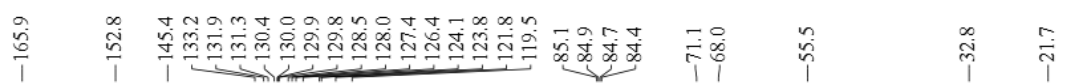

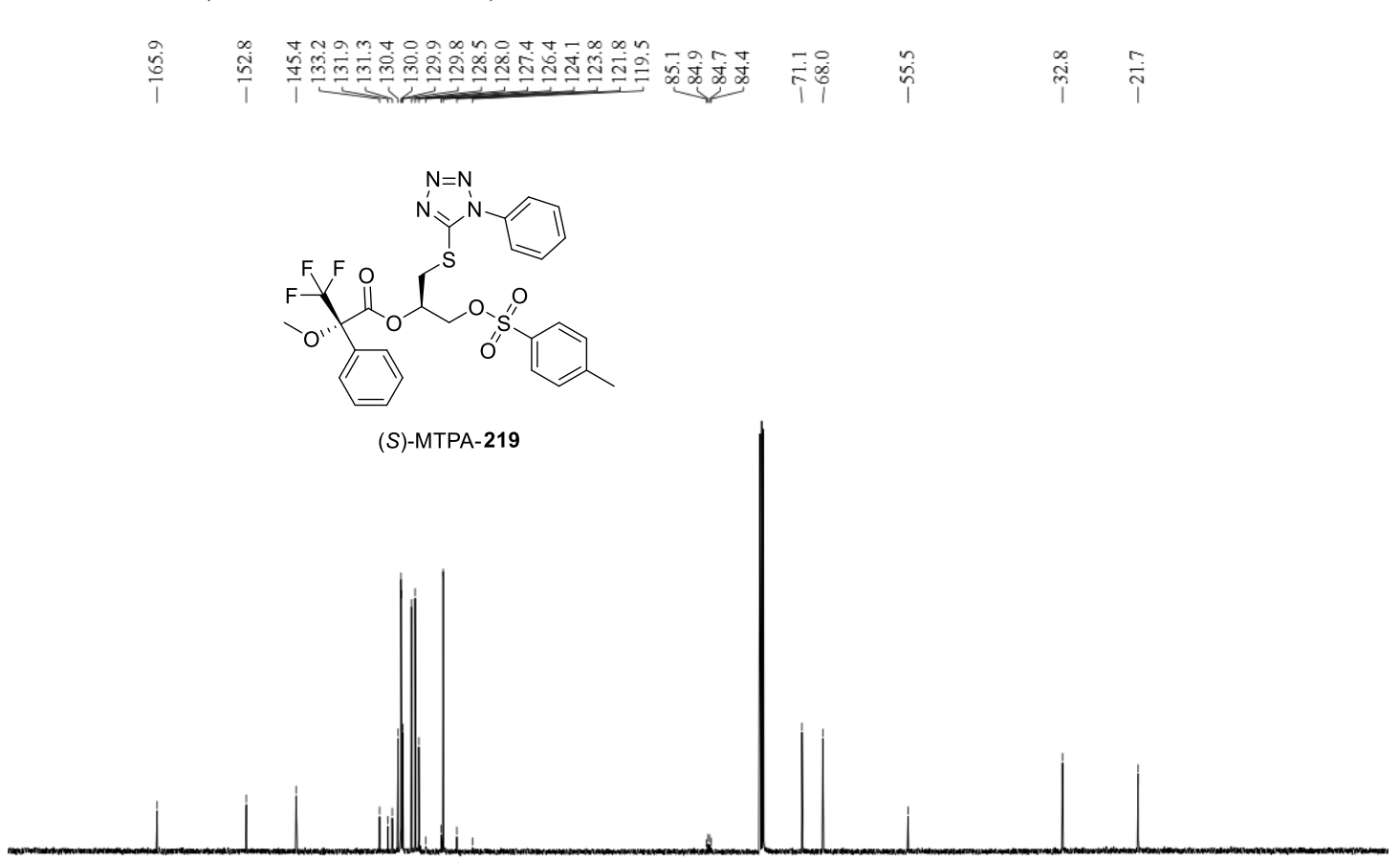

(S)-MTPA-219

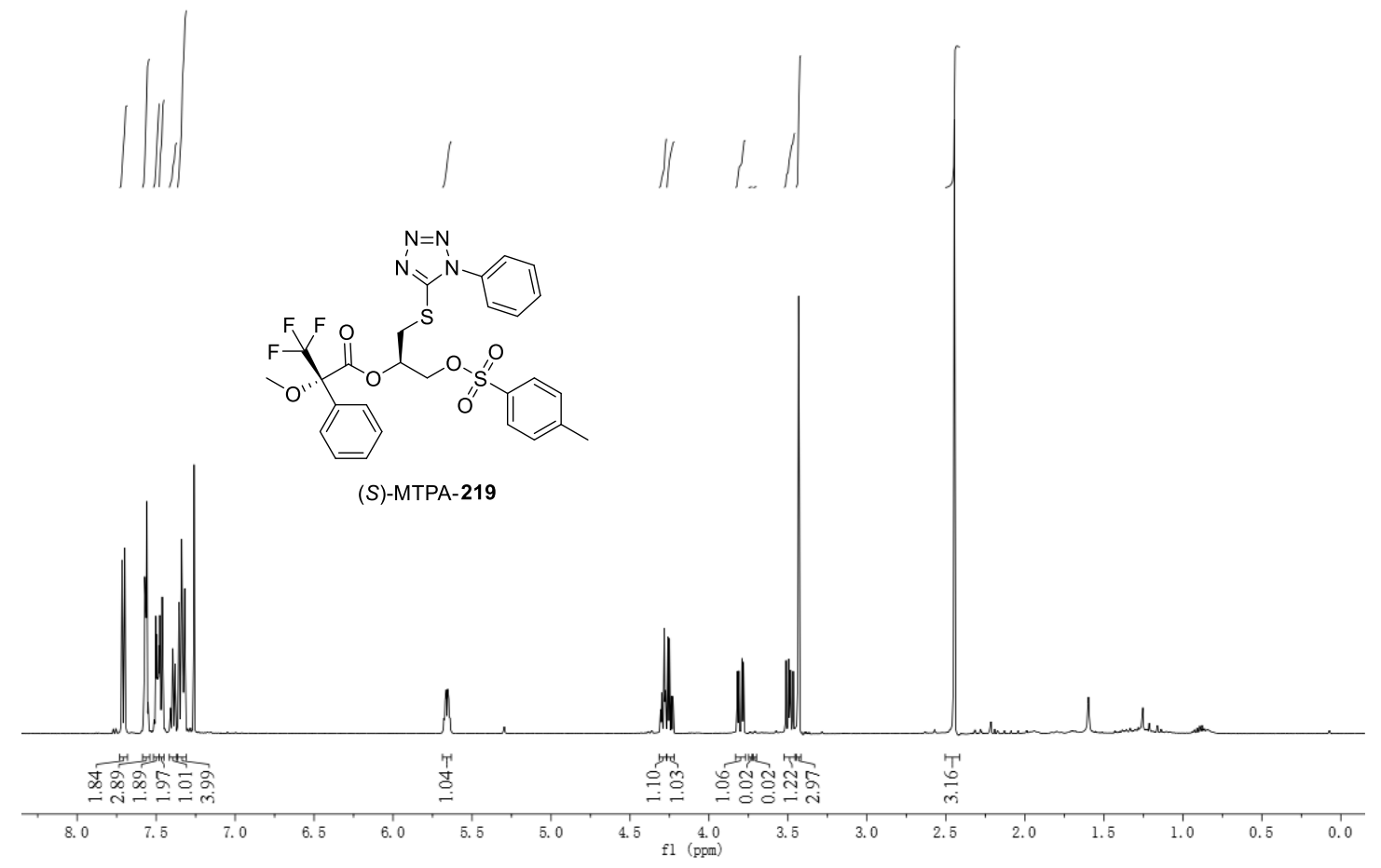

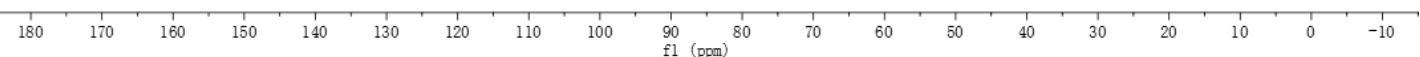


${ }^{1} \mathrm{H}$ NMR $\left(500 \mathrm{MHz}, \mathrm{CDCl}_{3}\right)$

$1 /$
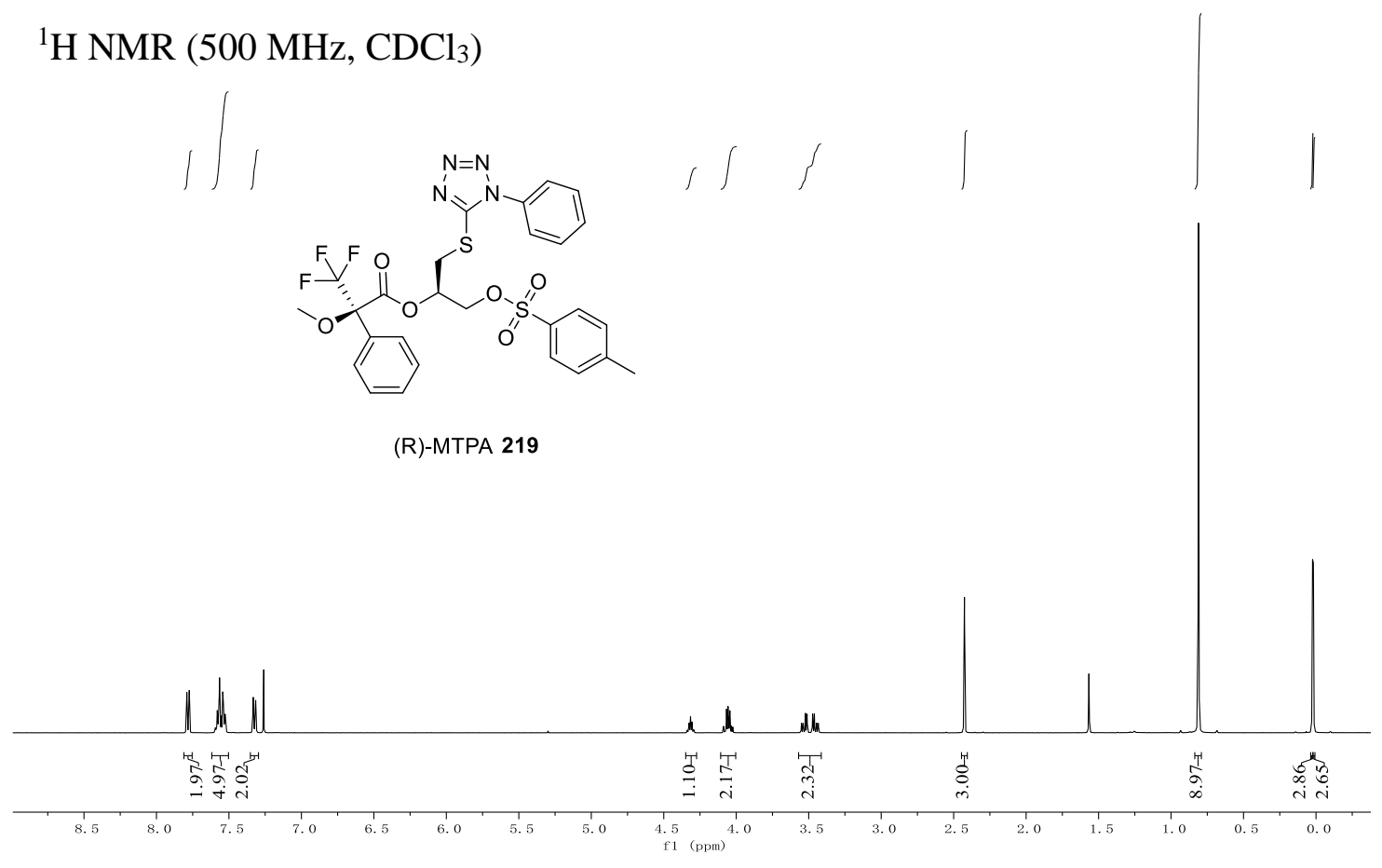

${ }^{13} \mathrm{C}$ NMR (125 MHz, $\left.\mathrm{CDCl}_{3}\right)$

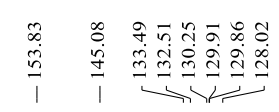

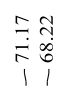

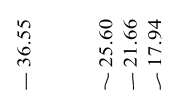

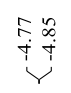

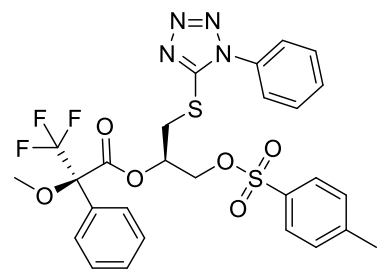

(R)-MTPA 219
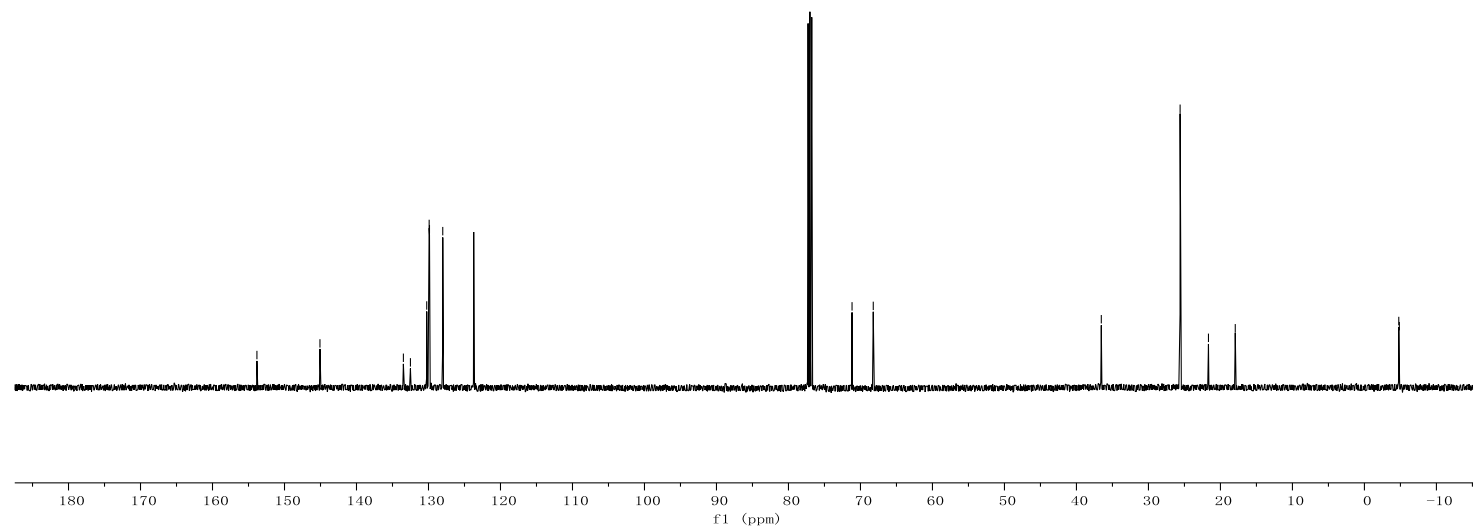

300 
${ }^{1} \mathrm{H}$ NMR (500 MHz, $\left.\mathrm{CDCl}_{3}\right)$<smiles>CCCCOS(=O)(=O)c1ccc(C)cc1</smiles>
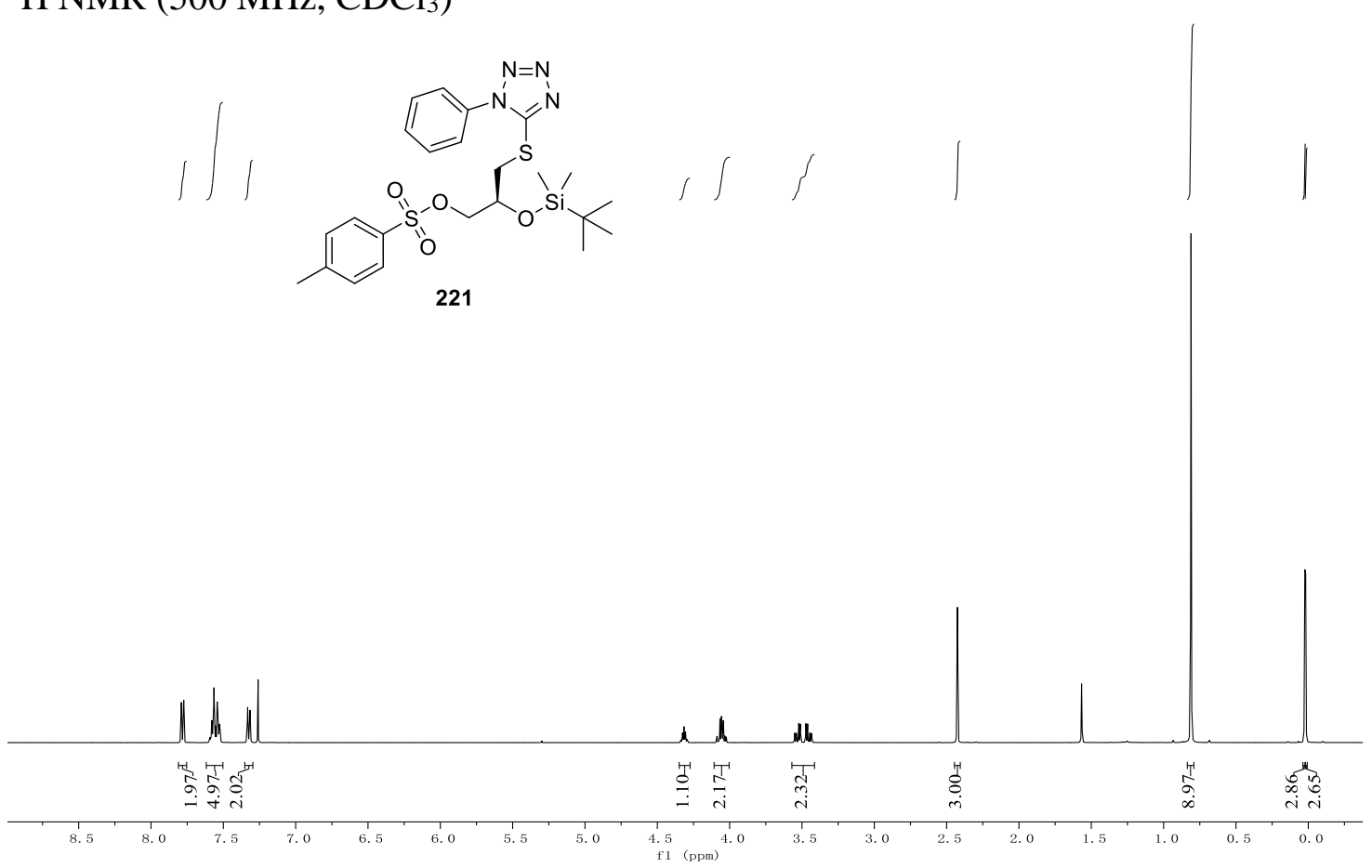

${ }^{13} \mathrm{C} \mathrm{NMR}\left(125 \mathrm{MHz}, \mathrm{CDCl}_{3}\right)$
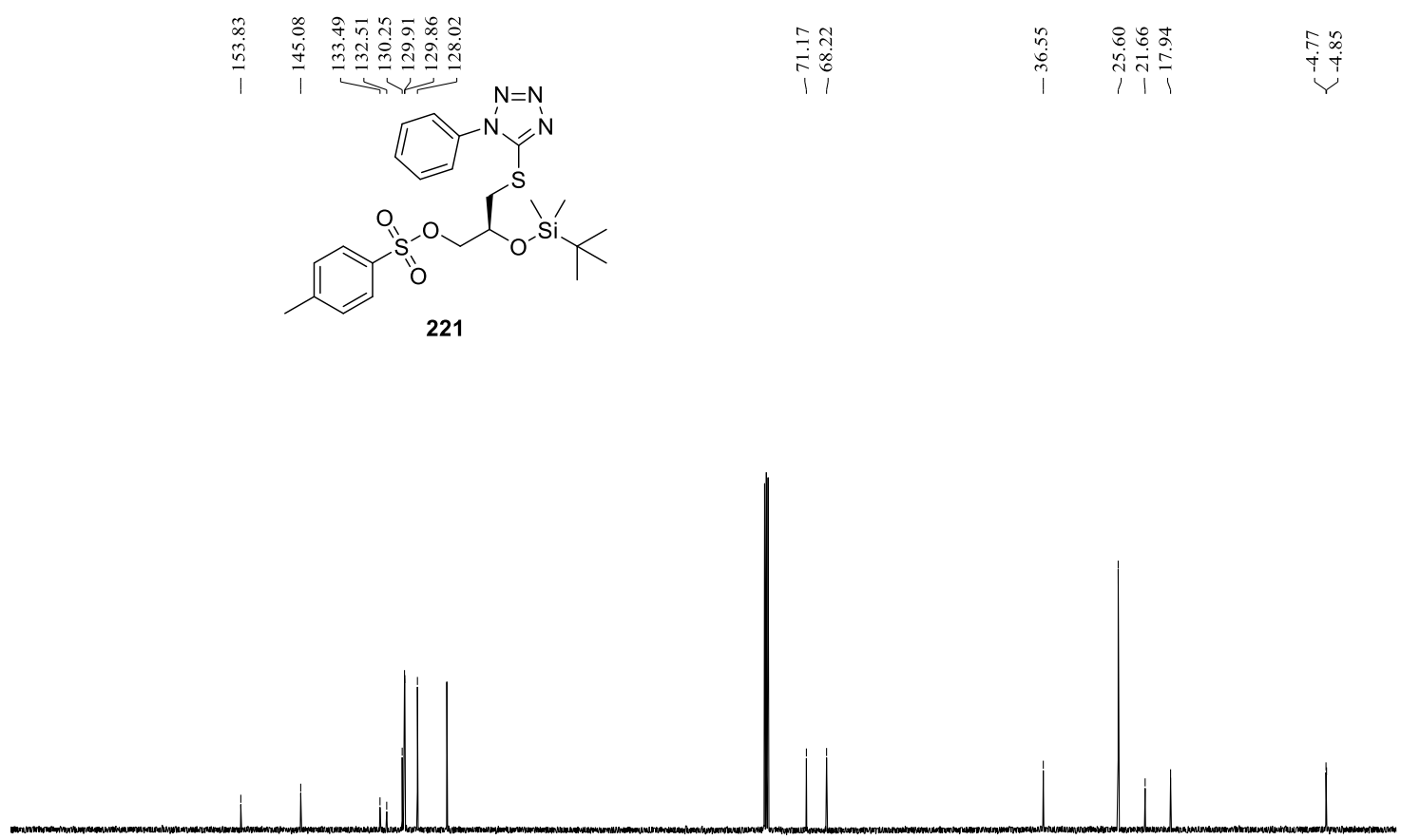
${ }^{1} \mathrm{H} \mathrm{NMR}\left(500 \mathrm{MHz}, \mathrm{CDCl}_{3}\right)$
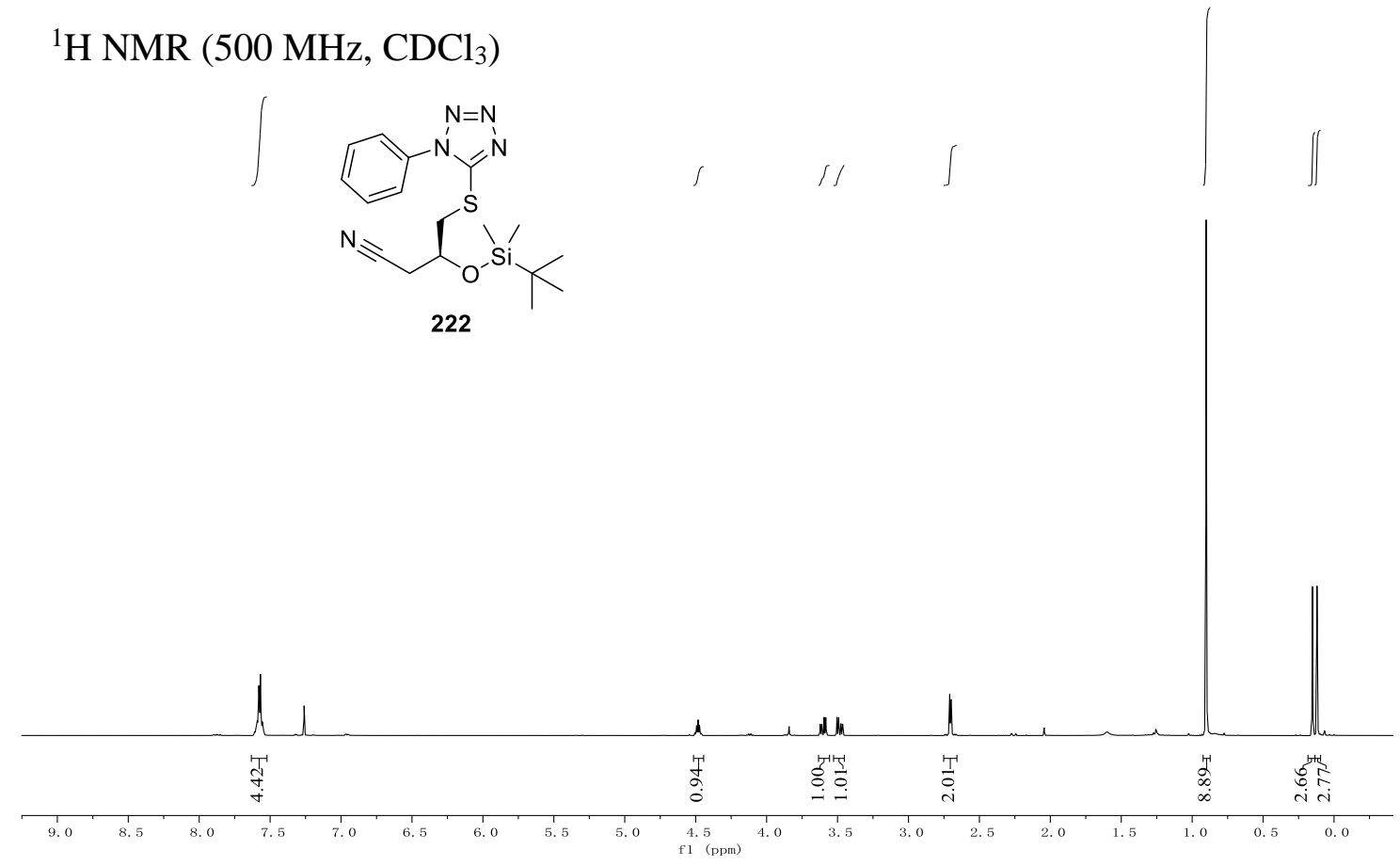

${ }^{13} \mathrm{C} \mathrm{NMR}\left(125 \mathrm{MHz}, \mathrm{CDCl}_{3}\right)$
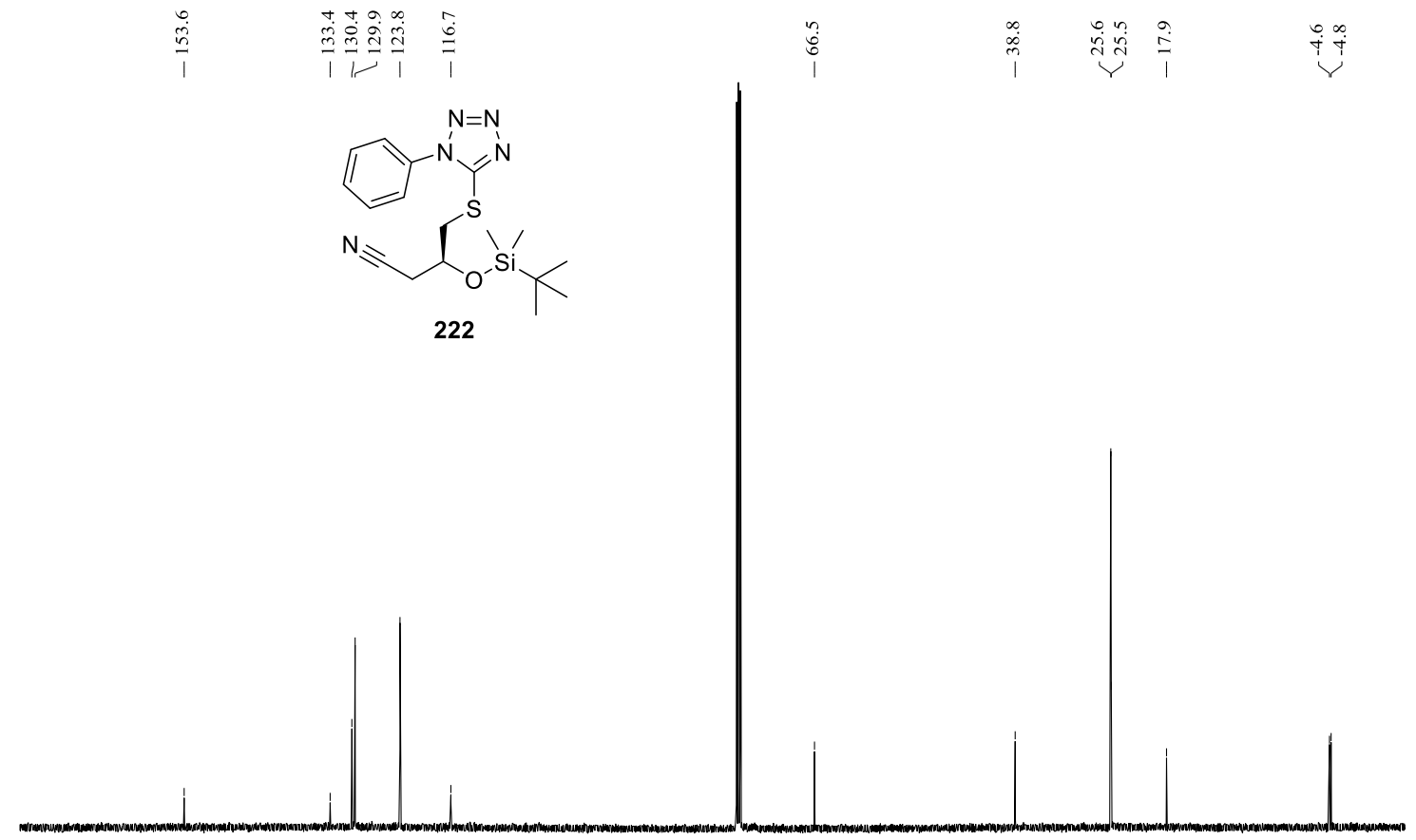

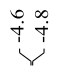


${ }^{1} \mathrm{H}$ NMR $\left(500 \mathrm{MHz}, \mathrm{CDCl}_{3}\right)$

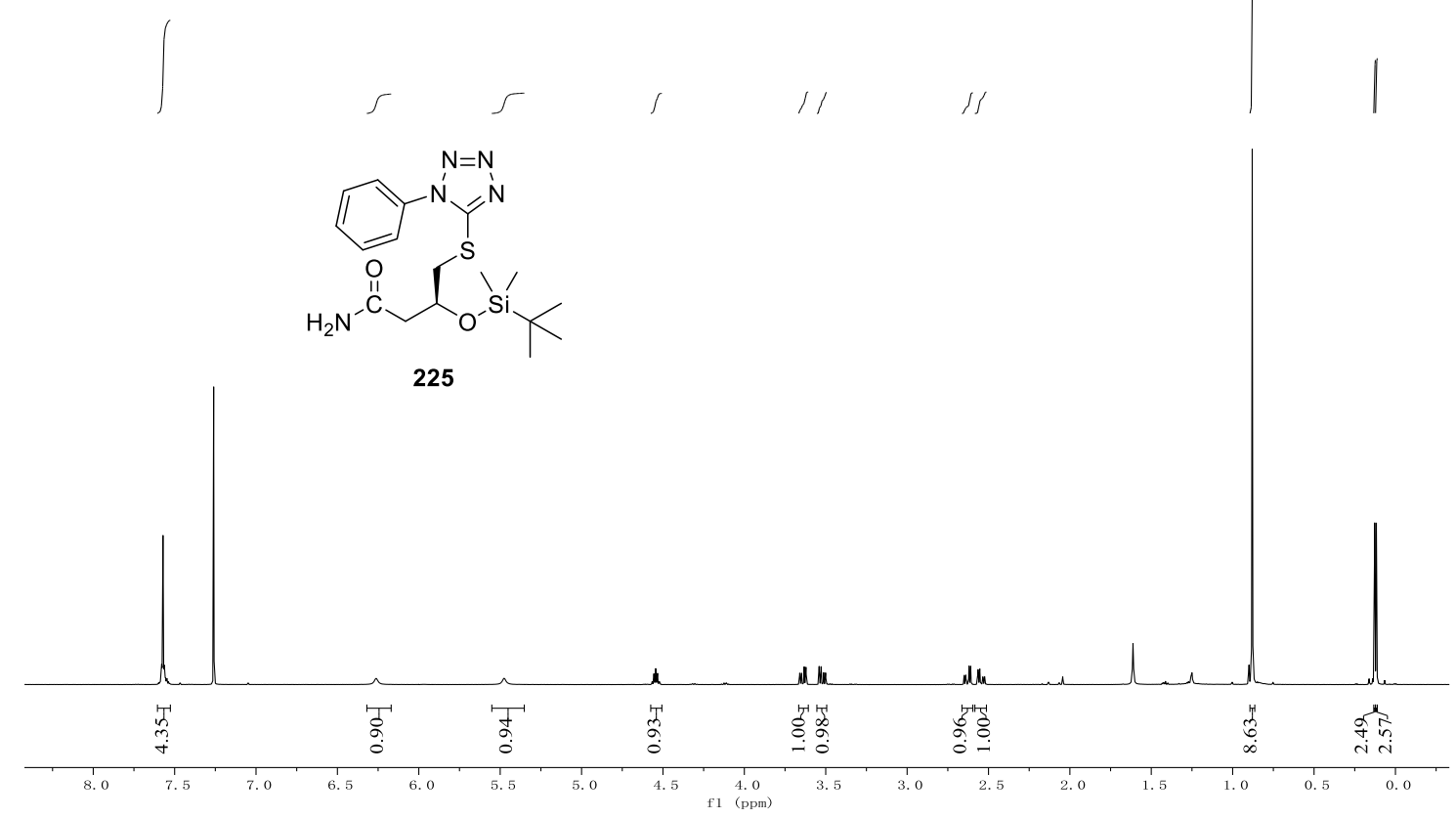

${ }^{13} \mathrm{C} \mathrm{NMR}\left(125 \mathrm{MHz}, \mathrm{CDCl}_{3}\right)$
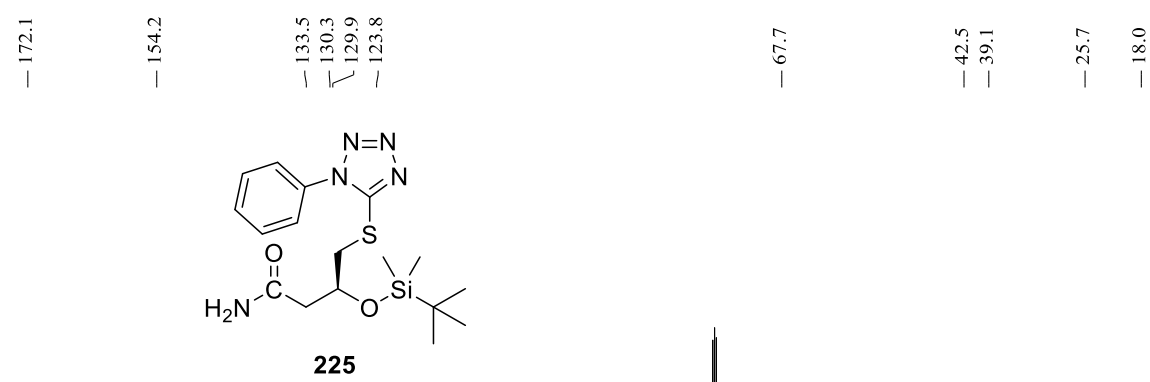

225
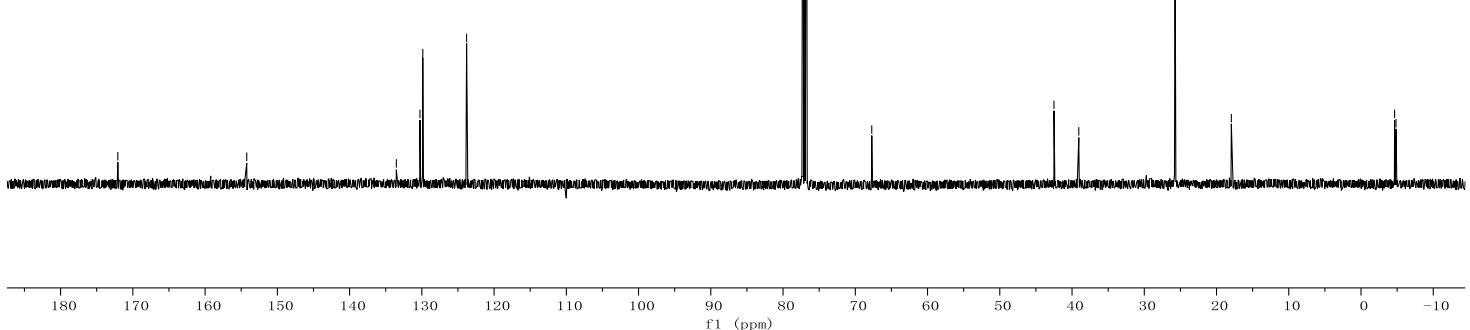
${ }^{1} \mathrm{H}$ NMR $\left(500 \mathrm{MHz}, \mathrm{CDCl}_{3}\right)$

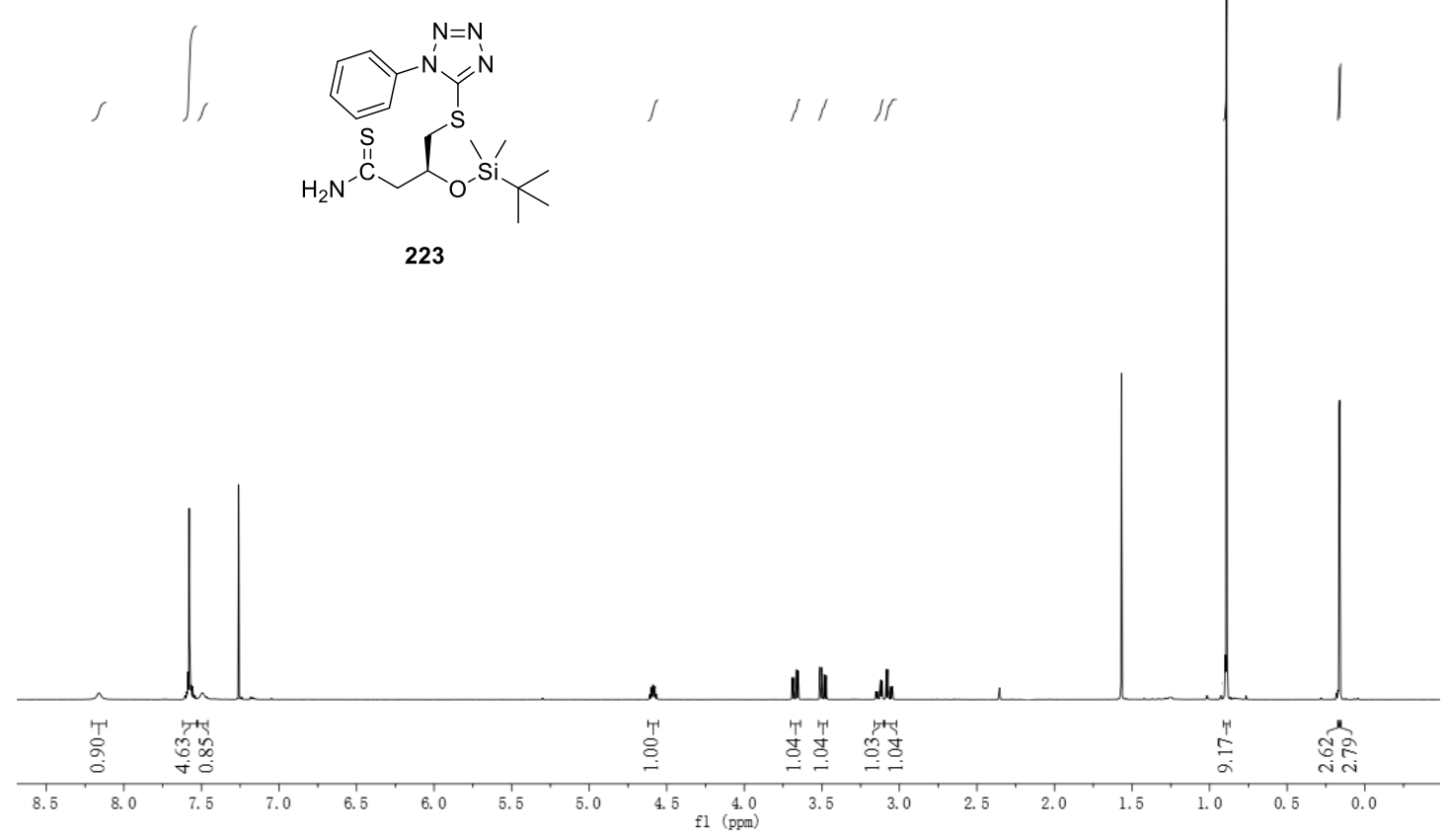

${ }^{13} \mathrm{C} \mathrm{NMR}\left(125 \mathrm{MHz}, \mathrm{CDCl}_{3}\right)$

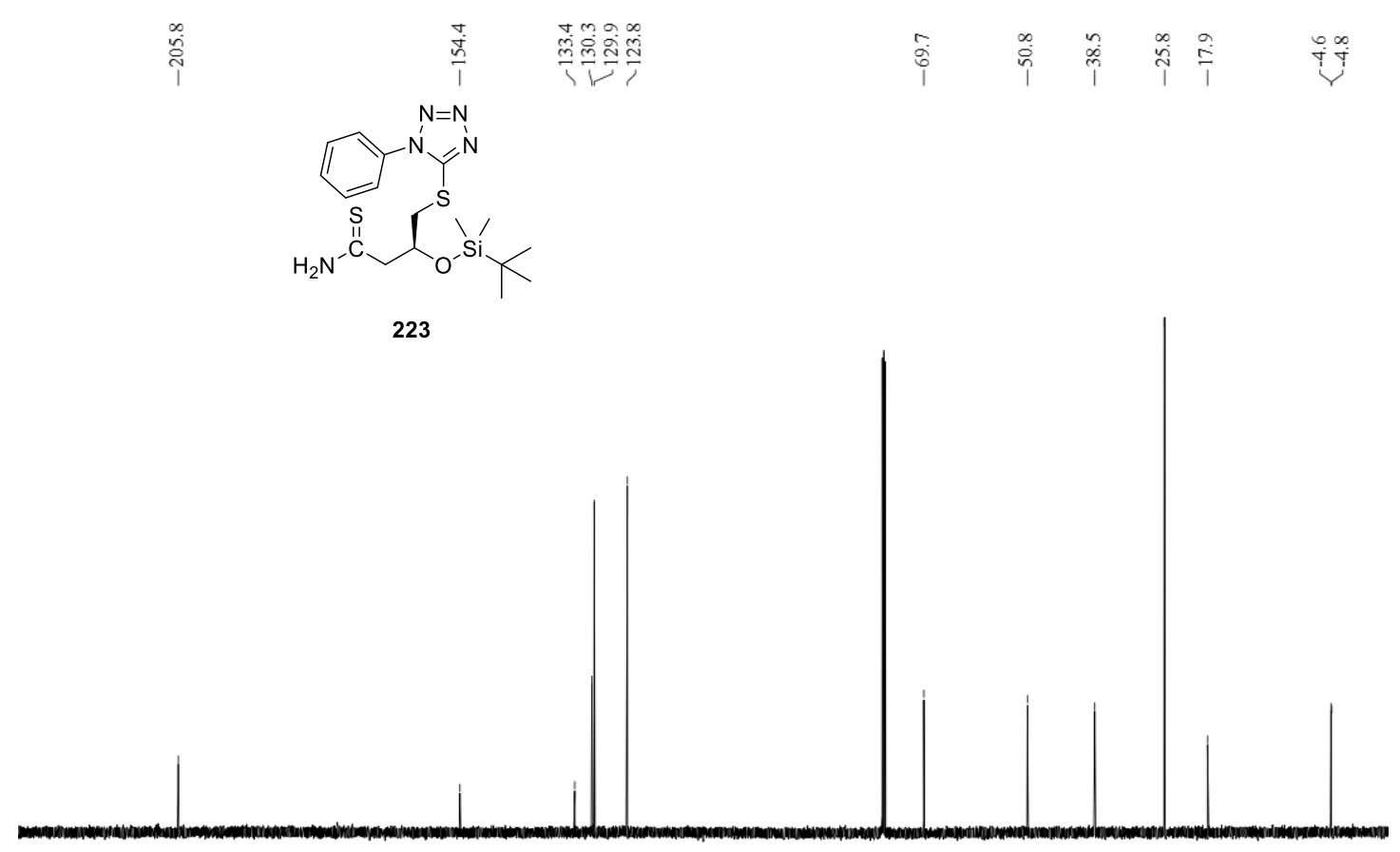

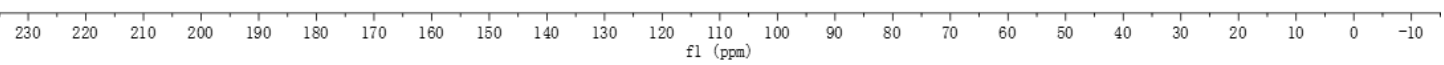


${ }^{1} \mathrm{H}$ NMR $\left(500 \mathrm{MHz}, \mathrm{CDCl}_{3}\right)$

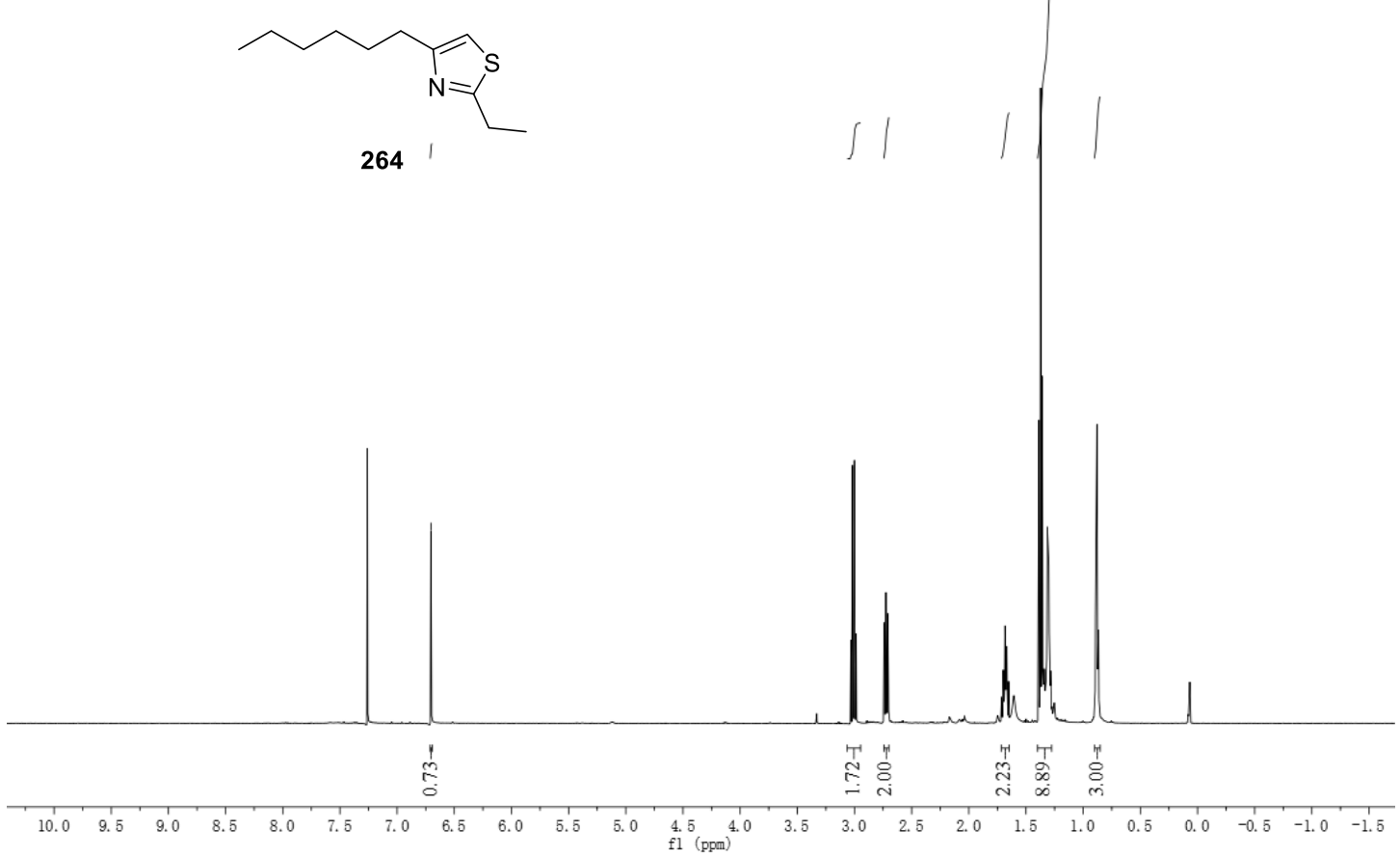

${ }^{13} \mathrm{C}$ NMR (125 MHz, $\left.\mathrm{CDCl}_{3}\right)$

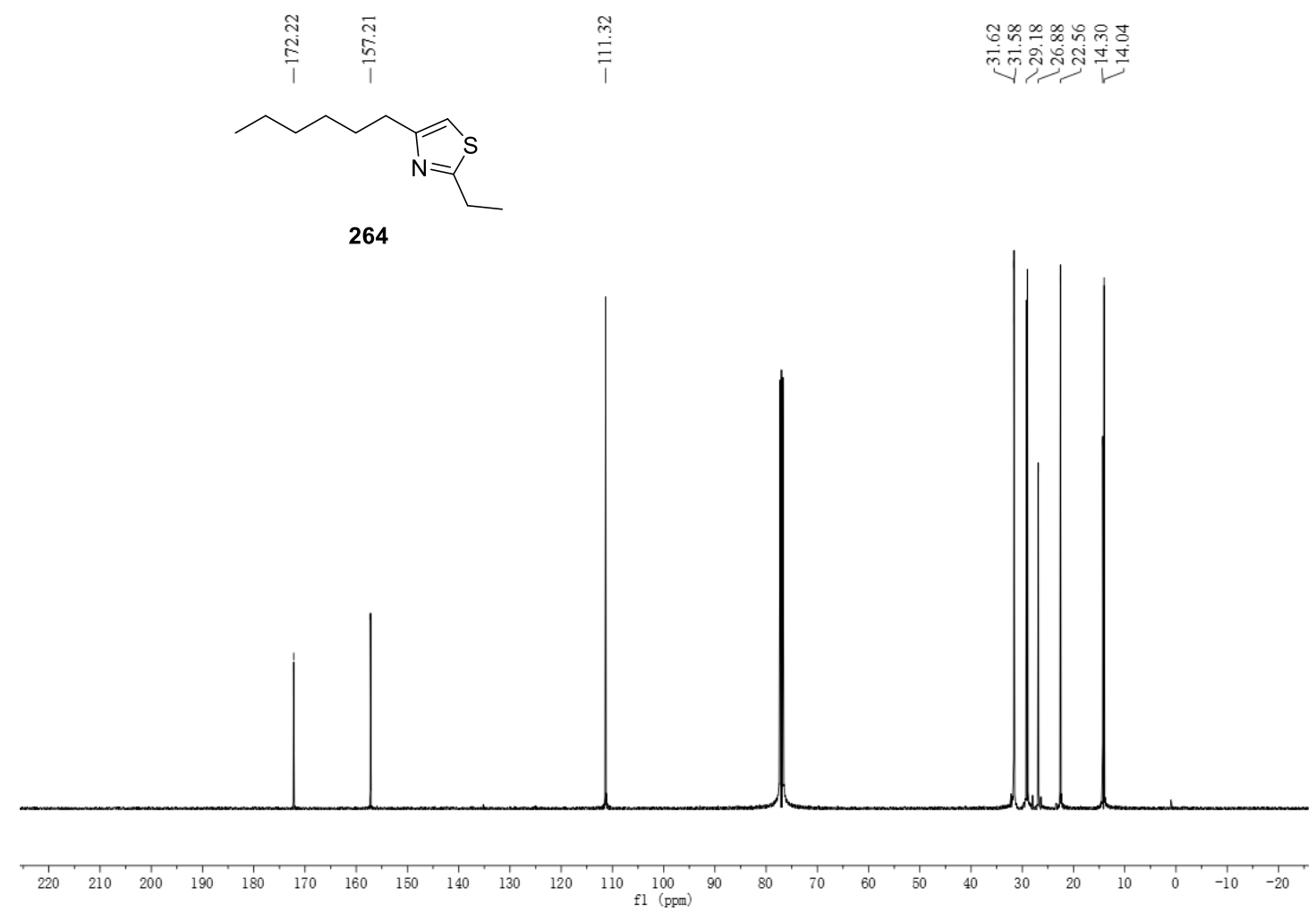


${ }^{1} \mathrm{H}$ NMR $\left(500 \mathrm{MHz}, \mathrm{CDCl}_{3}\right)$

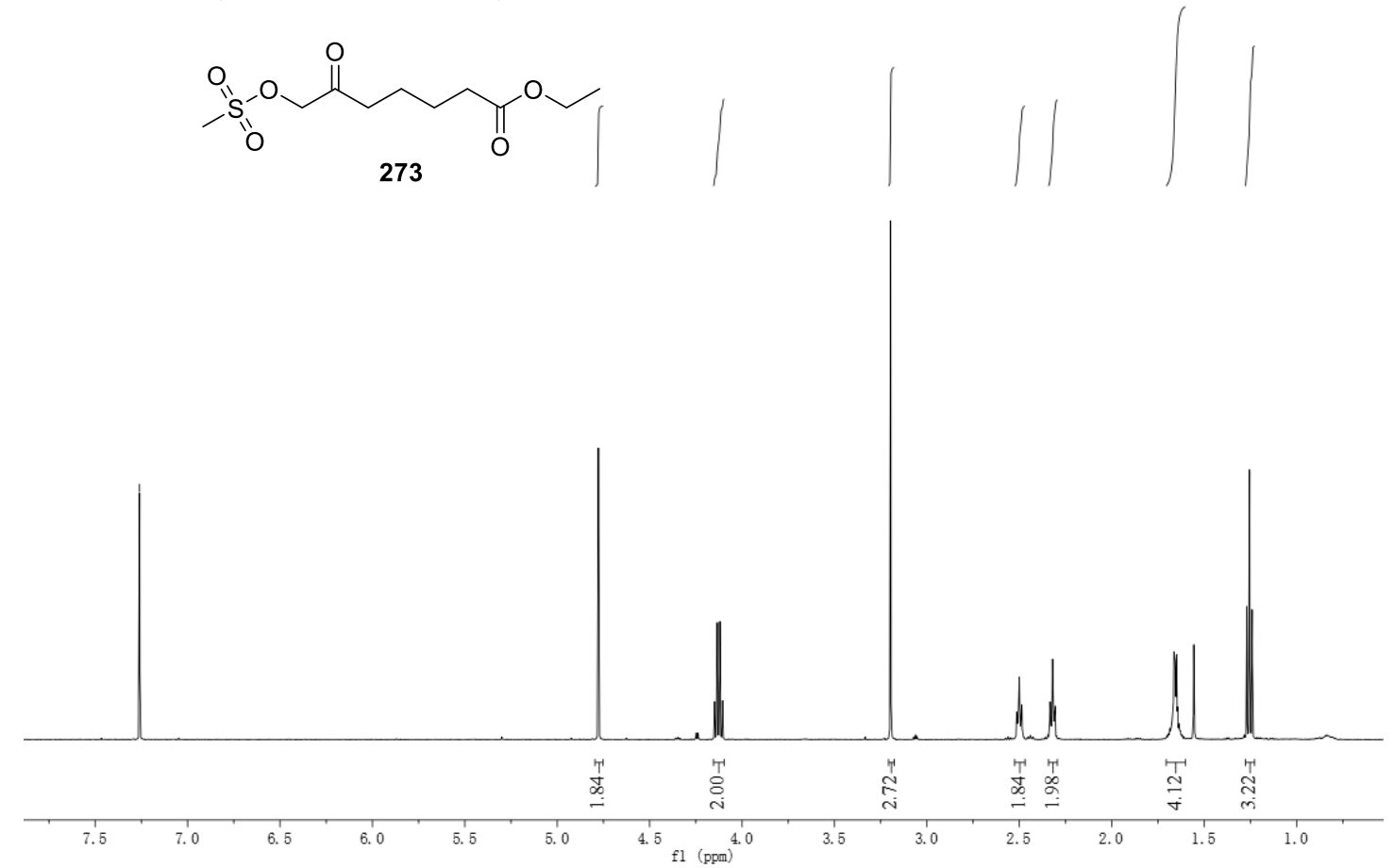

${ }^{13} \mathrm{C}$ NMR (125 MHz, $\left.\mathrm{CDCl}_{3}\right)$

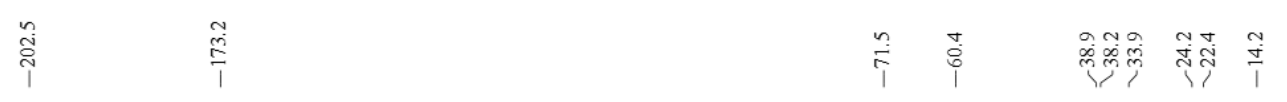<smiles>CCOC(=O)CCCCC(=O)COS(C)(=O)=O</smiles>

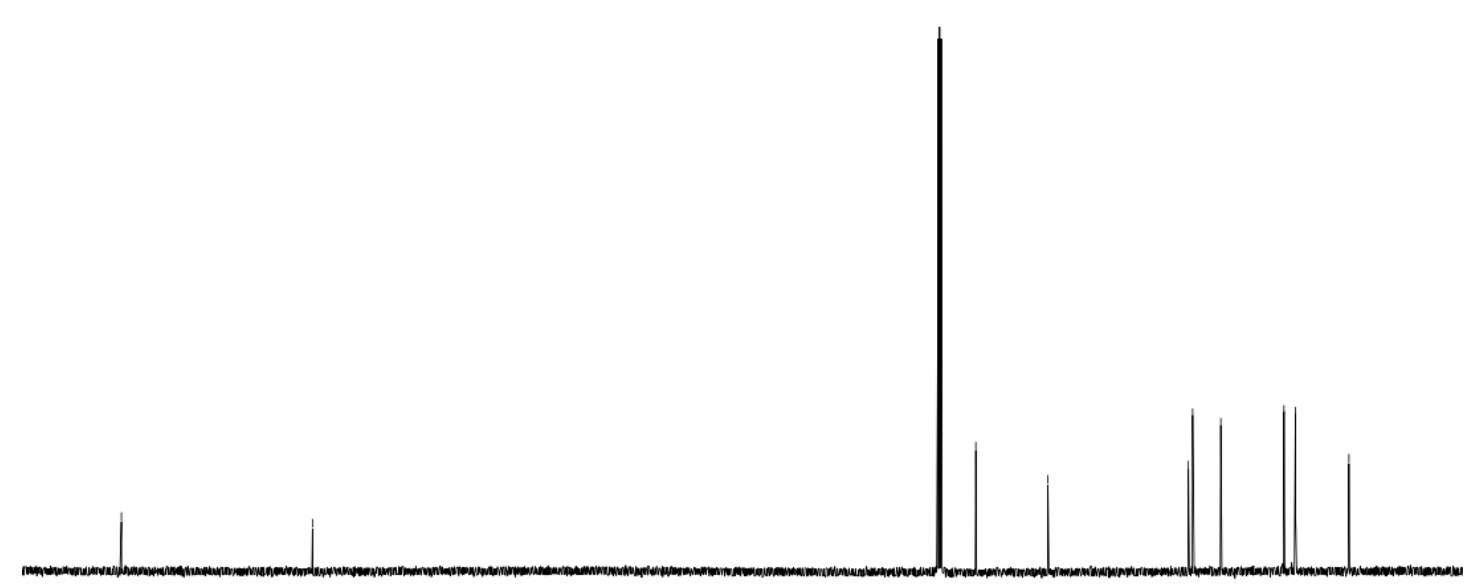

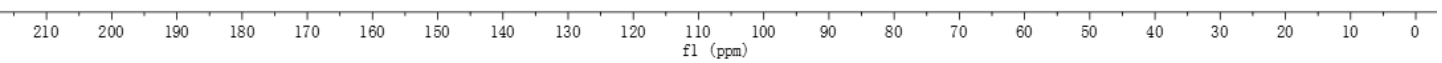


${ }^{1} \mathrm{H}$ NMR $\left(500 \mathrm{MHz}, \mathrm{CDCl}_{3}\right)$
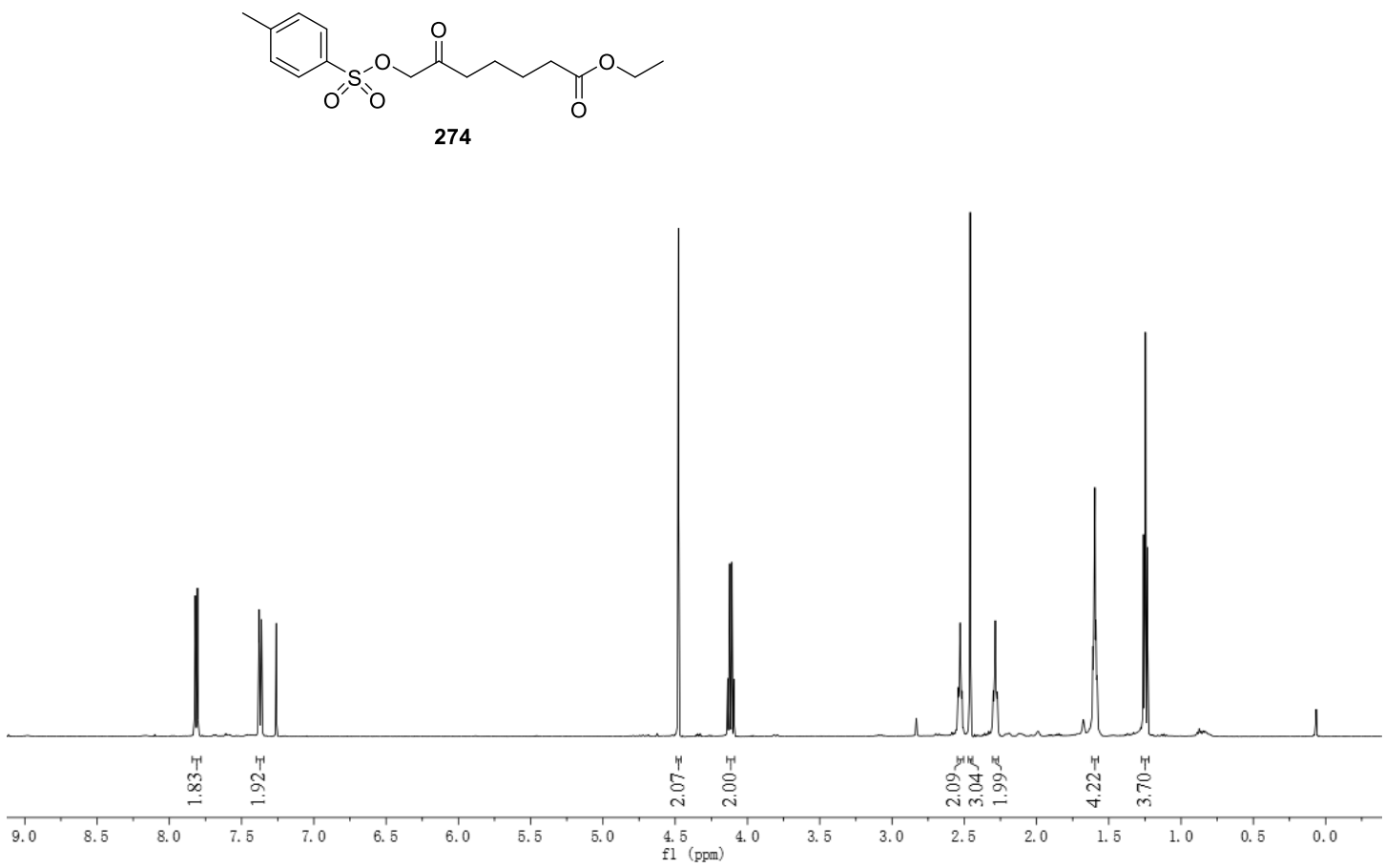

${ }^{13} \mathrm{C} \mathrm{NMR}\left(125 \mathrm{MHz}, \mathrm{CDCl}_{3}\right)$
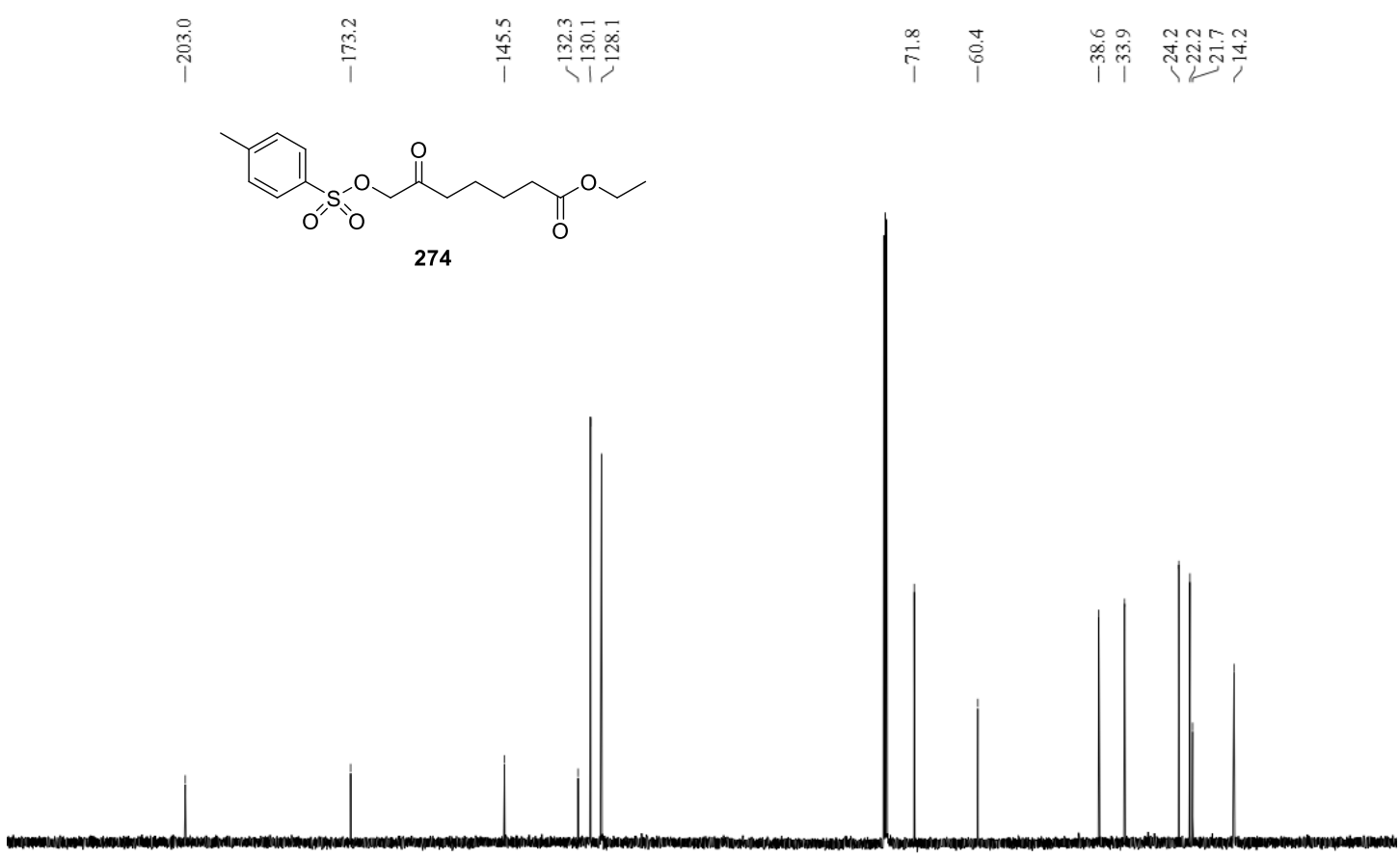

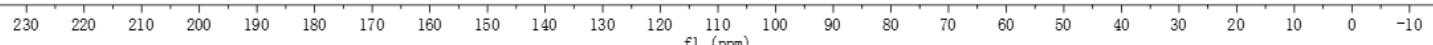


${ }^{1} \mathrm{H}$ NMR $\left(500 \mathrm{MHz}, \mathrm{CDCl}_{3}\right.$ )

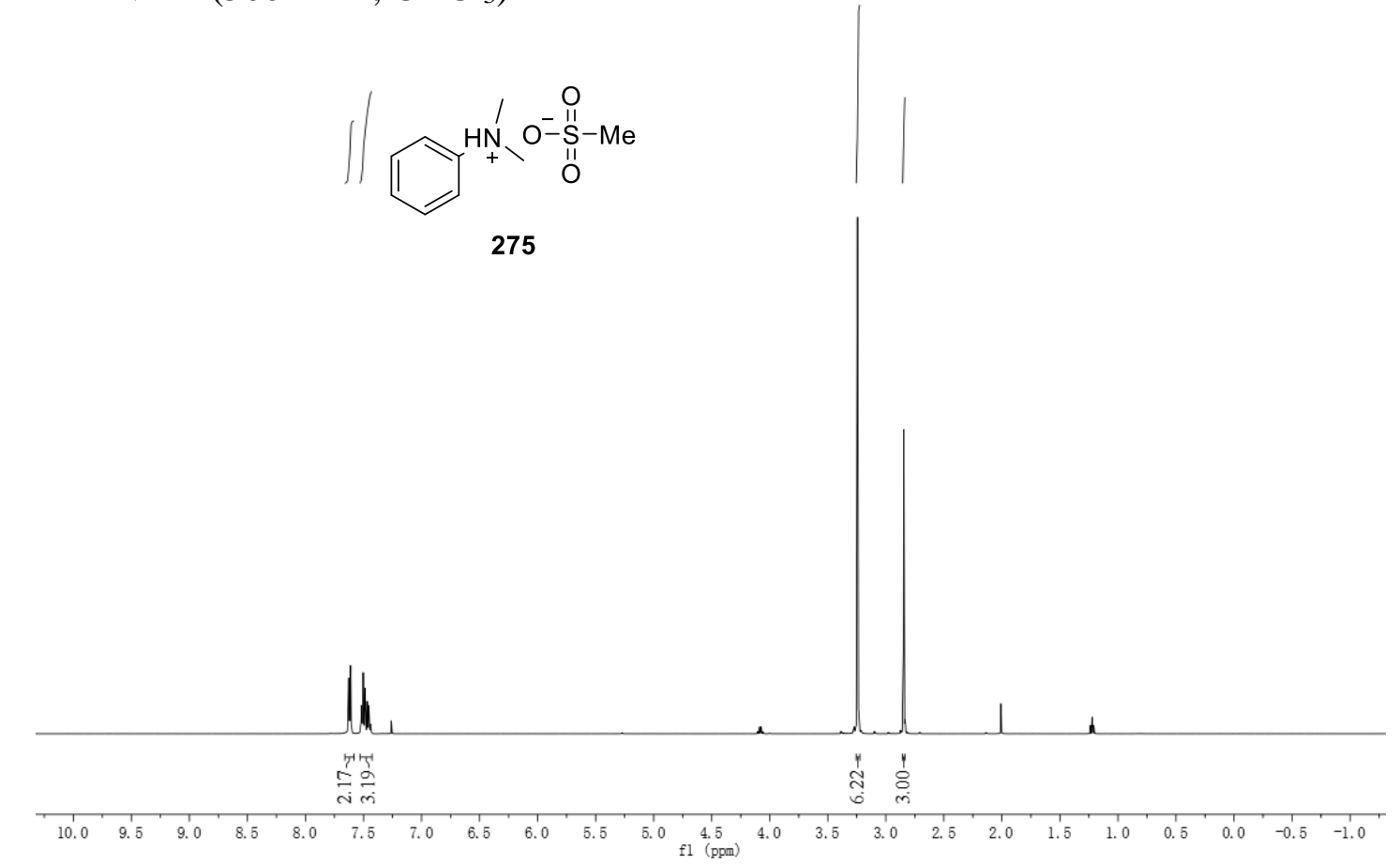

${ }^{13} \mathrm{C}$ NMR (125 MHz, $\left.\mathrm{CDCl}_{3}\right)$
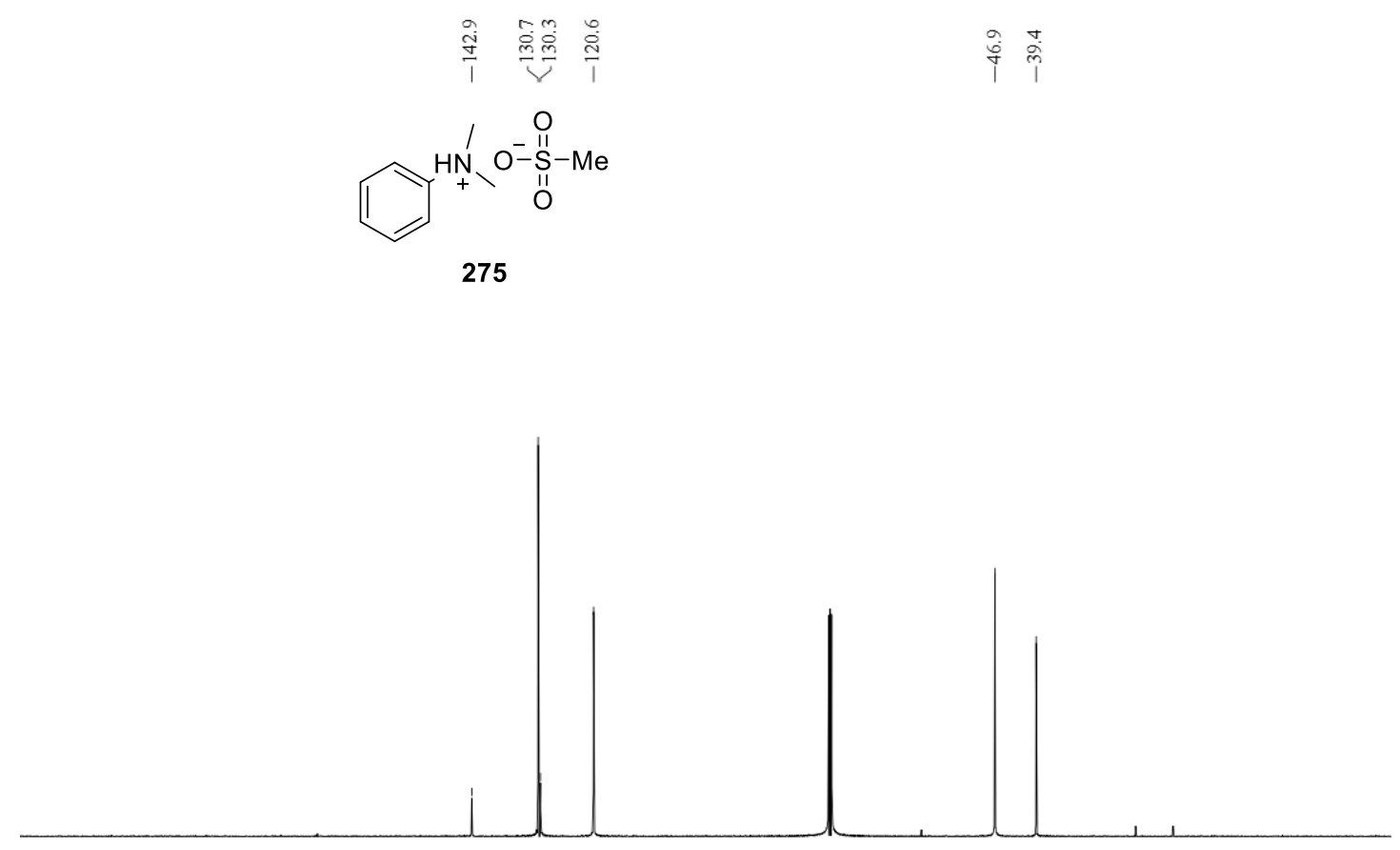

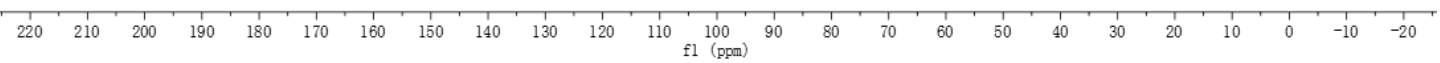


${ }^{1} \mathrm{H}$ NMR (500 MHz, $\mathrm{CDCl}_{3}$ )

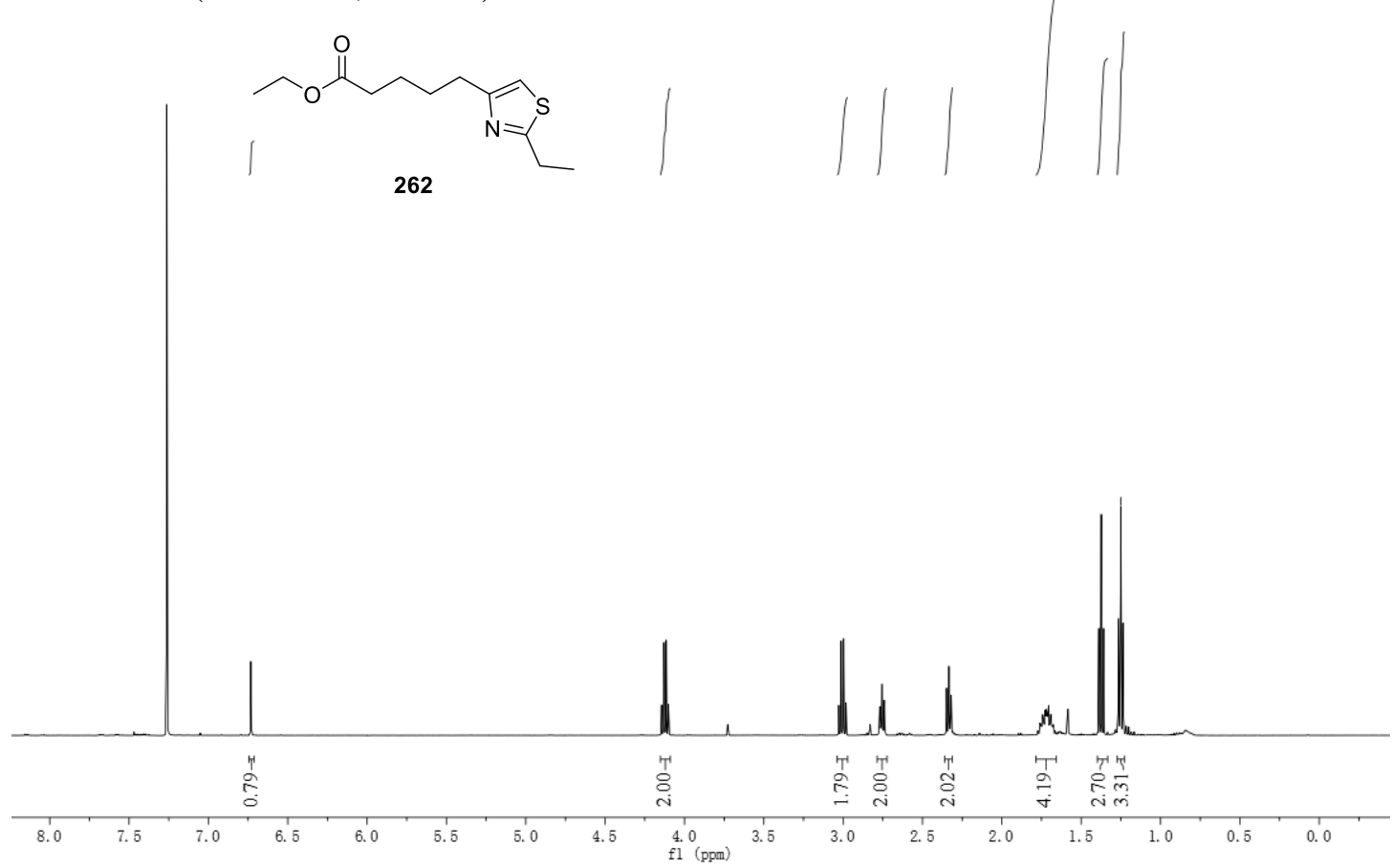

$\left.{ }^{13} \mathrm{C} \mathrm{NMR} \mathrm{(125} \mathrm{MHz,} \mathrm{CDCl}_{3}\right)$
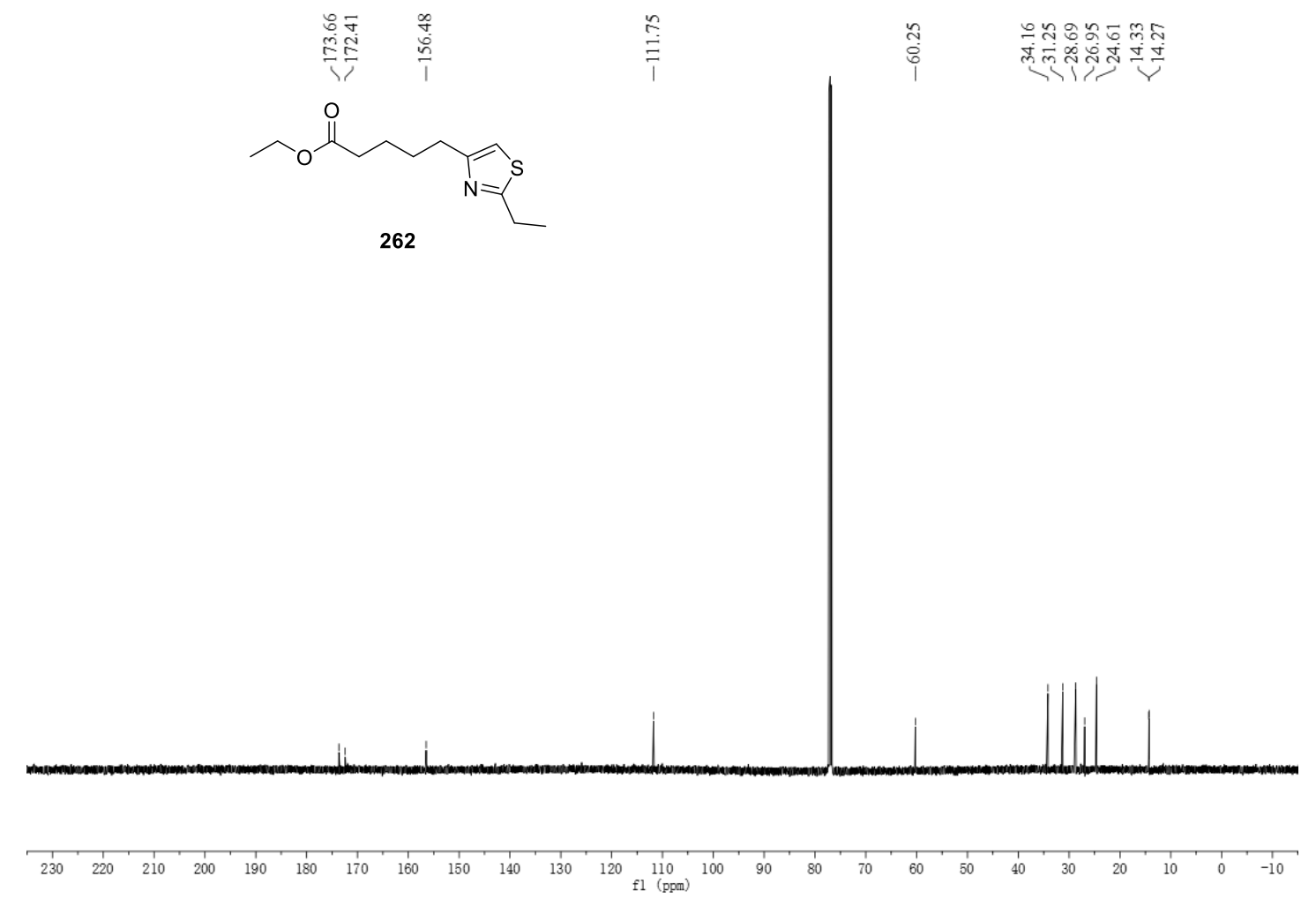
${ }^{1} \mathrm{H}$ NMR $\left(500 \mathrm{MHz}, \mathrm{CDCl}_{3}\right)$

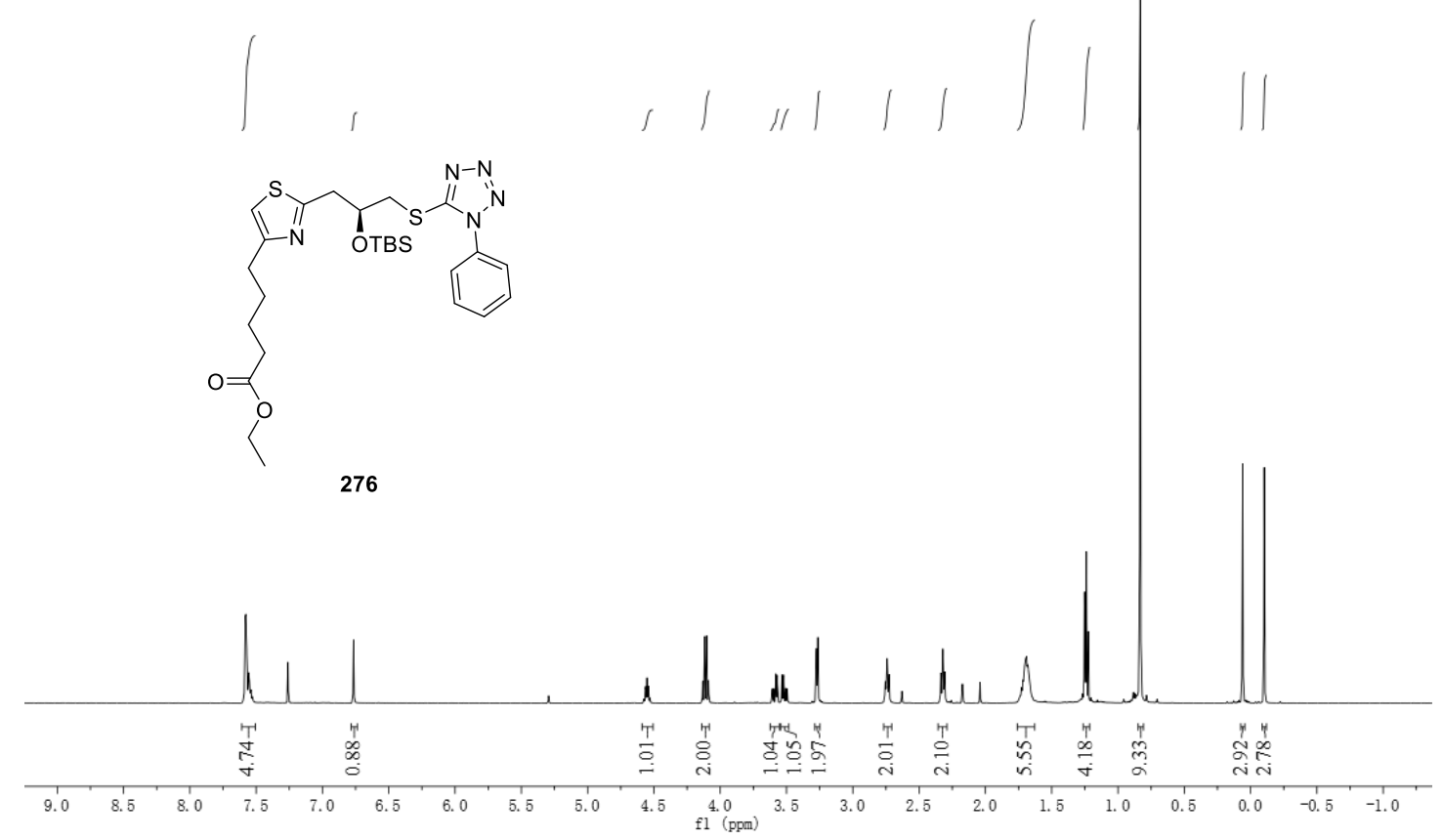

${ }^{13} \mathrm{C}$ NMR $\left(125 \mathrm{MHz}, \mathrm{CDCl}_{3}\right)$

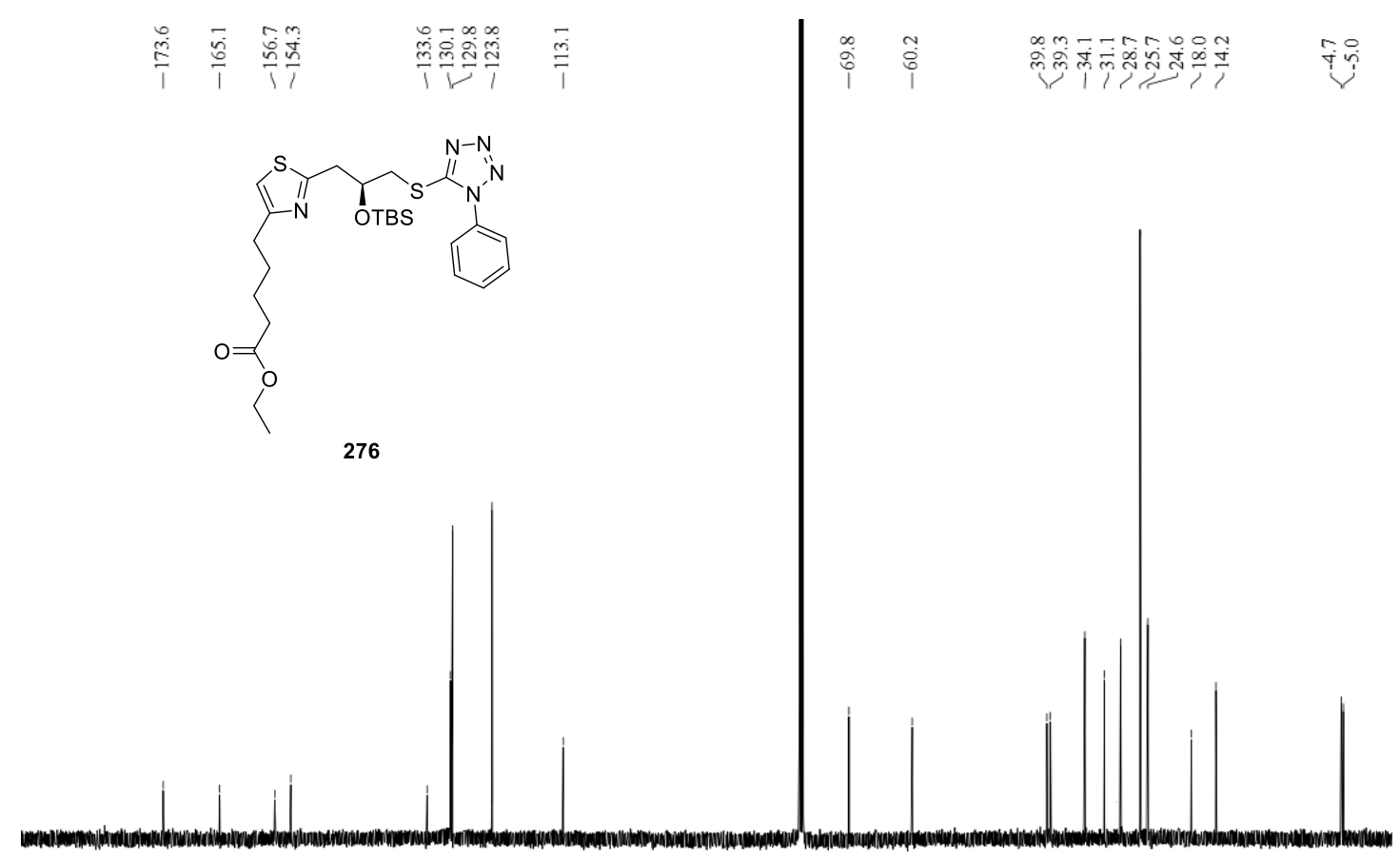

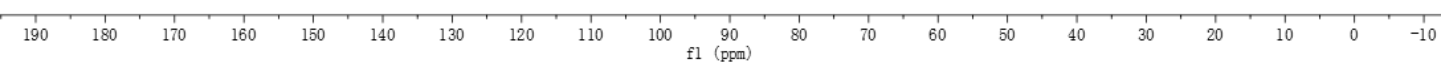


${ }^{1} \mathrm{H}$ NMR $\left(500 \mathrm{MHz}, \mathrm{CDCl}_{3}\right)$

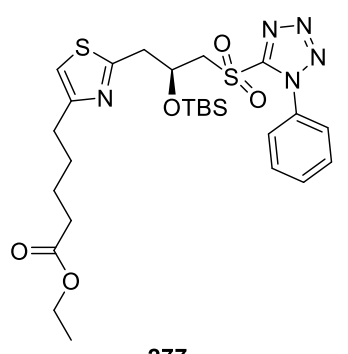

277

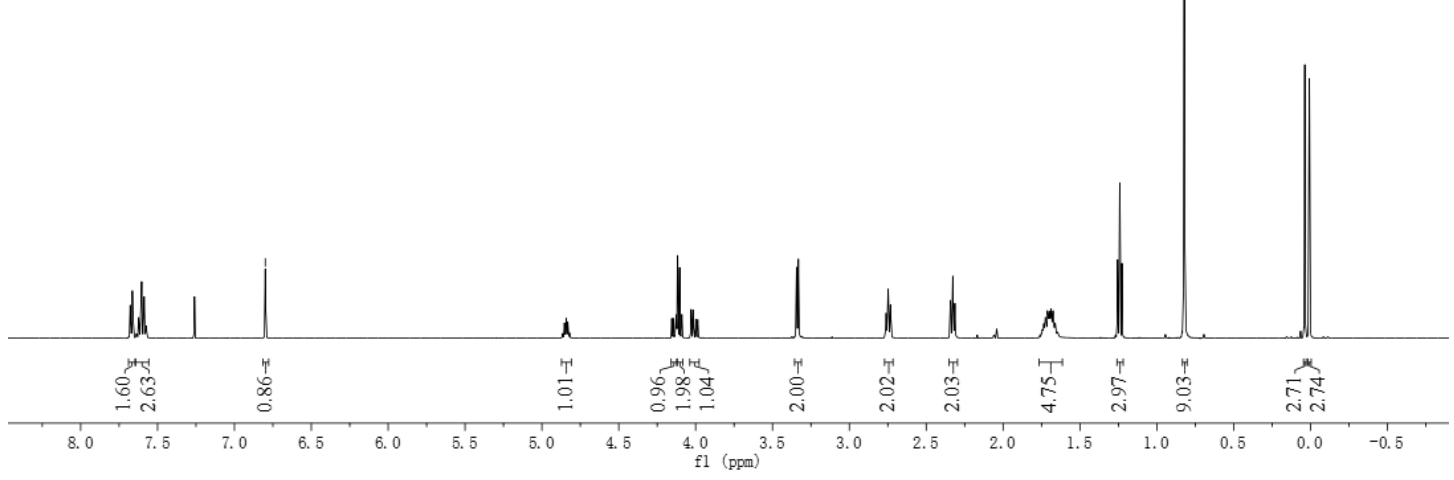

${ }^{13} \mathrm{C} \mathrm{NMR}\left(125 \mathrm{MHz}, \mathrm{CDCl}_{3}\right)$

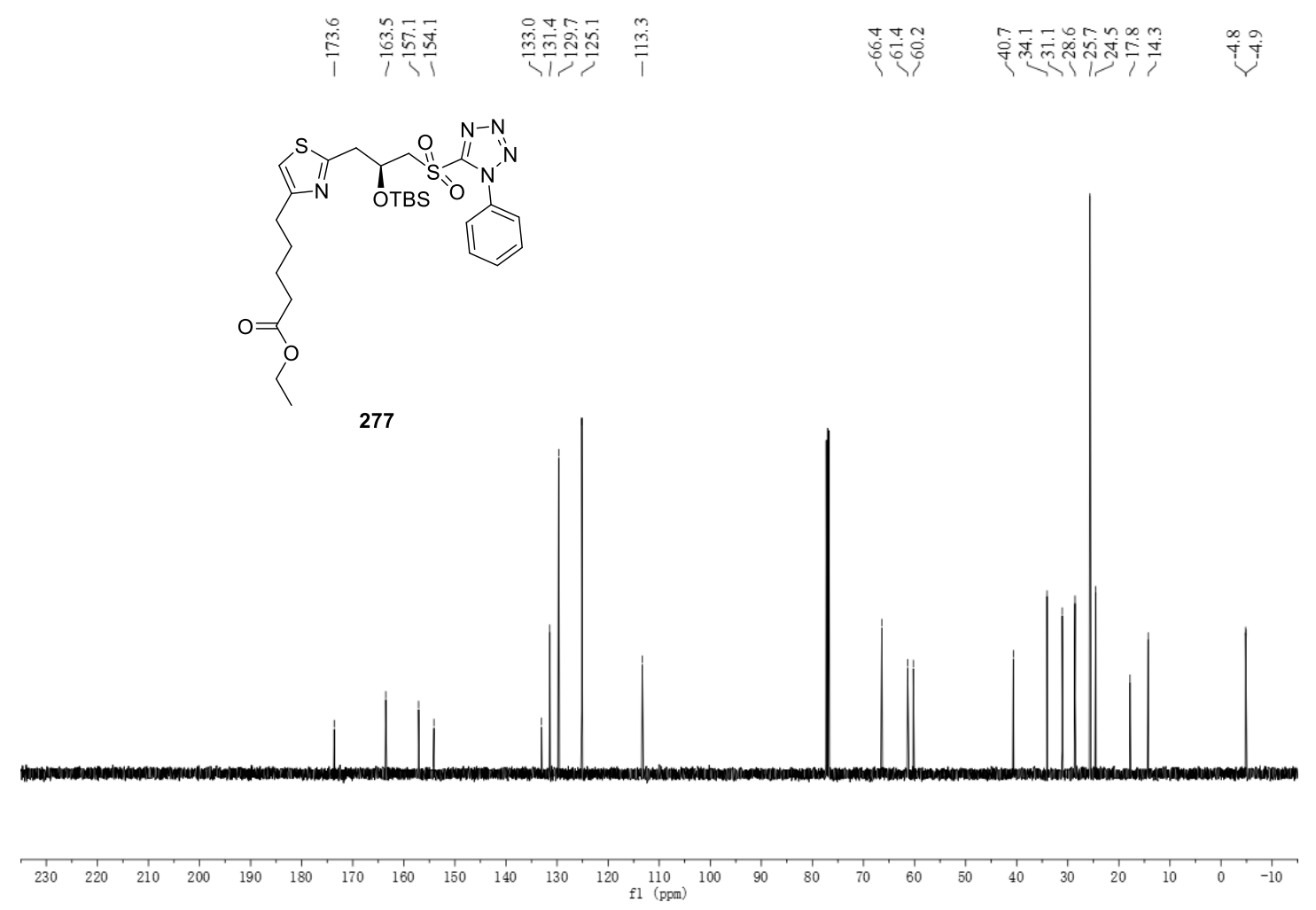


${ }^{1} \mathrm{H}$ NMR (500 MHz, $\mathrm{CDCl}_{3}$ )

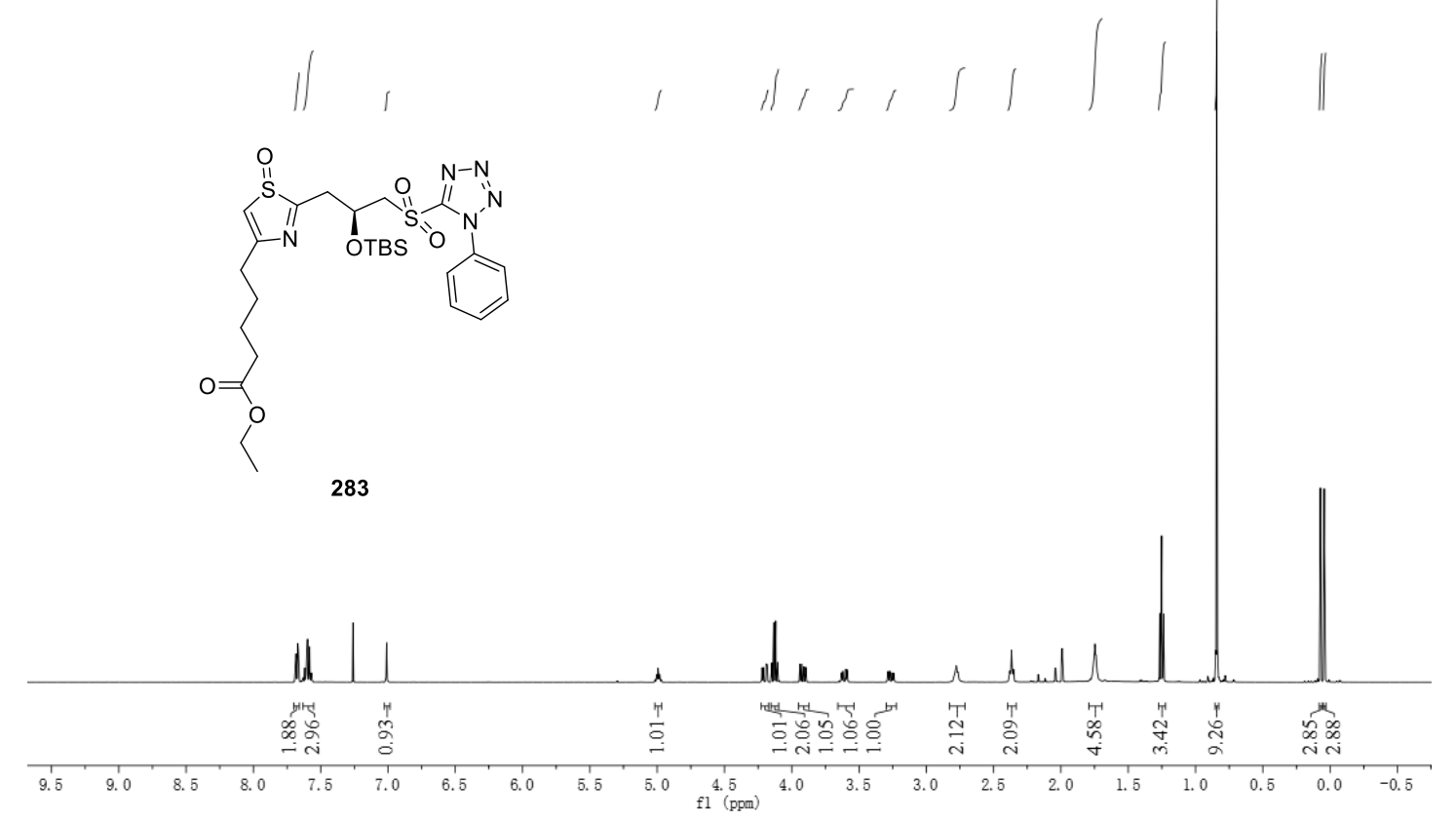

${ }^{13} \mathrm{C} \mathrm{NMR}\left(125 \mathrm{MHz}, \mathrm{CDCl}_{3}\right)$

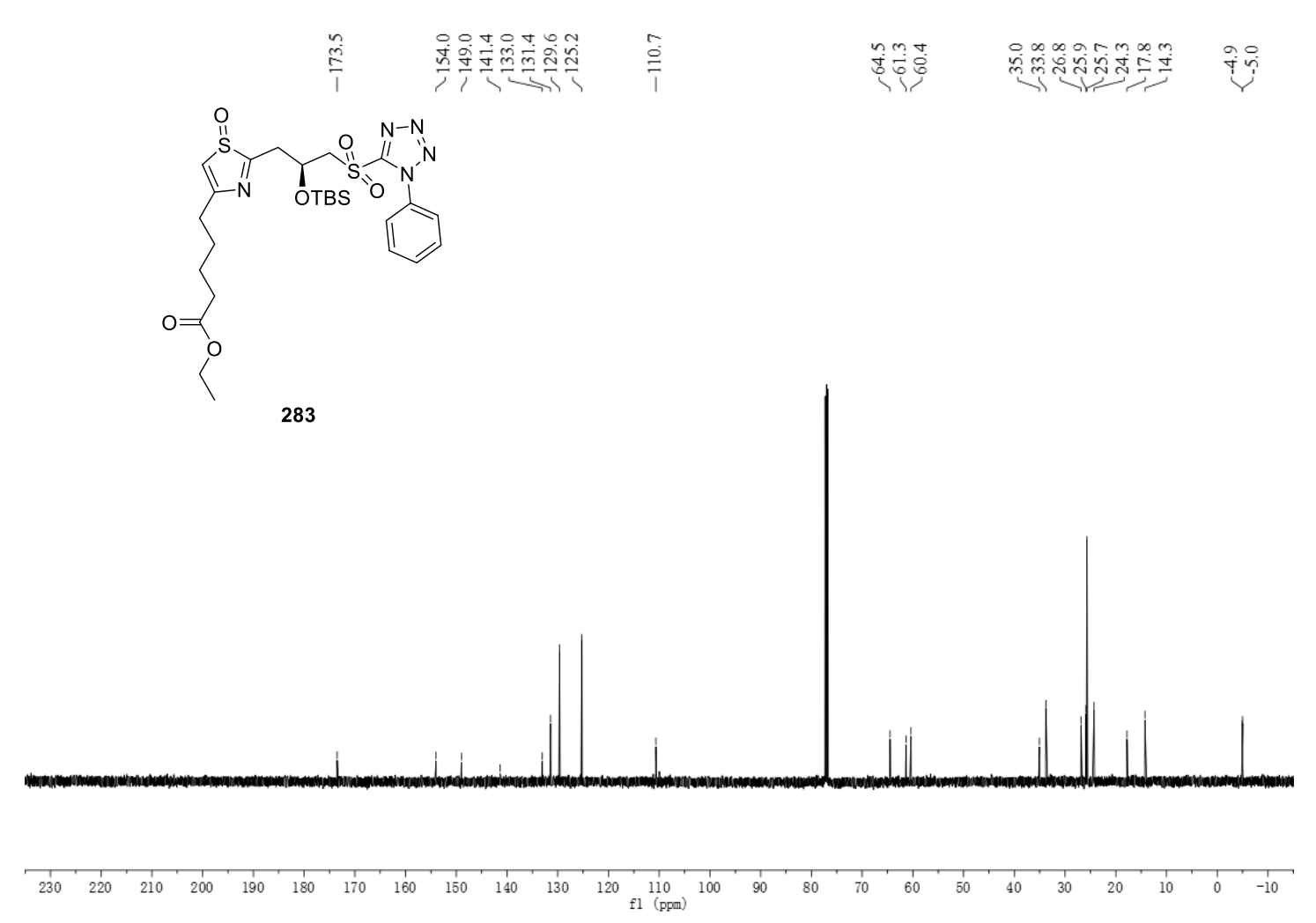


${ }^{1} \mathrm{H} \mathrm{NMR}\left(500 \mathrm{MHz}, \mathrm{CDCl}_{3}\right)$
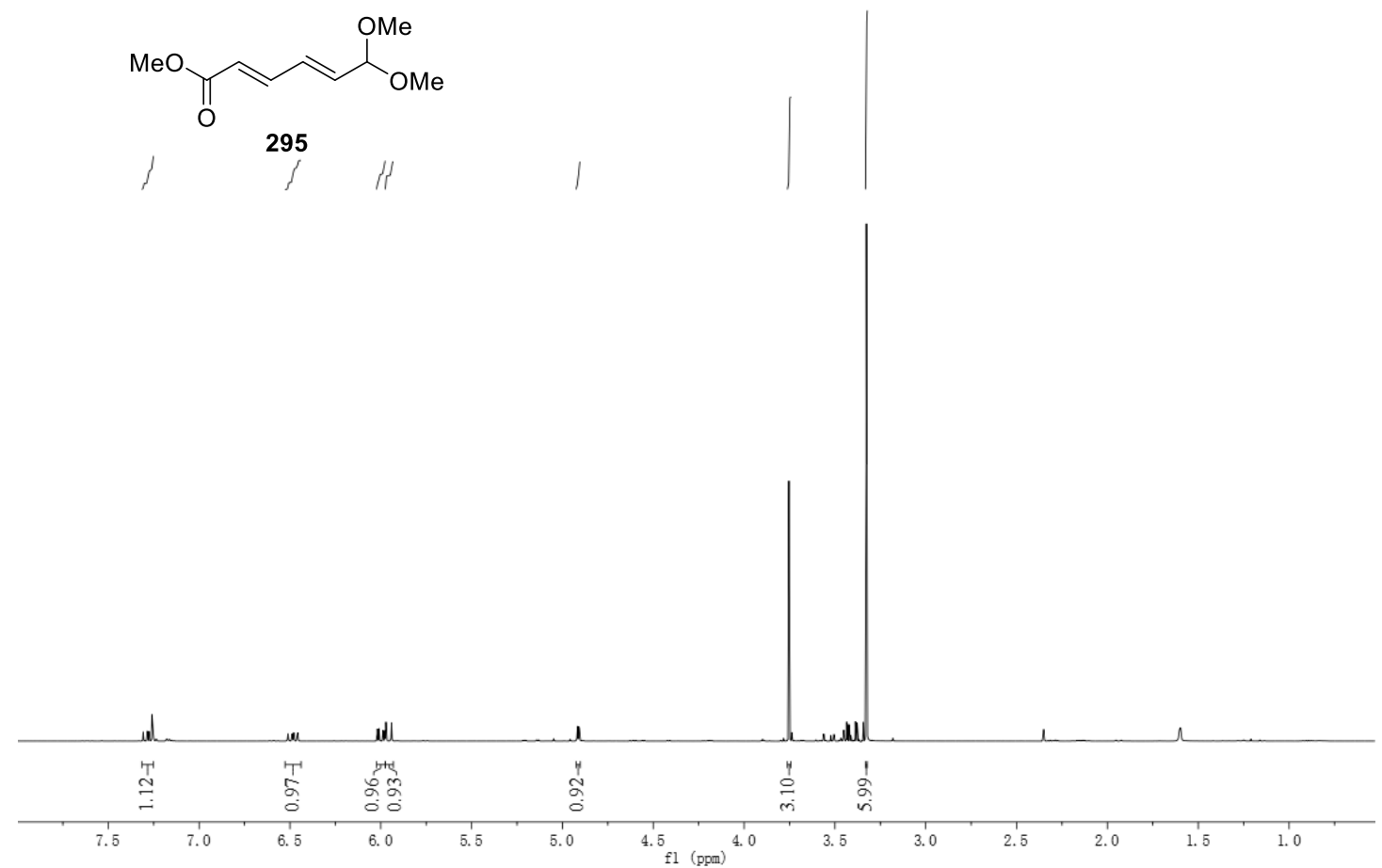

${ }^{13} \mathrm{C} \mathrm{NMR}\left(125 \mathrm{MHz}, \mathrm{CDCl}_{3}\right)$

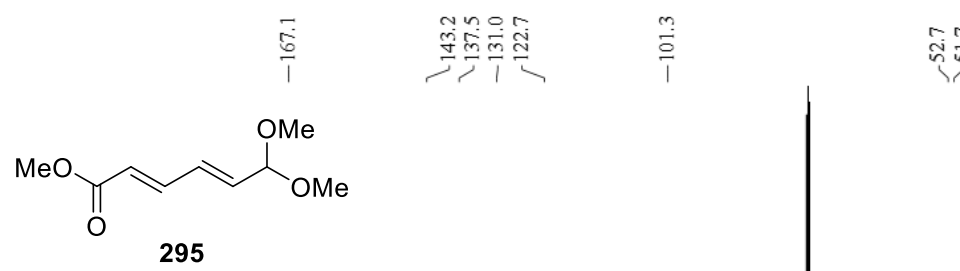

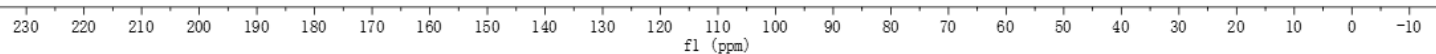


${ }^{1} \mathrm{H}$ NMR $\left(500 \mathrm{MHz}, \mathrm{CDCl}_{3}\right)$

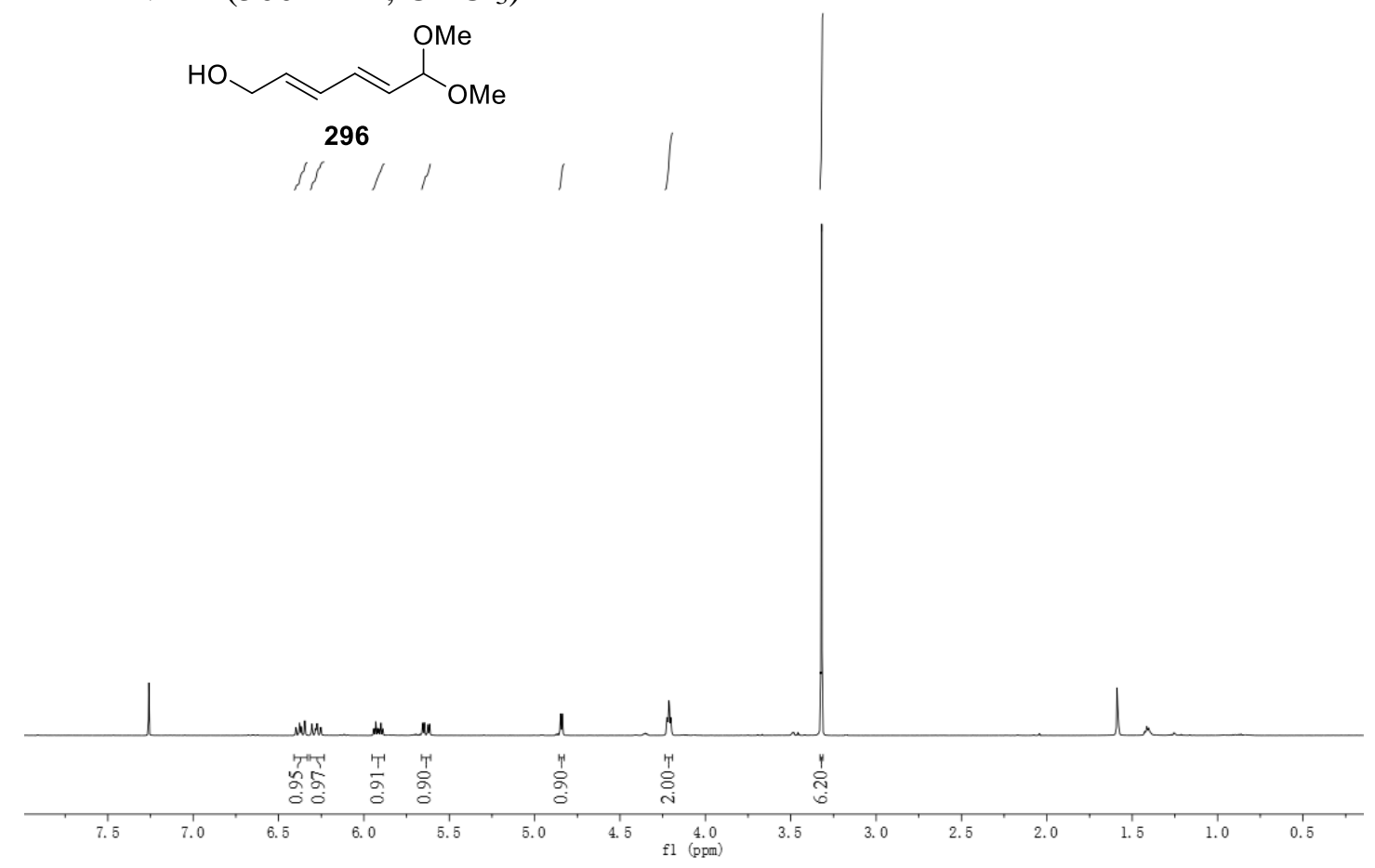

${ }^{13} \mathrm{C} \mathrm{NMR}\left(125 \mathrm{MHz}, \mathrm{CDCl}_{3}\right)$
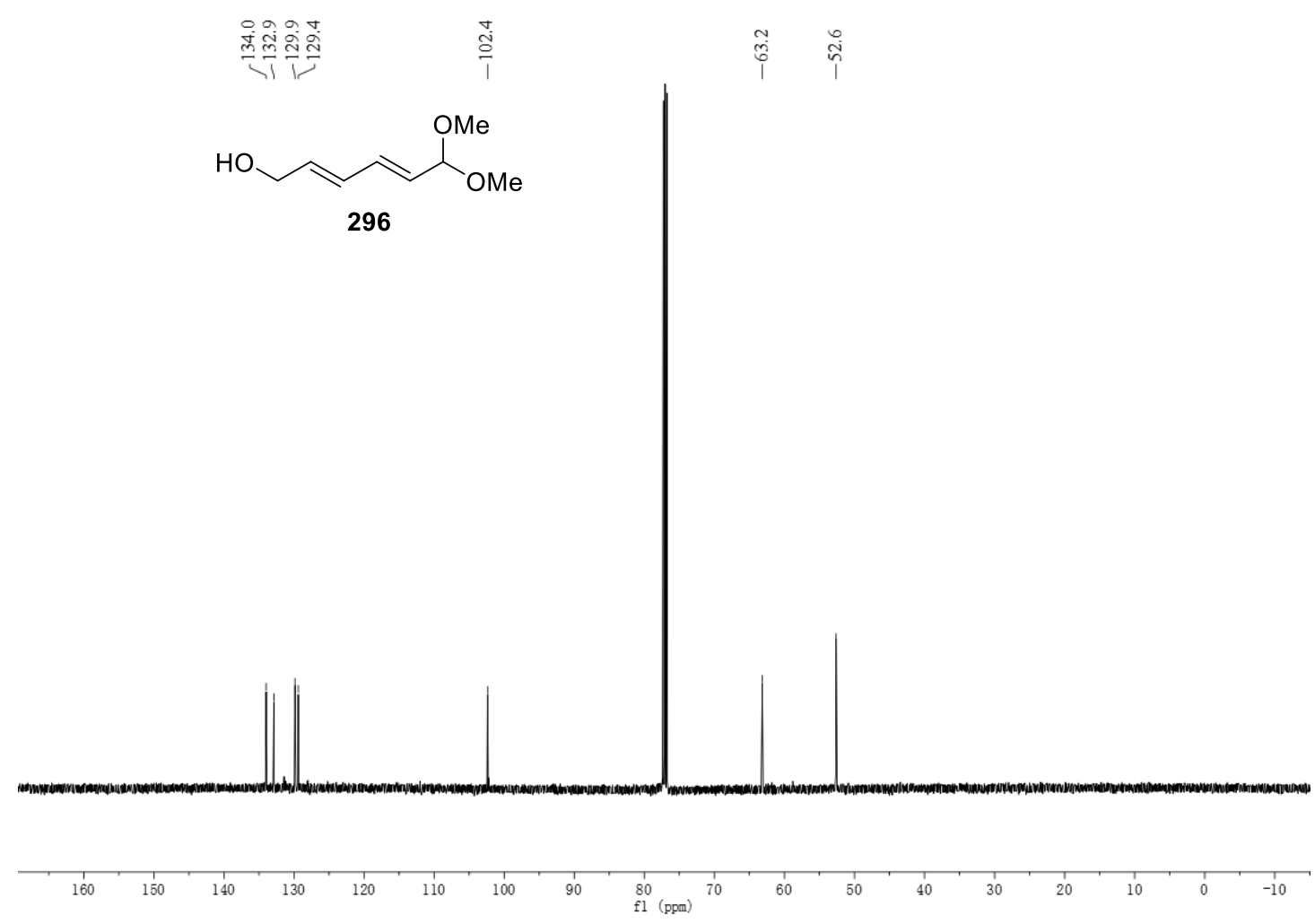


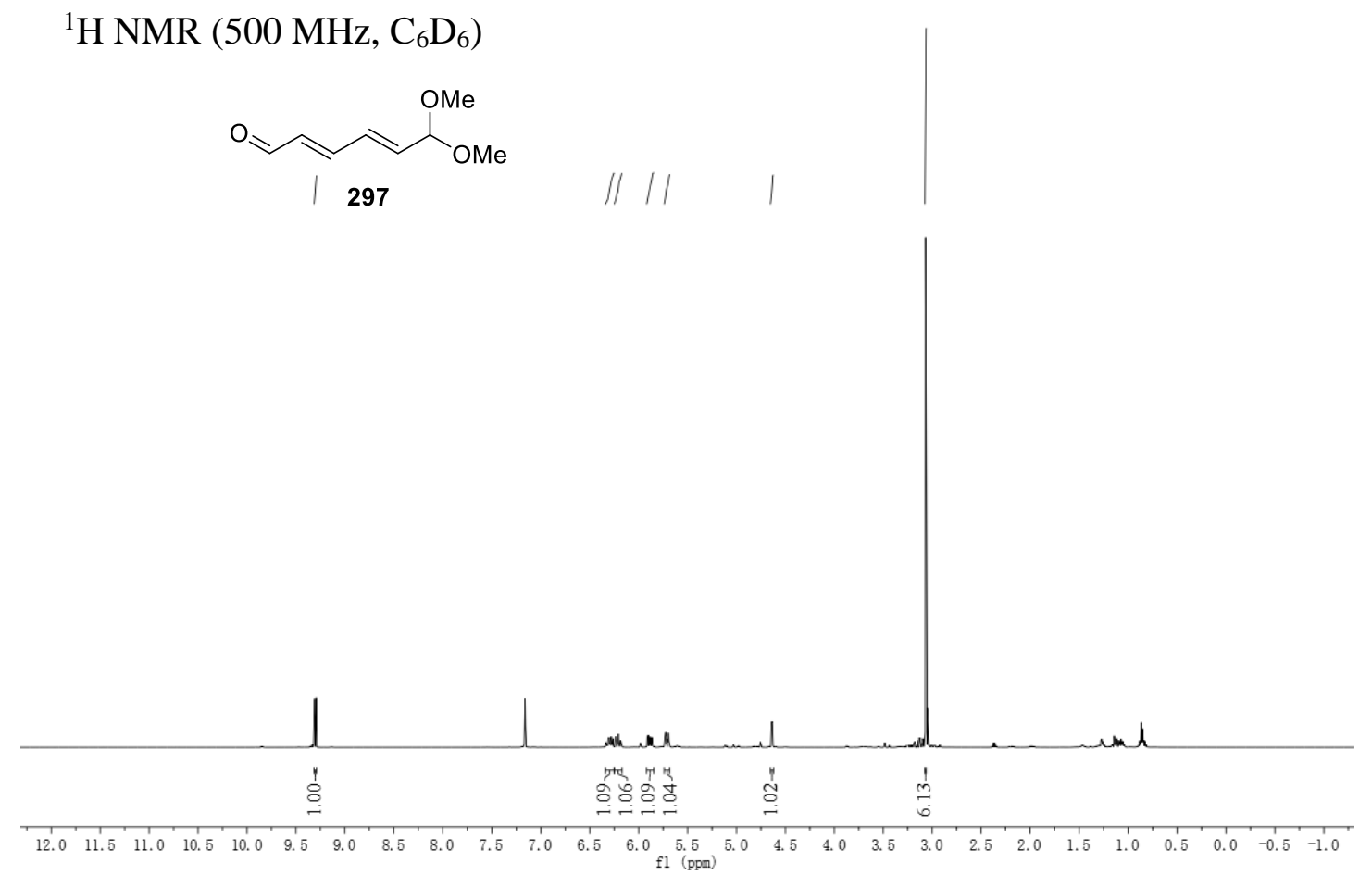

${ }^{13} \mathrm{C}$ NMR (125 MHz, $\left.\mathrm{C}_{6} \mathrm{D}_{6}\right)$
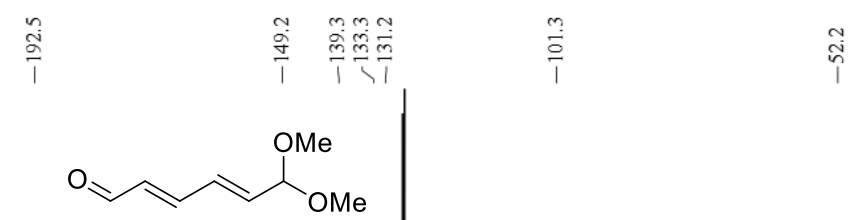

297

$\begin{array}{llllllllllllllll}1 & 1 & 1 & 1 & 1 & 1 & 1 & 1 & 1 & 1 & 1 & 1 & 1 & 1 & 1 & 1 \\ 220 & 210 & 200 & 190 & 180 & 170 & 160 & 150 & 140 & 130 & 120 & 110 & 100 & 90\end{array}$ 
${ }^{1} \mathrm{H}$ NMR $\left(500 \mathrm{MHz}, \mathrm{CDCl}_{3}\right)$
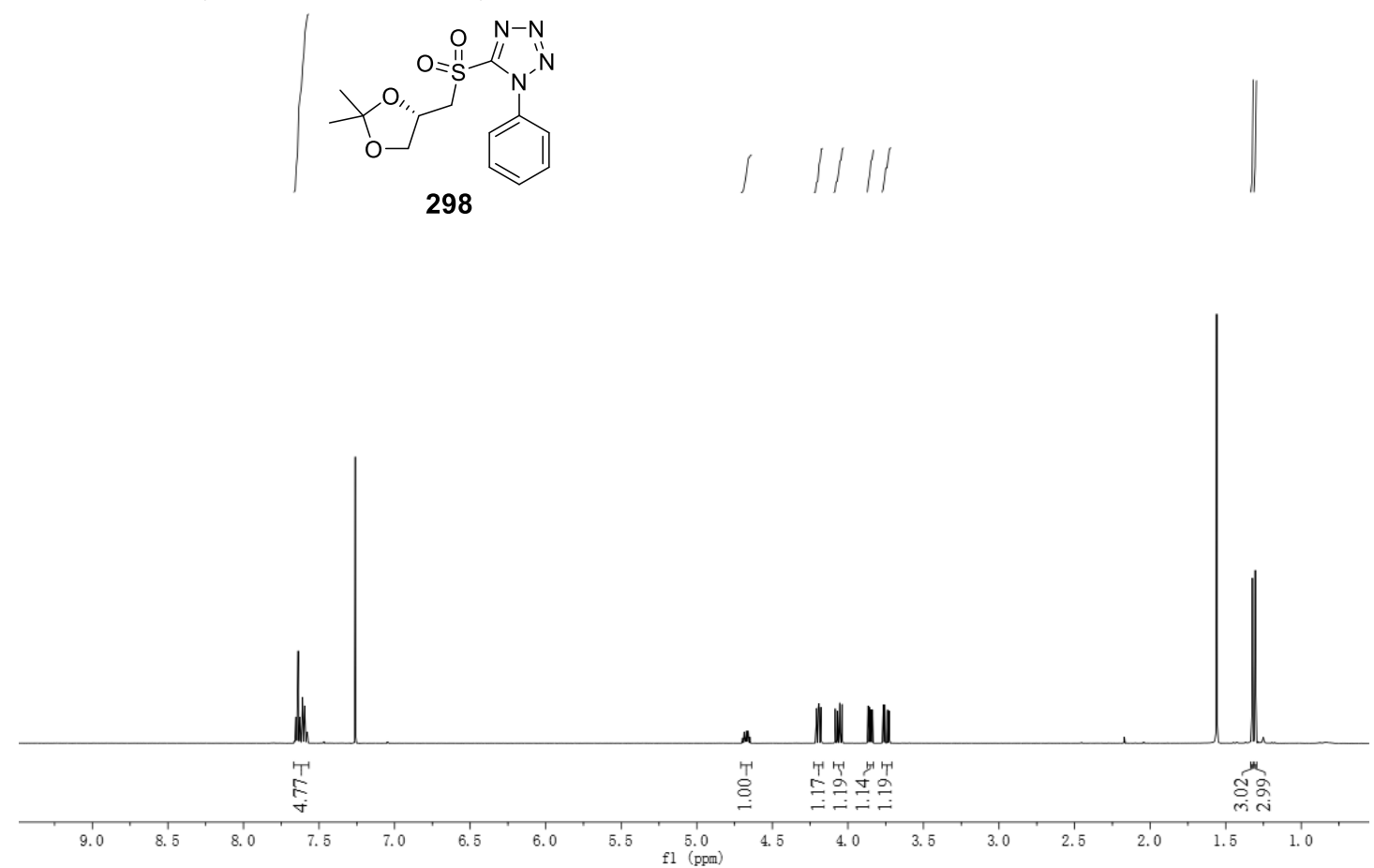

${ }^{13} \mathrm{C}$ NMR (125 MHz, $\left.\mathrm{CDCl}_{3}\right)$

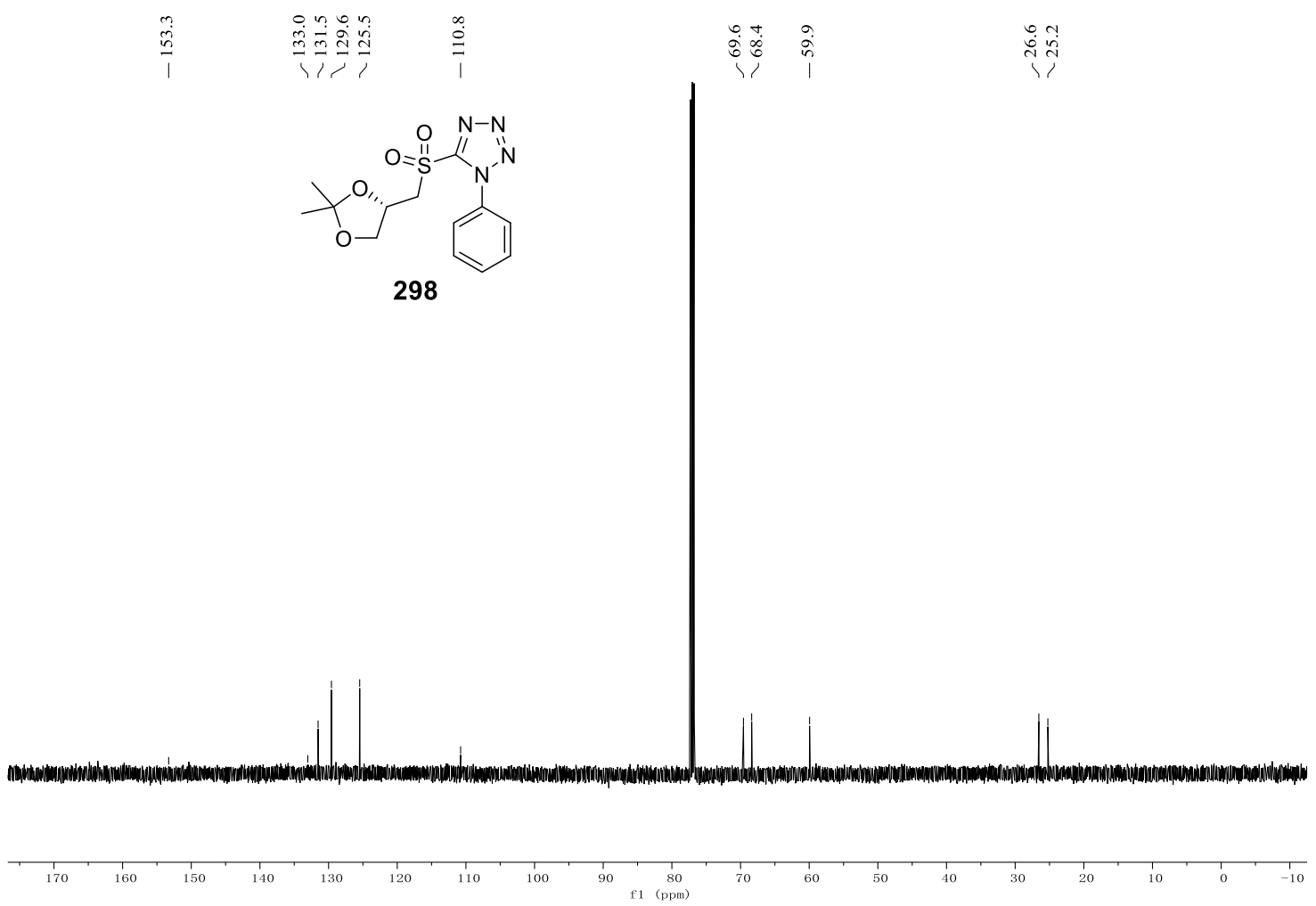


${ }^{1} \mathrm{H} \mathrm{NMR}\left(500 \mathrm{MHz}, \mathrm{CDCl}_{3}\right)$

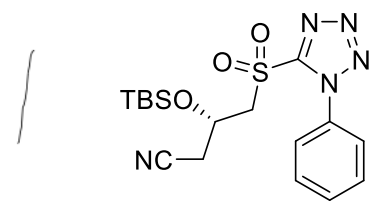

299

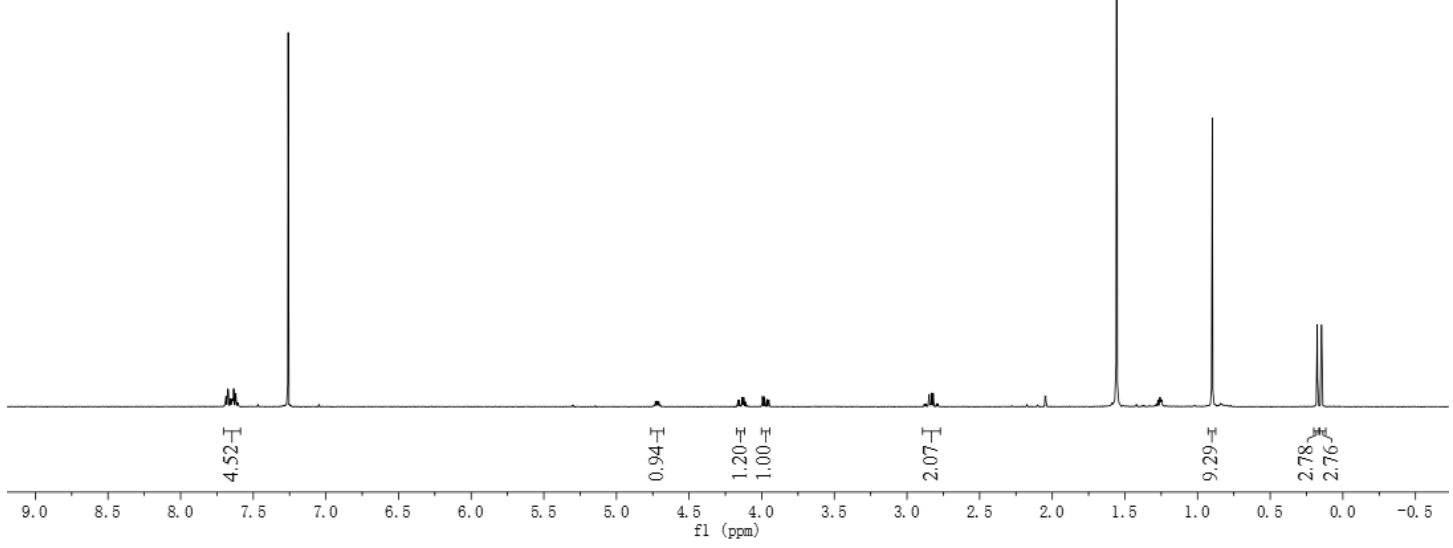

${ }^{13} \mathrm{C}$ NMR $\left(125 \mathrm{MHz}, \mathrm{CDCl}_{3}\right)$

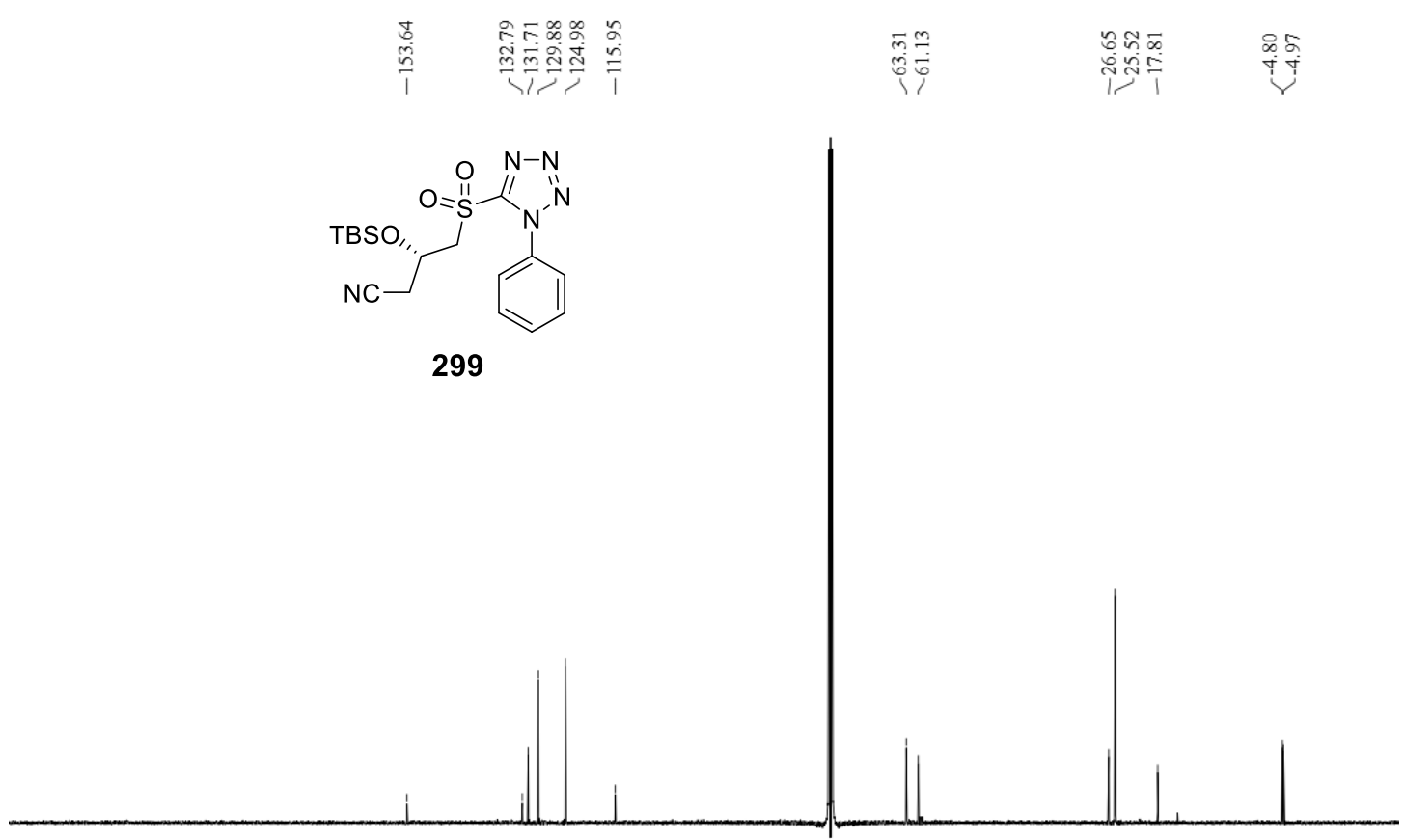

$\begin{array}{lllllllllllllllllllllllll}220 & 210 & 200 & 190 & 180 & 170 & 160 & 150 & 140 & 130 & 120 & 110 & 100 & 90 & 80 & 70 & 60 & 50 & 40 & 30 & 20 & 10 & 0 & -10 & -20\end{array}$ 
${ }^{1} \mathrm{H}$ NMR (500 MHz, $\left.\mathrm{CDCl}_{3}\right)$

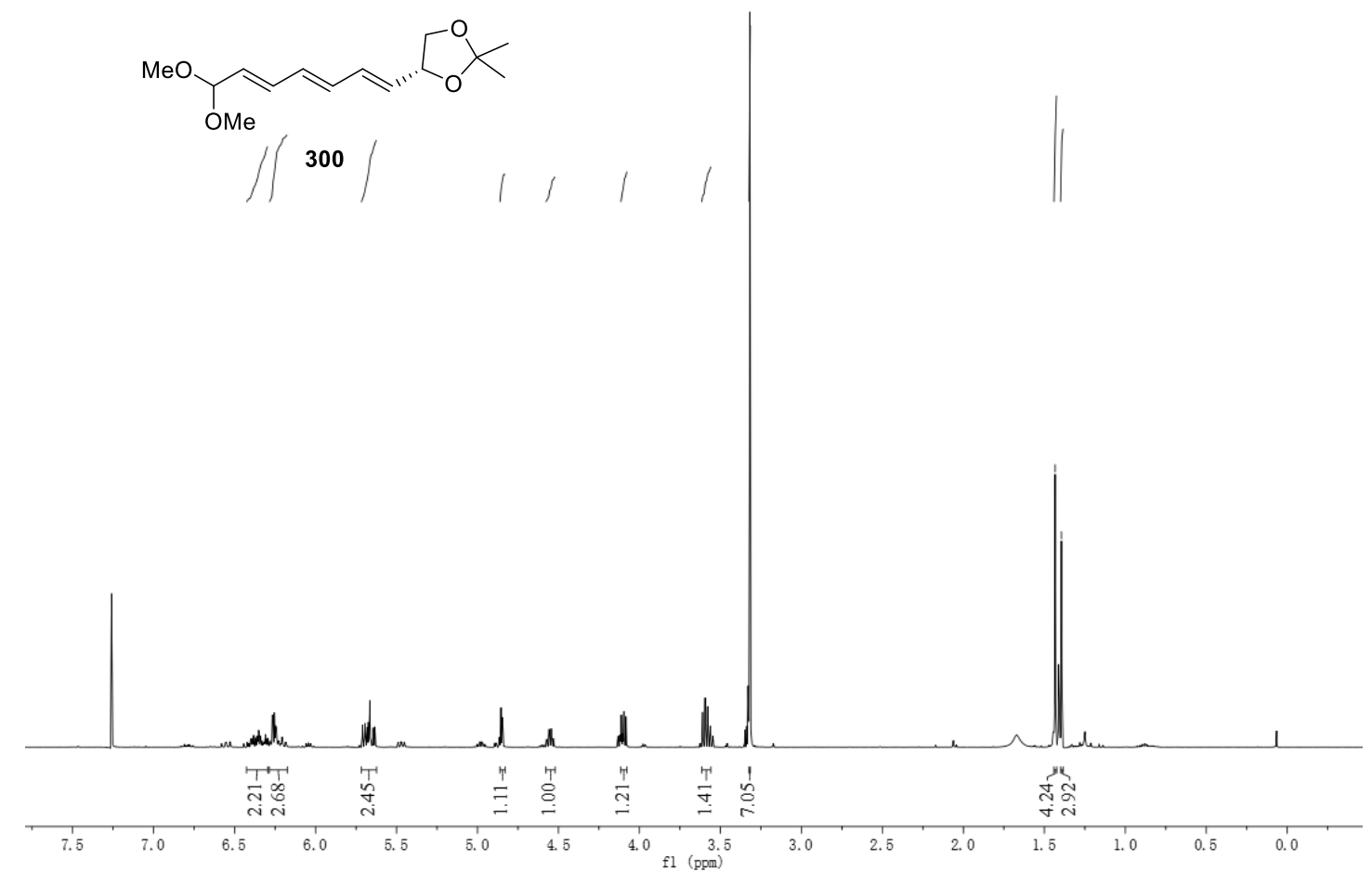

${ }^{13} \mathrm{C}$ NMR (125 MHz, $\left.\mathrm{CDCl}_{3}\right)$

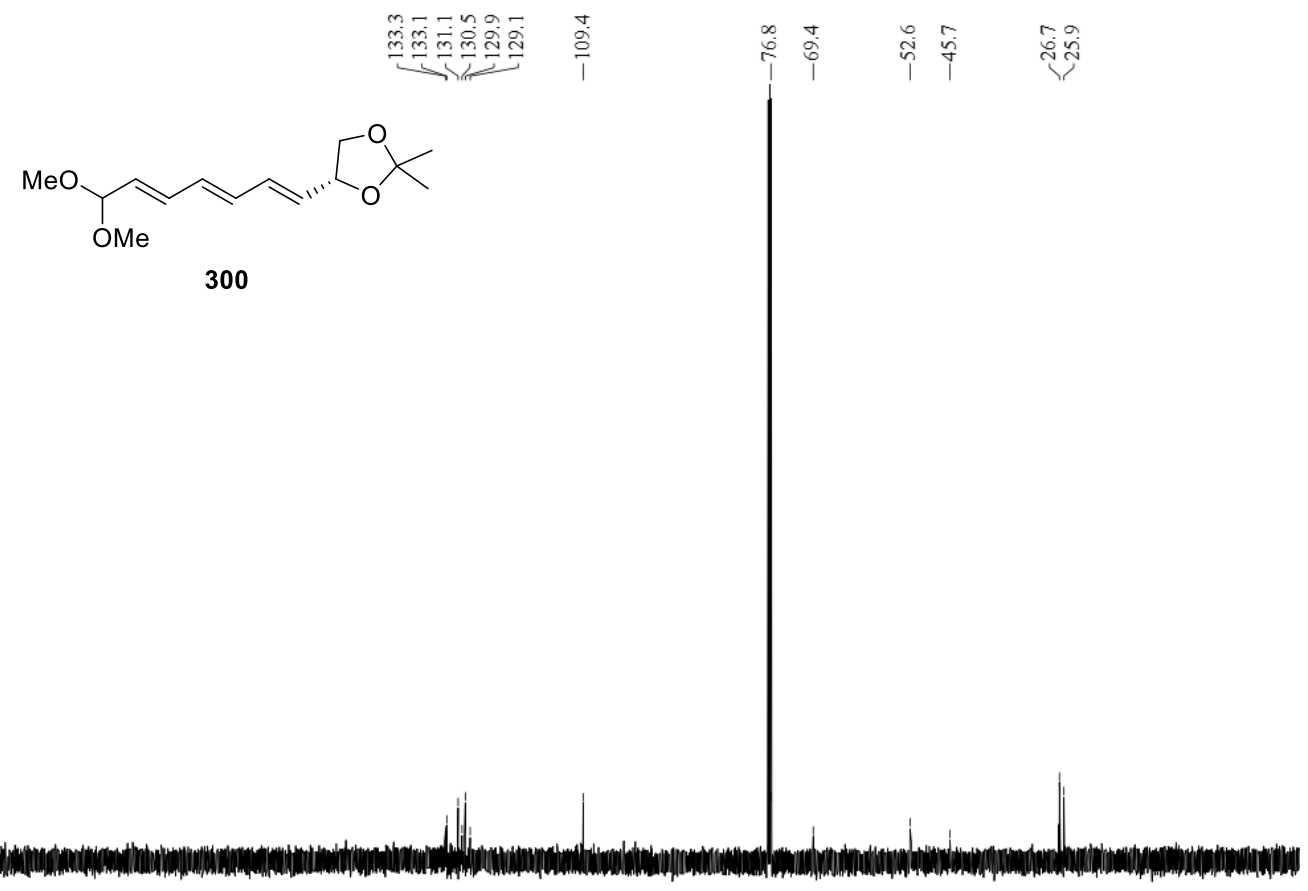

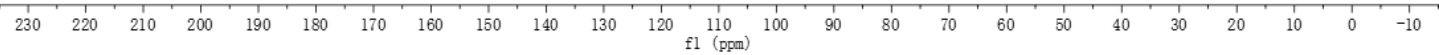




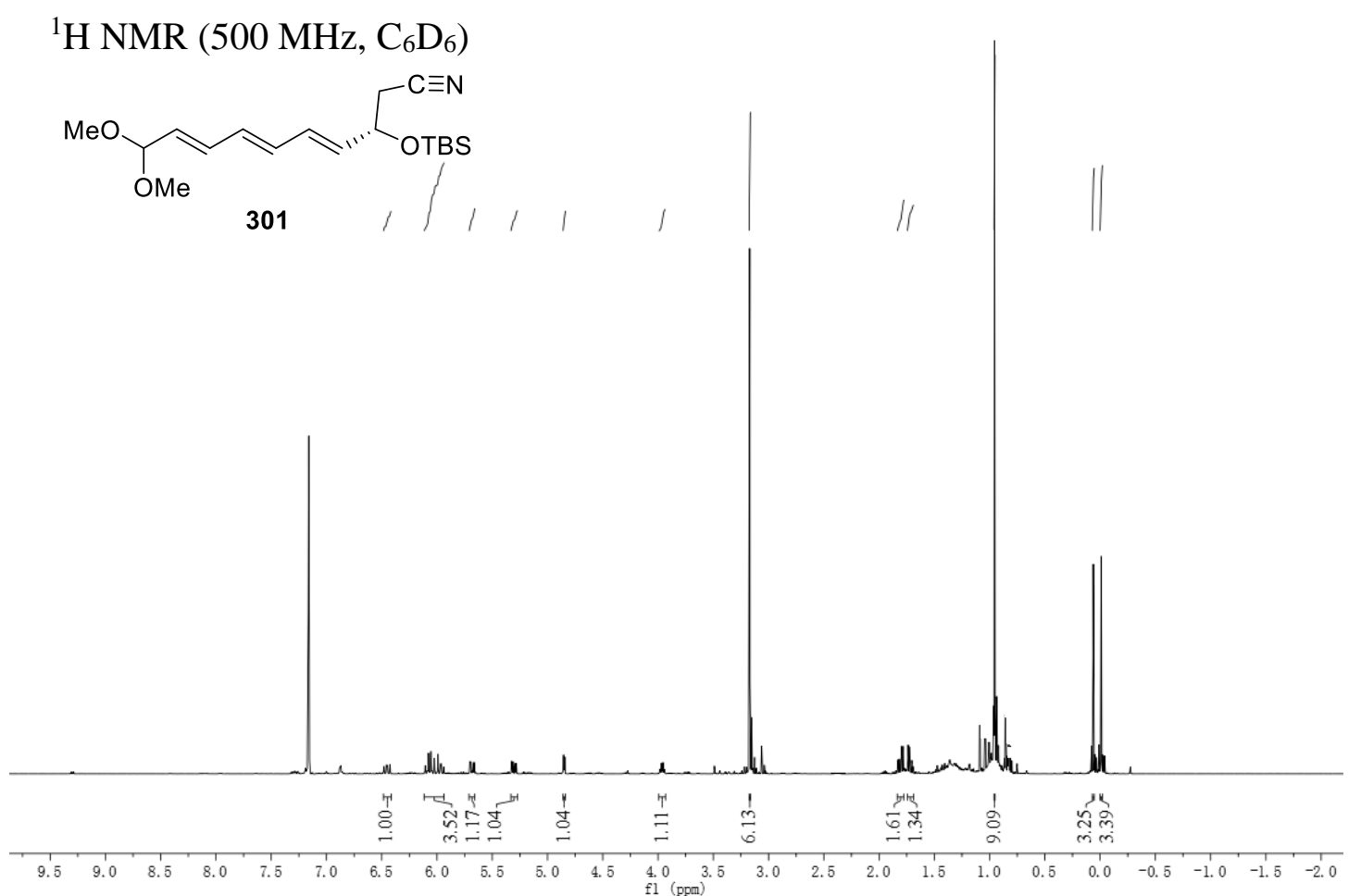

${ }^{13} \mathrm{C}$ NMR (125 MHz, C6 $\left.\mathrm{D}_{6}\right)$

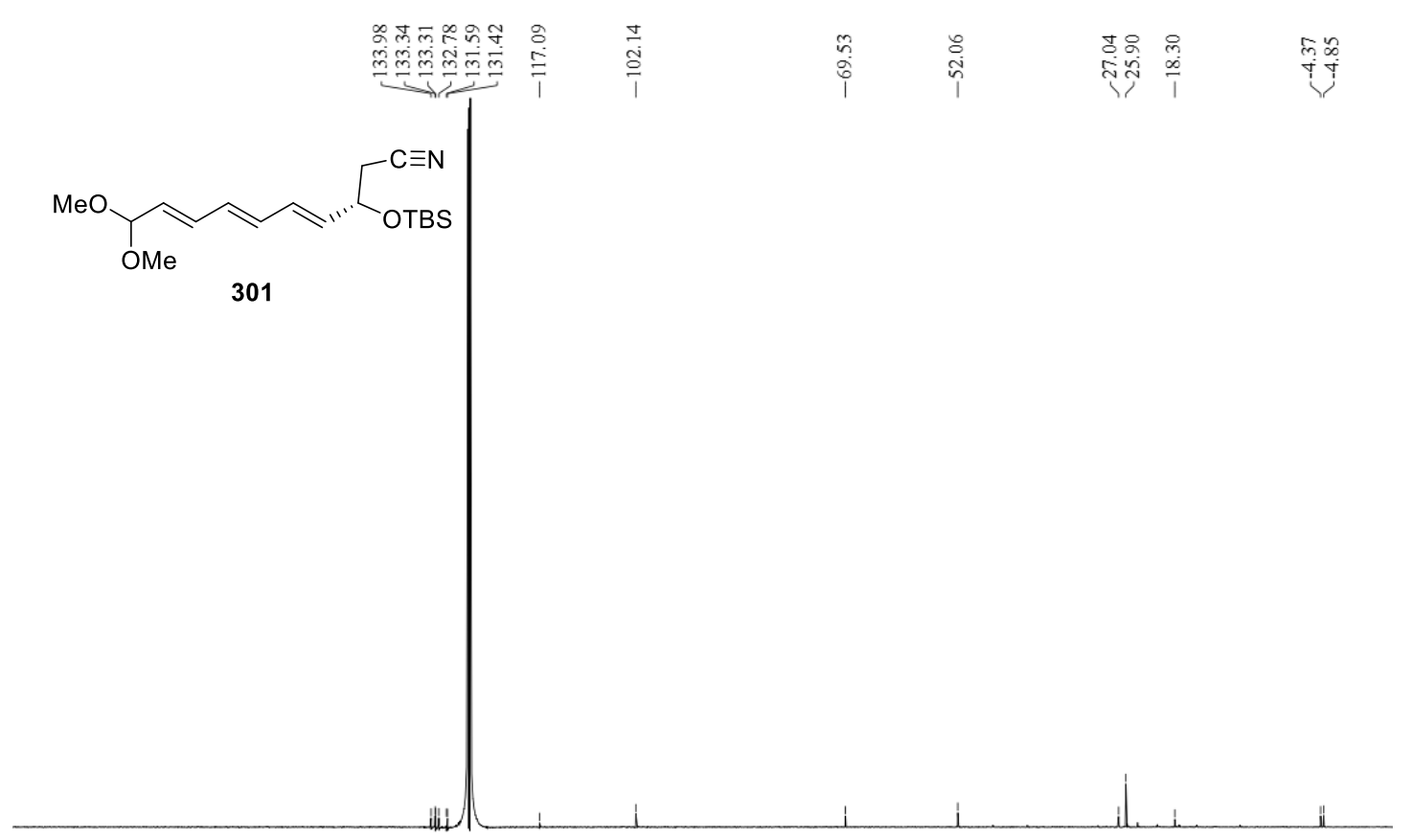


${ }^{1} \mathrm{H}$ NMR $\left(600 \mathrm{MHz}, \mathrm{CDCl}_{3}\right)$

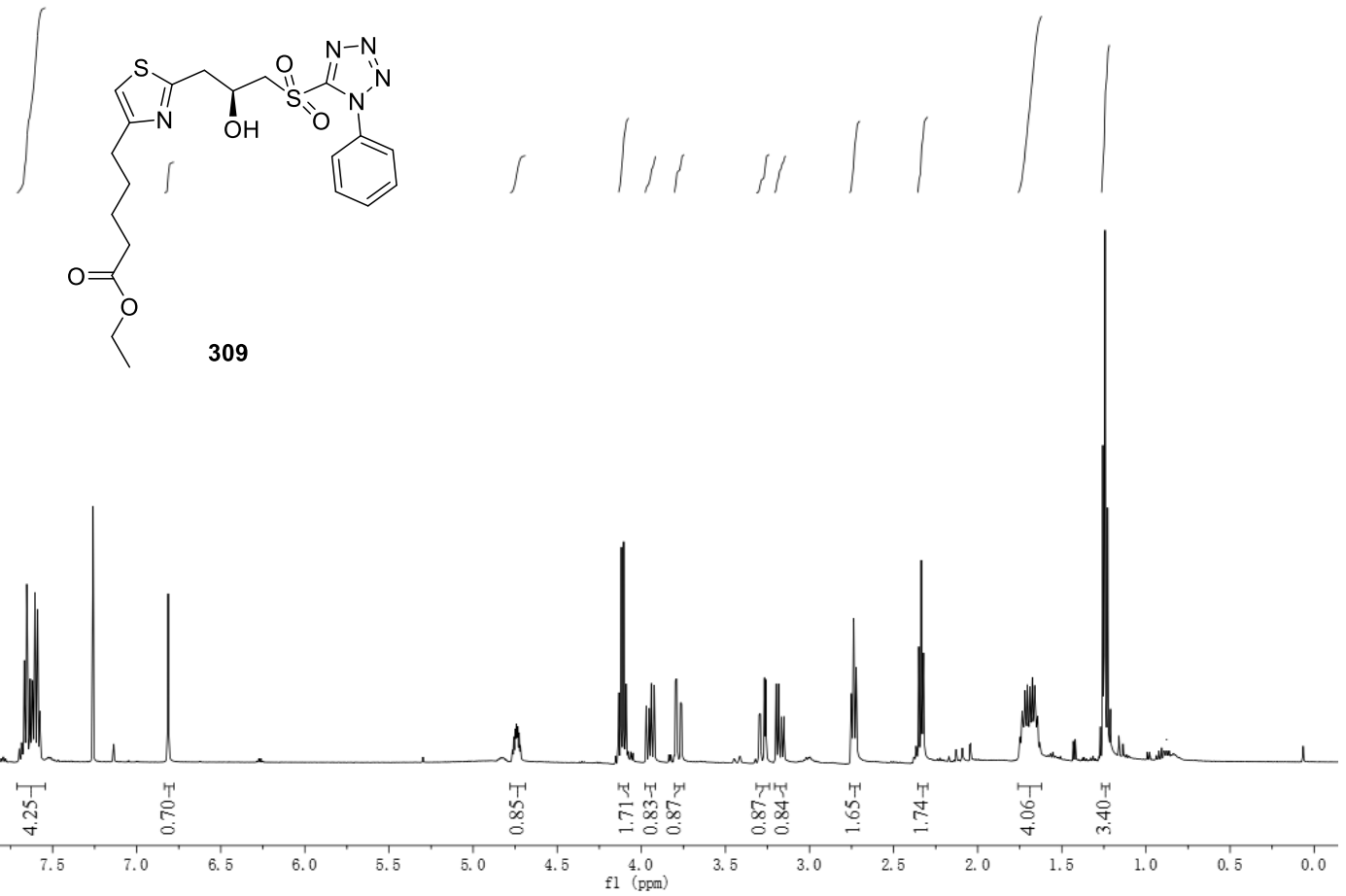

${ }^{13} \mathrm{C}$ NMR (150 MHz, $\left.\mathrm{CDCl}_{3}\right)$

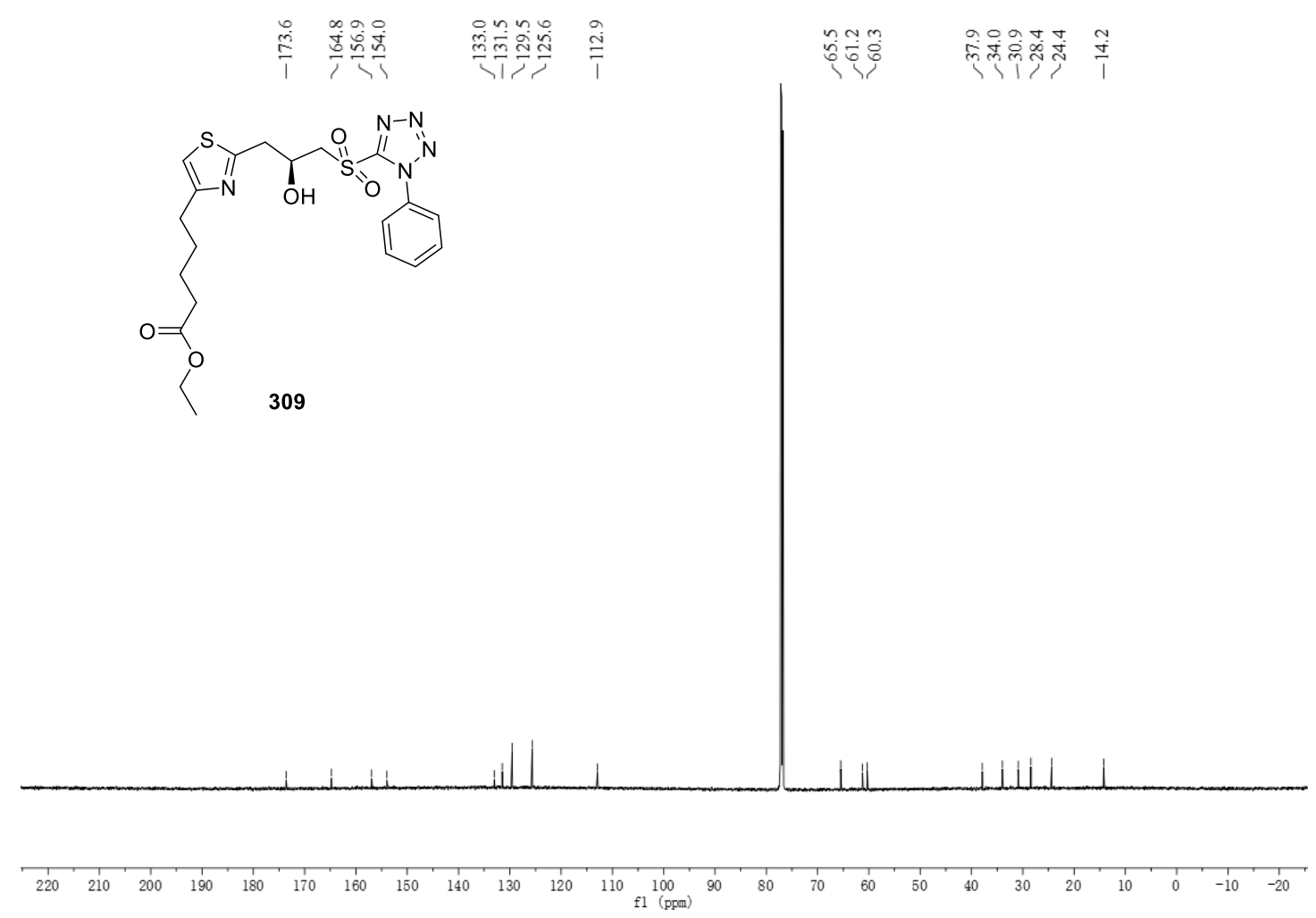




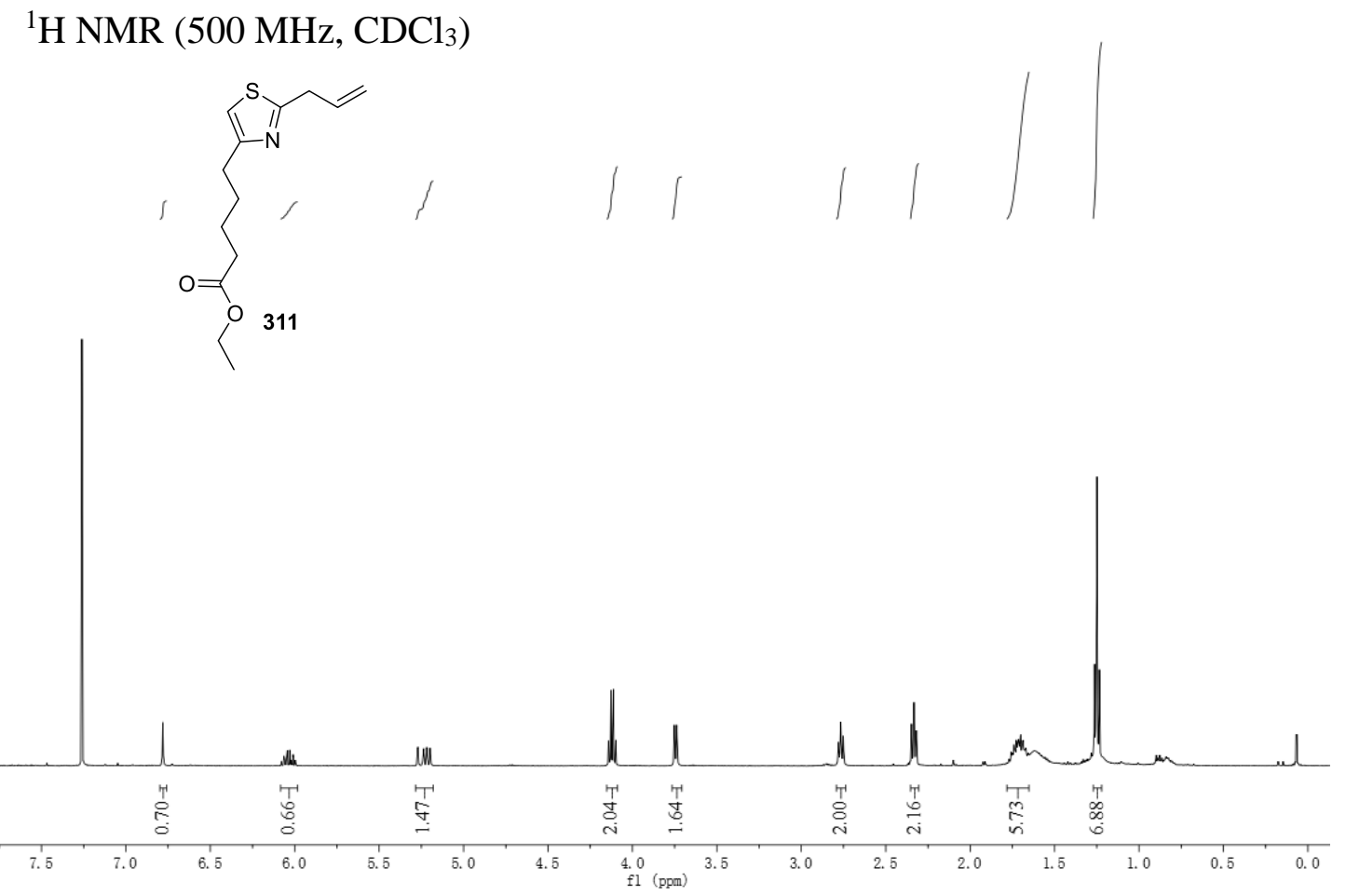

${ }^{13} \mathrm{C} \mathrm{NMR}\left(125 \mathrm{MHz}, \mathrm{CDCl}_{3}\right)$

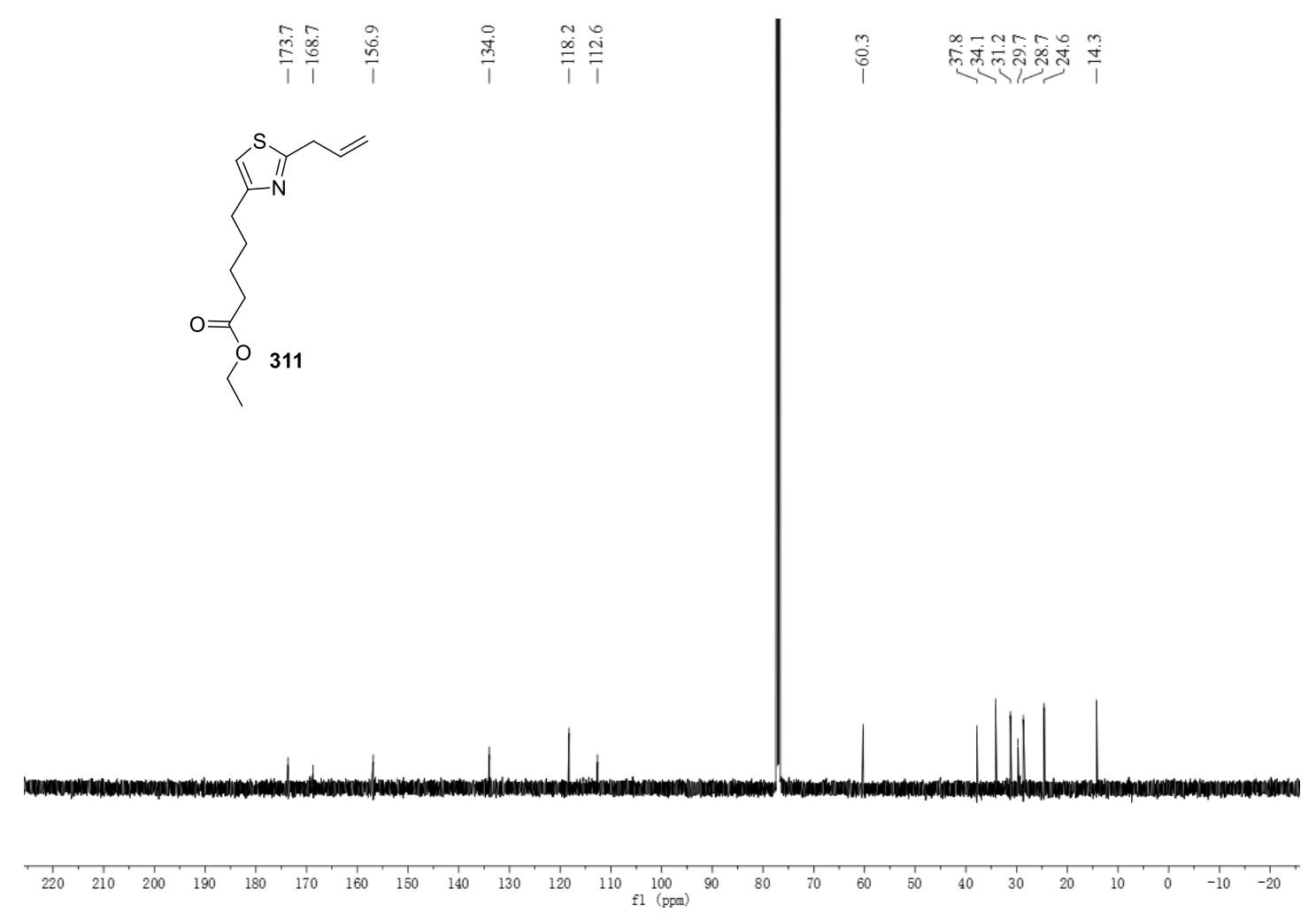


${ }^{1} \mathrm{H}$ NMR (500 MHz, $\left.\mathrm{CDCl}_{3}\right)$
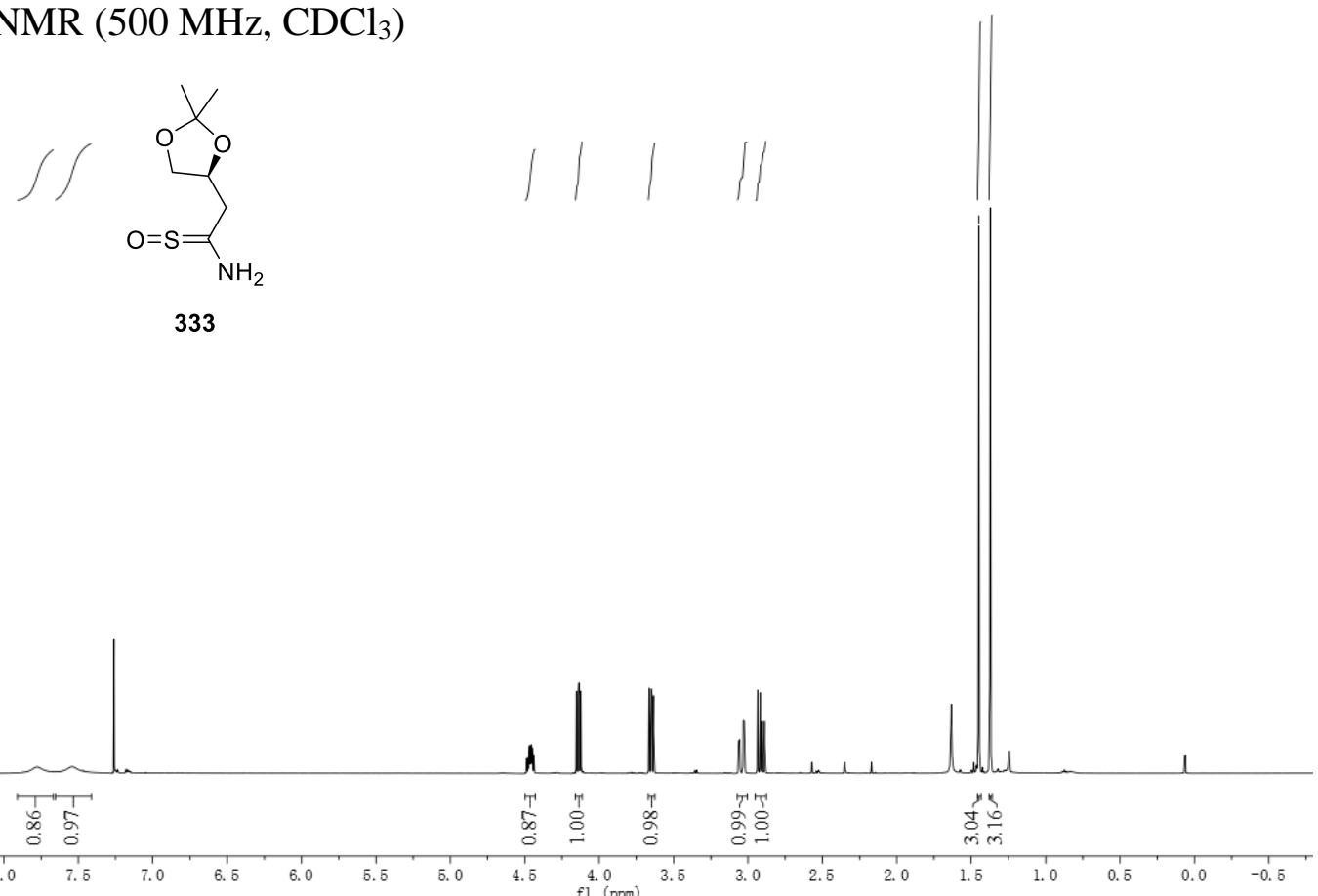

${ }^{13} \mathrm{C}$ NMR $\left(125 \mathrm{MHz}, \mathrm{CDCl}_{3}\right)$

iั
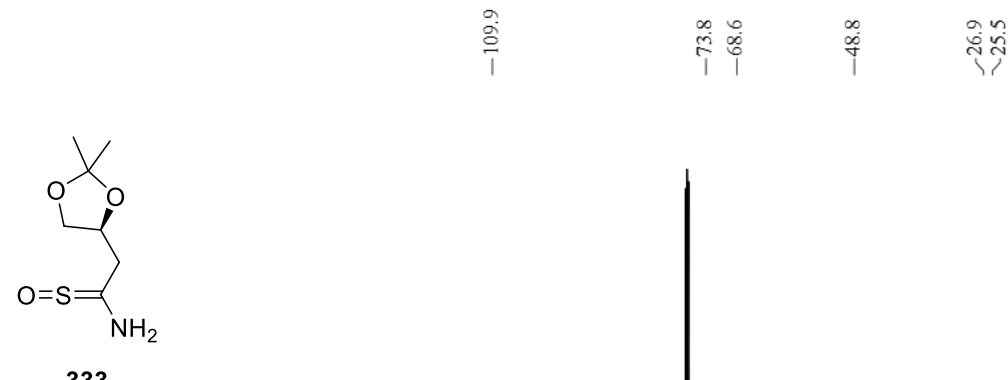

333

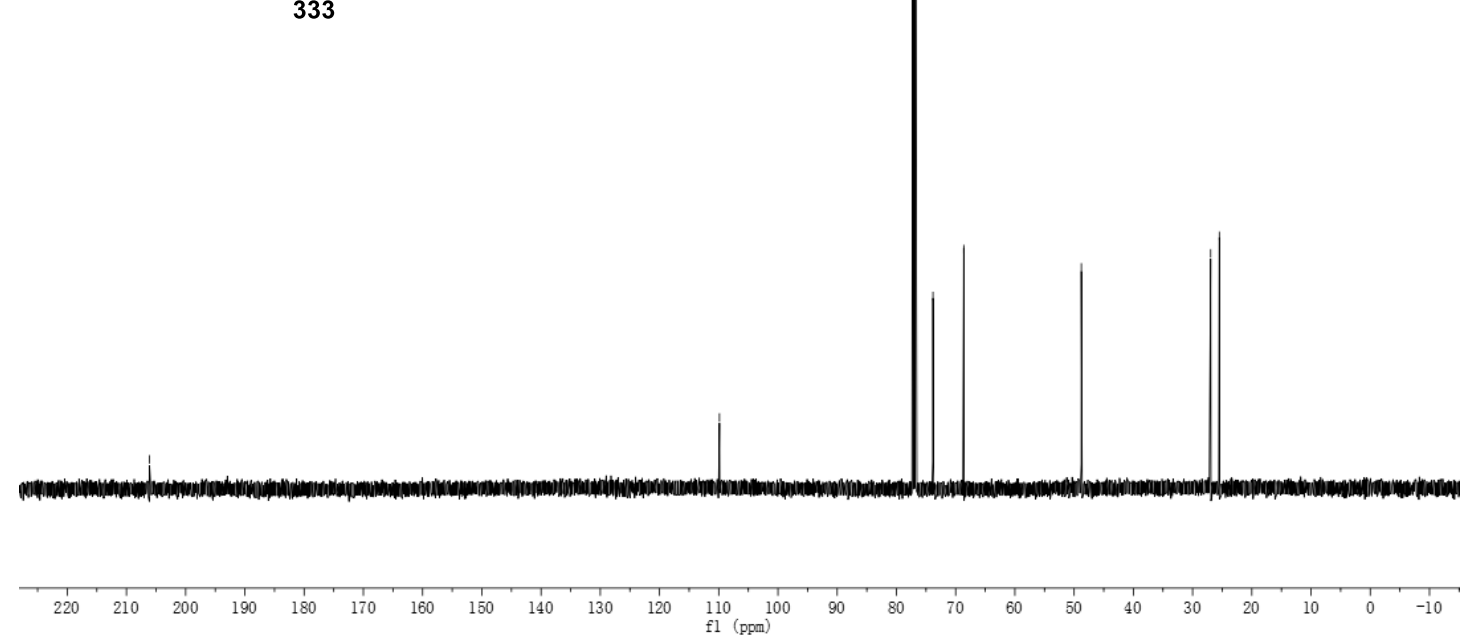


${ }^{1} \mathrm{H}$ NMR $\left(500 \mathrm{MHz}, \mathrm{CDCl}_{3}\right)$

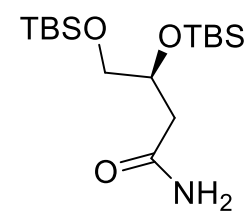

340

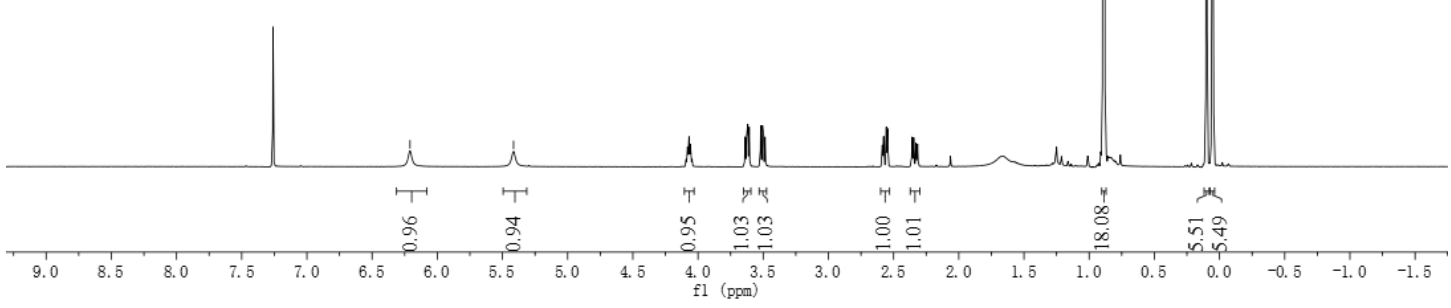

${ }^{13} \mathrm{C} \mathrm{NMR}\left(125 \mathrm{MHz}, \mathrm{CDCl}_{3}\right)$

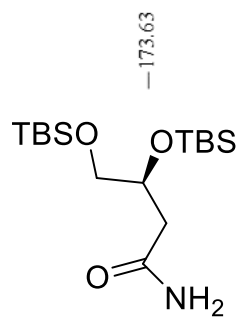

340

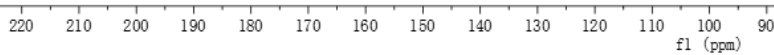


${ }^{1} \mathrm{H}$ NMR $\left(500 \mathrm{MHz}, \mathrm{CDCl}_{3}\right)$

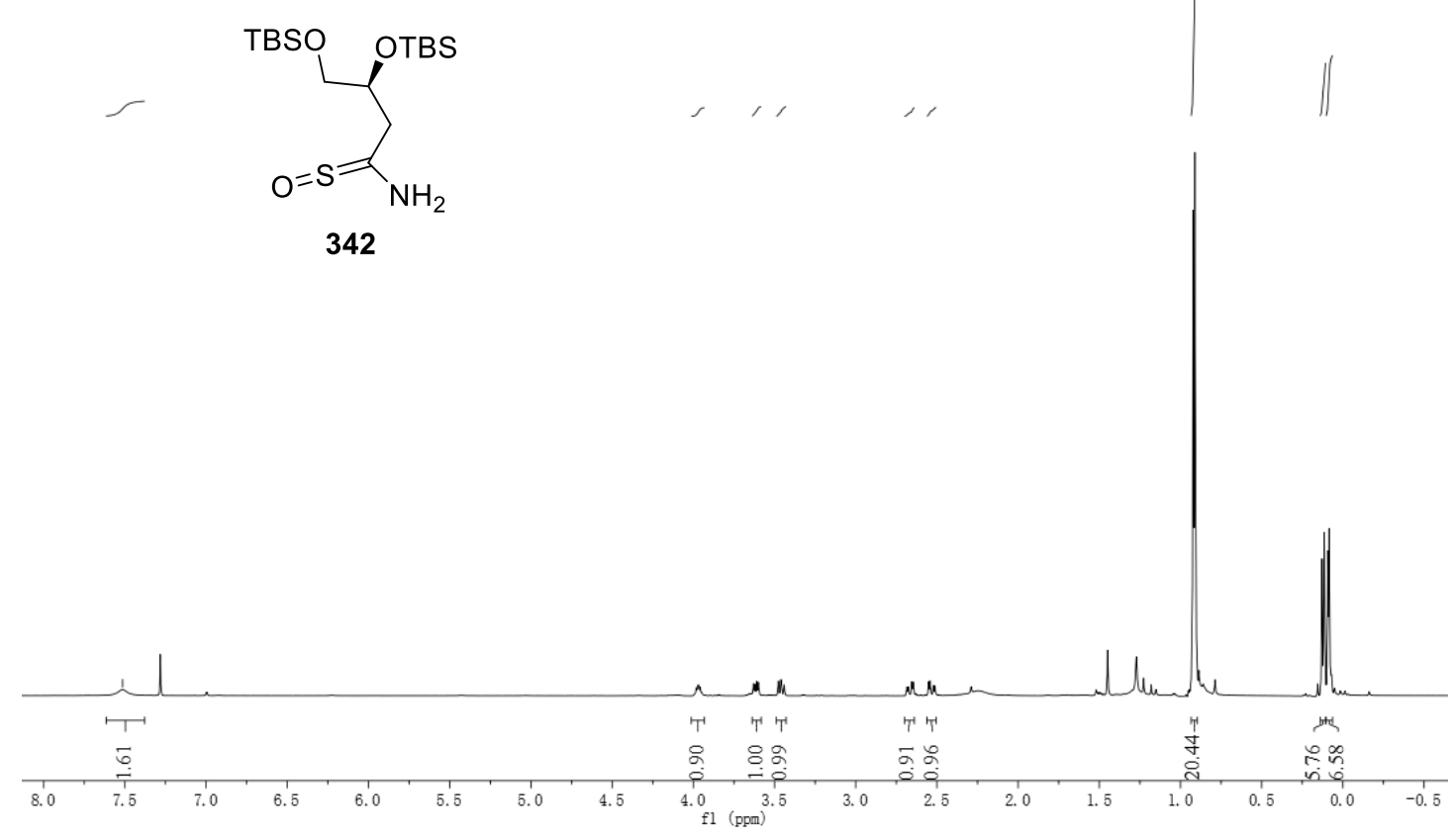

${ }^{13} \mathrm{C} \mathrm{NMR}\left(125 \mathrm{MHz}, \mathrm{CDCl}_{3}\right)$

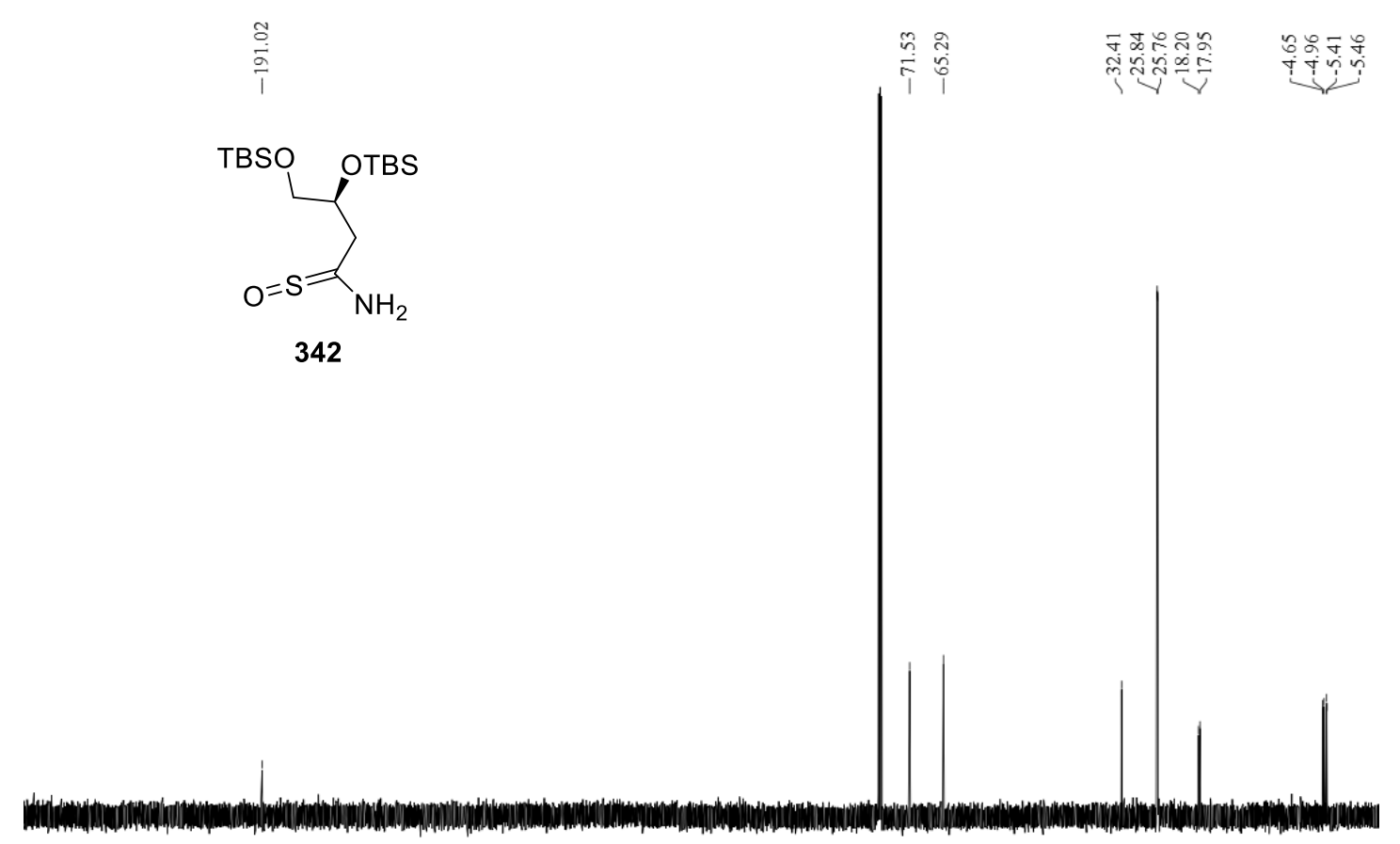

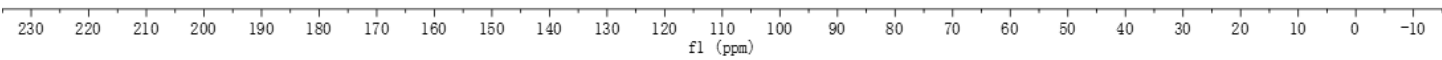




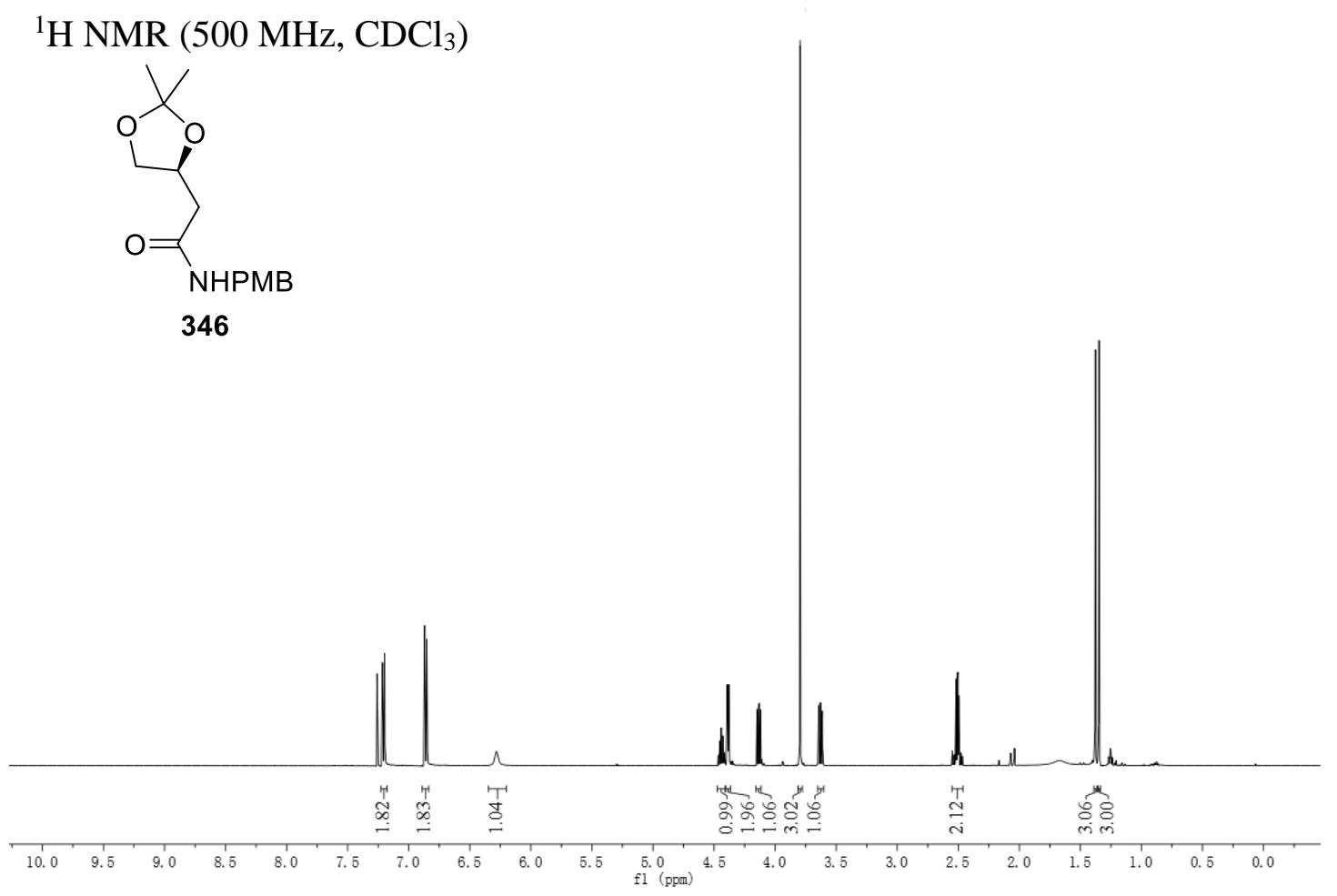

${ }^{13} \mathrm{C}$ NMR (125 MHz, $\left.\mathrm{CDCl}_{3}\right)$

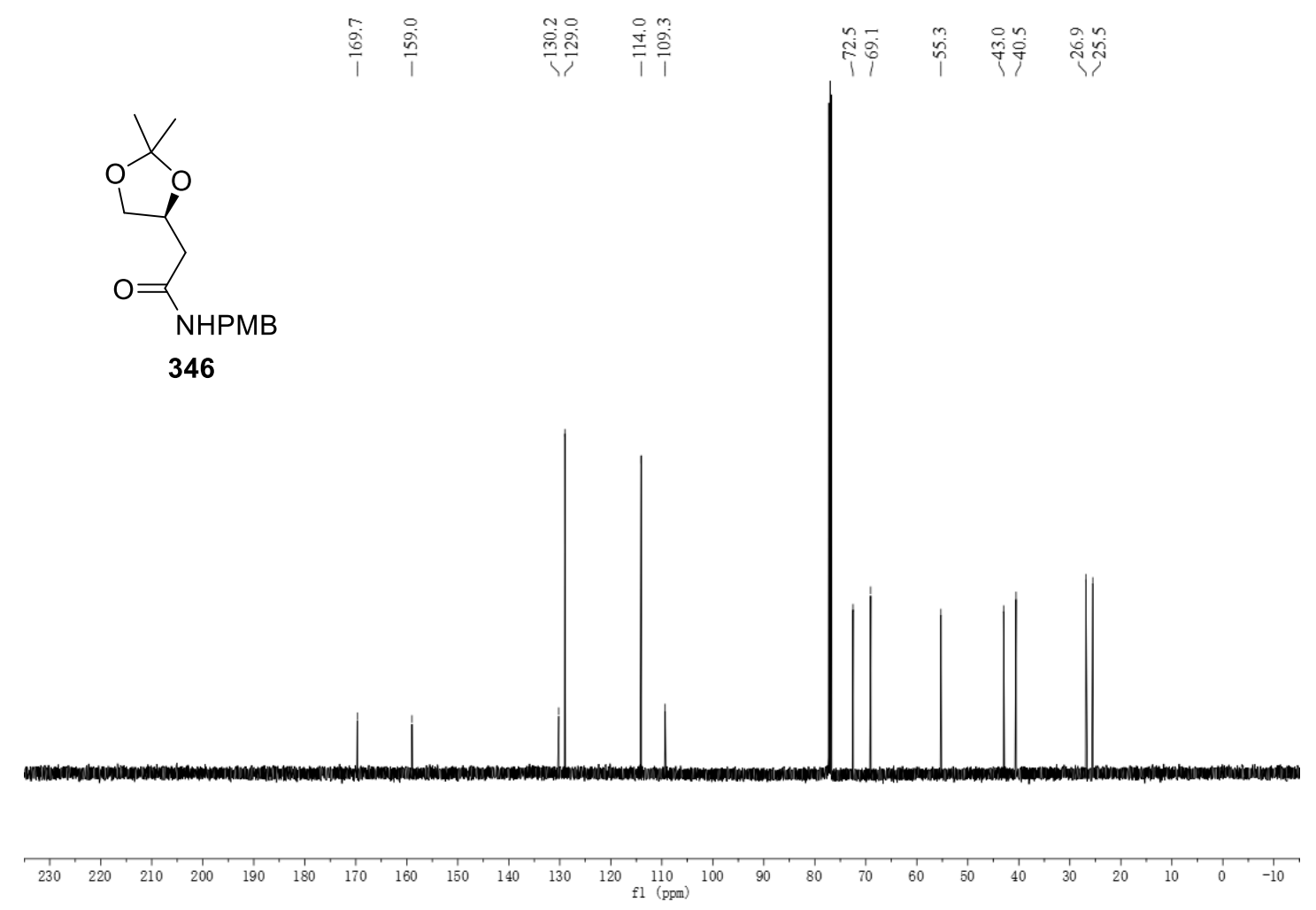


${ }^{1} \mathrm{H} \mathrm{NMR}\left(500 \mathrm{MHz}, \mathrm{CDCl}_{3}\right)$
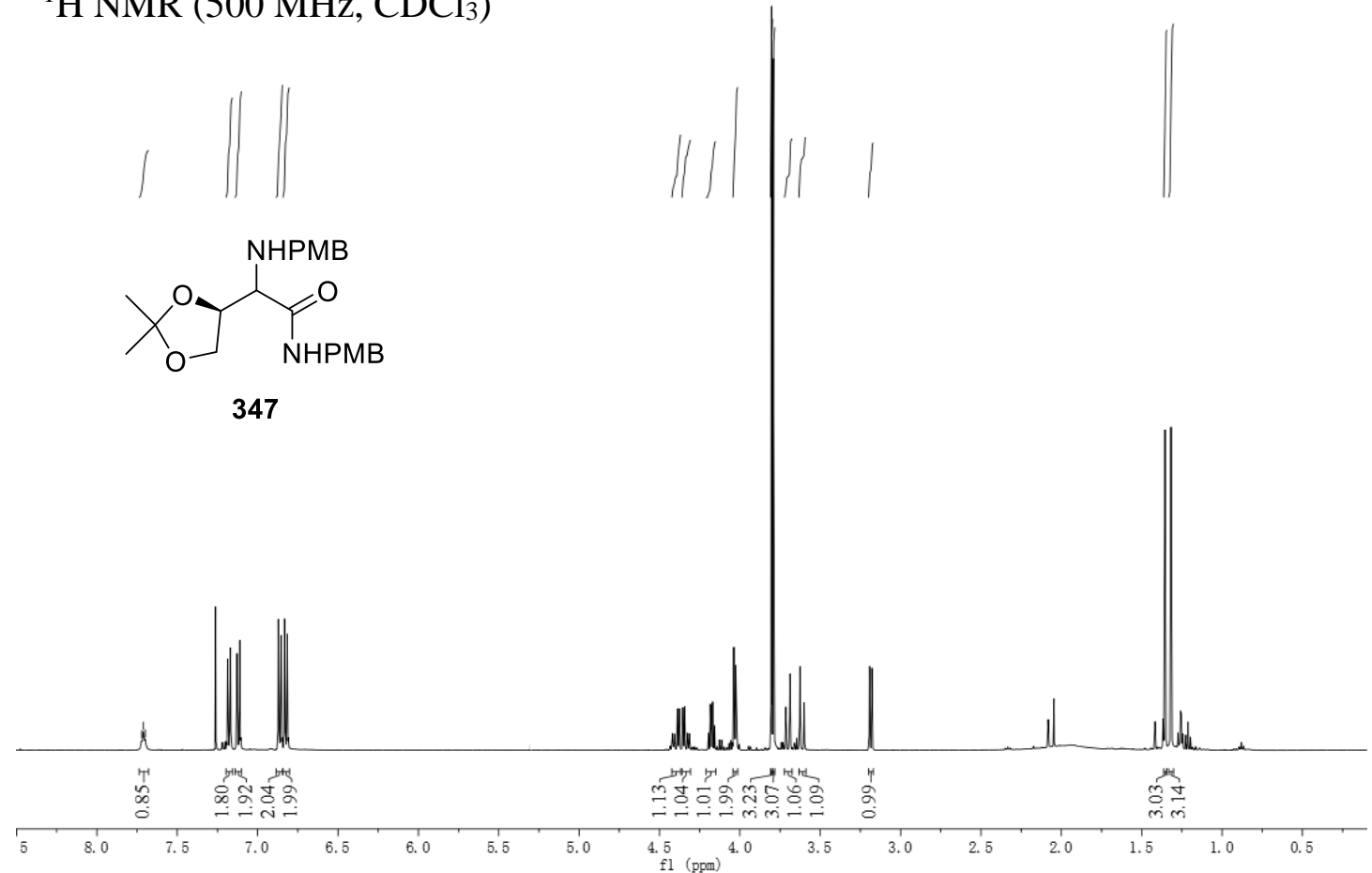

${ }^{13} \mathrm{C}$ NMR (125 MHz, $\left.\mathrm{CDCl}_{3}\right)$
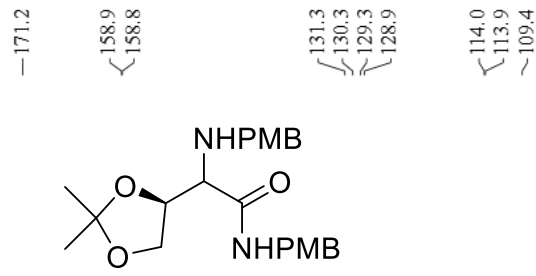

347

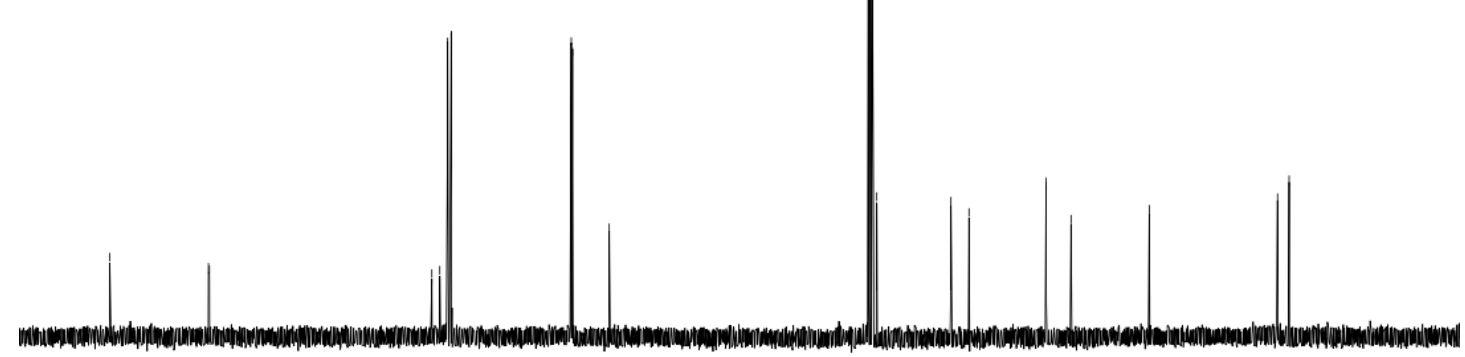

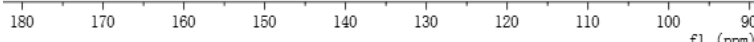




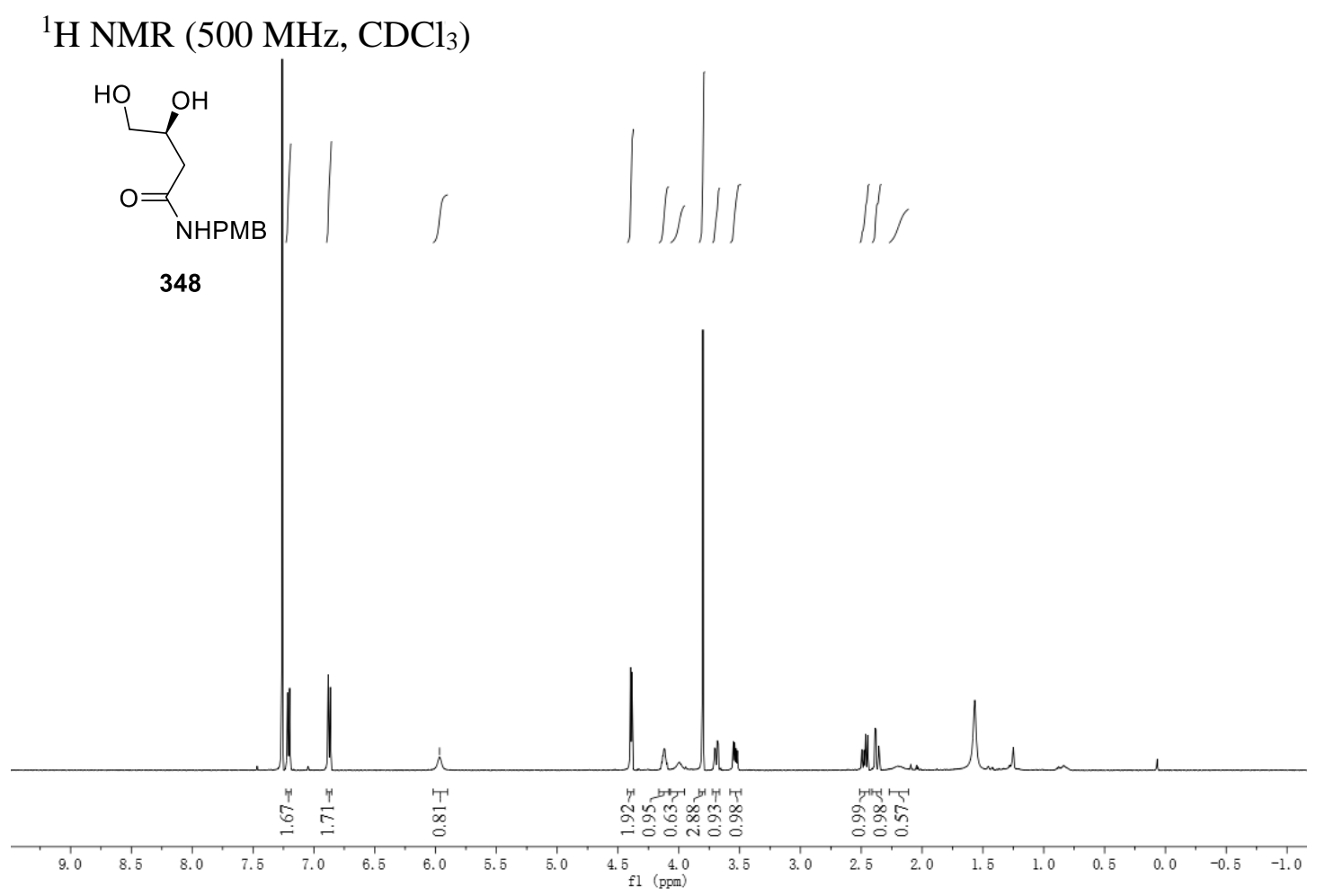

${ }^{13} \mathrm{C} \mathrm{NMR}\left(125 \mathrm{MHz}, \mathrm{CDCl}_{3}\right)$
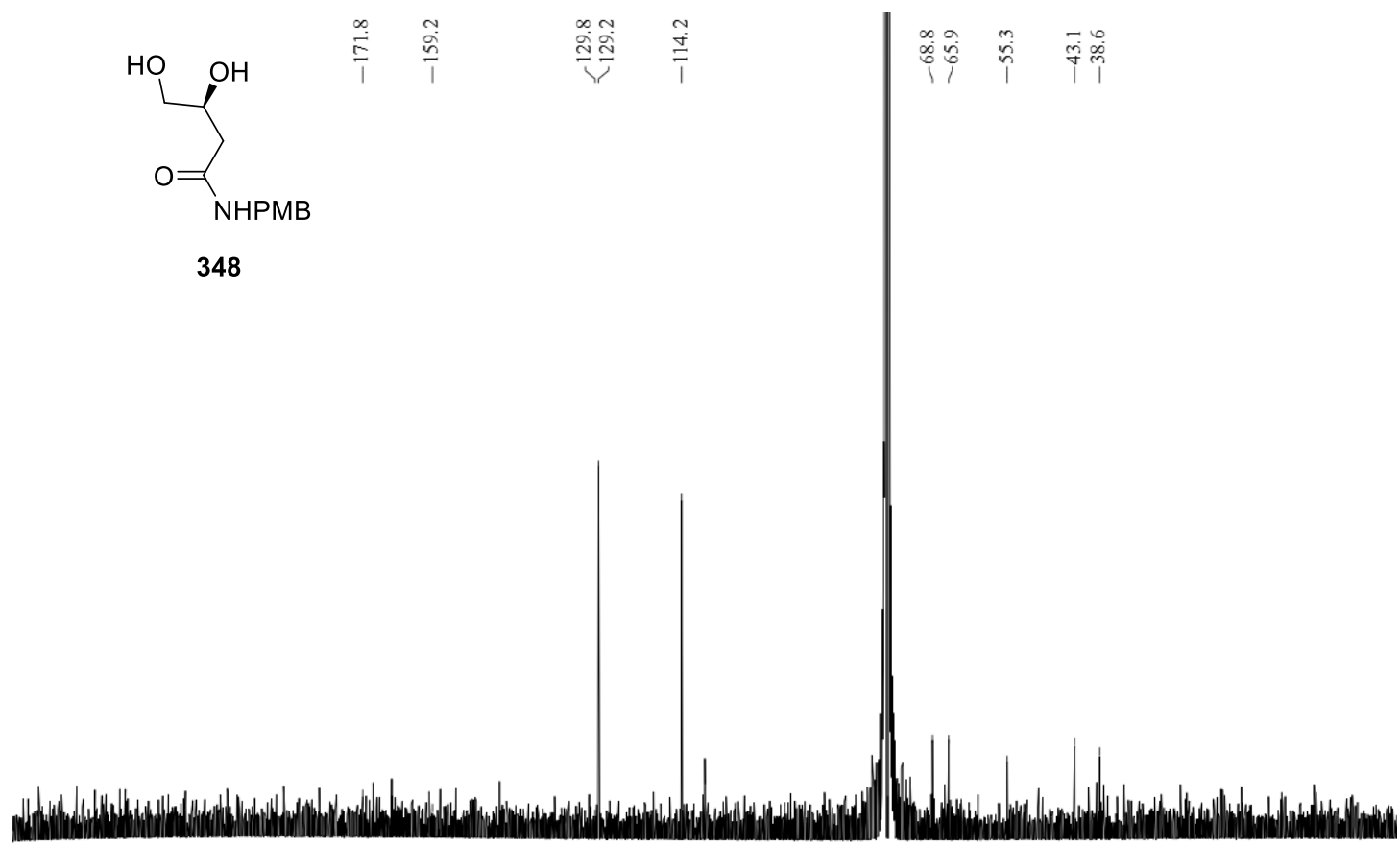

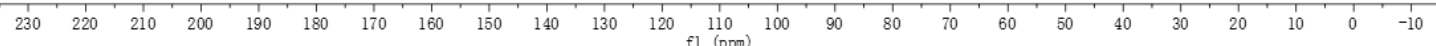


${ }^{1} \mathrm{H} \mathrm{NMR}\left(500 \mathrm{MHz}, \mathrm{CDCl}_{3}\right)$
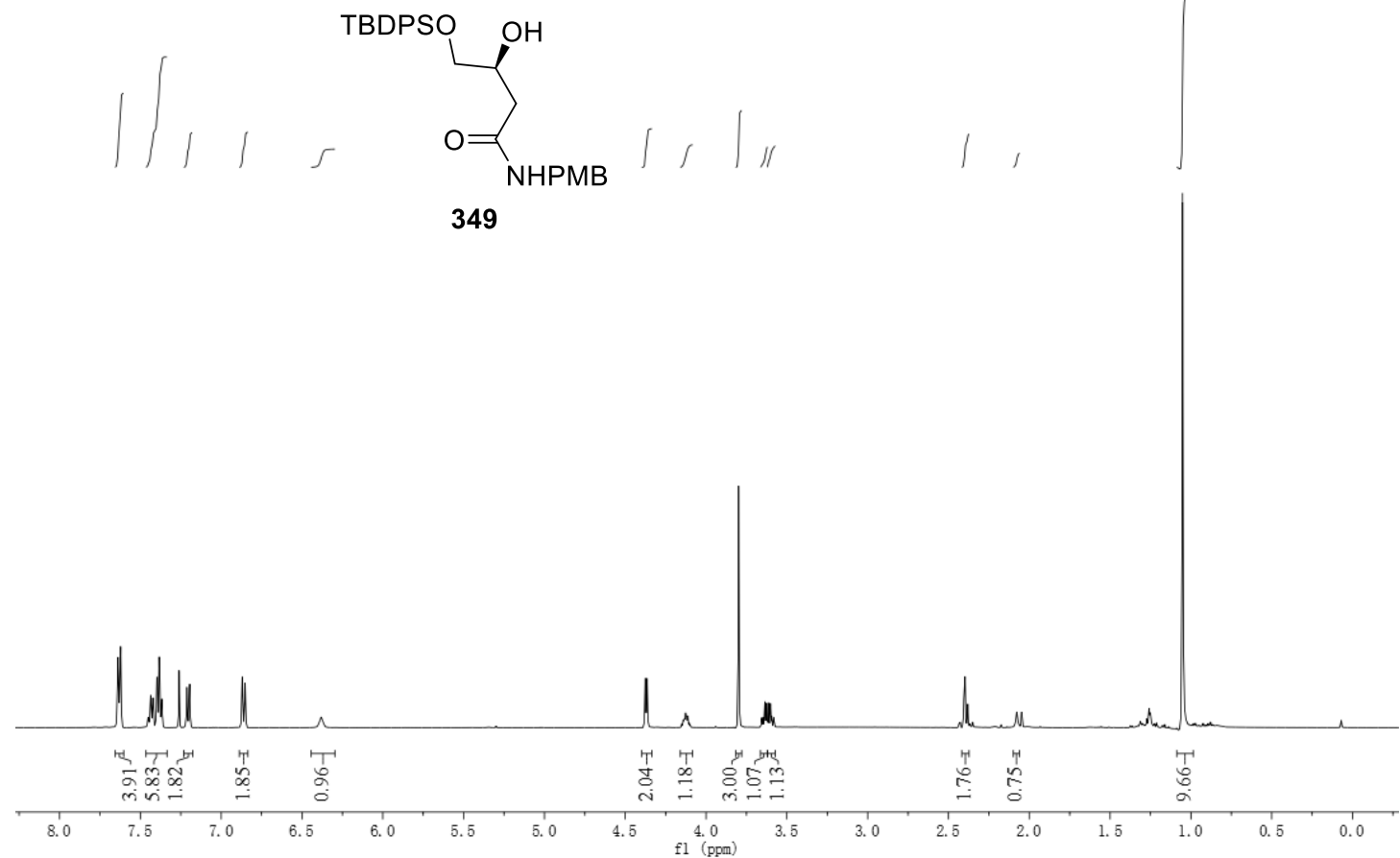

${ }^{13} \mathrm{C}$ NMR (125 MHz, $\mathrm{CDCl}_{3}$ )

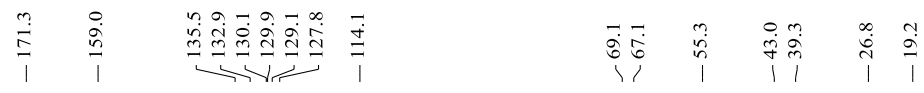

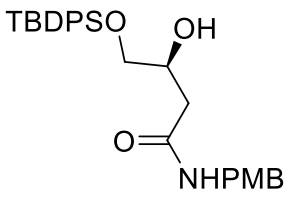

349

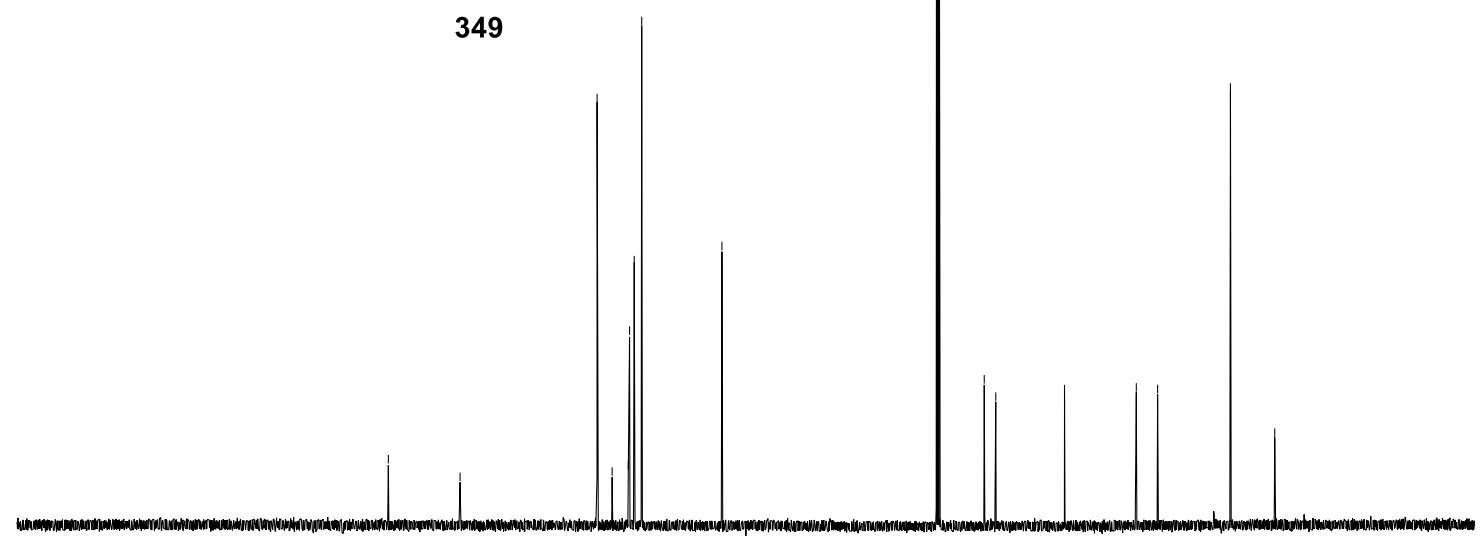

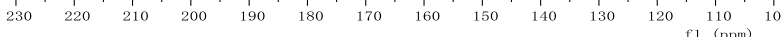


${ }^{1} \mathrm{H}$ NMR $\left(500 \mathrm{MHz}, \mathrm{CDCl}_{3}\right)$
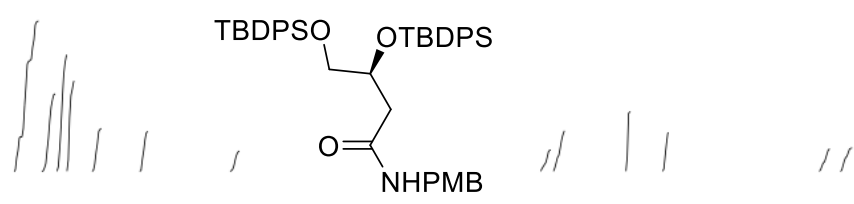

350

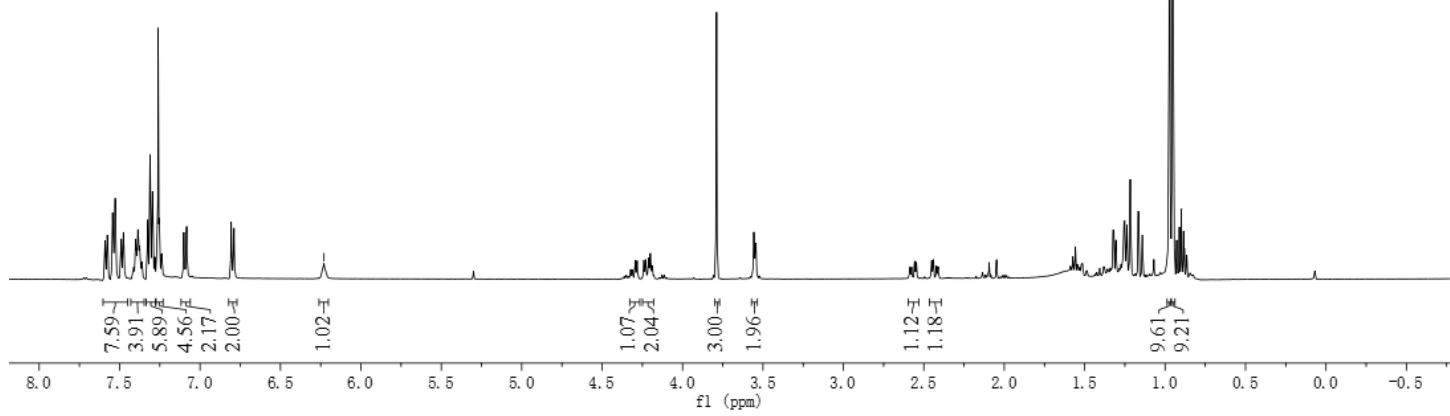

${ }^{13} \mathrm{C} \mathrm{NMR}\left(125 \mathrm{MHz}, \mathrm{CDCl}_{3}\right)$
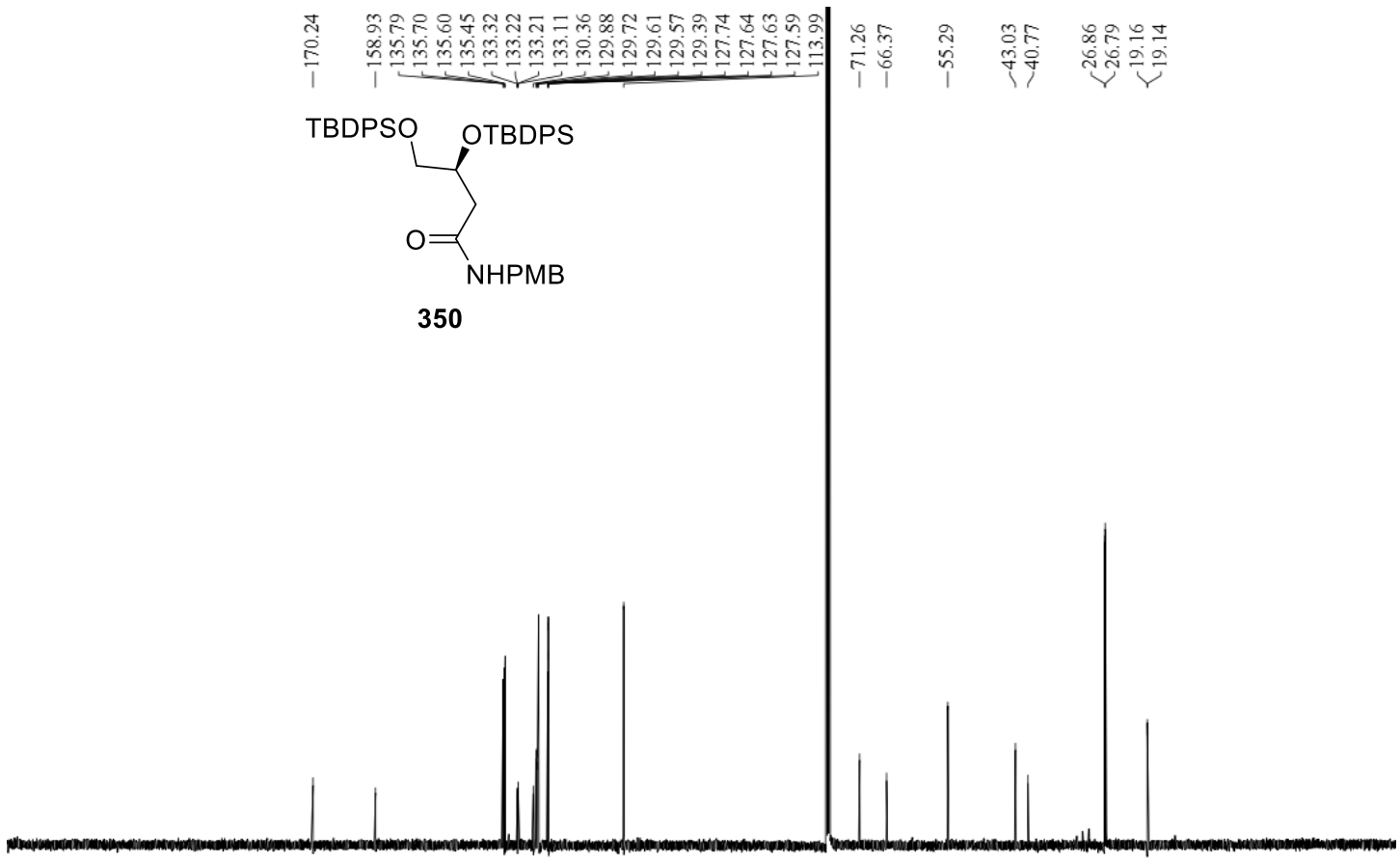

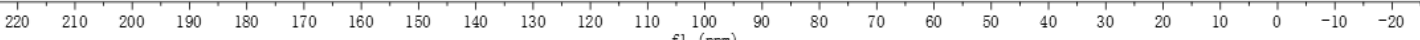


${ }^{1} \mathrm{H} \mathrm{NMR}\left(500 \mathrm{MHz}, \mathrm{CDCl}_{3}\right)$

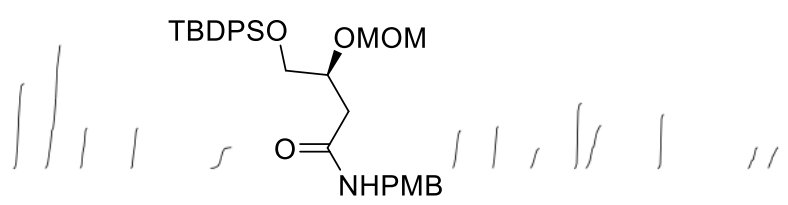

351

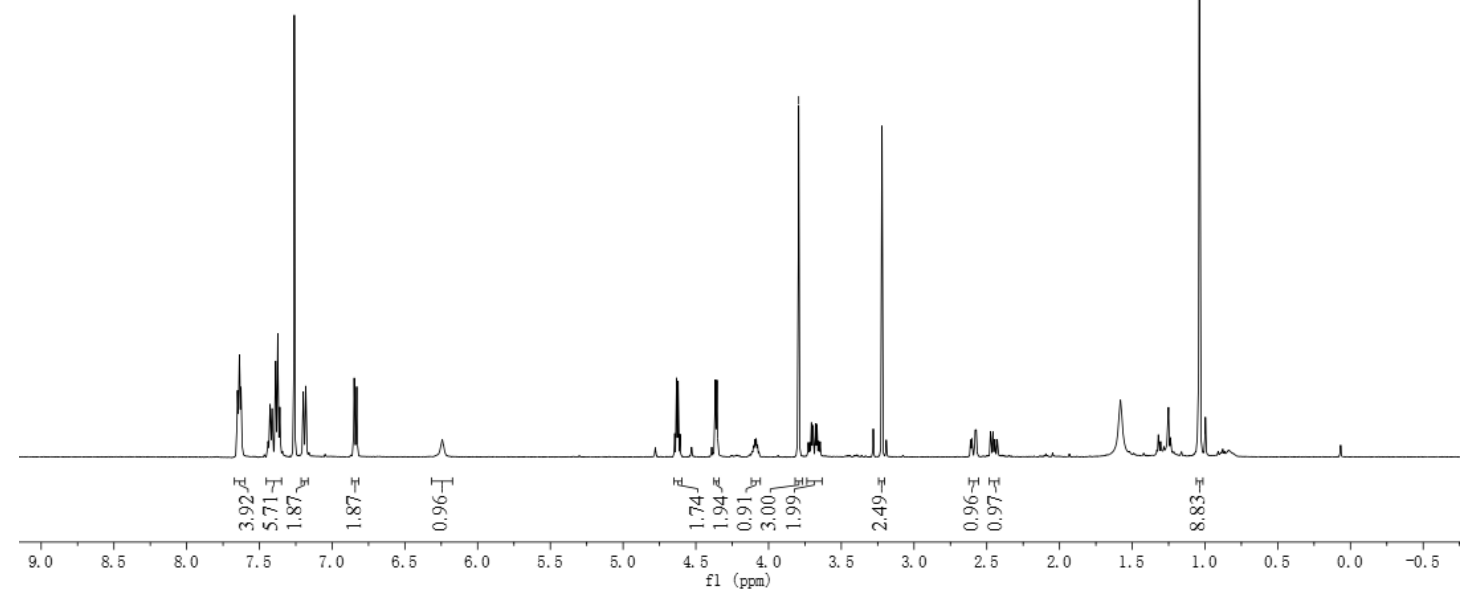

${ }^{13} \mathrm{C}$ NMR $\left(125 \mathrm{MHz}, \mathrm{CDCl}_{3}\right)$

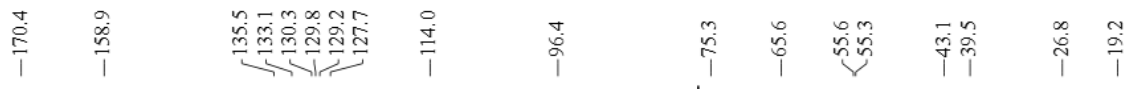

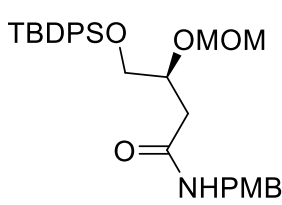

351

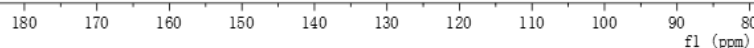




\section{Spectra of Chapter Three}

${ }^{1} \mathrm{H} \mathrm{NMR}\left(500 \mathrm{MHz}, \mathrm{CDCl}_{3}\right)$
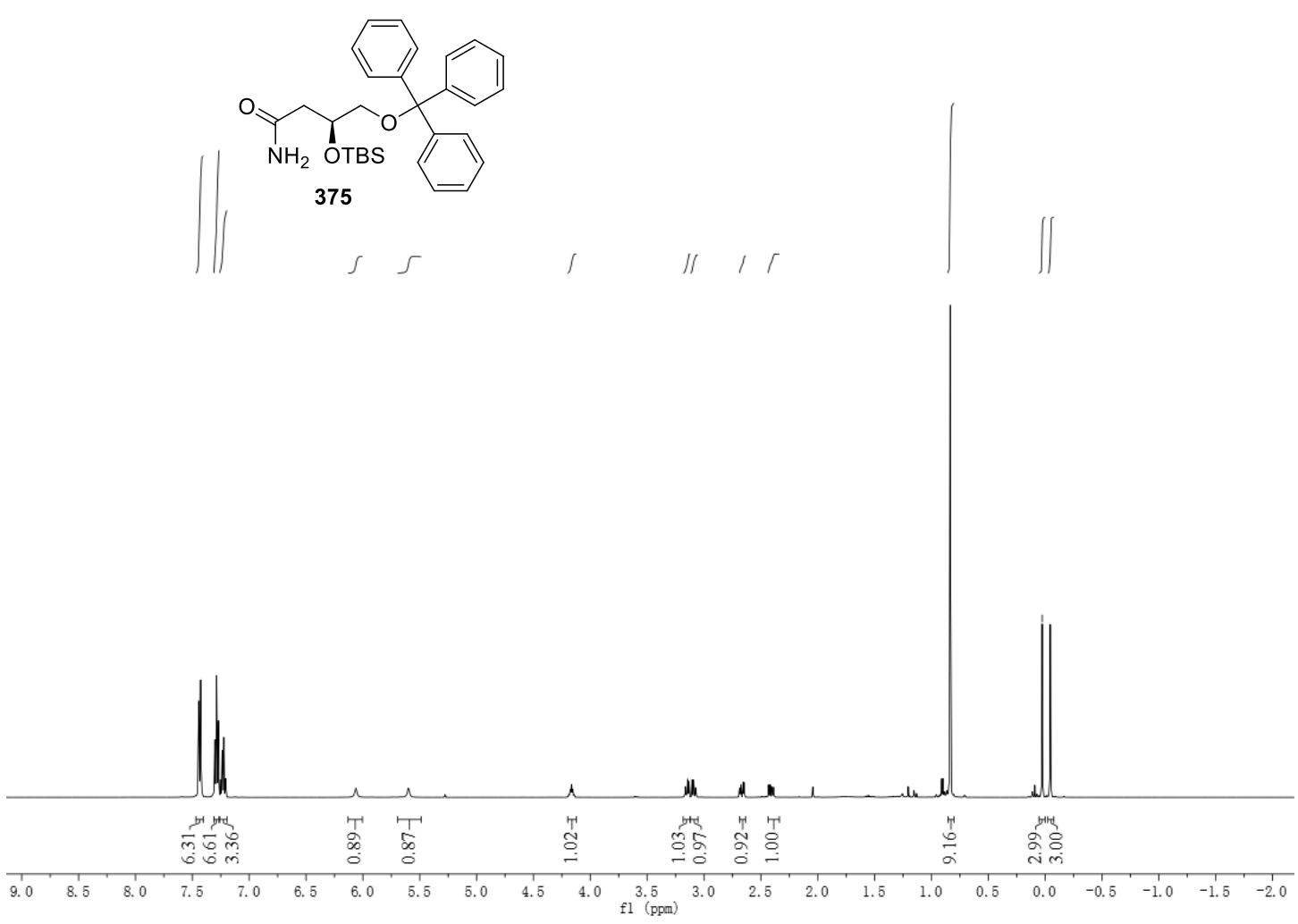

${ }^{13} \mathrm{C} \mathrm{NMR}\left(125 \mathrm{MHz}, \mathrm{CDCl}_{3}\right)$

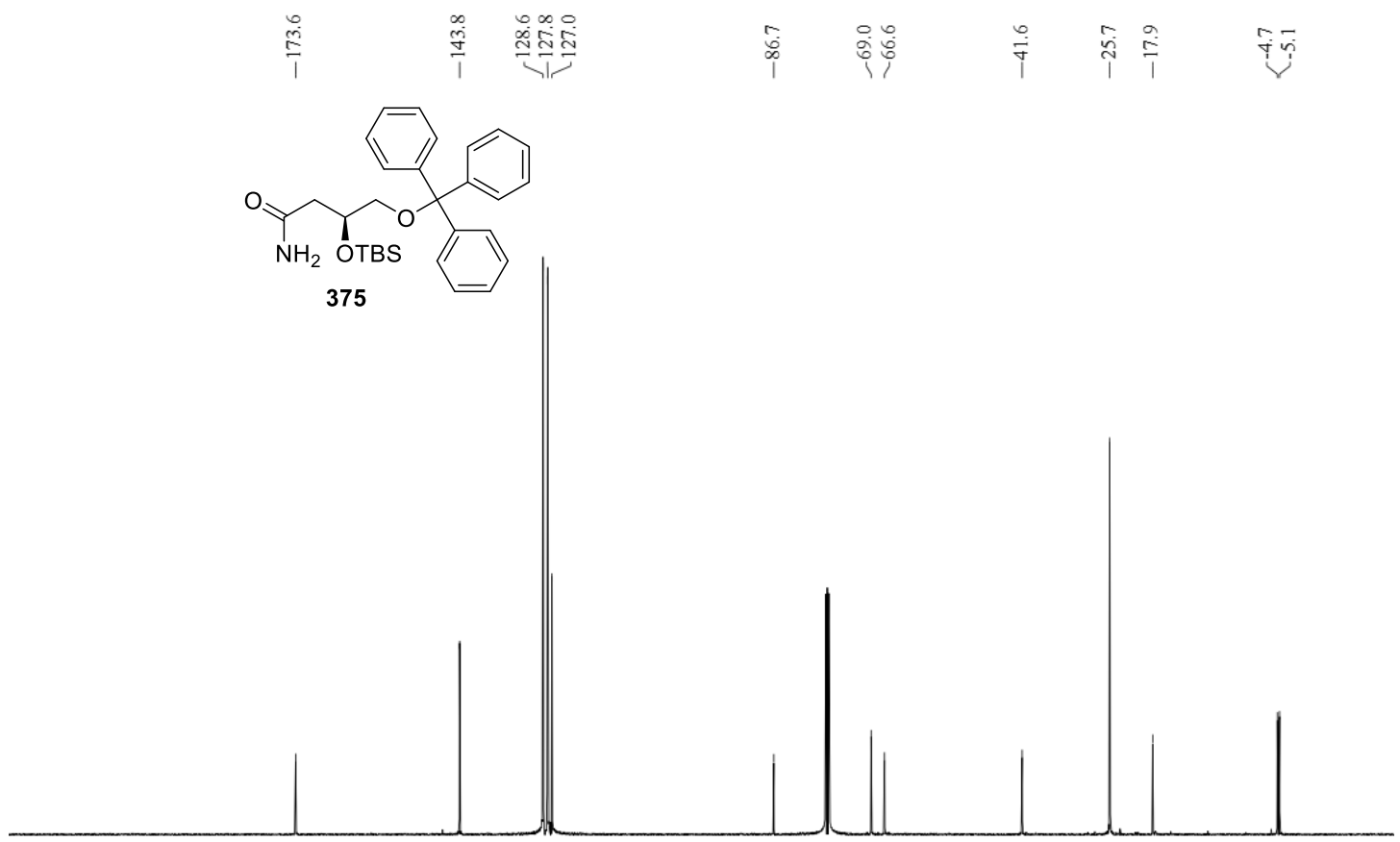

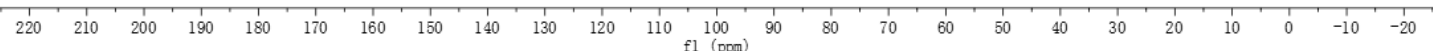


${ }^{1} \mathrm{H}$ NMR $\left(500 \mathrm{MHz}, \mathrm{CDCl}_{3}\right)$

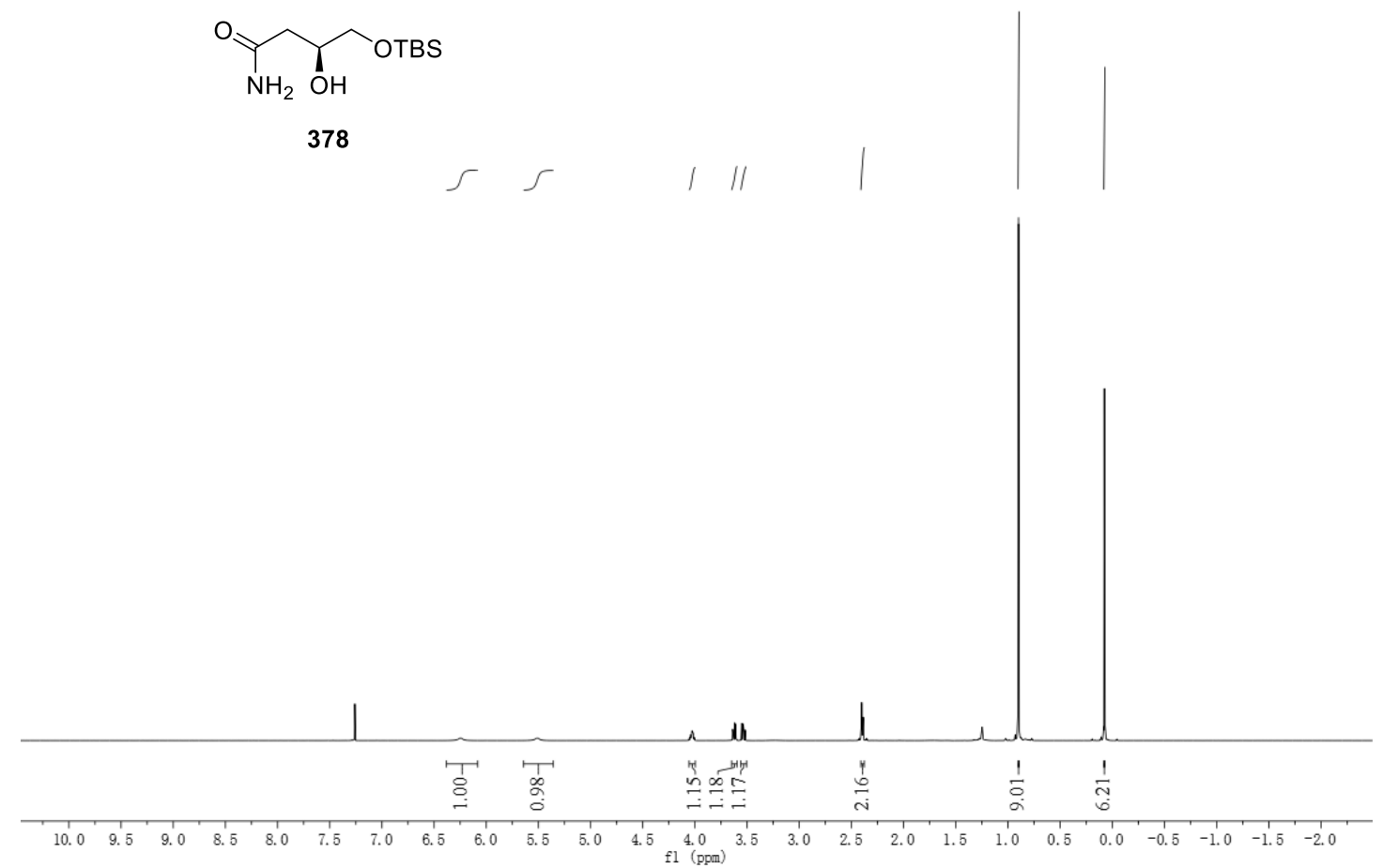

${ }^{13} \mathrm{C} \mathrm{NMR}\left(125 \mathrm{MHz}, \mathrm{CDCl}_{3}\right)$
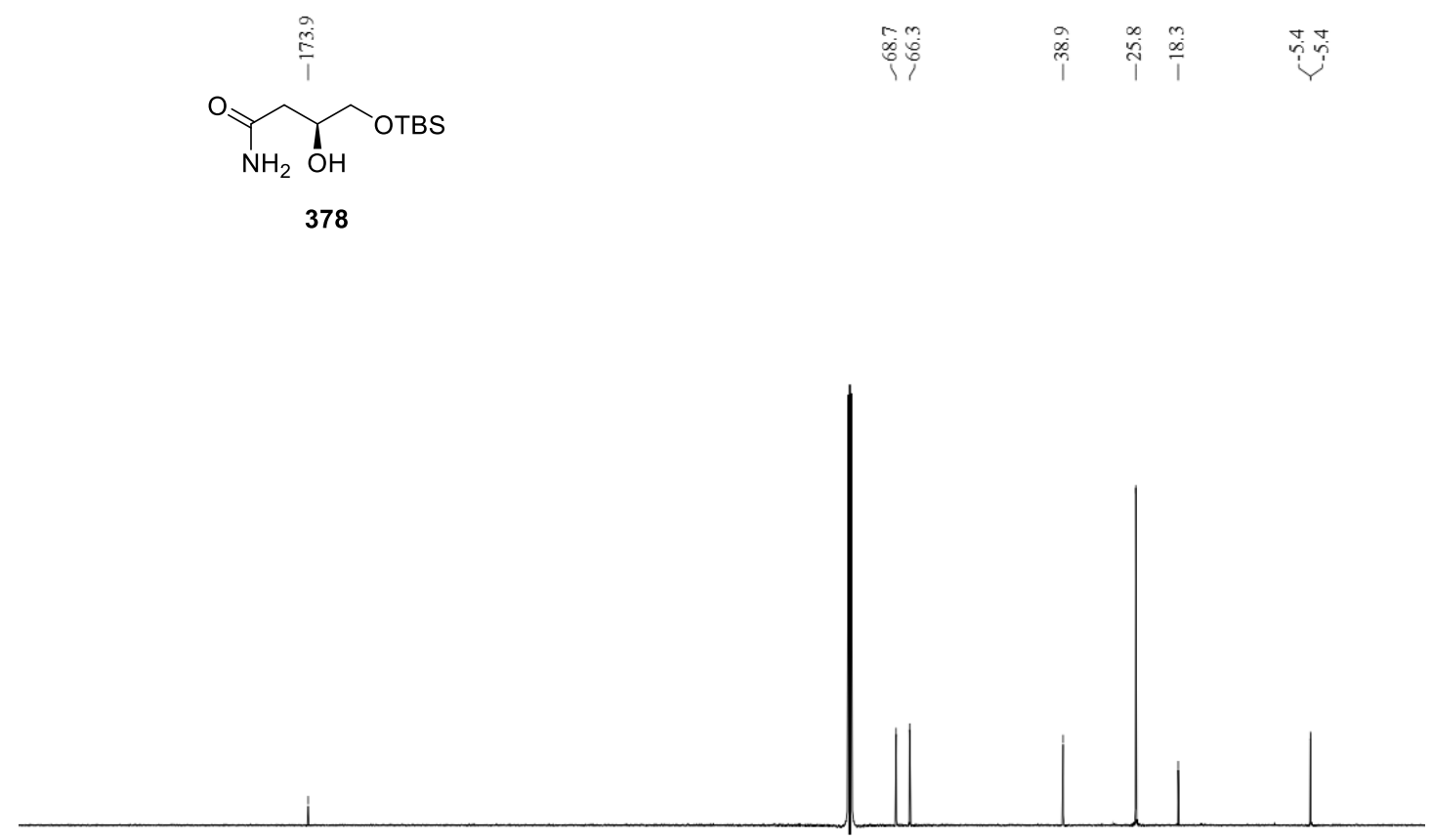

$\begin{array}{lllllllllllllllllllllllllllllll}1 & 1 & 210 & 200 & 190 & 180 & 170 & 160 & 150 & 140 & 130 & 120 & 110 & 100 & 90 & 80 & 70 & 60 & 50 & 40 & 30 & 20 & 10 & 0 & -10 & -20\end{array}$ 
${ }^{1} \mathrm{H}$ NMR $\left(500 \mathrm{MHz}, \mathrm{CDCl}_{3}\right)$

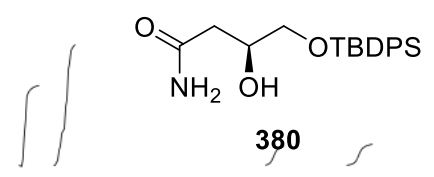

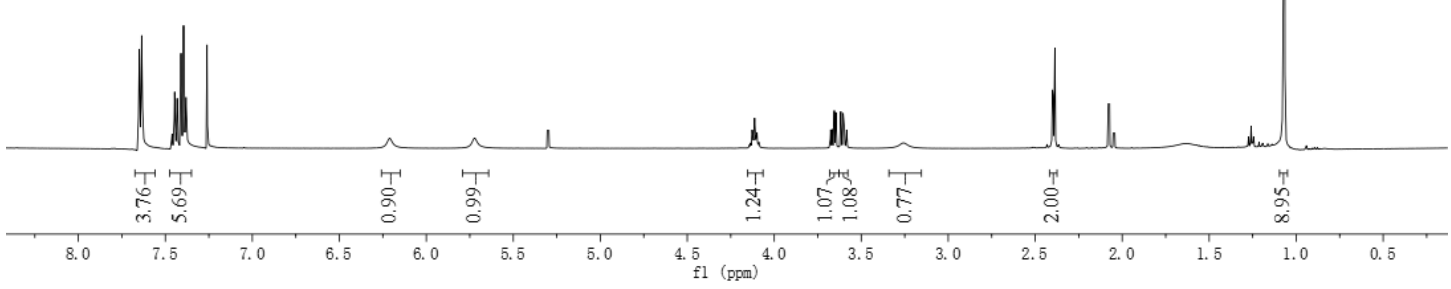

${ }^{13} \mathrm{C} \mathrm{NMR}\left(125 \mathrm{MHz}, \mathrm{CDCl}_{3}\right)$
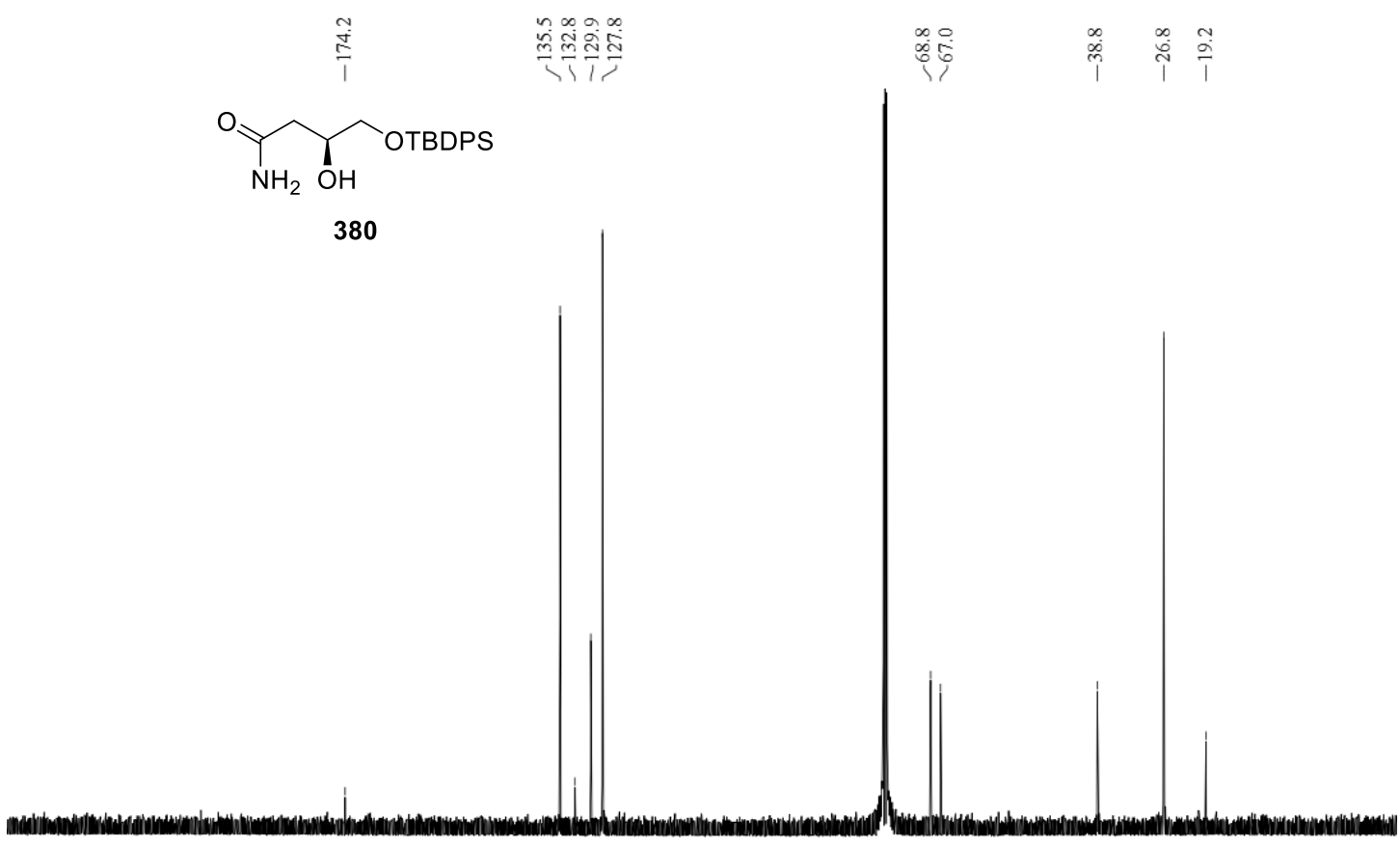

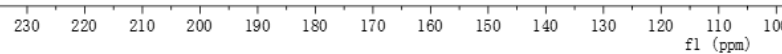




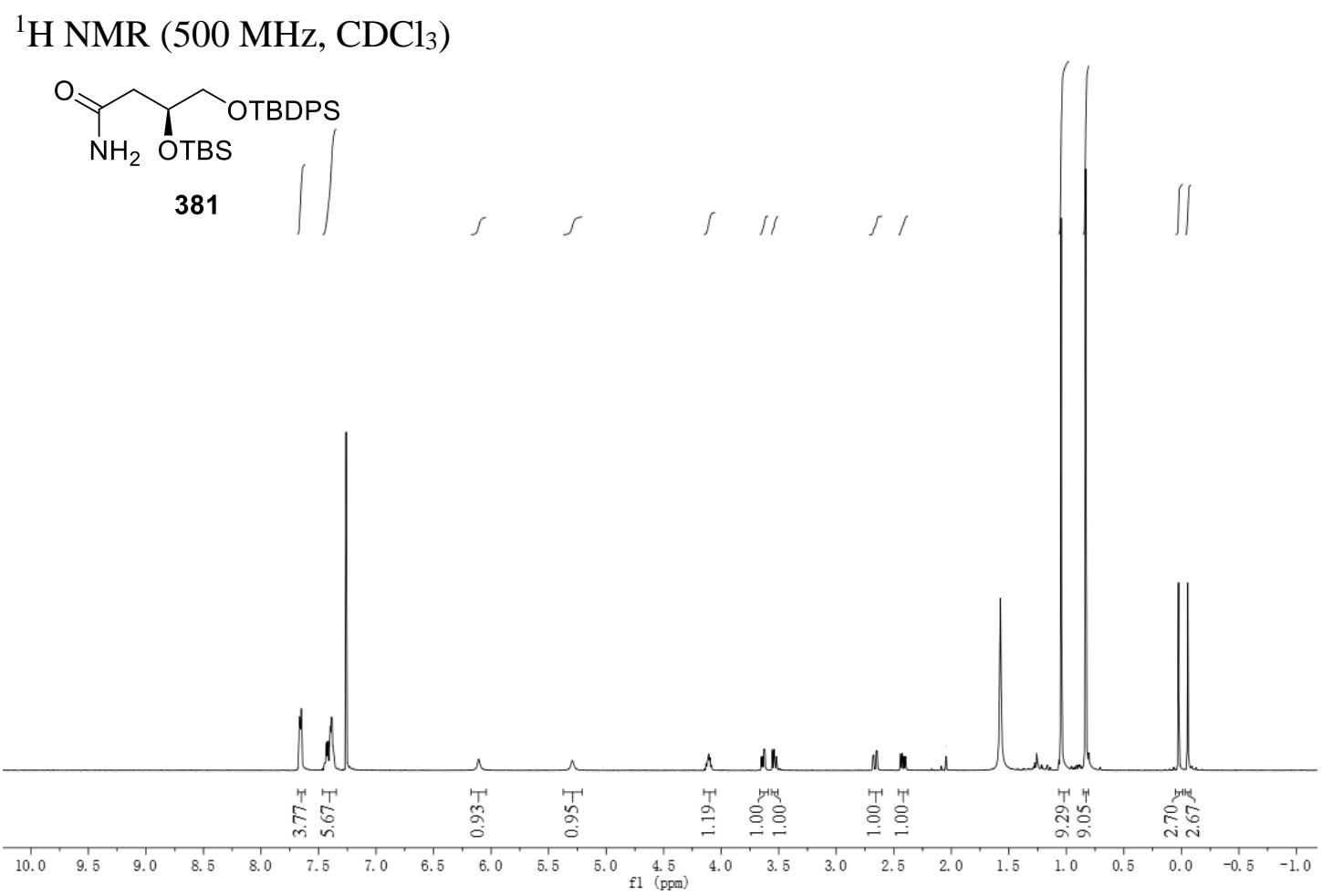

${ }^{13} \mathrm{C}$ NMR (125 MHz, $\left.\mathrm{CDCl}_{3}\right)$

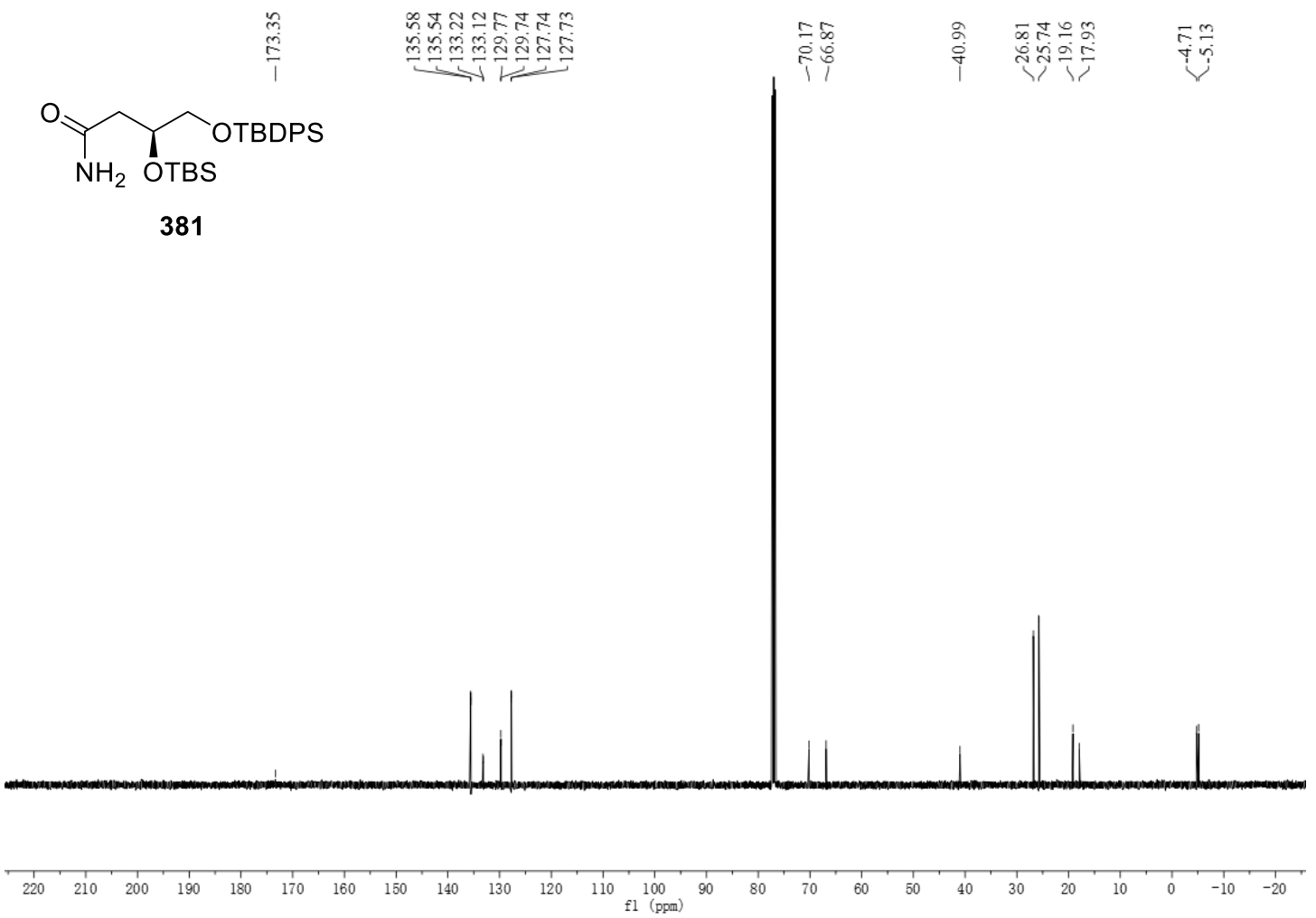




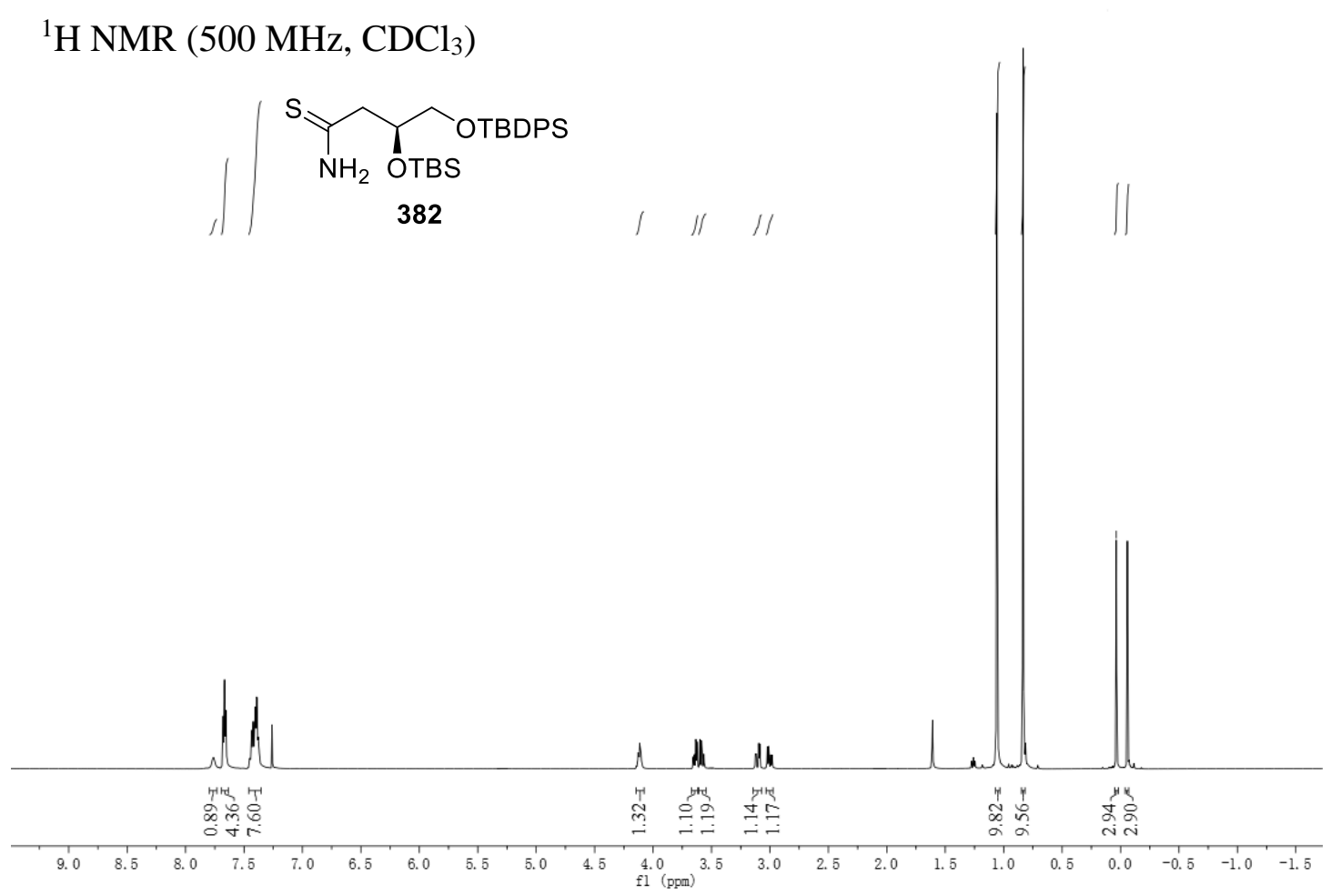

${ }^{13} \mathrm{C} \mathrm{NMR}\left(125 \mathrm{MHz}, \mathrm{CDCl}_{3}\right)$
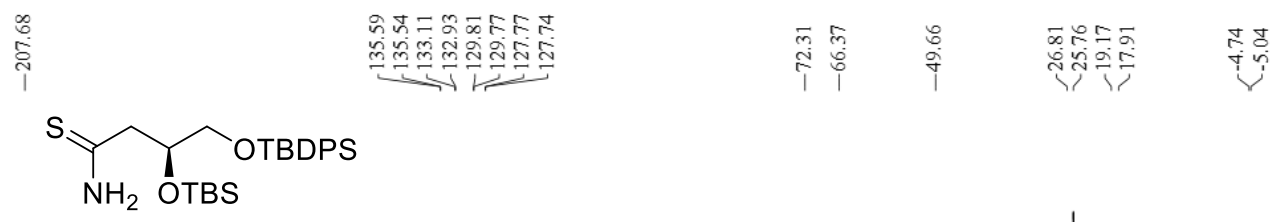

382

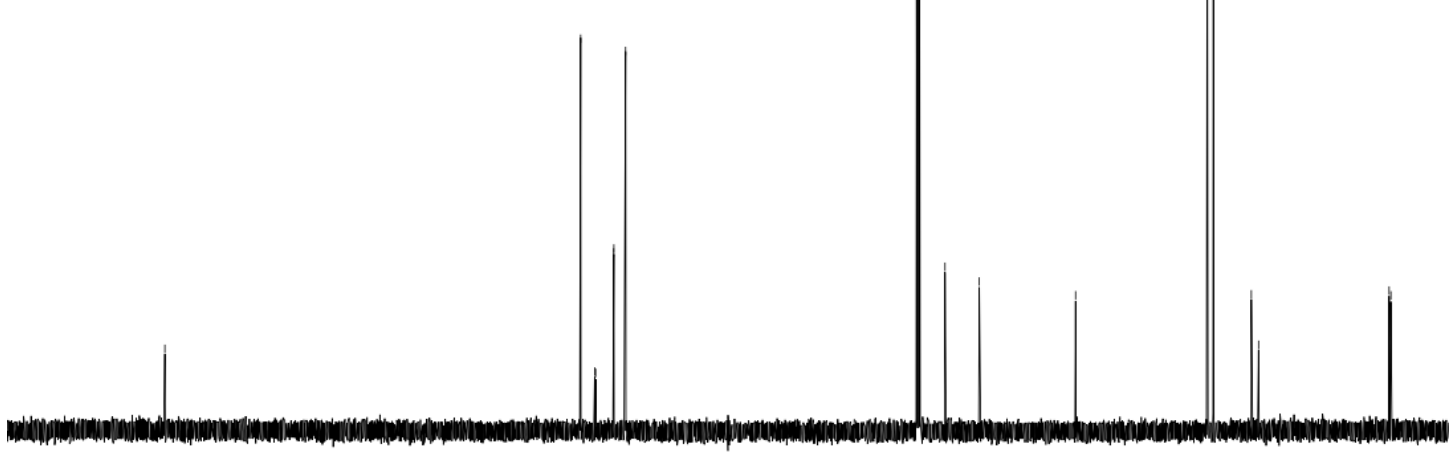

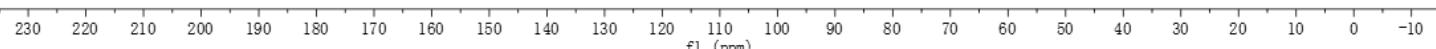


${ }^{1} \mathrm{H}$ NMR $\left(500 \mathrm{MHz}, \mathrm{CDCl}_{3}\right)$
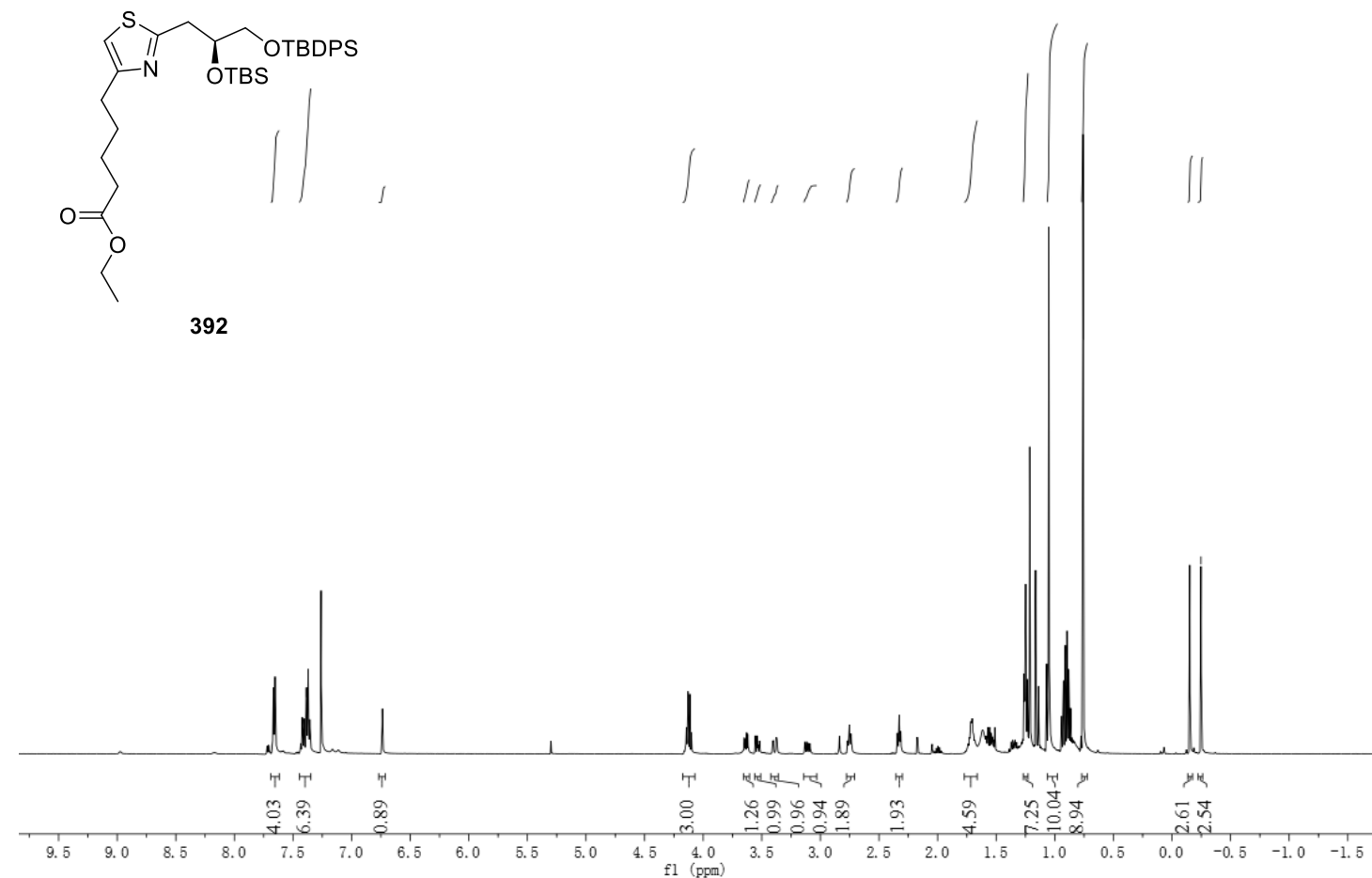

${ }^{13} \mathrm{C}$ NMR (125 MHz, $\left.\mathrm{CDCl}_{3}\right)$

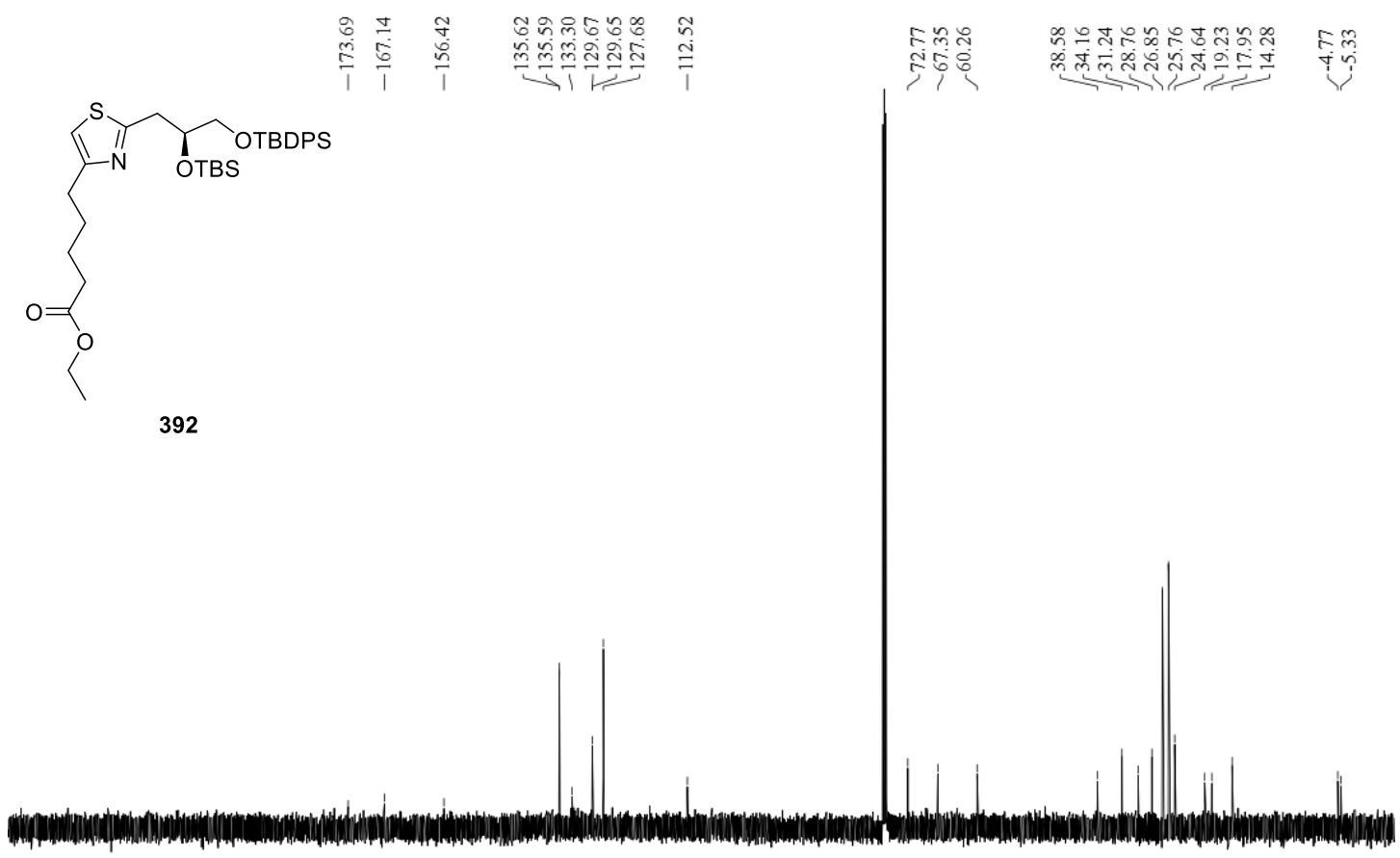

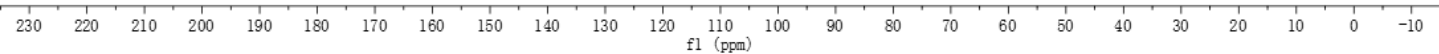


${ }^{1} \mathrm{H}$ NMR $\left(500 \mathrm{MHz}, \mathrm{CDCl}_{3}\right)$

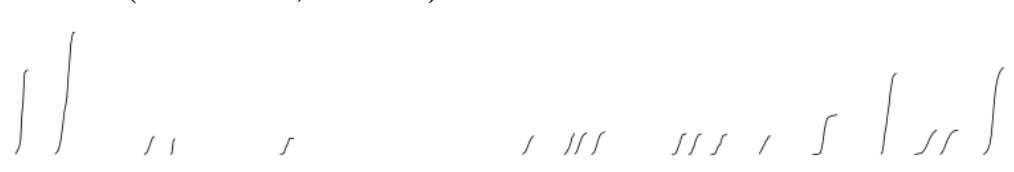<smiles>O=C1C=CCC(CCOC(=O)CCCCc2csc(CC(O)CO[18O])n2)O1</smiles>

393

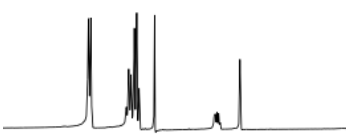

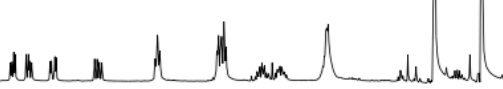

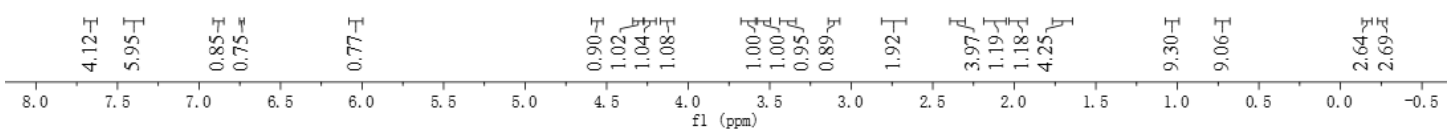

${ }^{13} \mathrm{C}$ NMR $\left(125 \mathrm{MHz}, \mathrm{CDCl}_{3}\right)$

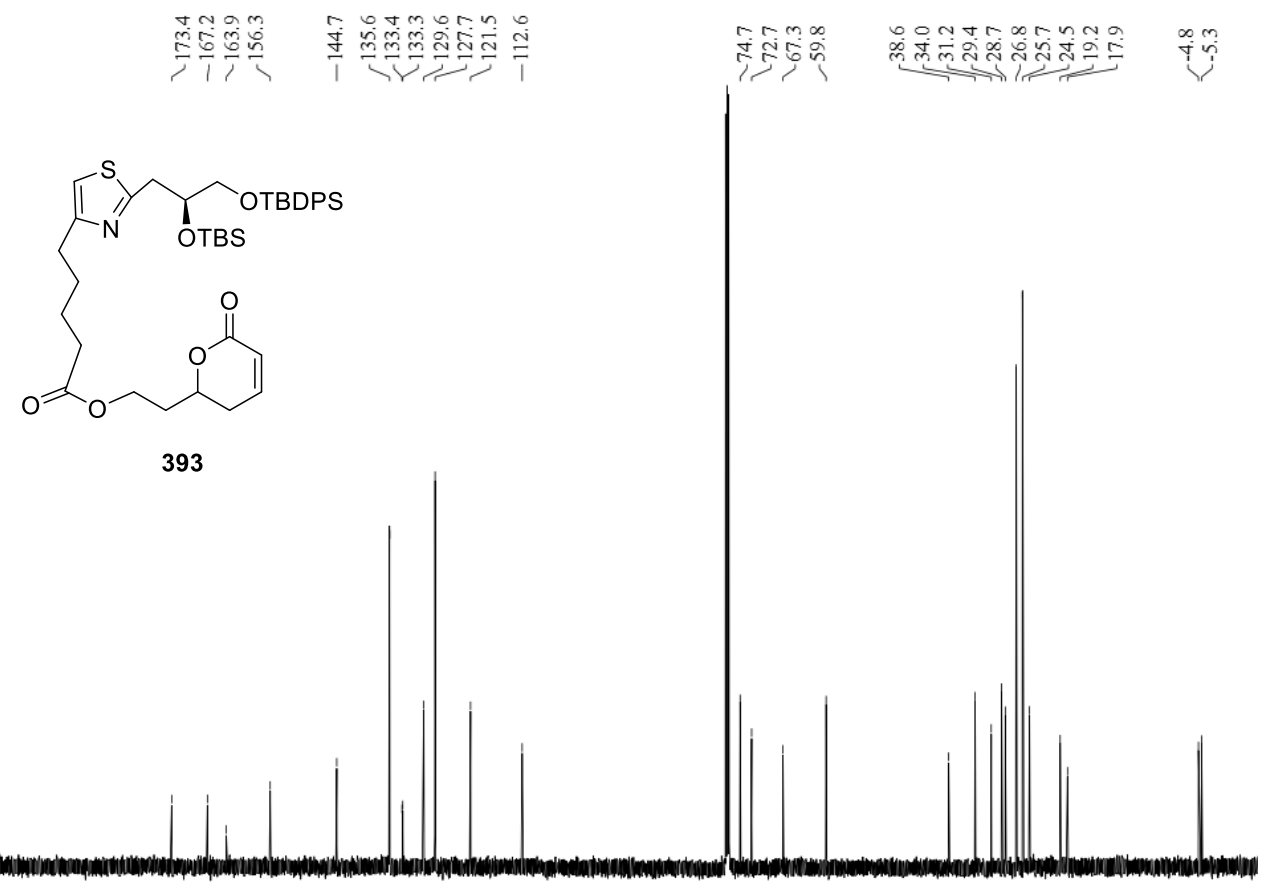

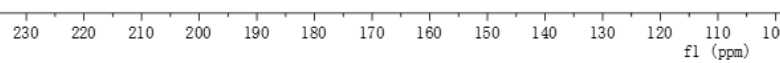




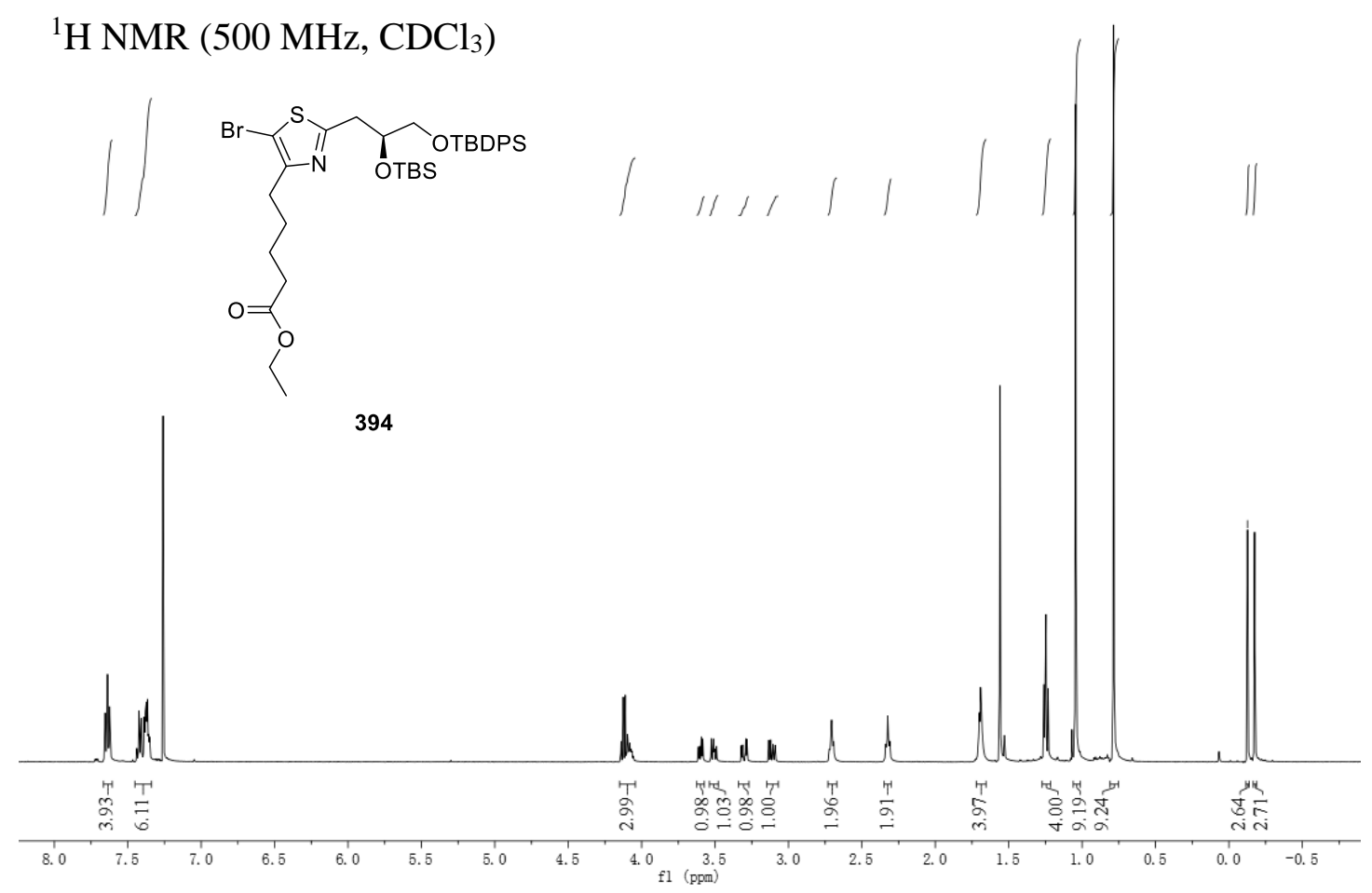

${ }^{13} \mathrm{C} \mathrm{NMR}\left(125 \mathrm{MHz}, \mathrm{CDCl}_{3}\right)$

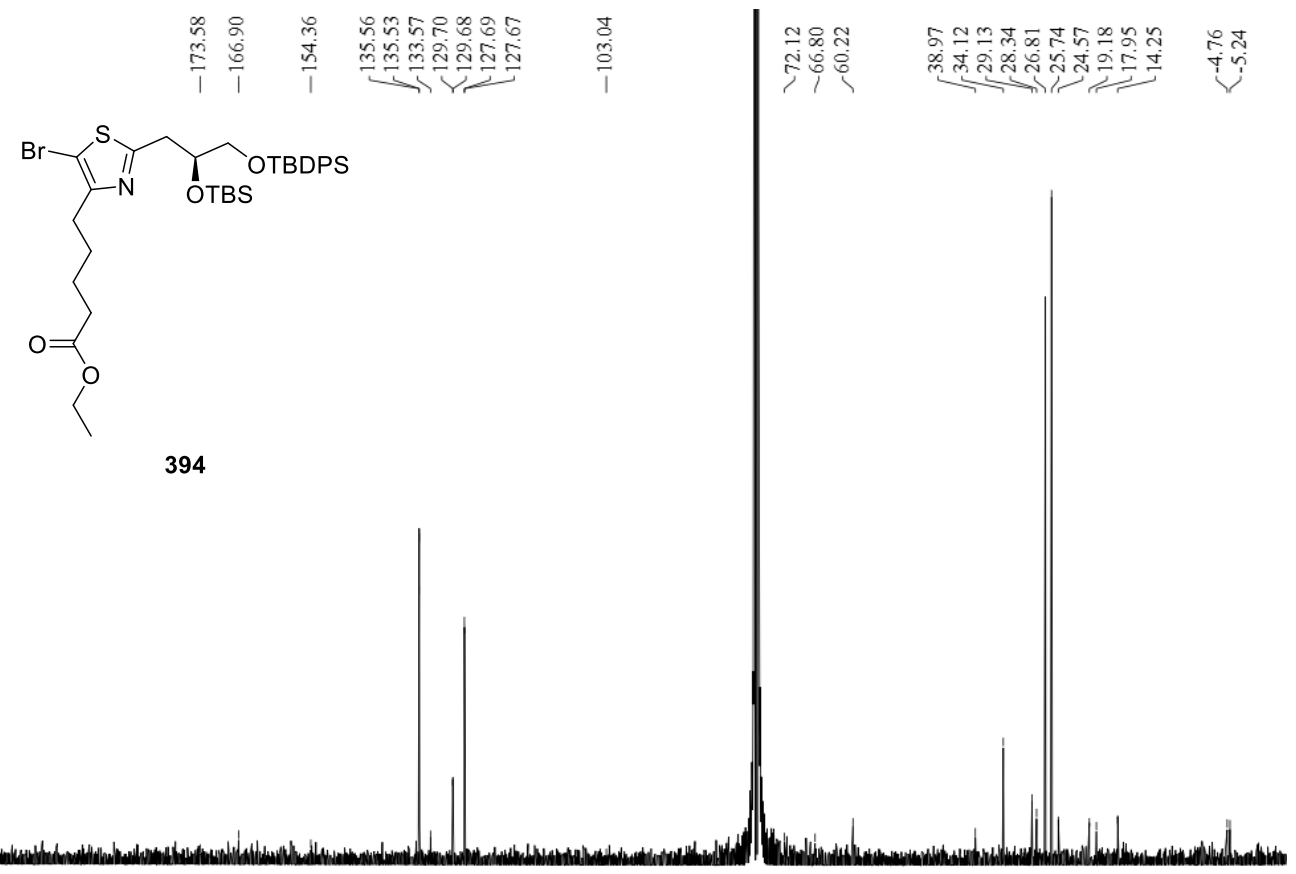

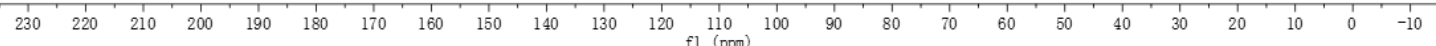




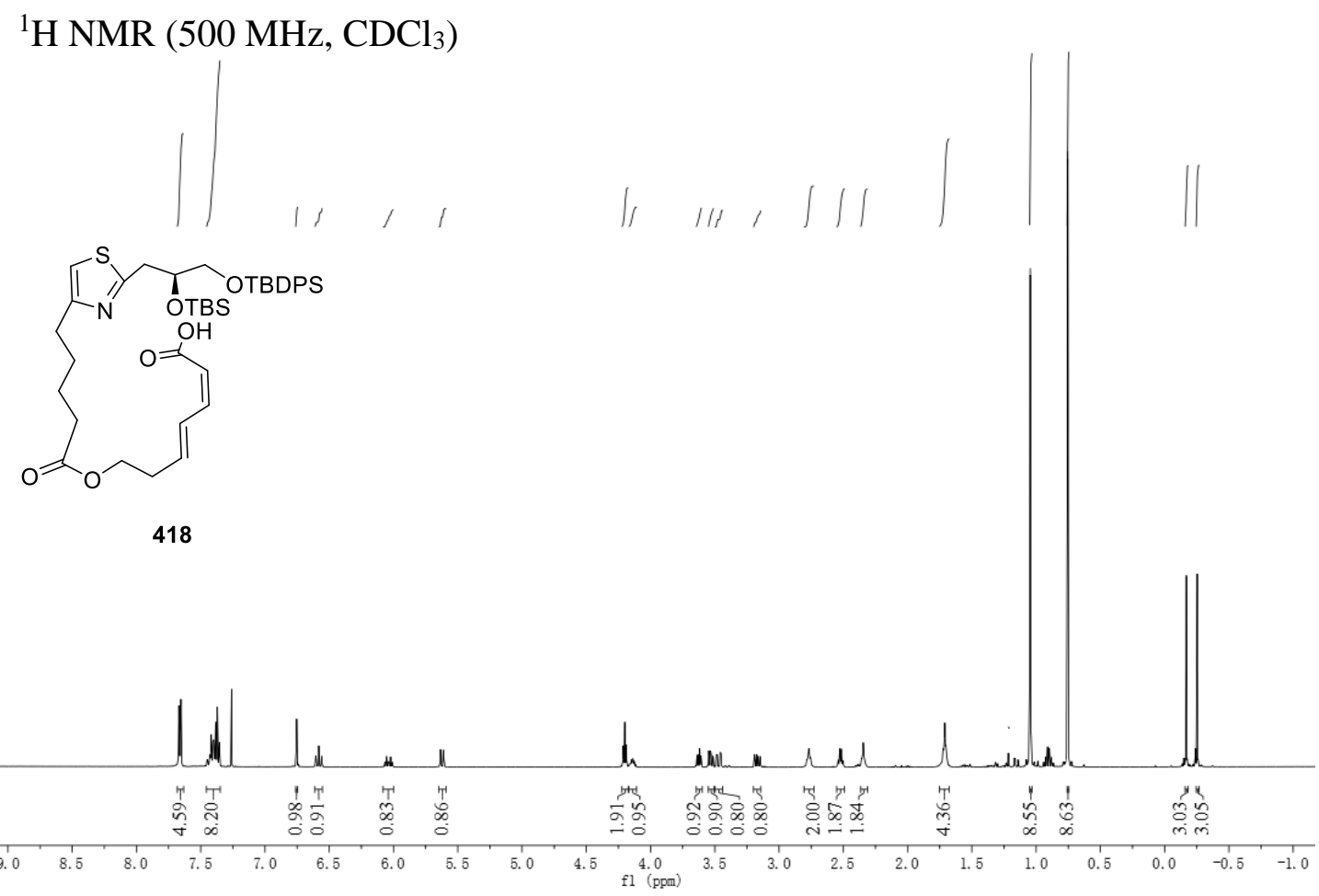

${ }^{13} \mathrm{C} \mathrm{NMR}\left(125 \mathrm{MHz}, \mathrm{CDCl}_{3}\right)$

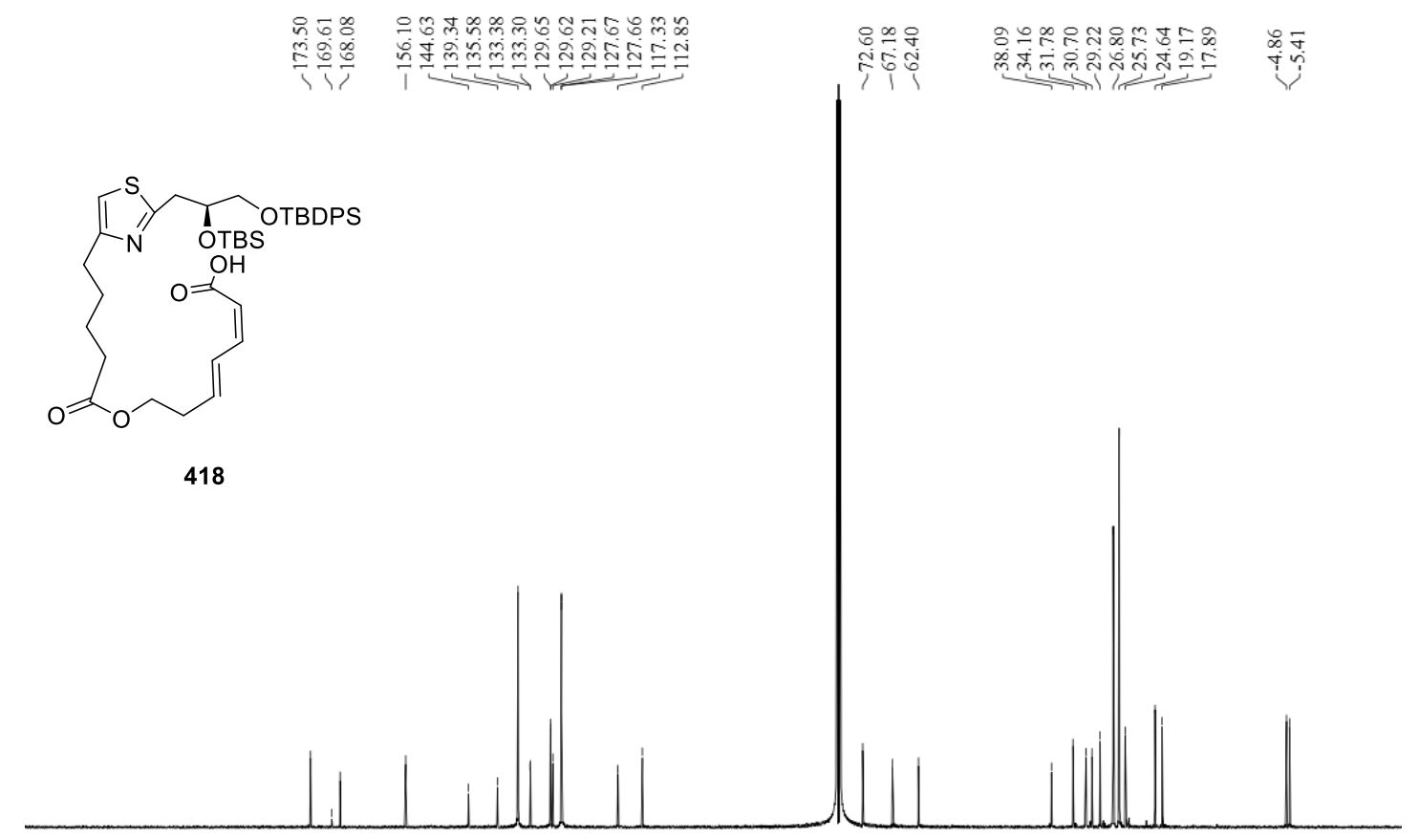

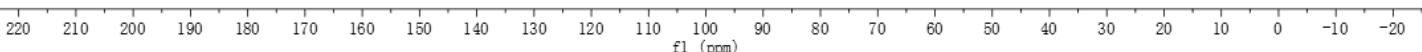


${ }^{1} \mathrm{H}$ NMR (500 MHz, $\left.\mathrm{CDCl}_{3}\right)$

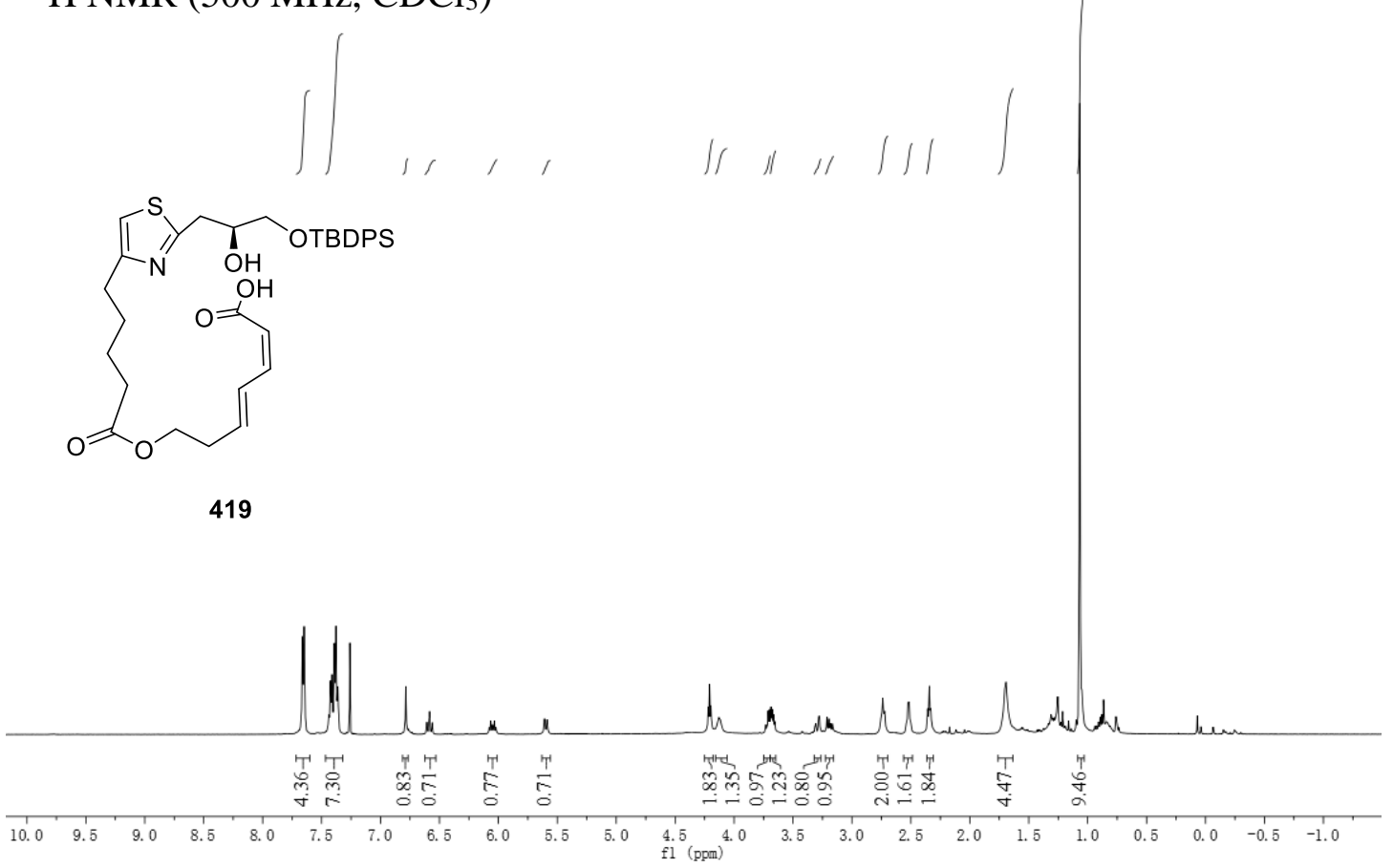

${ }^{13} \mathrm{C} \mathrm{NMR}\left(125 \mathrm{MHz}, \mathrm{CDCl}_{3}\right)$

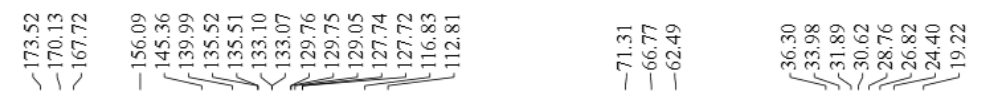

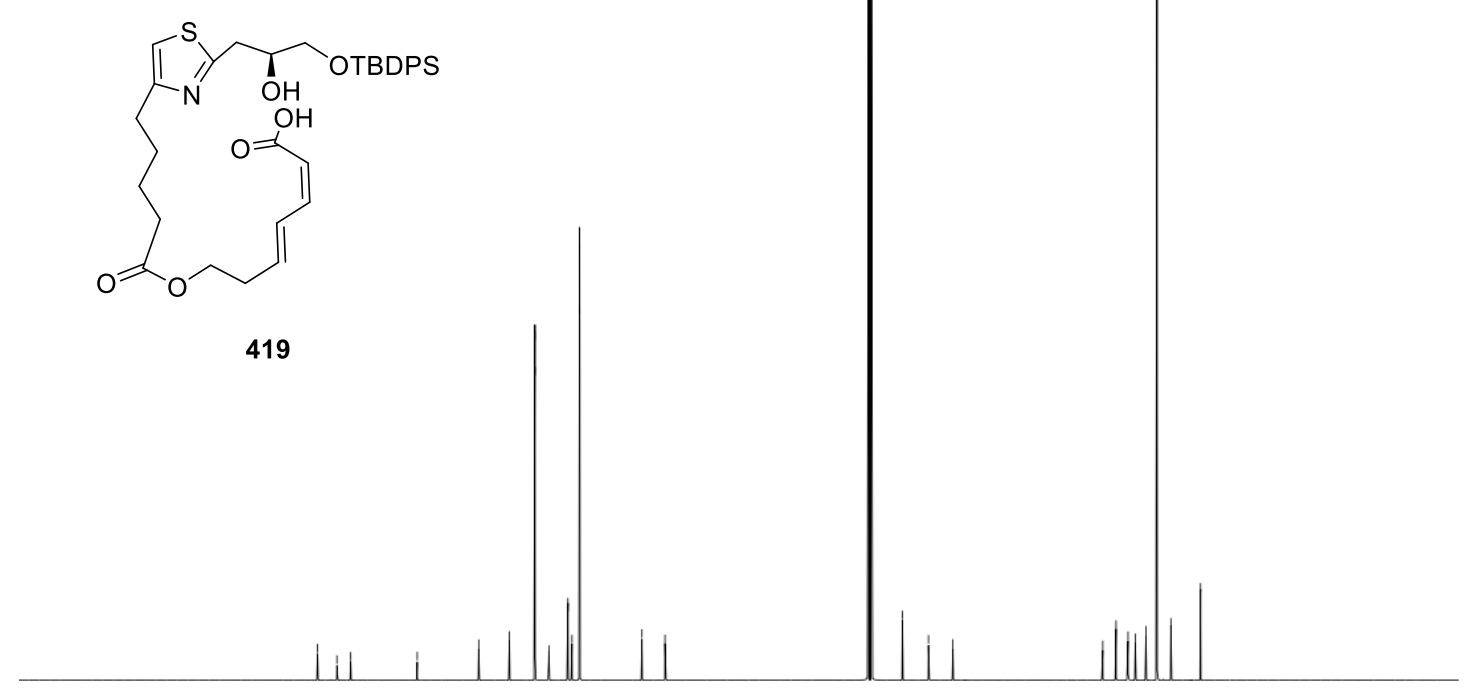

$\begin{array}{lllllllllllllllllllllllllllllll}1 & 1 & 210 & 200 & 190 & 180 & 170 & 160 & 150 & 140 & 130 & 120 & 110 & 100 & 90 & 80 & 70 & 60 & 50 & 40 & 30 & 20 & 10 & 0 & -10 & -20\end{array}$ 
${ }^{1} \mathrm{H}$ NMR (500 MHz, $\left.\mathrm{CDCl}_{3}\right)$
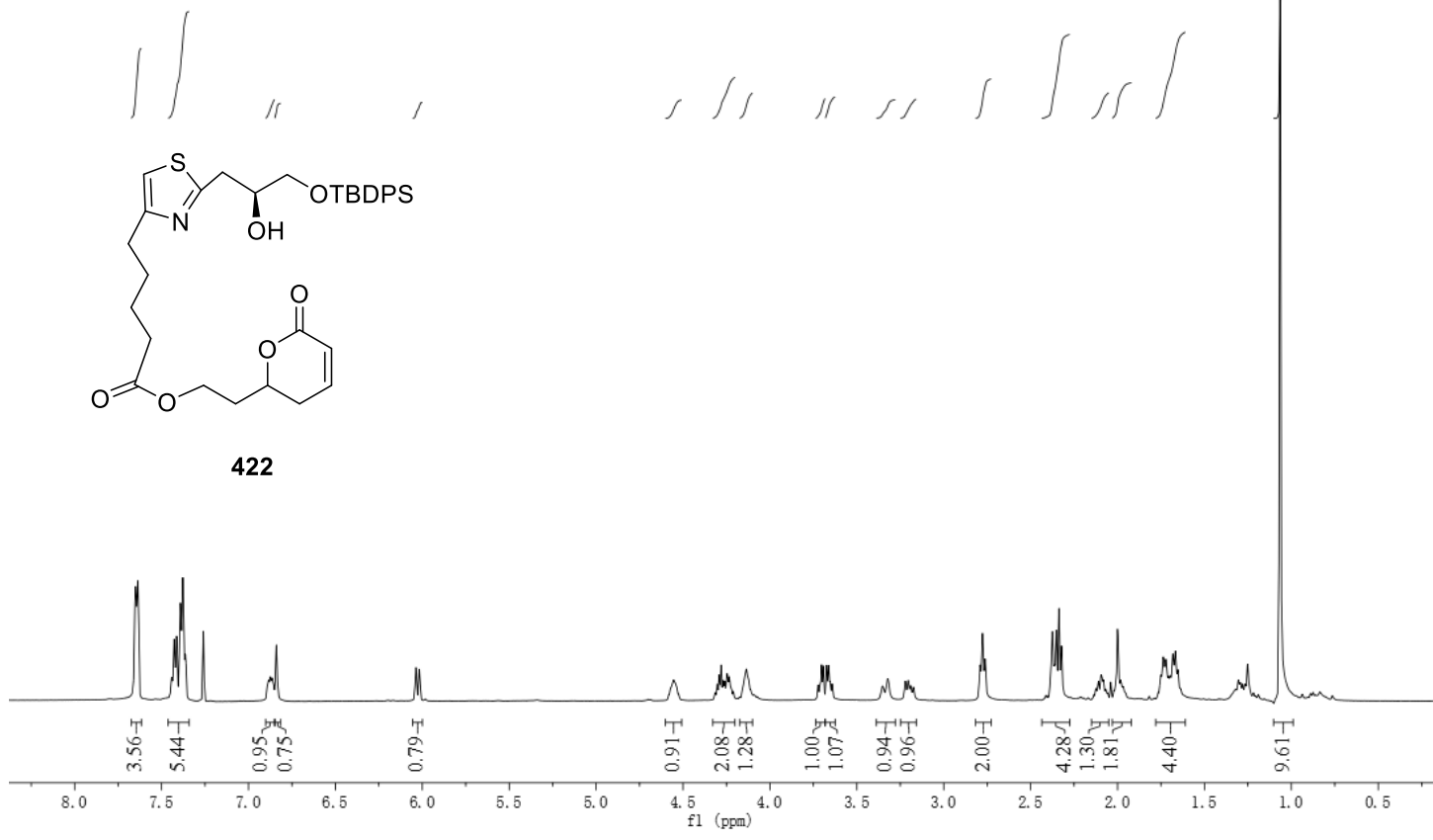

${ }^{13} \mathrm{C}$ NMR (125 MHz, $\left.\mathrm{CDCl}_{3}\right)$

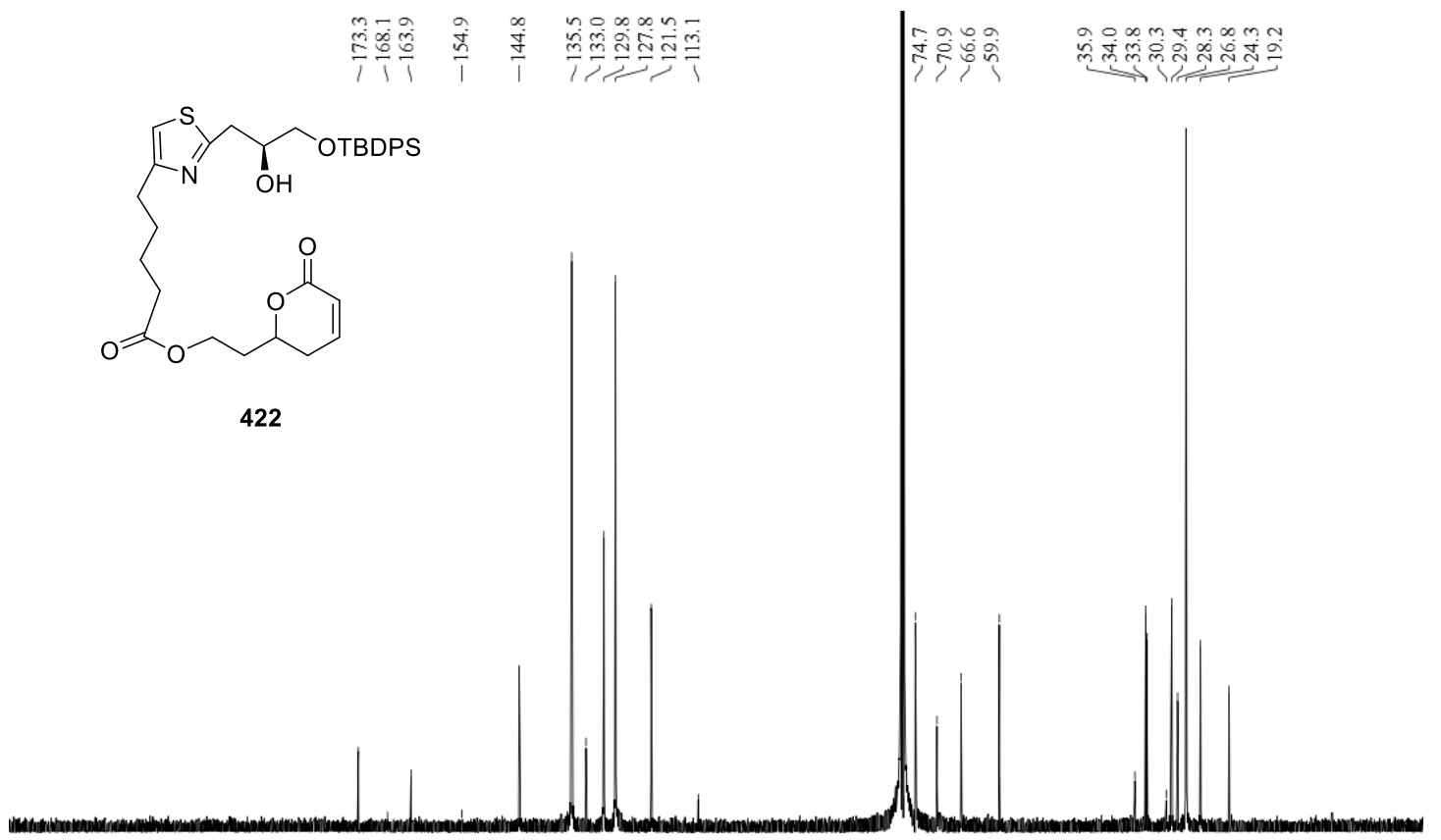

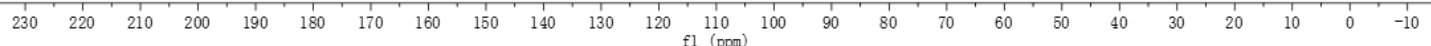


${ }^{1} \mathrm{H}$ NMR (500 MHz, $\left.\mathrm{CDCl}_{3}\right)$
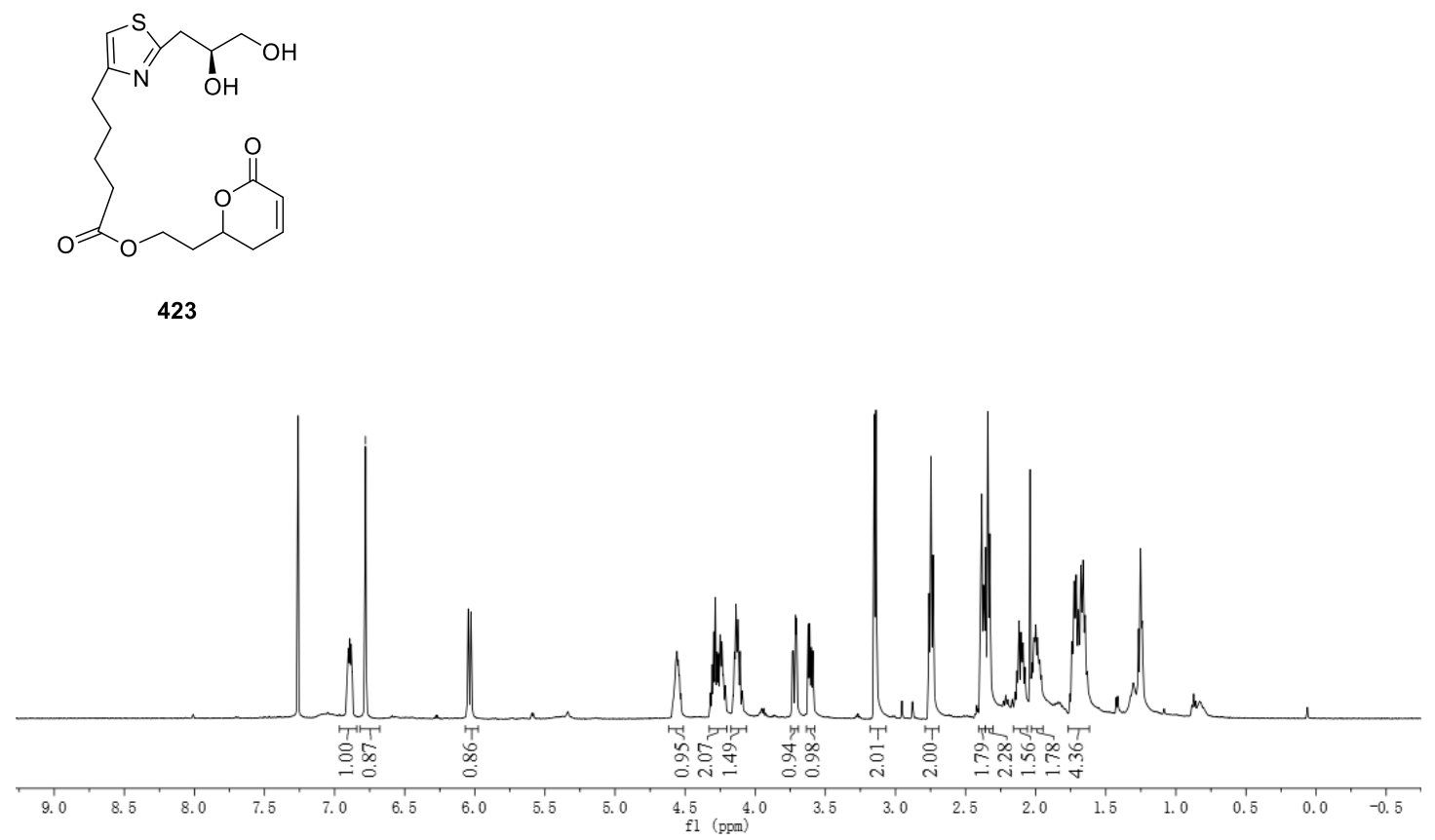

${ }^{13} \mathrm{C}$ NMR (125 MHz, $\left.\mathrm{CDCl}_{3}\right)$

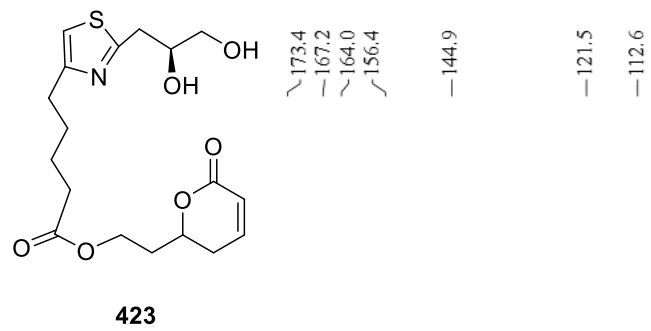

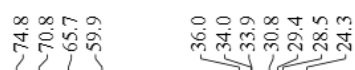

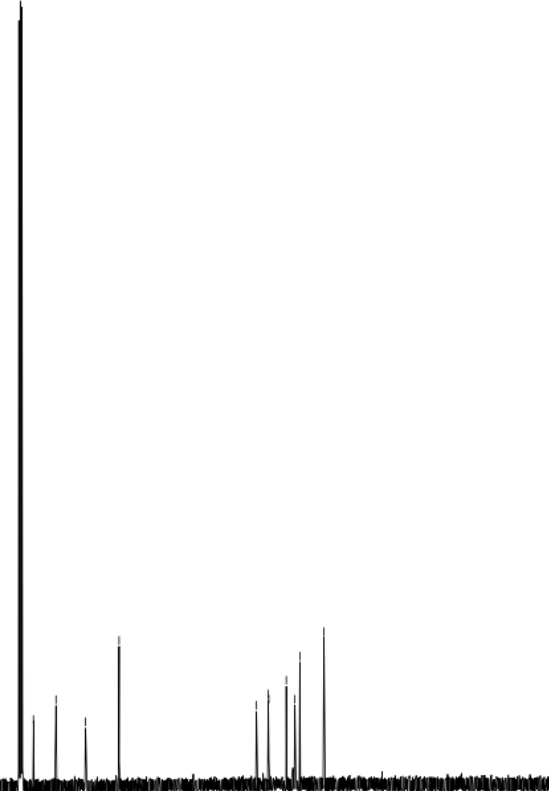

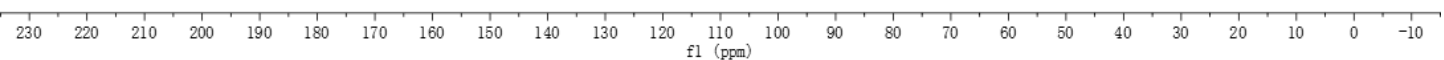




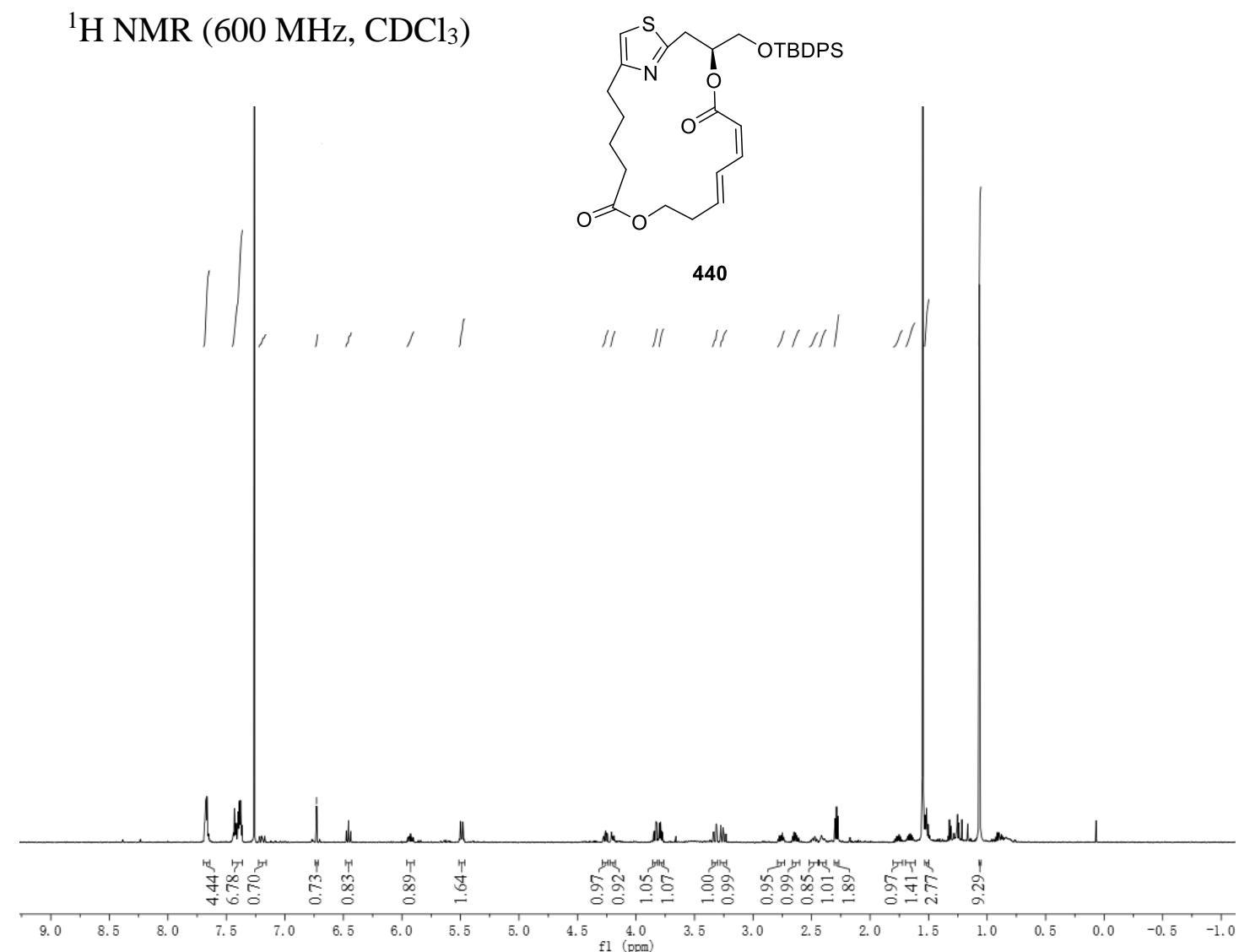

$\left.{ }^{13} \mathrm{C} \mathrm{NMR} \mathrm{(150} \mathrm{MHz,} \mathrm{CDCl}_{3}\right)$

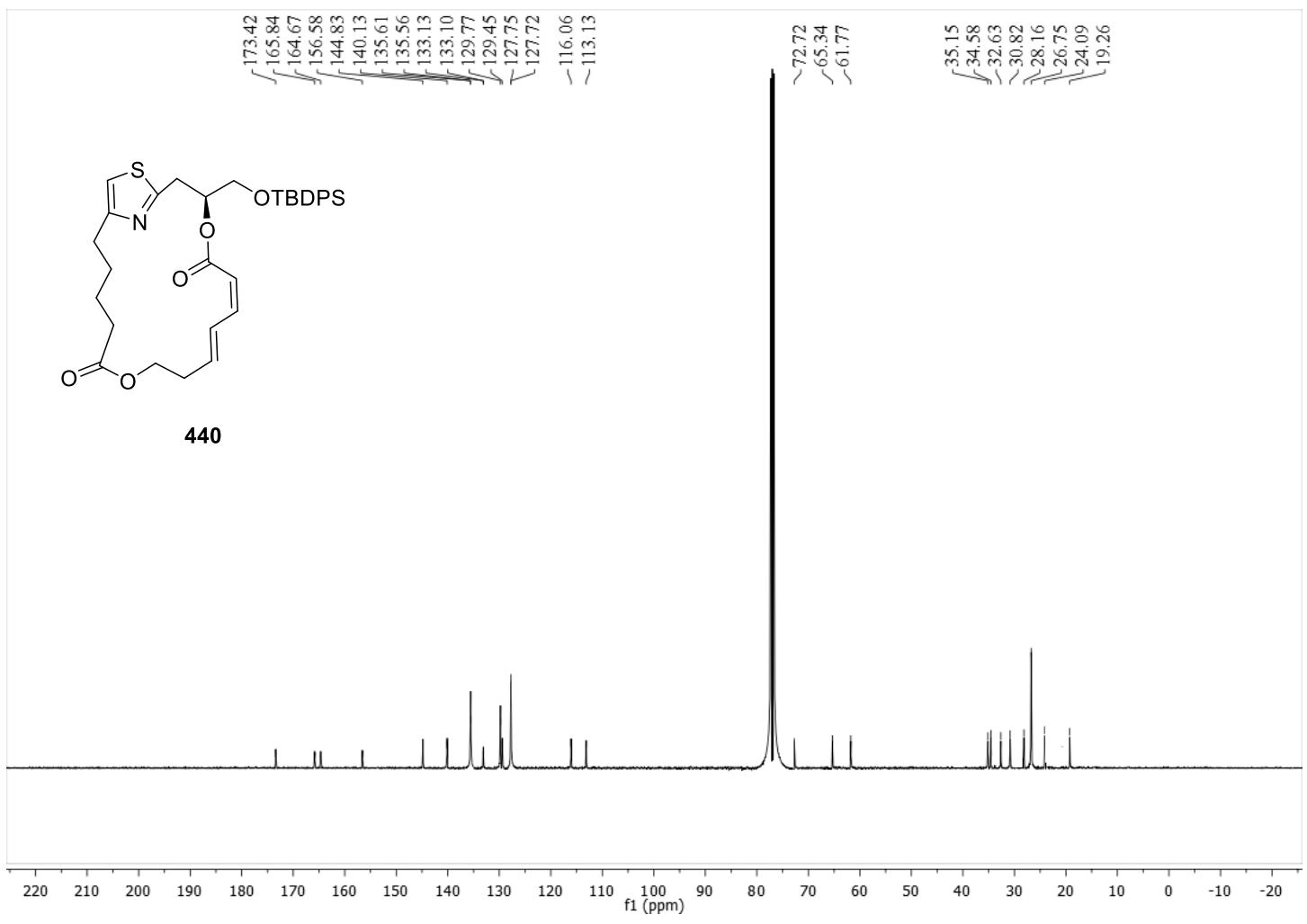


${ }^{1} \mathrm{H}$ NMR $\left(600 \mathrm{MHz}, \mathrm{CDCl}_{3}\right)$
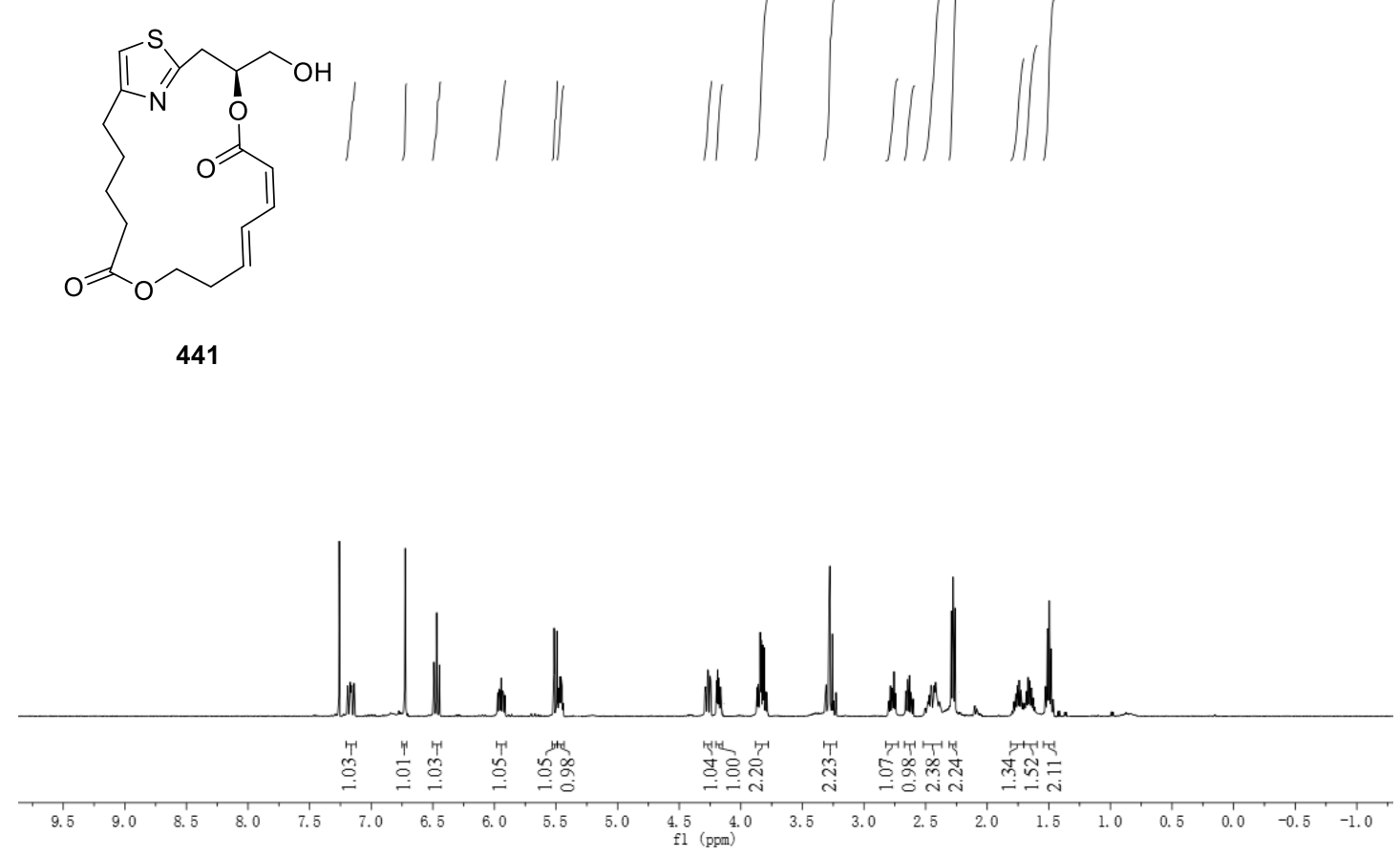

${ }^{13} \mathrm{C}$ NMR $\left(150 \mathrm{MHz}, \mathrm{CDCl}_{3}\right)$

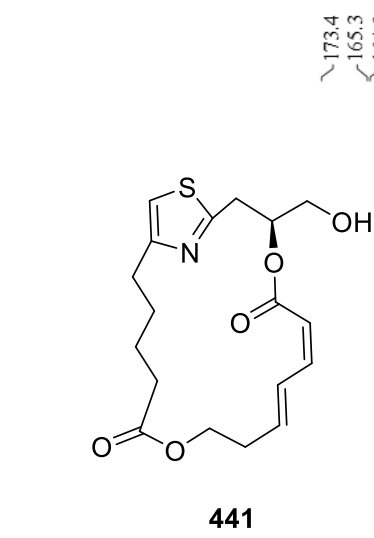

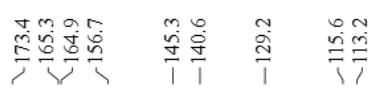

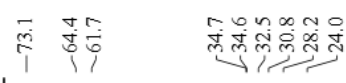

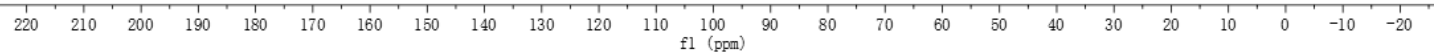


${ }^{1} \mathrm{H}$ NMR $\left(500 \mathrm{MHz}, \mathrm{CDCl}_{3}\right)$

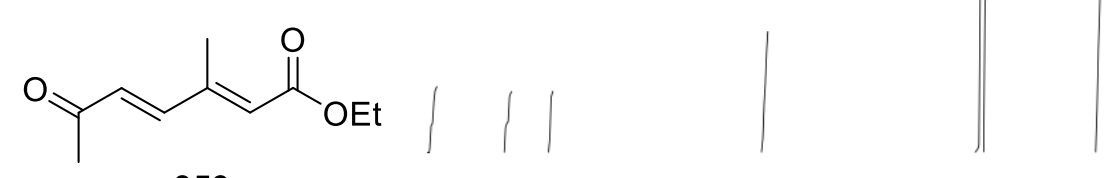

359

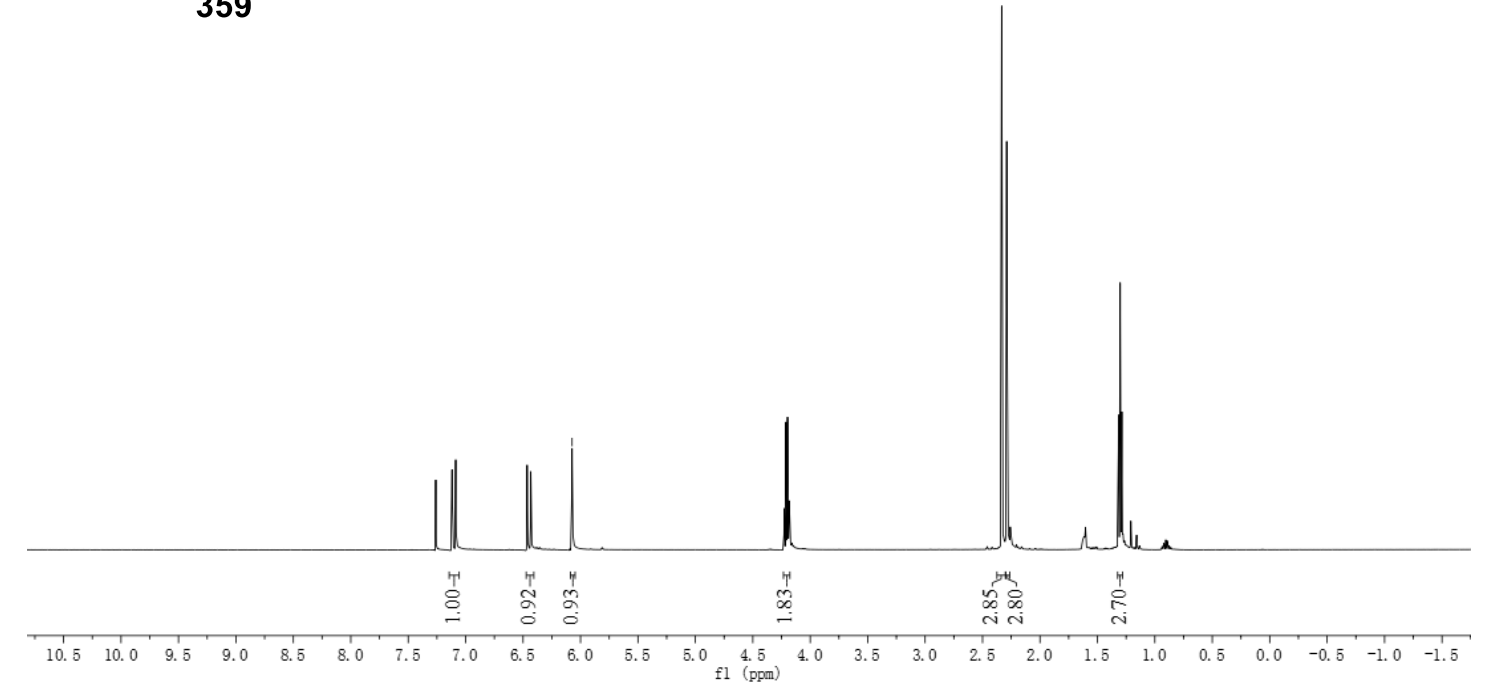

${ }^{13} \mathrm{C} \mathrm{NMR}\left(125 \mathrm{MHz}, \mathrm{CDCl}_{3}\right)$

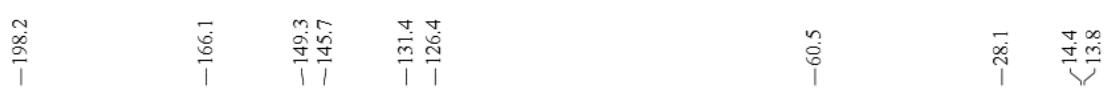<smiles>CCOC(=O)/C=C(C)/C=C/C(C)=O</smiles>

359

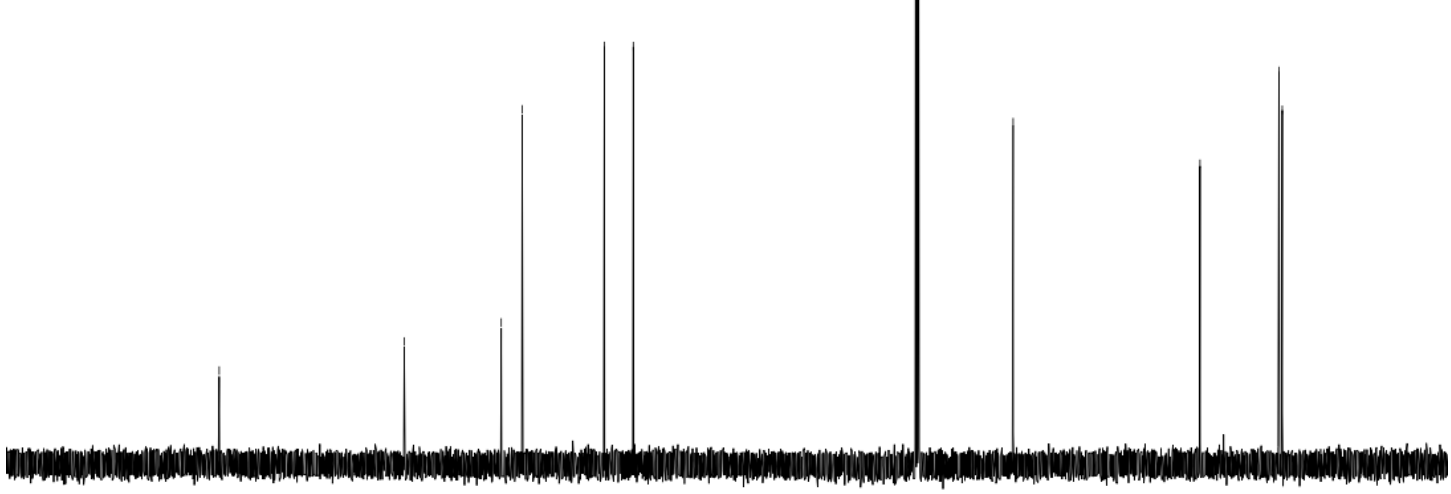

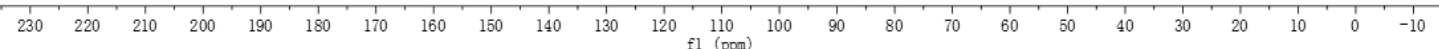


${ }^{1} \mathrm{H}$ NMR (500 MHz, $\mathrm{CDCl}_{3}$ )
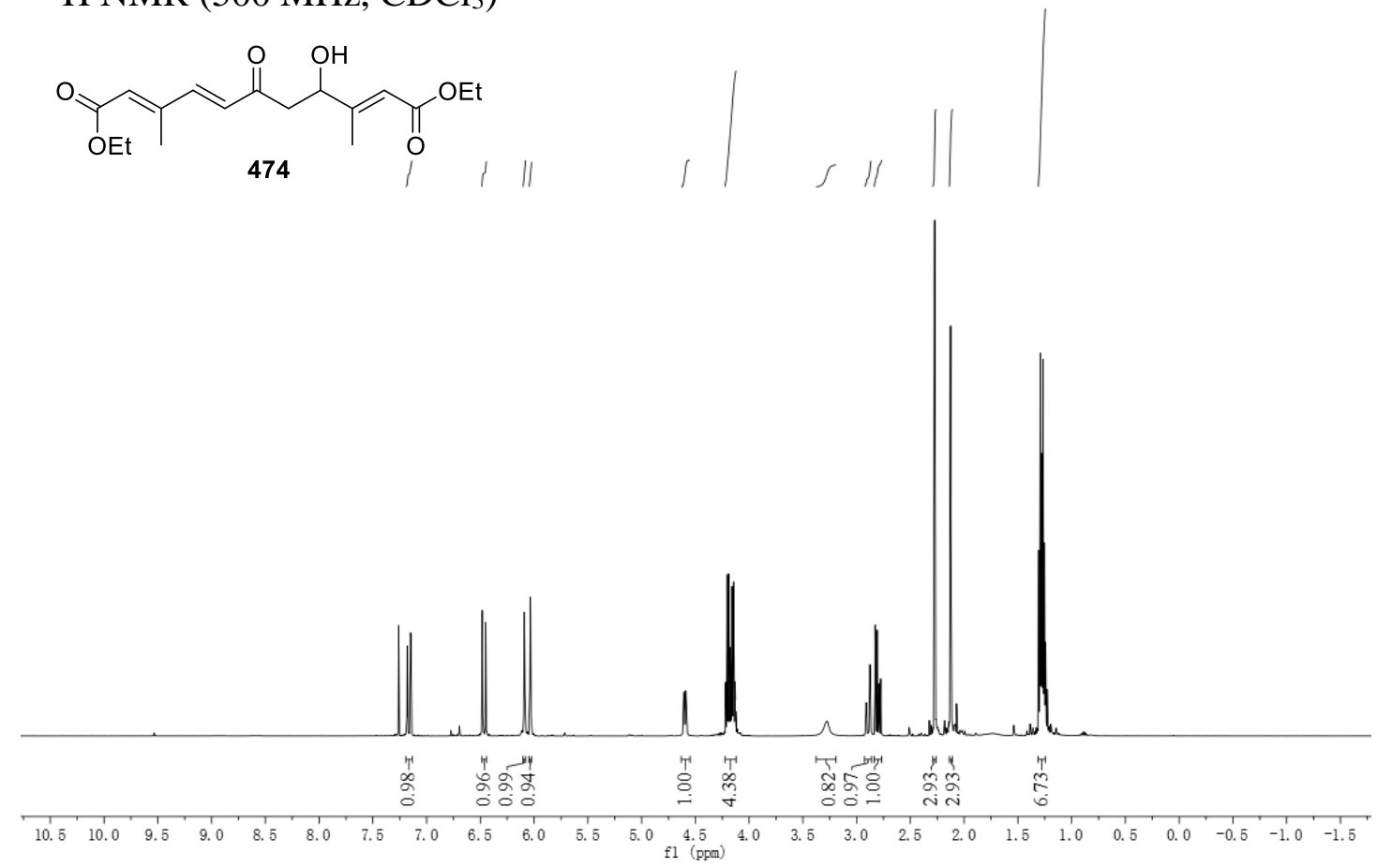

${ }^{13} \mathrm{C} \mathrm{NMR}\left(125 \mathrm{MHz}, \mathrm{CDCl}_{3}\right)$
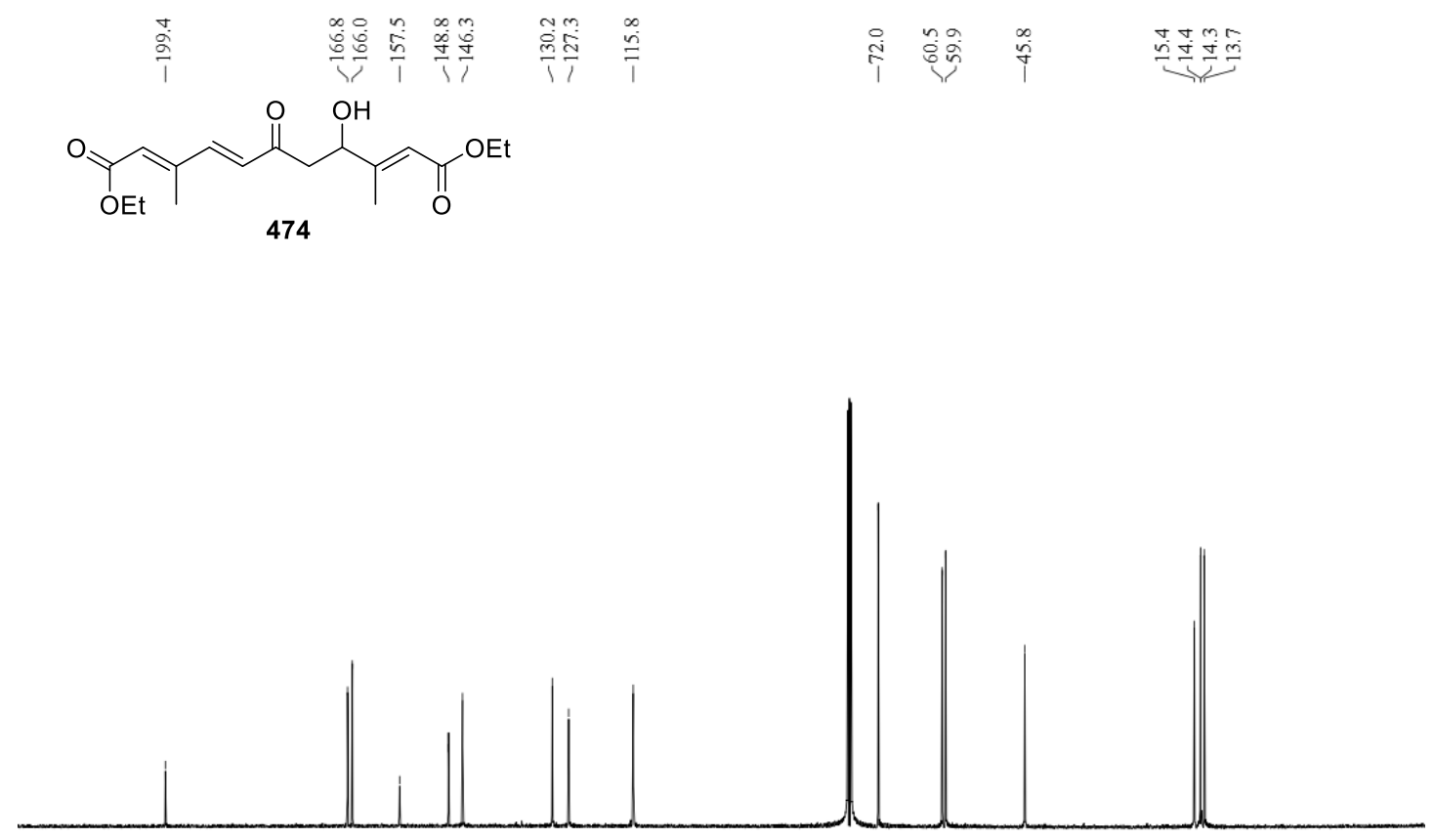

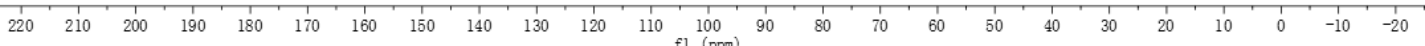


${ }^{1} \mathrm{H}$ NMR $\left(500 \mathrm{MHz}, \mathrm{CDCl}_{3}\right)$

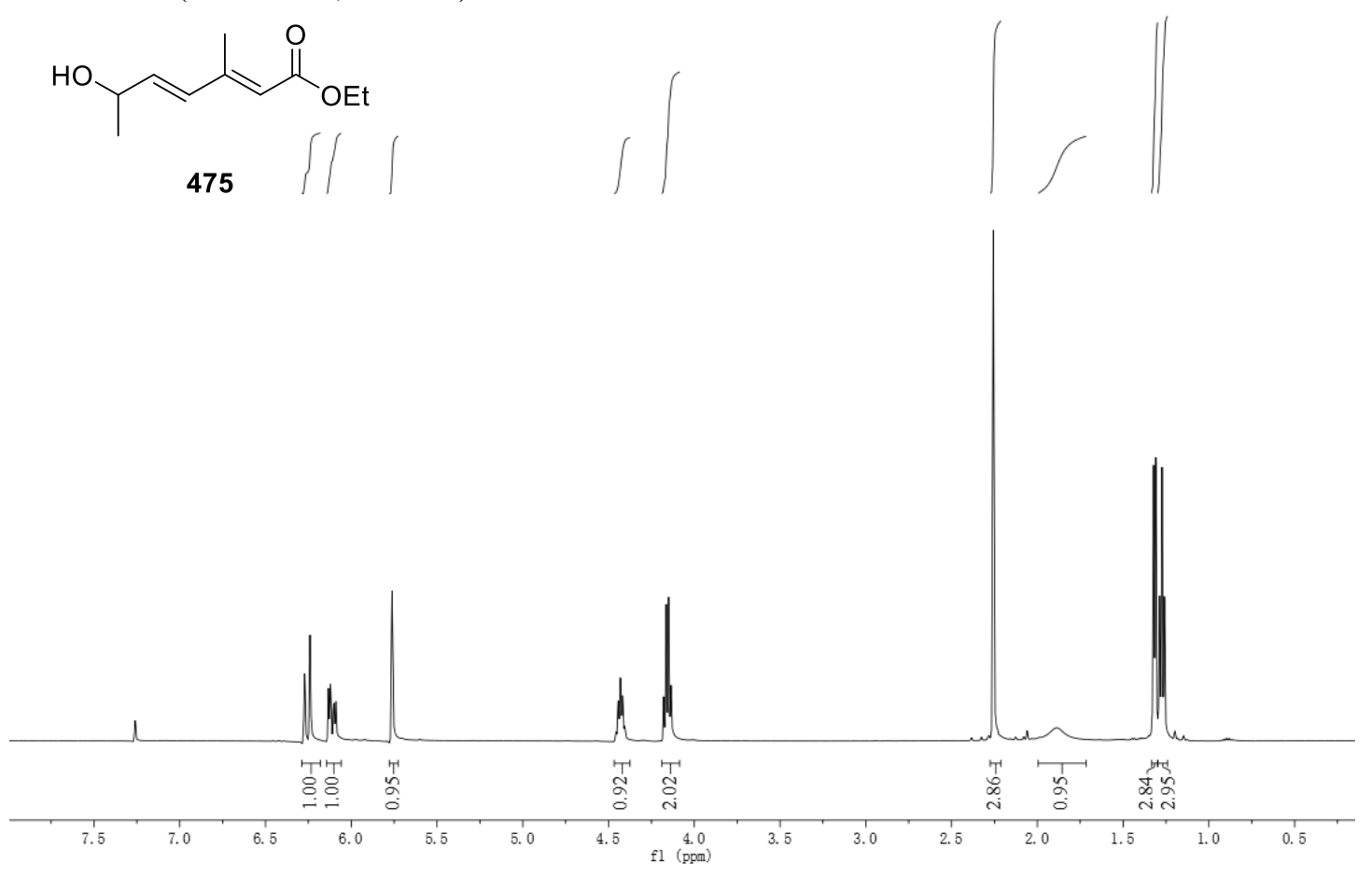

${ }^{13} \mathrm{C}$ NMR $\left(125 \mathrm{MHz}, \mathrm{CDCl}_{3}\right)$

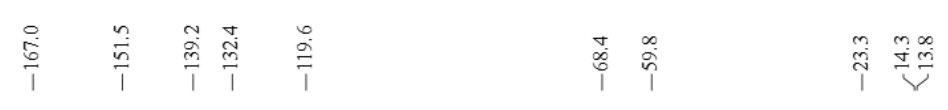<smiles>CCOC(=O)/C=C(C)/C=C/C(C)O</smiles>

475

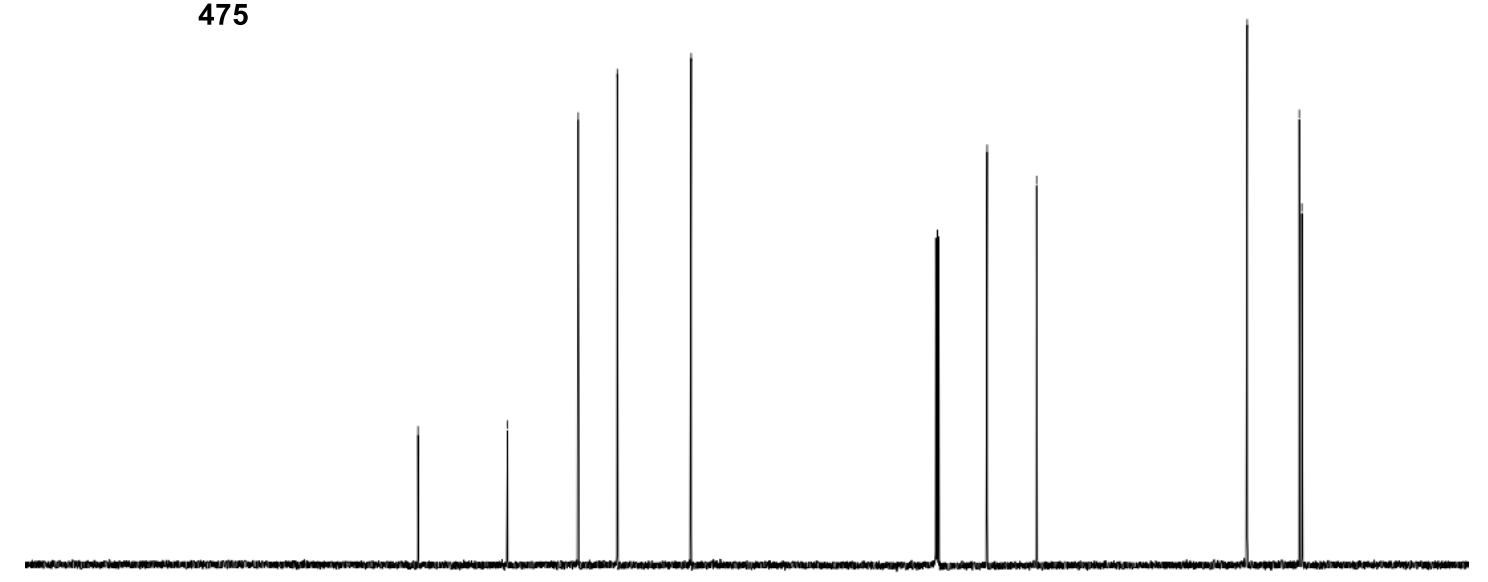

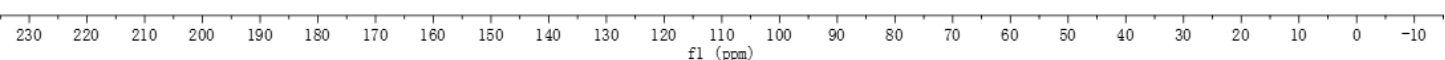


${ }^{1} \mathrm{H}$ NMR $\left(500 \mathrm{MHz}, \mathrm{CDCl}_{3}\right)$

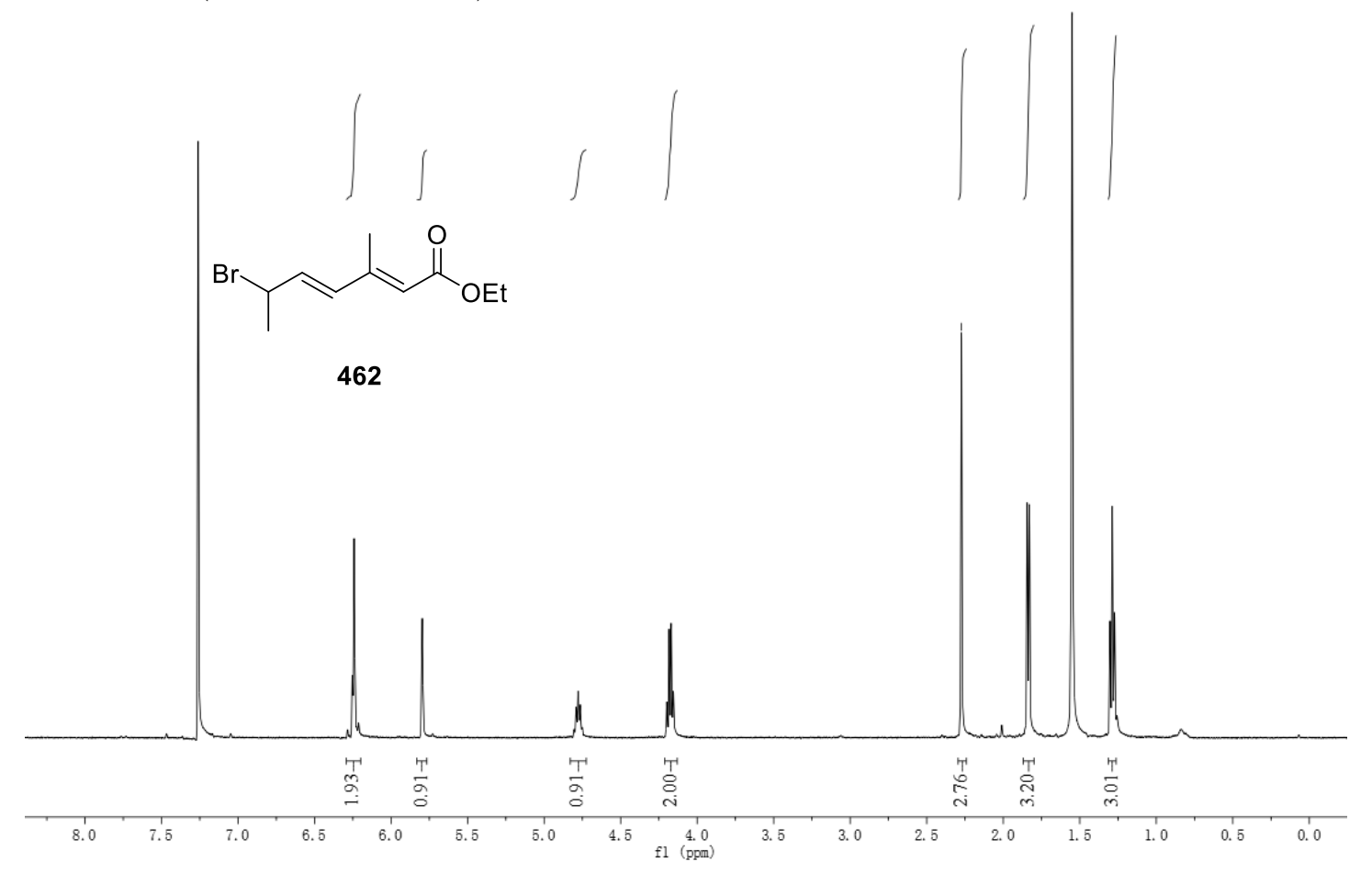

${ }^{13} \mathrm{C}$ NMR $\left(125 \mathrm{MHz}, \mathrm{CDCl}_{3}\right)$

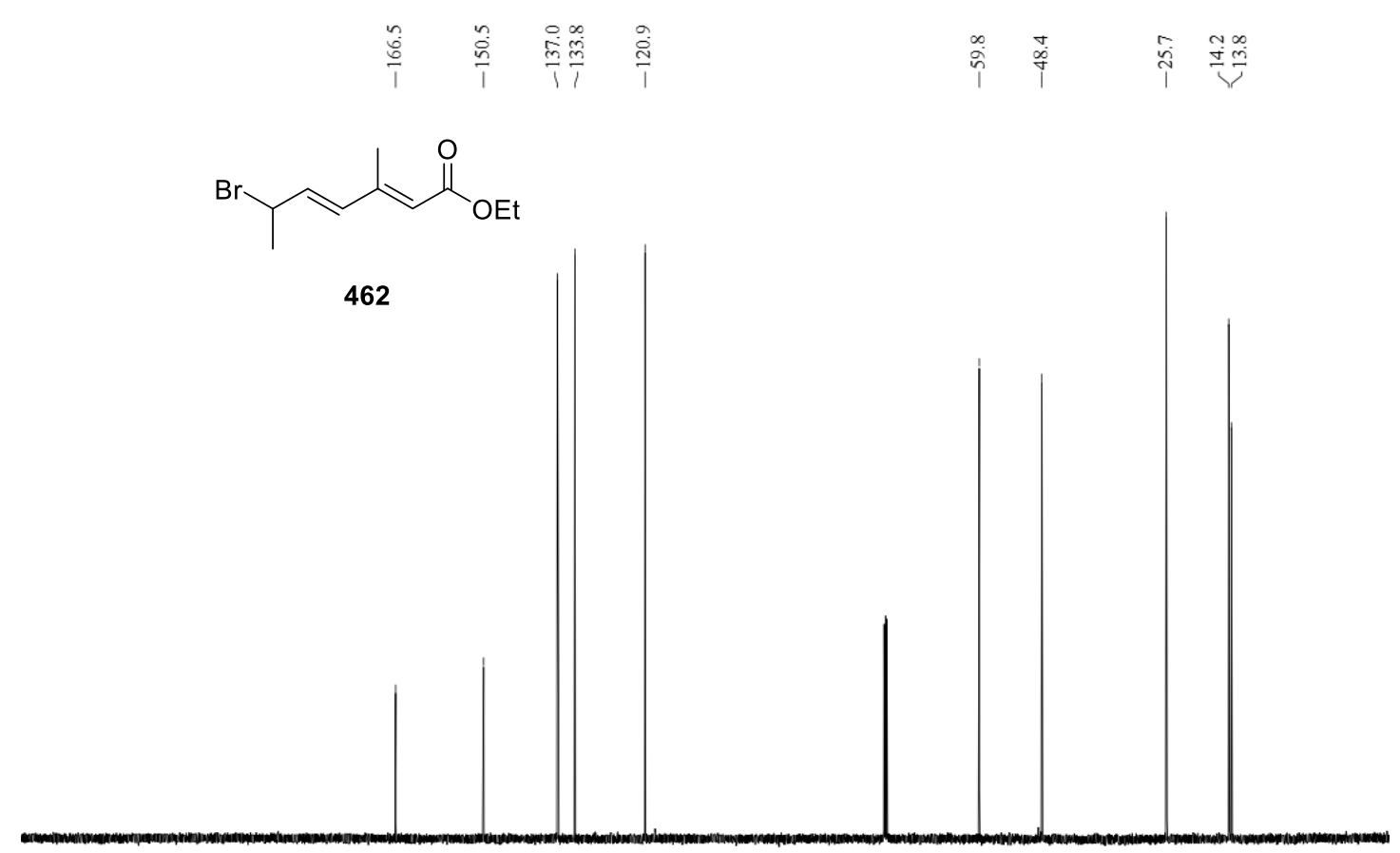

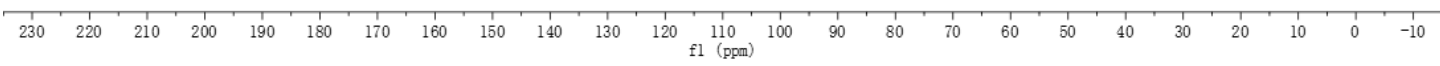


${ }^{1} \mathrm{H} \mathrm{NMR}\left(500 \mathrm{MHz}, \mathrm{CDCl}_{3}\right)$<smiles>CCOC(=O)/C=C(C)/C=C/C(C)[Pb](Br)c1ccccc1</smiles>

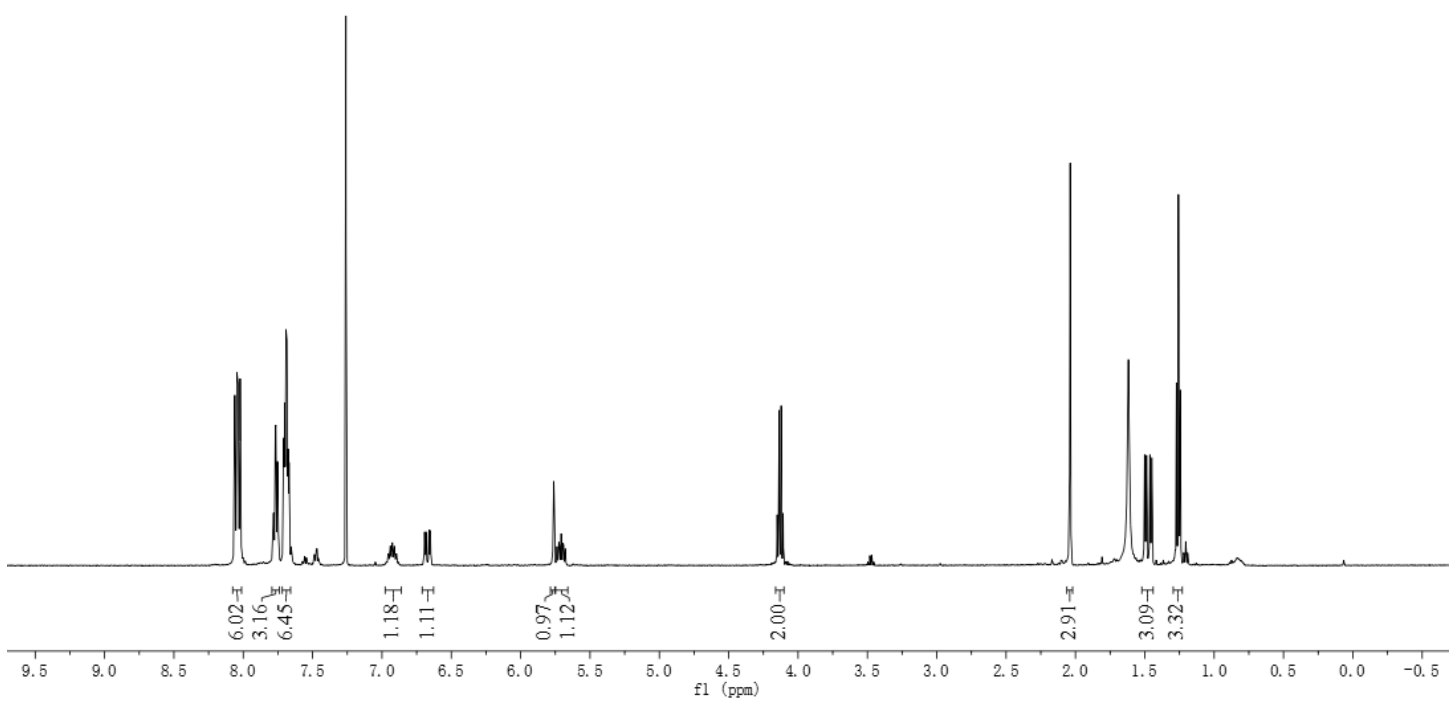

${ }^{13} \mathrm{C}$ NMR $\left(125 \mathrm{MHz}, \mathrm{CDCl}_{3}\right)$

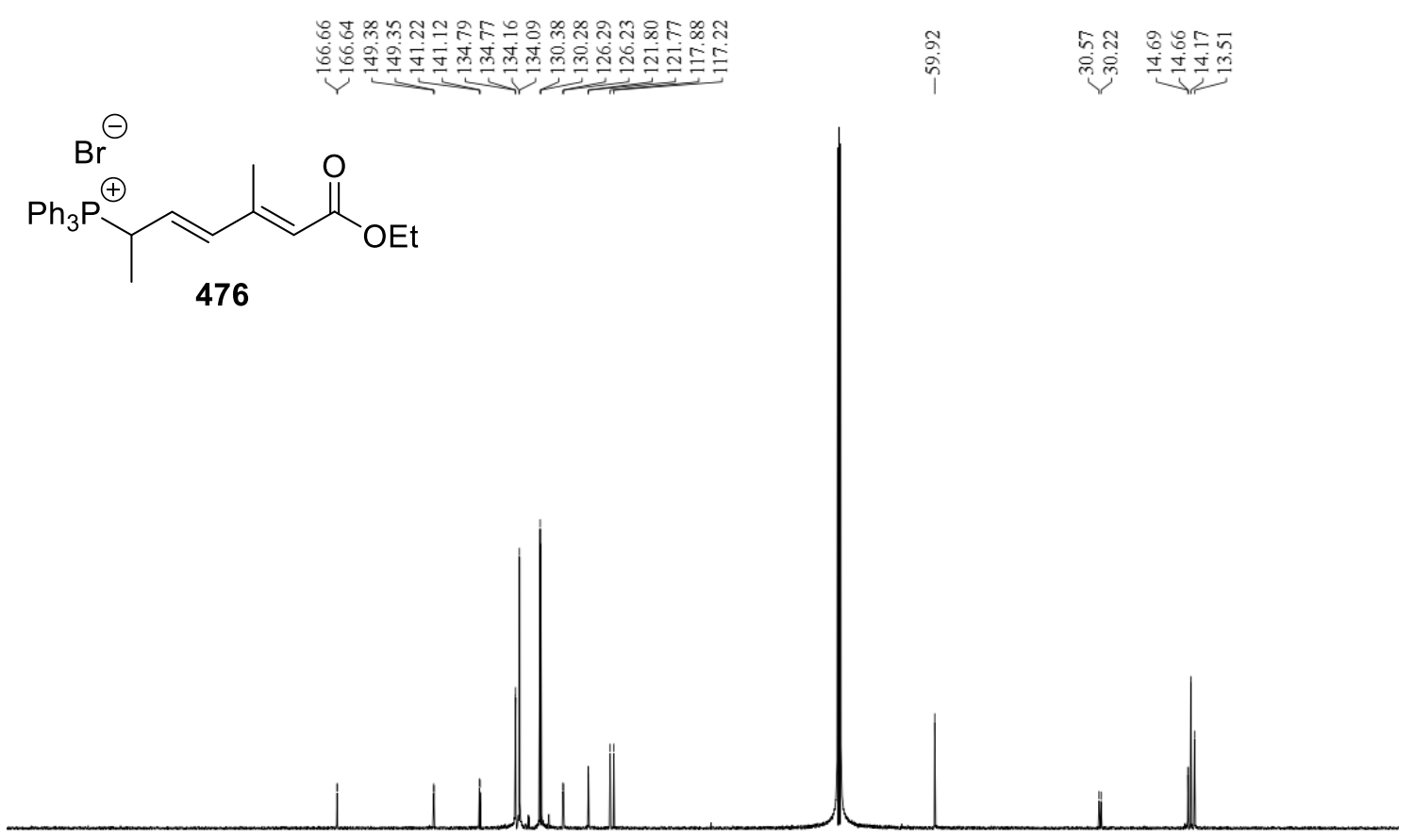

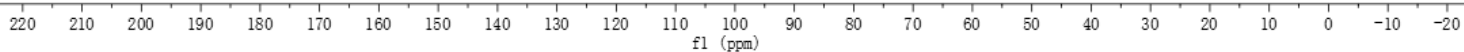


${ }^{1} \mathrm{H}$ NMR $\left(500 \mathrm{MHz}, \mathrm{CDCl}_{3}\right)$<smiles>CCOC(=O)/C=C(C)/C=C/C(C)=C/C1COC(C)(C)O1</smiles>

$E, E, E$-trienoate $\mathbf{4 7 7}$<smiles>CCOC(=O)/C=C(C)/C=C/C(C)=C\C1COC(C)(C)O1</smiles>

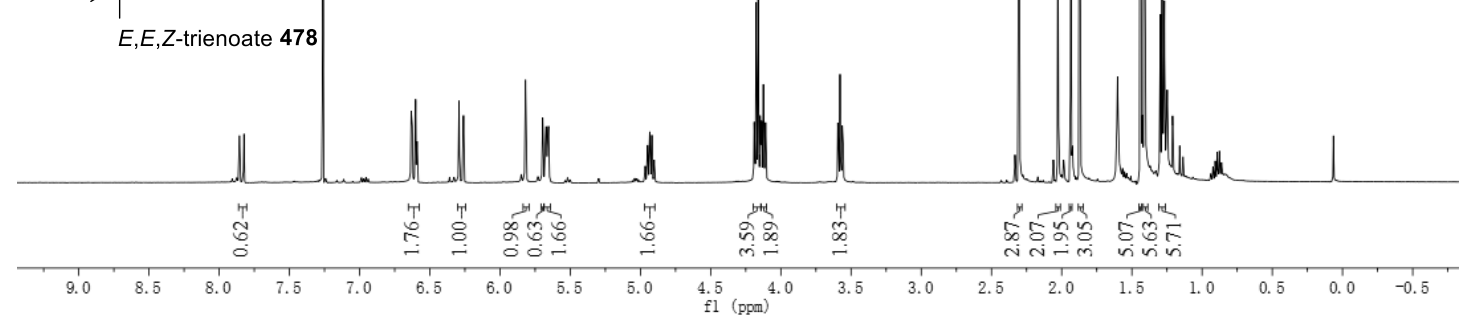

${ }^{13} \mathrm{C} \mathrm{NMR}\left(125 \mathrm{MHz}, \mathrm{CDCl}_{3}\right)$
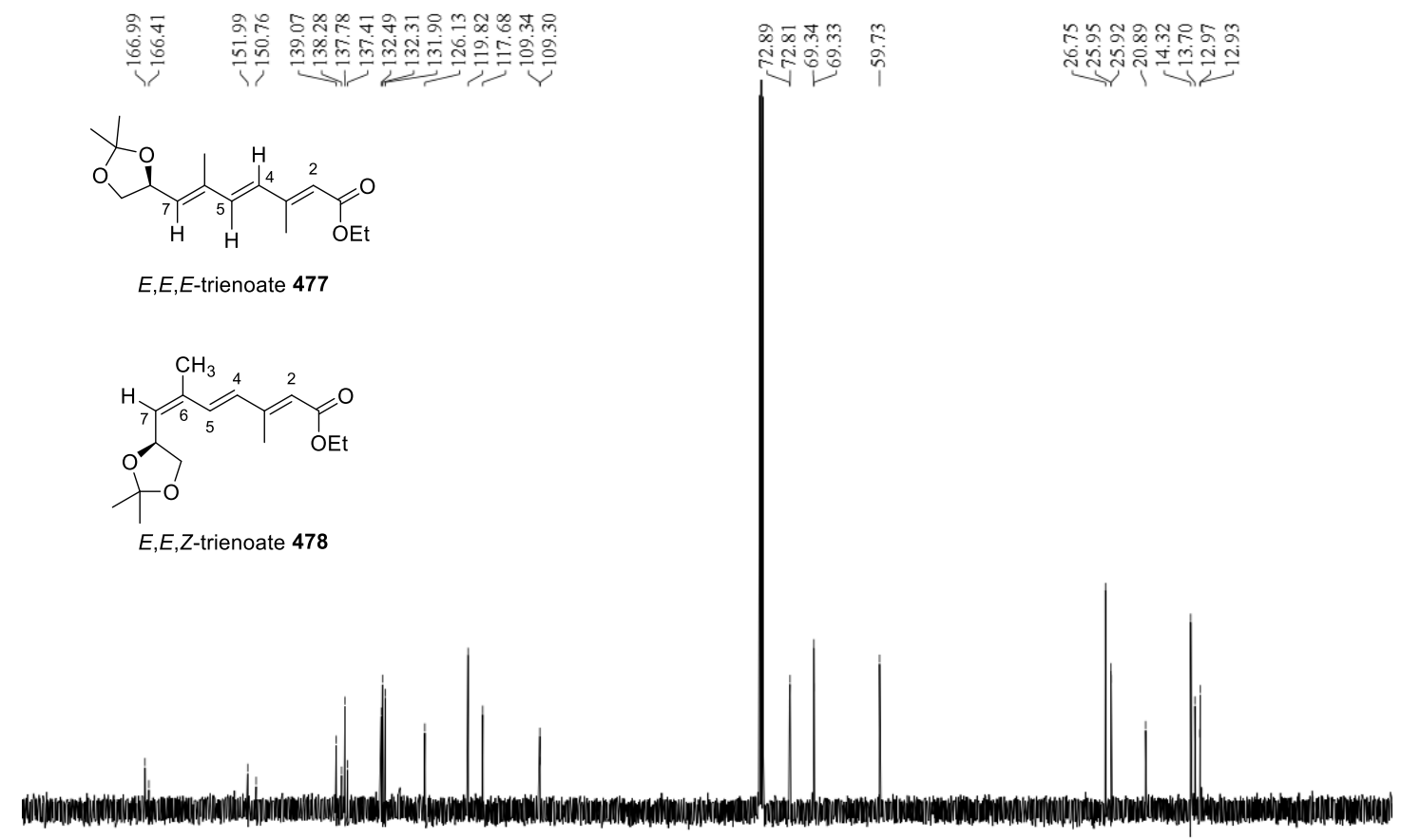

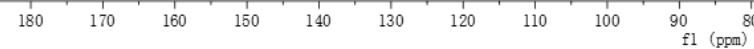


${ }^{1} \mathrm{H}$ NMR $\left(500 \mathrm{MHz}, \mathrm{CDCl}_{3}\right)$

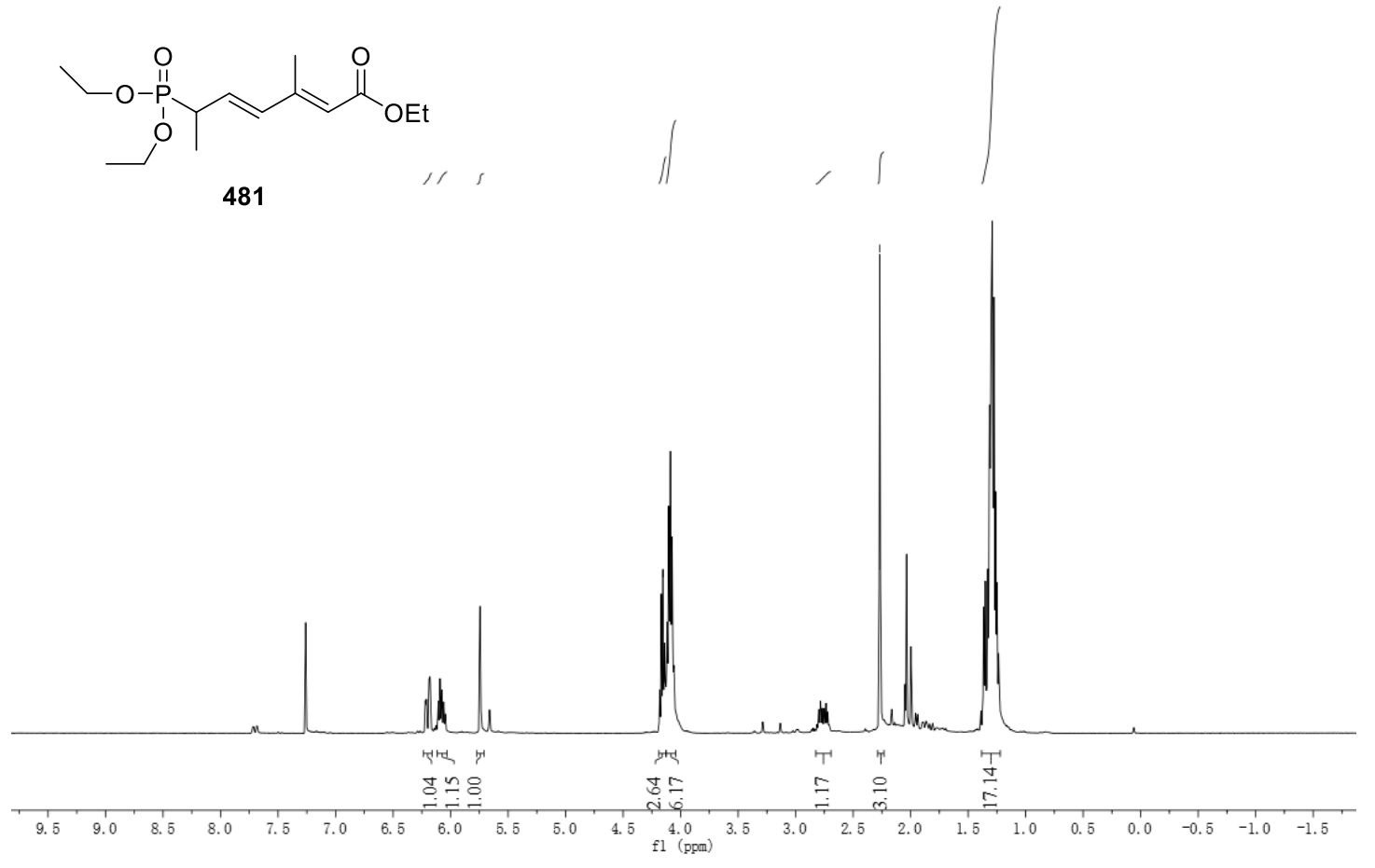

${ }^{13} \mathrm{C} \mathrm{NMR}\left(125 \mathrm{MHz}, \mathrm{CDCl}_{3}\right)$

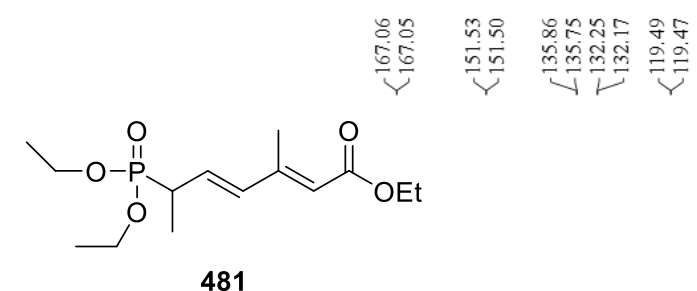

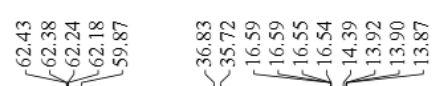

481

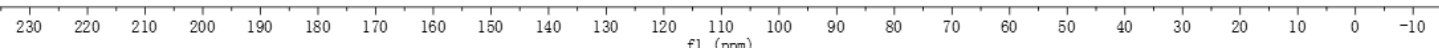


${ }^{1} \mathrm{H} \mathrm{NMR}\left(500 \mathrm{MHz}, \mathrm{CDCl}_{3}\right)$

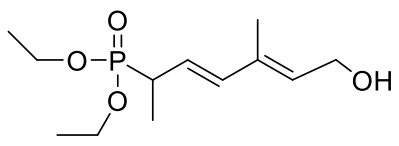

482
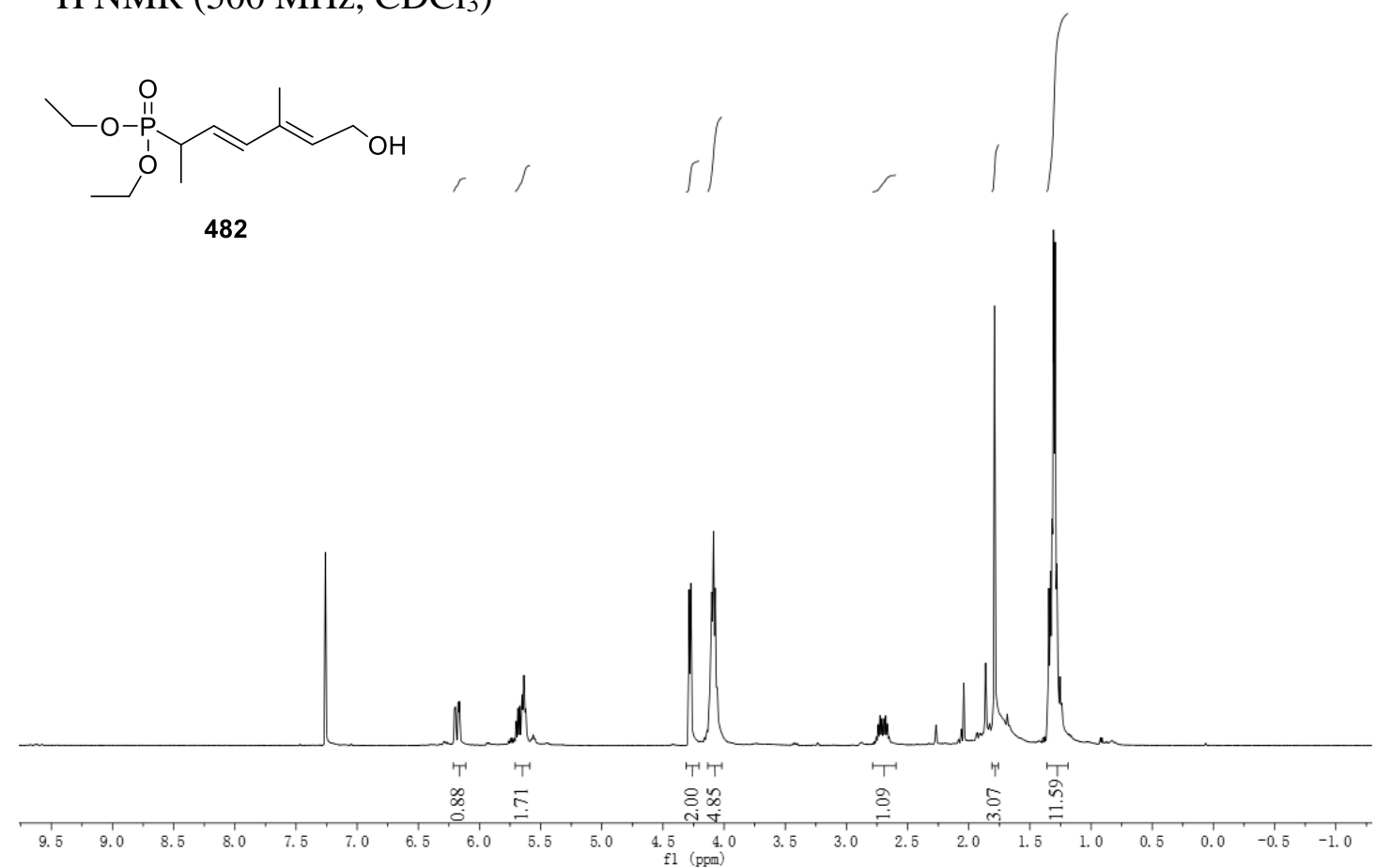

${ }^{13} \mathrm{C} \mathrm{NMR}\left(125 \mathrm{MHz}, \mathrm{CDCl}_{3}\right)$

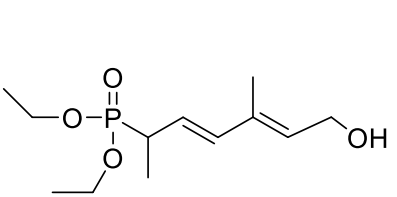

482
ซํㅠ용ำ

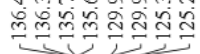

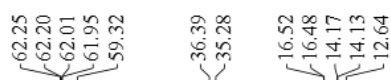

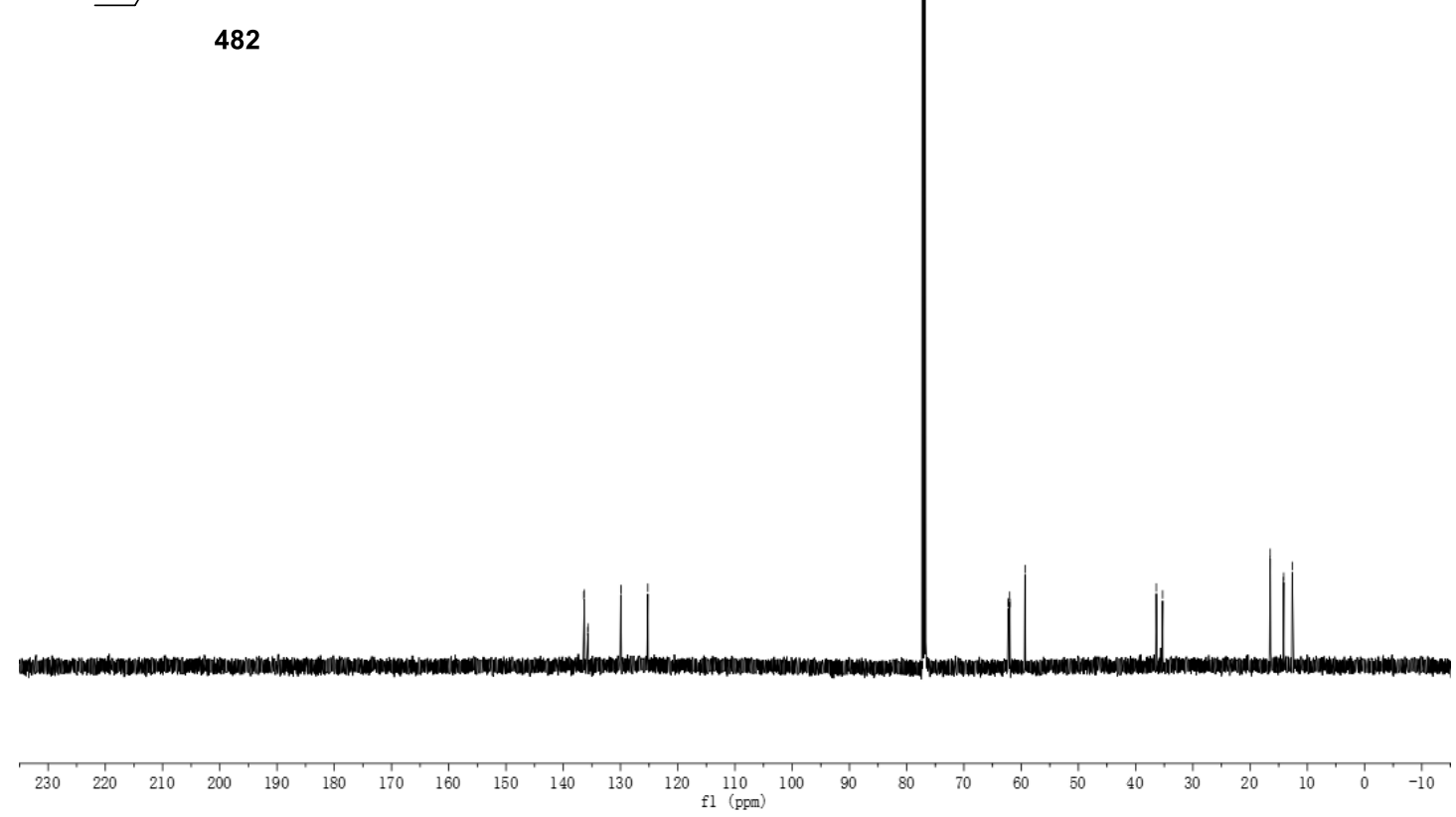


${ }^{1} \mathrm{H}$ NMR $\left(500 \mathrm{MHz}, \mathrm{CDCl}_{3}\right)$

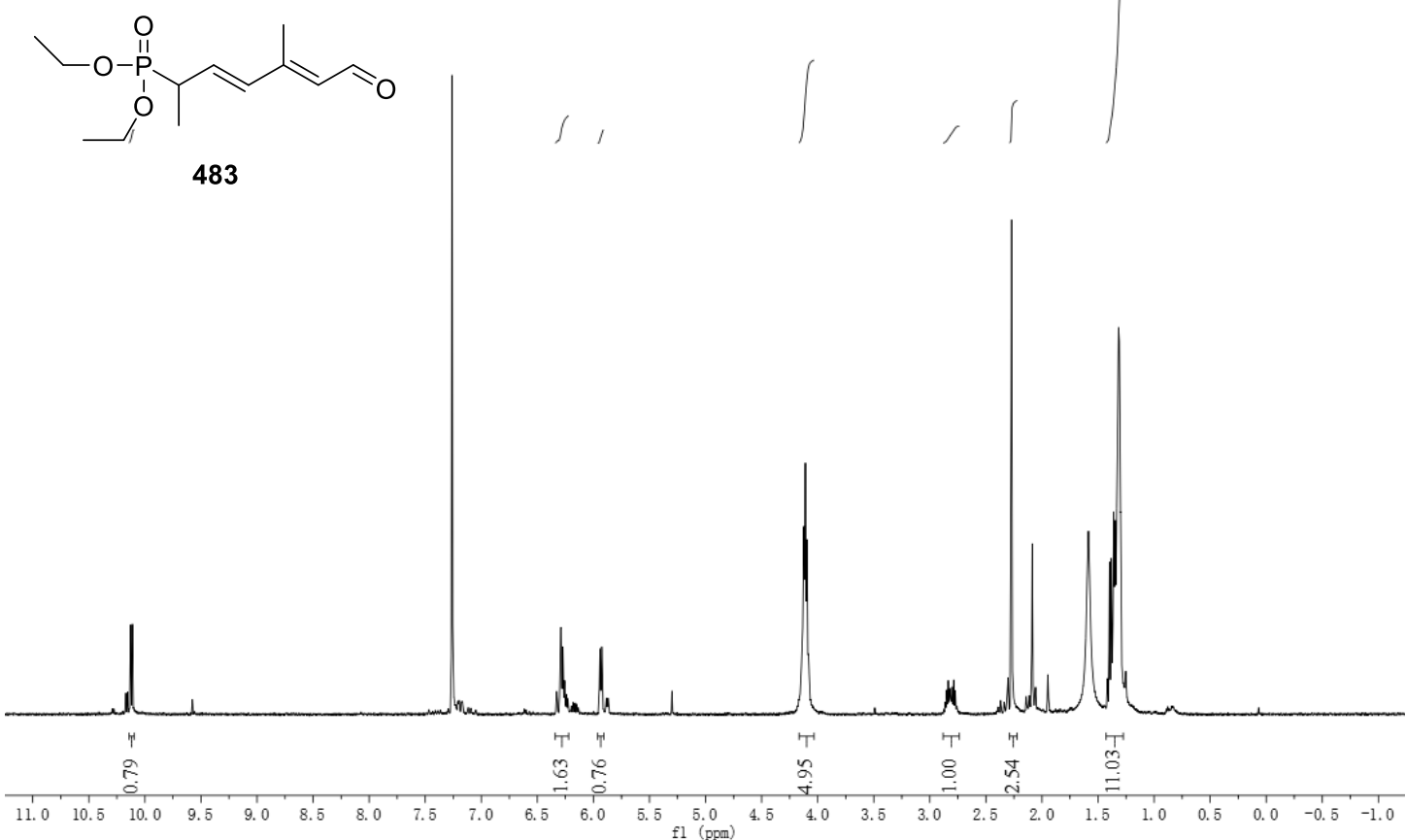

${ }^{13} \mathrm{C}$ NMR $\left(125 \mathrm{MHz}, \mathrm{CDCl}_{3}\right)$

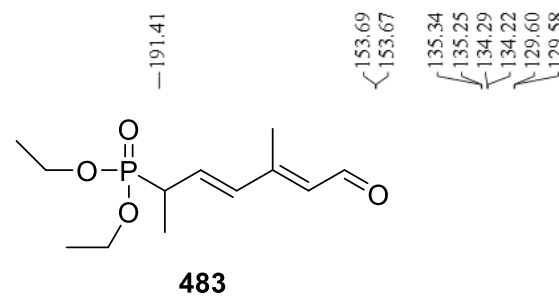

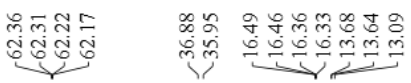

483

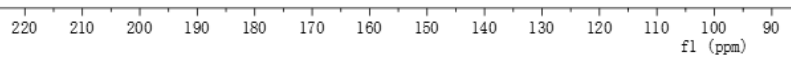


${ }^{1} \mathrm{H}$ NMR $\left(500 \mathrm{MHz}, \mathrm{CDCl}_{3}\right)$
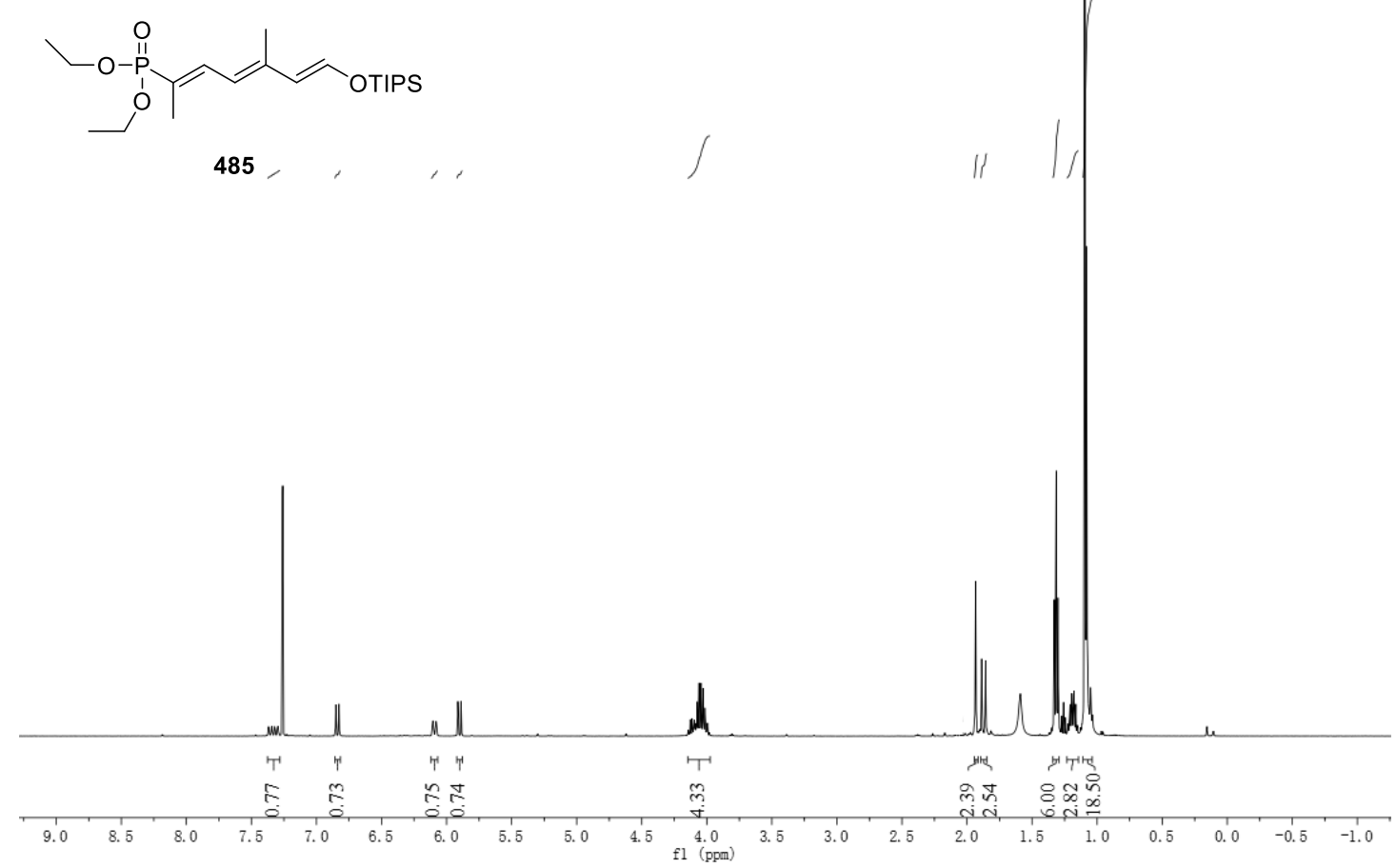

${ }^{13} \mathrm{C} \mathrm{NMR}\left(125 \mathrm{MHz}, \mathrm{CDCl}_{3}\right)$

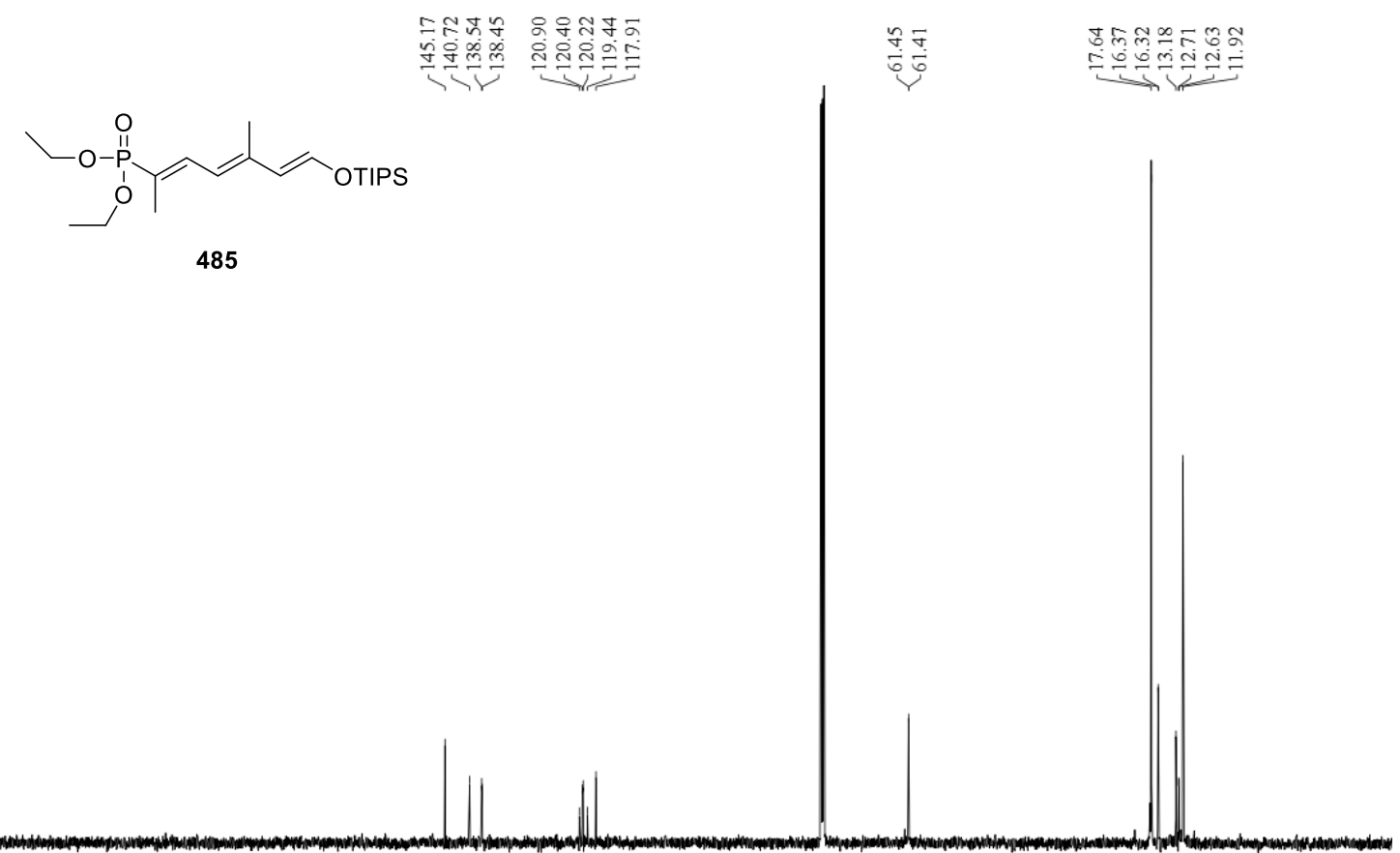

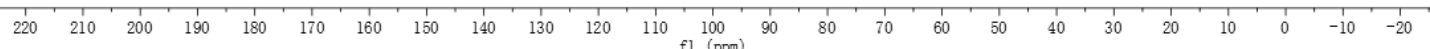




\section{Spectra of Chapter Four}

${ }^{1} \mathrm{H}$ NMR (500 MHz, $\left.\mathrm{CDCl}_{3}\right)$

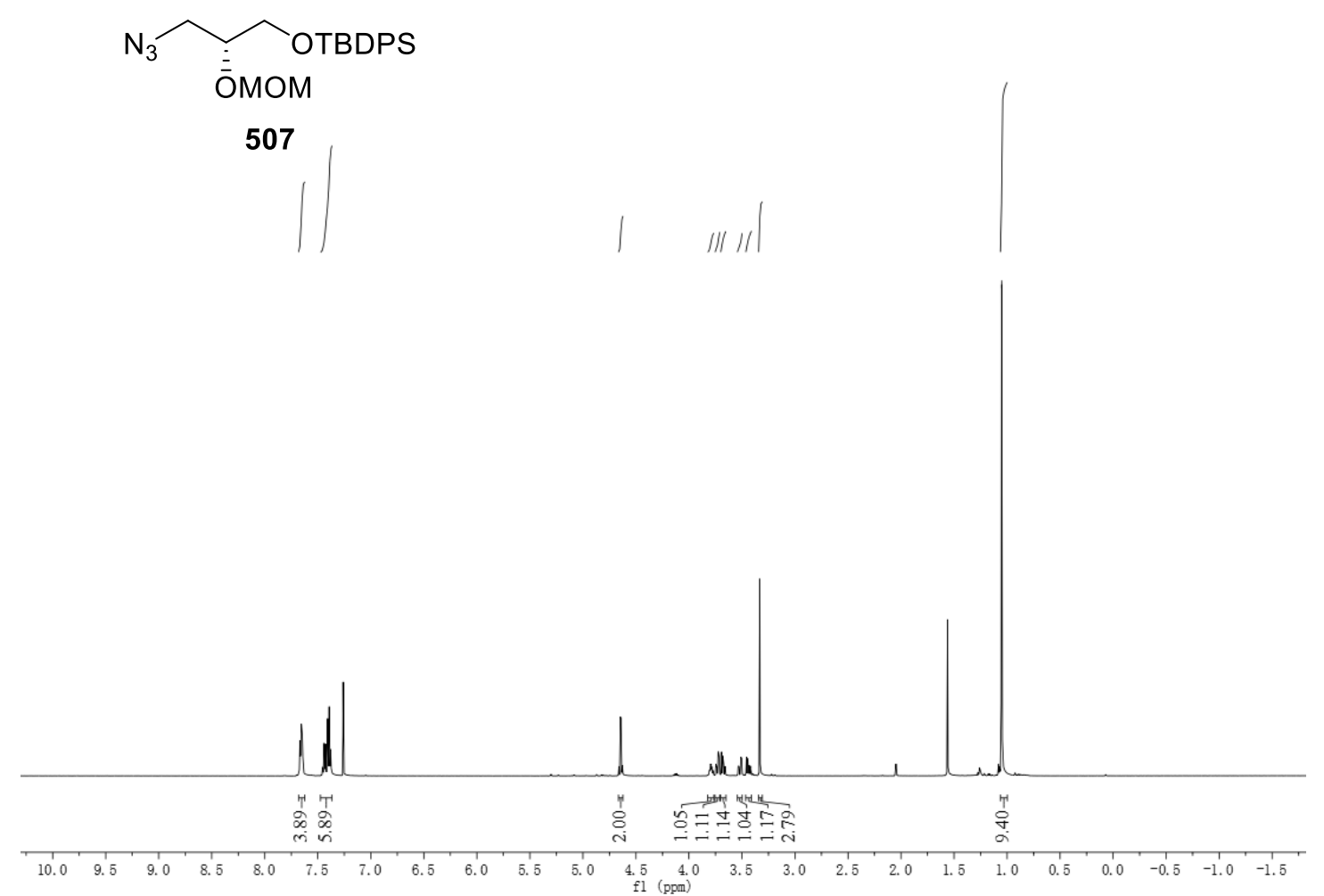

${ }^{13} \mathrm{C} \mathrm{NMR}\left(125 \mathrm{MHz}, \mathrm{CDCl}_{3}\right)$

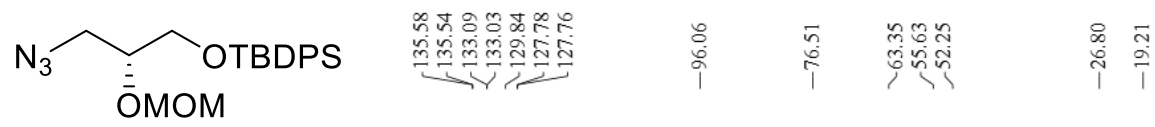

507

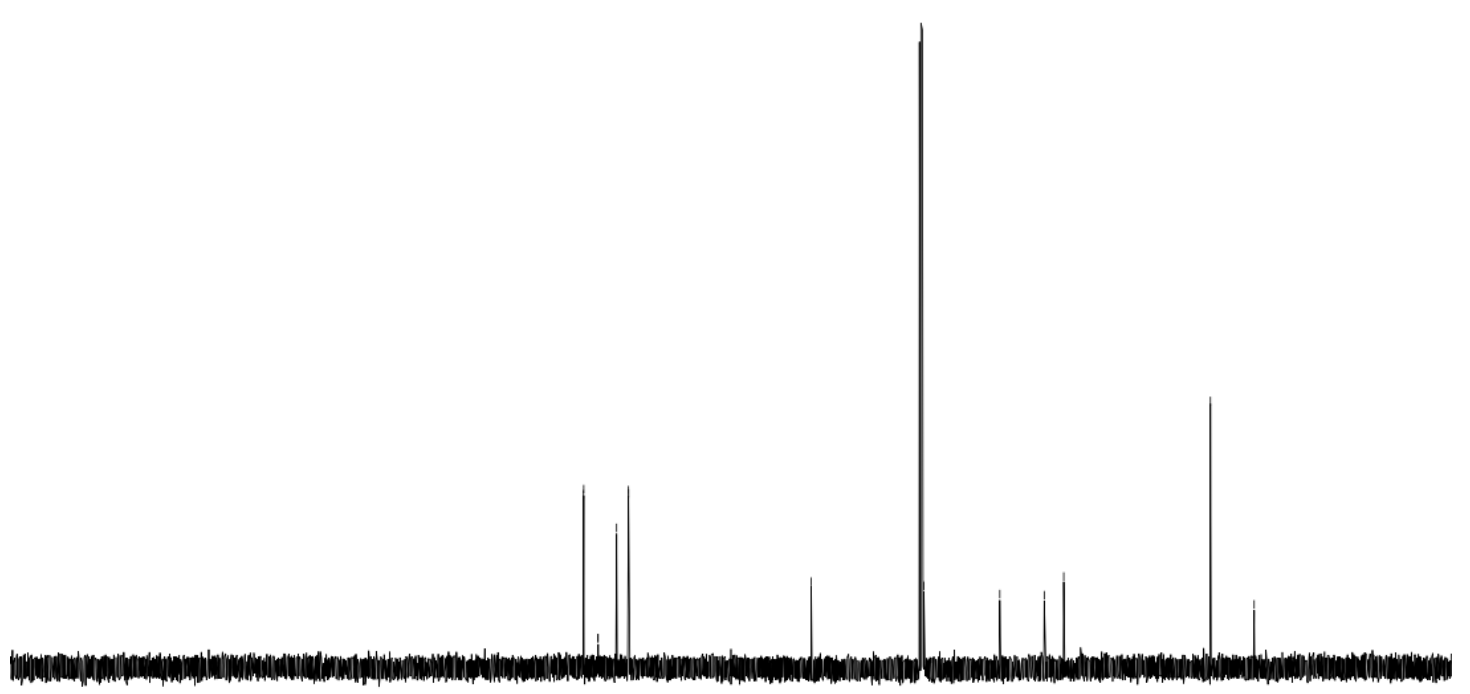

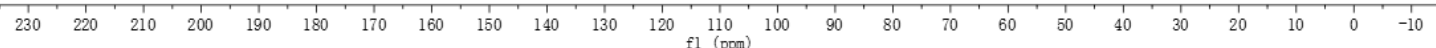


${ }^{1} \mathrm{H}$ NMR (500 MHz, $\mathrm{CDCl}_{3}$ )<smiles>CO[C@H](CN)CO</smiles>
508

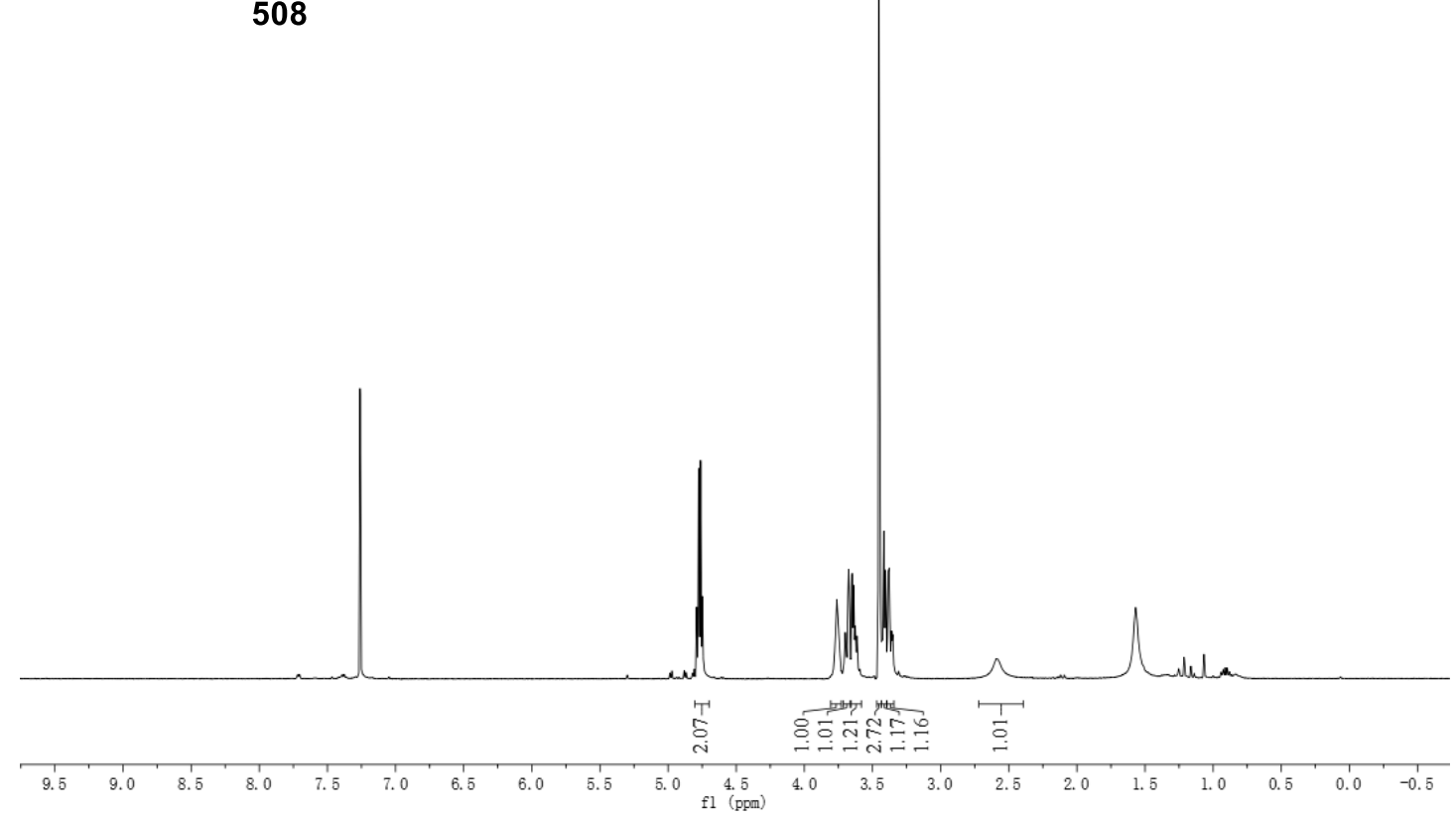

${ }^{13} \mathrm{C} \mathrm{NMR}\left(125 \mathrm{MHz}, \mathrm{CDCl}_{3}\right)$<smiles>NC[C@@H](CO)O[Na]</smiles> 

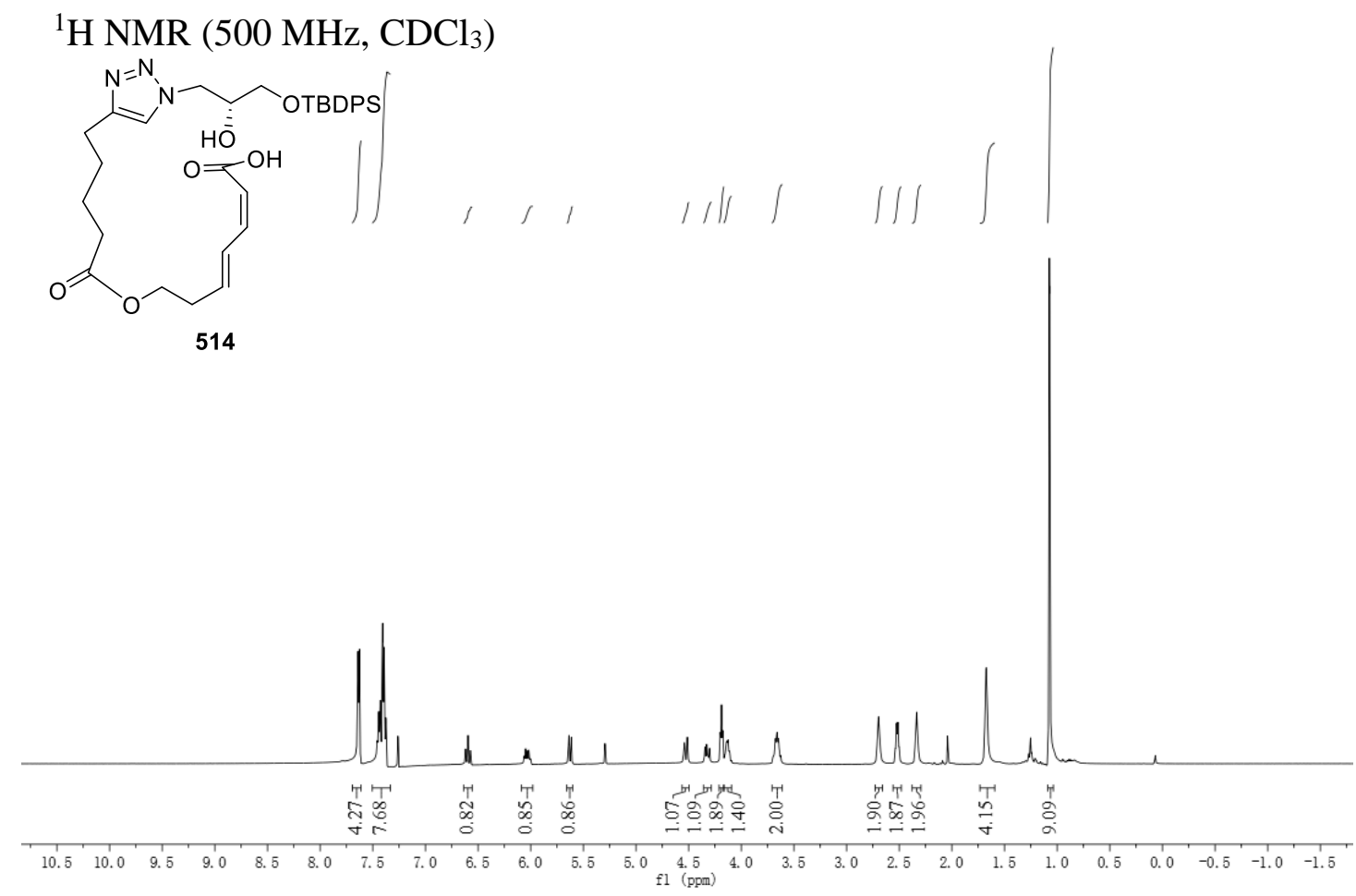

${ }^{13} \mathrm{C}$ NMR $\left(125 \mathrm{MHz}, \mathrm{CDCl}_{3}\right)$

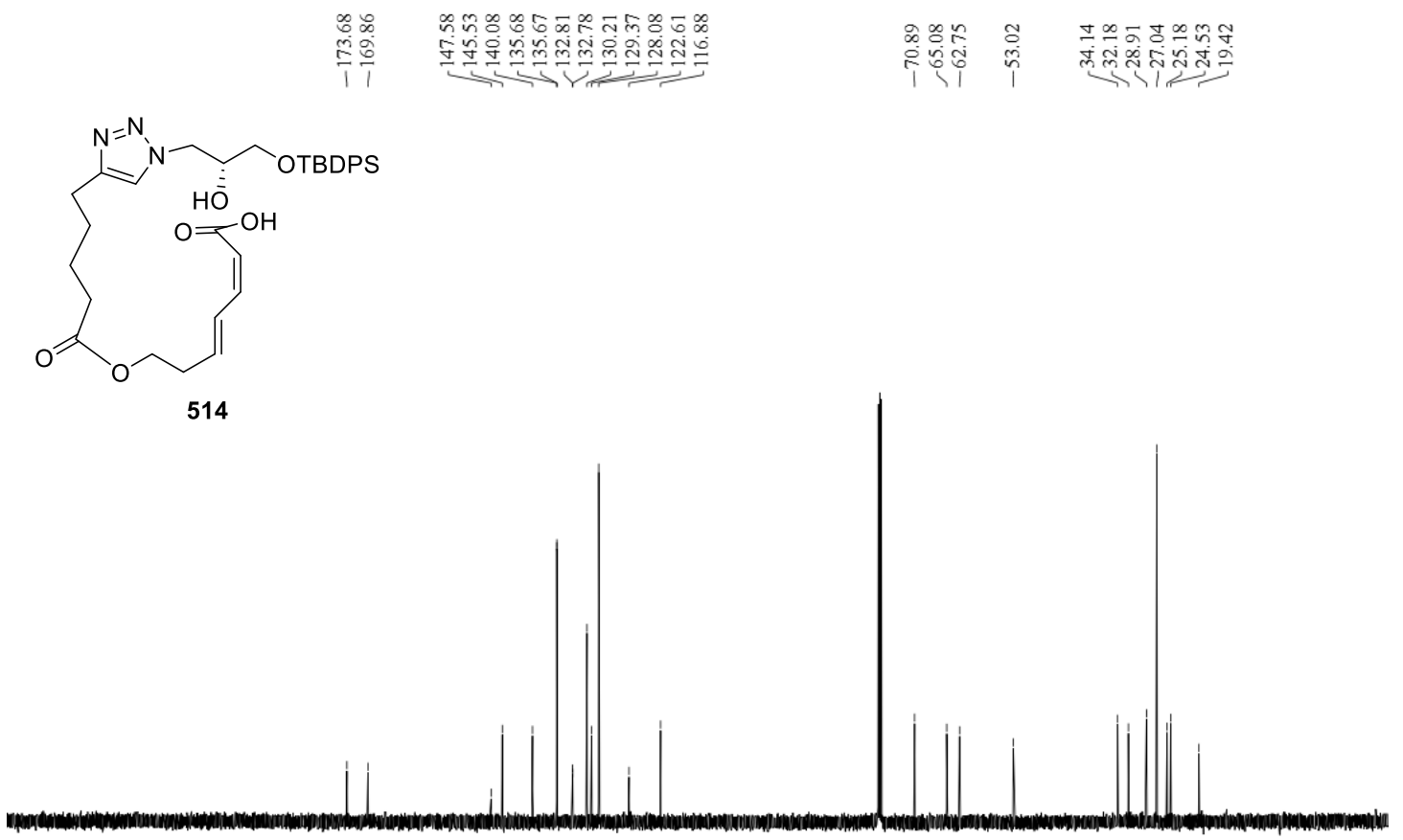

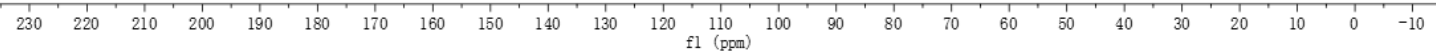


${ }^{1} \mathrm{H}$ NMR $\left(500 \mathrm{MHz}, \mathrm{CDCl}_{3}\right)$

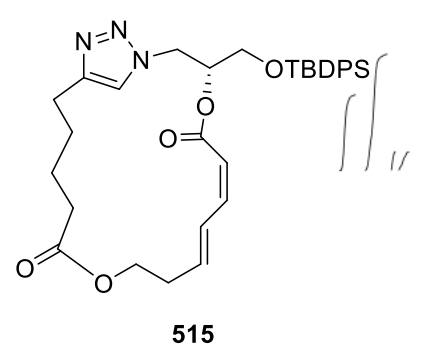
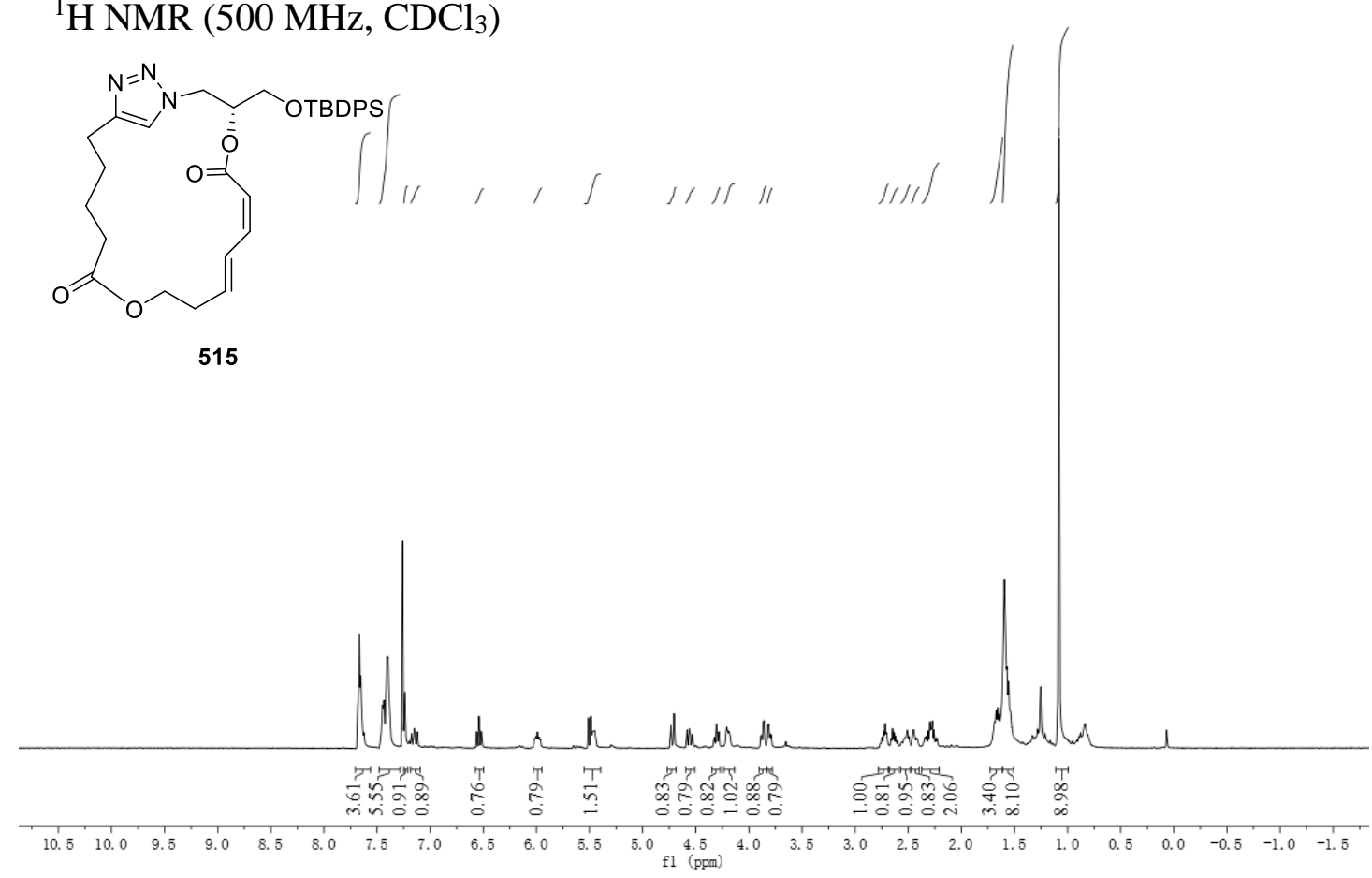

${ }^{13} \mathrm{C}$ NMR (125 MHz, $\left.\mathrm{CDCl}_{3}\right)$

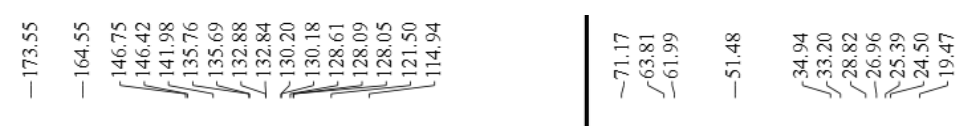

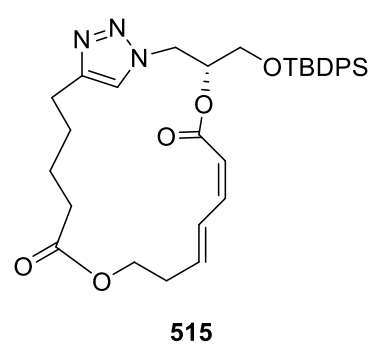

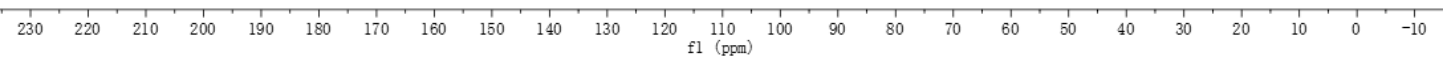


${ }^{1} \mathrm{H} \mathrm{NMR}\left(500 \mathrm{MHz}, \mathrm{CDCl}_{3}\right.$ )
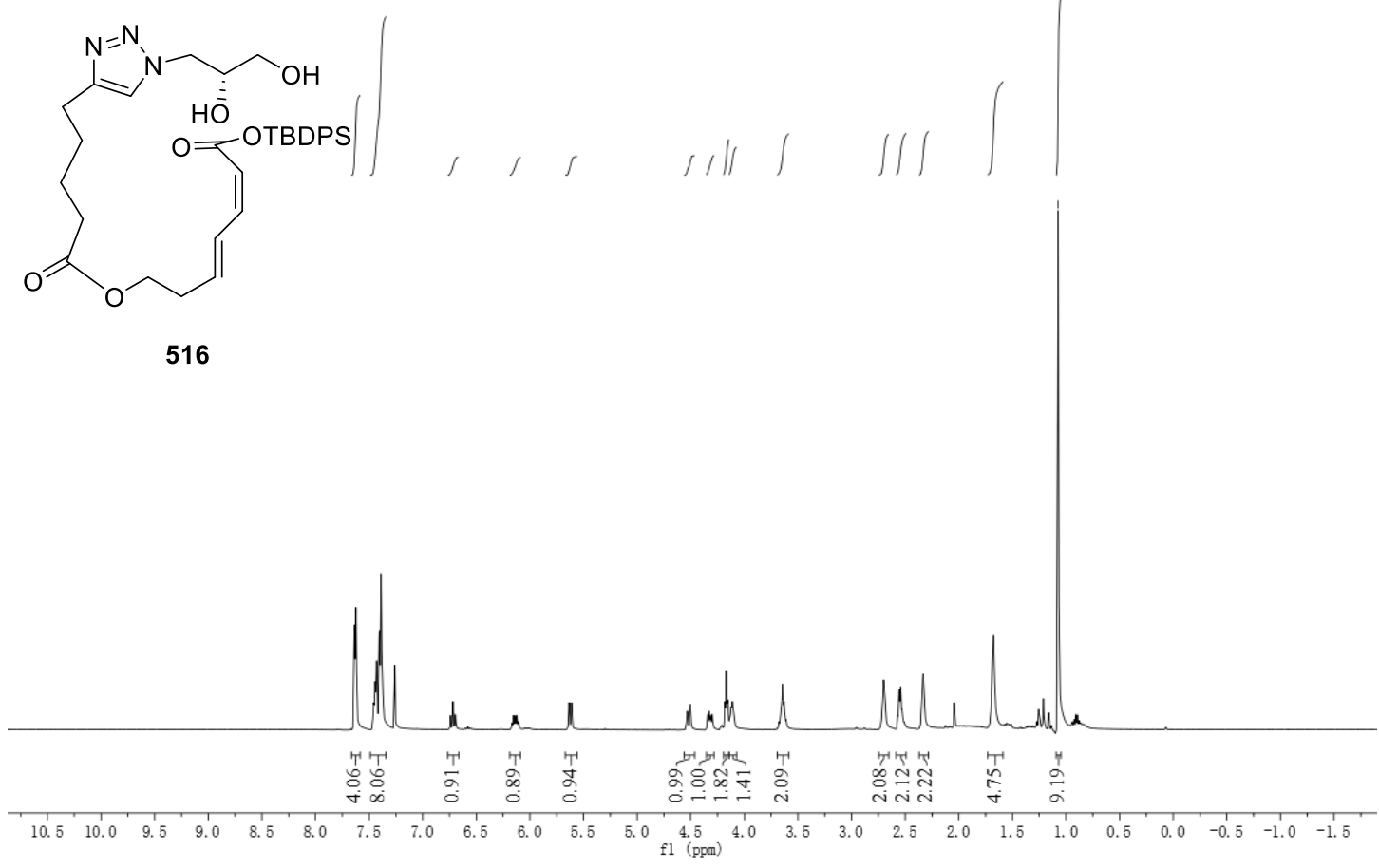

${ }^{13} \mathrm{C} \mathrm{NMR}\left(125 \mathrm{MHz}, \mathrm{CDCl}_{3}\right)$
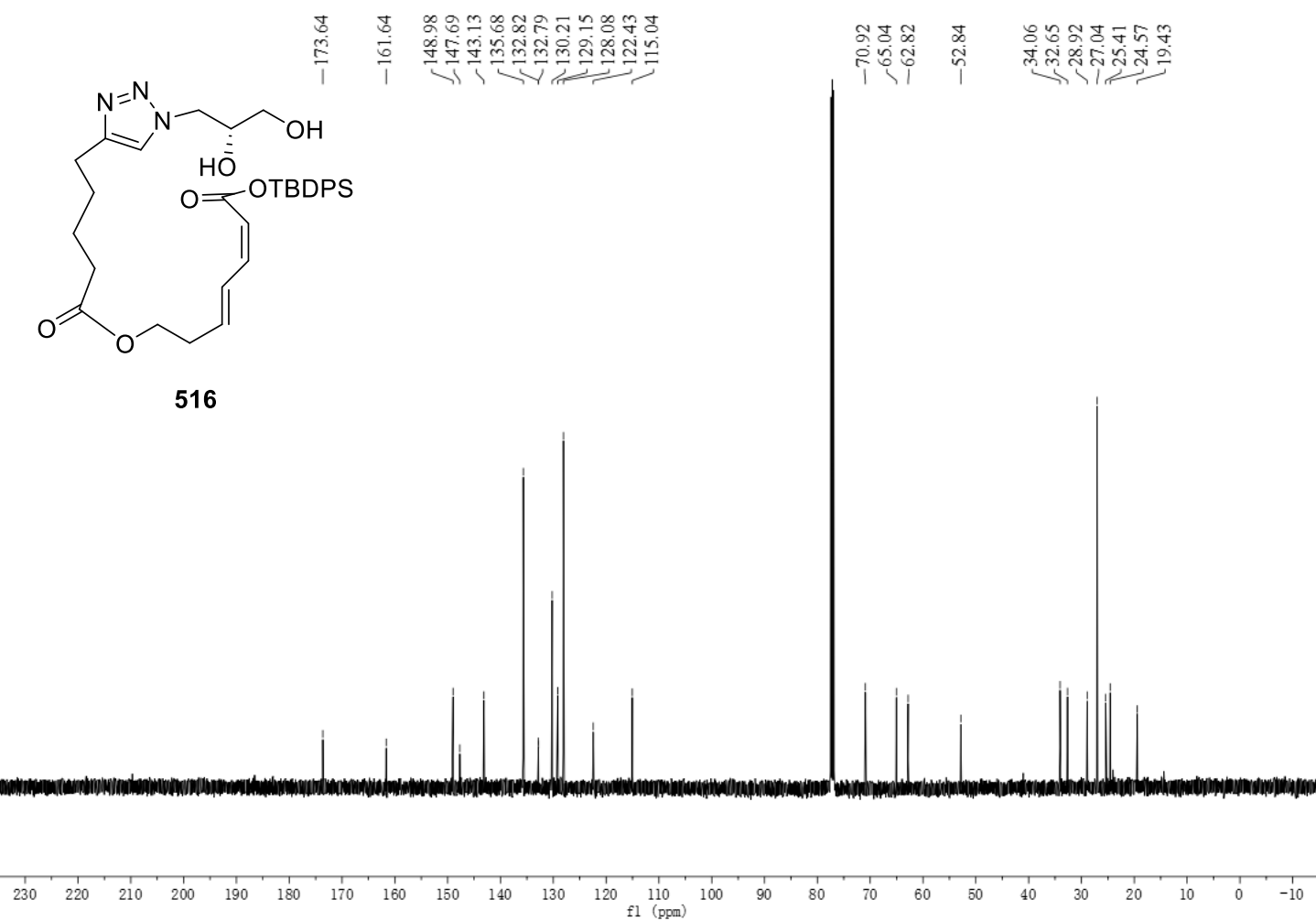
${ }^{1} \mathrm{H}$ NMR $\left(500 \mathrm{MHz}, \mathrm{CDCl}_{3}\right)$

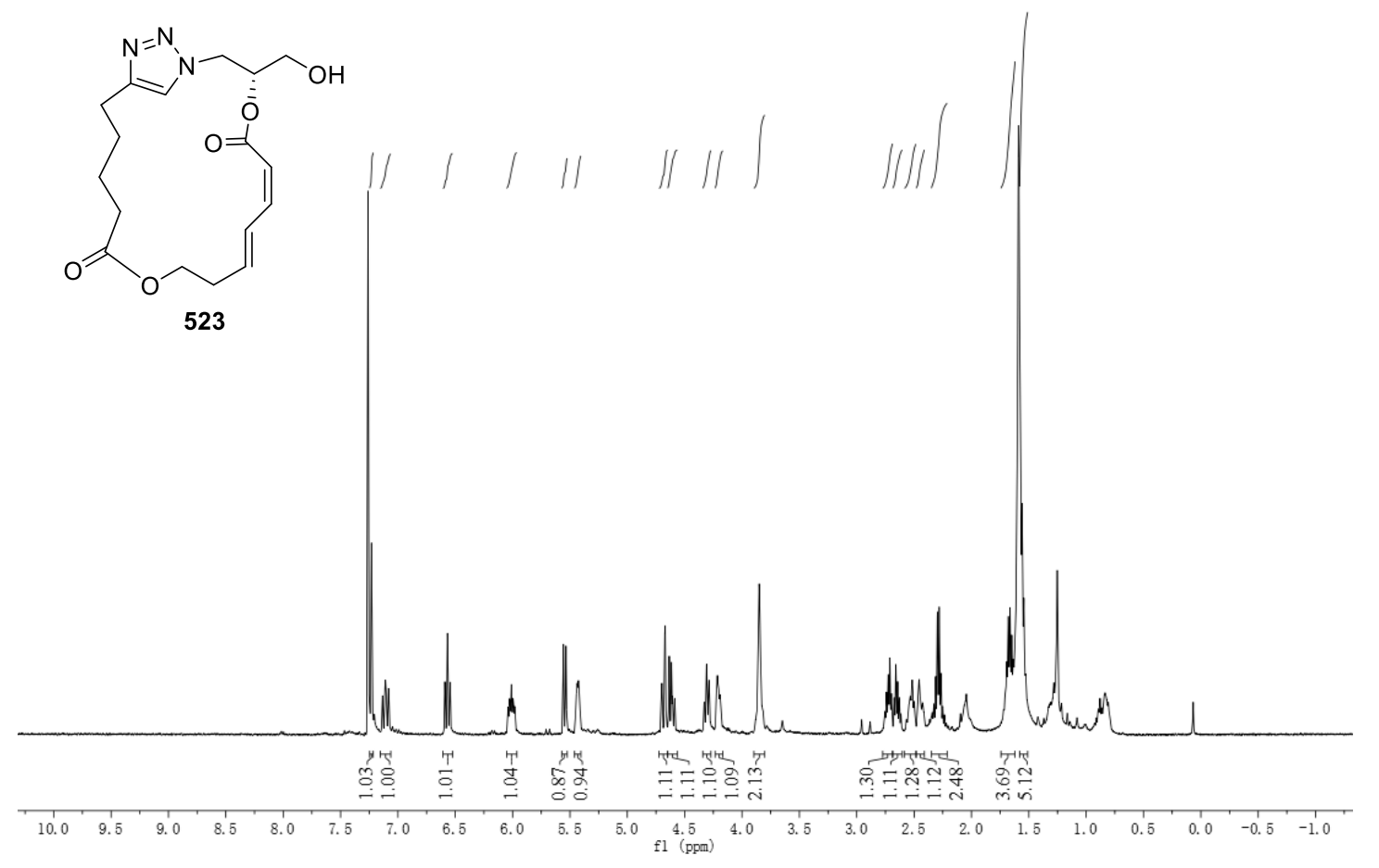

${ }^{13} \mathrm{C}$ NMR (125 MHz, $\left.\mathrm{CDCl}_{3}\right)$

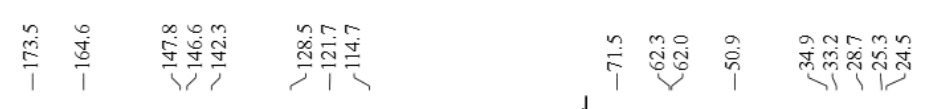

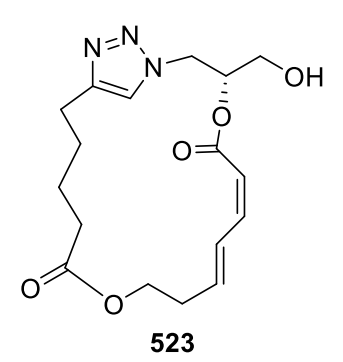

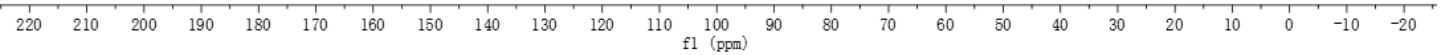




\section{Bibliography}

1. Dias, D. A.; Urban, S.; Roessner, U., A historical overview of natural products in drug discovery. Metabolites 2012, 2 (2), 303-336.

2. Hong, J., Role of natural product diversity in chemical biology. Curr. Opin. Chem. Biol. 2011, 15 (3), 350-354.

3. Ortholand, J.-Y.; Ganesan, A., Natural products and combinatorial chemistry: back to the future. Curr. Opin. Chem. Biol. 2004, 8 (3), 271-280.

4. Newman, D. J.; Cragg, G. M., Natural Products as Sources of New Drugs from 1981 to 2014. J. Nat. Prod. 2016, 79 (3), 629-661.

5. Molinski, T. F.; Dalisay, D. S.; Lievens, S. L.; Saludes, J. P., Drug development from marine natural products. Nat. Rev. Drug Discov. 2009, 8 (1), 69-85.

6. Simmons, T. L.; Andrianasolo, E.; McPhail, K.; Flatt, P.; Gerwick, W. H., Marine natural products as anticancer drugs. Mol. Cancer Ther. 2005, 4 (2), 333.

7. Rinehart, K. L.; Gloer, J. B.; Hughes, R. G.; Renis, H. E.; McGovren, J. P.; Swynenberg, E. B.; Stringfellow, D. A.; Kuentzel, S. L.; Li, L. H., Didemnins: antiviral and antitumor depsipeptides from a caribbean tunicate. Science 1981, 212 (4497), 933.

8. Rinehart, K. L.; Gloer, J. B.; Cook, J. C.; Mizsak, S. A.; Scahill, T. A., Structures of the didemnins, antiviral and cytotoxic depsipeptides from a Caribbean tunicate. J. Am. Chem. Soc. 1981, 103 (7), 1857-1859.

9. Andrew Dorr, F.; Kuhn, J. G.; Phillips, J.; Von Hoff, D. D., Phase I clinical and pharmacokinetic investigation of didemnin B, a cyclic depsipeptide. Eur. J. Cancer Clin. Oncol. 1988, 24 (11), 1699-1706.

10. Stewart, J. A.; Low, J. B.; Roberts, J. D.; Blow, A., A phase i clinical trial of didemnin B. Cancer 1991, 68 (12), 2550-2554.

11. Maroun, J. A.; Stewart, D.; Verma, S.; Eisenhauer, E., Phase I clinical study of didemnin B. Invest. New Drug 1998, 16 (1), 51-56.

12. Shin, D. M.; Holoye, P. Y.; Murphy, W. K.; Forman, A.; Papasozomenos, S. C., Phase I/II clinical trial of didemnin B in non-small-cell lung cancer: neuromuscular toxicity is doselimiting. Cancer Chemother. Pharmacol. 1991, 29 (2), 145-149.

13. Benvenuto, J. A.; Newman, R. A.; Bignami, G. S.; Raybould, T. J. G.; Raber, M. N.; Esparza, L.; Walters, R. S., Phase II clinical and pharmacological study of didemnin B in patients with metastatic breast cancer. Invest. New Drug 1992, 10 (2), 113-117.

14. Shin, D. M.; Holoye, P. Y.; Forman, A.; Winn, R.; Perez-Soler, R.; Dakhil, S.; Rosenthal, J.; Raber, M. N.; Hong, W. K., Phase II clinical trial of didemnin B in previously treated small cell lung cancer. Invest. New Drug 1994, 12 (3), 243-249. 
15. Goss, G.; Muldal, A.; Lohmann, R.; Taylor, M.; Lopez, P.; Armitage, G.; Steward, W. P., Didemnin B in favourable histology non-Hodgkin's lymphoma. Invest. New Drug 1995, 13 (3), 257-260.

16. Kucuk, O.; Young, M. L.; Habermann, T. M.; Wolf, B. C.; Jimeno, J.; Cassileth, P. A., Phase II Trial of didemnin B in previously treated non-Hodgkin's lymphoma: an Eastern Cooperative Oncology Group (ECOG) study. Am. J. Clin. Oncol. 2000, 23 (3).

17. Hochster, H.; Oratz, R.; Ettinger, D. S.; Borden, E., A phase II study of didemnin B (NSC 325319) in advanced malignant melanoma: an Eastern Cooperative Oncology Group study (PB687). Invest. New Drug 1998, 16 (3), 259-263.

18. Mittelman, A.; Chun, H. G.; Puccio, C.; Coombe, N.; Lansen, T.; Ahmed, T., Phase II clinical trial of didemnin B in patients with recurrent or refractory anaplastic astrocytoma or glioblastoma multiforme (NSC 325319). Invest. New Drug 1999, 17 (2), 179-182.

19. Taylor, S. A.; Giroux, D. J.; Jaeckle, K. A.; Panella, T. J.; Dakhil, S. R.; Schold, S. C., Phase II study of didemnin B in central nervous system tumors: A Southwest Oncology Group study. Invest. New Drug 1998, 16 (4), 331-332.

20. Rinehart, K. L.; Holt, T. G.; Fregeau, N. L.; Stroh, J. G.; Keifer, P. A.; Sun, F.; Li, L. H.; Martin, D. G., Ecteinascidins 729, 743, 745, 759A, 759B, and 770: potent antitumor agents from the Caribbean tunicate Ecteinascidia turbinata. J. Org. Chem. 1990, 55 (15), 4512-4515.

21. Wright, A. E.; Forleo, D. A.; Gunawardana, G. P.; Gunasekera, S. P.; Koehn, F. E.; McConnell, O. J., Antitumor tetrahydroisoquinoline alkaloids from the colonial ascidian Ecteinascidia turbinata. J. Org. Chem. 1990, 55 (15), 4508-4512.

22. Pommier, Y.; Kohlhagen, G.; Bailly, C.; Waring, M.; Mazumder, A.; Kohn, K. W., DNA sequence- and structure-selective alkylation of guanine N2 in the DNA minor groove by Ecteinascidin 743, a potent antitumor compound from the Caribbean tunicate Ecteinascidia turbinata. Biochemistry 1996, 35 (41), 13303-13309.

23. Leimgruber, W.; Stefanović, V.; Schenker, F.; Karr, A.; Berger, J., Isolation and characterization of anthramycin, a new antitumor antibiotic. J. Am. Chem. Soc. 1965, 87 (24), 5791-5793.

24. Harvey, A. L.; Edrada-Ebel, R.; Quinn, R. J., The re-emergence of natural products for drug discovery in the genomics era. Nat Rev Drug Discov 2015, 14 (2), 111-129.

25. Debbab, A.; Aly, A. H.; Lin, W. H.; Proksch, P., Bioactive compounds from marine bacteria and fungi. Microb. Biotechnol. 2010, 3 (5), 544-563.

26. Proksch, P.; Edrada-Ebel, R.; Ebel, R., Drugs from the sea-opportunities and obstacles. Mar. Drugs 2003, 1 (1), 5-17. 
27. Cuevas, C.; Pérez, M.; Martín, M. J.; Chicharro, J. L.; Fernández-Rivas, C.; Flores, M.; Francesch, A.; Gallego, P.; Zarzuelo, M.; de la Calle, F.; García, J.; Polanco, C.; Rodríguez, I.; Manzanares, I., Synthesis of ecteinascidin ET-743 and phthalascidin Pt-650 from cyanosafracin B. Org. Lett. 2000, 2 (16), 2545-2548.

28. He, W.; Zhang, Z.; Ma, D., A Scalable total synthesis of the antitumor agents ET-743 and lurbinectedin. Angew. Chem. Int. Ed. 2019, 58 (12), 3972-3975.

29. Searle, P. A.; Molinski, T. F.; Brzezinski, L. J.; Leahy, J. W., Absolute configuration of phorboxazoles A and B from the marine sponge Phorbas sp. 1. macrolide and hemiketal rings. $J$. Am. Chem. Soc. 1996, 118 (39), 9422-9423.

30. Uemura, D.; Takahashi, K.; Yamamoto, T.; Katayama, C.; Tanaka, J.; Okumura, Y.; Hirata, Y., Norhalichondrin A: an antitumor polyether macrolide from a marine sponge. J. Am. Chem. Soc. 1985, 107 (16), 4796-4798.

31. Hirata, Y.; Uemura, D., Halichondrins-antitumor polyether macrolides from a marine sponge. Pure Appl. Chem. 1986, 58 (5), 701-710.

32. Bai, R. L.; Paull, K. D.; Herald, C. L.; Malspeis, L.; Pettit, G. R.; Hamel, E., Halichondrin $\mathrm{B}$ and homohalichondrin $\mathrm{B}$, marine natural products binding in the vinca domain of tubulin. Discovery of tubulin-based mechanism of action by analysis of differential cytotoxicity data. $J$. Biol. Chem. 1991, 266 (24), 15882-15889.

33. Dabydeen, D. A.; Burnett, J. C.; Bai, R.; Verdier-Pinard, P.; Hickford, S. J. H.; Pettit, G. R.; Blunt, J. W.; Munro, M. H. G.; Gussio, R.; Hamel, E., Comparison of the activities of the truncated halichondrin B analog NSC 707389 (E7389) with those of the parent compound and a proposed binding site on tubulin. Mol. Pharmacol. 2006, 70 (6), 1866-1875.

34. Aicher, T. D.; Buszek, K. R.; Fang, F. G.; Forsyth, C. J.; Jung, S. H.; Kishi, Y.; Matelich, M. C.; Scola, P. M.; Spero, D. M.; Yoon, S. K., Total synthesis of halichondrin B and norhalichondrin B. J. Am. Chem. Soc. 1992, 114 (8), 3162-3164.

35. Munro, M. H. G.; Blunt, J. W.; Dumdei, E. J.; Hickford, S. J. H.; Lill, R. E.; Li, S.; Battershill, C. N.; Duckworth, A. R., The discovery and development of marine compounds with pharmaceutical potential. J. Biotechnol. 1999, 70 (1-3), 15-25.

36. Stamos, D. P.; Chen, S. S.; Kishi, Y., New synthetic route to the C.14-C.38 segment of halichondrins. J. Org. Chem. 1997, 62 (22), 7552-7553.

37. Wang, Y.; Habgood, G. J.; Christ, W. J.; Kishi, Y.; Littlefield, B. A.; Yu, M. J., Structureactivity relationships of halichondrin B analogues: modifications at $\mathrm{C} 30-\mathrm{C} 38$. Bioorg. Med. Chem. Lett. 2000, 10 (10), 1029-1032.

38. Littlefield, B. A.; Palme, M. H.; Seletsky, B. M.; Towle, M. J.; Yu, M. J.; Zheng, W., Macrocyclic analogs and methods of their use and preparation. Eisai R\&D Management Co. Ltd. 2001, US6214865B1. 
39. Towle, M. J.; Salvato, K. A.; Budrow, J.; Wels, B. F.; Kuznetsov, G.; Aalfs, K. K.; Welsh, S.; Zheng, W.; Seletsky, B. M.; Palme, M. H., In vitro and in vivo anticancer activities of synthetic macrocyclic ketone analogues of halichondrin B. Cancer Res. 2001, 61 (3), 1013-1021.

40. Swami, U.; Shah, U.; Goel, S., Eribulin in cancer treatment. Mar. Drugs 2015, 13 (8).

41. Pettit, G. R.; Herald, C. L.; Doubek, D. L.; Herald, D. L.; Arnold, E.; Clardy, J., Isolation and structure of bryostatin 1. J. Am. Chem. Soc. 1982, 104 (24), 6846-6848.

42. Sudek, S.; Lopanik, N. B.; Waggoner, L. E.; Hildebrand, M.; Anderson, C.; Liu, H.; Patel, A.; Sherman, D. H.; Haygood, M. G., Identification of the putative bryostatin polyketide synthase gene cluster from "Candidatus Endobugula sertula", the uncultivated microbial symbiont of the marine bryozoan Bugula neritina. J. Nat. Prod. 2007, 70 (1), 67-74.

43. Hennings, H.; Blumberg, P. M.; Pettit, G. R.; Herald, C. L.; Shores, R.; Yuspa, S. H., Bryostatin 1, an activator of protein kinase $\mathrm{C}$, inhibits tumor promotion by phorbol esters in SENCAR mouse skin. Carcinogenesis 1987, 8 (9), 1343-1346.

44. Sun, M.-K.; Hongpaisan, J.; Nelson, T. J.; Alkon, D. L., Poststroke neuronal rescue and synaptogenesis mediated in vivo by protein kinase $\mathrm{C}$ in adult brains. Proc. Natl. Acad. Sci. USA 2008, 105 (36), 13620-13625.

45. Way, K. J.; Katai, N.; King, G. L., Protein kinase C and the development of diabetic vascular complications. Diabet. Med. 2001, 18 (12), 945-959.

46. Schrott, L. M.; Jackson, K.; Yi, P.; Dietz, F.; Johnson, G. S.; Basting, T. F.; Purdum, G.; Tyler, T.; Rios, J. D.; Castor, T. P., Acute oral bryostatin-1 administration improves learning deficits in the APP/PS1 transgenic mouse model of Alzheimer's disease. Curr. Alzheimer Res. 2015, 12 (1), 22-31.

47. Schaufelberger, D. E.; Koleck, M. P.; Beutler, J. A.; Vatakis, A. M.; Alvarado, A. B.; Andrews, P.; Marzo, L. V.; Muschik, G. M.; Roach, J.; Ross, J. T.; Lebherz, W. B.; Reeves, M. P.; Eberwein, R. M.; Rodgers, L. L.; Testerman, R. P.; Snader, K. M.; Forenza, S., The largescale isolation of bryostatin 1 from Bugula neritina following current good manufacturing practices. J. Nat. Prod. 1991, 54 (5), 1265-1270.

48. Keck, G. E.; Poudel, Y. B.; Cummins, T. J.; Rudra, A.; Covel, J. A., Total synthesis of bryostatin 1. J. Am. Chem. Soc. 2011, 133 (4), 744-747.

49. Wender, P. A.; DeBrabander, J.; Harran, P. G.; Jimenez, J.-M.; Koehler, M. F. T.; Lippa, B.; Park, C.-M.; Siedenbiedel, C.; Pettit, G. R., The design, computer modeling, solution structure, and biological evaluation of synthetic analogs of bryostatin 1. Proc. Natl. Acad. Sci. USA 1998, 95 (12), 6624-6629.

50. Wender, P. A.; De Brabander, J.; Harran, P. G.; Jimenez, J.-M.; Koehler, M. F. T.; Lippa, B.; Park, C.-M.; Shiozaki, M., Synthesis of the first members of a new class of biologically active bryostatin analogues. J. Am. Chem. Soc. 1998, 120 (18), 4534-4535. 
51. Wender, P. A.; Baryza, J. L.; Brenner, S. E.; De Christopher, B. A.; Loy, B. A.; Schrier, A. J.; Verma, V. A., Design, synthesis, and evaluation of potent bryostatin analogs that modulate PKC translocation selectivity. Proc. Natl. Acad. Sci. USA 2011, 108 (17), 6721-6726.

52. Wender, P. A.; Cribbs, C. M.; Koehler, K. F.; Sharkey, N. A.; Herald, C. L.; Kamano, Y.; Pettit, G. R.; Blumberg, P. M., Modeling of the bryostatins to the phorbol ester pharmacophore on protein kinase C. Proc. Natl. Acad. Sci. USA 1988, 85 (19), 7197-7201.

53. Pettit, G. R.; Kamano, Y.; Herald, C. L.; Tuinman, A. A.; Boettner, F. E.; Kizu, H.; Schmidt, J. M.; Baczynskyj, L.; Tomer, K. B.; Bontems, R. J., The isolation and structure of a remarkable marine animal antineoplastic constituent: dolastatin 10. J. Am. Chem. Soc. 1987, 109 (22), 6883-6885.

54. Harrigan, G. G.; Luesch, H.; Yoshida, W. Y.; Moore, R. E.; Nagle, D. G.; Paul, V. J.; Mooberry, S. L.; Corbett, T. H.; Valeriote, F. A., Symplostatin 1: A dolastatin 10 analogue from the marine cyanobacterium Symploca hydnoides. J. Nat. Prod. 1998, 61 (9), 1075-1077.

55. Luesch, H.; Moore, R. E.; Paul, V. J.; Mooberry, S. L.; Corbett, T. H., Isolation of dolastatin 10 from the marine cyanobacterium Symploca species VP642 and total stereochemistry and biological evaluation of its analogue symplostatin 1. J. Nat. Prod. 2001, 64 (7), 907-910.

56. Pettit, G. R.; Singh, S. B.; Hogan, F.; Lloyd-Williams, P.; Herald, D. L.; Burkett, D. D.; Clewlow, P. J., Antineoplastic agents. Part 189. The absolute configuration and synthesis of natural (-)-dolastatin 10. J. Am. Chem. Soc. 1989, 111 (14), 5463-5465.

57. Bai, R.; Petit, G. R.; Hamel, E., Dolastatin 10, a powerful cytostatic peptide derived from a marine animal: inhibition of tubulin polymerization mediated through the vinca alkaloid binding domain. Biochem. Pharmacol. 1990, 39 (12), 1941-1949.

58. Bai, R. L.; Pettit, G. R.; Hamel, E., Binding of dolastatin 10 to tubulin at a distinct site for peptide antimitotic agents near the exchangeable nucleotide and vinca alkaloid sites. J. Biol. Chem. 1990, 265 (28), 17141-17149.

59. Ludueña, R. F.; Roach, M. C.; Prasad, V.; Pettit, G. R., Interaction of dolastatin 10 with bovine brain tubulin. Biochem. Pharmacol. 1992, 43 (3), 539-543.

60. Pitot, H. C.; McElroy, E. A.; Reid, J. M.; Windebank, A. J.; Sloan, J. A.; Erlichman, C.; Bagniewski, P. G.; Walker, D. L.; Rubin, J.; Goldberg, R. M., Phase I trial of dolastatin-10 (NSC 376128) in patients with advanced solid tumors. Clin. Cancer Res. 1999, 5 (3), 525-531.

61. Vaishampayan, U.; Glode, M.; Du, W.; Kraft, A.; Hudes, G.; Wright, J.; Hussain, M., Phase II study of dolastatin-10 in patients with hormone-refractory metastatic prostate adenocarcinoma. Clin. Cancer Res. 2000, 6 (11), 4205-4208.

62. Hoffman, M. A.; Blessing, J. A.; Lentz, S. S., A phase II trial of dolastatin-10 in recurrent platinum-sensitive ovarian carcinoma: a Gynecologic Oncology Group study. Gynecol. Oncol. 2003, 89 (1), 95-98. 
63. Miyazaki, K.; Kobayashi, M.; Natsume, T.; Gondo, M.; Mikami, T.; Sakakibara, K.; Tsukagoshi, S., Synthesis and antitumor activity of novel dolastatin 10 analogs. Chem. Pharm. Bull. (Tokyo) 1995, 43 (10), 1706-1718.

64. Kobayashi, M.; Natsume, T.; Tamaoki, S.; Watanabe, J.-i.; Asano, H.; Mikami, T.; Miyasaka, K.; Miyazaki, K.; Gondo, M.; Sakakibara, K.; Tsukagoshi, S., Antitumor activity of TZT-1027, a novel doiastatin 10 derivative. Jpn. J. Clin. Oncol. 1997, 88 (3), 316-327.

65. Tamura, K.; Nakagawa, K.; Kurata, T.; Satoh, T.; Nogami, T.; Takeda, K.; Mitsuoka, S.; Yoshimura, N.; Kudoh, S.; Negoro, S.; Fukuoka, M., Phase I study of TZT-1027, a novel synthetic dolastatin 10 derivative and inhibitor of tubulin polymerization, which was administered to patients with advanced solid tumors on days 1 and 8 in 3-week courses. Cancer Chemother. Pharmacol. 2007, 60 (2), 285-293.

66. Yamamoto, N.; Andoh, M.; Kawahara, M.; Fukuoka, M.; Niitani, H., Phase I study of TZT-1027, a novel synthetic dolastatin 10 derivative and inhibitor of tubulin polymerization, given weekly to advanced solid tumor patients for 3 weeks. Cancer Sci. 2009, 100 (2), 316-321. 67. Riely, G. J.; Gadgeel, S.; Rothman, I.; Saidman, B.; Sabbath, K.; Feit, K.; Kris, M. G.; Rizvi, N. A., A phase 2 study of TZT-1027, administered weekly to patients with advanced nonsmall cell lung cancer following treatment with platinum-based chemotherapy. Lung Cancer 2007, 55 (2), 181-185.

68. Horti, J.; Juhasz, E.; Monostori, Z.; Maeda, K.; Eckhardt, S.; Bodrogi, I., Phase I study of TZT-1027, a novel synthetic dolastatin 10 derivative, for the treatment of patients with nonsmall cell lung cancer. Cancer Chemother. Pharmacol. 2008, 62 (1), 173-180.

69. Miyazuki, K.; Kobayashi, M.; Natsume, T., Gondon, M., Mikami, T., Sakakibara, S., Synthesis and antitumor activity of novel dolastatin 10 analogs. Chem. Pharm. Bull. (Tokyo) 1995, 43 (10), 1706-1718.

70. Talpir, R.; Benayahu, Y.; Kashman, Y.; Pannell, L.; Schleyer, M., Hemiasterlin and geodiamolide TA; two new cytotoxic peptides from the marine sponge Hemiasterella minor (Kirkpatrick). Tetrahedron Lett. 1994, 35 (25), 4453-4456.

71. Coleman, J. E.; de Silva, E. D.; Kong, F.; Andersen, R. J.; Allen, T. M., Cytotoxic peptides from the marine sponge Cymbastela sp. Tetrahedron 1995, 51 (39), 10653-10662.

72. Nieman, J. A.; Coleman, J. E.; Wallace, D. J.; Piers, E.; Lim, L. Y.; Roberge, M.; Andersen, R. J., Synthesis and antimitotic/cytotoxic activity of hemiasterlin analogues. J. Nat. Prod. 2003, 66 (2), 183-199.

73. Loganzo, F.; Discafani, C. M.; Annable, T.; Beyer, C.; Musto, S.; Hari, M.; Tan, X.; Hardy, C.; Hernandez, R.; Baxter, M., HTI-286, a synthetic analogue of the tripeptide hemiasterlin, is a potent antimicrotubule agent that circumvents P-glycoprotein-mediated resistance in vitro and in vivo. Cancer Res. 2003, 63 (8), 1838-1845. 
74. Schwartz, R. E.; Hirsch, C. F.; Sesin, D. F.; Flor, J. E.; Chartrain, M.; Fromtling, R. E.; Harris, G. H.; Salvatore, M. J.; Liesch, J. M.; Yudin, K., Pharmaceuticals from cultured algae. $J$. Ind. Microbiol. 1990, 5 (2), 113-123.

75. Trimurtulu, G.; Ohtani, I.; Patterson, G. M. L.; Moore, R. E.; Corbett, T. H.; Valeriote, F. A.; Demchik, L., Total structures of cryptophycins, potent antitumor depsipeptides from the bluegreen alga Nostoc sp. strain GSV 224. J. Am. Chem. Soc. 1994, 116 (11), 4729-4737.

76. Golakoti, T.; Ogino, J.; Heltzel, C. E.; Le Husebo, T.; Jensen, C. M.; Larsen, L. K.; Patterson, G. M. L.; Moore, R. E.; Mooberry, S. L., Structure determination, conformational analysis, chemical stability studies, and antitumor evaluation of the cryptophycins. Isolation of 18 new analogs from Nostoc sp. strain GSV 224. J. Am. Chem. Soc. 1995, 117 (49), 12030-12049.

77. Wagner, M. M.; Paul, D. C.; Shih, C.; Jordan, M. A.; Wilson, L.; Williams, D. C., In vitro pharmacology of cryptophycin 52 (LY355703) in human tumor cell lines. Cancer Chemother. Pharmacol. 1999, 43 (2), 115-125.

78. Northcote, P. T.; Blunt, J. W.; Munro, M. H. G., Pateamine: a potent cytotoxin from the New Zealand Marine sponge, mycale sp. Tetrahedron Lett. 1991, 32 (44), 6411-6414.

79. Rzasa, R. M.; Romo, D.; Stirling, D. J.; Blunt, J. W.; Munro, M. H. G., Structural and synthetic studies of the pateamines: Synthesis and absolute configuration of the hydroxydienoate fragment. Tetrahedron Lett. 1995, 36 (30), 5307-5310.

80. Blincoe, S. N., Studies on some compounds from the Mycale species. Master's thesis, University of Canterbury, Christchurch, New Zealand. 1994.

81. Stirling, D. J. Studies on marine natural products. Doctoral dissertation, University of Canterbury, Christchurch, New Zealand. 1996.

82. Rzasa, R. M.; Shea, H. A.; Romo, D., Total synthesis of the novel immunosuppressive agent (-)-pateamine A from Mycale sp. employing a $\beta$-lactam-based macrocyclization. J. Am. Chem. Soc. 1998, 120 (3), 591-592.

83. Hood, K. A.; West, L. M.; Northcote, P. T.; Berridge, M. V.; Miller, J. H., Induction of apoptosis by the marine sponge (Mycale) metabolites, mycalamide A and pateamine. Apoptosis 2001, 6 (3), 207-219.

84. Romo, D.; Rzasa, R. M.; Shea, H. A.; Park, K.; Langenhan, J. M.; Sun, L.; Akhiezer, A.; Liu, J. O., Total synthesis and immunosuppressive activity of (-)-pateamine A and related compounds: implementation of a $\beta$-lactam-based macrocyclization. J. Am. Chem. Soc. 1998, 120 (47), 12237-12254.

85. Bordeleau, M.-E.; Matthews, J.; Wojnar, J. M.; Lindqvist, L.; Novac, O.; Jankowsky, E.; Sonenberg, N.; Northcote, P.; Teesdale-Spittle, P.; Pelletier, J., Stimulation of mammalian translation initiation factor eIF4A activity by a small molecule inhibitor of eukaryotic translation. Proc. Natl. Acad. Sci. USA 2005, 102 (30), 10460-10465. 
86. Low, W.-K.; Dang, Y.; Schneider-Poetsch, T.; Shi, Z.; Choi, N. S.; Merrick, W. C.; Romo, D.; Liu, J. O., Inhibition of eukaryotic translation initiation by the marine natural product pateamine A. Mol. Cell 2005, 20 (5), 709-722.

87. Pause, A.; Méthot, N.; Sonenberg, N., The HRIGRXXR region of the DEAD box RNA helicase eukaryotic translation initiation factor $4 \mathrm{~A}$ is required for RNA binding and ATP hydrolysis. Mol. Cell. Biol. 1993, 13 (11), 6789-6798.

88. Conroy, S. C.; Dever, T. E.; Owens, C. L.; Merrick, W. C., Characterization of the 46,000-Dalton subunit of eIF4F. Arch. Biochem Biophys. 1990, 282 (2), 363-371.

89. Yoder-Hill, J.; Pause, A.; Sonenberg, N.; Merrick, W. C., The p46 subunit of eukaryotic initiation factor eIF4F exchanges with eIF4A. J. Biol. Chem. 1993, 268 (8), 5566-5573.

90. Kapp, L. D.; Lorsch, J. R., The molecular mechanics of eukaryotic translation. Annu. Rev. Biochem. 2004, 73 (1), 657-704.

91. Merrick, W. C., Cap-dependent and cap-independent translation in eukaryotic systems. Gene 2004, 332, 1-11.

92. Shibuya, T.; Tange, T. Ø.; Sonenberg, N.; Moore, M. J., eIF4AIII binds spliced mRNA in the exon junction complex and is essential for nonsense-mediated decay. Nat. Struct. Mol. Biol. 2004, 11 (4), 346-351.

93. Palacios, I. M.; Gatfield, D.; St Johnston, D.; Izaurralde, E., An eIF4AIII-containing complex required for mRNA localization and nonsense-mediated mRNA decay. Nature 2004, 427 (6976), 753-757.

94. Hellen, C. U. T.; Sarnow, P., Internal ribosome entry sites in eukaryotic mRNA molecules. Gene Dev. 2001, 15 (13), 1593-1612.

95. Grifo, J. A.; Abramson, R. D.; Satler, C. A.; Merrick, W. C., RNA-stimulated ATPase activity of eukaryotic initiation factors. J. Biol. Chem. 1984, 259 (13), 8648-8654.

96. Rogers, G. W.; Richter, N. J.; Merrick, W. C., Biochemical and kinetic characterization of the RNA helicase activity of eukaryotic initiation factor 4A. J. Biol. Chem. 1999, 274 (18), 12236-12244.

97. Pestova, T. V.; Kolupaeva, V. G., The roles of individual eukaryotic translation initiation factors in ribosomal scanning and initiation codon selection. Gene Dev. 2002, 16 (22), 2906-2922. 98. Korneeva, N. L.; First, E. A.; Benoit, C. A.; Rhoads, R. E., Interaction between the NH2terminal domain of eIF4A and the central domain of eIF4G modulates RNA-stimulated ATPase activity. J. Biol.Chem. 2005, 280 (3), 1872-1881.

99. Bordeleau, M.-E.; Cencic, R.; Lindqvist, L.; Oberer, M.; Northcote, P.; Wagner, G.; Pelletier, J., RNA-mediated sequestration of the RNA helicase eIF4A by pateamine A inhibits translation initiation. Chem. Biol. 2006, 13 (12), 1287-1295. 
100. Stroupe, M. E.; Tange, T. Ø.; Thomas, D. R.; Moore, M. J.; Grigorieff, N., The threedimensional arcitecture of the EJC Core. J. Mol. Biol. 2006, 360 (4), 743-749.

101. Ballut, L.; Marchadier, B.; Baguet, A.; Tomasetto, C.; Séraphin, B.; Le Hir, H., The exon junction core complex is locked onto RNA by inhibition of eIF4AIII ATPase activity. Nat. Struct. Mol. Biol. 2005, 12 (10), 861-869.

102. Le Hir, H.; Izaurralde, E.; Maquat, L. E.; Moore, M. J., The spliceosome deposits multiple proteins 20 - 24 nucleotides upstream of mRNA exon-exon junctions. EMBO J. 2000, 19 (24), 6860-6869.

103. Dostie, J.; Dreyfuss, G., Translation is required to remove Y14 from mRNAs in the cytoplasm. Curr. Biol. 2002, 12 (13), 1060-1067.

104. Lejeune, F.; Ishigaki, Y.; Li, X.; Maquat, L. E., The exon junction complex is detected on CBP80-bound but not eIF4E-bound mRNA in mammalian cells: dynamics of mRNP remodeling. EMBO J. 2002, 21 (13), 3536.

105. Tange, T. Ø.; Nott, A.; Moore, M. J., The ever-increasing complexities of the exon junction complex. Curr. Opin. Cell. Biol. 2004, 16 (3), 279-284.

106. Le Hir, H.; Saulière, J.; Wang, Z., The exon junction complex as a node of posttranscriptional networks. Nat. Rev. Mol. 2015.

107. Chang, Y.-F.; Imam, J. S.; Wilkinson, M. F., The nonsense-mediated decay RNA surveillance pathway. Annu. Rev. Biochem. 2007, 76 (1), 51-74.

108. Schweingruber, C.; Rufener, S. C.; Zünd, D.; Yamashita, A.; Mühlemann, O., Nonsensemediated mRNA decay-mechanisms of substrate mRNA recognition and degradation in mammalian cells. BBA-Gene Regul. Mech. 2013, 1829 (6), 612-623.

109. Kervestin, S.; Jacobson, A., NMD: a multifaceted response to premature translational termination. Nat. Rev. Mol. 2012, 13 (11), 700-712.

110. Dang, Y.; Low, W.-K.; Xu, J.; Gehring, N. H.; Dietz, H. C.; Romo, D.; Liu, J. O., Inhibition of nonsense-mediated mRNA decay by the natural product pateamine A through eukaryotic initiation factor 4AIII. J. Biol. Chem. 2009, 284 (35), 23613-23621.

111. Ziehr, B.; Lenarcic, E.; Cecil, C.; Moorman, N. J., The eIF4AIII RNA helicase is a critical determinant of human cytomegalovirus replication. Virology 2016, 489, 194-201.

112. Clardy, J., Stopping trouble before it starts. ACS Chem. Biol. 2006, 1 (1), 17-19.

113. Aguilar, E.; Meyers, A. I., Reinvestigation of a modified Hantzsch thiazole synthesis. Tetrahedron Lett. 1994, 35 (16), 2473-2476.

114. Li, G.; Pattel, D.; Hruby, V. J., Asymmetric synthesis of $(2 R, 3 S)$ and $(2 S, 3 R)$ precursors of $\beta$-methyl-histidine, -phenylalanine and -tyrosine. Tetrahedron: Asymmetry 1993, 4 (11), 2315 2318.

115. Aksela, R.; Oehlschlager, A. C., Stannylmetallation of conjugated enynes. Tetrahedron 1991, 47 (7), 1163-1176. 
116. Corey, E. J.; Fuchs, P. L., A synthetic method for formyl $\rightarrow$ ethynyl conversion $\left(\mathrm{RCHO} \rightarrow \mathrm{RC}=\mathrm{CH}\right.$ or $\left.\mathrm{RC}=\mathrm{CR}^{\prime}\right)$. Tetrahedron Lett. 1972, 13 (36), 3769-3772.

117. Palomo, C.; Aizpurua, J. M.; Cuevas, C.; Mielgo, A.; Galarza, R., A mild method for the alcoholysis of $\beta$-lactams. Tetrahedron Lett. 1995, 36 (49), 9027-9030.

118. Remuiñán, M. J.; Pattenden, G., Total synthesis of (-)-pateamine, a novel polyene bismacrolide with immunosuppressive activity from the sponge Mycale sp. Tetrahedron Lett. 2000, 41 (38), 7367-7371.

119. Critcher, D. J.; Pattenden, G., Synthetic studies towards pateamine, a novel thiazolebased 19-membered bis-lactone from Mycale sp. Tetrahedron Lett. 1996, 37 (50), 9107-9110.

120. Zhuo, C.-X.; Fürstner, A., Catalysis-based total syntheses of pateamine A and DMDAPat A. J. Am. Chem. Soc. 2018, 140 (33), 10514-10523.

121. Jung, H.-Y.; Feng, X.; Kim, H.; Yun, J., Copper-catalyzed boration of activated alkynes. Chiral boranes via a one-pot copper-catalyzed boration and reduction protocol. Tetrahedron $\mathbf{2 0 1 2}$, 68 (17), 3444-3449.

122. Rupnicki, L.; Saxena, A.; Lam, H. W., Aromatic heterocycles as activating groups for asymmetric conjugate addition reactions. Enantioselective copper-catalyzed reduction of 2alkenylheteroarenes. J. Am. Chem. Soc. 2009, 131 (30), 10386-10387.

123. Smith, A. B.; Dong, S.; Brenneman, J. B.; Fox, R. J., Total synthesis of (+)-sorangicin A. J. Am. Chem. Soc. 2009, 131 (34), 12109-12111.

124. Fürstner, A.; Funel, J.-A.; Tremblay, M.; Bouchez, L. C.; Nevado, C.; Waser, M.; Ackerstaff, J.; Stimson, C. C., A versatile protocol for Stille-Migita cross coupling reactions. Chem. Commun. (Camb.) 2008, (25), 2873-2875.

125. Srogl, J.; Allred, G. D.; Liebeskind, L. S., Sulfonium salts. Participants par excellence in metal-catalyzed carbon-carbon bond-forming reactions. J. Am. Chem. Soc. 1997, 119 (50), 12376-12377.

126.

127. Low, W.-K.; Li, J.; Zhu, M.; Kommaraju, S. S.; Shah-Mittal, J.; Hull, K.; Liu, J. O.; Romo, D., Second-generation derivatives of the eukaryotic translation initiation inhibitor pateamine A targeting eIF4A as potential anticancer agents. Bioorg. Med. Chem. 2014, 22 (1), 116-125.

128. Zhuo, C.-X.; Fürstner, A., Concise synthesis of a pateamine A analogue with in vivo anticancer activity based on an iron-catalyzed pyrone ring opening/cross-coupling. Angew. Chem. Int. Ed. 2016, 55 (20), 6051-6056.

129. Maquat, L., Nonsense-Mediated mRNA Decay. CRC Press: 2006; pp 71-80.

130. Kuznetsov, G.; Xu, Q.; Rudolph-Owen, L.; TenDyke, K.; Liu, J.; Towle, M.; Zhao, N.; Marsh, J.; Agoulnik, S.; Twine, N., Potent in vitro and in vivo anticancer activities of des-methyl, des-amino pateamine A, a synthetic analogue of marine natural product pateamine A. Mol. 
Cancer Ther. 2009, 8 (5), 1250-1260.

131. Low, W.-K.; Dang, Y.; Bhat, S.; Romo, D.; Liu, J. O., Substrate-dependent targeting of eukaryotic translation initiation factor $4 \mathrm{~A}$ by pateamine $\mathrm{A}$ : negation of domain-linker regulation of activity. Chemistry \& Biology 2007, 14 (6), 715-727.

132. Mackay, I. R.; Rose, N. R., The Autoimmune Diseases. Elsevier Science: 2013; pp 429458.

133. Frischmeyer, P. A.; Dietz, H. C., Nonsense-mediated mRNA decay in health and disease. Hum. Mol. Genet. 1999, 8 (10), 1893-1900.

134. Cumming, A. H.; Brown, S. L.; Xu, T.; Cuyamendous, C.; Field, J. J.; Miller, J. H.; Harvey, J. E.; Teesdale-Spittle, P. H., Synthesis of a simplified triazole analogue of pateamine A. Org. Biomol.Chem. 2016, 14 (22), 5117-5127.

135. Cumming, A. H. Design and synthesis of simplified analogues of pateamine A. Doctoral dissertation, Victoria University of Wellington, New Zealand. 2014.

136. Burton, A. G.; Frampton, R. D.; Johnson, C. D.; Katritzky, A. R., The kinetics and mechanism of the electrophilic substitution of heteroaromatic compounds. Part XXVIII. The preparation and kinetic nitration of 2-, 3-, and 4-dimethylaminopyridines and their 1-oxides in sulphuric acid. J. Chem. Soc., Perkin Trans. 2 1972, (13), 1940-1949.

137. Jencks, W. P.; Regenstein, J., Ionization constants of acids and bases. In Handbook of Biochemistry and Molecular Biology, Fourth edition. CRC Press: 2010; pp 595-635.

138. Barreiro, E. J.; Kümmerle, A. E.; Fraga, C. A. M., The methylation effect in medicinal chemistry. Chem. Rev. 2011, 111 (9), 5215-5246.

139. Schönherr, H.; Cernak, T., Profound methyl effects in drug discovery and a call for new C-H methylation reactions. Angew. Chem. Int. Ed. 2013, 52 (47), 12256-12267.

140. Meldal, M.; Tornøe, C. W., Cu-catalyzed azide-alkyne cycloaddition. Chem. Rev. 2008, 108 (8), 2952-3015.

141. Wu, G.; Zheng, R.; Nelson, J.; Zhang, L., One-step synthesis of methanesulfonyloxymethyl ketones via gold-catalyzed oxidation of terminal alkynes: a combination of ligand and counter anion enables high efficiency and a one-pot synthesis of 2,4disubstituted thiazoles. Adv. Synth. Catal. 2014, 356 (6), 1229-1234.

142. Rostovtsev, V. V.; Green, L. G.; Fokin, V. V.; Sharpless, K. B., A stepwise Huisgen cycloaddition process: Copper(I)-catalyzed regioselective "ligation" of azides and terminal alkynes. Angew. Chem. Int. Ed. 2002, 41 (14), 2596-2599. 
143. Tornøe, C. W.; Christensen, C.; Meldal, M., Peptidotriazoles on solid phase: [1,2,3]triazoles by regiospecific copper(I)-catalyzed 1,3-dipolar cycloadditions of terminal alkynes to azides. J. Org. Chem. 2002, 67 (9), 3057-3064.

144. Cinellu, M. A., Gold-Alkene Complexes. In Modern Gold Catalyzed Synthesis, Wiley: 2012, pp 175-199.

145. Wiberg, K. B.; Rush, D. J., Solvent effects on the thioamide rotational barrier: an experimental and theoretical study. J. Am. Chem. Soc. 2001, 123 (9), 2038-2046.

146. Mykhaylychenko, S. S.; Pikun, N. V.; Shermolovich, Y. G., Acylation of primary polyfluoroalkanethioamides. J. Fluor.Chem. 2012, 140, 76-81.

147. Takahata, H.; Yamazaki, T., Synthesis of heterocycles using thioamide groups. J. Synth. Org. Chem Jpn. 1987, 45 (7), 682-690.

148. Satoh, T.; Miura, M., Catalytic direct arylation of heteroaromatic compounds. Chem. Lett. 2006, 36 (2), 200-205.

149. Xiong, Z.; Wang, N.; Dai, M.; Li, A.; Chen, J.; Yang, Z., Synthesis of novel palladacycles and their application in Heck and Suzuki reactions under aerobic conditions. Org. Lett. 2004, 6 (19), 3337-3340.

150. Kanchanadevi, A.; Ramesh, R.; Semeril, D., Efficient and recyclable Ru (II) arene thioamide catalysts for transfer hydrogenation of ketones: Influence of substituent on catalytic outcome. J. Organomet. Chem. 2016, 808, 68-77.

151. Hurd, R. N.; DeLaMater, G., The Preparation and chemical properties of thionamides. Chem. Rev. 1961, 61 (1), 45-86.

152. Willgerodt, C., Ueber die einwirkung von gelbem schwefelammonium auf ketone und chinone. Ber. Dtsch. Chem. Ges. 1887, 20 (2), 2467-2470.

153. Rekunge, D. S.; Khatri, C. K.; Chaturbhuj, G. U., Rapid and efficient protocol for Willgerodt-Kindler's thioacetamides catalyzed by sulfated polyborate. Monatsh. Chem. 2017, 148 (12), 2091-2095.

154. Nguyen, T. B.; Retailleau, P., Methyl ketone break-and-rebuild: a strategy for full $\alpha-$ heterofunctionalization of acetophenones. Green Chem. 2017, 19 (22), 5371-5374.

155. Murai, T., Synthesis of Thioamides. In Chemistry of Thioamides, Murai, T., Ed. Springer Singapore: Singapore, 2019; pp 45-73.

156. Demselben, Ueber die verbindungen des phosphors mit schwefel. Justus Liebigs Ann. Chem. 1843, 46 (3), 251-281.

157. Davy, H., A direct conversion of carboxylic acids into dithioesters. J. Chem. Soc., Chem. Commun. 1982, (8), 457-458.

158. Lecher, H. Z.; Greenwood, R. A.; Whitehouse, K. C.; Chao, T. H., The phosphonation of aromatic compounds with phosphorus pentasulfide. J. Am. Chem. Soc. 1956, 78 (19), 5018-5022. 
159. Choi, Y.; Ishikawa, H.; Velcicky, J.; Elliott, G. I.; Miller, M. M.; Boger, D. L., Total synthesis of (-)- and ent-(+)-vindoline. Org. Lett. 2005, 7 (20), 4539-4542.

160. Zhang, W.; Ding, M.; Li, J.; Guo, Z.; Lu, M.; Chen, Y.; Liu, L.; Shen, Y.-H.; Li, A., Total synthesis of hybridaphniphylline B. J. Am. Chem. Soc. 2018, 140 (12), 4227-4231.

161. Cherkasov, R. A.; Kutyrev, G. A.; Pudovik, A. N., Tetrahedron report number 186: Organothiophosphorus reagents in organic synthesis. Tetrahedron 1985, 41 (13), 2567-2624.

162. Cava, M. P.; Levinson, M. I., Thionation reactions of Lawesson's reagents. Tetrahedron 1985, 41 (22), 5061-5087.

163. Jesberger, M.; Davis, T. P.; Barner, L., Applications of Lawesson's reagent in organic and organometallic Syntheses. Synthesis 2003, 2003 (13), 1929-1958.

164. Bailey, P. D.; Mills, T. J.; Pettecrew, R.; Price, R. A., 5.06 - Amides. In Comprehensive Organic Functional Group Transformations II, Katritzky, A. R.; Taylor, R. J. K., Eds. Elsevier: Oxford, 2005; pp 201-294.

165. Oremus, V.; Heimgartner, H., Reaction of 1, 4-Dihydro-1-phenyltetrazole-5-thione with Epoxides; Formation of 1-[(1-Phenyl-1H-tetrazol-5-yl) thio] alkan-2-ols. J. Chem. Research (S) 1991, 10, 296-297.

166. Saikia, B.; Devi, T. J.; Barua, N. C., Stereoselective total synthesis of both $(6 R, 9 R, 10 S$, $7 E)$-and $(6 S, 9 R, 10 S, 7 E)$-epimers of oxylipin $(9 R, 10 S, 7 E)$-6, 9, 10-trihydroxyoctadec-7-enoic acid. Tetrahedron 2013, 69 (9), 2157-2166.

167. Pellissier, H., Catalytic non-enzymatic kinetic resolution. Adv. Synth. Catal. 2011, 353 (10), 1613-1666.

168. Schaus, S. E.; Brandes, B. D.; Larrow, J. F.; Tokunaga, M.; Hansen, K. B.; Gould, A. E.; Furrow, M. E.; Jacobsen, E. N., Highly selective hydrolytic kinetic resolution of terminal epoxides catalyzed by chiral (salen)Co ${ }^{\mathrm{III}}$ complexes. Practical synthesis of enantioenriched terminal epoxides and 1,2-diols. J. Am. Chem. Soc. 2002, 124 (7), 1307-1315.

169. Fleming, F. F.; Wang, Q.; Steward, O. W., Hydroxylated $\alpha, \beta$-unsaturated nitriles: stereoselective synthesis. J. Org. Chem. 2001, 66 (6), 2171-2174.

170. Mete, E.; Maraş, A.; Seçen, H., A short synthesis of 4-amino-3-hydroxybutyric acid (GABOB) via allyl cyanide. Russ. Chem. Bull. 2003, 52 (8), 1879-1881.

171. Herzberger, J.; Frey, H., Epicyanohydrin: polymerization by monomer activation gives access to nitrile-, amino-, and carboxyl-functional poly(ethylene glycol). Macromolecules 2015, 48 (22), 8144-8153.

172. Dale, J. A.; Mosher, H. S., Nuclear magnetic resonance enantiomer regents. Configurational correlations via nuclear magnetic resonance chemical shifts of diastereomeric mandelate, O-methylmandelate, and .alpha.-methoxy-alpha.-trifluoromethylphenylacetate (MTPA) esters. J. Am. Chem. Soc. 1973, 95 (2), 512-519. 
173. Torok, D. S.; Figueroa, J. J.; Scott, W. J., 1,3-Dioxolane formation via Lewis acidcatalyzed reaction of ketones with oxiranes. J. Org. Chem. 1993, 58 (25), 7274-7276.

174. Saha, S.; Mandal, S. K.; Roy, S. C., Fe(III) chloride catalyzed conversion of epoxides to acetonides. Tetrahedron Lett. 2008, 49 (41), 5928-5930.

175. Díez, D.; Beneitez, M. T.; Marcos, I. S.; Garrido, N. M.; Basabe, P.; Urones, J. G., Enantioselective synthesis of a 2,3,4-trisubstituted pyrrolidine from 1-hydroxymethyl-4phenylsulfonylbutadiene. Synlett 2001, 2001 (05), 0655-0657.

176. Meskens, F. A. J., Methods for the preparation of acetals from alcohols or oxiranes and carbonyl compounds. Synthesis 1981, 1981 (07), 501-522.

177. Martinelli, M. J.; Nayyar, N. K.; Moher, E. D.; Dhokte, U. P.; Pawlak, J. M.; Vaidyanathan, R., Dibutyltin oxide catalyzed selective sulfonylation of $\alpha$-chelatable primary alcohols. Org. Lett. 1999, 1 (3), 447-450.

178. Ohtani, I.; Kusumi, T.; Kashman, Y.; Kakisawa, H., High-field FT NMR application of Mosher's method. The absolute configurations of marine terpenoids. J. Am. Chem. Soc. 1991, 113 (11), 4092-4096.

179. Tsuruoka, A.; Negi, S.; Yanagisawa, M.; Nara, K., Practical oxirane ring opening with in situ prepared LiCN; synthesis of (2S,3R)-3-(2,4-difluorophenyl)-3-hydroxy-2-methyl-4-(1H1,2,4-triazol-1-yl)-1-butanenitrile. Synth. Commun. 1997, 27 (20), 3547-3557.

180. Jackman, L. M.; Lange, B. C., Methylation of lithioisobutyrophenone in weakly polar aprotic solvents. The effect of aggregation. J. Am. Chem. Soc. 1981, 103 (15), 4494-4499.

181. Spychała, J., Convenient syntheses of $N$-methylthioamides: A migration of the $\mathrm{H}_{2} \mathrm{~S}$ molecule in the thioamide-nitrile system. J. Sulfur Chem. 2006, 27 (3), 203-212.

182. Nagl, M.; Panuschka, C.; Barta, A.; Schmid, W., The $\mathrm{BF}_{3} \times \mathrm{OEt}_{2}$-assisted conversion of nitriles into thioamides with Lawesson's Reagent. Synthesis 2008, 2008 (24), 4012-4018.

183. Carey, F. A.; Sundberg, R. J., Functional Group Interconversion by Substitution, Including Protection and Deprotection. In Advanced Organic Chemistry: Part B: Reactions and Synthesis, Carey, F. A.; Sundberg, R. J., Eds. Springer US: Boston, MA, 2007; pp 215-288.

184. Lee, J.; Kim, M.; Chang, S.; Lee, H.-Y., Anhydrous hydration of nitriles to amides using aldoximes as the water Source. Org. Lett. 2009, 11 (24), 5598-5601.

185. Petrie, C. R.; Revankar, G. R.; Dalley, N. K.; George, R. D.; McKernan, P. A.; Hamill, R. L.; Robins, R. K., Synthesis and biological activity of certain nucleoside and nucleotide derivatives of pyrazofurin. J. Med. Chem. 1986, 29 (2), 268-278.

186. Lajoie, G.; Lépine, F.; Maziak, L.; Belleau, B., Facile regioselective formation of thiopeptide linkages from oligopeptides with new thionation reagents. Tetrahedron Lett. 1983, 24 (36), 3815-3818.

187. Charette, A. B.; Grenon, M., Mild method for the conversion of amides to thioamides. $J$. Org. Chem. 2003, 68 (14), 5792-5794. 
188. Voss, J.; Voss, J., 2,4-Bis(4-methoxyphenyl)-1,3,2,4-dithiadiphosphetane 2,4-disulfide. eEROS 2001.

189. Cava, M. P.; VanMeter, J. P., Condensed cyclobutane aromatic compounds. XXX. Synthesis of some unusual 2, 3-naphthoquinonoid heterocycles. A synthetic route to derivatives of naphtho [2,3-b] biphenylene and anthra[b]cyclobutene. J. Org. Chem. 1969, 34 (3), 538-545. 190. Thomsen, I.; Clausen, K.; Scheibye, S.; Lawesson, S. O., Thiation with 2,4-Bis(4methoxyphenyl)-1,3,2,4-dithiadiphosphetane 2,4-disulfide: $N$-methylthiopyrrolidone. Org. Synth. 1984, 158-158.

191. Arora, P.; Narang, R.; Nayak, S. K.; Singh, S. K.; Judge, V., 2,4-Disubstituted thiazoles as multitargated bioactive molecules. Med. Chem. Res. 2016, 25 (9), 1717-1743.

192. T Chhabria, M.; Patel, S.; Modi, P.S Brahmkshatriya, P., Thiazole: a review on chemistry, synthesis and therapeutic importance of its derivatives. Curr. Top. Med. Chem. 2016, 16 (26), 2841-2862.

193. Moradi, A. V.; Peyghan, A. A.; Hashemian, S.; Baei, M. T., Theoretical study of thiazole adsorption on the $(6,0)$ zigzag single-walled boron nitride nanotube. Bull.Korean Chem. Soc. 2012, 33 (10), 3285-3292.

194. Cui, S.; Wang, Y.; Lv, J.; Damu, G.; Zhou, C., Recent advances in application of thiazole compounds. Sci. Sin. Chim. 2012, 42 (8), 1105-1131.

195. de Souza, M. V. N., Synthesis and biological activity of natural thiazoles: An important class of heterocyclic compounds. J. Sulfur Chem. 2005, 26 (4-5), 429-449.

196. Gribble, G. W.; Joule, J. A., Five-Membered Ring Systems: With $N$ and $S$ Atom. In Progress in Heterocyclic Chemistry, Eds. Elsevier: 2015; pp 287-303.

197. Menche, D.; Hassfeld, J.; Li, J.; Rudolph, S., Total synthesis of archazolid A. J. Am. Chem. Soc. 2007, 129 (19), 6100-6101.

198. Zhu, B.; Panek, J. S., Total aynthesis of epothilone A. Org. Lett. 2000, 2 (17), 2575-2578.

199. Patt, W. C.; Massa, M. A., The total synthesis of the natural product endothelin converting enzyme (ECE) inhibitor, WS75624 B. Tetrahedron Lett. 1997, 38 (8), 1297-1300.

200. Merritt, E. A.; Bagley, M. C., Holzapfel-Meyers-Nicolaou modification of the Hantzsch thiazole synthesis. Synthesis 2007, 2007 (22), 3535-3541.

201. Bredenkamp, M. W.; Holzapfel, C. W.; van Zyl, W. J., The chiral synthesis of thiazole amino acid enantiomers. Synth. Commun. 1990, 20 (15), 2235-2249.

202. Nicolaou, K. C.; Safina, B. S.; Zak, M.; Lee, S. H.; Nevalainen, M.; Bella, M.; Estrada, A. A.; Funke, C.; Zécri, F. J.; Bulat, S., Total synthesis of thiostrepton. Retrosynthetic analysis and construction of key building blocks. J. Am. Chem. Soc. 2005, 127 (31), 11159-11175.

203. Bruno, P.; Peña, S.; Just-Baringo, X.; Albericio, F.; Álvarez, M., Total synthesis of aeruginazole A. Org. Lett. 2011, 13 (17), 4648-4651. 
204. Nicolaou, K. C.; Zak, M.; Safina, B. S.; Estrada, A. A.; Lee, S. H.; Nevalainen, M., Total synthesis of thiostrepton. Assembly of key building blocks and completion of the synthesis. $J$. Am. Chem. Soc. 2005, 127 (31), 11176-11183.

205. Kriek, M.; Martins, F.; Leonardi, R.; Fairhurst, S. A.; Lowe, D. J.; Roach, P. L., Thiazole synthase from Escherichia coli an investigation of the substrates and purified proteins required for activity in vitro. J. Biol. Chem. 2007, 282 (24), 17413-17423.

206. Xu, Z.; Ye, T., Thiazoline and thiazole and their Derivatives. In Heterocycles in Natural Product Synthesis, Wiley: 2011; pp 459-505.

207. Raman, P.; Razavi, H.; Kelly, J. W., Titanium (IV)-mediated tandem deprotectioncyclodehydration of protected cysteine $N$-amides: biomimetic syntheses of thiazoline-and thiazole-containing heterocycles. Org. Lett. 2000, 2 (21), 3289-3292.

208. Boyce, R. J.; Mulqueen, G. C.; Pattenden, G., Total synthesis of thiangazole, a novel inhibitor of HIV-1 from polyangium sp. Tetrahedron Lett. 1994, 35 (31), 5705-5708.

209. Sugiyama, H.; Yokokawa, F.; Shioiri, T., Asymmetric total synthesis of (-)-mycothiazole. Org. Lett. 2000, 2 (14), 2149-2152.

210. DeRoy, P. L.; Charette, A. B., Total synthesis of (+)-cystothiazole A. Org. Lett. 2003, 5 (22), 4163-4165.

211. Tang, X.; Yang, J.; Zhu, Z.; Zheng, M.; Wu, W.; Jiang, H., Access to thiazole via coppercatalyzed $[3+1+1]$-type condensation reaction under redox-neutral conditions. J. Org. Chem. 2016, 81 (22), 11461-11466.

212. Zhang, G.; Chen, B.; Guo, X.; Guo, S.; Yu, Y., Iron(II)-promoted synthesis of 2aminothiazoles via C-N bond formation from vinyl azides and potassium thiocyanate. Adv. Synth. Catal. 2015, 357 (5), 1065-1069.

213. Luo, Y.; Ji, K.; Li, Y.; Zhang, L., Tempering the reactivities of postulated $\alpha$-oxo gold carbenes using bidentate ligands: implication of tricoordinated gold intermediates and the development of an expedient bimolecular assembly of 2,4-disubstituted oxazoles. J. Am. Chem. Soc. 2012, 134 (42), 17412-17415.

214. Horneff, T.; Chuprakov, S.; Chernyak, N.; Gevorgyan, V.; Fokin, V. V., Rhodiumcatalyzed transannulation of 1,2,3-triazoles with nitriles. J. Am. Chem. Soc. 2008, 130 (45), 14972-14974.

215. He, W.; Xie, L.; Xu, Y.; Xiang, J.; Zhang, L., Electrophilicity of $\alpha$-oxo gold carbene intermediates: halogen abstractions from halogenated solvents leading to the formation of chloro/bromomethyl ketones. Org. Biomol.Chem. 2012, 10 (16), 3168-3171.

216. Ji, K.; Zhao, Y.; Zhang, L., Optimizing P,N-bidentate ligands for oxidative gold catalysis: efficient intermolecular trapping of $\alpha$-oxo gold carbenes by carboxylic acids. Angew. Chem. Int. Ed. 2013, 52 (25), 6508-6512. 
217. Rong, D.; Phillips, V. A.; Rubio, R. S.; Ángeles Castro, M.; Wheelhouse, R. T., A safe, convenient and efficient method for the preparation of heterocyclic $N$-oxides using urea-hydrogen peroxide. Tetrahedron Lett. 2008, 49 (48), 6933-6935.

218. Subbaraman, L. R.; Subbaraman, J.; Behrman, E. J., Reaction of nucleic acid components with m-chloroperoxybenzoic acid. Biochemistry 1969, 8 (7), 3059-3066.

219. Xu, M.; Ren, T.-T.; Li, C.-Y., Gold-catalyzed oxidative rearrangement of homopropargylic ether via oxonium ylide. Org. Lett. 2012, 14 (18), 4902-4905.

220. Kumar, D.; Kumar, N. M.; Patel, G.; Gupta, S.; Varma, R. S., A facile and eco-friendly synthesis of diarylthiazoles and diarylimidazoles in water. Tetrahedron Lett. 2011, 52 (16), 1983 1986.

221. Wu, G.; Wang, X.; Liu, H., Highly efficient one-pot synthesis of 2,4-disubstituted thiazoles using $\mathrm{Au}(\mathrm{I})$ catalyzed oxidation system at room temperature. Catalysts 2016, 6 (8), 126. 222. Zhang, L., A non-diazo approach to $\alpha$-oxo gold carbenes via gold-catalyzed alkyne oxidation. Acc. Chem. Res. 2014, 47 (3), 877-888.

223. Blakemore, P. R.; Cole, W. J.; Kocieński, P. J.; Morley, A., A stereoselective synthesis of trans-1,2-disubstituted alkenes based on the condensation of aldehydes with metallated 1phenyl-1H-tetrazol-5-yl sulfones. Synlett 1998, 1998 (01), 26-28.

224. Baudin, J. B.; Hareau, G.; Julia, S. A.; Lorne, R.; Ruel, O., Stereochemistry of direct olefin formation from carbonyl compounds and lithiated heterocyclic sulfones. Bull. Soc. Chim. Fr. 1993, 130 (6), 856-878.

225. Legnani, L.; Porta, A.; Caramella, P.; Toma, L.; Zanoni, G.; Vidari, G., Computational mechanistic study of the Julia-Kocieński reaction. J. Org. Chem. 2015, 80 (6), 3092-3100.

226. Chatterjee, B.; Bera, S.; Mondal, D., Julia-Kocienski olefination: a key reaction for the synthesis of macrolides. Tetrahedron: Asymmetry 2014, 25 (1), 1-55.

227. Blakemore, P. R.; Kocienski, P. J.; Marzcak, S.; Wicha, J., The modified Julia olefination in vitamin D2 synthesis. Synthesis 1999, 1999 (07), 1209-1215.

228. Grée, R.; Tourbah, H.; Carrié, R., Fumaraldehyde monodimethyl acetal: an easily accessible and versatile intermediate. Tetrahedron Lett. 1986, 27 (41), 4983-4986.

229. Valeev, R. F.; Bikzhanov, R. F.; Miftakhov, M. S., Synthesis of the C6-C21 fragment of epothilone analogues. Mendeleev Commun. 2014, 6 (24), 372-373.

230. Takano, D.; Nagamitsu, T.; Ui, H.; Shiomi, K.; Yamaguchi, Y.; Masuma, R.; Kuwajima, I.; Ōmura, S., Total synthesis of nafuredin, a selective NADH-fumarate reductase inhibitor. Org. Lett. 2001, 3 (15), 2289-2291.

231. Greene, T., W., Wuts, P., G., M., Protection for the Hydroxyl Group, Including 1,2- and 1,3-Diols. In Protective Groups in Organic Synthesis, Wiley: 1999; pp 17-245.

232. Gupta, M. K.; Li, Z.; Snowden, T. S., Preparation of one-carbon homologated amides from aldehydes or primary alcohols. Org. Lett. 2014, 16 (6), 1602-1605. 
233. Reeve, W.;Tsuk, R., Reactions of trihalomethylcarbinols with aqueous potassium hydroxide. J. Org. Chem. 1980, 45 (25), 5214-5215.

234. Schmid, C. R.; Bryant, J. D.; Dowlatzedah, M.; Phillips, J. L.; Prather, D. E.; Schantz, R. D.; Sear, N. L.; Vianco, C. S., Synthesis of 2,3-O-isopropylidene-D-glyceraldehyde in high chemical and optical purity: observations on the development of a practical bulk process. J. Org. Chem. 1991, 56 (12), 4056-4058.

235. Corey, E. J.; Link, J. O.; Shao, Y., Two effective procedures for the synthesis of trichloromethyl ketones, useful precursors of chiral $\alpha$-amino and $\alpha$-hydroxy acids. Tetrahedron Lett. 1992, 33 (24), 3435-3438.

236. Cafiero, L. R.; Snowden, T. S., General and practical conversion of aldehydes to homologated carboxylic acids. Org. Lett. 2008, 10 (17), 3853-3856.

237. Schmidt, A.-K. C.; Stark, C. B. W., TPAP-catalyzed direct oxidation of primary alcohols to carboxylic acids through stabilized aldehyde hydrates. Org. Lett. 2011, 13 (16), 4164-4167.

238. Li, Z.; Gupta, M. K.; Snowden, T. S., One-carbon homologation of primary alcohols and the reductive homologation of aldehydes involving a Jocic-Type reaction. Eur. J. Org. Chem. 2015, 2015 (32), 7009-7019.

239. Epp, J. B.; Widlanski, T. S., Facile preparation of nucleoside-5'-carboxylic Acids. J. Org. Chem. 1999, 64 (1), 293-295.

240. Shin, C.-g.; Nakamura, Y.; Yamada, Y.; Yonezawa, Y.; Umemura, K.; Yoshimura, J., Syntheses of 2-[(1S,3S)-1-amino-3-carboxy-3-hydroxypropyl] thiazole-4-carboxylic acid and the tripeptide skeleton of nosiheptide containing the acid. Bull. Chem. Soc. Jpn. 1995, 68 (11), 31513160.

241. Murray, R. W.; Agarwal, S. K., Singlet oxygen oxidation of substituted thiobenzamides. J. Photochem. 1984, 25 (2), 335-343.

242. Crank, G.; Mursyidi, A., Photochemical reactions of thioamides. J. Photochem. Photobiol. A: Chem. 1990, 53 (3), 301-310.

243. Jensen, F.; Greer, A.; Clennan, E. L., Reaction of organic sulfides with singlet oxygen: a revised mechanism. J. Am. Chem. Soc. 1998, 120 (18), 4439-4449.

244. Sarma, D.; Hanzlik, R. P., Synthesis of carbon-14, carbon-13 and deuterium labeled forms of thioacetamide and thioacetamide S-oxide. J. Labelled Compd. Radiopharm. 2011, 54 (13), 795-798.

245. Pattenden, G.; Critcher, D. J.; Remuiñán, M., Total synthesis of (-)-pateamine A, a novel immunosuppressive agent from Mycale sp. Can. J. Chem. 2004, 82 (2), 353-365.

246. Yamaura, M.; Suzuki, T.; Hashimoto, H.; Yoshimura, J.; Okamoto, T.; Shin, C.-G., Oxidative removal of $\mathrm{N}$-(4-methoxybenzyl) group on 2,5-piperazinediones with Cerium(IV) diammonium nitrate. Bull. Chem. Soc. Jpn. 1985, 58 (5), 1413-1420.

247. Allen, D. A.; Tomaso, A. E.; Priest, O. P.; Hindson, D. F.; Hurlburt, J. L., Mosher amides: 
determining the absolute stereochemistry of optically-active amines. J. Chem. Educ. 2008, 85 (5), 698.

248. Kojić-Prodić, B.; Molcanov, K., The nature of hydrogen bond: new insights into old theories. Acta. Chim. Slov. 2008, 55, 692-708.

249. Martinelli, M. J.; Vaidyanathan, R.; Pawlak, J. M.; Nayyar, N. K.; Dhokte, U. P.; Doecke, C. W.; Zollars, L. M. H.; Moher, E. D.; Khau, V. V.; Košmrlj, B., Catalytic regioselective sulfonylation of $\alpha$-chelatable alcohols: scope and mechanistic insight. J. Am. Chem. Soc. 2002, 124 (14), 3578-3585.

250. Newhouse, T.; Baran, P. S.; Hoffmann, R. W., The economies of synthesis. Chem. Soc. Rev. 2009, 38 (11), 3010-3021.

251. Moriyama, K.; Nakamura, Y.; Togo, H., Oxidative debenzylation of $N$-benzyl amides and $O$-benzyl ethers using alkali metal bromide. Org. Lett. 2014, 16 (14), 3812-3815.

252. Mulzer, J.; Schöllhorn, B., Multiple 1,2-O,O-Shift of tert-butyldiphenylsilyl groups in polyols. Angew. Chem. Int. Ed. 1990, 29 (4), 431-432.

253. Mosley, A. P., Acrylic Plastics. In Brydson's Plastics Materials (Eighth Edition), Gilbert, M., Ed. Butterworth-Heinemann: 2017; pp 441-456.

254. Srinivasan, S.; Lee, M. W.; Grady, M. C.; Soroush, M.; Rappe, A. M., Self-initiation mechanism in spontaneous thermal polymerization of ethyl and $n$-butyl acrylate: a theoretical study. J. Phys. Chem. A 2010, 114 (30), 7975-7983.

255. Ballard, N.; Asua, J. M., Radical polymerization of acrylic monomers: an overview. Prog. Polym. Sci. 2018, 79, 40-60.

256 Greene, T., W., Wuts, P., G., M., Protection for the Hydroxyl Group, Including 1,2- and 1,3-Diols. In Greene's Protective Groups in Organic Synthesis, Wiley: 2006; pp 123-130.

257. Ganapathi, K.; Kulkarni, K. D. Part V. Fine Structure and Orientation. In Chemistry of the Thiazoles, Springer: 1953; pp 45-57.

258. Pola, S., Significance of Thiazole-based Heterocycles for Bioactive Systems. In Scope of Selective Heterocycles from Organic and Pharmaceutical Perspective, InTech Rijeka, Croatia: 2016. DOI: $10.5772 / 62077$

259. Wang, J.; Sánchez-Roselló, M.; Aceña, J. L.; del Pozo, C.; Sorochinsky, A. E.; Fustero, S.; Soloshonok, V. A.; Liu, H., Fluorine in pharmaceutical industry: fluorine-containing drugs introduced to the market in the last decade (2001-2011). Chem. Rev. 2014, 114 (4), 2432-2506.

260. Cho, E. J.; Senecal, T. D.; Kinzel, T.; Zhang, Y.; Watson, D. A.; Buchwald, S. L., The palladium-catalyzed trifluoromethylation of aryl chlorides. Science 2010, 328 (5986), 1679-1681.

261. Alonso, C.; Martínez de Marigorta, E.; Rubiales, G.; Palacios, F., Carbon trifluoromethylation reactions of hydrocarbon derivatives and heteroarenes. Chem. Rev. 2015, 115 (4), 1847-1935.

262. Dürrenberger, F., Burgert; M., Burckhardt, S., Buhr, W., Kalogerakis, A., Reim, S., 
Manolova, V., Boyce, S., Yarnold, C., J., Pena, P., Shepherd, J., Lecci, C., Jarjes-Pike, R., Scott, J., Novel ferroportin inhibitors. 2017, WO2017068090.

263. Grushin, V. V.; Marshall, W. J., Unexpected $\mathrm{H}_{2} \mathrm{O}-$ Induced Ar-X Activation with trifluoromethylpalladium(II) aryls. J. Am. Chem. Soc. 2006, 128 (14), 4632-4641.

264. Grushin, V. V.; Marshall, W. J., Facile $\mathrm{Ar}-\mathrm{CF}_{3}$ bond formation at Pd. Strikingly different outcomes of reductive elimination from $\left[\left(\mathrm{Ph}_{3} \mathrm{P}\right)_{2} \mathrm{Pd}\left(\mathrm{CF}_{3}\right) \mathrm{Ph}\right]$ and [(Xantphos $\left.) \mathrm{Pd}\left(\mathrm{CF}_{3}\right) \mathrm{Ph}\right] . J . A m$. Chem. Soc. 2006, 128 (39), 12644-12645.

265. Mestre, J.; Lishchynskyi, A.; Castillón, S.; Boutureira, O., Trifluoromethylation of electron-rich alkenyl iodides with fluoroform-derived "ligandless" $\mathrm{CuCF}_{3}$. J. Org. Chem. 2018, 83 (15), 8150-8160.

266. Yu, X.; Sun, D., Macrocyclic drugs and synthetic methodologies toward macrocycles. Molecules 2013, 18 (6), 6230-6268.

267. Ridge, C. D.; Mazzola, E. P.; Coles, M. P.; Hinkley, S. F. R., Isolation and characterization of roridin E. Magn. Reson. Chem. 2017, 55 (4), 337-340.

268. Yadav, J. S.; Rajender, V., Studies directed towards the total synthesis of (-)-dictyostatin. Eur. J. Org. Chem. 2010, 2010 (11), 2148-2156.

269. Eisner, U.; Elvidge, J. A.; Linstead, R. P., 281. Polyene acids. Part VI. A new (cistrans-)isomer of sorbic acid and its relation to hexenolactones. J. Chem. Soc. (Resumed) 1953, (0), 1372-1379.

270. Cardillo, G.; Orena, M.; Sandri, S., Synthesis of compounds containing the isoprene unit: a stereospecific synthesis of dehydronerol isovalerate, a component of anthemis Montana L. Tetrahedron 1976, 32 (1), 107-108.

271. Masamune, S.; Imperiali, B.; Garvey, D. S., Synthesis of ansamycins: the ansa chain of rifamycin S. J. Am. Chem. Soc. 1982, 104 (20), 5528-5531.

272. Constantino, M. G.; Losco, P.; Castellano, E. E., A novel synthesis of ( \pm )-abscisic acid. J. Org. Chem. 1989, 54 (3), 681-683.

273. Feng, Y.; Liu, J.; Carrasco, Y. P.; MacMillan, J. B.; De Brabander, J. K., Rifamycin biosynthetic congeners: isolation and total Synthesis of rifsaliniketal and total synthesis of salinisporamycin and saliniketals A and B. J. Am. Chem. Soc. 2016, 138 (22), 7130-7142.

274. Nakata, T.; Hata, N.; Oishi, T., A stereoselective synthesis of $(2 Z, 4 E)$-dienoic acid involving masked functional groups: $n$-Bu $\mathrm{Bu}_{4} \mathrm{NF}$-induced ring opening of $\alpha, \beta$-unsaturated $\delta$-lactone. ChemInform 1990, 21 (34).

275. Bridgwood, K. A. Towards the total synthesis of leustroducsin B. Doctoral dissertation, University of Cambridge, Great Britain. 2009.

276. Li, H.-Y.; Sun, H.; DiMagno, S. G., Tetrabutylammonium fluoride. e-EROS 2007. 
277. Olofson, R. A.; Landesberg, J. M.; Houk, K. N.; Michelman, J. S., The deprotonation of thiazole and related bases. J. Am. Chem. Soc. 1966, 88 (18), 4265-4266.

278. Davies, J. S.; Higginbotham, C. L.; Tremeer, E. J.; Brown, C.; Treadgold, R. C., Protection of hydroxy groups by silylation: use in peptide synthesis and as lipophilicity modifiers for peptides. J. Chem. Soc., Perkin Trans. 1 1992, (22), 3043-3048.

279. Gillard, J. W.; Fortin, R.; Morton, H. E.; Yoakim, C.; Quesnelle, C. A.; Daignault, S.; Guindon, Y., Symmetrical alkoxysilyl ethers. A new class of alcohol-protecting groups. Preparation of tert-butoxydiphenylsilyl ethers. J. Org. Chem. 1988, 53 (11), 2602-2608.

280. Nicolaou, K. C.; Baker, T. M.; Nakamura, T., Synthesis of the WXYZA' domain of maitotoxin. J. Am. Chem. Soc. 2010, 133 (2), 220-226.

281. Yu, J.; Sun, J.; Niu, Y.; Li, R.; Liao, J.; Zhang, F.; Yu, B., Synthetic access toward the diverse ginsenosides. Chem. Sci. 2013, 4 (10), 3899-3905.

282. Smith Iii, A. B.; Smits, H.; Kim, D.-S., Spirastrellolide studies. Synthesis of the C(1)$\mathrm{C}(25)$ southern hemispheres of spirastrellolides A and B, exploiting anion relay chemistry. Tetrahedron 2010, 66 (33), 6597-6605.

283. Loh, T.-P.; Feng, L.-C., Progress towards the total synthesis of tedanolide: an efficient assembly of the C1-C11 subunit. Tetrahedron Lett. 2001, 42 (34), 6001-6005.

284. Prakash, C.; Saleh, S.; Blair, I. A., Selective de-protection of silyl ethers. Tetrahedron Lett. 1989, 30 (1), 19-22.

285. Ganapathi, K.; Kulkarni, K. D., Chemistry of the thiazoles. Proc. Natl. Acad. Sci. India. Sect. A 1953, 38 (1), 45-57.

286. Willwacher, J.; Heggen, B.; Wirtz, C.; Thiel, W.; Fürstner, A., Total Synthesis, stereochemical revision, and biological reassessment of mandelalide A: chemical mimicry of intrafamily relationships. Chem. Eur. J. 2015, 21 (29), 10416-10430.

287. Wang, M.; Li, C.; Yin, D.; Liang, X.-T., A mild and efficient approach for the deprotection of silyl ethers by sodium periodate. Tetrahedron Lett. 2002, 43 (48), 8727-8729.

288. Kawahara, S.-i.; Wada, T.; Sekine, M., 1:1 and 1:2 complexes of $\mathrm{Bu}_{4} \mathrm{NF}$ and $\mathrm{BF}_{3} \cdot \mathrm{Et}_{2} \mathrm{O}$ : unique properties as reagents for cleavage of silyl ethers. Tetrahedron Lett. 1996, 37 (4), 509-512. 289. Corey, E. J.; Venkateswarlu, A., Protection of hydroxyl groups as tert-butyldimethylsilyl derivatives. J. Am. Chem. Soc. 1972, 94 (17), 6190-6191.

290. Ouellette, R. J.; Rawn, J. D., Amines and Amides. In Organic Chemistry (Second Edition), Ouellette, R. J.; Rawn, J. D., Eds. Academic Press: 2018; pp 763-800.

291. Gille, A.; Hiersemann, M., (-)-Lytophilippine A: synthesis of a C1-C18 building block. Org. Lett. 2010, 12 (22), 5258-5261.

292. Frank, A. T.; Farina, N. S.; Sawwan, N.; Wauchope, O. R.; Qi, M.; Brzostowska, E. M.; Chan, W.; Grasso, F. W.; Haberfield, P.; Greer, A., Natural macrocyclic molecules have a possible limited structural diversity. Mol. Divers. 2007, 11 (3-4), 115-118. 
293. Wessjohann, L. A.; Ruijter, E.; Garcia-Rivera, D.; Brandt, W., What can a chemist learn from nature's macrocycles? - A brief, conceptual view. Mol. Divers. 2005, 9 (1), 171-186.

294. Lafontaine, J. A.; Provencal, D. P.; Gardelli, C.; Leahy, J. W., Enantioselective total synthesis of the antitumor macrolide rhizoxin D. J. Org. Chem. 2003, 68 (11), 4215-4234.

295. Prunet, J., Progress in metathesis through natural product synthesis. Eur. J. Org. Chem. 2011, 2011 (20-21), 3634-3647.

296. Peng, L.; Zhang, F.; Mei, T.; Zhang, T.; Li, Y., Studies on novel macrocyclization methods of cembrane-type diterpenoids: a Stille cyclization approach to $( \pm)$-isocembrene. Tetrahedron Lett. 2003, 44 (31), 5921-5923.

297. Kobayashi, K.; Fujii, Y.; Hirayama, Y.; Kobayashi, S.; Hayakawa, I.; Kigoshi, H., Design, synthesis, and biological evaluations of aplyronine A-mycalolide B hybrid compound. Org. Lett. 2012, 14 (5), 1290-1293.

298. Illuminati, G.; Mandolini, L., Ring closure reactions of bifunctional chain molecules. Acc. Chem. Res. 1981, 14 (4), 95-102.

299. Rossa, L.; Vögtle, F. In Synthesis of Medio- and Macrocyclic Compounds by High Dilution Principle Techniques, Cyclophanes I, Ed. Springer Berlin Heidelberg: 1983; pp 1-86.

300. Parenty, A.; Moreau, X.; Niel, G.; Campagne, J. M., Update 1 of: Macrolactonizations in the total synthesis of natural products. Chem. Rev. 2013, 113 (1), PR1-PR40.

301. Inanaga, J.; Hirata, K.; Saeki, H.; Katsuki, T.; Yamaguchi, M., A rapid esterification by means of mixed anhydride and its application to large-ring lactonization. Bull. Chem. Soc. Jpn. 1979, 52 (7), 1989-1993.

302. Kobayashi, Y.; Fukuda, A.; Kimachi, T.; Ju-ichi, M.; Takemoto, Y., Asymmetric synthetic study of macrolactin analogues. Tetrahedron 2005, 61 (10), 2607-2622.

303. Hikota, M.; Sakurai, Y.; Horita, K.; Yonemitsu, O., Synthesis of erythronolide a via a very efficient macrolactonization under usual acylation conditions with the Yamaguchi reagent. Tetrahedron Lett. 1990, 31 (44), 6367-6370.

304. Shin, Y.; Fournier, J.-H.; Brückner, A.; Madiraju, C.; Balachandran, R.; Raccor, B. S.; Edler, M. C.; Hamel, E.; Sikorski, Rachel P.; Vogt, A.; Day, B. W.; Curran, D. P., Synthesis and biological evaluation of (-)-dictyostatin and stereoisomers. Tetrahedron 2007, 63 (35), 85378562.

305. Schrof, R.; Altmann, K.-H., Studies toward the total synthesis of the marine macrolide salarin C. Org. Lett. 2018, 20 (23), 7679-7683.

306. Zhu, W.; Jiménez, M.; Jung, W.-H.; Camarco, D. P.; Balachandran, R.; Vogt, A.; Day, B. W.; Curran, D. P., Streamlined syntheses of (-)-dictyostatin, 16-desmethyl-25,26dihydrodictyostatin, and 6-epi-16-desmethyl-25,26-dihydrodictyostatin. J. Am. Chem. Soc. 2010, 132 (26), 9175-9187. 
307. Smith, A. B.; Dong, S.; Fox, R. J.; Brenneman, J. B.; Vanecko, J. A.; Maegawa, T., (+)Sorangicin A: evolution of a viable synthetic strategy. Tetrahedron 2011, 67 (51), 9809-9828.

308. Li, P.; Xu, J.-C., 1-Ethyl 2-halopyridinium salts, highly efficient coupling reagents for hindered peptide synthesis both in solution and the solid-phase. Tetrahedron 2000, 56 (41), 81198131.

309. Gu, Y.; Tian, S.-K., Olefination Reactions of Phosphorus-Stabilized Carbon Nucleophiles. In Stereoselective Alkene Synthesis, Wang, J., Ed. Springer Berlin Heidelberg: 2012; pp 197-238.

310. Vedejs, E.; Marth, C. F., Mechanism of the Wittig reaction: the role of substituents at phosphorus. J. Am. Chem. Soc. 1988, 110 (12), 3948-3958.

311. Vedejs, E.; Fleck, T. J., Kinetic (not equilibrium) factors are dominant in Wittig reactions of conjugated ylides. J. Am. Chem. Soc. 1989, 111 (15), 5861-5871.

312. Tian, X.-Y.; Han, J.-W.; Zhao, Q.; Wong, H. N. C., Asymmetric synthesis of 3,3,5,5tetrasubstituted 1,2-dioxolanes: total synthesis of epiplakinic acid F. Org. Biomol.Chem. 2014, 12 (22), 3686-3700.

313. Kauhl, U.; Andernach, L.; Opatz, T., Total Synthesis of epi-Trichosetin. J. Org. Chem. 2018, 83 (24), 15170-15177.

314. Tamura, R.; Saegusa, K.; Kakihana, M.; Oda, D., Stereoselective $E$ and $Z$ olefin formation by Wittig olefination of aldehydes with allylic phosphorus ylides. Stereochemistry. J. Org. Chem. 1988, 53 (12), 2723-2728.

315. Bhatt, U.; Christmann, M.; Quitschalle, M.; Claus, E.; Kalesse, M., The first total synthesis of (+)-ratjadone. J. Org. Chem. 2001, 66 (5), 1885-1893.

316. Edmonds, M.; Abell, A., The Wittig Reaction. In Modern Carbonyl Olefination Wiley: 2003, pp 1-17.

317. Wang, Z., Horner-Wadsworth-Emmons Olefination. In Comprehensive Organic Name Reactions and Reagents, Wiley: 2010, pp 1484-1490.

318. Blakemore, P. R., 1.15 Olefination of Carbonyl Compounds by Main-Group Element Mediators. In Comprehensive Organic Synthesis II (Second Edition), Knochel, P., Ed. Elsevier: Amsterdam, 2014; pp 516-608.

319. Simoni, D.; Rossi, M.; Rondanin, R.; Mazzali, A.; Baruchello, R.; Malagutti, C.; Roberti, M.; Invidiata, F. P., Strong bicyclic guanidine base-promoted Wittig and Horner-Wadsworth-Emmons Reactions. Org. Lett. 2000, 2 (24), 3765-3768.

320. Chintareddy, V. R.; Ellern, A.; Verkade, J. G., $\mathrm{P}\left[\mathrm{N}(i-\mathrm{Bu}) \mathrm{CH}_{2} \mathrm{CH}_{2}\right]_{3} \mathrm{~N}$ : Nonionic Lewis base for promoting the room-temperature synthesis of $\alpha, \beta$-unsaturated esters, fluorides, ketones, and nitriles using Wadsworth-Emmons phosphonates. J. Org. Chem. 2010, 75 (21), 7166-7174. 321. Iorga, B.; Eymery, F.; Mouriès, V.; Savignac, P., Phosphorylated aldehydes: preparations and synthetic uses. Tetrahedron 1998, 54 (49), 14637-14677. 
322. Haugan, J. A.; Lobkovskyb, E.; Liaaen-Jensena, S., Total synthesis of $\mathrm{C}_{31}$-methyl ketone apocarotenoids 3. On the structure of hopkinsiaxanthin: first total synthesis of (all-E)-(3S)-and (9Z)-(3S)-7'-apohopkinsiaxanthin. Acta Chem. Scand. 1997, 51, 1201-1216.

323. Zhao, Y.-J.; Loh, T.-P., Practical synthesis of 1,5-dimethyl substituted conjugated polyenes from geranyl acetate. Tetrahedron 2008, 64 (22), 4972-4978.

324. Mohamed-Hachi, A.; About-Jaudet, E.; Combret, J.-C.; Collignon, N., 4Diethoxyphosphonyl-2-methyl-1,3-dienolates and their O-acylated and O-silylated derivatives: new, efficient and highly stereoselective polyvinylogation reagents. Synthesis 1999, 1999 (07), 1188-1192.

325. Lengkeek, N. A.; Greenwood, P. F.; Nguyen, B.; Koutsantonis, G. A.; Piggott, M. J., Making mixtures to solve structures: structural elucidation via combinatorial synthesis. J. Comb. Chem. 2010, 12 (1), 141-150.

326. Luche, J.-L.; Rodriguez-Hahn, L.; Crabbé, P., Reduction of natural enones in the presence of cerium trichloride. J. Chem. Soc., Chem. Commun. 1978, (14), 601-602.

327. Arbusow, B. A., Michaelis-Arbusow-und perkow-reaktionen. Pure Appl. Chem. 1964, 9 (2), 307-336.

328. Bhattacharya, A. K.; Thyagarajan, G., Michaelis-Arbuzov rearrangement. Chem. Rev. 1981, 81 (4), 415-430.

329. Hari Babu, B.; Syam Prasad, G.; Naga Raju, C.; Venkata Basaveswara Rao, M., Synthesis of phosphonates via Michaelis-Arbuzov reaction. Curr. Org. Synth. 2017, 14 (6), 883-903.

330. Iranpoor, N.; Firouzabadi, H.; Rajabi Moghadam, K.; Etemadi-Davan, E., Triphenylphosphine/2,3-dichloro-5,6-dicyanobenzoquinone ( $\left.\mathrm{PPh}_{3} / \mathrm{DDQ}\right)$ system for conversion of alcohols and thiols into trialkyl phosphonates. Asian J. Org. Chem. 2015, 4 (11), 1289-1293.

331. Ma, X.; Xu, Q.; Li, H.; Su, C.; Yu, L.; Zhang, X.; Cao, H.; Han, L.-B., Alcohol-based Michaelis-Arbuzov reaction: an efficient and environmentally-benign method for C-P (O) bond formation. Green Chem. 2018, 20 (15), 3408-3413.

332. Thirumurugan, P.; Matosiuk, D.; Jozwiak, K., Click chemistry for drug development and diverse chemical-biology applications. Chem. Rev. 2013, 113 (7), 4905-4979.

333. Yang, M.; Li, J.; Chen, P. R., Transition metal-mediated bioorthogonal protein chemistry in living cells. Chem. Soc. Rev. 2014, 43 (18), 6511-6526.

334. Kempe, K.; Krieg, A.; Becer, C. R.; Schubert, U. S., "Clicking” on/with polymers: a rapidly expanding field for the straightforward preparation of novel macromolecular architectures. Chem. Soc. Rev. 2012, 41 (1), 176-191.

335. Kolb, H. C.; Finn, M. G.; Sharpless, K. B., Click chemistry: diverse chemical function from a few good reactions. Angew. Chem. Int. Ed. 2001, 40 (11), 2004-2021. 
336. Fürstner, A.; Bonnekessel, M.; Blank, J. T.; Radkowski, K.; Seidel, G.; Lacombe, F.; Gabor, B.; Mynott, R., Total synthesis of myxovirescin A1. Chem. Eur. J. 2007, 13 (31), 8762 8783.

337. De Luca, L.; Giacomelli, G.; Porcheddu, A., A very mild and chemoselective oxidation of alcohols to carbonyl compounds. Org. Lett. 2001, 3 (19), 3041-3043.

338. Denmark, S. E.; Edwards, M. G., On the mechanism of the selenolactonization reaction with selenenyl halides. J. Org. Chem. 2006, 71 (19), 7293-7306.

339. Meyers, S. R.; Juhn, F. S.; Griset, A. P.; Luman, N. R.; Grinstaff, M. W., Anionic amphiphilic dendrimers as antibacterial agents. J. Am. Chem. Soc. 2008, 130 (44), 14444-14445. 340. Dams, I.; Chodyński, M.; Krupa, M.; Pietraszek, A.; Zezula, M.; Cmoch, P.; Kosińska, M.; Kutner, A., A novel convergent synthesis of the potent antiglaucoma agent travoprost. Tetrahedron 2013, 69 (5), 1634-1648.

341. Lee, Y.-S.; Park, S. M.; Kim, B. H., Synthesis of 5-isoxazol-5-yl-2'-deoxyuridines exhibiting antiviral activity against HSV and several RNA viruses. Bioorg. Med. Chem. Lett. 2009, 19 (4), 1126-1128.

342. Ripperger, H., Zur darstellung und selektiven reduktion von geschützten endothiopeptiden. J. Prakt. Chem. 1987, 329 (6), 1039-1044.

343. Henrion, G.; Chavas, T. E. J.; Le Goff, X.; Gagosz, F., Biarylphosphonite gold(I) complexes as superior catalysts for oxidative cyclization of propynyl arenes into indan-2-ones. Angew. Chem. Int. Ed. 2013, 52 (24), 6277-6282.

344. Parsons, S. R.; Hooper, J. F.; Willis, M. C., O-Substituted alkyl aldehydes for rhodiumcatalyzed intermolecular alkyne hydroacylation: the utility of methylthiomethyl ethers. Org. Lett. 2011, 13 (5), 998-1000.

345. Mahammed, K. A.; Jayashankara, V. P.; Premsai Rai, N.; Mohana Raju, K.; Arunachalam, P. N., A mild and versatile synthesis of thioamides. Synlett 2009, 2009 (14), 2338-2340.

346. Frederico, D.; Donate, P. M.; Constantino, M. G.; Bronze, E. S.; Sairre, M. I., A short and efficient synthesis of crocetin-dimethylester and crocetindial. J. Org. Chem. 2003, 68 (23), 9126-9128.

347. Manley, D. W.; McBurney, R. T.; Miller, P.; Walton, J. C.; Mills, A.; O’Rourke, C., Titania-promoted carboxylic acid alkylations of alkenes and cascade addition-cyclizations. J. Org. Chem. 2014, 79 (3), 1386-1398.

348. Ella-Menye, J.-R.; Sharma, V.; Wang, G., New synthesis of chiral 1,3-oxazinan-2-ones from carbohydrate derivatives. J. Org. Chem. 2005, 70 (2), 463-469.

349. Hernández, D.; Lindsay, K. B.; Nielsen, L.; Mittag, T.; Bjerglund, K.; Friis, S.; Mose, R.; Skrydstrup, T., Further studies toward the stereocontrolled synthesis of silicon-containing peptide mimics. J. Org. Chem. 2010, 75 (10), 3283-3293. 
350. Gao, X.; Han, H.; Krische, M. J., Direct generation of acyclic polypropionate stereopolyads via double diastereo- and enantioselective iridium-catalyzed crotylation of 1,3diols: beyond stepwise carbonyl addition in polyketide construction. J. Am. Chem. Soc. 2011, 133 (32), 12795-12800.

351. Mohapatra, D. K.; Reddy, D. S.; Janaki Ramaiah, M.; Ghosh, S.; Pothula, V.; Lunavath, S.; Thomas, S.; Pushpa Valli, S. N. C. V. L.; Bhadra, M. P.; Yadav, J. S., Rugulactone derivatives act as inhibitors of NF- $\kappa \mathrm{B}$ activation and modulates the transcription of NF- $\kappa \mathrm{B}$ dependent genes in MDA-MB-231 cells. Bioorg. Med. Chem. Lett. 2014, 24 (5), 1389-1396.

352. Kirsch, S. F.; Klahn, P.; Menz, H., The use of COP-OAc in the catalyst-controlled syntheses of 1,3-polyols. Synthesis 2011, 2011 (22), 3592-3603.

353. Lengkeek, N. A.; Greenwood, P. F.; Nguyen, B.; Koutsantonis, G. A.; Piggott, M. J., Making mixtures to solve structures: structural elucidation via combinatorial synthesis. J. Comb. Chem. 2009, 12 (1), 141-150.

354. Magoulas, G. E.; Bariamis, S. E.; Athanassopoulos, C. M.; Haskopoulos, A.; Dedes, P. G.; Krokidis, M. G.; Karamanos, N. K.; Kletsas, D.; Papaioannou, D.; Maroulis, G., Syntheses, antiproliferative activity and theoretical characterization of acitretin-type retinoids with changes in the lipophilic part. Eur. J. Med. Chem. 2011, 46 (2), 721-737.

355. Kim, K.; Punna, V.; Karri, P.; Krishnamurthy, R., Synthesis of phosphoramidites of isoGNA, an isomer of glycerol nucleic acid. Beilstein J. Org. Chem. 2014, 10, 2131-2138.

356. Singh, S.; Gajulapati, V.; Kim, M.; Goo, J.-I.; Lee, J. K.; Lee, K.; Lee, C.-K.; Jeong, L. S.; Choi, Y., A divergent approach for the synthesis of d- and 1-4'-ethynyl dioxolane nucleosides with potent anti-HIV activity. Synthesis 2016, 48 (18), 3050-3056.

357. Leftheris, K.; Goodman, M., Synthesis and $\beta$-adrenergic antagonist activity of stereoisomeric practolol and propranolol derivatives. J. Med. Chem. 1990, 33 (1), 216-223.

358. Doboszewski, B.; Groaz, E.; Herdewijn, P., Synthesis of phosphonoglycine backbone units for the development of phosphono peptide nucleic acids. Eur. J. Org. Chem.2013, 2013 (22), 4804-4815. 